ORP-51438

Revision 0

\title{
Final Report Tests on the DuraMelter 1200 HLW Pilot Melter System Using AZ-101 HLW Simulants VSL-02R0100-2, Rev. 1, 2/17/03
}

Prepared for the U.S. Department of Energy Assistant Secretary for Environmental Management

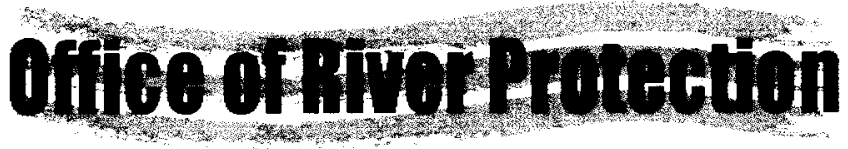

P.O. Box 450

Richland, Washington 99352 
ORP-51438

Revision 0

\section{Final Report Tests on the} DuraMelter 1200 HLW Pilot Melter System Using AZ-101 HLW

\section{Simulants}

VSL-02R0100-2, Rev. 1, 2/17/03

K. S. Matlack

Vitreous State Laboratory,

The Catholic University of America

A. A. Kruger

Department of Energy - Office of River Protection
W. K. Kot

T. Bardakci

W. Gong

N. A. D'Angelo

T. R. Schatz

I. L. Pegg

Vitreous State Laboratory,

The Catholic University of America

Date Published

December 2011

Prepared for the U.S. Department of Energy

Assistant Secretary for Environmental Management

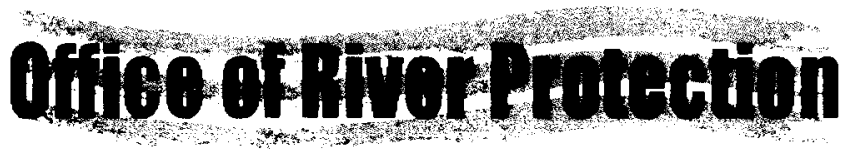

P.O. Box 450

Richland, Washington 99352

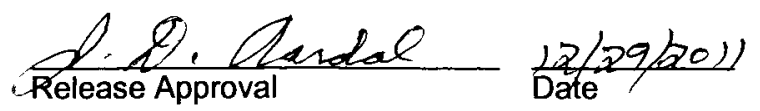

Approved for Public Reiease;

Further Dissemination Unlimited 
ORP-51438

Revision 0

TRADEMARK DISCLAIMER

Reference herein to any specific commercial product, process, or service by trade name, trademark, manufacturer, or

otherwise, does not necessarily constitute or imply its endorsement, recommendation, or favoring by the United

States Government or any agency thereof or its contractors or subcontractors.

This report has been reproduced from the best available copy.

Printed in the United States of America 


\section{Final Report - Tests on the DuraMelter 1200 HLW Pilot Melter System Using AZ-101 HLW Simulants}

\section{VSL-02R0100-2, Revision 1, dated 2/17/2003}

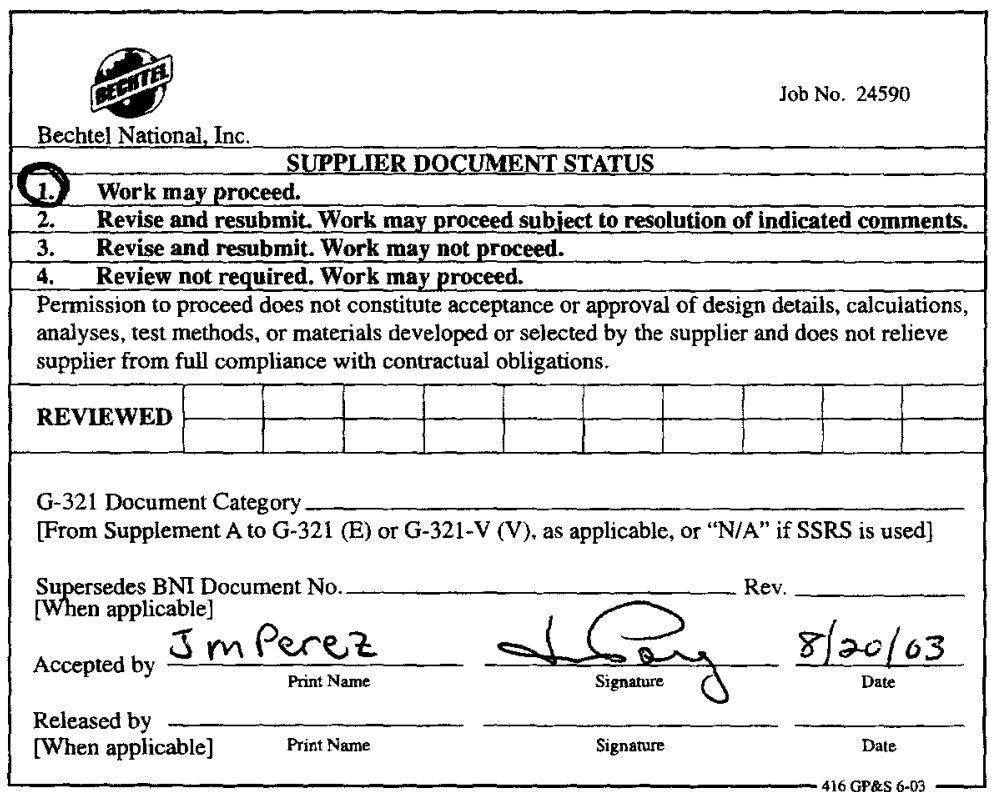

$8-13-030014103$

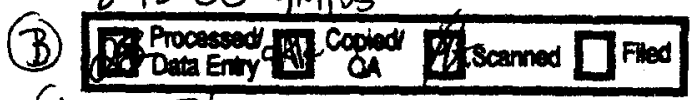

(4) K $8 / 13103$

(c) $5 \times 5 / 14 / 3$
$8-26-038727103$

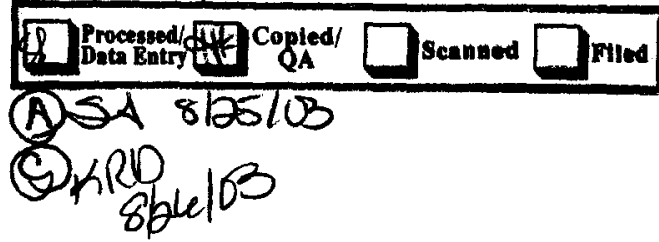




\section{Final Report}

Tests on the DuraMelter 1200

HLW Pilot Melter System Using AZ-101 HLW Simulants

prepared by

Keith S. Matlack, Wing K. Kot, Tevfik Bardakci, Weiliang Gong,

Nicholas A. D'Angelo, Timothy R. Schatz, and Ian L. Pegg

Vitreous State Laboratory

The Catholic University of America

Washington, DC 20064

for.

Duratek, Inc.

and

Bechtel National, Inc.

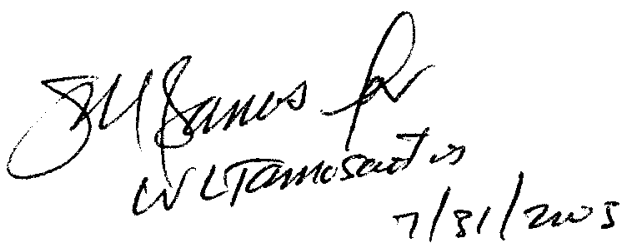

ACCEPTED FOR

WTP PROJECT USE

June 11, 2002

Rev. 1, 2/17/03 
Sent by: DURATEK INC CS

$05 / 28 / 2033 \quad 14: 28$
4102908154

ORP-51438, Rej 0
05/28/03 3:26PM; Jettex_ \#827;Page 2/2

PAGE $32 / 82$

\section{VSL Technical Report Review and Approval Form}

Report Title, Number, Revision, and Date: "Tests on the DuraMelter $1200 \mathrm{HZW}$ Pilor Melrer System Using A2-101 HLW Simulants." VSI-01R0100-2, Rev, 1, February 17, 2003.

\section{Relevant Background Dacuments:}

Contract: RPP.WTP Viarification Development (Duratel/Becbrel)

Task Specification: "Commissioning and HIW Throughpur Tests on the DM1200 Melier," Test Specification TSP-W375-00-0001 I: 'DM1200 Molres Integrated Off-Gas Treatonent Systern Tests," Test Specification, TSPW375-00-00020.

Test Plan: "Tests on the DM1200 HLW Pilor Melter System Using AZ-10I W aste Simulant5," K.S. Matlack, W.K. Kot, M. Brandys, and I.L. Pegg. Test Plan, VSL-01T0100-2, Rev 2, 7/3/01. "Tests on the DM1200 HLW Pilor Melter System Using AZ-101 Waste Simulants," K.S. Matlack, W.K. Kot, C.C. Chapman, M. Brandys and I.L. Pegg, Test Plan, VSL-01T0100-5̃, Rev 1., 9/11/01.

Other:

By signing below I certify that I have reviewed the subject report for correctness, rechnical adtequacy, completeness, accuracy, and compliance with established requirements and that all of my comments have been satisfactorily addressed by the authors in the revision listed above, which is therefore acceptable for final approval.

\begin{tabular}{|c|c|c|c|}
\hline Reviewer Nane & Signamure & Organization & Date \\
\hline Keith S. Maclack & & VSL & $.5 / 28 / 03$ \\
\hline Wing K. Kot & & VSL & $5 \mid 28103$ \\
\hline $\operatorname{Ian}$ C. Pegg & & VSI & 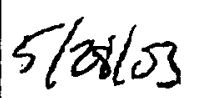 \\
\hline $\begin{array}{l}\text { modependar Revicwe } \\
\text { Femando Perez-Cardenas }\end{array}$ & & & $5 / 28 / 03$ \\
\hline $\begin{array}{l}\text { Ext-acd } \\
\text { Ianocent Joseph }\end{array}$ & & GTSD & \\
\hline
\end{tabular}

Report Approval:

VSL Principal In vestigator/Program Manager

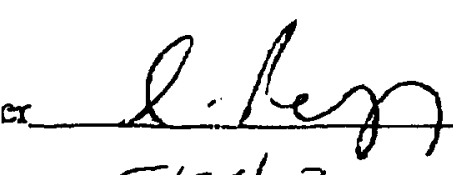

Date 
The Catholic University of America Vitreous State Laboratory
DuraMelter 1200 Tests with AZ-101HLW Simulants Final Report, VSL-02R0100-2, Rev.1

\section{Table of Contents}

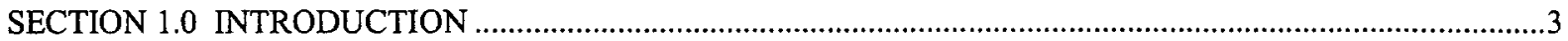

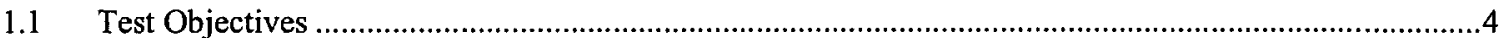

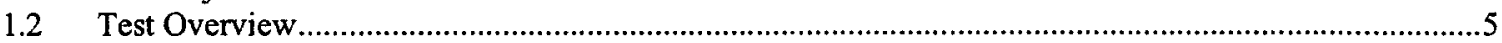

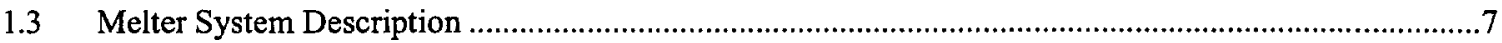

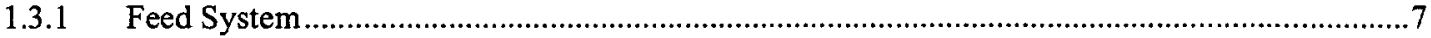

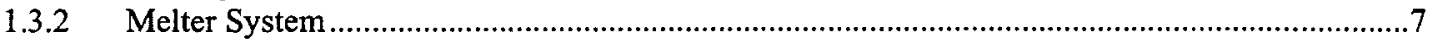

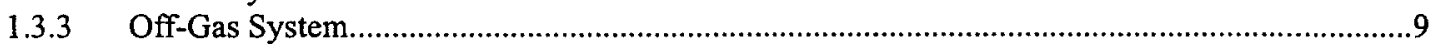

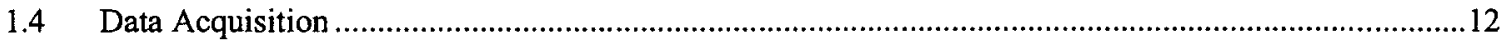

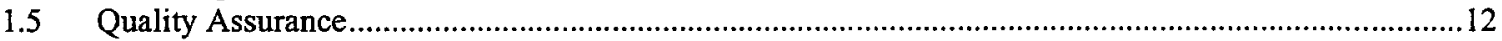

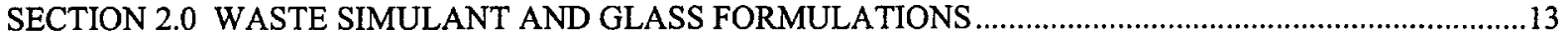

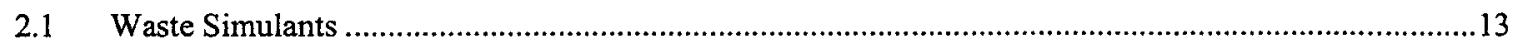

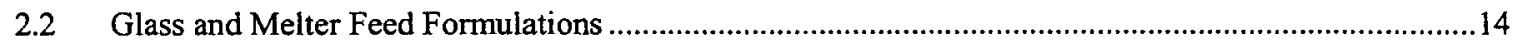

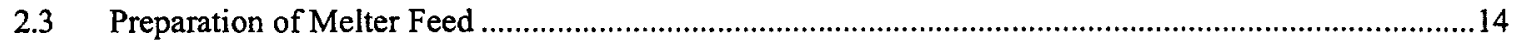

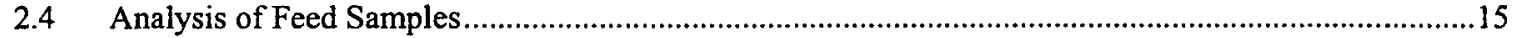

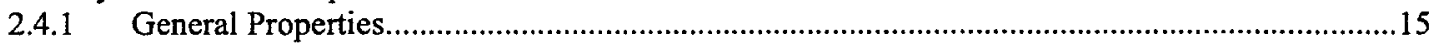

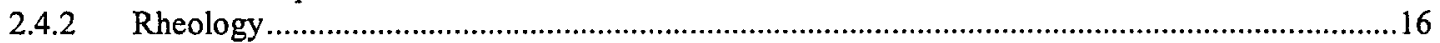

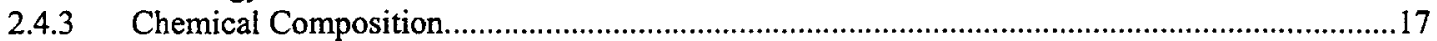

SECTION 3.0 MELTER OPERATIONS

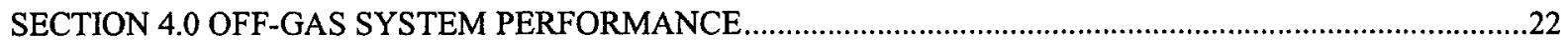

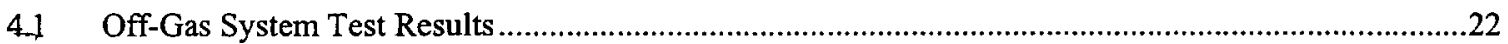

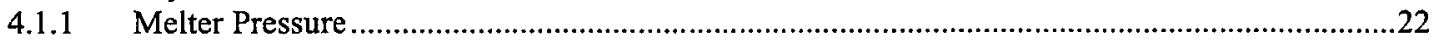

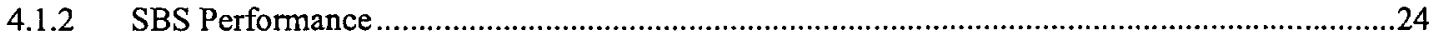

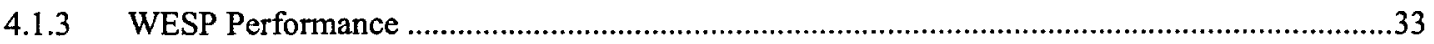

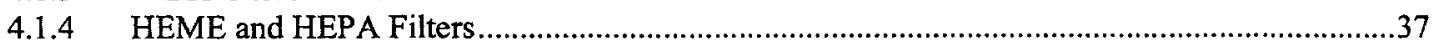

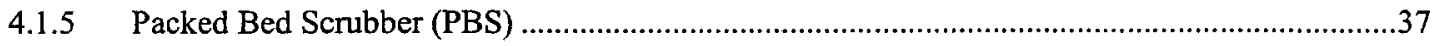

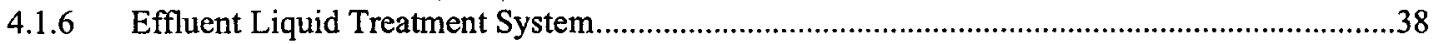

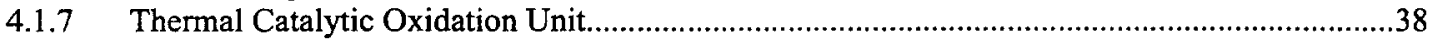

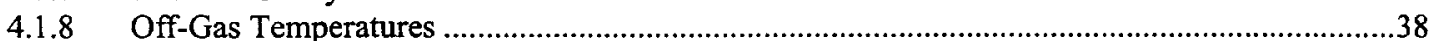

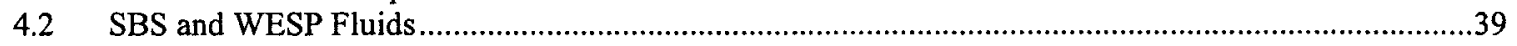

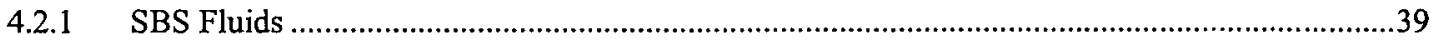

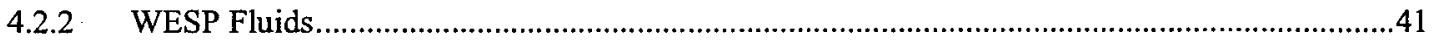

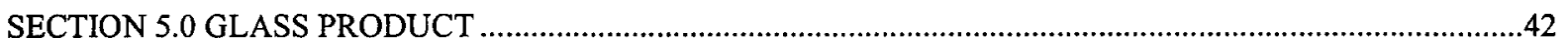

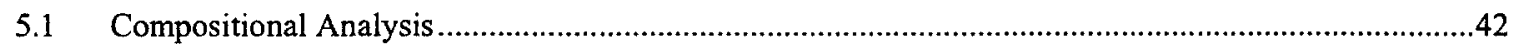

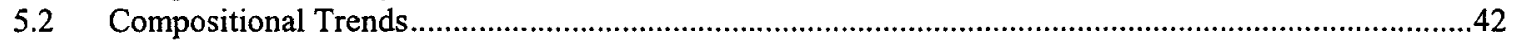

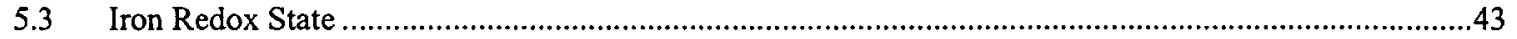

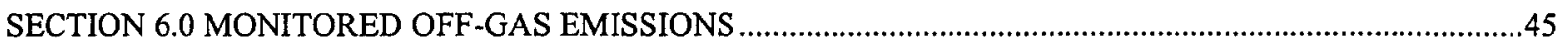

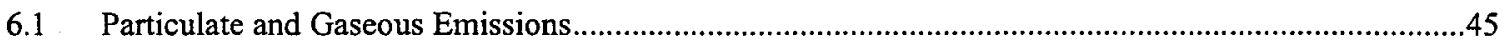

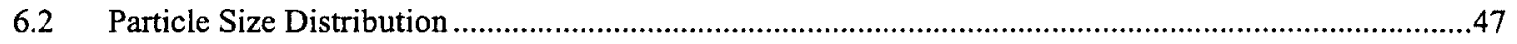

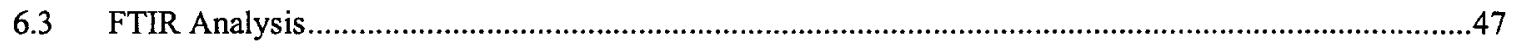

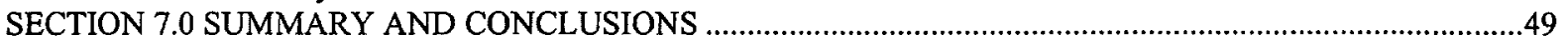

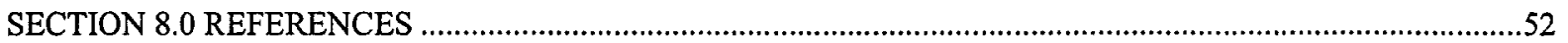

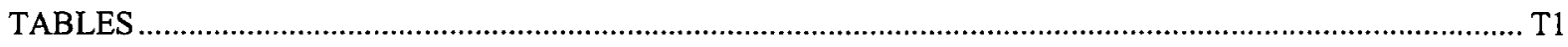

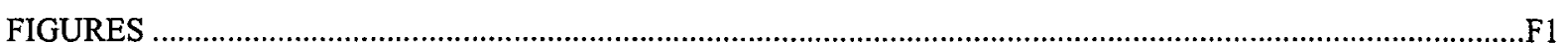

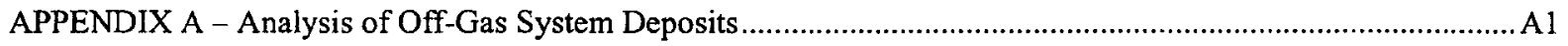

APPENDIX B - Viscosity and Particle Size Distribution of SBS Fluids................................................... B1 
The Catholic University of America

Vitreous State Laboratory
DuraMelter 1200 Tests with AZ-101HLW Simulants

Final Report, VSL-02R0100-2, Rev.I

\section{SECTION 1.0 INTRODUCTION}

This document provides the final report on data and results obtained from a series of nine tests performed on the one-third scale DuraMelter ${ }^{\mathrm{TM}} 1200$ (DM1200) HLW Pilot Melter system that has been installed at VSL with an integrated prototypical off-gas treatment system. That system has replaced the DM1000 system that was used for HLW throughput testing during Part B1 [1]. Both melters have similar melt surface areas $\left(1.2 \mathrm{~m}^{2}\right)$ but the DM1200 is prototypical of the present RPP-WTP HLW melter design whereas the DM1000 was not. These tests were performed under a corresponding RPP-WTP Test Specification [2] and associated Test Plans [3, 4]. The nine tests reported here were preceded by an initial series of short-duration tests conducted to support the start-up and commissioning of this system [5]. This report is a followup to the previously issued Preliminary Data Summary Reports $[6,7]$.

The DM1200 system was deployed for testing and confirmation of basic design, operability, flow sheet, and process control assumptions as well as for support of waste form qualification and permitting. These tests include data on processing rates, off-gas treatment system performance, recycle stream compositions, as well as process operability and reliability. Consequently, this system is a key component of the overall HLW vitrification development strategy. The primary objective of the present series of tests was to determine the effects of a variety of parameters on the glass production rate in comparison to the RPP-WTP HLW design basis of $400 \mathrm{~kg} / \mathrm{m}^{2} / \mathrm{d}$. Previous testing on the DM1000 system [1] concluded that achievement of that rate with simulants of projected WTP melter feeds (AZ-101 and C-106/AY-102) was unlikely without the use of bubblers. As part of those tests, the same feed that was used during the cold-commissioning of the West Valley Demonstration Project (WVDP) HLW vitrification system was run on the DM1000 system. The DM1000 tests reproduced the rates that were obtained at the larger WVDP facility, lending confidence to the tests results [1]. Since the inclusion or exclusion of a bubbler has significant design implications, the Project commissioned further tests to address this issue.

In an effort to identify factors that might increase the glass production rate for projected WTP melter feeds, a subsequent series of tests was performed on the DM100 system [9]. Several tests variables led to glass production rate increases to values significantly above the $400 \mathrm{~kg} / \mathrm{m}^{2} / \mathrm{d}$ requirement. However, while small-scale melter tests are useful for screening relative effects, they tend to overestimate absolute glass production rates, particularly for un-bubbled tests. Consequently, when scale-up effects were taken into account, it was not clear that any of the variables investigated would conclusively meet the $400 \mathrm{~kg} / \mathrm{m}^{2} / \mathrm{d}$ requirement without bubbling. The present series of tests was therefore performed on the DM1200 one-third scale HLW pilot melter system to provide the required basis for a final decision on whether bubblers would be 
included in the HLW melter. The present tests employed the same AZ-101 waste simulant and glass composition that was used for previous testing [1-3, 8,9] for consistency and comparability with the results from the earlier tests.

\section{$1.1 \quad$ Test Objectives}

The principal objectives of this work were to:

- Determine processing rates without bubbling on the DM1200 system in longer-duration tests than were performed on the DM1000 system [1] and at a larger scale than the tests performed on the DM100 system [9].

- If the design-basis rate of $400 \mathrm{~kg} / \mathrm{m}^{2} / \mathrm{d}$ is achieved without bubbling with a high-solids feed, determine the effect of solids content on processing rates from a similar test but with the nominal solids loading in the melter feed (this corresponds to $20 \mathrm{wt} \%$ solids in the Envelope D material from pretreatment).

- Determine the effect of continuous versus pulsed feed from two feed tubes instead of one on feed spreading and processing rates.

- Obtain processing data for a simulated, acidified AZ-101 HLW feed (Test 1 only), as employed at WVDP, using the DM1200 system at the Project nominal melter feed concentration (20 $\mathrm{wt} \%$ solids from pretreatment, which corresponds to approximately 400 grams of oxides per liter of feed), without bubbling, to determine the effect of nitrated melter feed on feed handling, foaming, cold-cap characteristics, feed spreading, and processing rates.

- Obtain processing data for a simulated AZ-101 HLW feed with frit instead of glass forming chemicals using the DM1200 system, without bubbling, to determine the effect on feed handling, foaming, cold-cap characteristics, and processing rates.

- Determine the amount of reductant (sugar) to be added to the feed to maintain part of the iron ( 5 to $15 \%$ ) in the $\mathrm{Fe}^{2+}$ state in order to prevent foaming incidents and determine the processing rate with this feed.

- Obtain processing data for a simulated AZ-101 HLW feed using the DM1200 system, without bubbling, at an operating temperature of between 1175 and $1200^{\circ} \mathrm{C}$, to determine the effect on feed handling, foaming, cold-cap characteristics, and processing rates. 
- Evaluate off-gas system component performance by sampling exhaust at various points in the off-gas system as well as sump solutions from the various off-gas system components.

- Collect data on mass balance across the melter.

- Identify possible processing problems such as foaming, secondary phase formation, and poor cold cap characteristics, particularly in comparison to previous small-scale and DM1000 melter tests.

- Collect data on product glass composition and product quality.

- Collect operating data with a simulated air-displacement slurry (ADS) pump melter feed system (i.e., intermittent, pulsed flow of feed to the melter), particularly with respect to offgas transients.

- Determine the effect of bubbling and the positioning of the bubblers (deep or shallow) on processing rates.

- Obtain processing data for a simulated AZ-101 HLW feed with frit instead of glass forming chemicals using the DM1200 system, with bubbling (standard " $L$ " shaped bubbler design), to determine the effect on feed handling, foaming, cold-cap characteristics, and processing rates.

\subsection{Test Overview}

The DM1200 system and the test matrices are described in the Test Plans [3, 4]. A synthesis of the two Test Plan matrices is compared to the tests conducted in Table 1.1. Three tests were not conducted at the direction of the Project based on the results of earlier tests. A detailed description of the off-gas treatment system has also been provided [10]. In addition, the results of extensive off-line testing of the submerged-bed scrubber (SBS) [11] and the commissioning tests [5] have also been reported. This set of nine tests generated a total of $26,725 \mathrm{~kg}$ of glass, as summarized below:

- Test 1 - High-solids feed (570 g glass/liter), without bubbling $(<4 \mathrm{lpm})$; 168 hours, $2301 \mathrm{~kg}$ glass produced.

- Test 2 - High-solids feed (570 g glass/liter) $+10 \mathrm{~g}$ sugar /liter, without bubbling (<4 lpm); 216 hours, $2594 \mathrm{~kg}$ glass produced. 
- Test 3 - Low-solids feed (400 g glass/liter), with deep bubbling ${ }^{1}$ (nominal $60 \mathrm{lpm}) ; 115$ hours, $6180 \mathrm{~kg}$ glass produced.

- Test 4 - Low-solids feed (400 g glass/liter), with shallow bubbling ${ }^{1}$ (nominal $60 \mathrm{lpm}) ; 122$ hours, $3465 \mathrm{~kg}$ glass produced.

- $\quad$ Test 5 - Nitrated feed ( 420 g glass/liter) with sugar ratio $=0.5$, without bubbling $(<4 \mathrm{lpm}) ; 144$ hours, $1659 \mathrm{~kg}$ glass produced.

- Test 6 - Frit-based feed ( $480 \mathrm{~g}$ glass/liter), without bubbling ( $<4 \mathrm{lpm}) ; 155$ hours, $1901 \mathrm{~kg}$ glass produced.

- Test 7 - Frit-based feed (480 g glass/liter), deep bubbling (60 lpm); 120 hours, $5976 \mathrm{~kg}$ glass produced.

- Test 8 - Nitrated feed ( 420 g glass/liter) with sugar ratio $=0.7$, without bubbling (<4 lpm); 100 hours, $944 \mathrm{~kg}$ glass produced.

- Test 9a - High-solids feed (570 g glass/liter) + $10 \mathrm{~g}$ sugar /liter, without bubbling $(<4 \mathrm{lpm}$ ); continuous feeding (as opposed to pulsed feeding) from two feed tubes instead of one, 69 hours, $859 \mathrm{~kg}$ glass produced.

- Test $9 \mathrm{~b}-$ High-solids feed ( $570 \mathrm{~g}$ glass/ liter) $+10 \mathrm{~g}$ sugar /liter, without bubbling $(<4 \mathrm{lpm})$; continuous feeding (as opposed to pulse) from two feed tubes instead of one, $1200^{\circ} \mathrm{C}$ glass temperature, 54 hours, $847 \mathrm{~kg}$ glass produced.

All of the tests were performed with the same waste simulant and glass composition, a nominal glass pool temperature of $1150^{\circ} \mathrm{C}$ (except Test $9 \mathrm{~b}$ ), essentially complete cold cap coverage, and a nominal plenum temperature between $400-600^{\circ} \mathrm{C}$. Side-to-bottom electrode firing was used for the unbubbled tests and for Test 4 with shallow bubbling. Side-to-side electrode firing was used (the bottom electrode was not powered) for the rest of the bubbled tests. One of the two installed feed tubes was used in order to more closely reflect the number of feed tubes per unit area planned for the full-scale HLW melter. The exception was Test 9, for which both feed tubes were used. Tables $1.2-1.4$ provide a summary of the test conditions and production rate data for each test.

1 All of the bubbled tests employed two "L"- shaped bubbler lances, one in each of two diagonally opposite corners. The normal immersion of the bubblers was such that their outlets were located 6 inches from the bottom of the melter floor, which is referred to as "deep." For the "shallow" bubbling test (Test 4), both bubblers were raised so that their outlets were located 13.5 inches from the melter floor. 
The Catholic University of America Vitreous State Laboratory
DuraMelter 1200 Tests with AZ-101HLW Simulants

Final Report, VSL-02R0100-2, Rev.1

\subsection{Melter System Description}

\subsubsection{Feed System}

The feed material for these tests was prepared by a chemical supplier, as detailed in Section 2. Each batch of feed slurry was shipped to VSL in lined 55-gallon drums (approximately 16 per shipment) and staged for unloading into the mix tank. Both the mix tank and the feed tank are 750-gallon polyethylene tanks with conical bottoms that are fitted with mechanical agitators; the feed tank also includes baffles to improve mixing, which were installed after Test 5 was completed. Five calibrated load cells directly mounted on the legs of the feed tank were used to measure additions to and removal from the feed tank and were electronically monitored to determine the feed rate to the melter. The requisite amount of feed is pumped to the feed tank from the mix tank; measured amounts of water were combined with the feed at this point for the high-water feed tests. The material in the feed tank is constantly recirculated from the feed tank discharge outlet, at the tank bottom, to the tank inlet at the top, which provides additional mixing.

The way in which the feed is introduced into the melter is designed to mimic the operation of an ADS pump, which is the present RPP-WTP baseline. The recirculation loop extends to the top of the melter where feed is diverted from the recirculation loop into the melter through two parallel Teflon-lined feed lines and water-cooled feed tubes. Three computeroperated pinch valves, one on each of the feed lines and one on the recirculation loop, are activated in a timed sequenced to introduce feed into the melter at the desired rate. The feed rate is regulated by adjusting the length of each pulse, the time between each pulse, and the pressure applied to the recirculation loop. A compressed air line is attached to each of the feed lines and can be used to automatically clear the feed lines into the melter after each pulse.

\subsubsection{Melter System}

The DuraMelter ${ }^{\mathrm{TM}} 1200$ is a Joule-heated melter with Inconel 690 electrodes and thus has an upper operating temperature of about $1200^{\circ} \mathrm{C}$. The melter shell is water-cooled and incorporates a jack-bolt thermal expansion system. The footprint of the melter is approximately $8 \mathrm{ft}$. by $6.5 \mathrm{ft}$. with a $4 \mathrm{ft}$. by $2.3 \mathrm{ft}$. air-lift discharge chamber appended to one end; the melter shell is almost $8 \mathrm{ft}$. tall. The melter surface area and the melt pool height are approximately 32 percent and 57 percent, respectively, of the corresponding values for the full-scale HLW melter. The discharge riser and trough are full-scale to verify pouring adequacy. Other aspects of the discharge system are also prototypical such as the chamber ventilation scheme. The glass contact refractory is Monofrax ${ }^{\mathrm{TM}} \mathrm{K}-3$ while the plenum area walls are constructed of Monofrax ${ }^{\mathrm{TM}} \mathrm{H}$ refractory. The surface of the glass pool is 34 " by 54 " with a glass depth of nominally $25^{\prime \prime}$. The resultant melt volume is approximately 45,000 cubic inches ( 735 liters), which represents a glass 
The Catholic University of America Vitreous State Laboratory
DuraMelter 1200 Tests with AZ-101HLW Simulants

Final Report, VSL-02R0100-2, Rev.1

tank capacity of more than 1.7 metric tons of glass; however, at the typical operating glass depth of about 29 inches, the inventory is closer to 2.0 MT. As compared to the two parallel pairs of flat plate electrodes in the former DuraMelter ${ }^{\mathrm{TM}} 1000$ [1], the DuraMelter ${ }^{\mathrm{TM}} 1200$ is fitted with one pair of electrodes placed high on opposite walls of the melter as well as one bottom electrode. The side electrodes are 11" by 34 " giving an electrode area for the pair of about 750 sq. in. Depending on the glass level, the plenum space extends about $33^{\prime \prime}$ to 36 " above the melt surface resulting in a plenum volume ranging from about 43 to $46 \mathrm{ft}^{3}$. Cross-sectional diagrams of the melter illustrating the discharge chamber and electrode configuration are provided in Figures 1.1 and 1.2.

All of the bubbled tests employed two "L"- shaped bubbler lances, one in each of two diagonally opposite corners. The normal immersion of the bubblers was such that their outlets were located 6 inches from the bottom of the melter floor, which is referred to as "deep." For the "shallow" bubbling test (Test 4), both bubblers were raised so that their outlets were located 13.5 inches from the melter floor.

The single-phase power supply to the melter electrodes ( $250 \mathrm{~kW}$ designed power) is derived from the DuraMelter ${ }^{\mathrm{TM}} 1000$ transformers by wiring them in parallel and using a single large silicon controlled rectifier (SCR). Current can be passed either from the side electrodes to the bottom electrode or between the two side electrodes only, by rearranging jumpers. Programmable process controllers are installed and can be used to control temperature or power. The melt temperature is controlled by configuring the process controller to maintain constant power and the power set-point is adjusted as needed to maintain the desired operating temperature. Alarms can be set to detect out-of-range temperatures or power in the melter. Backup process controllers are installed to be used in case of failure of the main controllers.

The DuraMelter ${ }^{\mathrm{TM}} 1200$ has several others features worth noting. The lid refractory is prototypic and also includes a two-piece construction, which simulates the seam that was part of the full-scale LAW lid, which was to have been fabricated in three pieces; however, that design element of the LAW lid has since been revised. Nozzles are provided for the off-gas film cooler, a standby off-gas port, discharge airlift, along with 11 ports available for top-entering bubblers, start-up heaters and other components as needed. In addition, a bubbler arrangement is installed in the bottom electrode with the objective of developing permanent bubblers for possible use on future melters. Another innovation is a fiber optic distributed temperature monitor that is cast into the Narcon ${ }^{\mathrm{TM}} 60 \mathrm{~A}$ castable on the discharge chamber side of the DM1200 that is intended to demonstrate the use of a distributed temperature sensor for refractory corrosion monitoring; however, this was not used for any of the present tests. 
The Catholic University of America

Vitreous State Laboratory
DuraMelter 1200 Tests with AZ-101HLW Simulants

Final Report, VSL-02R0100-2, Rev.1

\subsubsection{Off-Gas System}

The DM1200 off-gas system was designed to be prototypic of the off-gas treatment system planned for use with the HLW melters in the RPP-WTP facility. In addition, the system can be reconfigured to represent the LAW off-gas treatment train for testing with LAW feeds. A detailed description of the design and installation of the DM-1200 off-gas system was provided earlier [10]. A schematic process flow diagram showing the components of the system is provided in Figure 1.3.

\subsubsection{Functional Description}

The melter and the entire off-gas treatment system are maintained under slight negative pressure by external induced draft blowers. This negative pressure is necessary to minimize escape of gases from the system into the working spaces surrounding the melter and is prototypical of the full-scale system. The off-gas treatment system, shown schematically in Figure 1.3, consists of a submerged bed scrubber (SBS); a wet electrostatic precipitator (WESP); a high-efficiency mist eliminator (HEME); a high-efficiency particulate arrestor (HEPA); a packed-column caustic scrubber (PBS); a second HEME; a thermal catalytic oxidation unit (TCO); and a second HEPA as the primary treatment units. Differences between the DM1200 off-gas system and the RPP-WTP baseline design include the addition of intermediate blowers and a second High Efficiency Mist Eliminator (HEME) after the caustic scrubber in the pilot system; in addition, the melter pressure control air system was not in place for these tests. The second HEME is present in order to prevent undesirable salt carryover into the TCO unit. Several changes have been made to the RPP-WTP baseline design since the deployment of the DM1200 system, including the replacement of the packed-bed scrubber by a silver mordenite column for iodine removal, which is located after the TCO. The Project is evaluating the addition of a silver mordenite column to the DM1200 system; for the present series of test, however, the packed bed scrubber was not operated.

The system can be functionally subdivided into four subsystems:

Particulate Removal: $\quad$ Components from the submerged bed scrubber (SBS) to HEPA \#1 serve to remove essentially all of the particulate from the gas stream with an estimated removal efficiency of greater than $99.9999 \%$ for particles greater than $0.3 \mu \mathrm{m}$ in size. In the RPPWTP facility, this provision serves to segregate the radioactive from the non-radioactive components in the system for maintenance and handling purposes.

Acid Gas/VOC Control: $\quad$ The caustic scrubber and HEME \#2 provide acid gas scrubbing to 
Stack System:

Liquid Processing: protect the thermal catalytic oxidation (TCO) unit, which is designed to oxidize any hazardous organics that are present in the off-gas stream. However, as noted above, the caustic scrubber was by-passed for these tests. In addition, no catalyst was installed in the TCO for the present tests.

Components from HEPA \#2 to the stack outlet, including the emergency/bypass exhaust system, provide final filtration of the gas and serve to exhaust the treated gas stream to the atmosphere.

Components including the water spray lines, liquid sampling and water storage tanks, as well as the effluent evaporator, function to sample and process the system liquids for recycle or discharge.

Initial quenching of the gas stream from the melter takes place in the film cooler. Gas exits the film cooler at a temperature of 250 to $350^{\circ} \mathrm{C}$ flowing at a rate of $100-250 \mathrm{scfm}$, of which about $5-50 \mathrm{scfm}$ is water vapor. The off-gas is then rapidly quenched by direct liquid water contact in the Submerged Bed Scrubber (SBS), which also effects removal of most of the larger particulates. High superficial gas velocity in the piping between the film cooler and SBS minimizes particulate deposition. Gas leaves the SBS at a relatively low temperature (typically between 35 and $50^{\circ} \mathrm{C}$ ). Further mist and particulate removal continues in the WESP, HEPAs, and HEMEs. Water deluge nozzles are installed in WESP and HEMEs to wash down deposits into their respective collection sumps. The system components are fabricated from corrosion resistant materials including AL6XN in the SBS and 316L stainless steel and various plastics in other locations. There are extensive provisions for sampling both the gas and liquid streams throughout the system in order to collect mass balance information and removal efficiency data for each treatment stage.

\subsubsection{SBS Modifications}

Several modifications were made to the SBS as a result of data collected during initial stand-alone testing using a Landa evaporator as an off-gas/steam source simulator [11] and subsequent testing by feeding the DM1200 melter with water; these are briefly summarized here for background. Significant among the issues were:

- Fluctuations in melter pressure (about 2 in. W.C.) attributed to oscillations on the large gas bubble that forms underneath of the SBS diffuser plate at the exit of the down-comer pipe. 
- Rapid disintegration of the ceramic saddle packing media that was originally specified by the RPP-WTP Project.

- A need for supplemental cooling to handle the increased steam flow into the SBS at the higher processing rates that are realized when the glass pool is agitated by bubbling.

Several modifications were tested, including extension of the center inlet down-comer below the diffuser plate and alternative packing media. A perforated down-comer extension tube extending 12 inches below the diffuser plate was installed with holes along the tube wall with the intent of breaking up the gas bubble forming below the diffuser plate. This change reduced the amplitude of the pressure pulsations to within about 0.5 in W.C. Several other changes were also investigated, such as plugging weir tubes, plugging the standby gas nozzle, and constraining the inlet source of gas. However, the perforated down-comer extension appeared to have the most effect in mitigating the pressure fluctuations. In the down-comer extension used during the commissioning tests there were 32 half-inch diameter holes distributed along the tube in four rows of eight, $1 \frac{1 / 2}{2}$ inch apart.

Various packing media, including $1 / 2$ inch stainless steel saddles, metal balls, and ceramic balls were tested in place of the original ceramic Intallox saddle packing medium. Based on these tests, ceramic balls, approximately an inch in diameter, packed all the way up to the upper retaining screen were used during the commission testing. However, the original Intallox saddles were retained in the spaces between the liquid weirs that protruded through the diffuser plate to prevent jamming of the ceramic balls into crevices. A metal screen was laid on top of the weirs to eliminate intermixing of packing media.

\subsubsection{Modifications to SBS Cooling System}

A schematic diagram of the modified SBS cooling system is given in Figure 1.4. An external heat exchanger and an internal cooling coil were added in order to decrease the operating temperature of the SBS and minimize down-stream moisture condensation; the cooling coil is connected to the heat exchanger. This was in addition to the existing outer cooling jacket on the SBS, which alone proved insufficient for the higher glass production rate tests. 


\subsection{Data Acquisition}

During these tests, data from most measurement points were logged at two-minute intervals by a computer-based data acquisition system using LabVIEW $^{2}$ software. Redundant manual logging of many of these parameters was also performed by the operations staff. Other parameters were logged only manually. These measurements are discussed in Section 3.

\subsection{Quality Assurance}

This work was conducted under an NQA-1 based quality assurance program that is in place at VSL. This program is supplemented by a VSL Quality Assurance Project Plan (QAPP) for RPP-WTP work. Per RPP-WTP Project direction, the program was revised during the performance of this work. Accordingly, work performed before 8/1/01 was performed under an NQA-1 (1994) program and corresponding Quality Assurance Project Plan (QAPP) for RPPWTP work [12], while work performed after that date was performed under an NQA-1 (1989) and NQA-2a (1990) Part 2.7 based quality assurance program and corresponding QAPP [13]. The program is supported by VSL standard operating procedures that were used for this work [14]. This work was not subject to DOE/RW-0333P.

\footnotetext{
2 National Instruments Corporation, Austin Texas.
} 
The Catholic University of America

Vitreous State Laboratory
DuraMelter 1200 Tests with AZ-101HLW Simulants

Final Report, VSL-02R0100-2, Rev.1

\section{SECTION 2.0 \\ WASTE SIMULANT AND GLASS FORMULATIONS}

\subsection{Waste Simulants}

The AZ-101 HLW simulant that was used for these tests is based on waste compositions provided in the Tank Waste Remediation System Operation and Utilization Plan (TWRS-OUP) [15]. The TWRS-OUP provides inventory information on 24 major waste components, which is, for the most part identical to that found in the Best Basis Inventory (BBI) database [16]; the exceptions are mercury, which is omitted in the TWRS-OUP, and strontium, for which the higher of the two values was assumed in this work. The TWRS-OUP, in addition to total inventories, provides the information on the partitioning of those inventories into solid and supernatant fractions that is needed to define waste simulants. The chemical wash factors that are provided in the TWRS-OUP were applied to the solid fractions in defining the AZ-101 simulant. For waste components that are not tracked in the TWRS-OUP or the BBI, data from the HLW Feed Staging Plan [17] based on the recommended number of in-tank sludge washings were used. No radionuclides or noble metals were included and all constituents present at less than $0.05 \mathrm{wt} \%$ (waste oxide basis) were omitted. In addition, all of the TOC was assumed to be oxalate and the small amounts of boron, lithium, and zinc were omitted since much greater amounts are present in the glass forming additives. While it was recognized that more recent information was available (e.g., in the Tank Farm Contractor Operation and Utilization Plan [18]) the compositional changes for AZ-101 are not great. Furthermore, it was judged more important to keep exactly the same simulant and melter feed composition for these tests as those used previously $[1,5,8,9]$ in order to be able to directly compare the test results.

A total of 32 chemical components are present in the resulting simulant for the washed AZ-101 HLW (Table 2.1). This HLW material must then be blended with the projected products from LAW pretreatment to complete the waste simulant formulation. These pretreatment processes ( $\mathrm{Cs}$ and Tc removal by ion exchange and Sr/TRU removal by Sr/permanganate precipitation) lead to increases in the amounts of cesium, technetium, sodium, nitrate, strontium, and manganese in the HLW material. The pretreatment products from technetium removal, which contribute technetium, sodium, and nitrate, were neglected in the simulant since the impact on nitrate is relatively small ( 75 liters of Tc concentrate vs. 3,811 liters of Cs concentrate [19]) and sodium is used as a glass forming additive. The quantities of pretreatment products to be combined with the AZ-101 HLW material were calculated based on References [19] and [20]. In particular, $7.71 \%$ of the total $\mathrm{Sr} / \mathrm{TRU}$ precipitate from pretreatment of Envelope $\mathrm{C}$ waste was assumed to be added to AZ-101 waste, which is equivalent to $(78 \mathrm{MT} X 0.0771)=6.01 \mathrm{MT}$ of 
strontium and $(38 \mathrm{MT} X 0.0771)=2.93 \mathrm{MT}$ of manganese ${ }^{3}$. The strontium precipitate will probably consist of a combination of carbonate and hydroxide. We have assumed that the carbonate is predominant and have included $3 \mathrm{~g}$ of carbonate per $100 \mathrm{~g}$ of oxides in the simulant (no carbonate is present in the washed HLW material as a consequence of the assumed $100 \%$ wash factor for carbonate [15]). The composition of the resulting AZ-101 simulant mixed with pretreatment products is summarized in Table 2.1. Subsequent to the Turnover and prior to Test 1 , a spike of potassium iodide (at the expense of potassium nitrate) was added to the feed to achieve a target glass composition of 0.1 weight percent iodine if all of the iodine were retained.

\subsection{Glass and Melter Feed Formulations}

Glass formulations have been developed for the AZ-101 simulant that accommodate the replacement of iron by manganese for Sr/TRU removal and meet the processing and product quality requirements. The glass composition selected for these tests, HLW98-31, is presented in Table 2.1. On an oxide basis, this glass is composed of $27.0 \mathrm{wt} \%$ of simulated Envelope $\mathrm{D}$ waste and $3.5 \mathrm{wt} \%$ of pretreatment products; the resulting content of $\mathrm{MnO}$ is $3.03 \mathrm{wt} \%$. Crucible, DM10, DM100, and DM1000 tests have been conducted to determine that this glass meets all processing and performance requirements $[1,8,9]$. The measured viscosity and conductivity at $1150^{\circ} \mathrm{C}$ are $43 \mathrm{P}$ and $0.41 \mathrm{~S} / \mathrm{cm}$, respectively. Heat treatment of HLW98-31 at $950^{\circ} \mathrm{C}$ for 70 hours resulted in $0.26 \mathrm{vol} \%$ of spinel while the glass was completely homogeneous after 66 hours at $1050^{\circ} \mathrm{C}$. The glass also shows good TCLP performance: the Cd concentration in the TCLP leachate is $0.067 \mathrm{mg} / \mathrm{l}$, compared to the Universal Treatment Standard (UTS) level of 0.11 $\mathrm{mg} / \mathrm{l}$, while all other constituents are below their respective UTS levels by much wider margins.

The additional constituents required to form HLW98-31 glass from the AZ-101 simulant are boron, lithium, sodium, silicon, and zinc. The corresponding chemical additives that are the sources for these elements were selected based upon cost and compatibility with the vitrification process. The theoretical glass yield of the resulting feed is $0.39 \mathrm{~kg}$ of glass per $\mathrm{kg}$ of feed, which is equivalent to $0.55 \mathrm{~kg}$ of glass per liter of feed based on a density of the feed of $1.40 \mathrm{~g} / \mathrm{ml}$.

\subsection{Preparation of Melter Feed}

Early in B1 VSL solicited bids from vendors to collaborate in simulant and feed development and to supply material for the large-scale melter tests, which resulted in the selection of NOAH Technologies Corporation. NOAH Technologies' Quality Assurance program has been reviewed and approved by VSL. NOAH Technologies has produced the

3 Note that the WTP feed scheduling assumptions have since been revised so that, at present, no Sr/TRU precipitates will be available for blending with AZ-101 HLW material. 
simulant and feed samples used in development and screening tests conducted to date at VSL as well as larger-scale tests on the DM100, DM1000 and DM1200 melter systems.

Table 2.2 lists the starting materials and amounts required to generate the target AZ-101 simulant and feed; the data are based on a total of about 26 MT of feed, which would result in $10 \mathrm{MT}$ of glass. NOAH Technologies prepared the feed in batches based on their production capacity of about $4,000 \mathrm{~kg}$ per batch. The formulation was specified by VSL based on chemical assays of the raw materials provided by NOAH, or as necessary, assay samples of those materials performed by VSL. NOAH Technologies also prepared the nitrated feed by adding concentrated nitric acid to the feed. Addition was considered complete once the $\mathrm{pH}$ was between 3 and 4 , thereby mimicking the procedures used to produce the West Valley melter feed. The starting simulant and feed was prepared with sufficient oxide concentration so that after addition of nitric acid, the glass yield of the nitrated feed was about 400 grams per liter.

For the frit tests, the melter feed was prepared at NOAH Technologies by blending waste simulant produced by NOAH Technologies and frit produced by Ferro Corporation. The composition of the frit is provided in Table 2.3. The particle size of the frit was -200 mesh. The waste stimulant and frit were mixed such that the target glass composition defined in the last column of Table 2.1 was achieved. Tests using a feed prepared with -200 mesh glass frit showed that it had acceptable rheological properties at a concentration of about 570 grams of oxides per liter of feed. A feed prepared using HLW AZ-101 simulant and glass frit (instead of glass forming chemicals) did not show any adverse effects after aging for up to one week.

\subsection{Analysis of Feed Samples}

\section{General Properties}

Feed samples were analyzed from each distinct feed tank charging or at least once per day of operation to confirm chemical composition and physical properties. Sample names, sampling dates, sampling location, tank mass during sampling and measured properties are provided in Table 2.4. All samples were taken from the feed line immediately upstream of the entrance point to the melter. The measured feed sample properties for all four types of feed are summarized in Table 2.5. The target glass yields of $570 \mathrm{~g} / 1$ for Tests $1,2,9$ (Nominal), $400 \mathrm{~g} / 1$ for Tests 3-4 (High Water), $420 \mathrm{~g} / 1$ for Tests 5 and 8 (Nitrated Nominal), and $480 \mathrm{~g} / 1$ for Tests 6 and 7 (Frit Nominal) were approximated in all samples analyzed. The averages of glass yields for each type were close to their target values. The target values for glass yields in terms of $\mathrm{kg}$ glass $/ \mathrm{kg}$ feed were used in calculating production rates. Other measured parameters such as water percent and density fall into narrow ranges for similar feed types. The vast majority of measured feed $\mathrm{pH}$ values were between 9 and 10 , as expected, with the exception of samples from Tests 5 and 8 , in which the feed was acidified with nitric acid. The average measured $\mathrm{pH}$ 
was about 5 , which was slightly above the target $\mathrm{pH}$ range of 3-4 when the feed was prepared, which we attribute to aging effects: Laboratory tests conducted to determine the amount of acid required to reach the desired $\mathrm{pH}$ range, indicated that as the acidified feed ages, the $\mathrm{pH}$ gradually increases due to neutralization reactions with feed components.

\section{Rheology}

Samples of the melter feeds that were used for these tests were also subjected to rheological characterization. The results from rheological characterization of a variety of other melter feeds and waste simulants, as well as the effects of a range of test variables, are described in detail in a separate report [21]. Melter feeds were characterized using a Haake RS75 rheometer, which was equipped with either a Z40DIN or a FL22-SZ40 sensor. A typical set of measurements consists of identifying the flow characteristics of the slurry by measuring the shear stress on the slurry at controlled shear rates and temperatures. In these measurements, the shear rate values are preset and are increased stepwise from $0.01 \mathrm{~s}^{-1}$ to $200 \mathrm{~s}^{-1}\left(70 \mathrm{~s}^{-1}\right.$ for FL22SZ40) with a sufficient delay (typically 15 to 30 seconds) between steps to ensure that shear stress is allowed to fully relax and therefore measured at equilibrium. It should be noted that this approach is somewhat different than the "flow curve" approach that is often used in which the shear rate is ramped up to some maximum value and then ramped back down to produce a hysteresis curve that is dependent on the arbitrarily selected ramp rate. In contrast, the present measurements are equilibrium values of the shear stress at each measured shear rate. The viscosity of the sample as a function of the shear rate is then calculated as the ratio of the shear stress to the shear rate. All of the measurements in this work were made at $25^{\circ} \mathrm{C}$; previous work [21], which examined a range of temperatures, showed a relatively weak effect of temperature.

Rheograms for the melter feeds, which show the feed viscosity versus shear rate, are presented in Figure 2.1; measured values for viscosity at selected shear rates are compared in Table 2.4. The samples group into three distinct populations; the most viscous being the nominal feed, the least viscous being the nitrated feed, with both the high-water and the frit-based feeds falling in between. The observed and expected reduction in viscosity with increased water contents or decreased oxide loading has been observed in previous analysis of simulated HLW melter feeds $[9,21]$. Nominal feed has the highest oxide loading and lowest water content and thus the highest viscosity. The nitrated feed has the lowest viscosity even though the water content is comparable to the nominal feed. The lower $\mathrm{pH}$ promotes the dissolution of suspended particles thus decreasing viscosity. The high-water and frit-based feeds have similar viscosities even though the frit-based feed has a lower water content (61\% vs. 64\%). The similarity in viscosities may be due to less agglomeration of glass frit than hydroxide particles in high water feeds. 
The Catholic University of America

Vitreous State Laboratory
DuraMelter 1200 Tests with AZ-101HLW Simulants

Final Report, VSL-02R0100-2, Rev.1

\subsubsection{Chemical Composition}

A large number of feed samples was subjected to chemical analysis using $\mathrm{x}$-ray fluorescence (XRF) and direct-current plasma atomic emission spectroscopy (DCP). The chemical compositions of the feed samples from the nine tests were determined by first making a glass from the feed samples via crucible melt. The glass was subsequently crushed and either dissolved in an $\mathrm{HF} / \mathrm{HNO}_{3}$ acid mixture with the aid of a microwave oven to produce a solution for DCP analysis or analyzed directly by XRF. Target values for boron and lithium oxides were used for normalizing the XRF data since those two elements were not determined by XRF. Data from both methods are compared to the target composition and are listed in Tables 2.6 and 2.7 respectively. Average feed sample compositions obtained by DCP and XRF are compared to the average discharged glass analysis and target values in Table 2.8 .

The compositional analysis can be put into perspective by dividing the 31 elements into three categories; major elements with measured oxide concentrations greater than $3 \%$, intermediate elements with measured oxide concentrations between 0.5 and $3 \%$, with the remainder being minor elements. The major elements constitute the bulk of the glass and, therefore, largely determine its properties. Both XRF and DCP results for the major elements (Al, B, Fe, $\mathrm{Li}, \mathrm{Na}, \mathrm{Si}, \mathrm{Zr}$ ) are consistent and show good agreement with the target composition. One exception is lithium which is discussed further below. Elements in the intermediate concentration range $(\mathrm{Mg}, \mathrm{Mn}, \mathrm{Ni}, \mathrm{Sr}, \mathrm{Zn})$ vary in their similarity to target composition. Strontium values were consistently more than $15 \%$ below target whereas manganese, nickel, and zinc concentrations were much closer to target. Magnesium oxide concentrations were about 20 times higher than the $0.06 \mathrm{wt} \%$ target due to contamination during feed batching at NOAH. This error was reported to the WTP Project prior to proceeding; however, it was determined that it would have an inconsequential effect on the processing properties of the feed or glass. The large number of minor elements (As, $\mathrm{Ba}, \mathrm{Ca}, \mathrm{Cd}, \mathrm{Ce}, \mathrm{Cl}, \mathrm{Cr}, \mathrm{Cs}, \mathrm{Cu}, \mathrm{F}, \mathrm{K}, \mathrm{La}, \mathrm{P}, \mathrm{Pb}, \mathrm{Sb}, \mathrm{Se}, \mathrm{S}$, $\mathrm{Te}, \mathrm{Ti})$ are all inherited from the simulated waste. Deviations were not calculated for these oxides due to the high volatility of many of the constituents and the uncertainty associated with deviation calculations on very low concentrations. As expected, highly volatile elements such as selenium, sulfur, and chlorine are under-represented in the glasses. Conversely, common elements, which are typical impurities in bulk chemicals, such as calcium, phosphorus, and potassium are over-represented when the constituent is a minor element.

Lithium was the only major element to deviate from the target value and, therefore, an alternative analytical method was employed to determine if the discrepancy was actual or analytical in nature. Consequently, Atomic Absorption (AA) was used to supplement the results obtained by DCP. Several digested glass samples that had been analyzed by DCP were reanalyzed by $\mathrm{AA}$, the results of which are provided in Table 2.9. All measured lithium concentrations determined by both methods were below target, in some cases by over $25 \%$. The 
two methods provided comparable results with the DCP analysis being, on average, slightly higher and, therefore, closer to target. Based on this comparison we conclude that either the feed is actually slightly deficient in lithium or that there is a problem with the glass dissolution that only affects lithium; given the fact that a standard reference glass is included in each glass dissolution set and that no issues were observed with those lithium values, we consider the latter possibility unlikely. 


\section{SECTION 3.0 MELTER OPERATIONS}

Nine melter tests were conducted on the DM1200 with the AZ-101 simulant between $6 / 25 / 01$ and $11 / 10 / 01$, producing over $26,700 \mathrm{~kg}$ of glass. Feed type and system operating parameters, including glass bubbling rate, were varied, as shown in the test summary in Tables 1.2-1.4. Prior to beginning the tests, over $10,000 \mathrm{~kg}$ of glass was produced during the turnover of the melt pool composition and the commissioning tests that preceded the present tests [2].

The measured glass production rates are depicted in Figures 3.1 - 3.9 as cumulative and two-hour moving averages. Typically, by the end of each test, the cumulative production rate approximates the two-hour average rate, indicating that steady-state rates have been approached. The principal factor affecting glass production rates was the rate and mode of glass pool bubbling. The production rate with deep bubbling was about four times higher (Test 3: $980 \mathrm{~kg} / \mathrm{m}^{2} /$ day vs. Test $1: 255 \mathrm{~kg} / \mathrm{m}^{2} /$ day) than without bubbling. However, the actual effect of bubbling is even greater than this as a result of the difference in the feed solids content for these two tests: the feed for the bubbled test (Test 3 ) has a solids content corresponding to $400 \mathrm{~g}$ glass per liter, whereas the feed for the test without bubbling (Test 1) yielded $570 \mathrm{~g}$ glass per liter. Bubbling at a shallower depth resulted in an approximate two-fold increase in production rate over the test without bubbling (Test $4: 540 \mathrm{~kg} / \mathrm{m}^{2} /$ day vs. Test $1: 255 \mathrm{~kg} / \mathrm{m}^{2} /$ day), indicating that bubbler depth has a significant impact on glass production rate. It should be noted, however, that the average bubbling rate was slightly lower for Test 4 than for Test 3; the higher rate could not be sustained when the bubblers were raised to the shallow location due to pile-up of the feed. A four to five-fold increase in production rate attributable to bubbling was observed for frit-based feed (Test 7: $1100 \mathrm{~kg} / \mathrm{m}^{2} /$ day vs. Test $6: 210 \mathrm{~kg} / \mathrm{m}^{2} /$ day). The addition of sugar to these lownitrate feeds had a negative impact on glass production, as can be seen by comparing Test 1 (steady-state rate of $255 \mathrm{~kg} / \mathrm{m}^{2} /$ day) and Test 2 (steady-state rate of $205 \mathrm{~kg} / \mathrm{m}^{2} / \mathrm{day}$ ). Varying feed composition (nitration in combination with different amounts of sugar and the replacement of glass forming additives by glass frit) had no measurable effects on glass production rates. The general processing behavior varied little from tests to test; the appearance of the cold cap also showed little variation, with the exception of the test with nitrated feed, for which the cold cap appeared somewhat more fluid. The change to continuous feeding with two feed tubes using a peristaltic pump as opposed to pulsed feeding from a single feed tube using a simulated ADS pump also did not result in an increase in production rates, which is evident when comparing the results to comparable tests discussed above (Test $9 \mathrm{a}: 230 \mathrm{~kg} / \mathrm{m}^{2} /$ day vs. $205-255 \mathrm{~kg} / \mathrm{m}^{2} /$ day). As expected, increasing the glass pool temperature did result in an increase in the production rate (by about one third). 
A variety of operational measurements were recorded during these melter tests including temperatures throughout the melter system, as given in Tables 3.1-3. The target glass temperature of $1150^{\circ} \mathrm{C}$ was successfully maintained for most of the glass pool during each of the tests, as illustrated in Figures $3.10-3.18$. An exception is the intended increase to $1200^{\circ} \mathrm{C}$ in the latter portion of Test 9 (Figure 3.18). The coldest regions were between 27" and 30" from the floor as a result of those thermocouples being in or near the cold cap. In tests with bubbling, the $1150^{\circ} \mathrm{C}$ target temperature was averaged at more locations throughout the melt pool resulting in more uniform temperature profiles. This is most readily observed by comparing the thermocouple $1 "$ from the melter floor in tests with and without bubbling. Tests without bubbling have bottom glass pool temperatures about $50^{\circ} \mathrm{C}$ lower than the bulk glass temperature, whereas tests with bubbling have bottom glass temperatures similar to the remainder of the glass pool. Plenum temperatures between $300-500^{\circ} \mathrm{C}$ were obtained for the steady-state portions of the tests. Tests without bubbling typically resulted in plenum temperatures of $300-400^{\circ} \mathrm{C}$, whereas plenum temperatures for tests with bubbling were about $50-100^{\circ} \mathrm{C}$ higher due to additional heat transfer from the openings in the cold cap caused by bubbling. Despite the somewhat higher plenum temperatures for the bubbled test, visual observations of the cold cap indicated that the feed rates obtained were still close to the maximum that could be sustained for the given test conditions. The plenum temperature is a function of the extent to which the surface of the melt pool is covered by feed and examples of this relationship are shown in Figures $3.19-3.27$ as the plenum temperatures decreasing over the course of each test as the cold cap develops; similarly, the rise in plenum temperatures at about $90 \mathrm{hrs}$ into Test 3 (Figure 3.21) is a result of the drop in feed rate at that time (Figure 3.3).

Electrode temperatures were below $1100^{\circ} \mathrm{C}$ except for the East electrode in the bubbling tests and in the latter half of Test 9 . Electrode temperatures for the first half of Test 9 are typical of other tests; the bottom electrode being $40^{\circ} \mathrm{C}$ colder than the East electrode with the West electrode temperature being in-between (Figure 3.28). In the second half of Test 9, power was increased to raise the glass temperature to $1200^{\circ} \mathrm{C}$, which also raised the electrode temperatures about $50^{\circ} \mathrm{C}$. The discharge chamber and riser temperatures were maintained above $950^{\circ} \mathrm{C}$ throughout the tests. Gas temperatures after film cooler dilution typically averaged between 250 and $400^{\circ} \mathrm{C}$, depending on the plenum temperature, the amount of added film cooler air, and the temperature of the added film cooler air. As was expected from the plenum temperatures, tests with bubbling had film cooler outlet temperatures near the top of the range, whereas tests without bubbling had temperatures near the bottom of the range.

The amount of power supplied to the electrodes depended on the feed rate and the water content of the feed. Tests with higher feed rates (e.g., bubbled tests) required more power; however, when normalized to glass production, they had the lowest power utilization per unit glass produced (Tables 1.2-1.4). Typically, power utilization per unit mass of glass was inversely 
proportional to the glass production rate due to the constant amount of power required to maintain melter temperatures. An interesting illustration is provided in Test 9 as a result of the test being conducted at two temperatures resulting in two production rates using the same feed. Notice in Figure 3.28 and Table 1.4 that despite the increase in glass temperature and therefore power demand, the increased glass production rate more than compensates for the additional power usage. In tests without bubbling, power demand per unit glass production increased with increased feed water content as expected. 


\section{SECTION 4.0 OFF-GAS SYSTEM PERFORMANCE}

The off-gas treatment system, shown schematically in Figure 1.3, consists of a submerged bed scrubber (SBS), a wet electrostatic precipitator (WESP), a high efficiency mist eliminator (HEME), a high efficiency particulate arrestor (HEPA), a packed bed caustic scrubber (PBS), a second HEME, a thermal catalytic oxidation unit (TCO), and a second HEPA as the primary treatment units. The data on the off-gas system performance collected during the nine tests are presented and discussed in this section. Further details on post-test inspection results and characterization data for solids accumulations in various part of the system that were sampled are provided in Appendix A. Further details on SBS fluids (viscosity and particle size distribution) are provided in Appendix B.

\subsection{Off-Gas System Test Results}

Data for each of the off-gas system components, logged by LabVIEW, were imported into MS Excel files for data manipulation and plotting. Time " 0 " on the x-axis of each data plot corresponds to the start of water feed into the melter at the beginning of each test run. Where indicated, some data were smoothed by time averaging instantaneous measurements logged at two-minute intervals over ten-minute periods to reduce data scatter and the number of data points for the plots. The average, minimum, and maximum values of the measured off-gas system parameters are given in Table 4.1 for all nine tests.

The measured gas flow rates from the melter are presented in Section 6 (see Table 6.1), which includes the non-condensable flow rate and the water content. Of the non-condensable flow (variable, but typically about $160 \mathrm{dscfm}$ ), $70 \mathrm{scfm}$ is from the film cooler air and the majority of the remainder is from melter in-leakage (the bubblers and non-condensable feed decomposition gases are much smaller contributions). The film cooler air flow rate was held constant at $70 \mathrm{scfm}$ and the air temperature (about $75^{\circ} \mathrm{F}$ ) and supply pressure (about $2.2 \mathrm{psi}$ ) also varied little.

\subsubsection{Melter Pressure}

The computer logged melter pressure values for Test 1 at two-minute intervals are shown in Figure 4.1. As can be seen in the figure, the data show considerable scatter, most likely depending on when during the feed cycle the pressure was recorded. These data, smoothed by 
averaging over five consecutive measurements, are shown in Figure 4.2. The ten-minute time averaged melter pressure data for Tests 1 through 9 are shown in Figures 4.2 through 4.10. During most of the tests the melter pressure was in the range of -2 in. W.C. to -8 in W.C. The instantaneous melter pressure occasionally went positive. This occurred occasionally during initial feed introduction into the melter and during installation and removal of off-gas sampling equipment. Accumulation of feed on the cold-cap surface and subsequent break-up of the coldcap resulting in feed suddenly coming into contact with the hot glass surface can also cause melter pressure to increase.

During Test 1 , the melter pressure was controlled to within the negative pressure range for the duration of the test except for instantaneous positive pressure values during set-up and removal of the off-gas sampling equipment. Since the SBS immediately follows the melter in the off-gas train and because of the large pressure drop across the SBS, blow-down of the SBS (i.e., periodic removal of some of the accumulated fluids) also influences the melter pressure. Typically, after a blow-down the melter pressure goes from a slight negative value to a more negative value until the blower is adjusted to compensate for the effect. After the HLW tests reported here, a control-air system was installed in the DM1200 to better control the melter pressure and to minimize pressure variations.

During Test 2, the melter pressure was controlled within the negative pressure range for the duration of the test. Occasionally, instantaneous melter pressure values went positive during set-up and removal of the off-gas sampling equipment, during inspection of the film cooler, and possibly due to feed build up on the West side of the melter and subsequent sudden contact of feed with the hot glass surface.

The melter pressure as a function of time during Test 3 is shown in Figure 4.4. The instantaneous melter pressure occasionally went positive during set-up and removal of off-gas sampling equipment, during replacement of glass discharge can, and due to suspended portions of the cold-cap suddenly falling into the glass melt. The back-up off-gas system vent was activated several times during these pressure surges. During the tests, the SBS was blown-down to increase the melter vacuum.

The melter pressure during Test 4 is shown in Figure 4.5. Instantaneous positive pressure values were attributed to set-up and removal of off-gas sampling equipment.

The melter pressure during Test 5 is shown in Figure 4.6. Feeding was stopped from 24.0 hours to 24.7 hours from the start of the test due to a leak in the feed pump. The melter pressure was controlled to within acceptable limits during this test. 
Melter pressure data for Test 6 are shown in Figure 4.7. The melter pressure was controlled to within the negative pressure range except for occasional instantaneous positive pressure changes. The positive pressure changes occurred during camera maintenance, cleaning of view ports, dip sampling of the melt pool, and due to cold-cap breakage.

During Test 7 , feeding was interrupted briefly (from 38.5 to 42.7 hours) due to interruption of cooling water supply to the SBS. The off-gas was diverted to the back-up system during this time. This can be seen in Figure 4.8. Other than this interruption, the melter pressure was stable except for positive readings during set-up and removal of off-gas sampling equipment.

The melter pressure during Test 8 is shown in Figure 4.9. Instantaneous positive pressure values were attributed to set-up and removal of off-gas sampling equipment.

The melter pressure during Test 9 is given in Figure 4.10. The melter pressure was stable during the test. Instantaneous positive pressure values were attributed to set-up and removal of off-gas sampling equipment.

\subsubsection{SBS Performance}

Data on the performance of the SBS during the nine tests are presented in this section. Inlet and outlet gas temperatures, pressures and flow rates, pressure drop across the SBS, sump water temperature, heat exchanger inlet and outlet water temperatures, and flow rates were recorded during the tests. The amounts of heat removed by the SBS jacket cooling water and the cooling coil, which is connected to the plate heat exchanger, were calculated from these data. Post-test inspections of the SBS were also conducted, the results of which are summarized in this section with further details provided in Appendix A.

The performance of the SBS fluid handling system was uniformly good in that liquids and suspended solids were easily and reliably removed during routine blow-downs and, as discussed below, the amount of solids remaining in the SBS at the end of the tests was always very small. This suggests that the natural flows in the SBS sump in combination with the sparger lances serve to maintain good suspension of the collected solids.

After four tests, the SBS down-comer was redesigned and a new down-comer was installed at the request of the Project. The modification was done in response to solids deposition on the inside of the down-comer at the point at which it passes through the diffuser plate. A potential reason for solids accumulation at this location was assumed to be the slightly raised metal lugs used to position the down-comer when it was appended to the original SBS design. 
The Catholic University of America

Vitreous State Laboratory
DuraMelter 1200 Tests with AZ-101HLW Simulants

Final Report, VSL-02R0100-2, Rev.1

However, the new design, which provided a smooth, flush inside surface for the down-comer did not eliminate the solids deposition at that point. Since the pipe above that point is insulated, it is possible that the deposition is a result of condensation that begins at that point.

Data on the performance of the SBS with respect to solids removal are presented and discussed in Section 6.0. Results of the analysis of SBS sump fluids are presented and discussed in Section 4.2. The remainder of this section provides a discussion of the SBS operating parameters for each of the tests. At the end of each test, the SBS was blown down completely, the lower bowl removed, and the SBS bowl and down-comer inspected for deposits and photographed. The SBS sump was then refilled with city water before the start of each test. Specific observations and representative photographs are presented in the test-by-test discussion below.

\section{Test 1 SBS Data}

The SBS inlet and outlet gas temperatures for Test 1 are plotted in Figure 4.11. The inlet gas temperature peaked at $498^{\circ} \mathrm{C}$ and the outlet gas temperature peaked at $52.6^{\circ} \mathrm{C}$. The average inlet gas temperature was $228^{\circ} \mathrm{C}$. The average outlet gas temperature was $23.6^{\circ} \mathrm{C}$. The inlet, outlet, and differential pressures are shown in Figure 4.12. The inlet pressure averaged -8.2 in. W.C. and the outlet pressure averaged $-48.0 \mathrm{in}$. W.C. The apparent change in the measured value of the differential pressure after 100 hours into the test, seen in Figure 4.12, is an artifact that is due to clogging of the differential pressure sensor. This occurred several times during the present series of tests. In subsequent tests, this problem was alleviated by increasing the purge gas flow rate to the sensor.

Water temperatures in the SBS, the SBS chilled cooling water supply temperature, the water cooling jacket outlet temperature, and the water outlet temperature from the plate heat exchanger recorded during Test 1 are shown in Figure 4.13. These data are time-averaged over 10 minutes ( 5 measurements). There was very little difference in water temperatures measured at four depths $(48,60,72$ and 78 inches) within the SBS. During Test 1 , the liquid in the SBS was heated to a maximum temperature of $\approx 56^{\circ} \mathrm{C}$ during the initial period of water feeding and the average SBS water temperature was $\approx 24^{\circ} \mathrm{C}$. The average slurry feed rate to the melter was about $35.7 \mathrm{~kg} / \mathrm{hr}$. The nominal glass yield of this feed was $570 \mathrm{~g} /$ liter of feed $(0.38 \mathrm{~kg}$ glass $/ \mathrm{kg}$ feed $)$.

The amount of heat removed by the SBS jacket cooling water and the plate heat exchanger are shown in Figure 4.14. The heat-load data are calculated based on 10 minute timeaveraged cooling water temperature increases (outlet temperature minus supply temperature) across the cooling jacket and plate heat exchanger multiplied by the flow rate through each. The average flow through the jacket was $41.8 \mathrm{gpm}$ and the average cooling water flow through the 
plate heat exchanger was $50.6 \mathrm{gpm}$. For this test, heat removal averaged $52.7 \mathrm{~kW}$ by the plate heat exchanger and $14.7 \mathrm{~kW}$ by the cooling jacket. Consequently, during Test 1 , about $78 \%$ of the heat load to the SBS was removed by the plate heat exchanger and about $22 \%$ by the jacket.

When the volume of liquid in the SBS overflow tank exceeded 65 gallons, it was difficult to maintain a steady vacuum in the melter. Therefore, the liquid volume in the overflow tank was maintained below 60 gallons. About 141 hours into the test, the diaphragm pump used for blowdown in the SBS failed and was replaced. After about 100 hours of operation the differential pressure sensor for the SBS clogged and had to be cleaned.

\section{Test 2 SBS Data}

The SBS inlet and outlet gas temperatures for Test 2 are plotted in Figure 4.15. The inlet gas temperature peaked at $462^{\circ} \mathrm{C}$ and the outlet gas temperature peaked at $47.3^{\circ} \mathrm{C}$. The average inlet gas temperature was $228^{\circ} \mathrm{C}$. The average outlet gas temperature was $22.1^{\circ} \mathrm{C}$. The inlet, outlet, and differential pressures are shown in Figure 4.16. The inlet pressure averaged -7.9 in. W.C. and the outlet pressure averaged -48.4 in. W.C.

Water temperatures in the SBS, the SBS chilled cooling water supply temperature, the water cooling jacket outlet temperature, and the water outlet temperature from the plate heat exchanger recorded during Test 2 are shown in Figure 4.17. These data are time averaged over 10 minutes ( 5 measurements). There was very little difference in water temperatures measured at four depths $(48,60,72$ and 78 inches) within the SBS. During Test 2, the liquid in the SBS was heated to a maximum temperature of $\approx 51^{\circ} \mathrm{C}$ during the initial period of water feeding and the average SBS water temperature was $\approx 22^{\circ} \mathrm{C}$. The average slurry feed rate to the melter was about $31.8 \mathrm{~kg} / \mathrm{hr}$. The nominal glass yield of this feed was $570 \mathrm{~g} /$ liter of feed $(0.38 \mathrm{~kg}$ glass $/ \mathrm{kg}$ feed $)$.

The amount of heat removed by the SBS jacket cooling water and the plate heat exchanger are shown in Figure 4.18. The heat load data are calculated based on 10 minute timeaveraged cooling water temperature increases (outlet temperature minus supply temperature) across cooling jacket and plate heat exchanger multiplied by the flow rate through each. The average flow through the jacket was $45.5 \mathrm{gpm}$ and the average cooling water flow through the plate heat exchanger was $48.0 \mathrm{gpm}$. For this test, heat removal averaged $42.4 \mathrm{~kW}$ by the plate heat exchanger and $12.2 \mathrm{~kW}$ by the cooling jacket. Consequently, during Test \#2, about $78 \%$ of the heat load to the SBS was removed by the plate heat exchanger and about $22 \%$ by the jacket. 


\section{Test 3 SBS Data}

The SBS inlet and outlet gas temperatures for Test 3 are plotted in Figure 4.19. Inlet gas temperature peaked at $528^{\circ} \mathrm{C}$ and the outlet gas temperature peaked at $64.2^{\circ} \mathrm{C}$. The average inlet gas temperature was $312^{\circ} \mathrm{C}$. The average outlet gas temperature was $40.7^{\circ} \mathrm{C}$. The inlet, outlet, and differential pressures are shown in Figure 4.16. The inlet pressure averaged -7.6 in. W.C. and the outlet pressure averaged -58.2 in. W.C. The discontinuity in the differential pressure reading between 10 and 22 hours into the test is a result of clogging of the differential pressure sensor.

Water temperatures in the SBS, the SBS chilled cooling water supply temperature, the water cooling jacket outlet temperature, and the water outlet temperature from the plate heat exchanger recorded during Test 3 are shown in Figure 4.21. These data are time averaged over 10 minutes ( 5 measurements). There was very little difference in water temperatures measured at four depths (48, 60, 72 and 78 inches) within the SBS. During Test 3 the liquid in the SBS was heated to a maximum temperature of $\approx 55^{\circ} \mathrm{C}$ during the initial period of water feeding and the average SBS water temperature was $\approx 41^{\circ} \mathrm{C}$. The average slurry feed rate to the melter was about $168.3 \mathrm{~kg} / \mathrm{hr}$. The nominal glass yield of this feed was $400 \mathrm{~g} / \mathrm{liter}$ of feed $(0.29 \mathrm{~kg}$ glass $/ \mathrm{kg}$ feed $)$.

As expected, the high feed rate resulted in correspondingly high heat load on the SBS. The amount of heat removed by the SBS jacket cooling water and the plate heat exchanger are shown in Figure 4.22. The heat load data are calculated based on 10 minute time-averaged cooling water temperature increases (outlet temperature minus supply temperature) across cooling jacket and plate heat exchanger multiplied by the flow rate through each. The average flow through the jacket was $42.6 \mathrm{gpm}$ and the average cooling water flow through the plate heat exchanger was $48.7 \mathrm{gpm}$. For this test, heat removal averaged $153.6 \mathrm{~kW}$ by the plate heat exchanger and $47.7 \mathrm{~kW}$ by the cooling jacket. Consequently, during Test 3 about $76 \%$ of the heat load to the SBS was removed by the plate heat exchanger and about $24 \%$ by the jacket.

\section{Test 4 SBS Data}

The SBS inlet and outlet gas temperatures for Test 4 are plotted in Figure 4.23. The inlet gas temperature peaked at $487^{\circ} \mathrm{C}$ and the outlet gas temperature peaked at $57.3^{\circ} \mathrm{C}$. The average inlet gas temperature was $266^{\circ} \mathrm{C}$. The average outlet gas temperature was $34.3^{\circ} \mathrm{C}$. The inlet, outlet, and differential pressures are shown in Figure 4.24. The inlet pressure averaged -7.4 in. W.C. and the outlet pressure averaged -52.5 in. W.C.

Water temperatures in the SBS, the SBS chilled cooling water supply temperature, the water cooling jacket outlet temperature, and the water outlet temperature from the plate heat 
The Catholic University of America Vitreous State Laboratory
DuraMelter 1200 Tests with AZ-101HLW Simulants

Final Report, VSL-02R0100-2, Rev.I

exchanger recorded during Test 4 are shown in Figure 4.25. These data are time-averaged over 10 minutes ( 5 measurements). There was very little difference in water temperatures measured at four depths $(48,60,72$ and 78 inches) within the SBS. During Test 4 , the liquid in the SBS was heated to a maximum temperature of $\approx 62.7^{\circ} \mathrm{C}$ during the initial period of water feeding and the average SBS water temperature was $\approx 35^{\circ} \mathrm{C}$. The average slurry feed rate to the melter was about $96.7 \mathrm{~kg} / \mathrm{hr}$. The nominal glass yield of this feed was $400 \mathrm{~g} /$ liter of feed $(0.29 \mathrm{~kg}$ glass $/ \mathrm{kg}$ feed $)$.

The amount of heat removed by the SBS jacket cooling water and the plate heat exchanger are shown in Figure 4.26. The heat load data are calculated based on 10 minute timeaveraged cooling water temperature increases (outlet temperature minus supply temperature) across cooling jacket and plate heat exchanger multiplied by the flow rate through each. The average flow through the jacket was $41.8 \mathrm{gpm}$ and the average cooling water flow through the plate heat exchanger was $51.2 \mathrm{gpm}$. For this test, heat removal averaged $104.6 \mathrm{~kW}$ by the plate heat exchanger and $29.7 \mathrm{~kW}$ by the cooling jacket. Consequently, during Test 4 about $78 \%$ of the heat load to the SBS was removed by the plate heat exchanger and about $22 \%$ by the jacket.

Photographs of the solids deposits in the SBS bowl are shown in Figure 4.27. Photographs of the solids deposits inside the down-comer are shown in Figure 4.28. In order to alleviate the solids deposition and to provide better distribution of the gas bubbles, the downcomer was modified and re-installed in the SBS. The new down-comer, before and after installation, is shown in Figure 4.29. Additional views of the installed down-comer are given in Figure 4.30. The flat-bottom down-comer was used for Test 1-4, while the serrated-bottom down-comer was used for Test 5-9.

\section{Test 5 SBS Data}

The SBS inlet and outlet gas temperatures for Test 5 are plotted in Figure 4.31. The inlet gas temperature peaked at $408^{\circ} \mathrm{C}$ and the outlet gas temperature peaked at $42.9^{\circ} \mathrm{C}$. The average inlet gas temperature was $219^{\circ} \mathrm{C}$. The average outlet gas temperature was $25.0^{\circ} \mathrm{C}$. The inlet, outlet, and differential pressures are shown in Figure 4.32. The inlet pressure averaged -8.8 in. W.C. and the outlet pressure averaged -48.3 in. W.C.

Water temperatures in the SBS, the SBS chilled cooling water supply temperature, the water cooling jacket outlet temperature, and the water outlet temperature from the plate heat exchanger recorded during Test 5 are shown in Figure 4.33. These data are time-averaged over 10 minutes ( 5 measurements). There was very little difference in water temperatures measured at four depths (48, 60, 72 and 78 inches) within the SBS. During Test 5 , the liquid in the SBS was heated to a maximum temperature of $\approx 48.5^{\circ} \mathrm{C}$ during the initial period of water feeding and the average SBS water temperature was $\approx 25^{\circ} \mathrm{C}$. The average slurry feed rate to the melter was about 
$34.6 \mathrm{~kg} / \mathrm{hr}$. The nominal glass yield of this feed was $420 \mathrm{~g} / \mathrm{liter}$ of feed $(0.31 \mathrm{~kg}$ glass $/ \mathrm{kg}$ feed $)$.

The amount of heat removed by the SBS jacket cooling water and the plate heat exchanger are shown in Figure 4.34. The heat load data are calculated based on 10 minute timeaveraged cooling water temperature increases (outlet temperature minus supply temperature) across cooling jacket and plate heat exchanger multiplied by the flow rate through each. The average flow through the jacket was $38.7 \mathrm{gpm}$ and the average cooling water flow through the plate heat exchanger was $47.7 \mathrm{gpm}$. For this test, heat removal averaged $50.7 \mathrm{~kW}$ by the plate heat exchanger and $11.3 \mathrm{~kW}$ by the cooling jacket. Consequently, during Test 5 about $82 \%$ of the heat load to the SBS was removed by the plate heat exchanger and about $18 \%$ by the jacket.

Photographs of the solids deposits in the SBS bowl are shown in Figure 4.35. Photographs of the solids deposits inside the down-comer and a general view of the weir tubes are shown in Figure 4.36. A photograph of solids from the down-comer is shown in Figure 4.37.

\section{Test 6 SBS Data}

The SBS inlet and outlet gas temperatures for Test 6 are plotted in Figure 4.38. The inlet gas temperature peaked at $395^{\circ} \mathrm{C}$ and the outlet gas temperature peaked at $46.0^{\circ} \mathrm{C}$. The average inlet gas temperature was $182^{\circ} \mathrm{C}$. The average outlet gas temperature was $23.2^{\circ} \mathrm{C}$. The inlet, outlet, and differential pressures are shown in Figure 4.39. The inlet pressure averaged -8.4 in. W.C. and the outlet pressure averaged -49.3 in. W.C.

Water temperatures in the SBS, the SBS chilled cooling water supply temperature, the water cooling jacket outlet temperature, and the water outlet temperature from the plate heat exchanger recorded during Test 6 are shown in Figure 4.40. These data are time-averaged over 10 minutes ( 5 measurements). There was very little difference in water temperatures measured at four depths (48, 60, 72 and 78 inches) within the SBS. During Test 6 , the liquid in the SBS was heated to a maximum temperature of $\approx 52.2^{\circ} \mathrm{C}$ during the initial period of water feeding and the average SBS water temperature was $\approx 23.5^{\circ} \mathrm{C}$. The average slurry feed rate to the melter was about $35.5 \mathrm{~kg} / \mathrm{hr}$. The nominal glass yield of this feed was $480 \mathrm{~g} /$ liter of feed $(0.35 \mathrm{~kg}$ glass $/ \mathrm{kg}$ feed).

The amount of heat removed by the SBS jacket cooling water and the plate heat exchanger are shown in Figure 4.41. The heat load data are calculated based on 10 minute timeaveraged cooling water temperature increases (outlet temperature minus supply temperature) across cooling jacket and plate heat exchanger multiplied by the flow rate through each. The average flow through the jacket was $39.4 \mathrm{gpm}$ and the average cooling water flow through the plate heat exchanger was $49.7 \mathrm{gpm}$. For this test, heat removal averaged $52.6 \mathrm{~kW}$ by the plate 
The Catholic University of America Vitreous State Laboratory
DuraMelter 1200 Tests with AZ-101HLW Simulants

Final Report, VSL-02R0100-2, Rev.1

heat exchanger and $13.8 \mathrm{~kW}$ by the cooling jacket. Consequently, during Test 6 about $79 \%$ of the heat load to the SBS was removed by the plate heat exchanger and about $21 \%$ by the jacket.

Photographs of the solids deposits in the SBS bowl and a view of the down-comer are shown in Figure 4.42.

\section{Test 7 SBS Data}

The SBS inlet and outlet gas temperatures for Test 7 are plotted in Figure 4.43. The inlet gas temperature peaked at $424^{\circ} \mathrm{C}$ and the outlet gas temperature peaked at $49.4^{\circ} \mathrm{C}$. The average inlet gas temperature was $278^{\circ} \mathrm{C}$. The average outlet gas temperature was $37.6^{\circ} \mathrm{C}$. The inlet, outlet, and differential pressures are shown in Figure 4.44. The inlet pressure averaged -8.0 in. W.C. and the outlet pressure averaged -50.6 in. W.C. About 38 hours into the test, the cooling water to the SBS was interrupted and feed to the melter was stopped for about 5 hours. During this time the off-gas was diverted to the backup system. Feeding was restarted after the cooling water supply was restored.

Water temperatures in the SBS, the SBS chilled cooling water supply temperature, the water cooling jacket outlet temperature, and the water outlet temperature from the plate heat exchanger recorded during Test 7 are shown in Figure 4.45. These data are time-averaged over 10 minutes ( 5 measurements). There was very little difference in water temperatures measured at four depths $(48,60,72$ and 78 inches) within the SBS. During Test 7 , the liquid in the SBS was heated to a maximum temperature of $\approx 51.0^{\circ} \mathrm{C}$ during the initial period of water feeding and the average SBS water temperature was $\approx 39.5^{\circ} \mathrm{C}$. The average slurry feed rate to the melter was about $137.0 \mathrm{~kg} / \mathrm{hr}$. The nominal glass yield of this feed was $480 \mathrm{~g} /$ liter of feed $(0.35 \mathrm{~kg}$ glass $/ \mathrm{kg}$ feed).

The amount of heat removed by the SBS jacket cooling water and the plate heat exchanger are shown in Figure 4.46. The heat load data are calculated based on 10 minute timeaveraged cooling water temperature increases (outlet temperature minus supply temperature) across cooling jacket and plate heat exchanger multiplied by the flow rate through each. The average flow through the jacket was $40.0 \mathrm{gpm}$ and the average cooling water flow through the plate heat exchanger was $52.1 \mathrm{gpm}$. For this test, heat removal averaged $130.9 \mathrm{~kW}$ by the plate heat exchanger and $37.1 \mathrm{~kW}$ by the cooling jacket. Consequently, during Test 7 about $78 \%$ of the heat load to the SBS was removed by the plate heat exchanger and about $22 \%$ by the jacket.

Photographs of the solids deposits in the SBS bowl and a view of the down-comer are given in Figure 4.47. A close-up view of the SBS down-comer with the deposits is shown in Figure 4.48. 
The Catholic University of America Vitreous State Laboratory
DuraMelter 1200 Tests with AZ-101HLW Simulants

Final Report, VSL-02R0100-2, Rev.I

\section{Test 8 SBS Data}

The SBS inlet and outlet gas temperatures for Test 8 are plotted in Figure 4.49. The inlet gas temperature peaked at $368^{\circ} \mathrm{C}$ and the outlet gas temperature peaked at $41.1^{\circ} \mathrm{C}$. The average inlet gas temperature was $183^{\circ} \mathrm{C}$. The average outlet gas temperature was $23.3^{\circ} \mathrm{C}$. The inlet, outlet, and differential pressures are shown in Figure 4.50. The inlet pressure averaged -6.7 in. W.C. and the outlet pressure averaged -43.5 in. W.C.

Water temperatures in the SBS, the SBS chilled cooling water supply temperature, the water cooling jacket outlet temperature, and the water outlet temperature from the plate heat exchanger recorded during Test 8 are shown in Figure 4.51. These data are time-averaged over 10 minutes ( 5 measurements). Between 23 and 28 hours into the test the data indicate that the cooling water supply to the plate heat exchanger was interrupted. There was very little difference in water temperatures measured at four depths $(48,60,72$ and 78 inches) within the SBS. During Test 8 , the liquid in the SBS was heated to a maximum temperature of $\approx 46.7^{\circ} \mathrm{C}$ during the initial period of water feeding and the average SBS water temperature was $\approx 23.7^{\circ} \mathrm{C}$. The average slurry feed rate to the melter was about $34.6 \mathrm{~kg} / \mathrm{hr}$. The nominal glass yield of this feed was $420 \mathrm{~g} / \mathrm{liter}$ of feed $(0.31 \mathrm{~kg}$ glass $/ \mathrm{kg}$ feed).

The amount of heat removed by the SBS jacket cooling water and the plate heat exchanger are shown in Figure 4.52. The heat load data are calculated based on 10 minute timeaveraged cooling water temperature increases (outlet temperature minus supply temperature) across cooling jacket and plate heat exchanger multiplied by the flow rate through each. The average flow through the jacket was $40.8 \mathrm{gpm}$ and the average cooling water flow through the plate heat exchanger was $50.7 \mathrm{gpm}$. For this test, heat removal averaged $41.5 \mathrm{~kW}$ by the plate heat exchanger and $11.7 \mathrm{~kW}$ by the cooling jacket. Consequently, during Test 8 about $78 \%$ of the heat load to the SBS was removed by the plate heat exchanger and about $22 \%$ by the jacket.

\section{Test 9a and 9b SBS Data}

Test 9 was about 120 hours in duration. The first 66.7 hours of this test is referred to as Test $9 \mathrm{a}$ and the latter part as Test $9 \mathrm{~b}$. During Test $9 \mathrm{a}$ and $9 \mathrm{~b}$, a peristaltic pump was used to introduce feed into the melter through both feed tubes. During Test \# $9 \mathrm{~b}$ the nominal glass-melt temperature was increased to $1200^{\circ} \mathrm{C}$.

The SBS inlet and outlet gas temperatures for Test 9 are plotted in Figure 4.53. During Test $9 \mathrm{a}$, the inlet gas temperature peaked at $348^{\circ} \mathrm{C}$ and the outlet gas temperature peaked at $33.5^{\circ} \mathrm{C}$. The average inlet gas temperature was $198^{\circ} \mathrm{C}$. The average outlet gas temperature was $21.1^{\circ} \mathrm{C}$. The inlet, outlet, and differential pressures are shown in Figure 4.54. The inlet pressure 
The Catholic University of America Vitreous State Laboratory
DuraMelter 1200 Tests with AZ-101HLW Simulants

Final Report, VSL-02R0100-2, Rev.1

averaged -6.3 in. W.C. and the outlet pressure averaged -45.2 in. W.C. during Test 9 a.

During Test $9 \mathrm{~b}$, the inlet gas temperature peaked at $201^{\circ} \mathrm{C}$ and the outlet gas temperature peaked at $22.7^{\circ} \mathrm{C}$. The average inlet gas temperature was $178^{\circ} \mathrm{C}$. The average outlet gas temperature was $21.4^{\circ} \mathrm{C}$. The inlet, outlet, and differential pressures are shown in Figure 4.54. The inlet pressure averaged -5.9 in. W.C. and the outlet pressure averaged -45.2 in. W.C. during Test $9 \mathrm{~b}$. The differential pressure sensor was clogged for the first 17.5 hours of the test and again from hours 106 to 109 .

Water temperatures in the SBS, the SBS chilled cooling water supply temperature, the water cooling jacket outlet temperature, and the water outlet temperature from the plate heat exchanger recorded during Test 9 are shown in Figure 4.55. These data are time-averaged over 10 minutes ( 5 measurements). There was very little difference in water temperatures measured at four depths (48, 60, 72 and 78 inches) within the SBS. During Test 9a, the liquid in the SBS was heated to a maximum temperature of $\approx 39.1^{\circ} \mathrm{C}$ during the initial period of water feeding and the average SBS water temperature was $\approx 21.6^{\circ} \mathrm{C}$. The average slurry feed rate to the melter was about $30.0 \mathrm{~kg} / \mathrm{hr}$. The nominal glass yield of this feed was $570 \mathrm{~g} /$ liter of feed $(0.38 \mathrm{~kg}$ glass $/ \mathrm{kg}$ feed). During Test $9 \mathrm{~b}$, the liquid in the SBS was heated to a maximum temperature of $\approx 25.8^{\circ} \mathrm{C}$ and the average SBS water temperature was $\approx 21.9^{\circ} \mathrm{C}$. The average slurry feed rate to the melter was about $39.4 \mathrm{~kg} / \mathrm{hr}$. The nominal glass yield of this feed was $570 \mathrm{~g} /$ liter of feed $(0.38 \mathrm{~kg}$ glass $/ \mathrm{kg}$ feed).

The amount of heat removed by the SBS jacket cooling water and the plate heat exchanger are shown in Figure 4.56. The heat load data are calculated based on 10 minute timeaveraged cooling water temperature increases (outlet temperature minus supply temperature) across cooling jacket and plate heat exchanger multiplied by the flow rate through each. For Test $9 \mathrm{a}$, the average flow through the jacket was $41.4 \mathrm{gpm}$ and the average cooling water flow through the plate heat exchanger was $49.1 \mathrm{gpm}$. For Test $9 \mathrm{a}$, heat removal averaged $38.8 \mathrm{~kW}$ by the plate heat exchanger and $10.9 \mathrm{~kW}$ by the cooling jacket. Consequently, during Test $9 \mathrm{a}$ about $78 \%$ of the heat load to the SBS was removed by the plate heat exchanger and about $22 \%$ by the jacket. For Test $9 \mathrm{~b}$, the average flow through the jacket was $41.5 \mathrm{gpm}$ and the average cooling water flow through the plate heat exchanger was $49.0 \mathrm{gpm}$. For Test $9 \mathrm{~b}$, heat removal averaged $39.9 \mathrm{~kW}$ by the plate heat exchanger and $11.1 \mathrm{~kW}$ by the cooling jacket. Consequently, during Test $9 \mathrm{~b}$ about $78 \%$ of the heat load to the SBS was removed by the plate heat exchanger and about $22 \%$ by the jacket.

Post-test inspection of the SBS showed deposits in the bottom bowl and the down-comer. A photograph of the SBS bottom bowl is shown in Figure 4.57. A photograph of the down-comer with the deposits is shown in Figure 4.58. 


\subsubsection{WESP Performance}

The inlet and outlet gas temperatures and the differential pressure across the WESP were collected and recorded by the computerized data acquisition system. WESP current and voltage were recorded manually.

Data on the performance of the WESP with respect to solids removal are presented and discussed in Section 6.0. Results of the analysis of solids deposits in the WESP are presented and discussed in Section 4.2 and Appendix A.

\section{Test 1}

The WESP inlet and outlet gas temperatures and the differential pressure across the WESP during Test 1 are plotted in Figure 4.59. During this test, at a steady-state feed rate of $35.7 \mathrm{~kg} / \mathrm{hr}$, the WESP inlet gas temperature averaged $29.4^{\circ} \mathrm{C}$ and the outlet gas temperature averaged $36.0^{\circ} \mathrm{C}$, indicating a $6.6^{\circ} \mathrm{C}$ temperature increase across the WESP. The average WESP inlet temperature exceeded the average SBS outlet gas temperature by $5.8^{\circ} \mathrm{C}$ during this time; the increase is probably due to the higher ambient temperature in the vicinity of that elevated pipe run. The pressure differentials measured at 2-minute intervals across the WESP during this period averaged 1.5 in. W.C. with a standard deviation of 0.2 in. W.C. During Test 1 , the WESP inlet water spray was off. There were no WESP blow downs during the test. At the end of the test, the amount of liquid removed from the WESP sump was $217 \mathrm{~kg}$ which is essentially the same amount that was present at the beginning of the test; therefore, there was no water loss or gain during Test 1 .

\section{Test 2}

The WESP inlet and outlet gas temperatures and the differential pressure across the WESP during Test 2 are plotted in Figure 4.60. During this test, at a steady-state feed rate of $31.8 \mathrm{~kg} / \mathrm{hr}$, the WESP inlet gas temperature averaged $26.9^{\circ} \mathrm{C}$ and the outlet gas temperature averaged $31.2^{\circ} \mathrm{C}$, indicating a $4.3^{\circ} \mathrm{C}$ temperature increase across the WESP. The average WESP inlet temperature exceeded the average SBS outlet gas temperature by $4.8^{\circ} \mathrm{C}$ during this time. The pressure differentials measured at 2-minute intervals across the WESP during this period averaged 1.7 in. W.C. with a standard deviation of 0.3 in. W.C. During Test 2, the WESP inlet water spray was on at a rate of about 7-8 $\mathrm{lpm}$. During the test, water was added at a rate of about $1.1 \mathrm{lph}$ to compensate for water loss from the WESP. At the end of the test, $124 \mathrm{~kg}$ of liquid was removed from the WESP. 


\section{Test 3}

The WESP inlet and outlet gas temperatures and the differential pressure across the WESP during Test 3 are plotted in Figure 4.61. During this test, at a steady-state feed rate of $168.3 \mathrm{~kg} / \mathrm{hr}$, the WESP inlet gas temperature averaged $41.6^{\circ} \mathrm{C}$ and the outlet gas temperature averaged $45.0^{\circ} \mathrm{C}$, indicating a $3.4^{\circ} \mathrm{C}$ temperature increase across the WESP. The average WESP inlet temperature exceeded the average SBS outlet gas temperature by only $0.9^{\circ} \mathrm{C}$ during this time. The pressure differentials measured at 2-minute intervals across the WESP during this period averaged 2.1 in. W.C. with a standard deviation of 0.3 in. W.C. During Test 3 , the WESP inlet water spray was on at a rate of about $8 \mathrm{lpm}$. During the test, water was added at a rate of about $0.7 \mathrm{lph}$ to compensate for water loss from the WESP. At the end of the test, $121 \mathrm{~kg}$ of liquid was removed from the WESP.

\section{Test 4}

The WESP inlet and outlet gas temperatures and the differential pressure across the WESP during Test 4 are shown in Figure 4.62. During this test, at a steady-state feed rate of $96.7 \mathrm{~kg} / \mathrm{hr}$, the WESP inlet gas temperature averaged $36.2^{\circ} \mathrm{C}$ and the outlet gas temperature averaged $39.9^{\circ} \mathrm{C}$, indicating a $3.7^{\circ} \mathrm{C}$ temperature increase across the WESP. The average WESP inlet temperature exceeded the average SBS outlet gas temperature by $1.9^{\circ} \mathrm{C}$ during this time. The pressure differentials measured at 2-minute intervals across the WESP during this period averaged 1.9 in. W.C. with a standard deviation of 0.4 in. W.C. During Test 4 , the WESP inlet water spray was on at a rate of about $7-8 \mathrm{lpm}$. During the test, water was added at a rate of about $0.8 \mathrm{lph}$ to compensate for water loss from the WESP. At the end of the test, $126 \mathrm{~kg}$ of liquid was removed from the WESP.

At the end of the test, the WESP was inspected for deposits. Photographs of the rods and collector plates before and after wash-down are shown in Figures 4.63 and 4.64, respectively.

\section{Test 5}

The WESP inlet and outlet gas temperatures and the differential pressure across the WESP during Test 5 are shown in Figure 4.65. During this test, at a steady-state feed rate of $34.6 \mathrm{~kg} / \mathrm{hr}$, the WESP inlet gas temperature averaged $27.3^{\circ} \mathrm{C}$ and the outlet gas temperature averaged $31.1^{\circ} \mathrm{C}$, indicating a $3.8^{\circ} \mathrm{C}$ temperature increase across the WESP. The average WESP inlet temperature exceeded the average SBS outlet gas temperature by $2.3^{\circ} \mathrm{C}$ during this time. The pressure differentials measured at 2-minute intervals across the WESP during this period averaged 2.4 in. W.C. with a standard deviation of 0.4 in. W.C. During Test 5, the WESP inlet 
water spray was on at a rate of about $7-8 \mathrm{lpm}$. During the test, water was added at a rate of about $0.95 \mathrm{lph}$ to compensate for water loss from the WESP. At the end of the test, $126 \mathrm{~kg}$ of liquid was removed from the WESP.

\section{Test 6}

The WESP inlet and outlet gas temperatures and the differential pressure across the WESP during Test 6 are shown in Figure 4.66. During this test, at a steady-state feed rate of $35.5 \mathrm{~kg} / \mathrm{hr}$, the WESP inlet gas temperature averaged $25.3^{\circ} \mathrm{C}$ and the outlet gas temperature averaged $29.7^{\circ} \mathrm{C}$, indicating a $4.4^{\circ} \mathrm{C}$ temperature increase across the WESP. The average WESP inlet temperature exceeded the average SBS outlet gas temperature by $2.1^{\circ} \mathrm{C}$ during this time. The pressure differentials measured at 2-minute intervals across the WESP during this period averaged 2.5 in. W.C. with a standard deviation of 0.5 in. W.C. During Test 6 , the WESP inlet water spray was on at a rate of about $8 \mathrm{lpm}$. During the test, water was added at a rate of about $0.9 \mathrm{lph}$ to compensate for water loss from the WESP. At the end of the test, $121 \mathrm{~kg}$ of liquid was removed from the WESP.

- At the end of the test, the WESP was inspected for deposits. Photographs of the rods and collector plates before and after wash-down are shown in Figures 4.67 and 4.68, respectively.

\section{Test 7}

The WESP inlet and outlet gas temperatures and the differential pressure across the WESP during Test 7 are shown in Figure 4.69. During this test, at a steady-state feed rate of $137.0 \mathrm{~kg} / \mathrm{hr}$, the WESP inlet gas temperature averaged $37.5^{\circ} \mathrm{C}$ and the outlet gas temperature averaged $40.5^{\circ} \mathrm{C}$, indicating a $3.0^{\circ} \mathrm{C}$ temperature increase across the WESP. The average WESP inlet temperature was less than the average SBS outlet gas temperature by $0.1{ }^{\circ} \mathrm{C}$ during this time. The pressure differentials measured at 2-minute intervals across the WESP during this period averaged 2.3 in. W.C. with a standard deviation of 0.5 in. W.C. About 38 hours into the test, the cooling water to the SBS was interrupted and feed to the melter was stopped for about 5 hours. During this time, the off-gas was diverted to the backup system. Feeding was restarted after the cooling water supply was restored. During Test 7 , the WESP inlet water spray was off as a result of electrical instabilities in the WESP when the spray was on. During the test about 18.9 liters of water was removed at 87.4 hours and another 46.5 liters at 110.4 hours into the test. At the end of the test, $156 \mathrm{~kg}$ of liquid was removed from the WESP.

At the end of the test, the WESP was inspected for deposits. Photographs of the rods and collector plates before and after wash-down are shown in Figures 4.70 and 4.71, respectively. 
The Catholic University of America Vitreous State Laboratory
DuraMelter 1200 Tests with AZ-101HLW Simulants

Final Report, VSL-02R0100-2, Rev.1

\section{Test 8}

The WESP inlet and outlet gas temperatures and the differential pressure across the WESP during Test 8 are shown in Figure 4.72. During this test, at a steady-state feed rate of $34.6 \mathrm{~kg} / \mathrm{hr}$, the WESP inlet gas temperature averaged $25.3^{\circ} \mathrm{C}$ and the outlet gas temperature averaged $32.2^{\circ} \mathrm{C}$, indicating a $6.9^{\circ} \mathrm{C}$ temperature increase across the WESP. The average WESP inlet temperature exceeded the average SBS outlet gas temperature by $2.0^{\circ} \mathrm{C}$ during this time. The pressure differentials measured at 2-minute intervals across the WESP during this period averaged 1.8 in. W.C. with a standard deviation of 0.3 in. W.C. During Test 8 , the WESP inlet water spray was off. There were no WESP blow-downs during the test. At the end of the test, $151 \mathrm{~kg}$ of liquid was removed from the WESP. The liquid level in the WESP remained almost constant, between 12.1 " and 12.5", during the test.

At the end of the test the WESP was inspected for deposits. Photographs of the rods and collector plates before and after wash-down are given in Figures 4.73 and 4.74, respectively.

\section{Test 9}

Test 9 was about 120 hours in duration. The first 66.7 hours of this test are referred to as Test $9 \mathrm{a}$ and the latter part as Test $9 \mathrm{~b}$. During Test 9, a peristaltic pump was used to introduce feed into the melter through two feed tubes. During Test $9 b$, the nominal glass-melt temperature was increased to $1200^{\circ} \mathrm{C}$.

The WESP inlet and outlet gas temperatures and the differential pressure across the WESP during Test 9 are shown in Figure 4.75. During Test $9 \mathrm{a}$ at a steady-state feed rate of $30.0 \mathrm{~kg} / \mathrm{hr}$, the WESP inlet gas temperature averaged $24.6^{\circ} \mathrm{C}$ and the outlet gas temperature averaged $29.9^{\circ} \mathrm{C}$, indicating a $5.3^{\circ} \mathrm{C}$ temperature increase across the WESP. The average WESP inlet temperature exceeded the average SBS outlet gas temperature by $3.5^{\circ} \mathrm{C}$ during this time. The average differential pressure and the standard deviation were not calculated for this part of the test because the differential pressure sensor clogged 30 hours into the test.

During Test $9 \mathrm{~b}$, at a steady-state feed rate of $39.4 \mathrm{~kg} / \mathrm{hr}$, the WESP inlet gas temperature averaged $23.6^{\circ} \mathrm{C}$ and the outlet gas temperature averaged $29.7^{\circ} \mathrm{C}$, indicating a $6.1^{\circ} \mathrm{C}$ temperature increase across the WESP. The average WESP inlet temperature exceeded the average SBS outlet gas temperature by $2.2^{\circ} \mathrm{C}$ during this time. The pressure differentials measured at 2-minute intervals across the WESP during this period averaged $1.5 \mathrm{in}$. W.C. with a standard deviation of 0.2 in. W.C.

During Test 9, the WESP inlet water spray was off. There were no WESP blow-downs 
during the test. At the end of the test, $124 \mathrm{~kg}$ of liquid was removed from the WESP. The liquid level in the WESP changed very little, from 11.9" to 11.0 ", during the test.

At the end of the test, the WESP was inspected for deposits. Photographs of the rods and collector plates before and after wash-down are shown in Figures 4.76 and 4.77, respectively.

\subsubsection{WESP Electrical Data}

The average WESP current and voltage during the tests is shown graphically in Figure 4.78. The average WESP current and voltage before and after the wash-down are tabulated in Table 4.2. The data do not show any apparent trend. Additional data on WESP operating conditions are tabulated in Table 4.3.

\subsubsection{HEME and HEPA Filters}

The WESP is followed in the off-gas system by a Paxton blower (Blower \#1), a high efficiency mist eliminator (HEME \#1), a heater, and the first HEPA filter (HEPA \#1). The purpose of the HEME is to trap any entrained mist from the gas exiting the WESP. The purpose of the heater is to ensure that the water-saturated gas exiting the HEME is heated above its dew point before passing through the HEPA filter to prevent moisture condensation in the HEPA filter. The outlet temperature and the pressure differential across HEPA \#1 are the only two parameters that were monitored by the off-gas data acquisition system. The HEPA \#1 outlet temperature and pressure differential across HEPA \#1 are shown in Figures $4.79-4.87$ for the nine tests. The outlet temperatures ranged from 109 to $125^{\circ} \mathrm{C}$. The differential pressure across HEPA \#1 was in the range of 0.2 in. to 0.3 in. W.C. indicating that no significant particulate loading or moisture blinding of HEPA \#1 occurred during these tests.

\subsubsection{Packed Bed Scrubber (PBS)}

Following HEPA \#1 are a second blower and control damper, which are followed by a packed bed caustic scrubber. The PBS was not operated during any of these tests. Consequently, the only data collected was for gas inlet temperature. The inlet gas temperature was in the range of 74 to $88^{\circ} \mathrm{C}$ during these tests. 
The Catholic University of America Vitreous State Laboratory
DuraMelter 1200 Tests with AZ-101HLW Simulants

Final Report, VSL-02R0100-2, Rev.I

\subsubsection{Effluent Liquid Treatment System}

Effluent liquids from the SBS, WESP, HEME \#1, the caustic scrubber, and HEME \#2 (following the caustic scrubber) are all transferred to a series of sampling tanks that discharge into three 500-gallon storage tanks for neutralization, mixing, and storage. The largest effluent volume is the overflow (blow-down) from the SBS, which is pumped to one of two "SBS sampling" tanks. Caustic solution $(25-30 \% \mathrm{NaOH})$ from the same caustic tank that supplies the PBS can also be added to the 500-gallon storage tank that receives acidic effluents from SBS sampling tanks. Therefore, this storage tank is referred to as the "neutralization tank". Effluents collected in the storage tanks can be periodically pumped to the "Landa Evaporator" for concentration and either recycle or disposal. Salt residues from evaporation of the effluent liquids are periodically removed from the Landa Evaporator.

The various effluent liquid sampling and storage tanks are visually monitored during periodic rounds and effluent liquid transfers are made as needed. The only parameters of the effluent liquid treatment system monitored by the computerized data acquisition system during the post commissioning tests was the $\mathrm{pH}$ of the neutralization tank

\subsubsection{Thermal Catalytic Oxidation Unit}

A second HEME and a thermal catalytic Oxidation (TCO) unit follow the caustic scrubber in the off-gas treatment system. The HEME removes any water droplets that may be contained in the gas exiting the caustic scrubber. The TCO unit is equipped with a $80 \mathrm{~kW}$ heater at the front end to condition the incoming gas, as needed. The TCO was not active during these tests. However, the TCO heater was used to prevent moisture condensation within the building stack. The temperature of the gas at the outlet of the TCO was about $80^{\circ} \mathrm{C}$ for the first four tests and about $120^{\circ} \mathrm{C}$ for the last five tests.

\subsubsection{Off-Gas Temperatures}

A typical plot of the sequence of gas temperatures through the DM1200 off-gas system is given in Figure 4.88. 
The Catholic University of America Vitreous State Laboratory
DuraMelter 1200 Tests with AZ-101HLW Simulants Final Report, VSL-02R0100-2, Rev.1

\subsection{SBS and WESP Fluids}

\section{SBS Fluids}

One-liter samples were collected from the SBS and WESP sumps each time liquids were blown down and at the end of each test. Samples were subjected to total dissolved solids (TDS) and total suspended solids (TSS) determinations by gravimetric analysis of filtered material and the evaporated filtrate. Additional sample was filtered to generate solids and filtrate for complete chemical analysis, which included $\mathrm{pH}$ determination, direct current plasma emission spectroscopy (DCP) analysis for metals, atomic absorption (AA) for cesium, and ion chromatography for anions; the dried filtered solids underwent microwave-assisted acid dissolution prior to chemical analysis. The only anions determined in the filtered solids were sulfate and iodide due to interference from the acids required to dissolve the filtered solids. Due to the sheer volume of samples collected, not all samples were subject to each analysis. The results from additional characterization of selected samples with respect to viscosity and particle size distribution are presented in Appendix B.

All of the SBS and WESP sump samples that were taken throughout the DM1200 tests are listed in Tables 4.4 and 4.5; the middle letter in the sample name is "S" for the SBS samples and "W" for the WESP samples. The tables provide the TSS, TDS, and $\mathrm{pH}$ values for each sample, as well as the amount of glass that had been produced at the time the sample was taken. Also shown in Table 4.4 is the blow-down volume from which each SBS sample was taken and the cumulative SBS blow-down volume; as discussed earlier, the WESP was only blown down during Test 7. Detailed information on WESP water additions during testing are provided in Table 4.3. The volume of the SBS sump is about 500 gallons.

The TSS, TDS, and $\mathrm{pH}$ values for the SBS liquids are plotted in Figures 4.89 and 4.90 . The SBS TDS typically exceeded the TSS values and ranged from $60 \mathrm{mg} / 1$ to $6700 \mathrm{mg} / \mathrm{l}$. The higher values for both the TDS and TSS were from bubbling tests that featured higher production rates. One of the factors influencing the TDS was the solution $\mathrm{pH}$ since collected material was more likely to dissolve in the more acidic SBS solutions. The SBS solution $\mathrm{pH}$ was dependent on $\mathrm{NO}_{\mathrm{x}}$ melter emission rates, which were in turn a function of feed nitrate content, processing rate, and feed sugar content (see Section 6.3). The lowest measured $\mathrm{pH}$ values were about $\mathrm{pH} 1$, which occurred for Test 5 using nitrated feed. Tests 1 and 2 were identical except Test 2 was conducted with small amounts of sugar added to the feed resulting in substantially less nitrogen oxides being emitted. The decrease in emissions resulted in a SBS pH of about 8.5 instead of 3.7. During each test, the SBS solutions appeared to reach a steady-state $\mathrm{pH}$ with respect to melter emissions and the periodic blow-downs.

Figures 4.91-4.99 compare the amount of water fed to the total volumetric accumulations in the SBS over the course of each test. There is close agreement between these quantities for the 
tests without bubbling, indicating that nearly all of the feed water is condensed in the SBS as a result of its low operating temperature for those tests. In tests with bubbling (Tests 3, 4, and 7), the SBS sump temperatures rose to between 34 and $40^{\circ} \mathrm{C}$, resulting in a higher percentage of feed water being emitted. While this comparison illustrates these points, it could be further refined by taking into account the water feeding used to cool the melter plenum at the start of each test and by employing the instantaneous feed rate as opposed to the test average feed rate. Water was fed to the melter at the beginning of each test to create a cold cap and thereby minimize subsequent off-gas surges due to pulsed feeding onto bare glass (this is the same feed start-up protocol used at West Valley.) Consequently, the accumulation in the SBS is slightly greater than the amount of water that was introduced as part of the slurry feed.

Chemical analysis results for the SBS samples are provided in Table 4.6. Figures 4.1004.104 compare the SBS dissolved and suspended fractions from Tests 3 (sample 12J-S-142B) and Test 5 (sample 12L-S-62A) with the composition of the feed. As might be expected, the dissolved solids consist mainly of species such as alkali metals, halogens, boron, and sulfur, as well as significant amount of selenium. These constituents are also relatively volatile. The dissolved solids from Test 5 contain a larger proportion of metals that typically have limited solubility at higher $\mathrm{pH}$ values, such as alkaline earth metals and transition metals, as a result of the low solution $\mathrm{pH}$ of 1.37 . The suspended solids consist primarily of iron and silicon with significant amounts of selenium, tellurium, nickel, lead, chromium, and zirconium. Other metals such as aluminum, calcium, magnesium, cadmium, manganese, and zinc are present in both the suspended and dissolved fractions of the SBS samples ${ }^{4}$. Comparison of suspended solids from Tests 3 and 5 show that less of the alkaline earths, tellurium, selenium, and aluminum are observed in the suspended fraction of Test 5 due to the low $\mathrm{pH}$. Iodide was present only in the dissolved fraction.

The accumulations of elements in the SBS solutions (including blow-downs) over the course of Tests 1 and 2 are depicted in Figures 4.105-108. The total accumulations by constituent from these two tests are compared to their corresponding amounts in the target glass in Table 4.7. Over one kilogram each of selenium, iodine, and sulfate accumulated over the course of the tests, as well as tens of grams of metals such as cadmium, cesium, nickel, and tellurium. Also, more than 200 grams of both chlorine and fluorine were recovered in these fluids. However, the SBS liquids constitute a significant proportion of the elemental mass balance for only the halogens, sulfur, and selenium (Table 4.7), with up to 41 percent of these feed constituents reporting to the SBS fluids. It should be noted that the percentage of feed magnesium recovered in the SBS fluids is inflated as a result of the actual feed concentrations greatly exceeding the very low target value (see Section 2.4) that was used for these calculations. Several differences between the data for

${ }^{4}$ It should be noted that the filter pore size mandated by the standard methods that were used is 1.5 micron and therefore any particles less than this size could have contributed to the "dissolved" fraction instead of the suspended fraction. 
Test 1 and Test 2 are apparent, which is interesting given the fact that the only difference between these tests was the addition of $10 \mathrm{~g}$ of sugar per liter of feed for Test 2. Despite this seemingly small change, as discussed above, the increased amount of reductant has a significant impact on NOx emissions. The change in NOx emissions is then reflected in the SBS $\mathrm{pH}$, which, in turn, affects the scrubbing efficiency for various species. The $\mathrm{pH}$ also has a significant affect on the split between dissolved and suspended fractions for may elements. The greater rate of accumulation of iodine and sulfate in Test 2 (Figure 4.106) is probably due to increased scrubbing efficiency at higher solution $\mathrm{pH}(3.5 \mathrm{vs}$. 8.5). The increased rate of accumulation for cadmium (Figure 4.108) is mirrored by the significantly increased cadmium emission rates (based on off-gas sampling results; see Section 6.0, Tables 6.3 and 6.6) in Test 2. The instantaneous increase in accumulations of elements such as nickel, zirconium, calcium, manganese, and lithium at the end of Test 1 are probably related to the emptying and refilling of the SBS in between the tests. The wetting and drying cycle associated with dropping the SBS bowl and performing inspections may result in the spalling of deposits, while the addition of fresh water favors increased dissolution of material deposited within the SBS.

\section{WESP Fluids}

Results from the analysis of sump samples from the WESP are provided in Table 4.8 . These results indicate that the majority of the coarser, less-soluble species were removed by the SBS leaving predominantly highly soluble species for accumulation in the WESP. Notice that the concentrations of dissolved species in the WESP typically exceed the corresponding values in the SBS by greater than an order of magnitude. Conversely, the suspended solid concentration is substantially lower than that in the SBS. The WESP solutions are high in selenium, tellurium, alkali metals, boron, and sulfate, as depicted in Figures 4.109 and 4.110 for Tests 3 (sample 12K$\mathrm{W}-6 \mathrm{~A}$ ) and 5 (sample 12L-W-107A), respectively. These observations are generally consistent with the enhanced capture by the WESP of smaller particulates formed by the more volatile species, as is intended in the off-gas system design. The principal difference between the dissolved compositions in Tests 3 and 5 is the much larger proportion of tellurium in the Test 5 WESP solution. Between 120 and $220 \mathrm{~kg}$ of solution was removed from the WESP at the end of each test as compared to over $2000 \mathrm{~kg}$ removed from the SBS at the end of each test. This coupled with the lack of WESP blow downs during testing (only 18.9 liters was removed from the WESP during the nine tests) results in the WESP not being a significant factor with respect to elemental mass balances with the possible exception of selenium and tellurium. 
The Catholic University of America

Vitreous State Laboratory
DuraMelter 1200 Tests with AZ-101HLW Simulants

Final Report, VSL-02R0100-2, Rev.1

\section{SECTION 5.0 GLASS PRODUCT}

Over $26,700 \mathrm{~kg}$ of glass product was discharged from the melter using an airlift system into 55-gallon drums. The discharged product glass was sampled at the end of each test by removing sufficient glass from the top of the drums for total inorganic analysis and X-ray diffraction analysis (XRD). Product glass masses, discharge date, and the analyses performed for discharges during Tests 1 through 9 are listed in Table 5.1.

\subsection{Compositional Analysis}

Glass samples were crushed and either dissolved in $\mathrm{HF} / \mathrm{HNO}_{3}$ acid mixtures with the aid of a microwave oven to produce a solution for DCP analysis or analyzed directly by XRF. Target values for boron and lithium oxide concentrations were used for normalizing the XRF data since those elements were not determined by XRF. Digested glass samples were also analyzed for cesium using AA and ion chromatography for sulfate and iodide. XRF-analyzed compositions for discharged glass samples are provided in Table 5.2 for XRF data and in Table 5.3 for DCP data. Results of both DCP and XRF analysis are summarized for discharged glasses and compared to those for feed samples in Table 2.8. Generally, there was very good agreement with the target composition for the majority of oxides, in particular for the major oxides, as described for feed samples in Section 2.4. As was observed in the feed sample analysis, lithium and strontium are both about $15 \%$ below target, presumably as a result of actual deficiencies in the delivered feed. Lanthanum was consistently low in the product glass, as was the case for the feed samples (Section 2.4). The magnesium contamination (1.1-1.2 wt $\% \mathrm{MgO}$ vs. the $0.06 \mathrm{wt} \%$ target) present in the feed samples was also present in the discharged glasses. The loss of volatile elements such as sulfur, selenium, and tellurium, although still significant, was less pronounced in the discharged glasses due to the presence of a cold cap maintained in the melter but absent during crucible melting of feed samples. No iodine was detected in any of the samples by either method. The iodine detection limit is estimated at about $0.001 \mathrm{wt} \%$ for XRF but suitable glass standards are not available to confirm this. Data from the DCP and XRF analytical methods give comparable results for most constituents with XRF yielding results closer to target for some constituents (e.g., silica and iron) and DCP yielding results closer to target for others (e.g., zirconium).

\subsection{Compositional Trends}

Compositional trends over the course of the nine tests are plotted for selected elements in Figures 5.1-5.3 (XRF data) and 5.4-5.5 (DCP data). The figures illustrate many of the points 
apparent in the tabular summaries of the data; generally good agreement with target for most major oxides, deficiencies in lithium and strontium, significantly above-target concentrations of magnesium, and the loss of volatile elements. They also illustrate the compositional consistency for the majority of constituents over the course of testing. Exceptions occur during Test 6 and especially Test 7, both of which employed glass frit in place of chemicals for the glass formers. Notice the changes in aluminum, magnesium, calcium, and sulfur between $18000 \mathrm{~kg}$ and $24000 \mathrm{~kg}$ of production. Review of the feed data from individual tests (Table 2.6) shows many of the feed samples from these tests exhibit the same trends. All three elements are components in the simulant as opposed to the frit (see Tables 2.2 and 2.3); however, the trend cannot be attributed to a simple blending error in the ratio of simulant and frit because many other elements do not change in concentration over this period and aluminum concentrations increase while magnesium decreases. The most likely explanation appears to be contaminants in the glass frit but no impact on the glass production rates is anticipated from these variations. Fewer samples were analyzed using the DCP method and therefore trends are less obvious; however, two distinct boron populations can be discerned in Figure 5.4. Note that the glass poured during the first $12,000 \mathrm{~kg}$ of production had $\mathrm{B}_{2} \mathrm{O}_{3}$ concentrations averaging about $11 \mathrm{wt} \%$ (about $10 \%$ above target) whereas glasses produced in later tests have $\mathrm{B}_{2} \mathrm{O}_{3}$ concentrations close or slightly below the target value.

Volatile elements are of particular interest due to their often hazardous or radioactive nature and the implications for recycling off-gas system effluents. Iodine was the most volatile feed constituent given that none of the $0.1 \mathrm{wt} \%$ glass equivalent was measured in the product glass. As expected, selenium was also very volatile with less than a third of the feed selenium being incorporated into the product glass. Conversely, cesium, which is known to be volatile, was retained at concentrations at or near target levels. Another noteworthy observation is the increase in concentrations over feeding periods followed by dramatic decreases during idling periods for certain elements, most notably sulfur and selenium.

\subsection{Iron Redox State}

The iron oxidation state for glass samples from all nine tests was measured using colorimetric methods. Sample information including name, test, and the amount of glass produced are given in Table 5.4. The method detection limit of $0.4 \%$ divalent iron for these samples is a function of the lowest standard, the amount of glass dissolved, and the iron content of the glass.

One objective provided by the WTP Project for these melter tests was to add reductants (sugar) to yield a product glass with measurable amounts of divalent iron but not to exceed $20 \%$ of the total iron. This objective was intended to improve production rates by managing potential 
foaming. This objective was met in all tests except for Test 5 . This test featured feed acidified with nitric acid and sugar at a ratio of 0.5 ( 1 mole sucrose: 16 moles nitrate or 3 moles of carbon: 4 moles nitrate). Test 8 was a repeat of Test 5 with an increase in sugar ratio to 0.7 and resulted in between 1 and 2 percent measured $\mathrm{Fe}^{2+}$ content. Initial tests with extra sugar were monitored closely to ensure that the divalent iron did not exceed the stated upper limit. The results from three of the longer tests are depicted in Figure 5.6. Notice that the rate of increase with glass production is approximately the same for Tests 2 and 9. The drop in divalent iron concentration for the last sample for both tests is due to a lag in time between the end of feeding and final glass discharge. The similar results for the two tests with same feed composition, sugar level, and approximate feed rate were expected. The latter half of Test 9 was conducted at a melting temperature of $1200^{\circ} \mathrm{C}$ instead of $1150^{\circ} \mathrm{C}$, which may explain the higher divalent iron values reached in the latter portion of the test. The consistency of Test 7 divalent iron concentrations suggests a steady-state process with respect to iron reduction throughout the majority of the test. The lower divalent iron value is probably due to the increase in production rate (about 4 times higher than Test 2) and/or the air being bubbled through the melt pool. It is worth noting that all of the bubbled tests (Test 3, 4, and 7) as well as all of the tests without sugar additions (Tests 1, 3 , and 4) exhibited small but measurable amounts of divalent iron in the glass products. 


\section{SECTION 6.0 \\ MONITORED OFF-GAS EMISSIONS}

\subsection{Particulate and Gaseous Emissions}

Sixty-five exhaust samples were taken from the melter and various off-gas system components using 40-CFR-60 Methods 3, 5, and 29 to examine particulate and certain gaseous fluxes. The majority of the off-gas analyte concentrations were derived from laboratory data on solutions extracted from air samples (filters and various solutions) together with measurements of the volume of air sampled. The volume of air sampled and the rate at which it can be sampled are defined in 40-CFR-60 and SW-846. Isokinetic sampling, which entails removing gas from the exhaust at the same velocity that the air is flowing in the duct (40-CFR-60, Methods 1-5), was used. Typically, a sample size of $30 \mathrm{dscf}$ is taken at a rate of between 0.5 and $0.75 \mathrm{dscfm}$. Total particulate loading was determined by gravimetric analysis of the standard particle filter and of probe-rinse solutions. Downstream of the particulate filter in the sampling train are iced impingers with acidic (5\% concentrated nitric acid plus $10 \%$ hydrogen peroxide) and basic ( $0.2 \mathrm{~N}$ sodium hydroxide) solutions. The analysis of these solutions permits the determination of total gaseous emissions of several elements, notably halides and sulfur. A list of all isokinetic sampling taken is provided in Table 6.1 including sampling date, sampling location, air sample volume, air flow rates, particulate emission rates, and air moisture content. The particle loading on the WESP filters from Test 1 was so low that the three six-hour samples were combined into one 36-hour sample in the subsequent eight tests.

Average melter, SBS, and WESP particulate emission rates and associated decontamination factors (DFs, i.e., ratio of species inlet flux to species outlet flux) are summarized in Table 6.2 for all nine tests. Elemental emission rates and DFs are provided in Tables 6.3-6.29. Solids carry-over from the melter was minimal during these tests, never exceeding $0.5 \%$ of feed solids. As expected, the particulate emissions, both as flux and percent of feed, are far lower in tests without bubbling (Tests 1, 2, 6 and 9) than tests with bubbling (Tests 3, 4, and 7). DFs across the melter for total solids were around 200-400 with bubbling and around 500-2000 without bubbling. Exceptions were the two tests with nitrated feed without bubbling (Tests 5 and 8), which had the lowest DF values across the melter despite the low achieved production rates. Tests with frit additives as compared to comparable tests with chemicals as the source of glass formers, resulted in lower particle carryover for bubbled tests (Test 3 vs. Test 7) and higher particle carryover in tests without bubbling (Test 2 vs. Test 6). The average value given for Test 9 averages two samples taken while glass was produced at $1200^{\circ} \mathrm{C}$ and one while glass was produced at $1150^{\circ} \mathrm{C}$ (Table 6.27). As expected, the sample corresponding to $1150^{\circ} \mathrm{C}$ gave a higher melter DF (almost 800), whereas the samples corresponding to $1200^{\circ} \mathrm{C}$ gave a significantly lower DF (450). DFs across the SBS for total 
The Catholic University of America Vitreous State Laboratory
DuraMelter 1200 Tests with AZ-101HLW Simulants

Final Report, VSL-02R0100-2, Rev.1

particulate ranged from about 3 to 26 and were higher for the bubbled tests, for which the particulate flux from the melter was higher. Across the WESP, DF values ranged from about 50 to 300 with no clear trend relating to feed type or melter operating conditions. Cumulative DF values are calculated from feed fluxes into the melter and emissions from the WESP. Values exceeding one million were not obtained for any test however three tests had cumulative DFs greater than 800,000 .

The composition of the particles is similar to that observed in previous studies $[1,9]$ : high in volatile species such as boron, selenium, sulfur, and alkali, with lower concentrations of all other feed constituents. SBS particle emissions are even higher in selenium, sulfur, and alkali metals and even lower in other major feed constituents, such as silica, aluminum, and iron. Impinger solutions were analyzed for the all of the elements in the feed but only the halides, sulfur, selenium, and boron were detected. The presence of these elements in the gas fraction is consistent with observations from previous studies $[1,2,9]$. The average composition of feed, melter emissions, and SBS emissions (excluding oxygen, nitrate, nitrite) are displayed in Figures 6.1-6.3, respectively. Notice that the relative percentage of volatiles such as selenium, sulfur, and alkali increase down-stream as the major constituents decrease. These results are consistent with previously observed trends: melter emissions are the result of both volatile constituents and feed entrainment and the SBS removes all but some of the more volatile feed constituents, which form the majority of the finer particulate matter. The composition of WESP particle emissions are difficult to determine due to the very low particle loading. Gaseous WESP are similar to SBS emissions with smaller halogen fluxes.

Iodine was detected in significant quantities in samples taken at all three off-gas locations. Iodine was only detected in the impinger solution downstream of the particle filter, indicating that it was exclusively gaseous. Furthermore, greater than $90 \%$ of all the iodine detected was measured in the basic impinger $(0.2 \mathrm{~N} \mathrm{NaOH})$ downstream of the two acidic impingers (5\% nitric acid and 10\% hydrogen peroxide). This suggests that the iodine emitted is molecular $\left(\mathrm{I}_{2}\right)$ as opposed to $\mathrm{HI}$; however, the division between molecular and acid gas halogens based on impinger $\mathrm{pH}$ has only been verified for chlorine and fluorine. Iodine melter emissions were highly variable ranging from 5 to $68 \%$ of feed iodine with no clear trend with respect to melter operating conditions or feed type. This variability coupled with the lack of iodine detected in the glass product result in variable mass balance closure with respect to feed iodine. Significant iodine was measured in SBS emissions in only two tests (Tests 1 and 4) and in WESP emissions in Test 4. Iodine results from these nine tests are similar to the previous three Commissioning Tests [2] in that the majority of the iodine detected was in the basic impinger catch and melter emissions were variable; they are different in that little iodine was detected downstream of the WESP. 


\subsection{Particle Size Distribution}

Samples were also taken using a University of Washington cascade impactor, which separates particles into particle size ranges enabling the determination of particle size distributions; the limit of each size range is referred to as the "cut-point." The melter and SBS exhaust streams were sampled during each test. Data for the particle size distributions are provided in Tables 6.30 - 6.38 for melter emissions and Tables 6.39-6.40 for SBS emissions. The chemical compositions of each particle size fraction emitted from the melter from the first five tests are provided in Tables 6.41 - 6.45; typically, greater than about $65 \mathrm{wt} \%$ of the particulate is larger than about 14 microns. Enough material was collected on filters from the melter and SBS exhaust to determine size distributions; however, sufficient material for chemical analysis was only collected on melter exhaust samples. The particulate concentration in the WESP exhaust was insufficient for accurately determining particle size distributions and therefore no sampling was attempted. Particle emissions from the SBS were mainly sub-micron in size, whereas melter emissions were concentrated in the coarser size fractions. This is consistent with the design intent of the SBS, which is primarily to remove the larger particulate, leaving removal of the finer particulate to the WESP, which is much better suited to that function. The composition of the particles emitted from the melter varied with size as shown in Tables $6.41-6.45$. In the previous study [2], increases in mass percent for $\mathrm{Cd}, \mathrm{Cs}, \mathrm{K}, \mathrm{S}$, and $\mathrm{Te}$ were observed with decreasing particle size, whereas elemental mass percentages of $\mathrm{Al}, \mathrm{B}, \mathrm{Mn}$, $\mathrm{Si}, \mathrm{Sr}, \mathrm{Zn}$ and $\mathrm{Zr}$ decreased with decreasing particle size. Also, sodium and selenium were common in all size fractions and show some tendency towards the smaller fractions. This trend is roughly adhered to in all the samples analyzed except those from Test 2 (Table 6.42). Chemical analysis by particle size is complicated by the small accumulated masses on each individual filter.

\subsection{FTIR Analysis}

Off-gas analysis by Fourier Transform Infra-Red (FTIR) spectroscopy was performed using an On-Line Technologies Inc. Model 2010 Multi-Gas ${ }^{\mathrm{TM}}$ Analyzer. Data were recorded at $71 \mathrm{~s}$ intervals, corresponding to an average of 128 scans at $0.5 \mathrm{~cm}^{-1}$ spectral resolution. The melter off-gas supplied to the FTIR spectrometer was extracted using a heated sampling and transfer loop, which removed a gas sample stream from the off-gas system at 5 liters per minute. The sampling and transfer loop was maintained at $150 \mathrm{C}$ throughout in order to prevent analyte loss due to condensation.

Off-gas emissions were monitored by FTIR spectroscopy during each test for a set of selected species over discrete time intervals at specified off-gas system locations. Table 6.46 displays a summary of the average analyte concentrations measured over the course of each test. 
Real-time nitrogen oxide concentrations are presented in Figures 6.4-6.13 over the nine tests. As expected, $\mathrm{NO}$ and $\mathrm{NO}_{2}$ were observed at the highest concentrations among the nitrogen-bearing analytes. As expected, concentrations of these nitrogen species as well as carbon monoxide were orders of magnitude higher in Tests 5 and 8 due to the use of nitrated feed. The nitrogen dioxide concentration was below the nitrogen oxide concentration in these tests and neither constituent was removed by either the SBS or WESP. The increase in sugar from a ratio of 0.5 (Test 5) to 0.7 (Test 8) did not result in a reduction in nitrogen oxide concentrations but did result in significant increases in both carbon monoxide and carbon dioxide. In the other seven tests, nitrogen oxide concentrations were affected by feed rate and sugar additions to the feed. The only difference between Tests 1 and 2 is the addition of 10 grams of sugar per liter, which led to a reduction in nitrogen emissions by a factor of about 8 and an increase in carbon monoxide emissions by a factor of about 9 . Tests with bubbling (Tests 3, 4, and 7) had higher production rates and were conducted without sugar, resulting in higher relative nitrogen oxide emissions. Another aspect of the $\mathrm{NO}_{\mathrm{X}}$ emissions is the high degree of variability during each test as can be observed in Figures 6.4 - 6.13. Notice that even over short periods of time NO emissions vary over factors of 10. Other nitrogen species such as ammonia, hydrogen cyanide, and $\mathrm{N}_{2} \mathrm{O}$ were observed in small quantities in many of the tests. $\mathrm{HF}, \mathrm{HCl}$, and $\mathrm{SO}_{2}$ were detected at rather low concentrations, typically less than $1 \mathrm{ppm}$. Moisture percentages at the melter outlet were a function of feed rates and were greatly reduced downstream by condensation in the SBS unit. Nitrogen mass balances over tests are shown in Table 6.47 for the predominant $\mathrm{NO}_{\mathrm{X}}$ species (NO and $\mathrm{NO}_{2}$ ) compared to feed nitrogen. In tests without sugar (Tests $1,3,4,7$ ), all of the feed nitrate was emitted as nitrogen oxides. In the nitrated tests, about half the feed nitrates were emitted as nitrogen oxides in keeping with previous tests conducted with high nitrate feeds at sugar ratios of around 0.5. Although the amount of added sugar was small in Tests 2,6 and 9 the sugar ratio was well above 1.0 resulting in most of the feed nitrates being reduced to molecular nitrogen. 


\section{SECTION 7.0 SUMMARY AND CONCLUSIONS}

A series of nine tests was performed on the DM1200 HLW pilot melter system to collect data on glass production rates and system performance for a range of feed types and system operating parameters. The tests used the same AZ-101 HLW simulant and glass composition, which was selected to meet all product quality, waste loading, and processability requirements. Consequently, variation of the waste stimulant or the glass formulation was not within the scope of these tests. Within these constraints, factors that were varied included feed solids content; reductant additions; feed nitration; replacement of glass forming chemical by a glass frit; increased operating temperature; increased number of feed tubes; continuous instead of pulsed feed introduction; and inclusion of melt pool agitation by bubbling.

Bubbling gave by far the most significant impact on glass production rates, as summarized in Figure 7.1. This conclusion is consistent with the results of the previous tests with the same stimulant and glass composition that were conducted on the DM1000 [1] and DM100 melter systems [9]. Furthermore, the conclusion that the glass production rates with baseline AZ101 feed fall well short of the $400 \mathrm{~kg} / \mathrm{m}^{2} / \mathrm{d}$ WTP HLW melter design basis is also consistent with the previous tests performed at the same scale [1]. Previous smaller-scale tests performed on the DM100 system [9] also indicated generally rather modest increases in glass production rates for most of the variables investigated, although several tests gave rates significantly above the $400 \mathrm{~kg} / \mathrm{m}^{2} / \mathrm{d}$ design basis. However, while small-scale melter tests are useful for screening relative effects, they tend to overestimate absolute glass production rates, particularly for unbubbled tests. This effect is illustrated in Figure 7.2. Consequently, when scale-up effects were taken into account, it was not clear that any of the variables investigated in the DM100 tests would conclusively meet the $400 \mathrm{~kg} / \mathrm{m}^{2} / \mathrm{d}$ full-scale requirement without bubbling. The present series of test was therefore performed on the DM1200 one-third scale HLW pilot melter system to resolve this question. Notable differences between the results of the present tests and the screening tests conducted on the DM100 system [9] are that, as expected due to scale-up effects, the absolute rates are lower for the larger system, but also the effects of some of the seemingly more influential variables were significantly lower. For example, glass production rates increased from 430 to $650 \mathrm{~kg} / \mathrm{m}^{2} / \mathrm{d}$ in the DM100 tests when chemical glass formers were replaced by glass frit [9], whereas the same change in the DM1200 tests resulted in little, if any, improvement in the rate $\left(210 \mathrm{~kg} / \mathrm{m}^{2} / \mathrm{d}\right)$. It should be noted, however, that comparison of the results from these tests is complicated by the fact that the solids contents were not the same in the two sets of tests (higher for the DM100 tests).

In summary, while the glass production rate results from the present tests have extended the range of variables explored, they are consistent with our earlier analysis and recommendation to the RPP-WTP Project [22]. While it is possible that some combination of variables, which would represent significant departures from the baseline flow-sheet, design, and operating 
parameters, might meet the design basis rate, the data that are presently available suggest that such a solution would be unlikely to be robust with respect to variations in waste composition. Over and above the increase in absolute rates, this robustness is perhaps the strongest argument for the inclusion of bubblers into the HLW design, given the uncertainty and variability in the RPP-WTP HLW feed compositions.

In parallel with the glass production rate determinations, extensive data were collected on the performance of the DM1200 off-gas system over a wide range of feed types and operating conditions. The general performance of the system was good with minimal accumulations of solids in the film cooler and off-gas jumper, effective removal of larger solids by the SBS, and effective removal of smaller particulate by the WESP, which is consistent with the off-gas system design intent. The solids that were collected in the SBS were easily suspended and effectively removed in the liquid blow-downs, which resulted in minimal accumulation. In addition, the residual accumulation was soft and easily re-suspended. An important observation from these tests was the accumulation of deposits in the SBS down-comer at the point at which it passes through the diffuser plate; in one test, this lead to significant increases in the pressure drop across the SBS. Consequently, future testing should address this issue. The performance of the WESP used for these tests was generally good but, as a result of these tests, several design problems were identified that led to periods of electrical instability; these have since been rectified and the unit has been stable in subsequent tests.

Extensive data were collected from off-gas samples taken throughout the system during these tests. These data were reduced to DF values for total particulate across the melter, the SBS, and the WESP. In addition, across the melter and the SBS, where particle loads were sufficiently high, DFs by element, particle size distributions, and elemental compositions by particle size were determined. Cumulative DFs across the melter, SBS, and WESP were between about 170,000 and 980,000 , with several tests giving values towards the higher end of that range. Extensive data were also collected on the compositions of samples of sump fluids from the SBS and WESP that were collected throughout the tests, which are important for flow-sheet input and recycle closure. These data included $\mathrm{pH}$, dissolved and suspended solids fractions, and chemical composition. The SBS sump fluid TDS values typically exceeded the TSS values and ranged from $60 \mathrm{mg} / 1$ to $6700 \mathrm{mg} / 1$. The $\mathrm{pH}$ values ranged from 1.06 to 8.60 and were influenced by the melter emissions, particularly NOx, through the feed type, with the lowest $\mathrm{pH}$ values being for the nitrated feeds. The dissolved solids consisted mainly of species such as alkali metals, halogens, boron, and sulfur, as well as significant amount of selenium. The suspended solids consisted primarily of iron and silicon with significant amounts of selenium, tellurium, nickel, lead, chromium, and zirconium. Other metals such as aluminum, calcium, magnesium, cadmium, manganese, and zinc were present in both the suspended and dissolved fractions of the SBS samples. Iodide was present only in the dissolved fraction.

Results from the analysis of sump samples from the WESP indicate that the majority of the coarser, less-soluble species were removed by the SBS leaving predominantly highly soluble species for accumulation in the WESP. The concentrations of dissolved species in the WESP typically exceeded the corresponding values in the SBS by greater than an order of 
The Catholic University of America

Vitreous State Laboratory
DuraMelter 1200 Tests with AZ-10IHLW Simulants Final Report, VSL-02R0100-2, Rev.1

magnitude; however, the liquid accumulation rates in the WESP were also very much lower than for the SBS. Conversely, the suspended solid concentrations were substantially lower than those in the SBS. The WESP solutions were high in selenium, tellurium, alkali metals, boron, and sulfate. These observations are generally consistent with the enhanced capture by the WESP of smaller particulates formed by the more volatile species, as is intended in the off-gas system design. 


\section{SECTION 8.0 \\ REFERENCES}

[1] "Determination of Processing Rate of RPP-WTP HLW Simulants using a DuraMelter"TM 1000 Vitrification System," K.S. Matlack, W.K. Kot, F. Perez-Cardenas, and I.L. Pegg, VSL-00R2590-2, Rev. 0, 8/21/00.

[2] "HLW Melter Attainment Studies and Equipment Performance Evaluations," J.M. Perez, Test Specification, 24590-HLW-TSP-RT-01-001, Rev. 0, 8/6/01.

[3] "Tests on the DM1200 HLW Pilot Melter System Using AZ-101 Waste Simulants," K.S. Matlack, W.K. Kot, M. Brandys, and I.L. Pegg, Test Plan, VSL-01T0100-2, Rev.2, $7 / 3 / 01$.

[4] "Tests on the DM1200 HLW Pilot Melter System Using AZ-101 Waste Simulants," K.S. Matlack, W.K. Kot, C.C. Chapman, M. Brandys and I.L. Pegg, Test Plan, VSL-01T01003, Rev.1, 9/11/01.

[5] "Start-Up and Commissioning Tests on the DM1200 HLW Pilot Melter System Using HLW AZ-101 Simulants," K.S. Matlack, W.K. Kot, M. Brandys, C. N. Wilson, T. R. Schatz, W. Gong, and I.L. Pegg, VSL-01R0100-2, Rev. 0, 1/20/03.

[6] "Data Summary Report: Tests on the DM1200 HLW Pilot Melter System Using HLW AZ-101 Simulants: First Four Post-Commissioning Tests," K.S. Matlack, and I.L. Pegg, VSL-01S2100-1, Rev. 0, 10/17/01.

[7] "Data Summary Report: Tests on the DM1200 HLW Pilot Melter System Using HLW AZ-101 Simulants: Last Five Post-Commissioning Tests," K.S. Matlack, and I.L. Pegg, VSL-02S2100-1, Rev. 0, 3/1/02.

[8] "Tests on DuraMelter ${ }^{\mathrm{TM}} 10$ with AZ-101 Simulant in Support of DuraMelter ${ }^{\mathrm{TM}} 1000$ Throughput Tests," K. S. Matlack, W. K. Kot, F. Perez-Cardenas, and I. L. Pegg, VSL00R2501-1, Rev. 0, 4/21/00.

[9] "Melter Tests with AZ-101 HLW Simulants Using a DuraMelter" 100 Vitrification System," K.S. Matlack, W.K. Kot, and I.L. Pegg, VSL-01R10N0-1, 8/31/01; Rev. 1, $2 / 15 / 02$.

[10] "Design and Installation of a Prototypical Off-Gas Treatment System for the DM1200 RPP-WTP HLW Pilot Melter," R.T. Anderson, M. Brandys, and R. Jung, Final Report, VSL-01R2510-1, Rev. 0, 2/22/01. 
[11] "Submerged Bed Scrubber: Off-Line Component Testing of DM1200 SBS Unit," M. Brandys, R.T. Anderson, R. Jung, J. Rouse, and I.L. Pegg, Final Report, VSL01R2200-1, Rev. 0, 9/21/01.

[12] "Quality Assurance Project Plan for RPP-WTP Support Activities Conducted by VSL," Vitreous State Laboratory, QAPP Rev. 3, 8/28/00.

[13] "Quality Assurance Project Plan for RPP-WTP Support Activities Conducted by VSL," Vitreous State Laboratory, QAPP Rev. 4, 8/2/2001.

[14] "Master List of Controlled VSL Manuals and Standard Operating Procedures in Use," QA-MLCP, Rev. 0, August 14, 2001.

[15] "Tank Waste Remediation System Operation and Utilization Plan to Support Waste Feed Delivery," R.A. Kirkbride, G.K. Allen, R.M. Orme, R.S. Wittman, J.H. Baldwin, T.W. Crawford, J. Jo, L.J. Fergestrom, G.T. MacLean and D.L. Penwell, Volume I, HNF-SDWM-SP-012, Revision 1 (Draft), February 1999.

[16] Hanford Tank Waste Best Basis Inventory, Tank Waste Information Network System 2 (http://twins.pnl.gov:8001).

[17] "Phase I High-Level Waste Pretreatment and Feed Staging Plan," A.F. Manuel, S.L. Lambert and G.E. Stegen, WHC-SD-WM-ES-370, Revision1, September 1996.

[18] "Tank Farm Contractor Operation and Utilization Plan," R.A. Kirkbride, G.K. Allen, B.A. Highley, R.M. Orme, R.S. Wittman, J.H. Baldwin, T.W. Crawford, J. Jo, J. N. Strode, T.M. Hohl, S.L. Lambert, D.E. Place and J.A. Seidl, Volume I, HNF-SD-WMSP-012, Revision 2, April 2000.

[19] "Calculation of Lag Storage Requirements for Phase 1 Pretreatment Operations," BNFL, Inc. Memorandum \#001753, E. Slaathaug to I. Papp, February 17, 1999.

[20] "Using $\mathrm{MnO}_{4}$ for TRU Separations," M. Johnson, E-mail message to I.L. Pegg, May 17, 1999.

[21] "Physical and Rheological Properties of Waste Simulants and Melter Feeds for RPPWTP Vitrification," W.K. Kot, Hao Gan and I.L. Pegg, Final Report, VSL-00R2520-1, Oct. 31, 2000.

[22] "Notes in Response to HLW Glass Production Rate and Product Quality Control Questions," I.L. Pegg (VSL) to R. Voke (WTP), 2/19/01. 
The Catholic University of America

DuraMelter 1200 Tests with AZ-101HLW Simulants Vitreous State Laboratory

Table 1.1. Test Matrix.

\begin{tabular}{|c|c|c|c|c|c|c|c|}
\hline $\mathbf{T P}$ & $\begin{array}{c}\text { TP } \\
\text { Test } \\
\#\end{array}$ & $\begin{array}{l}\text { Actual } \\
\text { Test \# }\end{array}$ & Feed Type & Bubbling & Sugar & $\begin{array}{c}\text { Bulk Glass } \\
\text { Temperature, } \\
{ }^{\circ} \mathrm{C}\end{array}$ & $\begin{array}{l}\text { Type of } \\
\text { feeding }\end{array}$ \\
\hline \multirow{6}{*}{$\begin{array}{c}\text { VSL- } \\
01 \mathrm{~T} 01 \\
00-2\end{array}$} & 1 & 1 & High Solids & No & No & 1150 & $\begin{array}{c}\text { Pulsed } \\
\text { Simulated } \\
\text { ADS } \\
\end{array}$ \\
\hline & $1 \mathbf{a}$ & 2 & $\begin{array}{c}\text { High Solids }+10 \mathrm{~g} \text { sugar/ } \\
\text { liter feed }\end{array}$ & No & Yes & 1150 & $\begin{array}{c}\text { Pulsed } \\
\text { Simulated } \\
\text { ADS }\end{array}$ \\
\hline & 16 & $9 a$ & $\begin{array}{l}\text { High Solids }+10 \mathrm{~g} \text { sugar/ } \\
\text { liter feed }\end{array}$ & No & Yes & 1150 & $\begin{array}{l}\text { Continuous } \\
\text { Peristaltic }\end{array}$ \\
\hline & 2 & 4 & Nominal & $\begin{array}{c}\text { Yes; } \\
\text { Shallow, L- } \\
\text { shaped }\end{array}$ & Yes & 1150 & $\begin{array}{c}\text { Puised } \\
\text { Simulated } \\
\text { ADS }\end{array}$ \\
\hline & 3 & 3 & Nominal & $\begin{array}{l}\text { Yes; Deep, } \\
\text { L-shaped }\end{array}$ & Yes & 1150 & $\begin{array}{c}\text { Pulsed } \\
\text { Simulated } \\
\text { ADS }\end{array}$ \\
\hline & 4 & $\begin{array}{c}\text { Not } \\
\text { Conducted }\end{array}$ & Nominal & No & Yes & 1150 & $\begin{array}{c}\text { Pulsed } \\
\text { Simulated } \\
\text { ADS }\end{array}$ \\
\hline \multirow{7}{*}{$\begin{array}{l}\text { VSL- } \\
01 \mathrm{T01} \\
00-3\end{array}$} & \multirow{2}{*}{1} & 5 & Nitrated Sugar Ratio $=0.5$ & \multirow{2}{*}{ No } & \multirow{2}{*}{ Yes } & \multirow{2}{*}{1150} & \multirow{2}{*}{$\begin{array}{l}\text { Pulsed } \\
\text { Simulated } \\
\text { ADS }\end{array}$} \\
\hline & & 8 & Nitrated Sugar Ratio $=0.7$ & & & & \\
\hline & 2 & 6 & Frit $+7.5 \mathrm{~g} \mathrm{sugar} /$ liter & No & Yes & 1150 & $\begin{array}{c}\text { Pulsed } \\
\text { Simulated } \\
\text { ADS } \\
\end{array}$ \\
\hline & 3 & 7 & Frit +7.5 g sugar/ liter feed & $\begin{array}{l}\text { Yes; deep, } \\
\text { L-shaped }\end{array}$ & No & 1150 & $\begin{array}{c}\text { Pulsed } \\
\text { Simulated } \\
\text { ADS } \\
\end{array}$ \\
\hline & 4 & $9 b$ & $\begin{array}{c}\text { Nominal }+10 \text { g sugar/ liter } \\
\text { feed }\end{array}$ & No & Yes & 1175 to 1200 & $\begin{array}{l}\text { Continuous } \\
\text { Peristaltic }\end{array}$ \\
\hline & 5 & $\begin{array}{c}\text { Not } \\
\text { Conducted }\end{array}$ & Nominal & $\begin{array}{l}\text { Yes; deep, } \\
\text { simplified } \\
\text { straight-pipe }\end{array}$ & No & 1150 & $\begin{array}{c}\text { Pulsed } \\
\text { Simulated } \\
\text { ADS }\end{array}$ \\
\hline & 6 & $\begin{array}{c}\text { Not } \\
\text { Conducted }\end{array}$ & Nominal & $\begin{array}{c}\text { Yes; } \\
\text { shallow, } \\
\text { simplified } \\
\text { straight-pipe }\end{array}$ & No & 1150 & $\begin{array}{l}\text { Pulsed } \\
\text { Simulated } \\
\text { ADS }\end{array}$ \\
\hline
\end{tabular}

Notes:

- $\quad$ TP $=$ Test Plan

- All tests are with AZ-101.

- $\quad$ "Nominal" solids content corresponds to Envelope D feed from pretreatment of $20 \mathrm{wt} \%$ solids, which for the AZ101 formulation, gives a melter feed oxide content of about $400 \mathrm{~g} / \mathrm{l}$, based on the estimated density.

- Oxide content of "High solids" feed was about $570 \mathrm{~g} / \mathrm{l}$.

- Electrode firing patterns were side-to-bottom for the un-bubbled and shallow bubbler tests and side-to-side for the remaining bubbled tests.

- Tests 5 and 8 were conducted with an acidified (nitrated) feed. All other tests used alkaline feeds.

- Test 9 employed two feed tubes; all others employed one.

- Test $9 \mathrm{~b}$ was originally intended to be conducted with the pulsed, simulated ADS feed system. 
Table 1.2. Summary of Test Conditions and Results for Tests 1-4.

\begin{tabular}{|c|c|c|c|c|c|}
\hline \multicolumn{2}{|r|}{ Test \# } & 1 & 2 & 3 & 4 \\
\hline \multirow{3}{*}{$\stackrel{\mathscr{E}}{E}$} & Feed Start & $6 / 25 / 01 \quad 10: 45$ & $7 / 23 / 01 \quad 17: 47$ & $8 / 6 / 0116: 59$ & $8 / 20 / 0113: 08$ \\
\hline & Feed End & $7 / 2 / 01 \quad 10: 50$ & $8 / 1 / 0111: 47$ & $8 / 11 / 0111: 47$ & $8 / 25 / 011444$ \\
\hline & Interval & $168.1 \mathrm{hr}$ & $216 \mathrm{hr}$ & $114.8 \mathrm{hr}$ & $121.6 \mathrm{hr}$ \\
\hline \multicolumn{2}{|c|}{ Water Feeding for Cold Cap } & $2.25 \mathrm{hr}$ & $1.6 \mathrm{hr}$ & $0.8 \mathrm{hr}$ & $1.9 \mathrm{hr}$ \\
\hline \multicolumn{2}{|c|}{ Slurry Feeding } & $165.8 \mathrm{hr}$ & $214.4 \mathrm{hr}$ & $114.0 \mathrm{hr}$ & $119.7 \mathrm{hr}$ \\
\hline \multicolumn{2}{|c|}{ Cold cap burn } & $5.0 \mathrm{hr}$ & $10.0 \mathrm{hr}$ & $3.3 \mathrm{hr}$ & $3.3 \mathrm{hr}$ \\
\hline \multicolumn{2}{|c|}{ Total } & $173.1 \mathrm{hr}$ & $226.0 \mathrm{hr}$ & $118.1 \mathrm{hr}$ & $124.9 \mathrm{hr}$ \\
\hline \multicolumn{2}{|c|}{ Bubbling Rate } & $<4 \perp \mathrm{pm}$ & $<4 \mathrm{lpm}$ & Deep: $62 \mathrm{lpm}$ & Shallow: $50 \mathrm{lpm}$ \\
\hline \multicolumn{2}{|c|}{ Electrode Firing } & Side to Bottom & Side to Bottom & Side to Side & Side to Bottom \\
\hline \multirow{5}{*}{ 马्g } & Characteristics & High Solids & $\begin{array}{l}\text { High Solids }+ \\
10 \mathrm{~g} \mathrm{sugar} / 1\end{array}$ & High Water & High Water \\
\hline & Used & $5927 \mathrm{~kg}$ & $6816 \mathrm{~kg}$ & $19184 \mathrm{~kg}$ & $11573 \mathrm{~kg}$ \\
\hline & \multirow{2}{*}{ Glass yield (target) } & $570 \mathrm{~g} / 1$ & $570 \mathrm{~g} / \mathrm{l}$ & $400 \mathrm{~g} / 1$ & $400 \mathrm{~g} / \mathrm{l}$ \\
\hline & & $0.38 \mathrm{~kg} / \mathrm{kg}$ & $0.38 \mathrm{~kg} / \mathrm{kg}$ & $0.29 \mathrm{~kg} / \mathrm{kg}$ & $0.29 \mathrm{~kg} / \mathrm{kg}$ \\
\hline & Average Rate & $35.7 \mathrm{~kg} / \mathrm{hr}$ & $31.8 \mathrm{~kg} / \mathrm{hr}$ & $168.3 \mathrm{~kg} / \mathrm{hr}$ & $96.7 \mathrm{~kg} / \mathrm{hr}$ \\
\hline \multirow{5}{*}{ 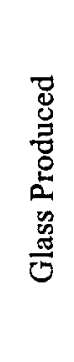 } & Poured & $2301 \mathrm{~kg}$ & $2594 \mathrm{~kg}$ & $6180 \mathrm{~kg}$ & $3465 \mathrm{~kg}$ \\
\hline & Average Rate ${ }^{s}$ & $278 \mathrm{~kg} / \mathrm{m}^{2} /$ day & $242 \mathrm{~kg} / \mathrm{m}^{2} /$ day & $1084 \mathrm{~kg} / \mathrm{m}^{2} /$ day & $579 \mathrm{~kg} / \mathrm{m}^{2} /$ day \\
\hline & Average Rate ${ }^{*}$ & $272 \mathrm{~kg} / \mathrm{m}^{2} /$ day & $242 \mathrm{~kg} / \mathrm{m}^{2} /$ day & $976 \mathrm{~kg} / \mathrm{m}^{2} /$ day & $561 \mathrm{~kg} / \mathrm{m}^{2} /$ day \\
\hline & Steady State Rate ${ }^{*}$ & $255 \mathrm{~kg} / \mathrm{m}^{2} /$ day & $205 \mathrm{~kg} / \mathrm{m}^{2} /$ day & $980 \mathrm{~kg} / \mathrm{m}^{2} /$ day & $540 \mathrm{~kg} / \mathrm{m}^{2} /$ day \\
\hline & Average Power Use & $\begin{array}{r}6.1 \mathrm{~kW} . \mathrm{hr} / \\
\mathrm{Kg} \text { glass }\end{array}$ & $\begin{array}{r}5.7 \mathrm{~kW} . \mathrm{hr} / \\
\mathrm{kg} \text { glass }\end{array}$ & $\begin{array}{r}3.9 \mathrm{~kW} . \mathrm{hr} / \\
\mathrm{kg} \text { glass }\end{array}$ & $\begin{array}{r}4.7 \mathrm{~kW} . \mathrm{hr} / \\
\mathrm{kg} \text { glass }\end{array}$ \\
\hline
\end{tabular}

$\$$ - Rates calculates from glass poured.

*- Rates calculated from feed data.

Note: Rates do not take into account the time for water feeding and cold cap burn-off. 
Table 1.3. Summary of Test Conditions and Results.

\begin{tabular}{|c|c|c|c|c|c|}
\hline \multicolumn{2}{|c|}{ Test \# } & 5 & 6 & 7 & 8 \\
\hline \multirow{3}{*}{$\stackrel{\mathscr{\Xi}}{\Xi}$} & Feed Start & $9 / 25 / 0113: 17$ & $10 / 11 / 0108: 21$ & $10 / 22 / 01 \quad 14: 12$ & $10 / 29 / 0113: 45$ \\
\hline & Feed End & $10 / 1 / 0113: 37$ & $10 / 17 / 0119: 24$ & $10 / 27 / 01 \quad 14: 12$ & $11 / 2 / 0117: 45$ \\
\hline & Interval & $144.5 \mathrm{hr}$ & $155.0 \mathrm{hr}$ & $120.0 \mathrm{hr}$ & $100.0 \mathrm{hr}$ \\
\hline \multicolumn{2}{|r|}{ Water Feeding for Cold Cap } & $2.0 \mathrm{hr}$ & $2.2 \mathrm{hr}$ & $1.5 \mathrm{hr}$ & $1.1 \mathrm{hr}$ \\
\hline \multicolumn{2}{|c|}{ Slurry Feeding } & $142.5 \mathrm{hr}$ & $152.8 \mathrm{hr}$ & $118.5 \mathrm{hr}$ & $98.9 \mathrm{hr}$ \\
\hline \multicolumn{2}{|c|}{ Cold cap burn } & $6.4 \mathrm{hr}$ & $14.5 \mathrm{hr}$ & $4.3 \mathrm{hr}$ & $10.3 \mathrm{hr}$ \\
\hline \multicolumn{2}{|c|}{ Total } & $150.9 \mathrm{hr}$ & $169.5 \mathrm{hr}$ & $124.3 \mathrm{hr}$ & $110.3 \mathrm{hr}$ \\
\hline \multicolumn{2}{|c|}{ Bubbling Rate } & $<4 \mathrm{lpm}$ & $<4 \mathrm{lpm}$ & $60 \mathrm{lpm}$ deep & $<4 \mathrm{lpm}$ \\
\hline \multicolumn{2}{|c|}{ Electrode Firing } & Side to Bottom & Side to Bottom & Side to Side & Side to Bottom \\
\hline \multirow{5}{*}{ 苞 } & Characteristics & $\begin{array}{l}\text { Nitrated + sugar } \\
\text { (sugar ratio }=0.5 \text { ) }\end{array}$ & $\begin{array}{l}\text { Frit }+7.5 \mathrm{~g} \\
\text { sugar } / 1\end{array}$ & $\begin{array}{l}\text { Frit }+7.5 \mathrm{~g} \\
\text { sugar } / 1\end{array}$ & $\begin{array}{l}\text { Nitrated + sugar } \\
\text { (sugar ratio }=0.7 \text { ) }\end{array}$ \\
\hline & Used & $4932 \mathrm{~kg}$ & $5417 \mathrm{~kg}$ & $16240 \mathrm{~kg}$ & $3426 \mathrm{~kg}$ \\
\hline & \multirow{2}{*}{ Glass yield (target) } & $420 \mathrm{~g} / 1 \mathrm{feed}$ & $480 \mathrm{~g} / \mathrm{l} \mathrm{feed}$ & $480 \mathrm{~g} / \mathrm{l} \mathrm{feed}$ & $420 \mathrm{~g} / \mathrm{l}$ feed \\
\hline & & $0.31 \mathrm{~kg} / \mathrm{kg}$ feed & $0.35 \mathrm{~kg} / \mathrm{kg}$ feed & $0.35 \mathrm{~kg} / \mathrm{kg}$ feed & $0.31 \mathrm{~kg} / \mathrm{kg}$ feed \\
\hline & Average Rate & $34.6 \mathrm{~kg} / \mathrm{hr}$ & $35.5 \mathrm{~kg} / \mathrm{hr}$ & $137.0 \mathrm{~kg} / \mathrm{hr}$ & $34.6 \mathrm{~kg} / \mathrm{hr}$ \\
\hline \multirow{5}{*}{ 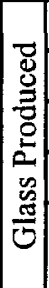 } & Poured & $1659 \mathrm{~kg}$ & $1901 \mathrm{~kg}$ & $5976 \mathrm{~kg}$ & $944 \mathrm{~kg}$ \\
\hline & Average Rate ${ }^{S}$ & $233 \mathrm{~kg} / \mathrm{m}^{2} /$ day & $249 \mathrm{~kg} / \mathrm{m}^{2} /$ day & $1009 \mathrm{~kg} / \mathrm{m}^{2} / \mathrm{day}$ & $191 \mathrm{~kg} / \mathrm{m}^{2} /$ day \\
\hline & Average Rate & $215 \mathrm{~kg} / \mathrm{m}^{2} /$ day & $248 \mathrm{~kg} / \mathrm{m}^{2} /$ day & $959 \mathrm{~kg} / \mathrm{m}^{2} /$ day & $215 \mathrm{~kg} / \mathrm{m}^{2} / \mathrm{day}$ \\
\hline & Steady State Rate & $220 \mathrm{~kg} / \mathrm{m}^{2} /$ day & $210 \mathrm{~kg} / \mathrm{m}^{2} /$ day & $1100 \mathrm{~kg} / \mathrm{m}^{2} /$ day & $200 \mathrm{~kg} / \mathrm{m}^{2} /$ day \\
\hline & Average Power Use & $\begin{array}{l}6.5 \mathrm{~kW} \cdot \mathrm{hr} / \mathrm{kg} \\
\text { glass }\end{array}$ & $\begin{array}{l}6.0 \mathrm{~kW} . \mathrm{hr} / \mathrm{kg} \\
\text { glass }\end{array}$ & $\begin{array}{l}3.3 \mathrm{~kW} . \mathrm{hr} / \mathrm{kg} \\
\text { glass }\end{array}$ & $\begin{array}{l}6.3 \mathrm{~kW} \cdot \mathrm{hr} / \mathrm{kg} \\
\text { glass }\end{array}$ \\
\hline
\end{tabular}

$\$$ - Rates calculated from glass poured.

*- Rates calculated from feed data.

Note: Rates do not take into account the time for water feeding and cold cap burn-off. 
The Catholic University of America

Vitreous State Laboratory
DuraMelter 1200 Tests with AZ-101HLW Simulants Final Report, VSL-02R0100-2, Rev.1

Table 1.4. Summary of Test Conditions and Results for Test 9 (Both Feed Tubes; Continuous Instead of Pulsed Feed).

\begin{tabular}{|c|c|c|c|}
\hline \multicolumn{2}{|c|}{ Test \# } & $9 \mathrm{a}$ & $9 b$ \\
\hline \multirow{3}{*}{ : } & Feed Start & $11 / 5 / 114: 20$ & $11 / 8 / 111: 00$ \\
\hline & Feed End & $11 / 8 / 111: 00$ & $11 / 10 / 117: 00$ \\
\hline & Interval & $68.6 \mathrm{hr}$ & $54.0 \mathrm{hr}$ \\
\hline \multicolumn{2}{|r|}{ Water Feeding for Cold Cap } & $1.1 \mathrm{hr}$ & NA \\
\hline \multicolumn{2}{|c|}{ Slurry Feeding } & $67.5 \mathrm{hr}$ & $54.0 \mathrm{hr}$ \\
\hline \multicolumn{2}{|c|}{ Cold cap burn } & NA & $6.9 \mathrm{hr}$ \\
\hline \multicolumn{2}{|c|}{ Total } & $68.6 \mathrm{hr}$ & $60.9 \mathrm{hr}$ \\
\hline \multicolumn{2}{|c|}{ Glass Temperature } & $1150^{\circ} \mathrm{C}$ & $1200^{\circ} \mathrm{C}$ \\
\hline \multicolumn{2}{|c|}{ Bubbling Rate } & \multicolumn{2}{|l|}{$<4 \mathrm{lpm}$} \\
\hline \multicolumn{2}{|c|}{ Electrode Firing } & \multicolumn{2}{|l|}{ Side to Side } \\
\hline \multirow{5}{*}{ ष्ठ } & Characteristics & \multicolumn{2}{|c|}{ Nominal + 10 g sugar $/ 1$} \\
\hline & \multirow{2}{*}{ Glass yield (target) } & \multicolumn{2}{|l|}{$570 \mathrm{~g} / \mathrm{l}$} \\
\hline & & \multicolumn{2}{|l|}{$0.38 \mathrm{~kg} / \mathrm{kg}$} \\
\hline & Used & $2028 \mathrm{~kg}$ & $2125 \mathrm{~kg}$ \\
\hline & Average Rate & $30.0 \mathrm{~kg} / \mathrm{hr}$ & $39.4 \mathrm{~kg} / \mathrm{hr}$ \\
\hline \multirow{5}{*}{ 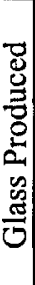 } & Poured & $859 \mathrm{~kg}$ & $847 \mathrm{~kg}$ \\
\hline & Average Rate ${ }^{S}$ & $255 \mathrm{~kg} / \mathrm{m}^{2} /$ day & $313 \mathrm{~kg} / \mathrm{m}^{2} /$ day \\
\hline & Average Rate $^{*}$ & $228 \mathrm{~kg} / \mathrm{m}^{2} /$ day & $299 \mathrm{~kg} / \mathrm{m}^{2} /$ day \\
\hline & Steady State Rate & $230 \mathrm{~kg} / \mathrm{m}^{2} /$ day & $310 \mathrm{~kg} / \mathrm{m}^{2} /$ day \\
\hline & Average Power Use & $\begin{array}{l}6.3 \mathrm{~kW} . \mathrm{hr} / \mathrm{kg} \\
\text { glass }\end{array}$ & $\begin{array}{l}5.4 \mathrm{~kW} \cdot \mathrm{hr} / \mathrm{kg} \\
\text { glass }\end{array}$ \\
\hline
\end{tabular}

$\$$ - Rates calculated from glass poured.

*- Rates calculated from feed data.

Note: Rates do not take into account the time for water feeding and cold cap burn. 
The Catholic University of America Vitreous State Laboratory
DuraMelter 1200 Tests with AZ-101HLW Simulants Final Report, VSL-02R0100-2, Rev.1

Table 2.1. Composition Summary of AZ-101 Waste, LAW Pretreatment Products, AZ-101 Simulant Plus Pretreatment Products, Glass Additives, and HLW98-31 Glass.

\begin{tabular}{|c|c|c|c|c|c|}
\hline & $\begin{array}{c}\text { AZ-101 Envelope } \\
\text { D Waste }\end{array}$ & $\begin{array}{c}\text { Pretreatment } \\
\text { Products(as wt } \% \\
\text { of total AZ-101 } \\
\text { oxides) }\end{array}$ & $\begin{array}{c}\text { AZ-101 Simulant } \\
\text { (Envelope D + } \\
\text { Pretreatment } \\
\text { Products) }\end{array}$ & $\begin{array}{l}\text { Additives (as wt } \% \\
\text { of glass) }\end{array}$ & $\begin{array}{l}\text { HLW98-31 Glass } \\
\text { Composition }\end{array}$ \\
\hline $\mathrm{Al}_{2} \mathrm{O}_{3}$ & $27.41 \%$ & & $24.27 \%$ & & $7.40 \%$ \\
\hline $\mathrm{As}_{2} \mathrm{O}_{3}$ & $0.15 \%$ & & $0.13 \%$ & & $0.04 \%$ \\
\hline $\mathrm{B}_{2} \mathrm{O}_{3}$ & & & & $10.00 \%$ & $10.00 \%$ \\
\hline $\mathrm{BaO}$ & $0.16 \%$ & & $0.14 \%$ & & $0.04 \%$ \\
\hline $\mathrm{CaO}$ & $0.91 \%$ & & $0.81 \%$ & & $0.25 \%$ \\
\hline $\mathrm{CdO}$ & $1.38 \%$ & & $1.22 \%$ & & $0.37 \%$ \\
\hline $\mathrm{CeO}_{2}$ & $0.31 \%$ & & $0.27 \%$ & & $0.08 \%$ \\
\hline $\mathrm{Cl}$ & $0.03 \%$ & & $0.02 \%$ & & $0.01 \%$ \\
\hline $\mathrm{Cr}_{2} \mathrm{O}_{3}$ & $0.16 \%$ & & $0.14 \%$ & & $0.04 \%$ \\
\hline $\mathrm{Cs}_{2} \mathrm{O}$ & $0.01 \%$ & $0.3 \%$ & $0.27 \%$ & & $0.08 \%$ \\
\hline $\mathrm{CuO}$ & $0.10 \%$ & & $0.09 \%$ & & $0.03 \%$ \\
\hline $\mathrm{F}$ & $0.14 \%$ & & $0.12 \%$ & & $0.04 \%$ \\
\hline $\mathrm{Fe}_{2} \mathrm{O}_{3}$ & $38.49 \%$ & & $34.08 \%$ & & $10.39 \%$ \\
\hline $\mathrm{K}_{2} \mathrm{O}$ & $0.63 \%$ & & $0.55 \%$ & & $0.17 \%$ \\
\hline $\mathrm{La}_{2} \mathrm{O}_{3}$ & $1.20 \%$ & & $1.06 \%$ & & $0.32 \%$ \\
\hline $\mathrm{Li}_{2} \mathrm{O}$ & & & & $6.00 \%$ & $6.00 \%$ \\
\hline $\mathrm{MgO}$ & $0.24 \%$ & & $0.21 \%$ & & $0.06 \%$ \\
\hline $\mathrm{MnO}$ & $7.76 \%$ & $3.5 \%$ & $9.94 \%$ & & $3.03 \%$ \\
\hline $\mathrm{Na}_{2} \mathrm{O}$ & $1.46 \%$ & $0.8 \%$ & $1.96 \%$ & $6.00 \%$ & $6.59 \%$ \\
\hline $\mathrm{NiO}$ & $1.99 \%$ & & $1.76 \%$ & & $0.54 \%$ \\
\hline $\mathrm{P}_{2} \mathrm{O}_{5}$ & $0.47 \%$ & & $0.42 \%$ & & $0.13 \%$ \\
\hline $\mathrm{PbO}$ & $0.56 \%$ & & $0.50 \%$ & & $0.15 \%$ \\
\hline $\mathrm{SO}_{3}$ & $0.93 \%$ & & $0.82 \%$ & & $0.25 \%$ \\
\hline $\mathrm{Sb}_{2} \mathrm{O}_{5}$ & $0.78 \%$ & & $0.69 \%$ & & $0.21 \%$ \\
\hline $\mathrm{SeO}_{2}$ & $0.55 \%$ & & $0.49 \%$ & & $0.15 \%$ \\
\hline $\mathrm{SiO}_{2}$ & $0.08 \%$ & & $0.07 \%$ & $45.51 \%$ & $45.53 \%$ \\
\hline $\mathrm{SrO}$ & $0.16 \%$ & $8.6 \%$ & $7.60 \%$ & & $2.32 \%$ \\
\hline $\mathrm{TeO}_{2}$ & $0.53 \%$ & & $0.47 \%$ & & $0.14 \%$ \\
\hline $\mathrm{TiO}_{2}$ & $0.23 \%$ & & $0.21 \%$ & & $0.06 \%$ \\
\hline $\mathrm{ZnO}$ & & & & $2.00 \%$ & $2.00 \%$ \\
\hline $\mathrm{ZrO}_{2}$ & $13.19 \%$ & & $11.68 \%$ & & $3.56 \%$ \\
\hline TOTAL & $100 \%$ & $13.16 \%$ & $100 \%$ & $69.51 \%$ & $100 \%$ \\
\hline Total Oxides (kg) & 82,997 & 10,920 & 93,917 & 214,108 & 308,025 \\
\hline \multicolumn{6}{|l|}{$\begin{array}{l}\text { Volatiles,g/100 g } \\
\text { oxides }\end{array}$} \\
\hline $\mathrm{CO}_{3}$ & 0.00 & $4.96^{*}$ & 3.00 & & \\
\hline $\mathrm{NO}_{2}$ & 1.08 & & 0.95 & & \\
\hline $\mathrm{NO}_{3}$ & 0.61 & 1.63 & 1.97 & & \\
\hline TOC & 1.50 & & 1.32 & & \\
\hline
\end{tabular}


The Catholic University of America Vitreous State Laboratory
DuraMelter 1200 Tests with AZ-101HLW Simulants Final Report, VSL-02R0100-2, Rev.1

Table 2.2. Chemical Compositions of the AZ-101 Simulant (Plus Pretreatment Products) and the Corresponding Melter Feed to Produce 10 MT of HLW98-31 Glass.

\begin{tabular}{|c|c|c|c|}
\hline \multicolumn{2}{|c|}{ AZ-101+ Pretreatment Products } & \multicolumn{2}{|c|}{ Glass-Forming Additives } \\
\hline Starting Materials & Target Weight, kg & Starting Materials & Target Weight, kg \\
\hline $\mathrm{Al}(\mathrm{OH})_{3}$ & 1131.6 & & \\
\hline $\mathrm{Na}_{2} \mathrm{HAsO}_{4}$ & 7.5 & & \\
\hline $\mathrm{Ba}(\mathrm{OH})_{2} * 8 \mathrm{H}_{2} \mathrm{O}$ & 8.7 & & \\
\hline $\mathrm{CaCO}_{3}$ & 44.1 & & \\
\hline $\mathrm{CdO}$ & 37.2 & & \\
\hline $\mathrm{Ce}(\mathrm{OH})_{4}$ & 10.1 & & \\
\hline $\mathrm{Cr}_{2} \mathrm{O}_{3} * 1.5 \mathrm{H}_{2} \mathrm{O}$ & 5.2 & & \\
\hline $\mathrm{CsOH}(50 \%$ Solution) & 17.5 & & \\
\hline $\mathrm{CuSO}_{4} * 5 \mathrm{H}_{2} \mathrm{O}$ & 8.8 & & \\
\hline $\mathrm{NaF}$ & 8.4 & & \\
\hline $\mathrm{Fe}(\mathrm{OH})_{3}$ Slurry & 10553.5 & & \\
\hline $\mathrm{KNO}_{3}$ & 28.3 & & \\
\hline $\mathrm{La}(\mathrm{OH})_{3} * 3 \mathrm{H}_{2} \mathrm{O}$ & 48.5 & & \\
\hline $\mathrm{Li}_{2} \mathrm{CO}_{3}$ & & $\mathrm{Li}_{2} \mathrm{CO}_{3}$ & 1484.1 \\
\hline$\left(\mathrm{MgCO}_{3}\right)_{4}\left(\mathrm{Mg}(\mathrm{OH})_{2} * 5 \mathrm{H}_{2} \mathrm{O}\right)$ & 15.1 & & \\
\hline $\mathrm{MnO}_{2}$ & 371.5 & & - \\
\hline \multirow[t]{2}{*}{$\mathrm{NaOH}(50 \%$ Solution $)$} & 12.8 & & \\
\hline & & $\mathrm{Na}_{2} \mathrm{~B}_{4} \mathrm{O}_{7}^{*} 10 \mathrm{H}_{2} \mathrm{O}$ & 2738.7 \\
\hline \multicolumn{4}{|l|}{$\mathrm{Ni}\left(\mathrm{NO}_{3}\right)_{2} * 6 \mathrm{H}_{2} \mathrm{O}$} \\
\hline $\mathrm{Ni}(\mathrm{OH})_{2}$ & 66.7 & & \\
\hline $\mathrm{FePO}_{4}(80 \%)$ & 33.8 & & \\
\hline $\mathrm{PbCO}_{3}{ }^{*} \mathrm{~Pb}(\mathrm{OH})_{2}$ & 17.3 & & \\
\hline $\mathrm{Na}_{2} \mathrm{SO}_{4}$ & 39.4 & & \\
\hline $\mathrm{Sb}_{2} \mathrm{O}_{5}$ & 21.1 & & \\
\hline $\mathrm{SeO}_{2}$ & 14.9 & & \\
\hline $\mathrm{SiO}_{2}$ & 2.2 & $\mathrm{SiO}_{2}$ & 4551.8 \\
\hline $\mathrm{Sr}\left(\mathrm{NO}_{3}\right)_{2}$ & 57.3 & & \\
\hline $\mathrm{Sr}(\mathrm{OH})_{2} * 8 \mathrm{H}_{2} \mathrm{O}$ & 522.2 & & \\
\hline $\mathrm{TeO}_{2}$ & 14.2 & & \\
\hline $\mathrm{TiO}_{2}$ & 6.3 & & \\
\hline $\mathrm{ZnO}$ & & $\mathrm{ZnO}$ & 200.0 \\
\hline $\mathrm{ZrOOH}\left(\mathrm{CO}_{3}\right) 0.5(50 \%)$ & 570.9 & & \\
\hline $\mathrm{Zr}(\mathrm{OH})_{4}(50 \%)$ & 330.8 & & \\
\hline $\mathrm{KCl}$ & 1.5 & & \\
\hline $\mathrm{KI}$ & 13.1 & & \\
\hline $\mathrm{Na}_{2} \mathrm{CO}_{3}$ & & $\mathrm{Na}_{2} \mathrm{CO}_{3}$ & 264.3 \\
\hline $\mathrm{NaNO}_{2}$ & 63.6 & & \\
\hline \multicolumn{4}{|l|}{$\mathrm{NaNO}_{3}$} \\
\hline $\mathrm{C}_{2} \mathrm{H}_{2} \mathrm{O}_{4} * 2 \mathrm{H}_{2} \mathrm{O}$ & 208.1 & & \\
\hline $\mathrm{H}_{2} \mathrm{O}$ & 1897.6 & & \\
\hline TOTAL & $16,189.9$ & TOTAL & 9238.9 \\
\hline & & FEED TOTAL & $25,428.8$ \\
\hline
\end{tabular}


The Catholic University of America

Vitreous State Laboratory
DuraMelter 1200 Tests with AZ-101HLW Simulants

Final Report, VSL-02R0100-2, Rev.1

Table 2.3. Composition and Properties of the Glass Frit Used in Tests 6 and 7.

\begin{tabular}{|c|c|}
\hline \multicolumn{2}{|c|}{ Composition } \\
\hline Oxide & Weight \% \\
\hline $\mathrm{B}_{2} \mathrm{O}_{3}$ & 14.39 \\
\hline $\mathrm{Li}_{2} \mathrm{O}$ & 8.63 \\
\hline $\mathrm{Na}_{2} \mathrm{O}$ & 8.63 \\
\hline $\mathrm{SiO}_{2}$ & 65.47 \\
\hline $\mathrm{ZnO}$ & 2.88 \\
\hline \multicolumn{2}{|c|}{ Viscosity } \\
\hline Temperature $\left({ }^{\circ} \mathrm{C}\right)$ & Viscosity (poise) \\
\hline 950 & 231 \\
\hline 1000 & 128 \\
\hline 1050 & 77 \\
\hline 1100 & 50 \\
\hline 1150 & 34 \\
\hline 1200 & 24 \\
\hline Glass Transitio & Ire $=448 \pm 5^{\circ} \mathrm{C}$ \\
\hline
\end{tabular}


The Catholic University of America

Vitreous State Laboratory
DuraMelter 1200 Tests with AZ-101HLW Simulants Final Report, VSL-02R0100-2, Rev.I

Table 2.4. Measured Feed Sample Properties.

\begin{tabular}{|c|c|c|c|c|c|c|c|c|c|c|}
\hline \multirow{2}{*}{$\stackrel{\stackrel{*}{*}}{\stackrel{*}{*}}$} & \multirow[b]{2}{*}{$\begin{array}{c}\text { Sampling } \\
\text { Date }\end{array}$} & \multirow[b]{2}{*}{ Sample Name } & \multirow{2}{*}{$\begin{array}{l}\text { Wt\% } \\
\text { Water }\end{array}$} & \multirow{2}{*}{$\begin{array}{c}\text { Density } \\
(\mathrm{g} / \mathrm{ml})\end{array}$} & \multirow{2}{*}{$\begin{array}{c}\text { Glass } \\
\text { Yield } \\
(\mathrm{kg} / \mathrm{kg})\end{array}$} & \multirow{2}{*}{$\begin{array}{l}\text { Glass } \\
\text { Yield } \\
(\mathrm{g} / \mathrm{l})\end{array}$} & \multirow[b]{2}{*}{$\mathrm{pH}$} & \multirow{2}{*}{$\begin{array}{c}\text { Yield } \\
\text { Stress } \\
(\mathrm{Pa})\end{array}$} & \multicolumn{2}{|c|}{ Viscosity (P) } \\
\hline & & & & & & & & & @1/s & (a) $10 / \mathrm{s}$ \\
\hline \multirow{9}{*}{1} & $6 / 25 / 01$ & $12 \mathrm{~F}-\mathrm{F}-81 \mathrm{~A}$ & 51.5 & 1.44 & 0.393 & 566 & 9.77 & 0.8 & $\mathrm{NA}$ & $\mathrm{NA}$ \\
\hline & $6 / 26 / 01$ & $12 F-F-97 A$ & 51.5 & 1.44 & 0.398 & 573 & 9.79 & 2.0 & 23.1 & 2.8 \\
\hline & $6 / 28 / 01$ & $12 \mathrm{~F}-\mathrm{F}-146 \mathrm{~A}$ & 51.3 & 1.47 & 0.404 & 594 & 9.77 & $<2.0$ & 43.7 & 5.9 \\
\hline & $6 / 29 / 01$ & $12 G-F-6 A$ & 52.5 & 1.42 & 0.385 & 547 & 9.76 & 0.7 & 30.1 & 2.9 \\
\hline & $6 / 30 / 01$ & $12 \mathrm{G}-\mathrm{F}-33 \mathrm{~A}$ & 50.9 & 1.46 & 0.404 & 590 & 9.78 & 0.3 & 30.8 & 3.6 \\
\hline & $7 / 1 / 01$ & $12 \mathrm{G}-\mathrm{F}-53 \mathrm{~A}$ & 51.0 & 1.45 & 0.398 & 577 & 9.76 & 2.5 & 33.8 & 3.4 \\
\hline & $7 / 2 / 01$ & $12 \mathrm{G}-\mathrm{F}-71 \mathrm{~A}$ & 50.9 & 1.46 & 0.400 & 584 & 9.78 & 0.6 & 27.2 & 3.1 \\
\hline & \multicolumn{2}{|c|}{ Average } & 51.4 & 1.45 & 0.397 & 576 & 9.77 & 1.4 & 31.5 & 3.6 \\
\hline & \multicolumn{2}{|c|}{ Standard Deviation } & 0.5 & 0.02 & 0.006 & 15 & 0.01 & $\mathrm{NA}$ & $\mathrm{NA}$ & NA \\
\hline \multirow{11}{*}{2} & $7 / 24 / 01$ & $12 \mathrm{G}-\mathrm{F}-130 \mathrm{~A}$ & 52.1 & 1.42 & 0.384 & 545 & 9.73 & 7.7 & 42.9 & 6.1 \\
\hline & $7 / 25 / 01$ & $12 \mathrm{G}-\mathrm{F}-149 \mathrm{~A}$ & 52.7 & 1.42 & 0.383 & 544 & 9.73 & NA & NA & NA \\
\hline & $7 / 26 / 01$ & $12 \mathrm{H}-\mathrm{F}-32 \mathrm{~A}$ & 52.0 & 1.47 & 0.394 & 579 & 9.76 & NA & NA & NA \\
\hline & $7 / 27 / 01$ & $12 \mathrm{H}-\mathrm{F}-43 \mathrm{~A}$ & 52.2 & 1.46 & 0.393 & 574 & 9.74 & NA & NA & NA \\
\hline & $7 / 28 / 01$ & $12 \mathrm{I}-\mathrm{F}-22 \mathrm{~A}$ & 51.8 & 1.47 & 0.359 & 528 & 9.78 & NA & NA & NA \\
\hline & $7 / 29 / 01$ & $12 I-F-40 A$ & 52.3 & 1.44 & 0.390 & 562 & 9.78 & NA & NA & $\mathrm{NA}$ \\
\hline & $7 / 30 / 01$ & $12 \mathrm{I}-\mathrm{F}-57 \mathrm{~A}$ & 53.1 & 1.45 & 0.381 & 552 & 9.79 & NA & $\mathrm{NA}$ & NA \\
\hline & $7 / 31 / 01$ & $12 \mathrm{I}-\mathrm{F}-66 \mathrm{~A}$ & 51.7 & 1.47 & 0.395 & 581 & 9.77 & NA & $\mathrm{NA}$ & NA \\
\hline & $8 / 01 / 01$ & $12 \mathrm{I}-\mathrm{F}-82 \mathrm{~A}$ & 51.6 & 1.46 & 0.389 & 568 & 9.77 & 8.3 & 31.7 & 5.9 \\
\hline & \multicolumn{2}{|c|}{ Average } & 52.5 & 1.45 & 0.385 & 559 & 9.76 & NA & $\mathrm{NA}$ & NA \\
\hline & \multicolumn{2}{|c|}{ Standard Deviation } & 0.5 & 0.02 & 0.010 & 17 & 0.02 & $\mathrm{NA}$ & NA & $\mathrm{NA}$ \\
\hline \multirow{9}{*}{3} & $8 / 07 / 01$ & $12 \mathrm{~J}-\mathrm{F}-53 \mathrm{~A}$ & 63.6 & 1.33 & 0.287 & 382 & 9.73 & 1.8 & 8.0 & 0.99 \\
\hline & $8 / 07 / 01$ & $12 J-F-62 A$ & 63.0 & 1.34 & 0.311 & 417 & 9.72 & NA & $\mathrm{NA}$ & $\mathrm{NA}$ \\
\hline & $8 / 08 / 01$ & $12 \mathrm{~J}-\mathrm{F}-81 \mathrm{~A}$ & 61.6 & 1.31 & 0.311 & 407 & 9.76 & NA & NA & NA \\
\hline & $8 / 09 / 01$ & $12 \mathrm{~J}-\mathrm{F}-99 \mathrm{~A}$ & 64.8 & 1.29 & 0.290 & 374 & 9.71 & $\mathrm{NA}$ & NA & NA \\
\hline & $8 / 09 / 01$ & $12 \mathrm{~J}-\mathrm{F}-112 \mathrm{~A}$ & 63.9 & 1.30 & 0.297 & 386 & 9.71 & $\mathrm{NA}$ & $\mathrm{NA}$ & $\mathrm{NA}$ \\
\hline & $8 / 10 / 01$ & $12 \mathrm{~J}-\mathrm{F}-131 \mathrm{~A}$ & 63.1 & 1.32 & 0.309 & 408 & 9.78 & NA & $\mathrm{NA}$ & NA \\
\hline & $8 / 11 / 01$ & $12 \mathrm{~J}-\mathrm{F}-149 \mathrm{~A}$ & 64.0 & 1.33 & 0.303 & 403 & 9.76 & 1.8 & 7.9 & 0.87 \\
\hline & \multicolumn{2}{|c|}{ Average } & 63.4 & 1.32 & 0.301 & 397 & 9.74 & NA & NA & NA \\
\hline & \multicolumn{2}{|c|}{ Standard Deviation } & 0.9 & 0.02 & 0.009 & 15 & 0.03 & NA & NA & $\mathrm{NA}$ \\
\hline \multirow[t]{6}{*}{4} & $8 / 21 / 01$ & $12 \mathrm{~K}-\mathrm{F}-54 \mathrm{~A}$ & 67.1 & 1.28 & 0.267 & 342 & 9.76 & 0.9 & 4.5 & 0.55 \\
\hline & $8 / 22 / 01$ & $12 \mathrm{~K}-\mathrm{F}-83 \mathrm{~A}$ & 66.6 & 1.32 & 0.296 & 391 & 9.77 & NA & $\overline{\mathrm{NA}}$ & $\mathrm{NA}$ \\
\hline & $8 / 23 / 01$ & $12 \mathrm{~K}-\mathrm{F}-104 \mathrm{~A}$ & 63.7 & 1.30 & 0.297 & 386 & 9.73 & NA & NA & NA \\
\hline & $8 / 24 / 01$ & $12 \mathrm{~K}-\mathrm{F}-125 \mathrm{~A}$ & 63.7 & 1.32 & 0.294 & 388 & 9.73 & 1.8 & 7.0 & 0.80 \\
\hline & \multicolumn{2}{|c|}{ Average } & 65.3 & 1.31 & 0.289 & 377 & 9.75 & NA & $\mathrm{NA}$ & NA \\
\hline & \multicolumn{2}{|c|}{ Standard Deviation } & 1.6 & 0.02 & 0.012 & 20 & 0.02 & NA & $\mathrm{NA}$ & NA \\
\hline
\end{tabular}


Table 2.4. Measured Feed Sample Properties (continued).

\begin{tabular}{|c|c|c|c|c|c|c|c|c|c|c|}
\hline \multirow{2}{*}{$\begin{array}{l}* \\
\stackrel{*}{*} \\
\stackrel{5}{*}\end{array}$} & \multirow[b]{2}{*}{$\begin{array}{l}\text { Sampling } \\
\text { Date }\end{array}$} & \multirow[b]{2}{*}{ Sample Name } & \multirow{2}{*}{$\begin{array}{l}\text { Wt\% } \\
\text { Water }\end{array}$} & \multirow{2}{*}{$\begin{array}{c}\text { Density } \\
(\mathrm{g} / \mathrm{ml})\end{array}$} & \multirow{2}{*}{$\begin{array}{c}\text { Glass } \\
\text { Yield } \\
(\mathrm{kg} / \mathrm{kg})\end{array}$} & \multirow{2}{*}{$\begin{array}{l}\text { Glass } \\
\text { Yield } \\
(\mathrm{g} / \mathrm{l}) \\
\end{array}$} & \multirow[b]{2}{*}{$\mathrm{pH}$} & \multirow{2}{*}{$\begin{array}{c}\text { Yield } \\
\text { Stress } \\
(\mathrm{Pa})\end{array}$} & \multicolumn{2}{|c|}{ Viscosity (P) } \\
\hline & & & & & & & & & $\stackrel{@}{1 / s}$ & $\begin{array}{c}@ \\
10 / \mathrm{s}\end{array}$ \\
\hline \multirow{10}{*}{5} & $9 / 24 / 01$ & $12 \mathrm{~K}-\mathrm{F}-144 \mathrm{~A}$ & 50.4 & 1.44 & 0.334 & 481 & 7.33 & 1.2 & 9.0 & 0.96 \\
\hline & $09 / 24 / 01$ & $12 \mathrm{~K}-\mathrm{F}-144 \mathrm{~A}$ & 50.4 & 1.44 & 0.334 & 481 & 7.33 & NA & NA & $\mathrm{NA}$ \\
\hline & $09 / 26 / 01$ & 12L-F-30A & 45.6 & 1.44 & 0.323 & 465 & 5.48 & NA & NA & $\mathrm{NA}$ \\
\hline & $09 / 27 / 01$ & $12 \mathrm{~L}-\mathrm{F}-49 \mathrm{~A}$ & 51.2 & 1.45 & 0.322 & 467 & 5.38 & NA & NA & NA \\
\hline & $09 / 28 / 01$ & 12L-F-70A & 50.8 & 1.43 & 0.320 & 457 & 5.32 & NA & $\mathrm{NA}$ & NA \\
\hline & $09 / 29 / 01$ & $12 \mathrm{~L}-\mathrm{F}-80 \mathrm{~A}$ & 54.1 & 1.40 & 0.291 & 407 & 5.32 & NA & NA & $\mathrm{NA}$ \\
\hline & $09 / 30 / 01$ & 12L-F-107A & 52.4 & 1.43 & 0.313 & 448 & 5.14 & NA & NA & NA \\
\hline & $10 / 01 / 01$ & $12 \mathrm{~L}-\mathrm{F}-119 \mathrm{~A}$ & 52.5 & 1.44 & 0.314 & 452 & 5.15 & $\mathrm{NA}$ & $\mathrm{NA}$ & NA \\
\hline & \multicolumn{2}{|c|}{ Average } & 51.6 & 1.43 & 0.317 & 454 & 5.59 & NA & NA & NA \\
\hline & \multicolumn{2}{|c|}{ Standard Deviation } & 1.3 & 0.02 & 0.012 & 22 & 0.72 & $\mathrm{NA}$ & NA & NA \\
\hline \multirow{24}{*}{6} & $10 / 11 / 01$ & $12 \mathrm{~L}-\mathrm{F}-147 \mathrm{~A}$ & 62.3 & 1.29 & 0.331 & 427 & 10.01 & 1.0 & 5.3 & 0.70 \\
\hline & $10 / 11 / 01$ & 12L-F-148A & 60.6 & 1.31 & 0.345 & 451 & 9.91 & NA & NA & NA \\
\hline & $10 / 11 / 01$ & 12L-F-155A & 62.0 & 1.31 & 0.352 & 461 & 9.90 & NA & NA & NA \\
\hline & $10 / 12 / 01$ & $12 M-F-6 A$ & 63.5 & 1.31 & 0.349 & 457 & 9.91 & $\mathrm{NA}$ & NA & NA \\
\hline & $10 / 12 / 01$ & $12 \mathrm{M}-\mathrm{F}-9 \mathrm{~A}$ & 60.0 & 1.31 & 0.337 & 442 & 9.91 & NA & NA & $\overline{\mathrm{NA}}$ \\
\hline & $10 / 12 / 01$ & $12 \mathrm{M}-\mathrm{F}-11 \mathrm{~A}$ & 60.3 & 1.31 & 0.347 & 454 & 9.81 & $\mathrm{NA}$ & NA & NA \\
\hline & $10 / 12 / 01$ & $12 \mathrm{M}-\mathrm{F}-19 \mathrm{~A}$ & 60.6 & 1.30 & 0.347 & 452 & 9.89 & NA & NA & NA \\
\hline & $10 / 12 / 01$ & $12 \mathrm{M}-\mathrm{F}-21 \mathrm{~A}$ & 60.3 & 1.30 & 0.347 & 451 & 9.94 & NA & NA & NA \\
\hline & $10 / 12 / 01$ & $12 \mathrm{M}-\mathrm{F}-22 \mathrm{~A}$ & 61.0 & 1.29 & 0.348 & 448 & 9.94 & NA & $\mathrm{NA}$ & NA \\
\hline & $10 / 12 / 01$ & $12 \mathrm{M}-\mathrm{F}-24 \mathrm{~A}$ & 60.0 & 1.30 & 0.314 & 408 & 9.92 & NA & NA & NA \\
\hline & $10 / 13 / 01$ & $12 \mathrm{M}-\mathrm{F}-30 \mathrm{~A}$ & 60.2 & 1.33 & 0.352 & 466 & 9.95 & NA & NA & NA \\
\hline & $10 / 13 / 01$ & $12 \mathrm{M}-\mathrm{F}-32 \mathrm{~A}$ & 61.1 & 1.29 & 0.340 & 439 & 9.96 & NA & NA & $\mathrm{NA}$ \\
\hline & $10 / 13 / 01$ & $12 \mathrm{M}-\mathrm{F}-33 \mathrm{~A}$ & 62.0 & 1.29 & 0.328 & 424 & 9.97 & $\mathrm{NA}$ & $\mathrm{NA}$ & $\mathrm{NA}$ \\
\hline & $10 / 13 / 01$ & $12 \mathrm{M}-\mathrm{F}-39 \mathrm{~A}$ & 61.0 & 1.30 & 0.339 & 440 & 9.94 & NA & NA & $\mathrm{NA}$ \\
\hline & $10 / 13 / 01$ & $12 \mathrm{M}-\mathrm{F}-40 \mathrm{~A}$ & 60.7 & 1.32 & 0.346 & 456 & 9.95 & $\mathrm{NA}$ & $\mathrm{NA}$ & $\mathrm{NA}$ \\
\hline & $10 / 13 / 01$ & $12 \mathrm{M}-\mathrm{F}-43 \mathrm{~A}$ & 60.5 & 1.30 & 0.344 & 447 & 9.92 & $\mathrm{NA}$ & $\mathrm{NA}$ & $\mathrm{NA}$ \\
\hline & $10 / 14 / 01$ & $12 \mathrm{M}-\mathrm{F}-49 \mathrm{~A}$ & 60.2 & 1.32 & 0.354 & 466 & 9.92 & $\mathrm{NA}$ & $\mathrm{NA}$ & $\mathrm{NA}$ \\
\hline & $10 / 14 / 01$ & $12 \mathrm{M}-\mathrm{F}-50 \mathrm{~A}$ & 62.1 & 1.30 & 0.332 & 431 & 9.92 & NA & $\mathrm{NA}$ & $\mathrm{NA}$ \\
\hline & $10 / 14 / 01$ & $12 \mathrm{M}-\mathrm{F}-54 \mathrm{~A}$ & 60.8 & 1.32 & 0.350 & 462 & 9.89 & $\mathrm{NA}$ & $\mathrm{NA}$ & $\mathrm{NA}$ \\
\hline & $10 / 15 / 01$ & $12 \mathrm{M}-\mathrm{F}-71 \mathrm{~A}$ & 60.7 & 1.29 & 0.340 & 440 & 9.85 & $\mathrm{NA}$ & $\mathrm{NA}$ & $\mathrm{NA}$ \\
\hline & $10 / 16 / 01$ & $12 \mathrm{M}-\mathrm{F}-87 \mathrm{~A}$ & 60.9 & 1.32 & 0.346 & 457 & 8.85 & $\mathrm{NA}$ & $\mathrm{NA}$ & $\mathrm{NA}$ \\
\hline & $10 / 17 / 01$ & $12 \mathrm{M}-\mathrm{F}-100 \mathrm{~A}$ & 60.8 & 1.33 & 0.351 & 467 & 8.66 & NA & $\mathrm{NA}$ & NA \\
\hline & \multicolumn{2}{|c|}{ Average } & 61.0 & 1.31 & 0.343 & 448 & 9.84 & NA & $\mathrm{NA}$ & $\mathrm{NA}$ \\
\hline & \multicolumn{2}{|c|}{ Standard Deviation } & 0.9 & 0.01 & 0.009 & 15 & 0.26 & NA & $\mathrm{NA}$ & $\mathrm{NA}$ \\
\hline
\end{tabular}


Table 2.4. Measured Feed Sample Properties (continued).

\begin{tabular}{|c|c|c|c|c|c|c|c|c|c|c|}
\hline \multirow{2}{*}{$\stackrel{\#}{\stackrel{\#}{*}}$} & \multirow[b]{2}{*}{$\begin{array}{l}\text { Sampling } \\
\text { Date }\end{array}$} & \multirow[b]{2}{*}{ Sample Name } & \multirow{2}{*}{$\begin{array}{l}\text { Wt\% } \\
\text { Water }\end{array}$} & \multirow{2}{*}{$\begin{array}{c}\text { Density } \\
(\mathrm{g} / \mathrm{ml})\end{array}$} & \multirow{2}{*}{$\begin{array}{c}\text { Glass } \\
\text { Yield } \\
(\mathrm{kg} / \mathrm{kg})\end{array}$} & \multirow{2}{*}{$\begin{array}{l}\text { Glass } \\
\text { Yield } \\
(\mathrm{g} / \mathrm{l})\end{array}$} & \multirow[b]{2}{*}{$\mathrm{pH}$} & \multirow{2}{*}{$\begin{array}{c}\text { Yield } \\
\text { Stress } \\
(\mathrm{Pa})\end{array}$} & \multicolumn{2}{|c|}{ Viscosity (P) } \\
\hline & & & & & & & & & $\begin{array}{c}@ \\
1 / \mathrm{s}\end{array}$ & $\begin{array}{c}(9) \\
10 / \mathrm{s}\end{array}$ \\
\hline \multirow{8}{*}{7} & $10 / 22 / 01$ & $12 \mathrm{M}-\mathrm{F}-143 \mathrm{~A}$ & 54.0 & 1.32 & 0.363 & 479 & 9.39 & 0.7 & 4.1 & 0.49 \\
\hline & $10 / 23 / 01$ & $12 \mathrm{~N}-\mathrm{F}-6 \mathrm{~A}$ & 59.9 & 1.35 & 0.358 & 483 & 9.38 & $\mathrm{NA}$ & NA & $\mathrm{NA}$ \\
\hline & $10 / 24 / 01$ & $12 \mathrm{~N}-\mathrm{F}-38 \mathrm{~A}$ & 60.4 & 1.34 & 0.344 & 461 & 9.51 & $\mathrm{NA}$ & $\mathrm{NA}$ & $\mathrm{NA}$ \\
\hline & $10 / 25 / 01$ & $12 \mathrm{~N}-\mathrm{F}-64 \mathrm{~A}$ & 60.0 & 1.34 & 0.356 & 477 & 9.00 & $\mathrm{NA}$ & $\mathrm{NA}$ & $\mathrm{NA}$ \\
\hline & $10 / 26 / 01$ & $12 \mathrm{~N}-\mathrm{F}-99 \mathrm{~A}$ & 60.2 & 1.33 & 0.353 & 470 & 9.00 & $\mathrm{NA}$ & $\mathrm{NA}$ & $\mathrm{NA}$ \\
\hline & $10 / 27 / 01$ & $12 \mathrm{~N}-\mathrm{F}-127 \mathrm{~A}$ & 61.6 & 1.32 & 0.350 & 462 & 8.58 & 0.7 & 4.0 & 0.53 \\
\hline & \multicolumn{2}{|c|}{ Average } & 60.2 & 1.33 & 0.354 & 472 & 9.14 & $\mathrm{NA}$ & NA & $\mathrm{NA}$ \\
\hline & \multicolumn{2}{|c|}{ Standard Deviation } & 0.8 & 0.01 & 0.006 & 9 & 0.32 & NA & NA & NA \\
\hline \multirow{6}{*}{8} & $10 / 30 / 01$ & $12 \mathrm{O}-\mathrm{F}-16 \mathrm{~A}$ & 51.7 & 1.42 & 0.309 & 439 & 4.94 & $\mathrm{ND}$ & 0.18 & 0.07 \\
\hline & $10 / 31 / 01$ & $12 \mathrm{O}-\mathrm{F}-31 \mathrm{~A}$ & 52.2 & 1.42 & 0.298 & 423 & 5.00 & $\mathrm{NA}$ & $\mathrm{NA}$ & $\mathrm{NA}$ \\
\hline & $11 / 01 / 01$ & $12 \mathrm{O}-\mathrm{F}-49 \mathrm{~A}$ & 54.2 & 1.40 & 0.288 & 403 & 4.95 & NA & $\mathrm{NA}$ & NA \\
\hline & $11 / 02 / 01$ & $120-F-68 A$ & 48.0 & 1.40 & 0.309 & 432 & 4.83 & ND & 0.11 & 0.05 \\
\hline & \multicolumn{2}{|c|}{ Average } & 51.5 & 1.41 & 0.301 & 424 & 4.93 & NA & $\mathrm{NA}$ & NA \\
\hline & \multicolumn{2}{|c|}{ Standard Deviation } & 2.3 & 0.01 & 0.009 & 14 & 0.06 & NA & NA & NA \\
\hline \multirow{7}{*}{9} & $11 / 06 / 01$ & $12 \mathrm{O}-\mathrm{F}-106 \mathrm{~A}$ & 50.7 & 1.47 & 0.404 & 593 & 9.74 & 6.3 & 28.6 & 4.3 \\
\hline & $11 / 07 / 01$ & $12 \mathrm{O}-\mathrm{F}-125 \mathrm{~A}$ & 50.6 & 1.46 & 0.390 & 569 & 9.78 & NA & NA & NA \\
\hline & $11 / 08 / 01$ & $12 \mathrm{O}-\mathrm{F}-144 \mathrm{~A}$ & 50.5 & 1.49 & 0.401 & 598 & 9.77 & $\mathrm{NA}$ & NA & $\mathrm{NA}$ \\
\hline & $11 / 09 / 01$ & 12P-F-13A & 50.6 & 1.48 & 0.395 & 584 & 9.81 & $\mathrm{NA}$ & $\mathrm{NA}$ & $\mathrm{NA}$ \\
\hline & $11 / 10 / 01$ & $12 \mathrm{P}-\mathrm{F}-41 \mathrm{~A}$ & 52.1 & 1.45 & 0.395 & 573 & 9.83 & 9.7 & 45.7 & 4.5 \\
\hline & \multicolumn{2}{|c|}{ Average } & 50.9 & 1.47 & 0.397 & 584 & 9.79 & $\mathrm{NA}$ & NA & $\mathrm{NA}$ \\
\hline & \multicolumn{2}{|c|}{ Standard Deviation } & 0.6 & 0.01 & 0.005 & 11 & 0.03 & $\mathrm{NA}$ & NA & NA \\
\hline
\end{tabular}

ND: Not Detected. NA: Not Analyzed.

Table 2.5. Averages for Measured Feed Sample Properties.

\begin{tabular}{|c|c|c|c|c|c|c|c|}
\hline \multirow{2}{*}{ Feed Type } & \multirow{2}{*}{ Test \# } & \multirow{2}{*}{$\begin{array}{l}\text { Wt\% } \\
\text { Water }\end{array}$} & \multirow{2}{*}{$\begin{array}{l}\text { Density } \\
(\mathrm{g} / \mathrm{ml})\end{array}$} & \multirow{2}{*}{$\mathrm{pH}$} & \multicolumn{2}{|c|}{ Glass Yield } & \multirow{2}{*}{$\frac{\text { Glass Yield }}{\text { Target, } \mathrm{kg} / \mathrm{kg}}$} \\
\hline & & & & & $g / 1$ & $\mathrm{~kg} / \mathrm{kg}$ & \\
\hline Nominal & $1,2,9$ & 51.6 & 1.45 & 9.77 & 571 & 0.39 & 0.38 \\
\hline High Water & 3,4 & 64.1 & 1.31 & 9.74 & 389 & 0.30 & 0.29 \\
\hline Frit Nominal & 6,7 & 60.6 & 1.31 & 9.67 & 453 & 0.35 & 0.35 \\
\hline Nitrated Nominal & 5,8 & 51.1 & 1.43 & 5.51 & 446 & 0.31 & 0.31 \\
\hline
\end{tabular}


The Catholic University of America

Vitreous State Laboratory
DuraMelter 1200 Tests with AZ-101HLW Simulants Final Report, VSL-02R0100-2, Rev.I

Table 2.6. XRF Analyzed Composition of Crucible-Melted Feed Samples (wt\%).

\begin{tabular}{|c|c|c|c|c|c|c|c|c|c|c|c|c|c|}
\hline Test \# & & \multicolumn{8}{|c|}{1} & \\
\hline $\begin{array}{l}\text { Sample } \\
\text { I.D. }\end{array}$ & Target & $\begin{array}{c}12 \mathrm{~F}-\mathrm{F}- \\
81 \mathrm{~A}\end{array}$ & $\begin{array}{c}12 \mathrm{~F}-\mathrm{F}- \\
97 \mathrm{~A}\end{array}$ & $\begin{array}{l}12 \mathrm{~F}-\mathrm{F} \\
146 \mathrm{~A}\end{array}$ & $\begin{array}{c}12 \mathrm{G}-\mathrm{F}- \\
6 \mathrm{~A}\end{array}$ & $\begin{array}{c}12 \mathrm{G}-\mathrm{F}- \\
33 \mathrm{~A}\end{array}$ & $\begin{array}{c}12 \mathrm{G}-\mathrm{F}- \\
53 \mathrm{~A}\end{array}$ & $\begin{array}{c}12 \mathrm{G}-\mathrm{F}- \\
71 \mathrm{~A}\end{array}$ & Avg. & $\begin{array}{l}12 \mathrm{G}-\mathrm{F}- \\
130 \mathrm{~A}\end{array}$ & $\begin{array}{c}12 \mathrm{G}-\mathrm{F}- \\
149 \mathrm{~A}\end{array}$ & $\begin{array}{c}12 \mathrm{H}-\mathrm{F} \\
32 \mathrm{~A}\end{array}$ & $\begin{array}{c}12 \mathrm{H}-\mathrm{F} \\
43 \mathrm{~A}\end{array}$ \\
\hline $\mathrm{Al}_{2} \mathrm{O}_{3}$ & 7.40 & 7.54 & 7.23 & 7.31 & 7.74 & 7.32 & 7.27 & 7.45 & 7.41 & 7.22 & 7.78 & 7.41 & 7.89 \\
\hline $\mathrm{As}_{2} \mathrm{O}_{3}$ & .04 & 0.04 & 0.04 & 0.04 & 0.04 & 0.04 & 0.04 & 0.04 & 0.04 & 0.04 & 0.03 & 0.03 & 0.04 \\
\hline $\mathrm{B}_{2} \mathrm{O}_{3}{ }^{*}$ & 10.00 & 10.00 & 10.00 & 10.00 & 10.00 & 10.00 & 10.00 & 10.00 & 10 & 10.00 & 10.00 & 10.00 & 10.00 \\
\hline $\mathrm{BaO}$ & .04 & $<0.05$ & $<0.05$ & $<0.05$ & $<0.05$ & $<0.05$ & $<0.05$ & $<0.05$ & $<0.05$ & $<0.05$ & $<0.05$ & $<0.05$ & $<0.05$ \\
\hline $\mathrm{CaO}$ & 25 & 0.29 & 0.29 & 0.30 & 0.28 & 0.29 & 0.29 & 0.29 & 0.29 & 0.30 & 0.29 & 0.30 & 0.28 \\
\hline $\mathrm{CdO}$ & 37 & 0.40 & 0.35 & 0.40 & 0.34 & 0.35 & 0.35 & 0.32 & 36 & 0.33 & 0.31 & 0.32 & 0.33 \\
\hline $\mathrm{CeO}_{2}$ & 08 & 0.07 & 0.06 & 0.06 & 0.07 & 0.06 & 0.07 & 0.07 & .07 & 0.07 & 0.06 & 0.07 & 0.09 \\
\hline $\mathrm{Cl}$ & 01 & 0.00 & 0.00 & 0.00 & 0.00 & 0.00 & 0.00 & 0.00 & .00 & 0.01 & 0.00 & 0.00 & 0.00 \\
\hline $\mathrm{Cr}_{2} \mathrm{O}_{3}$ & 04 & 0.05 & 0.05 & 0.05 & 0.04 & 0.04 & 0.04 & 0.04 & 0.04 & 0.05 & 0.04 & 0.05 & 0.05 \\
\hline $\mathrm{Cs}_{2} \mathrm{O}$ & 08 & 0.09 & 0.08 & 0.08 & 0.08 & 0.07 & 0.07 & 0.07 & .08 & 0.06 & 0.07 & 0.07 & 0.07 \\
\hline $\mathrm{CuO}$ & 03 & 0.03 & 0.04 & 0.03 & 0.03 & 0.03 & 0.04 & 0.04 & 0.03 & 0.03 & 0.02 & 0.03 & 0.03 \\
\hline F & 0.04 & NA & NA & $\mathrm{NA}$ & NA & $\mathrm{NA}$ & $\mathrm{NA}$ & NA & $\mathrm{NC}$ & NA & NA & NA & $\mathrm{NA}$ \\
\hline $\mathrm{Fe}_{2} \mathrm{O}_{3}$ & 10.39 & 10.28 & 10.76 & 10.54 & 9.69 & 10.35 & 10.38 & 10.35 & 10.34 & 10.29 & 9.80 & 10.35 & 10.57 \\
\hline $\mathrm{K}_{2} \mathrm{O}$ & 0.17 & 0.26 & 0.24 & 0.25 & 0.26 & 0.24 & 0.25 & 0.25 & 0.25 & 0.23 & 0.28 & 0.24 & 0.24 \\
\hline $\mathrm{La}_{2} \mathrm{O}_{3}$ & 0 & 0.12 & 0.06 & 0.13 & 0.14 & 0.15 & 0.11 & 0.11 & 0.12 & 0.11 & 0.12 & 0.12 & 0.11 \\
\hline $\mathrm{Li}_{2} \mathrm{O}^{*}$ & .00 & 6.00 & 6.00 & 6.00 & 6.00 & 6.00 & 6.00 & 6.00 & 6.00 & 6.00 & 6.00 & 6.00 & 6.00 \\
\hline $\mathrm{MgO}$ & 0 & 1.13 & 1.13 & 1.13 & 1.17 & 1.25 & 1.16 & 1.20 & 1.17 & 1.17 & 1.17 & 1.16 & 1.40 \\
\hline $\mathrm{MnO}$ & & 3.10 & 3.16 & 3.14 & 2.89 & 3.07 & 3.09 & 3.05 & 3.07 & 3.11 & 2.93 & 3.02 & 3.12 \\
\hline $\mathrm{Na}_{2} \mathrm{O}$ & 59 & 6.59 & 6.47 & 6.46 & 6.93 & 6.60 & 6.51 & 6.24 & 6.54 & 6.83 & 6.76 & 6.96 & 7.15 \\
\hline $\mathrm{NiO}$ & & 0.48 & 0.52 & 0.50 & 0.45 & 0.49 & 0.49 & 0.48 & 49 & 0.47 & 0.44 & 0.49 & 0.50 \\
\hline $\mathrm{P}_{2} \mathrm{O}_{5}$ & 0.13 & 0.34 & 0.34 & 0.33 & 0.35 & 0.33 & 0.34 & 0.34 & 0.34 & 0.42 & 0.32 & 0.32 & 0.36 \\
\hline $\mathrm{PbO}$ & 15 & 0.13 & 0.14 & 0.14 & 0.11 & 0.13 & 0.13 & 0.12 & 13 & 0.13 & 0.12 & 0.12 & 0.13 \\
\hline $\mathrm{Sb}_{2} \mathrm{O}_{3}$ & 0.21 & 0.18 & 0.17 & 0.18 & 0.18 & 0.18 & 0.19 & 0.18 & 0.18 & 0.15 & 0.16 & 0.17 & 0.16 \\
\hline $\mathrm{SeO}_{2}$ & 0.15 & 0.04 & 0.04 & 0.04 & 0.03 & 0.03 & 0.04 & 0.02 & 0.03 & 0.03 & 0.03 & 0.04 & 0.05 \\
\hline $\mathrm{SiO}_{2}$ & 45.54 & 44.86 & 44.50 & 44.33 & 45.60 & 44.77 & 44.88 & 45.25 & 44.88 & 44.88 & 45.70 & 44.69 & 47.71 \\
\hline $\mathrm{SO}_{3}$ & 0.25 & 0.21 & 0.25 & 0.20 & 0.22 & 0.24 & 0.20 & 0.17 & 0.21 & 0.17 & 0.17 & 0.17 & 0.20 \\
\hline SrO & & 1.94 & 2.02 & 2.15 & 1.88 & 2.04 & 2.06 & 2.03 & 2.01 & 2.04 & 1.89 & 1.99 & 1.42 \\
\hline $\mathrm{TeO}_{2}$ & 14 & 0.11 & 0.11 & 0.10 & 0.11 & 0.12 & 0.10 & 0.09 & 0.11 & 0.08 & 0.09 & 0.08 & 0.10 \\
\hline $\mathrm{TiO}_{2}$ & .06 & 0.09 & 0.08 & 0.08 & 0.08 & 0.08 & 0.07 & 0.07 & 0.08 & 0.07 & 0.07 & 0.08 & 0.07 \\
\hline $\mathrm{ZnO}$ & $\overline{2}$ & 1.80 & 1.89 & 1.90 & 1.69 & 1.82 & 1.84 & 1.82 & 1.82 & 1.85 & 1.72 & 1.86 & 1.97 \\
\hline $\mathrm{ZrO}_{2}$ & 3.56 & 3.87 & 4.02 & 4.16 & 3.64 & 3.94 & 4.03 & 3.95 & 3.95 & 3.90 & 3.67 & 3.90 & 0.00 \\
\hline Sum & 99.99 & 100.00 & 100.00 & 100.00 & 100.00 & 100.00 & 100.00 & 100.00 & 100.00 & 100.00 & 100.00 & 100.00 & 100.00 \\
\hline
\end{tabular}

\footnotetext{
* Target values. NA: Not Analyzed. NC: Not Calculated.
} 
Table 2.6. XRF Analyzed Composition of Crucible-Melted Feed Samples (wt\%).

\begin{tabular}{|c|c|c|c|c|c|c|c|c|c|c|c|c|}
\hline Test \# & & \multicolumn{6}{|c|}{2} & \multicolumn{5}{|c|}{3} \\
\hline $\begin{array}{c}\text { Sample } \\
\text { I.D. }\end{array}$ & Target & $\begin{array}{l}12 \mathrm{I}-\mathrm{F}- \\
22 \mathrm{~A}\end{array}$ & $\begin{array}{c}12 \mathrm{I}-\mathrm{F}- \\
40 \mathrm{~A}\end{array}$ & $\begin{array}{c}12 \mathrm{I}-\mathrm{F}- \\
57 \mathrm{~A}\end{array}$ & $\begin{array}{c}12 \mathrm{I}-\mathrm{F}- \\
66 \mathrm{~A}\end{array}$ & $\begin{array}{l}12 \mathrm{I}-\mathrm{F}- \\
82 \mathrm{~A}\end{array}$ & Avg. & $\begin{array}{c}12 \mathrm{~J}-\mathrm{F}- \\
53 \mathrm{~A}\end{array}$ & $\begin{array}{c}12 \mathrm{~J}-\mathrm{F}- \\
62 \mathrm{~A}\end{array}$ & $\begin{array}{c}12 \mathrm{~J}-\mathrm{F}- \\
81 \mathrm{~A}\end{array}$ & $\begin{array}{c}12 \mathrm{~J}-\mathrm{F}- \\
99 \mathrm{~A}\end{array}$ & $\begin{array}{l}12 \mathrm{~J}-\mathrm{F}- \\
112 \mathrm{~A}\end{array}$ \\
\hline $\mathrm{Al}_{2} \mathrm{O}_{3}$ & 7.40 & .55 & 7.48 & 7.41 & 7.41 & 77 & 7.50 & 7.53 & 7.46 & 7.23 & 7.71 & 7.32 \\
\hline $\mathrm{As}_{2} \mathrm{O}_{3}$ & 0.04 & 0.04 & 0.04 & 0.04 & 0.04 & 0.04 & 0.04 & 0.04 & 0.04 & 0.04 & 0.03 & 0.04 \\
\hline $\mathrm{B}_{2} \mathrm{O}_{3}{ }^{*}$ & 10.00 & 10.00 & 10.00 & 10.00 & 10.00 & 10.00 & 10.00 & 10.00 & 10.00 & 10.00 & 10.00 & 10.00 \\
\hline $\mathrm{BaO}$ & 0.04 & $<0.05$ & $<0.05$ & $<0.05$ & $<0.05$ & $<0.05$ & $<0.05$ & $<0.05$ & $<0.05$ & $<0.05$ & $<0.05$ & $<0.05$ \\
\hline $\mathrm{CaO}$ & & 0.29 & 0.29 & 0.29 & 0.29 & 0.30 & 0.29 & 0.30 & 0.34 & 0.30 & 0.28 & 0.30 \\
\hline $\mathrm{CdO}$ & .37 & 0.34 & 0.32 & 0.33 & 0.36 & 0.31 & 0.33 & 0.36 & 0.37 & 0.37 & 0.31 & 0.35 \\
\hline $\mathrm{CeO}_{2}$ & 0.08 & 0.07 & 0.06 & 0.07 & 0.06 & 0.06 & 0.07 & 0.08 & 0.07 & 0.07 & 0.06 & 0.07 \\
\hline $\mathrm{Cl}$ & 01 & .00 & 0.02 & 0.00 & 0.00 & 0.00 & 0.00 & 0.00 & 0.00 & 0.00 & 0.00 & 0.00 \\
\hline $\mathrm{Cr}_{2} \mathrm{O}_{3}$ & & .05 & 0.04 & 0.05 & 0.04 & & & 0.05 & .04 & 0.05 & 04 & 0.05 \\
\hline $\mathrm{Cs}_{2} \mathrm{O}$ & .08 & 0.08 & 0.08 & 0.09 & 0.08 & 0.07 & 0.07 & 0.07 & 0.07 & 0.07 & 0.06 & 0.06 \\
\hline $\mathrm{CuO}$ & 03 & .03 & 0.02 & 0.02 & 0.02 & 0.03 & 0.03 & 0.04 & 0.03 & 0.03 & 0.03 & 0.04 \\
\hline $\mathrm{F}$ & .04 & NA & $\mathrm{NA}$ & $\mathrm{NA}$ & $\mathrm{NA}$ & NA & $\mathrm{NC}$ & $\mathrm{NA}$ & NA & $\mathrm{NA}$ & $\mathrm{NA}$ & $\mathrm{NA}$ \\
\hline $\mathrm{Fe}_{2} \mathrm{O}_{3}$ & 10.39 & 9.91 & 10.33 & 10.17 & 10.29 & 10.33 & 10.23 & 10.81 & 10.65 & 10.71 & 9.77 & 10.49 \\
\hline $\mathrm{K}_{2} \mathrm{O}$ & 17 & 0.29 & 0.24 & 0.24 & 0.24 & 0.25 & 0.25 & 0.26 & 0.29 & 0.23 & 0.27 & 0.24 \\
\hline $\mathrm{La}_{2} \mathrm{O}_{3}$ & & 12 & 11 & 0.11 & 0.12 & 12 & & 0.14 & 0.17 & 0.14 & & 0.13 \\
\hline $\mathrm{Li}_{2} \mathrm{O}^{*}$ & & & .00 & 6.00 & & & & & 6.00 & 6.00 & & 6.00 \\
\hline $\mathrm{MgO}$ & & & 1.23 & 1.18 & 1.20 & & & 1.19 & 1.17 & 1.16 & 1.24 & 1.15 \\
\hline $\mathrm{MnO}$ & 03 & 2.89 & 2.99 & 2.95 & 3.02 & 2.99 & 3.00 & 3.06 & 3.12 & 3.16 & 79 & 3.05 \\
\hline $\mathrm{Na}_{2} \mathrm{O}$ & & & & & & & & & & 03 & & 6.49 \\
\hline $\mathrm{NiO}$ & & .48 & .45 & .46 & & & & & 0.54 & 0.51 & & 0.50 \\
\hline $\mathrm{P}_{2} \mathrm{O}_{5}$ & & 32 & 0.34 & 0.33 & 0.3 & & & 0.32 & 0.33 & 0.34 & 35 & 0.34 \\
\hline $\mathrm{PbO}$ & & 12 & 13 & & & & 12 & & 0.11 & 0.13 & & 0.12 \\
\hline $\mathrm{Sb}_{2} \mathrm{O}_{3}$ & & 18 & & & & & 0.17 & 0.17 & 0.17 & 0.18 & & 0.16 \\
\hline $\mathrm{SeO}_{2}$ & & 0.04 & 0.02 & 0.02 & 0.03 & 0.03 & 0.03 & 0.03 & 0.03 & 0.01 & 0.02 & 0.01 \\
\hline $\mathrm{SiO}_{2}$ & 45.54 & 44.75 & 44.93 & 44.97 & 44.77 & 44.64 & 45.23 & 44.47 & 44.50 & 44.93 & 46.11 & 45.11 \\
\hline $\mathrm{SO}_{3}$ & & 0.19 & 0.13 & 0.21 & 0.19 & 0.18 & 0.18 & 0.22 & 0.20 & 0.13 & 0.21 & 0.15 \\
\hline $\mathrm{SrO}$ & & 1.92 & 1.98 & 1.98 & & 2.11 & & & 1.91 & 2.10 & 1.83 & 2.00 \\
\hline $\mathrm{TeO}_{2}$ & 0.14 & 0.10 & 0.09 & 0.10 & 0.11 & 0.08 & 0.09 & 0.09 & 0.09 & 0.07 & 0.08 & 0.06 \\
\hline $\mathrm{TiO}_{2}$ & 0.06 & 0.07 & 0.07 & 0.07 & 0.07 & 0.07 & 0.07 & 0.08 & 0.09 & 0.07 & 0.07 & 0.08 \\
\hline $\mathrm{ZnO}$ & 2.00 & 1.76 & 1.81 & 1.78 & 1.84 & 1.89 & 1.83 & 1.92 & 2.04 & 1.93 & 1.71 & 1.88 \\
\hline $\mathrm{ZrO}_{2}$ & 3.56 & 3.78 & 3.90 & 3.91 & 3.96 & 4.10 & 3.46 & 3.88 & 3.82 & 4.04 & 3.47 & 3.84 \\
\hline Sum & 99.99 & 100.00 & 100.00 & 100.00 & 100.00 & 100.00 & 100.00 & 100.00 & 100.00 & 100.00 & 100.00 & 100.00 \\
\hline
\end{tabular}

* Target values. NA: Not Analyzed. NC: Not Calculated. 
The Catholic University of America

Vitreous State Laboratory
DuraMelter 1200 Tests with AZ-101HLW Simulants Final Report, VSL-02R0100-2, Rev.1

Table 2.6. XRF Analyzed Composition of Crucible-Melted Feed Samples (wt\%).

\begin{tabular}{|c|c|c|c|c|c|c|c|c|c|c|c|c|}
\hline Test \# & & \multicolumn{3}{|c|}{3} & \multicolumn{4}{|c|}{4} & \multicolumn{4}{|c|}{5} \\
\hline $\begin{array}{c}\text { Sample } \\
\text { I.D. }\end{array}$ & Target & $\begin{array}{l}12 \mathrm{~J}-\mathrm{F}- \\
131 \mathrm{~A}\end{array}$ & $\begin{array}{l}12 \mathrm{~J}-\mathrm{F}- \\
149 \mathrm{~A}\end{array}$ & Avg. & $\begin{array}{c}12 \mathrm{~K}-\mathrm{F}- \\
83 \mathrm{~A}\end{array}$ & $\begin{array}{c}12 \mathrm{~K}-\mathrm{F}- \\
104 \mathrm{~A}\end{array}$ & $\begin{array}{c}12 \mathrm{~K}-\mathrm{F}- \\
125 \mathrm{~A}\end{array}$ & Avg. & $\begin{array}{c}12 \mathrm{~K}-\mathrm{F}- \\
144 \mathrm{~A}\end{array}$ & $\begin{array}{c}12 \mathrm{~L}-\mathrm{F}- \\
30 \mathrm{~A}\end{array}$ & $\begin{array}{c}12 \mathrm{~L}-\mathrm{F}- \\
49 \mathrm{~A}\end{array}$ & $\begin{array}{c}12 \mathrm{~L}-\mathrm{F}- \\
70 \mathrm{~A}\end{array}$ \\
\hline $\mathrm{Al}_{2} \mathrm{O}_{3}$ & 7.40 & 7.29 & 7.60 & 7.45 & 7.36 & 7.77 & 7.51 & 7.54 & 7.66 & 7.23 & 7.36 & 7.48 \\
\hline $\mathrm{As}_{2} \mathrm{O}_{3}$ & 0.04 & 0.04 & 0.04 & 0.04 & 0.03 & 0.04 & 0.04 & 0.04 & 0.03 & 0.04 & 0.03 & 0.04 \\
\hline $\mathrm{B}_{2} \mathrm{O}_{3}{ }^{*}$ & 10.00 & 10.00 & 10.00 & 10.00 & 10.0 & 10.00 & 10.00 & 10.00 & 10.00 & 10.00 & 10.00 & 10.00 \\
\hline $\mathrm{BaO}$ & 0.04 & $<0.05$ & $<0.05$ & $<0.05$ & $<0.05$ & $<0.05$ & $<0.05$ & $<0.05$ & $<0.05$ & $<0.05$ & $<0.05$ & $<0.05$ \\
\hline $\mathrm{CaO}$ & 0.25 & 0.29 & 0.30 & 0.30 & 0.30 & 0.29 & 0.29 & 0.29 & 0.32 & 0.32 & 0.31 & 0.30 \\
\hline $\mathrm{CdO}$ & 0.37 & 0.39 & 0.34 & 0.36 & 0.33 & 0.36 & 0.34 & 0.34 & 0.35 & 0.42 & 0.33 & 0.33 \\
\hline $\mathrm{CeO}_{2}$ & 0.08 & 0.07 & 0.05 & 0.07 & 0.07 & 0.06 & 0.06 & 0.06 & 0.07 & 0.08 & 0.09 & 0.08 \\
\hline $\mathrm{Cl}$ & 0.01 & 0.00 & 0.00 & 0.00 & 0.00 & 0.00 & 0.00 & 0.00 & 0.00 & 0.01 & 0.00 & 0.01 \\
\hline $\mathrm{Cr}_{2} \mathrm{O}_{3}$ & 0.04 & 0.05 & 0.05 & 0.05 & 0.04 & 0.04 & 0.04 & 0.04 & 0.04 & 0.04 & 0.04 & 0.04 \\
\hline $\mathrm{Cs}_{2} \mathrm{O}$ & 0.08 & 0.08 & 0.07 & 0.07 & 0.05 & 0.08 & 0.08 & 0.07 & 0.06 & 0.07 & 0.06 & 0.05 \\
\hline $\mathrm{CuO}$ & 0.03 & 0.04 & 0.03 & 0.03 & 0.03 & 0.03 & 0.03 & 0.03 & 0.03 & 0.04 & 0.03 & 0.03 \\
\hline $\mathrm{F}$ & 0.04 & NA & $\mathrm{NA}$ & $\mathrm{NC}$ & NA & $\mathrm{NA}$ & $\mathrm{NA}$ & $\mathrm{NC}$ & $\mathrm{NA}$ & NA & NA & NA \\
\hline $\mathrm{Fe}_{2} \mathrm{O}_{3}$ & 10.39 & 10.56 & 10.11 & 10.44 & 10.45 & 10.24 & 10.40 & 10.37 & 10.13 & 10.07 & 10.09 & 10.35 \\
\hline $\mathrm{K}_{2} \mathrm{O}$ & 0.17 & 0.24 & 0.27 & 0.26 & 0.22 & 0.25 & 0.24 & 0.24 & 0.26 & 0.27 & 0.27 & 0.27 \\
\hline $\mathrm{La}_{2} \mathrm{O}_{3}$ & 0.32 & 0.12 & 0.11 & 0.13 & 0.13 & 0.13 & 0.15 & 0.14 & 0.11 & 0.13 & 0.13 & 0.13 \\
\hline $\mathrm{Li}_{2} \mathrm{O}^{*}$ & 6.00 & 6.00 & 6.00 & 6.00 & 6.00 & 6.00 & 6.00 & 6.00 & 6.00 & 6.00 & 6.00 & 6.00 \\
\hline $\mathrm{MgO}$ & 0.06 & 1.17 & 1.21 & 1.18 & 1.29 & 1.33 & 1.28 & 1.30 & 1.24 & 1.16 & 1.19 & 1.22 \\
\hline $\mathrm{MnO}$ & 3.03 & 3.05 & 2.83 & 3.01 & 3.01 & 2.83 & 2.98 & 2.94 & 2.88 & 2.97 & 2.90 & 2.86 \\
\hline $\mathrm{Na}_{2} \mathrm{O}$ & 6.59 & 6.56 & 6.64 & 6.43 & 6.78 & 6.48 & 6.21 & 6.49 & 7.25 & 6.63 & 6.54 & 6.68 \\
\hline $\mathrm{NiO}$ & 0.54 & 0.51 & 0.48 & 0.50 & 0.49 & 0.48 & 0.49 & 0.49 & 0.48 & 0.49 & 0.50 & 0.52 \\
\hline $\mathrm{P}_{2} \mathrm{O}_{5}$ & 0.13 & 0.32 & 0.35 & 0.34 & 0.33 & 0.32 & 0.33 & 0.33 & 0.34 & 0.31 & 0.31 & 0.31 \\
\hline $\mathrm{PbO}$ & 0.15 & 0.13 & 0.11 & 0.12 & 0.12 & 0.12 & 0.12 & 0.12 & 0.12 & 0.12 & 0.11 & 0.12 \\
\hline $\mathrm{Sb}_{2} \mathrm{O}_{3}$ & 0.21 & 0.18 & 0.17 & 0.17 & 0.15 & 0.17 & 0.18 & 0.17 & 0.17 & 0.18 & 0.14 & 0.13 \\
\hline $\mathrm{SeO}_{2}$ & 0.15 & 0.03 & 0.02 & 0.02 & 0.03 & 0.02 & 0.02 & 0.02 & 0.01 & 0.03 & 0.03 & 0.02 \\
\hline $\mathrm{SiO}_{2}$ & 45.54 & 44.68 & 45.53 & 45.05 & 45.06 & 45.34 & 45.30 & 45.23 & 44.68 & 45.11 & 45.90 & 45.95 \\
\hline $\mathrm{SO}_{3}$ & 0.25 & 0.19 & 0.22 & 0.19 & 0.21 & 0.24 & 0.22 & 0.22 & 0.08 & 0.25 & 0.20 & 0.18 \\
\hline $\mathrm{SrO}$ & 2.32 & 2.06 & 1.89 & 1.98 & 1.85 & 1.80 & 1.90 & 1.85 & 1.96 & 1.99 & 1.85 & 1.91 \\
\hline $\mathrm{TeO}_{2}$ & 0.14 & 0.10 & 0.09 & 0.08 & 0.09 & 0.10 & 0.10 & 0.10 & 0.05 & 0.12 & 0.08 & 0.07 \\
\hline $\mathrm{TiO}_{2}$ & 0.06 & 0.08 & 0.07 & 0.08 & 0.08 & 0.09 & 0.10 & 0.09 & 0.09 & 0.11 & 0.10 & 0.11 \\
\hline $\mathrm{ZnO}$ & 2.00 & 1.88 & 1.78 & 1.87 & 1.81 & 1.76 & 1.81 & 1.79 & 1.83 & 1.87 & 1.86 & 1.93 \\
\hline $\mathrm{ZrO}_{2}$ & 3.56 & 3.92 & 3.68 & 3.81 & 3.73 & 3.67 & 3.82 & 3.74 & 3.80 & 3.97 & 3.59 & 2.93 \\
\hline Sum & 99.99 & 100.00 & 100.00 & 100.00 & \begin{tabular}{|l|l|}
100.00 \\
\end{tabular} & 100.00 & 100.00 & 100.00 & \begin{tabular}{|c|}
100.00 \\
\end{tabular} & 100.00 & 100.00 & 100.00 \\
\hline
\end{tabular}


Table 2.6. XRF Analyzed Composition of Crucible-Melted Feed Samples (wt\%).

\begin{tabular}{|c|c|c|c|c|c|c|c|c|c|c|c|c|c|}
\hline Test \# & & \multicolumn{4}{|c|}{5} & \multicolumn{8}{|c|}{6} \\
\hline Sample & & $12 \mathrm{~L}-\mathrm{F}-$ & & $12 \mathrm{~L}-\mathrm{F}-$ & & 12L-F- & 12L-F- & $12 \mathrm{~L}-\mathrm{F}-$ & $12 \mathrm{M}-\mathrm{F}$ & $-12 \mathrm{M}-\mathrm{F}-$ & $12 \mathrm{M}-\mathrm{F}$ & $12 \mathrm{M}-\mathrm{F}$ & $12 \mathrm{M}-\mathrm{F}$ \\
\hline & Target & $80 \mathrm{~A}$ & $107 \mathrm{~A}$ & $119 \mathrm{~A}$ & Avg. & $147 \mathrm{~A}$ & $148 \mathrm{~A}$ & $155 \mathrm{~A}$ & $6 \mathrm{~A}$ & $9 \mathrm{~A}$ & $11 \mathrm{~A}$ & $19 \mathrm{~A}$ & $21 \mathrm{~A}$ \\
\hline $\mathrm{Al}_{2} \mathrm{O}_{3}$ & 7.40 & 8.04 & 7.14 & 7.12 & 7.43 & 7.47 & 7.43 & 7.14 & 7.65 & 7.52 & 7.68 & 7.60 & 7.67 \\
\hline $\mathrm{As}_{2} \mathrm{O}_{3}$ & 0.04 & 0.04 & 0.04 & 0.04 & 0.04 & 0.04 & 0.04 & 0.03 & 0.04 & 0.04 & 0.04 & 0.04 & 0.04 \\
\hline $\mathrm{B}_{2} \mathrm{O}_{3}{ }^{*}$ & 10.00 & 10.00 & 10.00 & 10.00 & 10.00 & 10.00 & 10.00 & 10.00 & 10.00 & 10.00 & 10.00 & 10.00 & 10.00 \\
\hline $\mathrm{BaO}$ & 0.04 & $<0.05$ & $<0.05$ & $<0.05$ & $<0.05$ & $<0.05$ & $<0.05$ & $<0.05$ & $<0.05$ & $<0.05$ & $<0.05$ & $<0.05$ & $<0.05$ \\
\hline $\mathrm{CaO}$ & 25 & 0.33 & 0.27 & 0.32 & 0.31 & 0.70 & 0.71 & 0.68 & 0.70 & 0.68 & 0.69 & \begin{tabular}{|l|}
0.69 \\
\end{tabular} & 0.67 \\
\hline$\overline{\mathrm{CdO}}$ & 37 & 0.37 & 0.33 & 0.31 & 0.35 & 0.34 & 0.39 & 0.38 & 0.35 & 0.35 & 0.34 & 0.36 & $\overline{0.34}$ \\
\hline $\mathrm{CeO}_{2}$ & 08 & 0.07 & 0.08 & 0.08 & 0.08 & 0.08 & 0.08 & 0.07 & 0.07 & 0.08 & 0.07 & 0.08 & 0.07 \\
\hline $\mathrm{Cl}$ & 0 & 0.00 & 0.01 & 0.01 & 0.01 & 0.01 & 0.00 & 0.00 & 0.00 & 0.00 & 0.00 & 0.01 & 0.00 \\
\hline $\mathrm{Cr}_{2} \mathrm{O}_{3}$ & 0.04 & 0.05 & 0.04 & 0.04 & 0.04 & 0.05 & 0.04 & 0.04 & 0.05 & 0.04 & 0.05 & 0.04 & 0.04 \\
\hline $\mathrm{Cs}_{2} \mathrm{O}$ & 0.08 & 0.08 & 0.06 & 0.05 & 0.06 & 0.07 & 0.09 & 0.08 & 0.07 & 0.07 & 0.08 & 0.08 & 0.08 \\
\hline $\mathrm{CuO}$ & 03 & 0.03 & 0.03 & 0.03 & 0.03 & 0.03 & 0.03 & 0.04 & 0.03 & 0.03 & 0.03 & 0.03 & 0.03 \\
\hline $\mathrm{F}$ & 0.04 & NA & NA & NA & $\mathrm{NC}$ & $\mathrm{NA}$ & $\mathrm{NA}$ & NA & NA & $\mathrm{NA}$ & $\mathrm{NA}$ & $\mathrm{NA}$ & NA \\
\hline $\mathrm{Fe}_{2} \mathrm{O}_{3}$ & 10.39 & 10.43 & 9.41 & 9.45 & 9.99 & 11.26 & 11.14 & 10.32 & 10.40 & 9.96 & 10.62 & 10.57 & 10.39 \\
\hline $\mathrm{K}_{2} \mathrm{O}$ & 0.17 & 0.29 & 0.26 & 0.26 & 0.27 & 0.25 & 0.24 & 0.23 & 0.26 & 0.26 & 0.26 & 0.25 & 0.26 \\
\hline $\mathrm{La}_{2} \mathrm{O}_{3}$ & 0.32 & 0.14 & 0.12 & 0.11 & 0.12 & 0.11 & 0.12 & 0.11 & 0.11 & 0.11 & 0.11 & 0.12 & 0.11 \\
\hline $\mathrm{Li}_{2} \mathrm{O}^{*}$ & 6.00 & 6.00 & 6.00 & 6.00 & 6.00 & 6.00 & 6.00 & 6.00 & 6.00 & 6.00 & 6.00 & 6.00 & 6.00 \\
\hline $\mathrm{MgO}$ & 0.06 & 1.36 & 1.16 & 1.06 & 1.20 & 1.22 & 1.23 & 1.21 & 1.28 & 1.19 & 1.28 & 1.21 & 1.28 \\
\hline $\mathrm{MnO}$ & 3.03 & 1.37 & 2.90 & 2.90 & 2.68 & 2.12 & 2.48 & 2.81 & 2.34 & 2.76 & 2.09 & 2.57 & 2.36 \\
\hline $\mathrm{Na}_{2} \mathrm{O}$ & 6.59 & 8.01 & 6.43 & 6.53 & 6.87 & 6.51 & 6.58 & 6.70 & 6.91 & 6.73 & 6.52 & 6.16 & 6.89 \\
\hline $\mathrm{NiO}$ & 0.54 & 0.55 & 0.45 & 0.44 & 0.49 & 0.54 & 0.53 & 0.48 & 0.49 & 0.46 & 0.50 & 0.50 & 0.50 \\
\hline $\mathrm{P}_{2} \mathrm{O}_{5}$ & 0.13 & 0.35 & 0.30 & 0.29 & 0.32 & 0.32 & 0.31 & 0.32 & 0.32 & 0.33 & 0.32 & 0.31 & 0.32 \\
\hline $\mathrm{PbO}$ & 0.15 & 0.07 & 0.12 & 0.12 & 0.11 & 0.12 & 0.13 & 0.12 & 0.12 & 0.12 & 0.11 & 0.13 & 0.11 \\
\hline $\mathrm{Sb}_{2} \mathrm{O}_{3}$ & 0.21 & 0.11 & 0.13 & 0.13 & 0.14 & 0.16 & 0.18 & 0.19 & 0.16 & 0.16 & 0.15 & 0.17 & 0.16 \\
\hline $\mathrm{SeO}_{2}$ & 0.15 & 0.02 & 0.03 & 0.03 & 0.02 & 0.05 & 0.04 & 0.05 & 0.03 & 0.03 & 0.04 & 0.05 & 0.04 \\
\hline $\mathrm{SiO}_{2}$ & 45.54 & 44.07 & 47.47 & 47.55 & 45.82 & 44.15 & 43.40 & 44.33 & 44.67 & 44.99 & 45.04 & 44.29 & 44.75 \\
\hline $\mathrm{SO}_{3}$ & 0.25 & 0.14 & 0.22 & 0.21 & 0.18 & 0.17 & 0.17 & 0.19 & 0.16 & 0.17 & 0.16 & 0.18 & 0.18 \\
\hline $\mathrm{SrO}$ & 2.32 & 2.08 & 1.71 & 1.65 & 1.88 & 1.95 & 2.04 & 1.97 & 1.85 & 1.91 & 1.82 & 2.01 & 1.82 \\
\hline $\mathrm{TeO}_{2}$ & 0.14 & 0.07 & 0.07 & 0.08 & 0.08 & 0.09 & 0.11 & 0.12 & 0.09 & 0.09 & 0.09 & 0.11 & 0.10 \\
\hline $\mathrm{TiO}_{2}$ & 0.06 & 0.10 & 0.10 & 0.12 & 0.10 & 0.21 & 0.22 & 0.21 & 0.21 & 0.20 & 0.20 & 0.23 & 0.19 \\
\hline $\mathrm{ZnO}$ & 2.00 & 1.99 & 1.73 & 1.75 & 1.85 & 2.08 & 2.11 & 2.08 & 1.95 & 1.95 & 1.96 & 2.08 & 1.94 \\
\hline $\mathrm{ZrO}_{2}$ & 3.56 & 3.88 & 3.43 & 3.36 & 3.57 & 3.94 & 4.21 & 4.14 & 3.73 & 3.81 & 3.80 & 4.19 & 3.71 \\
\hline Sum & 100.00 & 100.00 & 100.00 & 100.00 & 100.00 & 100.00 & 100.00 & 100.00 & 100.00 & 100.00 & 100.00 & 100.00 & 100.00 \\
\hline
\end{tabular}

* Target values. NA: Not Analyzed. NC: Not Calculated. 
The Catholic University of America

Vitreous State Laboratory
DuraMelter 1200 Tests with AZ-101HLW Simulants Final Report, VSL-02R0100-2, Rev.1

Table 2.6. XRF Analyzed Composition of Crucible-Melted Feed Samples (wt \%).

\begin{tabular}{|c|c|c|c|c|c|c|c|c|c|c|c|c|c|}
\hline Test \# & & \multicolumn{12}{|c|}{6} \\
\hline $\begin{array}{c}\text { Sample } \\
\text { I.D. }\end{array}$ & Target & $\begin{array}{l}12 \mathrm{M}- \\
\mathrm{F}-22 \mathrm{~A}\end{array}$ & $\begin{array}{c}12 \mathrm{M}- \\
\mathrm{F}-24 \mathrm{~A}\end{array}$ & $\begin{array}{c}12 \mathrm{M}- \\
\mathrm{F}-30 \mathrm{~A}\end{array}$ & $\begin{array}{l}12 \mathrm{M}- \\
\mathrm{F}-32 \mathrm{~A}\end{array}$ & $\begin{array}{l}\text { 12M- } \\
\text { F-33A }\end{array}$ & $\begin{array}{r}12 \mathrm{M}- \\
\mathrm{F}-39 \mathrm{~A}\end{array}$ & $\begin{array}{r}12 \mathrm{M}- \\
\mathrm{F}-40 \mathrm{~A}\end{array}$ & $\begin{array}{c}12 \mathrm{M}- \\
\mathrm{F}-43 \mathrm{~A}\end{array}$ & $\begin{array}{r}12 \mathrm{M}- \\
\mathrm{F}-49 \mathrm{~A}\end{array}$ & \begin{tabular}{|c|}
$12 \mathrm{M}-$ \\
$\mathrm{F}-50 \mathrm{~A}$
\end{tabular} & $\begin{array}{r}12 \mathrm{M}- \\
\mathrm{F}-54 \mathrm{~A}\end{array}$ & $\begin{array}{l}12 \mathrm{M}- \\
\mathrm{F}-71 \mathrm{~A}\end{array}$ \\
\hline $\mathrm{Al}_{2} \mathrm{O}_{3}$ & 7.40 & 7.36 & \begin{tabular}{|l|}
7.61 \\
\end{tabular} & \begin{tabular}{|l|}
7.30 \\
\end{tabular} & 7.68 & 7.81 & 7.58 & \begin{tabular}{|l|}
7.79 \\
\end{tabular} & \begin{tabular}{|l|}
7.77 \\
\end{tabular} & 7.72 & 7.74 & \begin{tabular}{|l|}
7.67 \\
\end{tabular} & 7.85 \\
\hline $\mathrm{As}_{2} \mathrm{O}_{3}$ & 0.04 & 0.04 & 0.04 & 0.04 & 0.03 & 0.03 & 0.03 & 0.03 & 0.03 & 0.03 & 0.03 & 0.03 & 0.03 \\
\hline $\mathrm{B}_{2} \mathrm{O}_{3}{ }^{*}$ & 10.00 & 10.00 & 10.00 & 10.00 & 10.00 & 10.00 & 10.00 & 10.00 & 10.00 & 10.00 & 10.00 & 10.00 & 10.00 \\
\hline $\mathrm{BaO}$ & 0.04 & $<0.05$ & $<0.05$ & $<0.05$ & $<0.05$ & $<0.05$ & $<0.05$ & $<0.05$ & $<0.05$ & $<0.05$ & $<0.05$ & $<0.05$ & $<0.05$ \\
\hline $\mathrm{CaO}$ & 0.25 & 0.69 & 0.69 & 0.68 & 0.70 & \begin{tabular}{|l|}
0.69 \\
\end{tabular} & 0.68 & 0.66 & 0.67 & \begin{tabular}{|l|}
0.69 \\
\end{tabular} & 0.68 & 0.67 & 0.64 \\
\hline $\mathrm{CdO}$ & 0.37 & 0.34 & 0.28 & 0.31 & 0.29 & 0.28 & 0.29 & 0.32 & 0.33 & 0.31 & 0.33 & 0.35 & 0.32 \\
\hline $\mathrm{CeO}_{2}$ & 0.08 & 0.08 & 0.07 & 0.08 & 0.07 & 0.07 & 0.07 & 0.08 & 0.08 & 0.08 & 0.06 & 0.07 & 0.08 \\
\hline $\mathrm{Cl}$ & 0.01 & 0.00 & 0.00 & 0.00 & 0.01 & 0.00 & 0.01 & 0.01 & 0.01 & 0.00 & 0.00 & 0.00 & 0.00 \\
\hline $\mathrm{Cr}_{2} \mathrm{O}_{3}$ & 0.04 & 0.05 & 0.05 & 0.04 & 0.05 & 0.05 & 0.04 & 0.04 & 0.04 & 0.04 & 0.04 & 0.04 & 0.04 \\
\hline $\mathrm{Cs}_{2} \mathrm{O}$ & 0.08 & 0.07 & 0.06 & 0.06 & 0.06 & 0.06 & 0.06 & 0.07 & 0.08 & 0.07 & 0.08 & 0.08 & 0.06 \\
\hline $\mathrm{CuO}$ & 0.03 & 0.03 & 0.03 & 0.03 & 0.03 & 0.03 & 0.03 & 0.03 & 0.03 & 0.03 & 0.03 & 0.03 & 0.03 \\
\hline$F$ & 0.04 & NA & NA & $\mathrm{NA}$ & $\mathrm{NA}$ & NA & $\mathrm{NA}$ & NA & NA & NA & $\mathrm{NA}$ & $\mathrm{NA}$ & $\mathrm{NA}$ \\
\hline $\mathrm{Fe}_{2} \mathrm{O}_{3}$ & 10.39 & 10.71 & 10.66 & 10.59 & 11.40 & 11.07 & 10.19 & 10.16 & 10.30 & 9.96 & 10.42 & 10.07 & 10.31 \\
\hline $\mathrm{K}_{2} \mathrm{O}$ & 0.17 & 0.24 & 0.24 & 0.24 & 0.25 & 0.25 & 0.27 & 0.26 & 0.26 & 0.26 & 0.26 & 0.26 & 0.26 \\
\hline$\overline{\mathrm{La}_{2} \mathrm{O}_{3}}$ & 0.32 & 0.11 & 0.12 & 0.12 & 0.13 & 0.11 & 0.12 & 0.10 & 0.11 & 0.11 & 0.10 & 0.11 & 0.12 \\
\hline $\mathrm{Li}_{2} \mathrm{O}^{*}$ & 6.00 & 6.00 & 6.00 & 6.00 & 6.00 & 6.00 & 6.00 & 6.00 & 6.00 & 6.00 & 6.00 & 6.00 & 6.00 \\
\hline $\mathrm{MgO}$ & 0.06 & 1.24 & 1.33 & 1.30 & 1.31 & 1.34 & 1.28 & 1.34 & 1.28 & 1.29 & 1.27 & 1.27 & 0.77 \\
\hline $\mathrm{MnO}$ & 3.03 & 2.52 & 2.50 & 2.53 & 1.84 & 1.63 & 1.84 & 2.03 & 2.35 & 2.28 & 1.28 & 1.94 & 2.83 \\
\hline $\mathrm{Na}_{2} \mathrm{O}$ & 6.59 & 6.76 & 6.81 & 6.67 & 6.83 & 7.00 & 7.04 & 7.06 & 6.58 & 6.57 & 6.61 & 6.71 & 6.50 \\
\hline $\mathrm{NiO}$ & 0.54 & 0.50 & 0.50 & 0.50 & 0.54 & 0.53 & 0.47 & 0.45 & 0.48 & 0.46 & 0.48 & 0.47 & 0.47 \\
\hline$\widehat{\mathrm{P}_{2} \mathrm{O}_{5}}$ & 0.13 & 0.32 & 0.32 & 0.33 & 0.32 & 0.33 & 0.32 & 0.34 & 0.33 & 0.33 & 0.33 & 0.32 & 0.25 \\
\hline $\mathrm{PbO}$ & 0.15 & 0.12 & 0.12 & 0.12 & 0.12 & 0.12 & 0.11 & 0.11 & 0.11 & 0.11 & 0.11 & 0.11 & 0.12 \\
\hline $\mathrm{Sb}_{2} \mathrm{O}_{3}$ & 0.21 & 0.16 & 0.12 & 0.15 & 0.12 & 0.12 & 0.11 & 0.15 & 0.15 & 0.16 & 0.13 & 0.16 & 0.17 \\
\hline $\mathrm{SeO}_{2}$ & 0.15 & 0.03 & 0.05 & 0.04 & 0.04 & 0.04 & 0.04 & 0.05 & 0.04 & 0.02 & 0.05 & 0.04 & 0.04 \\
\hline $\mathrm{SiO}_{2}$ & 45.54 & 44.21 & 44.25 & 44.43 & 43.91 & 45.11 & 45.32 & 45.20 & 45.04 & 45.81 & 45.85 & 45.50 & 44.60 \\
\hline $\mathrm{SO}_{3}$ & 0.25 & 0.15 & 0.16 & 0.14 & 0.13 & 0.14 & 0.16 & 0.18 & 0.14 & 0.09 & 0.20 & 0.19 & 0.14 \\
\hline $\mathrm{SrO}$ & 2.32 & 1.95 & 1.89 & 1.96 & 1.94 & 1.91 & 1.84 & 1.79 & 1.86 & 1.81 & 1.85 & 1.90 & 1.96 \\
\hline $\mathrm{TeO}_{2}$ & 0.14 & 0.10 & 0.08 & 0.09 & 0.08 & 0.07 & 0.09 & 0.10 & 0.09 & 0.06 & 0.10 & 0.10 & 0.10 \\
\hline $\mathrm{TiO}_{2}$ & 0.06 & 0.21 & 0.21 & 0.21 & 0.21 & 0.21 & 0.20 & 0.19 & 0.20 & 0.20 & 0.20 & 0.20 & 0.22 \\
\hline $\mathrm{ZnO}$ & 2.00 & 2.02 & 2.04 & 2.06 & 2.06 & 2.06 & 1.99 & 1.88 & 1.92 & 1.89 & 1.97 & 1.94 & 2.06 \\
\hline$\overline{\mathrm{ZrO}_{2}}$ & 3.56 & 4.03 & 3.81 & 4.02 & 3.90 & 2.98 & 3.87 & 3.62 & 3.76 & 3.67 & 3.85 & 3.81 & 4.07 \\
\hline Sum & 100.00 & 100.00 & 100.00 & 100.00 & 100.00 & 100.00 & 100.00 & 100.00 & 100.00 & 100.00 & 100.00 & 100.00 & 100.00 \\
\hline
\end{tabular}

* Target values. NA: Not Analyzed. NC: Not Calculated. 
The Catholic University of America Vitreous State Laboratory
DuraMelter 1200 Tests with AZ-101HLW Simulants

Final Report, VSL-02R0100-2, Rev.1

Table 2.6. XRF Analyzed Composition of Crucible-Melted Feed Samples (wt\%).

\begin{tabular}{|c|c|c|c|c|c|c|c|c|c|c|c|}
\hline Test \# & \multicolumn{4}{|c|}{6} & \multicolumn{7}{|c|}{7} \\
\hline $\begin{array}{c}\text { Sample } \\
\text { I.D. }\end{array}$ & Target & $\begin{array}{c}12 \mathrm{M}-\mathrm{F}- \\
87 \mathrm{~A}\end{array}$ & $\begin{array}{c}12 \mathrm{M}-\mathrm{F}- \\
100 \mathrm{~A}\end{array}$ & Avg. & $\begin{array}{c}12 \mathrm{M}-\mathrm{F}- \\
143 \mathrm{~A}\end{array}$ & $\begin{array}{c}12 \mathrm{~N}-\mathrm{F}- \\
6 \mathrm{~A}\end{array}$ & $\begin{array}{c}12 \mathrm{~N}-\mathrm{F}- \\
38 \mathrm{~A}\end{array}$ & $\begin{array}{c}12 \mathrm{~N}-\mathrm{F}- \\
64 \mathrm{~A}\end{array}$ & $\begin{array}{c}12 \mathrm{~N}-\mathrm{F}- \\
99 \mathrm{~A}\end{array}$ & $\begin{array}{l}12 \mathrm{~N}-\mathrm{F}- \\
127 \mathrm{~A}\end{array}$ & Avg. \\
\hline$\overline{\mathrm{Al}_{2} \mathrm{O}_{3}}$ & 7.40 & 7.83 & 7.76 & 7.62 & 8.49 & 8.41 & 8.54 & 7.70 & 8.04 & 8.50 & 8.28 \\
\hline $\mathrm{As}_{2} \mathrm{O}_{3}$ & 0.04 & 0.03 & 0.04 & 0.03 & 0.04 & 0.03 & 0.04 & 0.04 & 0.04 & 0.04 & 0.04 \\
\hline $\mathrm{B}_{2} \mathrm{O}_{3}{ }^{*}$ & 10.00 & 10.00 & 10.00 & 10.00 & 10.00 & 10.00 & 10.00 & 10.00 & 10.00 & 10.00 & 10.00 \\
\hline $\mathrm{BaO}$ & 0.04 & $<0.05$ & $<0.05$ & $<0.05$ & $<0.05$ & $<0.05$ & $<0.05$ & $<0.05$ & $<0.05$ & $<0.05$ & $<0.05$ \\
\hline $\mathrm{CaO}$ & 0.25 & 0.65 & 0.65 & 0.68 & 0.60 & 0.62 & 0.59 & 0.57 & 0.59 & 0.66 & 0.60 \\
\hline $\mathrm{CdO}$ & 0.37 & 0.36 & 0.38 & 0.33 & 0.30 & 0.33 & 0.30 & 0.38 & 0.40 & 0.39 & 0.35 \\
\hline $\mathrm{CeO}_{2}$ & 0.08 & 0.08 & 0.09 & 0.08 & 0.08 & 0.08 & 0.07 & 0.09 & 0.07 & 0.08 & 0.08 \\
\hline $\mathrm{Cl}$ & 0.01 & 0.00 & 0.00 & 0.00 & 0.01 & 0.01 & 0.00 & 0.00 & 0.00 & 0.00 & 0.00 \\
\hline $\mathrm{Cr}_{2} \mathrm{O}_{3}$ & 0.04 & 0.05 & 0.05 & 0.04 & 0.04 & 0.04 & 0.04 & 0.05 & 0.05 & 0.04 & 0.04 \\
\hline $\mathrm{Cs}_{2} \mathrm{O}$ & 0.08 & 0.08 & 0.08 & 0.07 & 0.07 & 0.08 & 0.07 & 0.06 & 0.08 & 0.07 & 0.07 \\
\hline $\mathrm{CuO}$ & 0.03 & 0.03 & 0.04 & 0.03 & 0.03 & 0.03 & 0.03 & 0.02 & 0.03 & 0.03 & 0.03 \\
\hline $\mathrm{F}=$ & 0.04 & NA & NA & $\mathrm{NC}$ & NA & $\mathrm{NA}$ & NA & $\mathrm{NA}$ & NA & NA & $\mathrm{NC}$ \\
\hline $\mathrm{Fe}_{2} \mathrm{O}_{3}$ & 10.39 & 10.59 & 10.99 & 10.55 & 10.10 & 10.90 & 10.62 & 10.62 & 10.54 & 10.73 & 10.59 \\
\hline $\mathrm{K}_{2} \mathrm{O}$ & 0.17 & 0.25 & 0.26 & 0.25 & 0.28 & 0.28 & 0.27 & 0.24 & 0.24 & 0.28 & 0.26 \\
\hline $\mathrm{La}_{2} \mathrm{O}_{3}$ & 0.32 & 0.12 & 0.13 & 0.11 & 0.13 & 0.13 & 0.13 & 0.13 & 0.12 & 0.12 & 0.13 \\
\hline $\mathrm{Li}_{2} \mathrm{O}^{*}$ & 6.00 & 6.00 & 6.00 & 6.00 & 6.00 & 6.00 & 6.00 & 6.00 & 6.00 & 6.00 & 6.00 \\
\hline $\mathrm{MgO}$ & 0.06 & 0.78 & 0.70 & 1.20 & 0.37 & 0.41 & 0.47 & 1.09 & 1.00 & 0.24 & 0.60 \\
\hline $\mathrm{MnO}$ & 3.03 & 2.78 & 2.44 & 2.28 & 2.78 & 2.71 & 2.56 & 3.02 & 2.59 & 2.11 & 2.63 \\
\hline $\mathrm{Na}_{2} \mathrm{O}$ & 6.59 & 6.63 & 5.99 & 6.67 & 6.38 & 6.10 & 6.84 & 6.57 & 6.81 & 6.54 & 6.54 \\
\hline $\mathrm{NiO}$ & 0.54 & 0.51 & 0.54 & 0.49 & 0.48 & 0.52 & 0.51 & 0.52 & 0.51 & 0.51 & 0.51 \\
\hline $\mathrm{P}_{2} \mathrm{O}_{5}$ & 0.13 & 0.26 & 0.25 & 0.31 & 0.21 & 0.19 & 0.21 & 0.31 & 0.28 & 0.18 & 0.23 \\
\hline $\mathrm{PbO}$ & 0.15 & 0.13 & 0.13 & 0.12 & 0.12 & 0.13 & 0.13 & 0.13 & 0.13 & 0.12 & 0.13 \\
\hline$\overline{\mathrm{Sb}_{2} \mathrm{O}_{3}}$ & 0.21 & 0.19 & 0.19 & 0.15 & 0.16 & 0.18 & 0.15 & 0.16 & 0.16 & 0.15 & 0.16 \\
\hline $\mathrm{SeO}_{2}$ & 0.15 & 0.03 & 0.04 & 0.04 & 0.04 & 0.04 & 0.04 & 0.03 & 0.03 & 0.05 & 0.04 \\
\hline$\overline{\mathrm{SiO}_{2}}$ & 45.54 & 44.05 & 44.25 & 44.69 & 45.34 & 43.98 & 44.08 & 43.67 & 43.78 & 44.43 & 44.22 \\
\hline $\mathrm{SO}_{3}$ & 0.25 & 0.12 & 0.17 & 0.16 & 0.19 & 0.16 & 0.16 & 0.18 & 0.13 & 0.17 & 0.16 \\
\hline SrO & 2.32 & 2.03 & 2.12 & 1.91 & 1.84 & 2.05 & 1.95 & 2.07 & 2.09 & 2.11 & 2.02 \\
\hline $\mathrm{TeO}_{2}$ & 0.14 & 0.08 & 0.11 & 0.09 & 0.10 & 0.09 & 0.09 & 0.10 & 0.09 & 0.09 & 0.09 \\
\hline $\mathrm{TiO}_{2}$ & 0.06 & 0.23 & 0.23 & 0.21 & 0.24 & 0.25 & 0.23 & 0.20 & 0.19 & 0.23 & 0.22 \\
\hline $\mathrm{ZnO}$ & 2.00 & 2.06 & 2.15 & 2.01 & 1.94 & 2.10 & 2.01 & 2.02 & 1.98 & 2.12 & 2.03 \\
\hline $\mathrm{ZrO}_{2}$ & 3.56 & 4.09 & 4.27 & 3.88 & 3.68 & 4.18 & 3.90 & 4.07 & 4.08 & 4.04 & 4.00 \\
\hline Sum & 99.99 & 100.00 & 100.00 & 100.00 & 100.00 & 100.00 & 100.00 & 100.00 & 100.00 & 100.00 & 100.00 \\
\hline
\end{tabular}

* Target values.

NA: Not Analyzed.

NC: Not Calculated. 
The Catholic University of America

Vitreous State Laboratory
DuraMelter 1200 Tests with AZ-101HLW Simulants

Final Report, VSL-02R0100-2, Rev.1

Table 2.6. XRF Analyzed Composition of Crucible-Melted Feed Samples (wt\%) (Continued).

\begin{tabular}{|c|c|c|c|c|c|c|c|c|c|c|c|c|c|}
\hline Test \# & & \multicolumn{5}{|c|}{8} & \multicolumn{6}{|c|}{9} & \multirow{2}{*}{$\begin{array}{c}\text { All } \\
\text { Tests } \\
\text { Avg. }\end{array}$} \\
\hline $\begin{array}{c}\text { Sample } \\
\text { I.D. }\end{array}$ & Target & $\begin{array}{c}12 \mathrm{O}-\mathrm{F}- \\
16 \mathrm{~A}\end{array}$ & $\begin{array}{c}120-F- \\
31 \mathrm{~A}\end{array}$ & $\begin{array}{c}12 \mathrm{O}-\mathrm{F}- \\
49 \mathrm{~A}\end{array}$ & $\begin{array}{c}12 \mathrm{O}-\mathrm{F} \\
68 \mathrm{~A}\end{array}$ & Avg. & $\begin{array}{c}12 \mathrm{O}-\mathrm{F}- \\
106 \mathrm{~A}\end{array}$ & $\begin{array}{c}12 \mathrm{O}-\mathrm{F}- \\
125 \mathrm{~A}\end{array}$ & $\begin{array}{c}12 \mathrm{O}-\mathrm{F}- \\
144 \mathrm{~A}\end{array}$ & $\begin{array}{c}12 \mathrm{P}-\mathrm{F}- \\
13 \mathrm{~A}\end{array}$ & $\begin{array}{c}12 \mathrm{P}-\mathrm{F}- \\
41 \mathrm{~A}\end{array}$ & Avg. & \\
\hline $\mathrm{Al}_{2} \mathrm{O}_{3}$ & 7.40 & 7.31 & 7.82 & 7.83 & 7.11 & 7.52 & 7.20 & 7.65 & 7.29 & 7.25 & 7.57 & 7.20 & 7.58 \\
\hline $\mathrm{As}_{2} \mathrm{O}_{3}$ & 0.04 & 0.03 & 0.04 & 0.04 & 0.04 & 0.04 & 0.04 & 0.04 & 0.04 & 0.04 & 0.04 & 0.04 & 0.04 \\
\hline $\mathrm{B}_{2} \mathrm{O}_{3}{ }^{*}$ & 10.00 & 10.00 & 10.00 & 10.00 & 10.00 & 10.00 & 10.00 & 10.00 & 10.00 & 10.00 & 10.00 & 10.00 & 10.00 \\
\hline $\mathrm{BaO}$ & 0.04 & $<0.05$ & $<0.05$ & $<0.05$ & $<0.05$ & $<0.05$ & $<0.05$ & $<0.05$ & $<0.05$ & $<0.05$ & $<0.05$ & $<0.05$ & $<0.05$ \\
\hline $\mathrm{CaO}$ & 0.25 & 0.30 & 0.31 & 0.31 & 0.30 & 0.30 & 0.29 & 0.29 & 0.29 & 0.28 & 0.29 & 0.29 & 0.44 \\
\hline $\mathrm{CdO}$ & 0.37 & 0.42 & 0.39 & 0.36 & 0.47 & 0.41 & 0.45 & 0.44 & 0.33 & 0.30 & 0.31 & 0.45 & 0.35 \\
\hline $\mathrm{CeO}_{2}$ & 0.08 & 0.08 & 0.08 & 0.07 & 0.09 & 0.08 & 0.07 & 0.06 & 0.06 & 0.06 & 0.06 & 0.07 & 0.07 \\
\hline $\mathrm{Cl}$ & 0.01 & 0.00 & 0.00 & 0.00 & 0.00 & 0.00 & 0.01 & 0.00 & 0.02 & 0.02 & 0.02 & 0.01 & 0.00 \\
\hline $\mathrm{Cr}_{2} \mathrm{O}_{3}$ & 0.04 & 0.04 & 0.04 & 0.04 & 0.04 & 0.04 & 0.04 & 0.05 & 0.05 & 0.04 & 0.04 & 0.04 & 0.04 \\
\hline $\mathrm{Cs}_{2} \mathrm{O}$ & 0.08 & 0.07 & 0.06 & 0.06 & 0.08 & 0.07 & 0.08 & 0.07 & 0.06 & 0.06 & 0.07 & 0.08 & 0.07 \\
\hline $\mathrm{CuO}$ & 0.03 & 0.03 & 0.03 & 0.03 & 0.03 & 0.03 & 0.03 & 0.03 & 0.03 & 0.03 & 0.03 & 0.03 & 0.03 \\
\hline $\mathrm{F}$ & 0.04 & NA & NA & NA & NA & $\mathrm{NC}$ & $\mathrm{NA}$ & NA & NA & NA & NA & $\mathrm{NC}$ & $\mathrm{NC}$ \\
\hline $\mathrm{Fe}_{2} \mathrm{O}_{3}$ & 10.39 & 9.66 & 9.67 & 9.90 & 10.13 & 9.84 & 10.43 & 9.93 & 10.44 & 10.19 & 10.18 & 10.43 & 10.35 \\
\hline $\mathrm{K}_{2} \mathrm{O}$ & 0.17 & 0.25 & 0.27 & 0.27 & 0.25 & 0.26 & 0.24 & 0.25 & 0.25 & 0.24 & 0.24 & 0.24 & 0.25 \\
\hline $\mathrm{La}_{2} \mathrm{O}_{3}$ & 0.32 & 0.12 & 0.13 & 0.13 & 0.13 & 0.13 & 0.12 & 0.12 & 0.12 & 0.13 & 0.13 & 0.12 & 0.12 \\
\hline $\mathrm{Li}_{2} \mathrm{O}^{*}$ & 6.00 & 6.00 & 6.00 & 6.00 & 6.00 & 6.00 & 6.00 & 6.00 & 6.00 & 6.00 & 6.00 & 6.00 & 6.00 \\
\hline $\mathrm{MgO}$ & 0.06 & 1.16 & 1.21 & 1.35 & 1.21 & 1.23 & 1.22 & 1.21 & 1.20 & 1.27 & 1.24 & 1.22 & 1.15 \\
\hline $\mathrm{MnO}$ & 3.03 & 2.85 & 2.51 & 1.65 & 3.38 & 2.59 & 3.27 & 3.19 & 3.38 & 3.18 & 2.98 & 3.27 & 2.71 \\
\hline $\mathrm{Na}_{2} \mathrm{O}$ & 6.59 & 6.42 & 6.68 & 7.35 & 6.29 & 6.68 & 6.58 & 6.69 & 6.52 & 7.16 & 6.69 & 6.58 & 6.67 \\
\hline $\mathrm{NiO}$ & 0.54 & 0.47 & 0.48 & 0.49 & 0.48 & 0.48 & 0.49 & 0.46 & 0.50 & 0.46 & 0.48 & 0.49 & 0.49 \\
\hline $\mathrm{P}_{2} \mathrm{O}_{5}$ & 0.13 & 0.30 & 0.33 & 0.33 & 0.31 & 0.32 & 0.32 & 0.31 & 0.32 & 0.31 & 0.33 & 0.32 & 0.32 \\
\hline $\mathrm{PbO}$ & 0.15 & 0.11 & 0.10 & 0.08 & 0.13 & 0.10 & 0.13 & 0.12 & 0.13 & 0.13 & 0.12 & 0.13 & 0.12 \\
\hline $\mathrm{Sb}_{2} \mathrm{O}_{3}$ & 0.21 & 0.18 & 0.15 & 0.11 & 0.21 & 0.16 & 0.19 & 0.18 & 0.16 & 0.15 & 0.16 & 0.19 & 0.16 \\
\hline $\mathrm{SeO}_{2}$ & 0.15 & 0.04 & 0.03 & 0.03 & 0.04 & 0.03 & 0.06 & 0.04 & 0.05 & 0.03 & 0.04 & 0.06 & 0.03 \\
\hline $\mathrm{SiO}_{2}$ & 45.54 & 46.29 & 45.95 & 45.81 & 45.09 & 45.78 & 44.37 & 45.10 & 44.61 & 44.76 & 45.03 & 44.37 & 44.98 \\
\hline $\mathrm{SO}_{3}$ & 0.25 & 0.20 & 0.18 & 0.16 & 0.18 & 0.18 & 0.23 & 0.19 & 0.23 & 0.22 & 0.23 & 0.23 & 0.18 \\
\hline $\mathrm{SrO}$ & 2.32 & 1.85 & 1.85 & 1.92 & 1.95 & 1.89 & 2.05 & 1.89 & 2.00 & 1.94 & 1.94 & 2.05 & 1.94 \\
\hline $\mathrm{TeO}_{2}$ & 0.14 & 0.11 & 0.08 & 0.07 & 0.11 & 0.09 & 0.13 & 0.10 & 0.09 & 0.07 & 0.09 & 0.13 & 0.09 \\
\hline $\mathrm{TiO}_{2}$ & 0.06 & 0.12 & 0.12 & 0.11 & 0.11 & 0.11 & 0.09 & 0.09 & 0.09 & 0.08 & 0.10 & 0.09 & 0.14 \\
\hline $\mathrm{ZnO}$ & 2.00 & 1.79 & 1.81 & 1.83 & 1.87 & 1.82 & 1.85 & 1.76 & 1.87 & 1.82 & 1.78 & 1.85 & 1.90 \\
\hline $\mathrm{ZrO}_{2}$ & 3.56 & 3.84 & 3.73 & 3.71 & 4.01 & 3.83 & 4.06 & 3.77 & 3.90 & 3.81 & 3.84 & 4.06 & 3.80 \\
\hline Sum & 100.00 & 100.00 & 100.00 & 100.00 & 100.00 & 100.00 & 100.00 & 100.00 & 100.00 & 100.00 & 100.00 & 100.00 & \begin{tabular}{|l|l|}
100.00 \\
\end{tabular} \\
\hline
\end{tabular}


The Catholic University of America Vitreous State Laboratory
DuraMelter 1200 Tests with AZ-J01HLW Simulants

Final Report, VSL-02R0100-2, Rev.1

Table 2.7. DCP Analyzed Compositions of Crucible-Melted Feed Samples (wt\%).

\begin{tabular}{|c|c|c|c|c|c|c|c|c|c|c|}
\hline Test\# & \multirow[b]{2}{*}{ Target } & \multicolumn{6}{|c|}{1} & \multicolumn{3}{|c|}{2} \\
\hline Oxide & & $\begin{array}{l}\text { 12F-F- } \\
146 A 1\end{array}$ & $\begin{array}{l}12 \mathrm{~F}-\mathrm{F}- \\
146 \mathrm{~A} 2\end{array}$ & $\begin{array}{c}12 \mathrm{G}-\mathrm{F}- \\
6 \mathrm{~A}\end{array}$ & $\begin{array}{c}12 \mathrm{G}-\mathrm{F}- \\
33 \mathrm{~A}\end{array}$ & $\begin{array}{c}12 \mathrm{G}-\mathrm{F}- \\
53 \mathrm{~A}\end{array}$ & $\begin{array}{c}12 \mathrm{G}-\mathrm{F}- \\
71 \mathrm{~A}\end{array}$ & $\begin{array}{c}12 \mathrm{G}-\mathrm{F}- \\
130 \mathrm{~A}\end{array}$ & $\begin{array}{c}12 \mathrm{G}-\mathrm{F}- \\
149 \mathrm{~A}\end{array}$ & $\begin{array}{l}12 \mathrm{I}-\mathrm{F}- \\
22 \mathrm{~A}\end{array}$ \\
\hline $\mathrm{Al}_{2} \mathrm{O}_{3}$ & 7.40 & 7.71 & 7.41 & 7.54 & 7.62 & 7.71 & 7.71 & 7.56 & 7.12 & 7.22 \\
\hline $\mathrm{As}_{2} \mathrm{O}_{3}$ & 0.04 & $\mathrm{NA}$ & NA & NA & NA & NA & NA & $\mathrm{NA}$ & 0.00 & 0.00 \\
\hline $\mathrm{B}_{2} \mathrm{O}_{3}$ & 10.00 & 10.91 & 10.72 & 10.65 & 10.36 & 10.87 & 10.32 & 10.55 & 10.11 & 10.42 \\
\hline $\mathrm{BaO}$ & 0.04 & 0.05 & 0.06 & 0.05 & 0.05 & 0.05 & 0.05 & 0.05 & 0.05 & 0.05 \\
\hline $\mathrm{CaO}$ & 0.25 & 0.31 & 0.31 & 0.27 & 0.27 & 0.27 & 0.29 & 0.27 & 0.29 & 0.29 \\
\hline $\mathrm{CdO}$ & 0.37 & NA & $\mathrm{NA}$ & $\mathrm{NA}$ & NA & NA & $\mathrm{NA}$ & $\mathrm{NA}$ & 0.28 & 0.28 \\
\hline $\mathrm{CeO}_{2}$ & 0.08 & $\overline{\mathrm{NA}}$ & $\mathrm{NA}$ & $\mathrm{NA}$ & $\mathrm{NA}$ & NA & $\mathrm{NA}$ & NA & $\mathrm{NA}$ & NA \\
\hline $\mathrm{Cl}$ & 0.01 & $\mathrm{NA}$ & $\mathrm{NA}$ & $\mathrm{NA}$ & $\mathrm{NA}$ & $\mathrm{NA}$ & $\mathrm{NA}$ & $\mathrm{NA}$ & $\mathrm{NA}$ & NA \\
\hline $\mathrm{Cr}_{2} \mathrm{O}_{3}$ & 0.04 & 0.05 & 0.04 & 0.04 & 0.04 & 0.04 & 0.05 & 0.05 & 0.04 & 0.04 \\
\hline $\mathrm{Cs}_{2} \mathrm{O}^{*}$ & $\overline{0.08}$ & 0.09 & $0.05 \mathrm{~A}$ & 0.08 & 0.08 & 0.09 & 0.08 & 0.08 & 0.08 & 0.08 \\
\hline $\mathrm{CuO}$ & 0.03 & NA & NA & $\mathrm{NA}$ & $\mathrm{NA}$ & $\mathrm{NA}$ & NA & NA & 0.02 & 0.03 \\
\hline $\mathrm{F}$ & 0.04 & NA & $\mathrm{NA}$ & $\mathrm{NA}$ & NA & $\mathrm{NA}$ & $\mathrm{NA}$ & NA & $\mathrm{NA}$ & NA \\
\hline $\mathrm{Fe}_{2} \mathrm{O}_{3}$ & 10.39 & 9.57 & 9.79 & 9.43 & 9.51 & 9.48 & 9.62 & 9.59 & 9.15 & 9.30 \\
\hline $\mathrm{K}_{2} \mathrm{O}$ & 0.17 & 0.21 & 0.20 & 0.19 & 0.18 & 0.17 & 0.19 & 0.17 & 0.23 & 0.25 \\
\hline $\mathrm{La}_{2} \mathrm{O}_{3}$ & 0.32 & NA & NA & $\mathrm{NA}$ & NA & NA & NA & NA & NA & NA \\
\hline $\mathrm{Li}_{2} \mathrm{O}$ & 6.00 & 5.26 & 5.04 & 5.15 & 5.15 & 5.18 & 4.92 & 5.38 & 4.94 & 4.74 \\
\hline $\mathrm{MgO}$ & 0.06 & 1.34 & 1.28 & 1.20 & 1.21 & 1.21 & 1.30 & 1.23 & 1.28 & 1.31 \\
\hline $\mathrm{MnO}$ & 3.03 & 2.84 & 2.77 & 2.79 & 2.79 & 2.82 & 2.83 & 2.66 & 2.45 & 2.41 \\
\hline $\mathrm{Na}_{2} \mathrm{O}$ & 6.59 & 6.66 & 6.42 & 6.46 & 6.41 & 6.55 & 6.49 & 6.53 & 6.10 & 6.68 \\
\hline $\mathrm{NiO}$ & 0.54 & 0.50 & 0.52 & 0.44 & 0.46 & 0.46 & 0.48 & 0.45 & 0.45 & 0.47 \\
\hline $\mathrm{P}_{2} \mathrm{O}_{5}$ & 0.13 & 0.22 & 0.26 & 0.10 & 0.26 & 0.23 & 0.29 & 0.45 & 0.37 & 0.32 \\
\hline $\mathrm{PbO}$ & 0.15 & $\mathrm{NA}$ & $\mathrm{NA}$ & $\mathrm{NA}$ & $\mathrm{NA}$ & NA & $\mathrm{NA}$ & $\mathrm{NA}$ & 0.18 & 0.18 \\
\hline $\mathrm{Sb}_{2} \mathrm{O}_{3}$ & 0.21 & NA & NA & NA & NA & NA & NA & $\mathrm{NA}$ & 0.15 & 0.19 \\
\hline $\mathrm{SeO}_{2}$ & 0.15 & $\mathrm{NA}$ & $\mathrm{NA}$ & $\mathrm{NA}$ & NA & NA & NA & NA & 0.01 & 0.03 \\
\hline $\mathrm{SiO}_{2}$ & 45.54 & 44.71 & 43.66 & 44.27 & 44.83 & 44.50 & 44.79 & 43.13 & 41.57 & 42.53 \\
\hline $\mathrm{SO}_{3}$ & 0.25 & NA & NA & $\mathrm{NA}$ & NA & NA & $\mathrm{NA}$ & NA & $0.09^{@}$ & $0.16^{@}$ \\
\hline SrO & 2.32 & 2.01 & 1.95 & 1.94 & 1.95 & 1.96 & 1.99 & 1.97 & 1.85 & 1.87 \\
\hline $\mathrm{TeO}_{2}$ & 0.14 & $\mathrm{NA}$ & $\mathrm{NA}$ & $\mathrm{NA}$ & NA & $\mathrm{NA}$ & $\mathrm{NA}$ & $\mathrm{NA}$ & 0.02 & 0.07 \\
\hline $\mathrm{TiO}_{2}$ & 0.06 & 0.11 & 0.10 & 0.09 & 0.09 & 0.09 & 0.10 & 0.09 & 0.09 & 0.10 \\
\hline $\mathrm{ZnO}$ & 2.00 & $\mathrm{NA}$ & $\mathrm{NA}$ & $\mathrm{NA}$ & $\mathrm{NA}$ & $\mathrm{NA}$ & $\mathrm{NA}$ & $\mathrm{NA}$ & 1.79 & 1.81 \\
\hline $\mathrm{ZrO}_{2}$ & 3.56 & 3.50 & 3.39 & 3.58 & 3.51 & 3.55 & 3.50 & 3.65 & 3.44 & 3.54 \\
\hline Sum & 99.99 & 96.05 & 93.96 & 94.26 & 94.77 & 95.24 & 95.01 & 93.88 & 92.16 & 94.38 \\
\hline
\end{tabular}

* Analyzed by AA.

NA: Not Analyzed.

@ - Analyzed by ion chromatography. 
The Catholic University of America

Vitreous State Laboratory
DuraMelter 1200 Tests with AZ-101HLW Simulants

Final Report, VSL-02R0100-2, Rev.1

Table 2.7. DCP Analyzed Compositions of Crucible-Melted Feed Samples (wt\%) (continued).

\begin{tabular}{|c|c|c|c|c|c|c|c|c|c|c|c|}
\hline Test\# & & 2 & 3 & \multicolumn{2}{|c|}{4} & \multicolumn{2}{|c|}{5} & \multicolumn{3}{|c|}{6} & 7 \\
\hline Oxide & Target & $\begin{array}{c}12 \mathrm{I}-\mathrm{F}- \\
66 \mathrm{~A}\end{array}$ & $\begin{array}{c}12 \mathrm{~J}-\mathrm{F}- \\
81 \mathrm{~A}\end{array}$ & \begin{tabular}{|c|}
$12 \mathrm{~K}-\mathrm{F}-$ \\
$54 \mathrm{~A}$
\end{tabular} & $\begin{array}{l}12 \mathrm{~K}-\mathrm{F}- \\
125 \mathrm{~A}\end{array}$ & $\begin{array}{c}12 \mathrm{~L}-\mathrm{F}- \\
30 \mathrm{~A}\end{array}$ & $\begin{array}{c}12 \mathrm{~L}-\mathrm{F}- \\
80 \mathrm{~A}\end{array}$ & $\begin{array}{l}\text { 12L-F- } \\
147 \mathrm{~A}\end{array}$ & $\begin{array}{c}12 \mathrm{M}-\mathrm{F}- \\
9 \mathrm{~A}\end{array}$ & $\begin{array}{c}12 \mathrm{M}-\mathrm{F}- \\
54 \mathrm{~A}\end{array}$ & $\begin{array}{c}12 \mathrm{~N}-\mathrm{F}- \\
6 \mathrm{Al}\end{array}$ \\
\hline $\mathrm{Al}_{2} \mathrm{O}_{3}$ & 7.40 & 7.16 & 7.26 & 7.89 & 7.31 & 6.81 & 7.51 & 7.14 & 7.16 & 7.37 & 8.29 \\
\hline $\mathrm{As}_{2} \mathrm{O}_{3}$ & .04 & 0.02 & .01 & 0.01 & 0.01 & 0.01 & 0.01 & 0.04 & 0.04 & 0.06 & 0.07 \\
\hline $\mathrm{B}_{2} \mathrm{O}_{3}$ & 10.00 & 10.39 & 9.77 & 8.84 & 8.52 & 9.77 & 10.73 & 9.24 & 9.38 & 9.62 & 9.50 \\
\hline $\mathrm{BaO}$ & 0.04 & 0.05 & 0.05 & 0.05 & 0.05 & 0.04 & 0.04 & 0.04 & 0.05 & 0.05 & 0.04 \\
\hline $\mathrm{CaO}$ & 25 & 0.27 & 0.28 & 0.29 & 0.26 & 0.26 & 0.28 & 0.68 & 0.65 & 0.66 & 0.59 \\
\hline $\mathrm{CdO}$ & 0.37 & 0.29 & 0.31 & 0.30 & 0.27 & 0.32 & 0.32 & 0.28 & 0.31 & 0.30 & 0.26 \\
\hline $\mathrm{CeO}_{2}$ & 0.08 & NA & NA & NA & NA & NA & NA & NA & $\mathrm{NA}$ & NA & NA \\
\hline $\mathrm{Cl}$ & 01 & NA & NA & NA & NA & NA & NA & NA & NA & NA & NA \\
\hline $\mathrm{Cr}_{2} \mathrm{O}_{3}$ & 0.04 & 0.05 & 0.04 & 0.05 & 0.04 & 0.04 & 0.05 & 0.05 & 0.04 & 0.04 & 0.04 \\
\hline $\mathrm{Cs}_{2} \mathrm{O}^{*}$ & 0.08 & 0.08 & 0.08 & 0.08 & 0.07 & 0.07 & 0.07 & 0.07 & 0.07 & 0.07 & 0.06 \\
\hline $\mathrm{CuO}$ & 0.03 & 0.02 & 0.03 & 0.03 & 0.03 & 0.03 & 0.03 & 0.03 & 0.03 & 0.03 & 0.03 \\
\hline $\bar{F}$ & 0.04 & NA & NA & NA & NA & NA & NA & NA & NA & NA & NA \\
\hline $\mathrm{Fe}_{2} \mathrm{O}_{3}$ & 10.39 & 9.30 & 9.61 & 9.94 & 10.38 & 9.60 & 9.71 & 10.11 & 9.42 & 9.89 & 9.80 \\
\hline $\mathrm{K}_{2} \mathrm{O}$ & & 0.19 & 0.19 & 0.18 & 0.19 & 0.20 & 0.22 & 0.19 & 0.19 & 0.19 & 0.22 \\
\hline $\mathrm{La}_{2} \mathrm{O}_{3}$ & & $\mathrm{NA}$ & NA & NA & NA & NA & NA & $\mathrm{NA}$ & $\mathrm{NA}$ & NA & NA \\
\hline $\mathrm{Li}_{2} \mathrm{O}$ & & 4.98 & 4.58 & 4.30 & 3.92 & 4.86 & 5.57 & 4.62 & 4.70 & 4.82 & 4.75 \\
\hline$\overline{\mathrm{Mg}}$ & & & 1.3 & & 1.28 & 1.23 & 1.39 & 1.35 & 1.27 & 1.30 & 0.48 \\
\hline$\overline{\mathrm{MnO}}$ & & 27 & 2.58 & & 3.09 & 3.02 & 1.45 & 2.09 & 2.96 & 2.10 & 2.73 \\
\hline $\mathrm{Na}_{2} \mathrm{O}$ & & .31 & 6.06 & 5.84 & 5.57 & 5.89 & 6.71 & 5.83 & 5.94 & 6.06 & 5.81 \\
\hline $\mathrm{NiO}$ & & 0.45 & 0.47 & 0.49 & 0.47 & 0.45 & 0.49 & 0.49 & 0.45 & 0.47 & 0.48 \\
\hline $\mathrm{P}_{2} \mathrm{O}_{5}$ & & 0.38 & 0.26 & 0.37 & 0.28 & 0.25 & 0.31 & 0.28 & 0.28 & 0.25 & 0.11 \\
\hline $\mathrm{PbO}$ & 0.15 & 0.18 & 0.18 & 0.18 & 0.15 & 0.15 & 0.11 & 0.16 & 0.18 & 0.18 & 0.20 \\
\hline $\mathrm{Sb}_{2} \mathrm{O}_{3}$ & 0.21 & 0.17 & 0.17 & 0.15 & 0.18 & 0.17 & 0.13 & 0.18 & 0.21 & 0.18 & 0.19 \\
\hline $\mathrm{SeO}_{2}$ & & 0.00 & 0.00 & 0.03 & -0.00 & 0.02 & 0.01 & 0.02 & 0.02 & 0.03 & 0.05 \\
\hline $\mathrm{SiO}_{2}$ & 45.54 & 42.68 & 43.69 & 42.94 & 45.43 & 45.70 & 42.28 & 43.03 & 43.28 & 44.56 & 43.47 \\
\hline $\mathrm{SO}_{3}$ & 0.25 & $0.16^{(a)}$ & $0.16^{\natural}$ & $0.18^{@}$ & NA & $\mathrm{NA}$ & NA & NA & $\mathrm{NA}$ & NA & NA \\
\hline $\mathrm{SrO}$ & & 1.91 & 1.94 & 1.94 & 1.87 & 1.71 & 1.92 & 1.78 & 1.87 & 1.92 & 1.86 \\
\hline $\mathrm{TeO}_{2}$ & 0.14 & 0.06 & 0.03 & 0.04 & 0.12 & 0.12 & 0.12 & 0.11 & 0.10 & 0.09 & 0.09 \\
\hline $\mathrm{TiO}_{2}$ & 0.06 & 0.10 & 0.10 & 0.12 & 0.13 & 0.13 & 0.12 & 0.23 & 0.23 & 0.23 & 0.27 \\
\hline $\mathrm{ZnO}$ & 2.00 & 1.78 & 1.84 & 1.89 & 1.94 & 1.84 & 1.93 & 1.99 & 2.03 & 2.05 & 2.05 \\
\hline $\mathrm{ZrO}_{2}$ & 3.56 & 3.59 & 3.60 & 3.76 & 3.64 & 3.38 & 3.51 & 3.54 & 3.68 & 3.75 & 3.66 \\
\hline Sum & 99.99 & 94.39 & 94.60 & 93.50 & 95.23 & 96.09 & 95.06 & 93.59 & 94.54 & 96.30 & 95.10 \\
\hline
\end{tabular}

* Analyzed by AA.

NA: Not Analyzed.

@- Analyzed by ion chromatography. 
The Catholic University of America Vitreous State Laboratory
DuraMelter 1200 Tests with AZ-101HLW Simulants Final Report, VSL-02R0100-2, Rev.1

Table 2.7. DCP Analyzed Compositions of Crucible-Melted Feed Samples (wt\%) (continued).

\begin{tabular}{|c|c|c|c|c|c|c|c|}
\hline Test\# & \multicolumn{2}{|c|}{7} & 8 & \multicolumn{2}{|c|}{9} & \multicolumn{2}{|c|}{ Total } \\
\hline Oxide & $\begin{array}{c}12 \mathrm{~N}-\mathrm{F}- \\
6 \mathrm{~A} 2 \\
\end{array}$ & $\begin{array}{c}12 \mathrm{~N}-\mathrm{F}- \\
99 \mathrm{~A}\end{array}$ & $\begin{array}{c}12 \mathrm{O}-\mathrm{F}- \\
16 \mathrm{~A}\end{array}$ & $\begin{array}{l}12 \mathrm{O}-\mathrm{F}- \\
125 \mathrm{Al}\end{array}$ & $\begin{array}{l}12 \mathrm{O}-\mathrm{F}- \\
125 \mathrm{~A} 2\end{array}$ & Target & Avg. \\
\hline $\mathrm{Al}_{2} \mathrm{O}_{3}$ & 7.91 & 7.79 & 7.17 & 6.57 & 6.52 & 7.40 & 7.39 \\
\hline $\mathrm{As}_{2} \mathrm{O}_{3}$ & 0.07 & 0.02 & 0.06 & 0.10 & 0.23 & 0.04 & 0.04 \\
\hline $\mathrm{B}_{2} \mathrm{O}_{3}$ & 9.26 & 9.49 & 10.01 & 9.67 & 9.58 & 10.00 & 9.95 \\
\hline $\mathrm{BaO}$ & 0.04 & 0.05 & 0.05 & 0.05 & 0.05 & 0.04 & 0.05 \\
\hline $\mathrm{CaO}$ & 0.55 & 0.55 & 0.27 & 0.26 & 0.24 & 0.25 & 0.36 \\
\hline $\mathrm{CdO}$ & 0.25 & 0.31 & 0.32 & 0.37 & 0.35 & 0.37 & 0.30 \\
\hline $\mathrm{CeO}_{2}$ & $\mathrm{NA}$ & $\mathrm{NA}$ & $\mathrm{NA}$ & NA & $\mathrm{NA}$ & 0.08 & NA \\
\hline $\mathrm{Cl}$ & NA & NA & $\mathrm{NA}$ & NA & NA & 0.01 & NA \\
\hline $\mathrm{Cr}_{2} \mathrm{O}_{3}$ & 0.04 & 0.04 & 0.04 & 0.04 & 0.04 & 0.04 & 0.04 \\
\hline $\mathrm{Cs}_{2} \mathrm{O}^{*}$ & 0.03 & 0.06 & $\overline{0.06}$ & 0.05 & 0.03 & 0.08 & 0.07 \\
\hline $\mathrm{CuO}$ & 0.03 & 0.02 & 0.03 & 0.03 & 0.03 & 0.03 & 0.03 \\
\hline F & NA & $\mathrm{NA}$ & NA & NA & NA & 0.04 & NA \\
\hline $\mathrm{Fe}_{2} \mathrm{O}_{3}$ & 9.70 & 9.49 & 8.63 & 9.34 & 8.94 & 10.39 & 9.55 \\
\hline $\mathrm{K}_{2} \mathrm{O}$ & 0.21 & 0.18 & 0.20 & 0.20 & 0.19 & 0.17 & 0.20 \\
\hline $\mathrm{La}_{2} \mathrm{O}_{3}$ & NA & $\mathrm{NA}$ & NA & NA & NA & 0.32 & NA \\
\hline $\mathrm{Li}_{2} \mathrm{O}$ & 4.55 & 4.82 & 5.08 & 4.60 & 4.51 & 6.00 & 4.85 \\
\hline $\mathrm{MgO}$ & 0.46 & 0.97 & 1.21 & 1.25 & 1.18 & 0.06 & 1.20 \\
\hline $\mathrm{MnO}$ & 2.75 & 2.57 & 2.97 & 3.18 & 3.16 & 3.03 & 2.66 \\
\hline $\mathrm{Na}_{2} \mathrm{O}$ & 5.60 & 5.94 & 6.04 & 5.62 & 5.53 & 6.59 & 6.13 \\
\hline $\mathrm{NiO}$ & 0.47 & 0.47 & 0.45 & 0.46 & 0.45 & 0.54 & 0.47 \\
\hline $\mathrm{P}_{2} \mathrm{O}_{5}$ & 0.14 & 0.21 & 0.33 & 0.25 & 0.23 & 0.13 & 0.27 \\
\hline $\mathrm{PbO}$ & 0.18 & 0.17 & 0.16 & 0.19 & 0.17 & 0.15 & 0.17 \\
\hline $\mathrm{Sb}_{2} \mathrm{O}_{3}$ & 0.22 & 0.19 & 0.19 & 0.20 & 0.21 & 0.21 & 0.18 \\
\hline $\mathrm{SeO}_{2}$ & 0.05 & 0.06 & 0.06 & -0.05 & -0.11 & 0.15 & 0.01 \\
\hline $\mathrm{SiO}_{2}$ & 42.82 & 42.61 & 45.67 & 44.48 & 45.07 & 45.54 & 43.82 \\
\hline $\mathrm{SO}_{3}$ & NA & NA & $\mathrm{NA}$ & NA & NA & $0.25^{\varrho}$ & $0.15^{@}$ \\
\hline $\mathrm{SrO}$ & 1.90 & 1.88 & 1.72 & 1.72 & 1.95 & 2.32 & 1.89 \\
\hline $\mathrm{TeO}_{2}$ & 0.12 & 0.06 & 0.08 & 0.06 & 0.08 & 0.14 & 0.08 \\
\hline $\mathrm{TiO}_{2}$ & 0.26 & 0.21 & 0.14 & 0.11 & 0.11 & 0.06 & 0.14 \\
\hline $\mathrm{ZnO}$ & 2.04 & 1.93 & 1.79 & 1.86 & 1.91 & 2.00 & 1.91 \\
\hline $\mathrm{ZrO}_{2}$ & 3.56 & 3.57 & 3.44 & 3.39 & 3.39 & 3.56 & 3.55 \\
\hline Sum & 93.21 & 93.66 & 96.16 & 93.99 & 94.05 & 99.99 & 94.55 \\
\hline
\end{tabular}

* Analyzed by AA.

NA: Not Analyzed

(a) - Analyzed by ion chromatography. 
The Catholic University of America Vitreous State Laboratory
DuraMelter 1200 Tests with AZ-101HLW Simulants

Final Report, VSL-02R0100-2, Rev.J

Table 2.8. Average Compositions and \% Deviation of Major Components From Target for Feed and Discharged Glass Samples.

\begin{tabular}{|c|c|c|c|c|c|c|c|c|c|}
\hline \multirow[b]{3}{*}{ Oxide } & \multirow{3}{*}{ Target } & \multicolumn{4}{|c|}{$\mathrm{XRF}$} & \multicolumn{4}{|c|}{ DCP } \\
\hline & & \multicolumn{2}{|c|}{ Feed } & \multicolumn{2}{|c|}{ Glass } & \multicolumn{2}{|c|}{ Feed } & \multicolumn{2}{|c|}{ Glass } \\
\hline & & Avg. & $\%$ Dev. & Avg. & $\%$ Dev. & Avg. & $\%$ Dev. & Avg. & $\%$ Dev. \\
\hline $\mathrm{Al}_{2} \mathrm{O}_{3}$ & 7.40 & 7.58 & 2.43 & 7.54 & 1.89 & 7.39 & -0.14 & 7.49 & 1.22 \\
\hline $\mathrm{As}_{2} \mathrm{O}_{3}$ & 0.04 & 0.04 & $\mathrm{NC}$ & 0.04 & $\mathrm{NC}$ & 0.04 & $\mathrm{NC}$ & 0.03 & $\mathrm{NC}$ \\
\hline $\mathrm{B}_{2} \mathrm{O}_{3}$ & 10.00 & $\mathrm{NA}$ & $\mathrm{NC}$ & NA & $\mathrm{NC}$ & 9.95 & -0.50 & 10.37 & 3.70 \\
\hline $\mathrm{BaO}$ & 0.04 & $<0.05$ & $\mathrm{NC}$ & $<0.05$ & $\mathrm{NC}$ & 0.05 & $\mathrm{NC}$ & 0.05 & $\mathrm{NC}$ \\
\hline$\overline{\mathrm{CaO}}$ & 0.25 & 0.44 & $\mathrm{NC}$ & 0.38 & $\mathrm{NC}$ & 0.36 & $\mathrm{NC}$ & 0.33 & $\overline{\mathrm{NC}}$ \\
\hline $\mathrm{CdO}$ & 0.37 & 0.35 & NC & 0.39 & NC & 0.30 & $\mathrm{NC}$ & 0.33 & $\overline{\mathrm{NC}}$ \\
\hline $\mathrm{CeO}_{2}$ & 0.08 & 0.07 & NC & 0.07 & NC & $\mathrm{NA}$ & $\mathrm{NC}$ & $\mathrm{NA}$ & $\mathrm{NC}$ \\
\hline $\mathrm{Cl}$ & 0.01 & $<0.01$ & $\mathrm{NC}$ & $<0.01$ & $\mathrm{NC}$ & NA & $\mathrm{NC}$ & NA & $\mathrm{NC}$ \\
\hline $\mathrm{Cr}_{2} \mathrm{O}_{3}$ & 0.04 & 0.04 & $\mathrm{NC}$ & 0.05 & NC & 0.04 & NC & 0.05 & $\mathrm{NC}$ \\
\hline $\mathrm{Cs}_{2} \mathrm{O}$ & 0.08 & 0.07 & $\mathrm{NC}$ & 0.07 & $\mathrm{NC}$ & $0.07^{\#}$ & $\mathrm{NC}$ & $0.08^{\#}$ & $\mathrm{NC}$ \\
\hline $\mathrm{CuO}$ & 0.03 & 0.03 & $\mathrm{NC}$ & 0.03 & $\mathrm{NC}$ & 0.03 & $\mathrm{NC}$ & 0.03 & $\mathrm{NC}$ \\
\hline $\mathrm{F}$ & 0.04 & $\mathrm{NA}$ & $\mathrm{NC}$ & NA & $\mathrm{NC}$ & NA & $\mathrm{NC}$ & NA & $\mathrm{NC}$ \\
\hline $\mathrm{Fe}_{2} \mathrm{O}_{3}$ & 10.39 & 10.35 & -0.38 & 10.19 & -1.92 & $9.5 \overline{5}$ & -8.08 & 9.51 & -8.47 \\
\hline $\mathrm{K}_{2} \mathrm{O}$ & 0.17 & 0.25 & $\mathrm{NC}$ & 0.25 & NC & 0.20 & $\mathrm{NC}$ & 0.19 & $\mathrm{NC}$ \\
\hline $\mathrm{La}_{2} \mathrm{O}_{3}$ & 0.32 & 0.12 & $\mathrm{NC}$ & 0.12 & NC & NA & NC & $\mathrm{NA}$ & $\overline{\mathrm{NC}}$ \\
\hline $\mathrm{Li}_{2} \mathrm{O}$ & 6.00 & NA & $\mathrm{NA}$ & $\mathrm{NA}$ & $\mathrm{NC}$ & 4.85 & -19.17 & 5.20 & -13.33 \\
\hline $\mathrm{MgO}$ & 0.06 & 1.15 & 1817 & 0.98 & 1533 & 1.20 & 1900 & 1.11 & 1750 \\
\hline $\mathrm{MnO}$ & 3.03 & 2.71 & -10.56 & 3.04 & 0.33 & 2.66 & -12.21 & 2.79 & -7.92 \\
\hline $\mathrm{Na}_{2} \mathrm{O}$ & 6.59 & 6.67 & 1.21 & 6.79 & 3.03 & 6.13 & -6.98 & 6.37 & -3.34 \\
\hline $\mathrm{NiO}$ & 0.54 & 0.49 & 9.26 & 0.48 & -11.11 & 0.47 & -12.96 & 0.47 & -12.96 \\
\hline $\mathrm{P}_{2} \mathrm{O}_{5}$ & 0.13 & 0.32 & $\mathrm{NC}$ & 0.30 & $\mathrm{NC}$ & 0.27 & $\mathrm{NC}$ & 0.34 & $\mathrm{NC}$ \\
\hline $\mathrm{PbO}$ & 0.15 & 0.12 & $\mathrm{NC}$ & 0.13 & $\mathrm{NC}$ & 0.17 & $\mathrm{NC}$ & 0.17 & $\mathrm{NC}$ \\
\hline $\mathrm{Sb}_{2} \mathrm{O}_{3}$ & 0.21 & 0.16 & $\mathrm{NC}$ & 0.17 & $\mathrm{NC}$ & 0.18 & $\mathrm{NC}$ & 0.17 & $\mathrm{NC}$ \\
\hline $\mathrm{SeO}_{2}$ & 0.15 & 0.03 & $\mathrm{NC}$ & 0.04 & $\mathrm{NC}$ & 0.01 & $\mathrm{NC}$ & 0.03 & $\mathrm{NC}$ \\
\hline $\mathrm{SiO}_{2}$ & 45.54 & 44.98 & -1.23 & 44.83 & -1.56 & 43.82 & -3.78 & 43.30 & -4.92 \\
\hline $\mathrm{SO}_{3}$ & 0.25 & 0.18 & $\mathrm{NC}$ & 0.21 & $\mathrm{NC}$ & $0.15^{@}$ & $\mathrm{NC}$ & $0.22^{@}$ & $\mathrm{NC}$ \\
\hline $\mathrm{SrO}$ & 2.32 & 1.94 & -16.38 & 2.00 & -13.79 & 1.89 & -18.53 & 1.95 & -15.95 \\
\hline $\mathrm{TeO}_{2}$ & 0.14 & 0.09 & $\mathrm{NC}$ & 0.11 & $\mathrm{NC}$ & 0.08 & $\mathrm{NC}$ & 0.12 & $\mathrm{NC}$ \\
\hline $\mathrm{TiO}_{2}$ & 0.06 & 0.14 & $\mathrm{NC}$ & 0.11 & $\mathrm{NC}$ & 0.14 & $\mathrm{NC}$ & 0.12 & $\mathrm{NC}$ \\
\hline $\mathrm{ZnO}$ & 2.00 & 1.90 & -5.00 & 1.88 & -6.00 & 1.91 & -4.50 & 1.86 & -7.00 \\
\hline $\mathrm{ZrO}_{2}$ & 3.56 & 3.80 & 6.74 & 3.82 & 7.30 & 3.55 & -0.28 & 3.61 & 1.40 \\
\hline
\end{tabular}

NA: Not Analyzed

NC: Not Calculated

$\%$ Dev. $=100 \times[($ target-measured $) /$ target $]$.

\#- Analyzed by AA.

(@) - Analyzed by ion chromatography. 
Table 2.9. DCP and AA Analyzed $\mathrm{Li}_{2} \mathrm{O}$ Concentrations for Feed and Glass Samples.

\begin{tabular}{|l|l|l|l|l|}
\hline \multicolumn{1}{|c|}{ Sample I.D. } & & Target & DCP & AA \\
\hline 12G-G-57A & Glass & 6.00 & 5.12 & 5.04 \\
\hline $12 \mathrm{G}-\mathrm{G}-79 \mathrm{~A}$ & Glass & 6.00 & 5.22 & 5.17 \\
\hline $12 \mathrm{~J}-\mathrm{G}-52 \mathrm{~B}$ & Glass & 6.00 & 5.50 & 5.38 \\
\hline $12 \mathrm{~J}-\mathrm{G}-52 \mathrm{~B}$, duplicate & Glass & 6.00 & 5.44 & 5.28 \\
\hline $12 \mathrm{~J}-\mathrm{G}-62 \mathrm{~A}$ & Glass & 6.00 & 5.27 & 5.23 \\
\hline $12 \mathrm{~J}-\mathrm{G}-100 \mathrm{C}$ & Glass & 6.00 & 5.51 & 5.16 \\
\hline $12 \mathrm{~J}-\mathrm{G}-125 \mathrm{~A}$ & Glass & 6.00 & 5.62 & 5.35 \\
\hline $12 \mathrm{~K}-\mathrm{G}-136 \mathrm{~B}$ & Glass & 6.00 & 5.09 & 5.08 \\
\hline $12 \mathrm{~K}-\mathrm{G}-138 \mathrm{~A}$ & Glass & 6.00 & 5.16 & 5.14 \\
\hline $12 \mathrm{~K}-\mathrm{G}-138 \mathrm{~A}$, duplicate & Glass & 6.00 & 4.93 & 4.89 \\
\hline $12 \mathrm{G}-\mathrm{F}-71 \mathrm{~A}$ & Feed & 6.00 & 4.92 & 4.71 \\
\hline $12 \mathrm{~J}-\mathrm{F}-81 \mathrm{~A}$ & Feed & 6.00 & 4.58 & 4.72 \\
\hline $12 \mathrm{~K}-\mathrm{F}-54 \mathrm{~A}$ & Feed & 6.00 & 4.30 & 4.32 \\
\hline
\end{tabular}


The Catholic University of America Vitreous State Laboratory
DuraMelter 1200 Tests with AZ-101HLW Simulants Final Report, VSL-02R0100-2, Rev.1

Table 3.1. DM1200 AZ-101 Melter Temperatures and Other Operational Parameters, Tests 1-4.

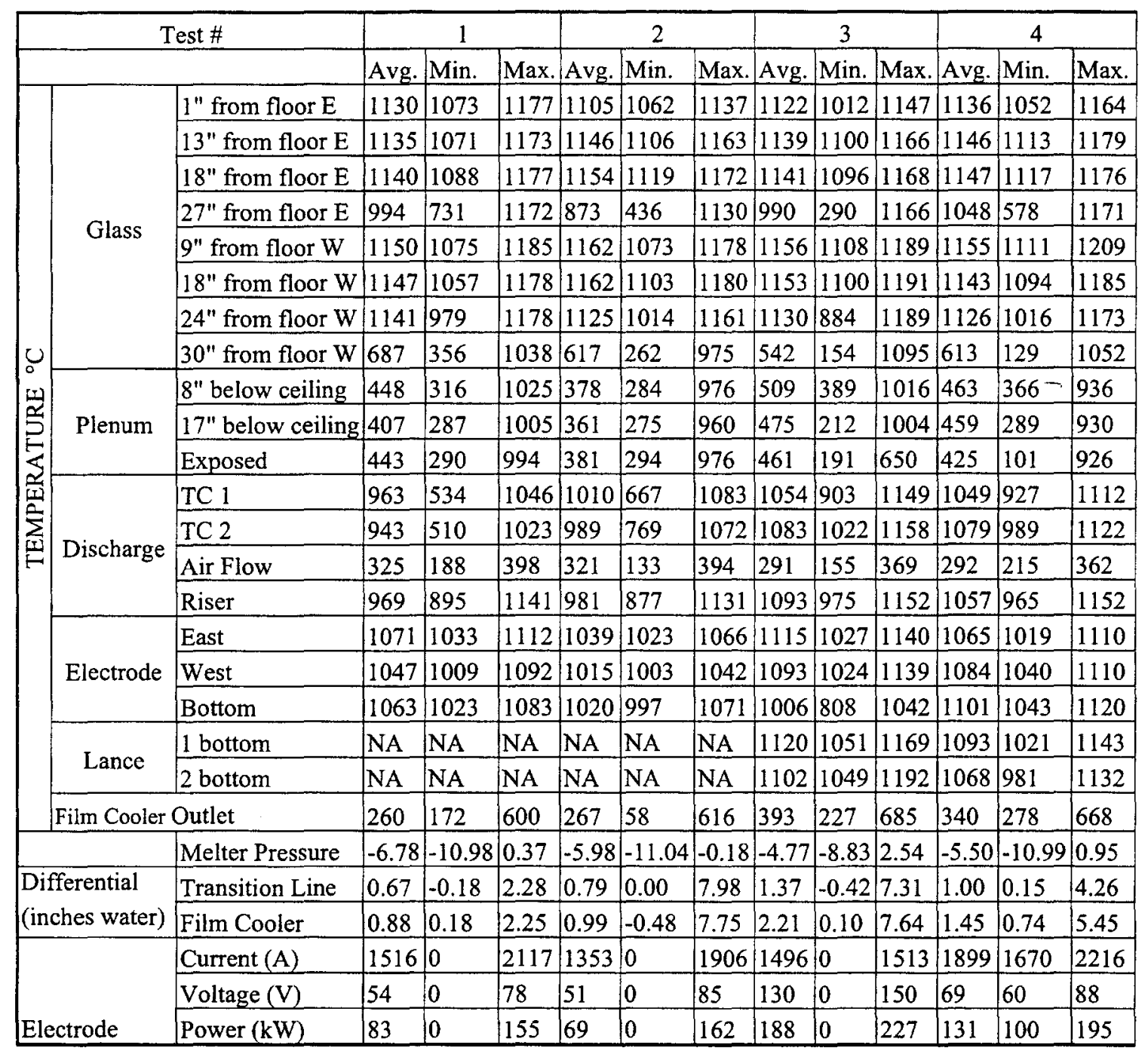


The Catholic University of America

Vitreous State Laboratory
DuraMelter 1200 Tests with AZ-101HLW Simulants Final Report, VSL-02R0100-2, Rev.1

Table 3.2. DM1200 AZ-101 Melter Temperatures and Other Operational Parameters, Tests 5-8.

\begin{tabular}{|c|c|c|c|c|c|c|c|c|c|c|c|c|c|}
\hline & & \multicolumn{3}{|c|}{ Test 5} & \multicolumn{3}{|c|}{ Test 6} & \multicolumn{3}{|c|}{ Test 7} & \multicolumn{3}{|c|}{ Test 8} \\
\hline & & Avg. & Min. & Max. & Avg. & Min. & Max. & Avg. & Min. & Max. & Avg. & Min. & Max. \\
\hline \multirow{8}{*}{ Glass } & 1 "from floor $E$ & 1096 & 1067 & 1118 & 1094 & 1038 & 1130 & 1158 & 1055 & 1204 & 1074 & 1027 & 1085 \\
\hline & 13 " from floor $\mathrm{E}$ & 1142 & 1107 & 1171 & 1138 & 1078 & 1165 & 1146 & 1082 & 1180 & 1152 & 1111 & 1167 \\
\hline & $18^{\prime \prime}$ from floor $\mathrm{E}$ & 1148 & 1109 & 1181 & 1142 & 1095 & 1170 & 1141 & 1063 & 1183 & 1158 & 1092 & 1173 \\
\hline & $27 "$ from floor $E$ & 932 & 780 & 1160 & 768 & 535 & 1090 & 982 & 424 & 1161 & 509 & 439 & 604 \\
\hline & $9^{\prime \prime}$ from floor $W$ & 1146 & 1107 & 1176 & 1157 & 1090 & 1177 & 1138 & 1088 & 1187 & 1144 & 1107 & 1159 \\
\hline & 18 " from floor $W$ & 1149 & 1119 & 1182 & 1158 & 1094 & 1179 & 1132 & 1078 & 1193 & 1147 & 1102 & 1163 \\
\hline & $24 "$ from floor $W$ & 1146 & 1042 & 1174 & 1114 & 962 & 1170 & 1052 & 849 & 1183 & 1135 & 997 & 1156 \\
\hline & $30^{\prime \prime}$ from floor $\mathrm{W}$ & 596 & 434 & 1000 & 513 & 207 & 820 & 452 & 187 & 794 & 490 & 341 & 703 \\
\hline \multirow{3}{*}{ Plenum } & $8^{\prime \prime}$ below ceiling & 417 & 320 & 664 & 336 & 246 & 642 & 480 & 347 & 731 & 349 & 296 & 566 \\
\hline & $17 "$ below ceiling & 407 & 319 & 650 & 307 & 28 & 2672 & 438 & 325 & 736 & 327 & 282 & 558 \\
\hline & Exposed & 411 & 118 & 647 & 225 & 75 & 354 & 458 & 277 & 752 & 339 & 272 & 565 \\
\hline \multirow{4}{*}{ Discharge } & TC 1 & 1027 & 963 & 1077 & 1064 & 825 & 3327 & 1078 & 1018 & 1101 & 1040 & 849 & 1073 \\
\hline & TC 2 & 1046 & 980 & 1083 & 1067 & 682 & 1103 & 1130 & 1049 & 1181 & 1081 & 832 & 1116 \\
\hline & Air Flow & 295 & 250 & 340 & 306 & 188 & 361 & 303 & 161 & 348 & 307 & 216 & 351 \\
\hline & Riser & 960 & 273 & 2694 & 988 & 935 & 1142 & 1111 & 938 & 1186 & 939 & 896 & 1122 \\
\hline \multirow{3}{*}{ Electrode } & East & 1048 & 1022 & 1072 & 1042 & 1008 & 1072 & 1101 & 1031 & 1147 & 1046 & 1019 & 1056 \\
\hline & West & 1058 & 1036 & 1087 & 1043 & 996 & 1075 & 1078 & 1006 & 1100 & 1028 & 1008 & 1045 \\
\hline & Bottom & 1038 & 990 & 2771 & 1013 & 981 & 1058 & 1013 & 913 & 1043 & 1018 & 986 & 1032 \\
\hline \multirow{2}{*}{ Lance } & 1 Bottom & NA & $\mathrm{NA}$ & $\mathrm{NA}$ & $\mathrm{NA}$ & $\mathrm{NA}$ & $\mathrm{NA}$ & 1104 & 1009 & 1189 & NA & $\mathrm{NA}$ & $\mathrm{NA}$ \\
\hline & 2 Bottom & NA & $\mathrm{NA}$ & $\mathrm{NA}$ & $\mathrm{NA}$ & $\mathrm{NA}$ & $\mathrm{NA}$ & 1117 & 1064 & 1172 & NA & $\mathrm{NA}$ & $\mathrm{NA}$ \\
\hline \multicolumn{2}{|c|}{ Film Cooler Outlet } & 284 & 210 & 470 & 232 & 172 & 425 & 348 & 42 & 510 & 239 & 182 & 441 \\
\hline \multicolumn{2}{|c|}{ Melter Pressure (" water) } & -6.17 & -12.40 & 1.48 & -5.43 & -13.21 & 15.17 & -4.63 & -11.07 & -0.19 & -5.71 & -12.80 & 0.10 \\
\hline \multirow{5}{*}{\begin{tabular}{|c|} 
Differential \\
Pressure
\end{tabular}} & Transition Line & 1.03 & 0.00 & 10.05 & 1.12 & 0.09 & 4.14 & 1.73 & 0.00 & 5.63 & 0.57 & -0.06 & 2.93 \\
\hline & Film Cooler & 1.29 & 0.41 & 10.05 & 1.36 & 0.42 & 4.34 & 2.16 & -0.01 & 6.37 & 0.99 & 0.32 & 3.81 \\
\hline & Current (A) & 1389 & 0 & 1645 & 1372 & 0 & 1568 & 1248 & 0 & 1333 & 1278 & 0 & 1530 \\
\hline & Voltage (V) & 54 & 0 & 68 & 54 & 0 & 71 & 128 & 0 & 153 & 53 & 0 & 68 \\
\hline & Power (kW) & 75 & 0 & 111 & 74 & 0 & 112 & 160 & 0 & 204 & 67 & 0 & 104 \\
\hline
\end{tabular}

NA: Not Analyzed 
The Catholic University of America

Vitreous State Laboratory
DuraMelter 1200 Tests with AZ-101HLW Simulants

Final Report, VSL-02R0100-2, Rev.I

Table 3.3. DM1200 AZ-101 Melter Temperatures and Other Operational Parameters, Test 9.

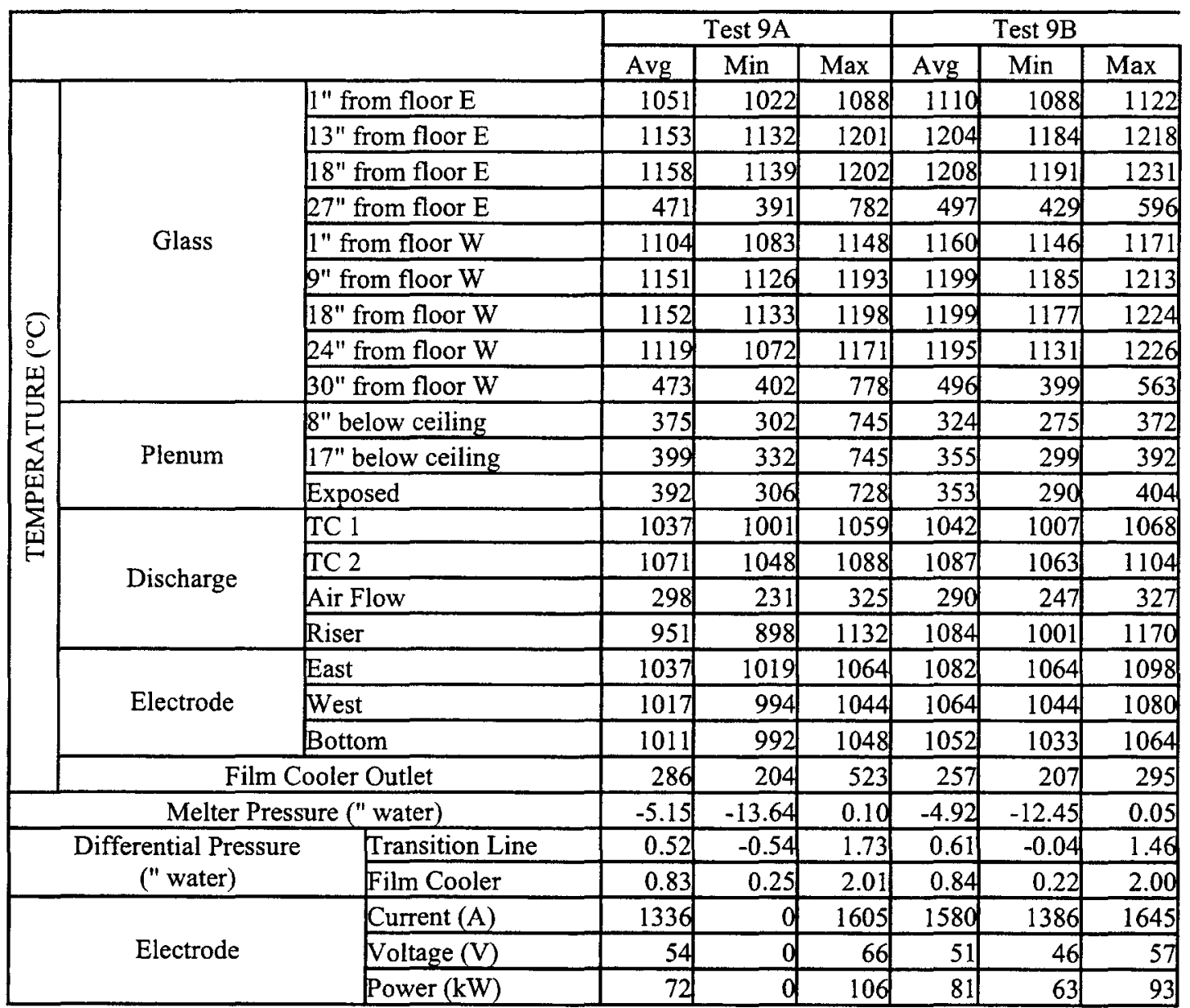


The Catholic University of America

Vitreous State Laboratory
DuraMelter 1200 Tests with AZ-101HLW Simulants Final Report, VSL-02R0100-2, Rev.1

Table 4.1. DM 1200 AZ-101 Tests Off-Gas System Measured Parameters.

\begin{tabular}{|c|c|c|c|c|c|c|c|c|c|c|c|c|c|}
\hline \multicolumn{2}{|l|}{ Test \# } & \multicolumn{3}{|c|}{1} & \multicolumn{3}{|c|}{2} & \multicolumn{3}{|c|}{3} & \multicolumn{3}{|c|}{4} \\
\hline & & Avg. & Min. & Max. & Avg. & Min. & Max. & Avg. & Min. & Max. & Avg. & Min. & Max. \\
\hline \multirow{16}{*}{ SBS } & Differential Pressure ("water) & 33.4 & 17.2 & 45.5 & 36.7 & 13.6 & 49.5 & 45.4 & $-6.5^{*}$ & 73.0 & 44.6 & 33.6 & 54.5 \\
\hline & Inlet gas pressure ("water) & -8.2 & -17.0 & -1.4 & -7.9 & -14.7 & -1.5 & -7.6 & -13.0 & 1.2 & -7.4 & -19.6 & 0.0 \\
\hline & Outlet gas pressure ("water) & -48.0 & -58.6 & -38.8 & -48.4 & -59.9 & -23.0 & -58.2 & -77.6 & -8.3 & -52.5 & -65.3 & -38.6 \\
\hline & Inlet gas Temp. $\left({ }^{\circ} \mathrm{C}\right)$ & 228.3 & 150.9 & 497.6 & 205.2 & 149.4 & 462.0 & 312.3 & 187.9 & 528.1 & 266.1 & 193.8 & 487.4 \\
\hline & Outlet gas Temp. $\left({ }^{\circ} \mathrm{C}\right)$ & 23.6 & 19.0 & 52.6 & 22.1 & 18.1 & 47.3 & 40.7 & 24.5 & 64.2 & 34.3 & 26.7 & 57.3 \\
\hline & Chilled Water Inlet Temp $\left({ }^{\circ} \mathrm{C}\right)$ & 12.5 & 7.5 & 18.2 & 12.6 & 8.1 & 24.1 & 15.9 & 11.0 & 30.8 & 14.7 & 10.3 & 26.1 \\
\hline & Chilled Water Outlet Temp $\left({ }^{\circ} \mathrm{C}\right)$ & 13.9 & 9.8 & 22.4 & 13.6 & 10.0 & 24.0 & 20.2 & 13.2 & 34.2 & 17.4 & 13.6 & 27.8 \\
\hline & Submerged $48^{\prime \prime}$ Temp $\left({ }^{\circ} \mathrm{C}\right)$ & 24.0 & 18.4 & 55.8 & 21.8 & 17.7 & 50.9 & 41.3 & 24.3 & 54.1 & 35.0 & 23.8 & 61.5 \\
\hline & Submerged $60^{\prime \prime} \operatorname{Temp}\left({ }^{\circ} \mathrm{C}\right)$ & 23.8 & 18.2 & 55.2 & 21.8 & 17.7 & 50.9 & 41.2 & 24.3 & 54.1 & 35.0 & 23.8 & 61.6 \\
\hline & Submerged $72 "$ Temp $\left({ }^{\circ} \mathrm{C}\right)$ & 25.0 & 18.8 & 56.4 & 22.5 & 17.8 & 50.9 & 41.5 & 24.9 & 54.7 & 36.0 & 25.6 & 62.7 \\
\hline & Submerged $78^{\prime \prime} \mathrm{Temp}\left({ }^{\circ} \mathrm{C}\right)$ & 24.0 & 18.2 & 55.8 & 21.8 & 17.6 & 50.9 & 41.3 & 24.9 & 54.1 & 35.0 & 23.8 & 61.6 \\
\hline & Recirc. pump discharge Temp $\left({ }^{\circ} \mathrm{C}\right)$ & 34.5 & 29.4 & 37.7 & 33.5 & 30.6 & 35.5 & 35.0 & 26.6 & 41.1 & 26.7 & 19.9 & 38.9 \\
\hline & Heat Exchanger Outlet Temp $\left({ }^{\circ} \mathrm{C}\right)$ & 16.5 & 12.5 & 32.0 & 16.0 & 12.8 & 29.6 & 27.9 & 16.9 & 40.3 & 22.5 & 16.7 & 35.7 \\
\hline & Chilled Water Flow (gal/min) & 41.8 & 28.8 & 43.0 & 45.5 & 2.8 & 48.1 & 42.6 & 29.4 & 45.7 & 41.8 & 6.0 & 45.6 \\
\hline & Heat Exchanger Flow (gal/min) & 50.6 & 41.6 & 54.3 & 48.0 & 15.2 & 54.3 & 48.7 & 31.3 & 54.3 & 51.2 & 41.0 & 54.3 \\
\hline & - pump discharge Pressure & 6.9 & -0.9 & 51.7 & 36.9 & -0.8 & 41.0 & 36.0 & -0.5 & 38.6 & 39.0 & 29.4 & 42.0 \\
\hline \multirow{3}{*}{ WESP } & Differential Pressure ("water) & 1.5 & 0.7 & 3.0 & 1.7 & 0.8 & 3.5 & 2.1 & -0.0 & 3.2 & 1.9 & 0.7 & 4.5 \\
\hline & Inlet gas Temp. $\left({ }^{\circ} \mathrm{C}\right)$ & 29.4 & 25.5 & 52.8 & 26.9 & 23.2 & 72.2 & 41.6 & 30.3 & 52.4 & 36.2 & 32.3 & 58.0 \\
\hline & Outlet gas Temp. $\left({ }^{\circ} \mathrm{C}\right)$ & 36.0 & 32.2 & 52.2 & 31.2 & 28.4 & 45.0 & 45.0 & 32.1 & 51.1 & 39.9 & 33.4 & 53.8 \\
\hline HEME 1 & Differential Pressures ("water) & 1.8 & 1.4 & 2.2 & 2.0 & 1.8 & 2.9 & 1.4 & 1.1 & 2.0 & 1.8 & 1.6 & 2.1 \\
\hline \multirow[b]{2}{*}{ HEPA 1} & Differential Pressure ("water) & 0.2 & -0.1 & 0.3 & 0.3 & -0.1 & 0.4 & 0.3 & 0.1 & 0.4 & 0.2 & -0.2 & 0.4 \\
\hline & Outlet Gas Temp. $\left({ }^{\circ} \mathrm{C}\right)$ & 122.0 & 113.8 & 123.8 & 117.8 & 106.1 & 123.6 & 124.6 & 79.0 & 129.0 & 119.9 & 100.3 & 121.9 \\
\hline \multicolumn{2}{|c|}{ Packed Bed Scrubber Inlet Gas Temp. $\left({ }^{\circ} \mathrm{C}\right)$} & 81.4 & 71.3 & 86.7 & 79.7 & 70.0 & 84.7 & 87.8 & 66.2 & 92.9 & 82.3 & 76.9 & 88.3 \\
\hline \multicolumn{2}{|c|}{ TCO Bypass Outlet Gas Temp. $\left({ }^{\circ} \mathrm{C}\right)$} & 79.7 & 69.5 & 83.5 & 75.9 & 53.8 & 80.6 & 80.2 & 46.3 & 84.3 & 79.0 & 66.3 & 82.7 \\
\hline
\end{tabular}

* Sensor error. 
Table 4.1. DM 1200 AZ-101 Tests Off-Gas System Measured Parameters (continued).

\begin{tabular}{|c|c|c|c|c|c|c|c|c|c|c|}
\hline \multicolumn{2}{|l|}{ Test \# } & \multicolumn{3}{|c|}{5} & \multicolumn{3}{|c|}{6} & \multicolumn{3}{|c|}{7} \\
\hline & & Avg. & Min. & Max. & Avg. & Min. & Max. & Avg. & Min. & Max. \\
\hline \multirow{7}{*}{ SBS } & Differential Pressure ("water) & 38.9 & 32.0 & 47.6 & 40.2 & 34.0 & 48.6 & 40.7 & 33.1 & 51.0 \\
\hline & Inlet gas pressure ("water) & -8.8 & -16.0 & -1.6 & -8.4 & -17.1 & -0.5 & -8.0 & -16.4 & -0.4 \\
\hline & Outlet gas pressure ("water) & -48.3 & -56.8 & -38.8 & -49.3 & -58.5 & -37.6 & -50.6 & -58.8 & -38.9 \\
\hline & Inlet gas Temp. $\left({ }^{\circ} \mathrm{C}\right)$ & 219 & 168 & 408 & 182 & 138 & 395 & 278 & 224 & 424 \\
\hline & Outlet gas Temp. $\left({ }^{\circ} \mathrm{C}\right)$ & 25.0 & 21.4 & 42.9 & 23.2 & 18.3 & 46.0 & 37.6 & 22.8 & 49.4 \\
\hline & Chilled Water Inlet Temp $\left({ }^{\circ} \mathrm{C}\right)$ & 13.5 & 9.0 & 18.8 & 12.8 & 7.8 & 17.6 & 14.7 & 10.6 & 19.3 \\
\hline & Chilled Water Outlet Temp $\left({ }^{\circ} \mathrm{C}\right)$ & 14.6 & 10.8 & 21.4 & 14.2 & 9.5 & 21.1 & 18.1 & 11.7 & 29.4 \\
\hline & Submerged $48^{\prime \prime}$ Temp $\left({ }^{\circ} \mathrm{C}\right)$ & 25.0 & 20.8 & 46.2 & 23.3 & 17.7 & 50.5 & 39.2 & 21.4 & 49.8 \\
\hline & Submerged $60^{\prime \prime} \mathrm{Temp}\left({ }^{\circ} \mathrm{C}\right)$ & 25.0 & 20.8 & 46.2 & 23.3 & 17.7 & 50.5 & 39.2 & 21.5 & 50.2 \\
\hline & Submerged $72^{\prime \prime} \mathrm{Temp}\left({ }^{\circ} \mathrm{C}\right)$ & 26.2 & 21.4 & 48.5 & 24.1 & 18.3 & 52.2 & 40.2 & 22.1 & 51.0 \\
\hline & Submerged $78^{\prime \prime}$ Temp $\left({ }^{\circ} \mathrm{C}\right)$ & 25.0 & 20.8 & 46.7 & 23.3 & 17.7 & 50.5 & 39.2 & 21.4 & 49.8 \\
\hline & Recirc. pump discharge Temp $\left({ }^{\circ} \mathrm{C}\right)$ & $*$ & "1 & * & 28.8 & 26.3 & 31.3 & 28.5 & 1.8 & 38.1 \\
\hline & Heat Exchanger Outlet Temp $\left({ }^{\circ} \mathrm{C}\right)$ & 17.6 & 13.8 & 28.2 & 16.9 & 13.2 & 29.5 & 24.5 & 14.8 & 42.6 \\
\hline & Chilled Water Flow (gal/min) & 38.7 & 13.7 & 42.4 & 39.4 & 23.9 & 42.3 & 40.0 & 3.0 & 43.3 \\
\hline & Heat Exchanger Flow (gal/min) & 47.7 & 40.3 & 54.3 & 49.7 & 40.8 & 54.3 & 52.1 & 3.1 & 54.3 \\
\hline & Recirc. pump discharge Pressure & 38.4 & 32.9 & 42.1 & 39.0 & 32.9 & 42.5 & 23.4 & -1.5 & 47.6 \\
\hline \multirow{3}{*}{ WESP } & Differential Pressure ("water) & 2.4 & 1.2 & 4.5 & 2.5 & 1.0 & 6.3 & 2.3 & 0.7 & 10.1 \\
\hline & Inlet gas Temp. $\left({ }^{\circ} \mathrm{C}\right)$ & 27.3 & 23.5 & 45.6 & 25.3 & 21.3 & 46.9 & 37.5 & 25.2 & 47.2 \\
\hline & Outlet gas Temp. $\left({ }^{\circ} \mathrm{C}\right)$ & 31.1 & 28.8 & 43.9 & 29.7 & 26.8 & 44.0 & 40.5 & 27.3 & 47.2 \\
\hline \multirow[t]{3}{*}{ HEME 1} & Differential Pressure ("water) & 2.3 & 2.0 & 2.9 & 2.3 & 2.0 & 2.9 & 1.8 & 1.4 & 2.3 \\
\hline & Differential Pressure ("water) & 0.2 & -0.2 & 0.4 & 0.2 & 0.1 & 0.4 & 0.2 & 0.1 & 0.4 \\
\hline & Outlet Gas Temp. $\left({ }^{\circ} \mathrm{C}\right)$ & 111 & 105 & 120 & 109 & 97 & 120 & 116 & 44 & 122 \\
\hline \multicolumn{2}{|c|}{ Packed Bed Scrubber Inlet Gas Temp. $\left({ }^{\circ} \mathrm{C}\right)$} & 77.6 & 74.3 & 84.6 & 77.8 & 74.3 & 83.6 & 80.9 & 41.4 & 87.4 \\
\hline \multicolumn{2}{|c|}{ TCO Bypass Outlet Gas Temp. $\left({ }^{\circ} \mathrm{C}\right)$} & 119 & 114 & 124 & 117 & 112 & 123 & 117 & 34 & 125 \\
\hline
\end{tabular}

* Sensor error. 
Table 4.1. DM 1200 AZ-101 Tests Off-Gas System Measured Parameters (continued).

\begin{tabular}{|c|c|c|c|c|c|c|c|c|c|c|}
\hline \multicolumn{2}{|l|}{ Test \# } & \multicolumn{3}{|c|}{8} & \multicolumn{3}{|c|}{$9 a$} & \multicolumn{3}{|c|}{$9 \mathrm{~b}$} \\
\hline & & Avg. & Min. & Max. & Avg. & Min. & Max. & Avg. & Min. & \\
\hline \multirow{5}{*}{ SBS } & Differential Pressure ("water) & 33.1 & 18.6 & 43.8 & 38.7 & 31.5 & 46.3 & 38.3 & 6.7 & 45.0 \\
\hline & Inlet gas pressure ("water) & -6.7 & -15.4 & 0.9 & -6.3 & -14.4 & 0.2 & -5.9 & -14.7 & 0.3 \\
\hline & Outlet gas pressure ("water) & -43.5 & 54.7 & -26.6 & -45.2 & -55.2 & -37.6 & -45.2 & -54.2 & -29.6 \\
\hline & Inlet gas Temp. $\left({ }^{\circ} \mathrm{C}\right)$ & 183 & 146 & 368 & 198 & 155 & 348 & 178 & 148 & 201 \\
\hline & Temp. $\left({ }^{\circ} \mathrm{C}\right)$ & 23.3 & 20.5 & 41.1 & 21.1 & 19.1 & 33.5 & 21.4 & 18.4 & 22.7 \\
\hline & Chill & 12.4 & 9.6 & 17.9 & 12.2 & 9.0 & 15.4 & 12.3 & 9.9 & 15.0 \\
\hline & Chilled Water $\mathrm{O}$ & 13.5 & 11.2 & 18.9 & 13.2 & 11.0 & 16.9 & 13.3 & 11.5 & 15. \\
\hline & \begin{tabular}{|l|} 
Submerged 48 \\
\end{tabular} & 23.3 & 20.4 & 45.0 & 21.2 & 18.8 & 37.4 & 21.5 & 17.4 & 23.4 \\
\hline & ged $60^{\prime \prime}$ Temp $\left({ }^{\circ} \mathrm{C}\right)$ & 23.3 & 20.4 & 45.0 & 21.2 & 19.2 & 36.8 & 21.6 & 17.4 & 23. \\
\hline & rged $72^{\prime \prime}$ Temp $\left({ }^{\circ} \mathrm{C}\right)$ & 25.0 & 20.8 & 46.7 & 22.7 & 20.4 & 39.1 & 23.0 & 18.7 & 25. \\
\hline & rged $78^{\prime \prime}$ Temp $\left({ }^{\circ} \mathrm{C}\right)$ & 23.2 & 19.8 & 45.0 & 21.2 & 18.8 & 36.8 & 21.5 & 17.4 & 23. \\
\hline & Recirc. pump discharge T & 40.0 & 24.7 & 44.9 & 26.4 & 21.3 & 30.1 & 26.3 & 24.5 & 29.1 \\
\hline & Heat Exchanger Outlet Te & 15.5 & 11.0 & 25.9 & 15.2 & 13.7 & 21.8 & 15.4 & 13.8 & 16.7 \\
\hline & Chilled Water Flow (gal/min) & 40.8 & 26.8 & 43.5 & 41.4 & 28.9 & 44.1 & 41.5 & 6.4 & 43.7 \\
\hline & Heat Exchanger $\mathrm{Fl}$ & 50.7 & 33.6 & 54.3 & 49.1 & 40.8 & 54.3 & 49.0 & 41.5 & 54. \\
\hline & & 39.7 & 37.1 & 42.1 & 39.5 & 37.1 & 41.8 & 39.9 & 38.1 & 42. \\
\hline \multirow{3}{*}{ WESP } & Differential Presst & 1.8 & -0.2 & 3.4 & 2.6 & 0.9 & 10.1 & 1.5 & 1.0 & 3. \\
\hline & Inlet g & 25.3 & 22.1 & 41.4 & 24.6 & 22.2 & 35.6 & 23.6 & 21.5 & 25. \\
\hline & Outlet gas $\mathrm{Te}$ & 32.2 & 28.9 & 38.9 & 29.9 & 25.4 & 33.0 & 29.7 & 27.8 & 31. \\
\hline \multirow[t]{3}{*}{ HEME 1} & Differential Pressure ("water) & 2.0 & 1.8 & 2.4 & 1.8 & 0.2 & 2.0 & 1.8 & 1.7 & 1.9 \\
\hline & Differe & 0.2 & 0.0 & 0.3 & 0.2 & 0.1 & 0.3 & 0.2 & 0.0 & 0.3 \\
\hline & Outlet Gas Temp. $\left({ }^{\circ} \mathrm{C}\right)$ & 117 & 109 & 120 & 118 & 108 & 118 & 118 & 114 & 119 \\
\hline \multicolumn{2}{|c|}{ Packed Bed Scrubber Inlet Gas Temp. $\left({ }^{\circ} \mathrm{C}\right)$} & 76.2 & 71.4 & 79.5 & 74.7 & 68.2 & 77.7 & 74.4 & 72.2 & 79. \\
\hline \multicolumn{2}{|c|}{ TCO Bypass Outlet Gas Temp. $\left({ }^{\circ} \mathrm{C}\right)$} & 120 & 103 & 128 & 121 & 97 & 125 & 122 & 119 & 125 \\
\hline
\end{tabular}


Table 4.2. WESP Current and Voltage Data Before and After Wash-Down.

\begin{tabular}{|c|c|c|c|}
\hline Test Number & WESP Wash-Down Date & $\begin{array}{c}\text { WESP Current, } \mathrm{mA} \\
\text { (WESP voltage, } \mathrm{kV} \text { )Before } \\
\text { Wash-Down }\end{array}$ & $\begin{array}{c}\text { WESP Current, mA } \\
\text { (WESP voltage, } \mathrm{kV} \text { ) } \\
\text { After Wash Down }\end{array}$ \\
\hline $\begin{array}{c}\text { Commissioning } \\
\text { Test \#3 }\end{array}$ & $5 / 23 / 01$ & $16.36(24.53)$ & $16.37(25.50)$ \\
\hline 3 & $8 / 13 / 01$ & $15.96(27.48)$ & $18.09(28.06)$ \\
\hline 4 & $8 / 30 / 01$ & $16.75(27.86)$ & $14.89(26.39)$ \\
\hline 5 & $10 / 02 / 01$ & $13.14(28.32)$ & $16.07(27.23)$ \\
\hline 6 & $10 / 17 / 01$ & $17.67(27.32)$ & $15.62(27.18)$ \\
\hline 7 & $10 / 27 / 01$ & $16.77(27.65)$ & $16.25(24.33)$ \\
\hline 8 & $11 / 02 / 01$ & $16.96(27.77)$ & $12.58(25.12)$ \\
\hline 9 & $11 / 14 / 01$ & $16.50(26.60)$ & $-\cdots---$ \\
\hline
\end{tabular}


Table 4.3. WESP Operating Conditions During DM 1200 AZ-101 Sampling Periods.

\begin{tabular}{|c|c|c|c|c|c|c|c|c|c|c|c|}
\hline Test \# & $\begin{array}{c}\text { Time Span } \\
\text { (Date - Time) }\end{array}$ & $\begin{array}{c}\text { Average } \\
\text { Inlet Gas } \\
\text { Temp' } \\
\left({ }^{\circ} \mathrm{C}\right)\end{array}$ & $\begin{array}{c}\text { Average } \\
\text { Outlet } \\
\text { Gas } \\
\text { Temp } \\
\left({ }^{\circ} \mathrm{C}\right)\end{array}$ & $\begin{array}{l}\text { Average } \\
\text { Diff. } \\
\text { Pressure }^{\mathrm{l}} \\
\text { (In-W.C.) }\end{array}$ & $\begin{array}{l}\text { Liquid } \\
\text { Level }^{2} \\
\text { (Inches) }\end{array}$ & $\begin{array}{l}\text { Blow } \\
\text { Down } \\
\text { Sample } \\
\text { Tank }^{2} \\
\text { (Gal) }\end{array}$ & $\begin{array}{c}\text { Voltage } \\
\text { Range }^{2}(\mathrm{kV})\end{array}$ & $\begin{array}{c}\text { Average } \\
\text { Voltage }^{2} \\
(\mathrm{kV})\end{array}$ & $\begin{array}{c}\text { Current } \\
\text { Range }^{2}(\mathrm{~mA})\end{array}$ & $\begin{array}{c}\text { Average } \\
\text { Current }^{2} \\
(\mathrm{~mA})\end{array}$ & $\begin{array}{l}\text { Recircula- } \\
\text { tion Pump } \\
\text { On-off/ } \\
\text { Recycle } \\
\text { Flow Rate } \\
\text { (lpm) }\end{array}$ \\
\hline 2 & $\begin{array}{c}7 / 29 / 01-10: 05 \\
7 / 31 / 01-19: 30\end{array}$ & 24.9 & 29.8 & 1.8 & 11.4 & 0 & $26.39-27.12$ & 26.74 & $15.54-19.31$ & 17.41 & 7.9 \\
\hline 3 & $\begin{array}{c}8 / 8 / 01-07: 52 \\
8 / 10 / 01-14: 36 \\
\end{array}$ & 42.2 & 45.9 & 2.1 & 11.3 & 0 & $26.94-28.09$ & 27.90 & $15.98-19.10$ & 17.27 & 8.0 \\
\hline 4 & $\begin{array}{l}8 / 22 / 01-07: 58 \\
8 / 24 / 01-19: 09\end{array}$ & 35.4 & 39.4 & 1.9 & 11.3 & 0 & $27.75-27.75$ & 27.75 & $16.03-18.18$ & 17.24 & 7.9 \\
\hline 5 & $\begin{array}{l}9 / 28-01-08: 47 \\
9 / 30 / 01-19: 09\end{array}$ & 26.6 & 30.9 & 2.3 & 11.2 & 0 & $27.00-28.32$ & 28.23 & $10.31-14.95$ & 13.39 & 7.8 \\
\hline 6 & $\begin{array}{l}10 / 15 / 01-08: 30 \\
10 / 17 / 01-19: 07\end{array}$ & 23.6 & 28.6 & 2.4 & 11.1 & 0 & $27.32-27.32$ & 27.32 & $16.02-18.78$ & 17.62 & 8.0 \\
\hline 7 & $\begin{array}{l}10 / 23 / 01-07: 55 \\
10 / 25 / 01-10: 12 \\
\end{array}$ & 36.4 & 39.5 & 2.2 & 13.1 & 0 & $26.93-28.58$ & 27.90 & $16.18-18.01$ & 16.89 & Off \\
\hline 8 & $\begin{array}{c}10 / 30 / 01-08: 13 \\
11 / 1 / 01-17: 35 \\
\end{array}$ & 24.6 & 31.6 & 1.8 & 12.4 & 0 & $27.79-28.28$ & 28.11 & $15.27-18.50$ & 16.73 & Off \\
\hline 9 & $\begin{array}{l}11 / 7 / 01-08: 00 \\
11 / 9 / 01-18: 30 \\
\end{array}$ & 23.4 & 29.7 & 1.5 & 11.0 & 0 & $26.60-27.01$ & 26.93 & $15.33-17.39$ & 16.39 & Off \\
\hline
\end{tabular}

Note: 'These are the average of the computer recorded values (taken at every 2 minutes), between the given time intervals.

'These values are derived from manually recorded logbook data (every 2 hours), between the given time intervals.

$7 / 29 / 01,1610$, Added 17 liters of water. Water level was raised to $12 "$.
$7 / 30 / 01,0440$, Added 22 liters of water. Water level was raised to $12 "$.

$7 / 30 / 01,1523$, Added 18 liters of water. Water level was raised to $12^{\prime \prime}$.

8/9/01, 2054, Added 22.7 liters of water. Water level was raised from 10.5" to 12".

$8 / 22 / 01,2224$, Replaced the WESP recirculation filter.

$8 / 23 / 01,0544$, Added 14.4 liters of water. Water level was raised from $11^{\text {" to }} 12^{\text {". }}$

$8 / 23 / 01,2009$, Added 16 liters of water. Water level was raised from 10.8 " to 12

8/24/01,0609, Added water. Water level was raised from 11 " to $12^{\prime \prime}$.

9/28/01, 1445, Added water. Water level was raised from 10.8" to 12".

9/29/01, 0545, Added 13.5 liters of water. Water level was raised from 10.5" to 12"

9/30/01, 0049, Added 13.5 liters of water. Water level was raised from 10.8" to 12".

$9 / 30 / 01,1416$, Added 19.6 liters of water. Water level was raised from 10.4" to 12.2

10/15/01, 1450, Added 18.6 liters of water. Water level was raised to 12

$10 / 16 / 01,2026$, Added water, Water level was raised from $10.5^{\prime \prime}$ to $12 "$.

11/17\%1, 1536, Added water. Water level was raised from $10.0^{\prime \prime}$ to $12^{\circ}$.

10/30/01, 0640, Restarted WESP recirculation pump. But at 0650, the current oscillated beten $19 \mathrm{~mA}$ to $3 \mathrm{~mA}$, and WESP pump was secured. 
The Catholic University of America Vitreous State Laboratory
DuraMelter 1200 Tests with AZ-101HLW Simulants

Final Report, VSL-02R0100-2, Rev.I

Table 4.4. Characteristics of SBS Fluid Samples from DM1200 Tests.

\begin{tabular}{|c|c|c|c|c|c|c|c|c|}
\hline Test & $\begin{array}{c}\text { Sampling } \\
\text { Date }\end{array}$ & Sample Name & $\begin{array}{c}\text { TSS } \\
(\mathrm{mg} / \mathrm{l})\end{array}$ & $\begin{array}{c}\text { TDS } \\
(\mathrm{mg} / \mathrm{l})\end{array}$ & $\begin{array}{c}\text { Filtrate } \\
\text { pH }\end{array}$ & $\begin{array}{l}\text { Cumulative } \\
\text { Glass (kg) }\end{array}$ & $\begin{array}{l}\text { Blowdown } \\
\text { Vol. (gal) }\end{array}$ & $\begin{array}{c}\text { Cumulative } \\
\text { Blowdown } \\
\text { Vol. (gal) } \\
\end{array}$ \\
\hline \multirow{26}{*}{$\# 1$} & \multirow{3}{*}{$06 / 25 / 01$} & $12 \mathrm{~F}-\mathrm{S}-84 \mathrm{~A}$ & 47 & 284 & 7.80 & 60 & 30.41 & 77.69 \\
\hline & & $12 \mathrm{~F}-\mathrm{S}-87 \mathrm{~A}$ & 69 & 354 & 7.30 & 140 & 31.64 & 109.33 \\
\hline & & $12 F-S-87 B$ & 88 & 408 & 5.96 & 220 & 30.84 & 140.17 \\
\hline & \multirow{4}{*}{$06 / 26 / 01$} & $12 \mathrm{~F}-\mathrm{S}-94 \mathrm{~A}$ & 92 & 300 & 4.08 & 300 & 26.38 & 166.55 \\
\hline & & $12 \mathrm{~F}-\mathrm{S}-98 \mathrm{~A}$ & 102 & 308 & 4.26 & 380 & 29.00 & 195.55 \\
\hline & & $12 \mathrm{~F}-\mathrm{S}-105 \mathrm{~A}$ & 45 & 296 & 4.10 & 440 & 23.28 & 218.83 \\
\hline & & $12 \mathrm{~F}-\mathrm{S}-107 \mathrm{~A}$ & 52 & 404 & 3.77 & 520 & 25.54 & 244.37 \\
\hline & \multirow{3}{*}{$6 / 27 / 01$} & $12 \mathrm{~F}-\mathrm{S}-116 \mathrm{~A}$ & 30 & 364 & 3.74 & 600 & 35.99 & 280.36 \\
\hline & & $12 \mathrm{~F}-\mathrm{S}-119 \mathrm{~A}$ & 120 & 392 & 3.67 & 700 & 27.79 & 308.15 \\
\hline & & $12 \mathrm{~F}-\mathrm{S}-126 \mathrm{~A}$ & 65 & 452 & 3.58 & 800 & 33.38 & 341.53 \\
\hline & \multirow{4}{*}{$06 / 28 / 01$} & $12 \mathrm{~F}-\mathrm{S}-136 \mathrm{~A}$ & 47 & 648 & 3.56 & 900 & 28.36 & 369.89 \\
\hline & & $12 \mathrm{~F}-\mathrm{S}-139 \mathrm{~A}$ & 50 & 630 & 3.61 & 978 & 31.74 & 401.63 \\
\hline & & $12 \mathrm{~F}-\mathrm{S}-141 \mathrm{~A}$ & 47 & 72 & 3.53 & 1080 & 30.16 & 431.79 \\
\hline & & $12 \mathrm{~F}-\mathrm{S}-148 \mathrm{~A}$ & 27 & 64 & 3.30 & 1180 & 30.83 & 462.62 \\
\hline & \multirow[b]{2}{*}{$06 / 29 / 01$} & 12G-S-14A & 27 & 820 & 3.32 & 1280 & 32.15 & 494.77 \\
\hline & & $12 \mathrm{G}-\mathrm{S}-20 \mathrm{~A}$ & 25 & 740 & 3.33 & 1380 & 33.50 & 528.27 \\
\hline & \multirow{4}{*}{$06 / 30 / 01$} & 12G-S-25A & 10 & 684 & 3.29 & 1483 & 30.99 & 559.26 \\
\hline & & $12 \mathrm{G}-\mathrm{S}-32 \mathrm{~A}$ & 20 & 704 & 3.32 & 1570 & 26.26 & 585.52 \\
\hline & & $12 \mathrm{G}-\mathrm{S}-40 \mathrm{~A}$ & 27 & 748 & 3.33 & 1650 & 29.08 & 614.60 \\
\hline & & $12 \mathrm{G}-\mathrm{S}-45 \mathrm{~A}$ & 20 & 420 & 3.34 & 1730 & 23.95 & 638.55 \\
\hline & \multirow{3}{*}{$07 / 01 / 01$} & $12 \mathrm{G}-\mathrm{S}-53 \mathrm{~A}$ & 23 & 772 & 3.45 & 1820 & 28.68 & 667.23 \\
\hline & & 12G-S-56A & 35 & 1048 & 3.72 & 1900 & 33.25 & 700.48 \\
\hline & & $12 \mathrm{G}-\mathrm{S}-58 \mathrm{~A}$ & 43 & 838 & 3.82 & 1980 & 34.65 & 735.13 \\
\hline & \multirow[b]{2}{*}{$07 / 02 / 01$} & 12G-S-64A & 72 & 804 & 3.74 & 2090 & 28.08 & 763.21 \\
\hline & & $12 \mathrm{G}-\mathrm{S}-73 \mathrm{~A}$ & 572 & 756 & 3.64 & 2200 & 0.00 & 763.21 \\
\hline & $07 / 03 / 01$ & $12 \mathrm{G}-\mathrm{S}-79 \mathrm{~A}$ & 252 & 680 & 3.85 & 2301 & 26.44 & 789.65 \\
\hline \multirow{19}{*}{ \#2 } & \multirow{3}{*}{$07 / 23 / 01$} & $12 \mathrm{G}-\mathrm{S}-116 \mathrm{~A}$ & 28 & 204 & 8.40 & 2350 & 0.00 & 789.65 \\
\hline & & 12G-S-116B & 62 & 304 & 8.48 & 2400 & 37.23 & 826.88 \\
\hline & & $12 \mathrm{G}-\mathrm{S}-124 \mathrm{~A}$ & 66 & 324 & 8.36 & 2460 & 35.04 & 861.92 \\
\hline & \multirow{4}{*}{$07 / 24 / 01$} & $12 \mathrm{G}-\mathrm{S}-126 \mathrm{~A}$ & 38 & 284 & 8.42 & 2510 & 33.53 & 895.45 \\
\hline & & $12 \mathrm{G}-\mathrm{S}-128 \mathrm{~A}$ & 38 & 424 & 8.48 & 2561 & 29.06 & 924.51 \\
\hline & & $12 \mathrm{G}-\mathrm{S}-135 \mathrm{~A}$ & 44 & 464 & 8.46 & 2650 & 31.22 & 955.73 \\
\hline & & $12 \mathrm{G}-\mathrm{S}-136 \mathrm{~A}$ & 96 & 506 & 8.46 & 2740 & 35.79 & 991.52 \\
\hline & \multirow{5}{*}{$07 / 25 / 01$} & $12 \mathrm{G}-\mathrm{S}-139 \mathrm{~A}$ & 104 & 496 & 8.56 & 2827 & 34.76 & 1026.28 \\
\hline & & $12 \mathrm{G}-\mathrm{S}-148 \mathrm{~A}$ & 84 & 504 & 8.60 & 2930 & 33.93 & 1060.21 \\
\hline & & 12G-S-151A & 130 & 400 & 8.60 & 3035 & 32.60 & 1092.81 \\
\hline & & $12 \mathrm{H}-\mathrm{S}-11 \mathrm{~A}$ & 122 & 436 & 8.51 & 3120 & 29.23 & 1122.04 \\
\hline & & $12 \mathrm{H}-\mathrm{S}-19 \mathrm{~A}$ & 130 & 420 & 8.57 & 3210 & 30.66 & 1152.70 \\
\hline & \multirow{2}{*}{$07 / 26 / 01$} & $12 \mathrm{H}-\mathrm{S}-24 \mathrm{~A}$ & 136 & 428 & 8.54 & 3298 & 28.26 & 1180.96 \\
\hline & & $12 \mathrm{H}-\mathrm{S}-33 \mathrm{~A}$ & 122 & 380 & 8.55 & 3360 & 33.00 & 1213.96 \\
\hline & \multirow{3}{*}{$07 / 27 / 01$} & $12 \mathrm{H}-\mathrm{S}-34 \mathrm{~A}$ & 128 & 436 & 8.56 & 3420 & 29.23 & 1243.19 \\
\hline & & $12 \mathrm{H}-\mathrm{S}-43 \mathrm{~A}$ & 130 & 468 & 8.52 & 3452 & 29.21 & 1272.40 \\
\hline & & $12 \mathrm{I}-\mathrm{S}-13 \mathrm{~A}$ & 118 & 480 & 8.47 & 3540 & 34.85 & 1307.25 \\
\hline & \multirow[b]{2}{*}{$07 / 28 / 01$} & $12 \mathrm{I}-\mathrm{S}-17 \mathrm{~A}$ & 106 & 472 & 8.50 & 3699 & 29.86 & 1337.11 \\
\hline & & $12 \mathrm{I}-\mathrm{S}-26 \mathrm{~A}$ & 101 & 554 & 8.53 & 3840 & 33.37 & 1370.48 \\
\hline
\end{tabular}


The Catholic University of America

Vitreous State Laboratory

DuraMelter 1200 Tests with AZ-101HLW Simulants

Final Report, VSL-02R0100-2, Rev.I

Table 4.4. Characteristics of SBS Samples from DM1200 Tests (continued).

\begin{tabular}{|c|c|c|c|c|c|c|c|c|}
\hline Test & $\begin{array}{l}\text { Sampling } \\
\text { Date }\end{array}$ & Sample Name & $\begin{array}{c}\text { TSS } \\
(\mathrm{mg} / \mathrm{l})\end{array}$ & $\begin{array}{c}\text { TDS } \\
(\mathrm{mg} / \mathrm{l})\end{array}$ & $\begin{array}{c}\text { Filtrate } \\
\text { pH }\end{array}$ & $\begin{array}{c}\text { Cumulative } \\
\text { Glass (kg) }\end{array}$ & $\begin{array}{c}\text { Blowdown } \\
\text { Vol. (gal) }\end{array}$ & $\begin{array}{l}\text { Cumulative } \\
\text { Blowdown } \\
\text { Vol. (gal) }\end{array}$ \\
\hline \multirow{8}{*}{$\# 2$} & \multirow{2}{*}{$07 / 29 / 01$} & $12 \mathrm{I}-\mathrm{S}-35 \mathrm{~A}$ & 80 & 260 & 8.50 & 3981 & 30.26 & 1400.74 \\
\hline & & $12 \mathrm{I}-\mathrm{S}-43 \mathrm{~A}$ & 78 & 308 & 8.49 & 4122 & 33.71 & 1434.45 \\
\hline & \multirow{2}{*}{$07 / 30 / 01$} & $12 \mathrm{I}-\mathrm{S}-50 \mathrm{~A}$ & 90 & 196 & 8.50 & 4250 & 32.89 & 1467.34 \\
\hline & & $12 \mathrm{I}-\mathrm{S}-58 \mathrm{~A}$ & 350 & 532 & 8.40 & 4379 & 33.18 & 1500.52 \\
\hline & \multirow{3}{*}{$07 / 31 / 01$} & $12 \mathrm{I}-\mathrm{S}-61 \mathrm{~A}$ & 230 & 600 & 8.51 & 4507 & 30.65 & 1531.17 \\
\hline & & $12 \mathrm{I}-\mathrm{S}-66 \mathrm{~A}$ & 254 & 568 & 8.49 & 4635 & 31.23 & 1562.40 \\
\hline & & $12 \mathrm{I}-\mathrm{S}-74 \mathrm{~A}$ & 268 & 688 & 8.46 & 4765 & 32.24 & 1594.64 \\
\hline & $08 / 01 / 01$ & $12 \mathrm{I}-\mathrm{S}-88 \mathrm{~A}$ & 268 & 672 & 8.45 & 4895 & 41.52 & 1636.16 \\
\hline \multirow{35}{*}{$\# 3$} & \multirow{4}{*}{$08 / 06 / 01$} & $12 \mathrm{~J}-\mathrm{S}-38 \mathrm{~A}$ & ND & ND & $\mathrm{ND}$ & 5004 & 48.00 & 1684.16 \\
\hline & & $12 \mathrm{~J}-\mathrm{S}-39 \mathrm{~A}$ & 208 & 292 & 7.08 & 5106 & 44.00 & 1728.16 \\
\hline & & $12 \mathrm{~J}-\mathrm{S}-40 \mathrm{~A}$ & ND & $\mathrm{ND}$ & $\mathrm{ND}$ & 5208 & 43.97 & 1772.13 \\
\hline & & $12 \mathrm{~J}-\mathrm{S}-42 \mathrm{~A}$ & $\mathrm{ND}$ & ND & ND & 5310 & 39.53 & 1811.66 \\
\hline & \multirow{13}{*}{$08 / 07 / 01$} & $12 \mathrm{~J}-\mathrm{S}-43 \mathrm{~A}$ & ND & ND & ND & 5412 & 38.19 & 1849.85 \\
\hline & & $12 \mathrm{~J}-\mathrm{S}-44 \mathrm{~A}$ & 488 & 956 & 5.72 & 5514 & 38.04 & 1887.89 \\
\hline & & $12 \mathrm{~J}-\mathrm{S}-45 \mathrm{~A}$ & $\mathrm{ND}$ & $\mathrm{ND}$ & ND & 5597 & 40.20 & 1928.09 \\
\hline & & $12 \mathrm{~J}-\mathrm{S}-45 \mathrm{~B}$ & $\mathrm{ND}$ & ND & $\mathrm{ND}$ & 5690 & 46.77 & 1974.86 \\
\hline & & $12 \mathrm{~J}-\mathrm{S}-51 \mathrm{~A}$ & $\mathrm{ND}$ & $\mathrm{ND}$ & ND & 5763 & 39.19 & 2014.05 \\
\hline & & $12 \mathrm{~J}-\mathrm{S}-52 \mathrm{~A}$ & ND & ND & ND & 5845 & 40.35 & 2054.40 \\
\hline & & $12 \mathrm{~J}-\mathrm{S}-52 \mathrm{~B}$ & ND & ND & ND & 5928 & 39.54 & 2093.94 \\
\hline & & $12 \mathrm{~J}-\mathrm{S}-53 \mathrm{~A}$ & 788 & 2080 & 4.89 & 6011 & 43.98 & 2137.92 \\
\hline & & $12 \mathrm{~J}-\mathrm{S}-55 \mathrm{~A}$ & ND & $\mathrm{ND}$ & ND & 6131 & 44.07 & 2181.99 \\
\hline & & $12 \mathrm{~J}-\mathrm{S}-55 \mathrm{~B}$ & ND & ND & ND & 6250 & 45.61 & 2227.60 \\
\hline & & $12 \mathrm{~J}-\mathrm{S}-56 \mathrm{~A}$ & $\mathrm{ND}$ & ND & ND & 6370 & 39.19 & 2266.79 \\
\hline & & $12 \mathrm{~J}-\mathrm{S}-62 \mathrm{~A}$ & ND & ND & ND & 6489 & 51.52 & 2318.31 \\
\hline & & $12 \mathrm{~J}-\mathrm{S}-64 \mathrm{~A}$ & $\mathrm{ND}$ & $\mathrm{ND}$ & $\mathrm{ND}$ & 6573 & 43.50 & 2361.81 \\
\hline & \multirow[t]{14}{*}{$08 / 08 / 01$} & $12 \mathrm{~J}-\mathrm{S}-64 \mathrm{~B}$ & 1138 & 2692 & 5.73 & 6657 & 39.88 & 2401.69 \\
\hline & & $12 \mathrm{~J}-\mathrm{S}-65 \mathrm{~A}$ & $\mathrm{ND}$ & ND & ND & 6741 & 41.75 & 2443.44 \\
\hline & & $12 \mathrm{~J}-\mathrm{S}-68 \mathrm{~A}$ & $\mathrm{ND}$ & ND & ND & 6825 & 44.39 & 2487.83 \\
\hline & & $12 \mathrm{~J}-\mathrm{S}-68 \mathrm{~B}$ & ND & $\mathrm{ND}$ & ND & 6909 & 36.94 & 2524.77 \\
\hline & & $12 \mathrm{~J}-\mathrm{S}-69 \mathrm{~A}$ & ND & ND & ND & 6993 & 41.18 & 2565.95 \\
\hline & & $12 \mathrm{~J}-\mathrm{S}-70 \mathrm{~A}$ & ND & $\mathrm{ND}$ & ND & 7084 & 45.87 & 2611.82 \\
\hline & & $12 \mathrm{~J}-\mathrm{S}-75 \mathrm{~A}$ & 1398 & 2720 & 6.11 & 7175 & 39.62 & 2651.44 \\
\hline & & $12 \mathrm{~J}-\mathrm{S}-75 \mathrm{~B}$ & ND & $\mathrm{ND}$ & ND & 7265 & 37.19 & 2688.63 \\
\hline & & $12 \mathrm{~J}-\mathrm{S}-76 \mathrm{~A}$ & ND & ND & ND & 7356 & 48.06 & 2736.69 \\
\hline & & $12 \mathrm{~J}-\mathrm{S}-80 \mathrm{~A}$ & ND & ND & ND & 7447 & 45.40 & 2782.09 \\
\hline & & $12 \mathrm{~J}-\mathrm{S}-82 \mathrm{~A}$ & $\mathrm{ND}$ & $\mathrm{ND}$ & $\mathrm{ND}$ & 7535 & 55.55 & 2837.64 \\
\hline & & $12 \mathrm{~J}-\mathrm{S}-84 \mathrm{~A}$ & ND & $\mathrm{ND}$ & ND & 7623 & 42.97 & 2880.61 \\
\hline & & $12 \mathrm{~J}-\mathrm{S}-86 \mathrm{~A}$ & $\mathrm{ND}$ & $\mathrm{ND}$ & ND & 7711 & 48.61 & 2929.22 \\
\hline & & $12 \mathrm{~J}-\mathrm{S}-86 \mathrm{~B}$ & 1626 & 2904 & 6.41 & 7798 & 53.06 & 2982.28 \\
\hline & \multirow{4}{*}{$08 / 09 / 01$} & $12 \mathrm{~J}-\mathrm{S}-91 \mathrm{~A}$ & 1696 & 2776 & 6.61 & 7886 & 50.12 & 3032.40 \\
\hline & & $12 \mathrm{~J}-\mathrm{S}-94 \mathrm{~A}$ & $\mathrm{ND}$ & ND & ND & 7974 & 43.86 & 3076.26 \\
\hline & & $12 \mathrm{~J}-\mathrm{S}-94 \mathrm{~B}$ & ND & $\mathrm{ND}$ & $\mathrm{ND}$ & 8098 & 50.94 & 3127.20 \\
\hline & & $12 \mathrm{~J}-\mathrm{S}-96 \mathrm{~A}$ & ND & ND & ND & 8221 & 45.75 & 3172.95 \\
\hline
\end{tabular}


The Catholic University of America Vitreous State Laboratory
DuraMelter 1200 Tests with AZ-101HLW Simulants

Final Report, VSL-02R0100-2, Rev.1

Table 4.4. Characteristics of SBS Fluid Samples from DM1200 Tests (continued).

\begin{tabular}{|c|c|c|c|c|c|c|c|c|}
\hline Test & $\begin{array}{c}\text { Sampling } \\
\text { Date }\end{array}$ & Sample Name & $\begin{array}{c}\text { TSS } \\
(\mathrm{mg} / \mathrm{l})\end{array}$ & $\begin{array}{c}\text { TDS } \\
\text { (mg/l) }\end{array}$ & $\begin{array}{c}\text { Filtrate } \\
\text { pH }\end{array}$ & $\begin{array}{l}\text { Cumulative } \\
\text { Glass (kg) }\end{array}$ & $\begin{array}{c}\text { Blowdown } \\
\text { Vol. (gal) }\end{array}$ & $\begin{array}{l}\text { Cumulative } \\
\text { Blowdown } \\
\text { Vol. (gal) }\end{array}$ \\
\hline \multirow{26}{*}{$\# 3$} & \multirow{8}{*}{$08 / 09 / 01$} & $12 \mathrm{~J}-\mathrm{S}-99 \mathrm{~A}$ & ND & ND & ND & 8345 & 57.04 & 3229.99 \\
\hline & & $12 \mathrm{~J}-\mathrm{S}-100 \mathrm{~A}$ & $\mathrm{ND}$ & ND & ND & 8468 & 51.08 & 3281.07 \\
\hline & & $12 \mathrm{~J}-\mathrm{S}-106 \mathrm{~A}$ & 1732 & 6692 & 6.62 & 8554 & 53.28 & 3334.35 \\
\hline & & $12 \mathrm{~J}-\mathrm{S}-107 \mathrm{~A}$ & ND & ND & ND & 8640 & 50.48 & 3384.83 \\
\hline & & $12 \mathrm{~J}-\mathrm{S}-108 \mathrm{~A}$ & ND & ND & ND & 8726 & 53.19 & 3438.02 \\
\hline & & $12 \mathrm{~J}-\mathrm{S}-109 \mathrm{~A}$ & $\mathrm{ND}$ & $\mathrm{ND}$ & ND & 8812 & 44.69 & 3482.71 \\
\hline & & $12 \mathrm{~J}-\mathrm{S}-111 \mathrm{~A}$ & ND & ND & ND & 8898 & 71.47 & 3554.18 \\
\hline & & $12 \mathrm{~J}-\mathrm{S}-113 \mathrm{~A}$ & ND & ND & ND & 8984 & 54.70 & 3608.88 \\
\hline & \multirow{10}{*}{$08 / 10 / 01$} & $12 \mathrm{~J}-\mathrm{S}-113 \mathrm{~B}$ & ND & ND & ND & 9088 & 55.79 & 3664.67 \\
\hline & & $12 \mathrm{~J}-\mathrm{S}-115 \mathrm{~A}$ & ND & ND & ND & 9192 & 65.77 & 3730.44 \\
\hline & & $12 \mathrm{~J}-\mathrm{S}-120 \mathrm{~A}$ & ND & ND & ND & 9296 & 40.32 & 3770.76 \\
\hline & & $12 \mathrm{~J}-\mathrm{S}-124 \mathrm{~A}$ & 2038 & 2656 & 6.94 & 9400 & 61.00 & 3831.76 \\
\hline & & $12 \mathrm{~J}-\mathrm{S}-126 \mathrm{~A}$ & $\mathrm{ND}$ & ND & ND & 9504 & 59.46 & 3891.22 \\
\hline & & $12 \mathrm{~J}-\mathrm{S}-128 \mathrm{~A}$ & $\mathrm{ND}$ & ND & ND & 9645 & 44.36 & 3935.58 \\
\hline & & $12 \mathrm{~J}-\mathrm{S}-131 \mathrm{~A}$ & ND & ND & ND & 9717 & 50.00 & 3985.58 \\
\hline & & $12 \mathrm{~J}-\mathrm{S}-136 \mathrm{~A}$ & ND & ND & ND & 9787 & 55.00 & 4040.58 \\
\hline & & $12 \mathrm{~J}-\mathrm{S}-136 \mathrm{~B}$ & 772 & 2784 & 4.72 & 10135 & 55.00 & 4095.58 \\
\hline & & $12 \mathrm{~J}-\mathrm{S}-138 \mathrm{~A}$ & ND & ND & ND & 10241 & 55.00 & 4150.58 \\
\hline & \multirow{8}{*}{$08 / 11 / 01$} & $12 \mathrm{~J}-\mathrm{S}-139 \mathrm{~A}$ & ND & ND & ND & 10347 & 55.00 & 4205.58 \\
\hline & & $12 \mathrm{~J}-\mathrm{S}-139 \mathrm{~B}$ & 3584 & 2720 & 5.47 & 10453 & 55.00 & 4260.58 \\
\hline & & $12 \mathrm{~J}-\mathrm{S}-141 \mathrm{~A}$ & $\mathrm{ND}$ & ND & ND & 10559 & 55.00 & 4315.58 \\
\hline & & $12 \mathrm{~J}-\mathrm{S}-142 \mathrm{~A}$ & ND & ND & ND & 10688 & 0.00 & 4315.58 \\
\hline & & $12 \mathrm{~J}-\mathrm{S}-142 \mathrm{~B}$ & 802 & 2476 & 4.90 & 10817 & 55.00 & 4370.58 \\
\hline & & $12 \mathrm{~J}-\mathrm{S}-149 \mathrm{~A}$ & ND & $\mathrm{ND}$ & ND & 10946 & 55.00 & 4425.58 \\
\hline & & $12 \mathrm{~J}-\mathrm{S}-147 \mathrm{~A}$ & ND & ND & ND & 10882 & 55.00 & 4480.58 \\
\hline & & $12 \mathrm{~J}-\mathrm{S}-149 \mathrm{~B}$ & 2182 & 2688 & 5.96 & 11075 & 55.00 & 4535.58 \\
\hline \multirow{20}{*}{$\# 4$} & \multirow{5}{*}{$08 / 20 / 01$} & $12 \mathrm{~K}-\mathrm{S}-36 \mathrm{~A}$ & 186 & 312 & 6.69 & 11145 & 57.34 & 4592.92 \\
\hline & & $12 \mathrm{~K}-\mathrm{S}-38 \mathrm{~A}$ & ND & ND & ND & 11214 & 45.36 & 4638.28 \\
\hline & & $12 \mathrm{~K}-\mathrm{S}-39 \mathrm{~A}$ & ND & ND & ND & 11284 & 48.79 & 4687.07 \\
\hline & & $12 \mathrm{~K}-\mathrm{S}-40 \mathrm{~A}$ & ND & ND & ND & 11353 & 46.68 & 4733.75 \\
\hline & & $12 \mathrm{~K}-\mathrm{S}-43 \mathrm{~A}$ & 326 & 760 & 6.82 & 11423 & 40.75 & 4774.50 \\
\hline & \multirow{8}{*}{$08 / 21 / 01$} & $12 \mathrm{~K}-\mathrm{S}-44 \mathrm{~A}$ & $\mathrm{ND}$ & ND & ND & 11481 & 39.56 & 4814.06 \\
\hline & & $12 \mathrm{~K}-\mathrm{S}-46 \mathrm{~A}$ & ND & ND & ND & 11539 & 54.01 & 4868.07 \\
\hline & & $12 \mathrm{~K}-\mathrm{S}-51 \mathrm{~A}$ & ND & ND & ND & 11597 & 38.73 & 4906.80 \\
\hline & & $12 \mathrm{~K}-\mathrm{S}-53 \mathrm{~A}$ & 440 & 1152 & 6.49 & 11656 & 44.24 & 4951.04 \\
\hline & & $12 \mathrm{~K}-\mathrm{S}-54 \mathrm{~A}$ & $\mathrm{ND}$ & ND & ND & 11714 & 38.02 & 4989.06 \\
\hline & & $12 \mathrm{~K}-\mathrm{S}-55 \mathrm{~A}$ & ND & ND & ND & 11772 & 39.37 & 5028.43 \\
\hline & & $12 \mathrm{~K}-\mathrm{S}-56 \mathrm{~A}$ & 514 & 1250 & 6.09 & 11830 & 36.38 & 5064.81 \\
\hline & & $12 \mathrm{~K}-\mathrm{S}-64 \mathrm{~A}$ & ND & ND & ND & 11888 & 38.42 & 5103.23 \\
\hline & \multirow{7}{*}{$08 / 22 / 01$} & $12 \mathrm{~K}-\mathrm{S}-67 \mathrm{~A}$ & ND & ND & ND & 11992 & 49.97 & 5153.20 \\
\hline & & $12 \mathrm{~K}-\mathrm{S}-70 \mathrm{~A}$ & ND & ND & ND & 12096 & 47.78 & 5200.98 \\
\hline & & $12 \mathrm{~K}-\mathrm{S}-71 \mathrm{~A}$ & 494 & 1656 & 5.11 & 12201 & 36.44 & 5237.42 \\
\hline & & $12 \mathrm{~K}-\mathrm{S}-77 \mathrm{~A}$ & ND & ND & ND & 12305 & 44.83 & 5282.25 \\
\hline & & $12 \mathrm{~K}-\mathrm{S}-80 \mathrm{~A}$ & ND & ND & ND & 12409 & 38.75 & 5321.00 \\
\hline & & $12 \mathrm{~K}-\mathrm{S}-83 \mathrm{~A}$ & ND & ND & ND & 12485 & 47.75 & 5368.75 \\
\hline & & $12 \mathrm{~K}-\mathrm{S}-84 \mathrm{~A}$ & 472 & 2948 & 4.64 & 12560 & 45.36 & 5414.11 \\
\hline
\end{tabular}


The Catholic University of America Vitreous State Laboratory
DuraMelter 1200 Tests with AZ-101HLW Simulants

Final Report, VSL-02R0100-2, Rev.1

Table 4.4. Characteristics of SBS Samples from DM1200 Tests (continued).

\begin{tabular}{|c|c|c|c|c|c|c|c|c|}
\hline Test & $\begin{array}{l}\text { Sampling } \\
\text { Date }\end{array}$ & Sample Name & $\begin{array}{c}\text { TSS } \\
(\mathrm{mg} / \mathrm{l})\end{array}$ & $\begin{array}{c}\text { TDS } \\
(\mathrm{mg} / \mathrm{l})\end{array}$ & Filtrate pH & $\begin{array}{c}\text { Cumulative } \\
\text { Glass (kg) }\end{array}$ & $\begin{array}{l}\text { Blowdown } \\
\text { Vol. (gal) }\end{array}$ & $\begin{array}{c}\text { Cumulative } \\
\text { Blowdown } \\
\text { Vol. (gal) }\end{array}$ \\
\hline \multirow{22}{*}{$\# 4$} & \multirow{8}{*}{$08 / 23 / 01$} & $12 \mathrm{~K}-\mathrm{S}-86 \mathrm{~A}$ & ND & $\mathrm{ND}$ & ND & 12636 & 44.32 & 5458.43 \\
\hline & & $12 \mathrm{~K}-\mathrm{S}-86 \mathrm{~B}$ & ND & ND & ND & 12711 & 40.56 & 5498.99 \\
\hline & & $12 \mathrm{~K}-\mathrm{S}-93 \mathrm{~A}$ & ND & ND & ND & 12787 & 37.14 & 5536.13 \\
\hline & & $12 \mathrm{~K}-\mathrm{S}-94 \mathrm{~A}$ & 448 & 1736 & 4.30 & 12862 & 41.28 & 5577.41 \\
\hline & & $12 \mathrm{~K}-\mathrm{S}-101 \mathrm{~A}$ & ND & $\mathrm{ND}$ & $\mathrm{ND}$ & 12938 & 41.85 & 5619.26 \\
\hline & & $12 \mathrm{~K}-\mathrm{S}-102 \mathrm{~A}$ & ND & $\mathrm{ND}$ & ND & 13043 & 49.43 & 5668.69 \\
\hline & & $12 \mathrm{~K}-\mathrm{S}-104 \mathrm{~A}$ & ND & ND & ND & 13147 & 35.55 & 5704.24 \\
\hline & & $12 \mathrm{~K}-\mathrm{S}-104 \mathrm{~B}$ & 462 & 1832 & 4.16 & 13252 & 38.63 & 5742.87 \\
\hline & \multirow{8}{*}{$08 / 24 / 01$} & $12 \mathrm{~K}-\mathrm{S}-105 \mathrm{~A}$ & ND & ND & $\mathrm{ND}$ & 13356 & 51.30 & 5794.17 \\
\hline & & $12 \mathrm{~K}-\mathrm{S}-110 \mathrm{~A}$ & $\mathrm{ND}$ & ND & ND & 13461 & 45.53 & 5839.70 \\
\hline & & $12 \mathrm{~K}-\mathrm{S}-113 \mathrm{~A}$ & ND & ND & ND & 13555 & 39.25 & 5878.95 \\
\hline & & $12 \mathrm{~K}-\mathrm{S}-114 \mathrm{~A}$ & 470 & 1816 & 4.23 & 13649 & 41.98 & 5920.93 \\
\hline & & $12 \mathrm{~K}-\mathrm{S}-121 \mathrm{~A}$ & ND & ND & $\mathrm{ND}$ & 13744 & 43.39 & 5964.32 \\
\hline & & $12 \mathrm{~K}-\mathrm{S}-123 \mathrm{~A}$ & ND & ND & ND & 13838 & 39.45 & 6003.77 \\
\hline & & $12 \mathrm{~K}-\mathrm{S}-125 \mathrm{~A}$ & $\mathrm{ND}$ & ND & ND & 13932 & 46.68 & 6050.45 \\
\hline & & $12 \mathrm{~K}-\mathrm{S}-127 \mathrm{~A}$ & 463 & 1836 & 4.17 & 14035 & 43.18 & 6093.63 \\
\hline & \multirow{5}{*}{$08 / 25 / 01$} & $12 \mathrm{~K}-\mathrm{S}-129 \mathrm{~A}$ & ND & $\mathrm{ND}$ & $\mathrm{ND}$ & 14139 & 46.40 & 6140.03 \\
\hline & & $12 \mathrm{~K}-\mathrm{S}-134 \mathrm{~A}$ & $\mathrm{ND}$ & ND & ND & 14242 & 42.43 & 6182.46 \\
\hline & & $12 \mathrm{~K}-\mathrm{S}-135 \mathrm{~A}$ & ND & ND & ND & 14346 & 42.45 & 6224.91 \\
\hline & & $12 \mathrm{~K}-\mathrm{S}-136 \mathrm{~A}$ & 448 & 1936 & ND & 14449 & 41.16 & 6266.07 \\
\hline & & $12 \mathrm{~K}-\mathrm{S}-138 \mathrm{~A}$ & ND & $\mathrm{ND}$ & ND & 14540 & 24.54 & 6290.61 \\
\hline & $08 / 29 / 01$ & $12 \mathrm{~K}-\mathrm{S}-144 \mathrm{~A}$ & $\mathrm{ND}$ & $\mathrm{ND}$ & $\mathrm{ND}$ & 14540 & 0.00 & 6290.61 \\
\hline \multirow{15}{*}{$\# 5$} & \multirow{2}{*}{$09 / 25 / 01$} & 12L-S-16A & 80 & 268 & 8.04 & 14643 & 49.45 & 6340.06 \\
\hline & & $12 \mathrm{~L}-\mathrm{S}-21 \mathrm{~A}$ & 110 & 528 & 2.78 & 14746 & 30.30 & 6370.36 \\
\hline & \multirow{2}{*}{$09 / 26 / 01$} & $12 \mathrm{~L}-\mathrm{S}-28 \mathrm{~A}$ & 78 & 688 & 2.47 & 14849 & 32.21 & 6402.57 \\
\hline & & $12 \mathrm{~L}-\mathrm{S}-37 \mathrm{~A}$ & 44 & 972 & 2.2 & 14952 & 37.54 & 6440.11 \\
\hline & \multirow{3}{*}{$09 / 27 / 01$} & 12L-S-41A & 68 & 1256 & 1.9 & 15056 & 33.23 & 6473.34 \\
\hline & & 12L-S-50A & 92 & 1872 & 1.69 & 15230 & 35.46 & 6508.80 \\
\hline & & $12 \mathrm{~L}-\mathrm{S}-58 \mathrm{~A}$ & 102 & 2172 & 1.47 & 15404 & 31.53 & 6540.33 \\
\hline & \multirow{2}{*}{$09 / 28 / 01$} & $12 \mathrm{~L}-\mathrm{S}-62 \mathrm{~A}$ & 86 & 2512 & 1.37 & 15578 & 28.01 & 6568.34 \\
\hline & & 12L-S-78A & 430 & 2628 & 1.13 & 15641 & 37.36 & 6605.70 \\
\hline & \multirow{2}{*}{$09 / 29 / 01$} & $12 \mathrm{~L}-\mathrm{S}-80 \mathrm{~A}$ & 470 & 2840 & 1.12 & 15704 & 27.04 & 6632.74 \\
\hline & & $12 \mathrm{~L}-\mathrm{S}-88 \mathrm{~A}$ & 501 & 3256 & 1.09 & 15767 & 35.31 & 6668.05 \\
\hline & \multirow{3}{*}{$09 / 30 / 01$} & $12 \mathrm{~L}-\mathrm{S}-95 \mathrm{~A}$ & 1004 & 3176 & 1.1 & 15829 & 29.42 & 6697.47 \\
\hline & & 12L-S-107A & 956 & 3176 & 1.04 & 15892 & 30.63 & 6728.10 \\
\hline & & $12 \mathrm{~L}-\mathrm{S}-109 \mathrm{~A}$ & 1024 & 1400 & 1.05 & 16049 & 29.57 & 6757.67 \\
\hline & $10 / 01 / 01$ & 12L-S-116A & 1076 & 3356 & 1.05 & 16199 & 26.85 & 6784.52 \\
\hline \multirow{7}{*}{ \#6 } & \multirow{3}{*}{$10 / 11 / 01$} & $12 \mathrm{~L}-\mathrm{S}-147 \mathrm{~A}$ & 50 & 284 & 4.27 & 16283 & 42.69 & 6827.21 \\
\hline & & $12 \mathrm{~L}-\mathrm{S}-150 \mathrm{~A}$ & 214 & 284 & 7.13 & 16367 & 36.58 & 6863.79 \\
\hline & & 12L-S-155A & 256 & 348 & 6.89 & 16452 & 33.99 & 6897.78 \\
\hline & \multirow{4}{*}{$10 / 12 / 01$} & $12 \mathrm{M}-\mathrm{S}-7 \mathrm{~A}$ & 260 & 288 & 6.71 & 16536 & 36.43 & 6934.21 \\
\hline & & $12 \mathrm{M}-\mathrm{S}-10 \mathrm{~A}$ & 230 & 368 & 6.47 & 16621 & 29.86 & 6964.07 \\
\hline & & $12 \mathrm{M}-\mathrm{S}-21 \mathrm{~A}$ & 248 & 368 & 6.55 & 16706 & 39.74 & 7003.81 \\
\hline & & $12 \mathrm{M}-\mathrm{S}-24 \mathrm{~A}$ & 5 & 888 & 2.2 & 16809 & 30.25 & 7034.06 \\
\hline
\end{tabular}

Values in italics are from interpolation. 
The Catholic University of America

Table 4.4. Characteristics of SBS Samples from DM1200 Tests (continued).

\begin{tabular}{|c|c|c|c|c|c|c|c|c|}
\hline Test & $\begin{array}{l}\text { Sampling } \\
\text { Date }\end{array}$ & Sample Name & TSS (mg/l) & $\begin{array}{c}\text { TDS } \\
(\mathrm{mg} / \mathrm{l})\end{array}$ & Filtrate $\mathbf{p H}$ & $\begin{array}{c}\text { Cumulative } \\
\text { Glass (kg) }\end{array}$ & $\begin{array}{l}\text { Blowdown } \\
\text { Vol. (gal) }\end{array}$ & $\begin{array}{l}\text { Cumulative } \\
\text { Blowdown } \\
\text { Vol. (gal) }\end{array}$ \\
\hline \multirow{12}{*}{ \#6 } & \multirow{3}{*}{$10 / 13 / 01$} & $12 \mathrm{M}-\mathrm{S}-29 \mathrm{~A}$ & 202 & 440 & 6.61 & 16912 & 31.55 & 7065.61 \\
\hline & & $12 \mathrm{M}-\mathrm{S}-33 \mathrm{~A}$ & 192 & 252 & 6.72 & 17015 & 39.77 & 7105.38 \\
\hline & & $12 \mathrm{M}-\mathrm{S}-40 \mathrm{~A}$ & 190 & 196 & 6.72 & 17118 & 33.56 & 7138.94 \\
\hline & \multirow{3}{*}{$10 / 14 / 01$} & $12 \mathrm{M}-\mathrm{S}-49 \mathrm{~A}$ & 204 & 214 & 6.54 & 17222 & 33.70 & 7172.64 \\
\hline & & $12 \mathrm{M}-\mathrm{S}-55 \mathrm{~A}$ & 206 & 264 & 6.50 & 17321 & 29.39 & 7202.03 \\
\hline & & $12 \mathrm{M}-\mathrm{S}-61 \mathrm{~A}$ & 202 & 274 & 6.33 & 17421 & 34.88 & 7236.91 \\
\hline & \multirow{3}{*}{$10 / 15 / 01$} & $12 \mathrm{M}-\mathrm{S}-63 \mathrm{~A}$ & 195 & 242 & 6.43 & 17521 & 28.77 & 7265.68 \\
\hline & & $12 \mathrm{M}-\mathrm{S}-78 \mathrm{~A}$ & 560 & 172 & 4.24 & 17620 & 38.25 & 7303.93 \\
\hline & & $12 \mathrm{M}-\mathrm{S}-82 \mathrm{~A}$ & 600 & 230 & 4.51 & 17720 & 29.31 & 7333.24 \\
\hline & $10 / 16 / 01$ & $12 \mathrm{M}-\mathrm{S}-90 \mathrm{~A}$ & 492 & 262 & 4.51 & 17820 & 33.15 & 7366.39 \\
\hline & \multirow{2}{*}{$10 / 17 / 01$} & $12 \mathrm{M}-\mathrm{S}-97 \mathrm{~A}$ & 576 & 234 & 4.36 & 17920 & 30.75 & 7397.14 \\
\hline & & $12 \mathrm{M}-\mathrm{S}-102 \mathrm{~A}$ & 536 & 211 & 4.24 & 18020 & 29.77 & 7426.91 \\
\hline \multirow{34}{*}{$\# 7$} & \multirow{2}{*}{$10 / 22 / 01$} & $12 \mathrm{M}-\mathrm{S}-142 \mathrm{~A}$ & 136 & 156 & 4.32 & 18100 & 35.97 & 7462.88 \\
\hline & & $12 \mathrm{M}-\mathrm{S}-144 \mathrm{~A}$ & 126 & 224 & 3.44 & 18233 & 31.11 & 7493.99 \\
\hline & \multirow{10}{*}{$10 / 23 / 01$} & $12 \mathrm{M}-\mathrm{S}-146 \mathrm{~A}$ & 156 & 268 & 3.1 & 18366 & 38.81 & 7532.80 \\
\hline & & $12 \mathrm{M}-\mathrm{S}-148 \mathrm{~A}$ & -158 & 770 & 2.84 & 18499 & 34.47 & 7567.27 \\
\hline & & $12 \mathrm{M}-\mathrm{S}-150 \mathrm{~A}$ & 202 & 572 & 2.7 & 18632 & 38.20 & 7605.47 \\
\hline & & $12 \mathrm{~N}-\mathrm{S}-6 \mathrm{~A}$ & 224 & 604 & 2.56 & 18760 & 38.23 & 7643.70 \\
\hline & & $12 \mathrm{~N}-\mathrm{S}-11 \mathrm{~A}$ & 240 & 774 & 2.51 & 18888 & 39.64 & 7683.34 \\
\hline & & $12 \mathrm{~N}-\mathrm{S}-12 \mathrm{~A}$ & 306 & 832 & 2.35 & 19016 & 43.46 & 7726.80 \\
\hline & & $12 \mathrm{~N}-\mathrm{S}-16 \mathrm{~A}$ & 342 & 920 & 2.29 & 19145 & 34.27 & 7761.07 \\
\hline & & $12 \mathrm{~N}-\mathrm{S}-17 \mathrm{~A}$ & 370 & 992 & 2.29 & 19272 & 34.84 & 7795.91 \\
\hline & & $12 \mathrm{~N}-\mathrm{S}-19 \mathrm{~A}$ & 412 & 1044 & 2.25 & 19399 & 36.08 & 7831.99 \\
\hline & & $12 \mathrm{~N}-\mathrm{S}-20 \mathrm{~A}$ & 442 & 1348 & 2.26 & 19526 & 46.51 & 7878.50 \\
\hline & \multirow{9}{*}{$10 / 24 / 01$} & $12 \mathrm{~N}-\mathrm{S}-21 \mathrm{~A}$ & 486 & 1300 & 2.26 & 19653 & 42.09 & 7920.59 \\
\hline & & No Sampling & ND & ND & ND & 19713 & 44.32 & 7964.91 \\
\hline & & $12 \mathrm{~N}-\mathrm{S}-36 \mathrm{~A}$ & 678 & 2136 & 2.30 & 19774 & 35.29 & 8000.20 \\
\hline & & $12 \mathrm{~N}-\mathrm{S}-38 \mathrm{~A}$ & 548 & 2048 & 2.30 & 19896 & 38.65 & 8038.85 \\
\hline & & $12 \mathrm{~N}-\mathrm{S}-39 \mathrm{~A}$ & 512 & 2136 & 2.31 & 20017 & 38.05 & 8076.90 \\
\hline & & $12 \mathrm{~N}-\mathrm{S}-46 \mathrm{~A}$ & 476 & 2116 & 2.26 & 20121 & 35.51 & 8112.41 \\
\hline & & $12 \mathrm{~N}-\mathrm{S}-48 \mathrm{~A}$ & 486 & 2360 & 2.28 & 20225 & 34.30 & 8146.71 \\
\hline & & $12 \mathrm{~N}-\mathrm{S}-50 \mathrm{~A}$ & 478 & 2104 & 2.31 & 20329 & 40.13 & 8186.84 \\
\hline & & $12 \mathrm{~N}-\mathrm{S}-51 \mathrm{~A}$ & 692 & 2128 & 2.30 & 20433 & 37.77 & 8224.61 \\
\hline & \multirow{11}{*}{$10 / 25 / 01$} & $12 \mathrm{~N}-\mathrm{S}-52 \mathrm{~A}$ & 492 & 2188 & 2.34 & 20538 & 42.87 & 8267.48 \\
\hline & & $12 \mathrm{~N}-\mathrm{S}-53 \mathrm{~A}$ & 540 & 2168 & 2.36 & 20663 & 37.51 & 8304.99 \\
\hline & & $12 \mathrm{~N}-\mathrm{S}-54 \mathrm{~A}$ & 428 & 1792 & 2.27 & 20788 & 38.96 & 8343.95 \\
\hline & & $12 \mathrm{~N}-\mathrm{S}-62 \mathrm{~A}$ & 420 & 1808 & 2.28 & 20913 & 39.29 & 8383.24 \\
\hline & & $12 \mathrm{~N}-\mathrm{S}-63 \mathrm{~A}$ & 400 & 1872 & 2.29 & 21038 & 43.24 & 8426.48 \\
\hline & & $12 \mathrm{~N}-\mathrm{S}-66 \mathrm{~A}$ & 424 & 1728 & 2.31 & 21157 & 45.07 & 8471.55 \\
\hline & & $12 \mathrm{~N}-\mathrm{S}-68 \mathrm{~A}$ & 396 & 1704 & 2.32 & 21276 & 43.24 & 8514.79 \\
\hline & & $12 \mathrm{~N}-\mathrm{S}-70 \mathrm{~A}$ & 444 & 1556 & 2.38 & 21395 & 36.01 & 8550.80 \\
\hline & & $12 \mathrm{~N}-\mathrm{S}-72 \mathrm{~A}$ & 344 & 1780 & 2.39 & 21514 & 49.10 & 8599.90 \\
\hline & & $12 \mathrm{~N}-\mathrm{S}-73 \mathrm{~A}$ & 292 & 1692 & 2.34 & 21617 & 40.29 & 8640.19 \\
\hline & & $12 \mathrm{~N}-\mathrm{S}-79 \mathrm{~A}$ & 350 & 1750 & 2.41 & 21720 & 42.51 & 8682.70 \\
\hline & \multirow{2}{*}{$10 / 26 / 01$} & $12 \mathrm{~N}-\mathrm{S}-81 \mathrm{~A}$ & 264 & 1992 & 2.41 & 21823 & 39.07 & 8721.77 \\
\hline & & $12 \mathrm{~N}-\mathrm{S}-85 \mathrm{~A}$ & 264 & 1632 & 2.38 & 21926 & 40.29 & 8762.06 \\
\hline
\end{tabular}

Values in italics are from interpolation. 
Table 4.4. Characteristics of SBS Fluid Samples from DM1200 Tests (continued).

\begin{tabular}{|c|c|c|c|c|c|c|c|c|}
\hline Test & $\begin{array}{l}\text { Sampling } \\
\text { Date }\end{array}$ & Sample Name & $\begin{array}{c}\text { TSS } \\
(\mathrm{mg} / \mathrm{l})\end{array}$ & $\begin{array}{c}\text { TDS } \\
(\mathrm{mg} / \mathrm{l})\end{array}$ & $\begin{array}{c}\text { Filtrate } \\
\text { pH }\end{array}$ & $\begin{array}{c}\text { Cumulative } \\
\text { Glass (kg) }\end{array}$ & $\begin{array}{l}\text { Blowdown } \\
\text { Vol. (gal) }\end{array}$ & $\begin{array}{c}\text { Cumulative } \\
\text { Blowdown } \\
\text { Vol. (gal) } \\
\end{array}$ \\
\hline \multirow{15}{*}{$\# 7$} & \multirow{15}{*}{$10 / 26 / 01$} & $12 \mathrm{~N}-\mathrm{S}-86 \mathrm{~A}$ & 236 & 1868 & 2.40 & 22029 & 45.55 & 8807.61 \\
\hline & & $12 \mathrm{~N}-\mathrm{S}-87 \mathrm{~A}$ & 248 & 1928 & 2.05 & 22260 & 39.00 & 8846.61 \\
\hline & & $12 \mathrm{~N}-\mathrm{S}-88 \mathrm{~A}$ & 228 & 2004 & 2.04 & 22492 & 40.25 & 8886.86 \\
\hline & & $12 \mathrm{~N}-\mathrm{S}-94 \mathrm{~A}$ & 224 & 2136 & 2.00 & 22580 & 40.64 & 8927.50 \\
\hline & & $12 \mathrm{~N}-\mathrm{S}-99 \mathrm{~A}$ & 184 & 1828 & 2.04 & 22669 & 40.04 & 8967.54 \\
\hline & & $12 \mathrm{~N}-\mathrm{S}-99 \mathrm{~B}$ & 188 & 1680 & 2.05 & 22757 & 44.64 & 9012.18 \\
\hline & & $12 \mathrm{~N}-\mathrm{S}-101 \mathrm{~A}$ & 234 & 2280 & 2.01 & 22845 & 38.65 & 9050.83 \\
\hline & & $12 \mathrm{~N}-\mathrm{S}-102 \mathrm{~A}$ & 192 & 2048 & 2.27 & 22934 & 37.78 & 9088.61 \\
\hline & & $12 \mathrm{~N}-\mathrm{S}-104 \mathrm{~A}$ & 132 & 1916 & 2.26 & 23022 & 43.64 & 9132.25 \\
\hline & & $12 \mathrm{~N}-\mathrm{S}-106 \mathrm{~A}$ & 352 & 2240 & 2.28 & 23202 & 53.67 & 9185.92 \\
\hline & & $12 \mathrm{~N}-\mathrm{S}-114 \mathrm{~A}$ & 260 & 2208 & 2.24 & 23372 & 78.35 & 9264.27 \\
\hline & & $12 \mathrm{~N}-\mathrm{S}-117 \mathrm{~A}$ & 384 & 2048 & 2.25 & 23563 & 34.44 & 9298.71 \\
\hline & & $12 \mathrm{~N}-\mathrm{S}-118 \mathrm{~A}$ & 596 & 2028 & 2.22 & 23734 & 50.89 & 9349.60 \\
\hline & & $12 \mathrm{~N}-\mathrm{S}-126 \mathrm{~A}$ & 232 & 2124 & 2.20 & 23905 & 53.55 & 9403.15 \\
\hline & & $12 \mathrm{~N}-\mathrm{S}-126 \mathrm{~B}$ & 448 & 2120 & 2.22 & 24076 & 39.27 & 9442.42 \\
\hline \multirow{13}{*}{$\# 8$} & \multirow{2}{*}{$10 / 29 / 01$} & $12 \mathrm{~N}-\mathrm{S}-152 \mathrm{~A}$ & 84 & 380 & 6.98 & 24144 & 31.27 & 9473.69 \\
\hline & & $12 \mathrm{~N}-\mathrm{S}-153 \mathrm{~A}$ & 32 & 556 & 6.63 & 24212 & 30.22 & 9503.91 \\
\hline & \multirow{3}{*}{$10 / 30 / 01$} & $12 \mathrm{O}-\mathrm{S}-13 \mathrm{~A}$ & 24 & 800 & 5.20 & 24280 & 28.73 & 9532.64 \\
\hline & & 120-S-14A & 16 & 988 & 4.83 & 24348 & 37.18 & 9569.82 \\
\hline & & $12 \mathrm{O}-\mathrm{S}-22 \mathrm{~A}$ & 26 & 1412 & 4.95 & 24416 & 34.63 & 9604.45 \\
\hline & \multirow{3}{*}{$10 / 31 / 01$} & $12 \mathrm{O}-\mathrm{S}-31 \mathrm{~A}$ & 24 & 1624 & 4.94 & 24484 & 41.45 & 9645.90 \\
\hline & & $120-\mathrm{S}-38 \mathrm{~A}$ & 16 & 1868 & 4.99 & 24561 & 22.10 & 9668.00 \\
\hline & & $120-\mathrm{S}-40 \mathrm{~A}$ & 24 & 2064 & 4.95 & 24637 & 21.98 & 9689.98 \\
\hline & \multirow{4}{*}{$11 / 01 / 01$} & $120-S-43 \mathrm{~A}$ & 12 & 2504 & 5.07 & 24714 & 29.98 & 9719.96 \\
\hline & & $12 \mathrm{O}-\mathrm{S}-43 \mathrm{~B}$ & 20 & 2732 & 5.02 & 24790 & 24.23 & 9744.19 \\
\hline & & 12O-S-51A & 0 & 2940 & 4.83 & 24867 & 19.17 & 9763.36 \\
\hline & & $120-\mathrm{S}-58 \mathrm{~A}$ & 8 & 3040 & 4.87 & 24943 & 19.27 & 9782.63 \\
\hline & $11 / 02 / 01$ & $120-S-60 \mathrm{~A}$ & 4 & 3148 & 4.90 & 25020 & 20.09 & 9802.72 \\
\hline \multirow{14}{*}{ \#9 } & $11 / 05 / 01$ & $120-\mathrm{S}-97 \mathrm{~A}$ & 88 & 412 & 7.83 & 25123 & 33.33 & 9836.05 \\
\hline & \multirow{2}{*}{$11 / 06 / 01$} & $120-\mathrm{S}-107 \mathrm{~A}$ & 70 & 316 & 8.06 & 25227 & 33.42 & 9869.47 \\
\hline & & $120-\mathrm{S}-115 \mathrm{~A}$ & 60 & 352 & 8.09 & 25331 & 27.46 & 9896.93 \\
\hline & \multirow{2}{*}{$11 / 07 / 01$} & $120-\mathrm{S}-118 \mathrm{~A}$ & 66 & 496 & 8.00 & 25469 & 21.19 & 9918.12 \\
\hline & & $120-\mathrm{S}-125 \mathrm{~A}$ & 66 & 408 & 7.91 & 25607 & 34.83 & 9952.95 \\
\hline & \multirow{3}{*}{$11 / 08 / 01$} & $12 \mathrm{O}-\mathrm{S}-135 \mathrm{~A}$ & 60 & 450 & 7.86 & 25743 & 29.92 & 9982.87 \\
\hline & & $12 \mathrm{O}-\mathrm{S}-145 \mathrm{~A}$ & 50 & 616 & 7.71 & 25879 & 33.82 & 10016.69 \\
\hline & & $12 \mathrm{O}-\mathrm{S}-146 \mathrm{~A}$ & 56 & 764 & 7.55 & 25986 & 32.95 & 10049.64 \\
\hline & \multirow{3}{*}{$11 / 09 / 01$} & $120-S-154 \mathrm{~A}$ & 52 & 890 & 6.62 & 26094 & 28.72 & 10078.36 \\
\hline & & $12 \mathrm{P}-\mathrm{S}-14 \mathrm{~A}$ & 50 & 844 & 4.80 & 26202 & 36.29 & 10114.65 \\
\hline & & 12P-S-21A & 96 & 955 & 6.11 & 26333 & 29.98 & 10144.63 \\
\hline & \multirow{3}{*}{$11 / 10 / 01$} & $12 \mathrm{P}-\mathrm{S}-28 \mathrm{~A}$ & 40 & 898 & 4.10 & 26464 & 31.01 & 10175.64 \\
\hline & & 12P-S-34A & 76 & 1172 & 3.74 & 26595 & 35.53 & 10211.17 \\
\hline & & $12 \mathrm{P}-\mathrm{S}-41 \mathrm{~A}$ & 72 & 1144 & 3.48 & 26726 & 35.16 & 10246.33 \\
\hline
\end{tabular}

Values in italics are from interpolation. 
The Catholic University of America

DuraMelter 1200 Tests with AZ-101HLW Simulants

Vitreous State Laboratory

Final Report, VSL-02R0100-2, Rev.l

Table 4.5. Characteristics of WESP Fluid Samples from DM1200 Tests.

\begin{tabular}{|c|c|c|c|c|c|c|}
\hline Test & $\begin{array}{c}\text { Sample } \\
\text { Date }\end{array}$ & Sample Name & TSS (mg/l) & TDS (mg/l) & Filtrate pH & Glass (kg) \\
\hline \multirow{4}{*}{ \#1 } & \multirow{4}{*}{$07 / 03 / 01$} & $12 \mathrm{~F}-\mathrm{W}-41 \mathrm{~A}$ & 334 & 31836 & 2.31 & 0 \\
\hline & & $12 \mathrm{~F}-\mathrm{W}-63 \mathrm{~A}$ & 137 & 32430 & 2.27 & 0 \\
\hline & & $12 \mathrm{~F}-\mathrm{W}-63 \mathrm{~B}$ & 43 & 752 & 3.20 & 0 \\
\hline & & $12 \mathrm{G}-\mathrm{W}-80 \mathrm{~A}$ & 40 & 116 & 6.13 & 60 \\
\hline \multirow{19}{*}{$\# 2$} & $07 / 23 / 01$ & $12 \mathrm{G}-\mathrm{W}-116 \mathrm{~A}$ & 24 & 1248 & 3.22 & 2350 \\
\hline & \multirow[b]{2}{*}{$07 / 24 / 01$} & $12 \mathrm{G}-\mathrm{W}-128 \mathrm{~A}$ & 8 & 1260 & 4.08 & 2561 \\
\hline & & $12 \mathrm{G}-\mathrm{W}-139 \mathrm{~A}$ & 2 & 1288 & 7.61 & 2827 \\
\hline & \multirow[b]{2}{*}{$07 / 25 / 01$} & $12 G-W-148 A$ & 146 & 1292 & 7.73 & 2930 \\
\hline & & $12 \mathrm{H}-\mathrm{W}-11 \mathrm{~A}$ & 12 & 1566 & 7.80 & 3120 \\
\hline & \multirow[b]{2}{*}{$07 / 26 / 01$} & $12 \mathrm{H}-\mathrm{W}-23 \mathrm{~A}$ & 0 & 1408 & 7.82 & 3298 \\
\hline & & $12 \mathrm{H}-\mathrm{W}-33 \mathrm{~A}$ & 12 & 1364 & 8.03 & 3360 \\
\hline & \multirow{3}{*}{$07 / 27 / 01$} & $12 \mathrm{H}-\mathrm{W}-40 \mathrm{~A}$ & 2 & 1580 & 7.97 & 3514 \\
\hline & & $12 \mathrm{H}-\mathrm{W}-43 \mathrm{~A}$ & 574 & 1556 & 7.89 & 3699 \\
\hline & & $12 \mathrm{I}-\mathrm{W}-13 \mathrm{~A}$ & 2 & 1408 & 7.95 & 3746 \\
\hline & \multirow[b]{2}{*}{$07 / 28 / 01$} & $12 \mathrm{I}-\mathrm{W}-22 \mathrm{~A}$ & 0 & 1420 & 7.89 & 3842 \\
\hline & & $12 \mathrm{I}-\mathrm{W}-26 \mathrm{~A}$ & 8 & 1308 & 7.92 & 3890 \\
\hline & \multirow{2}{*}{$07 / 29 / 01$} & $12 \mathrm{I}-\mathrm{W}-33 \mathrm{~A}$ & 6 & 1456 & 8.01 & 3981 \\
\hline & & $12 \mathrm{I}-\mathrm{W}-42 \mathrm{~A}$ & 12 & 1544 & 7.92 & 4122 \\
\hline & $07 / 30 / 01$ & $12 \mathrm{I}-\mathrm{W}-51 \mathrm{~A}$ & 2 & 1696 & 7.92 & 4250 \\
\hline & \multirow{3}{*}{$07 / 31 / 01$} & $12 I-W-61 A$ & 2 & 1976 & 7.91 & 4507 \\
\hline & & $12 \mathrm{I}-\mathrm{W}-68 \mathrm{~A}$ & 6 & 1904 & 7.89 & 4635 \\
\hline & & $12 \mathrm{I}-\mathrm{W}-73 \mathrm{~A}$ & 8 & 2080 & 7.90 & 4635 \\
\hline & $08 / 02 / 01$ & $12 \mathrm{~J}-\mathrm{W}-12 \mathrm{~A}$ & 50 & 2088 & 7.82 & 4635 \\
\hline \multirow{6}{*}{ \#3 } & \multirow[b]{2}{*}{$08 / 08 / 01$} & $12 \mathrm{~J}-\mathrm{W}-65 \mathrm{~A}$ & 0 & 2508 & 3.08 & 6657 \\
\hline & & $12 \mathrm{~J}-\mathrm{W}-86 \mathrm{~A}$ & 6 & 3800 & 3.00 & 7711 \\
\hline & $08 / 10 / 01$ & $12 \mathrm{~J}-\mathrm{W}-130 \mathrm{~A}$ & 333 & 428 & 7.07 & 9645 \\
\hline & \multirow{3}{*}{$08 / 13 / 01$} & $12 \mathrm{~K}-\mathrm{W}-6 \mathrm{~A}$ & 26 & 9492 & 2.75 & \\
\hline & & $12 \mathrm{~K}-\mathrm{W}-6 \mathrm{~B}$ & $\mathrm{NA}$ & NA & NA & \\
\hline & & $12 \mathrm{~K}-\mathrm{W}-6 \mathrm{C}$ & 92 & 2060 & 2.5 & 11100 \\
\hline \multirow{8}{*}{$\# 4$} & $08 / 21 / 01$ & $12 \mathrm{~K}-\mathrm{W}-55 \mathrm{~A}$ & 0 & 1328 & N/A & 11772 \\
\hline & \multirow[b]{2}{*}{$08 / 22 / 01$} & $12 \mathrm{~K}-\mathrm{W}-68 \mathrm{~A}$ & 0 & 1764 & N/A & 11992 \\
\hline & & $12 \mathrm{~K}-\mathrm{W}-83 \mathrm{~A}$ & 0 & 3072 & N/A & 12485 \\
\hline & \multirow{2}{*}{$08 / 23 / 01$} & $12 \mathrm{~K}-\mathrm{W}-92 \mathrm{~A}$ & 0 & 2440 & N/A & 12787 \\
\hline & & $12 \mathrm{~K}-\mathrm{W}-104 \mathrm{~A}$ & 4 & 3836 & $\mathrm{~N} / \mathrm{A}$ & 13147 \\
\hline & \multirow{2}{*}{$08 / 24 / 01$} & $12 \mathrm{~K}-\mathrm{W}-112 \mathrm{~A}$ & 0 & 3724 & $\mathrm{~N} / \mathrm{A}$ & 13555 \\
\hline & & $12 K-W-126 A$ & 2 & 4876 & N/A & 14035 \\
\hline & $08 / 25 / 01$ & $12 \mathrm{~K}-\mathrm{W}-143 \mathrm{~A}$ & 4 & 6360 & N/A & 14539 \\
\hline
\end{tabular}


Table 4.5. Characteristics of WESP Fluid Samples from DM1200 Tests (continued).

\begin{tabular}{|c|c|c|c|c|c|c|}
\hline Test & $\begin{array}{c}\text { Sample } \\
\text { Date }\end{array}$ & Sample Name & $\begin{array}{c}\text { TSS } \\
(\mathrm{mg} / \mathrm{l})\end{array}$ & $\begin{array}{c}\text { TDS } \\
(\mathrm{mg} / \mathrm{l})\end{array}$ & $\begin{array}{c}\text { Filtrate } \\
\mathrm{pH}\end{array}$ & $\begin{array}{c}\text { Glass } \\
(\mathrm{kg})\end{array}$ \\
\hline \multirow{9}{*}{$\# 5$} & $09 / 25 / 01$ & $12 \mathrm{~L}-\mathrm{W}-20 \mathrm{~A}$ & 0 & 592 & 2.00 & 14746 \\
\hline & $09 / 26 / 01$ & $12 \mathrm{~L}-\mathrm{W}-30 \mathrm{~A}$ & 0 & 1612 & 1.27 & 14849 \\
\hline & \multirow{2}{*}{$09 / 27 / 01$} & $12 \mathrm{~L}-\mathrm{W}-41 \mathrm{~A}$ & 4 & 2232 & 1.08 & 15056 \\
\hline & & $12 \mathrm{~L}-\mathrm{W}-50 \mathrm{~A}$ & 20 & 2880 & 0.91 & 15230 \\
\hline & $09 / 28 / 01$ & $12 \mathrm{~L}-\mathrm{W}-70 \mathrm{~A}$ & 86 & 3664 & 0.71 & 15641 \\
\hline & $09 / 29 / 01$ & $12 \mathrm{~L}-\mathrm{W}-80 \mathrm{~A}$ & 54 & 3964 & 0.64 & 15704 \\
\hline & \multirow{2}{*}{$09 / 30 / 01$} & $12 \mathrm{~L}-\mathrm{W}-95 \mathrm{~A}$ & 36 & 4056 & 0.57 & 15829 \\
\hline & & $12 \mathrm{~L}-\mathrm{W}-107 \mathrm{~A}$ & 140 & 4448 & 0.50 & 15892 \\
\hline & $10 / 01 / 01$ & $12 \mathrm{~L}-\mathrm{W}-124 \mathrm{~A}$ & 50 & 5860 & 0.42 & 16200 \\
\hline \multirow{10}{*}{$\# 6$} & \multirow{2}{*}{$10 / 12 / 01$} & $12 \mathrm{M}-\mathrm{W}-10 \mathrm{~A}$ & 6 & 752 & 2.15 & 16621 \\
\hline & & $12 \mathrm{M}-\mathrm{W}-24 \mathrm{~A}$ & 5 & 888 & 2.2 & 16809 \\
\hline & $10 / 13 / 01$ & $12 \mathrm{M}-\mathrm{W}-40 \mathrm{~A}$ & 0 & 824 & 2.45 & 17118 \\
\hline & $10 / 14 / 01$ & $12 \mathrm{M}-\mathrm{W}-61 \mathrm{~A}$ & 0 & 1168 & $\mathrm{~N} / \mathrm{A}$ & 17421 \\
\hline & $10 / 15 / 01$ & $12 \mathrm{M}-\mathrm{W}-72 \mathrm{~A}$ & 1396 & 6 & 2.65 & 17620 \\
\hline & \multirow{5}{*}{$10 / 16 / 01$} & $12 \mathrm{M}-\mathrm{W}-82 \mathrm{~A}$ & 1504 & 10 & -.2 .68 & 17720 \\
\hline & & $12 \mathrm{M}-\mathrm{W}-91 \mathrm{~A}$ & 1708 & 10 & 2.69 & 17820 \\
\hline & & $12 \mathrm{M}-\mathrm{W}-109 \mathrm{~A}$ & 12 & 2280 & 2.73 & 18019.5 \\
\hline & & $12 \mathrm{M}-\mathrm{W}-117 \mathrm{~A}$ & 38 & 2092 & 2.86 & 18019.5 \\
\hline & & $12 \mathrm{M}-\mathrm{W}-117 \mathrm{~B}$ & 88 & 536 & 3.82 & 18100 \\
\hline \multirow{3}{*}{$\# 7$} & \multirow{3}{*}{$10 / 27 / 01$} & $12 \mathrm{~N}-\mathrm{W}-115 \mathrm{~A}$ & 0 & 7392 & 2.47 & 23372 \\
\hline & & $12 \mathrm{~N}-\mathrm{W}-132 \mathrm{~A}$ & 40 & 7072 & 2.47 & 24075.5 \\
\hline & & $12 \mathrm{~N}-\mathrm{W}-132 \mathrm{~B}$ & 80 & 1452 & 2.68 & 24100 \\
\hline \multirow{2}{*}{$\# 8$} & & $120-W-75 \mathrm{~A}$ & 0 & 4704 & 1.41 & 25020 \\
\hline & & $12 \mathrm{O}-\mathrm{W}-75 \mathrm{~B}$ & 100 & 1916 & 2.68 & 25020 \\
\hline \multirow{2}{*}{$\# 9$} & & $12 \mathrm{P}-\mathrm{W}-47 \mathrm{~A}$ & 2 & 606 & 7.52 & 26725 \\
\hline & & $12 \mathrm{P}-\mathrm{W}-47 \mathrm{~B}$ & 1074 & 1890 & 3.91 & 26725 \\
\hline
\end{tabular}


The Catholic University of America Vitreous State Laboratory
DuraMelter 1200 Tests with AZ-101HLW Simulants

Final Report, VSL-02R0100-2, Rev.1

Table 4.6. Analytical Results for Samples of SBS Blow-Down Fluids (mg/l).

\begin{tabular}{|c|c|c|c|c|c|c|c|c|c|c|c|c|c|c|c|}
\hline Test & \multicolumn{15}{|c|}{1} \\
\hline I.D. & \multicolumn{3}{|c|}{$12 \mathrm{~F}-\mathrm{S}-84 \mathrm{~A}$} & \multicolumn{3}{|c|}{$12 \mathrm{~F}-\mathrm{S}-87 \mathrm{~A}$} & \multicolumn{3}{|c|}{$12 \mathrm{~F}-\mathrm{S}-87 \mathrm{~B}$} & \multicolumn{3}{|c|}{$12 \mathrm{~F}-\mathrm{S}-98 \mathrm{~A}$} & \multicolumn{3}{|c|}{$12 \mathrm{~F}-\mathrm{S}-105 \mathrm{~A}$} \\
\hline Glass (kg). & \multicolumn{3}{|c|}{60} & \multicolumn{3}{|c|}{140} & \multicolumn{3}{|c|}{220} & \multicolumn{3}{|c|}{380} & \multicolumn{3}{|c|}{440} \\
\hline $\mathrm{pH}$ & \multicolumn{3}{|c|}{7.80} & \multicolumn{3}{|c|}{7.30} & \multicolumn{3}{|c|}{5.96} & \multicolumn{3}{|c|}{4.26} & \multicolumn{3}{|c|}{4.10} \\
\hline & Sus* & Dis.\# & Total & Sus & Dis. & Total & Sus* & Dis.\# & Total & Sus* & Dis.\# & Total & Sus* & Dis.井 & Total \\
\hline Total & 47 & 284 & 331 & 69 & 354 & 432 & 88 & 408 & 496 & 102 & 308 & 410 & 45 & 296 & 341 \\
\hline $\mathrm{Al}$ & 2.37 & 0.43 & 2.80 & 3.76 & 0.35 & 4.11 & 3.85 & 2.67 & 6.52 & 3.70 & 6.12 & 9.82 & 1.39 & 6.34 & 7.73 \\
\hline As & 0.03 & 0.22 & 0.25 & 0.02 & 0.20 & 0.22 & 0.02 & 0.21 & 0.23 & 0.16 & 0.31 & 0.47 & 0.03 & 0.26 & 0.29 \\
\hline$B$ & 0.21 & 16.28 & 16.49 & 0.23 & 26.50 & 26.73 & 0.37 & 36.98 & 37.35 & 0.12 & 49.26 & 49.38 & 0.04 & 52.38 & 52.42 \\
\hline $\mathrm{Ba}$ & 0.03 & 0.19 & 0.22 & 0.04 & 0.19 & 0.23 & 0.02 & 0.24 & 0.26 & 0.01 & \begin{tabular}{|l|}
0.28 \\
\end{tabular} & \begin{tabular}{|l|}
0.29 \\
\end{tabular} & 0.01 & 0.29 & \begin{tabular}{|l|}
0.30 \\
\end{tabular} \\
\hline $\mathrm{Ca}$ & 0.23 & 32.96 & 33.19 & 0.27 & 29.49 & 29.76 & 0.342 & 28.02 & 28.36 & 0.06 & 25.61 & 25.67 & 0.03 & 25.41 & 25.44 \\
\hline $\mathrm{Cd}$ & 0.15 & 0.03 & 0.18 & 0.24 & 0.09 & 0.33 & 0.19 & 0.47 & 0.66 & 0.13 & \begin{tabular}{|l|}
0.95 \\
\end{tabular} & 1.08 & 0.07 & 1.00 & $\begin{array}{l}1.07 \\
\end{array}$ \\
\hline $\mathrm{Cr}$ & 0.05 & 0.12 & 0.17 & 0.10 & 0.14 & 0.24 & 0.13 & 0.12 & 0.25 & 0.14 & 0.10 & 0.24 & 0.08 & 0.12 & 0.20 \\
\hline Cs & NA & 0.27 & 0.27 & $<0.1$ & 0.40 & 0.40 & NA & 0.56 & 0.56 & $<0.1$ & 0.78 & 0.78 & $<0.1$ & 0.87 & 0.87 \\
\hline $\mathrm{Cu}$ & 0.02 & 0.01 & 0.03 & 0.03 & 0.01 & 0.04 & 0.03 & 0.02 & 0.05 & 0.02 & 0.08 & 0.10 & 0.01 & 0.08 & 0.09 \\
\hline $\mathrm{Fe}$ & 4.67 & 0.03 & 4.70 & 8.02 & 0.02 & 8.04 & 10.78 & 0.04 & 10.82 & 14.06 & 0.11 & 14.17 & 8.61 & 0.15 & 8.76 \\
\hline $\mathrm{K}$ & 0.09 & 3.14 & 3.23 & 0.13 & 2.52 & 2.65 & 0.16 & 2.50 & 2.66 & 0.07 & 2.67 & 2.74 & 0.04 & 2.72 & 2.76 \\
\hline $\mathrm{Li}$ & 0.10 & 1.45 & 1.55 & 0.17 & 2.09 & 2.26 & 0.23 & 2.93 & 3.16 & 0.11 & 4.77 & 4.88 & 0.05 & 4.96 & 5.01 \\
\hline $\mathrm{Mg}$ & 0.10 & 7.11 & 7.21 & 0.13 & 6.75 & 6.88 & 0.19 & 6.71 & 6.90 & 0.27 & 6.73 & 7.00 & 0.16 & 6.90 & 7.06 \\
\hline Mn & 0.55 & $<0.01$ & 0.55 & 0.56 & 0.02 & 0.58 & 0.58 & 0.18 & 0.76 & 0.72 & 0.79 & $1.5 \mathrm{I}$ & 0.21 & 0.78 & 0.99 \\
\hline $\mathrm{Na}$ & 0.42 & 21.82 & 22.24 & 0.52 & 15.10 & 15.62 & 0.96 & 18.61 & 19.57 & 0.23 & 24.31 & 24.54 & 0.12 & 25.28 & 25.4 \\
\hline $\mathrm{Ni}$ & 0.29 & 0.11 & 0.40 & 0.50 & 0.17 & 0.67 & 0.55 & 0.21 & 0.76 & 0.65 & 0.43 & 1.08 & 0.36 & 0.37 & 0.73 \\
\hline $\mathrm{P}$ & 0.30 & $<0.07$ & 0.30 & 0.18 & 0.40 & 0.58 & 0.14 & 0.10 & 0.24 & 0.11 & 0.17 & 0.28 & 0.05 & 0.51 & 0.56 \\
\hline $\mathrm{Pb}$ & 0.10 & 0.12 & 0.22 & 0.16 & 0.14 & 0.30 & 0.18 & 0.17 & 0.35 & 0.22 & 0.29 & 0.51 & 0.12 & 0.28 & 0.40 \\
\hline $\mathrm{Sb}$ & 0.12 & 0.20 & 0.32 & 0.16 & 0.20 & 0.36 & 0.16 & 0.14 & 0.30 & 0.20 & 0.11 & 0.31 & 0.06 & 0.27 & 0.33 \\
\hline $\mathrm{Se}$ & 0.62 & 17.29 & 17.91 & 1.11 & 26.80 & 27.91 & 1.33 & 40.34 & 41.67 & 1.78 & \begin{tabular}{|c|}
66.03 \\
\end{tabular} & $\mid 67.81$ & 0.90 & 65.41 & 66.31 \\
\hline $\mathrm{Si}$ & 9.06 & 3.14 & 12.20 & 12.73 & 3.94 & 16.67 & 17.28 & 5.50 & 22.78 & 20.82 & \begin{tabular}{|l|}
7.55 \\
\end{tabular} & 28.37 & 8.04 & 7.84 & 15.88 \\
\hline$\overline{\mathrm{Sr}}$ & 0.16 & 2.15 & 2.31 & 0.21 & 3.04 & 3.25 & 0.14 & 3.57 & 3.71 & $\begin{array}{l}0.08 \\
\end{array}$ & 5.26 & \begin{tabular}{|l|}
5.34 \\
\end{tabular} & 0.04 & 5.56 & 5.60 \\
\hline $\mathrm{Te}$ & 0.98 & 0.25 & 1.23 & 1.64 & 0.34 & 1.98 & 1.92 & 0.59 & 2.51 & 2.08 & 0.91 & 2.99 & 1.21 & 1.06 & 2.27 \\
\hline $\mathrm{Ti}$ & 0.04 & 0.01 & 0.05 & 0.05 & 0.01 & 0.06 & 0.11 & 0.01 & 0.12 & 0.08 & 0.01 & 0.09 & 0.04 & 0.01 & 0.05 \\
\hline $\mathrm{Zn}$ & 0.59 & 0.08 & 0.67 & 0.97 & 0.41 & 1.38 & 0.82 & 2.41 & 3.23 & 0.36 & 4.33 & 4.69 & 0.20 & 4.77 & 4.97 \\
\hline $\mathrm{Zr}_{\mathrm{r}}$ & 1.09 & 0.01 & 1.10 & 1.11 & 0.02 & 1.13 & 1.50 & 0.01 & 1.51 & 2.11 & 0.07 & 2.18 & 0.53 & 0.14 & 0.67 \\
\hline $\mathrm{Cl}$ & $\mathrm{NA}$ & 25.78 & 25.8 & NA & 24.3 & 24.3 & $\mathrm{NA}_{2}$ & 24.30 & 24.3 & $\mathrm{NA}$ & 24.77 & 24.8 & NA & \begin{tabular}{|l|}
24.93 \\
\end{tabular} & 24.9 \\
\hline $\mathrm{F}$ & NA & 6.02 & 6.02 & NA & 8.61 & 8.61 & $\mathrm{NA} 1$ & 11.04 & 11.04 & NA & 14.74 & \begin{tabular}{|l|}
14.74 \\
\end{tabular} & $\mathrm{NA}$ & \begin{tabular}{|l|}
15.18 \\
\end{tabular} & 15.18 \\
\hline I & $<0.1$ & 5.78 & 5.78 & $<0.1$ & 17.48 & 17.48 & $<0.13$ & 36.24 & 36.24 & $<0.1$ & 60.79 & 60.79 & $<0.1$ & 63.44 & 63.44 \\
\hline Nitrate & NA & 1.58 & 1.58 & NA & 4.28 & 4.28 & NA & 4.52 & 4.52 & $\mathrm{NA}$ ? & 24.33 & 24.33 & NA & \begin{tabular}{|l|}
33.68 \\
\end{tabular} & 33.68 \\
\hline Nitrite & $\mathrm{NA}$ & 0.64 & 0.64 & NA & 8.55 & 8.55 & NA 1 & 18.57 & 18.57 & NA & 11.90 & 11.90 & $\mathrm{NA}$ & \begin{tabular}{|l|}
7.25 \\
\end{tabular} & $\begin{array}{l}7.25 \\
\end{array}$ \\
\hline Sulfate & 0.49 & 56.91 & 57.40 & 0.20 & 56.61 & 56.81 & $0.66 / 6$ & 63.63 & 64.29 & $<0.1$ & \begin{tabular}{|l|}
74.47 \\
\end{tabular} & \begin{tabular}{|l|}
74.47 \\
\end{tabular} & $<0.1$ & $\mid 72.61$ & 72.61 \\
\hline
\end{tabular}


The Catholic University of America

Vitreous State Laboratory

Table 4.6. Analytical Results for Samples of SBS Blow-Down Fluids (mg/l), (continued).

\begin{tabular}{|c|c|c|c|c|c|c|c|c|c|c|c|c|c|c|}
\hline Test & \multicolumn{14}{|c|}{1} \\
\hline I.D. & \multicolumn{3}{|c|}{$12 \mathrm{~F}-\mathrm{S}-107 \mathrm{~A}$} & \multicolumn{3}{|c|}{$12 \mathrm{~F}-\mathrm{S}-116 \mathrm{~A}$} & \multicolumn{3}{|c|}{$12 \mathrm{~F}-\mathrm{S}-119 \mathrm{~A}$} & \multicolumn{3}{|c|}{$12 \mathrm{~F}-\mathrm{S}-126 \mathrm{~A}$} & \multicolumn{2}{|c|}{$12 \mathrm{~F}-\mathrm{S}-136 \mathrm{~A}$} \\
\hline Glass (kg) & \multicolumn{3}{|c|}{520} & \multicolumn{3}{|c|}{600} & \multicolumn{3}{|c|}{700} & \multicolumn{3}{|c|}{800} & \multicolumn{2}{|c|}{900} \\
\hline $\mathrm{pH}$ & \multicolumn{3}{|c|}{3.77} & \multicolumn{3}{|c|}{3.74} & \multicolumn{3}{|c|}{3.67} & \multicolumn{3}{|c|}{3.58} & \multicolumn{2}{|c|}{3.56} \\
\hline & Sus* & Dis.\# & Total & Sus* & Dis.\# & Aotal & Sus* & Dis.\# & Total & Sus* & Dis.\# & Total & \begin{tabular}{l|l} 
Sus* Dis.\# \\
\end{tabular} & Total \\
\hline Total & 52 & 404 & 456 & 30 & 364 & \begin{tabular}{|l|l|}
4 & 394 \\
\end{tabular} & 120 & 392 & 512 & 65 & 452 & 517 & \begin{tabular}{|l|l|}
47 & 648 \\
\end{tabular} & 695 \\
\hline $\mathrm{Al}$ & 1.29 & 7.09 & 8.38 & 0.57 & 7.40 & 7.97 & 3.80 & 12.02 & 15.82 & 1.54 & 10.65 & 12.19 & \begin{tabular}{|l|l|}
0.88 & 11.49 \\
\end{tabular} & 12.37 \\
\hline As & 0.04 & 0.41 & 0.45 & $\overline{0.06}$ & 0.38 & 0.44 & 0.03 & 0.36 & 0.39 & 0.04 & 0.34 & 0.38 & \begin{tabular}{l|l|}
0.05 & 0.35 \\
\end{tabular} & \begin{tabular}{|l|}
0.40 \\
\end{tabular} \\
\hline $\mathrm{B}$ & 0.05 & 58.73 & 58.78 & 0.03 & 62.07 & \begin{tabular}{l|l}
7 & 62.10 \\
\end{tabular} & 0.09 & \begin{tabular}{|l|}
72.49 \\
\end{tabular} & 72.58 & 0.07 & 80.73 & 80.80 & \begin{tabular}{|l|l|}
0.05 & 85.00 \\
\end{tabular} & 85.05 \\
\hline $\mathrm{Ba}$ & 0.01 & \begin{tabular}{|l|}
0.28 \\
\end{tabular} & \begin{tabular}{|l|}
0.29 \\
\end{tabular} & $<0.01$ & \begin{tabular}{|l|}
0.27 \\
\end{tabular} & \begin{tabular}{|l|l|}
7 & 0.27 \\
\end{tabular} & 0.01 & 0.27 & 0.28 & 0.01 & \begin{tabular}{|l|}
0.28 \\
\end{tabular} & 0.29 & \begin{tabular}{|l|l|}
0.01 & 0.27 \\
\end{tabular} & 0.28 \\
\hline $\mathrm{Ca}$ & 0.03 & 23.10 & 23.13 & 0.02 & 22.58 & \begin{tabular}{|l|l}
822.60 \\
8
\end{tabular} & 0.04 & 21.92 & 21.96 & 0.03 & 17.56 & 17.59 & \begin{tabular}{|l|l|}
0.02 & 16.57 \\
\end{tabular} & 16.59 \\
\hline $\mathrm{Cd}$ & 0.10 & 1.17 & \begin{tabular}{|l|}
1.27 \\
\end{tabular} & 0.06 & 1.25 & 1.31 & 0.16 & 1.51 & 1.67 & 0.12 & \begin{tabular}{|l|}
1.62 \\
\end{tabular} & 1.74 & \begin{tabular}{|l|l|}
0.09 & 1.70 \\
\end{tabular} & 1.79 \\
\hline $\mathrm{Cr}$ & 0.10 & 0.14 & 0.24 & 0.05 & 0.13 & 0.18 & 0.18 & 0.16 & 0.34 & 0.14 & 0.14 & 0.28 & \begin{tabular}{|l|l|}
0.11 & 0.16 \\
\end{tabular} & 0.27 \\
\hline Cs & $<0.1$ & 0.97 & 0.97 & $<0.1$ & 1.00 & 1.00 & $<0.1$ & 1.20 & 1.20 & $<0.1$ & 1.31 & 1.31 & $<0.1$ & 1.37 \\
\hline $\mathrm{Cu}$ & 0.01 & 0.10 & 0.11 & 0.01 & 0.09 & 0.10 & 0.02 & 0.09 & 0.11 & 0.01 & 0.11 & 0.12 & 0.01 & 0.12 \\
\hline $\mathrm{Fe}$ & 10.70 & 0.34 & \begin{tabular}{|l|}
11.04 \\
\end{tabular} & 6.16 & 0.41 & 6.57 & 21.42 & 0.40 & 21.82 & 15.41 & 0.62 & 16.03 & 11.54 & 12.25 \\
\hline $\mathrm{K}$ & 0.07 & 2.95 & 3.02 & 0.03 & 2.61 & 2.64 & 0.08 & 2.58 & 2.66 & 0.08 & 3.06 & 3.14 & 0.06 & -2.40 \\
\hline $\mathrm{Li}$ & 0.06 & 4.27 & 4.33 & 0.03 & 4.77 & 4.80 & 0.11 & 6.80 & 6.91 & 0.07 & 7.00 & 7.07 & 0.05 & 7.28 \\
\hline $\mathrm{Mg}$ & 0.17 & 6.86 & 7.03 & 0.09 & 6.94 & 7.03 & 0.35 & 7.94 & 8.29 & 0.21 & 7.23 & 7.44 & 7.19 & 7.30 \\
\hline $\mathrm{Mn}$ & 0.22 & 0.99 & 1.21 & 0.11 & 1.21 & 1.32 & 0.51 & 1.80 & 2.31 & 0.24 & 2.09 & 2.33 & \begin{tabular}{|l|l|}
0.17 & 2.32 \\
\end{tabular} & 2.49 \\
\hline $\mathrm{Na}$ & 0.12 & 28.02 & 28.14 & 0.06 & 29.39 & 29.45 & 0.16 & 33.95 & 34.11 & 0.09 & 35.36 & 35.45 & \begin{tabular}{|l|l|}
0.07 & 37.03 \\
\end{tabular} & 37.10 \\
\hline $\mathrm{Ni}$ & 0.41 & \begin{tabular}{|l|}
0.37 \\
\end{tabular} & \begin{tabular}{|l|}
0.78 \\
\end{tabular} & 0.21 & 0.35 & 0.56 & 0.85 & \begin{tabular}{|l|}
0.44 \\
\end{tabular} & 1.29 & 0.53 & \begin{tabular}{|l|}
0.48 \\
\end{tabular} & 1.01 & \begin{tabular}{|l|l|}
0.38 & 0.49 \\
\end{tabular} & 0.87 \\
\hline$P$ & 0.10 & 0.40 & 0.50 & 0.21 & 0.68 & 0.89 & 0.33 & 0.66 & 0.99 & 0.13 & 0.26 & 0.39 & $0.06<0.07$ & 0.06 \\
\hline$\overline{\mathrm{Pb}}$ & 0.13 & 0.29 & 0.42 & 0.08 & 0.29 & 0.37 & 0.26 & 0.34 & 0.60 & 0.17 & 0.15 & 0.32 & \begin{tabular}{|l|l|}
0.13 & 0.16 \\
\end{tabular} & 0.29 \\
\hline $\mathrm{Sb}$ & 0.13 & 0.19 & 0.32 & 0.09 & 0.25 & 0.34 & 0.22 & 0.16 & 0.38 & 0.20 & 0.06 & 0.26 & \begin{tabular}{|l|l|}
0.16 & 0.04 \\
\end{tabular} & 0.20 \\
\hline $\mathrm{Se}$ & 1.00 & 80.73 & \begin{tabular}{|l|}
81.73 \\
\end{tabular} & 0.55 & 78.64 & 79.19 & 2.15 & 104.8 & 107.0 & 1.17 & 115.8 & 117.0 & \begin{tabular}{|l|l|}
0.90 & 122.6 \\
\end{tabular} & 123.5 \\
\hline $\mathrm{Si}$ & 8.71 & \begin{tabular}{|l|}
8.32 \\
\end{tabular} & 17.03 & 4.60 & 8.37 & 12.97 & 23.06 & 9.71 & 32.77 & 9.49 & \begin{tabular}{|l|}
9.96 \\
\end{tabular} & 19.45 & \begin{tabular}{|l|l|}
7.89 & 10.27 \\
\end{tabular} & 18.16 \\
\hline$\overline{\mathrm{Sr}}$ & 0.04 & 6.33 & \begin{tabular}{|l|}
6.37 \\
\end{tabular} & 0.02 & 6.62 & 6.64 & 0.06 & 9.13 & 9.19 & 0.03 & 8.88 & 8.91 & \begin{tabular}{|l|l|}
0.02 & 9.40 \\
\end{tabular} & 9.42 \\
\hline $\mathrm{Te}$ & 1.32 & 1.47 & 2.79 & 0.78 & 1.55 & 2.33 & 2.34 & 1.55 & 3.89 & 1.49 & 1.50 & 2.99 & \begin{tabular}{l|l|}
1.15 & 1.65 \\
\end{tabular} & 2.80 \\
\hline$\overline{\mathrm{Ti}}$ & 0.05 & 0.01 & 0.06 & 0.03 & 0.01 & 0.04 & 0.09 & $<0.01$ & 0.09 & 0.06 & 0.02 & 0.08 & 0.02 & $0.0^{\prime}$ \\
\hline $\mathrm{Zn}$ & 0.20 & 5.42 & 5.62 & 0.13 & 5.86 & 5.99 & 0.32 & 7.28 & 7.60 & 0.19 & 8.64 & 8.83 & 0.15 & 9.28 \\
\hline $\mathrm{Zr}$ & 0.47 & \begin{tabular}{|l|}
0.24 \\
\end{tabular} & \begin{tabular}{|l|}
0.71 \\
\end{tabular} & 0.21 & 0.27 & 0.48 & 1.33 & 0.31 & 1.64 & 0.52 & 0.37 & 0.89 & \begin{tabular}{|l|l|}
0.36 & 0.39 \\
\end{tabular} & 0.75 \\
\hline $\mathrm{Cl}$ & $\mathrm{NA}$ & 26.22 & 26.22 & $\mathrm{NA}$ & 25.67 & 25.67 & $\mathrm{NA}$ & 26.90 & 26.90 & $\mathrm{NA}$ & 31.01 & 31.01 & \begin{tabular}{l|l|} 
NA & 34.64
\end{tabular} & 34.64 \\
\hline $\mathrm{F}$ & $\mathrm{NA}$ & 17.70 & 17.70 & NA & 18.71 & $\mid 18.71$ & $\mathrm{NA}$ & 23.08 & 23.08 & NA & 27.18 & 27.18 & \begin{tabular}{l|l|} 
NA 28.55 \\
\end{tabular} & 28.55 \\
\hline $\bar{I}$ & $<0.1$ & 78.60 & 78.60 & $<0.1$ & 77.23 & 77.23 & $<0.1$ & 80.44 & 80.44 & $<0.1$ & 95.69 & 95.69 & \begin{tabular}{l|l|}
$<0.1$ & 80.05 \\
\end{tabular} & 80.05 \\
\hline Nitrate & $\mathrm{NA}$ & \begin{tabular}{|l|}
45.88 \\
\end{tabular} & 45.88 & $\mathrm{NA}$ & 45.01 & 45.01 & $\mathrm{NA}$ & 43.96 & 43.96 & $\mathrm{NA}$ & 54.21 & 54.21 & NA 64.83 & 64.83 \\
\hline Nitrite & $\mathrm{NA}$ & $<0.1$ & $<0.1$ & NA & $<0.1$ & $\mid<0.1$ & $\mathrm{NA}$ & $<0.1$ & $<0.1$ & NA & \begin{tabular}{|c|}
$<.1$ \\
\end{tabular} & $<0.1$ & \begin{tabular}{l|l|}
$\mathrm{NA}$ & $<0.1$
\end{tabular} & $<0.1$ \\
\hline Sulfate & 0.61 & 76.33 & 76.94 & 1.29 & 83.11 & 84.40 & $<0.1$ & 94.80 & 94.80 & $<0.1$ & \begin{tabular}{|l|}
109.7 \\
\end{tabular} & 109.7 & \begin{tabular}{l|l|}
$<0.1$ & 125.7
\end{tabular} & 125.7 \\
\hline
\end{tabular}


Table 4.6. Analytical Results for Samples of SBS Blow-Down Fluids (mg/l), (continued).

\begin{tabular}{|c|c|c|c|c|c|c|c|c|c|c|c|c|c|c|c|}
\hline Test & \multicolumn{15}{|c|}{$\# 1$} \\
\hline I.D. & \multicolumn{3}{|c|}{$12 \mathrm{~F}-\mathrm{S}-139 \mathrm{~A}$} & \multicolumn{3}{|c|}{$12 \mathrm{~F}-\mathrm{S}-141 \mathrm{~A}$} & \multicolumn{3}{|c|}{$12 \mathrm{~F}-\mathrm{S}-148 \mathrm{~A}$} & \multicolumn{3}{|c|}{$12 \mathrm{G}-\mathrm{S}-14 \mathrm{~A}$} & \multicolumn{3}{|c|}{$12 \mathrm{G}-\mathrm{S}-20 \mathrm{~A}$} \\
\hline Glass $(\mathrm{kg})$ & \multicolumn{3}{|c|}{978} & \multicolumn{3}{|c|}{1080} & \multicolumn{3}{|c|}{1180} & \multicolumn{3}{|c|}{1280} & \multicolumn{3}{|c|}{1380} \\
\hline $\mathrm{pH}$ & \multicolumn{3}{|c|}{$3.6 \mathrm{I}$} & \multicolumn{3}{|c|}{3.53} & \multicolumn{3}{|c|}{3.30} & \multicolumn{3}{|c|}{3.32} & \multicolumn{3}{|c|}{3.33} \\
\hline & Sus* & Dis.\# & Total & Sus* & Dis.\# & Total & Sus* & Dis.\# & Total & Sus* & Dis.\# & Total & Sus* & Dis.\# & Total \\
\hline Total & 50 & 630 & 680 & 47 & 72 & 119 & 27 & 64 & 91 & 27 & 820 & 847 & 25 & 740 & 765 \\
\hline Al & 1.01 & 11.40 & 12.41 & 0.89 & 13.05 & 13.94 & 0.39 & 13.40 & 13.79 & 0.42 & 13.20 & 13.62 & 0.46 & 13.69 & 14.15 \\
\hline As & 0.15 & 0.30 & 0.45 & 0.07 & 0.40 & 0.47 & 0.06 & 0.35 & 0.41 & 0.07 & 0.41 & 0.48 & 0.04 & 0.40 & 0.44 \\
\hline B & 0.04 & 84.96 & 85.00 & 0.04 & 93.67 & 93.71 & 0.02 & 97.90 & 97.92 & 0.04 & 95.01 & 95.05 & 0.01 & 95.64 & 95.65 \\
\hline $\mathrm{Ba}$ & $<0.01$ & \begin{tabular}{|l|}
0.28 \\
\end{tabular} & \begin{tabular}{|l|}
0.28 \\
\end{tabular} & $<0.01$ & 0.28 & 0.28 & $<0.01$ & \begin{tabular}{|l|}
0.27 \\
\end{tabular} & 0.27 & $<0.01$ & 0.27 & 0.27 & 0.01 & 0.26 & 0.27 \\
\hline $\mathrm{Ca}$ & 0.03 & \begin{tabular}{|l|}
16.24 \\
\end{tabular} & 16.27 & 0.02 & 14.45 & 14.47 & 0.01 & 13.32 & 13.33 & 0.04 & 12.61 & 12.65 & 0.04 & 11.10 & 11.14 \\
\hline $\mathrm{Cd}$ & 0.09 & 1.73 & 1.82 & 0.10 & 1.92 & 2.02 & 0.08 & 1.97 & 2.05 & 0.04 & 1.97 & 2.01 & 0.04 & 1.97 & 2.01 \\
\hline $\mathrm{Cr}$ & 0.09 & 0.15 & 0.24 & 0.09 & 0.17 & 0.26 & 0.07 & 0.20 & 0.27 & 0.07 & 0.19 & 0.26 & 0.05 & 0.22 & 0.27 \\
\hline Cs & $<0.1$ & 1.50 & 1.50 & $<0.1$ & 1.61 & 1.61 & 0.10 & 1.67 & 1.77 & $<0.1$ & 1.59 & 1.59 & $<0.1$ & 1.57 & 1.57 \\
\hline $\mathrm{Cu}$ & 0.01 & 0.11 & 0.12 & 0.01 & 0.12 & 0.13 & $<0.01$ & 0.12 & 0.12 & $<0.01$ & 0.12 & 0.12 & $<0.01$ & 0.13 & 0.13 \\
\hline $\mathrm{Fe}$ & 11.08 & 0.58 & 11.66 & 12.26 & 0.81 & 13.07 & 7.86 & 1.28 & 9.14 & 7.02 & 1.08 & 8.10 & 7.05 & 1.12 & 8.17 \\
\hline $\mathrm{K}$ & 0.04 & 2.45 & 2.49 & 0.04 & 2.41 & 2.45 & 0.04 & 2.40 & 2.44 & 0.04 & 2.36 & 2.40 & 0.01 & 2.31 & 2.32 \\
\hline $\mathrm{Li}$ & 0.03 & 7.61 & 7.64 & 0.04 & 8.87 & 8.91 & 0.03 & 8.62 & 8.65 & 0.03 & 8.71 & 8.74 & 0.01 & 9.06 & 9.07 \\
\hline $\mathrm{Mg}$ & 0.13 & 7.39 & 7.52 & 0.11 & 7.67 & 7.78 & 0.04 & 7.48 & 7.52 & 0.05 & 7.57 & 7.62 & 0.05 & 7.50 & 7.55 \\
\hline $\mathrm{Mn}$ & 0.17 & 2.37 & 2.54 & 0.15 & 2.69 & 2.84 & 0.08 & 2.87 & 2.95 & 0.09 & 2.89 & 2.98 & 0.08 & 3.06 & 3.14 \\
\hline $\mathrm{Na}$ & 0.15 & 37.59 & 37.74 & 0.21 & 42.26 & 42.47 & 0.07 & 40.86 & 40.93 & 0.10 & 40.33 & 40.43 & 0.05 & 40.97 & 41.02 \\
\hline $\mathrm{Ni}$ & 0.35 & 0.52 & \begin{tabular}{|l|}
0.87 \\
\end{tabular} & 0.38 & \begin{tabular}{|c|}
0.55 \\
\end{tabular} & \begin{tabular}{|l|}
0.93 \\
\end{tabular} & 0.22 & 0.54 & 0.76 & 0.23 & 0.51 & 0.74 & 0.20 & 0.58 & 0.78 \\
\hline$P$ & 0.15 & 0.34 & 0.49 & 0.14 & 0.33 & 0.47 & 0.27 & 0.59 & 0.86 & 0.06 & 0.40 & 0.46 & 0.04 & 0.58 & 0.62 \\
\hline $\mathrm{Pb}$ & 0.13 & 0.17 & 0.30 & 0.13 & 0.19 & 0.32 & 0.09 & 0.22 & 0.31 & 0.07 & 0.20 & 0.27 & 0.08 & 0.20 & 0.28 \\
\hline $\mathrm{Sb}$ & 0.05 & 0.05 & 0.10 & 0.09 & 0.13 & 0.22 & 0.09 & 0.13 & 0.22 & 0.07 & 0.15 & 0.22 & 0.04 & 0.13 & 0.17 \\
\hline $\mathrm{Se}$ & 1.00 & 119.9 & 120.9 & 0.85 & 135.6 & 136.5 & 0.39 & 143.7 & 144.1 & 0.42 & 145.1 & 145.5 & 0.40 & 151.8 & 152.2 \\
\hline $\mathrm{Si}$ & 8.11 & 10.55 & 18.66 & 7.29 & 11.54 & 18.83 & 4.40 & 11.42 & 15.82 & 4.31 & 11.67 & 15.98 & 3.17 & 11.86 & 15.03 \\
\hline $\mathrm{Sr}$ & 0.02 & 9.42 & 9.44 & 0.02 & 10.86 & 10.88 & 0.01 & 10.59 & 10.60 & 0.02 & 10.25 & 10.27 & 0.01 & 10.65 & 10.66 \\
\hline $\mathrm{Te}$ & 1.13 & 1.55 & 2.68 & 1.09 & 1.93 & 3.02 & 0.55 & 2.91 & 3.46 & 0.59 & 2.24 & 2.83 & 0.58 & 2.12 & 2.70 \\
\hline $\mathrm{Ti}$ & 0.05 & 0.02 & 0.07 & 0.05 & 0.02 & 0.07 & 0.03 & 0.02 & 0.05 & 0.03 & 0.01 & 0.04 & 0.71 & 0.02 & 0.73 \\
\hline $\mathrm{Zn}$ & 0.16 & 9.27 & 9.43 & 0.14 & 10.66 & \begin{tabular}{|l|}
10.80 \\
\end{tabular} & 0.08 & 10.87 & 10.95 & 0.08 & 10.54 & 10.62 & 0.08 & 10.96 & 11.04 \\
\hline $\mathrm{Zr}$ & 0.39 & 0.35 & 0.74 & 0.27 & 0.36 & \begin{tabular}{|l|}
0.63 \\
\end{tabular} & 0.12 & 0.43 & 0.55 & 0.17 & 0.44 & 0.61 & 0.12 & 0.46 & 0.58 \\
\hline $\mathrm{Cl}$ & NA & 30.48 & 30.48 & NA & 32.14 & 32.14 & NA & 35.51 & 35.51 & $\mathrm{NA}$ & 34.05 & 34.05 & NA & 29.32 & 29.32 \\
\hline$F$ & NA & 29.48 & 29.48 & NA & 34.35 & 34.35 & NA & 39.15 & 39.15 & NA & 34.72 & 34.72 & NA & 34.35 & 34.35 \\
\hline I & $<0.1$ & 80.83 & 80.83 & $<0.1$ & 85.52 & 85.52 & $<0.1$ & 75.36 & 75.36 & $<0.1$ & 69.88 & 69.88 & $<0.1$. & 59.99 & 59.99 \\
\hline Nitrate & NA & 57.34 & 57.34 & NA & 66.66 & 66.66 & NA & 60.45 & 60.45 & $\mathrm{NA}$ & 56.71 & 56.71 & NA & 53.10 & 53.10 \\
\hline Nitrite & NA & $<0.1$ & $<0.1$ & NA & $<0.1$ & $<0.1$ & NA & $<0.1$ & $<0.1$ & $\mathrm{NA}$ & $<0.1$ & $<0.1$ & NA & $<0.1$ & $<0.1$ \\
\hline Sulfate & $<0.1$ & 104.6 & 104.6 & $<0.10$ & 127.2 & 127.2 & $<0.1$ & 137.2 & 137.2 & 0.49 & 123.1 & 123.6 & 0.28 & 128.3 & 128.6 \\
\hline
\end{tabular}


Table 4.6. Analytical Results for Samples of SBS Blow-Down Fluids (mg/l), (continued).

\begin{tabular}{|c|c|c|c|c|c|c|c|c|c|c|c|c|c|c|c|}
\hline Test & \\
\hline I.D. & \multicolumn{3}{|c|}{$12 \mathrm{G}-\mathrm{S}-25 \mathrm{~A}$} & \multicolumn{3}{|c|}{$12 \mathrm{G}-\mathrm{S}-32 \mathrm{~A}$} & \multicolumn{3}{|c|}{$12 \mathrm{G}-\mathrm{S}-40 \mathrm{~A}$} & \multicolumn{3}{|c|}{$12 \mathrm{G}-\mathrm{S}-45 \mathrm{~A}$} & \multicolumn{3}{|c|}{$12 \mathrm{G}-\mathrm{S}-53 \mathrm{~A}$} \\
\hline Glass (kg) & \multicolumn{3}{|c|}{1483} & \multicolumn{3}{|c|}{1570} & \multicolumn{3}{|c|}{1650} & \multicolumn{3}{|c|}{1730} & \multicolumn{3}{|c|}{1820} \\
\hline $\mathrm{pH}$ & \multicolumn{3}{|c|}{3.29} & \multicolumn{3}{|c|}{3.32} & \multicolumn{3}{|c|}{3.33} & \multicolumn{3}{|c|}{3.34} & \multicolumn{3}{|c|}{3.45} \\
\hline & Sus* & Dis.\# & $\mid$ Total & Sus* & Dis.\# & Total & Sus* & $\mid$ Dis.\#| & Total & Sus* & Dis.\# & Total & \begin{tabular}{|l|} 
Sus* \\
\end{tabular} & Dis.\# & Total \\
\hline Total & 10 & 684 & 694 & 20 & 704 & 724 & 27 & 748 & 775 & 20 & 420 & 440 & 23 & 772 & 795 \\
\hline $\mathrm{Al}$ & 0.19 & 13.43 & 13.62 & 0.38 & 13.58 & 13.96 & 0.52 & 13.43 & 13.95 & 0.40 & 14.17 & 14.57 & 0.44 & 15.21 & 15.65 \\
\hline As & 0.04 & \begin{tabular}{|l|}
0.37 \\
\end{tabular} & 0.41 & 0.02 & 0.39 & 0.41 & 0.14 & 0.40 & 0.54 & 0.03 & 0.35 & 0.38 & 0.05 & 0.36 & 0.41 \\
\hline $\mathrm{B}$ & 0.03 & 94.12 & 94.15 & 0.02 & $<0.15$ & 0.02 & 0.03 & 97.69 & 97.72 & 0.02 & 97.42 & 97.44 & 0.02 & 101.2 & 101.2 \\
\hline $\mathrm{Ba}$ & $<0.01$ & \begin{tabular}{|l|}
0.24 \\
\end{tabular} & 0.24 & $<0.01$ & 0.24 & 0.24 & $<0.01$ & 0.24 & 0.24 & $<0.01$ & 0.24 & \begin{tabular}{|l|}
0.24 \\
\end{tabular} & $<0.01$ & 0.24 & 0.24 \\
\hline $\mathrm{Ca}$ & 0.01 & 10.13 & 10.14 & 0.01 & 10.03 & 10.04 & 0.02 & $<0.01$ & 0.02 & 0.02 & 9.40 & 9.42 & 0.01 & 9.13 & 9.14 \\
\hline$\overline{\mathrm{Cd}}$ & 0.01 & 1.95 & 1.96 & 0.03 & 1.98 & 2.01 & 0.03 & 2.01 & 2.04 & 0.04 & 1.95 & 1.99 & 0.03 & 2.09 & 2.12 \\
\hline $\mathrm{Cr}$ & 0.05 & 0.21 & 0.26 & 0.06 & 0.20 & 0.26 & 0.08 & 0.21 & 0.29 & 0.06 & 0.22 & 0.28 & 0.04 & 0.25 & 0.29 \\
\hline Cs & $<0.1$ & 1.64 & 1.64 & $<0.1$ & 1.67 & 1.67 & $<0.1$ & 1.67 & 1.67 & $<0.1$ & 1.68 & 1.68 & $<0.1$ & 1.69 & 1.69 \\
\hline $\mathrm{Cu}$ & $<0.01$ & 0.12 & 0.12 & $<0.1$ & 0.12 & 0.12 & 0.01 & 0.13 & 0.14 & $<0.01$ & 0.13 & 0.13 & $<0.01$ & 0.14 & 0.16 \\
\hline $\mathrm{Fe}$ & 3.03 & 1.14 & 4.17 & 5.50 & 1.06 & 6.56 & 7.66 & 1.07 & $\overline{8.73}$ & 5.35 & 1.12 & 6.47 & 6.20 & 0.94 & 7.14 \\
\hline $\mathrm{K}$ & 0.07 & 2.19 & 2.26 & 0.02 & 2.17 & 2.19 & 0.10 & 2.11 & 2.21 & 0.06 & 2.00 & 2.06 & 0.03 & 2.15 & 2.18 \\
\hline$\overline{\mathrm{Li}}$ & 0.03 & 8.95 & 8.98 & 0.02 & 9.01 & 9.03 & 0.04 & 9.35 & 9.39 & 0.03 & 9.41 & 9.44 & 0.01 & 9.53 & 9.5 \\
\hline$\overline{M g}$ & 0.02 & 7.23 & 7.25 & 0.04 & 7.29 & 7.33 & 0.06 & 7.24 & 7.30 & 0.04 & 7.21 & 7.25 & 0.04 & 7.84 & 7.88 \\
\hline $\mathrm{Mn}$ & 0.03 & \begin{tabular}{|l|}
3.02 \\
\end{tabular} & 3.05 & 0.07 & 2.94 & 3.01 & 0.09 & 2.97 & 3.06 & 0.07 & 3.08 & 3.15 & 0.07 & 3.22 & 3.29 \\
\hline $\mathrm{Na}$ & 0.04 & \begin{tabular}{|l|}
39.63 \\
\end{tabular} & 39.67 & 0.03 & $<0.03$ & 0.03 & 0.04 & 40.72 & 40.76 & 0.03 & 40.94 & 40.97 & 0.03 & 45.01 & 45.04 \\
\hline $\mathrm{Ni}$ & 0.14 & \begin{tabular}{|l|}
0.54 \\
\end{tabular} & 0.68 & 0.19 & 0.54 & 0.73 & \begin{tabular}{l|l}
0.29 \\
\end{tabular} & \begin{tabular}{|l|}
0.56 \\
\end{tabular} & 0.85 & 0.21 & 0.58 & \begin{tabular}{|l|}
0.79 \\
\end{tabular} & 0.19 & 0.64 & 0.83 \\
\hline $\mathrm{P}$ & 0.15 & 0.46 & 0.61 & 0.09 & 0.53 & 0.62 & 0.14 & 0.41 & 0.55 & 0.05 & 0.56 & 0.61 & 0.05 & 0.64 & 0.69 \\
\hline $\mathrm{Pb}$ & 0.03 & 0.21 & 0.24 & 0.06 & 0.20 & 0.26 & 0.08 & 0.21 & 0.29 & 0.05 & 0.19 & 0.24 & 0.06 & 0.20 & 0.26 \\
\hline $\mathrm{Sb}$ & 0.02 & 0.15 & 0.17 & 0.04 & 0.11 & 0.15 & 0.06 & 0.13 & 0.19 & 0.06 & 0.10 & 0.16 & 0.02 & 0.09 & 0.11 \\
\hline $\mathrm{Se}$ & 0.13 & 157.5 & 157.6 & 0.33 & 154.5 & 154.8 & 0.41 & 159.4 & 159.8 & 0.29 & 160.0 & 160.3 & 0.45 & 171.8 & 172.3 \\
\hline $\mathrm{Si}$ & 1.62 & 11.54 & 13.16 & 3.27 & 11.56 & 14.83 & 4.08 & 11.75 & 15.83 & 3.43 & 11.73 & 15.16 & 3.53 & 12.98 & 16.51 \\
\hline $\mathrm{Sr}$ & $<0.01$ & 10.28 & 10.28 & 0.01 & \begin{tabular}{|l|}
10.46 \\
\end{tabular} & 10.47 & 0.01 & 10.35 & \begin{tabular}{|l|}
10.36 \\
\end{tabular} & 0.01 & 11.00 & 11.01 & 0.01 & 12.12 & 12.13 \\
\hline $\mathrm{Te}$ & 0.24 & \begin{tabular}{|l|}
2.42 \\
\end{tabular} & \begin{tabular}{|l|}
2.66 \\
\end{tabular} & 0.41 & 2.15 & 2.56 & 0.56 & \begin{tabular}{|l|}
2.14 \\
\end{tabular} & 2.70 & 0.40 & 2.06 & 2.46 & 0.43 & 1.88 & 2.31 \\
\hline $\mathrm{Ti}$ & 0.02 & \begin{tabular}{|l|}
0.01 \\
\end{tabular} & 0.03 & 0.03 & 0.01 & 0.04 & 0.04 & 0.01 & 0.05 & 0.03 & 0.03 & 0.06 & 0.02 & 0.03 & 0.05 \\
\hline $\mathrm{Zn}$ & 0.03 & 10.45 & 10.48 & 0.04 & 11.29 & 11.33 & 0.06 & 11.65 & 11.71 & 0.05 & 11.70 & 11.75 & 0.07 & 12.69 & 12.76 \\
\hline $\mathrm{Zr}$ & 0.04 & \begin{tabular}{|l|}
0.45 \\
\end{tabular} & \begin{tabular}{|l|}
0.49 \\
\end{tabular} & 0.13 & 0.44 & 0.57 & 0.16 & \begin{tabular}{|l|}
0.42 \\
\end{tabular} & 0.58 & 0.14 & \begin{tabular}{|l|}
0.43 \\
\end{tabular} & \begin{tabular}{|l|}
0.57 \\
\end{tabular} & 0.16 & \begin{tabular}{|l}
0.39 \\
\end{tabular} & 0.55 \\
\hline $\mathrm{Cl}$ & NA & 28.30 & 28.30 & NA & 29.32 & 29.32 & $\mathrm{NA}$ & 33.16 & 33.16 & NA & 28.05 & 28.05 & NA & 28.05 & 28.05 \\
\hline $\mathrm{F}$ & $\mathrm{NA}$ & 34.09 & 34.09 & $\mathrm{NA}$ & 33.33 & 33.33 & NA & \begin{tabular}{|l|}
33.33 \\
\end{tabular} & 33.33 & NA & 33.33 & \begin{tabular}{|l|}
33.33 \\
\end{tabular} & $\mathrm{NA}$ & 36.13 & 36.13 \\
\hline I & $<0.1$ & 48.74 & 48.74 & $<0.1$ & 48.74 & 48.74 & $<0.1$ & \begin{tabular}{|l|}
49.51 \\
\end{tabular} & 49.51 & $<0.1$ & 52.38 & 52.38 & $<0.1$ & 56.06 & 56.06 \\
\hline & $\mathrm{NA}$ & 53.10 & 53.10 & $\mathrm{NA}$ & 57.28 & 57.28 & NA & 67.41 & 67.41 & NA & 57.87 & \begin{tabular}{|l|}
57.87 \\
\end{tabular} & NA & 61.45 & 61.45 \\
\hline & & $<0.1$ & $<0.1$ & $\mathrm{NA}$ & $<0.1$ & $<0.1$ & NA & $<0.1$ & $<0.1$ & NA & $<0.1$ & $<0.1$ & NA & $<0.1$ & $<0.1$ \\
\hline Sulfate & $<0.1$ & \begin{tabular}{|l|}
127.3 \\
\end{tabular} & 127.3 & 0.11 & 129.2 & 129.3 & 0.65 & 122.5 & 123.2 & 0.86 & 125.4 & 126.3 & 0.15 & 137.9 & 0.15 \\
\hline
\end{tabular}

* Suspended

\# Dissolved

NA - Not Analyzed. 
The Catholic University of America

DuraMelter 1200 Tests with AZ-101HLW Simulants

Vitreous State Laboratory

Final Report, VSL-02R0100-2, Rev.1

Table 4.6. Analytical Results for Samples of SBS Blow-Down Fluids (mg/l), (continued).

\begin{tabular}{|c|c|c|c|c|c|c|c|c|c|c|c|c|c|c|c|}
\hline Test & \\
\hline I.D. & \multicolumn{3}{|c|}{$12 \mathrm{G}-\mathrm{S}-56 \mathrm{~A}$} & \multicolumn{3}{|c|}{$12 \mathrm{G}-\mathrm{S}-58 \mathrm{~A}$} & \multicolumn{3}{|c|}{$12 \mathrm{G}-\mathrm{S}-64 \mathrm{~A}$} & \multicolumn{3}{|c|}{ 12G-S-73A } & \multicolumn{3}{|c|}{$12 \mathrm{G}-\mathrm{S}-79 \mathrm{~A}$} \\
\hline$\overline{\text { Glass }(\mathrm{kg})}$ & \multicolumn{3}{|c|}{1900} & \multicolumn{3}{|c|}{1980} & \multicolumn{3}{|c|}{2090} & \multicolumn{3}{|c|}{2200} & \multicolumn{3}{|c|}{2301} \\
\hline $\mathrm{pH}$ & \multicolumn{3}{|c|}{3.72} & \multicolumn{3}{|c|}{3.82} & \multicolumn{3}{|c|}{3.74} & \multicolumn{3}{|c|}{3.64} & \multicolumn{3}{|c|}{3.85} \\
\hline & Sus* & Dis.\# & Total & Sus* & Dis.\# & Total & Sus* & Dis.\# & Total & Sus* & Dis.林 & Total & Sus* & Dis.\# & A Tota \\
\hline Total & 35 & 1048 & 1083 & 43 & 838 & 881 & 72 & 804 & 876 & 572 & 756 & 1328 & 252 & 680 & 932 \\
\hline $\mathrm{Al}$ & 1.13 & 15.66 & 16.79 & 0.99 & 15.45 & 16.44 & 1.90 & 16.26 & 18.16 & 15.29 & 19.95 & 35.24 & 6.81 & 19.89 & 26.70 \\
\hline As & 0.08 & 0.36 & 0.44 & 0.12 & 0.43 & 0.55 & 0.22 & \begin{tabular}{|l|}
0.44 \\
\end{tabular} & 0.66 & 0.66 & 0.41 & 1.07 & 0.14 & \begin{tabular}{l|l}
4 & 0.43 \\
\end{tabular} & 0.57 \\
\hline $\mathrm{B}$ & 0.05 & 105.8 & 105.9 & 0.04 & 107.9 & 107.9 & 0.05 & 109.3 & 109.4 & 0.56 & 111.4 & 112.0 & 0.22 & 2109.6 & \begin{tabular}{|l|l|}
5109.8 \\
\end{tabular} \\
\hline $\mathrm{Ba}$ & 0.01 & 0.25 & 0.26 & $<0.01$ & 0.26 & 0.26 & 0.01 & 0.25 & 0.26 & 0.08 & 0.29 & 0.37 & 0.03 & 0.28 & 0.31 \\
\hline $\mathrm{Ca}$ & 0.05 & 8.44 & 8.49 & 0.02 & 7.96 & 7.98 & 0.03 & 6.92 & 6.95 & 0.18 & 7.04 & 7.22 & 0.07 & 7.74 & 1.81 \\
\hline $\mathrm{Cd}$ & 0.05 & 2.28 & 2.33 & 0.07 & 2.36 & 2.43 & 0.06 & 2.35 & 2.41 & 0.78 & 2.50 & 3.28 & 0.27 & 2.43 & 2.70 \\
\hline $\mathrm{Cr}$ & 0.11 & 0.26 & 0.37 & 0.07 & 0.25 & 0.32 & 0.12 & 0.23 & 0.35 & 0.83 & 0.22 & 1.05 & 0.35 & 0.20 & 0.55 \\
\hline $\mathrm{Cs}$ & $<0.1$ & 1.82 & 1.82 & $<0.1$ & 2.03 & 2.03 & $<0.1$ & 2.06 & 2.06 & $<0.1$ & 1.90 & 1.90 & $<0.1$ & 1.90 & 1.90 \\
\hline $\mathrm{Cu}$ & 0.01 & 0.15 & 0.15 & $<0.1$ & 0.16 & 0.16 & 0.01 & 0.16 & 0.17 & 0.08 & 0.17 & 0.25 & 0.04 & 0.17 & 0.21 \\
\hline $\mathrm{Fe}$ & 3.99 & 0.58 & 14.57 & 12.03 & 0.48 & 12.51 & 18.46 & 0.58 & 19.04 & 99.36 & 0.49 & 99.85 & 39.87 & 0.22 & 40.09 \\
\hline $\bar{K}$ & 0.07 & 2.28 & 2.35 & 0.03 & 2.56 & 2.59 & 0.06 & 2.44 & 2.50 & \begin{tabular}{|l|}
0.34 \\
\end{tabular} & 3.24 & 3.58 & 0.14 & 3.10 & 3.24 \\
\hline$\overline{\mathrm{Li}}$ & 0.05 & 10.96 & 11.01 & 0.04 & 11.45 & 11.49 & 0.07 & 12.14 & 12.21 & 0.46 & 14.07 & 14.53 & 0.22 & 213.18 & 313.40 \\
\hline $\mathrm{Mg}$ & 0.18 & 7.66 & 7.84 & 0.17 & 7.62 & 7.79 & 0.26 & 7.90 & 8.16 & 1.05 & 8.91 & 9.96 & 0.51 & 8.74 & 9.25 \\
\hline $\mathrm{Mn}$ & 0.16 & \begin{tabular}{|l|}
3.35 \\
\end{tabular} & 3.51 & 0.11 & 3.29 & 3.40 & 0.19 & 3.30 & 3.49 & 4.26 & 3.95 & 8.21 & 1.77 & 3.75 & 5.52 \\
\hline $\mathrm{Na}$ & 0.09 & 50.84 & 50.93 & 0.06 & 54.45 & 54.51 & 0.20 & \begin{tabular}{|l|}
53.98 \\
\end{tabular} & 54.18 & 0.75 & 56.96 & 57.71 & 0.39 & 55.30 & 55.69 \\
\hline $\mathrm{Ni}$ & 0.45 & 0.70 & 1.15 & 0.37 & 0.66 & 1.03 & 0.61 & \begin{tabular}{|l|}
0.67 \\
\end{tabular} & 1.28 & 4.00 & 0.75 & 4.75 & 1.68 & $\begin{array}{ll}0.75 \\
\end{array}$ & 2.43 \\
\hline $\mathrm{P}$ & 0.20 & 0.90 & 1.10 & 0.35 & 0.84 & 1.19 & 0.10 & 0.93 & 1.03 & 1.18 & 0.99 & 2.17 & 0.36 & $5 \quad 1.04$ & 1.40 \\
\hline $\mathrm{Pb}$ & 0.12 & 0.23 & 0.35 & 0.08 & 0.24 & 0.32 & 0.14 & 0.24 & 0.38 & 1.20 & 0.24 & 1.44 & 0.51 & $\begin{array}{ll}0.24 \\
\end{array}$ & 0.75 \\
\hline $\mathrm{Sb}$ & 0.08 & 0.12 & 0.20 & 0.19 & 0.10 & 0.29 & 0.09 & 0.14 & 0.23 & 1.33 & 0.12 & 1.45 & $\begin{array}{l}0.47 \\
\end{array}$ & 0.16 & 0.63 \\
\hline $\mathrm{Se}$ & 1.18 & 178.1 & 179.3 & 0.96 & 183.6 & 184.6 & 1.37 & 187.6 & 189.0 & 13.75 & 200.4 & 214.2 & 5.46 & \begin{tabular}{|l|l|}
6 & 188.4 \\
\end{tabular} & 193.9 \\
\hline $\mathrm{Si}$ & 8.22 & \begin{tabular}{|l|}
13.99 \\
\end{tabular} & 22.21 & 6.43 & 14.71 & 21.14 & 11.33 & 14.92 & 26.25 & 120.2 & 16.02 & 136.2 & 52.75 & 515.80 & 68.55 \\
\hline $\mathrm{Sr}$ & 0.02 & 13.65 & 13.67 & 0.02 & 14.25 & 14.27 & \begin{tabular}{|l|}
0.03 \\
\end{tabular} & 15.26 & 15.29 & 0.32 & 18.17 & 18.49 & 0.15 & 517.35 & 17.50 \\
\hline $\mathrm{Te}$ & 1.10 & \begin{tabular}{|l|}
1.56 \\
\end{tabular} & 2.66 & 0.73 & 1.56 & 2.29 & 1.21 & 1.59 & 2.80 & 9.44 & \begin{tabular}{|l|}
1.40 \\
\end{tabular} & 10.84 & 4.49 & \begin{tabular}{|l|l|}
9 & 1.25 \\
\end{tabular} & 5.74 \\
\hline $\mathrm{Ti}$ & 0.05 & 0.04 & $\begin{array}{l}0.09 \\
\end{array}$ & 0.04 & 0.03 & 0.07 & 0.06 & 0.02 & 0.08 & 0.51 & 0.01 & $\begin{array}{l}0.52 \\
\end{array}$ & 0.18 & 0.01 & 0.19 \\
\hline $\mathrm{Zn}$ & 0.14 & 14.73 & 14.87 & 0.13 & 15.13 & 15.26 & 0.17 & 15.84 & 16.01 & 1.50 & 16.20 & 17.70 & 0.67 & 75.66 & 16.33 \\
\hline $\mathrm{Zr}$ & 0.42 & 0.25 & \begin{tabular}{|l|}
0.67 \\
\end{tabular} & 0.24 & \begin{tabular}{|l|}
0.18 \\
\end{tabular} & 0.42 & 0.37 & \begin{tabular}{|l|}
0.14 \\
\end{tabular} & 0.51 & \begin{tabular}{|l|l|}
14.17 \\
\end{tabular} & $\begin{array}{l}0.15 \\
\end{array}$ & \begin{tabular}{|l|}
14.32 \\
\end{tabular} & 5.32 & 0.05 & 5.37 \\
\hline $\mathrm{Cl}$ & $\mathrm{NA}$ & 32.14 & 32.14 & $\mathrm{NA}$ & 33.27 & 33.27 & NA & \begin{tabular}{|l|}
32.52 \\
\end{tabular} & 32.52 & NA & 32.80 & 32.80 & NA & 32.30 & 32.30 \\
\hline$F$ & NA & 36.89 & \begin{tabular}{|l|}
36.89 \\
\end{tabular} & $\mathrm{NA}$ & 34.93 & 34.93 & NA & 37.28 & 37.28 & NA & 37.96 & 37.96 & NA & 35.96 & 35.96 \\
\hline I & $<0.1$ & 69.16 & 69.16 & $<0.1$ & 82.05 & 82.05 & $<0.1$ & \begin{tabular}{|l|}
89.58 \\
\end{tabular} & 89.58 & $<0.1$ & 89.84 & 89.84 & $<0.1$ & 84.63 & 84.63 \\
\hline & $\mathrm{NA}$ & 75.17 & 75.17 & $\mathrm{NA}$ & 81.25 & 81.25 & NA & 91.28 & 91.28 & NA & 75.37 & 75.37 & NA & 73.14 & 73.14 \\
\hline & $\mathrm{NA}$ & $<0.1$ & $<0.1$ & $\mathrm{NA}$ & $<0.1$ & $<0.1$ & NA & $<0.1$ & $<0.1$ & NA & $<0.1$ & $<0.1$ & NA & $<<0.1$ & $<0.1$ \\
\hline Sulfate & $<0.1$ & 136.0 & 136.0 & 0.88 & 143.7 & 144.6 & 0.08 & \begin{tabular}{|l|}
143.7 \\
\end{tabular} & 143.8 & $<0.1$ & 160.0 & 160.0 & $<0.1$ & 139.4 & 139.4 \\
\hline
\end{tabular}

* Suspended

\# Dissolved

NA - Not Analyzed. 
Table 4.6. Analytical Results for Samples of SBS Blow-Down Fluids (mg/l), (continued).

\begin{tabular}{|c|c|c|c|c|c|c|c|c|c|c|c|c|c|c|c|}
\hline Test & \multicolumn{15}{|c|}{2} \\
\hline I.D. & \multicolumn{3}{|c|}{$12 \mathrm{G}-\mathrm{S}-116 \mathrm{~A}$} & \multicolumn{3}{|c|}{$12 \mathrm{G}-\mathrm{S}-116 \mathrm{~B}$} & \multicolumn{3}{|c|}{$12 \mathrm{G}-\mathrm{S}-124 \mathrm{~A}$} & \multicolumn{3}{|c|}{$12 \mathrm{G}-\mathrm{S}-126 \mathrm{~A}$} & \multicolumn{3}{|c|}{$12 \mathrm{G}-\mathrm{S}-128 \mathrm{~A}$} \\
\hline Glass (kg) & \multicolumn{3}{|c|}{2350} & \multicolumn{3}{|c|}{2400} & \multicolumn{3}{|c|}{2460} & \multicolumn{3}{|c|}{2510} & \multicolumn{3}{|c|}{2561} \\
\hline $\mathrm{pH}$ & \multicolumn{3}{|c|}{8.40} & \multicolumn{3}{|c|}{8.48} & \multicolumn{3}{|c|}{8.36} & \multicolumn{3}{|c|}{8.42} & \multicolumn{3}{|c|}{8.48} \\
\hline & Sus* & Dis.\# & Total & Sus* $^{*}$ & Dis.\# & Total & Sus* & Dis.\# & Total & Sus* & Dis.\# & Total & Sus* & Dis.\# & Total \\
\hline Total & 28 & 204 & 232 & 62 & 304 & 366 & 66 & 324 & 390 & 38 & 284 & 322 & 38 & 424 & 462 \\
\hline $\mathrm{AI}$ & 1.61 & 0.94 & 2.55 & 3.45 & 0.78 & 4.23 & 3.51 & 0.67 & 4.18 & 1.90 & 0.52 & 2.42 & 1.72 & 0.46 & 2.18 \\
\hline As & 0.04 & 0.17 & 0.21 & 0.04 & 0.16 & 0.20 & 0.11 & 0.23 & 0.34 & 0.06 & 0.22 & 0.28 & 0.05 & 0.28 & 0.33 \\
\hline $\mathrm{B}$ & 0.07 & 27.63 & 27.70 & 0.11 & 30.45 & 30.56 & 0.20 & 36.13 & 36.33 & 0.11 & 44.25 & 44.36 & 0.01 & 50.53 & 50.54 \\
\hline $\mathrm{Ba}$ & 0.01 & 0.03 & 0.04 & 0.02 & 0.03 & 0.05 & 0.02 & 0.04 & 0.06 & 0.01 & 0.05 & 0.06 & 0.01 & 0.05 & 0.06 \\
\hline $\mathrm{Ca}$ & 0.21 & 29.26 & 29.47 & 0.47 & 28.02 & 28.49 & 0.51 & 26.60 & 27.11 & 0.26 & 23.93 & 24.19 & 0.27 & 21.31 & 21.58 \\
\hline $\mathrm{Cd}$ & 0.04 & 0.03 & 0.07 & 0.12 & 0.03 & 0.15 & 0.22 & 0.08 & 0.30 & 0.44 & 0.23 & 0.67 & 1.10 & 0.24 & 1.34 \\
\hline $\mathrm{Cr}$ & 0.04 & 0.05 & 0.09 & 0.09 & 0.07 & 0.16 & 0.09 & 0.08 & 0.17 & 0.04 & 0.10 & 0.14 & 0.06 & 0.09 & 0.15 \\
\hline Cs & $<0.1$ & 0.40 & 0.40 & $<0.1$ & 0.40 & 0.40 & $<0.1$ & 0.50 & 0.50 & $<0.1$ & 0.60 & 0.60 & $<0.1$ & 0.70 & 0.70 \\
\hline $\mathrm{Cu}$ & 0.01 & $<0.01$ & 0.01 & 0.02 & $<0.01$ & 0.02 & 0.02 & 0.02 & 0.04 & 0.01 & 0.02 & 0.03 & 0.01 & 0.03 & 0.04 \\
\hline $\mathrm{Fe}$ & 3.72 & 0.01 & 3.73 & 9.58 & 0.01 & 9.59 & 10.18 & $<0.01$ & $\mid 10.18$ & 6.10 & 0.01 & 6.11 & 6.30 & $<0.01$ & 6.30 \\
\hline $\mathrm{K}$ & 0.03 & 3.27 & 3.30 & 0.06 & 3.19 & 3.25 & 0.08 & 3.36 & 3.44 & 0.04 & 3.17 & 3.21 & 0.01 & 3.08 & 3.09 \\
\hline $\mathrm{Li}$ & 0.10 & 12.23 & 12.33 & 0.23 & \begin{tabular}{|l|}
14.01 \\
\end{tabular} & 14.24 & 0.23 & 15.98 & 16.21 & 0.13 & 17.17 & 17.30 & 0.11 & 17.35 & 17.46 \\
\hline $\mathrm{Mg}$ & 0.13 & 7.78 & 7.91 & 0.31 & 7.63 & 7.94 & 0.33 & \begin{tabular}{|l|}
7.52 \\
\end{tabular} & 7.85 & 0.19 & 7.43 & 7.62 & 0.20 & 7.31 & 7.51 \\
\hline $\mathrm{Mn}$ & 0.19 & 0.03 & 0.22 & 0.28 & 0.04 & 0.32 & 0.28 & 0.04 & 0.32 & 0.14 & 0.06 & 0.20 & 0.14 & 0.06 & 0.20 \\
\hline $\mathrm{Na}$ & 0.10 & 26.94 & 27.04 & 0.19 & 27.93 & 28.12 & 0.23 & 30.98 & 31.21 & 0.14 & 34.19 & 34.33 & 0.15 & 35.72 & 35.87 \\
\hline $\mathrm{Ni}$ & 0.20 & 0.05 & \begin{tabular}{|l|}
0.25 \\
\end{tabular} & 0.47 & \begin{tabular}{|l|}
0.04 \\
\end{tabular} & 0.51 & 0.57 & \begin{tabular}{|l|}
0.08 \\
\end{tabular} & 0.65 & 0.26 & \begin{tabular}{|l|}
0.09 \\
\end{tabular} & 0.35 & 0.25 & \begin{tabular}{|l|}
0.11 \\
\end{tabular} & 0.36 \\
\hline $\bar{P}$ & 0.03 & 0.18 & 0.21 & 0.04 & $<0.07$ & 0.04 & 0.06 & 0.26 & 0.32 & 0.04 & 0.40 & 0.44 & 0.10 & 0.27 & 0.37 \\
\hline $\mathrm{Pb}$ & 0.05 & 0.05 & 0.10 & 0.11 & 0.05 & 0.16 & 0.13 & 0.07 & 0.20 & 0.14 & 0.08 & 0.22 & 0.21 & 0.09 & 0.30 \\
\hline $\mathrm{Sb}$ & 0.07 & 0.13 & 0.20 & 0.11 & 0.14 & 0.25 & 0.13 & 0.12 & 0.25 & 0.05 & 0.14 & 0.19 & 0.05 & 0.12 & 0.17 \\
\hline $\mathrm{Se}$ & 0.21 & |36.58 & 36.79 & 0.50 & 37.87 & 38.37 & 0.54 & 40.06 & 40.60 & 0.37 & 43.24 & \begin{tabular}{|l|}
43.61 \\
\end{tabular} & 0.45 & 47.51 & 47.96 \\
\hline $\mathrm{Si}$ & 5.73 & \begin{tabular}{|l|}
3.54 \\
\end{tabular} & \begin{tabular}{|l|}
9.27 \\
\end{tabular} & 10.87 & \begin{tabular}{|l|}
3.48 \\
\end{tabular} & 14.35 & 10.02 & 3.63 & 13.65 & 5.29 & 4.03 & 9.32 & 4.87 & \begin{tabular}{|l|}
4.29 \\
\end{tabular} & 9.16 \\
\hline $\mathrm{Sr}$ & 0.09 & 3.09 & 3.18 & 0.20 & 3.40 & 3.60 & 0.24 & 4.00 & 4.24 & 0.17 & 4.90 & 5.07 & 0.21 & 5.50 & 5.71 \\
\hline $\mathrm{Te}$ & 0.41 & 0.23 & 0.64 & 1.05 & 0.31 & 1.36 & 3.34 & 0.39 & 3.73 & 1.23 & 0.92 & 2.15 & 2.37 & 1.28 & 3.65 \\
\hline $\mathrm{Ti}$ & 0.03 & $<0.01$ & 0.03 & 0.05 & 0.01 & 0.06 & 0.09 & $<0.01$ & 0.09 & 0.02 & $<0.01$ & 0.02 & 0.03 & 0.01 & 0.04 \\
\hline $\mathrm{Zn}$ & 0.32 & \begin{tabular}{|l|}
0.02 \\
\end{tabular} & 0.34 & 0.81 & 0.02 & 0.83 & 0.96 & \begin{tabular}{|l|}
0.06 \\
\end{tabular} & 1.02 & 0.71 & \begin{tabular}{|l|}
0.06 \\
\end{tabular} & 0.77 & 0.85 & 0.04 & 0.89 \\
\hline $\mathrm{Zr}$ & 0.45 & 0.01 & 0.46 & 0.48 & 0.01 & 0.49 & 0.39 & $<0.01$ & 0.39 & 0.19 & 0.01 & 0.20 & 0.15 & $<0.01$ & 0.15 \\
\hline $\mathrm{Cl}$ & NA & 27.62 & 27.62 & NA & 29.10 & 29.10 & NA & 26.31 & 26.31 & NA & 25.60 & 25.60 & NA & 24.55 & 24.55 \\
\hline$F$ & NA & 7.86 & 7.86 & NA & 9.25 & 9.25 & NA & 9.77 & \begin{tabular}{|l|}
9.77 \\
\end{tabular} & $\mathrm{NA}$ & 11.61 & 11.61 & NA & 13.47 & 13.47 \\
\hline I & $<0.1$ & 1.80 & 1.80 & $<0.1$ & 2.19 & 2.19 & $<0.1$ & 4.88 & 4.88 & $<0.1$ & 16.23 & 16.23 & $<0.1$ & 25.82 & 25.82 \\
\hline Nitrate & NA & 3.82 & 3.82 & NA & 3.67 & 3.67 & NA & 3.77 & 3.77 & NA & 3.80 & 3.80 & NA & \begin{tabular}{|l|}
4.14 \\
\end{tabular} & 4.14 \\
\hline Nitrite & NA & $<0.1$ & $<0.1$ & $\mathrm{NA}$ & 0.87 & 0.87 & NA & 0.92 & 0.92 & NA & 1.51 & 1.51 & $\mathrm{NA}$ & 2.17 & 2.17 \\
\hline Sulfate & $<0.1$ & 13.17 & 13.17 & $<0.1$ & 62.50 & 62.50 & 1.95 & 67.28 & \begin{tabular}{|l|}
69.23 \\
\end{tabular} & $<0.1$ & 87.10 & 87.10 & $<0.1$ & 104.0 & 104.0 \\
\hline
\end{tabular}


Table 4.6. Analytical Results for Samples of SBS Blow-Down Fluids (mg/l), (continued).

\begin{tabular}{|c|c|c|c|c|c|c|c|c|c|c|c|c|c|c|c|}
\hline est & \\
\hline I.D. & \multicolumn{3}{|c|}{$12 \mathrm{G}-\mathrm{S}-135 \mathrm{~A}$} & \multicolumn{3}{|c|}{$12 \mathrm{G}-\mathrm{S}-136 \mathrm{~A}$} & \multicolumn{3}{|c|}{$12 \mathrm{G}-\mathrm{S}-139 \mathrm{~A}$} & \multicolumn{3}{|c|}{$12 \mathrm{G}-\mathrm{S}-148 \mathrm{~A}$} & \multicolumn{3}{|c|}{$12 \mathrm{G}-\mathrm{S}-151 \mathrm{~A}$} \\
\hline Glass (kg) & \multicolumn{3}{|c|}{2650} & \multicolumn{3}{|c|}{2740} & \multicolumn{3}{|c|}{2827} & \multicolumn{3}{|c|}{2930} & \multicolumn{3}{|c|}{3035} \\
\hline $\mathrm{pH}$ & \multicolumn{3}{|c|}{8.46} & \multicolumn{3}{|c|}{8.46} & \multicolumn{3}{|c|}{8.56} & \multicolumn{3}{|c|}{8.60} & \multicolumn{3}{|c|}{8.60} \\
\hline & Sus* & Dis.\# & \begin{tabular}{|l|} 
Total \\
\end{tabular} & Sus* & Dis.\# & Total & Sus* & Dis.\# & Total & Sus* & Dis.\# & Total & Sus* & Dis.\# & Tota \\
\hline Total & 44 & 464 & 508.0 & 96 & 506 & 602.0 & 104 & 496 & 600.0 & 84 & 504 & 588.0 & 130 & 400 & 530.0 \\
\hline $\mathrm{Al}$ & 1.72 & 0.44 & 2.16 & 4.55 & 0.38 & 4.93 & 4.71 & 0.67 & 5.38 & 3.30 & 0.40 & 3.70 & 5.19 & 0.36 & 5.55 \\
\hline As & .08 & 0.28 & 0.36 & 0.12 & 0.33 & 0.45 & 0.20 & 0.41 & 0.61 & 0.18 & 0.46 & 0.64 & 0.34 & 0.60 & 0.94 \\
\hline B & 0.14 & 55.61 & 55.75 & 0.21 & 63.18 & 63.39 & 0.20 & 69.39 & 69.59 & 0.03 & 75.32 & 75.35 & 0.24 & 79.65 & 79.85 \\
\hline $\mathrm{Ba}$ & 0.02 & 0.05 & 0.07 & 0.02 & 0.05 & 0.07 & 0.02 & 0.05 & 0.07 & 0.02 & 0.05 & 0.07 & 0.03 & 0.05 & 0.08 \\
\hline $\mathrm{Ca}$ & 0.28 & 19.30 & 19.58 & 0.44 & 17.52 & 17.96 & 0.43 & 15.17 & 15.60 & 0.38 & 13.32 & 13.70 & 0.45 & 12.34 & 12.79 \\
\hline $\mathrm{Cd}$ & 1.74 & 0.19 & 1.93 & 2.81 & 0.22 & 3.03 & 3.26 & 0.23 & 3.49 & 3.36 & 0.20 & 3.56 & 5.18 & 0.25 & $\overline{5.43}$ \\
\hline $\mathrm{Cr}$ & 0.07 & 0.09 & 0.16 & 0.10 & 0.08 & 0.18 & 0.14 & 0.08 & 0.22 & 0.11 & 0.07 & 0.18 & 0.16 & 0.07 & 0.2 \\
\hline Cs & $<0.1$ & 0.80 & 0.80 & $<0.1$ & 0.90 & 0.90 & $<0.1$ & 1.30 & 1.30 & $<0.1$ & 1.20 & 1.20 & 0.10 & 1.30 & 1.4 \\
\hline $\mathrm{Cu}$ & 0.01 & 0.04 & 0.05 & 0.01 & 0.05 & 0.06 & 0.02 & 0.06 & 0.08 & 0.02 & 0.05 & 0.07 & 0.03 & 0.06 & 0.09 \\
\hline $\mathrm{Fe}$ & 7.21 & 0.01 & 7.22 & 14.05 & 0.01 & 14.06 & 16.01 & 0.01 & 16.02 & 11.64 & $<0.01$ & 11.64 & 20.13 & $<0.01$ & 20.13 \\
\hline $\bar{K}$ & 0.03 & 3.65 & 3.68 & 0.10 & 3.96 & 4.06 & 0.08 & 3.04 & 3.12 & 0.05 & 2.98 & 3.03 & \begin{tabular}{|l|}
0.09 \\
\end{tabular} & 2.84 & 2.93 \\
\hline$\overline{\mathrm{Li}}$ & 0.12 & 17.24 & 17.36 & 0.25 & 17.30 & 17.55 & 0.26 & 16.65 & 16.91 & 0.20 & 16.67 & 16.87 & 0.28 & 16.16 & 16.44 \\
\hline $\mathrm{Mg}$ & 0.26 & 7.08 & 7.34 & 0.52 & 7.05 & 7.57 & 0.62 & 6.72 & 7.34 & 0.58 & 6.57 & 7.15 & 0.83 & \begin{tabular}{|c|}
6.28 \\
\end{tabular} & 7.11 \\
\hline $\mathrm{Mn}$ & 0.15 & 0.06 & 0.21 & 0.31 & 0.06 & 0.37 & 0.33 & 0.06 & 0.39 & 0.26 & 0.05 & 0.31 & 0.37 & 0.06 & 0.43 \\
\hline $\mathrm{Na}$ & 0.19 & 37.26 & 37.45 & 0.41 & 39.12 & 39.53 & 0.48 & 50.62 & 51.10 & 0.39 & 42.03 & 42.42 & 0.61 & 42.11 & 42.72 \\
\hline $\mathrm{Ni}$ & 0.30 & 0.13 & 0.43 & 0.56 & 0.14 & 0.70 & 0.63 & 0.14 & 0.77 & 0.51 & 0.16 & 0.67 & 0.71 & \begin{tabular}{|l|}
0.19 \\
\end{tabular} & 0.90 \\
\hline $\mathrm{P}$ & 0.04 & 0.27 & 0.31 & 0.03 & 0.13 & 0.16 & 0.03 & 0.41 & 0.44 & 0.06 & 0.10 & 0.16 & 0.03 & 0.40 & 0.43 \\
\hline $\mathrm{Pb}$ & 0.30 & 0.09 & 0.39 & 0.13 & 0.11 & 0.24 & 0.61 & 0.13 & 0.74 & 0.51 & 0.12 & 0.63 & 0.88 & 0.13 & 1.01 \\
\hline $\mathrm{Sb}$ & 0.08 & $\begin{array}{ll}0.08 \\
\end{array}$ & 0.16 & 0.14 & 0.12 & 0.26 & 0.18 & 0.10 & 0.28 & 0.12 & 0.13 & 0.25 & 0.19 & 0.13 & 0.32 \\
\hline $\mathrm{Se}$ & 0.57 & 51.87 & 52.44 & 0.83 & 57.40 & 58.23 & 1.25 & 62.76 & 64.01 & 1.05 & 64.41 & 65.46 & \begin{tabular}{|l|}
1.79 \\
\end{tabular} & 72.72 & 74.51 \\
\hline $\mathrm{Si}$ & 4.92 & 4.45 & 9.37 & 13.29 & 4.50 & 17.79 & 13.61 & 4.53 & 18.14 & 9.73 & 4.72 & 14.45 & 14.47 & 4.68 & 19.15 \\
\hline $\mathrm{Sr}$ & 0.27 & 5.91 & 6.18 & 0.59 & 6.58 & 7.17 & 0.71 & 6.88 & 7.59 & 0.70 & 7.00 & 7.70 & \begin{tabular}{|l|}
0.97 \\
\end{tabular} & 7.17 & 8.14 \\
\hline $\mathrm{Te}$ & 3.81 & 1.52 & 5.33 & 5.01 & 1.83 & 6.84 & 8.33 & 1.95 & 10.28 & 8.40 & 2.07 & 10.47 & 13.11 & 2.36 & 15.47 \\
\hline $\mathrm{Ti}$ & 0.02 & 0.01 & 0.03 & 0.04 & 0.01 & 0.05 & 0.05 & $<0.01$ & 0.05 & 0.04 & $<0.01$ & 0.04 & 0.05 & 0.01 & 0.06 \\
\hline $\mathrm{Zn}$ & 1.03 & 0.03 & 1.06 & 1.90 & 0.06 & 1.96 & 2.18 & 0.11 & 2.29 & 1.90 & 0.10 & 2.00 & 2.70 & 0.13 & 2.8 \\
\hline $\mathrm{Zr}$ & 0.16 & 0.01 & 0.17 & 0.54 & $<0.01$ & 0.54 & 0.56 & $<0.01$ & 0.56 & 0.34 & 0.01 & 0.35 & 0.63 & 0.01 & 0.6 \\
\hline $\mathrm{Cl}$ & NA & 25.46 & 25.46 & NA & 26.20 & 26.20 & NA & 23.84 & 23.84 & NA & 24.02 & 24.02 & $\mathrm{NA}$ & 23.76 & 23.7 \\
\hline$F$ & NA & 14.48 & 14.48 & NA & 16.47 & 16.47 & NA & 18.07 & 18.07 & NA & 19.05 & 19.05 & NA & 20.42 & 20.42 \\
\hline I & $<0.1$ & 37.73 & 37.73 & $<0.1$ & 53.91 & 53.91 & $<0.1$ & 72.13 & 72.13 & $<0.1$ & 99.18 & 99.18 & $<0.1$ & \begin{tabular}{|l|}
117.5 \\
\end{tabular} & 117.5 \\
\hline & NA & 4.90 & 4.90 & NA & 5.22 & 5.22 & NA & 15.97 & 15.97 & NA & 7.06 & 7.06 & $\mathrm{NA}$ & \begin{tabular}{|l|l|}
6.10 \\
\end{tabular} & 6.10 \\
\hline & NA & 2.78 & 2.78 & $\mathrm{NA}$ & 4.13 & 4.13 & NA & 9.50 & 9.50 & $\mathrm{NA}$ & 8.09 & 8.09 & NA & 9.37 & 9.37 \\
\hline ulfate & $<0.1$ & 123.9 & 123.9 & 0.01 & 170.3 & 170.3 & $<0.1$ & 180.7 & 180.7 & $<0.1$ & 194.6 & 194.6 & 0.07 & 226.8 & 226.9 \\
\hline
\end{tabular}

* Suspended

\# Dissolved

NA - Not Analyzed. 
Table 4.6. Analytical Results for Samples of SBS Blow-Down Fluids (mg/l), (continued).

\begin{tabular}{|c|c|c|c|c|c|c|c|c|c|c|c|c|c|c|c|}
\hline Test & \multicolumn{15}{|c|}{2} \\
\hline I.D. & & H-S-11 & & & $\mathrm{H}-\mathrm{S}-1 \mathrm{C}$ & & & H-S-24 & & & $2 \mathrm{H}-\mathrm{S}-33$ & & & $\mathrm{H}-\mathrm{S}$ & \\
\hline Glass $(\mathrm{kg})$ & & 3120 & & & 3210 & & & 3298 & & & 3360 & & & 3420 & \\
\hline $\mathrm{pH}$ & & 8.51 & & & 8.57 & & & 8.54 & & & 8.55 & & & 8.56 & \\
\hline & Sus* & Dis.\# & Total & Sus* & Dis.\# & Total & Sus* & Dis.\# & Total & Sus* & Dis.A & Total & Sus* & Dis.\# & Tota \\
\hline Total & 122 & 436 & 558 & 130 & 420 & 550 & 136 & 428 & 564 & 122 & 380 & 502 & 128 & 436 & 564 \\
\hline $\mathrm{Al}$ & 4.57 & 0.35 & 4.92 & 4.19 & 0.36 & 4.55 & 4.21 & 0.36 & 4.57 & 3.62 & 0.33 & 3.95 & 3.87 & 0.30 & 4.17 \\
\hline As & 0.31 & 0.64 & 0.95 & 0.57 & 0.72 & 1.29 & 0.62 & 0.84 & 1.46 & 0.68 & 0.93 & 1.61 & 0.59 & 0.88 & 1.47 \\
\hline B & 0.22 & 84.35 & 84.57 & 0.15 & 87.17 & 87.32 & 0.21 & 90.93 & 91.14 & 0.11 & 93.80 & 93.91 & 0.16 & 97.16 & 97.32 \\
\hline $\mathrm{Ba}$ & 0.02 & 0.05 & 0.07 & 0.03 & 0.05 & 0.08 & 0.02 & 0.05 & 0.07 & 0.03 & 0.05 & 0.08 & 0.03 & 0.05 & 0.08 \\
\hline $\mathrm{Ca}$ & 0.38 & 11.29 & 11.70 & 0.46 & 10.68 & 11.10 & 0.36 & 9.49 & 9.90 & 0.40 & 9.22 & 9.60 & 0.39 & 8.47 & 8.90 \\
\hline $\mathrm{Cd}$ & 5.98 & 0.25 & 6.23 & 8.84 & 0.26 & 9.10 & 9.09 & 0.30 & 9.39 & 8.58 & 0.24 & 8.82 & 8.11 & 0.24 & 8.35 \\
\hline $\mathrm{Cr}$ & 0.14 & 0.06 & 0.20 & 0.16 & 0.05 & 0.21 & 0.15 & 0.06 & 0.21 & 0.14 & 0.05 & 0.19 & 0.15 & 0.05 & 0.20 \\
\hline $\mathrm{Cs}$ & 0.12 & 1.30 & 1.42 & 0.13 & 1.30 & 1.43 & 0.15 & 1.40 & 1.55 & 0.13 & 1.40 & 1.53 & 0.15 & 1.60 & 1.75 \\
\hline $\mathrm{Cu}$ & 0.01 & 0.06 & 0.07 & 0.02 & 0.07 & 0.09 & 0.02 & 0.07 & 0.09 & 0.02 & 0.08 & 0.10 & 0.02 & 0.08 & 0.10 \\
\hline $\mathrm{Fe}$ & 17.16 & $<0.01$ & 17.16 & 17.52 & $<0.01$ & 17.52 & 17.20 & 0.01 & 17.21 & 15.18 & 0.01 & 15.19 & 16.13 & 0.01 & 16.14 \\
\hline $\mathrm{K}$ & 0.10 & 2.79 & 2.89 & 0.07 & 2.75 & 2.82 & 0.13 & 2.63 & 2.76 & 0.09 & 2.61 & 2.70 & 0.08 & 2.64 & 2.72 \\
\hline $\mathrm{Li}$ & 0.25 & 15.58 & 15.83 & 0.27 & 15.66 & 15.93 & 0.26 & 14.96 & 15.22 & 0.21 & 14.91 & 15.12 & 0.20 & 14.93 & 15.13 \\
\hline $\mathrm{Mg}$ & 0.70 & 6.04 & 6.74 & 0.85 & \begin{tabular}{|l|}
5.72 \\
\end{tabular} & 6.57 & 0.76 & \begin{tabular}{|l|}
5.57 \\
\end{tabular} & 6.33 & 0.77 & 5.33 & 6.10 & 0.88 & 5.23 & 6.11 \\
\hline Mn & 0.31 & 0.07 & 0.38 & 0.34 & 0.06 & 0.40 & 0.30 & 0.05 & 0.35 & 0.29 & 0.04 & 0.33 & 0.32 & 0.06 & 0.38 \\
\hline $\mathrm{Na}$ & 0.61 & 42.64 & 43.25 & 0.71 & 42.62 & 43.33 & 0.78 & 42.59 & 43.37 & 0.68 & 43.35 & 44.03 & 0.67 & 45.35 & 46.02 \\
\hline $\mathrm{Ni}$ & 0.61 & 0.21 & 0.82 & 0.69 & \begin{tabular}{|l|}
0.24 \\
\end{tabular} & 0.93 & 0.58 & 0.24 & 0.82 & 0.58 & \begin{tabular}{|l|l|} 
& 0.24 \\
\end{tabular} & \begin{tabular}{|l|}
0.82 \\
\end{tabular} & 0.62 & \begin{tabular}{|l|}
0.25 \\
\end{tabular} & 0.87 \\
\hline $\mathrm{P}$ & 0.05 & 0.28 & 0.33 & 0.06 & 0.35 & 0.41 & 0.15 & 0.31 & 0.46 & 0.06 & 0.33 & 0.39 & 0.12 & 0.21 & 0.33 \\
\hline $\mathrm{Pb}$ & 0.31 & 0.14 & 0.45 & 1.13 & 0.14 & 1.27 & 1.29 & 0.15 & 1.44 & 1.16 & 0.15 & 1.31 & 1.11 & 0.16 & 1.27 \\
\hline $\mathrm{Sb}$ & 0.18 & 0.13 & 0.31 & 0.18 & 0.14 & 0.32 & 0.24 & 0.14 & 0.38 & 0.18 & 0.21 & 0.39 & 0.17 & \begin{tabular}{|l|}
0.21 \\
\end{tabular} & 0.38 \\
\hline $\mathrm{Se}$ & 1.01 & 79.16 & 80.17 & 1.93 & 84.96 & 86.89 & 1.88 & 91.26 & 93.14 & 1.74 & 99.19 & 100.9 & 1.72 & 103.3 & 105.0 \\
\hline $\mathrm{Si}$ & 3.44 & 4.65 & 18.09 & 13.25 & \begin{tabular}{|l|}
4.62 \\
\end{tabular} & 17.87 & 12.52 & 4.50 & 17.02 & 11.52 & 4.35 & 15.87 & 12.43 & \begin{tabular}{|l|}
4.38 \\
\end{tabular} & 16.81 \\
\hline $\mathrm{Sr}$ & 0.86 & 7.18 & 8.04 & 1.12 & 6.99 & 8.11 & 0.93 & 7.03 & 7.96 & 0.99 & 6.98 & \begin{tabular}{|l|}
7.97 \\
\end{tabular} & 1.13 & 7.10 & 8.23 \\
\hline $\mathrm{Te}$ & 1.44 & 2.65 & 14.09 & 19.67 & 3.08 & 22.75 & 22.37 & 3.61 & 25.98 & 22.22 & 3.93 & 26.15 & 22.63 & 3.79 & 26.42 \\
\hline $\mathrm{T}$ & 0.05 & $<0.1$ & 0.05 & 0.05 & $<0.1$ & 0.05 & 0.05 & 0.01 & 0.06 & 0.05 & 0.01 & 0.06 & 0.05 & 0.01 & 0.06 \\
\hline $\mathrm{Zn}$ & 46 & 0.14 & 2.60 & 2.74 & \begin{tabular}{|l|}
0.13 \\
\end{tabular} & 2.87 & 2.55 & 0.14 & 2.69 & 2.32 & 0.11 & 2.43 & 2.49 & 0.14 & 2.6 \\
\hline $\mathrm{Zr}$ & 0.51 & 0.01 & 0.52 & 0.41 & $<0.01$ & 0.41 & 0.44 & 0.01 & 0.45 & 0.39 & $<0.01$ & 0.39 & 0.45 & \begin{tabular}{|l|}
0.01 \\
\end{tabular} & $\overline{0.46}$ \\
\hline $\mathrm{Cl}$ & $\mathrm{NA}$ & 25.19 & 25.19 & NA & 24.53 & 24.53 & NA & 25.39 & 25.39 & NA & 26.38 & 26.38 & $\mathrm{NA}$ & 26.13 & 26.13 \\
\hline $\bar{F}$ & NA & 23.18 & 23.18 & NA & 23.67 & 23.67 & NA & 25.29 & 25.29 & NA & 27.49 & 27.49 & NA & 28.13 & 28.13 \\
\hline I & $<0.1$ & 137.7 & 137.7 & $<0.1$ & 147.1 & 147.1 & $<0.1$ & 164.1 & 164.1 & $<0.1$ & \begin{tabular}{|l|}
189.7 \\
\end{tabular} & 189.7 & $<0.1$ & 204.0 & 204.0 \\
\hline & NA & 6.14 & 6.14 & NA & 5.98 & 5.98 & NA & 6.31 & 6.31 & NA & 6.75 & 6.75 & $\mathrm{NA}$ & 7.31 & 7.31 \\
\hline itrite & NA & 9.82 & 9.82 & NA & 9.89 & 9.89 & $\mathrm{NA}$ & 10.29 & 10.29 & $\mathrm{NA}$ & 10.34 & 10.34 & NA & 11.80 & 11.80 \\
\hline Sulfate & 0.27 & 241.8 & 242.1 & $<0.1$ & 266.4 & 266.4 & 0.47 & 311.7 & 312.2 & $<0.1$ & \begin{tabular}{|l|}
558.3 \\
\end{tabular} & 358.3 & $<0.1$ & 365.6 & 365.6 \\
\hline
\end{tabular}

* Suspended

\# Dissolved

NA - Not Analyzed. 
Table 4.6. Analytical Results for Samples of SBS Blow-Down Fluids (mg/l), (continued).

\begin{tabular}{|c|c|c|c|c|c|c|c|c|c|c|c|c|c|c|}
\hline Test & \multicolumn{14}{|c|}{2} \\
\hline I.D. & & H-S-43 & & & 2I-S-13 & & & 2I-S-17. & & & II-S-26 & & $12 \mathrm{I}-\mathrm{S}-35$ & \\
\hline Glass (kg) & & 3452 & & & 3540 & & & 3699 & & & 3840 & & 3981 & \\
\hline $\mathrm{pH}$ & & 8.52 & & & 8.47 & & & 8.50 & & & 8.53 & & 8.50 & \\
\hline & Sus* & Dis.\# & Total & Sus* & Dis.\# & 冓 Total & Sus* & Dis.\# & Total & Sus* & Dis.\# & Total & Sus* Dis.\# & Total \\
\hline Total & 130 & 468 & 598 & 118 & 480 & 598 & 106 & 472 & 578 & 101 & 554 & 655 & 260 & 340 \\
\hline $\mathrm{Al}$ & 3.74 & 0.30 & 4.04 & 3.14 & 0.26 & 3.40 & 2.68 & 0.28 & 2.96 & 2.27 & 0.32 & 2.59 & 0.26 & 2.31 \\
\hline As & 0.63 & 0.86 & 1.49 & 0.63 & 0.89 & 1.52 & 0.55 & 0.78 & 1.33 & 0.58 & 0.75 & 1.33 & 0.73 & 1.15 \\
\hline B & 0.15 & $<0.03$ & 0.15 & 0.10 & 102.1 & 102.2 & 0.14 & 102.7 & 102.8 & 0.21 & 103.8 & 104.0 & \begin{tabular}{|l|l|}
0.10 & 104.1 \\
\end{tabular} & 104.2 \\
\hline $\mathrm{Ba}$ & 0.03 & 0.05 & 0.08 & 0.02 & 0.07 & $\begin{array}{ll}7 & 0.09 \\
\end{array}$ & 0.02 & 0.07 & \begin{tabular}{|l|}
0.09 \\
\end{tabular} & 0.02 & 0.07 & 0.09 & \begin{tabular}{|l|l|}
0.02 & 0.07 \\
\end{tabular} & 0.09 \\
\hline $\mathrm{Ca}$ & 0.39 & $<0.01$ & 0.40 & 0.32 & 6.88 & 7.20 & 0.22 & 6.42 & 6.60 & 0.24 & 5.84 & 6.10 & 0.18 & 5.60 \\
\hline $\mathrm{Cd}$ & $\overline{8.82}$ & 0.25 & 9.07 & 9.75 & 0.22 & 9.97 & 8.86 & 0.26 & 9.12 & 9.92 & 0.27 & 10.19 & 6.67 & 6.94 \\
\hline $\mathrm{Cr}$ & 0.15 & 0.05 & 0.20 & 0.14 & 0.04 & 0.18 & 0.12 & 0.05 & 0.17 & 0.12 & 0.05 & 0.17 & 0.05 & 0.14 \\
\hline $\mathrm{Cs}$ & 0.15 & 1.70 & 1.85 & 0.15 & 1.50 & 1.65 & 0.13 & 1.30 & 1.43 & 0.14 & 1.30 & 1.44 & 1.40 & 1.51 \\
\hline $\mathrm{Cu}$ & 0.02 & 0.08 & 0.10 & 0.02 & 0.07 & 0.09 & 0.02 & 0.08 & \begin{tabular}{|l|}
0.10 \\
\end{tabular} & 0.02 & 0.06 & 0.08 & 0.05 & 0.07 \\
\hline $\mathrm{Fe}$ & 16.02 & $<0.01$ & 16.02 & 13.92 & 0.03 & 13.95 & 11.89 & 0.03 & 11.92 & 11.16 & 0.03 & 11.19 & 9.30 & 9.33 \\
\hline $\mathrm{K}$ & 0.08 & 2.69 & 2.77 & 0.08 & 2.43 & 2.51 & 0.12 & 2.31 & 2.43 & 0.07 & 2.27 & 2.34 & \begin{tabular}{|l|l|}
0.05 & 2.34 \\
\end{tabular} & 2.39 \\
\hline $\mathrm{Li}$ & 0.20 & $<0.02$ & 0.20 & 0.18 & 14.41 & 14.59 & 0.15 & 13.94 & 14.09 & 0.12 & 13.12 & 13.24 & \begin{tabular}{lll|}
0.10 & 13.52 \\
\end{tabular} & 13.62 \\
\hline $\mathrm{Mg}$ & 0.88 & $<0.01$ & 0.88 & 0.76 & 4.61 & 5.37 & 0.59 & 4.44 & \begin{tabular}{|l|}
5.03 \\
\end{tabular} & 0.61 & 4.21 & 4.82 & \begin{tabular}{|l|l|}
0.53 & 4.23 \\
\end{tabular} & 4.76 \\
\hline $\mathrm{Mn}$ & 0.30 & 0.05 & 0.35 & 0.25 & 0.05 & 0.30 & 0.19 & 0.04 & 0.23 & 0.19 & 0.05 & 0.24 & 0.04 & 0.21 \\
\hline $\mathrm{Na}$ & 0.68 & $<0.01$ & 0.68 & 0.63 & 43.94 & 44.57 & 0.55 & 42.04 & 42.59 & 0.53 & 41.35 & 41.88 & \begin{tabular}{|l|l|}
0.39 & 43.78 \\
\end{tabular} & 44.17 \\
\hline $\mathrm{Ni}$ & 0.60 & 0.25 & 0.85 & 0.52 & 0.19 & 0.71 & 0.41 & 0.18 & 0.59 & 0.43 & 0.15 & 0.58 & \begin{tabular}{|l|l|}
0.36 & 0.13 \\
\end{tabular} & 0.49 \\
\hline$P$ & 0.10 & 0.46 & 0.56 & 0.13 & 0.38 & 0.51 & 0.03 & 0.42 & 0.45 & 0.12 & 0.36 & 0.48 & 0.08 & 0.49 \\
\hline $\mathrm{Pb}$ & 1.16 & 0.17 & 1.33 & 1.13 & 0.18 & 1.31 & 1.20 & 0.18 & 1.38 & 1.31 & 0.18 & 1.49 & 0.92 & 1.1 \\
\hline $\mathrm{Sb}$ & 0.19 & 0.16 & \begin{tabular}{|l|} 
\\
\end{tabular} & 0.18 & 0.09 & 0.27 & 0.16 & 0.08 & 0.24 & 0.15 & 0.08 & 0.23 & 0.12 & 0.19 \\
\hline $\mathrm{Se}$ & 1.67 & 104.9 & 106.6 & 1.61 & 122.4 & 124.0 & 1.56 & 124.0 & 125.6 & 1.60 & 130.1 & 131.7 & \begin{tabular}{l|l|}
1.24 & 135.0 \\
\end{tabular} & 136.2 \\
\hline $\mathrm{Si}$ & 11.96 & 4.35 & 16.31 & 10.07 & 4.44 & 14.51 & 8.34 & \begin{tabular}{|l|}
4.35 \\
\end{tabular} & 12.69 & 7.39 & 4.36 & 11.75 & \begin{tabular}{l|l|}
6.40 & 4.41 \\
\end{tabular} & 10.8 \\
\hline $\mathrm{Sr}$ & 1.10 & $<0.01$ & 1.10 & 0.98 & 6.68 & 7.66 & 0.66 & 6.51 & 7.17 & 0.70 & 6.41 & 7.11 & 6.66 & 7.29 \\
\hline $\mathrm{Te}$ & 25.73 & 3.74 & 29.47 & 22.61 & 3.72 & 26.33 & 21.99 & 3.90 & 25.89 & 22.23 & 3.96 & 26.19 & 16.52 & 20.22 \\
\hline $\mathrm{Ti}$ & 0.05 & 0.01 & 0.06 & 0.05 & $<0.01$ & 0.05 & 0.04 & $<0.01$ & 0.04 & 0.04 & $<0.01$ & 0.04 & 0.03 & 0. \\
\hline $\mathrm{Zn}$ & 2.47 & 0.15 & 2.62 & 2.39 & 0.14 & 2.53 & 1.82 & 0.14 & 1.96 & 1.80 & 0.15 & 1.95 & 1.45 & 1. \\
\hline $\mathrm{Zr}$ & 0.40 & 0.01 & 0.41 & 0.31 & $<0.01$ & 0.31 & 0.25 & 0.01 & 0.26 & 0.24 & 0.01 & 0.25 & 0.22 & 0.23 \\
\hline $\mathrm{Cl}$ & $\mathrm{NA}$ & 26.87 & 26.87 & $\mathrm{NA}$ & 26.26 & 26.26 & NA & 25.03 & 25.03 & NA & NA & NA & \begin{tabular}{l|l|} 
NA 25.03 \\
\end{tabular} & 25.03 \\
\hline $\mathrm{F}$ & $\mathrm{NA}$ & 28.76 & 28.76 & $\mathrm{NA}$ & 28.78 & 28.78 & NA & 28.32 & 28.32 & NA & $\mathrm{NA}$ & NA & \begin{tabular}{l|l|} 
NA & 27.70 \\
\end{tabular} & 27.70 \\
\hline I & $<0.1$ & 213.9 & 213.9 & $<0.1$ & 219.5 & 219.5 & $<0.1$ & 228.2 & 228.2 & $<0.1$ & $\mathrm{NA}$ & $\mathrm{NC}$ & \begin{tabular}{l|l|}
$<0.1$ & 248.4 \\
\end{tabular} & 248.4 \\
\hline Nitrate & $\mathrm{NA}$ & 7.64 & 7.64 & $\mathrm{NA}$ & 7.51 & 7.51 & NA & 8.01 & 8.01 & NA & $\mathrm{NA}$ & $\mathrm{NA}$ & \begin{tabular}{l|l|} 
& NA \\
\end{tabular} & 8.01 \\
\hline Nitrite & $\mathrm{NA}$ & 12.08 & 12.08 & $\mathrm{NA}$ & 11.64 & 11.64 & NA & 12.80 & 12.80 & NA & $\mathrm{NA}$ & $\mathrm{NA}$ & \begin{tabular}{l|l|l|} 
NA & 14.07 \\
\end{tabular} & 14.07 \\
\hline Sulfate & $<0.1$ & 363.1 & 363.1 & 0.14 & 387.3 & \begin{tabular}{|l|}
387.4 \\
\end{tabular} & 0.42 & 351.5 & 351.9 & 0.27 & $\mathrm{NA}$ & $\mathrm{NQ}$ & \begin{tabular}{l|l|l|}
0.1979 .0 \\
\end{tabular} & 379.2 \\
\hline
\end{tabular}


Table 4.6. Analytical Results for Samples of SBS Blow-Down Fluids (mg/l), (continued).

\begin{tabular}{|c|c|c|c|c|c|c|c|c|c|c|c|c|c|c|}
\hline Test & \multicolumn{14}{|c|}{2} \\
\hline I.D. & & I-S-43 & & & II-S-50 & & & II-S-58 & & & $2 \mathrm{I}-\mathrm{S}-61$ & & 12I-S-66 & \\
\hline Glass (kg) & & 4122 & & & 4250 & & & 4379 & & & 4507 & & 4635 & \\
\hline $\mathrm{pH}$ & & 8.49 & & & & & & 8.40 & & & 8.51 & & 8.49 & \\
\hline & Sus* & Dis.\# & \begin{tabular}{|l|} 
Total \\
\end{tabular} & Sus* & Dis.\# & Total & Sus* & Dis.\# & Total & Sus* & Dis..\# & Total & Sus* Dis.\# & Tota \\
\hline Total & 78 & 308 & 386 & 90 & 196 & 286 & 350 & 532 & 882 & 230 & 600 & 830 & 254 & 822 \\
\hline $\mathrm{Al}$ & 2.07 & 0.22 & 2.29 & 2.23 & 0.21 & 2.44 & 14.41 & 0.19 & 14.6 & 8.10 & 0.27 & 8.37 & 8.67 & 8.91 \\
\hline As & 0.39 & 0.68 & 1.07 & 0.48 & 0.74 & 1.22 & 0.91 & 0.72 & 1.63 & 0.75 & 0.79 & 1.54 & 0.86 & 1.64 \\
\hline $\mathrm{B}$ & 0.09 & 105.8 & 105.9 & 0.10 & 108.5 & 108.6 & 0.63 & \begin{tabular}{|l|}
109.3 \\
\end{tabular} & 109.9 & 0.27 & 111.3 & 111.6 & \begin{tabular}{|l|l}
0.33 & 111.4 \\
\end{tabular} & 111.7 \\
\hline $\mathrm{Ba}$ & 0.01 & \begin{tabular}{|l|}
0.07 \\
\end{tabular} & 0.08 & 0.02 & 0.07 & 0.09 & 0.08 & 0.07 & \begin{tabular}{|l|}
0.15 \\
\end{tabular} & 0.05 & \begin{tabular}{|l|}
0.07 \\
\end{tabular} & 0.12 & \begin{tabular}{|l|l|}
0.05 & 0.07 \\
\end{tabular} & 0.12 \\
\hline $\mathrm{Ca}$ & 0.14 & 5.11 & 5.25 & 0.20 & 4.70 & 4.90 & 0.82 & 4.80 & 5.62 & 0.53 & 4.77 & 5.30 & 4.50 & 5.10 \\
\hline $\mathrm{Cd}$ & 6.07 & 0.27 & 6.34 & 7.37 & 0.27 & 7.64 & 14.73 & 0.33 & 15.06 & 12.55 & 0.32 & 12.87 & 13.98 & 14.28 \\
\hline $\mathrm{Cr}$ & 0.08 & 0.05 & 0.13 & 0.11 & 0.05 & 0.16 & 0.37 & 0.05 & 0.42 & 0.27 & 0.05 & \begin{tabular}{|l|}
0.32 \\
\end{tabular} & 0.29 & 0.34 \\
\hline $\mathrm{Cs}$ & $<0.1$ & 1.40 & 1.40 & 0.10 & 1.40 & 1.50 & 0.24 & 1.70 & 1.94 & 0.17 & 1.60 & 1.77 & 0.16 & 1.76 \\
\hline $\mathrm{Cu}$ & 0.02 & 0.06 & 0.08 & 0.02 & 0.06 & 0.08 & 0.06 & 0.07 & 0.13 & 0.06 & 0.06 & 0.12 & 0.06 & 0.14 \\
\hline $\mathrm{Fe}$ & 9.44 & 0.03 & 9.47 & 10.77 & 0.03 & 10.8 & 43.71 & 0.03 & 43.74 & 32.05 & 0.04 & 32.09 & 34.57 & 34.61 \\
\hline $\mathrm{K}$ & 0.07 & 2.43 & 2.50 & 0.07 & 2.33 & 2.40 & 0.21 & 2.33 & 2.54 & 0.13 & 2.44 & \begin{tabular}{|l|}
2.57 \\
\end{tabular} & 0.16 & 2.69 \\
\hline$\overline{\mathrm{Li}}$ & 0.09 & 14.44 & 14.53 & 0.11 & 14.15 & 14.26 & 0.47 & 13.56 & 14.03 & 0.32 & 14.54 & 14.86 & \begin{tabular}{l|l}
0.30 & 14.47 \\
\end{tabular} & 14.77 \\
\hline $\mathrm{Mg}$ & 0.49 & 4.35 & 4.84 & 0.64 & 4.28 & 4.92 & 2.26 & \begin{tabular}{|l|}
4.23 \\
\end{tabular} & 6.49 & 1.66 & 4.38 & 6.04 & \begin{tabular}{l|l|} 
NA & 4.39 \\
\end{tabular} & 4.39 \\
\hline $\mathrm{Mn}$ & 0.15 & 0.05 & 0.20 & 0.18 & 0.05 & \begin{tabular}{|l|}
0.23 \\
\end{tabular} & 1.58 & 0.05 & 1.63 & 0.70 & 0.04 & 0.74 & 0.72 & 0.76 \\
\hline $\mathrm{Na}$ & 0.35 & 47.45 & 47.8 & 0.46 & 47.78 & 48.24 & 1.30 & 46.99 & 48.29 & 0.91 & 49.90 & 50.81 & \begin{tabular}{ll|l}
0.96 & 51.93 \\
\end{tabular} & 52.89 \\
\hline $\mathrm{Ni}$ & 0.33 & 0.14 & 0.47 & 0.42 & 0.16 & 0.58 & 2.03 & 0.19 & 2.22 & 1.35 & 0.14 & 1.49 & 1.46 & 1.59 \\
\hline$P$ & 0.02 & 0.53 & 0.55 & 0.05 & 0.46 & 0.51 & 0.35 & 0.44 & 0.79 & 0.15 & 0.48 & 0.63 & 0.28 & 0.79 \\
\hline $\mathrm{Pb}$ & 0.81 & 0.20 & 1.01 & 0.95 & 0.19 & 1.14 & 1.85 & 0.20 & 2.05 & 1.57 & 0.20 & 1.77 & 1.74 & 1.95 \\
\hline $\mathrm{Sb}$ & 0.11 & 0.07 & 0.18 & 0.11 & 0.10 & 0.21 & 0.48 & 0.08 & 0.56 & 0.27 & 0.13 & 0.4 & 0.26 & 0.39 \\
\hline $\mathrm{Se}$ & 1.12 & \begin{tabular}{|l|}
136.7 \\
\end{tabular} & 137.8 & 1.42 & \begin{tabular}{|l|}
144.3 \\
\end{tabular} & 145.7 & 4.14 & 151.6 & 155.7 & 3.09 & 161.2 & 164.3 & \begin{tabular}{|l|l|l}
3.60 & 169.5 \\
\end{tabular} & 173.1 \\
\hline $\mathrm{Si}$ & 5.51 & 4.57 & 10.08 & 5.16 & 4.49 & 9.65 & 49.17 & 4.37 & 53.54 & 27.09 & 4.60 & 31.69 & 28.32 & 32.91 \\
\hline $\mathrm{Sr}$ & 0.58 & 6.97 & 7.55 & 0.83 & 6.87 & 7.70 & 3.13 & 6.94 & 10.07 & 1.99 & 7.73 & \begin{tabular}{|l|}
9.72 \\
\end{tabular} & 2.28 & 10.21 \\
\hline $\mathrm{Te}$ & 15.03 & 3.37 & 18.4 & 17.76 & 3.61 & 21.37 & 35.93 & 2.49 & 38.42 & 29.57 & 2.80 & 32.37 & 33.76 & 36.68 \\
\hline $\mathrm{Ti}$ & 0.03 & 0.01 & 0.04 & 0.03 & $<0.01$ & 0.03 & \begin{tabular}{l|l}
0.15 \\
\end{tabular} & 0.01 & 0.16 & 0.09 & 0.01 & 0.10 & $0.10<0.01$ & 0.10 \\
\hline $\mathrm{Zn}$ & 1.48 & 0.27 & 1.75 & 1.71 & 0.26 & 1.97 & 6.78 & 0.33 & 7.11 & 4.57 & 0.27 & 4.84 & \begin{tabular}{|l|l}
4.81 & 0.27 \\
\end{tabular} & 5.08 \\
\hline $\mathrm{Zr}$ & 0.21 & 0.01 & 0.22 & 0.24 & 0.01 & 0.25 & 2.96 & 0.01 & 2.97 & 1.12 & 0.01 & 1.13 & 1.21 & 1.22 \\
\hline $\mathrm{Cl}$ & $\mathrm{NA}$ & 25.28 & 25.28 & NA & 24.79 & 24.79 & NA & 24.30 & 24.3 & NA & 21.95 & 21.95 & \begin{tabular}{l|l|} 
NA & 24.44 \\
\end{tabular} & 24.44 \\
\hline $\mathrm{F}$ & $\mathrm{NA}$ & 27.70 & 27.7 & NA & 28.01 & 28.01 & $\mathrm{NA}$ & 28.47 & 28.47 & NA & 26.57 & 26.57 & \begin{tabular}{ll|} 
NA & 29.99 \\
\end{tabular} & 29.99 \\
\hline I & $<0.1$ & \begin{tabular}{|l|}
274.3 \\
\end{tabular} & 274.3 & $<0.1$ & 303.2 & 303.2 & $<0.1$ & 306.1 & 306.1 & $<0.1$ & 272.7 & 272,7 & \begin{tabular}{|c|c|c|}
$<0.126 .1$ \\
\end{tabular} & 326.1 \\
\hline Nitrate & NA & 8.39 & 8.39 & NA & 8.64 & 8.64 & NA & 8.41 & 8.41 & NA & 8.80 & 8.80 & 8.96 & 8.96 \\
\hline Nitrite & NA & 16.50 & 16.5 & $\mathrm{NA}$ & 18.29 & 18.29 & $\mathrm{NA}$ & 18.48 & 18.48 & NA & 17.87 & 17.87 & \begin{tabular}{|l|l|} 
NA & 18.12 \\
\end{tabular} & 18.12 \\
\hline Sulfate & 0.26 & 329.4 & 329.7 & 0.77 & 387.3 & 388.1 & 0.85 & 357.0 & 357.9 & 0.42 & 390.5 & 390.9 & \begin{tabular}{|l|l|}
0.42 & 407.6 \\
\end{tabular} & 408.0 \\
\hline
\end{tabular}


The Catholic University of America

Vitreous State Laboratory

DuraMelter 1200 Tests with AZ-101HLW Simulants Final Report, VSL-02R0100-2, Rev.1

Table 4.6. Analytical Results for Samples of SBS Blow-Down Fluids (mg/l), (continued).

\begin{tabular}{|c|c|c|c|c|c|c|c|c|c|c|c|c|c|c|}
\hline Test & \multicolumn{6}{|c|}{2} & \multicolumn{8}{|c|}{3} \\
\hline I.D. & & I-S-74t & & & $2 \mathrm{I}-\mathrm{S}-88$ & & & J-S-39 & & & $2 \mathrm{~J}-\mathrm{S}-44$ & & $12 \mathrm{~J}-\mathrm{S}-53$ & \\
\hline Glass $(\mathrm{kg})$ & & 4765 & & & 4895 & & & 5106 & & & 5514 & & 6011 & \\
\hline $\mathrm{pH}$ & & 8.46 & & & 8.45 & & & 7.08 & & & 5.72 & & 4.89 & \\
\hline & Sus* & Dis.\# & \begin{tabular}{|l|} 
Total \\
\end{tabular} & Sus* & Dis.刑 & \begin{tabular}{|l|} 
Total \\
\end{tabular} & Sus* & Dis.\# & A Total & Sus* & Dis.\# & Total & \begin{tabular}{|l|l|} 
Sus* & Dis.
\end{tabular} & Total \\
\hline Total & 268 & 688 & 956 & 268 & 672 & 940 & 208 & 292 & 500 & 488 & 956 & 1444 & \begin{tabular}{ll|l|l|}
788 & 2080 \\
\end{tabular} & 2868 \\
\hline $\mathrm{Al}$ & 9.76 & 0.24 & 10.00 & 10.59 & 0.26 & 10.85 & 11.34 & 0.44 & 11.78 & 24.27 & 22.22 & 46.49 & \begin{tabular}{|l|l}
38.85 & 49.81 \\
\end{tabular} & 88.66 \\
\hline As & 0.88 & 0.83 & \begin{tabular}{|l|}
1.71 \\
\end{tabular} & \begin{tabular}{|l|}
0.83 \\
\end{tabular} & 0.84 & \begin{tabular}{|l|}
1.67 \\
\end{tabular} & 0.23 & 0.13 & 0.36 & 0.75 & 0.22 & 0.97 & \begin{tabular}{|l|l|}
1.10 & 0.49 \\
\end{tabular} & 1.59 \\
\hline $\mathrm{B}$ & 0.43 & 110.3 & \begin{tabular}{|l|}
110.7 \\
\end{tabular} & 0.27 & 113.1 & 113.4 & 0.26 & 15.77 & 16.03 & 1.15 & 120.7 & 121.9 & \begin{tabular}{|l|l|}
2.08 & 251.8 \\
\end{tabular} & 253.9 \\
\hline $\mathrm{Ba}$ & 0.06 & 0.07 & \begin{tabular}{|l|}
0.13 \\
\end{tabular} & 0.06 & 0.07 & 0.13 & 0.04 & 0.11 & \begin{tabular}{|l|}
0.15 \\
\end{tabular} & 0.13 & 0.35 & 0.48 & \begin{tabular}{|l|l|}
0.38 & 0.33 \\
\end{tabular} & 0.71 \\
\hline $\mathrm{Ca}$ & 0.59 & 4.73 & 5.32 & 0.57 & 4.25 & 4.82 & 0.51 & 31.49 & 32.00 & 0.56 & 25.27 & 25.83 & \begin{tabular}{|l|l|}
0.44 .38 \\
\end{tabular} & 17.82 \\
\hline $\mathrm{Cd}$ & 14.17 & 0.29 & 14.46 & 12.89 & 0.28 & 13.17 & 1.44 & 0.90 & 2.34 & 2.96 & 4.79 & 7.75 & \begin{tabular}{|l|l|}
3.70 & 7.09 \\
\end{tabular} & 10.79 \\
\hline $\mathrm{Cr}$ & 0.30 & 0.05 & \begin{tabular}{|l|}
0.35 \\
\end{tabular} & \begin{tabular}{|l|}
0.29 \\
\end{tabular} & 0.04 & 0.33 & 0.18 & 0.02 & 0.20 & 0.73 & 0.04 & 0.77 & \begin{tabular}{l|l|}
1.40 & 0.09 \\
\end{tabular} & 1.49 \\
\hline $\mathrm{Cs}$ & 0.21 & 1.70 & 1.91 & 0.16 & $\mathrm{NA}$ & 0.16 & $<0.1$ & 0.37 & 0.37 & 0.26 & 3.20 & 3.46 & \begin{tabular}{|l|l|}
0.51 & 6.40 \\
\end{tabular} & 6.91 \\
\hline $\mathrm{Cu}$ & 0.05 & 0.07 & 0.12 & 0.05 & 0.07 & 0.12 & 0.05 & $<0.1$ & 0.05 & 0.16 & 0.04 & 0.20 & \begin{tabular}{l|l|}
0.24 & 0.16 \\
\end{tabular} & 0.40 \\
\hline $\mathrm{Fe}$ & 35.58 & 0.03 & 35.61 & 33.80 & 0.02 & 33.82 & 22.59 & 0.02 & 22.61 & 70.75 & 0.09 & 70.84 & \begin{tabular}{|l|l|}
121.9 & 0.15 \\
\end{tabular} & 122.1 \\
\hline $\mathrm{K}$ & 0.16 & 2.49 & 2.65 & 0.15 & 2.47 & 2.62 & 0.06 & 2.88 & 2.94 & 0.17 & 4.92 & 5.09 & \begin{tabular}{l|l|}
0.29 & 7.82 \\
\end{tabular} & 8.11 \\
\hline $\mathrm{Li}$ & 0.33 & 14.81 & 15.14 & 0.32 & 14.05 & 14.37 & 0.19 & 1.92 & 2.11 & 1.00 & 19.14 & 20.14 & \begin{tabular}{|l|l|}
1.89 & 42.56 \\
\end{tabular} & 44.45 \\
\hline $\mathrm{Mg}$ & 1.99 & 4.40 & 6.39 & 1.92 & 4.19 & 6.11 & 0.69 & 10.23 & 10.92 & 1.58 & 15.48 & 17.06 & \begin{tabular}{|l|l|}
2.63 & 23.82 \\
\end{tabular} & 26.45 \\
\hline $\mathrm{Mn}$ & 0.83 & 0.04 & 0.87 & 0.93 & 0.03 & 0.96 & 1.71 & 0.21 & \begin{tabular}{|l|}
1.92 \\
\end{tabular} & 3.20 & 3.81 & \begin{tabular}{|l|}
7.01 \\
\end{tabular} & \begin{tabular}{|l|l|}
5.33 & 10.42 \\
\end{tabular} & 15.75 \\
\hline $\mathrm{Na}$ & 1.00 & 52.27 & 53.27 & 0.96 & 49.91 & 50.87 & 0.39 & 17.93 & 18.32 & 1.85 & 72.03 & 73.88 & \begin{tabular}{|l|l|}
3.59 & 139.5 \\
\end{tabular} & 143.1 \\
\hline $\mathrm{Ni}$ & 1.54 & 0.15 & 1.69 & 1.46 & 0.11 & \begin{tabular}{|l|}
1.57 \\
\end{tabular} & 1.20 & 0.07 & 1.27 & 3.85 & \begin{tabular}{|l|}
0.51 \\
\end{tabular} & 4.36 & \begin{tabular}{|l|l|}
6.76 & 0.98 \\
\end{tabular} & 7.74 \\
\hline $\mathrm{P}$ & 0.27 & 0.46 & 0.73 & 0.21 & 0.39 & 0.60 & 0.27 & 0.08 & 0.35 & 0.94 & 0.41 & 1.35 & \begin{tabular}{|l|l|}
1.64 & 0.77 \\
\end{tabular} & 2.41 \\
\hline $\mathrm{Pb}$ & 1.69 & 0.20 & 1.89 & 1.55 & 0.20 & 1.75 & 0.41 & 0.05 & 0.46 & 1.18 & 0.35 & 1.53 & \begin{tabular}{|l|l|}
1.95 & 0.70 \\
\end{tabular} & 2.65 \\
\hline $\mathrm{Sb}$ & 0.27 & \begin{tabular}{|l|}
0.13 \\
\end{tabular} & 0.40 & 0.30 & 0.14 & 0.44 & 0.37 & $<0.03$ & 0.37 & 1.07 & 0.27 & 1.34 & \begin{tabular}{|l|l|}
1.87 & 0.40 \\
\end{tabular} & 2.27 \\
\hline $\mathrm{Se}$ & 3.76 & 166.8 & 170.6 & 3.61 & 165.0 & 168.6 & 3.08 & 44.97 & 48.05 & 15.24 & 109.9 & 125.1 & \begin{tabular}{|l|l|}
33.91 & 218.1 \\
\end{tabular} & 252.0 \\
\hline $\mathrm{Si}$ & 32.07 & 4.43 & 36.5 & 33.11 & 4.22 & 37.33 & 44.16 & 3.59 & 47.75 & 81.98 & 28.95 & 110.9 & \begin{tabular}{|l|l|}
120.9 & 62.18 \\
\end{tabular} & 183.1 \\
\hline $\mathrm{Sr}$ & 2.35 & 8.13 & 10.48 & 2.27 & 8.07 & 10.34 & 0.25 & 4.24 & 4.49 & 1.20 & 22.47 & 23.67 & \begin{tabular}{|l|l|}
2.66 & 42.95 \\
\end{tabular} & 45.61 \\
\hline Te & 34.57 & 3.23 & 37.8 & 34.04 & 3.50 & 37.54 & 5.54 & 0.41 & 5.95 & 17.13 & 1.37 & 18.5 & \begin{tabular}{|l|l|}
26.45 & 1.86 \\
\end{tabular} & 28.31 \\
\hline $\mathrm{Ti}$ & 0.11 & $<0.01$ & 0.11 & 0.11 & 0.01 & 0.12 & 0.12 & $<0.01$ & 0.12 & 0.26 & $<0.01$ & 0.26 & \begin{tabular}{|l|l|}
0.42 & 0.01 \\
\end{tabular} & 0.43 \\
\hline $\mathrm{Zn}$ & 5.30 & 0.26 & 5.56 & 5.18 & 0.23 & 5.41 & 1.90 & 1.27 & 3.17 & 4.09 & 16.46 & 20.55 & \begin{tabular}{|l|l|}
6.39 & 34.75 \\
\end{tabular} & 41.14 \\
\hline $\mathrm{Zr}$ & 1.46 & 0.01 & 1.47 & 1.87 & $<0.01$ & 1.87 & 4.30 & $<0.01$ & 4.30 & 7.29 & \begin{tabular}{|l|}
0.01 \\
\end{tabular} & 7.30 & \begin{tabular}{|l|l|}
12.11 & 0.02 \\
\end{tabular} & \begin{tabular}{|l|}
12.13 \\
\end{tabular} \\
\hline $\mathrm{Cl}$ & NA & 24.05 & 24.05 & $\mathrm{NA}$ & 23.94 & 23.94 & $\mathrm{NA}$ & 33.63 & 33.63 & NA & 54.88 & 54.88 & \begin{tabular}{|l|l|} 
NA & 81.70 \\
\end{tabular} & \begin{tabular}{|l|}
81.7 \\
\end{tabular} \\
\hline $\bar{F}$ & $\mathrm{NA}$ & 27.70 & 27.7 & $\mathrm{NA}$ & 29.18 & 29.18 & NA & 10.92 & 10.92 & NA & 62.28 & 62.28 & \begin{tabular}{l|l|} 
NA & 98.75
\end{tabular} & 98.75 \\
\hline I & $<0.1$ & 332.1 & $\mid 332.1$ & $<0.1$ & 334.9 & 334.9 & $<0.1$ & 13.50 & 13.5 & $<0.1$ & 258.1 & 258.1 & \begin{tabular}{|c|c|}
$<0.1$ & 450.2 \\
\end{tabular} & \begin{tabular}{|l|}
450.2 \\
\end{tabular} \\
\hline Nitrate & NA & 8.87 & 8.87 & NA & 9.12 & 9.12 & NA & 1.82 & 1.82 & NA & 12.00 & 12.00 & NA $19.06 \mid$ & \begin{tabular}{|l|}
19.06 \\
\end{tabular} \\
\hline Nitrite & NA & 18.74 & 18.74 & NA & 18.12 & 18.12 & NA & $<0.1$ & $<0.1$ & NA & 12.09 & 12.09 & NA $\mid 26.21$ & 26.21 \\
\hline Sulfate & 0.65 & 351.5 & 352.2 & 0.57 & 390.5 & 391.1 & NA & 94.32 & 94.32 & 1.48 & 245.3 & 246.8 & \begin{tabular}{|l|l|}
3.10 & 494.2 \\
\end{tabular} & 497.3 \\
\hline
\end{tabular}


Table 4.6. Analytical Results for Samples of SBS Blow-Down Fluids (mg/l), (continued).

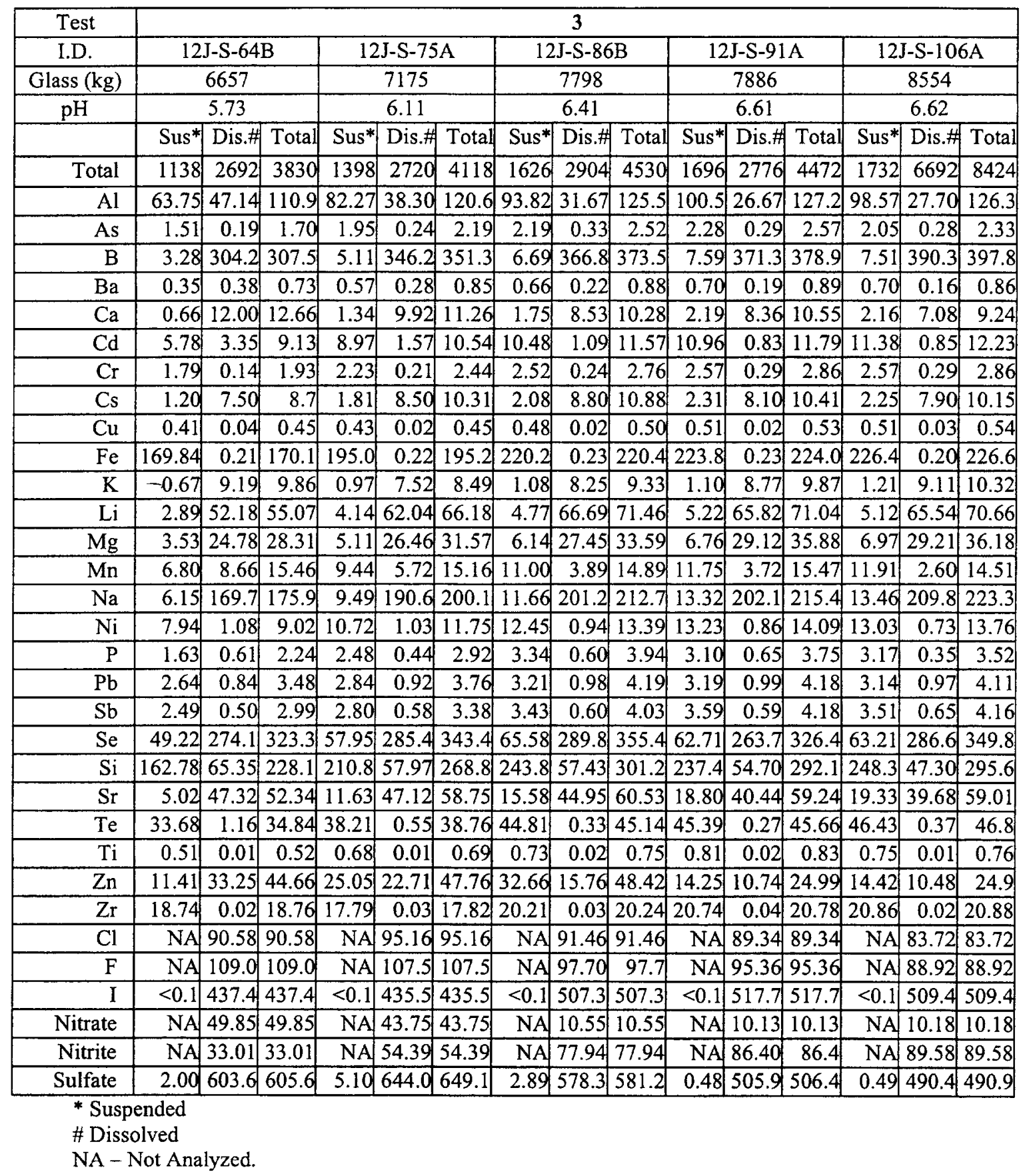


The Catholic University of America

DuraMelter 1200 Tests with AZ-101HLW Simulants

Vitreous State Laboratory

Final Report, VSL-02R0100-2, Rev.I

Table 4.6. Analytical Results for Samples of SBS Blow-Down Fluids (mg/l), (continued).

\begin{tabular}{|c|c|c|c|c|c|c|c|c|c|c|c|c|c|c|}
\hline Test & \multicolumn{14}{|c|}{3} \\
\hline I.D. & & J-S-124 & & & J-S-136 & & & J-S-13S & & & J-S-142 & & $12 \mathrm{~J}-\mathrm{S}-14 \mathrm{C}$ & 49B \\
\hline Glass (kg) & & 9400 & & & 10135 & & & 10453 & & & 10817 & & 11075 & \\
\hline $\mathrm{pH}$ & & 6.94 & & & 4.72 & & & 5.47 & & & 4.90 & & 5.96 & \\
\hline & Sus* & Dis.\# & Total & Sus* & Dis.\# & Total & Sus* & Dis.\# & Total & Sus* & Dis.\# & Total & \begin{tabular}{l|l|} 
Sus* & Dis. \#
\end{tabular} & Total \\
\hline Total & 2038 & 2656 & 4694 & 772 & 2784 & 3556 & 3584 & 2720 & 6304 & 802 & 2476 & 3278 & \begin{tabular}{|l|l|}
2182 & 2688 \\
\end{tabular} & 4870 \\
\hline $\mathrm{Al}$ & 116.41 & 30.50 & 146.9 & 37.91 & 54.18 & 92.09 & 173.8 & 57.00 & 230.8 & 35.96 & 58.03 & 93.99 & \begin{tabular}{|l|l|}
103.7 & 42.23 \\
\end{tabular} & 145.9 \\
\hline As & 3.02 & 0.44 & 3.46 & 1.20 & 0.92 & 2.12 & 3.55 & 0.69 & 4.24 & 1.18 & 1.06 & 2.24 & 2.35 & 2.90 \\
\hline $\mathrm{B}$ & 9.27 & 394.2 & 403.5 & 2.46 & 396.2 & 398.7 & 12.06 & 368.1 & 380.2 & 2.70 & 337.2 & 339.9 & \begin{tabular}{|l|l|l|}
7.61 & 336.1 \\
\end{tabular} & 343.7 \\
\hline $\mathrm{Ba}$ & 0.77 & 0.19 & 0.96 & 0.32 & 0.48 & 0.80 & 1.27 & 0.47 & 1.74 & 0.52 & 0.26 & 0.78 & \begin{tabular}{|l|l|}
0.85 & 0.29 \\
\end{tabular} & 1.14 \\
\hline$\overline{\mathrm{Ca}}$ & 2.05 & 7.22 & 9.27 & 0.39 & 9.15 & 9.54 & 1.71 & 9.42 & 11.13 & 0.34 & 8.83 & 9.17 & 1.52 & 10.32 \\
\hline $\mathrm{Cd}$ & 12.66 & 1.04 & 13.7 & 3.89 & 4.24 & 8.13 & 17.66 & 4.35 & 22.01 & 4.36 & 5.35 & 9.71 & 11.38 & 13.74 \\
\hline $\mathrm{Cr}$ & 2.80 & 0.44 & 3.24 & 1.35 & 0.22 & 1.57 & 4.87 & 0.17 & 5.04 & 1.42 & 0.18 & 1.60 & 2.46 & 2.69 \\
\hline Cs & 2.39 & 8.50 & 10.89 & 0.80 & 8.50 & 9.30 & 3.42 & 8.00 & 11.42 & 0.79 & 8.40 & 9.19 & 2.14 & 9.54 \\
\hline $\mathrm{Cu}$ & 0.60 & 0.03 & 0.63 & 0.24 & 0.12 & 0.36 & 1.08 & 0.09 & 1.17 & 0.26 & 0.16 & 0.42 & 0.68 & 0.73 \\
\hline $\mathrm{Fe}$ & 253.51 & 0.20 & 253.7 & 112.4 & 0.24 & 112.6 & 422.0 & 0.24 & 422.2 & \begin{tabular}{|l|}
107.7 \\
\end{tabular} & 0.24 & 107.9 & \begin{tabular}{|l|}
235.9 \\
\end{tabular} & 236.2 \\
\hline $\mathrm{K}$ & 1.17 & 8.94 & -10.11 & 0.41 & 9.34 & 9.75 & 1.48 & 8.92 & 10.40 & 0.40 & 9.60 & 10.00 & 1.02 & 10.01 \\
\hline $\mathrm{Li}$ & 5.92 & 66.24 & 72.16 & 2.12 & 65.29 & 67.41 & 8.85 & 61.62 & 70.47 & 2.06 & 58.34 & 60.4 & \begin{tabular}{|l|l|}
5.29 & 58.15 \\
\end{tabular} & 63.44 \\
\hline $\mathrm{Mg}$ & 7.34 & 28.38 & 35.72 & 2.74 & 28.99 & 31.73 & 10.74 & 28.61 & 39.35 & 2.69 & 26.58 & 29.27 & \begin{tabular}{|l|l|}
6.32 & 27.95 \\
\end{tabular} & 34.27 \\
\hline $\mathrm{Mn}$ & 15.10 & 2.47 & 17.57 & 5.73 & 5.05 & 10.78 & 39.26 & 7.12 & 46.38 & 6.33 & \begin{tabular}{|l|}
8.64 \\
\end{tabular} & 14.97 & \begin{tabular}{|l|l|}
31.93 & 6.83 \\
\end{tabular} & 38.76 \\
\hline $\mathrm{Na}$ & 15.46 & 198.6 & 214.1 & 4.97 & 205.7 & 210.7 & 21.24 & 188.4 & 209.6 & 4.74 & \begin{tabular}{|l|}
170.7 \\
\end{tabular} & 175.4 & \begin{tabular}{|l|l|}
13.89 & 173.1 \\
\end{tabular} & 187.0 \\
\hline $\mathrm{Ni}$ & 14.64 & 0.78 & 15.42 & 6.04 & 1.23 & \begin{tabular}{|l|}
7.27 \\
\end{tabular} & 24.35 & 1.41 & 25.76 & 6.24 & \begin{tabular}{|l|}
1.35 \\
\end{tabular} & 7.59 & \begin{tabular}{|l|l|}
13.35 & 1.23 \\
\end{tabular} & \begin{tabular}{|l|l|}
33 & 14.58 \\
\end{tabular} \\
\hline $\bar{P}$ & 3.38 & 0.41 & \begin{tabular}{|l|}
3.79 \\
\end{tabular} & 1.26 & 0.99 & 2.25 & 8.65 & 0.95 & 9.6 & 1.75 & 1.20 & 2.95 & \begin{tabular}{|l|}
3.96 \\
\end{tabular} & \begin{tabular}{|l|l|}
5 & 4.81 \\
\end{tabular} \\
\hline $\mathrm{Pb}$ & 3.68 & 0.98 & 4.66 & 1.69 & 1.01 & 2.70 & 7.20 & 1.03 & 8.23 & 1.77 & 1.08 & 2.85 & 4.39 & $\overline{5.46}$ \\
\hline $\mathrm{Sb}$ & 4.21 & 0.70 & 4.91 & 1.79 & 0.63 & \begin{tabular}{|l|}
2.42 \\
\end{tabular} & 8.47 & 0.64 & 9.11 & 1.74 & 0.72 & 2.46 & \begin{tabular}{|l|l|}
5.48 & 0.77 \\
\end{tabular} & 6.25 \\
\hline Se & 65.67 & 253.7 & 319.4 & 30.81 & 319.1 & 349.9 & 127.3 & 297.3 & 424.6 & 34.88 & 286.7 & 321.6 & \begin{tabular}{|l|l|}
74.44 & 260.1 \\
\end{tabular} & 1334.5 \\
\hline Si & 334.92 & 51.33 & 386.3 & 128.1 & 72.45 & 200.6 & 608.6 & 72.53 & 681.1 & 132.8 & 73.17 & 206.0 & \begin{tabular}{|l|l|}
433.63 .23 \\
\end{tabular} & 3496.8 \\
\hline $\mathrm{Sr}$ & 20.54 & 42.44 & 62.98 & 3.73 & 55.57 & 59.3 & 17.51 & 57.08 & 74.59 & 4.31 & 54.73 & 59.04 & \begin{tabular}{|l|l|}
14.63 & 49.30 \\
\end{tabular} & 63.93 \\
\hline $\mathrm{Te}$ & 52.48 & 0.44 & 52.92 & 22.33 & 1.81 & 24.14 & 85.43 & 1.62 & 87.05 & 23.53 & 2.14 & 25.67 & 45.72 & 347.05 \\
\hline $\mathrm{Ti}$ & 0.89 & 0.01 & 0.90 & 0.38 & 0.02 & 0.40 & 2.52 & 0.02 & 2.54 & 0.38 & 0.02 & 0.40 & 1.11 & 1.13 \\
\hline $\mathrm{Zn}$ & 40.87 & 13.23 & 54.1 & 7.60 & 37.41 & 45.01 & 38.64 & 40.90 & 79.54 & 8.02 & 42.35 & 50.37 & \begin{tabular}{|l|l|l|}
29.34 & 30.03 \\
\end{tabular} & 59.37 \\
\hline $\mathrm{Zr}$ & 29.05 & 0.03 & 29.08 & 12.94 & \begin{tabular}{|l|}
0.03 \\
\end{tabular} & 12.97 & 86.32 & 0.03 & 86.35 & 13.66 & 0.04 & 13.7 & \begin{tabular}{|l|l|}
66.60 & 0.04 \\
\end{tabular} & $4 \longdiv { 6 6 . 6 4 }$ \\
\hline $\mathrm{Cl}$ & $\mathrm{NA}$ & 68.56 & 68.56 & $\mathrm{NA}$ & 93.27 & 93.27 & NA & 87.09 & 87.09 & $\mathrm{NA}$ & 85.29 & 85.29 & \begin{tabular}{|l|l|} 
NA & 80.38 \\
\end{tabular} & 80.38 \\
\hline $\mathrm{F}$ & NA & 74.60 & 74.6 & NA & 106.8 & 106.8 & NA & 102.2 & 102.2 & $\mathrm{NA}$ & 107.1 & 107.1 & \begin{tabular}{l|l|} 
NA & 86.83 \\
\end{tabular} & 86.83 \\
\hline$I$ & $<0.1$ & 442.1 & 442.1 & $<0.1$ & 523.7 & 523.7 & $<0.1$ & 583.3 & 583.3 & $<0.1$ & 531.5 & 531.5 & \begin{tabular}{l|l|}
$<0.1$ & 494.6 \\
\end{tabular} & $6 \longdiv { 4 9 4 . 6 }$ \\
\hline Nitrate & $\mathrm{NA}$ & 23.48 & 23.48 & $\mathrm{NA}$ & 73.15 & 73.15 & NA & 23.62 & 23.62 & $\mathrm{NA}$ & 40.52 & 40.52 & NA 39.87 & 39.87 \\
\hline Nitrite & $\mathrm{NA}$ & 105.6 & 105.6 & NA & 76.55 & 76.55 & NA & 104.2 & 104.2 & $\mathrm{NA}$ & 65.56 & 65.56 & \begin{tabular}{l|l|l|} 
NA & 59.04 \\
\end{tabular} & 59.04 \\
\hline Sulfate & 2.43 & 488.0 & 490.4 & 0.24 & 587.5 & 587.7 & 8.67 & 587.5 & 596.2 & 1.95 & 581.7 & 583.7 & \begin{tabular}{|l|l|}
3.89 & 453.5 \\
\end{tabular} & \begin{tabular}{|l|l|}
457.4 \\
\end{tabular} \\
\hline
\end{tabular}


The Catholic University of America

Vitreous State Laboratory

DuraMelter 1200 Tests with AZ-101HLW Simulants

Final Report, VSL-02R0100-2, Rev.I

Table 4.6. Analytical Results for Samples of SBS Blow-Down Fluids (mg/l), (continued).

\begin{tabular}{|c|c|c|c|c|c|c|c|c|c|c|c|c|c|c|c|}
\hline Test & \multicolumn{15}{|c|}{4} \\
\hline I.D. & \multicolumn{3}{|c|}{$12 \mathrm{~K}-\mathrm{S}-36 \mathrm{~A}$} & \multicolumn{3}{|c|}{$12 \mathrm{~K}-\mathrm{S}-43 \mathrm{~A}$} & \multicolumn{3}{|c|}{$12 \mathrm{~K}-\mathrm{S}-53 \mathrm{~A}$} & \multicolumn{3}{|c|}{$12 \mathrm{~K}-\mathrm{S}-56 \mathrm{~A}$} & \multicolumn{3}{|c|}{$12 \mathrm{~K}-\mathrm{S}-71 \mathrm{~A}$} \\
\hline Glass (kg) & \multicolumn{3}{|c|}{11145} & \multicolumn{3}{|c|}{11423} & \multicolumn{3}{|c|}{11656} & \multicolumn{3}{|c|}{11830} & \multicolumn{3}{|c|}{12201} \\
\hline $\mathrm{pH}$ & \multicolumn{3}{|c|}{6.69} & \multicolumn{3}{|c|}{6.82} & \multicolumn{3}{|c|}{6.49} & \multicolumn{3}{|c|}{6.09} & \multicolumn{3}{|c|}{5.11} \\
\hline & Sus* & Dis.\# & Total & Sus* & \begin{tabular}{|l|l|} 
Dis.\# \\
\end{tabular} & Total & Sus* & Dis.\#伴 & \begin{tabular}{|l|} 
Total \\
\end{tabular} & Sus* & \begin{tabular}{|l|l|} 
Nis. Dis. \\
\end{tabular} & Total & Sus* & Dis.\# & Total \\
\hline Total & 186 & 313 & 499 & 326 & 760 & 1086 & 440 & 1152 & \begin{tabular}{|l|}
1592 \\
\end{tabular} & 514 & $\begin{array}{ll}41250 \\
\end{array}$ & 1764 & 494 & 1656 & 2150 \\
\hline $\mathrm{Al}$ & 10.27 & 0.68 & 10.95 & 18.14 & 4.13 & 22.27 & 21.82 & 12.13 & 33.95 & 24.06 & 6) 22.23 & 46.29 & 20.36 & 32.62 & 52.98 \\
\hline As & 0.35 & $<0.05$ & 0.35 & 0.49 & $<0.05$ & 0.49 & 0.63 & \begin{tabular}{|l|}
0.08 \\
\end{tabular} & \begin{tabular}{|l|}
0.71 \\
\end{tabular} & $<0.05$ & \begin{tabular}{|l|l|}
5 & 0.16 \\
\end{tabular} & 0.16 & 0.67 & 0.38 & 1.05 \\
\hline $\mathrm{B}$ & 0.62 & 22.18 & 22.8 & 1.18 & 98.21 & 99.39 & 1.40 & 143.7 & 145.1 & 1.44 & 4183.0 & 184.4 & 1.06 & 219.2 & 220.3 \\
\hline $\mathrm{Ba}$ & 0.07 & 0.08 & 0.15 & 0.11 & \begin{tabular}{|l|}
0.16 \\
\end{tabular} & 0.27 & 0.15 & \begin{tabular}{|l|}
0.22 \\
\end{tabular} & \begin{tabular}{|l|}
0.37 \\
\end{tabular} & 0.19 & \begin{tabular}{|l|}
90.29 \\
\end{tabular} & 0.48 & 0.16 & 0.48 & 0.64 \\
\hline $\mathrm{Ca}$ & 0.63 & 29.68 & 30.31 & 1.05 & 20.95 & 22.00 & 0.77 & 15.13 & 15.9 & 0.56 & 6) 12.68 & 13.24 & 0.20 & 10.53 & 10.73 \\
\hline $\mathrm{Cd}$ & 1.19 & 0.37 & 1.56 & 2.24 & 0.45 & 2.69 & 3.04 & 0.80 & 3.84 & 3.69 & $\begin{array}{l}9.57 \\
\end{array}$ & 5.26 & 2.56 & 4.04 & 6.60 \\
\hline $\mathrm{Cr}$ & 0.24 & 0.07 & 0.31 & 0.62 & 0.10 & 0.72 & 0.90 & 0.16 & 1.06 & 1.16 & 0.12 & 1.28 & 1.24 & 0.08 & 1.32 \\
\hline $\mathrm{Cs}$ & 0.14 & 0.75 & 0.89 & 0.21 & 2.40 & 2.61 & 0.28 & 4.20 & 4.48 & 0.28 & 5.00 & 5.28 & 0.26 & 5.90 & 6.16 \\
\hline $\mathrm{Cu}$ & 0.07 & 0.01 & 0.08 & 0.11 & 0.01 & 0.12 & 0.14 & 0.02 & 0.16 & 0.18 & 0.03 & 0.21 & 0.14 & 0.13 & 0.27 \\
\hline $\mathrm{Fe}$ & 19.86 & 0.05 & 19.91 & 48.14 & 0.09 & 48.23 & 71.59 & 0.12 & 71.71 & 89.04 & 0.08 & 89.12 & 95.91 & 0.11 & 96.02 \\
\hline $\mathrm{K}$ & 0.12 & 2.83 & 2.95 & 0.19 & 3.46 & 3.65 & 0.25 & 4.11 & 4.36 & 0.27 & 5.19 & 5.46 & 0.25 & 6.24 & 6.49 \\
\hline $\mathrm{Li}$ & 0.41 & 3.24 & 3.65 & 0.87 & 18.11 & 18.98 & 1.17 & 28.64 & 29.81 & 1.34 & 35.48 & 36.82 & 1.17 & 42.55 & 43.72 \\
\hline $\mathrm{Mg}$ & 1.91 & 8.33 & \begin{tabular}{|l|}
10.24 \\
\end{tabular} & 0.59 & 10.80 & 11.39 & 1.43 & 13.14 & 14.57 & 2.29 & 15.53 & 17.82 & 2.35 & 17.63 & 19.98 \\
\hline $\mathrm{Mn}$ & 1.85 & 0.56 & 2.41 & 1.75 & 0.80 & 2.55 & 2.11 & 1.10 & 3.21 & 2.28 & 2.03 & 4.31 & 2.01 & 3.97 & 5.98 \\
\hline $\mathrm{Na}$ & 1.08 & 19.74 & 20.82 & 2.04 & 53.30 & 55.34 & 2.39 & 82.85 & 85.24 & 2.60 & 99.70 & 102.3 & 1.94 & 122.9 & 124.8 \\
\hline $\mathrm{Ni}$ & 1.21 & 0.16 & 1.37 & 2.53 & 0.30 & 2.83 & 3.59 & \begin{tabular}{|l|}
0.48 \\
\end{tabular} & 4.07 & 4.40 & 0.58 & \begin{tabular}{|l|}
4.98 \\
\end{tabular} & 4.45 & 0.83 & 5.28 \\
\hline $\mathrm{P}$ & 0.38 & 0.48 & 0.86 & 0.67 & 0.52 & 1.19 & 0.97 & 0.60 & 1.57 & 1.12 & 0.22 & 1.34 & 1.13 & 0.48 & 1.61 \\
\hline $\mathrm{Pb}$ & 0.41 & 0.06 & 0.47 & 0.67 & 0.22 & 0.89 & 0.92 & 0.36 & 1.28 & 1.19 & 0.48 & 1.67 & 1.27 & 0.58 & 1.8 \\
\hline $\mathrm{Sb}$ & 0.34 & 0.05 & 0.39 & 0.60 & 0.17 & 0.77 & 0.81 & 0.29 & 1.10 & 0.97 & 0.46 & 1.43 & 0.94 & 0.42 & 1.36 \\
\hline $\mathrm{Se}$ & 5.80 & 25.51 & 31.31 & 7.72 & 63.92 & 71.64 & 11.40 & 97.21 & 108.6 & 16.98 & 118.9 & 135.9 & 20.52 & 166.5 & 187.0 \\
\hline $\mathrm{Si}$ & 33.05 & 5.77 & 38.82 & 48.62 & 16.26 & 64.88 & 62.90 & 24.99 & 87.89 & 68.51 & 35.27 & 103.8 & 63.61 & 45.40 & 109 \\
\hline $\mathrm{Sr}$ & 0.65 & 4.62 & 5.27 & $<0.1$ & 14.23 & 14.23 & 2.13 & 22.76 & 24.89 & 2.27 & 25.81 & 28.08 & 1.26 & 32.97 & 34.23 \\
\hline $\mathrm{Te}$ & 5.22 & $<0.20$ & 5.22 & 11.03 & $<0.20$ & 11.03 & 16.79 & $<0.20$ & 16.79 & 22.13 & 0.31 & 22.44 & 22.68 & 1.00 & 23.68 \\
\hline $\mathrm{Ti}$ & 0.10 & 0.01 & 0.11 & 0.19 & 0.01 & 0.20 & 0.27 & \begin{tabular}{l|}
0.01 \\
\end{tabular} & 0.28 & 0.34 & $\begin{array}{l}<<0.1 \\
\end{array}$ & 0.34 & 0.33 & 0.01 & \begin{tabular}{|l|}
0.34 \\
\end{tabular} \\
\hline $\mathrm{Zn}$ & 2.20 & 1.35 & 3.55 & 5.66 & 4.09 & 9.75 & 6.89 & 9.24 & 16.13 & 6.87 & 15.20 & 22.07 & 3.94 & 25.06 & 29.00 \\
\hline $\mathrm{Zr}$ & 4.39 & 0.02 & 4.41 & 3.65 & 0.03 & 3.68 & 4.38 & 0.03 & 4.41 & 5.09 & 0.01 & 5.10 & 4.98 & \begin{tabular}{|l|}
0.01 \\
\end{tabular} & 4.99 \\
\hline $\mathrm{Cl}$ & NA & 28.30 & 28.3 & NA & 31.23 & 31.23 & $\mathrm{NA}$ & 42.00 & 42.00 & NA & 52.27 & 52.27 & NA & 73.04 & 73.04 \\
\hline $\mathrm{F}$ & NA & 9.85 & 9.85 & NA & 28.21 & 28.21 & NA & 42.10 & 42.10 & NA & \begin{tabular}{|l|}
3.99 \\
\end{tabular} & 53.99 & $\mathrm{NA}$ & 73.52 & 73.52 \\
\hline I & $<0.1$ & 14.50 & 14.5 & $<0.1$ & 131.7 & 131.7 & $<0.1$ & 245.7 & 245.7 & $<0.1$ & 287.3 & \begin{tabular}{|l|}
287.3 \\
\end{tabular} & $<0.1$ & \begin{tabular}{|l|}
384.2 \\
\end{tabular} & 384.2 \\
\hline Nitrate & $\mathrm{NA}$ & 3.73 & 3.73 & NA & 6.67 & 6.67 & $\mathrm{NA}$ & \begin{tabular}{|l|}
8.43 \\
\end{tabular} & 8.43 & $\mathrm{NA}$ & 44.39 & 44.39 & NA & 16.57 & 16.5 \\
\hline Nitrite & NA & 0.93 & 0.93 & $\mathrm{NA}$ & 16.83 & 16.83 & $\mathrm{NA}$ & 40.18 & 40.18 & NA & 20.70 & 20.70 & NA & 31.05 & 31.05 \\
\hline Sulfate & 0.33 & 100.4 & 100.7 & $<0.1$ & 172.6 & 172.6 & 0.21 & 233.1 & 233.3 & $<0.1$ & 336.8 & 336.8 & 0.97 & 426.3 & 427. \\
\hline
\end{tabular}


Table 4.6. Analytical Results for Samples of SBS Blow-Down Fluids (mg/l), (continued).

\begin{tabular}{|c|c|c|c|c|c|c|c|c|c|c|c|c|c|c|c|}
\hline Test & \\
\hline I.D. & & K-S- 84 & & & $2 \mathrm{~K}-\mathrm{S}-94$ & & & $\overline{\mathrm{K}-\mathrm{S}-10}$ & & & $\overline{\mathrm{K}-\mathrm{S}-11}$ & & & $\overline{\mathrm{K}-\mathrm{S}-12}$ & $27 \mathrm{~A}$ \\
\hline Slass (kg) & & 12560 & & & 12862 & & & 13252 & & & 13649 & & & 14035 & \\
\hline $\mathrm{pH}$ & & 4.64 & & & 4.30 & & & 4.16 & & & 4.23 & & & 4.17 & \\
\hline & Sus* & Dis.\# & Total & Sus* & Dis. A & Total & Sus* & Dis.\# & Total & Sus* & Dis.\# & Total & Sus* & \begin{tabular}{|l|l} 
Dis.\# \\
\end{tabular} & Tota \\
\hline Total & 472 & 2948 & 3420 & 448 & 1736 & 2184 & 462 & 1832 & \begin{tabular}{|l|}
2294 \\
\end{tabular} & 470 & 1816 & 2286 & 463 & \begin{tabular}{|l|l}
1836 \\
\end{tabular} & 2299 \\
\hline $\mathrm{Al}$ & 17.79 & \begin{tabular}{|c|}
37.67 \\
\end{tabular} & 55.46 & 17.26 & 40.71 & \begin{tabular}{|l|}
57.97 \\
\end{tabular} & 17.74 & 42.36 & 60.1 & 19.25 & 42.10 & 61.35 & 19.13 & 42.61 & 61.74 \\
\hline$\overline{\text { As }}$ & 0.66 & \begin{tabular}{|l|}
0.49 \\
\end{tabular} & \begin{tabular}{|l|}
1.15 \\
\end{tabular} & \begin{tabular}{|l|}
0.63 \\
\end{tabular} & 0.50 & 1.13 & \begin{tabular}{|l|}
0.54 \\
\end{tabular} & \begin{tabular}{|l|}
0.65 \\
\end{tabular} & 1.19 & 0.54 & \begin{tabular}{|l|}
40.77 \\
\end{tabular} & 1.31 & 0.49 & 0.73 & 1.22 \\
\hline B & 0.81 & 226.0 & 226.8 & 0.72 & 233.3 & 234.0 & 0.76 & 246.2 & 247.0 & 0.86 & 242.6 & 243.5 & $\overline{0.84}$ & 239.7 & 240.5 \\
\hline$\overline{\mathrm{Ba}}$ & 0.24 & 0.36 & 0.60 & 0.27 & 0.31 & 0.58 & 0.29 & 0.28 & 0.57 & 0.28 & \begin{tabular}{|l|l|} 
& 0.27 \\
\end{tabular} & 0.55 & 0.24 & \begin{tabular}{|l|}
0.29 \\
\end{tabular} & 0.5 \\
\hline$\overline{\mathrm{Ca}}$ & 0.17 & 8.55 & 8.72 & 0.16 & 7.12 & 7.28 & 0.15 & 6.59 & 6.74 & 0.11 & 6.11 & 6.22 & 0.11 & 5.76 & 5.8 \\
\hline $\mathrm{Cd}$ & 1.78 & 5.22 & 7.00 & 1.52 & 5.63 & 7.15 & 1.47 & 5.95 & 7.42 & 1.44 & \begin{tabular}{|l|l|} 
& 5.68 \\
\end{tabular} & 7.12 & 1.41 & 5.56 & 6.97 \\
\hline$\overline{\mathrm{Cr}}$ & 1.22 & 0.13 & 1.35 & 1.16 & 0.15 & 1.31 & 1.16 & 0.18 & 1.34 & 1.14 & \begin{tabular}{|l|}
0.19 \\
\end{tabular} & 1.33 & 1.08 & 0.18 & 1.26 \\
\hline$\overline{\mathrm{Cs}}$ & 0.13 & 6.30 & 6.43 & 0.13 & 6.60 & 6.73 & 0.16 & 6.90 & 7.06 & 0.20 & 6.60 & 6.80 & 0.18 & 6.10 & 6.28 \\
\hline $\mathrm{Cu}$ & 0.09 & 0.22 & 0.31 & 0.08 & 0.25 & 0.33 & 0.08 & 0.27 & 0.35 & 0.08 & 0.28 & 0.36 & 0.08 & 0.29 & 0.3 \\
\hline $\mathrm{Fe}$ & 91.80 & 0.13 & \begin{tabular}{|l|}
91.93 \\
\end{tabular} & \begin{tabular}{|l|}
85.53 \\
\end{tabular} & 0.13 & 85.66 & \begin{tabular}{|l|}
85.85 \\
\end{tabular} & 0.14 & \begin{tabular}{|l|}
85.99 \\
\end{tabular} & 59.98 & 0.13 & 60.11 & 84.34 & 0.14 & 84.48 \\
\hline $\mathrm{K}$ & 0.20 & 6.12 & 6.32 & 0.20 & 6.20 & 6.40 & 0.18 & 6.25 & 6.43 & 0.18 & 6.18 & 6.36 & 0.21 & 6.37 & 6.58 \\
\hline$\overline{\mathrm{Li}}$ & 1.00 & 42.04 & 43.04 & 0.93 & 41.77 & 42.7 & 0.94 & 40.45 & 41.39 & 0.99 & 39.36 & 40.35 & 0.95 & 39.91 & 40.86 \\
\hline$\overline{\mathrm{Mg}}$ & 1.98 & \begin{tabular}{|l|}
17.31 \\
\end{tabular} & 19.29 & 1.73 & \begin{tabular}{|l|}
17.27 \\
\end{tabular} & 19.00 & 1.63 & 18.36 & 19.99 & 1.60 & 18.10 & \begin{tabular}{|l|}
19.7 \\
\end{tabular} & 1.64 & 17.44 & 19.0 \\
\hline $\mathrm{Mn}$ & 1.82 & 6.30 & 8.12 & 1.72 & 8.15 & \begin{tabular}{|l|}
9.87 \\
\end{tabular} & 1.75 & 10.20 & 11.95 & 1.82 & 11.02 & 12.84 & 1.73 & 10.48 & \begin{tabular}{|l|l}
12.2 \\
\end{tabular} \\
\hline $\mathrm{Na}$ & 1.44 & $\mid$\begin{tabular}{|l|}
121.1 \\
\end{tabular} & 122.5 & 1.26 & 122.0 & \begin{tabular}{|l|}
123.3 \\
\end{tabular} & 1.27 & 124.4 & 125.7 & 1.31 & 122.6 & 123.9 & 1.27 & 120.5 & 121.8 \\
\hline $\mathrm{Ni}$ & 4.24 & 0.88 & \begin{tabular}{|l|}
5.12 \\
\end{tabular} & 4.03 & 0.92 & 4.95 & 4.06 & 0.98 & \begin{tabular}{|l|}
5.04 \\
\end{tabular} & 4.06 & 0.97 & \begin{tabular}{|l|}
5.03 \\
\end{tabular} & 3.90 & 0.99 & 4.89 \\
\hline$P$ & 0.98 & 0.68 & 1.66 & 0.93 & 0.69 & 1.62 & 1.00 & 0.86 & 1.86 & 0.91 & \begin{tabular}{|l|}
0.82 \\
\end{tabular} & 1.73 & 0.92 & 0.80 & 1.72 \\
\hline$\overline{\mathrm{Pb}}$ & 1.24 & 0.58 & 1.82 & 1.20 & 0.59 & 1.79 & 1.24 & 0.61 & 1.85 & 1.14 & \begin{tabular}{|l|}
0.63 \\
\end{tabular} & 1.77 & 1.13 & 0.62 & 1.7 \\
\hline $\mathrm{Sb}$ & 0.89 & 0.40 & 1.29 & 0.89 & 0.40 & 1.29 & 0.97 & 0.35 & 1.32 & 1.09 & 0.36 & 1.45 & 1.00 & 0.34 & 1.3 \\
\hline $\mathrm{Se}$ & 22.16 & 189.0 & 211.2 & 22.66 & 192.8 & 215.5 & 26.01 & $\mid 206.1$ & 232.1 & 25.32 & 211.9 & 237.2 & 24.51 & 221.9 & 246. \\
\hline $\mathrm{Si}$ & 2.07 & 46.06 & \begin{tabular}{|l|}
108.1 \\
\end{tabular} & 57.20 & 47.20 & \begin{tabular}{|l|}
104.4 \\
\end{tabular} & \begin{tabular}{|l|}
58.23 \\
\end{tabular} & 49.92 & \begin{tabular}{|l|}
108.2 \\
\end{tabular} & 63.31 & 49.27 & 112.6 & 64.17 & 48.08 & 112.3 \\
\hline $\mathrm{Sr}$ & 1.00 & 32.49 & 33.49 & 1.04 & 32.91 & 33.95 & \begin{tabular}{|l|}
1.18 \\
\end{tabular} & 32.43 & 33.61 & 1.26 & 32.29 & 33.55 & 1.21 & 32.71 & 33.9 \\
\hline $\mathrm{Te}$ & 21.25 & \begin{tabular}{|l|}
1.34 \\
\end{tabular} & 22.59 & 20.07 & 1.51 & 21.58 & 20.08 & 1.67 & 21.75 & 19.05 & 1.83 & 20.88 & 18.43 & 1.95 & 20.3 \\
\hline $\mathrm{Ti}$ & 35 & $<0.01$ & \begin{tabular}{l|l}
0.35 \\
\end{tabular} & 0.32 & 0.01 & 0.33 & 0.37 & $<0.01$ & 0.37 & 0.38 & $<0.01$ & 0.38 & 0.38 & 0.01 & 0.3 \\
\hline $\mathrm{Zn}$ & 3.04 & \begin{tabular}{|l|}
26.47 \\
\end{tabular} & 29.51 & 2.75 & \begin{tabular}{|l|}
26.93 \\
\end{tabular} & 29.68 & 2.71 & \begin{tabular}{|l|}
27.87 \\
\end{tabular} & 30.58 & 2.90 & 28.33 & 31.23 & 2.96 & 28.40 & 31.36 \\
\hline $\mathrm{Zr}$ & 5.18 & 0.02 & 5.20 & 4.96 & 0.02 & 4.98 & 5.39 & 0.02 & 5.41 & 4.75 & 0.02 & 4.77 & 4.57 & 0.02 & 4.5 \\
\hline $\mathrm{Cl}$ & NA & 86.33 & 86.33 & $\mathrm{NA}$ & 99.61 & 99.61 & NA & 96.15 & 96.15 & $\mathrm{NA}$ & 113.5 & 113.5 & NA & \begin{tabular}{|l|}
85.75 \\
\end{tabular} & 85.7 \\
\hline $\mathrm{F}$ & NA & 78.55 & 78.55 & NA & 80.87 & 80.87 & NA & 78.16 & 78.16 & $\mathrm{NA}$ & 92.09 & 92.09 & $\mathrm{NA}$ & 65.78 & 65.7 \\
\hline I & $<0.1$ & 382.8 & 382.8 & $<0.1$ & 341.0 & 341 & NA & 322.9 & 322.9 & $<0.1$ & \begin{tabular}{|l|}
286.3 \\
\end{tabular} & 286.3 & $<0.1$ & 262.0 & 262 \\
\hline Nitrate & $\mathrm{NA}$ & 16.89 & 16.89 & $\mathrm{NA}$ & \begin{tabular}{|l|}
18.38 \\
\end{tabular} & \begin{tabular}{|c|}
18.38 \\
\end{tabular} & NA & 14.22 & 14.22 & $\mathrm{NA}$ & 23.28 & 23.28 & NA & 32.96 & 32.96 \\
\hline Nitrite & NA & 6.61 & 6.61 & NA & 4.95 & 4.95 & NA & 3.05 & 3.05 & NA & 4.71 & 4.71 & $\mathrm{NA}$ & 3.88 & 3.88 \\
\hline Sulfate & 0.64 & $\mid 450.1$ & \begin{tabular}{|l|}
450.7 \\
\end{tabular} & 1.01 & 465.0 & 466.0 & NA & 436.7 & 4 & 2.15 & 481.4 & \begin{tabular}{|l|}
483.5 \\
\end{tabular} & 2.28 & 350.4 & 352.7 \\
\hline
\end{tabular}

* Suspended

\# Dissolved

NA - Not Analyzed 
Table 4.6. Analytical Results for Samples of SBS Blow-Down Fluids (mg/l), (continued).

\begin{tabular}{|c|c|c|c|c|c|c|c|c|c|c|c|c|c|c|c|}
\hline Test & \multicolumn{3}{|c|}{4} & \multicolumn{12}{|c|}{5} \\
\hline I.D. & \multicolumn{3}{|c|}{$12 \mathrm{~K}-\mathrm{S}-136 \mathrm{~A}$} & \multicolumn{3}{|c|}{$12 \mathrm{~L}-\mathrm{S}-16 \mathrm{~A}$} & \multicolumn{3}{|c|}{$12 \mathrm{~L}-\mathrm{S}-21 \mathrm{~A}$} & \multicolumn{3}{|c|}{$12 \mathrm{~L}-\mathrm{S}-28 \mathrm{~A}$} & \multicolumn{3}{|c|}{$12 \mathrm{~L}-\mathrm{S}-37 \mathrm{~A}$} \\
\hline Glass (kg) & \multicolumn{3}{|c|}{14449} & \multicolumn{3}{|c|}{14643} & \multicolumn{3}{|c|}{14746} & \multicolumn{3}{|c|}{14849} & \multicolumn{3}{|c|}{14952} \\
\hline $\mathrm{pH}$ & \multicolumn{3}{|c|}{$\mathrm{NA}$} & \multicolumn{3}{|c|}{8.04} & \multicolumn{3}{|c|}{2.78} & \multicolumn{3}{|c|}{2.47} & \multicolumn{3}{|c|}{2.20} \\
\hline & Sus* & Dis.\# & Total & Sus* & Dis.\# & Total & Sus* & Dis.\# & Total & Sus* & Dis.\# & Total & Sus* & Dis.\# & Tot: \\
\hline Total & 448 & 1036 & 1484 & 80 & 268 & 348 & 110 & 528 & 638 & 78 & 688 & 766 & 44 & 972 & 1016 \\
\hline $\mathrm{Al}$ & 18.51 & 40.25 & 58.76 & 4.04 & 0.30 & 4.34 & 3.58 & 7.00 & 10.58 & 1.98 & 9.00 & 10.98 & 0.76 & 16.55 & 17.31 \\
\hline As & 0.53 & 0.78 & 1.31 & 0.25 & 0.14 & 0.39 & 0.16 & 0.13 & 0.29 & 0.03 & 0.27 & 0.30 & 0.09 & 0.14 & 0.23 \\
\hline B & 0.76 & 141.7 & 142.5 & 0.23 & 9.17 & 9.40 & 0.14 & 35.10 & 35.24 & 0.10 & 50.51 & 50.61 & 0.05 & 75.78 & 75.83 \\
\hline $\mathrm{Ba}$ & 0.24 & 0.06 & 0.30 & 0.03 & 0.05 & 0.08 & 0.01 & 0.26 & 0.27 & 0.01 & 0.34 & 0.35 & $<0.01$ & 0.51 & 0.51 \\
\hline $\mathrm{Ca}$ & 0.12 & 4.50 & 4.62 & $\overline{0.54}$ & 30.40 & 30.94 & 0.07 & 31.63 & 31.7 & 0.03 & 30.97 & 31.00 & 0.02 & 31.99 & 32.01 \\
\hline $\mathrm{Cd}$ & 1.48 & 5.26 & 6.74 & 0.30 & 0.04 & 0.34 & 0.07 & 2.08 & 2.15 & 0.07 & 2.95 & 3.02 & 0.03 & 4.49 & 4.52 \\
\hline $\mathrm{Cr}$ & 1.15 & 0.18 & 1.33 & 0.13 & 0.03 & 0.16 & 0.16 & 0.14 & 0.30 & 0.13 & 0.21 & 0.34 & 0.07 & 0.40 & 0.47 \\
\hline $\mathrm{Cs}$ & 0.15 & 6.80 & 6.95 & $<0.1$ & 0.54 & 0.54 & $<0.1$ & 1.09 & 1.09 & $<0.1$ & 1.34 & 1.34 & $<0.1$ & 2.22 & 2.22 \\
\hline $\mathrm{Cu}$ & 0.08 & 0.25 & 0.33 & 0.02 & $<0.1$ & 0.02 & 0.02 & 0.08 & 0.1 & 0.02 & 0.12 & 0.14 & 0.01 & 0.17 & 0.18 \\
\hline $\mathrm{Fe}$ & 88.19 & 0.05 & 88.24 & 8.97 & 0.01 & 8.98 & 18.02 & 0.99 & \begin{tabular}{|l|}
19.01 \\
\end{tabular} & 18.85 & 2.27 & 21.12 & 10.46 & 5.21 & 15.67 \\
\hline $\mathrm{K}$ & 0.19 & 4.83 & 5.02 & $\overline{0.04}$ & 3.63 & 3.67 & 0.04 & 4.73 & 4.77 & 0.04 & 4.07 & 4.11 & 0.03 & 7.96 & 1.9 \\
\hline $\mathrm{Li}$ & 0.94 & 35.78 & 36.72 & 0.15 & 4.48 & 4.63 & 0.26 & 13.04 & 13.3 & 0.19 & 16.40 & 16.59 & 0.09 & 25.34 & 25.43 \\
\hline $\mathrm{Mg}$ & 1.76 & 16.11 & 17.87 & 0.28 & 10.51 & 10.79 & 0.22 & 12.67 & 12.89 & 0.16 & 13.55 & 13.71 & 0.08 & 15.84 & 15.92 \\
\hline $\mathrm{Mn}$ & 2.09 & 8.59 & 10.68 & 0.78 & 0.31 & 1.09 & 0.35 & 2.84 & 3.19 & 0.17 & 4.45 & 4.62 & 0.08 & 7.30 & 7.38 \\
\hline $\mathrm{Na}$ & 1.35 & 64.93 & 66.28 & 0.36 & 27.10 & 27.46 & 0.20 & 43.81 & 44.01 & 0.14 & 50.10 & 50.24 & 0.06 & 88.37 & 88.43 \\
\hline $\mathrm{Ni}$ & 3.99 & 1.08 & 5.07 & 0.59 & 0.10 & 0.69 & 0.91 & 0.60 & 1.51 & 0.69 & 0.84 & 1.53 & 0.33 & 1.70 & 2.03 \\
\hline $\mathrm{P}$ & 0.88 & 1.09 & 1.97 & 0.17 & $<0.07$ & 0.17 & 0.27 & 0.21 & 0.48 & 0.31 & 0.33 & 0.64 & 0.16 & 0.29 & 0.45 \\
\hline $\mathrm{Pb}$ & 1.15 & 0.54 & 1.69 & 0.14 & 0.04 & 0.18 & 0.14 & 0.25 & 0.39 & 0.08 & 0.47 & 0.55 & 0.05 & 0.87 & 0.92 \\
\hline $\mathrm{Sb}$ & 0.92 & 0.18 & 1.10 & 0.19 & $<0.03$ & 0.19 & 0.23 & $<0.03$ & 0.23 & 0.17 & $<0.03$ & 0.17 & 0.08 & $<0.03$ & \\
\hline $\mathrm{Se}$ & 27.63 & 208.8 & 236.4 & 2.20 & 33.81 & 36.01 & 4.04 & 35.45 & 39.49 & 2.79 & 37.91 & 40.7 & 1.27 & 45.70 & 46.97 \\
\hline $\mathrm{Si}$ & 62.90 & 44.26 & 107.2 & 14.50 & 3.52 & 18.02 & 18.94 & 6.91 & 25.85 & 10.59 & 7.59 & 18.18 & 4.69 & 9.13 & 13.82 \\
\hline $\mathrm{Sr}$ & 1.47 & 28.96 & 30.43 & 0.20 & 3.01 & 3.21 & 0.07 & 7.60 & 7.67 & 0.04 & 9.78 & 9.82 & 0.02 & 14.86 & 14.88 \\
\hline $\mathrm{Te}$ & 19.43 & 2.33 & 21.76 & 2.28 & 0.26 & 2.54 & 2.72 & 1.06 & 3.78 & 1.87 & 2.14 & 4.01 & 0.86 & 4.24 & 5.1 \\
\hline$\overline{\mathrm{Ti}}$ & 0.43 & 0.03 & 0.46 & 0.07 & $<0.01$ & 0.07 & 0.12 & 0.01 & 0.13 & 0.09 & 0.01 & 0.10 & 0.06 & 0.01 & 0.07 \\
\hline $\mathrm{Zn}$ & 2.86 & 28.46 & 31.32 & 0.85 & 0.03 & 0.88 & 0.36 & 5.20 & 5.56 & 0.27 & 7.06 & 7.33 & 0.14 & 11.42 & 11.56 \\
\hline $\mathrm{Zr}$ & 4.66 & 0.02 & 4.68 & 1.60 & $<0.01$ & 1.60 & 3.15 & 0.07 & 3.22 & 2.57 & 0.16 & 2.73 & 1.59 & 0.73 & 2.32 \\
\hline $\mathrm{Cl}$ & NA & 116.4 & 116.4 & $\mathrm{NA}$ & 38.09 & 38.09 & NA & $\mathrm{NA}$ & NA & $\mathrm{NA}$ & NA & NA & $\mathrm{NA}$ & NA & \\
\hline $\mathrm{F}$ & NA & 82.34 & 82.34 & NA & 5.15 & 5.15 & $\mathrm{NA}$ & NA & NA & NA & NA & NA & NA & NA & \\
\hline I & NA & 224.0 & 224.0 & $\mathrm{NA}$ & 0.61 & 0.61 & NA & NA & NA & $\mathrm{NA}$ & NA & NA & NA & NA & \\
\hline Nitrate & NA & 48.99 & 48.99 & NA & 2.44 & 2.44 & NA & NA & NA & NA & NA & NA & NA & $\mathrm{NA}$ & \\
\hline Nitrite & NA & $<0.1$ & $<0.1$ & $\mathrm{NA}$ & $<0.1$ & $<0.1$ & NA & $\mathrm{NA}$ & NA & $\mathrm{NA}$ & NA & $\mathrm{NA}$ & NA & NA & $\overline{N A}$ \\
\hline Sulfate & NA & 446.7 & 446.7 & $\mathrm{NA}$ & 107.1 & 107.1 & NA & $\mathrm{NA}$ & $\mathrm{NA}$ & $\mathrm{NA}$ & $\mathrm{NA}$ & NA & NA & $\mathrm{NA}$ & \\
\hline
\end{tabular}

* Suspended

\# Dissolved

NA - Not Analyzed 
Table 4.6. Analytical Results for Samples of SBS Blow-Down Fluids (mg/l), (continued).

\begin{tabular}{|c|c|c|c|c|c|c|c|c|c|c|c|c|c|c|c|}
\hline Test & \multicolumn{15}{|c|}{5} \\
\hline I.D. & \multicolumn{3}{|c|}{$12 \mathrm{~L}-\mathrm{S}-41 \mathrm{~A}$} & \multicolumn{3}{|c|}{$12 \mathrm{~L}-\mathrm{S}-50 \mathrm{~A}$} & \multicolumn{3}{|c|}{$12 \mathrm{~L}-\mathrm{S}-58 \mathrm{~A}$} & \multicolumn{3}{|c|}{$12 \mathrm{~L}-\mathrm{S}-62 \mathrm{~A}$} & \multicolumn{3}{|c|}{$12 \mathrm{~L}-\mathrm{S}-78 \mathrm{~A}$} \\
\hline Glass (kg) & \multicolumn{3}{|c|}{15056} & \multicolumn{3}{|c|}{15230} & \multicolumn{3}{|c|}{15404} & \multicolumn{3}{|c|}{15578} & \multicolumn{3}{|c|}{15641} \\
\hline $\mathrm{pH}$ & \multicolumn{3}{|c|}{1.90} & \multicolumn{3}{|c|}{1.69} & \multicolumn{3}{|c|}{1.47} & \multicolumn{3}{|c|}{1.37} & \multicolumn{3}{|c|}{1.13} \\
\hline & Sus* & Dis.\# & Total & Sus* & Dis.\# & Total & Sus* & Dis.\# & Total & Sus* & Dis.\# & Total & Sus* & Dis.\# & Total \\
\hline Total & 68 & 1256 & 1324 & 92 & 1872 & 1964 & 102 & 2172 & 2274 & 86 & 2512 & 2598 & 430 & 2628 & 3058 \\
\hline $\mathrm{Al}$ & 1.07 & 21.21 & 22.28 & 1.30 & 30.00 & 31.3 & 1.42 & 33.02 & 34.44 & 1.15 & 38.97 & 40.12 & 9.61 & 54.57 & 64.18 \\
\hline As & 0.14 & 0.25 & 0.39 & 0.07 & 0.39 & 0.46 & 0.05 & 0.52 & 0.57 & 0.03 & 0.51 & 0.54 & 0.14 & 0.63 & 0.77 \\
\hline $\mathrm{B}$ & 0.07 & 106.8 & 106.9 & 0.08 & 135.6 & 135.7 & 0.07 & 165.5 & 165.6 & 0.05 & 187.4 & 187.5 & 0.27 & 233.4 & 233.7 \\
\hline $\mathrm{Ba}$ & $<0.01$ & 0.65 & 0.65 & $<0.01$ & 0.86 & 0.86 & $<0.01$ & 1.02 & 1.02 & $<0.01$ & 1.15 & 1.15 & 0.02 & 1.38 & 1.40 \\
\hline $\mathrm{Ca}$ & 0.03 & 30.92 & 30.95 & 0.03 & 29.64 & 29.67 & 0.03 & 28.16 & 28.19 & 0.03 & 25.63 & 25.66 & 0.11 & 27.56 & 27.67 \\
\hline $\mathrm{Cd}$ & 0.03 & 5.44 & 5.47 & 0.06 & 6.74 & 6.80 & 0.06 & 7.44 & 7.50 & 0.05 & 8.05 & 8.10 & 0.18 & 8.66 & 8.84 \\
\hline $\mathrm{Cr}$ & 0.10 & 0.61 & 0.71 & 0.12 & 0.79 & 0.91 & 0.13 & 0.89 & 1.02 & 0.10 & 0.94 & 1.04 & 0.44 & 0.98 & 1.42 \\
\hline$\overline{\mathrm{Cs}}$ & $<0.1$ & 2.40 & 2.40 & $<0.1$ & 3.00 & 3.00 & $<0.1$ & 3.30 & 3.30 & $<0.1$ & 3.50 & 3.50 & $<0.1$ & 4.00 & 4.00 \\
\hline$\overline{\mathrm{Cu}}$ & 0.02 & 0.23 & 0.25 & 0.02 & 0.29 & 0.31 & 0.03 & 0.33 & 0.36 & 0.02 & 0.36 & 0.38 & 0.04 & 0.41 & $\overline{0.45}$ \\
\hline $\mathrm{Fe}$ & 17.44 & 9.01 & 26.45 & 24.03 & 13.19 & 37.22 & 27.65 & 16.43 & 44.08 & 23.60 & 18.54 & 42.14 & 88.05 & 23.26 & 111.3 \\
\hline $\mathrm{K}$ & 0.04 & 5.45 & 5.49 & 0.05 & 6.39 & 6.44 & 0.05 & 7.02 & 7.07 & 0.04 & 7.29 & 7.33 & 0.16 & 18.07 & 18.23 \\
\hline $\mathrm{Li}$ & 0.12 & 29.95 & 30.07 & 0.15 & 41.05 & 41.2 & 0.15 & 49.23 & 49.38 & 0.11 & 56.37 & 56.48 & 0.47 & 66.24 & 66.71 \\
\hline $\mathrm{Mg}$ & 0.11 & \begin{tabular}{|l|}
18.18 \\
\end{tabular} & 18.29 & 0.13 & 21.66 & 21.79 & 0.12 & 23.87 & 23.99 & 0.09 & 24.87 & 24.96 & 0.35 & 27.27 & 27.62 \\
\hline $\mathrm{Mn}$ & 0.11 & 11.63 & 11.74 & 0.14 & 18.26 & 18.4 & 0.14 & 23.59 & 23.73 & 0.10 & 27.35 & 27.45 & 0.37 & 29.95 & 30.32 \\
\hline $\mathrm{Na}$ & 0.08 & 82.15 & 82.23 & 0.07 & 104.6 & 104.7 & 0.09 & 106.7 & 106.8 & 0.07 & 113.2 & 113.3 & 0.31 & 131.7 & 132.0 \\
\hline $\mathrm{Ni}$ & 0.42 & 2.40 & 2.82 & 0.45 & 3.45 & 3.90 & 0.38 & 4.45 & 4.83 & 0.25 & 4.67 & 4.92 & 1.09 & 7.32 & 8.41 \\
\hline$P$ & 0.30 & 0.62 & 0.92 & 0.36 & 0.89 & 1.25 & 0.36 & 1.13 & 1.49 & 0.27 & 1.45 & 1.72 & 1.14 & 1.85 & 2.99 \\
\hline $\mathrm{Pb}$ & 0.05 & 1.51 & 1.56 & 0.02 & 2.27 & 2.29 & 0.01 & 2.76 & 2.77 & $<0.01$ & 3.16 & 3.16 & 0.17 & 3.75 & 3.96 \\
\hline $\mathrm{Sb}$ & 0.11 & $<0.03$ & 0.11 & 0.11 & $<0.03$ & 0.11 & 0.07 & $<0.03$ & 0.07 & 0.05 & $<0.03$ & 0.05 & 0.56 & 0.11 & 0.67 \\
\hline $\mathrm{Se}$ & 1.90 & 51.88 & 53.78 & 2.16 & 64.45 & 66.61 & 2.19 & 74.68 & 76.87 & 1.88 & 84.16 & 86.04 & 7.37 & 102.9 & 110.3 \\
\hline $\mathrm{Si}$ & 7.20 & 10.21 & 17.41 & 9.65 & 12.10 & 21.75 & 11.29 & 13.33 & 24.62 & 9.63 & 13.90 & 23.53 & 78.79 & 15.95 & 94.74 \\
\hline $\mathrm{Sr}$ & 0.02 & 20.40 & 20.42 & 0.03 & 28.26 & 28.29 & 0.03 & 26.97 & 27.00 & 0.02 & 30.71 & 30.73 & 0.14 & 36.12 & 36.26 \\
\hline $\mathrm{Te}$ & 1.06 & 7.52 & 8.58 & 1.00 & 8.24 & 9.24 & 0.60 & 11.29 & 11.89 & 0.40 & 9.68 & 10.08 & 1.60 & 10.45 & 12.05 \\
\hline $\mathrm{Ti}$ & 0.09 & 0.03 & 0.12 & 0.12 & 0.06 & 0.18 & 0.13 & 0.08 & 0.21 & 0.10 & 0.11 & 0.21 & 0.46 & 0.18 & 0.64 \\
\hline $\mathrm{Zn}$ & 0.20 & 15.49 & 15.69 & 0.24 & 20.22 & 20.46 & 0.25 & 24.75 & 25.00 & 0.19 & 27.60 & 27.79 & 0.71 & 32.03 & 32.74 \\
\hline $\mathrm{Zr}$ & 2.41 & 1.98 & 4.39 & 2.95 & 4.22 & 7.17 & 2.57 & 6.84 & 9.41 & 1.94 & 9.31 & 11.25 & 9.29 & 16.82 & 26.11 \\
\hline $\mathrm{Cl}$ & NA & NA & NA & NA & $\mathrm{NA}$ & NA & NA & $\mathrm{NA}$ & NA & NA & 24.95 & 24.95 & NA & $\mathrm{NA}$ & $\mathrm{NC}$ \\
\hline$F$ & NA & NA & NA & NA & $\mathrm{NA}$ & NA & NA & NA & NA & $\mathrm{NA}$ & $<0.1$ & $<0.1$ & $\mathrm{NA}$ & NA & $\mathrm{NC}$ \\
\hline I & NA & NA & NA & NA & NA & NA & NA & $\mathrm{NA}$ & NA & NA & 11.83 & 11.83 & $<0.1$ & NA & $\mathrm{NC}$ \\
\hline Nitrate & NA & $\mathrm{NA}$ & $\mathrm{NA}$ & NA & $\mathrm{NA}$ & NA & $\mathrm{NA}$ & NA & $\mathrm{NA}$ & NA & 7175 & 7175 & NA & NA & $\overline{\mathrm{NA}}$ \\
\hline Nitrite & NA & NA & NA & NA & NA & NA & NA & NA & $\mathrm{NA}$ & NA & 2.97 & 2.97 & $\mathrm{NA}$ & NA & $\widehat{N A}$ \\
\hline Sulfate & NA & NA & NA & $\mathrm{NA}$ & $\mathrm{NA}$ & NA & $\mathrm{NA}$ & $\mathrm{NA}$ & NA & NA & 91.49 & 91.49 & NA & NA & $\mathrm{NC}$ \\
\hline Sum & NA & $\mathrm{NA}$ & NA & NA & $\mathrm{NA}$ & NA & $\mathrm{NA}$ & $\mathrm{NA}$ & NA & $\mathrm{NA}$ & 24.95 & 24.95 & 1.50 & $\mathrm{NA}$ & $\mathrm{NC}$ \\
\hline
\end{tabular}

* Suspended

\# Dissolved

NA - Not Analyzed

NC - Not Calculated 
Table 4.6. Analytical Results for Samples of SBS Blow-Down Fluids (mg/l), (continued).

\begin{tabular}{|c|c|c|c|c|c|c|c|c|c|c|c|c|c|c|c|}
\hline Test & \multicolumn{15}{|c|}{5} \\
\hline I.D. & \multicolumn{3}{|c|}{$12 \mathrm{~L}-\mathrm{S}-80 \mathrm{~A}$} & \multicolumn{3}{|c|}{$12 \mathrm{~L}-\mathrm{S}-88 \mathrm{~A}$} & \multicolumn{3}{|c|}{ 12L-S-95A } & \multicolumn{3}{|c|}{$12 \mathrm{~L}-\mathrm{S}-107 \mathrm{~A}$} & \multicolumn{3}{|c|}{$12 \mathrm{~L}-\mathrm{S}-109 \mathrm{~A}$} \\
\hline Glass (kg) & \multicolumn{3}{|c|}{15704} & \multicolumn{3}{|c|}{15767} & \multicolumn{3}{|c|}{15829} & \multicolumn{3}{|c|}{15892} & \multicolumn{3}{|c|}{16049} \\
\hline $\mathrm{pH}$ & \multicolumn{3}{|c|}{1.12} & \multicolumn{3}{|c|}{1.09} & \multicolumn{3}{|c|}{1.10} & \multicolumn{3}{|c|}{1.04} & \multicolumn{3}{|c|}{1.05} \\
\hline & Sus* & Dis.\# & Total & Sus* & Dis.\# & Total & Sus* & Dis. \# & Total & Sus* & Dis.\# & Total & Sus* & Dis.\# & 1010 \\
\hline Total & 470 & 2840 & 3310 & 501 & 3256 & 3757 & 1004 & 3176 & 4180 & 956 & 3176 & 4132 & 1024 & 1400 & 242 \\
\hline$\overline{\mathrm{Al}}$ & 9.76 & 60.84 & 70.6 & 10.16 & 2.04 & 12.2 & 19.43 & 72.26 & 91.69 & 17.32 & 75.84 & 93.16 & 19.09 & 80.20 & 99.29 \\
\hline As & 0.14 & 0.90 & 1.04 & 0.12 & 0.90 & 1.02 & 0.23 & 0.70 & 0.93 & $<0.01$ & 0.67 & 0.67 & 0.41 & 0.70 & 1.11 \\
\hline B & 0.30 & 256.6 & 256.9 & 0.32 & 15.66 & 15.98 & 0.70 & 301.9 & 302.6 & 0.59 & 296.7 & 297.3 & 0.64 & 391.5 & 392. \\
\hline $\mathrm{Ba}$ & 0.02 & 1.48 & 1.50 & 0.02 & 1.72 & 1.74 & 0.04 & 1.88 & 1.92 & 0.04 & 1.93 & 1.97 & 0.04 & 2.09 & 2.13 \\
\hline $\mathrm{Ca}$ & 0.10 & 27.06 & 27.16 & 0.12 & 23.73 & 23.85 & 0.19 & 25.87 & 26.06 & 0.19 & 24.32 & 24.51 & 0.21 & 23.90 & 24.1 \\
\hline $\mathrm{Cd}$ & 0.21 & 9.49 & 9.70 & 0.20 & 10.57 & 10.77 & 0.44 & 11.14 & 11.58 & 0.40 & 11.51 & 11.91 & 0.45 & 12.53 & 12.9 \\
\hline $\mathrm{Cr}$ & 0.55 & 1.02 & 1.57 & 0.54 & 1.10 & 1.64 & 1.11 & 1.13 & 2.24 & 1.10 & 1.16 & 2.26 & 1.08 & 1.17 & 2.2 \\
\hline $\mathrm{Cs}$ & $<0.1$ & 5.50 & 5.50 & $<0.1$ & 5.50 & 5.50 & $<0.1$ & 6.40 & 6.4 & $<0.1$ & 6.20 & 6.20 & $<0.1$ & 6.80 & 6.8 \\
\hline $\mathrm{Cu}$ & 0.05 & 0.47 & 0.52 & 0.08 & 0.51 & 0.59 & 0.13 & 0.51 & 0.64 & 0.16 & 0.52 & 0.68 & 0.14 & 0.56 & 0.7 \\
\hline $\mathrm{Fe}$ & 109.1 & 25.56 & 134.7 & 112.9 & 25.55 & 138.5 & 233.6 & 28.72 & 262.3 & 213.9 & 29.00 & 242.9 & 222.2 & 29.58 & 251. \\
\hline $\mathrm{K}$ & 0.18 & 8.45 & 8.63 & 0.18 & 9.39 & 9.57 & 0.36 & 11.00 & 11.36 & 0.30 & 11.61 & 11.91 & $0 . \overline{36}$ & 12.34 & 12. \\
\hline$\overline{\mathrm{Li}}$ & 0.53 & 75.28 & 75.81 & 0.58 & 3.01 & 3.59 & 1.19 & 92.19 & 93.38 & 1.13 & 97.21 & 98.34 & 1.21 & 103.9 & 105. \\
\hline$\overline{\mathrm{Mg}}$ & 0.38 & 24.36 & 24.74 & 0.40 & 1.53 & 1.93 & 0.81 & 26.62 & 27.43 & 0.75 & 27.27 & 28.02 & 0.79 & 28.14 & 28.93 \\
\hline $\mathrm{Mn}$ & 0.39 & 31.53 & 31.92 & 0.44 & 1.67 & 2.11 & 0.95 & 34.73 & 35.68 & 0.86 & 36.26 & 37.12 & 0.93 & 38.38 & 39.3 \\
\hline $\mathrm{Na}$ & 0.32 & 147.3 & 147.6 & 0.46 & 5.61 & 6.07 & 0.72 & 186.9 & 187.6 & 0.71 & 186.7 & 187.4 & 0.72 & 228.2 & 228.9 \\
\hline $\mathrm{Ni}$ & 1.18 & 8.36 & 9.54 & 1.19 & 8.89 & 10.08 & 2.52 & 10.14 & 12.66 & 2.40 & 10.46 & 12.86 & 2.68 & 10.91 & 13.59 \\
\hline $\mathrm{P}$ & 1.15 & 2.20 & 3.35 & 1.33 & 2.22 & 3.55 & 2.75 & 2.64 & 5.39 & 1.62 & 2.79 & 4.41 & 3.11 & 2.77 & 5.88 \\
\hline$\overline{\mathrm{Pb}}$ & 0.16 & 4.22 & 4.38 & 0.18 & 4.59 & 4.77 & 0.33 & 4.32 & 4.65 & 0.36 & 4.41 & 4.77 & 0.34 & 4.71 & 5.05 \\
\hline $\mathrm{Sb}$ & 0.57 & 0.12 & 0.69 & 0.71 & 0.15 & 0.86 & 1.06 & $<0.03$ & 1.06 & 0.94 & $<0.03$ & 0.94 & 0.58 & 0.08 & 0.66 \\
\hline $\mathrm{Se}$ & 7.67 & 117.3 & 125.0 & 8.37 & 121.1 & 129.5 & 16.97 & 125.4 & 142.4 & 16.26 & 135.0 & 151.3 & 17.93 & 135.4 & 153.3 \\
\hline $\mathrm{Si}$ & 9.53 & 17.75 & 97.28 & 86.45 & 19.86 & 106.3 & 165.6 & 21.78 & 187.4 & 174.8 & 22.06 & 196.9 & 160.6 & 23.31 & 183.9 \\
\hline $\mathrm{Sr}$ & 0.15 & 40.07 & 40.22 & 0.16 & 2.03 & 2.19 & 0.34 & 47.65 & 47.99 & 0.32 & 50.30 & 50.62 & 0.35 & 53.36 & 53.71 \\
\hline $\mathrm{Te}$ & 1.59 & 11.25 & 12.84 & 1.61 & 10.70 & 12.31 & 2.86 & 9.20 & 12.06 & 2.82 & 9.38 & 12.2 & 3.02 & 9.53 & 12.55 \\
\hline $\mathrm{Ti}$ & 0.54 & 0.24 & 0.78 & 0.55 & 0.26 & 0.81 & 1.13 & 0.28 & 1.41 & 1.08 & 0.29 & 1.37 & 1.16 & 0.29 & 1.45 \\
\hline $\mathrm{Zn}$ & 0.78 & 37.12 & 37.9 & 0.84 & 43.68 & 44.52 & 1.68 & 42.20 & 43.88 & 1.59 & 44.90 & 46.49 & 1.65 & 47.61 & 49.26 \\
\hline $\mathrm{Zr}$ & 9.92 & 21.39 & 31.31 & 11.10 & 21.40 & 32.5 & 22.03 & 24.97 & 47.00 & 21.20 & 25.34 & 46.54 & 23.70 & 26.81 & 50.51 \\
\hline $\mathrm{Cl}$ & NA & NA & NA & NA & $\mathrm{NA}$ & NA & NA & $\mathrm{NA}$ & NA & NA & NA & NA & $\mathrm{NA}$ & NA & $\mathrm{N}_{1}$ \\
\hline$F$ & $\mathrm{NA}$ & NA & NA & NA & $\mathrm{NA}$ & NA & NA & NA & NA & NA & $\mathrm{NA}$ & NA & NA & NA & $\mathrm{N}$ \\
\hline I & $<0.1$ & NA & $\mathrm{NQ}$ & $<0.1$ & $\mathrm{NA}$ & $\mathrm{NC}$ & $<0.1$ & NA & $\mathrm{NQ}$ & $<0.1$ & NA & $\mathrm{NQ}$ & $<0.1$ & NA & 18 \\
\hline Nitrate & NA & NA & NA & NA & NA & NA & $\mathrm{NA}$ & NA & NA & NA & NA & $\mathrm{NA}$ & NA & $\mathrm{NA}$ & \\
\hline Nitrite & NA & NA & NA & NA & $\mathrm{NA}$ & NA & NA & $\mathrm{NA}$ & NA & $\mathrm{NA}$ & NA & NA & NA & $\mathrm{NA}$ & $\mathrm{NA}$ \\
\hline Sulfate & 0.88 & $\mathrm{NA}$ & $\mathrm{NC}$ & 1.24 & $\mathrm{NA}$ & $\mathrm{NO}$ & 2.53 & $\mathrm{NA}$ & $\mathrm{NC}$ & $<0.1$ & $\mathrm{NA}$ & $\mathrm{NC}$ & $<0.1$ & $\mathrm{NA}$ & 170 \\
\hline
\end{tabular}

* Suspended

\# Dissolved

NA - Not Analyzed

NC - Not Calculated 
The Catholic University of America Vitreous State Laboratory
DuraMelter 1200 Tests with AZ-101HLW Simulants

Final Report, VSL-02R0100-2, Rev.1

Table 4.6. Analytical Results for Samples of SBS Blow-Down Fluids (mg/), (continued).

\begin{tabular}{|c|c|c|c|c|c|c|c|c|c|c|c|c|c|c|c|}
\hline Test & \multicolumn{3}{|c|}{5} & \\
\hline I.D. & & L-S-116 & & & C-S-14 & & & L-S-150 & & $12 \mathrm{~L}$ & -SS-15 & $5 A$ & & M-S-7 & \\
\hline Glass (kg) & & 16199 & & & 16283 & & & 16367 & & & 16452 & & & 16536 & \\
\hline $\mathrm{pH}$ & & 1.05 & & & 4.27 & & & 7.13 & & & 6.89 & & & 6.71 & \\
\hline & Sus* & Dis.\# & Total & Sus* & Dis.\# & \begin{tabular}{|l|} 
Total \\
\end{tabular} & Sus* & Dis.\# & Total & Sus* & Dis.肕 & Total & Sus* & Dis.井 & Tota \\
\hline Total & 1076 & 3356 & 4432 & 50 & 284 & \begin{tabular}{|l|}
334 \\
\end{tabular} & 214 & 284 & 498 & 256 & 348 & 604 & 260 & 288 & 54 \\
\hline $\mathrm{Al}$ & 19.56 & 82.36 & \begin{tabular}{|l|}
101.9 \\
\end{tabular} & 1.51 & 2.82 & 4.33 & 11.44 & 0.31 & 11.75 & 11.94 & 1.22 & 13.16 & 12.06 & 3.54 & 15. \\
\hline$\overline{\mathrm{As}}$ & 0.30 & 0.70 & 1.00 & 0.03 & $<0.05$ & 0.03 & $<0.01$ & $<0.05$ & $<0.05$ & 0.12 & $<0.05$ & 0.12 & 0.18 & $<0.05$ & 0.18 \\
\hline $\mathrm{B}$ & 0.70 & 393.6 & \begin{tabular}{|c|}
394.3 \\
\end{tabular} & 0.06 & 12.92 & 12.98 & 2.23 & 22.66 & 24.89 & 2.73 & 31.20 & 33.93 & 2.61 & 40.11 & 42.72 \\
\hline $\mathrm{Ba}$ & 0.05 & 2.06 & 2.11 & $<0.01$ & 0.07 & \begin{tabular}{|l|}
0.07 \\
\end{tabular} & 0.06 & 0.07 & 0.13 & 0.07 & 0.07 & 0.14 & 0.07 & 0.07 & 0.14 \\
\hline$\overline{\mathrm{Ca}}$ & 0.24 & 22.76 & 23.00 & 0.06 & 29.16 & 29.22 & 1.09 & 26.64 & 27.73 & 1.26 & 28.52 & 29.78 & 1.21 & 25.46 & 26.67 \\
\hline$\overline{\mathrm{Cd}}$ & 0.51 & 12.46 & 12.97 & 0.03 & 0.39 & 0.42 & 0.65 & \begin{tabular}{|l|}
0.40 \\
\end{tabular} & 1.05 & 0.89 & 0.61 & 1.50 & 1.11 & 1.00 & 2. \\
\hline $\mathrm{Cr}$ & 1.16 & \begin{tabular}{|l|}
1.14 \\
\end{tabular} & 2.30 & 0.06 & 0.09 & 0.15 & 0.22 & 0.02 & 0.24 & 0.24 & 0.02 & 0.26 & 0.28 & 0.03 & 0.3 \\
\hline $\mathrm{Cs}$ & $<0.1$ & 7.30 & 7.30 & $<0.1$ & 0.23 & 0.23 & $<0.1$ & 0.66 & 0.66 & $<0.1$ & 0.88 & 0.88 & $<0.1$ & 0.99 & 0.99 \\
\hline $\mathrm{Cu}$ & 0.16 & 0.56 & 0.72 & 0.02 & $\overline{0.01}$ & 0.03 & 0.06 & 0.01 & 0.07 & 0.07 & 0.02 & 0.09 & 0.07 & 0.02 & 0.09 \\
\hline$\overline{\mathrm{Fe}}$ & 246.0 & \begin{tabular}{|l|}
29.05 \\
\end{tabular} & 275.1 & 7.03 & 0.21 & 7.24 & 28.70 & 0.01 & 28.71 & 36.27 & 0.01 & 36.28 & 38.10 & 0.02 & $\overline{38.12}$ \\
\hline $\mathrm{K}$ & 0.40 & \begin{tabular}{|l|}
12.57 \\
\end{tabular} & 12.97 & 0.01 & 4.01 & 4.02 & \begin{tabular}{|l|}
0.16 \\
\end{tabular} & 4.51 & 4.67 & \begin{tabular}{|l|}
0.16 \\
\end{tabular} & 4.12 & 4.28 & 0.18 & 3.63 & \\
\hline$\overline{\mathrm{Li}}$ & 1.28 & \begin{tabular}{|l|}
89.84 \\
\end{tabular} & 91.12 & 0.06 & 3.69 & 3.75 & 1.76 & 5.64 & 7.40 & 2.18 & 6.29 & 8.47 & 2.09 & 6.48 & $\overline{8.57}$ \\
\hline $\mathrm{Mg}$ & 0.84 & 28.79 & 29.63 & 0.06 & 8.96 & 9.02 & 1.63 & 9.26 & 10.89 & 2.09 & 8.87 & 10.96 & 2.26 & 8.24 & $\overline{10}$ \\
\hline$\overline{M n}$ & 1.00 & 40.09 & 41.09 & 0.08 & 1.13 & 1.21 & 0.93 & 0.91 & 1.84 & 1.21 & 0.87 & 2.08 & 1.18 & 0.87 & 2.0 \\
\hline $\mathrm{Na}$ & 0.78 & 217.3 & 218.1 & 0.09 & 19.95 & 20.04 & 3.15 & \begin{tabular}{|l|}
25.12 \\
\end{tabular} & 28.27 & 3.44 & 26.46 & 29.9 & 3.68 & 25.97 & 29. \\
\hline$\overline{\mathrm{Ni}}$ & 2.89 & 11.04 & 13.93 & 0.24 & 0.51 & \begin{tabular}{|l|}
0.75 \\
\end{tabular} & 1.53 & \begin{tabular}{|l|}
0.37 \\
\end{tabular} & 1.90 & 1.78 & 0.39 & 2.17 & 1.95 & 0.44 & 2.39 \\
\hline $\mathrm{P}$ & 3.31 & 2.58 & 5.89 & 0.18 & $<0.07$ & 0.18 & 0.44 & $<0.07$ & 0.44 & 0.30 & $<0.07$ & 0.30 & 0.49 & 0.17 & 0. \\
\hline$\overline{\mathrm{Pb}}$ & 0.36 & 4.87 & 5.23 & $<0.01$ & 0.08 & 0.08 & 0.33 & \begin{tabular}{|l|}
0.09 \\
\end{tabular} & 0.42 & 0.44 & 0.12 & 0.56 & 0.54 & 0.14 & 0.6 \\
\hline$\overline{\mathrm{Sb}}$ & 0.73 & 0.10 & 0.83 & 0.05 & $<0.03$ & 0.05 & 0.37 & $<0.03$ & 0.37 & 0.24 & 0.06 & 0.30 & 0.36 & 0.06 & 0. \\
\hline $\mathrm{Se}$ & 9.94 & \begin{tabular}{|l|}
139.9 \\
\end{tabular} & 159.8 & 0.86 & 9.77 & \begin{tabular}{|l|}
10.63 \\
\end{tabular} & 2.64 & 26.53 & 29.17 & 2.74 & 39.44 & 42.18 & 3.06 & 53.66 & 56.7 \\
\hline $\mathrm{Si}$ & 164.3 & 23.32 & 187.6 & 9.98 & 2.97 & 12.95 & 33.91 & \begin{tabular}{|l|}
4.50 \\
\end{tabular} & 38.41 & 42.31 & 5.26 & 47.57 & 41.79 & 5.24 & 47.0 \\
\hline $\mathrm{Sr}$ & 0.37 & 55.37 & 55.74 & 0.02 & 2.22 & 2.24 & 0.54 & 6.39 & 6.93 & 0.74 & 7.53 & 8.27 & 0.73 & 7.79 & 8.3 \\
\hline $\mathrm{Te}$ & 3.09 & 9.49 & 12.58 & 0.29 & 0.31 & 0.60 & 1.58 & 0.31 & 1.89 & 2.62 & 0.30 & 2.92 & 3.78 & 0.49 & $\overline{4.2}$ \\
\hline $\mathrm{Ti}$ & 1.25 & \begin{tabular}{|c|}
0.28 \\
\end{tabular} & 1.53 & 0.06 & 0.01 & 0.07 & 0.25 & $<0.01$ & 0.25 & 0.30 & $<0.01$ & \begin{tabular}{l|}
0.3 \\
\end{tabular} & 0.33 & 0.01 & 0.3 \\
\hline $\mathrm{Zn}$ & 1.75 & 31.91 & 33.66 & 0.12 & 1.44 & 1.56 & 2.55 & \begin{tabular}{|c|}
1.03 \\
\end{tabular} & 3.58 & 2.71 & 1.23 & 3.94 & 2.77 & 1.61 & \\
\hline$\overline{\mathrm{Zr}}$ & 25.42 & 26.89 & 52.31 & 1.23 & 0.23 & 1.46 & 3.36 & 0.02 & 3.38 & 3.64 & 0.01 & 3.65 & 3.93 & 0.03 & 3.9 \\
\hline $\mathrm{Cl}$ & $\mathrm{NA}$ & NA & $\mathrm{NA}$ & NA & 31.98 & 31.98 & NA & NA & NA & NA & NA & $\mathrm{NA}$ & NA & NA & NA \\
\hline $\bar{F}$ & NA & NA & $\widehat{\mathrm{NA}}$ & $\overline{\mathrm{NA}}$ & 2.42 & \begin{tabular}{|l|}
2.42 \\
\end{tabular} & $\widehat{\mathrm{NA}}$ & NA & NA & NA & NA & $\mathrm{NA}$ & NA & NA & $\overline{N A}$ \\
\hline I & $<0.1$ & NA & $\mathrm{NC}$ & $\mathrm{NA}$ & 2.26 & 2.26 & $<0.1$ & NA & $\mathrm{NC}$ & $<0.1$ & NA & $\mathrm{NC}$ & $<0.1$ & NA & $\mathrm{NC}$ \\
\hline & $\mathrm{NA}$ & NA & $\mathrm{NA}$ & $\mathrm{NA}$ & 209.1 & 209.1 & NA & $\mathrm{NA}$ & NA & $\mathrm{NA}$ & $\overline{\mathrm{NA}}$ & $\overline{\mathrm{NA}}$ & NA & NA| & NA \\
\hline & NA & NA & NA & $\mathrm{NA}$ & $<0.1$ & $<0.1$ & $\mathrm{NA}$ & NA & NA & NA & NA & NA & NA & NA & $\mathrm{NA}$ \\
\hline Sulfate & 3.33 & NA & $\mathrm{NC}$ & $\mathrm{NA}$ & 56.32 & 56.32 & $<0.1$ & $\mathrm{NA}$ & $\mathrm{Nd}$ & 0.20 & NA & $\mathrm{NQ}$ & $<0.1$ & NA & $\mathrm{NC}$ \\
\hline
\end{tabular}

* Suspended

\# Dissolved

NA - Not Analyzed

NC - Not Calculated 
Table 4.6. Analytical Results for Samples of SBS Blow-Down Fluids (mg/l), (continued).

\begin{tabular}{|c|c|c|c|c|c|c|c|c|c|c|c|c|c|c|c|}
\hline Test & \multicolumn{15}{|c|}{6} \\
\hline I.D. & \multicolumn{3}{|c|}{$12 \mathrm{M}-\mathrm{S}-10 \mathrm{~A}$} & \multicolumn{3}{|c|}{$12 \mathrm{M}-\mathrm{S}-21 \mathrm{~A}$} & \multicolumn{3}{|c|}{$12 \mathrm{M}-\mathrm{S}-24 \mathrm{~A}$} & \multicolumn{3}{|c|}{$12 \mathrm{M}-\mathrm{S}-29 \mathrm{~A}$} & \multicolumn{3}{|c|}{$12 \mathrm{M}-\mathrm{S}-33 \mathrm{~A}$} \\
\hline Glass (kg) & \multicolumn{3}{|c|}{16621} & \multicolumn{3}{|c|}{16706} & \multicolumn{3}{|c|}{16809} & \multicolumn{3}{|c|}{16912} & \multicolumn{3}{|c|}{17015} \\
\hline $\mathrm{pH}$ & \multicolumn{3}{|c|}{6.47} & \multicolumn{3}{|c|}{6.55} & \multicolumn{3}{|c|}{2.2} & \multicolumn{3}{|c|}{6.61} & \multicolumn{3}{|c|}{6.72} \\
\hline & Sus* & Dis.\# & Total & Sus* & Dis.\# & Total & Sus* & Dis.\# & Total & Sus* & Dis.\# & Total & Sus* & Dis.\# & Tot \\
\hline Total & 230 & 368 & 598 & 248 & 368 & 616 & 5 & 888 & 893 & 202 & 440 & 642 & 192 & 252 & \\
\hline $\mathrm{Al}$ & 9.39 & 4.57 & 13.96 & 10.11 & 4.67 & 14.78 & 0.21 & 4.19 & $4.40^{4}$ & 8.22 & 4.35 & 12.57 & 7.40 & 4.55 & 11.95 \\
\hline As & 0.11 & $<0.05$ & 0.11 & 0.08 & 0.64 & 0.72 & $<0.05$ & 0.57 & 0.57 & 0.08 & 0.66 & 0.74 & 0.11 & 0.63 & 0.7 \\
\hline $\mathrm{B}$ & 2.23 & 46.65 & 48.88 & 2.42 & 50.62 & 53.04 & 0.05 & 52.02 & 52.07 & 1.75 & 54.48 & 56.23 & 1.63 & 53.95 & 55.58 \\
\hline $\mathrm{Ba}$ & 0.07 & 0.06 & 0.13 & 0.08 & 0.06 & 0.14 & $<0.01$ & 0.06 & 0.06 & 0.06 & 0.06 & 0.12 & 0.05 & 0.05 & 0.10 \\
\hline $\mathrm{Ca}$ & 1.02 & 22.87 & 23.89 & 1.05 & 21.07 & 22.12 & 0.02 & 18.95 & 18.97 & 0.76 & 17.81 & 18.57 & 0.65 & 16.09 & 16.7 \\
\hline Cd & 1.13 & 1.17 & 2.30 & 1.29 & 1.18 & \begin{tabular}{|l|}
2.47 \\
\end{tabular} & 0.03 & 1.06 & 1.09 & 1.26 & 1.22 & 2.48 & 1.23 & 1.18 & 2.4 \\
\hline $\mathrm{Cr}$ & 0.27 & 0.04 & 0.31 & 0.30 & 0.01 & 0.31 & 0.01 & 0.02 & 0.03 & 0.27 & 0.05 & 0.32 & 0.26 & 0.02 & 0.2 \\
\hline $\mathrm{Cs}$ & $<0.1$ & 1.10 & 1.10 & $<0.1$ & 1.20 & 1.20 & 0.11 & 1.20 & 1.31 & 0.16 & 1.30 & 1.46 & $<0.1$ & 1.30 & 1.3 \\
\hline $\mathrm{Cu}$ & 0.08 & 0.01 & 0.09 & 0.08 & 0.01 & 0.09 & $<0.01$ & 0.02 & 0.02 & 0.06 & 0.02 & 0.08 & 0.06 & 0.01 & 0.0 \\
\hline $\mathrm{Fe}$ & 37.51 & 0.03 & 37.54 & 41.21 & 0.02 & 41.23 & \begin{tabular}{|l|}
0.80 \\
\end{tabular} & 0.02 & 0.82 & 33.35 & 0.05 & 33.4 & 31.85 & $<0.01$ & 31.8 \\
\hline $\mathrm{K}$ & 0.15 & 3.22 & 3.37 & 0.17 & 3.11 & \begin{tabular}{|l|}
3.28 \\
\end{tabular} & $<0.01$ & 2.82 & 2.82 & 0.15 & 2.58 & 2.73 & \begin{tabular}{|l|}
0.14 \\
\end{tabular} & 2.51 & 2.6 \\
\hline $\mathrm{Li}$ & 1.78 & 6.25 & 8.03 & 1.93 & 6.41 & 8.34 & 0.04 & 6.05 & 6.09 & 1.44 & 5.85 & 7.29 & 1.28 & 5.72 & 7.0 \\
\hline $\mathrm{Mg}$ & 2.09 & 7.66 & 9.75 & 2.40 & 7.22 & 9.62 & 0.05 & 6.58 & 6.63 & 1.95 & 6.39 & 8.34 & 1.76 & 5.89 & 7.6 \\
\hline $\mathrm{Mn}$ & 1.06 & 0.87 & 1.93 & 1.17 & 0.83 & 2.00 & 0.02 & 0.76 & 0.78 & 0.94 & 0.74 & 1.68 & 0.99 & 0.70 & 1.6 \\
\hline $\mathrm{Na}$ & 2.81 & 24.14 & 26.95 & 3.04 & 24.09 & 27.13 & 0.06 & 22.76 & 22.82 & 2.40 & 21.61 & 24.01 & 2.21 & 20.83 & 23.04 \\
\hline$\overline{\mathrm{Ni}}$ & 1.82 & 0.43 & 2.25 & 1.99 & 0.39 & 2.38 & 0.04 & 0.37 & 0.41 & 1.68 & 0.41 & 2.09 & 1.52 & 0.31 & 1.8 \\
\hline $\mathrm{P}$ & 0.27 & 0.26 & 0.53 & 0.29 & $<0.07$ & 0.29 & 0.01 & $<0.07$ & 0.01 & 0.33 & 0.13 & 0.46 & 0.33 & 0.12 & 0.4 \\
\hline $\mathrm{Pb}$ & 0.54 & 0.14 & 0.68 & 0.59 & 0.13 & 0.72 & 0.01 & 0.13 & 0.14 & 0.53 & 0.16 & 0.69 & 0.45 & 0.09 & $\overline{0.5}$ \\
\hline $\mathrm{Sb}$ & 0.21 & 0.10 & 0.31 & 0.21 & 0.15 & 0.36 & 0.01 & 0.24 & 0.25 & 0.27 & 0.29 & 0.56 & 0.17 & 0.19 & 0.3 \\
\hline $\mathrm{Se}$ & 2.49 & 65.82 & 68.31 & 2.81 & 76.55 & 79.36 & 0.07 & 77.67 & 77.74 & 2.85 & 78.32 & 81.17 & 3.09 & 88.95 & 92.0 \\
\hline $\mathrm{Si}$ & 40.86 & 5.65 & 46.51 & 42.08 & 6.28 & 48.36 & 0.74 & 6.20 & 6.94 & 30.61 & 6.54 & 37.15 & 27.88 & 6.82 & 34. \\
\hline $\mathrm{Sr}$ & 0.64 & 7.72 & 8.36 & 0.75 & 8.30 & 9.05 & 0.02 & 7.98 & 8.00 & 0.57 & 7.91 & 8.48 & 0.50 & 7.76 & 8.2 \\
\hline $\mathrm{Te}$ & 4.47 & 0.77 & 5.24 & 4.76 & 1.01 & 5.77 & 0.10 & 1.29 & 1.39 & 4.82 & 1.67 & 6.49 & 4.96 & 1.40 & 6.3 \\
\hline $\mathrm{Ti}$ & 0.30 & 0.01 & 0.31 & 0.35 & $<0.01$ & 0.35 & 0.01 & 0.01 & 0.02 & 0.29 & 0.03 & 0.32 & 0.27 & $<0.01$ & 0.2 \\
\hline $\mathrm{Zn}$ & 2.29 & 1.61 & 3.90 & 2.49 & 1.57 & 4.06 & 0.05 & 1.33 & 1.38 & 1.96 & 1.47 & 3.43 & 1.87 & 1.41 & 3.2 \\
\hline$\overline{\mathrm{Zr}}$ & 3.19 & 0.03 & 3.22 & 3.32 & 0.01 & 3.33 & 0.07 & 0.01 & 0.08 & 2.53 & 0.04 & 2.57 & 2.36 & $<0.01$ & 2.36 \\
\hline $\mathrm{Cl}$ & NA & $\mathrm{NA}$ & NA & NA & NA & NA & NA & NA & NA & NA & NA & NA & NA & NA & \\
\hline $\mathrm{F}$ & NA & NA & NA & NA & NA & NA & $\mathrm{NA}$ & NA & $\mathrm{NA}$ & $\mathrm{NA}$ & $\mathrm{NA}$ & NA & NA & NA & \\
\hline I & $<0.1$ & NA & $\mathrm{Nd}$ & $<0.1$ & $\mathrm{NA}$ & $\mathrm{NC}$ & $<0.1$ & NA & $\mathrm{NC}$ & $<0.1$ & NA & $\mathrm{NC}$ & NA & NA & \\
\hline Nitrate & NA & $\mathrm{NA}$ & $\mathrm{NA}$ & NA & $\mathrm{NA}$ & $\mathrm{NA}$ & NA & $\mathrm{NA}$ & $\mathrm{NA}$ & NA & NA & NA & $\mathrm{NA}$ & NA & \\
\hline Nitrite & NA & NA & $\mathrm{NA}$ & $\mathrm{NA}$ & $\mathrm{NA}$ & NA & NA & $\mathrm{NA}$ & $\mathrm{NA}$ & $\mathrm{NA}$ & $\mathrm{NA}$ & $\mathrm{NA}$ & NA & NA & \\
\hline Sulfate & 0.18 & $\mathrm{NA}$ & $\mathrm{Nd}$ & $<0.1$ & $\mathrm{NA}$ & $\mathrm{NC}$ & $<0.1$ & NA & $\mathrm{NO}$ & $<0.1$ & NA & $\mathrm{NC}$ & NA & NA & \\
\hline
\end{tabular}

* Suspended

\# Dissolved

NA - Not Analyzed

NC - Not Calculated 
The Catholic University of America

DuraMelter 1200 Tests with AZ-101HLW Simulants

Vitreous State Laboratory

Final Report, VSL-02R0100-2, Rev.I

Table 4.6. Analytical Results for Samples of SBS Blow-Down Fluids (mg/), (continued).

\begin{tabular}{|c|c|c|c|c|c|c|c|c|c|c|c|c|c|c|c|}
\hline Test & \multicolumn{15}{|c|}{6} \\
\hline I.D. & \multicolumn{3}{|c|}{$12 \mathrm{M}-\mathrm{S}-40 \mathrm{~A}$} & \multicolumn{3}{|c|}{$12 \mathrm{M}-\mathrm{S}-49 \mathrm{~A}$} & \multicolumn{3}{|c|}{$12 \mathrm{M}-\mathrm{S}-55 \mathrm{~A}$} & \multicolumn{3}{|c|}{$12 \mathrm{M}-\mathrm{S}-61 \mathrm{~A}$} & \multicolumn{3}{|c|}{$12 \mathrm{M}-\mathrm{S}-63 \mathrm{~A}$} \\
\hline Glass (kg) & \multicolumn{3}{|c|}{17118} & \multicolumn{3}{|c|}{17222} & \multicolumn{3}{|c|}{17321} & \multicolumn{3}{|c|}{17421} & \multicolumn{3}{|c|}{17521} \\
\hline $\mathrm{pH}$ & \multicolumn{3}{|c|}{6.72} & \multicolumn{3}{|c|}{6.54} & \multicolumn{3}{|c|}{6.50} & \multicolumn{3}{|c|}{6.33} & \multicolumn{3}{|c|}{6.43} \\
\hline & Sus* & Dis.\# & Total & Sus* & Dis.\# & Total & Sus* & Dis.\# & Total & Sus* & Dis.\# & Total & Sus* & Dis.\# & Total \\
\hline Total & 190 & 196 & 386 & 204 & 214 & 418 & 206 & 264 & 470 & 202 & 274 & 476 & 195 & 242 & 437 \\
\hline $\mathrm{Al}$ & 7.52 & 4.25 & 11.77 & 8.21 & 4.25 & 12.46 & 8.07 & 4.85 & 12.92 & 7.91 & 4.69 & 12.6 & 7.21 & 5.52 & 12.73 \\
\hline As & 0.13 & 0.61 & 0.74 & 0.23 & 0.66 & 0.89 & 0.13 & 0.81 & 0.94 & 0.05 & 0.96 & 1.01 & 0.06 & 0.95 & 1.01 \\
\hline $\mathrm{B}$ & 1.71 & 53.96 & 55.67 & 1.88 & $<0.03$ & 1.88 & 1.79 & 57.85 & 59.64 & 1.68 & 58.94 & 60.62 & 1.58 & 60.90 & 62.48 \\
\hline $\mathrm{Ba}$ & 0.06 & 0.05 & 0.11 & 0.05 & 0.06 & 0.11 & 0.06 & 0.05 & 0.11 & 0.05 & \begin{tabular}{|l|}
0.05 \\
\end{tabular} & 0.10 & 0.05 & 0.06 & 0.11 \\
\hline $\mathrm{Ca}$ & 0.65 & 14.53 & \begin{tabular}{|c|}
15.18 \\
\end{tabular} & 0.61 & 2.34 & 2.95 & 0.55 & 12.55 & 13.1 & 0.51 & \begin{tabular}{|l|}
11.73 \\
\end{tabular} & 12.24 & 0.43 & 10.98 & 11.41 \\
\hline $\mathrm{Cd}$ & 1.32 & 1.06 & 2.38 & 1.46 & 1.19 & 2.65 & 1.39 & 1.39 & 2.78 & 1.45 & 1.52 & 2.97 & 1.34 & 1.84 & 3.18 \\
\hline $\mathrm{Cr}$ & 0.27 & 0.01 & 0.28 & 0.26 & 0.03 & 0.29 & 0.29 & 0.03 & 0.32 & 0.29 & 0.04 & 0.33 & 0.30 & 0.06 & 0.36 \\
\hline Cs & $<0.1$ & 1.30 & 1.30 & $<0.1$ & 1.30 & 1.30 & $<0.1$ & 1.30 & 1.30 & $<0.1$ & 1.40 & 1.40 & $<0.1$ & 1.40 & 1.40 \\
\hline $\mathrm{Cu}$ & 0.06 & 0.01 & 0.07 & 0.06 & 0.01 & 0.07 & 0.06 & 0.02 & 0.08 & 0.05 & 0.02 & 0.07 & 0.06 & 0.02 & 0.08 \\
\hline $\mathrm{Fe}$ & 32.00 & 0.01 & 32.01 & 34.74 & 0.03 & 34.77 & 34.06 & 0.01 & 34.07 & 35.14 & 0.03 & 35.17 & 34.19 & 0.03 & 34.22 \\
\hline $\mathrm{K}$ & 0.14 & 2.42 & 2.56 & 0.15 & 2.30 & 2.45 & 0.15 & 2.30 & 2.45 & 0.16 & 2.05 & 2.21 & 0.14 & 1.90 & 2.04 \\
\hline $\mathrm{Li}$ & 1.31 & 5.46 & 6.77 & 1.41 & 2.54 & 3.95 & 1.45 & 5.61 & 7.06 & 1.38 & 5.55 & 6.93 & 1.24 & 5.70 & 6.94 \\
\hline $\mathrm{Mg}$ & 1.87 & 5.43 & 7.30 & 1.98 & 1.34 & 3.32 & 2.00 & 5.13 & 7.13 & 1.93 & 4.90 & 6.83 & 1.67 & 4.78 & 6.45 \\
\hline $\mathrm{Mn}$ & 0.96 & 0.66 & 1.62 & 1.00 & 0.61 & 1.61 & 0.87 & 0.64 & 1.51 & 0.85 & 0.60 & 1.45 & 0.95 & 0.62 & 1.57 \\
\hline $\mathrm{Na}$ & 2.26 & 19.80 & 22.06 & 2.55 & 0.11 & 2.66 & 2.46 & 19.78 & 22.24 & 2.34 & 19.45 & 21.79 & 2.15 & 19.50 & 21.65 \\
\hline $\mathrm{Ni}$ & 1.56 & 0.28 & \begin{tabular}{|l|}
1.84 \\
\end{tabular} & 1.61 & 0.31 & 1.92 & 1.65 & 0.32 & 1.97 & 1.69 & 0.33 & 2.02 & 1.65 & 0.37 & 2.02 \\
\hline$P$ & 0.37 & 0.22 & 0.59 & 0.35 & 0.63 & 0.98 & 0.40 & 0.47 & 0.87 & 0.26 & 0.32 & 0.58 & 0.25 & 0.42 & 0.67 \\
\hline $\mathrm{Pb}$ & 0.49 & 0.11 & 0.6 & 0.47 & 0.12 & 0.59 & 0.53 & 0.11 & 0.64 & 0.54 & 0.14 & 0.68 & 0.48 & 0.14 & 0.62 \\
\hline $\mathrm{Sb}$ & 0.14 & 0.36 & 0.5 & 0.19 & 0.47 & 0.66 & 0.16 & 0.48 & 0.64 & 0.25 & 0.47 & 0.72 & 0.21 & 0.45 & 0.66 \\
\hline $\mathrm{Se}$ & 3.61 & 91.45 & 95.06 & 4.08 & 100.5 & 104.6 & 4.64 & 109.8 & 114.4 & 4.79 & 110.2 & 115.0 & 5.17 & 112.1 & 117.3 \\
\hline $\mathrm{Si}$ & 27.50 & 6.89 & 34.39 & 29.82 & 2.05 & 31.87 & 28.52 & 7.71 & 36.23 & 28.60 & \begin{tabular}{|l|}
7.88 \\
\end{tabular} & 36.48 & 25.85 & 8.45 & 34.3 \\
\hline $\mathrm{Sr}$ & 0.53 & 7.67 & 8.20 & 0.55 & 2.05 & 2.60 & 0.54 & 8.26 & 8.80 & 0.52 & 8.30 & 8.82 & 0.45 & 8.34 & 8.79 \\
\hline $\mathrm{Te}$ & 4.93 & 1.76 & 6.69 & 5.04 & 2.03 & 7.07 & 5.12 & 2.35 & 7.47 & 5.26 & 2.66 & 7.92 & 5.32 & 2.68 & 8.00 \\
\hline $\mathrm{Ti}$ & 0.27 & 0.01 & 0.28 & 0.29 & 0.01 & 0.30 & 0.30 & 0.02 & 0.32 & 0.31 & 0.02 & 0.33 & 0.33 & 0.03 & $\overline{0.36}$ \\
\hline $\mathrm{Zn}$ & 1.92 & 1.24 & 3.16 & 1.94 & 1.38 & 3.32 & 1.84 & 1.52 & 3.36 & 1.75 & 1.57 & 3.32 & 1.56 & 1.77 & 3.3 \\
\hline $\mathrm{Zr}$ & 2.20 & 0.01 & 2.21 & 2.33 & 0.02 & 2.35 & 2.20 & 0.02 & 2.22 & 2.06 & 0.02 & 2.08 & 2.06 & 0.03 & 2.09 \\
\hline $\mathrm{Cl}$ & $\mathrm{NA}$ & NA & NA & NA & NA & NA & NA & NA & NA & NA & NA & NA & NA & NA & NA \\
\hline $\mathrm{F}$ & $\mathrm{NA}$ & $\mathrm{NA}$ & NA & NA & NA & $\mathrm{NA}$ & NA & NA & $\mathrm{NA}$ & NA & NA & NA & NA & NA & NA \\
\hline $\mathrm{I}$ & $\mathrm{NA}$ & $\mathrm{NA}$ & NA & NA & NA & $\mathrm{NA}$ & $<0.1$ & NA & $\mathrm{NC}$ & $<0.1$ & NA & $\mathrm{NC}$ & NA & NA & NA \\
\hline Nitrate & $\mathrm{NA}$ & $\mathrm{NA}$ & NA & $\mathrm{NA}$ & NA & $\mathrm{NA}$ & $\mathrm{NA}$ & $\mathrm{NA}$ & $\mathrm{NA}$ & $\mathrm{NA}$ & NA & NA & $\mathrm{NA}$ & NA & $\mathrm{NA}$ \\
\hline Nitrite & $\mathrm{NA}$ & $\mathrm{NA}$ & NA & $\mathrm{NA}$ & $\mathrm{NA}$ & $\mathrm{NA}$ & NA & $\mathrm{NA}$ & NA & $\mathrm{NA}$ & NA & NA & NA & NA & NA \\
\hline Sulfate & $\mathrm{NA}$ & NA & NA & NA & NA & NA & 0.15 & NA & $\mathrm{NC}$ & $<0.1$ & NA & $\mathrm{NC}$ & NA & NA & $\mathrm{NA}$ \\
\hline
\end{tabular}


The Catholic University of America

Vitreous State Laboratory

Table 4.6. Analytical Results for Samples of SBS Blow-Down Fluids (mg/l), (continued).

\begin{tabular}{|c|c|c|c|c|c|c|c|c|c|c|c|c|c|c|c|}
\hline Test & \multicolumn{15}{|c|}{6} \\
\hline I.D. & \multicolumn{3}{|c|}{$12 \mathrm{M}-\mathrm{S}-78 \mathrm{~A}$} & \multicolumn{3}{|c|}{$12 \mathrm{M}-\mathrm{S}-82 \mathrm{~A}$} & \multicolumn{3}{|c|}{$12 \mathrm{M}-\mathrm{S}-90 \mathrm{~A}$} & \multicolumn{3}{|c|}{$12 \mathrm{M}-\mathrm{S}-97 \mathrm{~A}$} & \multicolumn{3}{|c|}{$12 \mathrm{M}-\mathrm{S}-102 \mathrm{~A}$} \\
\hline Glass (kg) & \multicolumn{3}{|c|}{17620} & \multicolumn{3}{|c|}{17720} & \multicolumn{3}{|c|}{17820} & \multicolumn{3}{|c|}{17920} & \multicolumn{3}{|c|}{18020} \\
\hline $\mathrm{pH}$ & \multicolumn{3}{|c|}{4.24} & \multicolumn{3}{|c|}{4.51} & \multicolumn{3}{|c|}{4.51} & \multicolumn{3}{|c|}{4.36} & \multicolumn{3}{|c|}{4.24} \\
\hline & Sus* & Dis.井 & Total & Sus* & Dis.\# & Total & Sus* & Dis.\# & Total & Sus* & Dis.\# & Total & Sus* & Dis.\# & Tot \\
\hline Total & 560 & 172 & 732 & 600 & 230 & 830 & 492 & 262 & 754 & 576 & 234 & 810 & 536 & 211 & \\
\hline$\overline{\mathrm{Al}}$ & 14.21 & 9.64 & 23.85 & 18.30 & 10.16 & 28.46 & 15.68 & 10.82 & 26.5 & 16.00 & 11.44 & 27.44 & 13.48 & 11.46 & 24.9 \\
\hline As & 0.24 & 0.99 & 1.23 & 0.21 & 1.19 & 1.40 & 0.24 & 1.26 & 1.50 & 0.28 & 1.05 & 1.33 & $<0.01$ & 1.22 & 1.2 \\
\hline B & 3.07 & 71.87 & 74.94 & 3.62 & 73.36 & 76.98 & 3.03 & 74.22 & 77.25 & 3.11 & 76.31 & 79.42 & 3.36 & 74.60 & 77.9 \\
\hline $\mathrm{Ba}$ & 0.11 & 0.11 & 0.22 & 0.14 & 0.11 & 0.25 & 0.11 & 0.12 & 0.23 & 0.13 & 0.09 & 0.22 & 0.12 & 0.08 & \\
\hline $\mathrm{Ca}$ & 0.59 & 10.99 & 11.58 & 0.72 & 10.39 & 11.11 & 0.57 & 9.79 & 10.36 & 0.62 & 8.96 & 9.58 & 0.54 & 8.02 & \\
\hline$\overline{C d}$ & 0.99 & 3.67 & 4.66 & 1.29 & 3.77 & 5.06 & 1.61 & 4.21 & 5.82 & 1.72 & 4.42 & 6.14 & 1.42 & 4.75 & 6.1 \\
\hline $\mathrm{Cr}$ & 0.92 & 0.05 & 0.97 & 0.80 & 0.04 & 0.84 & 0.93 & 0.04 & 0.97 & 1.18 & 0.05 & 1.23 & 1.11 & 0.06 & 1.1 \\
\hline $\mathrm{Cs}$ & $<0.1$ & 1.40 & 1.40 & 0.15 & 1.50 & 1.65 & $<0.1$ & 2.30 & 2.3 & $<0.1$ & 2.30 & 2.30 & 0.15 & 2.40 & \\
\hline$\overline{\mathrm{Cu}}$ & 0.12 & 0.05 & 0.17 & 0.15 & 0.05 & 0.20 & 0.10 & 0.06 & 0.16 & 0.12 & 0.06 & 0.18 & 0.12 & 0.06 & 0. \\
\hline $\mathrm{Fe}$ & 128.1 & 0.39 & 128.5 & 120.8 & 0.25 & 121.1 & 96.46 & 0.14 & 96.6 & 119.8 & 0.18 & 112.0 & 110.1 & 0.26 & 110 \\
\hline $\mathrm{K}$ & 0.44 & 2.25 & 2.69 & 0.42 & 2.40 & 2.82 & 0.36 & 2.85 & 3.21 & 0.45 & 2.01 & 2.46 & 0.37 & 1.79 & 2.1 \\
\hline $\mathrm{Li}$ & 2.44 & 6.18 & 8.62 & 2.89 & 6.94 & 9.83 & 2.42 & 7.76 & 10.18 & 2.49 & 7.37 & 9.86 & 2.00 & 7.13 & 9. \\
\hline $\mathrm{Mg}$ & 0.93 & 6.54 & 7.47 & 1.67 & 6.41 & 8.08 & 1.52 & 6.28 & 7.8 & 1.23 & 6.09 & 7.32 & 0.88 & 5.65 & b. \\
\hline $\mathrm{Mn}$ & 2.99 & 0.85 & 3.84 & 3.04 & 1.02 & 4.06 & 2.16 & 1.13 & 3.29 & 2.55 & 0.96 & 3.51 & 2.18 & 0.96 & 3. \\
\hline $\mathrm{Na}$ & 4.43 & 19.96 & 24.39 & 5.36 & 21.38 & 26.74 & 4.38 & 24.09 & 28.47 & 4.54 & 22.13 & 26.67 & 4.62 & 20.93 & 25.5 \\
\hline $\mathrm{Ni}$ & 5.80 & 0.33 & 6.13 & 5.54 & 0.34 & 5.88 & 4.49 & 0.37 & 4.86 & 5.46 & 0.30 & 5.76 & 5.04 & 0.31 & 5.3 \\
\hline$P$ & 0.77 & 0.81 & 1.58 & 0.88 & 0.78 & 1.66 & 1.10 & 0.96 & 2.06 & 1.23 & 0.45 & 1.68 & 0.81 & 0.81 & 1.6 \\
\hline $\mathrm{Pb}$ & 1.52 & 0.15 & 1.67 & 1.42 & 0.17 & 1.59 & 1.50 & 0.20 & 1.70 & 1.89 & 0.18 & 2.07 & 1.77 & 0.21 & 1.5 \\
\hline $\mathrm{Sb}$ & 0.71 & 0.19 & 0.90 & 0.58 & 0.32 & 0.90 & 0.45 & 0.48 & 0.93 & 0.25 & 0.09 & 0.34 & 0.59 & 0.33 & 0.9 \\
\hline $\mathrm{Se}$ & 20.24 & 120.8 & 141.0 & 19.47 & 131.8 & 151.3 & 16.64 & 133.4 & 150.0 & 23.10 & 135.8 & 158.9 & 23.91 & 135.7 & 159 \\
\hline $\mathrm{Si}$ & 80.71 & 13.84 & 94.55 & 86.45 & 14.08 & 100.5 & 67.75 & 14.56 & 82.31 & 75.83 & 14.78 & 90.61 & 80.92 & 14.87 & 95.7 \\
\hline $\mathrm{Sr}$ & 0.61 & 8.92 & 9.53 & 0.86 & 10.08 & 10.94 & 0.83 & 10.69 & 11.52 & 0.90 & 10.00 & 10.9 & 0.74 & 9.37 & 10.1 \\
\hline $\mathrm{Te}$ & 13.80 & 5.18 & 18.98 & 14.25 & 3.67 & 17.92 & 18.93 & 4.65 & 23.58 & 22.60 & 5.58 & 28.18 & 3.47 & 6.56 & 10.0 \\
\hline $\mathrm{Ti}$ & 1.01 & 0.03 & 1.04 & 1.02 & 0.02 & 1.04 & 0.88 & 0.02 & 0.90 & 1.05 & 0.02 & 1.07 & 0.92 & 0.03 & 0.9 \\
\hline $\mathrm{Zn}$ & 2.36 & 2.94 & 5.30 & 2.83 & 3.57 & 6.40 & 2.31 & 3.83 & 6.14 & 2.38 & 4.07 & 6.45 & 1.96 & 4.10 & 6.0 \\
\hline $\mathrm{Zr}$ & 3.05 & 2.22 & 5.27 & 4.93 & 2.10 & 7.03 & 4.26 & 1.87 & 6.13 & 4.18 & 1.58 & 5.76 & 2.76 & 1.83 & 4.5 \\
\hline $\mathrm{Cl}$ & NA & NA & NA & NA & 54.66 & 54.66 & NA & NA & NA & NA & NA & NA & NA & NA & \\
\hline $\mathrm{F}$ & NA & NA & NA & NA & 84.48 & 84.48 & NA & NA & NA & NA & NA & NA & NA & NA & \\
\hline I & NA & NA & NA & NA & 57.52 & 57.52 & $<0.1$ & NA & $\mathrm{NC}$ & $<0.1$ & NA & $\mathrm{NO}$ & $<0.1$ & NA & \\
\hline Nitrate & NA & NA & NA & NA & 115.9 & 115.9 & NA & $\mathrm{NA}$ & NA & NA & NA & NA & NA & NA & \\
\hline Nitrite & NA & NA & NA & NA & $<0.1$ & $<0.1$ & NA & $\mathrm{NA}$ & NA & NA & NA & NA & NA & NA & \\
\hline Sulfate & NA & NA & $\mathrm{NA}$ & NA & 312.8 & 312.8 & $<0.1$ & NA & $\mathrm{NC}$ & 0.42 & NA & $\mathrm{NC}$ & $<0.1$ & $\mathrm{NA}$ & \\
\hline
\end{tabular}

* Suspended

\# Dissolved

NA - Not Analyzed

NC - Not Calculated 
Table 4.6. Analytical Results for Samples of SBS Blow-Down Fluids (mg/l), (continued).

\begin{tabular}{|c|c|c|c|c|c|c|c|c|c|c|c|c|c|c|c|}
\hline Test & \multicolumn{15}{|c|}{7} \\
\hline I.D. & \multicolumn{3}{|c|}{$12 \mathrm{M}-\mathrm{S}-142 \mathrm{~A}$} & \multicolumn{3}{|c|}{$12 \mathrm{M}-\mathrm{S}-144 \mathrm{~A}$} & \multicolumn{3}{|c|}{$12 \mathrm{M}-\mathrm{S}-146 \mathrm{~A}$} & \multicolumn{3}{|c|}{$12 \mathrm{M}-\mathrm{S}-148 \mathrm{~A}$} & \multicolumn{3}{|c|}{$12 \mathrm{M}-\mathrm{S}-150 \mathrm{~A}$} \\
\hline Glass (kg) & \multicolumn{3}{|c|}{18100} & \multicolumn{3}{|c|}{18233} & \multicolumn{3}{|c|}{18366} & \multicolumn{3}{|c|}{18499} & \multicolumn{3}{|c|}{18632} \\
\hline $\mathrm{pH}$ & \multicolumn{3}{|c|}{4.32} & \multicolumn{3}{|c|}{3.44} & \multicolumn{3}{|c|}{3.10} & \multicolumn{3}{|c|}{2.84} & \multicolumn{3}{|c|}{2.70} \\
\hline & Sus* & Dis.\# & Total & Sus* & Dis.\# & Total & Sus* & Dis.\# & Total & Sus* & Dis.\# & Total & Sus* & 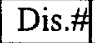 & Tota \\
\hline Total & 136 & 156 & 292 & 126 & 224 & 350 & 156 & 268 & 424 & 158 & 770 & 928 & 202 & \begin{tabular}{|l|}
572 \\
\end{tabular} & 774 \\
\hline $\mathrm{Al}$ & 3.53 & 7.05 & 10.58 & 2.25 & 11.32 & 13.57 & 2.37 & 14.94 & 17.31 & 2.02 & 18.00 & 20.02 & 2.23 & 20.91 & 23.1 \\
\hline As & 0.03 & 0.48 & 0.51 & 0.18 & 0.67 & 0.85 & 0.06 & 0.88 & 0.94 & $<0.01$ & 1.27 & 1.27 & $<0.01$ & 1.48 & 1.4 \\
\hline $\mathrm{B}$ & 1.06 & 16.58 & 17.64 & 0.95 & 28.75 & 29.7 & 1.15 & \begin{tabular}{|l|}
44.83 \\
\end{tabular} & 45.98 & 1.06 & 66.21 & 67.27 & 1.16 & \begin{tabular}{|l|}
83.88 \\
\end{tabular} & 85.0 \\
\hline $\mathrm{Ba}$ & 0.02 & 0.10 & 0.12 & 0.02 & \begin{tabular}{|l|} 
\\
\end{tabular} & 0.13 & 0.02 & \begin{tabular}{|l|}
0.13 \\
\end{tabular} & 0.15 & 0.03 & 0.15 & 0.18 & 0.04 & \begin{tabular}{|l|}
0.15 \\
\end{tabular} & 0.19 \\
\hline $\mathrm{Ca}$ & 0.24 & 39.43 & 39.67 & 0.22 & 34.60 & 34.82 & 0.25 & 34.15 & 34.4 & 0.24 & 29.66 & 29.9 & 0.26 & 27.48 & 27.7 \\
\hline $\mathrm{Cd}$ & 0.19 & 1.22 & 1.41 & 0.14 & 2.28 & 2.42 & 0.14 & 3.59 & 3.73 & 0.15 & 4.90 & 5.05 & 0.16 & 5.52 & 5.6 \\
\hline $\mathrm{Cr}$ & 0.15 & 0.05 & 0.20 & 0.15 & 0.11 & 0.26 & 0.18 & 0.17 & 0.35 & 0.19 & 0.22 & 0.41 & 0.23 & 0.26 & $\overline{0.4}$ \\
\hline $\mathrm{Cs}$ & $<0.1$ & 0.64 & 0.64 & $<0.1$ & 0.37 & 0.37 & $<0.1$ & 1.70 & 1.70 & $<0.1$ & 2.20 & 2.20 & $<0.1$ & 2.80 & $\overline{2.8}$ \\
\hline $\mathrm{Cu}$ & 0.04 & 0.03 & 0.07 & 0.04 & 0.06 & 0.10 & 0.04 & 0.08 & 0.12 & 0.04 & 0.09 & 0.13 & 0.04 & 0.11 & 0.13 \\
\hline $\mathrm{Fe}$ & 20.34 & 0.25 & 20.59 & 25.73 & 1.20 & 26.93 & 33.67 & 2.10 & 35.77 & 33.86 & 3.13 & 36.99 & 41.77 & 4.00 & 45.77 \\
\hline $\mathrm{K}$ & 0.08 & 3.23 & 3.31 & 0.08 & 2.91 & 2.99 & 0.10 & 3.17 & 3.27 & 0.10 & 3.26 & 3.36 & 0.10 & 3.26 & 3.3 \\
\hline $\mathrm{Li}$ & 0.81 & 3.64 & 4.45 & 0.73 & 4.43 & 5.16 & 0.91 & 5.41 & 6.32 & 0.89 & 6.71 & 7.60 & 0.95 & 8.00 & 8.9 \\
\hline$\overline{\mathrm{Mg}}$ & 0.26 & 11.30 & 11.56 & 0.20 & 10.81 & $\mid 11.01$ & 0.24 & 10.19 & 10.43 & 0.24 & 9.39 & 9.63 & 0.29 & 8.96 & 9.25 \\
\hline $\mathrm{Mn}$ & 0.56 & 0.82 & 1.38 & 0.25 & 1.72 & 1.97 & 0.16 & 2.88 & 3.04 & 0.08 & 4.10 & 4.18 & 0.09 & 4.65 & 4.74 \\
\hline $\mathrm{Na}$ & 1.50 & 24.12 & 25.62 & 1.34 & 24.69 & 26.03 & 1.64 & 26.05 & 27.69 & 1.57 & 28.27 & 29.84 & 1.71 & 37.68 & 39.39 \\
\hline $\mathrm{Ni}$ & 1.13 & \begin{tabular}{|l|}
0.18 \\
\end{tabular} & 1.31 & 1.36 & \begin{tabular}{|l|} 
\\
\end{tabular} & 1.59 & 1.73 & \begin{tabular}{|l|}
0.28 \\
\end{tabular} & 2.01 & 1.68 & 0.33 & 2.01 & 1.98 & 0.35 & 2.3 \\
\hline $\mathrm{P}$ & 0.12 & $<0.07$ & 0.12 & 0.08 & 0.22 & 0.3 & 0.11 & 0.73 & 0.84 & 0.05 & 0.50 & 0.55 & 0.08 & 0.57 & 0.6 \\
\hline $\mathrm{Pb}$ & 0.34 & 0.10 & 0.44 & 0.27 & 0.36 & $\begin{array}{ll}0.63 \\
\end{array}$ & 0.28 & 0.64 & 0.92 & 0.27 & 0.91 & 1.18 & 0.28 & 1.14 & 1.4 \\
\hline $\mathrm{Sb}$ & 0.20 & 0.22 & 0.42 & 0.20 & 0.27 & 0.47 & 0.29 & 0.43 & 0.72 & 0.27 & 0.93 & 1.20 & 0.29 & 0.54 & 0.8 ? \\
\hline $\mathrm{Se}$ & 2.72 & 50.30 & 53.02 & 3.54 & 73.54 & 77.08 & 5.71 & \begin{tabular}{|l|}
87.79 \\
\end{tabular} & 93.5 & 17.44 & 91.74 & 109.2 & 45.56 & 98.66 & 144. \\
\hline $\mathrm{Si}$ & 25.29 & \begin{tabular}{|l|}
4.62 \\
\end{tabular} & 29.91 & 23.04 & 10.52 & 33.56 & 26.68 & \begin{tabular}{|l|}
15.91 \\
\end{tabular} & 42.59 & 23.18 & 21.88 & 45.06 & 27.90 & 27.14 & 55.0 \\
\hline $\mathrm{Sr}$ & 0.19 & 3.56 & 3.75 & 0.13 & 4.69 & 4.82 & 0.18 & 5.68 & 5.86 & 0.20 & 6.70 & 6.90 & 0.25 & 7.68 & 7.9 \\
\hline $\mathrm{Te}$ & 2.84 & 2.79 & 5.63 & 2.54 & 7.88 & 10.42 & 2.86 & \begin{tabular}{|l|}
12.75 \\
\end{tabular} & 15.61 & 2.23 & 17.80 & 20.03 & 2.49 & 22.18 & 24.67 \\
\hline $\mathrm{Ti}$ & 0.25 & 0.02 & 0.27 & 0.22 & 0.08 & 0.30 & 0.27 & \begin{tabular}{|l|}
0.11 \\
\end{tabular} & 0.38 & 0.27 & 0.16 & 0.43 & 0.30 & 0.18 & 0.48 \\
\hline $\mathrm{Zn}$ & 0.80 & 1.53 & 2.33 & 0.75 & 2.11 & 2.86 & 0.90 & 2.68 & 3.58 & 0.88 & 3.22 & 4.10 & 0.93 & 4.56 & 5.4 \\
\hline $\mathrm{Zr}$ & 1.55 & 0.85 & 2.40 & 0.55 & 3.04 & 3.59 & 0.56 & 4.98 & 5.54 & 0.49 & 6.99 & 7.48 & 0.54 & 8.34 & 8.8 \\
\hline $\mathrm{Cl}$ & NA & 48.53 & 48.53 & NA & NA & NA & NA & NA & NA & $\mathrm{NA}$ & $\mathrm{NA}$ & NA & NA & NA & $\overline{\mathrm{N}} /$ \\
\hline $\mathrm{F}$ & NA & 38.72 & 38.72 & NA & $\overline{\mathrm{NA}}$ & NA & NA & NA & NA & $\overline{\mathrm{NA}}$ & NA & NA & NA & NA & $\overline{\mathrm{NA}}$ \\
\hline I & NA & 16.17 & 16.17 & NA & $\mathrm{NA}$ & NA & $\mathrm{NA}$ & NA & NA & $<0.1$ & NA & $\mathrm{NQ}$ & $<0.1$ & NA & NC \\
\hline rate & NA & 9.29 & 9.29 & $\mathrm{NA}$ & NA & NA & NA & NA & $\mathrm{NA}$ & $\mathrm{NA}$ & $\mathrm{NA}$ & $\mathrm{NA}$ & $\mathrm{NA}$ & NA & $\mathrm{NA}$ \\
\hline & NA & $<0.1$ & $<0.1$ & $\mathrm{NA}$ & $\mathrm{NA}$ & NA & NA & NA & $\mathrm{NA}$ & NA & $\mathrm{NA}$ & NA & $\mathrm{NA}$ & $\mathrm{NA}$ & $\mathrm{N} A$ \\
\hline Sulfate & NA & 143.0 & 143.0 & $\mathrm{NA}$ & NA & NA & NA & NA & NA & $<0.1$ & $\mathrm{NA}$ & $\mathrm{NC}$ & $<0.1$ & NA & $\overline{\mathrm{NC}}$ \\
\hline
\end{tabular}

* Suspended

\# Dissolved

NA - Not Analyzed

NC - Not Calculated 
Table 4.6. Analytical Results for Samples of SBS Blow-Down Fluids (mg/l), (continued).

\begin{tabular}{|c|c|c|c|c|c|c|c|c|c|c|c|c|c|c|c|}
\hline Test & \multicolumn{15}{|c|}{7} \\
\hline I.D. & \multicolumn{3}{|c|}{$12 \mathrm{~N}-\mathrm{S}-6 \mathrm{~A}$} & \multicolumn{3}{|c|}{$12 \mathrm{~N}-\mathrm{S}-11 \mathrm{~A}$} & \multicolumn{3}{|c|}{$12 \mathrm{~N}-\mathrm{S}-12 \mathrm{~A}$} & \multicolumn{3}{|c|}{$12 \mathrm{~N}-\mathrm{S}-16 \mathrm{~A}$} & \multicolumn{3}{|c|}{$12 \mathrm{~N}-\mathrm{S}-17 \mathrm{~A}$} \\
\hline Glass $(\mathrm{kg})$ & \multicolumn{3}{|c|}{18760} & \multicolumn{3}{|c|}{18888} & \multicolumn{3}{|c|}{19016} & \multicolumn{3}{|c|}{19145} & \multicolumn{3}{|c|}{19272} \\
\hline $\mathrm{pH}$ & \multicolumn{3}{|c|}{2.56} & \multicolumn{3}{|c|}{2.51} & \multicolumn{3}{|c|}{2.35} & \multicolumn{3}{|c|}{2.29} & \multicolumn{3}{|c|}{2.29} \\
\hline & Sus* & Dis.\# & Total & Sus* & Dis.\# & Total & Sus* & Dis.\# & Total & Sus* & Dis.\# & Total & Sus* & Dis.\# & Total \\
\hline Total & 224 & 604 & 828 & 240 & 774 & 1014 & 306 & 832 & 1138 & 342 & 920 & 1262 & 370 & 992 & 1362 \\
\hline $\mathrm{Al}$ & 2.13 & 23.26 & 25.39 & 1.94 & 23.70 & 25.64 & 2.18 & 25.47 & 27.65 & 2.37 & 27.12 & 29.49 & 2.53 & 29.23 & 31.76 \\
\hline As & 0.06 & 2.11 & 2.17 & $<0.01$ & 2.26 & 2.26 & 0.32 & 2.65 & 2.97 & $<0.01$ & \begin{tabular}{|l|}
2.83 \\
\end{tabular} & 2.83 & $<0.01$ & 3.27 & 3.27 \\
\hline B & 1.07 & 90.21 & 91.28 & 1.06 & 100.1 & 101.2 & 1.23 & 111.6 & 112.8 & 1.34 & 121.2 & 122.5 & 1.37 & 127.8 & 129.2 \\
\hline $\mathrm{Ba}$ & 0.04 & 0.19 & 0.23 & 0.04 & 0.18 & 0.22 & 0.05 & 0.17 & 0.22 & 0.06 & 0.17 & 0.23 & 0.07 & 0.16 & 0.23 \\
\hline $\mathrm{Ca}$ & 0.24 & 25.40 & 25.64 & 0.22 & 23.17 & 23.39 & 0.24 & 21.00 & 21.24 & 0.27 & $\mid 19.31$ & 19.58 & 0.28 & $\mid 17.87$ & 18.15 \\
\hline $\mathrm{Cd}$ & 0.15 & 7.34 & 7.49 & 0.15 & 8.66 & 8.81 & 0.17 & 9.79 & 9.96 & 0.19 & 11.27 & 11.46 & 0.20 & 12.58 & 12.78 \\
\hline $\mathrm{Cr}$ & 0.21 & 0.28 & 0.49 & 0.21 & 0.31 & 0.52 & 0.24 & 0.33 & 0.57 & 0.25 & 0.36 & \begin{tabular}{|l|}
0.61 \\
\end{tabular} & 0.28 & 0.40 & 0.68 \\
\hline Cs & $<0.1$ & 3.30 & 3.30 & $<0.1$ & 3.70 & 3.70 & $<0.1$ & 4.00 & 4.00 & $<0.1$ & 4.40 & 4.40 & 0.10 & 4.70 & 4.80 \\
\hline$\overline{\mathrm{Cu}}$ & 0.03 & 0.12 & 0.15 & 0.02 & 0.13 & 0.15 & 0.04 & 0.15 & 0.19 & 0.07 & 0.15 & 0.22 & 0.04 & 0.17 & $\overline{0.21}$ \\
\hline $\mathrm{Fe}$ & 38.22 & 4.65 & 42.87 & 37.01 & 4.92 & 41.93 & 45.15 & 5.29 & 50.44 & 50.71 & 5.71 & 56.42 & \begin{tabular}{|l|}
55.70 \\
\end{tabular} & 6.26 & 61.96 \\
\hline $\mathrm{K}$ & 0.10 & 4.52 & 4.62 & 0.10 & 4.42 & 4.52 & 0.11 & 4.49 & 4.6 & 0.12 & 4.35 & 4.47 & 0.14 & 4.65 & 4.79 \\
\hline $\mathrm{Li}$ & 0.90 & 9.34 & 10.24 & 0.86 & 9.74 & 10.6 & 0.94 & 10.51 & 11.45 & 1.03 & 11.29 & 12.32 & 1.04 & 12.14 & 13.18 \\
\hline $\mathrm{Mg}$ & 0.26 & 8.53 & 8.79 & 0.25 & 7.88 & 8.13 & 0.28 & 7.34 & 7.62 & 0.32 & 6.93 & 7.25 & 0.35 & 6.62 & 6.97 \\
\hline $\mathrm{Mn}$ & 0.08 & 5.78 & 5.86 & 0.07 & 6.31 & 6.38 & 0.09 & 7.02 & 7.11 & 0.08 & 7.54 & 7.62 & 0.10 & 8.07 & 8.17 \\
\hline $\mathrm{Na}$ & 1.55 & 32.69 & 34.24 & 1.49 & 32.69 & 34.18 & 1.65 & 33.34 & \begin{tabular}{|l|}
34.99 \\
\end{tabular} & 1.88 & 34.85 & \begin{tabular}{|l|}
36.73 \\
\end{tabular} & 1.88 & 35.29 & 37.17 \\
\hline $\mathrm{Ni}$ & 1.82 & 0.47 & 2.29 & 1.74 & 0.52 & 2.26 & 2.02 & 0.62 & 2.64 & 2.20 & 0.69 & 2.89 & 2.41 & 0.79 & 3.20 \\
\hline$P$ & 0.06 & 0.79 & 0.85 & 0.10 & 0.86 & 0.96 & 0.21 & 1.02 & 1.23 & 0.06 & 0.95 & 1.01 & $<0.01$ & 0.88 & 0.88 \\
\hline $\mathrm{Pb}$ & 0.23 & 1.43 & 1.66 & 0.21 & 1.59 & 1.80 & 0.22 & 1.82 & 2.04 & 0.27 & 1.96 & 2.23 & 0.31 & 2.04 & 2.35 \\
\hline $\mathrm{Sb}$ & 0.30 & 0.69 & 0.99 & 0.31 & 0.78 & 1.09 & 0.41 & 0.83 & 1.24 & 0.33 & 0.86 & 1.19 & 0.50 & 0.94 & 1.44 \\
\hline $\mathrm{Se}$ & 78.52 & \begin{tabular}{|l|}
95.98 \\
\end{tabular} & 174.5 & 102.8 & 91.92 & 194.7 & 134.6 & 82.33 & 216.9 & 77.76 & 75.10 & 152.9 & 192.8 & 67.21 & 260.0 \\
\hline $\mathrm{Si}$ & 23.78 & 33.72 & 57.5 & 19.78 & 36.47 & 56.25 & 23.30 & 39.64 & 62.94 & 28.45 & 42.82 & 71.27 & 27.25 & 45.80 & 73.05 \\
\hline $\mathrm{Sr}$ & 0.24 & 8.40 & 8.64 & 0.24 & \begin{tabular}{|l|}
8.53 \\
\end{tabular} & \begin{tabular}{|l|}
8.77 \\
\end{tabular} & 0.27 & 8.97 & $\begin{array}{c}9.24 \\
\end{array}$ & 0.33 & 9.40 & \begin{tabular}{|c|}
9.73 \\
\end{tabular} & \begin{tabular}{|l|}
0.38 \\
\end{tabular} & 10.02 & 10.4 \\
\hline $\mathrm{Te}$ & 2.15 & 29.89 & 32.04 & 1.96 & 31.27 & 33.23 & 2.31 & 34.90 & 37.21 & 4.05 & 37.73 & 41.78 & 2.67 & 41.68 & 44.35 \\
\hline$\overline{\mathrm{Ti}}$ & 0.28 & 0.21 & 0.49 & 0.28 & 0.23 & 0.51 & 0.32 & 0.26 & 0.58 & 0.35 & 0.28 & 0.63 & 0.38 & 0.30 & 0.68 \\
\hline $\mathrm{Zn}$ & 0.92 & 4.52 & 5.44 & 0.90 & 4.71 & 5.61 & 1.03 & 4.94 & 5.97 & 1.10 & 5.26 & 6.36 & 1.13 & 5.65 & 6.78 \\
\hline $\mathrm{Zr}$ & 0.47 & 9.72 & 10.19 & 0.44 & 10.15 & 10.59 & 0.49 & 10.76 & 11.25 & 0.55 & 11.44 & 11.99 & \begin{tabular}{l|}
0.57 \\
\end{tabular} & 12.05 & 12.62 \\
\hline$\overline{\mathrm{Cl}}$ & NA & NA & NA & NA & NA & NA & NA & NA & NA & NA & NA & NA & $\mathrm{NA}$ & NA & NA \\
\hline$F$ & NA & $\mathrm{NA}$ & NA & NA & $\mathrm{NA}$ & NA & $\mathrm{NA}$ & NA & NA & NA & NA & NA & $\mathrm{NA}$ & NA & $\overline{\mathrm{NA}}$ \\
\hline I & $<0.1$ & NA & $\mathrm{NC}$ & $<0.1$ & $\mathrm{NA}$ & NC & NA & NA & $\mathrm{NA}$ & $<0.1$ & NA & $\mathrm{Nd}$ & $<0.1$ & NA & $\mathrm{NC}$ \\
\hline Nitrate & $\mathrm{NA}$ & NA & NA & $\mathrm{NA}$ & $\mathrm{NA}$ & NA & $\mathrm{NA}$ & NA & NA & NA & NA & NA & NA & NA & $\mathrm{NA}$ \\
\hline Nitrite & NA & $\mathrm{NA}$ & NA & NA & $\mathrm{NA}$ & NA & NA & NA & $\mathrm{NA}$ & NA & NA & $\mathrm{NA}$ & NA & NA & $\overline{\mathrm{NA}}$ \\
\hline Sulfate & $<0.1$ & NA & $\mathrm{NC}$ & $<0.1$ & $\mathrm{NA}$ & $\mathrm{Nd}$ & NA & NA & NA & 0.26 & $\mathrm{NA}$ & $\mathrm{NC}$ & $<0.1$ & NA & $\mathrm{NC}$ \\
\hline
\end{tabular}


Table 4.6. Analytical Results for Samples of SBS Blow-Down Fluids (mg/l), (continued).

\begin{tabular}{|c|c|c|c|c|c|c|c|c|c|c|c|c|c|c|c|}
\hline Test & \multicolumn{15}{|c|}{7} \\
\hline I.D. & \multicolumn{3}{|c|}{$12 \mathrm{~N}-\mathrm{S}-19 \mathrm{~A}$} & \multicolumn{3}{|c|}{$12 \mathrm{~N}-\mathrm{S}-20 \mathrm{~A}$} & \multicolumn{3}{|c|}{$12 \mathrm{~N}-\mathrm{S}-21 \mathrm{~A}$} & \multicolumn{3}{|c|}{$12 \mathrm{~N}-\mathrm{S}-36 \mathrm{~A}$} & \multicolumn{3}{|c|}{$12 \mathrm{~N}-\mathrm{S}-38 \mathrm{~A}$} \\
\hline Glass (kg) & \multicolumn{3}{|c|}{19399} & \multicolumn{3}{|c|}{19526} & \multicolumn{3}{|c|}{19653} & \multicolumn{3}{|c|}{19774} & \multicolumn{3}{|c|}{19896} \\
\hline $\mathrm{pH}$ & \multicolumn{3}{|c|}{2.25} & \multicolumn{3}{|c|}{2.26} & \multicolumn{3}{|c|}{2.26} & \multicolumn{3}{|c|}{2.30} & \multicolumn{3}{|c|}{2.30} \\
\hline & Sus* & Dis.\# & Total & Sus* & Dis.\# & Total & Sus* & Dis. \# & Total & Sus* & Dis.\# & Total & Sus* & Dis.\# & Total \\
\hline Total & 412 & 1044 & 1456 & 442 & 1348 & 1790 & 486 & 1300 & 1786 & 678 & 2136 & 2814 & 548 & 2048 & 2596 \\
\hline $\mathrm{Al}$ & 2.71 & 29.52 & 32.23 & 2.89 & 32.00 & 34.89 & 2.99 & 31.99 & 34.98 & 4.05 & 36.54 & 40.59 & 2.95 & 36.58 & 39.53 \\
\hline As & 0.12 & 3.54 & 3.66 & 0.52 & 3.88 & 4.40 & 0.52 & 4.32 & 4.84 & 0.36 & 4.94 & 5.30 & 0.47 & 4.87 & 5.34 \\
\hline $\mathrm{B}$ & 1.49 & 139.6 & $\mid 141.1$ & 1.57 & 141.7 & 143.3 & 1.77 & 148.8 & 150.6 & 2.18 & 164.0 & 166.2 & 1.72 & \begin{tabular}{|l|}
166.7 \\
\end{tabular} & 168.4 \\
\hline $\mathrm{Ba}$ & 0.08 & 0.12 & 0.20 & 0.09 & 0.10 & 0.19 & 0.10 & 0.08 & 0.18 & 0.17 & 0.10 & 0.27 & 0.13 & 0.10 & 0.23 \\
\hline $\mathrm{Ca}$ & 0.30 & 16.60 & 16.9 & 0.30 & 15.40 & 15.7 & 0.31 & 13.70 & 14.01 & 0.44 & 11.89 & 12.33 & 0.30 & 11.55 & 11.85 \\
\hline $\mathrm{Cd}$ & 0.21 & 13.77 & 13.98 & 0.23 & 15.02 & 15.25 & 0.26 & 12.35 & 12.61 & 0.44 & 14.26 & 14.7 & 0.29 & 15.51 & 15.8 \\
\hline $\mathrm{Cr}$ & 0.30 & 0.40 & 0.7 & 0.33 & 0.42 & 0.75 & 0.33 & 0.41 & 0.74 & 0.58 & 0.50 & 1.08 & 0.37 & 0.52 & 0.89 \\
\hline Cs & 0.11 & 5.00 & 5.11 & $<0.1$ & 5.20 & 5.20 & $<0.1$ & 5.40 & 5.40 & $<0.1$ & 5.40 & 5.40 & $<0.1$ & 5.80 & 5.80 \\
\hline $\mathrm{Cu}$ & 0.04 & 0.17 & 0.21 & 0.04 & 0.17 & 0.21 & 0.05 & 0.17 & 0.22 & 0.03 & 0.18 & 0.21 & 0.05 & 0.18 & 0.23 \\
\hline $\mathrm{Fe}$ & 55.13 & 6.64 & |61.77 & 65.44 & 7.18 & \begin{tabular}{|l|l|}
72.62 \\
\end{tabular} & 68.76 & 7.32 & 76.08 & 94.00 & 8.61 & 102.6 & 68.56 & 8.72 & 77.28 \\
\hline $\mathrm{K}$ & 0.15 & 4.46 & 4.61 & 0.17 & 4.54 & 4.71 & 0.17 & 4.59 & 4.76 & 0.27 & 4.72 & 4.99 & 0.18 & 4.86 & 5.04 \\
\hline $\mathrm{Li}$ & 1.20 & 12.80 & 14.00 & 1.18 & 13.53 & \begin{tabular}{|l|}
14.71 \\
\end{tabular} & 1.29 & 13.76 & 15.05 & 1.73 & 16.34 & 18.07 & 1.26 & 16.97 & 18.23 \\
\hline $\mathrm{Mg}$ & 0.37 & 6.27 & 6.64 & 0.40 & 6.02 & 6.42 & 0.41 & 5.61 & \begin{tabular}{|l|}
6.02 \\
\end{tabular} & 0.55 & 5.38 & 5.93 & 0.38 & 5.36 & 5.74 \\
\hline $\mathrm{Mn}$ & 0.11 & 8.59 & 8.70 & 0.13 & 9.11 & 9.24 & 0.13 & 9.09 & 9.22 & 0.17 & \begin{tabular}{|l|}
10.98 \\
\end{tabular} & 11.15 & 0.16 & 11.17 & 11.33 \\
\hline $\mathrm{Na}$ & 2.11 & 36.37 & 38.48 & 2.07 & 38.09 & 40.16 & 2.27 & 37.88 & 40.15 & 3.01 & 42.18 & 45.19 & 2.19 & 42.54 & 44.73 \\
\hline $\mathrm{Ni}$ & 2.57 & 0.83 & 3.40 & 3.12 & \begin{tabular}{|l|}
0.95 \\
\end{tabular} & \begin{tabular}{|l|}
4.07 \\
\end{tabular} & 3.49 & 0.98 & 4.47 & 4.98 & 1.34 & 6.32 & 3.23 & \begin{tabular}{|l|}
1.29 \\
\end{tabular} & 4.52 \\
\hline $\mathrm{P}$ & 0.14 & 1.03 & 1.17 & 0.24 & 0.98 & 1.22 & 0.16 & 0.97 & 1.13 & 0.25 & 1.16 & 1.41 & 0.16 & 1.01 & 1.17 \\
\hline $\mathrm{Pb}$ & 0.32 & 2.03 & 2.35 & 0.36 & 2.02 & 2.38 & 0.38 & 2.13 & 2.51 & 0.66 & 2.38 & 3.04 & 0.50 & 2.35 & 2.85 \\
\hline $\mathrm{Sb}$ & 0.46 & 0.99 & 1.45 & 0.50 & 1.10 & 1.6 & 0.57 & 1.07 & 1.64 & 1.18 & 1.24 & 2.42 & 0.68 & 1.27 & 1.95 \\
\hline $\mathrm{Se}$ & 208.9 & 54.99 & 263.9 & 198.4 & 43.03 & 241.4 & 234.5 & 40.59 & \begin{tabular}{|l|}
275.1 \\
\end{tabular} & 328.3 & \begin{tabular}{|l|}
53.88 \\
\end{tabular} & 382.2 & 278.1 & \begin{tabular}{|l|}
53.25 \\
\end{tabular} & 331.4 \\
\hline $\mathrm{Si}$ & 28.15 & 46.89 & 75.04 & 30.56 & 51.14 & \begin{tabular}{|l|}
81.7 \\
\end{tabular} & 32.22 & 52.03 & 84.25 & 41.95 & 57.99 & 99.94 & 32.81 & 58.61 & 91.42 \\
\hline $\mathrm{Sr}$ & 0.42 & 10.27 & 10.69 & 0.45 & \begin{tabular}{|l|}
10.83 \\
\end{tabular} & 11.28 & 0.52 & 10.90 & 11.42 & 1.02 & 11.82 & 12.84 & 0.72 & 12.36 & 13.08 \\
\hline $\mathrm{Te}$ & 2.99 & 43.07 & 46.06 & 3.23 & 43.97 & 47.2 & 3.93 & 47.29 & 51.22 & 5.28 & 52.04 & 57.32 & 4.26 & 50.76 & 55.02 \\
\hline $\mathrm{Ti}$ & 0.41 & 0.32 & 0.73 & 0.45 & \begin{tabular}{|l|}
0.32 \\
\end{tabular} & 0.77 & 0.47 & 0.33 & \begin{tabular}{|l|}
0.80 \\
\end{tabular} & 0.74 & 0.35 & 1.09 & 0.51 & 0.37 & 0.88 \\
\hline $\mathrm{Zn}$ & 1.26 & 5.95 & 7.21 & 1.37 & 6.25 & 7.62 & 1.50 & 6.39 & 7.89 & 1.97 & 7.30 & 9.27 & 1.49 & 7.57 & 9.06 \\
\hline $\mathrm{Zr}$ & 0.58 & 12.60 & 13.18 & 0.63 & 13.10 & 13.73 & 0.71 & 13.28 & 13.99 & 1.29 & 14.46 & 15.75 & 0.84 & 15.06 & 15.9 \\
\hline $\mathrm{Cl}$ & NA & NA & $\mathrm{NA}$ & NA & NA & NA & NA & $\mathrm{NA}$ & NA & NA & $\mathrm{NA}$ & $\mathrm{NA}$ & NA & $\mathrm{NA}$ & NA \\
\hline$F$ & $\mathrm{NA}$ & NA & NA & NA & NA & NA & NA & $\mathrm{NA}$ & $\mathrm{NA}$ & $\mathrm{NA}$ & NA & NA & NA & NA & NA \\
\hline I & $<0.1$ & NA & $\mathrm{NC}$ & $\mathrm{NA}$ & NA & NA & NA] & $\mathrm{NA}$ & NA & $<0.1$ & NA & $\mathrm{NC}$ & NA & NA & $\mathrm{NA}$ \\
\hline Nitrate & $\mathrm{NA}$ & NA & NA & NA & NA & NA & NA & $\mathrm{NA}$ & NA & NA & NA & NA & $\mathrm{NA}$ & NA & $\mathrm{NA}$ \\
\hline Nitrite & NA & $\mathrm{NA}$ & NA & $\mathrm{NA}$ & NA & NA & $\mathrm{NA}$ & $\mathrm{NA}$ & $\mathrm{NA}$ & $\mathrm{NA}$ & NA & $\mathrm{NA}$ & $\mathrm{NA}$ & $\mathrm{NA}$ & NA \\
\hline Sulfate & 1.01 & NA & $\mathrm{NQ}$ & NA & NA & NA & NA & NA & NA & $<0.1$ & NA & NC & $\mathrm{NA}$ & NA & $\mathrm{NA}$ \\
\hline
\end{tabular}


Table 4.6. Analytical Results for Samples of SBS Blow-Down Fluids (mg/l), (continued).

\begin{tabular}{|c|c|c|c|c|c|c|c|c|c|c|c|c|c|c|}
\hline Test & \multicolumn{14}{|c|}{7} \\
\hline I.D. & \multicolumn{3}{|c|}{$12 \mathrm{~N}-\mathrm{S}-39 \mathrm{~A}$} & \multicolumn{3}{|c|}{$12 \mathrm{~N}-\mathrm{S}-46 \mathrm{~A}$} & \multicolumn{3}{|c|}{$12 \mathrm{~N}-\mathrm{S}-48 \mathrm{~A}$} & \multicolumn{2}{|c|}{$12 \mathrm{~N}-\mathrm{S}-50 \mathrm{~A}$} & \multicolumn{3}{|c|}{$12 \mathrm{~N}-\mathrm{S}-51 \mathrm{~A}$} \\
\hline Glass (kg) & \multicolumn{3}{|c|}{20017} & \multicolumn{3}{|c|}{20121} & \multicolumn{3}{|c|}{20225} & \multicolumn{2}{|c|}{20329} & \multicolumn{3}{|c|}{20433} \\
\hline $\mathrm{pH}$ & \multicolumn{3}{|c|}{2.31} & \multicolumn{3}{|c|}{2.26} & \multicolumn{3}{|c|}{2.28} & \multicolumn{2}{|l|}{2.31} & \multicolumn{3}{|c|}{2.30} \\
\hline & Sus* & Dis.\# & Total & \begin{tabular}{|l} 
Sus* \\
(
\end{tabular} & Dis.\# & Total & Sus* & Dis.井 & Total & Sus* Dis.\# & Total & Sus* & Dis.\# & \begin{tabular}{|l|} 
Total \\
\end{tabular} \\
\hline Total & 512 & 2136 & 2648 & 476 & 2116 & 2592 & 486 & 2360 & 2846 & \begin{tabular}{|l|l|}
478 & 2104 \\
\end{tabular} & 2582 & 692 & 2128 & 2820 \\
\hline $\mathrm{Al}$ & 2.73 & \begin{tabular}{|l}
37.48 \\
\end{tabular} & 40.21 & 2.73 & 35.88 & 38.61 & 2.77 & 36.36 & 39.13 & \begin{tabular}{|l|l|}
2.72 & 35.17 \\
\end{tabular} & 37.89 & 3.83 & 33.76 & 37.59 \\
\hline As & 0.19 & 5.01 & \begin{tabular}{|l|}
5.20 \\
\end{tabular} & $\overline{0.19}$ & 4.98 & 5.17 & $\overline{0.10}$ & 4.97 & 5.07 & \begin{tabular}{|l|l|}
0.68 & 5.07 \\
\end{tabular} & \begin{tabular}{|l|}
5.75 \\
\end{tabular} & 1.06 & \begin{tabular}{|l|}
5.08 \\
\end{tabular} & 6.14 \\
\hline B & 1.48 & 170.9 & 172.4 & 1.48 & 174.0 & 175.5 & 1.51 & \begin{tabular}{|l|}
168.1 \\
\end{tabular} & 169.6 & \begin{tabular}{|l|l|}
1.46 & 168.5 \\
\end{tabular} & 170.0 & 1.97 & 171.0 & 173.0 \\
\hline $\mathrm{Ba}$ & 0.12 & 0.08 & 0.20 & $\overline{0.12}$ & \begin{tabular}{|l|}
0.08 \\
\end{tabular} & 0.20 & $\overline{0.13}$ & 0.08 & 0.21 & \begin{tabular}{|l|l|}
0.11 & 0.08 \\
\end{tabular} & \begin{tabular}{|l|}
0.19 \\
\end{tabular} & 0.15 & \begin{tabular}{|l|}
0.08 \\
\end{tabular} & 0.23 \\
\hline$\overline{\mathrm{Ca}}$ & 0.27 & 10.28 & 10.55 & $\overline{0.27}$ & 9.19 & 9.46 & $\overline{0.26}$ & 9.62 & 9.88 & 0.26 & 8.67 & 0.35 & 7.69 & 8.04 \\
\hline$\overline{\mathrm{Cd}}$ & 0.24 & 15.66 & 15.9 & $\overline{0.24}$ & 15.74 & 15.98 & $\overline{0.23}$ & 15.60 & 15.83 & \begin{tabular}{|l|l|}
0.24 & 16.63 \\
\end{tabular} & \begin{tabular}{|l|}
16.87 \\
\end{tabular} & 0.32 & \begin{tabular}{|l|}
15.97 \\
\end{tabular} & 16.29 \\
\hline $\mathrm{Cr}$ & 0.34 & 0.53 & 0.87 & $\overline{0.34}$ & \begin{tabular}{|l|}
0.52 \\
\end{tabular} & 0.86 & 0.33 & \begin{tabular}{|l|}
0.53 \\
\end{tabular} & 0.86 & \begin{tabular}{|c|c|}
0.30 & 0.51
\end{tabular} & \begin{tabular}{|l|}
0.81 \\
\end{tabular} & 0.47 & \begin{tabular}{|c|}
0.51 \\
\end{tabular} & 0.98 \\
\hline Cs & $<0.1$ & 6.30 & 6.30 & $<0.1$ & 5.70 & 5.70 & 0.14 & 6.20 & 6.34 & $<0.1$ & 5.70 & $<0.1$ & 6.10 & 6.10 \\
\hline $\mathrm{Cu}$ & 0.04 & 0.18 & 0.22 & 0.04 & 0.17 & 0.21 & 0.04 & 0.17 & 0.21 & 0.04 & 0.21 & 0.06 & 0.17 & 0.23 \\
\hline $\mathrm{Fe}$ & 57.32 & $\overline{8.61}$ & 65.93 & 57.32 & 8.38 & 65.7 & 61.27 & 8.50 & 69.77 & 61.25 & 69.34 & 87.14 & 7.81 & 94.95 \\
\hline $\mathrm{K}$ & 0.18 & 4.99 & 5.17 & 0.18 & 4.82 & 5.00 & 0.07 & 4.93 & 5.00 & 4.72 & 4.90 & 0.24 & 4.53 & 4.77 \\
\hline$\overline{\mathrm{Li}}$ & 1.15 & 16.88 & \begin{tabular}{|l|}
18.03 \\
\end{tabular} & 1.15 & 16.65 & 17.8 & 1.13 & 16.68 & 17.81 & \begin{tabular}{l|l|}
1.06 & 16.26 \\
\end{tabular} & 17.32 & 1.45 & 15.64 & 17.09 \\
\hline $\mathrm{Mg}$ & 0.35 & 5.00 & \begin{tabular}{|l|}
5.35 \\
\end{tabular} & 0.35 & \begin{tabular}{|l|}
4.67 \\
\end{tabular} & 5.02 & 0.34 & \begin{tabular}{|l|}
4.73 \\
\end{tabular} & 5.07 & \begin{tabular}{|l|l|}
0.34 & 4.85 \\
\end{tabular} & 5.19 & 0.48 & \begin{tabular}{|l|}
4.65 \\
\end{tabular} & \begin{tabular}{|l|}
5.13 \\
\end{tabular} \\
\hline Mn & 0.12 & 10.99 & \begin{tabular}{|l|}
11.11 \\
\end{tabular} & $\overline{0.12}$ & 10.68 & 10.8 & $\overline{0.10}$ & 10.68 & 10.78 & \begin{tabular}{|l|l|}
0.13 & 10.32 \\
\end{tabular} & 10.45 & 0.19 & 9.72 & 9.91 \\
\hline $\mathrm{Na}$ & 2.26 & 43.10 & 45.36 & 2.26 & 41.29 & 43.55 & 2.02 & 42.06 & \begin{tabular}{|l|}
44.08 \\
\end{tabular} & \begin{tabular}{|l|l|}
1.87 & 40.67 \\
\end{tabular} & 42.54 & 2.54 & \begin{tabular}{|l|}
39.48 \\
\end{tabular} & 42.02 \\
\hline $\mathrm{Ni}$ & 2.57 & 1.24 & \begin{tabular}{|l|}
3.81 \\
\end{tabular} & 2.57 & \begin{tabular}{|l|}
1.17 \\
\end{tabular} & 3.74 & 2.59 & 1.21 & 3.80 & \begin{tabular}{|l|l|}
2.75 & 1.16 \\
\end{tabular} & 3.91 & 3.91 & 1.16 & 5.07 \\
\hline $\mathrm{P}$ & 0.21 & 1.12 & 1.33 & 0.21 & 1.01 & 1.22 & $<0.01$ & 1.09 & 1.09 & 0.14 & 1.02 & 0.34 & 1.00 & 1.34 \\
\hline $\mathrm{Pb}$ & 0.46 & $\overline{2.39}$ & 2.85 & $\overline{0.46}$ & 2.37 & 2.83 & 0.53 & 2.31 & 2.84 & 0.42 & 2.75 & 0.60 & 2.26 & 2.86 \\
\hline$\overline{\mathrm{Sb}}$ & 0.70 & 1.28 & 1.98 & 0.70 & 1.34 & 2.04 & 0.58 & 1.27 & 1.85 & 0.66 & 1.96 & 0.88 & 1.34 & 2.22 \\
\hline $\mathrm{Se}$ & 253.1 & 45.18 & 298.3 & 253.1 & 36.36 & 289.5 & 258.4 & 38.04 & 296.4 & \begin{tabular}{|l|l|}
271.4 & 31.05 \\
\end{tabular} & 302.5 & 372.1 & 28.51 & 400.6 \\
\hline$\overline{\mathrm{Si}}$ & 29.34 & 59.76 & 89.1 & 29.34 & \begin{tabular}{|l|}
57.73 \\
\end{tabular} & \begin{tabular}{|l|}
87.07 \\
\end{tabular} & 30.84 & \begin{tabular}{|l|}
58.67 \\
\end{tabular} & 89.51 & \begin{tabular}{|l|l|}
28.45 & 57.06 \\
\end{tabular} & 85.51 & 36.29 & \begin{tabular}{|l|}
56.72 \\
\end{tabular} & 93.01 \\
\hline $\mathrm{Sr}$ & 0.65 & 12.13 & \begin{tabular}{|l|}
12.78 \\
\end{tabular} & 0.65 & \begin{tabular}{|l|}
11.90 \\
\end{tabular} & 12.55 & 0.66 & 11.99 & 12.65 & \begin{tabular}{|l|l|}
0.62 & 11.64 \\
\end{tabular} & 12.26 & 0.87 & 11.16 & 12.03 \\
\hline $\mathrm{Te}$ & 3.25 & 49.50 & 52.75 & 3.25 & 52.60 & 55.85 & $\overline{3.33}$ & 52.80 & 56.13 & \begin{tabular}{|l|l|}
3.87 & 53.17 \\
\end{tabular} & 57.04 & 5.85 & 53.54 & 59.39 \\
\hline $\mathrm{Ti}$ & 0.43 & 0.38 & \begin{tabular}{|l|}
0.81 \\
\end{tabular} & 0.43 & \begin{tabular}{|l|}
0.36 \\
\end{tabular} & 0.79 & 0.42 & \begin{tabular}{|l|}
0.37 \\
\end{tabular} & 0.79 & \begin{tabular}{|l|l|}
0.43 & 0.35 \\
\end{tabular} & 0.78 & 0.59 & 0.35 & 0.94 \\
\hline $\mathrm{Zn}$ & 1.30 & 7.47 & 8.77 & 1.30 & 7.19 & 8.49 & 1.28 & 7.22 & 8.50 & \begin{tabular}{ll|}
1.24 & 6.96 \\
\end{tabular} & 8.20 & 1.65 & 6.83 & 8.48 \\
\hline $\mathrm{Zr}$ & 0.65 & 14.99 & \begin{tabular}{|l|}
15.64 \\
\end{tabular} & 0.65 & \begin{tabular}{|l|}
14.67 \\
\end{tabular} & 15.32 & 0.67 & 14.72 & 15.39 & \begin{tabular}{|l|l|}
0.62 & 14.21 \\
\end{tabular} & 14.83 & 0.84 & 13.58 & 14.42 \\
\hline $\mathrm{Cl}$ & NA & $\mathrm{NA}$ & NA & $\mathrm{NA}$ & $\mathrm{NA}$ & $\mathrm{NA}$ & NA & $\mathrm{NA}$ & $\mathrm{NA}$ & NA $\mathrm{NA}$ & NA & $\mathrm{NA}$ & NA & NA \\
\hline $\mathrm{F}$ & $\mathrm{NA}$ & $\mathrm{NA}$ & NA & $\mathrm{NA}$ & NA & $\mathrm{NA}$ & NA & NA & $\mathrm{NA}$ & NA & NA & $\mathrm{NA}$ & NA & NA \\
\hline I & $<0.1$ & $\mathrm{NA}$ & $\mathrm{Nq}$ & $<0.1$ & NA & $\mathrm{NQ}$ & $<0.1$ & $\mathrm{NA}$ & $\mathrm{NQ}$ & NA & NA & $\mathrm{NA}$ & $\mathrm{NA}$ & $\overrightarrow{\mathrm{NA}}$ \\
\hline Nitrate & $\mathrm{NA}$ & $\overline{\mathrm{NA}}$ & NA & NA & NA & NA & NA & NA & NA & NA & NA & NA & NA & $\mathrm{NA}$ \\
\hline Nitrite & NA & $\mathrm{NA}$ & NA & NA & NA & $\mathrm{NA}$ & NA & $\mathrm{NA}$ & NA & NA & NA & NA & NA & $\mathrm{NA}$ \\
\hline Sulfate & $<0.1$ & NA & NO & $<0.1$ & NA & Nd & $<0.1$ & NA & $\mathrm{NC}$ & NA & NA & NA & NA & $\mathrm{NA}$ \\
\hline
\end{tabular}


The Catholic University of America

Table 4.6. Analytical Results for Samples of SBS Blow-Down Fluids (mg/l), (continued).

\begin{tabular}{|c|c|c|c|c|c|c|c|c|c|c|c|c|c|c|c|}
\hline Test & \\
\hline I.D. & \multicolumn{3}{|c|}{$12 \mathrm{~N}-\mathrm{S}-52 \mathrm{~A}$} & \multicolumn{3}{|c|}{$12 \mathrm{~N}-\mathrm{S}-53 \mathrm{~A}$} & \multicolumn{3}{|c|}{$12 \mathrm{~N}-\mathrm{S}-54 \mathrm{~A}$} & \multicolumn{3}{|c|}{$12 \mathrm{~N}-\mathrm{S}-62 \mathrm{~A}$} & \multicolumn{3}{|c|}{$12 \mathrm{~N}-\mathrm{S}-63 \mathrm{~A}$} \\
\hline Ilass $(\mathrm{kg})$ & \multicolumn{3}{|c|}{20538} & \multicolumn{3}{|c|}{20663} & \multicolumn{3}{|c|}{20788} & \multicolumn{3}{|c|}{20913} & \multicolumn{3}{|c|}{21038} \\
\hline $\mathrm{pH}$ & \multicolumn{3}{|c|}{2.34} & \multicolumn{3}{|c|}{2.36} & \multicolumn{3}{|c|}{2.27} & \multicolumn{3}{|c|}{2.28} & \multicolumn{3}{|c|}{2.29} \\
\hline & Sus* & Dis.册 & Total & Sus* & Dis.\# & Total & Sus* & Dis.\# & Total & Sus* & Dis.\# & Total & Sus* & Dis.\# & Total \\
\hline Total & 492 & 2188 & 2680 & 540 & 2168 & 2708 & 428 & 1792 & 2220 & 420 & 1808 & 2228 & 400 & 1872 & 2272 \\
\hline $\mathrm{Al}$ & 3.07 & 32.98 & 36.05 & 3.37 & 32.19 & 35.56 & 2.57 & 31.62 & 34.19 & 2.47 & 31.67 & 34.14 & 2.05 & 31.52 & 33.57 \\
\hline As & 0.10 & 5.31 & 5.41 & 0.75 & 5.23 & 5.98 & $<0.01$ & 5.48 & 5.48 & 0.04 & 5.50 & 5.54 & $<0.01$ & 5.55 & 5.5 \\
\hline $\bar{B}$ & 1.42 & 172.6 & 174.0 & 1.44 & 171.0 & 172.4 & 1.36 & 173.4 & 174.8 & 1.58 & 175.9 & 177.5 & 1.13 & 177.1 & 178. \\
\hline $\mathrm{Ba}$ & 0.12 & 0.08 & 0.20 & 0.13 & 0.08 & \begin{tabular}{|l|}
0.21 \\
\end{tabular} & 0.13 & 0.07 & 0.20 & 0.12 & 0.08 & 0.20 & 0.11 & 0.09 & $0.2 C$ \\
\hline $\mathrm{Ca}$ & 0.28 & 6.90 & 7.18 & 0.32 & 6.36 & 6.68 & 0.24 & 6.10 & 6.34 & 0.23 & 5.81 & 6.04 & 0.21 & 5.35 & 5.56 \\
\hline $\mathrm{Cd}$ & 0.27 & 16.34 & 16.61 & 0.27 & 16.71 & 16.98 & 0.24 & 17.31 & 17.55 & 0.24 & 17.75 & 17.99 & 0.19 & 17.09 & 17.28 \\
\hline $\mathrm{Cr}$ & 0.40 & \begin{tabular}{|l|}
0.48 \\
\end{tabular} & \begin{tabular}{|l|}
0.88 \\
\end{tabular} & 0.41 & 0.49 & 0.90 & 0.33 & 0.48 & 0.81 & 0.28 & 0.48 & 0.76 & 0.25 & \begin{tabular}{|l|l|} 
& 0.49 \\
\end{tabular} & \begin{tabular}{|l|}
0.7 \\
\end{tabular} \\
\hline Cs & $<0.1$ & 6.00 & 6.00 & $<0.1$ & 5.40 & 5.40 & $<0.1$ & 5.70 & 5.70 & $<0.1$ & 6.10 & 6.10 & $<0.1$ & 5.70 & 5.7 \\
\hline$\overline{\mathrm{Cu}}$ & 0.04 & 0.17 & 0.21 & 0.05 & 0.17 & 0.22 & 0.04 & 0.16 & 0.20 & 0.03 & 0.16 & 0.19 & 0.03 & 0.15 & 0.1 \\
\hline $\mathrm{Fe}$ & 66.97 & 7.58 & 74.55 & 76.21 & 7.46 & 83.67 & 68.51 & 7.23 & 75.74 & 59.95 & 6.94 & 66.89 & \begin{tabular}{|l|}
48.07 \\
\end{tabular} & 6.65 & 54.72 \\
\hline $\mathrm{K}$ & 0.19 & 4.30 & 4.49 & 0.22 & 4.42 & 4.64 & 0.20 & 4.35 & 4.55 & 0.18 & 4.31 & 4.49 & 0.10 & 4.33 & 4.43 \\
\hline$\overline{\mathrm{Li}}$ & 1.10 & 15.22 & 16.32 & 1.07 & 15.05 & 16.12 & 1.03 & 15.21 & 16.24 & 1.18 & 15.11 & 16.29 & 0.85 & 14.94 & 15.7 \\
\hline $\mathrm{Mg}$ & 0.37 & 4.62 & 4.99 & 0.38 & 4.82 & 5.20 & 0.31 & 4.47 & 4.78 & 0.28 & 4.57 & 4.85 & 0.22 & 4.54 & 4.7 \\
\hline $\mathrm{Mn}$ & 0.12 & 9.30 & 9.42 & 0.17 & 9.24 & 9.41 & 0.13 & 9.23 & 9.36 & 0.09 & 9.11 & 9.20 & 0.08 & 8.81 & $\overline{8.8}$ \\
\hline $\mathrm{Na}$ & 1.86 & 38.95 & 40.81 & 1.93 & 37.97 & 39.9 & 1.79 & 37.65 & 39.44 & 2.09 & 37.99 & 40.08 & 1.49 & 38.10 & 39.59 \\
\hline $\mathrm{Ni}$ & 3.12 & 1.13 & \begin{tabular}{|l|}
4.25 \\
\end{tabular} & 3.42 & 1.16 & 4.58 & 2.85 & \begin{tabular}{|l|}
1.14 \\
\end{tabular} & 3.99 & 2.43 & 1.06 & 3.49 & 2.02 & 1.08 & 3.10 \\
\hline $\mathrm{P}$ & $<0.01$ & 0.95 & 0.95 & 0.31 & 0.93 & 1.24 & 0.16 & 1.04 & 1.20 & $<0.01$ & 0.91 & 0.91 & $<0.01$ & 1.07 & 1. \\
\hline $\mathrm{Pb}$ & 0.48 & 2.28 & 2.76 & 0.50 & 2.29 & 2.79 & 0.47 & 2.24 & 2.71 & 0.50 & 2.23 & 2.73 & 0.42 & 2.30 & 2.7 \\
\hline $\mathrm{Sb}$ & 0.50 & 1.36 & 1.86 & 0.61 & 1.47 & 2.08 & \begin{tabular}{|c|}
0.51 \\
\end{tabular} & \begin{tabular}{|c|}
1.43 \\
\end{tabular} & 1.94 & 0.52 & 1.37 & 1.89 & 0.22 & 1.39 & 1.6 \\
\hline $\mathrm{Se}$ & 66.6 & 24.77 & 291.4 & 293.5 & 23.47 & 317.0 & 207.9 & 20.07 & 228.0 & 206.6 & 21.81 & 228.4 & 237.1 & 25.78 & 262.9 \\
\hline $\mathrm{Si}$ & 32.07 & 55.95 & 88.02 & 28.14 & 55.61 & 83.75 & 26.02 & 55.06 & 81.08 & 28.47 & 56.01 & 84.48 & 25.21 & 56.10 & 81.3 \\
\hline $\mathrm{Sr}$ & 0.70 & 10.68 & 11.38 & 0.72 & 10.58 & 11.30 & 0.59 & 10.66 & 11.25 & \begin{tabular}{|c|}
0.58 \\
\end{tabular} & 10.49 & 11.07 & 0.46 & 10.26 & 10.7 \\
\hline $\mathrm{Te}$ & 4.74 & 56.38 & 61.12 & 7.07 & 52.13 & 59.2 & 6.17 & 53.66 & 59.83 & 6.19 & 53.37 & 59.56 & 4.81 & 54.42 & 59.2 \\
\hline $\mathrm{Ti}$ & 0.49 & 0.33 & \begin{tabular}{|l|}
0.82 \\
\end{tabular} & 0.52 & \begin{tabular}{|l|}
0.33 \\
\end{tabular} & 0.85 & 0.44 & 0.32 & 0.76 & 0.40 & \begin{tabular}{|c|}
0.31 \\
\end{tabular} & $\begin{array}{r}0.71 \\
\end{array}$ & 0.34 & \begin{tabular}{|c|}
0.31 \\
\end{tabular} & 0.6 \\
\hline $\mathrm{Zn}$ & 1.19 & 6.67 & 7.86 & 1.27 & 6.50 & 7.77 & 1.14 & 6.67 & 7.81 & 1.32 & 6.46 & 7.78 & 0.95 & 6.33 & 7.2 \\
\hline$\overline{\mathrm{Zr}}$ & 0.61 & 13.20 & 13.81 & 0.79 & 13.06 & 13.85 & 0.55 & 13.05 & 13.6 & 0.61 & 12.86 & 13.47 & 0.47 & 12.60 & $13.0^{7}$ \\
\hline $\mathrm{Cl}$ & NA & NA & NA & NA & NA & NA & NA & NA & NA & NA & NA & $\mathrm{NA}$ & NA & NA & $\mathrm{N}$ \\
\hline$F$ & NA & NA & NA & NA & NA & NA & NA & NA & NA & NA & NA & NA & NA & NA & $\overline{\mathrm{NA}}$ \\
\hline$I$ & $<0.1$ & NA & $\mathrm{NQ}$ & $\mathrm{NA}$ & $\mathrm{NA}$ & NA & $\mathrm{NA}$ & $\mathrm{NA}$ & NA & $<0.1$ & $\mathrm{NA}$ & $\mathrm{NQ}$ & $<0.1$ & NA & $\overline{\mathrm{NC}}$ \\
\hline Nitrate & NA & NA & NA & $\mathrm{NA}$ & $\mathrm{NA}$ & NA & NA & NA & NA & NA & NA & NA & NA & NA & $\overline{\mathrm{NA}}$ \\
\hline & $\mathrm{NA}$ & NA & NA & $\mathrm{NA}$ & NA & $\mathrm{NA}$ & NA & $\mathrm{NA}$ & $\mathrm{NA}$ & NA & $\mathrm{NA}$ & $\mathrm{NA}$ & NA & NA & $\overline{\mathrm{NA}}$ \\
\hline Sulfate & 1.20 & NA & $\mathrm{NQ}$ & NA & $\mathrm{NA}$ & NAC & NA & $\mathrm{NA}$ & NA & $<0.1$ & NA & $\mathrm{NG}$ & $<0.1$ & NA & 180 \\
\hline
\end{tabular}

* Suspended

\# Dissolved

NA - Not Analyzed

NC - Not Calculated 
The Catholic University of America Vitreous State Laboratory
DuraMelter 1200 Tests with AZ-101HLW Simulants

Final Report, VSL-02R0100-2, Rev.1

Table 4.6. Analytical Results for Samples of SBS Blow-Down Fluids (mg/), (continued).

\begin{tabular}{|c|c|c|c|c|c|c|c|c|c|c|c|c|c|c|c|}
\hline Test & \multicolumn{15}{|c|}{7} \\
\hline I.D. & \multicolumn{3}{|c|}{$12 \mathrm{~N}-\mathrm{S}-66 \mathrm{~A}$} & \multicolumn{3}{|c|}{$12 \mathrm{~N}-\mathrm{S}-68 \mathrm{~A}$} & \multicolumn{3}{|c|}{$12 \mathrm{~N}-\mathrm{S}-70 \mathrm{~A}$} & \multicolumn{3}{|c|}{$12 \mathrm{~N}-\mathrm{S}-72 \mathrm{~A}$} & \multicolumn{3}{|c|}{$12 \mathrm{~N}-\mathrm{S}-73 \mathrm{~A}$} \\
\hline Glass (kg) & \multicolumn{3}{|c|}{21157} & \multicolumn{3}{|c|}{21276} & \multicolumn{3}{|c|}{21395} & \multicolumn{3}{|c|}{21514} & \multicolumn{3}{|c|}{21617} \\
\hline $\mathrm{pH}$ & \multicolumn{3}{|c|}{2.31} & \multicolumn{3}{|c|}{2.32} & \multicolumn{3}{|c|}{2.38} & \multicolumn{3}{|c|}{2.39} & \multicolumn{3}{|c|}{2.34} \\
\hline & Sus* & Dis.\# & Total & Sus* & Dis.\# & Total & Sus* & Dis.\# & Total & Sus* & Dis.\# & Tota & Sus* & * Dis.\# & Total \\
\hline Total & 424 & 1728 & 2152 & 396 & 1704 & 2100 & 444 & 1556 & 2000 & 344 & 1780 & 2124 & 292 & \begin{tabular}{|l|}
21692 \\
21
\end{tabular} & \\
\hline $\mathrm{Al}$ & 1.88 & 30.94 & 32.82 & 1.97 & 31.06 & 33.03 & 2.54 & 30.02 & 32.56 & 1.62 & 28.99 & 30.61 & 1.37 & $7 \quad 28.32$ & 29.69 \\
\hline As & $<0.01$ & 5.22 & 5.22 & 0.24 & \begin{tabular}{|l|}
4.87 \\
\end{tabular} & 5.11 & 0.10 & 4.97 & 5.07 & 0.33 & \begin{tabular}{|l|}
5.09 \\
\end{tabular} & 5.42 & 0.31 & 4.98 & 5.29 \\
\hline $\mathrm{B}$ & 1.18 & $\mid 177.1$ & \begin{tabular}{|l|}
178.3 \\
\end{tabular} & 1.22 & 175.6 & 176.8 & 1.76 & | 176.8 & 178.6 & 1.20 & 176.4 & 177.6 & 0.68 & \begin{tabular}{|l|l|}
8 & 173.5 \\
\end{tabular} & $\widehat{174.2}$ \\
\hline $\mathrm{Ba}$ & 0.12 & 0.08 & 0.20 & 0.11 & 0.09 & 0.20 & 0.13 & 0.08 & 0.21 & 0.08 & 0.09 & 0.17 & 0.07 & 0.09 & $\overline{0.16}$ \\
\hline $\mathrm{Ca}$ & 0.18 & 5.17 & 5.35 & 0.19 & 4.77 & $\overline{4.96}$ & 0.25 & 4.33 & 4.58 & 0.14 & 4.25 & 4.39 & 0.12 & 5.00 & 5.1 \\
\hline$\overline{\mathrm{Cd}}$ & 0.18 & 18.13 & 18.31 & 0.24 & 18.17 & 18.41 & 0.26 & 17.34 & 17.6 & 0.18 & 18.10 & 18.28 & 0.12 & 17.28 & 1 \\
\hline $\mathrm{Cr}$ & 0.22 & 0.45 & \begin{tabular}{|l|}
0.67 \\
\end{tabular} & 0.23 & 0.46 & 0.69 & 0.35 & 0.43 & 0.78 & 0.18 & 0.43 & 0.61 & 0.14 & 0.40 & 0.5 \\
\hline $\mathrm{Cs}$ & $<0.1$ & 5.60 & 5.60 & $<0.1$ & 6.20 & 6.20 & $<0.1$ & 6.00 & 6.00 & $<0.1$ & 6.00 & 6.00 & $<0.1$ & 6.10 & 6.1 \\
\hline$\overline{\mathrm{Cu}}$ & 0.03 & 0.15 & 0.18 & 0.02 & 0.15 & 0.17 & 0.04 & 0.15 & 0.19 & 0.01 & 0.15 & 0.16 & 0.03 & 0.14 & 0.17 \\
\hline $\mathrm{Fe}$ & 49.44 & 6.41 & 55.85 & 56.12 & 6.40 & 62.52 & 63.05 & 5.87 & 68.92 & 39.11 & 5.80 & 44.91 & 30.66 & 5.02 & 35.6 \\
\hline $\mathrm{K}$ & 0.14 & 4.03 & 4.17 & 0.15 & 4.17 & 4.32 & 0.14 & 3.95 & 4.09 & 0.13 & 3.94 & 4.07 & 0.09 & 3.83 & 3.9 \\
\hline $\mathrm{Li}$ & 0.86 & 15.17 & 16.03 & 0.89 & 15.18 & 16.07 & 1.33 & 14.61 & 15.94 & 0.89 & 14.38 & 15.27 & 0.50 & 13.68 & 14.1 \\
\hline$\overline{\mathrm{Mg}}$ & 0.22 & 4.72 & \begin{tabular}{|l|}
4.94 \\
\end{tabular} & 0.23 & 4.87 & 5.10 & 0.28 & 4.75 & 5.03 & 0.17 & 4.82 & \begin{tabular}{|l|}
4.99 \\
\end{tabular} & 0.13 & \begin{tabular}{|l|}
3.63 \\
\end{tabular} & 4.7 \\
\hline $\mathrm{Mn}$ & 0.10 & 8.74 & 8.84 & 0.11 & 8.94 & 9.05 & 0.11 & 8.43 & 8.54 & 0.09 & 8.17 & 8.26 & 0.05 & 7.43 & 7.48 \\
\hline $\mathrm{Na}$ & 1.72 & 37.56 & 39.28 & 1.64 & 37.38 & 39.02 & 2.32 & 36.31 & 38.63 & 1.60 & 35.25 & 36.85 & 0.92 & 35.07 & 35.99 \\
\hline $\mathrm{Ni}$ & 2.02 & 1.01 & \begin{tabular}{|l|}
3.03 \\
\end{tabular} & 2.14 & \begin{tabular}{|l|}
1.01 \\
\end{tabular} & 3.15 & 2.86 & 0.98 & 3.84 & 1.71 & \begin{tabular}{|l|}
0.90 \\
\end{tabular} & 2.61 & 1.26 & 0.82 & 2.0 \\
\hline $\mathrm{P}$ & 0.21 & 1.14 & 1.35 & 0.14 & 1.18 & 1.32 & $<0.01$ & 1.13 & 1.13 & 0.06 & 0.97 & 1.03 & $<0.01$ & 1.09 & 1.0 \\
\hline $\mathrm{Pb}$ & 0.42 & 2.25 & 2.67 & 0.31 & 2.30 & 2.61 & 0.51 & 2.24 & 2.75 & 0.28 & 2.10 & 2.38 & 0.26 & 2.07 & $2.3 ?$ \\
\hline $\mathrm{Sb}$ & 0.37 & 1.50 & 1.87 & 0.51 & 1.43 & 1.94 & 0.54 & 1.41 & 1.95 & 0.43 & 1.37 & 1.8 & 0.28 & 1.36 & 1.6 \\
\hline $\mathrm{Se}$ & 238.3 & 28.03 & 266.3 & 237.6 & 28.69 & 266.3 & 232.0 & 29.11 & 261.1 & 199.0 & 28.02 & 227.0 & \begin{tabular}{|l|}
140.3 \\
\end{tabular} & 27.94 & 168.2 \\
\hline $\mathrm{Si}$ & 20.83 & 55.54 & \begin{tabular}{|l|}
76.37 \\
\end{tabular} & 24.60 & \begin{tabular}{|l|}
55.73 \\
\end{tabular} & 80.33 & 29.18 & 55.74 & 84.92 & 20.37 & 53.17 & 73.54 & 12.72 & 49.55 & 62.27 \\
\hline $\mathrm{Sr}$ & 0.42 & 10.34 & 10.76 & 0.42 & 10.35 & 10.77 & 0.56 & 9.89 & 10.45 & 0.31 & \begin{tabular}{|c|}
9.99 \\
\end{tabular} & $\begin{array}{l}10.3 \\
\end{array}$ & \begin{tabular}{|l|}
0.21 \\
\end{tabular} & 9.53 & 9.7 \\
\hline $\mathrm{Te}$ & 6.66 & 50.09 & 56.75 & 5.33 & 48.86 & 54.19 & 5.53 & 48.57 & 54.1 & 4.24 & 46.95 & 51.19 & 2.20 & 47.01 & 49.21 \\
\hline $\mathrm{Ti}$ & 0.33 & 0.29 & 0.62 & 0.34 & \begin{tabular}{|l|}
0.29 \\
\end{tabular} & 0.63 & 0.51 & 0.26 & 0.77 & 0.30 & \begin{tabular}{|l|}
0.25 \\
\end{tabular} & $\begin{array}{l}0.55 \\
\end{array}$ & 0.22 & 0.24 & 0.46 \\
\hline $\mathrm{Zn}$ & 0.96 & 6.30 & 7.26 & 1.00 & 6.20 & 7.20 & 1.38 & 6.30 & 7.68 & 0.99 & 6.12 & 7.11 & 0.57 & 5.95 & $\overline{6.52}$ \\
\hline $\mathrm{Zr}$ & 0.43 & 12.52 & 12.95 & 0.45 & 12.48 & 12.93 & 0.73 & 11.90 & 12.63 & 0.45 & \begin{tabular}{|l|}
11.87 \\
\end{tabular} & 12.32 & 0.31 & 11.17 & 11.48 \\
\hline $\mathrm{Cl}$ & NA & NA & $\mathrm{NA}$ & NA & NA & $\mathrm{NA}$ & $\mathrm{NA}$ & $\mathrm{NA}$ & $\mathrm{NA}$ & $\mathrm{NA}$ & NA & $\mathrm{NA}$ & $\mathrm{NA}$ & NA & $\mathrm{N} A$ \\
\hline $\bar{F}$ & NA & NA & NA & NA & $\mathrm{NA}$ & $\mathrm{NA}$ & $\mathrm{NA}$ & NA & NA & NA & NA & $\mathrm{NA}$ & $\mathrm{NA}$ & NA & $\overline{\mathrm{NA}}$ \\
\hline I & NA & NA & $\mathrm{NA}$ & NA & $\mathrm{NA}$ & NA & NA & $\mathrm{NA}$ & $\mathrm{NA}$ & NA & NA & NA & $<0.1$ & NA & NC \\
\hline rate & NA & NA & NA & NA & NA & $\mathrm{NA}$ & NA & $\mathrm{NA}$ & NA & NA & $\mathrm{NA}$ & $\mathrm{NA}$ & $\mathrm{NA}$ & NA & $\overline{N A}$ \\
\hline & NA & NA & NA & NA & $\mathrm{NA}$ & $\mathrm{NA}$ & NA & NA & $\mathrm{NA}$ & $\mathrm{NA}$ & $\mathrm{NA}$ & NA & $\mathrm{NA}$ & NA & $\overline{\mathrm{N} A}$ \\
\hline Sulfate & NA & $\mathrm{NA}$ & $\mathrm{NA}$ & NA & NA & NA & NA & $\mathrm{NA}$ & $\mathrm{NA}$ & $\mathrm{NA}$ & $\mathrm{NA}$ & NA & \begin{tabular}{|c|}
$<0.1$ \\
\end{tabular} & NA & $\overline{\mathrm{NC}}$ \\
\hline
\end{tabular}

* Suspended

\# Dissolved

NA - Not Analyzed

NC - Not Calculated 
The Catholic University of America

Vitreous State Laboratory

DuraMelter 1200 Tests with AZ-101HLW Simulants

Final Report, VSL-02R0100-2, Rev.1

Table 4.6. Analytical Results for Samples of SBS Blow-Down Fluids (mg/l), (continued).

\begin{tabular}{|c|c|c|c|c|c|c|c|c|c|c|c|c|c|c|c|}
\hline Test & \multicolumn{15}{|c|}{$\# 7$} \\
\hline I.D. & \multicolumn{3}{|c|}{$12 \mathrm{~N}-\mathrm{S}-79 \mathrm{~A}$} & \multicolumn{3}{|c|}{$12 \mathrm{~N}-\mathrm{S}-81 \mathrm{~A}$} & \multicolumn{3}{|c|}{$12 \mathrm{~N}-\mathrm{S}-85 \mathrm{~A}$} & \multicolumn{3}{|c|}{$12 \mathrm{~N}-\mathrm{S}-86 \mathrm{~A}$} & \multicolumn{3}{|c|}{$12 \mathrm{~N}-\mathrm{S}-87 \mathrm{~A}$} \\
\hline Glass (kg) & \multicolumn{3}{|c|}{21720} & \multicolumn{3}{|c|}{21823} & \multicolumn{3}{|c|}{21926} & \multicolumn{3}{|c|}{22029} & \multicolumn{3}{|c|}{22260} \\
\hline $\mathrm{pH}$ & \multicolumn{3}{|c|}{2.41} & \multicolumn{3}{|c|}{2.41} & \multicolumn{3}{|c|}{2.38} & \multicolumn{3}{|c|}{2.40} & \multicolumn{3}{|c|}{2.05} \\
\hline & Sus* & Dis.\# & Total & Sus* & Dis. & Total & Sus* & Dis.\# & \begin{tabular}{|l|} 
Total \\
\end{tabular} & Sus* & Dis.\# & Total & Sus* & Dis.\# & Tota \\
\hline Total & 350 & 1750 & 2100 & 264 & 1992 & 2256 & -264 & 1632 & 1896 & 236 & 1868 & 2104 & 248 & 1928 & 217 \\
\hline $\mathrm{Al}$ & 2.38 & 29.98 & 32.36 & 1.66 & 29.22 & 30.88 & 2.40 & 29.44 & 31.84 & 1.58 & 29.59 & 31.17 & 1.39 & 29.03 & 30.42 \\
\hline As & 0.11 & 4.86 & 4.97 & 0.08 & 5.43 & 5.51 & 0.03 & 5.39 & 5.42 & 0.07 & 5.40 & 5.47 & 0.04 & 5.34 & 5.38 \\
\hline $\bar{B}$ & 1.67 & 173.0 & 174.7 & 1.03 & 173.4 & 174.4 & 1.48 & \begin{tabular}{|l|}
176.7 \\
\end{tabular} & 178.2 & 1.14 & 174.2 & 175.3 & 0.82 & 178.2 & 179. \\
\hline$\overline{\mathrm{Ba}}$ & 0.09 & 0.09 & 0.18 & 0.07 & 0.09 & 0.16 & 0.08 & 0.09 & \begin{tabular}{|l|}
0.17 \\
\end{tabular} & 0.07 & 0.10 & 0.17 & 0.07 & 0.10 & 0.1 \\
\hline $\mathrm{Ca}$ & 0.20 & 4.83 & 5.03 & 0.15 & 4.68 & 4.83 & 0.17 & 4.63 & 4.80 & $\overline{0.14}$ & 4.49 & 4.63 & 0.12 & 4.15 & 4.27 \\
\hline$\overline{\mathrm{Cd}}$ & 0.25 & 17.19 & 17.44 & 0.18 & 17.02 & 17.2 & 0.25 & 17.28 & 17.53 & 0.20 & 16.84 & 17.04 & 0.18 & 17.57 & 17.75 \\
\hline $\mathrm{Cr}$ & 0.26 & 0.41 & 0.67 & 0.19 & 0.38 & 0.57 & 0.24 & 0.38 & 0.62 & 0.17 & 0.37 & 0.54 & 0.16 & 0.35 & 0.51 \\
\hline$\overline{\mathrm{Cs}}$ & $<0.1$ & 5.80 & 5.80 & $<0.1$ & 5.80 & 5.80 & $<0.1$ & 5.20 & 5.20 & $<0.1$ & 5.70 & 5.70 & $<0.1$ & 5.50 & 5.5 \\
\hline$\overline{\mathrm{Cu}}$ & 0.03 & 0.14 & 0.17 & 0.02 & 0.14 & 0.16 & 0.03 & 0.14 & 0.17 & 0.02 & 0.14 & 0.16 & 0.02 & 0.14 & 0.16 \\
\hline $\mathrm{Fe}$ & 48.27 & $\overline{5.14}$ & 53.41 & 35.51 & 5.21 & 40.72 & 41.91 & 4.89 & 46.8 & 31.07 & $\overrightarrow{4.84}$ & 35.91 & 29.18 & 4.65 & 33.83 \\
\hline $\mathrm{K}$ & 0.07 & 3.86 & 3.93 & 0.10 & 3.69 & 3.79 & 0.11 & 3.80 & 3.91 & 0.11 & 3.74 & 3.85 & 0.04 & 3.56 & 3.6 \\
\hline $\mathrm{Li}$ & 1.20 & 13.84 & 15.04 & 0.77 & 13.60 & 14.37 & 1.11 & 13.44 & 14.55 & 0.86 & 13.59 & 14.45 & 0.60 & 13.49 & 14.05 \\
\hline $\mathrm{Mg}$ & 0.24 & 4.74 & 4.98 & 0.18 & 4.87 & 5.05 & 0.24 & 4.77 & 5.01 & 0.19 & 4.86 & 5.05 & 0.15 & 4.82 & 4.9 \\
\hline $\mathrm{Mn}$ & 0.10 & 7.41 & 7.51 & 0.06 & 7.15 & 7.21 & 0.08 & 6.77 & 6.85 & 0.06 & 6.92 & 6.98 & 0.05 & 6.43 & 6.4 \\
\hline $\mathrm{Na}$ & 2.21 & 35.34 & 37.55 & 1.36 & 34.73 & 36.09 & 2.01 & 34.78 & 36.79 & 1.61 & 34.61 & 36.22 & 1.09 & 33.80 & 34.85 \\
\hline $\mathrm{Ni}$ & 2.03 & 0.83 & 2.86 & 1.64 & 0.80 & 2.44 & 2.04 & 0.76 & 2.80 & 1.53 & 0.75 & 2.28 & 1.36 & 0.71 & 2.07 \\
\hline $\mathrm{P}$ & 0.22 & 1.28 & 1.50 & $<0.01$ & 1.05 & 1.05 & $<0.01$ & 1.07 & 1.07 & 0.06 & 1.00 & 1.06 & 0.07 & 1.04 & 1.1 \\
\hline $\mathrm{Pb}$ & 0.41 & 2.04 & 2.45 & 0.29 & 2.15 & 2.44 & 0.39 & 2.02 & 2.41 & 0.27 & 2.02 & 2.29 & 0.28 & 2.04 & 2.3 \\
\hline $\mathrm{Sb}$ & 0.62 & 1.39 & 2.01 & 0.31 & 1.38 & 1.69 & 0.58 & 1.39 & 1.97 & 0.49 & 1.35 & 1.84 & 0.33 & 1.31 & 1.6 \\
\hline $\mathrm{Se}$ & 172.8 & 31.08 & 203.9 & 132.1 & 29.26 & 161.4 & 98.43 & 32.60 & 131.0 & 113.2 & 35.42 & 148.6 & 157.4 & 44.51 & 201.9 \\
\hline $\mathrm{Si}$ & 32.05 & 52.56 & 84.61 & 18.07 & 51.51 & 69.58 & 25.88 & 49.84 & 75.72 & 18.10 & 50.29 & 68.39 & 14.59 & 47.17 & $61.7 t$ \\
\hline $\mathrm{Sr}$ & 0.35 & 9.51 & 9.86 & 0.24 & 9.61 & 9.85 & 0.29 & 9.59 & 9.88 & 0.22 & 9.87 & 10.09 & \begin{tabular}{|l|}
0.19 \\
\end{tabular} & \begin{tabular}{|l|}
9.97 \\
\end{tabular} & 10.16 \\
\hline $\mathrm{Te}$ & 3.61 & 52.43 & 56.04 & 2.98 & 50.97 & 53.95 & 3.21 & 51.86 & 55.07 & 2.80 & 49.41 & 52.21 & 2.65 & 48.80 & 51.45 \\
\hline $\mathrm{Ti}$ & 0.42 & 0.23 & 0.65 & 0.30 & 0.23 & 0.53 & 0.39 & 0.22 & 0.61 & 0.28 & 0.22 & 0.50 & 0.25 & 0.21 & 0.4 \\
\hline $\mathrm{Zn}$ & 1.24 & 6.17 & 7.41 & 0.80 & 6.04 & 6.84 & 1.10 & 6.00 & 7.10 & 0.90 & 5.98 & 6.88 & 0.65 & \begin{tabular}{|l|}
5.87 \\
\end{tabular} & 6.52 \\
\hline $\mathrm{Zr}$ & 0.63 & 11.33 & 11.96 & 0.42 & 11.19 & 11.61 & 0.61 & 10.98 & 11.59 & 0.41 & 11.26 & 11.67 & 0.33 & \begin{tabular}{|l|}
10.93 \\
\end{tabular} & 11.26 \\
\hline $\mathrm{Cl}$ & NA & $\mathrm{NA}$ & NA & NA & NA & NA & NA & $\mathrm{NA}$ & NA & $\mathrm{NA}$ & NA & $\mathrm{NA}$ & NA & NA & $\mathrm{NA}$ \\
\hline$F$ & $\mathrm{NA}$ & $\mathrm{NA}$ & $\mathrm{NA}$ & $\mathrm{NA}$ & $\mathrm{NA}$ & NA & $\mathrm{NA}$ & NA & NA & $\mathrm{NA}$ & $\mathrm{NA}$ & NA & NA & $\mathrm{NA}$ & NA \\
\hline I & $<0.1$ & NA & $\mathrm{NQ}$ & $<0.1$ & $\mathrm{NA}$ & $\mathrm{NC}$ & $<0.1$ & NA & $\mathrm{NQ}$ & $<0.1$ & NA & $\mathrm{NC}$ & $<0.1$ & NA & $\mathrm{NC}$ \\
\hline Nitrate & $\mathrm{NA}$ & NA & NA & NA & NA & NA & $\mathrm{NA}$ & NA & $\mathrm{NA}$ & NA & $\mathrm{NA}$ & $\mathrm{NA}$ & NA & NA & $\overline{N A}$ \\
\hline rite & $\mathrm{NA}$ & NA & NA & NA & NA & NA & NA & $\mathrm{NA}$ & $\mathrm{NA}$ & NA & $\mathrm{NA}$ & $\mathrm{NA}$ & NA & NA & $\mathrm{NA}$ \\
\hline Sulfate & $<0.1$ & NA & $\mathrm{NQ}$ & 0.37 & $\mathrm{NA}$ & $\mathrm{NC}$ & $<0.1$ & NA & $\mathrm{NC}$ & $<0.1$ & NA & $\mathrm{NQ}$ & $<0.1$ & $\mathrm{NA}$ & NC \\
\hline
\end{tabular}

* Suspended

\# Dissolved

NA - Not Analyzed

NC - Not Calculated 
Table 4.6. Analytical Results for Samples of SBS Blow-Down Fluids (mg/l), (continued).

\begin{tabular}{|c|c|c|c|c|c|c|c|}
\hline Test & \multicolumn{7}{|c|}{$\# 7$} \\
\hline I.D. & $12 \mathrm{~N}-\mathrm{S}-8$ & $38 \mathrm{~A}$ & $12 \mathrm{~N}-\mathrm{S}-94 \mathrm{~A}$ & $12 \mathrm{~N}-\mathrm{S}-99 \mathrm{~A}$ & $12 \mathrm{~N}-\mathrm{S}-99 \mathrm{~B}$ & $12 \mathrm{~N}-\mathrm{S}-10$ & $01 \mathrm{~A}$ \\
\hline lass $(\mathrm{kg})$ & 22492 & & 22580 & 22669 & 22757 & 22845 & \\
\hline $\mathrm{pH}$ & 2.04 & & 2.00 & 2.04 & 2.05 & 2.01 & \\
\hline 2280 & Sus*|Dis.\# & Total & Sus* Dis.\#l Total & Sus*|Dis.\#Total & Sus*|Dis.\#|Total & Sus*|Dis.\#| & Tota \\
\hline Total & \begin{tabular}{|l|l|}
228 & 2004 \\
\end{tabular} & 2232 & \begin{tabular}{|l|l|l|}
224 & 2136 & 2360 \\
\end{tabular} & \begin{tabular}{|l|l|l|}
184 & 1828 & 2012 \\
\end{tabular} & \begin{tabular}{|l|l|l|}
188 & 1680 & 1868 \\
\end{tabular} & \begin{tabular}{l|l|}
234 & 2280 \\
\end{tabular} & 2514 \\
\hline $\mathrm{Al}$ & 1.4829 .83 & 31.31 & 1.1228 .0429 .16 & 0.9627 .6828 .64 & 0.7628 .5129 .27 & 0.9328 .352 & 529.28 \\
\hline As & \begin{tabular}{|l|l|}
0.23 & 5.47 \\
\end{tabular} & 5.70 & \begin{tabular}{|l|l|l|}
0.17 & 5.32 & 5.49 \\
\end{tabular} & \begin{tabular}{|l|l|l|l|}
0.02 & 5.50 & 5.52 \\
\end{tabular} & \begin{tabular}{|c|c|c|}
0.34 & 5.34 & 5.68 \\
\end{tabular} & 5.87 & $5.8^{\prime}$ \\
\hline B & \begin{tabular}{l|l|}
1.09 & 184.0 \\
\end{tabular} & 185.09 & \begin{tabular}{|l|l|l|}
0.83 & 184.8 & 185.6 \\
\end{tabular} & \begin{tabular}{|l|l|l|}
0.480 .781 .2 \\
\end{tabular} & \begin{tabular}{|l|l|l|l|}
0.23 & 195.86 .0 \\
\end{tabular} & $0.31|188.5|$ & 188 \\
\hline $\mathrm{Ba}$ & \begin{tabular}{|l|l|}
0.07 & 0.10 \\
\end{tabular} & 0.17 & \begin{tabular}{|l|l|l|}
0.06 & 0.07 & 0.13 \\
\end{tabular} & \begin{tabular}{|l|l|}
0.06 & 0.08 \\
\end{tabular} & \begin{tabular}{|l|l|}
0.06 & 0.08 \\
\end{tabular} & 0.09 & J.1 \\
\hline $\mathrm{Ca}$ & \begin{tabular}{l|l|}
0.12 & 4.15 \\
\end{tabular} & 4.27 & \begin{tabular}{|l|l|l|}
0.10 & 3.67 & 3.77 \\
\end{tabular} & \begin{tabular}{|l|l|l|}
0.09 & 3.76 & 3.85 \\
\end{tabular} & \begin{tabular}{|l|l|}
0.07 & 4.31 \\
\end{tabular} & 3.88 & 3. \\
\hline $\mathrm{Cd}$ & \begin{tabular}{l|l|}
0.23 & 17.54 \\
\end{tabular} & 17.77 & \begin{tabular}{|l|l|l|}
0.18 & 17.92 & 18.1 \\
\end{tabular} & \begin{tabular}{l|l|l|l|}
0.15 & 18.52 & 18.67 \\
\end{tabular} & \begin{tabular}{|l|l|l|}
0.13 & 18.12 & 18.25 \\
\end{tabular} & \begin{tabular}{l|l|}
0.16 & 18.78 \\
\end{tabular} & 18.94 \\
\hline $\mathrm{Cr}$ & \begin{tabular}{|l|l|}
0.16 & 0.35 \\
\end{tabular} & 0.51 & \begin{tabular}{|l|l|l|}
0.13 & 0.39 & 0.52 \\
\end{tabular} & \begin{tabular}{ll|l|}
0.40 & 0.53 \\
\end{tabular} & \begin{tabular}{|l|l|}
0.10 & 0.38 \\
\end{tabular} & \begin{tabular}{|l|l|}
0.12 & 0.39 \\
\end{tabular} & 0.5 \\
\hline Cs & \begin{tabular}{l|l|}
$<0.1$ & 6.10 \\
\end{tabular} & 6.10 & \begin{tabular}{|l|l|}
5.80 & 5.80 \\
\end{tabular} & \begin{tabular}{|l|l|}
5.60 & 5.60 \\
\end{tabular} & \begin{tabular}{|l|l|}
5.80 & 5.80 \\
\end{tabular} & 5.80 & 5. \\
\hline $\mathrm{Cu}$ & \begin{tabular}{|l|l|}
0.01 & 0.14 \\
\end{tabular} & 0.15 & \begin{tabular}{|l|l|l|}
0.01 & 0.13 & 0.14 \\
\end{tabular} & \begin{tabular}{|l|l|}
0.13 & 0.14 \\
\end{tabular} & \begin{tabular}{l|l|}
0.15 & 0.16 \\
\end{tabular} & 0.01 & 0. \\
\hline $\mathrm{Fe}$ & \begin{tabular}{|l|l|}
34.35 & 4.67 \\
\end{tabular} & 39.02 & \begin{tabular}{|l|l|l|}
26.57 & 4.63 & 31.2 \\
\end{tabular} & 4.5727 .88 & \begin{tabular}{|l|l|l|}
19.71 & 4.6824 .39 \\
\end{tabular} & $5.00 \sqrt[3]{3}$ & 31. \\
\hline$\overline{\mathrm{K}}$ & \begin{tabular}{|l|l|}
0.10 & 3.61 \\
\end{tabular} & 3.71 & \begin{tabular}{|l|l|l|}
0.08 & 3.13 & 3.21 \\
\end{tabular} & \begin{tabular}{|l|l|l|}
0.04 & 3.20 & 3.24 \\
\end{tabular} & \begin{tabular}{|l|l|l|}
0.05 & 3.79 & 3.84 \\
\end{tabular} & 3.54 & 3.62 \\
\hline$\overline{\mathrm{Li}}$ & \begin{tabular}{l|l|l|}
0.81 & 13.71
\end{tabular} & 14.52 & $0.60|14.94| 15.54$ & $0.36|14.57| 14.93$ & $0.18|15.94| 16.12$ & \begin{tabular}{ll|l|l|}
0.2314 .601 &
\end{tabular} & $\longdiv { 1 4 . 8 }$ \\
\hline $\mathrm{Mg}$ & \begin{tabular}{|l|l|}
0.19 & 4.85 \\
\end{tabular} & 5.04 & \begin{tabular}{|l|l|l|}
0.14 & 4.75 & 4.89 \\
\end{tabular} & \begin{tabular}{|l|l|l|}
0.11 & 4.78 & 4.89 \\
\end{tabular} & \begin{tabular}{|l|l|l|}
0.09 & 5.09 & 5.18 \\
\end{tabular} & 0.11 & 5.0 \\
\hline $\mathrm{Mn}$ & \begin{tabular}{|l|l|}
0.08 & 6.41 \\
\end{tabular} & 6.49 & \begin{tabular}{|l|l|l|}
0.06 & 6.49 \\
\end{tabular} & \begin{tabular}{|l|l|l|}
0.05 & 6.17 & 6.22 \\
\end{tabular} & \begin{tabular}{|l|l|l|}
0.03 & 6.29 & 6.32 \\
\end{tabular} & \begin{tabular}{l|l|}
0.05 & 6.18 \\
\end{tabular} & 6. \\
\hline $\mathrm{Na}$ & $1.46 / 34.70$ & 36.16 & \begin{tabular}{|l|l|l|}
1.1034 .30 & 35.4 \\
\end{tabular} & 0.6433 .9034 .54 & $0.32 / 37.9938 .31$ & 0.4134 .813 & 35.2 \\
\hline $\mathrm{Ni}$ & \begin{tabular}{|l|l|}
1.59 & 0.73 \\
\end{tabular} & 2.32 & \begin{tabular}{|l|l|l|}
1.27 & 0.76 & 2.03 \\
\end{tabular} & \begin{tabular}{|l|l|l|}
1.09 & 0.80 & 1.89 \\
\end{tabular} & \begin{tabular}{|l|l|l|}
0.91 & 0.79 & 1.70 \\
\end{tabular} & \begin{tabular}{l|l|}
1.15 & 0.76 \\
\end{tabular} & 1.9 \\
\hline $\mathbf{P}$ & \begin{tabular}{l|l|}
0.02 & 1.10 \\
\end{tabular} & 1.12 & \begin{tabular}{|l|l|l|}
0.07 & 1.31 & 1.38 \\
\end{tabular} & \begin{tabular}{l|l|}
0.03 & 1.41 \\
\end{tabular} & \begin{tabular}{|l|l|}
1.38 & 1.38 \\
\end{tabular} & \begin{tabular}{l|l|}
0.15 & 1.45 \\
\end{tabular} & 1.6 \\
\hline $\mathrm{Pb}$ & \begin{tabular}{|l|l|}
0.27 & 2.03 \\
\end{tabular} & 2.30 & \begin{tabular}{|l|l|l|}
0.23 & 2.11 & 2.34 \\
\end{tabular} & \begin{tabular}{|l|l|}
0.24 & 2.14 \\
\end{tabular} & \begin{tabular}{|l|l|}
2.13 & 2.35 \\
\end{tabular} & 0.31 & 2.4 \\
\hline $\mathrm{Sb}$ & \begin{tabular}{l|l|}
0.47 & 1.37 \\
\end{tabular} & 1.84 & \begin{tabular}{|l|l|l|}
0.38 & 1.32 & 1.70 \\
\end{tabular} & \begin{tabular}{|l|l|l|}
0.29 & 1.32 & 1.61 \\
\end{tabular} & \begin{tabular}{l|l|}
1.26 & 1.47 \\
\end{tabular} & \begin{tabular}{l|l|}
0.26 & 1.21 \\
\end{tabular} & 1.4 \\
\hline Se & 99.5049 .16 & 148.7 & 136.748 .99185 .7 & \begin{tabular}{|l|l|l|}
110.454 .29 & 164.7 \\
\end{tabular} & 122.672 .53195 .1 & 140.963 .812 & 204. \\
\hline $\mathrm{Si}$ & 19.7348 .05 & 67.78 & 13.9947 .2761 .26 & \begin{tabular}{|l|l|l|}
8.83 & 46.22 & 55.05 \\
\end{tabular} & \begin{tabular}{|l|l|}
5.8844 .3950 .27 \\
\end{tabular} & $7.27 / 43.995$ & $\sqrt[51.2]{51}$ \\
\hline$\overline{\mathrm{Sr}}$ & \begin{tabular}{|l|l|}
0.21 & 10.07 \\
\end{tabular} & 10.28 & $0.18|10.15| 10.33$ & 0.1610 .3110 .47 & 0.1612 .1612 .32 & \begin{tabular}{l|l|l|}
0.19 & 10.93 \\
\end{tabular} & 11.1 \\
\hline$\overline{\mathrm{Te}}$ & 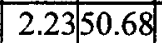 & 52.91 & 1.69449 .3951 .08 & \begin{tabular}{|l|l|l|}
1.51 & 53.3054 .81 \\
\end{tabular} & \begin{tabular}{l|l|l|}
1.335 .7357 .06 \\
\end{tabular} & 1.4358 .005 & 59.4 \\
\hline$\overline{\mathrm{Ti}}$ & \begin{tabular}{|l|l|}
0.27 & 0.20 \\
\end{tabular} & 0.47 & \begin{tabular}{|l|l|l|}
0.20 & 0.22 & 0.42 \\
\end{tabular} & \begin{tabular}{|l|l|l|}
0.16 & 0.22 & 0.38 \\
\end{tabular} & \begin{tabular}{|l|l|l|}
0.12 & 0.19 & 0.31 \\
\end{tabular} & \begin{tabular}{l|l|}
0.15 & 0.20
\end{tabular} & 0.3 \\
\hline $\mathrm{Zn}$ & \begin{tabular}{l|l|}
0.84 & 6.05 \\
\end{tabular} & 6.89 & \begin{tabular}{l|l|l}
0.62 & 5.95 & 6.57 \\
\end{tabular} & \begin{tabular}{|l|l|l|}
0.39 & 7.95 & 8.34 \\
\end{tabular} & \begin{tabular}{|l|l|l|}
0.23 & 6.61 & 6.84 \\
\end{tabular} & \begin{tabular}{l|l|}
0.30 & 6.70 \\
\end{tabular} & 7.0 \\
\hline$\overline{Z r}$ & \begin{tabular}{l|l|l|}
0.371 .09 \\
\end{tabular} & 11.46 & 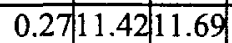 & \begin{tabular}{|l|l|}
0.1911 .2411 .43 \\
\end{tabular} & \begin{tabular}{|l|l|l|}
0.12 & 11.38 & 11.5 \\
\end{tabular} & \begin{tabular}{l|l|l|}
0.1211 .721 \\
\end{tabular} & 11.8 \\
\hline $\mathrm{Cl}$ & $\mathrm{NA}$ & NA & NA & NA & NA NA & NA & $\mathrm{N}$ \\
\hline $\bar{F}$ & NA & $\mathrm{NA}$ & NA & NA & \begin{tabular}{l|l|} 
NA & NA \\
\end{tabular} & NA & $\mathrm{N}$ \\
\hline $\mathrm{I}$ & NA & NA & NA & $<0.1$ & \begin{tabular}{l|l|}
$\mathrm{NA}$ & $\mathrm{NQ}$ \\
\end{tabular} & NA & $\mathrm{N}$ \\
\hline Nitrate & NA NA & NA & \begin{tabular}{l|l|}
$\mathrm{NA}$ & $\mathrm{NA}$ \\
\end{tabular} & NA & \begin{tabular}{l|l|}
$\mathrm{NA}$ & $\mathrm{NA}$ \\
\end{tabular} & NA & $\overline{\mathrm{NA}}$ \\
\hline Nitrite & NA & $\mathrm{NA}$ & $\mathrm{NA}$ & $\mathrm{NA}$ & \begin{tabular}{l|l|} 
NA & NA \\
\end{tabular} & NA & $\overline{\mathrm{N}} \mathrm{z}$ \\
\hline Sulfate & \begin{tabular}{l|l|}
$\mathrm{NA}$ & $\mathrm{NA}$ \\
\end{tabular} & $\mathrm{NA}$ & \begin{tabular}{l|l|l|}
$\mathrm{NA}$ & $\mathrm{NA}$ & $\mathrm{NA}$ \\
\end{tabular} & \begin{tabular}{|l|l|l}
$<0.1$ & NA & NO \\
\end{tabular} & \begin{tabular}{l|l|l}
0.15 & $\mathrm{NA}$ & $\mathrm{NQ}$ \\
\end{tabular} & \begin{tabular}{l|l|}
$\mathrm{NA}$ & $\mathrm{NA}$ \\
\end{tabular} & $\mathrm{N}$ \\
\hline
\end{tabular}


Table 4.6. Analytical Results for Samples of SBS Blow-Down Fluids (mg/l), (continued).

\begin{tabular}{|c|c|c|c|c|c|c|c|c|c|c|c|c|c|c|c|}
\hline Test & \multicolumn{15}{|c|}{$\# 7$} \\
\hline I.D. & \multicolumn{3}{|c|}{$12 \mathrm{~N}-\mathrm{S}-102 \mathrm{~A}$} & \multicolumn{3}{|c|}{$12 \mathrm{~N}-\mathrm{S}-104 \mathrm{~A}$} & \multicolumn{3}{|c|}{$12 \mathrm{~N}-\mathrm{S}-106 \mathrm{~A}$} & \multicolumn{3}{|c|}{$12 \mathrm{~N}-\mathrm{S}-114 \mathrm{~A}$} & \multicolumn{3}{|c|}{$12 \mathrm{~N}-\mathrm{S}-117 \mathrm{~A}$} \\
\hline$\overline{\text { Glass (kg) }}$ & \multicolumn{3}{|c|}{22934} & \multicolumn{3}{|c|}{23022} & \multicolumn{3}{|c|}{23202} & \multicolumn{3}{|c|}{23372} & \multicolumn{3}{|c|}{23563} \\
\hline $\mathrm{pH}$ & \multicolumn{3}{|c|}{2.27} & \multicolumn{3}{|c|}{2.26} & \multicolumn{3}{|c|}{2.28} & \multicolumn{3}{|c|}{2.24} & \multicolumn{3}{|c|}{2.25} \\
\hline & Sus* & Dis.\# & Total & \begin{tabular}{|l} 
Sus* \\
\end{tabular} & Dis.\# & Total & Sus* & Dis.\# & Total & Sus* & Dis.\# & Total & Sus* & Dis.\# & Total \\
\hline Total & 192 & 2048 & 2240 & 132 & 1916 & 2048 & 352 & 2240 & 2592 & 260 & 2208 & 2468 & 384 & 2048 & 2432 \\
\hline $\mathrm{Al}$ & 0.52 & 29.79 & 30.31 & 0.53 & 34.91 & 35.44 & 2.75 & 42.95 & 45.7 & 2.26 & 43.11 & 45.37 & 3.61 & 53.30 & 56.9 \\
\hline As & $<0.01$ & 5.62 & 5.62 & $<0.01$ & 5.59 & 5.59 & 0.09 & 5.75 & 5.84 & 0.09 & 6.03 & 6.12 & $<0.01$ & 5.96 & 5.9 \\
\hline B & 0.11 & 190.1 & 190.2 & 0.13 & 193.6 & \begin{tabular}{|l|}
193.7 \\
\end{tabular} & 1.40 & 195.3 & 196.7 & $\overline{0.90}$ & 194.2 & $\mid 195.1$ & 1.37 & 198.9 & 200.3 \\
\hline $\mathrm{Ba}$ & 0.06 & 0.06 & 0.12 & 0.07 & 0.06 & 0.13 & 0.14 & 0.06 & 0.20 & 0.15 & 0.06 & 0.21 & 0.21 & 0.06 & 0.27 \\
\hline $\mathrm{Ca}$ & 0.05 & 3.40 & 3.45 & $\overline{0.05}$ & 3.66 & 3.71 & 0.27 & 3.54 & 3.81 & 0.19 & 3.70 & 3.89 & 0.36 & 4.25 & 4.6 \\
\hline $\mathrm{Cd}$ & 0.08 & 18.63 & 18.71 & $\overline{0.09}$ & 19.10 & 19.19 & 0.37 & 19.46 & 19.83 & 0.27 & 20.49 & 20.76 & 0.58 & 21.21 & 21.79 \\
\hline $\mathrm{Cr}$ & 0.08 & 0.38 & 0.46 & $\overline{0.08}$ & 0.38 & \begin{tabular}{|l|}
0.46 \\
\end{tabular} & 0.32 & 0.39 & 0.71 & 0.26 & 0.38 & 0.64 & 0.49 & 0.42 & 0.9 \\
\hline Cs & $<0.1$ & 5.50 & 5.50 & $<0.1$ & 5.40 & 5.40 & $<0.1$ & 6.00 & 6.00 & $<0.1$ & 6.40 & 6.40 & 0.10 & 6.10 & 6.2 \\
\hline $\mathrm{Cu}$ & 0.01 & 0.15 & 0.16 & 0.01 & 0.16 & 0.17 & 0.02 & 0.16 & 0.18 & 0.03 & 0.18 & 0.21 & 0.05 & 0.18 & $\overline{0.23}$ \\
\hline $\mathrm{Fe}$ & 14.05 & 4.95 & 19.00 & 13.95 & 5.00 & 18.95 & 57.07 & 6.06 & 63.13 & 47.37 & 6.05 & 53.42 & 83.16 & 7.04 & 90.20 \\
\hline $\mathrm{K}$ & 0.01 & 2.78 & 2.79 & 0.03 & 2.93 & 2.96 & 0.17 & 3.03 & 3.20 & 0.10 & 3.10 & 3.20 & 0.21 & 3.37 & 3.5 \\
\hline$\overline{\mathrm{Li}}$ & 0.10 & 14.18 & 14.28 & 0.11 & 14.18 & 14.29 & 1.15 & 16.02 & 17.17 & $\overline{0.76}$ & 14.99 & 15.75 & 1.16 & 16.76 & 17.9 \\
\hline$\overline{M g}$ & 0.06 & \begin{tabular}{|l|}
5.27 \\
\end{tabular} & 5.33 & $<0.01$ & 5.04 & \begin{tabular}{|l|}
5.04 \\
\end{tabular} & 0.35 & 5.01 & 5.36 & 0.26 & 4.44 & 4.70 & 0.53 & 4.59 & 5.12 \\
\hline $\mathrm{Mn}$ & 0.02 & 6.31 & 6.33 & 0.03 & 6.48 & 6.51 & 0.12 & 7.55 & 7.67 & 0.08 & 7.42 & 7.50 & 0.17 & 9.74 & 9.9 \\
\hline $\mathrm{Na}$ & 0.21 & 38.62 & \begin{tabular}{|c|}
38.83 \\
\end{tabular} & 0.20 & 41.20 & 41.40 & 1.97 & 47.04 & 49.01 & 1.26 & 45.71 & 46.97 & 1.89 & 51.11 & $\overline{53.00}$ \\
\hline $\mathrm{Ni}$ & 0.59 & 0.73 & \begin{tabular}{|l|}
1.32 \\
\end{tabular} & 0.64 & \begin{tabular}{|l|}
0.73 \\
\end{tabular} & \begin{tabular}{|l|}
1.37 \\
\end{tabular} & 3.03 & \begin{tabular}{|l|}
0.91 \\
\end{tabular} & 3.94 & 2.29 & \begin{tabular}{|l|}
0.88 \\
\end{tabular} & 3.17 & 4.87 & 1.26 & 6.1 \\
\hline$P$ & $<0.01$ & 0.95 & 0.95 & 0.06 & 0.92 & 0.98 & 0.18 & 1.07 & 1.25 & $<0.01$ & 1.00 & 1.00 & 0.16 & 0.90 & 1.0 \\
\hline$\overline{\mathrm{Pb}}$ & 0.21 & 1.97 & 2.18 & 0.23 & 2.13 & 2.36 & 0.53 & 2.16 & 2.69 & 0.51 & 2.12 & 2.63 & 0.72 & 2.33 & $\overline{3.0}$ \\
\hline $\mathrm{Sb}$ & 0.14 & 1.36 & 1.50 & $\overline{0.11}$ & 1.40 & 1.51 & 0.74 & 1.53 & 2.27 & 0.50 & 1.45 & 1.95 & 1.12 & 1.31 & 2.4 \\
\hline $\mathrm{Se}$ & 155.4 & 55.75 & 211.2 & 88.03 & 51.22 & 139.3 & 145.6 & 47.75 & 193.4 & 101.1 & 54.22 & \begin{tabular}{|l|}
155.3 \\
\end{tabular} & \begin{tabular}{|l|}
113.3 \\
\end{tabular} & 63.27 & 176. \\
\hline $\mathrm{Si}$ & 2.89 & 44.98 & \begin{tabular}{|l|}
47.87 \\
\end{tabular} & 3.44 & 47.38 & 50.82 & 27.06 & 60.29 & 87.35 & 22.52 & 57.65 & 80.17 & 33.95 & 67.32 & 101. \\
\hline $\mathrm{Sr}$ & 0.11 & 10.98 & 11.09 & 0.12 & 11.16 & 11.28 & 0.58 & 12.13 & 12.71 & 0.57 & 12.36 & 12.93 & 1.17 & 13.52 & 14.6 \\
\hline $\mathrm{Te}$ & 1.23 & 54.99 & 56.22 & 1.10 & 55.24 & 56.34 & 3.66 & 56.16 & 59.82 & $\overline{2.34}$ & 55.30 & 57.64 & 4.85 & 55.26 & 60.11 \\
\hline $\mathrm{Ti}$ & 0.08 & 0.20 & \begin{tabular}{|l|}
0.28 \\
\end{tabular} & 0.09 & 0.20 & \begin{tabular}{|l|}
0.29 \\
\end{tabular} & 0.46 & \begin{tabular}{|l|}
0.22 \\
\end{tabular} & 0.68 & 0.34 & \begin{tabular}{|l|}
0.24 \\
\end{tabular} & \begin{tabular}{|l|}
0.58 \\
\end{tabular} & 0.65 & \begin{tabular}{|l|}
0.24 \\
\end{tabular} & 0.89 \\
\hline $\mathrm{Zn}$ & 0.12 & 6.75 & 6.87 & 0.14 & 6.68 & 6.82 & 1.23 & 7.15 & 8.38 & 0.80 & 7.56 & 8.36 & 1.20 & 8.65 & 9.8 \\
\hline $\mathrm{Zr}$ & 0.06 & 10.68 & \begin{tabular}{|l|}
10.74 \\
\end{tabular} & 0.06 & 10.82 & 10.88 & 0.56 & 11.55 & 12.11 & 0.39 & 11.57 & 11.96 & 0.63 & 12.89 & 13.52 \\
\hline $\mathrm{Cl}$ & NA & NA & $\mathrm{NA}$ & $\mathrm{NA}$ & NA & NA & NA & $\mathrm{NA}$ & $\mathrm{NA}$ & $\mathrm{NA}$ & NA & NA & NA & NA & $\overline{N A}$ \\
\hline$F$ & NA & $\mathrm{NA}$ & NA & $\mathrm{NA}$ & NA & NA & NA & $\mathrm{NA}$ & NA & $\mathrm{NA}$ & $\mathrm{NA}$ & NA & $\mathrm{NA}$ & NA & $\mathrm{NA}$ \\
\hline I & $<0.1$ & NA & $\mathrm{NC}$ & NA & NA & NA & $<0.1$ & $\mathrm{NA}$ & $\mathrm{NQ}$ & $<0.1$ & NA & NC & $<0.1$ & NA & NC \\
\hline rate & NA & $\mathrm{NA}$ & NA & $\mathrm{NA}$ & NA & NA & NA & NA & NA & NA & NA & NA & NA & NA & NA \\
\hline Nitrite & NA & NA & $\mathrm{NA}$ & $\mathrm{NA}$ & NA & NA & NA & NA & $\mathrm{NA}$ & $\mathrm{NA}$ & NA & NA & NA & NA & NA \\
\hline Sulfate & 0.15 & NA & $\mathrm{NQ}$ & $\mathrm{NA}$ & NA & NA & 0.88 & NA & $\mathrm{NC}$ & $<0.1$ & NA & $\mathrm{NC}$ & 0.54 & $\mathrm{NA}$ & $\overline{\mathrm{NC}}$ \\
\hline
\end{tabular}

* Suspended

\# Dissolved

NA - Not Analyzed

NC - Not Calculated 
The Catholic University of America

Vitreous State Laboratory

Table 4.6. Analytical Results for Samples of SBS Blow-Down Fluids (mg/l), (continued).

\begin{tabular}{|c|c|c|c|c|c|c|c|c|c|c|c|c|c|c|c|}
\hline Test & \multicolumn{9}{|c|}{ \#7 } & \multicolumn{6}{|c|}{$\# 8$} \\
\hline I.D. & \multicolumn{3}{|c|}{$12 \mathrm{~N}-\mathrm{S}-118 \mathrm{~A}$} & \multicolumn{3}{|c|}{$12 \mathrm{~N}-\mathrm{S}-126 \mathrm{~A}$} & \multicolumn{3}{|c|}{$12 \mathrm{~N}-\mathrm{S}-126 \mathrm{~B}$} & \multicolumn{3}{|c|}{$12 \mathrm{~N}-\mathrm{S}-152 \mathrm{~A}$} & \multicolumn{3}{|c|}{$12 \mathrm{~N}-\mathrm{S}-153 \mathrm{~A}$} \\
\hline$\overline{\text { Glass }(\mathrm{kg})}$ & \multicolumn{3}{|c|}{23734} & \multicolumn{3}{|c|}{23905} & \multicolumn{3}{|c|}{24076} & \multicolumn{3}{|c|}{24144} & \multicolumn{3}{|c|}{24212} \\
\hline $\mathrm{pH}$ & \multicolumn{3}{|c|}{2.22} & \multicolumn{3}{|c|}{2.20} & \multicolumn{3}{|c|}{2.22} & \multicolumn{3}{|c|}{6.98} & \multicolumn{3}{|c|}{6.63} \\
\hline & Sus* & Dis.\# & Total & Sus* & Dis. & Total & Sus* & Dis.\# & Total & Sus* & Dis. \# & Total & Sus* & Dis.\# & Total \\
\hline Total & 596 & 2028 & 2624 & 232 & 2124 & 2356 & 448 & 2120 & 2568 & 84 & 380 & 464 & 32 & 556 & 588 \\
\hline $\mathrm{Al}$ & 5.07 & 43.96 & 49.03 & 1.56 & 39.79 & 41.35 & 3.23 & 46.33 & 49.56 & 2.96 & 0.71 & 3.67 & 0.67 & 3.51 & 4.18 \\
\hline As & $<0.01$ & 6.01 & 6.01 & 0.03 & 5.87 & 5.90 & 0.39 & 6.00 & 6.39 & 0.22 & 0.11 & 0.33 & 0.07 & 0.28 & 0.35 \\
\hline B & 1.95 & 198.6 & 200.6 & 0.47 & 199.1 & 199.6 & 1.08 & 200.9 & 202.0 & 0.34 & 16.50 & 16.84 & 0.07 & 25.89 & 25.96 \\
\hline $\mathrm{Ba}$ & 0.32 & 0.06 & 0.38 & 0.13 & 0.06 & 0.19 & 0.21 & 0.06 & 0.27 & 0.03 & 0.10 & 0.13 & 0.01 & 0.14 & 0.15 \\
\hline $\mathrm{Ca}$ & 0.46 & 4.72 & 5.18 & 0.14 & 4.56 & 4.70 & 0.26 & 4.66 & 4.92 & 0.58 & 39.65 & 40.23 & 0.18 & 42.89 & 43.07 \\
\hline $\mathrm{Cd}$ & 0.77 & 22.19 & 22.96 & 0.22 & 21.61 & 21.83 & 0.48 & 21.72 & 22.2 & 0.30 & 0.95 & 1.25 & 0.14 & 1.15 & 1.2 \\
\hline $\mathrm{Cr}$ & 0.59 & 0.41 & 1.00 & 0.18 & 0.40 & 0.58 & 0.37 & 0.41 & 0.78 & 0.10 & 0.03 & 0.13 & 0.06 & 0.03 & 0.0 \\
\hline Cs & 0.13 & 6.10 & 6.23 & $<0.1$ & 6.10 & 6.10 & 0.10 & 6.30 & 6.40 & $<0.1$ & 0.49 & 0.49 & $<0.1$ & 0.82 & 0.8 \\
\hline$\overline{\mathrm{Cu}}$ & 0.07 & 0.18 & 0.25 & 0.02 & 0.18 & 0.20 & 0.03 & 0.18 & $\overline{0.21}$ & 0.02 & $<0.01$ & 0.02 & 0.02 & 0.01 & 0.0 \\
\hline $\mathrm{Fe}$ & 116.5 & 6.57 & 123.1 & 37.67 & 6.52 & 44.19 & 84.88 & 6.92 & 91.8 & 14.28 & 0.01 & 14.29 & 6.51 & 0.02 & 6.53 \\
\hline $\mathrm{K}$ & 0.24 & 3.27 & 3.51 & 0.06 & 3.23 & 3.29 & 0.21 & 3.26 & 3.47 & 0.06 & 3.30 & 3.36 & 0.03 & 3.99 & 4.02 \\
\hline$\overline{\mathrm{Li}}$ & 1.63 & 15.46 & 17.09 & 0.42 & 15.01 & 15.43 & 0.92 & 15.61 & 16.53 & 0.26 & 3.43 & 3.69 & 0.07 & 7.41 & 7.4 \\
\hline $\mathrm{Mg}$ & 0.61 & 4.26 & 4.87 & 0.17 & 3.75 & 3.92 & 0.38 & 3.69 & 4.07 & 0.12 & 11.62 & 11.74 & 0.05 & 11.82 & 11.8 \\
\hline $\mathrm{Mn}$ & 0.23 & 9.15 & 9.38 & 0.06 & \begin{tabular}{|l|}
8.98 \\
\end{tabular} & 9.04 & 0.19 & 9.65 & 9.84 & 0.10 & 1.37 & 1.47 & 0.05 & 1.28 & 1.3 \\
\hline $\mathrm{Na}$ & 2.69 & 45.37 & 48.06 & 0.65 & 42.06 & 42.71 & 1.54 & 46.24 & 47.78 & 0.47 & 25.05 & 25.52 & 0.12 & $\mid 40.87$ & 40.99 \\
\hline $\mathrm{Ni}$ & 5.34 & 1.03 & 6.37 & 1.62 & 0.90 & 2.52 & 3.34 & 1.03 & 4.37 & 0.76 & 0.14 & 0.90 & 0.29 & 0.23 & 0.52 \\
\hline $\bar{P}$ & $<0.01$ & 0.87 & 0.87 & 0.08 & 1.01 & 1.09 & $<0.01$ & 1.21 & 1.21 & 0.12 & $<0.07$ & 0.12 & 0.06 & $<0.07$ & $\overline{0.0}$ \\
\hline $\mathrm{Pb}$ & 1.05 & 2.34 & 3.39 & 0.42 & 2.32 & 2.74 & 0.49 & 2.36 & 2.85 & 0.13 & 0.08 & 0.21 & 0.08 & 0.13 & 0.2 \\
\hline$\overline{\mathrm{Sb}}$ & 1.50 & 1.31 & 2.81 & 0.34 & 1.34 & 1.68 & 1.04 & $\overline{1.43}$ & 2.47 & 0.12 & 0.19 & 0.31 & 0.08 & 0.15 & 0.23 \\
\hline $\mathrm{Se}$ & 258.3 & 64.98 & 323.3 & 121.8 & 65.84 & 187.6 & 266.6 & 65.43 & 332.0 & 16.40 & 15.57 & 31.97 & 6.71 & \begin{tabular}{|l|}
16.68 \\
\end{tabular} & 23.39 \\
\hline $\mathrm{Si}$ & 41.27 & 58.07 & 99.34 & 11.06 & 51.35 & 62.41 & 25.49 & 58.14 & 83.63 & 8.00 & 3.77 & $11.7 \pi$ & 2.89 & \begin{tabular}{|l|}
3.73 \\
\end{tabular} & 6.62 \\
\hline$\overline{\mathrm{Sr}}$ & 1.64 & 12.98 & 14.62 & 0.56 & 12.99 & 13.55 & 1.04 & 13.13 & 14.17 & 0.12 & 2.78 & 2.9 & 0.05 & 4.47 & 4.52 \\
\hline $\mathrm{Te}$ & 5.71 & 58.66 & 64.37 & 1.58 & 57.43 & 59.01 & 3.70 & 57.09 & 60.79 & 1.80 & 0.28 & 2.08 & 0.87 & 0.29 & 1.1 \\
\hline $\mathrm{Ti}$ & 0.80 & 0.26 & 1.06 & 0.22 & 0.27 & $\overline{0.49}$ & 0.53 & 0.27 & 0.80 & 0.12 & 0.01 & 0.13 & 0.05 & 0.01 & 0.0 \\
\hline $\mathrm{Zn}$ & 1.71 & 8.15 & 9.86 & 0.43 & 7.18 & 7.61 & $\begin{array}{l}0.93 \\
\end{array}$ & 7.71 & 8.64 & 0.61 & 0.57 & 1.18 & 0.18 & 1.83 & 2.0 \\
\hline $\mathrm{Zr}$ & 0.88 & 12.19 & 13.07 & 0.24 & 12.02 & 12.26 & 0.49 & 11.97 & 12.46 & 0.96 & 0.01 & 0.97 & 0.54 & 0.02 & 0.56 \\
\hline $\mathrm{Cl}$ & NA & NA & NA & $\mathrm{NA}$ & NA & $\mathrm{NA}$ & NA & 116.5 & 116.5 & NA & 42.56 & 42.56 & NA & $\mathrm{NA}$ & NA \\
\hline $\mathrm{F}$ & NA & NA & NA & NA & $\mathrm{NA}$ & NA & NA & 315.4 & 315.4 & $\mathrm{NA}$ & 28.23 & 28.23 & NA & NA & $\overline{\mathrm{NA}}$ \\
\hline I & $<0.1$ & NA & $\mathrm{NQ}$ & $<0.1$ & NA & $\mathrm{NQ}$ & NA & 323.9 & 323.9 & $\mathrm{NA}$ & 22.29 & 22.29 & $<0.1$ & NA & $\mathrm{NC}$ \\
\hline Nitrate & $\mathrm{NA}$ & NA & NA & NA & NA & NA & NA & 7.83 & 7.83 & NA & 4.95 & 4.95 & NA & NA & NA \\
\hline & NA & NA & $\mathrm{NA}$ & $\mathrm{NA}$ & NA & $\mathrm{NA}$ & NA & $<0.1$ & $<0.1$ & $\mathrm{NA}$ & 5.08 & 5.08 & $\mathrm{NA}$ & $\mathrm{NA}$ & $\mathrm{NA}$ \\
\hline Sulfate & $<0.1$ & NA & $\mathrm{NO}$ & $<0.1$ & NA & $\mathrm{NC}$ & $\mathrm{NA}$ & 1045 & 1045 & NA & 149.1 & 149.1 & $<0.1$ & $\mathrm{NA}$ & $\mathrm{NC}$ \\
\hline
\end{tabular}

* Suspended

\# Dissolved

NA - Not Analyzed

NC - Not Calculated 
Table 4.6. Analytical Results for Samples of SBS Blow-Down Fluids (mg/l), (continued).

\begin{tabular}{|c|c|c|c|c|c|c|c|c|c|c|c|c|c|c|c|}
\hline Test & \multicolumn{15}{|c|}{$\# 8$} \\
\hline I.D. & \multicolumn{3}{|c|}{$12 \mathrm{O}-\mathrm{S}-13 \mathrm{~A}$} & \multicolumn{3}{|c|}{$120-S-14 A$} & \multicolumn{3}{|c|}{$12 \mathrm{O}-\mathrm{S}-22 \mathrm{~A}$} & \multicolumn{3}{|c|}{$12 \mathrm{O}-\mathrm{S}-31 \mathrm{~A}$} & \multicolumn{3}{|c|}{$12 \mathrm{O}-\mathrm{S}-38 \mathrm{~A}$} \\
\hline Glass $(\mathrm{kg})$ & \multicolumn{3}{|c|}{24280} & \multicolumn{3}{|c|}{24348} & \multicolumn{3}{|c|}{24416} & \multicolumn{3}{|c|}{24484} & \multicolumn{3}{|c|}{24561} \\
\hline $\mathrm{pH}$ & \multicolumn{3}{|c|}{5.20} & \multicolumn{3}{|c|}{4.83} & \multicolumn{3}{|c|}{4.95} & \multicolumn{3}{|c|}{4.94} & \multicolumn{3}{|c|}{4.99} \\
\hline & Sus* & Dis.\# & \begin{tabular}{|l|} 
Total \\
\end{tabular} & Sus* & Dis.\# & \begin{tabular}{|l|} 
Total \\
\end{tabular} & Sus* & Dis.\# & \begin{tabular}{|l|} 
Total \\
\end{tabular} & Sus* & Dis.\# & Total & Sus* & Dis.\# & Total \\
\hline Total & 24 & 800 & 824 & 16 & \begin{tabular}{|c|}
988 \\
\end{tabular} & 1004 & 26 & 1412 & 1438 & 24 & 1624 & 1648 & 16 & 1868 & 1884 \\
\hline $\mathrm{Al}$ & 0.32 & 5.21 & 5.53 & 0.20 & 6.87 & 7.07 & 0.36 & 7.74 & 8.10 & 0.28 & 8.68 & 8.96 & 0.19 & 9.49 & 9.68 \\
\hline As & 0.03 & 0.32 & 0.35 & $<0.01$ & 0.27 & 0.27 & 0.02 & 0.28 & 0.3 & $<0.01$ & 0.26 & 0.26 & 0.04 & 0.31 & $\overline{0.35}$ \\
\hline B & 0.03 & 56.40 & 56.43 & 0.02 & 87.43 & 87.45 & 0.03 & 102.5 & 102.5 & 0.02 & 132.5 & 132.5 & 0.01 & 155.3 & 155.3 \\
\hline $\mathrm{Ba}$ & 0.01 & 0.25 & 0.26 & 0.01 & 0.31 & 0.32 & 0.01 & 0.39 & 0.4 & 0.01 & 0.47 & 0.48 & 0.01 & 0.53 & 0.54 \\
\hline$\overline{\mathrm{Ca}}$ & 0.11 & 45.10 & 45.21 & 0.07 & 41.36 & \begin{tabular}{|l|}
41.43 \\
\end{tabular} & 0.11 & 42.14 & 42.25 & 0.09 & 37.59 & 37.68 & 0.06 & 34.27 & 34.33 \\
\hline $\mathrm{Cd}$ & 0.06 & 2.12 & 2.18 & 0.04 & 3.31 & 3.35 & 0.07 & 4.68 & \begin{tabular}{|l|}
4.75 \\
\end{tabular} & 0.07 & 5.52 & 5.59 & 0.05 & 6.64 & 6.69 \\
\hline $\mathrm{Cr}$ & 0.05 & 0.05 & 0.1 & 0.03 & 0.09 & 0.12 & 0.05 & 0.11 & 0.16 & 0.04 & 0.17 & 0.21 & 0.03 & 0.21 & 0.24 \\
\hline $\mathrm{Cs}$ & $<0.1$ & 1.70 & 1.70 & $<0.1$ & 3.10 & 3.10 & $<0.1$ & 5.50 & 5.50 & $<0.1$ & 6.60 & 6.60 & $<0.1$ & 7.30 & 7.30 \\
\hline $\mathrm{Cu}$ & 0.02 & 0.02 & 0.04 & 0.02 & 0.03 & 0.05 & 0.03 & 0.03 & 0.06 & 0.03 & 0.05 & 0.08 & 0.02 & 0.05 & 0.07 \\
\hline $\mathrm{Fe}$ & 5.02 & 0.06 & 5.08 & 3.34 & 0.19 & 3.53 & 5.61 & 0.28 & 5.89 & 4.98 & 0.52 & 5.50 & 3.39 & 0.67 & 4.06 \\
\hline $\bar{K}$ & 0.02 & 4.84 & 4.86 & 0.02 & 6.83 & 6.85 & 0.02 & 8.51 & 8.53 & 0.01 & 10.92 & 10.93 & 0.01 & 12.78 & 12.79 \\
\hline$\overline{\mathrm{Li}}$ & 0.04 & 14.16 & 14.2 & 0.03 & 23.76 & 23.79 & 0.05 & 34.82 & 34.87 & 0.04 & 43.28 & 43.32 & 0.03 & 48.77 & 48.8 \\
\hline $\mathrm{Mg}$ & 0.03 & \begin{tabular}{|l|}
12.53 \\
\end{tabular} & 12.56 & 0.03 & 14.22 & 14.25 & 0.04 & 14.38 & 14.42 & 0.04 & 14.71 & 14.75 & 0.03 & 15.18 & 15.21 \\
\hline$\overline{M n}$ & 0.04 & 1.73 & 1.77 & 0.03 & 2.17 & 2.20 & 0.06 & 2.67 & 2.73 & 0.06 & 3.35 & 3.41 & 0.04 & 3.82 & 3.86 \\
\hline$\overline{\mathrm{Na}}$ & 0.05 & 72.97 & 73.02 & 0.06 & 93.61 & 93.67 & 0.04 & 125.3 & 125.3 & 0.04 & 163.3 & 163.3 & \begin{tabular}{l|l}
0.03 \\
\end{tabular} & 189.4 & 189.4 \\
\hline $\mathrm{Ni}$ & 0.19 & 0.37 & 0.56 & 0.12 & 0.51 & \begin{tabular}{|l|}
0.63 \\
\end{tabular} & 0.19 & 0.62 & 0.81 & 0.17 & \begin{tabular}{|l|}
0.71 \\
\end{tabular} & \begin{tabular}{|c|}
0.88 \\
\end{tabular} & $\begin{array}{l}0.11 \\
\end{array}$ & $\begin{array}{l}0.78 \\
\end{array}$ & 0.89 \\
\hline $\bar{P}$ & 0.03 & 0.14 & 0.17 & 0.04 & 0.22 & 0.26 & 0.15 & $<0.07$ & 0.15 & 0.01 & 0.48 & 0.49 & 0.04 & 0.56 & $\overline{0.6}$ \\
\hline $\mathrm{Pb}$ & 0.02 & 0.17 & 0.19 & 0.03 & 0.25 & 0.28 & 0.08 & 0.33 & 0.41 & 0.12 & 0.45 & 0.57 & $<0.01$ & 0.52 & 0.52 \\
\hline $\mathrm{Sb}$ & 0.05 & 0.22 & 0.27 & $<0.03$ & 0.22 & 0.22 & $<0.03$ & 0.08 & 0.08 & $<0.03$ & 0.12 & 0.12 & $<0.03$ & 0.20 & 0.20 \\
\hline $\mathrm{Se}$ & 4.56 & 19.00 & 23.56 & 2.24 & 22.97 & 25.21 & 2.85 & 27.60 & 30.45 & 1.53 & 36.19 & 37.72 & 0.79 & 41.45 & 42.24 \\
\hline $\mathrm{Si}$ & 1.71 & 3.96 & \begin{tabular}{|c|}
5.67 \\
\end{tabular} & 1.17 & 3.86 & 5.03 & 1.83 & 4.14 & 5.97 & 1.67 & 4.54 & 6.21 & 1.07 & 4.80 & 5.87 \\
\hline$\overline{\mathrm{Sr}}$ & 0.04 & 7.26 & 7.3 & 0.03 & 11.20 & 11.23 & 0.06 & \begin{tabular}{|c|}
13.62 \\
\end{tabular} & 13.68 & 0.05 & 16.41 & 16.46 & 0.04 & 18.77 & 18.81 \\
\hline $\mathrm{Te}$ & 0.54 & 0.38 & 0.92 & 0.32 & 0.35 & 0.67 & 0.57 & 0.39 & 0.96 & 0.51 & 0.58 & 1.09 & 0.34 & 0.50 & 0.84 \\
\hline $\mathrm{Ti}$ & 0.04 & 0.01 & 0.05 & 0.03 & 0.01 & 0.04 & 0.04 & 0.02 & 0.06 & 0.07 & 0.02 & 0.09 & 0.04 & 0.02 & 0.06 \\
\hline $\mathrm{Zn}$ & 0.08 & 3.91 & 3.99 & 0.06 & 5.60 & 5.66 & 0.12 & 7.03 & 7.15 & 0.10 & 7.92 & 8.02 & 0.07 & 8.57 & 8.64 \\
\hline $\mathrm{Zr}$ & 0.44 & 0.21 & 0.65 & 0.33 & 0.45 & 0.78 & 0.60 & 0.56 & 1.16 & 0.57 & 0.85 & 1.42 & 0.40 & 1.05 & 1.45 \\
\hline $\mathrm{Cl}$ & $\mathrm{NA}$ & $\mathrm{NA}$ & NA & NA & $\mathrm{NA}$ & $\mathrm{NA}$ & NA & NA & $\mathrm{NA}$ & NA & $\mathrm{NA}$ & NA & NA & $\mathrm{NA}$ & NA \\
\hline$F$ & NA & $\mathrm{NA}$ & NA & NA & NA & NA & NA & NA & NA & NA & NA & NA & NA & NA & NA \\
\hline I & NA & NA & NA & NA & $\mathrm{NA}$ & NA & NA & NA & NA & $\mathrm{NA}$ & NA & NA & $\mathrm{NA}$ & $\mathrm{NA}$ & $\mathrm{NA}$ \\
\hline Nitrate & NA & NA & $\mathrm{NA}$ & NA & $\mathrm{NA}$ & $\mathrm{NA}$ & $\mathrm{NA}$ & $\mathrm{NA}$ & NA & NA & NA & NA & NA & NA & NA \\
\hline Nitrite & $\mathrm{NA}$ & NA & $\mathrm{NA}$ & NA & $\mathrm{NA}$ & $\mathrm{NA}$ & NA & NA & NA & NA & NA & NA & NA & $\mathrm{NA}$ & NA \\
\hline Sulfate & $\mathrm{NA}$ & NA & NA & NA & NA & NA & $\mathrm{NA}$ & NA & $\mathrm{NA}$ & NA & NA & NA & NA & NA & NA \\
\hline
\end{tabular}


Table 4.6. Analytical Results for Samples of SBS Blow-Down Fluids (mg/l), (continued).

\begin{tabular}{|c|c|c|c|c|c|c|c|c|c|c|c|c|c|c|c|}
\hline Test & \multicolumn{15}{|c|}{$\# 8$} \\
\hline I.D. & \multicolumn{3}{|c|}{$120-S-40 A$} & \multicolumn{3}{|c|}{$12 \mathrm{O}-\mathrm{S}-43 \mathrm{~A}$} & \multicolumn{3}{|c|}{$12 \mathrm{O}-\mathrm{S}-43 \mathrm{~B}$} & \multicolumn{3}{|c|}{$12 \mathrm{O}-\mathrm{S}-58 \mathrm{~A}$} & \multicolumn{3}{|c|}{$12 \mathrm{O}-\mathrm{S}-60 \mathrm{~A}$} \\
\hline Glass (kg) & \multicolumn{3}{|c|}{24637} & \multicolumn{3}{|c|}{24714} & \multicolumn{3}{|c|}{24790} & \multicolumn{3}{|c|}{24943} & \multicolumn{3}{|c|}{25020} \\
\hline $\mathrm{pH}$ & \multicolumn{3}{|c|}{4.95} & \multicolumn{3}{|c|}{5.07} & \multicolumn{3}{|c|}{5.02} & \multicolumn{3}{|c|}{4.87} & \multicolumn{3}{|c|}{4.90} \\
\hline & Sus* & Dis.\# & Total & Sus* & Dis.\# & Total & Sus* & Dis. & Total & Sus* & Dis.\# & Total & Sus* & Dis.\# & Tot: \\
\hline Total & 24 & 2064 & 2088 & 12 & 2504 & 2516 & 20 & 2732 & 2752 & 8 & 3040 & 3048 & & 3148 & נונד \\
\hline $\mathrm{Al}$ & 0.29 & 9.42 & 9.71 & 0.17 & 10.38 & 10.55 & 0.29 & 11.34 & 11.63 & 0.11 & 12.25 & 12.36 & 0.05 & 12.21 & 12.2 \\
\hline As & 0.02 & 0.17 & 0.19 & 0.01 & 0.24 & 0.25 & 0.02 & 0.25 & 0.27 & 0.01 & 0.24 & 0.25 & $<0.01$ & 0.19 & 0.1 \\
\hline $\mathrm{B}$ & 0.02 & 169.7 & 169.7 & 0.01 & 190.8 & 190.8 & 0.01 & 208.2 & 208.2 & 0.01 & 234.7 & 234.7 & $<0.01$ & 244.2 & 244 \\
\hline$\overline{\mathrm{Ba}}$ & 0.01 & 0.60 & 0.61 & $<0.01$ & 0.66 & 0.66 & 0.01 & 0.71 & 0.72 & $<0.01$ & 0.80 & 0.80 & $<0.01$ & 0.85 & 0. \\
\hline $\mathrm{Ca}$ & 0.09 & 31.95 & 32.04 & 0.04 & 29.25 & 29.29 & 0.06 & 29.24 & 29.3 & 0.02 & 26.78 & 26.8 & 0.01 & 24.93 & 24.9 \\
\hline$\overline{\mathrm{Cd}}$ & 0.09 & 7.51 & 7.60 & 0.04 & 8.16 & 8.20 & 0.07 & 8.48 & 8.55 & 0.03 & 9.82 & 9.85 & 0.02 & 10.19 & 10.2 \\
\hline $\mathrm{Cr}$ & 0.04 & 0.24 & 0.28 & 0.02 & 0.28 & 0.30 & 0.03 & 0.31 & 0.34 & 0.01 & 0.38 & 0.39 & 0.01 & 0.41 & 0.4 \\
\hline $\mathrm{Cs}$ & $<0.1$ & 8.00 & 8.00 & $<0.1$ & 8.80 & 8.80 & $<0.1$ & 9.10 & 9.10 & $<0.1$ & 11.10 & 11.10 & $<0.1$ & 11.40 & 11.4 \\
\hline $\mathrm{Cu}$ & 0.03 & 0.05 & 0.08 & 0.01 & 0.06 & 0.07 & 0.02 & 0.07 & 0.09 & 0.01 & \begin{tabular}{|l|}
0.08 \\
\end{tabular} & 0.09 & $<0.01$ & 0.08 & 0.0 \\
\hline $\mathrm{Fe}$ & 5.19 & 0.84 & 6.03 & 2.64 & 1.03 & 3.67 & 4.47 & 1.23 & $5.70^{+}$ & 1.85 & 1.67 & 3.52 & 0.91 & 1.85 & 2.1 \\
\hline $\mathrm{K}$ & 0.02 & 13.19 & 13.21 & 0.01 & 14.97 & 14.98 & 0.01 & 16.03 & 16.04 & 0.01 & 18.17 & $\overline{18} .18$ & 0.01 & 18.57 & 18.5 \\
\hline$\overline{\mathrm{Li}}$ & 0.05 & 51.83 & 51.88 & 0.03 & 61.69 & 61.72 & 0.04 & 60.14 & 60.18 & 0.02 & 67.98 & 68.00 & 0.01 & 70.62 & 70.6 \\
\hline $\mathrm{Mg}$ & 0.04 & 14.64 & 14.68 & 0.02 & 15.05 & 15.07 & 0.04 & 15.84 & 15.88 & 0.01 & 16.35 & 16.36 & 0.01 & 15.76 & 15.7 \\
\hline $\mathrm{Mn}$ & 0.07 & 4.31 & 4.38 & 0.04 & 4.99 & 5.03 & 0.05 & 4.98 & 5.03 & 0.02 & 5.78 & 5.80 & 0.01 & 5.92 & 5.9 \\
\hline $\mathrm{Na}$ & 0.04 & 208.4 & 208.4 & 0.02 & 233.4 & 233.4 & 0.03 & 248.7 & 248.7 & 0.01 & 277.3 & 277.3 & 0.01 & 289.8 & 289 \\
\hline $\mathrm{Ni}$ & 0.17 & 0.85 & 1.02 & 0.09 & 0.95 & 1.04 & 0.15 & 1.02 & 1.17 & 0.06 & 1.14 & 1.20 & 0.03 & 1.20 & 1.2 \\
\hline $\mathrm{P}$ & 0.05 & 0.41 & 0.46 & 0.04 & 0.64 & 0.68 & 0.05 & 0.47 & 0.52 & 0.02 & 0.61 & 0.63 & 0.01 & 0.88 & 0.8 \\
\hline $\mathrm{Pb}$ & 0.12 & 0.55 & 0.67 & 0.06 & 0.58 & 0.64 & 0.11 & 0.68 & 0.79 & 0.04 & 0.80 & 0.84 & 0.02 & 0.83 & 0.8 \\
\hline $\mathrm{Sb}$ & 0.03 & 0.11 & 0.14 & 0.01 & $<0.03$ & 0.01 & $<0.01$ & $<0.03$ & $<0.03$ & $<0.01$ & $<0.03$ & $<0.03$ & $<0.01$ & $<0.03$ & $<0.0$ \\
\hline $\mathrm{Se}$ & 1.04 & 43.82 & 44.86 & 0.45 & 43.68 & 44.13 & 0.66 & 48.53 & 49.19 & 0.23 & 53.10 & 53.33 & 0.12 & 63.00 & 63.1 \\
\hline$\overline{\mathrm{Si}}$ & 1.68 & 4.94 & 6.62 & 0.88 & 5.41 & 6.29 & 1.52 & 5.83 & 7.35 & 0.58 & 6.32 & 6.90 & 0.27 & 6.21 & $\overline{6.4}$ \\
\hline $\mathrm{Sr}$ & 0.06 & 19.17 & 19.23 & 0.03 & 22.09 & 22.12 & 0.06 & 24.97 & 25.03 & 0.02 & 28.39 & 28.41 & 0.01 & 28.16 & 28.1 \\
\hline $\mathrm{Te}$ & 0.45 & 0.53 & 0.98 & 0.23 & 0.51 & 0.74 & 0.38 & 0.49 & 0.87 & 0.13 & 0.67 & 0.8 & 0.07 & 0.73 & 0 \\
\hline $\mathrm{Ti}$ & 0.04 & 0.01 & 0.05 & 0.02 & 0.02 & 0.04 & 0.03 & 0.02 & 0.05 & 0.01 & 0.02 & 0.03 & 0.01 & 0.02 & 0.0 \\
\hline $\mathrm{Zn}$ & 0.12 & 9.08 & 9.20 & 0.06 & 10.49 & 10.55 & 0.09 & 11.85 & 11.94 & 0.03 & 12.93 & 12.96 & 0.02 & 13.58 & 13. \\
\hline $\mathrm{Zr}$ & 0.62 & 1.30 & 1.92 & 0.33 & 1.59 & 1.92 & 0.54 & 1.91 & 2.45 & 0.22 & 2.50 & 2.72 & 0.11 & 2.79 & 2.9 \\
\hline $\mathrm{Cl}$ & NA & $\mathrm{NA}$ & NA & NA & NA & NA & $\mathrm{NA}$ & NA & NA & NA & NA & NA & $\mathrm{NA}$ & 22.77 & 22.7 \\
\hline $\mathrm{F}$ & NA & NA & NA & $\mathrm{NA}$ & $\mathrm{NA}$ & NA & $\mathrm{NA}$ & NA & NA & NA & NA & NA & $\mathrm{NA}$ & $<0.1$ & $<0.1$ \\
\hline I & NA & $\mathrm{NA}$ & NA & NA & $\mathrm{NA}$ & NA & NA & $\mathrm{NA}$ & NA & NA & NA & NA & $\mathrm{NA}$ & 115.9 & 115. \\
\hline Nitrate & NA & NA & NA & NA & NA & NA & NA & $\mathrm{NA}$ & NA & NA & NA & $\mathrm{NA}$ & NA & 3913 & 391 \\
\hline Nitrite & NA & NA & NA & $\mathrm{NA}$ & NA & NA & NA & $\mathrm{NA}$ & NA & $\mathrm{NA}$ & NA & $\mathrm{NA}$ & $\mathrm{NA}$ & 164.7 & 164. \\
\hline Sulfate & NA & NA & $\mathrm{NA}$ & $\mathrm{NA}$ & NA & NA & $\mathrm{NA}$ & $\mathrm{NA}$ & NA & $\mathrm{NA}$ & $\mathrm{NA}$ & $\mathrm{NA}$ & $\mathrm{NA}$ & 99.87 & 99.8 \\
\hline
\end{tabular}

* Suspended

\# Dissolved

NA - Not Analyzed 
Table 4.6. Analytical Results for Samples of SBS Blow-Down Fluids (mg/l), (continued).

\begin{tabular}{|c|c|c|c|c|c|c|c|c|c|c|c|c|c|c|c|}
\hline Test & \multicolumn{15}{|c|}{$\# 9$} \\
\hline I.D. & \multicolumn{3}{|c|}{ 120-S-97A } & \multicolumn{3}{|c|}{$120-S-107 \mathrm{~A}$} & \multicolumn{3}{|c|}{$12 \mathrm{O}-\mathrm{S}-115 \mathrm{~A}$} & \multicolumn{3}{|c|}{$12 \mathrm{O}-\mathrm{S}-118 \mathrm{~A}$} & \multicolumn{3}{|c|}{$12 \mathrm{O}-\mathrm{S}-125 \mathrm{~A}$} \\
\hline Glass $(\mathrm{kg})$ & \multicolumn{3}{|c|}{25123} & \multicolumn{3}{|c|}{25227} & \multicolumn{3}{|c|}{25331} & \multicolumn{3}{|c|}{25469} & \multicolumn{3}{|c|}{25607} \\
\hline $\mathrm{pH}$ & \multicolumn{3}{|c|}{7.83} & \multicolumn{3}{|c|}{8.06} & \multicolumn{3}{|c|}{8.09} & \multicolumn{3}{|c|}{8.00} & \multicolumn{3}{|c|}{7.91} \\
\hline & Sus & Dis.\# & Total & Sus* & Dis.\# & Total & Sus* & Dis.\# & Total & Sus* & Dis.\# & Total & Sus* & Dis.\# & \\
\hline Total & 88 & 412 & 500 & 70 & 316 & 386 & 60 & 352 & 714 & 66 & 496 & 562 & 00 & 700 & \\
\hline $\mathrm{Al}$ & 4.03 & 0.15 & 4.18 & 3.01 & 0.16 & 3.17 & 2.37 & 0.14 & 2.51 & 2.41 & 0.14 & 2.55 & 2.11 & 0.15 & \\
\hline As & 0.11 & 0.18 & 0.29 & 0.11 & 0.21 & 0.32 & 0.12 & 0.32 & 0.44 & 0.16 & 0.44 & 0.60 & 0.19 & 0.53 & ... \\
\hline $\mathrm{B}$ & 0.25 & 20.50 & 20.75 & 0.15 & 27.83 & 27.98 & 0.11 & 33.91 & 34.02 & 0.11 & 41.56 & 41.67 & 0.10 & 44.13 & 44.2 \\
\hline $\mathrm{Ba}$ & 0.02 & 0.07 & 0.09 & 0.02 & 0.06 & 0.08 & 0.02 & 0.06 & 0.08 & 0.02 & 0.06 & 0.08 & 0.01 & 0.05 & \\
\hline $\mathrm{Ca}$ & 0.59 & 38.70 & 39.29 & 0.50 & 34.82 & 35.32 & 0.42 & 31.96 & 32.38 & 0.43 & 33.60 & 34.03 & 0.40 & 28.48 & 28.8 \\
\hline $\mathrm{Cd}$ & 0.56 & 0.20 & 0.76 & 1.21 & 0.20 & 1.41 & 1.68 & 0.22 & 1.90 & 2.43 & 0.22 & 2.65 & 3.14 & 0.15 & \\
\hline $\mathrm{Cr}$ & 0.10 & 0.03 & 0.13 & 0.09 & 0.03 & 0.12 & 0.08 & 0.03 & 0.11 & 0.09 & 0.03 & 0.12 & 0.09 & 0.02 & \\
\hline $\mathrm{Cs}$ & $<0.1$ & 0.45 & 0.45 & $<0.1$ & 0.54 & 0.54 & $<0.1$ & 0.59 & 0.59 & $<0.1$ & 0.67 & 0.67 & $<0.1$ & 0.77 & 0.1 \\
\hline $\mathrm{Cu}$ & 0.03 & 0.02 & 0.05 & 0.04 & 0.01 & 0.05 & 0.04 & $<0.01$ & 0.04 & 0.04 & 0.02 & 0.06 & 0.04 & 0.01 & \\
\hline $\mathrm{Fe}$ & 0.50 & 0.02 & 10.52 & 9.53 & 0.02 & 9.55 & 7.98 & 0.02 & 8.00 & 8.72 & 0.03 & 8.75 & 8.07 & 0.02 & \\
\hline $\mathrm{K}$ & 0.05 & 3.65 & 3.7 & 0.05 & 3.12 & 3.17 & 0.04 & 2.88 & 2.92 & 0.05 & 2.77 & 2.82 & 0.05 & $\overline{2} .61$ & \\
\hline $\mathrm{Li}$ & 0.28 & 4.40 & 4.68 & 0.17 & 4.51 & 4.68 & 0.12 & 4.71 & 4.83 & 0.13 & 4.74 & 4.87 & 0.11 & 4.95 & \\
\hline $\mathrm{Mg}$ & 0.19 & 12.99 & 13.18 & 0.22 & 11.81 & 12.03 & 0.22 & 11.13 & 11.35 & 0.25 & 10.39 & 10.64 & 0.27 & 9.58 & \\
\hline $\mathrm{Mn}$ & 0.46 & 0.33 & 0.79 & 0.34 & 0.24 & 0.58 & 0.26 & 0.21 & 0.47 & 0.26 & 0.21 & 0.47 & 0.24 & 0.15 & 0.0 \\
\hline $\mathrm{Na}$ & 0.34 & 31.69 & 32.03 & 0.21 & 30.46 & 30.67 & 0.18 & 29.17 & 29.35 & 0.20 & 28.30 & 28.5 & 0.27 & 27.94 & 28.2 \\
\hline $\mathrm{Ni}$ & 0.61 & 0.13 & 0.74 & 0.52 & 0.10 & 0.62 & 0.42 & 0.09 & 0.51 & 0.45 & 0.09 & 0.54 & 0.41 & 0.10 & 0.5 \\
\hline $\mathrm{P}$ & 0.12 & 0.26 & 0.38 & 0.16 & 0.17 & 0.33 & 0.10 & $<0.07$ & 0.10 & 0.09 & 0.31 & 0.40 & 0.13 & 0.54 & 0.6 \\
\hline $\mathrm{Pb}$ & 0.26 & 0.09 & 0.35 & 0.30 & 0.09 & 0.39 & 0.29 & 0.10 & 0.39 & 0.31 & 0.10 & 0.41 & 0.36 & 0.11 & 0.4 \\
\hline $\mathrm{Sb}$ & 0.13 & 0.46 & 0.59 & 0.05 & 0.11 & 0.16 & 0.04 & 0.05 & 0.09 & 0.02 & 0.05 & 0.07 & 0.11 & 0.06 & 0.1 \\
\hline $\mathrm{Se}$ & 1.66 & 20.10 & 21.76 & 1.81 & 37.26 & 39.07 & 1.71 & 53.88 & 55.59 & 1.89 & 59.35 & 61.24 & 2.79 & 73.74 & 76.5 \\
\hline$\overline{\mathrm{Si}}$ & 4.65 & 2.29 & 16.94 & 10.05 & 2.21 & 12.26 & 7.74 & 2.23 & 9.97 & 8.17 & 2.25 & 10.42 & 7.18 & 2.31 & 9.4 \\
\hline $\mathrm{Sr}$ & 0.16 & 3.20 & 3.36 & 0.22 & 3.18 & 3.40 & 0.22 & 3.19 & 3.41 & 0.24 & 3.14 & 3.38 & 0.25 & 3.20 & 3.4 \\
\hline $\mathrm{Te}$ & .81 & 0.36 & 2.17 & 3.77 & 0.95 & 4.72 & 4.91 & 1.93 & 6.84 & 7.67 & 2.79 & 10.46 & 8.99 & 3.81 & 12.8 \\
\hline $\mathrm{Ti}$ & .09 & 0.01 & 0.1 & 0.07 & 0.01 & 0.08 & 0.05 & 0.01 & 0.06 & 0.06 & 0.01 & 0.07 & 0.05 & 0.01 & \\
\hline $\mathrm{Zn}$ & 83 & 0.19 & 1.02 & 0.91 & 0.13 & 1.04 & 0.84 & 0.10 & 0.94 & 0.96 & 0.10 & 1.06 & 1.00 & 0.08 & \\
\hline $\mathrm{Zr}$ & 1.61 & 0.02 & 1.63 & 1.03 & 0.01 & 1.04 & 0.77 & 0.01 & 0.78 & 0.76 & 0.01 & 0.77 & 0.63 & 0.01 & 0.6 \\
\hline $\mathrm{Cl}$ & NA & 40.73 & 40.73 & $\mathrm{NA}$ & NA & NA & NA & NA & NA & NA & NA & NA & NA & NA & $\mathrm{N}$ \\
\hline $\mathrm{F}$ & NA & 12.01 & 12.01 & NA & NA & NA & NA & NA & $\mathrm{NA}$ & NA & NA & NA & NA & NA & $\mathrm{N}$ \\
\hline$I$ & NA & 15.39 & 15.39 & NA & NA & NA & NA & NA & NA & NA & NA & NA & NA & NA & \\
\hline Nitrate & NA & 52.05 & 52.05 & NA & $\mathrm{NA}$ & NA & NA & $\mathrm{NA}$ & NA & NA & $\mathrm{NA}$ & NA & NA & NA & \\
\hline Nitrite & $\mathrm{NA}$ & 15.75 & 15.75 & NA & $\mathrm{NA}$ & NA & NA & NA & NA & NA & NA & NA & NA & $\mathrm{NA}$ & \\
\hline Sulfate & $\mathrm{NA}$ & 108.1 & 108.1 & NA & $\mathrm{NA}$ & NA & NA & NA & NA & $\mathrm{NA}$ & $\mathrm{NA}$ & NA & NA & NA & $\mathrm{N}$ \\
\hline
\end{tabular}

* Suspended

\# Dissolved

NA - Not Analyzed 
Table 4.6. Analytical Results for Samples of SBS Blow-Down Fluids (mg/l), (continued).

\begin{tabular}{|c|c|c|c|c|c|c|c|c|c|c|c|c|c|c|c|}
\hline Test & \multicolumn{15}{|c|}{ \#9 } \\
\hline I.D. & \multicolumn{3}{|c|}{$120-S-135 \mathrm{~A}$} & \multicolumn{3}{|c|}{$12 \mathrm{O}-\mathrm{S}-145 \mathrm{~A}$} & \multicolumn{3}{|c|}{$12 \mathrm{O}-\mathrm{S}-146 \mathrm{~A}$} & \multicolumn{3}{|c|}{$12 \mathrm{O}-\mathrm{S}-154 \mathrm{~A}$} & \multicolumn{3}{|c|}{$12 \mathrm{P}-\mathrm{S}-14 \mathrm{~A}$} \\
\hline Glass (kg) & \multicolumn{3}{|c|}{25743} & \multicolumn{3}{|c|}{25879} & \multicolumn{3}{|c|}{25986} & \multicolumn{3}{|c|}{26094} & \multicolumn{3}{|c|}{26202} \\
\hline $\mathrm{pH}$ & \multicolumn{3}{|c|}{7.86} & \multicolumn{3}{|c|}{7.71} & \multicolumn{3}{|c|}{7.55} & \multicolumn{3}{|c|}{6.62} & \multicolumn{3}{|c|}{4.80} \\
\hline & Sus* & Dis.\# & Total & Sus* & Dis.\# & Total & Sus* & Dis.\# & Total & Sus* & Dis.\# & Total & Sus* & Dis.\# & Total \\
\hline Total & 60 & 450 & 510 & 50 & 616 & 666 & 56 & 764 & 820 & 52 & 890 & 942 & 50 & 844 & 894 \\
\hline $\mathrm{Al}$ & 1.80 & 0.17 & 1.97 & 1.17 & 0.28 & 1.45 & 1.06 & 0.52 & 1.58 & 0.56 & 1.56 & 2.12 & 0.34 & 2.22 & 2.56 \\
\hline As & 0.18 & 0.74 & 0.92 & 0.22 & 0.94 & 1.16 & 0.32 & 1.24 & 1.56 & 0.32 & 1.57 & 1.89 & 0.21 & 2.16 & 2.37 \\
\hline $\mathrm{B}$ & 0.08 & 52.90 & 52.98 & 0.05 & 57.17 & 57.22 & 0.04 & 63.74 & 63.78 & 0.03 & 70.95 & 70.98 & 0.02 & 76.08 & $\overline{76.1}$ \\
\hline $\mathrm{Ba}$ & 0.01 & 0.05 & 0.06 & 0.01 & 0.06 & 0.07 & 0.01 & 0.06 & 0.07 & $<0.01$ & 0.07 & 0.07 & $<0.01$ & 0.07 & 0.07 \\
\hline $\mathrm{Ca}$ & 0.33 & 24.33 & 24.66 & 0.23 & 22.27 & 22.5 & 0.21 & 20.23 & 20.44 & 0.08 & 19.15 & 19.23 & 0.04 & 17.56 & 17.60 \\
\hline $\mathrm{Cd}$ & 4.45 & 0.14 & 4.59 & 3.12 & 0.16 & 3.28 & 4.73 & 0.18 & 4.91 & 4.33 & 0.98 & 5.31 & 3.27 & 6.66 & 9.93 \\
\hline $\mathrm{Cr}$ & 0.08 & 0.02 & 0.10 & 0.06 & 0.01 & 0.07 & 0.06 & 0.01 & 0.07 & 0.05 & 0.01 & 0.06 & 0.04 & 0.01 & 0.05 \\
\hline Cs & $<0.1$ & 0.82 & 0.82 & $<0.1$ & 0.93 & 0.93 & $<0.1$ & 1.16 & 1.16 & $<0.1$ & 1.21 & 1.21 & $<0.1$ & 1.33 & 1.33 \\
\hline $\mathrm{Cu}$ & 0.03 & 0.01 & 0.04 & 0.03 & 0.01 & 0.04 & 0.03 & 0.01 & 0.04 & 0.02 & 0.01 & 0.03 & 0.02 & 0.02 & 0.04 \\
\hline $\mathrm{Fe}$ & 6.59 & 0.01 & 6.60 & 4.95 & $<0.01$ & 4.95 & 4.86 & 0.01 & 4.87 & 3.66 & 0.02 & 3.68 & 3.31 & 0.03 & 3.34 \\
\hline $\mathrm{K}$ & 0.05 & 2.97 & 3.02 & 0.04 & 2.78 & 2.82 & 0.04 & 2.65 & 2.69 & 0.04 & 2.53 & 2.57 & 0.03 & 2.35 & 2.38 \\
\hline$\overline{\mathrm{Li}}$ & 0.09 & 4.58 & 4.67 & 0.06 & 4.52 & 4.58 & 0.06 & 4.60 & 4.66 & 0.04 & 4.66 & 4.70 & 0.04 & 4.61 & 4.65 \\
\hline $\mathrm{Mg}$ & 0.26 & 8.00 & 8.26 & 0.20 & 7.50 & 7.70 & 0.19 & 6.92 & 7.11 & 0.13 & 6.58 & 6.71 & 0.07 & 6.32 & 6.39 \\
\hline $\mathrm{Mn}$ & 0.17 & 0.10 & 0.27 & 0.13 & \begin{tabular}{|l|}
0.11 \\
\end{tabular} & 0.24 & 0.12 & 0.14 & 0.26 & 0.08 & 0.18 & 0.26 & 0.06 & 0.23 & 0.29 \\
\hline $\mathrm{Na}$ & 0.21 & 28.60 & 28.81 & 0.17 & 27.35 & 27.52 & 0.22 & 27.18 & 27.4 & 0.21 & 26.70 & 26.91 & 0.19 & 25.42 & 25.61 \\
\hline $\mathrm{Ni}$ & 0.33 & \begin{tabular}{|l|}
0.07 \\
\end{tabular} & 0.40 & 0.24 & \begin{tabular}{|l|}
0.07 \\
\end{tabular} & 0.31 & 0.22 & 0.08 & 0.30 & 0.16 & 0.11 & 0.27 & 0.14 & 0.11 & 0.25 \\
\hline $\mathrm{P}$ & 0.08 & $<0.07$ & 0.08 & 0.05 & $<0.07$ & 0.05 & 0.05 & $<0.07$ & 0.05 & 0.04 & $<0.07$ & 0.04 & 0.02 & 0.13 & 0.15 \\
\hline $\mathrm{Pb}$ & 0.39 & 0.10 & 0.49 & 0.35 & 0.11 & 0.46 & 0.39 & \begin{tabular}{|l|}
0.13 \\
\end{tabular} & 0.52 & 0.39 & 0.15 & 0.54 & 0.39 & 0.20 & 0.59 \\
\hline $\mathrm{Sb}$ & 0.16 & 0.20 & 0.36 & 0.08 & 0.14 & 0.22 & 0.04 & 0.25 & 0.29 & 0.05 & 0.32 & 0.37 & 0.03 & 0.39 & 0.42 \\
\hline $\mathrm{Se}$ & 4.50 & 90.72 & 95.22 & 5.67 & 98.16 & 103.8 & 6.64 & 119.0 & 125.6 & 10.85 & 115.5 & 126.4 & 14.15 & 120.2 & 134.4 \\
\hline $\mathrm{Si}$ & 5.62 & 2.44 & 8.06 & 4.06 & 2.50 & 6.56 & 3.84 & 2.62 & 6.46 & 2.67 & 2.98 & 5.65 & 2.28 & 3.30 & 5.58 \\
\hline $\mathrm{Sr}$ & 0.21 & 3.57 & 3.78 & 0.13 & 3.64 & 3.77 & 0.08 & 3.91 & 3.99 & 0.03 & 4.15 & 4.18 & 0.02 & 4.15 & $\overline{4.17}$ \\
\hline $\mathrm{Te}$ & 8.89 & 5.59 & 14.48 & 4.09 & 6.98 & 11.07 & 12.34 & 7.89 & 20.23 & 13.25 & 3.27 & 16.52 & 12.22 & 2.90 & 15.12 \\
\hline $\mathrm{Ti}$ & 0.04 & $<0.01$ & 0.04 & 0.03 & $<0.01$ & 0.03 & 0.03 & 0.01 & 0.04 & 0.02 & 0.01 & 0.03 & 0.02 & 0.01 & 0.03 \\
\hline $\mathrm{Zn}$ & 0.85 & 0.05 & 0.9 & 0.65 & \begin{tabular}{|l|}
0.09 \\
\end{tabular} & 0.74 & 0.64 & 0.12 & 0.76 & 0.35 & 0.74 & 1.09 & 0.17 & 1.38 & 1.55 \\
\hline $\mathrm{Zr}$ & 0.47 & $<0.01$ & 0.47 & 0.31 & 0.01 & 0.32 & 0.28 & 0.02 & 0.30 & 0.20 & 0.02 & 0.22 & 0.16 & 0.06 & 0.22 \\
\hline $\mathrm{Cl}$ & NA & 40.73 & 40.73 & NA & NA & NA & NA & NA & NA & NA & NA & NA & NA & NA & NA \\
\hline $\mathrm{F}$ & NA & 12.01 & 12.01 & $\mathrm{NA}$ & $\mathrm{NA}$ & NA & $\mathrm{NA}$ & NA & $\mathrm{NA}$ & $\mathrm{NA}$ & $\mathrm{NA}$ & NA & NA & NA & NA \\
\hline I & $<0.1$ & 15.39 & 15.39 & NA & NA & $\mathrm{NA}$ & NA & NA & NA & NA & NA & NA & NA & $\mathrm{NA}$ & NA \\
\hline Nitrate & $\mathrm{NA}$ & 52.05 & 52.05 & NA & $\mathrm{NA}$ & NA & NA & $\mathrm{NA}$ & NA & $\mathrm{NA}$ & NA & NA & $\mathrm{NA}$ & $\mathrm{NA}$ & NA \\
\hline Nitrite & NA & 15.75 & 15.75 & NA & $\mathrm{NA}$ & $\mathrm{NA}$ & NA & $\mathrm{NA}$ & NA & $\mathrm{NA}$ & $\mathrm{NA}$ & NA & $\mathrm{NA}$ & $\mathrm{NA}$ & NA \\
\hline Sulfate & $<0.1$ & 108.1 & 108.1 & NA & NA & NA & NA & NA & NA & NA & NA & NA & $\mathrm{NA}$ & NA & $\mathrm{NA}$ \\
\hline
\end{tabular}


The Catholic University of America Vitreous State Laboratory
DuraMelter 1200 Tests with AZ-101HLW Simulants

Final Report, VSL-02R0100-2, Rev.l

Table 4.6. Analytical Results for Samples of SBS Blow-Down Fluids (mg/l), (continued).

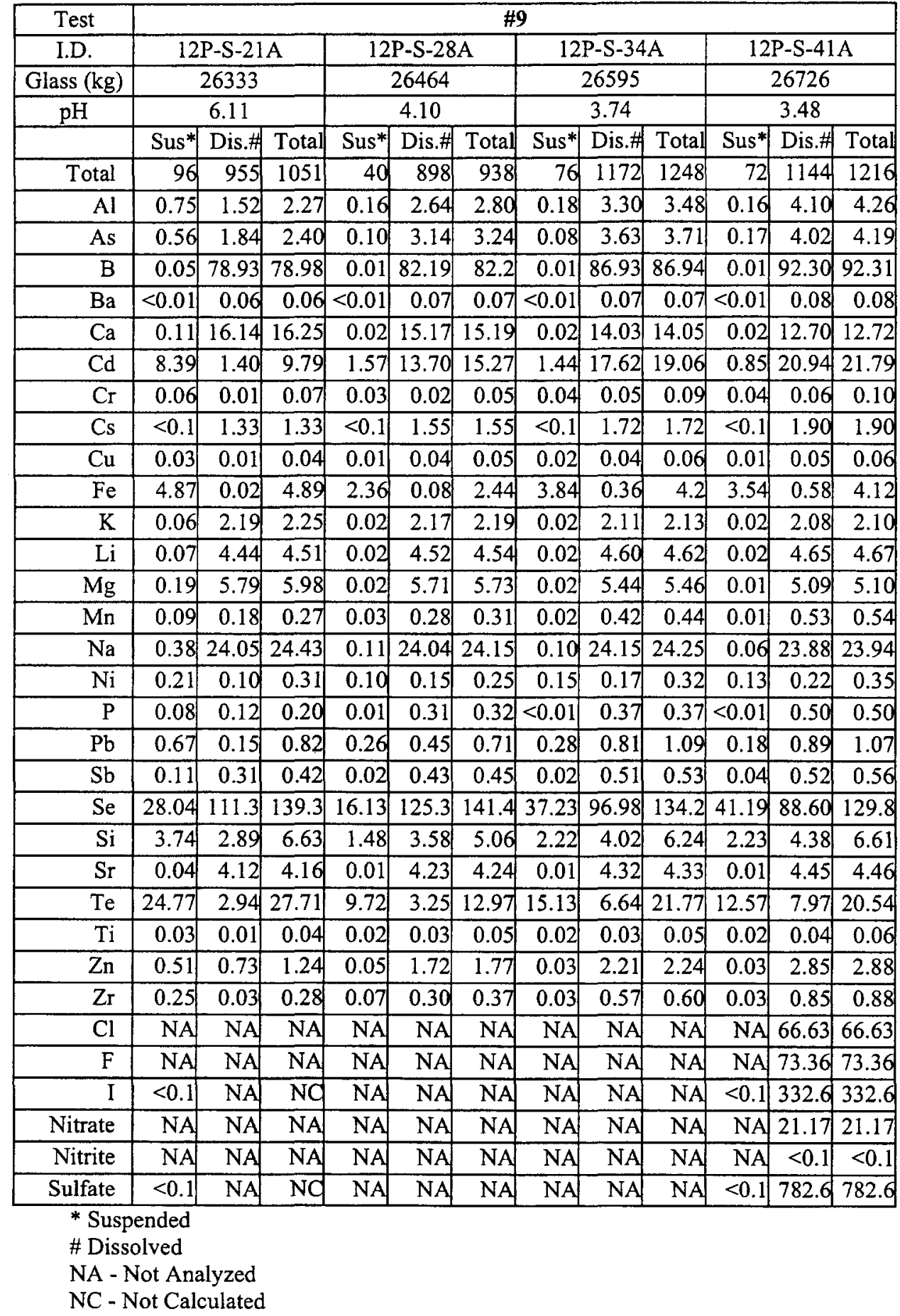


Table 4.7. Total Accumulation in SBS from Tests 1 and 2.

\begin{tabular}{|c|c|c|c|}
\hline & $\begin{array}{c}\text { Total in SBS } \\
\text { (g) }\end{array}$ & $\begin{array}{c}\text { Total from } \\
\text { Target Glass } \\
(\mathrm{kg})\end{array}$ & $\%$ in SBS \\
\hline $\mathrm{Al}$ & 103.63 & 191.60 & 0.05 \\
\hline As & 7.96 & 1.48 & 0.54 \\
\hline $\mathrm{B}$ & 787.79 & 151.92 & 0.52 \\
\hline $\mathrm{Ba}$ & 1.68 & 1.75 & 0.10 \\
\hline $\mathrm{Ca}$ & 106.13 & 8.74 & 1.22 \\
\hline $\mathrm{Cd}$ & 51.09 & 15.84 & 0.32 \\
\hline $\mathrm{Cr}$ & 2.71 & 1.34 & 0.20 \\
\hline $\mathrm{Cs}$ & 11.01 & 3.69 & 0.30 \\
\hline $\mathrm{Cu}$ & 1.06 & 1.17 & 0.09 \\
\hline $\mathrm{Fe}$ & 191.58 & 355.63 & 0.05 \\
\hline $\mathrm{K}$ & 25.35 & 6.90 & 0.37 \\
\hline $\mathrm{Li}$ & 107.56 & 136.31 & 0.08 \\
\hline $\mathrm{Mg}$ & 63.38 & $33.98^{5}$ & 0.19 \\
\hline $\mathrm{Mn}$ & 17.30 & 108.63 & 0.02 \\
\hline $\mathrm{Na}$ & 385.28 & 239.12 & 0.16 \\
\hline $\mathrm{Ni}$ & 10.96 & 20.75 & 0.05 \\
\hline $\mathrm{P}$ & 6.07 & 2.78 & 0.22 \\
\hline $\mathrm{Pb}$ & 7.99 & 6.81 & 0.11 \\
\hline $\mathrm{Sb}$ & 3.24 & 7.73 & 0.04 \\
\hline $\mathrm{Se}$ & 1188.22 & 5.22 & 22.76 \\
\hline $\mathrm{Si}$ & 266.30 & 1041.50 & 0.03 \\
\hline $\mathrm{Sr}$ & 88.79 & 95.94 & 0.09 \\
\hline $\mathrm{Te}$ & 135.07 & 5.47 & 2.47 \\
\hline $\mathrm{Ti}$ & 0.91 & 1.76 & 0.05 \\
\hline $\mathrm{Zn}$ & 64.80 & 78.57 & 0.08 \\
\hline $\mathrm{Zr}$ & 14.91 & 128.89 & 0.01 \\
\hline $\mathrm{Cl}$ & 245.31 & 0.49 & 50.16 \\
\hline $\mathrm{F}$ & 239.97 & 1.96 & 12.27 \\
\hline I & 1314.16 & 4.89 & 26.87 \\
\hline Nitrate & 278.30 & 29.40 & 0.95 \\
\hline Nitrite & 65.62 & 14.18 & 0.46 \\
\hline Sulfate & 1916.42 & 14.67 & 13.06 \\
\hline Total & $31344^{*}$ & 4895 & 0.64 \\
\hline
\end{tabular}

* Based on gravimetric analysis for total dissolved and suspended solids, not chemical analysis.

${ }^{\$}$ Value used is analyzed instead of target.

NA - Not Analyzed

NC - Not Calculated 
Table 4.8. Analytical Results for Samples of WESP Blowdown Fluids (mg/l).

\begin{tabular}{|c|c|c|c|c|c|c|c|c|c|c|c|c|c|c|c|}
\hline Test & \multicolumn{12}{|c|}{$\# 1$} & \multirow{2}{*}{\multicolumn{3}{|c|}{$\frac{\# 2}{12 \mathrm{G}-\mathrm{W}-116 \mathrm{~A}}$}} \\
\hline I.D. & \multicolumn{3}{|c|}{$12 \mathrm{~F}-\mathrm{W}-41 \mathrm{~A}$} & \multicolumn{3}{|c|}{$12 \mathrm{~F} \cdot \mathrm{W}-63 \mathrm{~A}$} & \multicolumn{3}{|c|}{$12 \mathrm{~F}-\mathrm{W}-63 \mathrm{~B}$} & \multicolumn{3}{|c|}{$12 \mathrm{G}-\mathrm{W}-80 \mathrm{~A}$} & & & \\
\hline Glass $(\mathrm{kg})$ & \multicolumn{3}{|c|}{0} & \multicolumn{3}{|c|}{0} & \multicolumn{3}{|c|}{0} & \multicolumn{3}{|c|}{60} & \multicolumn{3}{|c|}{2350} \\
\hline $\mathrm{pH}$ & \multicolumn{3}{|c|}{2.31} & \multicolumn{3}{|c|}{2.27} & \multicolumn{3}{|c|}{3.20} & \multicolumn{3}{|c|}{6.13} & \multicolumn{3}{|c|}{3.22} \\
\hline & Sus* & Dis.\# & Total & Sus* & Dis.\# & Total & Sus* & Dis.\# & Total & Sus* & Dis.\# & Total & Sus* & Dis.\# & Total \\
\hline Total & 334 & 31836 & 32170 & 137 & 32430 & 32567 & 43 & 753 & 796 & 40 & 116 & 156 & 24 & 1248 & 1272 \\
\hline Al & 1.23 & 10.06 & 11.29 & 0.26 & 13.00 & 13.25 & 0.09 & 0.47 & 0.56 & 0.04 & 0.05 & 0.10 & 0.02 & 0.81 & 0.82 \\
\hline As & 0.23 & 62.80 & 63.03 & $<0.01$ & 83.92 & 83.92 & $<0.01$ & 3.01 & 3.01 & 0.07 & 0.23 & 0.30 & $<0.01$ & 4.18 & 4.18 \\
\hline $\mathrm{B}$ & 0.45 & 913.4 & 913.8 & 0.12 & 1321 & 1321 & $<0.01$ & 26.15 & 26.15 & 0.01 & 0.75 & 0.76 & 2.93 & 5.46 & 8.39 \\
\hline $\mathrm{Ba}$ & 0.03 & 0.11 & 0.14 & 0.01 & 0.12 & 0.13 & $<0.01$ & 0.04 & 0.05 & 0.01 & 0.02 & 0.03 & $<0.01$ & 0.07 & 0.07 \\
\hline $\mathrm{Ca}$ & 0.52 & 26.16 & 26.68 & 0.05 & 27.43 & 27.48 & 0.05 & 35.23 & 35.28 & 0.30 & 35.92 & 36.22 & 0.02 & 40.82 & 40.84 \\
\hline $\mathrm{Cd}$ & 2.35 & 130.5 & 132.7 & 1.07 & 148.4 & 149.4 & 0.36 & 5.76 & 6.12 & 1.11 & 0.05 & 1.16 & $<0.01$ & 5.70 & 5.70 \\
\hline $\mathrm{Cr}$ & 17.67 & 26.37 & 44.04 & 7.44 & 25.84 & 33.28 & 1.37 & 0.46 & 1.83 & 1.07 & 0.01 & 1.08 & $<0.01$ & 0.95 & 0.95 \\
\hline $\mathrm{Cs}$ & $<0.1$ & 4.82 & 4.82 & $<0.1$ & 2.56 & 2.56 & $<0.1$ & 1.73 & 1.73 & $<0.1$ & 0.90 & 0.90 & $<0.1$ & 16.80 & 16.80 \\
\hline $\mathrm{Cu}$ & 0.05 & 3.72 & 3.77 & 0.02 & 4.30 & 4.32 & 0.01 & 0.21 & 0.22 & 0.03 & 0.01 & 0.03 & $<0.01$ & 0.63 & 0.63 \\
\hline $\mathrm{Fe}$ & 11.55 & 0.64 & 12.19 & 3.11 & 0.95 & 4.06 & 1.35 & 0.03 & 1.38 & 1.62 & 0.01 & 1.63 & 0.02 & 0.04 & 0.07 \\
\hline $\bar{K}$ & 1.46 & 478.1 & 479.6 & 0.54 & 529.1 & 529.6 & 0.06 & 12.94 & 13.00 & 0.03 & 3.01 & 3.03 & 0.01 & 11.16 & 11.16 \\
\hline $\mathrm{Li}$ & 0.12 & 379.1 & 379.2 & 0.07 & 486.7 & 486.8 & $<0.01$ & 16.72 & 16.72 & $<0.01$ & 0.19 & 0.19 & $<0.01$ & 19.94 & 19.94 \\
\hline $\mathrm{Mg}$ & 0.23 & 6.49 & 6.71 & 0.03 & 7.15 & 7.18 & \begin{tabular}{|l|}
0.02 \\
\end{tabular} & 7.32 & 7.33 & 0.02 & 7.41 & 7.43 & $<0.01$ & 10.34 & 10.34 \\
\hline $\mathrm{Mn}$ & 0.06 & 2.63 & 2.68 & 0.01 & 3.54 & 3.55 & 0.01 & 0.15 & 0.16 & 0.02 & $<0.01$ & 0.02 & $<0.01$ & 0.20 & 0.20 \\
\hline $\mathrm{Na}$ & 1.34 & 2226 & 2227 & 0.65 & 3206 & 3207 & 0.07 & 85.52 & 85.59 & 0.06 & 10.66 & 10.72 & 0.02 & 94.08 & 94.10 \\
\hline $\mathrm{Ni}$ & 0.54 & 14.05 & 14.60 & 0.07 & 15.44 & 15.51 & \begin{tabular}{l|l}
0.03 \\
\end{tabular} & 2.37 & 2.40 & 0.07 & 0.05 & 0.11 & 0.02 & 1.03 & 1.05 \\
\hline $\bar{P}$ & $<0.01$ & 4.59 & 4.59 & $<0.01$ & 5.11 & 5.11 & $<0.01$ & $<0.07$ & $<0.07$ & 0.04 & 0.21 & 0.24 & $<0.01$ & $<0.07$ & $<0.01$ \\
\hline $\mathrm{Pb}$ & 1.84 & 1.61 & 3.45 & 0.92 & 1.92 & 2.84 & 0.33 & 0.05 & 0.37 & 0.93 & $<0.02$ & 0.93 & $<0.01$ & $<0.02$ & $<0.01$ \\
\hline $\mathrm{Sb}$ & 0.76 & 5.90 & 6.66 & 0.40 & 6.48 & 6.88 & 0.29 & 0.09 & 0.38 & 0.81 & 0.25 & 1.05 & $<0.01$ & 0.31 & 0.31 \\
\hline $\mathrm{Se}$ & 68.32 & 11775 & 11843 & 29.46 & 13455 & 13485 & 5.14 & 304.8 & 309.9 & 2.04 & 22.54 & \begin{tabular}{|l|}
24.58 \\
\end{tabular} & 0.02 & 464.1 & 464.2 \\
\hline $\mathrm{Si}$ & 5.77 & 12.74 & 18.52 & 1.06 & 15.41 & 16.47 & 0.63 & 2.54 & 3.17 & 0.46 & 2.76 & 3.22 & $<0.01$ & 2.57 & 2.63 \\
\hline $\mathrm{Sr}$ & 0.04 & 6.88 & 6.91 & 0.02 & 7.44 & 7.45 & $<0.01$ & 0.87 & 0.87 & 0.01 & 0.19 & 0.19 & $<0.01$ & 0.94 & $\overline{0.94}$ \\
\hline $\mathrm{Te}$ & 30.98 & 466.4 & 497.4 & 13.54 & \begin{tabular}{l|}
546.5 \\
\end{tabular} & 560.0 & 6.36 & 18.30 & 24.66 & 17.28 & 9.97 & 27.25 & $<0.01$ & 25.36 & 25.36 \\
\hline $\mathrm{Ti}$ & 0.28 & 0.09 & 0.37 & 0.07 & 0.10 & 0.17 & 0.02 & 0.01 & 0.03 & 0.02 & 0.01 & 0.02 & $<0.01$ & $<0.01$ & $<0.01$ \\
\hline $\mathrm{Zn}$ & 0.13 & 46.01 & 46.14 & 0.05 & 54.32 & 54.37 & 0.03 & 1.57 & 1.60 & 0.18 & 0.04 & 0.22 & 0.01 & 2.26 & 2.27 \\
\hline $\mathrm{Zr}$ & 0.06 & 0.04 & 11.29 & \begin{tabular}{l|}
0.01 \\
\end{tabular} & 0.02 & 0.03 & $<0.01$ & 0.01 & 0.01 & 0.01 & 0.01 & 0.02 & $<0.01$ & $<0.01$ & $<0.01$ \\
\hline $\mathrm{Cl}$ & $\mathrm{NA}$ & NA & NA & $\mathrm{NA}$ & $\mathrm{NA}$ & NA & NA & NA & NA & NA & 26.74 & 26.74 & $\mathrm{NA}$ & 30.58 & 30.58 \\
\hline $\bar{F}$ & NA & NA & NA & NA & NA & $\mathrm{NA}$ & NA & NA & $\mathrm{NA}$ & NA & 1.19 & 1.19 & NA & 2.03 & 2.03 \\
\hline I & $<0.1$ & NA & $\mathrm{Nd}$ & $<0.1$ & NA & $\mathrm{NC}$ & $<0.1$ & NA & $\mathrm{NQ}$ & NA & 5.64 & 5.64 & $<0.1$ & 0.65 & 0.65 \\
\hline Nitrate & NA & NA & NA & $\mathrm{NA}$ & NA & NA & $\mathrm{NA}$ & $\mathrm{NA}$ & NA & NA & 6.19 & 6.19 & NA & 43.62 & 43.62 \\
\hline Nitrite & NA & NA & NA & NA & NA & NA & NA & NA & NA & NA & 35.68 & 35.68 & NA & $<0.1$ & $<0.1$ \\
\hline Sulfate & $<0.1$ & NA & Nd & $<0.1$ & NA & $\mathrm{NC}$ & $<0.1$ & NA & $\mathrm{Nd}$ & NA & \begin{tabular}{|c|}
53.18 \\
\end{tabular} & \begin{tabular}{|c|}
53.18 \\
\end{tabular} & 0.29 & 245.8 & 246.1 \\
\hline
\end{tabular}


Table 4.8. Analytical Results for Samples of WESP Blowdown Fluids (mg/l), (continued).

\begin{tabular}{|c|c|c|c|c|c|c|c|c|c|c|c|c|c|c|c|}
\hline Test & \multicolumn{15}{|c|}{$\# 2$} \\
\hline I.D. & \multicolumn{3}{|c|}{ 12G-W-128A } & \multicolumn{3}{|c|}{$12 \mathrm{G}-\mathrm{W}-139 \mathrm{~A}$} & \multicolumn{3}{|c|}{$12 \mathrm{G}-\mathrm{W}-148 \mathrm{~A}$} & \multicolumn{3}{|c|}{$12 \mathrm{H}-\mathrm{W}-11 \mathrm{~A}$} & \multicolumn{3}{|c|}{$12 \mathrm{H}-\mathrm{W}-33 \mathrm{~A}$} \\
\hline lass $(\mathrm{kg})$. & \multicolumn{3}{|c|}{2561} & \multicolumn{3}{|c|}{2827} & \multicolumn{3}{|c|}{2930} & \multicolumn{3}{|c|}{3120} & \multicolumn{3}{|c|}{3360} \\
\hline $\mathrm{pH}$ & \multicolumn{3}{|c|}{4.08} & \multicolumn{3}{|c|}{7.61} & \multicolumn{3}{|c|}{7.73} & \multicolumn{3}{|c|}{7.80} & \multicolumn{3}{|c|}{8.03} \\
\hline & Sus* & Dis.\# & Total & Sus* & Dis.\# & Total & Sus* & Dis.\#佛 & Total & Sus* & Dis.\# & Total & Sus* & Dis. & Total \\
\hline Total & & 1260 & 1268 & 2 & 1288 & 1290 & 146 & 1292 & 1438 & NA & 1566 & 1578 & 12 & \begin{tabular}{|l|l|}
2 & 1364 \\
\end{tabular} & 1376 \\
\hline $\mathrm{Al}$ & NA & 0.91 & 0.91 & NA & 0.11 & 0.11 & 0.05 & 0.13 & 0.18 & NA & 0.13 & 0.13 & $<0.01$ & 0.15 & 0.15 \\
\hline As & $\mathrm{NA}$ & 5.14 & 5.14 & NA & 4.69 & 4.69 & $<0.01$ & 5.53 & 5.53 & NA & 6.66 & $6.66 \mathrm{~F}$ & k0.01 & 8.22 & 8.2 \\
\hline $\mathrm{B}$ & $\overline{N A}$ & 9.08 & 9.08 & NA & 11.75 & 11.75 & $<0.01$ & 17.26 & 17.26 & NA & 22.96 & 22.96 & K0.01 & 34.43 & 34.4 \\
\hline $\mathrm{Ba}$ & $\mathrm{NA}$ & 0.07 & 0.07 & NA & 0.06 & 0.06 & $<0.01$ & 0.06 & 0.06 & NA & 0.06 & $0.06 \mathrm{~F}$ & k0.01 & 0.06 & 0.0 \\
\hline $\mathrm{Ca}$ & $\mathrm{NA}$ & 46.42 & 46.42 & NA & 49.70 & 49.70 & 0.10 & 54.74 & 54.84 & NA & 59.41 & \begin{tabular}{|l|}
59.41 \\
\end{tabular} & 0.01 & 68.87 & 68.88 \\
\hline $\mathrm{Cd}$ & NA & \begin{tabular}{|l|}
8.72 \\
\end{tabular} & 8.72 & NA & 0.49 & 0.49 & 0.17 & \begin{tabular}{|l|}
0.58 \\
\end{tabular} & 0.76 & NA & 0.40 & 0.40 & 0.01 & 0.44 & 0.4 \\
\hline $\mathrm{Cr}$ & $\mathrm{NA}$ & 0.31 & 0.31 & NA & 0.01 & 0.01 & 0.01 & 0.02 & 0.03 & NA & 0.02 & 0.02 & $<0.01$ & 0.02 & 0.02 \\
\hline Cs & $\mathrm{NA}$ & 20.50 & 20.50 & NA & 20.00 & 20.00 & $<0.1$ & 21.90 & 21.90 & NA & 24.60 & 24.60 & $<0.1$ & 27.40 & 27.40 \\
\hline $\mathrm{Cu}$ & NA & 0.84 & 0.84 & NA & 0.13 & 0.13 & 0.02 & 0.14 & 0.16 & NA & \begin{tabular}{|l|}
0.21 \\
\end{tabular} & $0.21 k$ & $<0.01$ & 0.35 & 0.35 \\
\hline $\mathrm{Fe}$ & NA & $<0.01$ & $<0.01$ & $\mathrm{NA}$ & 0.01 & 0.01 & 0.05 & $<0.01$ & 0.05 & $\mathrm{NA}$ & $<0.01$ & $<0.01$ & $<0.01$ & $<0.01$ & $<0.01$ \\
\hline $\mathrm{K}$ & $\mathrm{NA}$ & 14.20 & 14.20 & NA & 12.87 & 12.87 & $<0.01$ & 14.60 & 14.60 & NA & 15.32 & 15.32 & $<0.01$ & 17.05 & 17.05 \\
\hline $\mathrm{Li}$ & $\mathrm{NA}$ & 24.26 & 24.26 & NA & 22.13 & 22.13 & $<0.01$ & 25.20 & 25.20 & NA & 25.79 & 25.79 & $<0.01$ & 28.08 & 28.08 \\
\hline $\mathrm{Mg}$ & NA & 11.66 & 11.66 & NA & 12.57 & 12.57 & 0.03 & 14.01 & 14.05 & NA & 15.17 & 15.17 & $<0.01$ & 18.57 & 18.57 \\
\hline $\mathrm{Mn}$ & NA & 0.22 & 0.22 & $\mathrm{NA}$ & 0.13 & 0.13 & $<0.01$ & 0.09 & 0.09 & $\mathrm{NA}$ & 0.07 & 0.07 & $<0.01$ & 0.06 & 0.06 \\
\hline $\mathrm{Na}$ & NA & 108.9 & 108.9 & NA & 104.6 & 104.6 & 0.05 & \begin{tabular}{|l|}
116.1 \\
\end{tabular} & 116.2 & NA & \begin{tabular}{|l|}
120.9 \\
\end{tabular} & 120.9 & $<0.01$ & 123.3 & 123.3 \\
\hline $\mathrm{Ni}$ & $\mathrm{NA}$ & 1.19 & 1.19 & NA & 0.88 & 0.88 & $<0.01$ & \begin{tabular}{|l|}
0.99 \\
\end{tabular} & 0.99 & NA & \begin{tabular}{|l|}
0.99 \\
\end{tabular} & 0.99 & $<0.01$ & $1 \quad 1.00$ & 1.00 \\
\hline $\mathrm{P}$ & NA & $<0.07$ & $<0.07$ & NA & $<0.07$ & $<0.07$ & 0.17 & $<0.07$ & 0.17 & NA & $<0.01$ & $<0.01$ & \begin{tabular}{|l|}
0.02 \\
\end{tabular} & $2<0.07$ & 0.02 \\
\hline $\mathrm{Pb}$ & $\mathrm{NA}$ & $\begin{array}{l}0.03 \\
\end{array}$ & $\begin{array}{l}0.03 \\
\end{array}$ & NA & 0.04 & 0.04 & $<0.01$ & \begin{tabular}{|l|}
0.05 \\
\end{tabular} & 0.05 & NA & \begin{tabular}{|l|}
0.08 \\
\end{tabular} & 0.08 & $<0.01$ & 0.11 & 0.1 \\
\hline $\mathrm{Sb}$ & $\mathrm{NA}$ & 0.25 & 0.25 & NA & 0.27 & 0.27 & $<0.01$ & 0.31 & 0.31 & $\mathrm{NA}$ & 0.41 & 0.41 & $<0.01$ & 0.47 & 0.47 \\
\hline $\mathrm{Se}$ & NA & 558.4 & 558.4 & NA & 504.4 & 504.4 & $<0.01$ & \begin{tabular}{|l|}
482.8 \\
\end{tabular} & 482.8 & $\mathrm{NA}$ & 558.0 & 558.0 & $\begin{array}{l}0.01 \\
\end{array}$ & \begin{tabular}{|l|}
589.7 \\
\end{tabular} & 589.7 \\
\hline $\mathrm{Si}$ & NA & 2.96 & 2.96 & NA & 3.15 & 3.15 & 0.38 & 3.65 & 4.03 & NA & 4.08 & 4.08 & 0.01 & 4.89 & 4.90 \\
\hline $\mathrm{Sr}$ & NA & 1.15 & 1.15 & NA & 1.06 & 1.06 & $<0.01$ & 1.18 & 1.18 & NA & \begin{tabular}{|l|}
1.22 \\
\end{tabular} & 1.22 & $<0.01$ & 1.29 & 1.29 \\
\hline $\mathrm{Te}$ & NA & 25.77 & 25.77 & NA & 17.89 & 17.89 & $<0.01$ & 20.39 & 20.39 & NA & 21.94 & 21.94 & $<0.01$ & 22.95 & $\overline{22.95}$ \\
\hline $\mathrm{Ti}$ & NA & $<0.01$ & $<0.01$ & NA & $<0.01$ & $<0.01$ & $<0.01$ & $<0.01$ & $<0.01$ & NA & $<0.01$ & $<0.01$ & $<0.01$ & $<0.01$ & $<0.01$ \\
\hline $\mathrm{Zn}$ & NA & $2.61]$ & 2.61 & $\mathrm{NA}$ & 0.10 & 0.10 & 0.02 & 0.12 & 0.13 & NA & 0.04 & 0.04 & $<0.01$ & 0.03 & 0.03 \\
\hline $\mathrm{Zr}$ & NA & $<0.01$ & $<0.01$ & NA & 0.01 & 0.01 & $<0.01$ & $<0.01$ & $<0.01$ & NA & $<0.01$ & $<0.01$ & $<0.01$ & $<0.01$ & $<0.01$ \\
\hline $\mathrm{Cl}$ & $\mathrm{NA}$ & 38.63 & 38.63 & NA & 42.19 & 42.19 & NA & 47.95 & 47.95 & NA & \begin{tabular}{|l|}
53.53 \\
\end{tabular} & 53.53 & NA & 70.51 & 70.51 \\
\hline$F$ & NA & 3.45 & 3.45 & NA & 4.56 & 4.56 & NA & \begin{tabular}{|l|}
6.53 \\
\end{tabular} & 6.53 & NA & \begin{tabular}{|l|}
9.38 \\
\end{tabular} & 9.38 & $\mathrm{NA}$ & 14.04 & 14.04 \\
\hline I & NA & 2.37 & 2.37 & NA & 10.23 & 10.23 & $<0.1$ & 22.24 & 22.24 & NA & 36.40 & 36.40 & $<0.1$ & 87.44 & 87.44 \\
\hline Nitrate & $\mathrm{NA}$ & 45.80 & 45.80 & NA & 42.66 & 42.66 & NA & 45.90 & 45.90 & NA & 47.59 & 47.59 & $\mathrm{NA}$ & 44.72 & 44.72 \\
\hline Nitrite & NA & $<0.1$ & $<0.1$ & NA & 1.96 & 1.96 & NA & 3.85 & 3.85 & NA & \begin{tabular}{|l|}
5.88 \\
\end{tabular} & 5.88 & $\mathrm{NA}$ & 7.91 & \begin{tabular}{|l|}
7.91 \\
\end{tabular} \\
\hline Sulfate & NA & 319.7 & 319.7 & NA & 292.0 & 292.0 & 0.35 & 342.4 & 342.8 & NA & 355.0 & 355.0 & 0.01 & 377.7 & $\sqrt{377.7}$ \\
\hline
\end{tabular}


Table 4.8. Analytical Results for Samples of WESP Blowdown Fluids (mg/l), (continued).

\begin{tabular}{|c|c|c|c|c|c|c|c|c|c|c|c|c|c|c|c|}
\hline Test & \multicolumn{15}{|c|}{$\# 2$} \\
\hline I.D. & \multicolumn{3}{|c|}{$12 \mathrm{H}-\mathrm{W}-40 \mathrm{~A}$} & \multicolumn{3}{|c|}{$12 \mathrm{H}-\mathrm{W}-43 \mathrm{~A}$} & \multicolumn{3}{|c|}{$12 \mathrm{I}-\mathrm{W}-13 \mathrm{~A}$} & \multicolumn{3}{|c|}{$12 \mathrm{I}-\mathrm{W}-22 \mathrm{~A}$} & \multicolumn{3}{|c|}{$12 \mathrm{I}-\mathrm{W}-26 \mathrm{~A}$} \\
\hline ass $(\mathrm{kg})$. & \multicolumn{3}{|c|}{3452} & \multicolumn{3}{|c|}{3452} & \multicolumn{3}{|c|}{3540} & \multicolumn{3}{|c|}{3699} & \multicolumn{3}{|c|}{3840} \\
\hline $\mathrm{pH}$ & \multicolumn{3}{|c|}{7.97} & \multicolumn{3}{|c|}{7.89} & \multicolumn{3}{|c|}{7.95} & \multicolumn{3}{|c|}{7.89} & \multicolumn{3}{|c|}{7.92} \\
\hline & Sus* & Dis.\# & Tota & Sus* & Dis.\# & \begin{tabular}{|l|} 
Total \\
\end{tabular} & Sus* & Dis.\# & Total & Sus* & Dis.\# & Total & Sus* & Dis.\# & Total \\
\hline Total & & 1580 & 1582 & 574 & 1556 & 2130 & 2 & 1408 & 1410 & & 1420 & 1420 & & 1308 & 1316 \\
\hline $\mathrm{Al}$ & $<0.01$ & 0.16 & 0.16 & 2.76 & 0.15 & 2.91 & NA & 0.16 & 0.16 & NA & 0.16 & 0.1 & NA & 0.17 & 0.1 \\
\hline As & $<0.01$ & 8.61 & 8.61 & 3.23 & 8.52 & 11.75 & $\mathrm{NA}$ & 9.20 & 9.20 & NA & 8.47 & 8.47 & $\mathrm{NA}$ & 8.76 & 8.7 \\
\hline B & $<0.01$ & 37.94 & 37.94 & 0.30 & 39.43 & 39.72 & NA & 42.34 & 42.34 & NA & \begin{tabular}{|l|}
44.67 \\
\end{tabular} & 44.67 & NA & 46.29 & 46.2 \\
\hline $\mathrm{Ba}$ & $<0.01$ & 0.07 & 0.07 & 0.21 & 0.06 & \begin{tabular}{|l|}
0.26 \\
\end{tabular} & $\mathrm{NA}$ & 0.07 & \begin{tabular}{|l|}
0.07 \\
\end{tabular} & $\mathrm{NA}$ & 0.08 & 0.08 & $\mathrm{NA}$ & 0.08 & 0.08 \\
\hline $\mathrm{Ca}$ & $<0.01$ & 74.57 & 74.57 & 7.10 & 74.29 & 81.39 & NA & 78.05 & 78.05 & NA & 77.71 & 77.71 & NA & 83.34 & 83.3 \\
\hline $\mathrm{Cd}$ & $<0.01$ & 0.44 & 0.44 & 48.34 & 0.30 & 48.64 & NA & 0.43 & \begin{tabular}{|l|}
0.43 \\
\end{tabular} & $\mathrm{NA}$ & \begin{tabular}{|l|}
0.41 \\
\end{tabular} & $\mid$\begin{tabular}{|l|}
0.41 \\
\end{tabular} & NA & 0.28 & 0.2 \\
\hline $\mathrm{Cr}$ & $<0.01$ & 0.03 & 0.03 & 11.66 & 0.04 & 11.69 & NA & 0.04 & 0.04 & NA & 0.04 & 0.04 & $\mathrm{NA}$ & \begin{tabular}{|l|}
0.05 \\
\end{tabular} & 0.0 \\
\hline Cs & $<0.1$ & 28.80 & 28.80 & 0.46 & 26.00 & 26.46 & NA & 26.40 & 26.40 & NA & 27.10 & 27.10 & NA & 26.70 & 26.7 \\
\hline$\overline{\mathrm{Cu}}$ & $<0.01$ & 0.42 & 0.42 & 2.52 & 0.45 & \begin{tabular}{|l|}
2.97 \\
\end{tabular} & NA & 0.47 & 0.47 & $\mathrm{NA}$ & 0.49 & 0.49 & $\mathrm{NA}$ & 0.45 & 0.45 \\
\hline $\mathrm{Fe}$ & $<0.01$ & $<0.01$ & $<0.01$ & 17.88 & 0.01 & 17.88 & NA & $<0.01$ & $<0.01$ & NA & 0.01 & 0.01 & $\widehat{\mathrm{NA}}$ & $<0.01$ & $<0.01$ \\
\hline $\bar{K}$ & $<0.01$ & 17.16 & 17.16 & 0.29 & 19.46 & 19.75 & $\mathrm{NA}$ & 17.64 & 17.64 & $\mathrm{NA}$ & 18.09 & 18.09 & $\overline{\mathrm{NA}}$ & 18.98 & 18.9 \\
\hline$\overline{\mathrm{Li}}$ & $<0.01$ & 27.70 & 27.70 & 0.29 & 26.84 & 27.13 & NA & 27.56 & 27.56 & $\mathrm{NA}$ & 27.77 & 27.77 & NA & 28.62 & 28.6 \\
\hline $\mathrm{Mg}$ & $<0.01$ & 19.55 & 19.55 & 0.41 & 20.18 & 20.59 & NA & 21.11 & 21.11 & NA & 22.43 & 22.43 & NA & 24.15 & 24.15 \\
\hline $\mathrm{Mn}$ & $<0.01$ & 0.06 & 0.06 & 0.64 & 0.04 & 0.68 & $\mathrm{NA}$ & 0.06 & 0.06 & $\mathrm{NA}$ & 0.05 & 0.05 & $\mathrm{NA}$ & 0.04 & 0.0 \\
\hline $\mathrm{Na}$ & $<0.01$ & 124.3 & 124.3 & 1.95 & 123.1 & 125.0 & NA & 125.0 & 125.0 & $\mathrm{NA}$ & 125.9 & 125.9 & NA & 130.2 & 130.2 \\
\hline $\mathrm{Ni}$ & $<0.01$ & \begin{tabular}{|l|}
0.99 \\
\end{tabular} & 0.99 & 1.02 & 0.90 & 1.91 & NA & 0.99 & 0.99 & $\mathrm{NA}$ & 0.96 & 0.96 & NA & 0.95 & 0.9 \\
\hline $\mathrm{P}$ & $<0.01$ & $<0.07$ & $<0.07$ & $<0.01$ & $<0.07$ & $<0.07$ & NA & $<0.01$ & $<0.01$ & $\mathrm{NA}$ & $<0.01$ & $<0.01$ & NA & $<0.01$ & $\leq 0.01$ \\
\hline $\mathrm{Pb}$ & $<0.01$ & 0.11 & 0.11 & 8.92 & 0.12 & 9.04 & NA & 0.12 & 0.12 & $\mathrm{NA}$ & 0.16 & 0.16 & NA & 0.15 & 0.15 \\
\hline $\mathrm{Sb}$ & $<0.01$ & 0.43 & $\overline{0.43}$ & 5.27 & 0.54 & 5.81 & NA & 0.40 & 0.40 & NA & 0.46 & 0.46 & NA & 0.54 & 0.54 \\
\hline $\mathrm{Se}$ & $<0.01$ & 628.9 & 628.9 & 42.52 & 768.8 & 811.3 & NA & 662.0 & 662.0 & $\mathrm{NA}$ & 607.4 & 607.4 & NA & 655.0 & 655.0 \\
\hline$\overline{\mathrm{Si}}$ & 0.01 & \begin{tabular}{|l|}
5.51 \\
\end{tabular} & 5.52 & 6.29 & 5.54 & \begin{tabular}{|l|}
11.83 \\
\end{tabular} & NA & 5.66 & 5.66 & NA & 6.07 & 6.07 & NA & 6.51 & \begin{tabular}{|l}
6.5 \\
\end{tabular} \\
\hline $\mathrm{Sr}$ & $<0.01$ & \begin{tabular}{|l|}
1.32 \\
\end{tabular} & 1.32 & 0.25 & \begin{tabular}{|l|}
1.31 \\
\end{tabular} & 1.56 & NA & 1.36 & 1.36 & $\mathrm{NA}$ & 1.37 & 1.37 & NA & 1.43 & 1.4 \\
\hline $\mathrm{Te}$ & $<0.01$ & 23.91 & 23.91 & 177.1 & 23.85 & 200.9 & NA & 24.98 & 24.98 & $\mathrm{NA}$ & 24.24 & 24.24 & $\mathrm{NA}$ & 23.21 & 23.2 \\
\hline $\mathrm{Ti}$ & $<0.01$ & $<0.01$ & $<0.01$ & 0.48 & $<0.01$ & \begin{tabular}{|l|}
0.48 \\
\end{tabular} & NAK & $<0.01$ & $<0.01$ & NA & $<0.01$ & $<0.01$ & NA & $<0.01$ & $<0.01$ \\
\hline $\mathrm{Zn}$ & $<0.01$ & 0.04 & 0.04 & 6.88 & \begin{tabular}{|l|}
0.04 \\
\end{tabular} & 6.91 & NA & 0.03 & \begin{tabular}{|l|}
0.03 \\
\end{tabular} & $\mathrm{NA}$ & 0.03 & 0.03 & NA & 0.02 & 0.02 \\
\hline $\mathrm{Zr}$ & $<0.01$ & $<0.01$ & $<0.01$ & 0.33 & $<0.01$ & 0.33 & $\mathrm{NA}$ & 0.01 & 0.01 & $\mathrm{NA}$ & $<0.01$ & $<0.01$ & NA & 0.01 & 0.0 \\
\hline $\mathrm{Cl}$ & $\mathrm{NA}$ & 74.44 & 74.44 & NA & 80.99 & 80.99 & $\mathrm{NA}$ & 82.03 & 82.03 & $\mathrm{NA}$ & 80.45 & 80.45 & NA & 88.42 & 88.42 \\
\hline $\mathrm{F}$ & NA & 15.37 & 15.37 & $\mathrm{NA}$ & 16.85 & 16.85 & NA & 17.69 & 17.69 & $\mathrm{NA}$ & 14.09 & 14.09 & NA & 18.46 & 18.46 \\
\hline$I$ & $<0.1$ & 100.9 & 100.9 & $<0.01$ & 116.4 & 116.4 & NA & 122.3 & \begin{tabular}{|l|}
122.3 \\
\end{tabular} & $\mathrm{NA}$ & 128.8 & 128.8 & NA & 143.5 & 143.5 \\
\hline trate & $\mathrm{NA}$ & 46.63 & 46.63 & NA & 56.95 & 56.95 & NA & 45.68 & \begin{tabular}{|l|}
45.68 \\
\end{tabular} & $\mathrm{NA}$ & 43.30 & 43.30 & NA & 43.35 & 43.35 \\
\hline & NA & 8.69 & 8.69 & NA & 8.56 & 8.56 & NA & 9.85 & \begin{tabular}{|l|}
9.85 \\
\end{tabular} & $\mathrm{NA}$ & 10.93 & 10.93 & NA & 14.36 & 14.36 \\
\hline Sulfate & .02 & \begin{tabular}{|l|}
395.7 \\
\end{tabular} & 395.7 & 0.35 & 384.5 & 384.8 & NA. & 529.2 & 529.2 & $\mathrm{NA}$ & 386.8 & 386.8 & $\mathrm{NA}$ & 423.5 & 423. \\
\hline
\end{tabular}

Sus* - Suspended

Dis\# - Dissolved

NA - Not Analyzed 
The Catholic University of America

Vitreous State Laboratory

DuraMelter 1200 Tests with AZ-101HLW Simulants

Final Report, VSL-02R0100-2, Rev.1

Table 4.8. Analytical Results for Samples of WESP Blowdown Fluids (mg/l), (continued).

\begin{tabular}{|c|c|c|c|c|c|c|c|c|c|c|c|c|c|c|c|}
\hline est & \multicolumn{15}{|c|}{ \#2 } \\
\hline I.D. & \multicolumn{3}{|c|}{$12 I-W-33 A$} & \multicolumn{3}{|c|}{$12 \mathrm{I}-\mathrm{W}-42 \mathrm{~A}$} & \multicolumn{3}{|c|}{$12 \mathrm{I}-\mathrm{W}-51 \mathrm{~A}$} & \multicolumn{3}{|c|}{$12 \mathrm{I}-\mathrm{W}-61 \mathrm{~A}$} & \multicolumn{3}{|c|}{$12 \mathrm{I}-\mathrm{W}-68 \mathrm{~A}$} \\
\hline Glass $(\mathrm{kg})$. & \multicolumn{3}{|c|}{3981} & \multicolumn{3}{|c|}{4122} & \multicolumn{3}{|c|}{4250} & \multicolumn{3}{|c|}{4507} & \multicolumn{3}{|c|}{4635} \\
\hline $\mathrm{pH}$ & \multicolumn{3}{|c|}{8.01} & \multicolumn{3}{|c|}{7.92} & \multicolumn{3}{|c|}{7.92} & \multicolumn{3}{|c|}{7.91} & \multicolumn{3}{|c|}{7.89} \\
\hline & Sus* & Dis.\# & Total & Sus* & Dis.\# & Total & Sus* & Dis.\# & Total & Sus* & Dis.\# & Total & Sus*1 & Dis.\# & Total \\
\hline otal & & 1456 & 1462 & 12 & 1544 & 1556 & 2 & 1696 & 1698 & 2 & \begin{tabular}{|l|}
1976 \\
\end{tabular} & 1978 & 6 & 1904 & 1910 \\
\hline 1 & 0.01 & 0.17 & 0.17 & NA & 0.17 & 0.17 & $\mathrm{NA}$ & 0.17 & 0.17 & $<0.01$ & 0.18 & & NA & 0.18 & 0.1 \\
\hline is & 0.01 & 9.04 & 9.04 & $\mathrm{NA}$ & 9.64 & 9.64 & $\mathrm{NA}$ & 10.43 & 10.43 & $<0.01$ & 11.29 & 11.29 & NA & 10.59 & 10. \\
\hline $\bar{B}$ & $<0.01$ & 47.20 & 47.20 & NA & 50.97 & 50.97 & $\mathrm{NA}$ & 52.84 & 52.84 & $<0.01$ & 58.29 & 58.29 & $\mathrm{NA}$ & 55.16 & 55.1 \\
\hline $\mathrm{Ba}$ & $<0.01$ & 0.08 & 0.08 & $\mathrm{NA}$ & 0.09 & 0.09 & NA & 0.09 & 0.09 & $<0.01$ & 0.09 & 0.09 & NA & 0.09 & 0.09 \\
\hline $\mathrm{Ca}$ & $<0.01$ & 87.61 & 87.61 & NA & 95.04 & 95.04 & $\mathrm{NA}$ & 92.96 & 92.96 & $<0.01$ & 99.70 & 99.70 & NA & 95.81 & 95.81 \\
\hline $\mathrm{d}$ & 0.01 & 0.28 & \begin{tabular}{|l|}
0.28 \\
\end{tabular} & NA & 0.22 & 0.22 & $\mathrm{NA}$ & 0.28 & 0.28 & $<0.01$ & \begin{tabular}{|l|}
0.28 \\
\end{tabular} & 0.28 & NA & 0.28 & 0.28 \\
\hline $\mathrm{rr}$ & 0.01 & 0.05 & 0.05 & $\mathrm{NA}$ & 0.06 & 0.06 & NA & 0.07 & 0.07 & $<0.01$ & 0.07 & 0.07 & $\mathrm{NA}$ & 0.07 & 0.6 \\
\hline $\mathrm{Cs}$ & $<0.1$ & 26.50 & 26.50 & $\mathrm{NA}$ & 22.14 & 22.14 & $\mathrm{NA}$ & 29.90 & 29.90 & $<0.1$ & 31.50 & 31.50 & $\mathrm{NA}$ & 29.20 & 29.20 \\
\hline$\overline{\mathrm{Cu}}$ & $<0.01$ & 0.45 & 0.45 & $\mathrm{NA}$ & 0.43 & 0.43 & NA & 0.48 & 0.48 & $<0.01$ & 0.48 & 0.48 & $\mathrm{NA}$ & 0.45 & 0.45 \\
\hline $\mathrm{Fe}$ & $<0.01$ & $<0.01$ & $<0.01$ & $\mathrm{NA}$ & 0.02 & 0.02 & NA & 0.02 & 0.02 & $<0.01$ & 0.03 & 0.03 & NA & 0.03 & 0.03 \\
\hline K & $<0.01$ & 18.47 & 18.47 & $\mathrm{NA}$ & 19.61 & 19.61 & $\mathrm{NA}$ & 20.02 & 20.02 & $<0.01$ & 20.61 & 20.61 & $\mathrm{NA}$ & 20.16 & 20.1 \\
\hline $\mathrm{Li}$ & $<0.01$ & 27.61 & 27.61 & $\mathrm{NA}$ & 28.83 & 28.83 & NA & 28.88 & 28.88 & $<0.01$ & 29.11 & 29.11 & NA & 28.41 & 28. \\
\hline$\overline{M g}$ & $<0.01$ & 24.77 & 24.77 & $\mathrm{NA}$ & 26.41 & 26.41 & NA & 28.25 & 28.25 & $<0.01$ & 29.37 & 29.37 & $\mathrm{NA}$ & 28.65 & 28.65 \\
\hline$\overline{\mathrm{Mn}}$ & $<0.01$ & 0.05 & $\begin{array}{l}0.05 \\
\end{array}$ & $\mathrm{NA}$ & 0.06 & 0.06 & $\mathrm{NA}$ & 0.05 & 0.05 & $<0.01$ & 0.04 & 0.04 & NA & 0.06 & 0.06 \\
\hline $\mathrm{Na}$ & $<0.01$ & 127.0 & 127.0 & $\mathrm{NA}$ & 134.0 & 134.0 & $\mathrm{NA}$ & 136.0 & 136.0 & $<0.01$ & 140.5 & 140.5 & NA & 136.8 & 136.8 \\
\hline $\mathrm{Ji}$ & $<0.01$ & 0.90 & 0.90 & $\mathrm{NA}$ & 0.88 & 0.88 & NA & 0.84 & 0.84 & $<0.01$ & 0.82 & 0.82 & $\mathrm{NA}$ & 0.82 & 0.82 \\
\hline & $<0.01$ & $<0.01$ & $<0.01$ & $\mathrm{NA}$ & 0.08 & 0.08 & NA & 0.08 & 0.08 & $<0.01$ & 0.29 & 0.29 & $\mathrm{NA}$ & 0.32 & 0.3 \\
\hline $\mathrm{pb}$ & $<0.01$ & 0.16 & 0.16 & $\mathrm{NA}$ & 0.17 & 0.17 & NA & 0.18 & 0.18 & $<0.01$ & 0.18 & 0.18 & $\mathrm{NA}$ & 0.18 & 0.18 \\
\hline $\mathrm{sb}$ & $<0.01$ & 0.52 & 0.52 & NA & 0.61 & 0.61 & NA & 0.65 & 0.65 & $<0.01$ & 0.58 & 0.58 & NA & 0.59 & 0.59 \\
\hline $\mathrm{Se}$ & $<0.01$ & 676.8 & 676.8 & $\mathrm{NA}$ & 698.1 & 698.1 & $\mathrm{NA}$ & 704.8 & 704.8 & $<0.01$ & 723.4 & 723.4 & NA & 725.8 & 725.8 \\
\hline$\overline{\mathrm{i}}$ & $<0.01$ & \begin{tabular}{|l|}
6.48 \\
\end{tabular} & 6.48 & NA & 7.10 & 7.10 & NA & 7.19 & 7.19 & $<0.01$ & 7.58 & $\begin{array}{l}7.59 \\
\end{array}$ & NA & 7.44 & \begin{tabular}{|l|}
7.44 \\
\end{tabular} \\
\hline Sr & $<0.01$ & 1.40 & 1.40 & $\mathrm{NA}$ & 1.42 & 1.42 & $\mathrm{NA}$ & 1.46 & 1.46 & $<0.01$ & 1.49 & 1.49 & NA & 1.43 & 1.43 \\
\hline $\mathrm{Te}$ & $<0.01$ & 24.47 & 24.47 & NA & 24.54 & 24.54 & NA & 27.05 & 27.05 & $<0.01$ & 29.69 & 29.69 & NA & 26.19 & 26.19 \\
\hline Ti & $<0.01$ & $<0.01$ & $<0.01$ & NA & 0.01 & 0.01 & NA & 0.01 & 0.01 & $<0.01$ & \begin{tabular}{|l|}
0.01 \\
\end{tabular} & 0.01 & NA & 0.01 & 0.01 \\
\hline $\mathrm{Zn}$ & $<0.01$ & 0.02 & 0.02 & $\mathrm{NA}$ & 0.02 & 0.02 & NA & 0.02 & 0.02 & $<0.01$ & 0.02 & 0.02 & $\mathrm{NA}$ & 0.03 & 0.03 \\
\hline $\mathrm{Zr}$ & $<0.01$ & 0.01 & 0.01 & NA & 0.02 & 0.02 & $\mathrm{NA}$ & 0.02 & 0.02 & $<0.01$ & 0.02 & 0.02 & NA & 0.02 & 0.02 \\
\hline $\mathrm{Cl}$ & $\mathrm{NA}$ & 94.40 & 94.40 & NA & 90.73 & 90.73 & $\mathrm{NA}$ & 105.7 & 105.7 & NA & 113.0 & 113.0 & NA & 116.4 & 116.4 \\
\hline$F$ & $\mathrm{NA}$ & \begin{tabular}{|l|}
19.31 \\
\end{tabular} & 19.31 & NA & 20.38 & 20.38 & NA & 22.86 & 22.86 & NA & 26.34 & 26.34 & $\mathrm{NA}$ & 24.80 & 24.80 \\
\hline & $<0.1$ & 156.0 & 156.0 & NA & 153.4 & 153.4 & NA & 171.1 & 171.1 & $<0.1$ & 198.9 & 198.9 & $\mathrm{NA}$ & 192.6 & 192.6 \\
\hline & $\mathrm{NA}$ & 45.57 & 45.57 & $\mathrm{NA}$ & 45.60 & 45.60 & NA & 45.99 & 45.99 & $\mathrm{NA}$ & 47.84 & 47.84 & $\mathrm{NA}$ & 45.27 & 45.27 \\
\hline & $\mathrm{NA}$ & 16.27 & 16.27 & NA & 18.06 & 18.06 & NA & 20.82 & 20.82 & $\mathrm{NA}$ & 23.70 & 23.70 & NA & 21.70 & 21.70 \\
\hline Sulfate & $<0.1$ & 470.4 & 470.4 & NA & 439.2 & 439.2 & $\mathrm{NA}$ & 478.4 & 478.4 & $<0.1$ & [494.7] & 494.7 & $\mathrm{NA}$ & 464.0 & 464.0 \\
\hline
\end{tabular}

Sus* - Suspended

Dis\# - Dissolved

NA - Not Analyzed 
The Catholic University of America Vitreous State Laboratory
DuraMelter 1200 Tests with AZ-101HLW Simulants Final Report, VSL-02R0100-2, Rev.1

Table 4.8. Analytical Results for Samples of WESP Blowdown Fluids (mg/l), (continued).

\begin{tabular}{|c|c|c|c|c|c|c|c|c|c|c|c|c|c|c|}
\hline Test & \multicolumn{8}{|c|}{$\# 2$} & \multicolumn{6}{|c|}{$\# 3$} \\
\hline I.D. & \multicolumn{3}{|c|}{$12 \mathrm{I}-\mathrm{W}-73 \mathrm{~A}$} & \multicolumn{3}{|c|}{$12 \mathrm{~J}-\mathrm{W}-12 \mathrm{~A}$} & \multicolumn{3}{|c|}{$12 \mathrm{~J}-\mathrm{W}-13 \mathrm{~A}$} & \multicolumn{3}{|c|}{$12 \mathrm{~J}-\mathrm{W}-65 \mathrm{~A}$} & \multicolumn{2}{|c|}{$12 \mathrm{~J}-\mathrm{W}-86 \mathrm{~A}$} \\
\hline lass $(\mathrm{kg})$. & \multicolumn{3}{|c|}{4765} & \multicolumn{3}{|c|}{4765} & \multicolumn{3}{|c|}{4900} & \multicolumn{3}{|c|}{6657} & \multicolumn{2}{|c|}{7711} \\
\hline $\mathrm{pH}$ & \multicolumn{3}{|c|}{7.90} & \multicolumn{3}{|c|}{7.82} & \multicolumn{3}{|c|}{6.99} & \multicolumn{3}{|c|}{3.08} & \multicolumn{2}{|l|}{3.00} \\
\hline & Sus* & Dis.\# & Total & Sus* & Dis.\# & Total & Sus* & Dis.\# & Total & Sus* & Dis.\# & Total & Sus* Dis.\# & Total \\
\hline Total & & 2080 & 2088 & 50 & 2088 & 2138 & 288 & 604 & \begin{tabular}{|l|}
892 \\
\end{tabular} & 0 & 2508 & 2508 & 63800 & 3806 \\
\hline $\mathrm{Al}$ & $\mathrm{NA}$ & 0.18 & 0.18 & 0.11 & 0.18 & 0.29 & 0.12 & 0.07 & 0.19 & NA & 1.28 & 1.28 & \begin{tabular}{l|l|} 
NA & 2.28 \\
\end{tabular} & 2.28 \\
\hline As & NA & 11.99 & 11.99 & 0.41 & 13.26 & 13.67 & 2.43 & 9.86 & 12.30 & NA & 19.53 & 19.53 & \begin{tabular}{l|l|} 
NA & 25.15 \\
\end{tabular} & 25.15 \\
\hline B & $\mathrm{NA}$ & 61.57 & 61.57 & $<0.01$ & 72.29 & 72.29 & $<0.01$ & 14.28 & 14.28 & $\mathrm{NA}$ & 71.29 & 71.29 & \begin{tabular}{l|l|}
$\mathrm{NA}$ & 115.0 \\
\end{tabular} & 115.0 \\
\hline $\mathrm{Ba}$ & $\mathrm{NA}$ & 0.10 & 0.10 & 0.01 & 0.10 & 0.11 & \begin{tabular}{|l|}
0.04 \\
\end{tabular} & \begin{tabular}{|l|}
0.03 \\
\end{tabular} & 0.07 & NA & 0.14 & 0.14 & \begin{tabular}{|l|l|}
$\mathrm{NA}$ & 0.19 \\
\end{tabular} & 0.19 \\
\hline$\overline{\mathrm{Ca}}$ & NA & 74.86 & 74.86 & 0.53 & \begin{tabular}{|l|}
82.87 \\
\end{tabular} & 83.40 & 0.92 & 38.01 & \begin{tabular}{|c|}
38.93 \\
\end{tabular} & NA & 60.81 & 60.81 & \begin{tabular}{l|l|} 
NA & 65.84 \\
\end{tabular} & 65.84 \\
\hline$\overline{\mathrm{Cd}}$ & NA & \begin{tabular}{|l|}
0.26 \\
\end{tabular} & 0.26 & 6.37 & \begin{tabular}{|l|}
0.28 \\
\end{tabular} & 6.65 & 42.62 & 0.32 & 42.94 & NA & \begin{tabular}{|l|}
64.82 \\
\end{tabular} & 64.82 & \begin{tabular}{l|l|}
$\mathrm{NA}$ & 76.31
\end{tabular} & 76.31 \\
\hline $\mathrm{Cr}$ & $\mathrm{NA}$ & 0.08 & 0.08 & 0.34 & 0.10 & 0.44 & \begin{tabular}{|l|}
1.61 \\
\end{tabular} & 0.03 & 1.64 & $\mathrm{NA}$ & 0.22 & 0.22 & \begin{tabular}{l|l|} 
NA & 0.40 \\
\end{tabular} & 0.40 \\
\hline $\mathrm{Cs}$ & $\mathrm{NA}$ & 31.30 & 31.30 & 0.10 & 34.30 & 34.40 & 0.64 & 11.50 & 12.14 & $\mathrm{NA}$ & 56.26 & 56.26 & \begin{tabular}{l|l|} 
NA & 81.22 \\
\end{tabular} & 81.22 \\
\hline$\overline{\mathrm{Cu}}$ & NA & \begin{tabular}{|l|}
0.48 \\
\end{tabular} & 0.48 & 0.08 & \begin{tabular}{|l|}
0.24 \\
\end{tabular} & 0.32 & 0.13 & 0.02 & 0.14 & NA & 1.61 & 1.61 & \begin{tabular}{l|l|}
$\mathrm{NA}$ & 2.17
\end{tabular} & 2.17 \\
\hline $\mathrm{Fe}$ & $\mathrm{NA}$ & 0.03 & 0.03 & 1.20 & 0.03 & 1.23 & 2.25 & 0.02 & 2.26 & NA & 0.04 & 0.04 & 0.06 & 0.06 \\
\hline $\mathrm{K}$ & $\mathrm{NA}$ & 21.63 & 21.63 & 0.03 & 23.36 & 23.39 & 0.20 & 7.10 & 7.31 & $\mathrm{NA}$ & 23.89 & 23.89 & \begin{tabular}{l|l|} 
NA & 36.65 \\
\end{tabular} & 36.65 \\
\hline$\overline{\mathrm{Li}}$ & $\mathrm{NA}$ & 25.29 & 25.29 & 0.04 & 27.42 & 27.47 & 0.23 & 7.57 & 7.81 & NA & 53.76 & 53.76 & \begin{tabular}{|l|l|} 
NA & 84.75 \\
\end{tabular} & 84.75 \\
\hline $\mathrm{Mg}$ & NA & 28.93 & 28.93 & 0.03 & 32.72 & 32.75 & 0.05 & 12.28 & 12.32 & $\mathrm{NA}$ & 17.80 & 17.80 & \begin{tabular}{|l|l|} 
NA & 19.62 \\
\end{tabular} & 19.62 \\
\hline $\mathrm{Mn}$ & $\mathrm{NA}$ & \begin{tabular}{|l|}
0.05 \\
\end{tabular} & 0.05 & 0.13 & 0.05 & 0.18 & 0.04 & 0.03 & 0.07 & $\mathrm{NA}$ & \begin{tabular}{|l|}
0.49 \\
\end{tabular} & 0.49 & \begin{tabular}{|l|l|}
$\mathrm{NA}$ & 0.79 \\
\end{tabular} & 0.79 \\
\hline $\mathrm{Na}$ & $\mathrm{NA}$ & 107.3 & 107.3 & 0.32 & 116.4 & 116.4 & 2.02 & 48.38 & 50.39 & NA & 293.2 & 293.2 & \begin{tabular}{l|l|} 
NA & 429.0
\end{tabular} & 429.0 \\
\hline $\mathrm{Ni}$ & NA & \begin{tabular}{|l|}
0.77 \\
\end{tabular} & 0.77 & 0.08 & \begin{tabular}{|l|}
0.70 \\
\end{tabular} & 0.78 & 0.17 & \begin{tabular}{|l|}
0.29 \\
\end{tabular} & 0.46 & $\mathrm{NA}$ & 1.12 & 1.12 & NA 1.55 & 1.55 \\
\hline $\mathrm{P}$ & NA & 0.27 & 0.27 & $<0.01$ & 0.24 & 0.24 & $<0.01$ & 0.11 & 0.11 & $\mathrm{NA}$ & 0.60 & 0.60 & 0.65 & 0.65 \\
\hline $\mathrm{Pb}$ & $\mathrm{NA}$ & 0.20 & 0.20 & 0.97 & 0.23 & 1.21 & 5.94 & $<0.01$ & 5.94 & $\mathrm{NA}$ & 0.96 & 0.96 & $\mathrm{NA}$ & 1.18 \\
\hline $\mathrm{Sb}$ & NA & 0.62 & 0.62 & 0.24 & 0.73 & 0.96 & 1.37 & \begin{tabular}{|l|}
0.37 \\
\end{tabular} & 1.74 & NA & 0.33 & 0.33 & 0.25 & 0.25 \\
\hline $\mathrm{Se}$ & NA & 898.4 & 898.4 & 2.38 & 1008 & 1011 & 12.85 & \begin{tabular}{|l|}
414.9 \\
\end{tabular} & 427.8 & NA & \begin{tabular}{|l|}
899.2 \\
\end{tabular} & 899.2 & \begin{tabular}{l|l} 
NA & 913.6
\end{tabular} & 913.6 \\
\hline $\mathrm{Si}$ & NA & 8.07 & 8.07 & 0.69 & 9.00 & 9.69 & \begin{tabular}{|l|}
0.79 \\
\end{tabular} & 2.57 & 3.36 & $\mathrm{NA}$ & 4.61 & 4.61 & \begin{tabular}{|l|l|}
$\mathrm{NA}$ & 5.94 \\
\end{tabular} & 5.94 \\
\hline $\mathrm{Sr}$ & $\mathrm{NA}$ & 1.54 & 1.54 & 0.02 & 1.75 & 1.77 & 0.03 & 0.36 & 0.39 & $\mathrm{NA}$ & 1.66 & 1.66 & 2.51 & 2.51 \\
\hline $\mathrm{Te}$ & NA & 27.72 & 27.72 & 20.15 & 34.04 & 54.18 & \begin{tabular}{|l|}
115.7 \\
\end{tabular} & 15.30 & 131.0 & $\mathrm{NA}$ & 48.09 & 48.09 & \begin{tabular}{l|l|}
$\mathrm{NA}$ & 63.16 \\
\end{tabular} & 63.16 \\
\hline $\mathrm{Ti}$ & NA & \begin{tabular}{|l|}
0.01 \\
\end{tabular} & 0.01 & 0.01 & \begin{tabular}{|l|}
0.01 \\
\end{tabular} & 0.03 & 0.02 & $<0.01$ & \begin{tabular}{|l|}
0.02 \\
\end{tabular} & NA & 0.01 & 0.01 & \begin{tabular}{|l|l|}
$\mathrm{NA}$ & 0.03 \\
\end{tabular} & 0.03 \\
\hline $\mathrm{Zn}$ & $\mathrm{NA}$ & 0.02 & 0.02 & 0.13 & 0.02 & 0.15 & 0.41 & \begin{tabular}{|l|}
0.01 \\
\end{tabular} & 0.42 & NA & 4.75 & 4.75 & 6.88 & 6.88 \\
\hline $\mathrm{Zr}$ & $\mathrm{NA}$ & 0.01 & 0.01 & 0.06 & \begin{tabular}{|l|}
0.03 \\
\end{tabular} & 0.08 & 0.02 & 0.01 & 0.03 & NA & 0.02 & 0.02 & 0.04 & $\overline{0.04}$ \\
\hline $\mathrm{Cl}$ & $\mathrm{NA}$ & 128.9 & 128.9 & NA & 154.1 & 154.1 & NA & 82.03 & 82.03 & $\mathrm{NA}$ & 83.40 & 83.40 & \begin{tabular}{l|l|}
$\mathrm{NA}$ & 102.2 \\
\end{tabular} & 102.2 \\
\hline $\bar{F}$ & $\mathrm{NA}$ & 27.23 & 27.23 & NA & 31.00 & 31.00 & NA & 17.69 & 17.69 & $\mathrm{NA}$ & 7.92 & \begin{tabular}{|l|}
7.92 \\
\end{tabular} & \begin{tabular}{l|l|}
$\mathrm{NA}$ & 8.74 \\
\end{tabular} & 8.74 \\
\hline I & $\mathrm{NA}$ & 214.2 & 214.2 & $<0.1$ & 274.1 & 274.1 & $<0.1$ & 122.3 & 122.3 & $\mathrm{NA}$ & \begin{tabular}{|l|}
6.13 \\
\end{tabular} & 6.13 & \begin{tabular}{l|l}
$\mathrm{NA}$ & 1.56 \\
\end{tabular} & 1.56 \\
\hline Nitrate & $\mathrm{NA}$ & 48.19 & 48.19 & NA & 52.86 & 52.86 & NA & 45.68 & 45.68 & $\mathrm{NA}$ & 28.55 & 28.55 & \begin{tabular}{l|l|} 
NA & 44.59 \\
\end{tabular} & \begin{tabular}{|l|l|}
44.59 \\
\end{tabular} \\
\hline Nitrite & $\mathrm{NA}$ & 24.19 & 24.19 & $\mathrm{NA}$ & 35.75 & 35.75 & NA & \begin{tabular}{|l|}
9.85 \\
\end{tabular} & \begin{tabular}{|l|}
9.85 \\
\end{tabular} & $\mathrm{NA}$ & $<0.1$ & \begin{tabular}{|c|}
$<0.1$ \\
\end{tabular} & \begin{tabular}{l|l|}
$\mathrm{NA}$ & $<0.1$ \\
\end{tabular} & $<0.1$ \\
\hline Sulfate & $\mathrm{NA}$ & 517.5 & 517.5 & 0.04 & 559.4 & 559.5 & $<0.1$ & 429.2 & 429.2 & $\mathrm{NA}$ & 1140 & 1140 & \begin{tabular}{l|l|} 
NA & 1492 \\
\end{tabular} & 1492 \\
\hline
\end{tabular}


Table 4.8. Analytical Results for Samples of WESP Blowdown Fluids (mg/l), (continued).

\begin{tabular}{|c|c|c|c|c|c|c|c|c|c|c|c|c|c|}
\hline Test & \multicolumn{7}{|c|}{$\# 3$} & \multicolumn{6}{|c|}{$\# 5$} \\
\hline I.D. & $12 \mathrm{~J}-\mathrm{W}-13$ & & & $2 \mathrm{~K}-\mathrm{W}-\mathrm{t}$ & & & $\mathrm{K}-\mathrm{W}-6$ & & & $\mathrm{~L}-\mathrm{W}-2 \mathrm{C}$ & & $12 \mathrm{~L}-\mathrm{W}-30$ & \\
\hline Glass $(\mathrm{kg})$ & 9645 & & & 9645 & & & 11100 & & & 14746 & & 14849 & \\
\hline $\mathrm{pH}$ & 7.07 & & & 2.75 & & & 2.5 & & & 2.00 & & 1.27 & \\
\hline & Sus* Dis.\# & \begin{tabular}{|l|} 
Total \\
\end{tabular} & \begin{tabular}{|l|} 
Sus* \\
\end{tabular} & Dis.\# & Total & Sus* & Dis.\# & Total & Sus* & Dis.\# & Total & 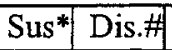 & Tota \\
\hline Total & \begin{tabular}{|l|l|}
333 & 428 \\
\end{tabular} & 761 & 26 & 949 & 2518 & 92 & 2060 & 2152 & 0 & 592 & 592 & \begin{tabular}{l|l|}
0 & 1612 \\
\end{tabular} & 1612 \\
\hline $\mathrm{Al}$ & \begin{tabular}{l|l|}
$\mathrm{NA}$ & 3.71 \\
\end{tabular} & 3.71 & 0.02 & 4.94 & 4.96 & 0.56 & 0.88 & 1.44 & $\mathrm{NA}$ & 0.53 & 0.53 & \begin{tabular}{l|l|}
$\mathrm{NA}$ & 2.61 \\
\end{tabular} & 2.61 \\
\hline As & \begin{tabular}{l|l|} 
NA & 49.79 \\
\end{tabular} & \begin{tabular}{|l|}
49.79 \\
\end{tabular} & 0.10 & 60.56 & 60.66 & 0.32 & 9.94 & 10.25 & NA & 1.88 & 1.88 & 3.88 & $\overline{3.88}$ \\
\hline B & \begin{tabular}{l|l|} 
NA & 212.7 \\
\end{tabular} & 212.7 & 0.31 & 281.7 & 282.0 & 0.09 & 22.17 & 22.26 & NA & 15.53 & 15.53 & \begin{tabular}{l|l|} 
NA & 47.31 \\
\end{tabular} & 47.31 \\
\hline $\mathrm{Ba}$ & \begin{tabular}{l|l}
$\mathrm{NA}$ & 0.25 \\
\end{tabular} & 0.25 & $<0.01$ & 0.30 & 0.30 & 0.01 & \begin{tabular}{|l|}
0.07 \\
\end{tabular} & 0.07 & NA & \begin{tabular}{|l|}
0.07 \\
\end{tabular} & 0.07 & \begin{tabular}{l|l} 
NA & 0.15 \\
\end{tabular} & 0.15 \\
\hline $\mathrm{Ca}$ & \begin{tabular}{l|l}
$\mathrm{NA}$ & 91.27
\end{tabular} & 91.27 & 0.02 & 77.34 & 77.36 & 0.06 & 39.48 & 39.54 & NA & 43.40 & 43.40 & \begin{tabular}{l|l|} 
NA & 54.10 \\
\end{tabular} & 54.10 \\
\hline $\mathrm{Cd}$ & \begin{tabular}{l|l} 
NA & 99.70
\end{tabular} & 99.70 & 1.76 & 117.0 & 119.2 & 2.27 & 46.58 & 48.85 & NA & 14.75 & 14.75 & \begin{tabular}{l|l|} 
NA & 51.84 \\
\end{tabular} & 51.84 \\
\hline $\mathrm{Cr}$ & \begin{tabular}{l|l|} 
NA & 0.64 \\
\end{tabular} & 0.64 & 0.18 & 1.35 & 1.52 & 2.80 & 2.90 & 5.70 & NA & 4.88 & 4.88 & \begin{tabular}{|l|l|} 
NA & 24.84 \\
\end{tabular} & 24.84 \\
\hline Cs & \begin{tabular}{l|l|}
$\mathrm{NA}$ & 163.7 \\
\end{tabular} & 163.7 & $<0.1$ & 200.6 & 200.6 & $<0.1$ & 23.56 & 23.56 & NA & 5.15 & 5.15 & \begin{tabular}{l|l|} 
NA & 10.49 \\
\end{tabular} & 10.49 \\
\hline $\mathrm{Cu}$ & \begin{tabular}{|l|l|} 
NA & 5.57 \\
\end{tabular} & 5.57 & 0.01 & 8.43 & 8.44 & 0.02 & 0.65 & 0.67 & NA & 2.40 & 2.40 & \begin{tabular}{|l|l|}
$\mathrm{NA}$ & 5.24 \\
\end{tabular} & 5.24 \\
\hline $\mathrm{Fe}$ & 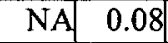 & 0.08 & 0.64 & 0.12 & 0.76 & 4.51 & 0.20 & 4.71 & NA & 0.89 & 0.89 & 9.91 & 9.91 \\
\hline $\mathrm{K}$ & \begin{tabular}{l|l|} 
NA & 74.88 \\
\end{tabular} & 74.88 & 0.11 & 100.4 & 100.5 & 0.02 & \begin{tabular}{|l|}
12.92 \\
\end{tabular} & 12.94 & $\mathrm{NA}$ & 5.15 & 5.15 & 8.14 & 8.14 \\
\hline $\mathrm{Li}$ & \begin{tabular}{l|l|} 
NA & 141.4 \\
\end{tabular} & 141.4 & 0.01 & 185.8 & 185.8 & 0.01 & 24.71 & 24.72 & NA & 6.03 & 6.03 & \begin{tabular}{l|l|} 
NA & 13.08 \\
\end{tabular} & 13.08 \\
\hline $\mathrm{Mg}$ & \begin{tabular}{l|l|} 
NA & 28.95 \\
\end{tabular} & 28.95 & 0.02 & 29.89 & 29.91 & 0.07 & 10.26 & 10.32 & NA & \begin{tabular}{|l|}
13.27 \\
\end{tabular} & 13.27 & \begin{tabular}{l|l|}
$\mathrm{NA}$ & 15.90 \\
\end{tabular} & 15.90 \\
\hline $\mathrm{Mn}$ & \begin{tabular}{l|l}
$\mathrm{NA}$ & 1.36 \\
\end{tabular} & 1.36 & 0.13 & 1.75 & 1.88 & 0.51 & 0.66 & 1.16 & NA & 0.24 & 0.24 & \begin{tabular}{l|l|} 
NA & 0.86 \\
\end{tabular} & 0.86 \\
\hline $\mathrm{Na}$ & \begin{tabular}{l|l|} 
NA & 787.7 \\
\end{tabular} & 787.7 & 0.11 & 982.3 & 982.4 & 0.18 & \begin{tabular}{|l|}
113.8 \\
\end{tabular} & 114.0 & NA & 41.78 & \begin{tabular}{|l|}
41.78 \\
\end{tabular} & \begin{tabular}{l|l|}
$\mathrm{NA}$ & 70.83 \\
\end{tabular} & 70.83 \\
\hline $\mathrm{Ni}$ & \begin{tabular}{l|l|}
$\mathrm{NA}$ & 2.45 \\
\end{tabular} & 2.45 & 0.01 & 2.90 & 2.91 & 0.11 & 2.92 & \begin{tabular}{|l|}
3.03 \\
\end{tabular} & NA & 0.31 & \begin{tabular}{|l|}
0.31 \\
\end{tabular} & \begin{tabular}{|l|l|}
$\mathrm{NA}$ & 1.33 \\
\end{tabular} & 1.33 \\
\hline $\mathrm{P}$ & \begin{tabular}{l|l|} 
NA & 1.32 \\
\end{tabular} & 1.32 & 0.04 & 1.65 & 1.69 & 0.29 & 0.38 & 0.67 & NA & 0.26 & 0.26 & \begin{tabular}{l|l|}
$\mathrm{NA}$ & 0.52 \\
\end{tabular} & 0.52 \\
\hline $\mathrm{Pb}$ & 1.29 & 1.29 & 0.51 & 1.40 & 1.90 & 1.41 & 0.11 & 1.53 & NA & 2.48 & 2.48 & \begin{tabular}{l|l|} 
NA & 14.09 \\
\end{tabular} & 14.09 \\
\hline $\mathrm{Sb}$ & 0.27 & 0.27 & 0.18 & 0.45 & 0.63 & 1.12 & 0.23 & 1.35 & NA & 0.06 & 0.06 & \begin{tabular}{|l|l|}
$\mathrm{NA}$ & 0.48 \\
\end{tabular} & 0.48 \\
\hline $\mathrm{Se}$ & \begin{tabular}{l|l|} 
NA & 2538 \\
\end{tabular} & 2538 & 1.01 & 3432 & 3433 & 13.86 & 1338 & 1352 & NA & 134.0 & 134.0 & \begin{tabular}{l|l|} 
NA & 291.4 \\
\end{tabular} & 291.4 \\
\hline $\mathrm{Si}$ & \begin{tabular}{l|l|} 
NA & 10.09 \\
\end{tabular} & 10.09 & 0.44 & 12.39 & 12.83 & 4.93 & 2.91 & 7.83 & NA & 2.61 & \begin{tabular}{|l|}
2.61 \\
\end{tabular} & \begin{tabular}{ll|}
$\mathrm{NA}$ & 3.37 \\
\end{tabular} & \begin{tabular}{|l|}
3.37 \\
\end{tabular} \\
\hline $\mathrm{Sr}$ & \begin{tabular}{|l|l|}
$\mathrm{NA}$ & 4.94 \\
\end{tabular} & 4.94 & $<0.01$ & 4.29 & 4.29 & 0.01 & 0.85 & 0.86 & NA & 0.59 & 0.59 & 1.13 & 1.13 \\
\hline $\mathrm{Te}$ & \begin{tabular}{l|l|} 
NA & 102.7 \\
\end{tabular} & 102.7 & 13.19 & 117.9 & 131.1 & 22.38 & 46.96 & 69.34 & NA & 75.70 & 75.70 & \begin{tabular}{l|l|} 
NA & 280.1 \\
\end{tabular} & 280.1 \\
\hline $\mathrm{Ti}$ & \begin{tabular}{l|l|} 
NA & 0.04 \\
\end{tabular} & 0.04 & 0.01 & 0.05 & 0.06 & 0.04 & 0.01 & 0.05 & NA & 0.01 & 0.01 & \begin{tabular}{|l|l|} 
NA & 0.05 \\
\end{tabular} & \begin{tabular}{|l|}
0.05 \\
\end{tabular} \\
\hline $\mathrm{Zn}$ & \begin{tabular}{l|l|} 
NA & 11.34 \\
\end{tabular} & 11.34 & 0.03 & 14.44 & 14.46 & 0.14 & 2.89 & 3.03 & NA & 1.00 & 1.00 & 3.44 & 3.44 \\
\hline $\mathrm{Zr}$ & \begin{tabular}{l|l|} 
NA & 0.04 \\
\end{tabular} & 0.04 & 0.03 & 0.05 & 0.08 & 0.83 & 0.01 & 0.84 & NA & $<0.01$ & $<0.01$ & 0.05 & 0.05 \\
\hline $\mathrm{Cl}$ & \begin{tabular}{l|l|} 
NA & 160.4 \\
\end{tabular} & 160.4 & NA & 196.8 & 196.8 & $\mathrm{NA}$ & 35.53 & 35.53 & NA & \begin{tabular}{|l|}
43.05 \\
\end{tabular} & 43.05 & \begin{tabular}{|l|l|} 
NA & 47.33 \\
\end{tabular} & 47.33 \\
\hline $\mathrm{F}$ & NA 10.31 & 10.31 & $\mathrm{NA}$ & 10.72 & 10.72 & NA & 1.17 & 1.17 & NA & 2.14 & 2.14 & \begin{tabular}{l|l|}
$\mathrm{NA}$ & 6.18 \\
\end{tabular} & 6.18 \\
\hline$I$ & \begin{tabular}{l|l|}
$\mathrm{NA}$ & 0.70 \\
\end{tabular} & 0.70 & NA & 12.13 & 12.13 & NA & 16.34 & 16.34 & NA & 1.20 & 1.20 & \begin{tabular}{l|l|} 
NA & 2.68 \\
\end{tabular} & 2.68 \\
\hline Nitrate & NA 94.01 & 94.01 & $\mathrm{NA}$ & 149.3 & 149.3 & NA & 47.68 & 47.68 & NA & 1130 & 1130 & \begin{tabular}{l|l|} 
NA & 5633 \\
\end{tabular} & 5633 \\
\hline Nitrite & $<0.1$ & $<0.1$ & NA & $<0.1$ & $<0.1$ & $\mathrm{NA}$ & $<0.1$ & $<0.1$ & NA & $<0.1$ & $<0.1$ & \begin{tabular}{l|l}
$N A$ & $<0.1$ \\
\end{tabular} & $<0.1$ \\
\hline Sulfate & NA 2967 & 2967 & $\mathrm{NA}$ & 3817 & 3817 & $\mathrm{NA}$ & 401.7 & 401.7 & NA & 142.6 & 142.6 & \begin{tabular}{l|l|l} 
& NA & 179.0
\end{tabular} & 179.0 \\
\hline
\end{tabular}


Table 4.8. Analytical Results for Samples of WESP Blowdown Fluids (mg/l), (continued).

\begin{tabular}{|c|c|c|c|c|c|c|c|c|c|c|c|c|c|c|}
\hline Test & \multicolumn{14}{|c|}{$\# 5$} \\
\hline I.D. & \multicolumn{3}{|c|}{$12 \mathrm{~L}-\mathrm{W}-41 \mathrm{~A}$} & \multicolumn{3}{|c|}{$12 \mathrm{~L}-\mathrm{W}-50 \mathrm{~A}$} & \multicolumn{3}{|c|}{$12 \mathrm{~L}-\mathrm{W}-70 \mathrm{~A}$} & \multicolumn{3}{|c|}{$12 \mathrm{~L}-\mathrm{W}-80 \mathrm{~A}$} & \multicolumn{2}{|c|}{$12 \mathrm{~L}-\mathrm{W}-95 \mathrm{~A}$} \\
\hline lass (kg). & \multicolumn{3}{|c|}{15056} & \multicolumn{3}{|c|}{15230} & \multicolumn{3}{|c|}{15641} & \multicolumn{3}{|c|}{15704} & \multicolumn{2}{|c|}{15829} \\
\hline $\mathrm{pH}$ & \multicolumn{3}{|c|}{1.08} & \multicolumn{3}{|c|}{0.91} & \multicolumn{3}{|c|}{0.71} & \multicolumn{3}{|c|}{0.64} & \multicolumn{2}{|l|}{0.57} \\
\hline & Sus* & Dis.\# & Total & Sus* & Dis.\# & Total & Sus* & Dis.\# & Total & Sus* & Dis.\# & Total & Sus* ${ }^{*}$ Dis.\# & Tota \\
\hline Total & & 2232 & 2236 & 20 & 2880 & 2900 & 86 & 3664 & 3750 & 54 & 3964 & 4018 & \begin{tabular}{l|l}
36 & 4056 \\
\end{tabular} & 409 \\
\hline $\mathrm{Al}$ & NA & 6.40 & 6.40 & $\overline{N A}$ & 6.39 & \begin{tabular}{|l|}
6.39 \\
\end{tabular} & NA & 8.23 & 8.23 & $\mathrm{NA}$ & 9.92 & 9.92 & \begin{tabular}{l|l}
$\mathrm{NA}$ & 11.07
\end{tabular} & 11.07 \\
\hline As & NA & 5.28 & 5.28 & $\mathrm{NA}$ & 5.78 & 5.78 & $\overrightarrow{\mathrm{NA}}$ & 7.66 & 7.66 & NA & 8.16 & 8.16 & \begin{tabular}{l|l} 
NA & 7.50
\end{tabular} & 7.50 \\
\hline$B$ & NA & \begin{tabular}{|l|}
79.63 \\
\end{tabular} & \begin{tabular}{|l|}
79.63 \\
\end{tabular} & $\overline{\mathrm{NA}}$ & 104.4 & \begin{tabular}{|l|}
104.4 \\
\end{tabular} & NA & 164.8 & 164.8 & NA & 196.8 & 196.8 & \begin{tabular}{l|l} 
NA & 247.1
\end{tabular} & 247. \\
\hline $\mathrm{Ba}$ & NA & \begin{tabular}{|l|}
0.19 \\
\end{tabular} & \begin{tabular}{|l|}
0.19 \\
\end{tabular} & NA & 0.22 & 0.22 & NA & 0.29 & 0.29 & $\mathrm{NA}$ & 0.31 & 0.31 & \begin{tabular}{l|l} 
NA & 0.38
\end{tabular} & 0.38 \\
\hline $\mathrm{Ca}$ & NA & 60.95 & 60.95 & $\overline{\mathrm{NA}}$ & 64.57 & 64.57 & NA & 80.05 & 80.05 & $\mathrm{NA}$ & \begin{tabular}{|l|}
87.62 \\
\end{tabular} & 87.62 & \begin{tabular}{l|l} 
NA & 94.31
\end{tabular} & 94.31 \\
\hline $\mathrm{Cd}$ & NA & \begin{tabular}{|l|}
66.17 \\
\end{tabular} & 66.17 & NA & 74.22 & 74.22 & NA & 86.61 & 86.61 & NA & 87.52 & 87.52 & \begin{tabular}{l|l} 
NA & 89.95 \\
\end{tabular} & 89.95 \\
\hline $\mathrm{Cr}$ & $\mathrm{NA}$ & 36.23 & 36.23 & NA & 41.32 & 41.32 & NA & 53.09 & 53.09 & NA & 54.51 & 54.51 & \begin{tabular}{l|l} 
NA & 55.35
\end{tabular} & 55.35 \\
\hline Cs & NA & 14.13 & \begin{tabular}{|l|}
14.13 \\
\end{tabular} & $\overline{\mathrm{NA}}$ & 15.96 & 15.96 & NA & 20.25 & 20.25 & $\mathrm{NA}$ & 21.95 & 21.95 & \begin{tabular}{l|l|} 
NA & 25.71
\end{tabular} & 25.7 \\
\hline $\mathrm{Cu}$ & NA & 7.22 & \begin{tabular}{|l|}
7.22 \\
\end{tabular} & NA & 9.26 & 9.26 & NA & 13.24 & 13.24 & $\mathrm{NA}$ & 14.61 & 14.61 & \begin{tabular}{l|l}
$\mathrm{NA}$ & 17.46
\end{tabular} & 17.4 \\
\hline $\mathrm{Fe}$ & NA & 23.02 & 23.02 & NA & 34.51 & 34.51 & NA & 67.54 & 67.54 & NA & 84.40 & 84.40 & \begin{tabular}{l|l} 
NA & 101.6
\end{tabular} & 101.6 \\
\hline$\overline{\mathrm{K}}$ & NA & 10.52 & 10.52 & NA & 11.61 & 11.61 & NA & 14.95 & 14.95 & NA & 17.19 & 17.19 & NA 21.33 & 21.33 \\
\hline$\overline{\mathrm{Li}}$ & NA & 19.02 & 19.02 & $\overline{\mathrm{NA}}$ & 22.60 & 22.60 & NA & 27.17 & 27.17 & NA & 32.78 & 32.78 & \begin{tabular}{l|l} 
NA & 44.47
\end{tabular} & 44.47 \\
\hline$\overline{\mathrm{Mg}}$ & NA & 19.40 & 19.40 & $\overline{\mathrm{NA}}$ & 20.06 & 20.06 & $\mathrm{NA}$ & 24.10 & 24.10 & $\mathrm{NA}$ & 26.77 & 26.77 & \begin{tabular}{l|l} 
NA & 29.26 \\
\end{tabular} & 29.26 \\
\hline $\mathrm{Mn}$ & NA & 1.49 & 1.49 & $\overline{\mathrm{NA}}$ & 1.97 & 1.97 & NA & 2.98 & 2.98 & NA & 4.17 & 4.17 & \begin{tabular}{l|l}
$\mathrm{NA}$ & 5.01
\end{tabular} & 5.0 \\
\hline $\mathrm{Na}$ & NA & 89.02 & \begin{tabular}{|l|}
89.02 \\
\end{tabular} & NA & 99.24 & 99.24 & NA & 118.9 & 118.9 & NA & 134.1 & 134.1 & NA 189.6 & 189.6 \\
\hline $\mathrm{Ni}$ & NA & \begin{tabular}{|l|}
2.13 \\
\end{tabular} & \begin{tabular}{|l|}
2.13 \\
\end{tabular} & $\overline{\mathrm{NA}}$ & 2.73 & 2.73 & NA & 3.84 & 3.84 & NA & 4.48 & \begin{tabular}{|l|}
4.48 \\
\end{tabular} & \begin{tabular}{l|l} 
NA & 5.09
\end{tabular} & 5.09 \\
\hline $\mathrm{P}$ & $\mathrm{NA}$ & 0.57 & 0.57 & NA & 0.81 & 0.81 & NA & 1.00 & 1.00 & $\mathrm{NA}$ & 1.15 & 1.15 & \begin{tabular}{l|l} 
NA & 1.30
\end{tabular} & 1.30 \\
\hline$\overline{\mathrm{Pb}}$ & $\mathrm{NA}$ & 22.52 & 22.52 & $\overline{\mathrm{NA}}$ & 28.26 & 28.26 & NA & 37.63 & \begin{tabular}{|l|}
37.63 \\
\end{tabular} & $\mathrm{NA}$ & 39.19 & 39.19 & \begin{tabular}{l|l} 
NA & 42.15
\end{tabular} & 42.15 \\
\hline $\mathrm{Sb}$ & NA & \begin{tabular}{|l|}
1.01 \\
\end{tabular} & 1.01 & NA & 1.59 & 1.59 & NA & 2.56 & 2.56 & $\mathrm{NA}$ & 3.87 & 3.87 & \begin{tabular}{l|l} 
NA & 5.18 \\
\end{tabular} & 5.18 \\
\hline $\mathrm{Se}$ & $\mathrm{NA}$ & 384.4 & 384.4 & $\overline{\mathrm{NA}}$ & 410.5 & 410.5 & NA & 403.3 & 403.3 & $\mathrm{NA}$ & 528.0 & 528.0 & \begin{tabular}{l|l} 
NA & 625.7
\end{tabular} & 625.7 \\
\hline $\mathrm{Si}$ & NA & \begin{tabular}{|l|}
4.13 \\
\end{tabular} & \begin{tabular}{|l|}
4.13 \\
\end{tabular} & $\overline{\mathrm{NA}}$ & 4.42 & 4.42 & NA & 5.47 & 5.47 & NA & 6.09 & 6.09 & \begin{tabular}{l|l}
$\mathrm{NA}$ & 6.54
\end{tabular} & 6.54 \\
\hline $\mathrm{Sr}$ & NA & 1.70 & 1.70 & $\overline{\mathrm{NA}}$ & 2.22 & 2.22 & NA & 3.30 & 3.30 & $\mathrm{NA}$ & 4.81 & 4.81 & \begin{tabular}{|l|l|} 
NA & 4.81 \\
\end{tabular} & 4.81 \\
\hline$\overline{\mathrm{Te}}$ & NA & 571.6 & 571.6 & $\overline{\mathrm{NA}}$ & 670.5 & 670.5 & NA & 713.1 & 713.1 & NA & 726.6 & 726.6 & \begin{tabular}{l|l} 
NA & 776.4 \\
\end{tabular} & 776.4 \\
\hline $\mathrm{Ti}$ & NA & 0.07 & 0.07 & NA & 0.09 & 0.09 & $\mathrm{NA}$ & 0.14 & 0.14 & $\mathrm{NA}$ & 0.16 & 0.16 & \begin{tabular}{|l|l|}
$\mathrm{NA}$ & 0.18 \\
\end{tabular} & 0.18 \\
\hline $\mathrm{Zn}$ & NA & 5.75 & 5.75 & NA & 7.03 & 7.03 & $\mathrm{NA}$ & 8.80 & 8.80 & NA & 9.80 & 9.80 & \begin{tabular}{l|l}
$\mathrm{NA}$ & 11.12 \\
\end{tabular} & $\overline{11.12}$ \\
\hline $\mathrm{Zr}$ & NA & 0.11 & 0.11 & NA & 0.19 & 0.19 & NA & 0.41 & 0.41 & NA & 0.69 & 0.69 & \begin{tabular}{|l|l|} 
NA & 0.98 \\
\end{tabular} & 0.98 \\
\hline $\mathrm{Cl}$ & NA & 52.99 & 52.99 & NA & 58.51 & 58.51 & NA & 65.55 & 65.55 & NA & 69.75 & 69.75 & \begin{tabular}{|l|l|} 
NA & 69.21 \\
\end{tabular} & $\longdiv { 6 9 . 2 1 }$ \\
\hline$F$ & NA & 9.60 & \begin{tabular}{|l|}
9.60 \\
\end{tabular} & $\mathrm{NA}$ & $<0.1$ & $<0.1$ & NA & $<0.1$ & $<0.1$ & $\mathrm{NA}$ & $<0.1$ & $<0.1$ & \begin{tabular}{l|l|}
$\mathrm{NA}$ & $<0.1$ \\
\end{tabular} & $<0.1$ \\
\hline I & NA & 3.61 & 3.61 & NA & 4.60 & 4.60 & NA & 6.13 & 6.13 & NA & 6.79 & 6.79 & \begin{tabular}{l|l|}
$\mathrm{NA}$ & 4.70
\end{tabular} & 4.70 \\
\hline Nitrate & NA & 10027 & 10027 & $\mathrm{NA}$ & 13353 & 13353 & NA2 2 & 21305 & 21305 & $\overrightarrow{\mathrm{NA}}$ & 27111 & 27111 & \begin{tabular}{|l|l|}
$\mathrm{NA}$ & 33113 \\
\end{tabular} & 33113 \\
\hline Nitrite & NA & $<0.1$ & $<0.1$ & NA & 3.75 & 3.75 & $\mathrm{NA}$ & 4.96 & 4.96 & $\mathrm{NA}$ & 7.87 & $\begin{array}{l}7.87 \\
\end{array}$ & \begin{tabular}{l|l|} 
NA & 19.88 \\
\end{tabular} & 19.88 \\
\hline Sulfate & NA & 206.5 & 206.5 & $\mathrm{NA}$ & 234.0 & 234.0 & NA & 268.3 & 268.3 & $\overline{\mathrm{NA}}$ & 292.7 & 292.7 & \begin{tabular}{|l|l|} 
NA & 295.2 \\
\end{tabular} & 295.2 \\
\hline
\end{tabular}

Sus* - Suspended

Dis\# - Dissolved

NA - Not Analyzed 
Table 4.8. Analytical Results for Samples of WESP Blowdown Fluids (mg/l), (continued).

\begin{tabular}{|c|c|c|c|c|c|c|c|c|c|c|c|c|c|c|c|}
\hline Test & \multicolumn{6}{|c|}{$\# 5$} & \multicolumn{9}{|c|}{$\# 6$} \\
\hline I.D. & \multicolumn{3}{|c|}{$12 \mathrm{~L}-\mathrm{W}-107 \mathrm{~A}$} & \multicolumn{3}{|c|}{$12 \mathrm{~L}-\mathrm{W}-124 \mathrm{~A}$} & \multicolumn{3}{|c|}{$12 \mathrm{M}-\mathrm{W}-10 \mathrm{~A}$} & \multicolumn{3}{|c|}{$12 \mathrm{M}-\mathrm{W}-24 \mathrm{~A}$} & \multicolumn{3}{|c|}{$12 \mathrm{M}-\mathrm{W}-40 \mathrm{~A}$} \\
\hline ilass $(\mathrm{kg})$. & \multicolumn{3}{|c|}{15892} & \multicolumn{3}{|c|}{16200} & \multicolumn{3}{|c|}{16621} & \multicolumn{3}{|c|}{16809} & \multicolumn{3}{|c|}{17118} \\
\hline $\mathrm{pH}$ & \multicolumn{3}{|c|}{0.50} & \multicolumn{3}{|c|}{0.42} & \multicolumn{3}{|c|}{2.15} & \multicolumn{3}{|c|}{2.20} & \multicolumn{3}{|c|}{2.45} \\
\hline & Sus* & Dis.\# & Total & Sus* & Dis.\# & Total & Sus* & Dis.\# & Total & Sus* & Dis.\# & Total & Sus* & Dis.\# & \\
\hline Total & 140 & 4448 & 4588 & 50 & 5860 & 5910 & 6 & 752 & 758 & 5 & 888 & 893 & & 824 & \\
\hline$\overline{\mathrm{Al}}$ & $\mathrm{NA}$ & 10.73 & 10.73 & $\mathrm{NA}$ & 13.64 & 13.64 & $\overrightarrow{\mathrm{NA}}$ & 2.38 & 2.38 & NA & 2.47 & 2.47 & $\mathrm{NA}$ & 2.20 & 7 \\
\hline As & $\mathrm{NA}$ & 8.22 & 8.22 & NA & 9.15 & 9.15 & $\mathrm{NA}$ & 3.70 & 3.70 & $\mathrm{NA}$ & 5.20 & 5.20 & NA & 6.35 & 62 \\
\hline $\mathrm{B}$ & NA & 297.0 & 297.0 & $\mathrm{NA}$ & 402.8 & 402.8 & $\mathrm{NA}$ & 36.96 & 36.96 & $\mathrm{NA}$ & 43.04 & 43.04 & $\mathrm{NA}$ & 49.37 & 49.3 \\
\hline $\mathrm{Ba}$ & NA & 0.43 & \begin{tabular}{|l|}
0.43 \\
\end{tabular} & $\mathrm{NA}$ & 0.56 & 0.56 & NA & 0.09 & 0.09 & NA & 0.10 & 0.10 & $\mathrm{NA}$ & 0.10 & 0.10 \\
\hline$\overline{\mathrm{Ca}}$ & $\mathrm{NA}$ & 100.2 & 100.2 & $\mathrm{NA}$ & 119.8 & 119.8 & $\mathrm{NA}$ & 59.54 & 59.54 & NA & 64.77 & 64.77 & $\mathrm{NA}$ & 69.80 & 69.8 \\
\hline $\mathrm{Cd}$ & NA & 99.69 & 99.69 & NA & 112.6 & 112.6 & $\mathrm{NA}$ & 11.05 & 11.05 & NA & 14.44 & 14.44 & NA & 16.80 & 16.8 \\
\hline $\mathrm{Cr}$ & NA & 54.07 & 54.07 & NA & 61.73 & 61.73 & $\overline{\mathrm{NA}}$ & 2.52 & 2.52 & $\mathrm{NA}$ & 2.43 & 2.43 & $\mathrm{NA}$ & 2.05 & \\
\hline $\mathrm{Cs}$ & NA & 26.86 & 26.86 & $\mathrm{NA}$ & 34.19 & 34.19 & $\overline{\mathrm{NA}}$ & 4.80 & 4.80 & NA & 6.90 & 6.90 & $\mathrm{NA}$ & 7.90 & \\
\hline $\mathrm{Cu}$ & NA & 19.59 & 19.59 & $\mathrm{NA}$ & 23.00 & 23.00 & NA & 14.27 & 14.27 & $\mathrm{NA}$ & 14.19 & 14.19 & $\mathrm{NA}$ & 12.68 & \\
\hline $\mathrm{Fe}$ & NA & 110.1 & 110.1 & $\mathrm{NA}$ & 149.7 & 149.7 & $\overline{\mathrm{NA}}$ & 8.54 & 8.54 & NA & $\begin{array}{l}7.97 \\
\end{array}$ & 7.97 & $\mathrm{NA}$ & 6.40 & \\
\hline $\mathrm{K}$ & NA & 22.37 & 22.31 & $\mathrm{NA}$ & 25.94 & 25.94 & $\mathrm{NA}$ & 6.54 & 6.54 & NA & 7.51 & 7.51 & $\mathrm{NA}$ & 8.66 & \\
\hline$\overline{\mathrm{Li}}$ & NA & 50.38 & 50.38 & $\mathrm{NA}$ & 71.95 & \begin{tabular}{|l|}
71.95 \\
\end{tabular} & $\overline{\mathrm{NA}}$ & 7.48 & 7.48 & NA & 9.46 & 9.46 & NA & 11.80 & \\
\hline$\overline{\mathrm{Mg}}$ & NA & 28.20 & 28.20 & $\mathrm{NA}$ & 31.43 & 31.43 & $\mathrm{NA}$ & 13.15 & 13.15 & NA & 14.40 & 14.40 & $\mathrm{NA}$ & 16.06 & \\
\hline $\mathrm{Mn}$ & $\mathrm{NA}$ & 4.12 & 4.12 & $\mathrm{NA}$ & 5.39 & 5.39 & $\overline{\mathrm{NA}}$ & 0.82 & 0.82 & $\mathrm{NA}$ & 0.80 & 0.80 & $\mathrm{NA}$ & 0.74 & \\
\hline $\mathrm{Na}$ & NA & \begin{tabular}{|l|}
238.2 \\
\end{tabular} & \begin{tabular}{|l|}
238.2 \\
\end{tabular} & $\mathrm{NA}$ & 334.0 & 334.0 & $\overline{\mathrm{NA}}$ & 45.90 & \begin{tabular}{|l|}
45.90 \\
\end{tabular} & NA & \begin{tabular}{|l|}
54.72 \\
\end{tabular} & 54.72 & $\mathrm{NA}$ & 61.86 & \\
\hline $\mathrm{Ni}$ & NA & 4.59 & 4.59 & $\mathrm{NA}$ & 5.77 & 5.77 & $\mathrm{NA}$ & 0.85 & \begin{tabular}{|l|}
0.85 \\
\end{tabular} & $\mathrm{NA}$ & 0.87 & 0.87 & $\mathrm{NA}$ & 0.80 & \\
\hline $\mathrm{P}$ & $\mathrm{NA}$ & 1.55 & 1.55 & $\mathrm{NA}$ & 1.94 & 1.94 & $\mathrm{NA}$ & 0.17 & 0.17 & NA & 0.15 & 0.1 & NA & 0.37 & \\
\hline $\mathrm{Pb}$ & NA & 41.79 & 41.79 & $\mathrm{NA}$ & 46.34 & 46.34 & $\mathrm{NA}$ & 1.37 & 1.37 & NA & 1.36 & 1.36 & NA & 1.07 & \\
\hline $\mathrm{Sb}$ & NA & 5.51 & 5.51 & $\mathrm{NA}$ & 8.03 & \begin{tabular}{|l|}
8.03 \\
\end{tabular} & $\overline{\mathrm{NA}}$ & 0.78 & 0.78 & NA & 0.89 & 0.89 & NA & 0.85 & \\
\hline $\mathrm{Se}$ & NA & 676.1 & 676.1 & $\mathrm{NA}$ & 792.5 & 792.5 & $\overline{\mathrm{NA}}$ & 167.4 & 167.4 & NA & 241.8 & 241.8 & NA & 278.6 & 278 \\
\hline$\overline{\mathrm{Si}}$ & NA & 6.22 & 6.22 & $\mathrm{NA}$ & 7.81 & 7.81 & $\mathrm{NA}$ & 2.76 & 2.76 & NA & 3.06 & 3.06 & NA & 3.37 & \\
\hline $\mathrm{Sr}$ & NA & 5.33 & 5.33 & NA & 7.89 & 7.89 & $\mathrm{NA}$ & 0.97 & 0.97 & NA & 1.03 & 1.03 & NA & 1.03 & \\
\hline $\mathrm{Te}$ & NA & 839.9 & 839.9 & NA & 952.6 & 952.6 & $\mathrm{NA}$ & 17.46 & 17.46 & $\mathrm{NA}$ & 18.70 & 18.70 & NA & 14.17 & 14.1 \\
\hline$\overline{\mathrm{Ti}}$ & NA & 0.18 & 0.18 & NA & 0.22 & 0.22 & $\mathrm{NA}$ & 0.01 & 0.01 & NA & 0.01 & 0.01 & NA & 0.01 & 0.0 \\
\hline $\mathrm{Zn}$ & NA & 12.15 & 12.15 & NA & 15.34 & 15.34 & $\mathrm{NA}$ & 3.48 & 3.48 & NA & 3.93 & 3.93 & $\mathrm{NA}$ & 3.94 & 3.5 \\
\hline $\mathrm{Zr}$ & $\mathrm{NA}$ & 1.06 & 1.06 & NA & 1.78 & 1.78 & $\mathrm{NA}$ & 0.08 & 0.08 & NA & 0.07 & 0.07 & $\mathrm{NA}$ & 0.07 & $0 . \mathrm{C}$ \\
\hline$\overline{\mathrm{Cl}}$ & $\mathrm{NA}$ & 73.07 & 73.07 & $\mathrm{NA}$ & 78.42 & 78.42 & $\mathrm{NA}$ & 72.56 & 72.56 & $\mathrm{NA}$ & 94.46 & 94.46 & NA & 115.1 & 115 . \\
\hline$F$ & $\mathrm{NA}$ & $<0.1$ & $<0.1$ & $\mathrm{NA}$ & $<0.1$ & $<0.1$ & $\mathrm{NA}$ & 4.49 & 4.49 & NA & 6.45 & 6.45 & NA & 11.02 & 11.0 \\
\hline I & NA & 4.15 & 4.15 & $\mathrm{NA}$ & 4.63 & 4.63 & $\mathrm{NA}$ & 1.41 & 1.41 & $\mathrm{NA}$ & 1.73 & 1.73 & $\mathrm{NA}$ & 1.41 & 1.4 \\
\hline Nitrate & NA & 42164 & 42164 & $\mathrm{NA}$ & 39004 & 39004 & $\mathrm{NA}$ & 1103 & 1103 & NA & 1097 & 1097 & NA & 1011 & 101 \\
\hline Nitrite & NA & 28.10 & 28.10 & $\mathrm{NA}$ & 30.48 & 30.48 & $\mathrm{NA}$ & $<0.1$ & $<0.1$ & $\mathrm{NA}$ & $<0.1$ & $<0.1$ & $\mathrm{NA}$ & $<0.1$ & $<0$. \\
\hline Sulfate & NA & 312.9 & 312.9 & $\mathrm{NA}$ & 397.8 & 397.8 & $\mathrm{NA}$ & 97.91 & \begin{tabular}{|c|}
97.91 \\
\end{tabular} & NA & 112.2 & 112.2 & NA & 122.3 & 122 \\
\hline
\end{tabular}

Sus* - Suspended

Dis\# - Dissolved

NA - Not Analyzed 
Table 4.8. Analytical Results for Samples of WESP Blowdown Fluids (mg/l), (continued).

\begin{tabular}{|c|c|c|c|c|c|c|c|c|c|c|}
\hline Test & \multicolumn{10}{|c|}{ \#6 } \\
\hline I.D. & \multicolumn{3}{|c|}{$12 \mathrm{M}-\mathrm{W}-61 \mathrm{~A}$} & \multicolumn{2}{|c|}{$12 \mathrm{M}-\mathrm{W}-72 \mathrm{~A}$} & \multicolumn{2}{|c|}{$12 \mathrm{M}-\mathrm{W}-82 \mathrm{~A}$} & \multicolumn{2}{|c|}{$12 \mathrm{M}-\mathrm{W}-91 \mathrm{~A}$} & $12 \mathrm{M}-\mathrm{W}-109 \mathrm{~A}$ \\
\hline Glass $(\mathrm{kg})$ & \multicolumn{3}{|c|}{17421} & \multicolumn{2}{|c|}{17620} & \multicolumn{2}{|c|}{17720} & \multicolumn{2}{|c|}{17820} & 18020 \\
\hline $\mathrm{pH}$ & \multicolumn{3}{|c|}{ NA } & \multicolumn{2}{|l|}{2.65} & \multicolumn{2}{|l|}{2.68} & \multicolumn{2}{|l|}{2.69} & 2.73 \\
\hline & Sus* & Dis.\# & Total & Sus* Dis.\# & Total & Sus*|Dis.\# & Total & Sus*Dis.\# & Total & Sus* Dis.\#Total \\
\hline Total & 0 & 1168 & 1168 & $1396 \quad 6$ & 1402 & \begin{tabular}{|l|l|}
1504 & 10 \\
\end{tabular} & 1514 & \begin{tabular}{|l|l|}
1708 & 10 \\
\end{tabular} & 1718 & \begin{tabular}{l|l|l|}
12 & 2280 & 2292 \\
\end{tabular} \\
\hline $\mathrm{Al}$ & NA & 2.37 & 2.37 & 2.27 & $\mathrm{NC}$ & \begin{tabular}{|l|l|} 
NA & 2.34 \\
\end{tabular} & $\mathrm{NC}$ & \begin{tabular}{l|l|} 
NA & 2.64 \\
\end{tabular} & $\mathrm{NC}$ & \begin{tabular}{|l|l|l|} 
NA & 2.82 & 2.82 \\
\end{tabular} \\
\hline As & NA & 8.71 & 8.71 & \begin{tabular}{l|l|} 
NA & 9.80 \\
\end{tabular} & $\mathrm{NC}$ & NA 11.03 & $\mathrm{NC}$ & NA|14.00 & $\mathrm{NC}$ & 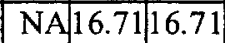 \\
\hline $\mathrm{B}$ & NA & 66.74 & 66.74 & NA 71.42 & $\overrightarrow{\mathrm{NQ}}$ & \begin{tabular}{|l|l|} 
NA 81.11 \\
\end{tabular} & $\mathrm{NC}$ & NA94.99 & $\mathrm{NC}$ & NA 117.0117 .0 \\
\hline $\mathrm{Ba}$ & NA & 0.12 & 0.12 & \begin{tabular}{|l|l|}
$\mathrm{NA}$ & 0.12 \\
\end{tabular} & $\mathrm{NC}$ & \begin{tabular}{|l|l|} 
NA & 0.12 \\
\end{tabular} & $\mathrm{NC}$ & \begin{tabular}{|l|l|}
$\mathrm{NA}$ & 0.14 \\
\end{tabular} & $\mathrm{NC}$ & \begin{tabular}{|l|l|l|l|} 
NA & 0.16 & 0.16 \\
\end{tabular} \\
\hline$\overline{\mathrm{Ca}}$ & NA & 82.16 & 82.16 & \begin{tabular}{|l|l|} 
NA & 84.43 \\
\end{tabular} & $\mathrm{NG}$ & NAp0.38 & $\mathrm{NC}$ & NA|101.5 & $\overline{\mathrm{NC}}$ & \begin{tabular}{|l|l|l|} 
NA 124.8 & 124.8 \\
\end{tabular} \\
\hline $\mathrm{Cd}$ & NA & 24.11 & 24.11 & \begin{tabular}{l|l|} 
NA & 25.95 \\
\end{tabular} & NC & NA29.31 & $\overline{\mathrm{NC}}$ & NA35.79 & $\mathrm{NC}$ & NA 44.3444 .34 \\
\hline $\mathrm{Cr}$ & $\mathrm{NA}$ & 2.02 & 2.02 & \begin{tabular}{l|l|} 
NA & 1.73 \\
\end{tabular} & $\mathrm{NC}$ & \begin{tabular}{|l|l|}
$\mathrm{NA}$ & 1.74 \\
\end{tabular} & $\mathrm{NC}$ & \begin{tabular}{l|l|}
$\mathrm{NA}$ & 1.87 \\
\end{tabular} & $\mathrm{NQ}$ & \begin{tabular}{|l|l|l|}
$\mathrm{NA}$ & 1.89 & 1.89 \\
\end{tabular} \\
\hline $\mathrm{Cs}$ & $\mathrm{NA}$ & 12.30 & 12.30 & \begin{tabular}{l|l|} 
NA & 12.70 \\
\end{tabular} & $\mathrm{NQ}$ & NA 14.90 & $\mathrm{NC}$ & NA $\mid 16.70$ & $\mathrm{NC}$ & \begin{tabular}{|l|l|l|} 
NA 19.6019 .60 \\
\end{tabular} \\
\hline$\overline{\mathrm{Cu}}$ & NA & 14.40 & 14.40 & \begin{tabular}{l|l|} 
NA & 12.58 \\
\end{tabular} & $\mathrm{NC}$ & NA 14.78 & $\mathrm{NC}$ & NA 15.96 & $\mathrm{NC}$ & NA 16.5616 .56 \\
\hline $\mathrm{Fe}$ & $\mathrm{NA}$ & 3.63 & 3.63 & \begin{tabular}{l|l|}
$\mathrm{NA}$ & 1.91 \\
\end{tabular} & $\mathrm{NC}$ & \begin{tabular}{l|l|} 
NA & 2.03 \\
\end{tabular} & $\mathrm{NC}$ & \begin{tabular}{l|l|}
$\mathrm{NA}$ & 1.92 \\
\end{tabular} & $\mathrm{NG}$ & \begin{tabular}{|l|l|l|l|}
$\mathrm{NA}$ & 1.57 & 1.57 \\
\end{tabular} \\
\hline $\mathrm{K}$ & NA & 11.03 & 11.03 & NA 11.40 & $\mathrm{NC}$ & NA 12.20 & $\mathrm{NC}$ & NA 14.03 & $\mathrm{NC}$ & \begin{tabular}{|l|l|} 
NA 16.57 & 16.57 \\
\end{tabular} \\
\hline $\mathrm{Li}$ & $\mathrm{NA}$ & 17.03 & 17.03 & \begin{tabular}{l|l|} 
NA & 17.50 \\
\end{tabular} & $\mathrm{NC}$ & NA 19.50 & $\mathrm{NC}$ & NA 23.24 & $\mathrm{NC}$ & NA27.8627.86 \\
\hline $\mathrm{Mg}$ & $\mathrm{NA}$ & 19.25 & 19.25 & NA 19.91 & $\mathrm{Nd}$ & NA21.40 & $\mathrm{NC}$ & NA24.19 & $\mathrm{NQ}$ & NA27.7227.72 \\
\hline Mn & $\mathrm{NA}$ & 0.83 & 0.83 & \begin{tabular}{l|l} 
NA & 0.78 \\
\end{tabular} & $\mathrm{NC}$ & \begin{tabular}{|l|l|} 
NA & 0.78 \\
\end{tabular} & $\mathrm{NC}$ & \begin{tabular}{l|l|} 
NA & 0.90 \\
\end{tabular} & $\mathrm{NC}$ & \begin{tabular}{|l|l|l|} 
NA & 0.96 & 0.96 \\
\end{tabular} \\
\hline$\overline{\mathrm{Na}}$ & NA & 82.69 & 82.69 & \begin{tabular}{|l|l|} 
NA & 85.41 \\
\end{tabular} & NC & NA94.57 & $\overline{\mathrm{NC}}$ & NA 112.8 & $\mathrm{NC}$ & \begin{tabular}{|l|l|l|} 
NA 134.4134 .4 \\
\end{tabular} \\
\hline$\overline{\mathrm{Ni}}$ & NA & 0.93 & 0.93 & \begin{tabular}{l|l|} 
NA & 0.85 \\
\end{tabular} & $\mathrm{NQ}$ & \begin{tabular}{l|l|} 
NA & 0.92 \\
\end{tabular} & $\mathrm{NC}$ & \begin{tabular}{l|l|} 
NA & 1.04
\end{tabular} & $\mathrm{NC}$ & \begin{tabular}{|l|l|l} 
NA & 1.10 & 1.10 \\
\end{tabular} \\
\hline $\bar{P}$ & NA & 0.39 & 0.39 & \begin{tabular}{l|l|}
$\mathrm{NA}$ & 0.22 \\
\end{tabular} & $\mathrm{NC}$ & \begin{tabular}{l|l|}
$\mathrm{NA}$ & 0.21 \\
\end{tabular} & $\widehat{\mathrm{NC}}$ & \begin{tabular}{|l|l|} 
NA & 0.43 \\
\end{tabular} & $\mathrm{NC}$ & \begin{tabular}{|l|l|l|} 
NA & 0.55 & 0.55 \\
\end{tabular} \\
\hline$\overline{\mathrm{Pb}}$ & NA & 0.23 & 0.23 & \begin{tabular}{l|l|} 
NA & 0.19 \\
\end{tabular} & $\mathrm{NO}$ & \begin{tabular}{|l|l|}
$\mathrm{NA}$ & 0.22 \\
\end{tabular} & $\overline{\mathrm{NC}}$ & \begin{tabular}{l|l|} 
NA & 0.27
\end{tabular} & $\mathrm{NC}$ & \begin{tabular}{|l|l|l|} 
NA & 0.33 & 0.33 \\
\end{tabular} \\
\hline $\mathrm{Sb}$ & NA & 0.99 & 0.99 & \begin{tabular}{l|l|} 
NA & 0.94 \\
\end{tabular} & Nd & \begin{tabular}{|l|l|} 
NA & 1.07 \\
\end{tabular} & $\mathrm{NC}$ & \begin{tabular}{l|l|} 
NA & 1.10 \\
\end{tabular} & $\mathrm{NQ}$ & \begin{tabular}{|l|l|l|} 
NA & 1.22 & 1.22 \\
\end{tabular} \\
\hline $\mathrm{Se}$ & NA & 488.9 & 488.9 & NA 543.1 & $\mathrm{NC}$ & NA 848.1 & $\mathrm{NC}$ & NA 593.6 & $\mathrm{NQ}$ & \begin{tabular}{|l|l|l|} 
NA & 812.7 & 812.7 \\
\end{tabular} \\
\hline$\overline{\mathrm{Si}}$ & NA & 4.29 & 4.29 & \begin{tabular}{l|l} 
NA & 4.50 \\
\end{tabular} & $\mathrm{NC}$ & \begin{tabular}{l|l|} 
NA & 5.16 \\
\end{tabular} & $\mathrm{NC}$ & \begin{tabular}{l|l|}
$\mathrm{NA}$ & 6.06
\end{tabular} & $\mathrm{NC}$ & \begin{tabular}{|l|l|l|} 
NA & 7.19 & 7.19 \\
\end{tabular} \\
\hline$\overline{\mathrm{Sr}}$ & NA & 1.23 & 1.23 & \begin{tabular}{|l|l|}
$\mathrm{NA}$ & 1.21 \\
\end{tabular} & $\mathrm{NC}$ & \begin{tabular}{|l|l|}
$\mathrm{NA}$ & 1.28 \\
\end{tabular} & $\mathrm{NC}$ & \begin{tabular}{l|l|} 
NA & 1.47 \\
\end{tabular} & $\mathrm{NC}$ & \begin{tabular}{|l|l|l|} 
NA & 1.62 & 1.62 \\
\end{tabular} \\
\hline $\mathrm{Te}$ & $\mathrm{NA}$ & 11.28 & 11.28 & \begin{tabular}{l|l|} 
NA & 24.53 \\
\end{tabular} & $\mathrm{NQ}$ & NA25.73 & $\mathrm{NC}$ & NA29.87 & $\mathrm{NC}$ & NA29.4629.46 \\
\hline$\overline{\mathrm{Ti}}$ & NA & 0.01 & 0.01 & \begin{tabular}{|l|l|} 
NA & 0.01 \\
\end{tabular} & $\mathrm{NC}$ & \begin{tabular}{|l|l|} 
NA & 0.02 \\
\end{tabular} & $\mathrm{NC}$ & \begin{tabular}{l|l|} 
NA & 0.02 \\
\end{tabular} & $\mathrm{NC}$ & \begin{tabular}{|l|l|l|} 
NA & 0.03 & 0.03 \\
\end{tabular} \\
\hline$\overline{Z n}$ & NA & 4.76 & 4.76 & 4.46 & NQ & \begin{tabular}{l|l|} 
NA & 4.79 \\
\end{tabular} & $\mathrm{NC}$ & \begin{tabular}{l|l|} 
NA & 5.45 \\
\end{tabular} & $\mathrm{NC}$ & \begin{tabular}{|l|l|l|} 
NA & 5.81 & 5.81 \\
\end{tabular} \\
\hline$\overline{\mathrm{Zr}}$ & $\mathrm{NA}$ & 0.08 & 0.08 & \begin{tabular}{l|l|} 
NA & 0.08 \\
\end{tabular} & NQ & \begin{tabular}{|l|l|}
$\mathrm{NA}$ & 0.08 \\
\end{tabular} & $\mathrm{NC}$ & \begin{tabular}{l|l|} 
NA & 0.10
\end{tabular} & $\overrightarrow{\mathrm{NC}}$ & \begin{tabular}{|l|l|l|} 
NA & 0.10 & 0.10 \\
\end{tabular} \\
\hline $\mathrm{Cl}$ & NA & 157.1 & 157.1 & \begin{tabular}{|l|l|} 
NA & 170.2 \\
\end{tabular} & $\mathrm{Nd}$ & NA186.9 & $\mathrm{NC}$ & NA228.3 & $\mathrm{NC}$ & \begin{tabular}{|l|l|} 
NA 250.4250 .4 \\
\end{tabular} \\
\hline$F$ & NA & 17.59 & 17.59 & NA 21.45 & NC & NA23.19 & $\mathrm{NC}$ & NA25.91 & $\mathrm{NC}$ & NA33.7833.78 \\
\hline I & $\mathrm{NA}$ & 2.51 & 2.51 & \begin{tabular}{|l|l|} 
NA & 1.83 \\
\end{tabular} & NC & \begin{tabular}{|l|l|} 
NA & 2.44 \\
\end{tabular} & $\mathrm{NC}$ & \begin{tabular}{|l|l|}
$\mathrm{NA}$ & 3.64 \\
\end{tabular} & $\mathrm{NC}$ & \begin{tabular}{|l|l|l|}
$\mathrm{NA}$ & 4.31 & 4.31 \\
\end{tabular} \\
\hline Nitrate & NA & 1131 & 1131 & \begin{tabular}{l|l|} 
NA & 999.5 \\
\end{tabular} & $\mathrm{NC}$ & \begin{tabular}{|l|l|} 
NA & 1045 \\
\end{tabular} & $\overrightarrow{\mathrm{NC}}$ & \begin{tabular}{l|l|} 
NA & 1168 \\
\end{tabular} & $\mathrm{NC}$ & \begin{tabular}{|l|l|l|} 
NA & 1201 & 1201 \\
\end{tabular} \\
\hline Nitrite & NA & $<0.1$ & $<0.1$ & \begin{tabular}{l|l|}
$\mathrm{NA}$ & $<0.1$ \\
\end{tabular} & NC & \begin{tabular}{|l|l|}
$\mathrm{NA}$ & $<0.1$ \\
\end{tabular} & Nd & \begin{tabular}{l|l|} 
NA & $<0.1$ \\
\end{tabular} & $\mathrm{NC}$ & \begin{tabular}{|l|l|l|}
$\mathrm{NA}$ & $<0.1$ & $<0.1$ \\
\end{tabular} \\
\hline Sulfate & NA. & 143.04 & 143.04 & \begin{tabular}{l|l|} 
NA & 170.5 \\
\end{tabular} & $\mathrm{NC}$ & NA 175.8 & $\mathrm{NC}$ & NA|200.8 & $\mathrm{NC}$ & \begin{tabular}{l|l|l|} 
NA23.1 & \\
\end{tabular} \\
\hline
\end{tabular}


Table 4.8. Analytical Results for Samples of WESP Blowdown Fluids (mg/l), (continued).

\begin{tabular}{|c|c|c|c|c|c|c|c|c|c|c|c|c|c|c|}
\hline Test & \multicolumn{5}{|c|}{ \#6 } & \multicolumn{9}{|c|}{$\# 7$} \\
\hline I.D. & $12 \mathrm{M}-\mathrm{W}-11$ & $17 \mathrm{~A}$ & & M-W-11 & & & $\mathrm{J}-\mathrm{W}-11$ & & & $\mathrm{~V}-\mathrm{W}-13$ & & $12 \mathrm{~N}$ & $\mathrm{~J}-\mathrm{W}-13$ & $32 \mathrm{~B}$ \\
\hline Glass (kg). & 18020 & & & 18100 & & & 23372 & & & 24076 & & & 24100 & \\
\hline $\mathrm{pH}$ & 2.86 & & & 3.82 & & & 2.47 & & & 2.47 & & & 2.68 & \\
\hline & Sus* Dis.\# & Total & Sus* & Dis. \# & Total & Sus* & Dis.\# & Total & Sus* & \begin{tabular}{|l|} 
Dis..\# \\
\end{tabular} & Total & Sus* & Dis.\# & Total \\
\hline Total & 2092 & 2130 & 88 & 536 & 624 & 0 & 7392 & 7392 & 40 & 7072 & 7112 & 80 & 1452 & 1532 \\
\hline $\mathrm{Al}$ & \begin{tabular}{l|l|} 
NA & 3.16 \\
\end{tabular} & 3.16 & 0.05 & 0.58 & 0.64 & $\mathrm{NA}$ & 6.94 & 6.94 & NA & 7.62 & 7.62 & 0.02 & 0.76 & 0.78 \\
\hline As & \begin{tabular}{l|l|} 
NA & 16.42 \\
\end{tabular} & \begin{tabular}{|l|}
16.42 \\
\end{tabular} & 0.44 & 5.14 & 5.59 & $\mathrm{NA}$ & 110.7 & 110.7 & NA & 129.2 & 129.2 & 0.23 & 28.60 & 28.82 \\
\hline B & \begin{tabular}{l|l|l|} 
NA & 120.6 \\
\end{tabular} & 120.6 & 0.04 & 13.98 & 14.02 & $\mathrm{NA}$ & 380.1 & 380.1 & NA & 411.4 & 411.4 & 0.03 & 68.36 & 68.39 \\
\hline $\mathrm{Ba}$ & \begin{tabular}{l|l|} 
NA & 0.16 \\
\end{tabular} & 0.16 & 0.01 & 0.05 & 0.06 & $\mathrm{NA}$ & 0.08 & 0.08 & NA & 0.09 & 0.09 & $<0.01$ & 0.05 & 0.05 \\
\hline$\overline{\mathrm{Ca}}$ & \begin{tabular}{l|l|}
$\mathrm{NA}$ & 131.2 \\
\end{tabular} & 131.2 & 0.06 & 50.70 & 50.75 & $\mathrm{NA}$ & \begin{tabular}{|l|}
42.87 \\
\end{tabular} & 42.87 & NA & 41.12 & 41.12 & 0.01 & 46.95 & 46.96 \\
\hline$\overline{\mathrm{Cd}}$ & \begin{tabular}{l|l|} 
NA & 46.96 \\
\end{tabular} & 46.96 & 2.57 & 22.94 & 25.51 & $\mathrm{NA}$ & 351.1 & 351.1 & NA & 449.0 & 449.0 & 0.65 & \begin{tabular}{|l|}
85.13 \\
\end{tabular} & 85.78 \\
\hline $\mathrm{Cr}$ & NA 1.89 & 1.89 & 1.24 & 0.31 & 1.55 & NA & 1.42 & 1.42 & NA & 1.34 & 1.34 & 0.22 & 0.86 & 1.07 \\
\hline Cs & \begin{tabular}{l|l|} 
NA & 21.00 \\
\end{tabular} & 21.00 & $<0.1$ & 5.40 & 5.40 & NA & 93.20 & 93.20 & NA & 105.6 & 105.6 & $<0.1$ & 17.70 & 17.70 \\
\hline $\mathrm{Cu}$ & NA 15.91 & 15.91 & 0.08 & 0.95 & 1.03 & $\mathrm{NA}$ & 2.67 & 2.67 & $\mathrm{NA}$ & 2.89 & 2.89 & 0.01 & \begin{tabular}{|l|}
0.49 \\
\end{tabular} & 0.50 \\
\hline $\mathrm{Fe}$ & \begin{tabular}{l|l|}
$\mathrm{NA}$ & 1.54 \\
\end{tabular} & 1.54 & 6.47 & 0.03 & 6.51 & NA & 2.10 & 2.10 & NA & 2.10 & 2.10 & 1.20 & 1.98 & 3.19 \\
\hline $\mathrm{K}$ & \begin{tabular}{|l|l|} 
NA & 17.42 \\
\end{tabular} & 17.42 & 0.05 & 5.51 & 5.56 & $\mathrm{NA}$ & 43.63 & 43.63 & NA & 45.39 & 45.39 & 0.01 & 11.18 & 11.19 \\
\hline $\mathrm{Li}$ & \begin{tabular}{l|l|} 
NA & 29.24 \\
\end{tabular} & 29.24 & 0.01 & 6.59 & 6.61 & NA & 121.4 & 121.4 & $\mathrm{NA}$ & 129.8 & 129.8 & 0.01 & 25.17 & 25.19 \\
\hline $\mathrm{Mg}$ & \begin{tabular}{l|l|} 
NA & 27.84 \\
\end{tabular} & 27.84 & 0.08 & 12.53 & 12.60 & NA & 12.92 & 12.92 & $\mathrm{NA}$ & 12.66 & 12.66 & 0.01 & 12.57 & 12.59 \\
\hline $\mathrm{Mn}$ & \begin{tabular}{l|l|}
$\mathrm{NA}$ & 1.01 \\
\end{tabular} & 1.01 & 0.04 & 0.13 & 0.17 & $\mathrm{NA}$ & 1.64 & 1.64 & NA & \begin{tabular}{|l|}
1.79 \\
\end{tabular} & 1.79 & $<0.01$ & 0.26 & 0.26 \\
\hline $\mathrm{Na}$ & \begin{tabular}{l|l|} 
NA & 140.3 \\
\end{tabular} & 140.3 & 0.05 & 42.46 & 42.51 & NA & 443.1 & 443.1 & NA & 458.1 & 458.1 & 0.05 & 94.57 & 94.62 \\
\hline $\mathrm{Ni}$ & \begin{tabular}{l|l|}
$\mathrm{NA}$ & 1.12 \\
\end{tabular} & 1.12 & 0.05 & 2.08 & 2.12 & NA & 1.98 & 1.98 & NA & 2.09 & 2.09 & 0.01 & 1.06 & 1.07 \\
\hline$P$ & 0.52 & 0.52 & $<0.01$ & $<0.07$ & $<0.07$ & NA & 1.63 & 1.63 & NA & 1.69 & 1.69 & 0.02 & 0.33 & 0.35 \\
\hline $\mathrm{Pb}$ & \begin{tabular}{l|l|} 
NA & 0.37
\end{tabular} & 0.37 & 1.53 & $<0.02$ & 1.53 & $\mathrm{NA}$ & 3.68 & 3.68 & $\mathrm{NA}$ & 3.81 & 3.81 & 0.88 & 0.43 & 1.31 \\
\hline$\overline{S b}$ & \begin{tabular}{l|l|} 
NA & 1.33 \\
\end{tabular} & 1.33 & 0.98 & 0.23 & 1.21 & NA & 5.24 & 5.24 & $\mathrm{NA}$ & 5.90 & 5.90 & 0.54 & 1.50 & 2.04 \\
\hline $\mathrm{Se}$ & \begin{tabular}{l|l|} 
NA & 844.6 \\
\end{tabular} & 844.6 & 14.47 & 365.7 & 380.1 & $\mathrm{NA}$ & 3764 & 3764 & NA & 4018 & 4018 & 14.31 & 1169 & \begin{tabular}{|l|}
1183 \\
\end{tabular} \\
\hline Si & \begin{tabular}{l|l|} 
NA & 8.43 \\
\end{tabular} & 8.43 & 0.63 & 1.47 & 2.10 & NA & 16.61 & 16.61 & NA & 19.52 & 19.52 & 0.36 & 3.46 & 3.82 \\
\hline $\mathrm{Sr}$ & \begin{tabular}{|l|l|}
$\mathrm{NA}$ & 2.13 \\
\end{tabular} & 2.13 & $<0.01$ & 0.36 & 0.37 & NA & 1.04 & 1.04 & $\mathrm{NA}$ & 1.24 & 1.24 & $<0.01$ & 0.37 & 0.37 \\
\hline $\mathrm{Te}$ & \begin{tabular}{l|l|} 
NA & 27.55 \\
\end{tabular} & 27.55 & 31.04 & 13.38 & 44.42 & $\mathrm{NA}$ & 104.5 & 104.5 & $\mathrm{NA}$ & 116.9 & 116.9 & 11.11 & 46.97 & 58.07 \\
\hline $\mathrm{Ti}$ & \begin{tabular}{l|l|}
$\mathrm{NA}$ & 0.02 \\
\end{tabular} & 0.02 & 0.02 & $<0.01$ & 0.02 & NA & 0.07 & 0.07 & $\mathrm{NA}$ & 0.08 & 0.08 & 0.01 & 0.01 & 0.02 \\
\hline $\mathrm{Zn}$ & 6.26 & 6.26 & 0.06 & 1.23 & 1.29 & $\mathrm{NA}$ & 12.41 & 12.41 & NA & 13.55 & 13.55 & 0.03 & 2.96 & 2.98 \\
\hline $\mathrm{Zr}$ & \begin{tabular}{|l|r|}
$\mathrm{NA}$ & 0.23 \\
\end{tabular} & 0.23 & 0.04 & 0.01 & 0.05 & NA & 1.13 & 1.13 & NA & 1.24 & 1.24 & 0.02 & 0.07 & 0.09 \\
\hline $\mathrm{Cl}$ & \begin{tabular}{l|l|} 
NA & 292.7 \\
\end{tabular} & 292.7 & NA & 87.15 & 87.15 & $\mathrm{NA}$ & 737.8 & 737.8 & NA & 870.8 & 870.8 & NA & 143.3 & 143.3 \\
\hline $\mathrm{F}$ & \begin{tabular}{l|l|l} 
NA & 37.19 \\
\end{tabular} & 37.19 & NA & 9.67 & 9.67 & NA & 389.9 & 389.9 & $\mathrm{NA}$ & 470.0 & 470.0 & $\mathrm{NA}$ & 91.04 & 91.04 \\
\hline I & 3.82 & 3.82 & $\mathrm{NA}$ & $<0.1$ & $<0.1$ & $\mathrm{NA}$ & 71.99 & 71.99 & NA & 83.71 & 83.71 & NA & \begin{tabular}{|l|}
4.53 \\
\end{tabular} & 4.53 \\
\hline Nitrate & \begin{tabular}{l|l|} 
NA & 1107 \\
\end{tabular} & 1107 & $\mathrm{NA}$ & 143.1 & 143.1 & NA & 125.2 & 125.2 & NA & 126.3 & 126.3 & $\mathrm{NA}$ & 35.75 & 35.75 \\
\hline Nitrite & \begin{tabular}{l|l|}
$\mathrm{NA}$ & $<0.1$ \\
\end{tabular} & $<0.1$ & $\mathrm{NA}$ & $<0.1$ & $<0.1$ & NA & $<0.1$ & $<0.1$ & NA & $<0.1$ & $<0.1$ & NA & $<0.1$ & $<0.1$ \\
\hline Sulfate & \begin{tabular}{l|l|} 
NA & 281.0 \\
\end{tabular} & 281.0 & NA & 105.9 & 105.9 & NA & 2770 & 2770 & $\mathrm{NA}$ & 3001 & 3001 & NA & \begin{tabular}{|l|}
549.3 \\
\end{tabular} & 549.3 \\
\hline
\end{tabular}


Table 4.8. Analytical Results for Samples of WESP Blowdown Fluids (mg/l), (continued).

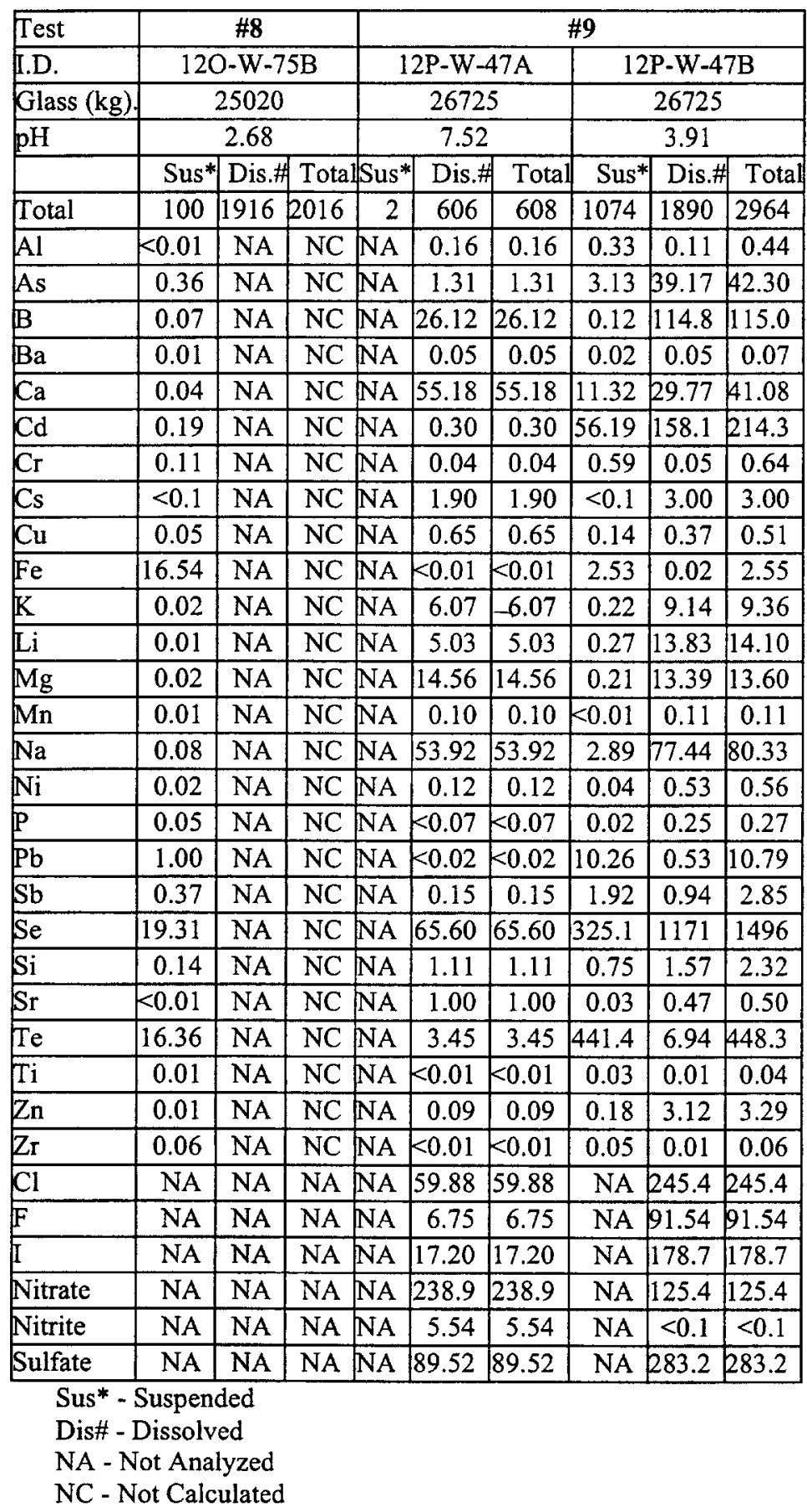


The Catholic University of America Vitreous State Laboratory
DuraMelter 1200 Tests with AZ-101HLW Simulants

Final Report, VSL-02R0100-2, Rev.1

Table 5.1. Discharged Glass Samples and Analyses Performed.

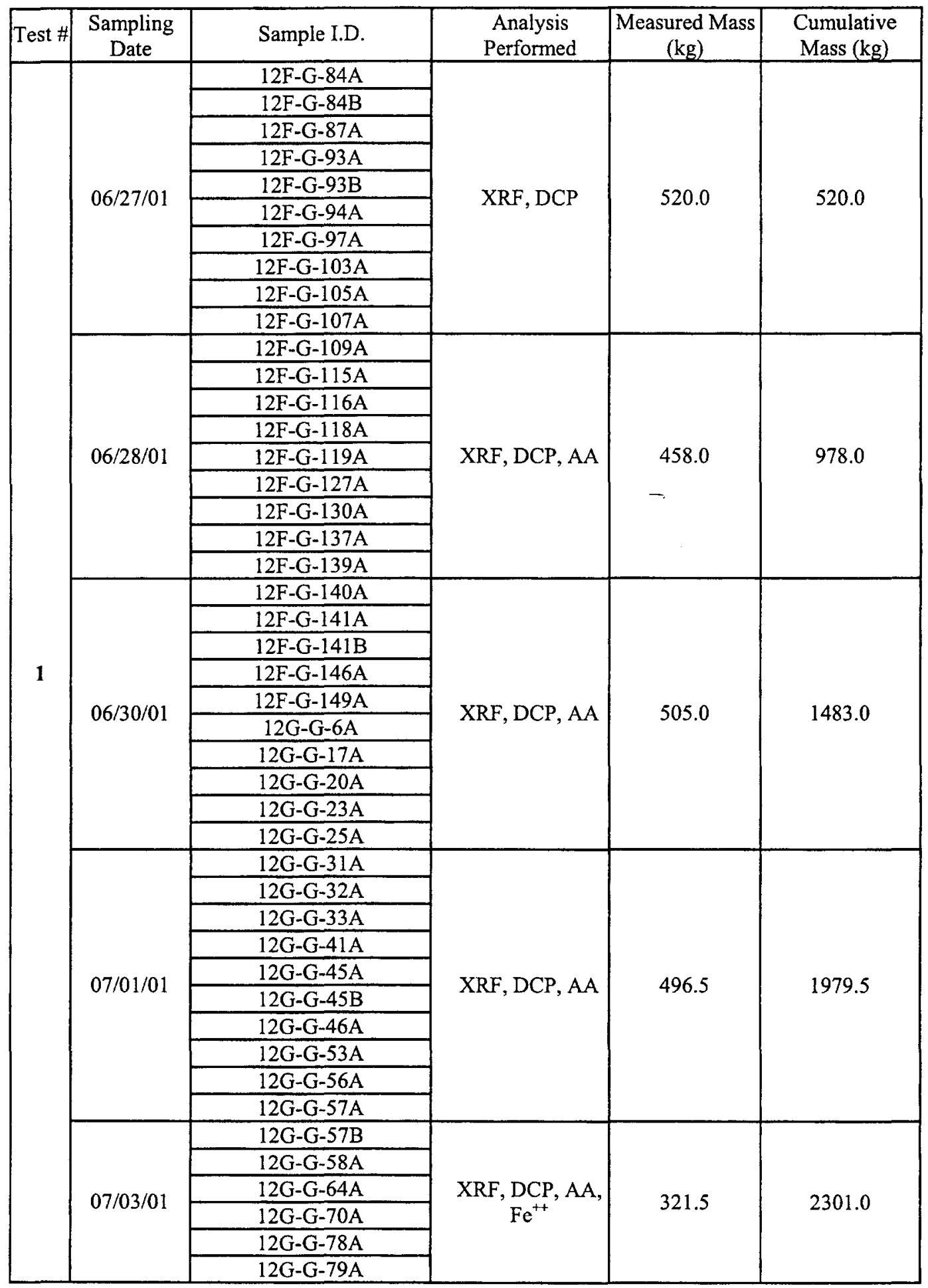


The Catholic University of America Vitreous State Laboratory
DuraMelter 1200 Tests with AZ-101HLW Simulants

Final Report, VSL-02R0100-2, Rev.I

Table 5.1. Discharged Glass Samples and Analyses Performed, (continued).

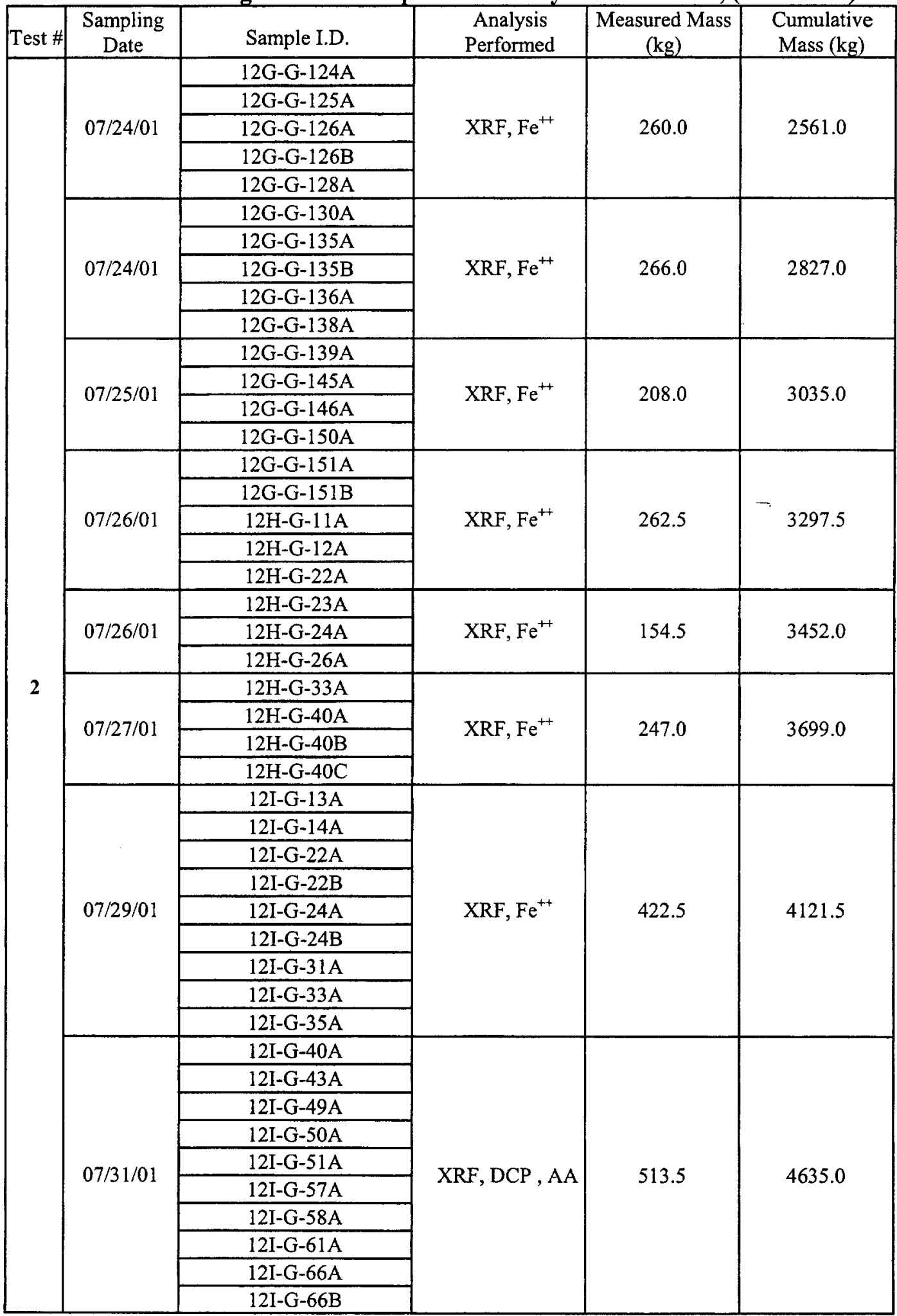


The Catholic University of America Vitreous State Laboratory
DuraMelter 1200 Tests with AZ-101HLW Simulants

Final Report, VSL-02R0100-2, Rev.1

Table 5.1. Discharged Glass Samples and Analyses Performed, (continued).

\begin{tabular}{|c|c|c|c|c|c|}
\hline Test \# & $\begin{array}{l}\text { Sampling } \\
\text { Date }\end{array}$ & Sample I.D. & $\begin{array}{c}\text { Analysis } \\
\text { Performed }\end{array}$ & $\begin{array}{c}\text { Measured Mass } \\
(\mathrm{kg})\end{array}$ & $\begin{array}{c}\text { Cumulative } \\
\text { Mass (kg) }\end{array}$ \\
\hline \multirow{5}{*}{2} & \multirow{5}{*}{$08 / 02 / 01$} & $12 \mathrm{I}-\mathrm{G}-74 \mathrm{~A}$ & \multirow{5}{*}{$\mathrm{XRF}, \mathrm{DCP}, \mathrm{AA}$} & \multirow{5}{*}{260.0} & \multirow{5}{*}{4895.0} \\
\hline & & $12 \mathrm{I}-\mathrm{G}-82 \mathrm{~A}$ & & & \\
\hline & & $12 \mathrm{I}-\mathrm{G}-82 \mathrm{~B}$ & & & \\
\hline & & $12 \mathrm{I}-\mathrm{G}-88 \mathrm{~A}$ & & & \\
\hline & & $12 \mathrm{~J}-\mathrm{G}-7 \mathrm{~A}$ & & & \\
\hline \multirow{36}{*}{3} & \multirow[b]{2}{*}{$08 / 06 / 01$} & $12 \mathrm{~J}-\mathrm{G}-37 \mathrm{~A}$ & \multirow[b]{2}{*}{$\mathrm{XRF}, \mathrm{DCP}, \mathrm{AA}$} & \multirow[b]{2}{*}{109.0} & \multirow[b]{2}{*}{5004.0} \\
\hline & & $12 \mathrm{~J}-\mathrm{G}-37 \mathrm{~B}$ & & & \\
\hline & \multirow{9}{*}{$08 / 07 / 01$} & $12 \mathrm{~J}-\mathrm{G}-39 \mathrm{~A}$ & \multirow{9}{*}{$\mathrm{XRF}, \mathrm{DCP}, \mathrm{AA}$} & \multirow{9}{*}{510.5} & \multirow{9}{*}{5514.5} \\
\hline & & $12 \mathrm{~J}-\mathrm{G}-39 \mathrm{~B}$ & & & \\
\hline & & $12 \mathrm{~J}-\mathrm{G}-40 \mathrm{~A}$ & & & \\
\hline & & $12 \mathrm{~J}-\mathrm{G}-40 \mathrm{~B}$ & & & \\
\hline & & $12 \mathrm{~J}-\mathrm{G}-42 \mathrm{~A}$ & & & \\
\hline & & $12 \mathrm{~J}-\mathrm{G}-43 \mathrm{~A}$ & & & \\
\hline & & $12 \mathrm{~J}-\mathrm{G}-43 \mathrm{~B}$ & & & \\
\hline & & $12 \mathrm{~J}-\mathrm{G}-43 \mathrm{C}$ & & & \\
\hline & & $12 \mathrm{~J}-\mathrm{G}-44 \mathrm{~A}$ & & & \\
\hline & \multirow{7}{*}{$08 / 07 / 01$} & $12 \mathrm{~J}-\mathrm{G}-45 \mathrm{~A}$ & \multirow{7}{*}{$\mathrm{XRF}, \mathrm{DCP}, \mathrm{AA}$} & \multirow{7}{*}{496.5} & \multirow{7}{*}{6011.0} \\
\hline & & $12 \mathrm{~J}-\mathrm{G}-45 \mathrm{~B}$ & & & \\
\hline & & $12 \mathrm{~J}-\mathrm{G}-51 \mathrm{~A}$ & & & \\
\hline & & 12J-G-51B & & & \\
\hline & & $12 \mathrm{~J}-\mathrm{G}-51 \mathrm{C}$ & & & \\
\hline & & $12 \mathrm{~J}-\mathrm{G}-52 \mathrm{~A}$ & & & \\
\hline & & $12 \mathrm{~J}-\mathrm{G}-52 \mathrm{~B}$ & & & \\
\hline & \multirow{9}{*}{$08 / 07 / 01$} & $12 \mathrm{~J}-\mathrm{G}-52 \mathrm{C}$ & \multirow{9}{*}{$\mathrm{XRF}, \mathrm{DCP}, \mathrm{AA}$} & \multirow{9}{*}{477.5} & \multirow{9}{*}{6488.5} \\
\hline & & $12 \mathrm{~J}-\mathrm{G}-53 \mathrm{~A}$ & & & \\
\hline & & $12 \mathrm{~J}-\mathrm{G}-53 \mathrm{~B}$ & & & \\
\hline & & $12 \mathrm{~J}-\mathrm{G}-55 \mathrm{~A}$ & & & \\
\hline & & $12 \mathrm{~J}-\mathrm{G}-56 \mathrm{~A}$ & & & \\
\hline & & $12 J-G-56 B$ & & & \\
\hline & & $12 \mathrm{~J}-\mathrm{G}-56 \mathrm{C}$ & & & \\
\hline & & $12 \mathrm{~J}-\mathrm{G}-56 \mathrm{D}$ & & & \\
\hline & & $12 \mathrm{~J}-\mathrm{G}-62 \mathrm{~A}$ & & & \\
\hline & \multirow{9}{*}{$08 / 08 / 01$} & 12J-G-62B & \multirow{9}{*}{$\mathrm{XRF}, \mathrm{DCP}, \mathrm{AA}$} & \multirow{9}{*}{504.5} & \\
\hline & & $12 \mathrm{~J}-\mathrm{G}-64 \mathrm{~A}$ & & & \\
\hline & & $12 \mathrm{~J}-\mathrm{G}-65 \mathrm{~A}$ & & & \\
\hline & & $12 \mathrm{~J}-\mathrm{G}-65 \mathrm{~B}$ & & & \\
\hline & & $12 \mathrm{~J}-\mathrm{G}-65 \mathrm{C}$ & & & 6993.0 \\
\hline & & $12 \mathrm{~J}-\mathrm{G}-68 \mathrm{~A}$ & & & \\
\hline & & 12J-G-68B & & & \\
\hline & & $12 \mathrm{~J}-\mathrm{G}-69 \mathrm{~A}$ & & & \\
\hline & & $12 \mathrm{~J}-\mathrm{G}-69 \mathrm{~B}$ & & & \\
\hline
\end{tabular}


The Catholic University of America Vitreous State Laboratory
DuraMelter 1200 Tests with AZ-101HLW Simulants

Final Report, VSL-02R0100-2, Rev.1

Table 5.1. Discharged Glass Samples and Analyses Performed, (continued).

\begin{tabular}{|c|c|c|c|c|c|}
\hline Test \# & $\begin{array}{c}\text { Sampling } \\
\text { Date } \\
\end{array}$ & Sample I.D. & $\begin{array}{c}\text { Analysis } \\
\text { Performed }\end{array}$ & $\begin{array}{c}\text { Measured Mass } \\
(\mathrm{kg})\end{array}$ & $\begin{array}{l}\text { Cumulative } \\
\text { Mass (kg) }\end{array}$ \\
\hline \multirow{42}{*}{3} & \multirow{9}{*}{ 08/08/01 } & $12 \mathrm{~J}-\mathrm{G}-70 \mathrm{~A}$ & \multirow{9}{*}{$\mathrm{XRF}, \mathrm{DCP}, \mathrm{AA}$} & \multirow{9}{*}{454.0} & \multirow{9}{*}{7447.0} \\
\hline & & $12 \mathrm{~J}-\mathrm{G}-70 \mathrm{~B}$ & & & \\
\hline & & $12 \mathrm{~J}-\mathrm{G}-75 \mathrm{~A}$ & & & \\
\hline & & $12 \mathrm{~J}-\mathrm{G}-75 \mathrm{~B}$ & & & \\
\hline & & $12 \mathrm{~J}-\mathrm{G}-76 \mathrm{~A}$ & & & \\
\hline & & $12 \mathrm{~J}-\mathrm{G}-76 \mathrm{~B}$ & & & \\
\hline & & $12 \mathrm{~J}-\mathrm{G}-76 \mathrm{C}$ & & & \\
\hline & & $12 \mathrm{~J}-\mathrm{G}-76 \mathrm{D}$ & & & \\
\hline & & $12 \mathrm{~J}-\mathrm{G}-78 \mathrm{~A}$ & & & \\
\hline & \multirow{9}{*}{$08 / 09 / 01$} & $12 \mathrm{~J}-\mathrm{G}-82 \mathrm{~A}$ & \multirow{9}{*}{$\mathrm{XRF}$} & \multirow{9}{*}{527.0} & \multirow{9}{*}{7974.0} \\
\hline & & $12 \mathrm{~J}-\mathrm{G}-82 \mathrm{~B}$ & & & \\
\hline & & $12 \mathrm{~J}-\mathrm{G}-84 \mathrm{~A}$ & & & \\
\hline & & $12 \mathrm{~J}-\mathrm{G}-84 \mathrm{~B}$ & & & \\
\hline & & $12 \mathrm{~J}-\mathrm{G}-86 \mathrm{~A}$ & & & \\
\hline & & $12 \mathrm{~J}-\mathrm{G}-86 \mathrm{~B}$ & & & \\
\hline & & $12 \mathrm{~J}-\mathrm{G}-91 \mathrm{~A}$ & & & \\
\hline & & $12 \mathrm{~J}-\mathrm{G}-94 \mathrm{~A}$ & & & \\
\hline & & $12 \mathrm{~J}-\mathrm{G}-94 \mathrm{~B}$ & & & \\
\hline & \multirow{8}{*}{ 08/09/01 } & $12 \mathrm{~J}-\mathrm{G}-94 \mathrm{C}$ & \multirow{8}{*}{$\mathrm{XRF}, \mathrm{DCP}, \mathrm{AA}$} & \multirow{8}{*}{494.0} & \multirow{8}{*}{8468.0} \\
\hline & & $12 \mathrm{~J}-\mathrm{G}-94 \mathrm{D}$ & & & \\
\hline & & $12 \mathrm{~J}-\mathrm{G}-96 \mathrm{~A}$ & & & \\
\hline & & $12 \mathrm{~J}-\mathrm{G}-96 \mathrm{~B}$ & & & \\
\hline & & $12 \mathrm{~J}-\mathrm{G}-97 \mathrm{~A}$ & & & \\
\hline & & $12 \mathrm{~J}-\mathrm{G}-100 \mathrm{~A}$ & & & \\
\hline & & $12 \mathrm{~J}-\mathrm{G}-100 \mathrm{~B}$ & & & \\
\hline & & $12 \mathrm{~J}-\mathrm{G}-100 \mathrm{C}$ & & & \\
\hline & \multirow{9}{*}{ 08/09/01 } & $12 \mathrm{~J}-\mathrm{G}-106 \mathrm{~A}$ & \multirow{9}{*}{ XRF, DCP . AA } & \multirow{9}{*}{515.5} & \multirow{9}{*}{8983.5} \\
\hline & & $12 \mathrm{~J}-\mathrm{G}-107 \mathrm{~A}$ & & & \\
\hline & & $12 \mathrm{~J}-\mathrm{G}-108 \mathrm{~A}$ & & & \\
\hline & & $12 \mathrm{~J}-\mathrm{G}-108 \mathrm{~B}$ & & & \\
\hline & & $12 \mathrm{~J}-\mathrm{G}-109 \mathrm{~A}$ & & & \\
\hline & & $12 \mathrm{~J}-\mathrm{G}-109 \mathrm{~B}$ & & & \\
\hline & & $12 \mathrm{~J}-\mathrm{G}-111 \mathrm{~A}$ & & & \\
\hline & & $12 \mathrm{~J}-\mathrm{G}-111 \mathrm{~B}$ & & & \\
\hline & & $12 \mathrm{~J}-\mathrm{G}-112 \mathrm{~A}$ & & & \\
\hline & \multirow{7}{*}{$08 / 10 / 01$} & $12 \mathrm{~J}-\mathrm{G}-112 \mathrm{~B}$ & \multirow{7}{*}{$\mathrm{XRF}, \mathrm{DCP}, \mathrm{AA}$} & \multirow{7}{*}{520.0} & \multirow{7}{*}{9503.5} \\
\hline & & $12 \mathrm{~J}-\mathrm{G}-113 \mathrm{~A}$ & & & \\
\hline & & $12 \mathrm{~J}-\mathrm{G}-115 \mathrm{~A}$ & & & \\
\hline & & $12 \mathrm{~J}-\mathrm{G}-120 \mathrm{~A}$ & & & \\
\hline & & $12 \mathrm{~J}-\mathrm{G}-120 \mathrm{~B}$ & & & \\
\hline & & $12 \mathrm{~J}-\mathrm{G}-123 \mathrm{~A}$ & & & \\
\hline & & $12 \mathrm{~J}-\mathrm{G}-125 \mathrm{~A}$ & & & \\
\hline
\end{tabular}


The Catholic University of America Vitreous State Laboratory
DuraMelter 1200 Tests with AZ-101HLW Simulants

Final Report, VSL-02R0100-2, Rev.1

Table 5.1. Discharged Glass Samples and Analyses Performed, (continued).

\begin{tabular}{|c|c|c|c|c|c|}
\hline Test \# & $\begin{array}{c}\text { Sampling } \\
\text { Date }\end{array}$ & Sample I.D. & $\begin{array}{l}\text { Analysis } \\
\text { Performed }\end{array}$ & $\begin{array}{c}\text { Measured Mass } \\
(\mathrm{kg})\end{array}$ & $\begin{array}{c}\text { Cumulative } \\
\text { Mass (kg) }\end{array}$ \\
\hline \multirow{24}{*}{3} & \multirow{4}{*}{$08 / 10 / 01$} & $12 J-G-125 B$ & \multirow{4}{*}{$\mathrm{XRF}, \mathrm{DCP}, \mathrm{AA}$} & \multirow{4}{*}{283.0} & \multirow{4}{*}{9786.5} \\
\hline & & $12 \mathrm{~J}-\mathrm{G}-126 \mathrm{~A}$ & & & \\
\hline & & $12 \mathrm{~J}-\mathrm{G}-126 \mathrm{~B}$ & & & \\
\hline & & $12 \mathrm{~J}-\mathrm{G}-129 \mathrm{~A}$ & & & \\
\hline & \multirow{5}{*}{$08 / 10 / 01$} & $12 \mathrm{~J}-\mathrm{G}-129 \mathrm{~B}$ & \multirow{5}{*}{$\mathrm{XRF}, \mathrm{DCP}, \mathrm{AA}$} & \multirow{5}{*}{348.5} & \multirow{5}{*}{10135.0} \\
\hline & & $12 \mathrm{~J}-\mathrm{G}-130 \mathrm{~A}$ & & & \\
\hline & & $12 \mathrm{~J}-\mathrm{G}-130 \mathrm{~B}$ & & & \\
\hline & & $12 \mathrm{~J}-\mathrm{G}-131 \mathrm{~A}$ & & & \\
\hline & & $12 \mathrm{~J}-\mathrm{G}-136 \mathrm{~A}$ & & & \\
\hline & \multirow{8}{*}{$08 / 11 / 01$} & $12 \mathrm{~J}-\mathrm{G}-136 \mathrm{~B}$ & \multirow{8}{*}{ XRF, DCP, AA } & \multirow{8}{*}{423.5} & \multirow{8}{*}{10558.5} \\
\hline & & $12 \mathrm{~J}-\mathrm{G}-138 \mathrm{~A}$ & & & \\
\hline & & $12 \mathrm{~J}-\mathrm{G}-138 \mathrm{~B}$ & & & \\
\hline & & $12 \mathrm{~J}-\mathrm{G}-138 \mathrm{C}$ & & & \\
\hline & & $12 \mathrm{~J}-\mathrm{G}-139 \mathrm{~A}$ & & & \\
\hline & & $12 \mathrm{~J}-\mathrm{G}-139 \mathrm{~B}$ & & & \\
\hline & & $12 \mathrm{~J}-\mathrm{G}-139 \mathrm{C}$ & & & \\
\hline & & $12 \mathrm{~J}-\mathrm{G}-141 \mathrm{~A}$ & & & \\
\hline & \multirow{7}{*}{$08 / 11 / 01$} & $12 \mathrm{~J}-\mathrm{G}-142 \mathrm{~A}$ & \multirow{7}{*}{ 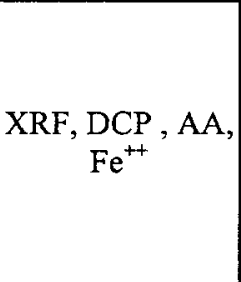 } & \multirow{7}{*}{516.0} & \multirow{7}{*}{11074.5} \\
\hline & & $12 \mathrm{~J}-\mathrm{G}-147 \mathrm{~A}$ & & & \\
\hline & & $12 \mathrm{~J}-\mathrm{G}-148 \mathrm{~A}$ & & & \\
\hline & & $12 \mathrm{~J}-\mathrm{G}-149 \mathrm{~A}$ & & & \\
\hline & & $12 \mathrm{~J}-\mathrm{G}-150 \mathrm{~A}$ & & & \\
\hline & & $12 \mathrm{~J}-\mathrm{G}-152 \mathrm{~A}$ & & & \\
\hline & & $12 \mathrm{~J}-\mathrm{G}-152 \mathrm{~B}$ & & & \\
\hline \multirow{22}{*}{4} & \multirow{5}{*}{$08 / 20 / 01$} & $12 \mathrm{~K}-\mathrm{G}-37 \mathrm{~A}$ & \multirow{5}{*}{ XRF, DCP, AA } & \multirow{5}{*}{348.5} & \multirow{5}{*}{11423.0} \\
\hline & & $12 \mathrm{~K}-\mathrm{G}-38 \mathrm{~A}$ & & & \\
\hline & & $12 \mathrm{~K}-\mathrm{G}-39 \mathrm{~A}$ & & & \\
\hline & & $12 \mathrm{~K}-\mathrm{G}-40 \mathrm{~A}$ & & & \\
\hline & & $12 \mathrm{~K}-\mathrm{G}-42 \mathrm{~A}$ & & & \\
\hline & \multirow{7}{*}{$08 / 21 / 01$} & $12 \mathrm{~K}-\mathrm{G}-46 \mathrm{~A}$ & & & \\
\hline & & $12 \mathrm{~K}-\mathrm{G}-51 \mathrm{~A}$ & & & \\
\hline & & $12 \mathrm{~K}-\mathrm{G}-53 \mathrm{~A}$ & & & \\
\hline & & $12 \mathrm{~K}-\mathrm{G}-53 \mathrm{~B}$ & XRF & 465.0 & 11888.0 \\
\hline & & $12 \mathrm{~K}-\mathrm{G}-54 \mathrm{~A}$ & & & \\
\hline & & $12 \mathrm{~K}-\mathrm{G}-63 \mathrm{~A}$ & & & \\
\hline & & $12 \mathrm{~K}-\mathrm{G}-64 \mathrm{~A}$ & & & \\
\hline & & $12 \mathrm{~K}-\mathrm{G}-65 \mathrm{~A}$ & & & \\
\hline & & $12 \mathrm{~K}-\mathrm{G}-66 \mathrm{~A}$ & & & \\
\hline & & $12 \mathrm{~K}-\mathrm{G}-66 \mathrm{~B}$ & & & \\
\hline & & $12 \mathrm{~K}-\mathrm{G}-67 \mathrm{~A}$ & & & \\
\hline & $08 / 22 / 1$ & $12 \mathrm{~K}-\mathrm{G}-70 \mathrm{~A}$ & YPF & 5210 & 124000 \\
\hline & $00 / 22101$ & $12 \mathrm{~K}-\mathrm{G}-71 \mathrm{~A}$ & AKr & 321.0 & 12409.0 \\
\hline & & $12 \mathrm{~K}-\mathrm{G}-76 \mathrm{~A}$ & & & \\
\hline & & $12 \mathrm{~K}-\mathrm{G}-76 \mathrm{~B}$ & & & \\
\hline & & $12 \mathrm{~K}-\mathrm{G}-78 \mathrm{~A}$ & & & \\
\hline & & $12 \mathrm{~K}-\mathrm{G}-78 \mathrm{~B}$ & & & \\
\hline
\end{tabular}


The Catholic University of America Vitreous State Laboratory
DuraMelter 1200 Tests with AZ-101HLW Simulants Final Report, VSL-02R0100-2, Rev.1

Table 5.1. Discharged Glass Samples and Analyses Performed, (continued).

\begin{tabular}{|c|c|c|c|c|c|}
\hline Test \# & $\begin{array}{c}\text { Sampling } \\
\text { Date }\end{array}$ & Sample I.D. & Analysis Performed & $\begin{array}{l}\text { Measured } \\
\text { Mass (kg) } \\
\end{array}$ & $\begin{array}{l}\text { Cumulative } \\
\text { Mass (kg) }\end{array}$ \\
\hline \multirow{38}{*}{4} & \multirow{11}{*}{$08 / 23 / 01$} & $12 \mathrm{~K}-\mathrm{G}-80 \mathrm{~A}$ & \multirow{11}{*}{$\mathrm{XRF}$} & \multirow{11}{*}{528.5} & \multirow{11}{*}{12937.5} \\
\hline & & $12 \mathrm{~K}-\mathrm{G}-80 \mathrm{~B}$ & & & \\
\hline & & $12 \mathrm{~K}-\mathrm{G}-83 \mathrm{~A}$ & & & \\
\hline & & $12 \mathrm{~K}-\mathrm{G}-84 \mathrm{~A}$ & & & \\
\hline & & $12 \mathrm{~K}-\mathrm{G}-84 \mathrm{~B}$ & & & \\
\hline & & $12 \mathrm{~K}-\mathrm{G}-86 \mathrm{~A}$ & & & \\
\hline & & $12 \mathrm{~K}-\mathrm{G}-86 \mathrm{~B}$ & & & \\
\hline & & $12 \mathrm{~K}-\mathrm{G}-92 \mathrm{~A}$ & & & \\
\hline & & $12 \mathrm{~K}-\mathrm{G}-92 \mathrm{~B}$ & & & \\
\hline & & $12 \mathrm{~K}-\mathrm{G}-93 \mathrm{~A}$ & & & \\
\hline & & $12 \mathrm{~K}-\mathrm{G}-96 \mathrm{~A}$ & & & \\
\hline & \multirow{9}{*}{$08 / 24 / 01$} & $12 \mathrm{~K}-\mathrm{G}-96 \mathrm{~B}$ & \multirow{9}{*}{$\mathrm{XRF}$} & \multirow{9}{*}{523.0} & \multirow{9}{*}{13460.5} \\
\hline & & $12 \mathrm{~K}-\mathrm{G}-101 \mathrm{~A}$ & & & \\
\hline & & $12 \mathrm{~K}-\mathrm{G}-101 \mathrm{~B}$ & & & \\
\hline & & $12 \mathrm{~K}-\mathrm{G}-102 \mathrm{~A}$ & & & \\
\hline & & $12 \mathrm{~K}-\mathrm{G}-104 \mathrm{~A}$ & & & \\
\hline & & $12 \mathrm{~K}-\mathrm{G}-104 \mathrm{~B}$ & & & \\
\hline & & $12 \mathrm{~K}-\mathrm{G}-105 \mathrm{~A}$ & & & \\
\hline & & $12 \mathrm{~K}-\mathrm{G}-110 \mathrm{~A}$ & & & \\
\hline & & $12 \mathrm{~K}-\mathrm{G}-110 \mathrm{~B}$ & & & \\
\hline & \multirow{8}{*}{$08 / 24 / 01$} & $12 \mathrm{~K}-\mathrm{G}-112 \mathrm{~A}$ & \multirow{8}{*}{ XRF, DCP, AA } & \multirow{8}{*}{471.0} & \multirow{8}{*}{13931.5} \\
\hline & & $12 \mathrm{~K}-\mathrm{G}-112 \mathrm{~B}$ & & & \\
\hline & & $12 \mathrm{~K}-\mathrm{G}-113 \mathrm{~A}$ & & & \\
\hline & & $12 \mathrm{~K}-\mathrm{G}-114 \mathrm{~A}$ & & & \\
\hline & & $12 \mathrm{~K}-\mathrm{G}-115 \mathrm{~A}$ & & & \\
\hline & & $12 \mathrm{~K}-\mathrm{G}-121 \mathrm{~A}$ & & & \\
\hline & & $12 \mathrm{~K}-\mathrm{G}-123 \mathrm{~A}$ & & & \\
\hline & & $12 \mathrm{~K}-\mathrm{G}-125 \mathrm{~A}$ & & & \\
\hline & \multirow{9}{*}{$08 / 25 / 01$} & $12 \mathrm{~K}-\mathrm{G}-125 \mathrm{~B}$ & \multirow{9}{*}{ XRF, DCP, AA } & \multirow{9}{*}{517.5} & \multirow{9}{*}{14449.0} \\
\hline & & $12 \mathrm{~K}-\mathrm{G}-126 \mathrm{~A}$ & & & \\
\hline & & $12 \mathrm{~K}-\mathrm{G}-129 \mathrm{~A}$ & & & \\
\hline & & $12 \mathrm{~K}-\mathrm{G}-134 \mathrm{~A}$ & & & \\
\hline & & $12 \mathrm{~K}-\mathrm{G}-134 \mathrm{~B}$ & & & \\
\hline & & $12 \mathrm{~K}-\mathrm{G}-135 \mathrm{~A}$ & & & \\
\hline & & $12 \mathrm{~K}-\mathrm{G}-135 \mathrm{~B}$ & & & \\
\hline & & $12 \mathrm{~K}-\mathrm{G}-136 \mathrm{~A}$ & & & \\
\hline & & $12 \mathrm{~K}-\mathrm{G}-136 \mathrm{~B}$ & & & \\
\hline & $08 / 25 / 01$ & $12 \mathrm{~K}-\mathrm{G}-138 \mathrm{~A}$ & $\mathrm{XRF}, \mathrm{DCP}, \mathrm{AA}, \mathrm{Fe}^{++}$ & 90.5 & 14539.5 \\
\hline \multirow{6}{*}{5} & \multirow{6}{*}{$09 / 27 / 01$} & 12L-G-16A & & & \\
\hline & & 12L-G-17A & & & \\
\hline & & 12L-G-21A & & & \\
\hline & & $12 \mathrm{~L}-\mathrm{G}-28 \mathrm{~A}$ & & & \\
\hline & & $12 \mathrm{~L}-\mathrm{G}-28 \mathrm{~B}$ & & & \\
\hline & & 12L-G-28C & & & \\
\hline
\end{tabular}


The Catholic University of America Vitreous State Laboratory
DuraMelter 1200 Tests with AZ-101HLW Simulants

Final Report, VSL-02R0100-2, Rev.1

Table 5.1. Discharged Glass Samples and Analyses Performed, (continued).

\begin{tabular}{|c|c|c|c|c|c|}
\hline Test \# & $\begin{array}{c}\text { Sampling } \\
\text { Date }\end{array}$ & Sample I.D. & $\begin{array}{l}\text { Analysis } \\
\text { Performed }\end{array}$ & $\begin{array}{c}\text { Measured Mass } \\
(\mathrm{kg})\end{array}$ & $\begin{array}{c}\text { Cumulative } \\
\text { Mass (kg) }\end{array}$ \\
\hline \multirow{27}{*}{5} & & 12L-G-29A & \multirow{5}{*}{$\mathrm{XRF}, \mathrm{Fe}^{++}$} & \multirow{5}{*}{516.0} & \multirow{5}{*}{15055.5} \\
\hline & & $12 \mathrm{~L}-\mathrm{G}-30 \mathrm{~A}$ & & & \\
\hline & & $12 \mathrm{~L}-\mathrm{G}-39 \mathrm{~A}$ & & & \\
\hline & & $12 \mathrm{~L}-\mathrm{G}-41 \mathrm{~A}$ & & & \\
\hline & & $12 \mathrm{~L}-\mathrm{G}-46 \mathrm{~A}$ & & & \\
\hline & \multirow{10}{*}{$09 / 29 / 01$} & $12 \mathrm{~L}-\mathrm{G}-47 \mathrm{~A}$ & \multirow{10}{*}{$\begin{array}{c}\text { XRF, } \\
\mathrm{Fe}^{++}\end{array}$} & \multirow{10}{*}{522.5} & \multirow{10}{*}{15578.0} \\
\hline & & $12 \mathrm{~L}-\mathrm{G}-49 \mathrm{~A}$ & & & \\
\hline & & $12 \mathrm{~L}-\mathrm{G}-50 \mathrm{~A}$ & & & \\
\hline & & 12L-G-59A & & & \\
\hline & & $12 \mathrm{~L}-\mathrm{G}-61 \mathrm{~A}$ & & & \\
\hline & & $12 \mathrm{~L}-\mathrm{G}-68 \mathrm{~A}$ & & & \\
\hline & & 12L-G-68B & & & \\
\hline & & 12L-G-73A & & & \\
\hline & & 12L-G-79A & & & \\
\hline & & 12L-G-80A & & & \\
\hline & \multirow{6}{*}{$09 / 30 / 01$} & $12 \mathrm{~L}-\mathrm{G}-83 \mathrm{~A}$ & \multirow{6}{*}{$\mathrm{XRF}, \mathrm{Fe}^{++}$} & \multirow{6}{*}{314.0} & \multirow{6}{*}{15892.0} \\
\hline & & $12 \mathrm{~L}-\mathrm{G}-88 \mathrm{~A}$ & & & \\
\hline & & 12L-G-90A & & & \\
\hline & & 12L-G-95A & & & \\
\hline & & $12 \mathrm{~L}-\mathrm{G}-99 \mathrm{~A}$ & & & \\
\hline & & $12 \mathrm{~L}-\mathrm{G}-101 \mathrm{~A}$ & & & \\
\hline & \multirow{6}{*}{$10 / 01 / 01$} & $12 \mathrm{~L}-\mathrm{G}-108 \mathrm{~A}$ & \multirow{6}{*}{$\begin{array}{l}\text { XRF, } \\
\mathrm{Fe}^{++}\end{array}$} & \multirow{6}{*}{306.5} & \multirow{6}{*}{16198.5} \\
\hline & & 12L-G-109A & & & \\
\hline & & $12 \mathrm{~L}-\mathrm{G}-116 \mathrm{~A}$ & & & \\
\hline & & $12 \mathrm{~L}-\mathrm{G}-116 \mathrm{~B}$ & & & \\
\hline & & $12 \mathrm{~L}-\mathrm{G}-118 \mathrm{~A}$ & & & \\
\hline & & $12 \mathrm{~L}-\mathrm{G}-119 \mathrm{~A}$ & & & \\
\hline \multirow{17}{*}{6} & \multirow{9}{*}{$10 / 12 / 01$} & $12 \mathrm{~L}-\mathrm{G}-147 \mathrm{~A}$ & \multirow{9}{*}{$\mathrm{XRF}, \mathrm{Fe}^{++}$} & \multirow{9}{*}{507.0} & \multirow{9}{*}{16705.5} \\
\hline & & $12 \mathrm{~L}-\mathrm{G}-148 \mathrm{~A}$ & & & \\
\hline & & $12 \mathrm{~L}-\mathrm{G}-150 \mathrm{~A}$ & & & \\
\hline & & $12 \mathrm{~L}-\mathrm{G}-150 \mathrm{~B}$ & & & \\
\hline & & $12 \mathrm{~L}-\mathrm{G}-155 \mathrm{~A}$ & & & \\
\hline & & $12 \mathrm{M}-\mathrm{G}-6 \mathrm{~A}$ & & & \\
\hline & & $12 M-G-9 A$ & & & \\
\hline & & $12 \mathrm{M}-\mathrm{G}-10 \mathrm{~A}$ & & & \\
\hline & & $12 \mathrm{M}-\mathrm{G}-19 \mathrm{~A}$ & & & \\
\hline & & $12 \mathrm{M}-\mathrm{G}-20 \mathrm{~A}$ & \multirow{8}{*}{$\mathrm{XRF}, \mathrm{Fe}^{++}$} & \multirow{8}{*}{516.0} & \multirow{8}{*}{17221.5} \\
\hline & & $12 \mathrm{M}-\mathrm{G}-21 \mathrm{~A}$ & & & \\
\hline & & $12 \mathrm{M}-\mathrm{G}-24 \mathrm{~A}$ & & & \\
\hline & $10 / 14 / 01$ & $12 \mathrm{M}-\mathrm{G}-30 \mathrm{~A}$ & & & \\
\hline & $10 / 14 / 01$ & $12 \mathrm{M}-\mathrm{G}-32 \mathrm{~A}$ & & & \\
\hline & & $12 \mathrm{M}-\mathrm{G}-40 \mathrm{~A}$ & & & \\
\hline & & $12 \mathrm{M}-\mathrm{G}-48 \mathrm{~A}$ & & & \\
\hline & & $12 \mathrm{M}-\mathrm{G}-50 \mathrm{~A}$ & & & \\
\hline
\end{tabular}


Table 5.1. Discharged Glass Samples and Analyses Performed, (continued).

\begin{tabular}{|c|c|c|c|c|c|}
\hline Test \# & $\begin{array}{l}\text { Sampling } \\
\text { Date }\end{array}$ & Sample I.D. & $\begin{array}{l}\text { Analysis } \\
\text { Performed }\end{array}$ & $\begin{array}{c}\text { Measured Mass } \\
(\mathrm{kg})\end{array}$ & $\begin{array}{c}\text { Cumulative } \\
\text { Mass (kg) }\end{array}$ \\
\hline \multirow{12}{*}{6} & \multirow{5}{*}{$10 / 15 / 01$} & $12 \mathrm{M}-\mathrm{G}-54 \mathrm{~A}$ & \multirow{5}{*}{$\begin{array}{c}\text { XRF, } \\
\mathrm{Fe}^{++}\end{array}$} & \multirow{5}{*}{257.0} & \multirow{5}{*}{17478.5} \\
\hline & & $12 \mathrm{M}-\mathrm{G}-55 \mathrm{~A}$ & & & \\
\hline & & $12 \mathrm{M}-\mathrm{G}-62 \mathrm{~A}$ & & & \\
\hline & & $12 \mathrm{M}-\mathrm{G}-63 \mathrm{~A}$ & & & \\
\hline & & $12 \mathrm{M}-\mathrm{G}-70 \mathrm{~A}$ & & & \\
\hline & \multirow{6}{*}{$10 / 16 / 01$} & 12M-G-72A & \multirow{6}{*}{$\mathrm{XRF}$} & \multirow{6}{*}{541.0} & \multirow{6}{*}{18019.5} \\
\hline & & $12 \mathrm{M}-\mathrm{G}-79 \mathrm{~A}$ & & & \\
\hline & & $12 \mathrm{M}-\mathrm{G}-80 \mathrm{~A}$ & & & \\
\hline & & $12 \mathrm{M}-\mathrm{G}-87 \mathrm{~A}$ & & & \\
\hline & & $12 \mathrm{M}-\mathrm{G}-109 \mathrm{~A}$ & & & \\
\hline & & $12 \mathrm{M}-\mathrm{G}-111 \mathrm{~A}$ & & & \\
\hline & $10 / 18 / 01$ & $12 \mathrm{M}-\mathrm{G}-117 \mathrm{~A}$ & XRF, DCP, AA & 80.0 & 18099.5 \\
\hline \multirow{34}{*}{7} & \multirow{10}{*}{$10 / 23 / 01$} & $12 \mathrm{M}-\mathrm{G}-140 \mathrm{~A}$ & \multirow{10}{*}{ XRF } & \multirow{10}{*}{532.0} & \multirow{10}{*}{18631.5} \\
\hline & & $12 \mathrm{M}-\mathrm{G}-141 \mathrm{~A}$ & & & \\
\hline & & $12 \mathrm{M}-\mathrm{G}-143 \mathrm{~A}$ & & & \\
\hline & & $12 \mathrm{M}-\mathrm{G}-143 \mathrm{~B}$ & & & \\
\hline & & $12 \mathrm{M}-\mathrm{G}-145 \mathrm{~A}$ & & & \\
\hline & & $12 \mathrm{M}-\mathrm{G}-146 \mathrm{~A}$ & & & \\
\hline & & 12M-G-146B & & & \\
\hline & & $12 \mathrm{M}-\mathrm{G}-148 \mathrm{~A}$ & & & \\
\hline & & $12 \mathrm{M}-\mathrm{G}-148 \mathrm{~B}$ & & & \\
\hline & & $12 \mathrm{M}-\mathrm{G}-149 \mathrm{~A}$ & & & \\
\hline & \multirow{8}{*}{$10 / 23 / 01$} & $12 \mathrm{M}-\mathrm{G}-150 \mathrm{~A}$ & \multirow{8}{*}{$\mathrm{XRF}$} & \multirow{8}{*}{513.0} & \multirow{8}{*}{19144.5} \\
\hline & & $12 \mathrm{M}-\mathrm{G}-155 \mathrm{~A}$ & & & \\
\hline & & $12 \mathrm{M}-\mathrm{G}-155 \mathrm{~B}$ & & & \\
\hline & & $12 \mathrm{~N}-\mathrm{G}-11 \mathrm{~A}$ & & & \\
\hline & & $12 \mathrm{~N}-\mathrm{G}-11 \mathrm{~B}$ & & & \\
\hline & & $12 \mathrm{~N}-\mathrm{G}-12 \mathrm{~A}$ & & & \\
\hline & & $12 \mathrm{~N}-\mathrm{G}-12 \mathrm{~B}$ & & & \\
\hline & & $12 \mathrm{~N}-\mathrm{G}-12 \mathrm{C}$ & & & \\
\hline & \multirow{9}{*}{$10 / 24 / 01$} & $12 \mathrm{~N}-\mathrm{G}-16 \mathrm{~A}$ & \multirow{9}{*}{ XRF } & \multirow{9}{*}{508.0} & \multirow{9}{*}{19652.5} \\
\hline & & $12 \mathrm{~N}-\mathrm{G}-16 \mathrm{~B}$ & & & \\
\hline & & $12 \mathrm{~N}-\mathrm{G}-19 \mathrm{~A}$ & & & \\
\hline & & $12 \mathrm{~N}-\mathrm{G}-19 \mathrm{~B}$ & & & \\
\hline & & $12 \mathrm{~N}-\mathrm{G}-19 \mathrm{C}$ & & & \\
\hline & & $12 \mathrm{~N}-\mathrm{G}-20 \mathrm{~A}$ & & & \\
\hline & & $12 \mathrm{~N}-\mathrm{G}-21 \mathrm{~A}$ & & & \\
\hline & & $12 \mathrm{~N}-\mathrm{G}-21 \mathrm{~B}$ & & & \\
\hline & & $12 \mathrm{~N}-\mathrm{G}-21 \mathrm{C}$ & & & \\
\hline & & $12 \mathrm{~N}-\mathrm{G}-27 \mathrm{~A}$ & \multirow{7}{*}{$\mathrm{XRF}, \mathrm{Fe}^{++}$} & & \\
\hline & & $12 \mathrm{~N}-\mathrm{G}-27 \mathrm{~B}$ & & & \\
\hline & & $12 \mathrm{~N}-\mathrm{G}-36 \mathrm{~A}$ & & & \\
\hline & $10 / 24 / 01$ & $12 \mathrm{~N}-\mathrm{G}-36 \mathrm{~B}$ & & 364.5 & 20017.0 \\
\hline & & $12 N-G-38 A$ & & & \\
\hline & & $12 \mathrm{~N}-\mathrm{G}-38 \mathrm{~B}$ & & & \\
\hline & & $12 \mathrm{~N}-\mathrm{G}-38 \mathrm{C}$ & & & \\
\hline
\end{tabular}


Table 5.1. Discharged Glass Samples and Analyses Performed, (continued).

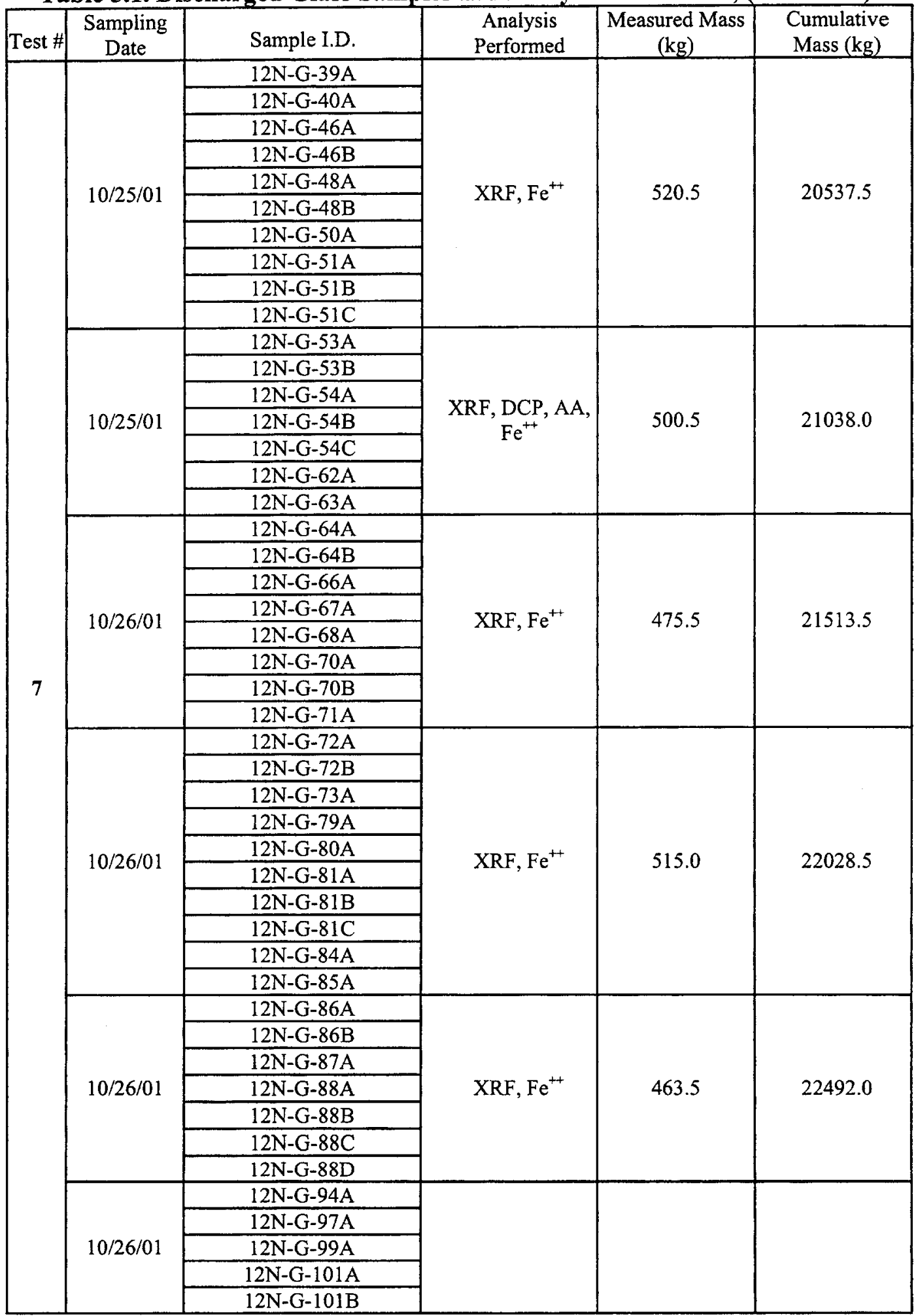


The Catholic University of America Vitreous State Laboratory
DuraMelter 1200 Tests with AZ-10IHLW Simulants

Final Report, VSL-02R0100-2, Rev.I

Table 5.1. Discharged Glass Samples and Analyses Performed, (continued).

\begin{tabular}{|c|c|c|c|c|c|}
\hline Test \# & $\begin{array}{l}\text { Sampling } \\
\text { Date }\end{array}$ & Sample I.D. & $\begin{array}{c}\text { Analysis } \\
\text { Performed }\end{array}$ & $\begin{array}{c}\text { Measured Mass } \\
(\mathrm{kg})\end{array}$ & $\begin{array}{l}\text { Cumulative } \\
\text { Mass (kg) }\end{array}$ \\
\hline \multirow{17}{*}{7} & \multirow{3}{*}{$10 / 26 / 01$} & $12 \mathrm{~N}-\mathrm{G}-102 \mathrm{~A}$ & \multirow{3}{*}{$\mathrm{XRF}$} & \multirow{3}{*}{530.0} & \multirow{3}{*}{23022.0} \\
\hline & & $12 \mathrm{~N}-\mathrm{G}-103 \mathrm{~A}$ & & & \\
\hline & & $12 \mathrm{~N}-\mathrm{G}-103 \mathrm{~B}$ & & & \\
\hline & \multirow{7}{*}{$10 / 27 / 01$} & $12 \mathrm{~N}-\mathrm{G}-104 \mathrm{~A}$ & \multirow{7}{*}{ XRF } & \multirow{7}{*}{540.5} & \multirow{7}{*}{23562.5} \\
\hline & & $12 \mathrm{~N}-\mathrm{G}-104 \mathrm{~B}$ & & & \\
\hline & & $12 \mathrm{~N}-\mathrm{G}-106 \mathrm{~A}$ & & & \\
\hline & & $12 \mathrm{~N}-\mathrm{G}-113 \mathrm{~A}$ & & & \\
\hline & & $12 \mathrm{~N}-\mathrm{G}-114 \mathrm{~A}$ & & & \\
\hline & & $12 \mathrm{~N}-\mathrm{G}-115 \mathrm{~A}$ & & & \\
\hline & & $12 \mathrm{~N}-\mathrm{G}-115 \mathrm{~B}$ & & & \\
\hline & \multirow{7}{*}{$10 / 27 / 01$} & $12 \mathrm{~N}-\mathrm{G}-117 \mathrm{~A}$ & \multirow{7}{*}{ XRF, DCP, AA } & \multirow{7}{*}{513.0} & \multirow{7}{*}{24075.5} \\
\hline & & $12 \mathrm{~N}-\mathrm{G}-117 \mathrm{~B}$ & & & \\
\hline & & $12 \mathrm{~N}-\mathrm{G}-118 \mathrm{~A}$ & & & \\
\hline & & $12 \mathrm{~N}-\mathrm{G}-119 \mathrm{~A}$ & & & \\
\hline & & $12 \mathrm{~N}-\mathrm{G}-119 \mathrm{~B}$ & & & \\
\hline & & $12 \mathrm{~N}-\mathrm{G}-126 \mathrm{~A}$ & & & \\
\hline & & $12 \mathrm{~N}-\mathrm{G}-126 \mathrm{~B}$ & & & \\
\hline \multirow{18}{*}{8} & \multirow{8}{*}{$10 / 31 / 01$} & $12 \mathrm{~N}-\mathrm{G}-153 \mathrm{~A}$ & \multirow{8}{*}{$\mathrm{XRF}, \mathrm{Fe}^{++}$} & \multirow{8}{*}{408.5} & \multirow{8}{*}{24484.0} \\
\hline & & $12 \mathrm{~N}-\mathrm{G}-154 \mathrm{~A}$ & & & \\
\hline & & $12 \mathrm{O}-\mathrm{G}-13 \mathrm{~A}$ & & & \\
\hline & & $120-G-14 A$ & & & \\
\hline & & $120-\mathrm{G}-16 \mathrm{~A}$ & & & \\
\hline & & $12 \mathrm{O}-\mathrm{G}-22 \mathrm{~A}$ & & & \\
\hline & & $120-G-24 \mathrm{~A}$ & & & \\
\hline & & $120-G-31 A$ & & & \\
\hline & \multirow{10}{*}{$11 / 02 / 01$} & $12 \mathrm{O}-\mathrm{G}-32 \mathrm{~A}$ & \multirow{10}{*}{$\begin{array}{c}\text { XRF, } \\
\mathrm{Fe}^{++}\end{array}$} & \multirow{10}{*}{535.5} & \multirow{10}{*}{25019.5} \\
\hline & & $120-G-38 A$ & & & \\
\hline & & $120-\mathrm{G}-40 \mathrm{~A}$ & & & \\
\hline & & $12 \mathrm{O}-\mathrm{G}-43 \mathrm{~A}$ & & & \\
\hline & & $120-\mathrm{G}-49 \mathrm{~A}$ & & & \\
\hline & & $120-G-51 A$ & & & \\
\hline & & $12 \mathrm{O}-\mathrm{G}-58 \mathrm{~A}$ & & & \\
\hline & & $120-G-60 A$ & & & \\
\hline & & $120-G-66 A$ & & & \\
\hline & & $12 \mathrm{O}-\mathrm{G}-68 \mathrm{~A}$ & & & \\
\hline \multirow{11}{*}{9} & & $12 \mathrm{O}-\mathrm{G}-95 \mathrm{~A}$ & & & \\
\hline & & $120-G-96 A$ & & & \\
\hline & & $120-G-97 A$ & & & \\
\hline & $11 / 06 / 01$ & $120-G-99 A$ & $\mathrm{XRF}, \mathrm{Fe}$ & 311.0 & 25330.5 \\
\hline & & $12 \mathrm{O}-\mathrm{G}-106 \mathrm{~A}$ & & & \\
\hline & & $12 \mathrm{O}-\mathrm{G}-107 \mathrm{~A}$ & & & \\
\hline & & $12 \mathrm{O}-\mathrm{G}-108 \mathrm{~A}$ & & & \\
\hline & & $12 \mathrm{O}-\mathrm{G}-116 \mathrm{~A}$ & & & \\
\hline & $11 / 07 / 01$ & $12 \mathrm{O}-\mathrm{G}-118 \mathrm{~A}$ & XKF, $\underset{F_{e}^{++}}{\mathrm{DCP}}, A \mathrm{AA}$, & 276.5 & 25607.0 \\
\hline & & $12 \mathrm{O}-\mathrm{G}-124 \mathrm{~A}$ & & & \\
\hline & & $12 \mathrm{O}-\mathrm{G}-125 \mathrm{~A}$ & & & \\
\hline
\end{tabular}


The Catholic University of America Vitreous State Laboratory
DuraMelter 1200 Tests with AZ-101HLW Simulants Final Report, VSL-02R0100-2, Rev.1

Table 5.1. Discharged Glass Samples and Analyses Performed, (continued).

\begin{tabular}{|c|c|c|c|c|c|}
\hline Test \# & $\begin{array}{c}\text { Sampling } \\
\text { Date }\end{array}$ & Sample I.D. & $\begin{array}{c}\text { Analysis } \\
\text { Performed }\end{array}$ & $\begin{array}{l}\text { Measured } \\
\text { Mass(kg) }\end{array}$ & $\begin{array}{l}\text { Cumulative } \\
\text { Mass (kg) }\end{array}$ \\
\hline \multirow{20}{*}{9} & \multirow{5}{*}{$11 / 08 / 01$} & $12 \mathrm{O}-\mathrm{G}-126 \mathrm{~A}$ & \multirow{5}{*}{$\mathrm{XRF}, \mathrm{Fe}^{++}$} & \multirow{5}{*}{271.5} & \multirow{5}{*}{25878.5} \\
\hline & & $120-\mathrm{G}-134 \mathrm{~A}$ & & & \\
\hline & & $120-\mathrm{G}-135 \mathrm{~A}$ & & & \\
\hline & & $120-\mathrm{G}-136 \mathrm{~A}$ & & & \\
\hline & & $120-\mathrm{G}-144 \mathrm{~A}$ & & & \\
\hline & \multirow{6}{*}{$11 / 09 / 01$} & $12 \mathrm{O}-\mathrm{G}-146 \mathrm{~A}$ & \multirow{6}{*}{$\mathrm{XRF}, \mathrm{Fe}^{++}$} & \multirow{6}{*}{323.0} & \multirow{6}{*}{26201.5} \\
\hline & & $120-\mathrm{G}-146 \mathrm{~B}$ & & & \\
\hline & & $12 \mathrm{O}-\mathrm{G}-153 \mathrm{~A}$ & & & \\
\hline & & $120-\mathrm{G}-154 \mathrm{~A}$ & & & \\
\hline & & $120-G-155 \mathrm{~A}$ & & & \\
\hline & & $12 \mathrm{P}-\mathrm{G}-12 \mathrm{~A}$ & & & \\
\hline & \multirow{9}{*}{$11 / 10 / 01$} & $12 \mathrm{P}-\mathrm{G}-15 \mathrm{~A}$ & \multirow{9}{*}{$\begin{array}{l}\text { XRF, } \\
\mathrm{Fe}^{++}\end{array}$} & \multirow{9}{*}{524.0} & \multirow{9}{*}{26725.5} \\
\hline & & $12 \mathrm{P}-\mathrm{G}-15 \mathrm{~B}$ & & & \\
\hline & & $12 \mathrm{P}-\mathrm{G}-21 \mathrm{~A}$ & & & \\
\hline & & $12 \mathrm{P}-\mathrm{G}-28 \mathrm{~A}$ & & & \\
\hline & & $12 \mathrm{P}-\mathrm{G}-31 \mathrm{~A}$ & & & \\
\hline & & $12 \mathrm{P}-\mathrm{G}-32 \mathrm{~A}$ & & & \\
\hline & & $12 \mathrm{P}-\mathrm{G}-34 \mathrm{~A}$ & & & \\
\hline & & $12 \mathrm{P}-\mathrm{G}-41 \mathrm{~A}$ & & & \\
\hline & & $12 \mathrm{P}-\mathrm{G}-42 \mathrm{~A}$ & & & \\
\hline
\end{tabular}


Table 5.2. XRF Analyzed Compositions for Discharged Glasses (wt \%).

\begin{tabular}{|c|c|c|c|c|c|c|c|c|c|c|c|c|}
\hline Test \# & & \multicolumn{5}{|c|}{1} & \multicolumn{6}{|c|}{2} \\
\hline Glass (kg) & & 520 & 978 & 1483 & 1979 & 2301 & 2561 & 2827 & 3035 & 3297 & 3452 & 3699 \\
\hline Sample & Гarget & $12 F-G-$ & $12 \mathrm{~F}-\mathrm{G}-$ & $12 \mathrm{G}-\mathrm{G}-$ & $12 \mathrm{G}-\mathrm{G}-$ & $12 \mathrm{G}-\mathrm{G}-$ & 2G-G- & $12 \mathrm{G}-\mathrm{G}$ & $12 \mathrm{G}-\mathrm{G}-$ & $12 \mathrm{H}-\mathrm{G}-$ & $12 \mathrm{H}-\mathrm{G}-$ & $12 \mathrm{H}-\mathrm{G}-$ \\
\hline I.D. & & $107 \mathrm{~A}$ & $139 \mathrm{~A}$ & $25 \mathrm{~A}$ & $57 \mathrm{~A}$ & $79 \mathrm{~A}$ & $128 \mathrm{~A}$ & $138 \mathrm{~A}$ & $150 \mathrm{~A}$ & $22 \mathrm{~A}$ & $26 \mathrm{~A}$ & $40 \mathrm{C}$ \\
\hline $\mathrm{Al}_{2} \mathrm{O}_{3}$ & 7.40 & 7.69 & 7.55 & 7.64 & 7.48 & 7.76 & 7.42 & 7.45 & 7.47 & 7.53 & 7.49 & 7.47 \\
\hline$\overline{\mathrm{As}_{2} \mathrm{O}_{3}}$ & 0.04 & 0.04 & 0.04 & 0.04 & 0.04 & 0.04 & 0.04 & 0.04 & 0.04 & 0.04 & 0.04 & 0.04 \\
\hline$\overline{\mathrm{B}_{2} \mathrm{O}_{3}{ }^{*}}$ & 10.00 & 10.00 & 10.00 & 10.00 & 10.00 & 10.00 & 10.00 & 10.00 & 10.00 & 10.00 & 10.00 & 10.00 \\
\hline$\overline{\mathrm{BaO}}$ & 0.04 & $<0.05$ & $<0.05$ & $<0.05$ & $<0.05$ & $<0.05$ & $<0.05$ & $<0.05$ & $<0.05$ & $<0.05$ & $<0.05$ & $<0.05$ \\
\hline $\mathrm{CaO}$ & 0.25 & 0.37 & 0.37 & 0.33 & 0.32 & 0.31 & 0.32 & 0.32 & 0.31 & 0.31 & 0.30 & 0.30 \\
\hline $\mathrm{CdO}$ & 0.37 & 0.43 & 0.42 & 0.40 & 0.38 & 0.35 & 0.37 & 0.41 & 0.38 & 0.38 & 0.34 & 0.38 \\
\hline $\mathrm{CeO}_{2}$ & 0.08 & 0.07 & 0.07 & 0.08 & 0.06 & 0.07 & 0.07 & 0.07 & 0.07 & 0.07 & 0.07 & 0.06 \\
\hline $\mathrm{Cl}$ & 0.01 & 0.00 & 0.00 & 0.00 & 0.00 & 0.00 & 0.00 & 0.00 & 0.00 & 0.00 & 0.00 & 0.00 \\
\hline $\mathrm{Cr}_{2} \mathrm{O}_{3}$ & 0.04 & 0.05 & 0.05 & 0.05 & 0.05 & 0.05 & 0.05 & 0.05 & 0.04 & 0.05 & 0.05 & 0.05 \\
\hline $\mathrm{Cs}_{2} \mathrm{O}$ & 0.08 & 0.09 & 0.08 & 0.08 & 0.08 & 0.07 & 0.08 & 0.09 & 0.08 & 0.08 & 0.07 & 0.08 \\
\hline $\mathrm{CuO}$ & 0.03 & 0.04 & 0.04 & 0.03 & 0.03 & 0.03 & 0.04 & 0.03 & 0.04 & 0.03 & 0.03 & 0.03 \\
\hline $\mathrm{F}$ & 0.04 & NA & NA & NA & NA & $\mathrm{NA}$ & $\mathrm{NA}$ & NA & NA & NA & $\mathrm{NA}$ & 0.00 \\
\hline $\mathrm{Fe}_{2} \mathrm{O}_{3}$ & 10.39 & 9.98 & 10.25 & 9.93 & 9.98 & 9.98 & 10.20 & 10.11 & 10.09 & 9.99 & 10.06 & 10.03 \\
\hline $\mathrm{K}_{2} \mathrm{O}$ & 0.17 & 0.25 & 0.26 & 0.24 & 0.24 & 0.26 & 0.26 & 0.24 & 0.25 & 0.25 & 0.25 & 0.25 \\
\hline $\mathrm{La}_{2} \mathrm{O}_{3}$ & 0.32 & 0.15 & 0.13 & 0.12 & 0.11 & 0.11 & 0.12 & 0.12 & 0.12 & 0.11 & 0.10 & 0.12 \\
\hline $\mathrm{Li}_{2} \mathrm{O}^{*}$ & 6.00 & 6.00 & 6.00 & 6.00 & 6.00 & 6.00 & 6.00 & 6.00 & 6.00 & 6.00 & 6.00 & 6.00 \\
\hline $\mathrm{MgO}$ & 0.06 & 0.31 & 0.41 & 0.62 & 0.79 & 0.86 & 0.83 & 0.92 & 0.96 & 0.96 & 0.95 & 0.96 \\
\hline $\mathrm{MnO}$ & 3.03 & 3.14 & 3.17 & 3.08 & 3.06 & 3.04 & 3.12 & 3.09 & 3.09 & 3.07 & 3.07 & 3.04 \\
\hline $\mathrm{Na}_{2} \mathrm{O}$ & 6.59 & 6.93 & 6.83 & 7.15 & 7.18 & 6.92 & 6.68 & 6.72 & 6.95 & 6.94 & 6.87 & 6.82 \\
\hline $\mathrm{NiO}$ & 0.54 & 0.49 & 0.50 & 0.48 & 0.47 & 0.47 & 0.47 & 0.46 & 0.46 & 0.45 & 0.45 & 0.45 \\
\hline $\mathrm{P}_{2} \mathrm{O} 5$ & 0.13 & 0.21 & 0.25 & 0.26 & 0.29 & 0.28 & 0.30 & 0.30 & 0.29 & 0.30 & 0.30 & 0.34 \\
\hline $\mathrm{PbO}$ & 0.15 & 0.14 & 0.13 & 0.13 & 0.13 & 0.13 & 0.12 & 0.13 & 0.13 & 0.13 & 0.13 & 0.13 \\
\hline $\mathrm{Sb}_{2} \mathrm{O}_{3}$ & 0.21 & 0.19 & 0.19 & 0.17 & 0.18 & 0.17 & 0.16 & 0.19 & 0.17 & 0.18 & 0.15 & 0.18 \\
\hline $\mathrm{SeO}_{2}$ & 0.15 & 0.02 & 0.03 & 0.04 & 0.05 & 0.06 & 0.02 & 0.03 & 0.03 & 0.03 & 0.03 & 0.04 \\
\hline $\mathrm{SiO}_{2}$ & 45.54 & 45.01 & 44.69 & 44.99 & 45.00 & 44.91 & 45.02 & 44.98 & 44.83 & 45.00 & 45.28 & 45.07 \\
\hline $\mathrm{SO}_{3}$ & 0.25 & 0.14 & 0.17 & 0.20 & 0.23 & 0.25 & 0.21 & 0.21 & 0.22 & 0.21 & 0.22 & 0.24 \\
\hline $\mathrm{SrO}$ & 2.32 & 2.13 & 2.14 & 2.02 & 2.00 & 2.02 & 2.07 & 2.05 & 2.04 & 2.02 & 1.99 & 2.03 \\
\hline $\mathrm{TeO}_{2}$ & 0.14 & 0.11 & 0.12 & 0.12 & 0.13 & 0.11 & 0.11 & 0.13 & 0.12 & 0.12 & 0.10 & 0.11 \\
\hline $\mathrm{TiO}_{2}$ & 0.06 & 0.07 & 0.07 & 0.07 & 0.07 & 0.08 & 0.08 & 0.07 & 0.07 & 0.07 & 0.07 & 0.07 \\
\hline $\mathrm{ZnO}$ & 2.00 & 1.89 & 1.92 & 1.83 & 1.80 & 1.82 & 1.88 & 1.84 & 1.84 & 1.81 & 1.84 & 1.81 \\
\hline $\mathrm{ZrO}_{2}$ & 3.56 & 4.09 & 4.15 & 3.94 & 3.89 & 3.89 & 3.99 & 3.99 & 3.95 & 3.91 & 3.78 & 3.93 \\
\hline Sum & 99.99 & 100.00 & 100.00 & 100.00 & 100.00 & 100.00 & 100.00 & 100.00 & 100.00 & 100.00 & 100.00 & 100.00 \\
\hline
\end{tabular}

* Target values. NA: Not Analyzed. 
The Catholic University of America Vitreous State Laboratory
DuraMelter 1200 Tests with AZ-101HLW Simulants

Final Report, VSL-02R0100-2, Rev.1

Table 5.2. XRF Analyzed Compositions for Discharged Glasses (wt\%) (continued).

\begin{tabular}{|c|c|c|c|c|c|c|c|c|c|c|c|c|}
\hline Test \# & & \multicolumn{3}{|c|}{2} & \multicolumn{8}{|c|}{3} \\
\hline Glass $(\mathrm{kg})$ & & 4121.5 & 4635 & 4895 & 5004 & 5514.5 & 6011 & 6488.5 & 6993 & 7447 & 7974 & 8468 \\
\hline $\begin{array}{c}\text { Sample } \\
\text { I.D. }\end{array}$ & Target & \begin{tabular}{|c|}
$12 \mathrm{I}-\mathrm{G}-$ \\
$35 \mathrm{~A}$
\end{tabular} & $\begin{array}{c}12 \mathrm{I}-\mathrm{G}- \\
66 \mathrm{~B}\end{array}$ & $\begin{array}{c}12 \mathrm{~J}-\mathrm{G}- \\
7 \mathrm{~A}\end{array}$ & $\begin{array}{c}12 \mathrm{~J}-\mathrm{G}- \\
37 \mathrm{~B}\end{array}$ & $\begin{array}{c}12 \mathrm{~J}-\mathrm{G}- \\
44 \mathrm{~A}\end{array}$ & $\begin{array}{c}12 \mathrm{~J}-\mathrm{G}- \\
52 \mathrm{~B}\end{array}$ & $\begin{array}{c}12 \mathrm{~J}-\mathrm{G}- \\
62 \mathrm{~A}\end{array}$ & $\begin{array}{c}12 \mathrm{~J}-\mathrm{G}- \\
69 \mathrm{~A}\end{array}$ & $\begin{array}{c}12 \mathrm{~J}-\mathrm{G}- \\
78 \mathrm{~A}\end{array}$ & $\begin{array}{c}12 \mathrm{~J}-\mathrm{G}- \\
94 \mathrm{~B}\end{array}$ & $\begin{array}{c}12 \mathrm{~J}-\mathrm{G}- \\
100 \mathrm{C}\end{array}$ \\
\hline $\mathrm{Al}_{2} \mathrm{O}_{3}$ & 7.40 & 7.58 & 7.37 & 7.60 & 7.51 & 8.00 & 7.25 & 7.32 & 7.21 & 7.19 & 7.38 & 7.36 \\
\hline$\overline{\mathrm{As}_{2} \mathrm{O}_{3}}$ & 0.04 & 0.04 & 0.04 & 0.04 & 0.04 & 0.04 & 0.04 & 0.04 & 0.04 & 0.04 & 0.04 & 0.04 \\
\hline $\mathrm{B}_{2} \mathrm{O}_{3}{ }^{*}$ & 10.00 & 0.00 & 10.00 & 10.00 & 10.00 & 10.00 & 10.00 & 10.00 & 10.00 & 10.00 & 10.00 & 10.00 \\
\hline $\mathrm{BaO}$ & 0.04 & $<0.05$ & $<0.05$ & $<0.05$ & $<0.05$ & $<0.05$ & $<0.05$ & $<0.05$ & $<0.05$ & $<0.05$ & $<0.05$ & $<0.05$ \\
\hline $\mathrm{CaO}$ & 0.25 & 0.31 & 0.31 & 0.31 & 0.30 & 0.30 & 0.30 & 0.29 & 0.30 & 0.30 & 0.29 & 0.29 \\
\hline $\mathrm{CdO}$ & 0.37 & 0.36 & 0.38 & 0.35 & 0.36 & 0.36 & 0.45 & 0.47 & 0.42 & 0.46 & 0.46 & 0.44 \\
\hline $\mathrm{CeO}_{2}$ & 0.08 & 0.07 & 0.07 & 0.07 & 0.07 & 0.07 & 0.07 & 0.06 & 0.06 & 0.07 & 0.08 & 0.07 \\
\hline $\mathrm{Cl}$ & 0.01 & 0.00 & 0.00 & 0.01 & 0.00 & 0.00 & 0.00 & 0.00 & 0.00 & 0.00 & 0.00 & 0.00 \\
\hline $\mathrm{Cr}_{2} \mathrm{O}_{3}$ & 0.04 & 0.05 & 0.05 & 0.05 & 0.05 & 0.05 & 0.05 & 0.05 & 0.05 & 0.05 & 0.05 & 0.05 \\
\hline $\mathrm{Cs}_{2} \mathrm{O}$ & 0.08 & 0.09 & 0.08 & 0.07 & 0.08 & 0.08 & 0.09 & 0.08 & 0.06 & 0.08 & 0.09 & 0.08 \\
\hline $\mathrm{CuO}$ & 0.03 & 0.03 & 0.03 & 0.03 & 0.03 & 0.03 & 0.03 & 0.03 & 0.03 & 0.03 & 0.03 & 0.03 \\
\hline $\mathrm{F}$ & 0.04 & 0.00 & NA & $\mathrm{NA}$ & NA & NA & NA & NA & NA & $\mathrm{NA}$ & NA & NA \\
\hline$\overline{\mathrm{Fe}_{2} \mathrm{O}_{3}}$ & 10.39 & 10.18 & 10.10 & 10.21 & 10.16 & 9.83 & 10.29 & 10.26 & 10.51 & 10.46 & 10.23 & 10.18 \\
\hline $\mathrm{K}_{2} \mathrm{O}$ & 0.17 & 0.27 & 0.24 & 0.27 & 0.26 & 0.28 & 0.24 & 0.24 & 0.25 & 0.26 & 0.24 & 0.25 \\
\hline $\mathrm{La}_{2} \mathrm{O}_{3}$ & 0.32 & 0.10 & 0.11 & 0.12 & 0.12 & 0.12 & 0.12 & 0.12 & 0.12 & 0.13 & 0.13 & 0.12 \\
\hline $\mathrm{Li}_{2} \mathrm{O}^{*}$ & 6.00 & 0.00 & 6.00 & 6.00 & 6.00 & 6.00 & 6.00 & 6.00 & 6.00 & 6.00 & 6.00 & 6.00 \\
\hline $\mathrm{MgO}$ & 0.06 & 0.99 & 1.08 & 1.05 & 1.07 & 1.10 & 1.14 & 1.07 & 1.09 & 1.18 & 1.12 & 1.13 \\
\hline $\mathrm{MnO}$ & 3.03 & 3.08 & 3.06 & 3.10 & 3.04 & 2.93 & 3.07 & 3.10 & 3.15 & 3.14 & 3.07 & 3.05 \\
\hline $\mathrm{Na}_{2} \mathrm{O}$ & 6.59 & 6.13 & 7.07 & 6.72 & 6.55 & 7.15 & 7.14 & 6.92 & 6.82 & 6.71 & 6.82 & 7.04 \\
\hline $\mathrm{NiO}$ & 0.54 & 0.47 & 0.46 & 0.46 & 0.46 & 0.44 & 0.48 & 0.49 & 0.50 & 0.50 & 0.49 & 0.48 \\
\hline$\overline{\mathrm{P}_{2} \mathrm{O}_{5}}$ & 0.13 & 0.32 & 0.33 & 0.32 & 0.43 & 0.33 & 0.37 & 0.38 & 0.33 & 0.34 & 0.32 & 0.35 \\
\hline$\overline{\mathrm{PbO}}$ & 0.15 & 0.13 & 0.13 & 0.13 & 0.12 & 0.12 & 0.13 & 0.13 & 0.13 & 0.13 & 0.13 & 0.12 \\
\hline $\mathrm{Sb}_{2} \mathrm{O}_{3}$ & 0.21 & 0.18 & 0.18 & 0.16 & 0.17 & 0.17 & 0.19 & 0.18 & 0.16 & 0.18 & 0.19 & 0.19 \\
\hline $\mathrm{SeO}_{2}$ & 0.15 & 0.05 & 0.05 & 0.05 & 0.04 & 0.03 & 0.03 & 0.02 & 0.03 & 0.02 & 0.03 & 0.02 \\
\hline $\mathrm{SiO}_{2}$ & 45.54 & 45.34 & 44.68 & 44.63 & 44.94 & 44.58 & 44.22 & 44.49 & 44.39 & 44.31 & 44.60 & 44.68 \\
\hline $\mathrm{SO}_{3}$ & 0.25 & 0.24 & 0.26 & 0.25 & 0.25 & 0.21 & 0.23 & 0.22 & 0.22 & 0.21 & 0.21 & 0.21 \\
\hline $\mathrm{SrO}$ & 2.32 & 2.04 & 2.02 & 2.04 & 2.04 & 2.01 & 2.09 & 2.09 & 2.11 & 2.13 & 2.07 & 2.01 \\
\hline $\mathrm{TeO}_{2}$ & 0.14 & 0.11 & 0.12 & 0.10 & 0.11 & 0.11 & 0.12 & 0.12 & 0.11 & 0.12 & 0.13 & 0.13 \\
\hline $\mathrm{TiO}_{2}$ & 0.06 & 0.08 & 0.07 & 0.07 & 0.07 & 0.09 & 0.07 & 0.07 & 0.08 & 0.08 & 0.08 & 0.08 \\
\hline $\mathrm{ZnO}$ & 2.00 & 1.85 & 1.82 & 1.87 & 1.83 & 1.80 & 1.85 & 1.85 & 1.92 & 1.90 & 1.84 & 1.81 \\
\hline $\mathrm{ZrO}_{2}$ & 3.56 & 3.95 & 3.93 & 3.95 & 3.93 & 3.81 & 3.99 & 3.95 & 3.94 & 4.02 & 3.91 & 3.83 \\
\hline Sum & 99.99 & 100.00 & 100.00 & 100.00 & 100.00 & 100.00 & 100.00 & 100.00 & \begin{tabular}{|l|}
100.00 \\
\end{tabular} & 100.00 & 100.00 & 100.00 \\
\hline
\end{tabular}


Table 5.2. XRF Analyzed Compositions for Discharged Glasses (wt\%) (continued).

\begin{tabular}{|c|c|c|c|c|c|c|c|c|c|c|c|c|}
\hline Test \# & & \multicolumn{5}{|c|}{3} & \multicolumn{6}{|c|}{4} \\
\hline Glass (kg) & & 8983.5 & 9503.5 & 9787 & 10135 & 10559 & 11423 & 11888 & 12409 & 12938 & 13461 & 13932 \\
\hline Sample & Target & $12 \mathrm{~J}-\mathrm{G}-$ & $12 \mathrm{~J}-\mathrm{G}-$ & $12 \mathrm{~J}-\mathrm{G}-$ & $12 \mathrm{~J}-\mathrm{G}-$ & $12 \mathrm{~J}-\mathrm{G}-$ & $12 \mathrm{~K}-\mathrm{G}-$ & $12 \mathrm{~K}-\mathrm{G}$ & $-12 \mathrm{~K}-\mathrm{G}-$ & $12 \mathrm{~K}-\mathrm{G}$ & $12 \mathrm{~K}-\mathrm{G}$ & $2 \mathrm{~K}-\mathrm{G}$ \\
\hline I.D. & & $112 \mathrm{~A}$ & $125 \mathrm{~A}$ & $129 \mathrm{~A}$ & $136 \mathrm{~A}$ & $141 \mathrm{~A}$ & $42 \mathrm{~A}$ & $64 \mathrm{~A}$ & $78 \mathrm{~B}$ & $96 \mathrm{~A}$ & $110 \mathrm{~B}$ & $125 \mathrm{~A}$ \\
\hline $\mathrm{Al}_{2} \mathrm{O}_{3}$ & 7.40 & 6.67 & 7.31 & 7.25 & 7.37 & 7.23 & 7.71 & 7.29 & 7.17 & 7.27 & 7.51 & 7.49 \\
\hline $\mathrm{As}_{2} \mathrm{O}_{3}$ & 0.04 & 0.05 & 0.04 & 0.04 & 0.04 & 0.03 & 0.04 & 0.04 & 0.04 & 0.04 & 0.04 & 0.03 \\
\hline $\mathrm{B}_{2} \mathrm{O}_{3}{ }^{*}$ & 10.00 & 10.00 & 10.00 & 10.00 & 10.00 & 10.00 & 10.00 & 10.00 & 10.00 & 10.00 & 10.00 & 10.00 \\
\hline $\mathrm{BaO}$ & 0.04 & $<0.05$ & $<0.05$ & $<0.05$ & $<0.05$ & $<0.05$ & $<0.05$ & $<0.05$ & $<0.05$ & $<0.05$ & $<0.05$ & $<0.05$ \\
\hline $\mathrm{CaO}$ & 0.25 & 0.30 & 0.30 & 0.29 & 0.29 & 0.29 & 0.28 & 0.30 & \begin{tabular}{|l|}
0.29 \\
\end{tabular} & 0.29 & 0.29 & 0.29 \\
\hline $\mathrm{CdO}$ & 0.37 & 0.46 & 0.40 & 0.39 & 0.42 & 0.37 & 0.43 & 0.46 & 0.41 & 0.44 & 0.35 & 0.41 \\
\hline $\mathrm{CeO}_{2}$ & 0.08 & 0.07 & 0.06 & 0.08 & 0.06 & 0.07 & 0.07 & 0.06 & 0.07 & 0.06 & 0.08 & 0.06 \\
\hline $\mathrm{Cl}$ & 0.01 & 0.01 & 0.00 & 0.00 & 0.00 & 0.00 & 0.00 & 0.00 & 0.00 & 0.00 & 0.00 & 0.00 \\
\hline $\mathrm{Cr}_{2} \mathrm{O}_{3}$ & 0.04 & 0.24 & 0.05 & 0.05 & 0.05 & 0.05 & 0.05 & 0.05 & 0.05 & 0.05 & 0.05 & 0.05 \\
\hline $\mathrm{Cs}_{2} \mathrm{O}$ & 0.08 & 0.08 & 0.07 & 0.07 & 0.08 & 0.07 & 0.08 & 0.08 & 0.07 & 0.08 & 0.07 & 0.07 \\
\hline $\mathrm{CuO}$ & 0.03 & 0.04 & 0.03 & 0.04 & 0.04 & 0.04 & 0.03 & 0.03 & 0.03 & 0.03 & 0.04 & 0.04 \\
\hline $\bar{F}$ & 0.04 & NA & NA & NA & NA & NA & NA & NA & NA & NA & NA & NA \\
\hline $\mathrm{Fe}_{2} \mathrm{O}_{3}$ & 10.39 & 11.28 & 10.28 & 10.41 & 10.31 & 10.38 & 9.82 & 10.30 & 10.50 & 10.26 & 10.47 & 10.31 \\
\hline $\mathrm{K}_{2} \mathrm{O}$ & 0.17 & 0.23 & 0.24 & 0.25 & 0.26 & 0.24 & 0.24 & 0.23 & 0.23 & 0.24 & 0.25 & 0.23 \\
\hline $\mathrm{La}_{2} \mathrm{O}_{3}$ & 0.32 & 0.13 & 0.13 & 0.13 & 0.12 & 0.11 & 0.11 & 0.12 & 0.13 & 0.13 & 0.13 & 0.12 \\
\hline $\mathrm{Li}_{2} \mathrm{O}^{*}$ & 6.00 & 6.00 & 6.00 & 6.00 & 6.00 & 6.00 & 6.00 & 6.00 & 6.00 & 6.00 & 6.00 & 6.00 \\
\hline $\mathrm{MgO}$ & 0.06 & 1.15 & 1.13 & 1.14 & 1.09 & 1.19 & 1.12 & 1.18 & 1.15 & 1.20 & 1.23 & 1.28 \\
\hline $\mathrm{MnO}$ & 3.03 & 3.25 & 3.09 & 3.14 & 3.10 & 3.13 & 2.99 & 3.09 & 3.16 & 3.07 & 3.14 & 3.08 \\
\hline $\mathrm{Na}_{2} \mathrm{O}$ & 6.59 & 6.63 & 6.94 & 6.70 & 6.55 & 6.98 & 7.08 & 6.94 & 6.89 & 6.69 & 7.10 & 6.96 \\
\hline $\mathrm{NiO}$ & 0.54 & 0.60 & 0.49 & 0.50 & 0.49 & 0.51 & 0.45 & 0.49 & 0.50 & 0.49 & 0.51 & 0.48 \\
\hline $\mathrm{P}_{2} \mathrm{O}_{5}$ & 0.13 & 0.31 & 0.33 & 0.33 & 0.33 & 0.32 & 0.33 & 0.33 & 0.33 & 0.33 & 0.35 & 0.32 \\
\hline $\mathrm{PbO}$ & 0.15 & 0.14 & 0.13 & 0.13 & 0.13 & 0.13 & 0.12 & 0.13 & 0.13 & 0.13 & 0.14 & 0.12 \\
\hline$\overline{\mathrm{Sb}_{2} \mathrm{O}_{3}}$ & 0.21 & 0.19 & 0.16 & 0.15 & 0.17 & 0.15 & 0.17 & 0.18 & 0.16 & 0.19 & 0.14 & 0.17 \\
\hline $\mathrm{SeO}_{2}$ & 0.15 & 0.03 & 0.02 & 0.03 & 0.03 & 0.03 & 0.02 & 0.03 & 0.03 & 0.03 & 0.03 & 0.03 \\
\hline $\mathrm{SiO}_{2}$ & 45.54 & 43.31 & 44.65 & 44.75 & 44.86 & 44.53 & 45.24 & 44.54 & 44.52 & 45.06 & 45.97 & 45.36 \\
\hline $\mathrm{SO}_{3}$ & 0.25 & 0.21 & 0.22 & 0.23 & 0.22 & 0.23 & 0.21 & 0.22 & 0.22 & 0.22 & 0.23 & 0.23 \\
\hline $\mathrm{SrO}$ & 2.32 & 2.22 & 2.06 & 2.06 & 2.07 & 2.05 & 1.89 & 2.01 & 2.01 & 1.93 & 1.94 & 1.91 \\
\hline $\mathrm{TeO}_{2}$ & 0.14 & 0.14 & 0.12 & 0.10 & 0.12 & 0.11 & 0.12 & 0.13 & 0.12 & 0.13 & 0.07 & 0.12 \\
\hline $\mathrm{TiO}_{2}$ & 0.06 & 0.08 & 0.07 & 0.08 & 0.07 & 0.07 & 0.07 & 0.08 & 0.08 & 0.08 & 0.08 & 0.08 \\
\hline $\mathrm{ZnO}$ & 2.00 & 1.99 & 1.86 & 1.87 & 1.86 & 1.88 & 1.74 & 1.84 & 1.87 & 1.81 & 1.93 & 1.83 \\
\hline $\mathrm{ZrO}_{2}$ & 3.56 & 4.22 & 3.86 & 3.84 & 3.90 & 3.84 & 3.63 & 3.89 & 3.87 & 3.79 & 1.93 & 2.98 \\
\hline Sum & 99.99 & 100.00 & 100.00 & 100.00 & 100.00 & 100.00 & 100.00 & 100.00 & 100.00 & 100.00 & 100.00 & 100.00 \\
\hline
\end{tabular}


Table 5.2. XRF Analyzed Compositions for Discharged Glasses (wt \%) (continued).

\begin{tabular}{|c|c|c|c|c|c|c|c|c|c|c|c|c|}
\hline Test \# & & \multicolumn{2}{|c|}{4} & \multicolumn{4}{|c|}{5} & \\
\hline Glass (kg) & & 14449 & 14540 & 15056 & 15578 & 15892 & 16199 & 16706 & 17222 & $\mid$\begin{tabular}{|l|}
17479 \\
\end{tabular} & 18020 & 18100 \\
\hline Sample & Target & $12 \mathrm{~K}-\mathrm{G}-$ & $12 \mathrm{~K}-\mathrm{G}-$ & 12L-G- & 12L-G- & 12L-G- & 12L-G. & $12 \mathrm{M}-$ & $12 \mathrm{M}-$ & $12 \mathrm{M}-$ & $12 \mathrm{M}-\mathrm{G}$ & $12 \mathrm{M}-\mathrm{C}$ \\
\hline I.D. & & $136 \mathrm{~B}$ & $138 \mathrm{~A}$ & $46 \mathrm{~A}$ & $80 \mathrm{~A}$ & $101 \mathrm{~A}$ & $119 \mathrm{~A}$ & G-19A & G-50A & G-70A & $111 \mathrm{~A}$ & $117 \mathrm{~A}$ \\
\hline $\mathrm{Al}_{2} \mathrm{O}_{3}$ & 7.40 & 7.22 & 7.16 & 7.40 & 7.47 & 7.30 & 7.26 & 7.28 & 7.26 & 7.08 & 7.43 & 7.37 \\
\hline$\overline{\mathrm{As}_{2} \mathrm{O}_{3}}$ & 0.04 & 0.04 & 0.05 & 0.04 & 0.04 & 0.04 & 0.04 & 0.04 & 0.04 & 0.04 & 0.04 & 0.04 \\
\hline $\mathrm{B}_{2} \mathrm{O}_{3}{ }^{*}$ & 10.00 & 10.00 & 10.00 & 10.00 & 10.00 & 10.00 & 10.00 & 10.00 & 10.00 & 10.00 & 10.00 & 10.00 \\
\hline $\mathrm{BaO}$ & 0.04 & $<0.05$ & $<0.05$ & $<0.05$ & $<0.05$ & $<0.05$ & $<0.05$ & $<0.05$ & $<0.05$ & $<0.05$ & $<0.05$ & $<0.05$ \\
\hline $\mathrm{CaO}$ & 0.25 & 0.29 & 0.29 & 0.29 & 0.29 & 0.29 & 0.30 & 0.37 & 0.45 & 0.47 & 0.51 & 0.51 \\
\hline $\mathrm{CdO}$ & 0.37 & 0.41 & 0.39 & 0.38 & 0.31 & 0.41 & 0.32 & 0.41 & 0.38 & 0.41 & 0.40 & 0.39 \\
\hline $\mathrm{CeO}_{2}$ & 0.08 & 0.06 & 0.06 & 0.07 & 0.07 & 0.07 & 0.07 & 0.07 & 0.07 & 0.07 & 0.08 & 0.08 \\
\hline $\mathrm{Cl}$ & 0.01 & 0.01 & 0.00 & 0.00 & 0.00 & 0.01 & 0.01 & 0.01 & 0.00 & 0.01 & 0.01 & 0.01 \\
\hline $\mathrm{Cr}_{2} \mathrm{O}_{3}$ & 0.04 & 0.05 & 0.05 & 0.05 & 0.05 & 0.04 & 0.04 & 0.04 & 0.04 & 0.04 & 0.04 & 0.04 \\
\hline $\mathrm{Cs}_{2} \mathrm{O}$ & 0.08 & 0.07 & 0.07 & 0.07 & 0.05 & 0.07 & 0.05 & 0.08 & 0.07 & 0.08 & 0.08 & 0.08 \\
\hline $\mathrm{CuO}$ & $\mathrm{c}$ & 0.03 & 0.03 & 0.03 & 0.04 & 0.03 & 0.03 & 0.03 & 0.03 & 0.03 & 0.03 & 0.03 \\
\hline & $\mathrm{c}$ & NA & NA & NA & NA & NA & NA & NA & NA & NA & $\mathrm{NA}$ & NA \\
\hline $\mathrm{Fe}_{2} \mathrm{O}_{3}$ & 1.39 & 10.52 & 10.63 & 10.19 & 10.04 & 10.04 & 9.99 & 9.86 & 10.12 & 10.27 & 10.20 & 10.26 \\
\hline $\mathrm{K}_{2} \mathrm{O}$ & 17 & 0.25 & 0.24 & 0.25 & 0.26 & 0.25 & 0.26 & 0.25 & 0.24 & 0.25 & 0.26 & 0.26 \\
\hline $\mathrm{La}_{2} \mathrm{O}_{3}$ & & 14 & 13 & 0.12 & 0.13 & 0.13 & 0.12 & 0.13 & 0.11 & 0.12 & 0.11 & 0.12 \\
\hline $\mathrm{Li}_{2} \mathrm{O}^{*}$ & & 00 & & 6.00 & 6.00 & 6.00 & 6.00 & 6.00 & 6.00 & 6.00 & 6.00 & 6.00 \\
\hline $\mathrm{MgO}$ & & 19 & 14 & 1.18 & 1.16 & 1.16 & 1.21 & 1.15 & 1.25 & 1.15 & 1.02 & 1.04 \\
\hline $\mathrm{nO}$ & & 14 & & 3.06 & 2.98 & 2.96 & .00 & 2.97 & 2.95 & 3.00 & 2.93 & 2.99 \\
\hline $\mathrm{Na}_{2} \mathrm{O}$ & & 6.64 & & 6.62 & 6.87 & 6.85 & 7.09 & 6.82 & 7.02 & 6.41 & 6.43 & 6.32 \\
\hline $\mathrm{NiO}$ & & 0.50 & & 0.44 & 0.45 & 0.45 & 0.45 & 0.44 & 0.46 & 0.47 & 0.47 & 0.48 \\
\hline $\mathrm{P}_{2} \mathrm{O}_{5}$ & & 0.32 & & 0.33 & 0.32 & 0.32 & 0.31 & 0.31 & 0.31 & 0.31 & 0.30 & 0.30 \\
\hline $\mathrm{PbO}$ & & 13 & & 0.12 & 0.12 & 0.12 & 0.12 & 0.13 & 0.12 & 0.13 & 0.12 & 0.13 \\
\hline${ }_{2} \mathrm{O}_{3}$ & & 17 & 16 & 0.15 & 0.13 & 0.17 & 0.13 & 0.16 & 0.16 & 0.17 & 0.17 & 0.16 \\
\hline $\mathrm{SeO}_{2}$ & & 03 & 0.04 & 02 & .04 & 0.04 & 0.04 & 0.05 & 0.06 & 0.06 & 0.06 & 0.07 \\
\hline $\mathrm{SiO}_{2}$ & 54 & .65 & 4.34 & 45.29 & 45.73 & 45.42 & 45.59 & 45.51 & 45.30 & \begin{tabular}{|l|l|}
44.94 \\
\end{tabular} & 44.99 & 44.90 \\
\hline S & & & & 16 & 0.19 & 0.21 & 0.22 & 0.23 & 0.24 & 0.24 & 0.25 & 0.24 \\
\hline $\mathrm{Sr}$ & & & & 95 & 1.82 & 1.89 & 1.83 & 1.90 & 1.95 & 2.04 & 2.00 & 2.01 \\
\hline & & 0.12 & & 0.10 & 0.08 & 0.11 & 0.09 & 0.12 & 0.12 & 0.12 & 0.13 & 0.12 \\
\hline & & 0.09 & & 10 & 0.09 & 0.10 & 0.10 & 0.12 & 0.14 & 0.14 & 0.16 & 0.17 \\
\hline $\mathrm{ZnO}$ & & 1.86 & & 1.83 & 1.82 & 1.80 & 1.82 & 1.82 & 1.90 & 1.96 & 1.96 & 1.98 \\
\hline $\mathrm{ZrO}_{2}$ & & 3.89 & 3.91 & 3.80 & 3.49 & 3.75 & 3.55 & 3.74 & 3.25 & 4.03 & 3.85 & 3.94 \\
\hline Sum & 99.99 & 100.00 & 100.00 & 100.00 & 100.00 & 100.00 & 100.00 & 100.00 & 100.00 & 100.00 & 100.00 & 100.00 \\
\hline
\end{tabular}

* Target values. NA: Not Analyzed. 
Table 5.2. XRF Analyzed Compositions for Discharged Glasses (wt\%) (continued).

\begin{tabular}{|c|c|c|c|c|c|c|c|c|c|c|c|c|}
\hline Test \# & & \\
\hline ass $(\mathrm{kg})$ & \multirow{3}{*}{ Target } & 18632 & 19653 & 20017 & 20538 & 21038 & 21514 & 22029 & 22492 & 23022 & 23563 & 24076 \\
\hline Sample & & $12 \mathrm{M}-\mathrm{G}$ & $12 \mathrm{~N}-\mathrm{G}$ & $2 \mathrm{~N}-\mathrm{G}$ & $12 \mathrm{~N}-\mathrm{G}-$ & $12 \mathrm{~N}-\mathrm{G}-$ & $2 \mathrm{~N}-\mathrm{G}-$ & $2 \mathrm{~N}-\mathrm{G}-$ & $12 \mathrm{~N}-\mathrm{G}-$ & $12 \mathrm{~N}-\mathrm{G}-$ & $2 \mathrm{~N}-\mathrm{G}$ & $12 \mathrm{~N}-\mathrm{G}$ \\
\hline I.D. & & $149 \mathrm{~A}$ & $21 \mathrm{C}$ & $38 \mathrm{C}$ & $51 \mathrm{C}$ & $63 \mathrm{~A}$ & $71 \mathrm{~A}$ & $85 \mathrm{~A}$ & 88D & & & $126 \mathrm{~B}$ \\
\hline $\mathrm{Al}_{2} \mathrm{O}_{3}$ & 7.40 & 7.68 & 7.96 & 8.03 & 7.96 & 8.02 & 8.22 & 8.05 & 8.16 & 8.22 & 7.94 & 8.17 \\
\hline $\mathrm{As}_{2} \mathrm{O}_{3}$ & 0.04 & 0.04 & 0.03 & 0.03 & 0.03 & 0.04 & 0.04 & 0.03 & 0.04 & 0.03 & 0.03 & 0.04 \\
\hline $\mathrm{B}_{2} \mathrm{O}_{3}{ }^{*}$ & 10.00 & 10.00 & 10.00 & 10.00 & 10.00 & 10.00 & 10.00 & 10.00 & 10.00 & 10.00 & 10.00 & 10.00 \\
\hline $\mathrm{BaO}$ & 0.04 & $<0.05$ & $<0.05$ & $<0.05$ & $<0.05$ & $<0.05$ & $<0.05$ & $<0.05$ & $<0.05$ & $<0.05$ & $<0.05$ & $<0.05$ \\
\hline $\mathrm{CaO}$ & & 0.54 & 0.56 & 0.56 & \begin{tabular}{|l|}
0.58 \\
\end{tabular} & 0.56 & 0.56 & 0.56 & 0.57 & 0.56 & 0.59 & 0.59 \\
\hline$\overline{\mathrm{CdO}}$ & 37 & $\overline{38}$ & 36 & 0.37 & 41 & 0.36 & 38 & 0.41 & .35 & 35 & .37 & .37 \\
\hline$\overline{\mathrm{CeO}_{2}}$ & & 08 & 08 & 0.08 & 0.09 & 0.08 & 0.07 & 0.08 & 0.08 & .10 & 1.08 & 8.08 \\
\hline $\mathrm{Cl}$ & 01 & $\overline{01}$ & 01 & 0.00 & 0.00 & 0.00 & 01 & 0.00 & 0.00 & .00 & 0.00 & 1.00 \\
\hline $\mathrm{Cr}_{2} \mathrm{O}_{3}$ & 0.04 & 04 & 0.04 & 0.04 & 0.04 & 0.05 & 0.04 & 0.05 & 0.04 & 0.05 & 0.05 & .05 \\
\hline $\mathrm{Cs}_{2} \mathrm{O}$ & & 07 & 0.06 & 0.07 & 0.08 & 0.07 & 0.08 & 0.08 & 0.07 & 0.07 & 0.06 & .06 \\
\hline $\mathrm{CuO}$ & & 03 & 0.03 & 0.03 & 0.03 & 0.03 & .03 & 0.03 & 0.02 & .03 & 0.03 & 0.03 \\
\hline $\mathrm{F}$ & 04 & NA & NA & NA & NA & NA & $\mathrm{NA}$ & NA & $\mathrm{NA}$ & NA & NA & NA \\
\hline $\mathrm{Fe}_{2} \mathrm{O}_{3}$ & .39 & 9.97 & 10.04 & 10.08 & 10.34 & -10.20 & 9.90 & 10.32 & 10.11 & 9.98 & 10.24 & 10.17 \\
\hline $\mathrm{K}_{2} \mathrm{O}$ & & 0.28 & & 0.29 & 0.27 & 0.26 & 0.27 & 0.26 & 0.27 & 0.27 & 0.25 & 0.27 \\
\hline $\mathrm{La}_{2} \mathrm{O}_{3}$ & & 0.11 & 0. & 0.12 & 0.12 & 0.12 & 0.11 & 0.11 & 0.12 & 0.11 & 0.11 & 0.12 \\
\hline $\mathrm{Li}_{2} \mathrm{O}^{*}$ & 6.00 & 6.00 & 6.00 & 6.00 & 6.00 & 6.00 & 6.00 & 6.00 & 6.00 & 6.00 & 6.00 & 5.00 \\
\hline $\mathrm{MgO}$ & 0.06 & 0.95 & 0.71 & 0.68 & 0.67 & 0.69 & 0.82 & 0.81 & 0.87 & 0.85 & 0.69 & .59 \\
\hline $\mathrm{MnO}$ & & 2.93 & 2.95 & 3.00 & 2.99 & 3.03 & 2.91 & 3.01 & 3.03 & 2.90 & 3.07 & 3.09 \\
\hline $\mathrm{Na}_{2} \mathrm{O}$ & & 6. & 6.56 & 6.52 & 6.46 & 6.74 & 6.92 & 6.42 & 6.89 & 7.04 & 6.77 & 6.88 \\
\hline $\mathrm{NiO}$ & & 0.46 & 0.48 & 0.48 & 0.49 & 0.48 & 0.48 & 0.49 & 0.49 & 0.48 & 0.49 & 0.48 \\
\hline $\mathrm{P}_{2} \mathrm{O}_{5}$ & & 0. & 0.26 & 0.23 & 0.23 & 0.25 & 0.25 & 0.27 & 0.25 & 0.27 & 0.25 & 0.25 \\
\hline $\mathrm{PbO}$ & 0.15 & 0.12 & 0.12 & 0.12 & 0.12 & 0.13 & 0.12 & 0.13 & 0.12 & 0.13 & 0.12 & 0.13 \\
\hline $\mathrm{Sb}_{2} \mathrm{O}_{3}$ & 0.21 & 0.16 & 0.15 & 0.16 & 0.19 & 0.15 & 0.17 & 0.18 & 0.15 & 0.14 & 0.15 & 0.15 \\
\hline $\mathrm{SeO}_{2}$ & & 04 & 0.03 & .03 & .03 & 0.04 & 0.04 & 0.04 & 0.04 & 0.04 & 0.03 & 0.03 \\
\hline $\mathrm{SiO}_{2}$ & 45.54 & 44.89 & 45.57 & 44.88 & 44.30 & 44.45 & 44.53 & 44.10 & 44.17 & 44.36 & 44.41 & 44.20 \\
\hline $\mathrm{SO}_{3}$ & 0.2 & 0.22 & 0.17 & 0.17 & 0.16 & 0.16 & 0.16 & 0.16 & 0.16 & 0.16 & 0.15 & 0.15 \\
\hline $\mathrm{SrO}$ & & 1.95 & 1.94 & 1.96 & 2.05 & 1.99 & 1.94 & 2.07 & 1.99 & 1.95 & 2.01 & 2.00 \\
\hline $\mathrm{TeO}_{2}$ & 0.14 & 0.12 & 0.11 & 0.12 & 0.13 & 0.10 & 0.11 & 0.12 & 0.10 & 0.10 & 0.10 & 0.10 \\
\hline $\mathrm{TiO}_{2}$ & 0.06 & 0.19 & 0.19 & 0.20 & 0.22 & 0.21 & 0.21 & 0.21 & 0.20 & 0.20 & 0.20 & 0.21 \\
\hline $\mathrm{ZnO}$ & 2.00 & 1.92 & 1.94 & 1.96 & 2.03 & 1.97 & 1.89 & 1.99 & 1.95 & 1.91 & 2.01 & 2.01 \\
\hline $\mathrm{ZrO}_{2}$ & 3.56 & 3.79 & 3.29 & 3.82 & 4.01 & 3.85 & 3.78 & 4.06 & 3.80 & 3.74 & 3.84 & 3.81 \\
\hline $\mathrm{MII}$ & 99.99 & 100.00 & 100.00 & 100.00 & 100.00 & 100.00 & 100.00 & 100.00 & 100.0 & 100.00 & 100.00 & 100.00 \\
\hline
\end{tabular}

* Target values. NA: Not Analyzed. 
The Catholic University of America Vitreous State Laboratory
DuraMelter 1200 Tests with AZ-101HLW Simulants

Final Report, VSL-02R0100-2, Rev.I

Table 5.2. XRF Analyzed Compositions for Discharged Glasses (wt\%) (continued).

\begin{tabular}{|c|c|c|c|c|c|c|c|c|c|}
\hline Test \# & & \multicolumn{2}{|c|}{8} & \multicolumn{5}{|c|}{9} & \multirow{3}{*}{$\begin{array}{c}\text { Overall } \\
\text { Avg. }\end{array}$} \\
\hline Glass (kg) & \multirow[b]{2}{*}{ Target } & 24484 & 25020 & 25331 & 25607 & 26155 & 26478 & 27002 & \\
\hline $\begin{array}{c}\text { Sample } \\
\text { I.D. }\end{array}$ & & $\begin{array}{c}120-\mathrm{G}- \\
31 \mathrm{~A}\end{array}$ & $\begin{array}{c}12 \mathrm{O}-\mathrm{G}- \\
68 \mathrm{~A}\end{array}$ & $\begin{array}{c}12 \mathrm{O}-\mathrm{G}- \\
107 \mathrm{~A}\end{array}$ & $\begin{array}{c}12 \mathrm{O}-\mathrm{G}- \\
125 \mathrm{~A}\end{array}$ & $\begin{array}{c}12 \mathrm{O}-\mathrm{G}- \\
144 \mathrm{~A}\end{array}$ & $\begin{array}{c}12 \mathrm{P}-\mathrm{G}- \\
12 \mathrm{~A}\end{array}$ & $\begin{array}{c}12 \mathrm{P}-\mathrm{G}- \\
42 \mathrm{~A}\end{array}$ & \\
\hline $\mathrm{Al}_{2} \mathrm{O}_{3}$ & 7.40 & 8.10 & 7.88 & 7.66 & 7.64 & 7.87 & 7.45 & 7.46 & 7.54 \\
\hline $\mathrm{As}_{2} \mathrm{O}_{3}$ & 0.04 & 0.03 & 0.04 & 0.04 & 0.04 & 0.03 & 0.04 & 0.03 & 0.04 \\
\hline $\mathrm{B}_{2} \mathrm{O}_{3}{ }^{*}$ & 10.00 & 10.00 & 10.00 & 10.00 & 10.00 & 10.00 & 10.00 & 10.00 & 10.00 \\
\hline $\mathrm{BaO}$ & 0.04 & $<0.05$ & $<0.05$ & $<0.05$ & $<0.05$ & $<0.05$ & $<0.05$ & $<0.05$ & $<0.05$ \\
\hline $\mathrm{CaO}$ & 0.25 & 0.54 & 0.49 & 0.46 & 0.44 & 0.42 & 0.41 & 0.37 & 0.38 \\
\hline $\mathrm{CdO}$ & 0.37 & 0.38 & 0.37 & 0.38 & 0.36 & 0.40 & 0.36 & 0.36 & 0.39 \\
\hline $\mathrm{CeO}_{2}$ & 0.08 & 0.09 & 0.09 & 0.08 & 0.08 & 0.08 & 0.08 & 0.08 & 0.07 \\
\hline $\mathrm{Cl}$ & 0.01 & 0.01 & 0.01 & 0.01 & 0.01 & 0.01 & 0.01 & 0.01 & 0.00 \\
\hline $\mathrm{Cr}_{2} \mathrm{O}_{3}$ & 0.04 & 0.04 & 0.04 & 0.04 & 0.04 & 0.04 & 0.04 & 0.05 & 0.05 \\
\hline $\mathrm{Cs}_{2} \mathrm{O}$ & 0.08 & 0.07 & 0.07 & 0.07 & 0.07 & 0.08 & 0.07 & 0.07 & 0.07 \\
\hline $\mathrm{CuO}$ & 0.03 & 0.03 & 0.03 & 0.03 & 0.03 & 0.03 & 0.03 & 0.03 & 0.03 \\
\hline $\mathrm{F}$ & 0.04 & NA & NA & NA & NA & NA & NA & NA & NA \\
\hline $\mathrm{Fe}_{2} \mathrm{O}_{3}$ & 10.39 & 10.15 & 10.06 & 10.16 & 10.23 & 9.86 & 10.23 & 10.12 & 10.19 \\
\hline $\mathrm{K}_{2} \mathrm{O}$ & 0.17 & 0.29 & 0.28 & 0.26 & 0.26 & 0.26 & 0.26 & 0.25 & 0.25 \\
\hline $\mathrm{La}_{2} \mathrm{O}_{3}$ & 0.32 & 0.11 & 0.12 & 0.12 & 0.12 & 0.11 & 0.12 & 0.11 & 0.12 \\
\hline $\mathrm{Li}_{2} \mathrm{O}^{*}$ & 6.00 & 6.00 & 6.00 & 6.00 & 6.00 & 6.00 & 6.00 & 6.00 & 6.00 \\
\hline $\mathrm{MgO}$ & 0.06 & 0.68 & 0.79 & 0.79 & 0.88 & 0.96 & 1.03 & 1.01 & 0.98 \\
\hline $\mathrm{MnO}$ & 3.03 & 2.98 & 2.87 & 2.91 & 2.97 & 2.89 & 3.03 & 3.07 & 3.04 \\
\hline $\mathrm{Na}_{2} \mathrm{O}$ & 6.59 & 6.50 & 6.47 & 6.63 & 6.43 & 6.52 & 6.89 & 6.96 & 6.79 \\
\hline $\mathrm{NiO}$ & 0.54 & 0.49 & 0.48 & 0.48 & 0.48 & 0.46 & 0.48 & 0.48 & 0.48 \\
\hline $\mathrm{P}_{2} \mathrm{O}_{5}$ & 0.13 & 0.26 & 0.26 & 0.27 & 0.28 & 0.28 & 0.29 & 0.29 & 0.30 \\
\hline$\overline{\mathrm{PbO}}$ & 0.15 & 0.13 & 0.12 & 0.12 & 0.12 & 0.12 & 0.13 & 0.12 & 0.13 \\
\hline $\mathrm{Sb}_{2} \mathrm{O}_{3}$ & 0.21 & 0.16 & 0.16 & 0.15 & 0.15 & 0.17 & 0.16 & 0.16 & 0.17 \\
\hline $\mathrm{SeO}_{2}$ & 0.15 & 0.05 & 0.06 & 0.06 & 0.07 & 0.05 & 0.04 & 0.04 & 0.04 \\
\hline $\mathrm{SiO}_{2}$ & 45.54 & 44.50 & 45.05 & 44.96 & 44.97 & 45.32 & 44.59 & 44.85 & 44.83 \\
\hline $\mathrm{SO}_{3}$ & 0.25 & 0.20 & 0.22 & 0.24 & 0.24 & 0.23 & 0.22 & 0.21 & 0.21 \\
\hline $\mathrm{SrO}$ & 2.32 & 2.03 & 1.98 & 2.00 & 2.01 & 1.94 & 2.00 & 1.97 & 2.00 \\
\hline $\mathrm{TeO}_{2}$ & 0.14 & 0.11 & 0.11 & 0.11 & 0.11 & 0.12 & 0.10 & 0.10 & 0.11 \\
\hline $\mathrm{TiO}_{2}$ & 0.06 & 0.20 & 0.18 & 0.16 & 0.15 & 0.14 & 0.13 & 0.12 & 0.11 \\
\hline $\mathrm{ZnO}$ & 2.00 & 1.99 & 1.94 & 1.96 & 1.95 & 1.85 & 1.94 & 1.87 & 1.88 \\
\hline $\mathrm{ZrO}_{2}$ & 3.56 & 3.91 & 3.86 & 3.89 & 3.90 & 3.80 & 3.90 & 3.85 & 3.82 \\
\hline Sum & 99.99 & 100.00 & 100.00 & 100.00 & 100.00 & 100.00 & 100.00 & 100.00 & \\
\hline
\end{tabular}

* - Target values.

NA - Not Analyzed. 
Table 5.3. DCP Analyzed Composition of Discharged Glass Samples (wt\%).

\begin{tabular}{|c|c|c|c|c|c|c|c|c|}
\hline Test\# & & \multicolumn{4}{|c|}{1} & \multicolumn{3}{|c|}{2} \\
\hline Glass (kg) & & 978 & 1483 & 1979 & 2301 & 4635 & 4895 & 5004 \\
\hline Oxide & Target & $\begin{array}{c}12 \mathrm{~F}-\mathrm{G}- \\
139 \mathrm{~A}\end{array}$ & $\begin{array}{c}12 \mathrm{G}-\mathrm{G}- \\
25 \mathrm{~A}\end{array}$ & $\begin{array}{c}12 \mathrm{G}-\mathrm{G}- \\
57 \mathrm{~A}\end{array}$ & $\begin{array}{c}12 \mathrm{G}-\mathrm{G}- \\
79 \mathrm{~A}\end{array}$ & $\begin{array}{c}12 \mathrm{I}-\mathrm{G}- \\
66 \mathrm{~B}\end{array}$ & $\begin{array}{c}12 \mathrm{~J}-\mathrm{G}- \\
7 \mathrm{~A}\end{array}$ & $\begin{array}{c}12 \mathrm{~J}-\mathrm{G}- \\
37 \mathrm{~B}\end{array}$ \\
\hline $\mathrm{Al}_{2} \mathrm{O}_{3}$ & 7.40 & 7.55 & $\begin{array}{l}7.84 \\
\end{array}$ & \begin{tabular}{|l|}
7.45 \\
\end{tabular} & \begin{tabular}{|l|}
7.67 \\
\end{tabular} & 7.60 & 8.07 & 7.84 \\
\hline $\mathrm{As}_{2} \mathrm{O}_{3}$ & 0.04 & 0.05 & NA & $\mathrm{NA}$ & NA & NA & NA & $\mathrm{NA}$ \\
\hline $\mathrm{B}_{2} \mathrm{O}_{3}$ & 10.00 & 9.80 & 11.03 & 11.16 & 10.84 & 10.65 & 10.81 & 10.70 \\
\hline $\mathrm{BaO}$ & 0.04 & 0.05 & 0.05 & 0.05 & 0.06 & 0.05 & 0.05 & 0.06 \\
\hline $\mathrm{CaO}$ & 0.25 & 0.31 & 0.32 & 0.32 & 0.34 & 0.29 & 0.31 & 0.31 \\
\hline $\mathrm{CdO}$ & 0.37 & 0.31 & NA & NA & $\mathrm{NA}$ & $\mathrm{NA}$ & $\mathrm{NA}$ & $\mathrm{NA}$ \\
\hline $\mathrm{CeO}_{2}$ & 0.08 & $\mathrm{NA}$ & $\mathrm{NA}$ & NA & NA & $\mathrm{NA}$ & $\mathrm{NA}$ & $\mathrm{NA}$ \\
\hline $\mathrm{Cl}$ & 0.01 & $\mathrm{NA}$ & $\mathrm{NA}$ & $\mathrm{NA}$ & $\mathrm{NA}$ & $\mathrm{NA}$ & NA & $\mathrm{NA}$ \\
\hline $\mathrm{Cr}_{2} \mathrm{O}_{3}$ & 0.04 & 0.04 & 0.04 & 0.05 & 0.05 & 0.05 & 0.05 & 0.05 \\
\hline $\mathrm{Cs}_{2} \mathrm{O}^{*}$ & 0.08 & 0.07 & 0.09 & 0.10 & 0.10 & 0.07 & 0.08 & 0.09 \\
\hline $\mathrm{CuO}$ & 0.03 & 0.03 & NA & $\mathrm{NA}$ & NA & NA & NA & NA \\
\hline $\mathrm{F}$ & .04 & NA & $\mathrm{NA}$ & $\mathrm{NA}$ & $\mathrm{NA}$ & $\overline{\mathrm{NA}}$ & $\mathrm{NA}$ & $\overline{N A}$ \\
\hline $\mathrm{Fe}_{2} \mathrm{O}_{3}$ & 10.39 & 8.82 & 9.36 & 9.22 & 9.33 & 9.57 & -9.39 & 9.44 \\
\hline $\mathrm{K}_{2} \mathrm{O}$ & 0.17 & 0.19 & 0.19 & 0.19 & 0.22 & 0.18 & 0.20 & 0.20 \\
\hline $\mathrm{La}_{2} \mathrm{O}_{3}$ & 0.32 & $\mathrm{NA}$ & NA & $\mathrm{NA}$ & $\mathrm{NA}$ & $\mathrm{NA}$ & $\mathrm{NA}$ & NA \\
\hline $\mathrm{Li}_{2} \mathrm{O}$ & 6.00 & 5.38 & 5.29 & 5.12 & 5.22 & 5.29 & 5.46 & 5.34 \\
\hline $\mathrm{MgO}$ & 0.06 & 0.52 & 0.69 & 0.81 & 0.96 & 1.13 & 1.18 & 1.19 \\
\hline $\mathrm{MnO}$ & & 2.62 & 2.89 & 2.76 & 2.82 & 2.65 & 2.81 & 2.73 \\
\hline $\mathrm{Na}_{2} \mathrm{O}$ & 6.59 & 6.44 & 6.71 & 6.42 & 6.55 & 6.56 & 6.76 & 6.65 \\
\hline $\mathrm{NiO}$ & 0.54 & 0.46 & 0.46 & 0.46 & 0.50 & 0.45 & 0.48 & 0.51 \\
\hline $\mathrm{P}_{2} \mathrm{O}_{5}$ & 0.13 & 0.38 & 0.21 & 0.17 & 0.32 & 0.38 & 0.34 & 0.38 \\
\hline $\mathrm{PbO}$ & .15 & 0.19 & $\mathrm{NA}$ & $\mathrm{NA}$ & $\mathrm{NA}$ & $\mathrm{NA}$ & NA & $\mathrm{NA}$ \\
\hline $\mathrm{Sb}_{2} \mathrm{O}_{3}$ & 21 & 0.20 & NA & NA & NA & NA & NA & NA \\
\hline $\mathrm{SeO}_{2}$ & 0.15 & 0.02 & NA & NA & NA & NA & NA & NA \\
\hline $\mathrm{SiO}_{2}$ & 45.54 & 41.51 & 44.83 & 44.42 & 40.42 & 44.77 & 43.89 & 44.31 \\
\hline $\mathrm{SO}_{3}$ & 0.25 & NA & NA & NA & NA & $\mathrm{NA}$ & NA & $\overline{\mathrm{NA}}$ \\
\hline $\mathrm{SrO}$ & 2.32 & 2.02 & 2.03 & 1.93 & 1.99 & 1.97 & 2.04 & 2.02 \\
\hline $\mathrm{TeO}_{2}$ & 0.14 & 0.12 & $\mathrm{NA}$ & $\mathrm{NA}$ & $\mathrm{NA}$ & $\mathrm{NA}$ & $\mathrm{NA}$ & NA \\
\hline $\mathrm{TiO}_{2}$ & 0.06 & 0.10 & 0.09 & 0.09 & 0.11 & 0.10 & 0.11 & 0.10 \\
\hline $\mathrm{ZnO}$ & 2.00 & 1.86 & $\mathrm{NA}$ & $\mathrm{NA}$ & NA & $\mathrm{NA}$ & $\mathrm{NA}$ & $\mathrm{NA}$ \\
\hline $\mathrm{ZrO}_{2}$ & 3.56 & 3.87 & 3.71 & 3.47 & 3.51 & 3.71 & 3.89 & 3.81 \\
\hline$\overline{S U M}$ & 99.99 & 92.89 & 95.85 & 94.20 & 90.99 & 95.46 & 95.92 & 95.73 \\
\hline
\end{tabular}

* Analyzed by AA. NA: Not Analyzed. 
Table 5.3. DCP Analyzed Composition of Discharged Glass Samples (wt\%) (continued).

\begin{tabular}{|c|c|c|c|c|c|c|c|c|c|c|c|c|}
\hline Test\# & & \multicolumn{11}{|c|}{3} \\
\hline$\overline{\text { Glass }(\mathrm{kg})}$ & & \multicolumn{2}{|c|}{6011} & 6489 & 6993 & 7447 & 8468 & 8984 & 9504 & 9787 & 10 & 135 \\
\hline Oxide & Target & $\begin{array}{c}12 \mathrm{~J}-\mathrm{G}- \\
52 \mathrm{~B} 1\end{array}$ & $\begin{array}{l}12 \mathrm{~J}-\mathrm{G} \\
52 \mathrm{~B} 2 \\
\end{array}$ & $\begin{array}{c}12 \mathrm{~J}-\mathrm{G}- \\
62 \mathrm{~A}\end{array}$ & $\begin{array}{c}12 \mathrm{~J}-\mathrm{G}- \\
69 \mathrm{~B}\end{array}$ & $\begin{array}{c}12 \mathrm{~J}-\mathrm{G}- \\
78 \mathrm{~A} \\
\end{array}$ & $\begin{array}{c}12 \mathrm{~J}-\mathrm{G}- \\
100 \mathrm{C}\end{array}$ & $\begin{array}{c}12 \mathrm{~J}-\mathrm{G}- \\
112 \mathrm{~A} \\
\end{array}$ & $\begin{array}{l}12 \mathrm{~J}-\mathrm{G}- \\
125 \mathrm{~A}\end{array}$ & \begin{tabular}{|c|}
$12 \mathrm{~J}-\mathrm{G}-$ \\
$129 \mathrm{~A}$ \\
\end{tabular} & $\begin{array}{l}2 \mathrm{~J}-\mathrm{G}- \\
136 \mathrm{Al}\end{array}$ & $\begin{array}{l}12 \mathrm{~J}-\mathrm{G}- \\
136 \mathrm{~A} 2 \\
\end{array}$ \\
\hline $\mathrm{Al}_{2} \mathrm{O}_{3}$ & 7.40 & 7.74 & 7.69 & 7.38 & 7.50 & 7.48 & 7.88 & 7.60 & 7.99 & \begin{tabular}{|l|}
7.88 \\
\end{tabular} & \begin{tabular}{|l|}
8.07 \\
\end{tabular} & 7.91 \\
\hline $\mathrm{As}_{2} \mathrm{O}_{3}$ & 0.04 & NA & NA & $\mathrm{NA}$ & NA & NA & NA & NA & NA & NA & NA & NA \\
\hline $\mathrm{B}_{2} \mathrm{O}_{3}$ & 10.00 & 10.99 & 10.85 & 10.53 & 10.89 & 10.72 & 10.73 & 10.52 & 10.61 & 10.62 & 11.15 & 10.94 \\
\hline $\mathrm{BaO}$ & 0.04 & 0.06 & 0.05 & 0.06 & 0.05 & 0.05 & 0.05 & 0.05 & 0.05 & 0.05 & 0.06 & 0.05 \\
\hline $\mathrm{CaO}$ & & 0.31 & 0.28 & 0.31 & 0.27 & 0.27 & 0.27 & 0.28 & 0.28 & 0.29 & 0.30 & 0.28 \\
\hline $\mathrm{CdO}$ & & $\overline{\mathrm{NA}}$ & $\mathrm{NA}$ & $\mathrm{NA}$ & NA & $\mathrm{NA}$ & NA & NA & $\mathrm{NA}$ & $\mathrm{NA}$ & $\mathrm{NA}$ & NA \\
\hline $\mathrm{CeO}_{2}$ & & NA & NA & $\mathrm{NA}$ & NA & NA & NA & $\overline{\mathrm{NA}}$ & NA & NA & NA & NA \\
\hline $\mathrm{Cl}$ & & NA & NA & NA & NA & NA & NA & NA & NA & NA & $\mathrm{NA}$ & NA \\
\hline $\mathrm{Cr}_{2} \mathrm{O}_{3}$ & & .05 & 0.06 & 0.05 & 0.05 & 0.05 & 0.05 & 0.21 & 0.05 & 0.05 & 0.05 & 0.05 \\
\hline $\mathrm{Cs}_{2} \mathrm{O}^{*}$ & & .07 & 0.04 & 0.10 & 0.08 & 0.09 & 0.10 & 0.08 & 0.08 & 0.09 & 0.08 & 0.05 \\
\hline $\mathrm{CuO}$ & & NA & NA & NA & NA & NA & NA & NA & NA & NA & $\mathrm{NA}$ & NA \\
\hline $\bar{F}$ & 04 & NA & NA & NA & $\mathrm{NA}$ & NA & NA & NA & NA & NA & NA & NA \\
\hline $\mathrm{Fe}_{2} \mathrm{O}_{3}$ & 0.39 & .51 & 9.99 & 9.39 & 9.54 & 9.53 & 9.44 & 9.94 & 9.43 & 9.46 & 9.49 & 10.10 \\
\hline $\mathrm{K}_{2} \mathrm{O}$ & 17 & 0.19 & 0.17 & 0.21 & 0.18 & 0.18 & 0.19 & 0.18 & 0.19 & 0.20 & 0.21 & 0.17 \\
\hline $\mathrm{La}_{2} \mathrm{O}_{3}$ & 32 & NA & $\mathrm{NA}$ & NA & $\mathrm{NA}$ & $\mathrm{NA}$ & $\mathrm{NA}$ & $\mathrm{NA}$ & $\mathrm{NA}$ & $\mathrm{NA}$ & NA & NA \\
\hline $\mathrm{Li}_{2} \mathrm{O}$ & 6.00 & 5.50 & 5.44 & 5.27 & 5.33 & 5.29 & 5.51 & 5.48 & 5.62 & 5.58 & 5.66 & 5.51 \\
\hline $\mathrm{MgO}$ & $\overline{06}$ & 1.22 & 1.14 & 1.28 & 1.20 & 1.18 & 1.25 & 1.27 & 1.28 & 1.30 & 1.28 & 1.21 \\
\hline $\mathrm{MnO}$ & & 2.82 & 2.70 & 2.77 & 2.82 & 2.77 & 2.81 & 2.78 & 2.81 & 2.81 & 2.80 & 2.84 \\
\hline $\mathrm{Na}_{2} \mathrm{O}$ & $\overline{59}$ & 6.72 & 6.73 & 6.39 & 6.53 & 6.48 & 6.75 & 6.73 & 6.85 & 6.78 & 6.90 & 6.78 \\
\hline $\mathrm{NiO}$ & & 0.50 & 0.48 & 0.49 & 0.47 & 0.46 & 0.47 & 0.56 & 0.49 & 0.52 & 0.52 & 0.48 \\
\hline $\mathrm{P}_{2} \mathrm{O}_{5}$ & 13 & 0.41 & 0.57 & 0.28 & 0.43 & 0.33 & 0.41 & 0.35 & 0.40 & 0.33 & 0.34 & 0.50 \\
\hline $\mathrm{PbO}$ & 15 & NA & NA & NA & NA & NA & NA & NA & NA & NA & $\mathrm{NA}$ & NA \\
\hline $\mathrm{Sb}_{2} \mathrm{O}_{3}$ & & NA & NA & NA & $\mathrm{NA}$ & NA & NA & NA & $\mathrm{NA}$ & NA & $\mathrm{NA}$ & $\overline{\mathrm{NA}}$ \\
\hline $\mathrm{SeO}_{2}$ & .15 & NA & NA & $\mathrm{NA}$ & NA & $\mathrm{NA}$ & NA & NA & $\mathrm{NA}$ & NA & NA & NA \\
\hline $\mathrm{SiO}_{2}$ & 45.54 & 44.48 & 44.45 & 44.91 & 44.50 & 44.44 & 44.64 & 43.66 & 44.33 & 37.87 & 44.18 & 45.18 \\
\hline $\mathrm{SO}_{3}$ & 0.25 & $\mathrm{NA}$ & $\mathrm{NA}$ & $\mathrm{NA}$ & NA & $\mathrm{NA}$ & NA & NA & NA & $\mathrm{NA}$ & $\mathrm{NA}$ & $\mathrm{NA}$ \\
\hline $\mathrm{SrO}$ & 2.32 & 2.04 & 2.02 & 1.95 & 2.02 & 1.97 & 2.06 & 2.02 & 2.06 & 2.05 & 2.08 & 2.08 \\
\hline $\mathrm{TeO}_{2}$ & 14 & NA & NA & NA & NA & $\mathrm{NA}$ & NA & NA & NA & $\mathrm{NA}$ & NA & NA \\
\hline $\mathrm{TiO}_{2}$ & & 0.10 & 0.09 & 0.10 & 0.09 & 0.09 & 0.10 & 0.10 & 0.10 & 0.10 & 0.11 & 0.10 \\
\hline & & $\mathrm{NA}$ & $\mathrm{NA}$ & $\mathrm{NA}$ & NA & $\mathrm{NA}$ & NA & $\mathrm{NA}$ & $\mathrm{NA}$ & $\mathrm{NA}$ & $\mathrm{NA}$ & $\mathrm{NA}$ \\
\hline $\mathrm{ZrO}_{2}$ & & 3.79 & 3.69 & 3.35 & 3.44 & 3.45 & 3.76 & 3.72 & 3.75 & 3.76 & 3.77 & 3.84 \\
\hline SUM & 99.99 & 96.48 & 96.45 & 94.80 & 95.39 & 94.82 & 96.50 & 95.54 & 96.38 & 89.75 & 97.04 & 98.05 \\
\hline
\end{tabular}

*Analyzed by AA. 
Table 5.3. DCP Analyzed Composition of Discharged Glass Samples (wt\%) (continued).

\begin{tabular}{|c|c|c|c|c|c|c|c|c|c|c|c|c|}
\hline Test\# & & \multicolumn{3}{|c|}{3} & \multicolumn{4}{|c|}{4} & \multicolumn{2}{|c|}{5} & \multicolumn{2}{|c|}{6} \\
\hline Glass $(\mathrm{kg})$ & & 10559 & 110 & & 13932 & 14449 & 145 & 540 & 15578 & 16199 & 17479 & 18100 \\
\hline Oxide & Target & $\begin{array}{c}12 \mathrm{~J}-\mathrm{G}- \\
141 \mathrm{~A}\end{array}$ & \begin{tabular}{|l|}
$12 \mathrm{~J}-\mathrm{G}-$ \\
$152 \mathrm{~B} 1$
\end{tabular} & $\begin{array}{l}12 \mathrm{~J}-\mathrm{G}- \\
152 \mathrm{~B} 2\end{array}$ & $\begin{array}{c}12 \mathrm{~K}-\mathrm{G}- \\
125 \mathrm{~A}\end{array}$ & $12 \mathrm{~K}-\mathrm{G}-$ & $12 \mathrm{~K}-\mathrm{G}-$ & $\begin{array}{l}12 \mathrm{~K}-\mathrm{G}- \\
138 \mathrm{~A}\end{array}$ & $\begin{array}{c}12 \mathrm{~L}-\mathrm{G}- \\
80 \mathrm{~A}\end{array}$ & $\begin{array}{c}12 \mathrm{~L}-\mathrm{G}- \\
119 \mathrm{~A}\end{array}$ & $\begin{array}{c}12 \mathrm{M}-\mathrm{G}- \\
70 \mathrm{~A}\end{array}$ & $\begin{array}{c}12 \mathrm{M}-\mathrm{G}- \\
117 \mathrm{~A}\end{array}$ \\
\hline $\mathrm{Al}_{2} \mathrm{O}_{3}$ & 7.40 & 7.32 & 7.48 & 7.50 & \begin{tabular}{|l|}
7.21 \\
\end{tabular} & 7.14 & 7.22 & 6.91 & 7.37 & 6.87 & 6.90 & 6.96 \\
\hline $\mathrm{As}_{2} \mathrm{O}_{3}$ & 0.04 & NA & NA & NA & 0.02 & 0.04 & 0.01 & 0.00 & 0.02 & 0.00 & 0.02 & 0.00 \\
\hline $\mathrm{B}_{2} \mathrm{O}_{3}$ & 10.00 & 10.68 & 10.73 & 11.32 & 10.13 & 10.20 & 10.21 & 9.97 & 10.26 & 9.75 & 9.63 & 9.46 \\
\hline $\mathrm{BaO}$ & 0.04 & 0.05 & 0.05 & 0.05 & 0.05 & 0.05 & 0.05 & 0.05 & 0.05 & 0.05 & 0.05 & 0.05 \\
\hline $\mathrm{CaO}$ & 0.25 & 0.28 & 0.27 & 0.28 & 0.27 & 0.27 & 0.28 & 0.26 & 0.28 & 0.27 & 0.45 & 0.50 \\
\hline $\mathrm{CdO}$ & 0.37 & $\mathrm{NA}$ & $\overline{\mathrm{NA}}$ & $\mathrm{NA}$ & 0.36 & 0.35 & 0.33 & 0.36 & 0.33 & 0.33 & 0.34 & 0.34 \\
\hline $\mathrm{CeO}_{2}$ & 0.08 & NA & NA & NA & NA & $\mathrm{NA}$ & $\mathrm{NA}$ & NA & $\mathrm{NA}$ & $\mathrm{NA}$ & NA & $\mathrm{NA}$ \\
\hline $\mathrm{Cl}$ & 0.01 & NA & NA & NA & NA & NA & NA & NA & NA & $\mathrm{NA}$ & NA & NA \\
\hline $\mathrm{Cr}_{2} \mathrm{O}_{3}$ & 0.04 & 0.06 & 0.06 & 0.06 & 0.05 & 0.05 & 0.05 & 0.05 & 0.05 & 0.04 & 0.04 & 0.04 \\
\hline $\mathrm{Cs}_{2} \mathrm{O}^{* *}$ & 0.08 & 0.08 & 0.09 & 0.04 & 0.08 & 0.08 & 0.08 & 0.05 & 0.07 & 0.07 & 0.07 & 0.07 \\
\hline $\mathrm{CuO}$ & 0.03 & $\overline{\mathrm{NA}}$ & $\mathrm{NA}$ & $\mathrm{NA}$ & 0.03 & 0.03 & 0.03 & 0.02 & 0.03 & 0.03 & 0.03 & 0.03 \\
\hline $\mathrm{F}$ & 0.04 & NA & $\overline{\mathrm{NA}}$ & NA & NA & $\mathrm{NA}$ & $\mathrm{NA}$ & NA & NA & NA & $\mathrm{NA}$ & $\mathrm{NA}$ \\
\hline $\mathrm{Fe}_{2} \mathrm{O}_{3}$ & 10.39 & 9.62 & 9.60 & 9.97 & 9.33 & 9.21 & 9.28 & 9.37 & 9.56 & 9.26 & 9.85 & 9.94 \\
\hline $\mathrm{K}_{2} \mathrm{O}$ & 0.17 & 0.18 & 0.18 & 0.17 & 0.19 & 0.19 & 0.20 & 0.19 & 0.20 & 0.19 & 0.19 & 0.21 \\
\hline $\mathrm{La}_{2} \mathrm{O}_{3}$ & 0.32 & $\mathrm{NA}$ & NA & NA & $\mathrm{NA}$ & NA & NA & NA & NA & NA & NA & $\mathrm{NA}$ \\
\hline $\mathrm{Li}_{2} \mathrm{O}$ & 6.00 & 5.23 & 5.22 & 5.25 & 5.18 & 5.09 & 5.16 & 4.93 & 5.19 & 4.87 & 4.78 & 4.69 \\
\hline $\mathrm{MgO}$ & 0.06 & 1.27 & 1.24 & 1.22 & 1.29 & 1.29 & 1.32 & 1.21 & 1.30 & 1.25 & 1.26 & 1.15 \\
\hline $\mathrm{MnO}$ & 3.03 & 2.65 & 2.68 & 2.71 & 2.49 & 2.51 & 2.52 & 2.47 & 3.09 & 2.94 & 2.99 & 2.91 \\
\hline $\mathrm{Na}_{2} \mathrm{O}$ & 6.59 & 6.38 & 6.47 & 6.51 & 6.25 & 6.15 & 6.25 & 6.00 & 6.33 & 5.94 & 5.88 & 5.78 \\
\hline $\mathrm{NiO}$ & 0.54 & 0.48 & 0.47 & 0.50 & 0.46 & 0.46 & 0.47 & 0.45 & 0.43 & 0.43 & 0.45 & 0.45 \\
\hline $\mathrm{P}_{2} \mathrm{O}_{5}$ & 0.13 & 0.43 & 0.42 & 0.48 & 0.35 & 0.32 & 0.34 & 0.39 & 0.38 & 0.33 & 0.29 & 0.36 \\
\hline $\mathrm{PbO}$ & 0.15 & $\mathrm{NA}$ & $\mathrm{NA}$ & $\mathrm{NA}$ & 0.18 & 0.18 & 0.18 & 0.18 & 0.17 & 0.16 & 0.17 & 0.16 \\
\hline $\mathrm{Sb}_{2} \mathrm{O}_{3}$ & 0.21 & NA & NA & NA & 0.18 & 0.14 & 0.11 & 0.05 & 0.18 & 0.18 & 0.19 & 0.18 \\
\hline $\mathrm{SeO}_{2}$ & 0.15 & NA & $\mathrm{NA}$ & $\mathrm{NA}$ & 0.04 & 0.01 & 0.03 & 0.00 & 0.01 & 0.07 & 0.05 & 0.04 \\
\hline $\mathrm{SiO}_{2}$ & 45.54 & 40.72 & 44.33 & 45.65 & 43.01 & 38.99 & 43.71 & 42.96 & 43.35 & 44.64 & 44.56 & 44.61 \\
\hline $\mathrm{SO}_{3} * * *$ & 0.25 & NA & NA & NA & 0.18 & 0.21 & 0.31 & 0.16 & $\mathrm{NA}$ & NA & NA & NA \\
\hline $\mathrm{SrO}$ & 2.32 & 1.97 & 1.99 & 1.99 & 1.81 & 1.82 & 1.82 & 1.94 & 1.85 & 1.73 & 1.81 & 1.81 \\
\hline $\mathrm{TeO}_{2}$ & 0.14 & NA & NA & NA & 0.08 & 0.09 & 0.05 & 0.00 & 0.17 & 0.15 & 0.17 & 0.13 \\
\hline $\mathrm{TiO}_{2}$ & 0.06 & 0.10 & 0.10 & 0.10 & 0.11 & 0.11 & 0.12 & 0.11 & 0.13 & 0.13 & 0.17 & 0.19 \\
\hline $\mathrm{ZnO}$ & 2.00 & NA & NA & $\mathrm{NA}$ & 1.75 & 1.75 & 1.73 & 1.73 & 1.80 & 1.77 & 1.92 & 1.95 \\
\hline $\mathrm{ZrO}_{2}$ & 3.56 & 3.66 & 3.69 & 3.75 & 3.43 & 3.48 & 3.48 & 3.41 & 3.52 & 3.34 & 3.48 & 3.53 \\
\hline SUM & 99.99 & 91.16 & 95.06 & 97.57 & 94.51 & 90.22 & 95.32 & 93.23 & 96.13 & 94.78 & 95.76 & 95.54 \\
\hline
\end{tabular}

** Analyzed by AA.

*** Analyzed by IC. 
The Catholic University of America Vitreous State Laboratory
DuraMelter 1200 Tests with AZ-101HLW Simulants

Final Report, VSL-02R0100-2, Rev.1

Table 5.3. DCP Analyzed Composition of Discharged Glass Samples (wt\%) (continued).

\begin{tabular}{|c|c|c|c|c|c|c|c|c|}
\hline Test\# & & 7 & & 8 & & 9 & & \\
\hline Glass (kg) & & 21038 & 24076 & 25020 & 25607 & 267 & & \\
\hline Oxide & Target & $\begin{array}{c}12 \mathrm{~N}-\mathrm{G}- \\
63 \mathrm{~A}\end{array}$ & $\begin{array}{c}12 \mathrm{~N}-\mathrm{G}- \\
126 \mathrm{~B}\end{array}$ & $\begin{array}{c}120-\mathrm{G}- \\
68 \mathrm{~A}\end{array}$ & $\begin{array}{c}12 \mathrm{O}-\mathrm{G}- \\
125 \mathrm{~A}\end{array}$ & $\begin{array}{c}12 \mathrm{P}-\mathrm{G}- \\
42 \mathrm{Al}\end{array}$ & $\begin{array}{c}12 \mathrm{P}-\mathrm{G}- \\
42 \mathrm{~A} 2\end{array}$ & $\begin{array}{c}\text { Overall } \\
\text { Avg. }\end{array}$ \\
\hline $\mathrm{Al}_{2} \mathrm{O}_{3}$ & 7.40 & 7.50 & 7.74 & 7.22 & 7.06 & 7.11 & 7.14 & 7.49 \\
\hline $\mathrm{As}_{2} \mathrm{O}_{3}$ & 0.04 & 0.01 & 0.02 & 0.03 & 0.03 & 0.00 & 0.02 & 0.03 \\
\hline $\mathrm{B}_{2} \mathrm{O}_{3}$ & 10.00 & 9.39 & 9.31 & 9.47 & 9.51 & 9.84 & 9.75 & 10.37 \\
\hline $\mathrm{BaO}$ & 0.04 & 0.05 & 0.05 & 0.05 & 0.05 & 0.05 & 0.05 & 0.05 \\
\hline $\mathrm{CaO}$ & 0.25 & 0.56 & 0.57 & 0.46 & 0.42 & 0.36 & 0.33 & 0.33 \\
\hline$\overline{\mathrm{CdO}}$ & 0.37 & 0.33 & 0.32 & 0.34 & 0.33 & 0.30 & 0.29 & 0.33 \\
\hline $\mathrm{CeO}_{2}$ & 0.08 & NA & $\mathrm{NA}$ & NA & NA & NA & NA & $\mathrm{NC}$ \\
\hline$\overline{\mathrm{Cl}}$ & 0.01 & NA & NA & NA & NA & NA & NA & $\mathrm{NC}$ \\
\hline $\mathrm{Cr}_{2} \mathrm{O}_{3}$ & 0.04 & 0.05 & 0.05 & 0.04 & 0.04 & 0.04 & 0.04 & 0.05 \\
\hline $\mathrm{Cs}_{2} \mathrm{O}^{* *}$ & 0.08 & 0.07 & 0.07 & 0.07 & 0.07 & $\mathrm{NA}$ & NA & 0.08 \\
\hline $\mathrm{CuO}$ & 0.03 & 0.03 & 0.02 & 0.02 & 0.03 & 0.03 & 0.02 & 0.03 \\
\hline $\bar{F}$ & 0.04 & $\mathrm{NA}$ & NA & $\mathrm{NA}$ & $\mathrm{NA}$ & $\mathrm{NA}$ & $\mathrm{NA}$ & $\mathrm{NC}$ \\
\hline$\overline{\mathrm{Fe}_{2} \mathrm{O}_{3}}$ & 10.39 & 9.94 & 9.64 & 9.84 & 9.52 & 9.72 & 9.25 & 9.51 \\
\hline $\mathrm{K}_{2} \mathrm{O}$ & 0.17 & 0.21 & 0.21 & 0.20 & 0.20 & 0.20 & 0.18 & 0.19 \\
\hline $\mathrm{La}_{2} \mathrm{O}_{3}$ & 0.32 & NA & $\overline{\mathrm{NA}}$ & NA & $\mathrm{NA}$ & NA & $\mathrm{NA}$ & $\mathrm{NC}$ \\
\hline $\mathrm{Li}_{2} \mathrm{O}$ & 6.00 & 4.71 & 4.79 & 4.69 & 4.73 & 4.87 & 4.82 & 5.20 \\
\hline $\mathrm{MgO}$ & 0.06 & 0.81 & 0.65 & 0.86 & 0.97 & 1.12 & 1.04 & 1.11 \\
\hline $\mathrm{MnO}$ & 3.03 & 3.02 & 3.14 & 2.87 & 2.87 & 3.08 & 3.10 & 2.79 \\
\hline $\mathrm{Na}_{2} \mathrm{O}$ & 6.59 & 5.78 & 5.84 & 5.68 & 5.74 & 5.93 & 5.97 & 6.37 \\
\hline $\mathrm{NiO}$ & 0.54 & 0.48 & 0.46 & 0.44 & 0.44 & 0.46 & 0.46 & 0.47 \\
\hline$\overline{\mathrm{P}_{2} \mathrm{O}_{5}}$ & 0.13 & 0.23 & 0.19 & 0.32 & 0.29 & 0.33 & 0.16 & 0.34 \\
\hline $\mathrm{PbO}$ & 0.15 & 0.17 & 0.16 & 0.16 & 0.16 & 0.16 & 0.14 & 0.17 \\
\hline $\mathrm{Sb}_{2} \mathrm{O}_{3}$ & 0.21 & 0.19 & 0.19 & 0.18 & 0.19 & 0.18 & 0.20 & 0.17 \\
\hline $\mathrm{SeO}_{2}$ & 0.15 & 0.01 & 0.02 & 0.05 & 0.03 & 0.01 & 0.00 & 0.03 \\
\hline $\mathrm{SiO}_{2}$ & 45.54 & 45.22 & 43.71 & 45.08 & 44.25 & 44.88 & 45.07 & 43.30 \\
\hline $\mathrm{SO}_{3}$ & 0.25 & NA & NA & NA & NA & NA & NA & $\mathrm{NC}$ \\
\hline SrO & 2.32 & 1.86 & 1.88 & 1.79 & 1.78 & 1.81 & 1.93 & 1.95 \\
\hline $\mathrm{TeO}_{2}$ & 0.14 & 0.11 & 0.13 & 0.13 & 0.14 & 0.10 & 0.19 & 0.12 \\
\hline $\mathrm{TiO}_{2}$ & 0.06 & 0.24 & 0.23 & 0.20 & 0.18 & 0.15 & 0.16 & 0.12 \\
\hline $\mathrm{ZnO}$ & 2.00 & 2.00 & 1.98 & 1.90 & 1.91 & 1.89 & 1.91 & 1.86 \\
\hline$\overline{\mathrm{ZrO}}$ & 3.56 & 3.55 & 3.52 & 3.48 & 3.43 & 3.46 & 3.47 & 3.61 \\
\hline SUM & 99.99 & 96.51 & 94.89 & 95.56 & 94.36 & 96.08 & 95.68 & 94.51 \\
\hline
\end{tabular}

** Analyzed by AA.

NC: Not Calculated. 
Table 5.4. Measured Iron Redox of Discharged Glasses.

\begin{tabular}{|c|c|c|c|c|}
\hline Test \# & $\begin{array}{l}\text { Discharge } \\
\text { Date }\end{array}$ & Sample I.D. & $\begin{array}{l}\text { Cumulative } \\
\text { Mass (kg) }\end{array}$ & $\begin{array}{c}\mathrm{Fe}^{2+} / \mathrm{Fe}_{\mathrm{tot}} \\
(\%)\end{array}$ \\
\hline 1 & 07/03/01 & $12 \mathrm{G}-\mathrm{G}-79 \mathrm{~A}$ & 2301.0 & 1.9 \\
\hline \multirow{7}{*}{2} & $07 / 24 / 01$ & $12 \mathrm{G}-\mathrm{G}-128 \mathrm{~A}$ & 2561.0 & 3.0 \\
\hline & $07 / 24 / 01$ & $12 \mathrm{G}-\mathrm{G}-138 \mathrm{~A}$ & 2827.0 & 6.3 \\
\hline & $07 / 25 / 01$ & $12 \mathrm{G}-\mathrm{G}-150 \mathrm{~A}$ & 3035.0 & 9.1 \\
\hline & $07 / 26 / 01$ & $12 \mathrm{H}-\mathrm{G}-22 \mathrm{~A}$ & 3297.5 & 11.3 \\
\hline & $07 / 26 / 01$ & $12 \mathrm{H}-\mathrm{G}-26 \mathrm{~A}$ & 3452.0 & 12.7 \\
\hline & $07 / 27 / 01$ & $12 \mathrm{H}-\mathrm{G}-40 \mathrm{C}$ & 3699.0 & 13.7 \\
\hline & $07 / 29 / 01$ & $12 \mathrm{I}-\mathrm{G}-35 \mathrm{~A}$ & 4121.5 & 9.4 \\
\hline 3 & $08 / 11 / 01$ & $12 \mathrm{~J}-\mathrm{G}-152 \mathrm{~B}$ & 11074.5 & 1.6 \\
\hline 4 & $08 / 25 / 01$ & $12 \mathrm{~K}-\mathrm{G}-138 \mathrm{~A}$ & 14539.5 & 2.1 \\
\hline \multirow{4}{*}{5} & $09 / 27 / 01$ & $12 \mathrm{~L}-\mathrm{G}-46 \mathrm{~A}$ & 15055.5 & $<0.4$ \\
\hline & $09 / 29 / 01$ & $12 \mathrm{~L}-\mathrm{G}-80 \mathrm{~A}$ & 15578.0 & $<0.4$ \\
\hline & $09 / 30 / 01$ & $12 \mathrm{~L}-\mathrm{G}-101 \mathrm{~A}$ & 15892.0 & $<0.4$ \\
\hline & $10 / 01 / 01$ & $12 \mathrm{~L}-\mathrm{G}-119 \mathrm{~A}$ & 16198.5 & $<0.4$ \\
\hline \multirow{3}{*}{6} & $10 / 12 / 01$ & $12 \mathrm{M}-\mathrm{G}-19 \mathrm{~A}$ & 16705.5 & 1.7 \\
\hline & $10 / 14 / 01$ & $12 \mathrm{M}-\mathrm{G}-50 \mathrm{~A}$ & 17221.5 & 3.5 \\
\hline & $10 / 15 / 01$ & $12 \mathrm{M}-\mathrm{G}-70 \mathrm{~A}$ & 17478.5 & 4.5 \\
\hline \multirow{6}{*}{7} & $10 / 24 / 01$ & $12 \mathrm{~N}-\mathrm{G}-38 \mathrm{C}$ & 20017.0 & 5.1 \\
\hline & $10 / 25 / 01$ & $12 \mathrm{~N}-\mathrm{G}-51 \mathrm{C}$ & 20537.5 & 6.5 \\
\hline & $10 / 25 / 01$ & $12 N-G-63 A$ & 21038.0 & 5.8 \\
\hline & $10 / 26 / 01$ & $12 \mathrm{~N}-\mathrm{G}-71 \mathrm{~A}$ & 21513.5 & 6.0 \\
\hline & $10 / 26 / 01$ & $12 \mathrm{~N}-\mathrm{G}-85 \mathrm{~A}$ & 22028.5 & 5.8 \\
\hline & $10 / 26 / 01$ & $12 \mathrm{~N}-\mathrm{G}-88 \mathrm{D}$ & 22492.0 & 4.8 \\
\hline \multirow[b]{2}{*}{8} & $10 / 31 / 01$ & $12 \mathrm{O}-\mathrm{G}-31 \mathrm{~A}$ & 24484.0 & 2.0 \\
\hline & $11 / 02 / 01$ & $120-\mathrm{G}-68 \mathrm{~A}$ & 25019.5 & 1.2 \\
\hline \multirow{5}{*}{9} & $11 / 06 / 01$ & $120-\mathrm{G}-107 \mathrm{~A}$ & 25330.5 & 2.9 \\
\hline & $11 / 07 / 01$ & $120-\mathrm{G}-125 \mathrm{~A}$ & 25607.0 & 8.6 \\
\hline & $11 / 08 / 01$ & $120-G-144 \mathrm{~A}$ & 25878.5 & 11.1 \\
\hline & $11 / 09 / 01$ & $12 \mathrm{P}-\mathrm{G}-12 \mathrm{~A}$ & 26201.5 & 17.5 \\
\hline & $11 / 10 / 01$ & 12P-G-42A & 26725.5 & 11.9 \\
\hline
\end{tabular}


The Catholic University of America Vitreous State Laboratory
DuraMelter 1200 Tests with AZ-101HLW Simulants Final Report, VSL-02R0100-2, Rev.I

Table 6.1. Summary of Off-Gas Particulate Sampling Results During DM 1200 AZ-101 Tests.

\begin{tabular}{|c|c|c|c|c|c|c|c|c|c|}
\hline Test \# & Date & Location & Total PM (grams) & $\begin{array}{c}\text { Meter Volume } \\
\text { (dscf) }\end{array}$ & $\begin{array}{c}\text { Concentration } \\
\text { (mg/dscf) }\end{array}$ & Flow Rate (dscfm) & $\begin{array}{l}\text { Particulate Emission } \\
\text { Rate }(\mathrm{mg} / \mathrm{min})\end{array}$ & $\begin{array}{c}\text { Moisture } \\
(\% \text { vol. })\end{array}$ & $\begin{array}{c}\% \\
\text { Isokinteic }\end{array}$ \\
\hline \multirow{9}{*}{1} & $6 / 29 / 01$ & Melter Outlet & 0.0929 & 60.670 & 1.5311 & 158.68 & 243.0 & 13.00 & 104 \\
\hline & $6 / 29 / 01$ & Scrubber Outlet & 0.0046 & 79.768 & 0.0580 & 197.30 & 11.5 & 2.99 & 102 \\
\hline & $6 / 29 / 01$ & WESP Outlet & 0.0022 & 101.724 & 0.0218 & 160.66 & 3.51 & 2.75 & 102 \\
\hline & $6 / 29 / 01$ & Melter Outlet & 0.0537 & 53.850 & 0.9980 & 142.39 & 142.1 & 13.84 & 103 \\
\hline & $6 / 29 / 01$ & Scrubber Outlet & 0.0081 & 64.561 & 0.1255 & 161.35 & 20.2 & 2.86 & 101 \\
\hline & $6 / 29 / 01$ & WESP Outlet & 0.0033 & 110.421 & 0.0295 & 180.73 & 5.34 & 2.90 & 99 \\
\hline & $6 / 30 / 01$ & Melter Outlet & 0.0296 & 54.083 & 0.5475 & 147.59 & 80.8 & 9.22 & 100 \\
\hline & $6 / 30 / 01$ & Scrubber Outlet & 0.0047 & 69.519 & 0.0672 & 171.88 & 11.5 & 2.53 & 102 \\
\hline & $6 / 30 / 01$ & WESP Outlet & 0.0036 & 104.712 & 0.0345 & 169.62 & 5.85 & 2.63 & 100 \\
\hline \multirow{7}{*}{2} & $7 / 29 / 01$ & Melter Outlet & 0.0745 & 48.815 & 1.5252 & 160.77 & 245.2 & 9.16 & 87 \\
\hline & $7 / 29 / 01$ & Scrubber Outlet & 0.0633 & 204.385 & 0.3097 & 187.80 & 58.2 & 2.50 & 109 \\
\hline & $7 / 29-31 / 01$ & WESP Outlet & 0.0023 & 1862.443 & 0.0012 & 214.48 & 0.27 & 2.7 & 97 \\
\hline & $7 / 30 / 01$ & Melter Outlet & 0.0507 & 46.429 & 1.0913 & 154.71 & 168.8 & 9.59 & 86 \\
\hline & $7 / 30 / 01$ & Scrubber Outlet & 0.0456 & 221.418 & 0.2059 & 212.22 & 43.7 & 2.17 & 105 \\
\hline & $7 / 31 / 01$ & Melter Outlet & 0.0596 & 49.837 & 1.1964 & 160.39 & 191.9 & 8.43 & 85 \\
\hline & $7 / 30 / 01$ & Scrubber Outlet & 0.0248 & 146.001 & 0.1698 & 214.40 & 36.4 & 2.36 & 105 \\
\hline \multirow{8}{*}{3} & $8 / 8 / 01$ & Melter Outlet & 0.7240 & 34.847 & 20.7765 & 163.33 & 3393.4 & 29.32 & 59 \\
\hline & $8 / 8 / 01$ & Scrubber Outlet & 0.1388 & 151.39 & 0.9168 & 210.12 & 192.6 & 8.13 & 102 \\
\hline & $8 / 9 / 01$ & Melter Outlet & 1.4912 & 34.433 & 43.3069 & 132.85 & 5753.3 & 40.03 & 111 \\
\hline & $8 / 9 / 01$ & Scrubber Outlet & 0.0986 & 98.818 & 0.9974 & 195.49 & 195.0 & 12.24 & 108 \\
\hline & $8 / 10 / 01$ & Melter Outlet & 0.7026 & 18.24 & 38.5173 & 102.39 & 3943.8 & 41.16 & 114 \\
\hline & $8 / 10 / 01$ & Scrubber Outlet & 0.1269 & 183.744 & 0.6906 & 189.49 & 130.9 & 7.55 & 97 \\
\hline & $8 / 8-10 / 01$ & WESP Outlet & 0.0083 & 1632.622 & 0.0051 & 207.216 & 1.06 & 8.14 & 102 \\
\hline & $8 / 10 / 01$ & Melter Outlet & 0.5403 & 18.079 & 29.8872 & 129.28 & 3863.8 & 33.49 & 102 \\
\hline \multirow{7}{*}{4} & $8 / 22 / 01$ & Melter Outlet & 0.4105 & 35.058 & 11.71 & 151.14 & 1770 & 23.8 & 99 \\
\hline & $8 / 22 / 01$ & Scrubber Outlet & 0.0602 & 97.783 & 0.6208 & 200.10 & 124.2 & 5.25 & 98 \\
\hline & $8 / 23 / 01$ & Melter Outlet & 0.2631 & 23.701 & 11.10 & 149.85 & 1664 & 25.2 & 101 \\
\hline & $8 / 23 / 01$ & Scrubber Outlet & 0.0476 & 72.412 & 0.6573 & 195.61 & 128.6 & 5.27 & 99 \\
\hline & $8 / 24 / 01$ & Melter Outlet & 0.4159 & 35.163 & 11.83 & 153.00 & 1810 & 25.4 & 98 \\
\hline & $8 / 24 / 01$ & Scrubber Outlet & 0.0508 & 94.582 & 0.5371 & 193.42 & 103.9 & 5.14 & 98 \\
\hline & $8 / 22-24 / 01$ & WESP Outlet & 0.0060 & 1752.331 & 0.0034 & 192.59 & 0.66 & 5.46 & 102 \\
\hline
\end{tabular}


The Catholic University of America Vitreous State Laboratory
DuraMelter 1200 Tests with AZ-101 HLW Simulants

Final Report, VSL-02R0100-2, Rev.I

Table 6.1. Summary of Off-Gas Particulate Sampling Results During DM 1200 AZ-101 Tests (continued).

\begin{tabular}{|c|c|c|c|c|c|c|c|c|c|}
\hline Test & Date & Location & $\begin{array}{c}\text { Total } \\
\text { PM (g) }\end{array}$ & $\begin{array}{c}\text { Meter } \\
\text { Volume } \\
\text { (dscf) }\end{array}$ & $\begin{array}{l}\text { Concentration } \\
\text { (mg/dscf) }\end{array}$ & $\begin{array}{c}\text { Flow } \\
\text { Rate } \\
\text { (dscfm) }\end{array}$ & $\begin{array}{l}\text { Particulate } \\
\text { Emission } \\
\text { Rate } \\
\text { (mg/min) }\end{array}$ & $\begin{array}{l}\text { Moisture } \\
\text { (\% vol.) }\end{array}$ & $\begin{array}{c}\% \\
\text { Isokinetic }\end{array}$ \\
\hline \multirow{7}{*}{5} & $9 / 28 / 01$ & Melter Outlet & 0.2223 & 54.496 & 4.097 & 178 & 730 & 8.14 & 96 \\
\hline & $9 / 28 / 01$ & Scrubber Outlet & 0.0351 & 156.7 & 0.224 & 209 & 46.8 & 2.45 & 100 \\
\hline & $9 / 29 / 01$ & Melter Outlet & 0.2427 & 43.943 & 5.524 & 174 & 958 & 9.79 & 100 \\
\hline & $9 / 29 / 01$ & Scrubber Outlet & 0.0346 & 70.374 & 0.491 & 208 & 102 & 3.73 & 102 \\
\hline & $9 / 30 / 01$ & Melter Outlet & 0.2700 & 40.915 & 6.598 & 175 & 1161 & 9.42 & 98 \\
\hline & $9 / 30 / 01$ & Scrubber Outlet & 0.0163 & 57.389 & 0.491 & 211 & 59.9 & 2.64 & 109 \\
\hline & $9 / 28-30 / 01$ & WESP Outlet & 0.0076 & 1989.5 & 0.0038 & 212 & 0.81 & 3.43 & 104 \\
\hline \multirow{7}{*}{6} & $10 / 15 / 01$ & Melter Outlet & 0.1066 & 88.28 & 1.2075 & 189 & 228.2 & 6.16 & 91 \\
\hline & $10 / 15 / 01$ & Scrubber Outlet & 0.0286 & 214 & 0.1336 & 218 & 29.2 & 2.11 & 98 \\
\hline & $10 / 16 / 01$ & Melter Outlet & 0.1029 & 75.631 & 1.3606 & 168 & 229.0 & 9.27 & 94 \\
\hline & $10 / 16 / 01$ & Scrubber Outlet & 0.0272 & 73.319 & 0.3710 & 207 & 77.0 & 2.73 & 94 \\
\hline & $10 / 17 / 01$ & Melter Outlet & 0.0735 & 77.913 & 0.9434 & 172 & 162.0 & 7.1 & 95 \\
\hline & $10 / 17 / 01$ & Scrubber Outlet & 0.0134 & 105.971 & 0.1264 & 199.8 & 25.3 & 2.18 & 98 \\
\hline & $10 / 15-17 / 01$ & WESP Outlet & 0.0071 & 2083 & 0.0034 & 227 & 0.77 & 2.5 & 103 \\
\hline \multirow{7}{*}{7} & $10 / 23 / 01$ & Melter Outlet & 0.4596 & 35.823 & 12.83 & 179 & 2294 & 26.08 & 120 \\
\hline & $10 / 23 / 01$ & Scrubber Outlet & 0.0762 & 39.672 & 1.921 & 271 & 320 & 6.54 & 103 \\
\hline & $10 / 24 / 01$ & Melter Outlet & 0.4522 & 33.568 & 13.471 & 163 & 2206 & 30.96 & 144 \\
\hline & $10 / 24 / 01$ & Scrubber Outlet & 0.0713 & 33.792 & 2.11 & 269 & 568 & 7.42 & 88 \\
\hline & $10 / 25 / 01$ & Melter Outlet & 0.4070 & 33.352 & 12.203 & 175 & 2135 & 32.85 & 134 \\
\hline & $10 / 25 / 01$ & Scrubber Outlet & 0.0749 & 43.417 & 1.725 & 287 & 496 & 7.63 & 106 \\
\hline & $10 / 23-25 / 01$ & WESP Outlet & 0.0134 & 1875 & 0.0071 & 215 & 1.53 & 6.0 & 106 \\
\hline \multirow{7}{*}{8} & $10 / 30 / 01$ & Melter Outlet & 0.3402 & 40.326 & 8.436 & 160.5 & 1354 & 11.05 & 101 \\
\hline & $10 / 30 / 01$ & Scrubber Outlet & 0.1065 & 94.384 & 1.128 & 193.9 & 218.8 & 3.43 & 98 \\
\hline & $10 / 31 / 01$ & Melter Outlet & 0.2536 & 40.420 & 6.274 & 170 & 1066.4 & 8.67 & 95 \\
\hline & $10 / 31 / 01$ & Scrubber Outlet & 0.0332 & 35.312 & 0.9402 & 194.2 & 182.6 & 3.26 & 87 \\
\hline & $11 / 1 / 01$ & Melter Outlet & 0.2902 & 34.405 & 8.435 & 172.3 & 1453.4 & 10.58 & 96 \\
\hline & \begin{tabular}{|l|}
$11 / 1 / 01$ \\
\end{tabular} & Scrubber Outlet & 0.0466 & 43.622 & 1.068 & 199.7 & 213.3 & 2.85 & 88 \\
\hline & $10 / 30-11 / 1 / 01$ & WESP Outlet & 0.0104 & 1927.4 & 0.0054 & 203 & 1.09 & 2.75 & 106 \\
\hline \multirow{2}{*}{$9 \mathrm{a}$} & $11 / 7 / 01$ & Melter Outlet & 0.1185 & 56.838 & 2.085 & 169.86 & 354.1 & 9.36 & 101 \\
\hline & $11 / 7 / 01$ & Scrubber Outlet & 0.0581 & 97.648 & 0.595 & 200.39 & 119.2 & 2.5 & 98 \\
\hline \multirow{2}{*}{$9 a-b$} & $11 / 8 / 01$ & Scrubber Outlet & 0.0553 & 56.574 & 0.9775 & 199.18 & 194.7 & 2.59 & 98 \\
\hline & $11 / 7-9 / 01$ & WESP Outlet & 0.0163 & 1856.4 & 0.0088 & 196 & 1.72 & 2.09 & 105 \\
\hline \multirow{3}{*}{$9 b$} & $11 / 8 / 01$ & Melter Outlet & 0.1524 & 40.874 & 3.729 & 150.19 & 560 & 11.39 & 109 \\
\hline & $11 / 9 / 01$ & Melter Outlet & 0.1719 & 53.308 & 3.225 & 215.01 & 693.3 & 8.41 & 99 \\
\hline & $11 / 9 / 01$ & Scrubber Outlet & 0.1335 & 97.838 & 1.365 & 196.6 & 268.3 & 2.52 & 100 \\
\hline
\end{tabular}


The Catholic University of America Vitreous State Laboratory
DuraMelter 1200 Tests with AZ-101HLW Simulants Final Report, VSL-02R0100-2, Rev.I

Table 6.2. Average Carryover and DF Values from Melter, SBS, and WESP.

\begin{tabular}{|c|c|c|c|c|c|c|c|c|c|c|c|}
\hline \multirow[b]{2}{*}{ Test } & \multirow{2}{*}{$\begin{array}{c}\text { Solids } \\
\text { Feed } \\
\text { Flux* } \\
\text { (g/min) }\end{array}$} & \multicolumn{3}{|c|}{ Melter } & \multicolumn{3}{|c|}{ SBS } & \multicolumn{3}{|c|}{ WESP } & \multirow{2}{*}{$\begin{array}{c}\text { Cumulative } \\
\text { DF across } \\
\text { Melter, } \\
\text { SBS and } \\
\text { WESP }\end{array}$} \\
\hline & & $\begin{array}{l}\text { Outlet } \\
\text { Flux } \\
\text { (mg/min) }\end{array}$ & DF & $\begin{array}{c}\% \\
\text { emitted }\end{array}$ & $\begin{array}{l}\text { Outlet } \\
\text { Flux } \\
\text { (mg/min }\end{array}$ & $\mathrm{DF}$ & $\begin{array}{c}\% \\
\text { emitted }\end{array}$ & $\begin{array}{c}\text { Outlet } \\
\text { Flux } \\
\text { (mg/min) }\end{array}$ & DF & $\begin{array}{c}\% \\
\text { emitted }\end{array}$ & \\
\hline 1 & 317.2 & 155 & 2042 & 0.05 & 14.4 & 11.0 & 9.3 & $\mathrm{NC}$ & $\mathrm{NC}$ & $\mathrm{NC}$ & $\mathrm{NC}$ \\
\hline 2 & 232.2 & 202 & 1149 & 0.09 & 46.1 & 4.0 & 22.8 & 0.27 & 171 & 0.58 & 859878 \\
\hline 3 & 1036.3 & 4520 & 229 & 0.44 & 172.8 & 26.0 & 3.8 & 1.06 & 163 & 0.61 & 977673 \\
\hline 4 & 536.7 & 1748 & 307 & 0.33 & 118.9 & 14.7 & 6.8 & 0.66 & 180 & 0.56 & 813132 \\
\hline 5 & 275.3 & 950 & 290 & 0.34 & 69.6 & 13.6 & 7.3 & 0.81 & 85.9 & 1.2 & 344166 \\
\hline 6 & 160.3 & 206 & 777 & 0.13 & 43.8 & 4.7 & 21.3 & 0.77 & 56.9 & 1.8 & 208225 \\
\hline 7 & 957.7 & 2212 & 433 & 0.23 & 461.3 & 4.8 & 20.9 & 1.53 & 302 & 0.33 & 625926 \\
\hline 8 & 285.2 & 1291 & 221 & 0.45 & 204.9 & 6.3 & 15.9 & 1.09 & 188 & 0.53 & 259243 \\
\hline $9 \mathrm{ab}$ & 281.8 & 536 & 526 & 0.19 & 194.1 & 2.8 & 36.2 & 1.72 & 113 & 0.89 & 165784 \\
\hline
\end{tabular}

* - Calculated from average feed rate during sampling periods only (as opposed to the entire test) and the percent solids after drying at $110^{\circ} \mathrm{C}$.

$\mathrm{NC}-$ Not Calculated due to insufficient sampling time. 
Table 6.3. Melter Emission Rates for DM 1200 AZ101 Test 1.

\begin{tabular}{|c|c|c|c|c|c|c|c|}
\hline & $\begin{array}{l}\text { Average } \\
\text { Feed Flux } \\
(\mathrm{mg} / \mathrm{min})\end{array}$ & $\begin{array}{c}\text { Run } 1 \\
(\mathrm{mg} / \mathrm{min})\end{array}$ & $\begin{array}{c}\text { Run } 2 \\
(\mathrm{mg} / \mathrm{min})\end{array}$ & $\begin{array}{c}\text { Run } 3 \\
(\mathrm{mg} / \mathrm{min})\end{array}$ & $\begin{array}{l}\text { Average } \\
(\mathrm{mg} / \mathrm{min})\end{array}$ & $\begin{array}{c}\text { DF } \\
\text { Across } \\
\text { Melter }\end{array}$ \\
\hline \multirow{29}{*}{ 畩 } & Total $^{\$}$ & 317167 & 243.0 & 142.1 & 80.8 & 155.3 & 2042 \\
\hline & $\mathrm{Al}$ & 12425 & 7.38 & 0.69 & 1.05 & 3.04 & 4090 \\
\hline & As & 96 & 0.84 & 0.30 & $<0.09$ & $<0.41$ & $>234$ \\
\hline & $\mathrm{B}$ & 9843 & 6.28 & 0.22 & 84.10 & 30.20 & 326 \\
\hline & $\mathrm{Ba}$ & 114 & 0.12 & $<0.09$ & $<0.09$ & $<0.10$ & $>1140$ \\
\hline & $\mathrm{Ca}$ & 567 & 2.06 & $<0.09$ & 11.24 & $<4.46$ & $>127$ \\
\hline & $\mathrm{Cd}$ & 1051 & 1.18 & 0.68 & 0.15 & 0.67 & 1572 \\
\hline & $\mathrm{Cr}$ & 87 & 0.15 & 0.15 & $<0.09$ & $<0.13$ & $>669$ \\
\hline & $\mathrm{Cs}$ & 239 & 0.55 & 0.50 & 0.14 & 0.40 & 599 \\
\hline & $\mathrm{Cu}$ & 76 & 0.31 & 0.21 & 0.36 & 0.29 & 258 \\
\hline & $\mathrm{Fe}$ & 23043 & 14.89 & 1.87 & 2.40 & 6.38 & 3609 \\
\hline & $I$ & 317 & $<0.09$ & $<0.09$ & $<0.09$ & $<0.09$ & $>5286$ \\
\hline & $\mathrm{K}$ & 448 & 0.61 & 0.21 & 0.64 & 0.48 & 925 \\
\hline & $\mathrm{Li}$ & 8840 & 3.36 & 0.63 & 0.48 & 1.49 & 5932 \\
\hline & $\mathrm{Mg \#}$ & 2208 & 2.78 & 0.39 & 0.37 & 1.18 & 1871 \\
\hline & $\mathrm{Mn}$ & 7443 & 1.92 & 0.22 & 0.20 & 0.78 & 9587 \\
\hline & $\mathrm{Na}$ & 15507 & 17.42 & 2.68 & 3.02 & 7.71 & 2012 \\
\hline & $\mathrm{Ni}$ & 1346 & 0.69 & $<0.09$ & 0.13 & $<0.30$ & $\geq 4487$ \\
\hline & $P$ & 180 & 4.30 & $<0.09$ & 0.17 & $<1.52$ & $>118$ \\
\hline & $\mathrm{Pb}$ & 442 & 0.48 & 0.30 & 0.36 & 0.38 & 1156 \\
\hline & $S$ & 318 & 0.68 & 1.53 & 35.46 & 12.56 & 25 \\
\hline & $\mathrm{Sb}$ & 501 & 0.64 & $<0.09$ & 0.17 & $<0.30$ & $>1670$ \\
\hline & $\mathrm{Se}$ & 339 & 44.41 & 14.54 & 253.80 & 104.25 & 3 \\
\hline & $\mathrm{Si}$ & 67505 & 20.85 & 1.38 & 7.09 & 9.78 & 6906 \\
\hline & $\mathrm{Sr}$ & 6222 & 5.70 & 0.94 & 0.48 & 2.37 & 2624 \\
\hline & $\mathrm{Te}$ & 355 & 2.79 & 3.09 & 1.82 & 2.56 & 138 \\
\hline & $\mathrm{Ti}$ & 114 & $<0.09$ & $<0.09$ & $<0.09$ & $<0.09$ & $>1901$ \\
\hline & $\mathrm{Zn}$ & 5096 & 7.80 & 1.58 & 1.28 & 3.55 & 1434 \\
\hline & $\mathrm{Zr}$ & 8359 & 1.78 & $<0.09$ & 0.15 & $<0.67$ & $>12476$ \\
\hline \multirow{6}{*}{ 心 } & $\mathrm{B}$ & 9843 & 23.41 & 33.25 & 0.32 & 18.99 & 518 \\
\hline & $\mathrm{Cl}$ & 32 & 4.58 & 7.58 & $<0.17$ & $<4.05$ & $>8$ \\
\hline & $\mathrm{F}$ & 127 & 4.37 & 8.64 & $<0.17$ & $<4.33$ & $>29$ \\
\hline & $I$ & 317 & 189.70 & 208.54 & 249.85 & 216.03 & 1 \\
\hline & $\mathrm{S}$ & 318 & 13.02 & 18.46 & $<0.17$ & $<10.49$ & $>30$ \\
\hline & $\mathrm{Se}$ & 339 & 26.66 & 22.97 & 0.55 & 16.73 & 20 \\
\hline
\end{tabular}

$\mathrm{NC}$ - Not Calculated

$\$$ - From gravimetric analysis of filters and rinse dry-downs

\# - The analyzed MgO concentration of $1.15 \mathrm{wt} \%$ instead of the target value of $0.06 \mathrm{wt} \%$ was used to calculate the feed flux 
The Catholic University of America

Table 6.4. SBS Emission Rates for DM 1200 AZ101 Test 1.

\begin{tabular}{|c|c|c|c|c|c|c|c|}
\hline & $\begin{array}{c}\text { Average } \\
\text { Melter } \\
\text { Emissions } \\
\text { Flux } \\
\text { (mg/min) }\end{array}$ & $\begin{array}{c}\text { Run } 1 \\
(\mathrm{mg} / \mathrm{min})\end{array}$ & $\begin{array}{c}\operatorname{Run} 2 \\
(\mathrm{mg} / \mathrm{min})\end{array}$ & $\begin{array}{c}\operatorname{Run} 3 \\
(\mathrm{mg} / \mathrm{min})\end{array}$ & $\begin{array}{l}\text { Average } \\
(\mathrm{mg} / \mathrm{min})\end{array}$ & $\begin{array}{c}\text { DF } \\
\text { Across } \\
\text { SBS }\end{array}$ \\
\hline \multirow{29}{*}{$\frac{\mathscr{y}}{0}$} & Total $^{\text {s }}$ & 155.3 & 11.5 & 20.2 & 11.5 & 14.4 & 11 \\
\hline & $\mathrm{Al}$ & 3.04 & 0.45 & $<0.09$ & 0.21 & 0.22 & 14 \\
\hline & As & 0.38 & $<0.09$ & 0.10 & $<0.09$ & $<0.09$ & $>6$ \\
\hline & $\mathrm{B}$ & 30.20 & 0.41 & 0.20 & 0.14 & 0.25 & 121 \\
\hline & $\mathrm{Ba}$ & $<0.09$ & $<0.09$ & $<0.09$ & $<0.09$ & $<0.09$ & $\mathrm{NC}$ \\
\hline & $\mathrm{Ca}$ & 4.43 & 0.60 & 0.44 & 0.67 & 0.57 & 8 \\
\hline & $\mathrm{Cd}$ & 0.67 & 0.10 & 0.24 & $<0.09$ & $<0.14$ & $>5$ \\
\hline & $\mathrm{Cr}$ & 0.10 & $<0.09$ & $<0.09$ & $<0.09$ & $<0.09$ & $>2$ \\
\hline & $\mathrm{Cs}$ & 0.40 & 0.10 & 0.21 & $<0.09$ & $<0.13$ & $>3$ \\
\hline & $\mathrm{Cu}$ & 0.29 & 0.10 & 0.10 & 0.13 & 0.11 & 3 \\
\hline & $\mathrm{Fe}$ & 6.38 & 0.12 & $<0.09$ & $<0.09$ & $<0.10$ & $>64$ \\
\hline & $\mathrm{I}$ & $<0.09$ & $<0.09$ & $<0.09$ & $<0.09$ & $<0.09$ & $\mathrm{NC}$ \\
\hline & $\mathrm{K}$ & 0.48 & 0.26 & 0.54 & 0.41 & 0.40 & 1 \\
\hline & $\mathrm{Li}$ & 1.49 & 0.09 & 0.25 & $<0.09$ & $<0.14$ & $>11$ \\
\hline & $\mathrm{Mg}$ & 1.18 & $<0.09$ & $<0.09$ & 0.09 & 0.03 & 20 \\
\hline & $\mathrm{Mn}$ & 0.78 & $<0.09$ & $<0.09$ & $<0.09$ & $<0.09$ & $>13$ \\
\hline & $\mathrm{Na}$ & 7.71 & 2.30 & 1.64 & 1.42 & 1.79 & 4 \\
\hline & $\mathrm{Ni}$ & 0.27 & $<0.09$ & $<0.09$ & $<0.09$ & $<0.09$ & $>5$ \\
\hline & $\mathrm{P}$ & 1.49 & 0.79 & 1.14 & 0.57 & 0.83 & 2 \\
\hline & $\mathrm{Pb}$ & 0.38 & 0.12 & 0.13 & 0.17 & 0.14 & 3 \\
\hline & $\mathrm{S}$ & 12.56 & 0.33 & 0.55 & $<0.09$ & $<0.32$ & $>43$ \\
\hline & $\mathrm{Sb}$ & 0.27 & 0.54 & $<0.09$ & $<0.09$ & $<0.24$ & $>1$ \\
\hline & $\mathrm{Se}$ & 104.25 & 1.24 & 3.47 & 1.00 & 1.90 & 55 \\
\hline & $\mathrm{Si}$ & 9.78 & 0.29 & 0.21 & 0.85 & 0.45 & 22 \\
\hline & $\mathrm{Sr}$ & 2.37 & $<0.09$ & $<0.09$ & $<0.09$ & $<0.09$ & $>40$ \\
\hline & $\mathrm{Te}$ & 2.56 & 0.70 & 1.64 & 0.21 & 0.85 & 3 \\
\hline & $\mathrm{Ti}$ & $<0.09$ & $<0.09$ & $<0.09$ & $<0.09$ & $<0.09$ & $\mathrm{NC}$ \\
\hline & $\mathrm{Zn}$ & 3.55 & 0.18 & 0.10 & $<0.09$ & $<0.12$ & $>30$ \\
\hline & $\mathrm{Zr}$ & 0.64 & $<0.09$ & $<0.09$ & $<0.09$ & $<0.09$ & $>11$ \\
\hline \multirow{6}{*}{ ర్జ } & $\mathrm{B}$ & 18.99 & 0.39 & 0.70 & 0.41 & 0.50 & 38 \\
\hline & $\mathrm{Cl}$ & 4.05 & $<0.17$ & $<0.17$ & $<0.17$ & $<0.17$ & $>68$ \\
\hline & $F$ & 4.33 & $<0.17$ & $<0.17$ & $<0.17$ & $<0.17$ & $>72$ \\
\hline & I & 216.03 & 208.36 & 178.99 & 422.54 & 269.96 & 1 \\
\hline & $\mathrm{S}$ & 10.49 & $<0.17$ & $<0.17$ & $<0.17$ & $<0.17$ & $>175$ \\
\hline & $\mathrm{Se}$ & 16.73 & 2.33 & 5.61 & 6.84 & 4.93 & 3 \\
\hline
\end{tabular}

NC - Not Calculated

${ }^{s}$ - From gravimetric analysis of filters and rinse dry-downs 
Table 6.5. WESP Emission Rates for DM 1200 AZ101 Test 1.

\begin{tabular}{|c|c|c|c|c|c|c|c|}
\hline & $\begin{array}{l}\text { Average } \\
\text { SBS } \\
\text { Emissions } \\
\text { Flux } \\
\text { (mg/min) }\end{array}$ & $\begin{array}{c}\text { Run } 1 \\
(\mathrm{mg} / \mathrm{min})\end{array}$ & $\begin{array}{c}\text { Run } 2 \\
(\mathrm{mg} / \mathrm{min})\end{array}$ & $\begin{array}{c}\text { Run } 3 \\
(\mathrm{mg} / \mathrm{min})\end{array}$ & $\begin{array}{l}\text { Average } \\
(\mathrm{mg} / \mathrm{min})\end{array}$ & $\begin{array}{c}\text { DF } \\
\text { Across } \\
\text { WESP }\end{array}$ \\
\hline \multirow{29}{*}{$\frac{\mathscr{e}}{e}$} & Total $^{\$}$ & 14.4 & 3.51 & 5.34 & 5.85 & 4.9 & 3 \\
\hline & $\mathrm{Al}$ & 0.22 & $<0.09$ & $<0.09$ & $<0.09$ & $<0.09$ & $>2.4$ \\
\hline & As & $<0.09$ & 0.44 & $<0.09$ & $<0.09$ & $<0.15$ & $\mathrm{NC}$ \\
\hline & B & 0.25 & $<0.09$ & $<0.09$ & $<0.09$ & $<0.09$ & $>2.8$ \\
\hline & $\mathrm{Ba}$ & $<0.09$ & $<0.09$ & $<0.09$ & $<0.09$ & $<0.09$ & $\mathrm{NC}$ \\
\hline & $\mathrm{Ca}$ & 0.57 & 0.19 & 0.15 & 1.11 & 0.48 & 2 \\
\hline & $\mathrm{Cd}$ & 0.11 & $<0.09$ & $<0.09$ & $<0.09$ & $<0.09$ & $>1.2$ \\
\hline & $\mathrm{Cr}$ & $<0.09$ & $<0.09$ & $<0.09$ & $<0.09$ & $<0.09$ & $\mathrm{NC}$ \\
\hline & Cs & 0.11 & $<0.09$ & $<0.09$ & $<0.09$ & $<0.09$ & $>1.2$ \\
\hline & $\mathrm{Cu}$ & 0.11 & $<0.09$ & 0.09 & 0.14 & 0.08 & 1 \\
\hline & $\mathrm{Fe}$ & 0.04 & $<0.09$ & $<0.09$ & 0.10 & $<0.09$ & $>0.4$ \\
\hline & I & $<0.09$ & $<0.09$ & $<0.09$ & $<0.09$ & $<0.09$ & $\mathrm{NC}$ \\
\hline & $\mathrm{K}$ & 0.40 & 0.22 & 0.37 & 0.19 & 0.26 & 2 \\
\hline & $\overline{\mathrm{Li}}$ & 0.12 & $<0.09$ & $<0.09$ & $<0.09$ & $<0.09$ & $>1.3$ \\
\hline & $\mathrm{Mg}$ & 0.03 & $<0.09$ & $<0.09$ & $<0.09$ & $<0.09$ & $>0.3$ \\
\hline & $\mathrm{Mn}$ & $<0.09$ & $<0.09$ & $<0.09$ & $<0.09$ & $<0.09$ & $\mathrm{NC}$ \\
\hline & $\mathrm{Na}$ & 1.79 & 0.35 & 0.51 & 3.31 & 1.39 & 3 \\
\hline & $\mathrm{Ni}$ & $<0.09$ & $<0.09$ & $<0.09$ & $<0.09$ & $<0.09$ & $\mathrm{NC}$ \\
\hline & $\mathrm{P}$ & 0.83 & 0.79 & 0.10 & $<0.09$ & 0.30 & 3 \\
\hline & $\mathrm{Pb}$ & 0.14 & 0.16 & 0.15 & 0.17 & 0.16 & 1 \\
\hline & $\mathrm{S}$ & 0.30 & 0.37 & $<0.09$ & $<0.09$ & $<0.12$ & $>1$ \\
\hline & $\mathrm{Sb}$ & 0.18 & 0.19 & 0.42 & $<0.09$ & $<0.20$ & $>1$ \\
\hline & $\mathrm{Se}$ & 1.90 & $<0.09$ & $<0.09$ & $<0.09$ & $<0.09$ & $>21.1$ \\
\hline & $\mathrm{Si}$ & 0.45 & 0.31 & 0.14 & 0.39 & 0.28 & 2 \\
\hline & $\mathrm{Sr}$ & $<0.09$ & $<0.09$ & $<0.09$ & $<0.09$ & $<0.09$ & $\mathrm{NC}$ \\
\hline & $\mathrm{Te}$ & 0.85 & 0.25 & 0.37 & 0.43 & 0.35 & 3 \\
\hline & $\mathrm{Ti}$ & $<0.09$ & $<0.09$ & $<0.09$ & $<0.09$ & $<0.09$ & $\mathrm{NC}$ \\
\hline & $\mathrm{Zn}$ & 0.10 & $<0.09$ & $<0.09$ & 0.09 & $<0.09$ & $>1$ \\
\hline & $\mathrm{Zr}$ & $<0.09$ & $<0.09$ & $<0.09$ & $<0.09$ & $<0.09$ & $\mathrm{NC}$ \\
\hline \multirow{6}{*}{$\mathbb{0}$} & $\mathrm{B}$ & 0.50 & 0.44 & 0.65 & 0.54 & 0.55 & 1 \\
\hline & $\mathrm{Cl}$ & $<0.17$ & $<0.17$ & $<0.17$ & $<0.17$ & $<0.17$ & $\mathrm{NC}$ \\
\hline & $\mathrm{F}$ & $<0.17$ & $<0.17$ & 0.33 & $<0.17$ & $<0.17$ & $\mathrm{NC}$ \\
\hline & I & 269.96 & 144.07 & 360.80 & 227.81 & 244.23 & 1 \\
\hline & $S$ & $<0.17$ & $<0.17$ & $<0.17$ & $<0.17$ & $<0.17$ & $\mathrm{NC}$ \\
\hline & $\mathrm{Se}$ & 4.93 & $<0.17$ & 0.25 & 0.22 & $<0.17$ & 14 \\
\hline
\end{tabular}

NC - Not Calculated

s - From gravimetric analysis of filters and rinse dry-downs 
Table 6.6. Melter Emission Rates for DM 1200 AZ101 Test 2.

\begin{tabular}{|c|c|c|c|c|c|c|c|}
\hline & $\begin{array}{l}\text { Average } \\
\text { Feed Flux } \\
(\mathrm{mg} / \mathrm{min})\end{array}$ & $\begin{array}{c}\text { Run } 1 \\
\text { (mg/min) }\end{array}$ & $\begin{array}{c}\text { Run } 2 \\
\text { (mg/min) }\end{array}$ & $\begin{array}{c}\text { Run } 3 \\
(\mathrm{mg} / \mathrm{min})\end{array}$ & $\begin{array}{l}\text { Average } \\
(\mathrm{mg} / \mathrm{min})\end{array}$ & $\begin{array}{c}\text { DF } \\
\text { Across } \\
\text { Melter }\end{array}$ \\
\hline \multirow{29}{*}{. } & Total $^{\S}$ & 232167 & 245.2 & 168.8 & 191.9 & 202 & 1149 \\
\hline & $\mathrm{Al}$ & 9095 & 4.88 & 7.83 & 4.75 & 5.82 & 1563 \\
\hline & As & 70 & 2.78 & 0.87 & 1.09 & 1.58 & 45 \\
\hline & $B$ & 7205 & 4.15 & 9.39 & 4.67 & 6.07 & 1187 \\
\hline & $\mathrm{Ba}$ & 83 & $<0.06$ & 0.11 & 0.13 & $<0.10$ & $>830$ \\
\hline & $\mathrm{Ca}$ & 415 & 0.83 & 0.84 & 0.51 & 0.72 & 573 \\
\hline & $\mathrm{Cd}$ & 769 & 23.02 & 2.97 & 14.38 & 13.45 & 57 \\
\hline & $\mathrm{Cr}$ & 64 & 0.11 & 0.14 & 0.13 & 0.13 & 503 \\
\hline & $\mathrm{Cs}$ & 175 & 0.64 & 0.44 & 0.31 & 0.46 & 380 \\
\hline & $\mathrm{Cu}$ & 56 & 0.50 & 0.10 & 0.12 & 0.24 & 232 \\
\hline & $\mathrm{Fe}$ & 16867 & 9.09 & 13.18 & 8.53 & 10.27 & 1643 \\
\hline & I & 232 & $<0.06$ & $<0.06$ & $<0.06$ & $<0.06$ & $>3869$ \\
\hline & $\mathrm{K}$ & 328 & 0.67 & 0.55 & 0.48 & 0.57 & 578 \\
\hline & $\mathrm{Li}$ & 6471 & 1.78 & 3.22 & 2.04 & 2.35 & 2755 \\
\hline & $\mathrm{Mg \#}$ & 1613 & 1.25 & 2.25 & 1.58 & 1.69 & 954 \\
\hline & $\mathrm{Mn}$ & 5448 & 0.90 & 2.89 & 1.39 & 1.72 & 3159 \\
\hline & $\mathrm{Na}$ & 11351 & 7.09 & 8.64 & 6.76 & 7.50 & 1514 \\
\hline & $\mathrm{Ni}$ & 985 & 0.42 & 0.53 & 0.54 & 0.50 & 1976 \\
\hline & $\mathrm{P}$ & 132 & 0.18 & 0.11 & 0.67 & 0.32 & 412 \\
\hline & $\mathrm{Pb}$ & 323 & 2.98 & 0.46 & 1.70 & 1.71 & 189 \\
\hline & $\mathrm{S}$ & 233 & 1.32 & 0.83 & 0.48 & 0.88 & 265 \\
\hline & $\mathrm{Sb}$ & 367 & 0.20 & 0.82 & 0.63 & 0.55 & 665 \\
\hline & $\mathrm{Se}$ & 248 & 34.38 & 30.06 & 24.48 & 29.64 & 8 \\
\hline & $\mathrm{Si}$ & 49414 & 12.48 & 22.26 & 11.39 & 15.38 & 3213 \\
\hline & $\mathrm{Sr}$ & 4555 & 2.48 & 4.82 & 3.64 & 3.64 & 1250 \\
\hline & $\mathrm{Te}$ & 260 & 62.65 & 5.61 & 44.72 & 37.66 & 7 \\
\hline & $\mathrm{Ti}$ & 84 & 0.10 & 0.07 & $<0.06$ & $<0.08$ & $>1050$ \\
\hline & $\mathrm{Zn}$ & 3730 & 3.14 & 4.77 & 3.73 & 3.88 & 961 \\
\hline & $\mathrm{Zr}$ & 6119 & 0.72 & 1.18 & 0.69 & 0.86 & 7074 \\
\hline \multirow{6}{*}{ 苞 } & $\mathrm{B}$ & 7205 & 38.42 & 17.29 & 17.87 & 24.53 & 294 \\
\hline & $\mathrm{Cl}$ & 23 & 6.60 & 6.05 & 3.30 & 5.31 & 4 \\
\hline & $\mathrm{F}$ & 93 & 5.71 & 7.69 & 2.23 & 5.21 & 18 \\
\hline & $\mathrm{I}$ & 232 & 47.60 & 78.72 & 64.65 & 63.66 & 4 \\
\hline & $\mathrm{S}$ & 233 & 38.87 & 9.99 & 20.11 & 22.99 & 10 \\
\hline & $\mathrm{Se}$ & 248 & 9.10 & 42.67 & 15.03 & 22.27 & 11 \\
\hline
\end{tabular}

NC - Not Calculated

$\$$ - From gravimetric analysis of filters and rinse dry-downs

\# - The analyzed $\mathrm{MgO}$ concentration of $1.15 \mathrm{wt} \%$ instead of the target value of $0.06 \mathrm{wt} \%$ was used to calculate the feed flux 
The Catholic University of America

Vitreous State Laboratory
DuraMelter 1200 Tests with AZ-101HLW Simulants Final Report, VSL-02R0100-2, Rev.1

Table 6.7. SBS Emission Rates for DM 1200 AZ101 Test 2.

\begin{tabular}{|c|c|c|c|c|c|c|c|}
\hline & $\begin{array}{c}\text { Average } \\
\text { Melter } \\
\text { Emissions } \\
\text { Flux } \\
\text { (mg/min) }\end{array}$ & $\begin{array}{c}\text { Run } 1 \\
(\mathrm{mg} / \mathrm{min})\end{array}$ & $\begin{array}{c}\text { Run } 2 \\
(\mathrm{mg} / \mathrm{min})\end{array}$ & $\begin{array}{c}\text { Run } 3 \\
(\mathrm{mg} / \mathrm{min})\end{array}$ & $\begin{array}{l}\text { Average } \\
\text { (mg/min) }\end{array}$ & $\begin{array}{c}\text { DF } \\
\text { Across } \\
\text { SBS }\end{array}$ \\
\hline \multirow{29}{*}{$\frac{\mathscr{D}}{0}$} & Total $^{\text {s }}$ & 202 & 58.2 & 43.7 & 36.4 & 46.1 & 4 \\
\hline & $\mathrm{Al}$ & 5.82 & $<0.06$ & $<0.06$ & $<0.06$ & $<0.06$ & $>97$ \\
\hline & As & 1.58 & 0.89 & 0.44 & 0.41 & 0.58 & 3 \\
\hline & $\mathrm{B}$ & 6.07 & $<0.06$ & $<0.06$ & $<0.06$ & $<0.06$ & $>101$ \\
\hline & $\mathrm{Ba}$ & 0.08 & $<0.06$ & $<0.06$ & $<0.06$ & $<0.06$ & $>1$ \\
\hline & $\mathrm{Ca}$ & 0.72 & $<0.06$ & 0.07 & 0.09 & $<0.06$ & $>12$ \\
\hline & $\mathrm{Cd}$ & 13.45 & 9.78 & 4.03 & 4.03 & 5.95 & 2 \\
\hline & $\mathrm{Cr}$ & 0.13 & $<0.06$ & $<0.06$ & $<0.06$ & $<0.06$ & $>2$ \\
\hline & $\mathrm{Cs}$ & 0.46 & 0.28 & 0.26 & 0.16 & 0.23 & 2 \\
\hline & $\mathrm{Cu}$ & 0.24 & $<0.06$ & $<0.06$ & $<0.06$ & $<0.06$ & $>4$ \\
\hline & $\mathrm{Fe}$ & 10.27 & $<0.06$ & $<0.06$ & $<0.06$ & $<0.06$ & $>171$ \\
\hline & $I$ & $<0.06$ & $<0.06$ & $<0.06$ & $<0.06$ & $<0.06$ & $\mathrm{NC}$ \\
\hline & $\mathrm{K}$ & 0.57 & 0.13 & 0.15 & 0.11 & 0.13 & 4 \\
\hline & $\mathrm{Li}$ & 2.35 & 0.15 & 0.17 & 0.11 & 0.14 & 16 \\
\hline & $\mathrm{Mg}$ & 1.69 & $<0.06$ & $<0.06$ & $<0.06$ & $<0.06$ & $>28$ \\
\hline & $\mathrm{Mn}$ & 1.72 & $<0.06$ & $<0.06$ & $<0.06$ & $<0.06$ & $>29$ \\
\hline & $-\mathrm{Na}$ & 7.50 & 0.97 & 1.09 & 0.69 & 0.92 & 8 \\
\hline & $\mathrm{Ni}$ & 0.50 & $<0.06$ & $<0.06$ & $<0.06$ & $<0.06$ & $>8$ \\
\hline & $\mathrm{P}$ & 0.32 & 0.18 & $<0.06$ & 0.27 & $<0.17$ & $>2$ \\
\hline & $\mathrm{Pb}$ & 1.71 & 1.18 & 0.39 & 0.42 & 0.66 & 3 \\
\hline & $S$ & 0.88 & 0.92 & $\mathrm{NA}$ & 0.23 & 0.58 & 2 \\
\hline & $\mathrm{Sb}$ & 0.55 & 0.09 & 0.17 & 0.07 & 0.11 & 5 \\
\hline & $\mathrm{Se}$ & 29.64 & 3.92 & 5.51 & 3.20 & 4.21 & 7 \\
\hline & $\mathrm{Si}$ & 15.38 & 0.15 & 0.10 & 0.17 & 0.14 & 108 \\
\hline & $\mathrm{Sr}$ & 3.64 & $<0.06$ & $<0.06$ & $<0.06$ & $<0.06$ & $>61$ \\
\hline & $\mathrm{Te}$ & 37.66 & 25.12 & 13.51 & 18.24 & 18.96 & 2 \\
\hline & $\mathrm{Ti}$ & 0.06 & $<0.06$ & $<0.06$ & $<0.06$ & $<0.06$ & $>1$ \\
\hline & $\mathrm{Zn}$ & 3.88 & $<0.06$ & $<0.06$ & 0.07 & $<0.06$ & $>65$ \\
\hline & $\mathrm{Zr}$ & 0.86 & $<0.06$ & $<0.06$ & $<0.06$ & $<0.06$ & $>14$ \\
\hline \multirow{6}{*}{$\underset{5}{5}$} & $\mathrm{~B}$ & 24.53 & 1.27 & 1.78 & 0.45 & 1.17 & 21 \\
\hline & $\mathrm{Cl}$ & 5.31 & 0.63 & 0.93 & $<0.07$ & $<0.54$ & $>10$ \\
\hline & $F$ & 5.21 & $<0.07$ & $<0.07$ & $<0.07$ & $<0.06$ & $>87$ \\
\hline & $I$ & 63.66 & 4.53 & 11.26 & 2.12 & 5.97 & 11 \\
\hline & $\mathrm{S}$ & 22.99 & $<0.07$ & $<0.07$ & $<0.07$ & $<0.06$ & $>383$ \\
\hline & $\mathrm{Se}$ & 22.27 & 4.37 & 14.54 & 1.68 & 6.86 & 3 \\
\hline
\end{tabular}

$\mathrm{NC}$ - Not Calculated

$\$$ - From gravimetric analysis of filters and rinse dry-downs

NA - Not Analyzed 
The Catholic University of America

Table 6.8. WESP Emission Rates for DM 1200 AZ101 Test 2.

\begin{tabular}{|c|c|c|c|c|c|c|c|c|}
\hline & $\begin{array}{l}\text { Average } \\
\text { Feed Flux } \\
(\mathrm{mg} / \mathrm{min})\end{array}$ & $\begin{array}{l}\text { Average } \\
\text { Melter } \\
\text { Emissions } \\
\text { Flux } \\
(\mathrm{mg} / \mathrm{min}) \\
\end{array}$ & $\begin{array}{c}\text { Average } \\
\text { SBS } \\
\text { Emissions } \\
\text { Flux } \\
\text { (mg/min) }\end{array}$ & $\begin{array}{l}\text { WESP } \\
\text { Emissions } \\
\text { Flux } \\
(\mathrm{mg} / \mathrm{min})\end{array}$ & $\begin{array}{l}\text { DF Across } \\
\text { WESP }\end{array}$ & $\begin{array}{l}\text { DF Across } \\
\text { SBS, } \\
\text { WESP }\end{array}$ & $\begin{array}{c}\text { Cumulative DF } \\
\text { Across Melter, } \\
\text { SBS, WESP }\end{array}$ \\
\hline \multirow{29}{*}{ 总 } & Total $^{\$}$ & 232167 & 202 & 46.1 & 0.27 & 171 & 673 & 773890 \\
\hline & $\mathrm{Al}$ & 9095 & 5.82 & $<0.06$ & $<0.06$ & $\mathrm{NC}$ & $>97$ & $>151591$ \\
\hline & As & 70 & 1.58 & 0.58 & $<0.06$ & $>9.1$ & $>26$ & $>1172$ \\
\hline & $B$ & 7205 & 6.07 & $<0.06$ & $<0.06$ & $\mathrm{NC}$ & $>101$ & $>120086$ \\
\hline & $\mathrm{Ba}$ & 83 & 0.08 & $<0.06$ & $<0.06$ & $\mathrm{NC}$ & $>1$ & $>1386$ \\
\hline & $\mathrm{Ca}$ & 415 & 0.72 & $<0.06$ & $<0.06$ & $>0.9$ & $>12$ & $>6915$ \\
\hline & $\mathrm{Cd}$ & 769 & 13.45 & 5.95 & $<0.06$ & $>92.9$ & $>224$ & $>12820$ \\
\hline & $\mathrm{Cr}$ & 64 & 0.13 & $<0.06$ & $<0.06$ & $\mathrm{NC}$ & $>2$ & $>1059$ \\
\hline & $\mathrm{Cs}$ & 175 & 0.46 & 0.23 & $<0.06$ & $>3.7$ & $>8$ & $>2920$ \\
\hline & $\mathrm{Cu}$ & 56 & 0.24 & $<0.06$ & $<0.06$ & $\mathrm{NC}$ & $>4$ & $>927$ \\
\hline & $\mathrm{Fe}$ & 16867 & 10.27 & $<0.06$ & $<0.06$ & $\mathrm{NC}$ & $>171$ & $>281122$ \\
\hline & I & 232 & $<0.06$ & $<0.06$ & $<0.06$ & $\mathrm{NC}$ & $\mathrm{NC}$ & $>3869$ \\
\hline & $\mathrm{K}$ & 328 & 0.57 & 0.13 & $<0.06$ & $>2.0$ & $>9$ & $>5461$ \\
\hline & $\mathrm{Li}$ & 6471 & 2.35 & 0.14 & $<0.06$ & $>2.2$ & $>39$ & $>107847$ \\
\hline & $\mathrm{Mg \#}$ & 1613 & 1.69 & $<0.06$ & $<0.06$ & $\mathrm{NC}$ & $>28$ & $>26933$ \\
\hline & $\mathrm{Mn}$ & 5448 & 1.72 & $<0.06$ & $<0.06$ & $\mathrm{NC}$ & $>29$ & $>90801$ \\
\hline & $\mathrm{Na}$ & 11351 & -7.50 & 0.92 & $<0.06$ & $>14.3$ & $>125$ & $>189191$ \\
\hline & $\mathrm{Ni}$ & 985 & 0.50 & $<0.06$ & $<0.06$ & $\mathrm{NC}$ & $>8$ & $>16419$ \\
\hline & $\mathrm{P}$ & 132 & 0.32 & 0.15 & $<0.06$ & $>2.3$ & $>5$ & $>2196$ \\
\hline & $\mathrm{Pb}$ & 323 & 1.71 & 0.66 & $<0.06$ & $>10.3$ & $\geq 29$ & $>5388$ \\
\hline & $S$ & 233 & 0.88 & 0.58 & $<0.06$ & $>9.6$ & $>15$ & $>3877$ \\
\hline & $\mathrm{Sb}$ & 367 & 0.55 & 0.11 & $<0.06$ & $>1.7$ & $>9$ & $>6117$ \\
\hline & $\mathrm{Se}$ & 248 & 29.64 & 4.21 & $<0.06$ & $>65.8$ & $>494$ & $>4130$ \\
\hline & $\mathrm{Si}$ & 49414 & 15.38 & 0.14 & $<0.06$ & $>2.2$ & $\geq 256$ & $>823561$ \\
\hline & $\mathrm{Sr}$ & 4555 & 3.64 & $<0.06$ & $<0.06$ & $\mathrm{NC}$ & $>61$ & $>75910$ \\
\hline & $\mathrm{Te}$ & 260 & 37.66 & 18.96 & $<0.06$ & $\geq 296.2$ & $>628$ & $>4331$ \\
\hline & $\mathrm{Ti}$ & 84 & 0.06 & $<0.06$ & $<0.06$ & $\mathrm{NC}$ & $>1$ & $>1392$ \\
\hline & $\mathrm{Zn}$ & 3730 & 3.88 & 0.02 & $<0.06$ & $>0.3$ & $>65$ & $>62174$ \\
\hline & $\mathrm{Zr}$ & 6119 & 0.86 & $<0.06$ & $<0.06$ & $\mathrm{NC}$ & $>14$ & $>101978$ \\
\hline \multirow{6}{*}{ 象 } & $\mathrm{B}$ & 7205 & 24.53 & 1.17 & 0.10 & 12 & 251 & 73797 \\
\hline & $\mathrm{Cl}$ & 23 & 5.31 & 0.52 & $<0.07$ & $>7.4$ & $>89$ & $>387$ \\
\hline & $\mathrm{F}$ & 93 & 5.21 & $<0.06$ & $<0.07$ & $\mathrm{NC}$ & $>87$ & $>1548$ \\
\hline & I & 232 & 63.66 & 5.97 & $<0.07$ & $>85.3$ & $>1061$ & $>3869$ \\
\hline & $S$ & 233 & 22.99 & $<0.06$ & $<0.07$ & $\mathrm{NC}$ & $>383$ & $>3877$ \\
\hline & $\mathrm{Se}$ & 248 & 22.27 & 6.86 & $<0.07$ & $>98.1$ & $>371$ & $>4130$ \\
\hline
\end{tabular}

$\mathrm{NC}-$ Not Calculated

$\$$ - From gravimetric analysis of filters and rinse dry-downs

\# - The analyzed $\mathrm{MgO}$ concentration of $1.15 \mathrm{wt} \%$ instead of the target value of $0.06 \mathrm{wt} \%$ was used to calculate the feed flux 
Table 6.9. Melter Emission Rates for DM 1200 AZ101 Test 3.

\begin{tabular}{|c|c|c|c|c|c|c|c|}
\hline & $\begin{array}{l}\text { Average } \\
\text { Feed Flux } \\
(\mathrm{mg} / \mathrm{min})\end{array}$ & $\begin{array}{c}\text { Run 1 } \\
(\mathrm{mg} / \mathrm{min})\end{array}$ & $\begin{array}{c}\text { Run } 2 \\
(\mathrm{mg} / \mathrm{min})\end{array}$ & $\begin{array}{c}\text { Run } 3 \\
(\mathrm{mg} / \mathrm{min})\end{array}$ & $\begin{array}{l}\text { Average } \\
(\mathrm{mg} / \mathrm{min})\end{array}$ & $\begin{array}{c}\text { DF } \\
\text { Across } \\
\text { Melter }\end{array}$ \\
\hline \multirow{29}{*}{. } & Total $^{\$}$ & 1036333 & 5753 & 3944 & 3864 & 4520 & 229 \\
\hline & $\mathrm{Al}$ & 40600 & 226.23 & 164.76 & 117.46 & 169.48 & 240 \\
\hline & As & 314 & 7.72 & 4.93 & 4.85 & 5.83 & 54 \\
\hline & $\mathrm{B}$ & 32162 & 220.74 & 121.33 & 79.40 & 140.49 & 229 \\
\hline & $\mathrm{Ba}$ & 371 & 2.08 & 1.18 & 1.10 & 1.46 & 255 \\
\hline & $\mathrm{Ca}$ & 1852 & 12.27 & 8.21 & 6.75 & 9.08 & 204 \\
\hline & $\mathrm{Cd}$ & 3433 & 27.28 & 20.20 & 16.32 & 21.27 & 161 \\
\hline & $\mathrm{Cr}$ & 284 & 6.76 & 4.45 & 3.03 & 4.75 & 60 \\
\hline & $\mathrm{Cs}$ & 782 & 22.48 & 14.20 & 13.16 & 16.61 & 47 \\
\hline & $\mathrm{Cu}$ & 248 & 1.24 & 0.92 & 0.74 & 0.97 & 257 \\
\hline & $\mathrm{Fe}$ & 75292 & 452.63 & 294.49 & 191.32 & 312.81 & 241 \\
\hline & $\mathrm{I}$ & 1036 & $<0.06$ & $<0.06$ & $<0.06$ & $<0.06$ & $\geq 17272$ \\
\hline & $\mathrm{K}$ & 1463 & 15.79 & 8.87 & $<0.06$ & 11.55 & 127 \\
\hline & $\mathrm{Li}$ & 28884 & 93.35 & 66.62 & 53.72 & 71.23 & 405 \\
\hline & $\mathrm{Mg \#}$ & 7200 & 49.75 & 35.86 & 24.90 & 36.84 & 195 \\
\hline & $\mathrm{Mn}$ & 24319 & 35.88 & 24.80 & 37.46 & 32.71 & 743 \\
\hline & $\mathrm{Na}$ & 50670 & 314.32 & 193.73 & 161.33 & 223.13 & 227 \\
\hline & $\mathrm{Ni}$ & 4398 & 25.92 & 18.50 & 12.01 & 18.81 & 234 \\
\hline & $\mathrm{P}$ & 588 & 9.26 & 4.56 & 4.80 & 6.21 & 95 \\
\hline & $\mathrm{Pb}$ & 1443 & -9.22 & 6.09 & 6.38 & 7.23 & 200 \\
\hline & $\mathrm{S}$ & 1038 & 112.15 & 87.00 & 108.27 & 102.47 & 10 \\
\hline & $\mathrm{Sb}$ & 1638 & 8.73 & 4.92 & 4.68 & 6.11 & 268 \\
\hline & $\mathrm{Se}$ & 1106 & 300.38 & 431.85 & 723.54 & 485.26 & 2 \\
\hline & $\mathrm{Si}$ & 220570 & 600.12 & 408.96 & 368.81 & 459.30 & 480 \\
\hline & $\mathrm{Sr}$ & 20330 & 92.44 & 69.25 & 50.50 & 70.73 & 287 \\
\hline & $\mathrm{Te}$ & 1160 & 113.04 & 71.25 & 61.36 & 81.88 & 14 \\
\hline & $\mathrm{Ti}$ & 373 & 1.59 & 0.94 & 0.98 & 1.17 & 319 \\
\hline & $\mathrm{Zn}$ & 16652 & 90.96 & 66.60 & 46.20 & 67.92 & 245 \\
\hline & $\mathrm{Zr}$ & 27312 & 53.46 & 25.72 & 33.60 & 37.59 & 727 \\
\hline \multirow{6}{*}{ : } & $\mathrm{B}$ & 32162 & 361.41 & 303.03 & 334.82 & 333.09 & 97 \\
\hline & $\mathrm{Cl}$ & 104 & 26.73 & 58.38 & 136.21 & 73.78 & 1 \\
\hline & $\mathrm{F}$ & 415 & 90.69 & 99.35 & 204.56 & 131.53 & 3 \\
\hline & $\mathrm{I}$ & 1036 & 435.05 & 508.64 & 582.72 & 508.80 & 2 \\
\hline & $\mathrm{S}$ & 1038 & 149.33 & 124.73 & 220.70 & 164.92 & 6 \\
\hline & $\mathrm{Se}$ & 1106 & 2.07 & 7.09 & 139.77 & 49.64 & 22 \\
\hline
\end{tabular}

NC - Not Calculated

$\$$ - From gravimetric analysis of filters and rinse dry-downs

\# - The analyzed $\mathrm{MgO}$ concentration of $1.15 \mathrm{wt} \%$ instead of the target value of $0.06 \mathrm{wt} \%$ was used to calculate the feed flux 
The Catholic University of America

Table 6.10. SBS Emission Rates for DM 1200 AZ101 Test 3.

\begin{tabular}{|c|c|c|c|c|c|c|c|}
\hline & $\begin{array}{l}\text { Average } \\
\text { Melter } \\
\text { Emissions } \\
\text { Flux } \\
\text { (mg/min) }\end{array}$ & $\begin{array}{c}\text { Run } 1 \\
(\mathrm{mg} / \mathrm{min})\end{array}$ & $\begin{array}{c}\text { Run } 2 \\
(\mathrm{mg} / \mathrm{min})\end{array}$ & $\begin{array}{c}\text { Run } 3 \\
(\mathrm{mg} / \mathrm{min})\end{array}$ & $\begin{array}{l}\text { Average } \\
(\mathrm{mg} / \mathrm{min})\end{array}$ & $\begin{array}{c}\text { DF } \\
\text { Across } \\
\text { SBS }\end{array}$ \\
\hline \multirow{29}{*}{$\frac{\mathscr{d}}{0}$} & Total $^{s}$ & 4520 & 192.6 & 195.0 & 130.9 & 172.8 & 26.2 \\
\hline & $\mathrm{Al}$ & 169.48 & 0.37 & 0.41 & 0.32 & 0.37 & 462 \\
\hline & As & 5.83 & 1.31 & 1.72 & 0.73 & 1.25 & 5 \\
\hline & $\mathrm{B}$ & 140.49 & 0.14 & 0.17 & 0.11 & 0.14 & 995 \\
\hline & $\mathrm{Ba}$ & 1.46 & $<0.06$ & $<0.06$ & $<0.06$ & $<0.06$ & $>24$ \\
\hline & $\mathrm{Ca}$ & 9.08 & $<0.06$ & 0.07 & $<0.06$ & $<0.06$ & $>151$ \\
\hline & $\mathrm{Cd}$ & 21.27 & 1.43 & 1.79 & 0.66 & 1.29 & 16 \\
\hline & $\mathrm{Cr}$ & 4.75 & 0.62 & 0.61 & 0.30 & 0.51 & 9 \\
\hline & $\mathrm{Cs}$ & 16.61 & 5.34 & 5.07 & 2.92 & 4.44 & 4 \\
\hline & $\mathrm{Cu}$ & 0.97 & $<0.06$ & $<0.06$ & $<0.06$ & $<0.06$ & $>16$ \\
\hline & $\mathrm{Fe}$ & 312.81 & 0.47 & 0.66 & 0.49 & 0.54 & 581 \\
\hline & $\mathrm{I}$ & $<0.06$ & $<0.06$ & $<0.06$ & $<0.06$ & $<0.06$ & $\mathrm{NC}$ \\
\hline & $\mathrm{K}$ & 11.55 & 2.00 & 1.99 & 1.18 & 1.72 & 7 \\
\hline & $\mathrm{Li}$ & 71.23 & 4.83 & 4.67 & 2.97 & 4.16 & 17 \\
\hline & $\mathrm{Mg}$ & 36.84 & 0.12 & 0.17 & 0.11 & 0.14 & 273 \\
\hline & $\mathrm{Mn}$ & 32.71 & 0.09 & 0.11 & 0.15 & 0.12 & 279 \\
\hline & $\mathrm{Na}$ & 223.13 & 22.87 & 24.17 & 14.85 & 20.63 & 11 \\
\hline & $\mathrm{Ni}$ & 18.81 & 0.07 & 0.09 & $<0.06$ & $<0.07$ & $>269$ \\
\hline & $\mathrm{P}$ & 6.21 & $<0.06$ & $<0.06$ & $<0.06$ & $<0.06$ & $>103$ \\
\hline & $\mathrm{Pb}$ & 7.23 & 0.42 & 0.32 & 0.26 & 0.33 & 22 \\
\hline & $\mathrm{S}$ & 102.47 & 31.36 & 31.79 & 20.75 & 27.97 & 4 \\
\hline & $\mathrm{Sb}$ & 6.11 & 0.46 & 0.55 & 0.22 & 0.41 & 15 \\
\hline & $\mathrm{Se}$ & 485.26 & 25.33 & 17.94 & 18.40 & 20.56 & 24 \\
\hline & $\mathrm{Si}$ & 459.30 & 0.54 & 0.97 & 0.83 & 0.78 & 590 \\
\hline & $\mathrm{Sr}$ & 70.73 & 0.18 & 0.15 & 0.16 & 0.16 & 429 \\
\hline & $\mathrm{Te}$ & 81.88 & 5.44 & 5.79 & 2.71 & 4.64 & 18 \\
\hline & $\mathrm{Ti}$ & 1.17 & $<0.06$ & $<0.06$ & $<0.06$ & $<0.06$ & $>19$ \\
\hline & $\mathrm{Zn}$ & 67.92 & 0.44 & 0.49 & 0.31 & 0.41 & 165 \\
\hline & $\mathrm{Zr}$ & 37.59 & $<0.06$ & $<0.06$ & $<0.06$ & $<0.06$ & $>627$ \\
\hline \multirow{6}{*}{ 晃 } & $\mathrm{B}$ & 333.09 & 8.42 & 7.50 & 9.57 & 8.50 & 39 \\
\hline & $\mathrm{Cl}$ & 73.78 & 1.90 & 1.02 & 1.98 & 1.63 & 45 \\
\hline & $\mathrm{F}$ & 131.53 & 0.54 & 0.83 & 0.53 & 0.63 & 209 \\
\hline & $I$ & 508.80 & 8.26 & 3.76 & 4.76 & 5.59 & 91 \\
\hline & $S$ & 164.92 & 13.30 & 32.96 & 19.11 & 21.79 & 8 \\
\hline & $\mathrm{Se}$ & 49.64 & 67.03 & 17.61 & 74.09 & 52.91 & 1 \\
\hline
\end{tabular}

NC - Not Calculated

$\$$ - From gravimetric analysis of filters and rinse dry-downs 
The Catholic University of America

DuraMelter 1200 Tests with AZ-101HLW Simulants Vitreous State Laboratory

Table 6.11. WESP Emission Rates for DM 1200 AZ101 Test 3.

\begin{tabular}{|c|c|c|c|c|c|c|c|c|}
\hline & $\begin{array}{l}\text { Average } \\
\text { Feed Flux } \\
(\mathrm{mg} / \mathrm{min})\end{array}$ & $\begin{array}{c}\text { Average } \\
\text { Melter } \\
\text { Emissions } \\
\text { Flux } \\
\text { (mg/min) }\end{array}$ & $\begin{array}{c}\text { Average } \\
\text { SBS } \\
\text { Emissions } \\
\text { Flux } \\
\text { (mg/min) }\end{array}$ & $\begin{array}{l}\text { WESP } \\
\text { Emissions } \\
\text { Flux } \\
\text { (mg/min) }\end{array}$ & $\begin{array}{c}\text { DF } \\
\text { Across } \\
\text { WESP }\end{array}$ & $\begin{array}{c}\text { DF Across } \\
\text { SBS, } \\
\text { WESP }\end{array}$ & $\begin{array}{c}\text { Cumulative DF } \\
\text { Across Melter, } \\
\text { SBS, WESP }\end{array}$ \\
\hline \multirow{29}{*}{$\frac{\mathscr{Q}}{0}$} & Total $^{S}$ & 1036333 & 4520 & 172.8 & 1.06 & 163 & 4264 & 977673 \\
\hline & $\mathrm{Al}$ & 40600 & 169.48 & 0.37 & $<0.06$ & $>5.7$ & $>2825$ & $>676665$ \\
\hline & As & 314 & 5.83 & 1.25 & $<0.06$ & $>19.6$ & $>97$ & $>5232$ \\
\hline & $\mathrm{B}$ & 32162 & 140.49 & 0.14 & $<0.06$ & $>2.2$ & $>2342$ & $>536034$ \\
\hline & $\mathrm{Ba}$ & 371 & 1.46 & $<0.06$ & $<0.06$ & $\mathrm{NC}$ & $>24$ & $>6188$ \\
\hline & $\mathrm{Ca}$ & 1852 & 9.08 & 0.02 & $<0.06$ & $>0.4$ & $>151$ & $>30865$ \\
\hline & $\mathrm{Cd}$ & 3433 & 21.27 & 1.29 & $<0.06$ & $>20.2$ & $>354$ & $>57224$ \\
\hline & $\mathrm{Cr}$ & 284 & 4.75 & 0.51 & $<0.06$ & $>8.0$ & $>79$ & $>4727$ \\
\hline & $\mathrm{Cs}$ & 782 & 16.61 & 4.44 & $<0.06$ & $>69.4$ & $>277$ & $>13034$ \\
\hline & $\mathrm{Cu}$ & 248 & 0.97 & $<0.06$ & $<0.06$ & $\mathrm{NC}$ & $>16$ & $>4139$ \\
\hline & $\mathrm{Fe}$ & 75292 & 312.81 & 0.54 & $<0.06$ & $>8.4$ & $>5214$ & $>1254859$ \\
\hline & I & 1036 & $<0.06$ & $<0.06$ & $<0.06$ & $\mathrm{NC}$ & $\mathrm{NC}$ & $>17272$ \\
\hline & $\mathrm{K}$ & 1463 & 11.55 & 1.72 & $<0.06$ & $>26.9$ & $>193$ & $>24375$ \\
\hline & $\mathrm{Li}$ & 28884 & 71.23 & 4.16 & $<0.06$ & $>65.0$ & $>1187$ & $>481402$ \\
\hline & $\mathrm{Mg \#}$ & 7200 & 36.84 & 0.14 & $<0.06$ & $>2.1$ & $>614$ & $>120000$ \\
\hline & $\mathrm{Mn}$ & 24319 & 32.71 & 0.12 & $<0.06$ & $>1.8$ & $>545$ & $>405311$ \\
\hline & $\mathrm{Na}$ & 50670 & 223.13 & 20.63 & 0.06 & $-\quad 319$ & 3447 & 782786 \\
\hline & $\mathrm{Ni}$ & 4398 & 18.81 & 0.05 & $<0.06$ & $>0.8$ & $>314$ & $>73292$ \\
\hline & $\mathrm{P}$ & 588 & 6.21 & $<0.06$ & $<0.06$ & $\mathrm{NC}$ & $\geq 103$ & $>9804$ \\
\hline & $\mathrm{Pb}$ & 1443 & 7.23 & 0.33 & $<0.06$ & $>5.2$ & $>121$ & $>24051$ \\
\hline & $\mathrm{S}$ & 1038 & 102.47 & 27.97 & $<0.06$ & $>437.0$ & $\geq 1708$ & $>17305$ \\
\hline & $\mathrm{Sb}$ & 1638 & 6.11 & 0.41 & $<0.06$ & $>6.4$ & $>102$ & $>27305$ \\
\hline & $\mathrm{Se}$ & 1106 & 485.26 & 20.56 & $<0.06$ & $>321.2$ & $>8088$ & $>18437$ \\
\hline & $\mathrm{Si}$ & 220570 & 459.30 & 0.78 & $<0.06$ & $>12.2$ & $>7655$ & $>3676168$ \\
\hline & $\mathrm{Sr}$ & 20330 & 70.73 & 0.16 & $<0.06$ & $>2.6$ & $>1179$ & $>338841$ \\
\hline & $\mathrm{Te}$ & 1160 & 81.88 & 4.64 & $<0.06$ & $>72.6$ & $>1365$ & $>19333$ \\
\hline & $\mathrm{Ti}$ & 373 & 1.17 & $<0.06$ & $<0.06$ & $\mathrm{NC}$ & $>19$ & $>6213$ \\
\hline & $\mathrm{Zn}$ & 16652 & 67.92 & 0.41 & $<0.06$ & $>6.4$ & $>1132$ & $>277527$ \\
\hline & $\mathrm{Zr}$ & 27312 & 37.59 & $<0.06$ & $<0.06$ & $\mathrm{NC}$ & $>627$ & $>455205$ \\
\hline \multirow{6}{*}{$\stackrel{\mathscr{J}}{0}$} & B & 32162 & 333.09 & 8.50 & 0.53 & 16 & 626 & 60443 \\
\hline & $\mathrm{Cl}$ & 104 & 73.78 & 1.63 & $<0.07$ & $>23.3$ & $>1230$ & $>1727$ \\
\hline & $\mathrm{F}$ & 415 & 131.53 & 0.63 & 0.44 & 1 & 299 & 941 \\
\hline & I & 1036 & 508.80 & 5.59 & 6.25 & 1 & 81 & 166 \\
\hline & $\mathrm{S}$ & 1038 & 164.92 & 21.79 & 2.85 & 8 & 58 & 364 \\
\hline & $\mathrm{Se}$ & 1106 & 49.64 & 52.91 & 0.15 & 356 & 334 & 7436 \\
\hline
\end{tabular}

NC - Not Calculated

$\$$ - From gravimetric analysis of filters and rinse dry-downs

\# - The analyzed $\mathrm{MgO}$ concentration of $1.15 \mathrm{wt} \%$ instead of the target value of $0.06 \mathrm{wt} \%$ was used to calculate the feed flux 
Table 6.12. Melter Emission Rates for DM 1200 AZ101 Test 4.

\begin{tabular}{|c|c|c|c|c|c|c|c|}
\hline & $\begin{array}{l}\text { Average } \\
\text { Feed Flux } \\
\text { (mg/min) }\end{array}$ & $\begin{array}{c}\text { Run } 1 \\
(\mathrm{mg} / \mathrm{min})\end{array}$ & $\begin{array}{c}\operatorname{Run} 2 \\
(\mathrm{mg} / \mathrm{min})\end{array}$ & $\begin{array}{c}\text { Run } 3 \\
(\mathrm{mg} / \mathrm{min})\end{array}$ & $\begin{array}{l}\text { Average } \\
\text { (mg/min) }\end{array}$ & $\begin{array}{c}\text { DF Across } \\
\text { Melter }\end{array}$ \\
\hline \multirow{29}{*}{$\frac{2}{0}$} & Total $^{\$}$ & 536667 & 1770 & 1664 & 1810 & 1748 & 307.4 \\
\hline & $\mathrm{Al}$ & 21025 & 74.14 & 78.56 & 76.40 & 76.37 & 275 \\
\hline & As & 163 & 1.77 & 1.39 & 1.52 & 1.56 & 104 \\
\hline & $\mathrm{B}$ & 16655 & 49.19 & 52.86 & 45.75 & 49.26 & 338 \\
\hline & $\mathrm{Ba}$ & 192 & 0.64 & 0.60 & 0.67 & 0.64 & 300 \\
\hline & $\mathrm{Ca}$ & 959 & 3.55 & 3.58 & 3.90 & 3.68 & 261 \\
\hline & $\mathrm{Cd}$ & 1778 & 9.34 & 8.38 & 7.85 & 8.53 & 209 \\
\hline & $\mathrm{Cr}$ & 147 & 2.07 & 2.17 & 2.05 & 2.10 & 70 \\
\hline & $\mathrm{Cs}$ & 405 & 8.41 & 7.87 & 8.68 & 8.32 & 49 \\
\hline & $\mathrm{Cu}$ & 129 & 0.50 & 0.47 & 0.49 & 0.49 & 263 \\
\hline & $\mathrm{Fe}$ & 38990 & 118.41 & 116.88 & 124.22 & 119.84 & 325 \\
\hline & $I$ & 537 & $<0.06$ & $<0.06$ & $<0.06$ & $<0.06$ & $>8944$ \\
\hline & $\mathrm{K}$ & 757 & 4.40 & 6.16 & 5.82 & 5.46 & 139 \\
\hline & $\mathrm{Li}$ & 14958 & 32.70 & 32.37 & 33.49 & 32.85 & 455 \\
\hline & Mg\# & 3725 & 15.13 & 15.76 & 16.15 & 15.68 & 238 \\
\hline & $\mathrm{Mn}$ & 12593 & 16.80 & 11.41 & 14.09 & 14.10 & 893 \\
\hline & $\mathrm{Na}$ & 26240 & 89.75 & 92.95 & 96.65 & 93.11 & 282 \\
\hline & $\mathrm{Ni}$ & 2277 & 7.38 & 7.73 & 7.51 & 7.54 & 302 \\
\hline & $\mathrm{P}$ & 305 & 1.64 & 1.23 & 1.77 & 1.54 & 197 \\
\hline & $\mathrm{Pb}$ & 747 & 3.36 & 2.94 & 2.88 & 3.06 & 244 \\
\hline & $\mathrm{S}$ & 538 & 33.46 & 35.20 & 34.35 & 34.34 & 16 \\
\hline & $\mathrm{Sb}$ & 848 & 1.99 & 1.67 & 2.22 & 1.96 & 433 \\
\hline & $\mathrm{Se}$ & 573 & 308.93 & 265.95 & 259.47 & 278.12 & 2 \\
\hline & $\mathrm{Si}$ & 114223 & 173.00 & 153.20 & 189.47 & 171.89 & 664 \\
\hline & $\mathrm{Sr}$ & 10528 & 30.26 & 30.62 & 33.01 & 31.30 & 336 \\
\hline & $\mathrm{Te}$ & 601 & 44.75 & 44.10 & 37.37 & 42.07 & 14 \\
\hline & $\mathrm{Ti}$ & 193 & 1.33 & 1.73 & 0.98 & 1.35 & 143 \\
\hline & $\mathrm{Zn}$ & 8623 & 30.23 & 29.80 & 30.74 & 30.26 & 285 \\
\hline & $\mathrm{Zr}$ & 14144 & 14.09 & 9.38 & 14.49 & 12.65 & 1118 \\
\hline \multirow{6}{*}{ 包 } & $\mathrm{B}$ & 16655 & 136.38 & 152.31 & 151.04 & 146.58 & 114 \\
\hline & $\mathrm{Cl}$ & 54 & 72.91 & 68.99 & 58.23 & 66.71 & 1 \\
\hline & $\mathrm{F}$ & 215 & 63.91 & 63.67 & 57.24 & 61.61 & 3 \\
\hline & 1 & 537 & 500.39 & 407.69 & 437.61 & 448.56 & 1 \\
\hline & $\mathrm{S}$ & 538 & 100.72 & 93.55 & 89.98 & 94.75 & 6 \\
\hline & $\mathrm{Se}$ & 573 & 37.28 & 41.17 & 33.22 & 37.22 & 15 \\
\hline
\end{tabular}

NC - Not Calculated

$s$ - From gravimetric analysis of filters and rinse dry-downs

\# - The analyzed $\mathrm{MgO}$ concentration of $1.15 \mathrm{wt} \%$ instead of the target value of $0.06 \mathrm{wt} \%$ was used to calculate the feed flux 
Table 6.13. SBS Emission Rates for DM 1200 AZ101 Test 4.

\begin{tabular}{|c|c|c|c|c|c|c|c|}
\hline & $\begin{array}{c}\text { Average } \\
\text { Melter } \\
\text { Emissions } \\
\text { Flux } \\
(\mathrm{mg} / \mathrm{min})\end{array}$ & $\begin{array}{c}\text { Run } 1 \\
(\mathrm{mg} / \mathrm{min})\end{array}$ & $\begin{array}{c}\text { Run } 2 \\
(\mathrm{mg} / \mathrm{min})\end{array}$ & $\begin{array}{c}\text { Run } 3 \\
(\mathrm{mg} / \mathrm{min})\end{array}$ & $\begin{array}{l}\text { Average } \\
(\mathrm{mg} / \mathrm{min})\end{array}$ & $\begin{array}{c}\text { DF Across } \\
\text { SBS }\end{array}$ \\
\hline \multirow{29}{*}{$\frac{\mathscr{Q}}{0}$} & Total $^{\text {s }}$ & 1748 & 124.2 & 128.6 & 103.9 & 118.9 & 14.7 \\
\hline & $\mathrm{Al}$ & 76.37 & 0.19 & 0.34 & 0.10 & 0.21 & 361 \\
\hline & As & 1.56 & 0.48 & 0.42 & 0.27 & 0.39 & 4 \\
\hline & $\mathrm{B}$ & 49.26 & 0.37 & 0.38 & 0.14 & 0.29 & 167 \\
\hline & $\mathrm{Ba}$ & 0.64 & $<0.06$ & $<0.06$ & $<0.06$ & $<0.06$ & $>11$ \\
\hline & $\mathrm{Ca}$ & 3.68 & 0.14 & 0.21 & 0.13 & 0.16 & 23 \\
\hline & $\mathrm{Cd}$ & 8.53 & 1.12 & 1.13 & 0.77 & 1.01 & 8 \\
\hline & $\mathrm{Cr}$ & 2.10 & 0.46 & 0.45 & 0.35 & 0.42 & 5 \\
\hline & $\mathrm{Cs}$ & 8.32 & 2.28 & 2.45 & 1.95 & 2.23 & 4 \\
\hline & $\mathrm{Cu}$ & 0.49 & $<0.06$ & $<0.06$ & $<0.06$ & $<0.06$ & $>8$ \\
\hline & $\mathrm{Fe}$ & 119.84 & 0.17 & 0.24 & 0.18 & 0.20 & 610 \\
\hline & $I$ & $<0.06$ & $<0.06$ & $<0.06$ & $<0.06$ & $<0.06$ & $\mathrm{NC}$ \\
\hline & $\mathrm{K}$ & 5.46 & 0.91 & 1.38 & 0.93 & 1.07 & 5 \\
\hline & $\mathrm{Li}$ & 32.85 & 2.27 & 2.65 & 2.09 & 2.33 & 14 \\
\hline & $\mathrm{Mg}$ & 15.68 & 0.08 & 0.12 & $<0.06$ & $<0.09$ & $>174$ \\
\hline & $\mathrm{Mn}$ & 14.10 & $<0.06$ & 0.21 & $<0.06$ & 0.07 & 200 \\
\hline & $\mathrm{Na}$ & 93.11 & 9.64 & 11.28 & 8.80 & 9.90 & -9 \\
\hline & $\mathrm{Ni}$ & 7.54 & $<0.06$ & 0.08 & 0.07 & 0.05 & 126 \\
\hline & $\mathrm{P}$ & 1.54 & $<0.06$ & 0.08 & $<0.06$ & $<0.07$ & $>22$ \\
\hline & $\mathrm{Pb}$ & 3.06 & 0.25 & 0.25 & 0.20 & 0.23 & 13 \\
\hline & $\mathrm{S}$ & 34.34 & 10.88 & 11.97 & 9.70 & 10.85 & 3 \\
\hline & $\mathrm{Sb}$ & 1.96 & 0.23 & 0.29 & 0.17 & 0.23 & 8 \\
\hline & $\mathrm{Se}$ & 278.12 & 20.46 & 21.87 & 15.21 & 19.18 & 14 \\
\hline & $\mathrm{Si}$ & 171.89 & 0.48 & 0.53 & 0.30 & 0.43 & 396 \\
\hline & $\mathrm{Sr}$ & 31.30 & 0.09 & 0.27 & $<0.06$ & $<0.15$ & $>209$ \\
\hline & $\mathrm{Te}$ & 42.07 & 9.31 & 9.04 & 6.46 & 8.27 & 5 \\
\hline & $\mathrm{Ti}$ & 1.35 & 0.38 & 0.11 & 0.08 & 0.19 & 7 \\
\hline & $\mathrm{Zn}$ & 30.26 & 0.28 & 0.52 & 0.15 & 0.32 & 96 \\
\hline & $\mathrm{Zr}$ & 12.65 & $<0.06$ & $<0.06$ & $<0.06$ & $<0.06$ & $>211$ \\
\hline \multirow{6}{*}{ שூ } & B & 146.58 & 4.17 & 5.20 & 4.77 & 4.72 & 31 \\
\hline & $\mathrm{Cl}$ & 66.71 & 0.93 & 1.28 & 0.88 & 1.03 & 65 \\
\hline & $\mathrm{F}$ & 61.61 & 0.41 & 0.54 & 0.58 & 0.51 & 121 \\
\hline & $\mathrm{I}$ & 448.56 & 77.00 & 352.47 & 203.04 & 210.84 & 2 \\
\hline & $\mathrm{S}$ & 94.75 & $<0.07$ & $<0.07$ & $<0.07$ & $<0.07$ & $>1579$ \\
\hline & $\mathrm{Se}$ & 37.22 & 17.27 & 24.13 & 26.93 & 22.78 & 2 \\
\hline
\end{tabular}

NC - Not Calculated

$\$$ - From gravimetric analysis of filters and rinse dry-downs 
Table 6.14. WESP Emission Rates for DM 1200 AZ101 Test 4.

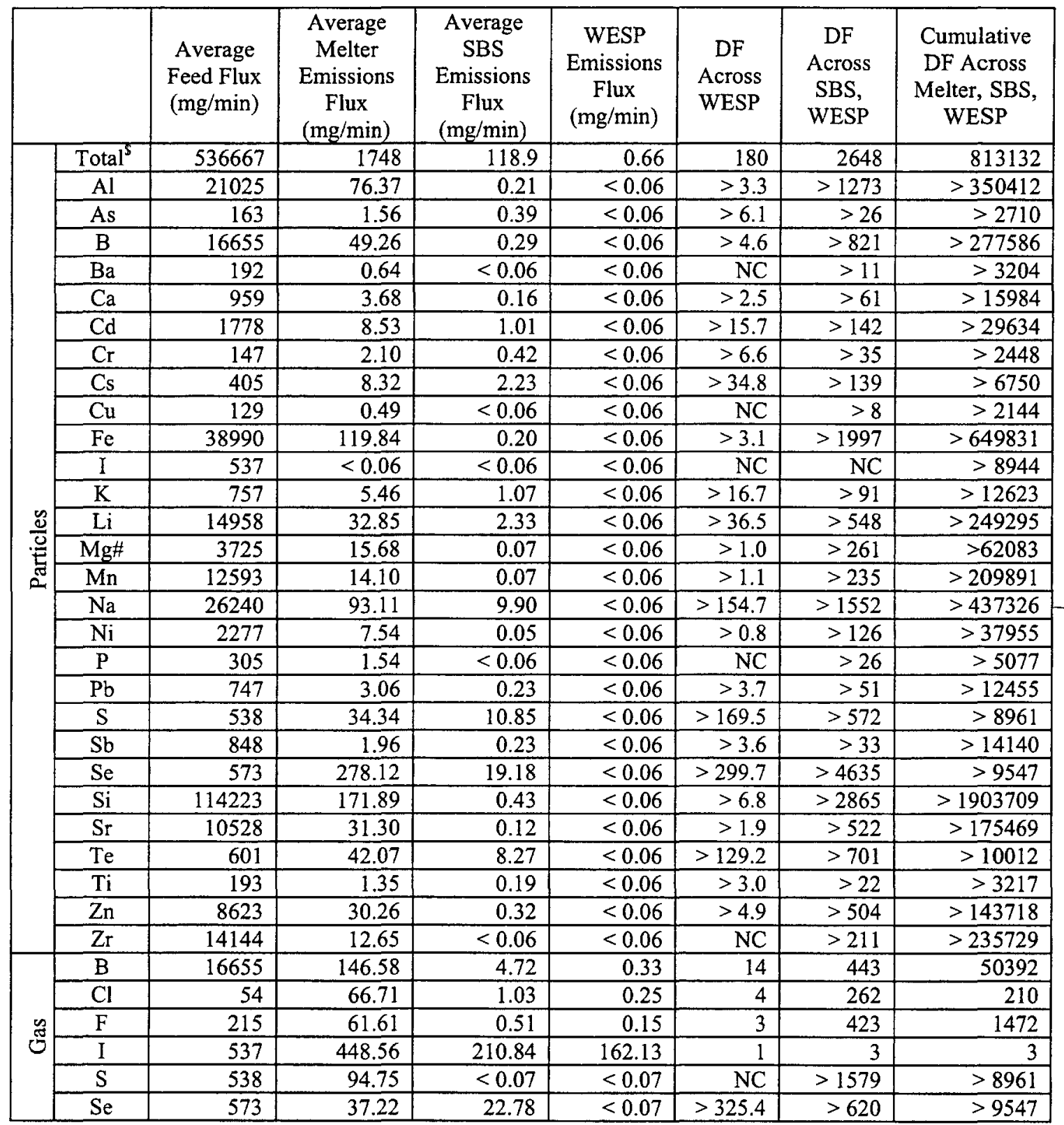

NC - Not Calculated

$s$ - From gravimetric analysis of filters and rinse dry-downs

\# - The analyzed MgO concentration of $1.15 \mathrm{wt} \%$ instead of the target value of $0.06 \mathrm{wt} \%$ was used to calculate the feed flux 
Table 6.15. Melter Emission Rates for DM 1200 AZ101 Test 5.

\begin{tabular}{|c|c|c|c|c|c|c|c|}
\hline & $\begin{array}{l}\text { Average } \\
\text { Feed Flux } \\
(\mathrm{mg} / \mathrm{min})\end{array}$ & $\begin{array}{c}\text { Run } 1 \\
(\mathrm{mg} / \mathrm{min})\end{array}$ & $\begin{array}{c}\text { Run } 2 \\
(\mathrm{mg} / \mathrm{min})\end{array}$ & $\begin{array}{c}\text { Run } 3 \\
(\mathrm{mg} / \mathrm{min})\end{array}$ & $\begin{array}{l}\text { Average } \\
\text { (mg/min) }\end{array}$ & $\begin{array}{c}\text { DF Across } \\
\text { Melter }\end{array}$ \\
\hline \multirow{29}{*}{ : } & Total $^{\text {S }}$ & 275333 & 730 & 958 & 1161 & 949.7 & 290 \\
\hline & $\mathrm{Al}$ & 10787 & 13.43 & 10.66 & 21.25 & 15.11 & 714 \\
\hline & As & 83 & 0.38 & 0.10 & 0.65 & 0.38 & 221 \\
\hline & $\mathrm{B}$ & 8545 & 13.71 & 34.64 & 30.22 & 26.19 & 326 \\
\hline & $\mathrm{Ba}$ & 99 & 0.14 & 0.19 & 0.25 & 0.19 & 514 \\
\hline & $\mathrm{Ca}$ & 492 & 1.11 & 1.19 & 1.81 & 1.37 & 359 \\
\hline & $\mathrm{Cd}$ & 912 & 1.44 & 2.78 & 2.68 & 2.30 & 397 \\
\hline & $\mathrm{Cr}$ & 75 & 0.23 & 0.25 & 0.30 & 0.26 & 289 \\
\hline & $\mathrm{Cs}$ & 208 & 0.86 & 2.12 & 1.40 & 1.46 & 142 \\
\hline & $\mathrm{Cu}$ & 66 & 0.08 & 0.22 & 0.24 & 0.18 & 361 \\
\hline & $\mathrm{Fe}$ & 20003 & 25.10 & 20.16 & 39.17 & 28.15 & 711 \\
\hline & I & 275 & $<0.06$ & $<0.06$ & $<0.06$ & $<0.06$ & $>4589$ \\
\hline & $\mathrm{K}$ & 389 & 1.36 & 4.21 & 2.81 & 2.80 & 139 \\
\hline & $\mathrm{Li}$ & 7674 & 12.77 & 19.14 & 22.69 & 18.20 & 422 \\
\hline & $\mathrm{Mg \#}$ & 1920 & 3.26 & 3.10 & 5.56 & 3.97 & 105 \\
\hline & $\mathrm{Mn}$ & 6461 & 2.94 & 3.11 & 4.23 & 3.43 & 1886 \\
\hline & $\mathrm{Na}$ & 13462 & 29.45 & 66.48 & 57.85 & 51.26 & 263 \\
\hline & $\mathrm{Ni}$ & 1168 & 1.48 & 1.22 & 2.39 & 1.70 & 689 \\
\hline & $\mathrm{P}$ & 156 & 0.52 & 0.28 & 1.12 & 0.64 & 245 \\
\hline & $\mathrm{Pb}$ & 383 & 0.42 & 0.58 & 0.81 & 0.60 & 634 \\
\hline & $\mathrm{S}$ & 276 & 1.06 & 1.79 & 1.63 & 1.49 & 185 \\
\hline & $\mathrm{Sb}$ & 435 & 0.14 & 0.09 & 0.11 & 0.11 & 3892 \\
\hline & $\mathrm{Se}$ & 294 & 32.36 & 40.78 & 43.14 & 38.76 & 8 \\
\hline & $\mathrm{Si}$ & 58601 & 33.56 & 32.16 & 49.43 & 38.38 & 1527 \\
\hline & $\mathrm{Sr}$ & 5401 & 5.87 & 6.76 & 11.07 & 7.90 & 684 \\
\hline & $\mathrm{Te}$ & 308 & 0.97 & 1.23 & 1.28 & 1.16 & 266 \\
\hline & $\mathrm{Ti}$ & 99 & 0.21 & 0.24 & 0.41 & 0.29 & 346 \\
\hline & $\mathrm{Zn}$ & 4424 & 4.74 & 4.22 & 7.62 & 5.53 & 801 \\
\hline & $\mathrm{Zr}$ & 7256 & 6.62 & 5.32 & 9.98 & 7.30 & 993 \\
\hline \multirow{6}{*}{ ఝु } & $\mathrm{B}$ & 8545 & 47.13 & 73.35 & 75.57 & 65.35 & 131 \\
\hline & $\mathrm{Cl}$ & 28 & $<0.07$ & $<0.07$ & $<0.07$ & $<0.07$ & $>459$ \\
\hline & $\mathrm{F}$ & 110 & 140.87 & 142.14 & 135.78 & 139.60 & 1 \\
\hline & $\mathrm{I}$ & 275 & 7.52 & 16.81 & 14.52 & 12.95 & 21 \\
\hline & $S$ & 276 & 7.63 & 11.55 & 2.74 & 7.31 & 38 \\
\hline & $\mathrm{Se}$ & 294 & 0.26 & 0.33 & 0.98 & 0.52 & 562 \\
\hline
\end{tabular}

NC - Not Calculated

$\$$ - From gravimetric analysis of filters and rinse dry-downs

\# - The analyzed $\mathrm{MgO}$ concentration of $1.15 \mathrm{wt} \%$ instead of the target value of $0.06 \mathrm{wt} \%$ was used to calculate the feed flux 
Table 6.16. SBS Emission Rates for DM 1200 AZ101 Test 5.

\begin{tabular}{|c|c|c|c|c|c|c|c|}
\hline & $\begin{array}{c}\text { Average Melter } \\
\text { Emissions Flux } \\
(\mathrm{mg} / \mathrm{min})\end{array}$ & $\begin{array}{c}\text { Run } 1 \\
(\mathrm{mg} / \mathrm{min})\end{array}$ & $\begin{array}{c}\text { Run } 2 \\
\text { (mg/min) }\end{array}$ & $\begin{array}{c}\text { Run } 3 \\
(\mathrm{mg} / \mathrm{min})\end{array}$ & $\begin{array}{l}\text { Average } \\
(\mathrm{mg} / \mathrm{min})\end{array}$ & $\begin{array}{l}\text { DF } \\
\text { Across } \\
\text { SBS }\end{array}$ \\
\hline \multirow{29}{*}{ 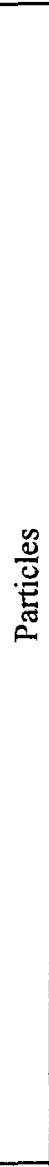 } & Total $^{8}$ & 949.7 & 46.8 & 102 & 59.9 & 69.6 & 14 \\
\hline & $\mathrm{Al}$ & 15.11 & 0.08 & 0.19 & 0.21 & 0.16 & 95 \\
\hline & As & 0.38 & 0.08 & 0.22 & 0.17 & 0.16 & 2 \\
\hline & $\mathrm{B}$ & 26.19 & 0.22 & 0.15 & 0.13 & 0.17 & 156 \\
\hline & $\mathrm{Ba}$ & 0.19 & $<0.06$ & $<0.06$ & $<0.06$ & $<0.06$ & $>3$ \\
\hline & $\mathrm{Ca}$ & 1.37 & 0.16 & 0.35 & 0.31 & 0.27 & 5 \\
\hline & $\mathrm{Cd}$ & 2.30 & 0.33 & 0.31 & 0.24 & 0.29 & 8 \\
\hline & $\mathrm{Cr}$ & 0.26 & $<0.06$ & $<0.06$ & $<0.06$ & $<0.06$ & $>4$ \\
\hline & $\mathrm{Cs}$ & 1.46 & 0.25 & 0.26 & 0.22 & 0.24 & 6 \\
\hline & $\mathrm{Cu}$ & 0.18 & $<0.06$ & $<0.06$ & $<0.06$ & $<0.06$ & $>3$ \\
\hline & $\mathrm{Fe}$ & 28.15 & 0.35 & 0.15 & 0.21 & 0.24 & 118 \\
\hline & I & $<0.06$ & $<0.06$ & $<0.06$ & $<0.06$ & $<0.06$ & $\mathrm{NC}$ \\
\hline & $\mathrm{K}$ & 2.80 & 0.18 & 0.33 & 0.25 & 0.25 & 11 \\
\hline & $\mathrm{Li}$ & 18.20 & 0.55 & 0.94 & 0.76 & 0.75 & 24 \\
\hline & $\mathrm{Mg}$ & 3.97 & $<0.06$ & 0.10 & 0.10 & 0.07 & 61 \\
\hline & $\mathrm{Mn}$ & 3.43 & $<0.06$ & $<0.06$ & $<0.06$ & $<0.06$ & $>57$ \\
\hline & $\mathrm{Na}$ & 51.26 & 2.32 & 4.81 & 3.54 & 3.56 & 14 \\
\hline & $\mathrm{Ni}$ & 1.70 & $<0.06$ & $<0.06$ & $<0.06$ & $<0.06$ & $>28$ \\
\hline & $\mathrm{P}$ & 0.64 & $<0.06$ & 0.07 & 0.13 & $<0.09$ & $>7$ \\
\hline & $\mathrm{Pb}$ & 0.60 & 0.08 & 0.09 & 0.09 & 0.09 & 7 \\
\hline & $\mathrm{S}$ & 1.49 & 0.77 & 0.34 & 0.52 & 0.54 & 3 \\
\hline & $\mathrm{Sb}$ & 0.11 & $<0.06$ & $<0.06$ & $<0.06$ & $<0.06$ & $>2$ \\
\hline & $\mathrm{Se}$ & 38.76 & 5.65 & 4.10 & 4.64 & 4.80 & 8 \\
\hline & $\mathrm{Si}$ & 38.38 & 0.46 & 1.79 & 1.07 & 1.10 & 35 \\
\hline & $\mathrm{Sr}$ & 7.90 & $<0.06$ & 0.07 & 0.07 & 0.05 & 132 \\
\hline & $\mathrm{Te}$ & 1.16 & 0.34 & 0.36 & 0.41 & 0.37 & 3 \\
\hline & $\mathrm{Ti}$ & 0.29 & $<0.06$ & $<0.06$ & $<0.06$ & $<0.06$ & $>5$ \\
\hline & $\mathrm{Zn}$ & 5.53 & $<0.06$ & 0.08 & 0.09 & 0.06 & 92 \\
\hline & $\mathrm{Zr}$ & 7.30 & $<0.06$ & $<0.06$ & $<0.06$ & $<0.06$ & $>122$ \\
\hline \multirow{6}{*}{ 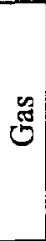 } & $B$ & 65.35 & 5.85 & 9.01 & 6.11 & 6.99 & 9 \\
\hline & $\mathrm{Cl}$ & $<0.06$ & $<0.07$ & $<0.07$ & $<0.07$ & $<0.07$ & $>0$ \\
\hline & $F$ & 139.60 & 18.24 & 31.46 & 23.67 & 24.46 & 6 \\
\hline & I & 12.95 & 4.89 & 13.13 & 8.89 & 8.97 & 1 \\
\hline & $S$ & 7.31 & 3.11 & 10.32 & 2.00 & 5.14 & 1 \\
\hline & $\mathrm{Se}$ & 0.52 & 1.17 & 0.41 & 0.24 & 0.61 & 1 \\
\hline
\end{tabular}

$\mathrm{NC}-$ Not calculated

$s$ - From gravimetric analysis of filters and rinse dry-downs 
The Catholic University of America Vitreous State Laboratory
DuraMelter 1200 Tests with AZ-101HLW Simulants

Final Report, VSL-02R0100-2, Rev.I

Table 6.17. WESP Emission Rates for DM 1200 AZ101 Test 5.

\begin{tabular}{|c|c|c|c|c|c|c|c|c|}
\hline & $\begin{array}{c}\text { Average } \\
\text { Feed Flux } \\
(\mathrm{mg} / \mathrm{min})\end{array}$ & $\begin{array}{c}\text { Average } \\
\text { Melter } \\
\text { Emissions } \\
\text { Flux } \\
(\mathrm{mg} / \mathrm{min}) \\
\end{array}$ & $\begin{array}{c}\text { Average } \\
\text { SBS } \\
\text { Emissions } \\
\text { Flux } \\
\text { (mg/min) }\end{array}$ & $\begin{array}{l}\text { WESP } \\
\text { Emission } \\
\text { s Flux } \\
\text { (mg/min) }\end{array}$ & $\begin{array}{c}\text { DF } \\
\text { Across } \\
\text { WESP }\end{array}$ & $\begin{array}{c}\text { DF Across } \\
\text { SBS, } \\
\text { WESP }\end{array}$ & $\begin{array}{c}\text { Cumulative DF } \\
\text { Across Melter, } \\
\text { SBS, WESP }\end{array}$ \\
\hline \multirow{29}{*}{$\frac{\mathscr{E}}{\mathscr{E}}$} & Total $^{\$}$ & 275333 & 949.7 & 69.6 & 0.8 & 86 & 1187 & 344166 \\
\hline & $\mathrm{Al}$ & 10787 & 15.11 & 0.16 & $<0.06$ & $>2.5$ & $>252$ & $>179776$ \\
\hline & As & 83 & 0.38 & 0.16 & $<0.06$ & $>2.5$ & $>6$ & $>1390$ \\
\hline & $\mathrm{B}$ & 8545 & 26.19 & 0.17 & $<0.06$ & $>2.6$ & $>436$ & $>142414$ \\
\hline & $\mathrm{Ba}$ & 99 & 0.19 & $<0.06$ & $<0.06$ & $\mathrm{NC}$ & $>3$ & $>1644$ \\
\hline & $\mathrm{Ca}$ & 492 & 1.37 & 0.27 & $<0.06$ & $>4.3$ & $>23$ & $>8200$ \\
\hline & $\mathrm{Cd}$ & 912 & 2.30 & 0.29 & $<0.06$ & $>4.6$ & $>38$ & $>15203$ \\
\hline & $\mathrm{Cr}$ & 75 & 0.26 & $<0.06$ & $<0.06$ & $\mathrm{NC}$ & $>4$ & $>1256$ \\
\hline & Cs & 208 & 1.46 & 0.24 & $<0.06$ & $>3.8$ & $>24$ & $>3463$ \\
\hline & $\mathrm{Cu}$ & 66 & 0.18 & $<0.06$ & $<0.06$ & $\mathrm{NC}$ & $>3$ & $>1100$ \\
\hline & $\mathrm{Fe}$ & 20003 & 28.15 & 0.24 & $<0.06$ & $>3.7$ & $>469$ & $>333391$ \\
\hline & $\mathrm{I}$ & 275 & $<0.06$ & $<0.06$ & $<0.06$ & $\mathrm{NC}$ & $\mathrm{NC}$ & $>4589$ \\
\hline & $\mathrm{K}$ & 389 & 2.80 & 0.25 & $<0.06$ & $>3.9$ & $>47$ & $>6476$ \\
\hline & $\mathrm{Li}$ & 7674 & 18.20 & 0.75 & $<0.06$ & $>11.7$ & $\geq 303$ & $>127899$ \\
\hline & $\mathrm{Mg \#}$ & 1920 & 3.97 & 0.07 & $<0.06$ & $>1.0$ & $>66$ & $>32000$ \\
\hline & $\mathrm{Mn}$ & 6461 & 3.43 & $<0.06$ & $<0.06$ & $\mathrm{NC}$ & $>57$ & $>107683$ \\
\hline & $\mathrm{Na}$ & 13462 & 51.26 & 3.56 & $<0.06$ & $>55.6$ & $>854$ & $>224367$ \\
\hline & $\mathrm{Ni}$ & 1168 & 1.70 & $<0.06$ & $<0.06$ & $\mathrm{NC}$ & $>28$ & $>19472$ \\
\hline & $\mathrm{P}$ & 156 & 0.64 & 0.07 & $<0.06$ & $>1.0$ & $>11$ & $>2605$ \\
\hline & $\mathrm{Pb}$ & 383 & 0.60 & 0.09 & $<0.06$ & $>1.3$ & $>10$ & $>6390$ \\
\hline & $\mathrm{S}$ & 276 & 1.49 & 0.54 & $<0.06$ & $>8.5$ & $>25$ & $>4597$ \\
\hline & $\mathrm{Sb}$ & 435 & 0.11 & $<0.06$ & $<0.06$ & $\mathrm{NC}$ & $>2$ & $>7254$ \\
\hline & $\mathrm{Se}$ & 294 & 38.76 & 4.80 & $<0.06$ & $>75.0$ & $>646$ & $>4898$ \\
\hline & $\mathrm{Si}$ & 58601 & 38.38 & 1.10 & $<0.06$ & $>17.3$ & $>640$ & $>976685$ \\
\hline & $\mathrm{Sr}$ & 5401 & 7.90 & 0.05 & $<0.06$ & $>0.7$ & $>132$ & $>90023$ \\
\hline & $\mathrm{Te}$ & 308 & 1.16 & 0.37 & $<0.06$ & $>5.7$ & $>19$ & $>5136$ \\
\hline & $\mathrm{Ti}$ & 99 & 0.29 & $<0.06$ & $<0.06$ & $\mathrm{NC}$ & $>5$ & $>1651$ \\
\hline & $\mathrm{Zn}$ & 4424 & 5.53 & 0.06 & $<0.06$ & $>0.9$ & $>92$ & $>73733$ \\
\hline & $\mathrm{Zr}$ & 7256 & 7.30 & $<0.06$ & $<0.06$ & $\mathrm{NC}$ & $>122$ & $>120939$ \\
\hline \multirow{6}{*}{ : } & $\mathrm{B}$ & 8545 & 65.35 & 6.99 & 0.44 & 16 & 150 & 19629 \\
\hline & $\mathrm{Cl}$ & 28 & $<0.06$ & $<0.07$ & $<0.07$ & $\mathrm{NC}$ & $\mathrm{NC}$ & $>459$ \\
\hline & $\mathrm{F}$ & 110 & 139.60 & 24.46 & 31.93 & 1 & 4 & 3 \\
\hline & I & 275 & 12.95 & 8.97 & 2.96 & 3 & 4 & 93 \\
\hline & $\mathrm{S}$ & 276 & 7.31 & 5.14 & 1.15 & 4 & 6 & 240 \\
\hline & $\mathrm{Se}$ & 294 & 0.52 & 0.61 & 0.11 & 6 & 5 & 2776 \\
\hline
\end{tabular}

NC - Not Calculated

$\$$ - From gravimetric analysis of filters and rinse dry-downs

\# - The analyzed $\mathrm{MgO}$ concentration of $1.15 \mathrm{wt} \%$ instead of the target value of $0.06 \mathrm{wt} \%$ was used to calculate the feed flux 
The Catholic University of America

Vitreous State Laboratory
DuraMelter 1200 Tests with AZ-101HLW Simulants

Final Report, VSL-02R0100-2, Rev.I

Table 6.18. Melter Emission Rates for DM 1200 AZ101 Test 6.

\begin{tabular}{|c|c|c|c|c|c|c|c|}
\hline & $\begin{array}{l}\text { Average } \\
\text { Feed Flux } \\
\text { (mg/min) }\end{array}$ & $\begin{array}{c}\text { Run } 1 \\
(\mathrm{mg} / \mathrm{min})\end{array}$ & $\begin{array}{c}\text { Run } 2 \\
\text { (mg/min) }\end{array}$ & $\begin{array}{c}\text { Run } 3 \\
(\mathrm{mg} / \mathrm{min})\end{array}$ & $\begin{array}{c}\text { Melter } \\
\text { Average } \\
\text { (mg/min) }\end{array}$ & $\begin{array}{c}\text { DF } \\
\text { Across } \\
\text { Melter }\end{array}$ \\
\hline \multirow{29}{*}{$\frac{\mathscr{0}}{0}$} & Total $^{\$}$ & 160333 & 228.2 & 229.0 & 162.0 & 206.4 & 797 \\
\hline & $\mathrm{Al}$ & 6281 & 7.32 & 3.83 & 2.66 & 4.60 & 1364 \\
\hline & As & 49 & 0.19 & 1.04 & 0.47 & 0.57 & 85 \\
\hline & B & 4976 & 1.87 & 1.32 & 1.24 & 1.48 & 3367 \\
\hline & $\mathrm{Ba}$ & 57 & $<0.06$ & $<0.06$ & $<0.06$ & $<0.06$ & $>957$ \\
\hline & $\mathrm{Ca}$ & 287 & 4.51 & 0.92 & 0.51 & 1.98 & 145 \\
\hline & $\mathrm{Cd}$ & 531 & 0.74 & 3.80 & 1.38 & 1.97 & 269 \\
\hline & $\mathrm{Cr}$ & 44 & 0.16 & 0.17 & 0.11 & 0.15 & 302 \\
\hline & $\mathrm{Cs}$ & 121 & 0.26 & 0.68 & 0.35 & 0.43 & 282 \\
\hline & $\mathrm{Cu}$ & 38 & 0.09 & $<0.06$ & $<0.06$ & $<0.07$ & $>543$ \\
\hline & $\mathrm{Fe}$ & 11649 & 7.28 & 5.74 & 6.45 & 6.49 & 1795 \\
\hline & I & 160 & $<0.06$ & $<0.06$ & $<0.06$ & $<0.06$ & $>2672$ \\
\hline & $\mathrm{K}$ & 226 & 0.32 & 0.45 & 0.30 & 0.36 & 632 \\
\hline & $\mathrm{Li}$ & 4469 & 1.41 & 1.63 & 1.22 & 1.42 & 3149 \\
\hline & $\mathrm{Mg \#}$ & 1114 & 1.05 & 0.48 & 0.45 & 0.66 & 1687 \\
\hline & $\mathrm{Mn}$ & 3762 & 0.43 & 0.35 & 0.29 & 0.36 & 10536 \\
\hline & $\mathrm{Na}$ & 7839 & 3.47 & 4.48 & 3.27 & 3.74 & 2096 \\
\hline & $\mathrm{Ni}$ & 680 & 0.49 & 0.29 & 0.30 & 0.36 & 1884 \\
\hline & $\mathrm{P}$ & 91 & 0.10 & $<0.06$ & 0.12 & $<0.08$ & $>1240$ \\
\hline & $\mathrm{Pb}$ & 223 & 0.18 & 0.37 & 0.20 & 0.25 & 896 \\
\hline & $\mathrm{S}$ & 161 & 0.95 & 1.45 & 1.12 & 1.17 & 137 \\
\hline & $\mathrm{Sb}$ & 253 & $<0.06$ & 0.25 & 0.08 & $<0.13$ & $>1946$ \\
\hline & $\mathrm{Se}$ & 171 & 20.39 & 37.55 & 27.24 & 28.39 & 6 \\
\hline & $\mathrm{Si}$ & 34125 & 20.07 & 9.34 & 7.94 & 12.45 & 2742 \\
\hline & $\mathrm{Sr}$ & 3145 & 1.24 & 0.95 & 0.93 & 1.04 & 3026 \\
\hline & $\mathrm{Te}$ & 179 & 2.25 & 14.10 & 5.22 & 7.19 & 25 \\
\hline & $\mathrm{Ti}$ & 58 & 1.53 & 0.19 & 0.09 & 0.60 & 96 \\
\hline & $\mathrm{Zn}$ & 2576 & 0.89 & 0.74 & 0.65 & 0.76 & 3397 \\
\hline & $\mathrm{Zr}$ & 4226 & 0.68 & 0.57 & 0.44 & 0.56 & 7501 \\
\hline \multirow{6}{*}{ రृ } & $\mathrm{B}$ & 4976 & 6.24 & 23.09 & 13.74 & 14.36 & 347 \\
\hline & $\mathrm{Cl}$ & 16 & 3.99 & 12.35 & 8.47 & 8.27 & 2 \\
\hline & $\mathrm{F}$ & 64 & 4.99 & 34.68 & 22.52 & 20.73 & 3 \\
\hline & I & 160 & 30.12 & 22.67 & 30.80 & 27.86 & 6 \\
\hline & $S$ & 161 & 9.05 & 32.92 & 16.60 & 19.52 & 8 \\
\hline & $\mathrm{Se}$ & 171 & 9.74 & 6.50 & 14.01 & 10.08 & 17 \\
\hline
\end{tabular}

$\mathrm{NC}$ - Not Calculated

$\$$ - From gravimetric analysis of filters and rinse dry-downs

\# - The analyzed $\mathrm{MgO}$ concentration of $1.15 \mathrm{wt} \%$ instead of the target value of $0.06 \mathrm{wt} \%$ was used to calculate the feed flux 
Table 6.19. SBS Emission Rates for DM 1200 AZ101Test 6.

\begin{tabular}{|c|c|c|c|c|c|c|c|}
\hline & $\begin{array}{c}\text { Average } \\
\text { Melter } \\
\text { Emissions } \\
\text { Flux } \\
(\mathrm{mg} / \mathrm{min}) \\
\end{array}$ & $\begin{array}{c}\text { Run } 1 \\
(\mathrm{mg} / \mathrm{min})\end{array}$ & $\begin{array}{c}\text { Run } 2 \\
(\mathrm{mg} / \mathrm{min})\end{array}$ & $\begin{array}{c}\text { Run } 3 \\
\text { (mg/min) }\end{array}$ & $\begin{array}{l}\text { Average } \\
\text { (mg/min) }\end{array}$ & $\begin{array}{c}\text { DF Across } \\
\text { SBS }\end{array}$ \\
\hline \multirow{29}{*}{$\frac{\frac{\mathscr{Q}}{0}}{\mathrm{E}}$} & Total $^{\$}$ & 206.4 & 29.2 & 77.0 & 25.3 & 43.8 & 6 \\
\hline & $\mathrm{Al}$ & 4.60 & $<0.06$ & 0.15 & $<0.06$ & $<0.09$ & $>51$ \\
\hline & As & 0.57 & 0.20 & 0.71 & 0.12 & 0.34 & 2 \\
\hline & $\mathrm{B}$ & 1.48 & 0.19 & 0.19 & $<0.06$ & $<0.15$ & $>10$ \\
\hline & $\mathrm{Ba}$ & $<0.06$ & $<0.06$ & $<0.06$ & $<0.06$ & $<0.06$ & $\mathrm{NC}$ \\
\hline & $\mathrm{Ca}$ & 1.98 & 0.09 & 0.25 & 0.14 & 0.16 & 12 \\
\hline & $\mathrm{Cd}$ & 1.97 & 0.70 & 2.54 & 0.29 & 1.18 & 2 \\
\hline & $\mathrm{Cr}$ & 0.15 & 0.08 & 0.09 & 0.10 & 0.09 & 2 \\
\hline & Cs & 0.43 & 0.20 & 0.43 & 0.11 & 0.25 & 2 \\
\hline & $\mathrm{Cu}$ & 0.03 & $<0.06$ & $<0.06$ & $<0.06$ & $<0.06$ & $>1$ \\
\hline & $\mathrm{Fe}$ & 6.49 & 0.07 & 0.08 & $<0.06$ & $<0.07$ & $>108$ \\
\hline & I & $<0.06$ & $<0.06$ & $<0.06$ & $<0.06$ & $<0.06$ & $\mathrm{NC}$ \\
\hline & $\mathrm{K}$ & 0.36 & 0.11 & 0.19 & 0.09 & 0.13 & 3 \\
\hline & $\mathrm{Li}$ & 1.42 & 0.25 & 0.48 & 0.20 & 0.31 & 5 \\
\hline & $\mathrm{Mg}$ & 0.66 & $<0.06$ & $<0.06$ & $<0.06$ & $<0.06$ & $>11$ \\
\hline & $\mathrm{Mn}$ & 0.36 & $<0.06$ & $<0.06$ & $<0.06$ & $<0.06$ & $>6$ \\
\hline & $\mathrm{Na}$ & 3.74 & 0.91 & 1.65 & 0.71 & 1.09 & 3 \\
\hline & $\mathrm{Ni}$ & 0.36 & $<0.06$ & $<0.06$ & $<0.06$ & $<0.06$ & $>6$ \\
\hline & $\mathrm{P}$ & 0.07 & 0.23 & $<0.06$ & 0.42 & 0.22 & $\mathrm{NC}$ \\
\hline & $\mathrm{Pb}$ & 0.25 & 0.10 & 0.24 & 0.08 & 0.14 & 2 \\
\hline & $\mathrm{S}$ & 1.17 & 0.57 & 0.43 & 0.22 & 0.41 & 3 \\
\hline & $\mathrm{Sb}$ & 0.11 & $<0.06$ & 0.21 & $<0.06$ & $<0.11$ & $>1$ \\
\hline & $\mathrm{Se}$ & 28.39 & 5.85 & 11.58 & 3.16 & 6.86 & 4 \\
\hline & $\mathrm{Si}$ & 12.45 & 0.51 & 1.11 & 0.25 & 0.62 & 20 \\
\hline & $\mathrm{Sr}$ & 1.04 & $<0.06$ & $<0.06$ & $<0.06$ & $<0.06$ & $>17$ \\
\hline & $\mathrm{Te}$ & 7.19 & 2.51 & 9.07 & 1.50 & 4.36 & 2 \\
\hline & $\mathrm{Ti}$ & 0.60 & $<0.06$ & $<0.06$ & $<0.06$ & $<0.06$ & $>10$ \\
\hline & $\mathrm{Zn}$ & 0.76 & $<0.06$ & $<0.06$ & $<0.06$ & $<0.06$ & $>13$ \\
\hline & $\mathrm{Zr}$ & 0.56 & $<0.06$ & $<0.06$ & $<0.06$ & $<0.06$ & $>9$ \\
\hline \multirow{6}{*}{ 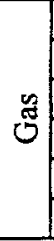 } & $\mathrm{B}$ & 14.36 & 1.23 & 2.23 & 0.62 & 1.36 & 11 \\
\hline & $\mathrm{Cl}$ & 8.27 & 1.35 & 2.71 & 0.23 & 1.43 & 6 \\
\hline & $\mathrm{F}$ & 20.73 & 0.98 & 1.96 & 0.92 & 1.29 & 16 \\
\hline & $\mathrm{I}$ & 27.86 & $<0.07$ & $<0.07$ & $<0.07$ & $<0.07$ & $>464$ \\
\hline & $\mathrm{S}$ & 19.52 & $<0.07$ & $<0.07$ & $<0.07$ & $<0.07$ & $>325$ \\
\hline & $\mathrm{Se}$ & 10.08 & 8.35 & 6.34 & 1.42 & 5.37 & 2 \\
\hline
\end{tabular}

NC - Not Calculated

$s$ - From gravimetric analysis of filters and rinse dry-downs 
The Catholic University of America

DuraMelter 1200 Tests with AZ-101HLW Simulants

Vitreous State Laboratory

Final Report, VSL-02R0100-2, Rev.1

Table 6.20. WESP Emission Rates for DM 1200 AZ101 Test 6.

\begin{tabular}{|c|c|c|c|c|c|c|c|c|}
\hline & $\begin{array}{l}\text { Average } \\
\text { Feed Flux } \\
(\mathrm{mg} / \mathrm{min})\end{array}$ & $\begin{array}{c}\text { Average } \\
\text { Melter } \\
\text { Emissions } \\
\text { Flux } \\
\text { (mg/min) }\end{array}$ & $\begin{array}{l}\text { Average } \\
\text { SBS } \\
\text { Emissions } \\
\text { Flux } \\
\text { (mg/min) }\end{array}$ & $\begin{array}{c}\text { WESP } \\
\text { Emissions } \\
\text { Flux } \\
\text { (mg/min) }\end{array}$ & $\begin{array}{c}\text { DF } \\
\text { Across } \\
\text { WESP }\end{array}$ & $\begin{array}{l}\text { DF Across } \\
\text { SBS, WESP }\end{array}$ & $\begin{array}{c}\text { Cumulative DF } \\
\text { Across Melter, } \\
\text { SBS, WESP }\end{array}$ \\
\hline \multirow{29}{*}{ 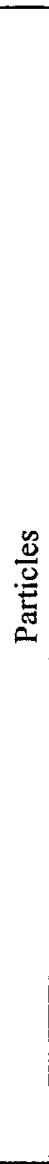 } & Total $^{\$}$ & 160333 & 206.4 & 43.8 & 0.8 & 55 & 258 & 208225 \\
\hline & $\mathrm{Al}$ & 6281 & 4.60 & 0.05 & $<0.06$ & $>0.8$ & $>77$ & $>104688$ \\
\hline & As & 49 & 0.57 & 0.34 & $<0.06$ & $>5.4$ & $>9$ & $>810$ \\
\hline & $\mathrm{B}$ & 4976 & 1.48 & 0.13 & $<0.06$ & $>2.0$ & $>25$ & $>82931$ \\
\hline & $\mathrm{Ba}$ & 57 & $<0.06$ & $<0.06$ & $<0.06$ & $\mathrm{NC}$ & $\mathrm{NC}$ & $>957$ \\
\hline & $\mathrm{Ca}$ & 287 & 1.98 & 0.16 & $<0.06$ & $>2.5$ & $>33$ & $>4775$ \\
\hline & $\mathrm{Cd}$ & 531 & 1.97 & 1.18 & $<0.06$ & $>18.4$ & $>33$ & $>8853$ \\
\hline & $\mathrm{Cr}$ & 44 & 0.15 & 0.09 & $<0.06$ & $>1.4$ & $>2$ & $>731$ \\
\hline & $\mathrm{Cs}$ & 121 & 0.43 & 0.25 & $<0.06$ & $>3.8$ & $>7$ & $>2017$ \\
\hline & $\mathrm{Cu}$ & 38 & 0.03 & $<0.06$ & $<0.06$ & $\mathrm{NC}$ & $>1$ & $>640$ \\
\hline & $\mathrm{Fe}$ & 11649 & 6.49 & 0.05 & $<0.06$ & $>0.8$ & $>108$ & $>194142$ \\
\hline & I & 160 & $<0.06$ & $<0.06$ & $<0.06$ & $\mathrm{NC}$ & $\mathrm{NC}$ & $>2672$ \\
\hline & $\mathrm{K}$ & 226 & 0.36 & 0.13 & $<0.06$ & $>2.1$ & $>6$ & $>3771$ \\
\hline & $\mathrm{Li}$ & 4469 & 1.42 & 0.31 & $<0.06$ & $>4.8$ & $>24$ & $>74479$ \\
\hline & $\mathrm{Mg \#}$ & 1114 & 0.66 & $<0.06$ & $<0.06$ & $\mathrm{NC}$ & $>11$ & $>18560$ \\
\hline & $\mathrm{Mn}$ & 3762 & 0.36 & $<0.06$ & $<0.06$ & $\mathrm{NC}$ & $>6$ & $>62707$ \\
\hline & $\mathrm{Na}$ & 7839 & 3.74 & 1.09 & $<0.06$ & $>17.0$ & $>62$ & $>130654$ \\
\hline & $\mathrm{Ni}$ & 680 & 0.36 & $<0.06$ & $<0.06$ & $\mathrm{NC}$ & $>6$ & $>11339$ \\
\hline & $\mathrm{P}$ & 91 & 0.07 & 0.22 & $<0.06$ & $>3.4$ & $>1$ & $>1517$ \\
\hline & $\mathrm{Pb}$ & 223 & 0.25 & 0.14 & $<0.06$ & $>2.2$ & $>4$ & $>3721$ \\
\hline & $\mathrm{S}$ & 161 & 1.17 & 0.41 & $<0.06$ & $>6.3$ & $>20$ & $>2677$ \\
\hline & $\mathrm{Sb}$ & 253 & 0.11 & 0.07 & $<0.06$ & $>1.1$ & $>2$ & $>4224$ \\
\hline & $\mathrm{Se}$ & 171 & 28.39 & 6.86 & $<0.06$ & $>107.2$ & $>473$ & $>2852$ \\
\hline & $\mathrm{Si}$ & 34125 & 12.45 & 0.62 & $<0.06$ & $>9.8$ & $>207$ & $>568748$ \\
\hline & $\mathrm{Sr}$ & 3145 & 1.04 & $<0.06$ & $<0.06$ & $\mathrm{NC}$ & $>17$ & $>52423$ \\
\hline & $\mathrm{Te}$ & 179 & 7.19 & 4.36 & $<0.06$ & $>68.1$ & $>120$ & $>2991$ \\
\hline & $\mathrm{Ti}$ & 58 & 0.60 & $<0.06$ & $<0.06$ & $\mathrm{NC}$ & $>10$ & $>961$ \\
\hline & $\mathrm{Zn}$ & 2576 & 0.76 & $<0.06$ & $<0.06$ & $\mathrm{NC}$ & $>13$ & $>42937$ \\
\hline & $\mathrm{Zr}$ & 4226 & 0.56 & $<0.06$ & $<0.06$ & $\mathrm{NC}$ & $>9$ & $>70426$ \\
\hline \multirow{6}{*}{$\stackrel{\mathscr{0}}{0}$} & $\mathrm{~B}$ & 4976 & 14.36 & 1.36 & 0.16 & 8 & 89 & 30724 \\
\hline & $\mathrm{Cl}$ & 16 & 8.27 & 1.43 & $<0.07$ & $>20.4$ & $>138$ & $>267$ \\
\hline & $\mathrm{F}$ & 64 & 20.73 & 1.29 & 0.67 & 2 & 31 & 96 \\
\hline & $\mathrm{I}$ & 160 & 27.86 & $<0.07$ & 0.17 & $\mathrm{NC}$ & 167 & 961 \\
\hline & $\mathrm{S}$ & 161 & 19.52 & $<0.07$ & $<0.07$ & $\mathrm{NC}$ & $>325$ & $>2677$ \\
\hline & $\mathrm{Se}$ & 171 & 10.08 & 5.37 & $<0.07$ & $>76.7$ & $>168$ & $>2852$ \\
\hline
\end{tabular}

NC - Not Calculated

$\$$ - From gravimetric analysis of filters and rinse dry-downs

\# - The analyzed $\mathrm{MgO}$ concentration of $1.15 \mathrm{wt} \%$ instead of the target value of $0.06 \mathrm{wt} \%$ was used to calculate the feed flux 
The Catholic University of America

Vitreous State Laboratory
DuraMelter 1200 Tests with AZ-101HLW Simulants

Final Report, VSL-02R0100-2, Rev.I

Table 6.21. Melter Emission Rates for DM 1200 AZ101 Test 7.

\begin{tabular}{|c|c|c|c|c|c|c|c|}
\hline & $\begin{array}{l}\text { Average } \\
\text { Feed Flux } \\
\text { (mg/min) }\end{array}$ & $\begin{array}{c}\text { Run } 1 \\
\text { (mg/min) }\end{array}$ & $\begin{array}{c}\text { Run } 2 \\
(\mathrm{mg} / \mathrm{min})\end{array}$ & $\begin{array}{c}\text { Run } 3 \\
(\mathrm{mg} / \mathrm{min})\end{array}$ & $\begin{array}{l}\text { Average } \\
(\mathrm{mg} / \mathrm{min})\end{array}$ & $\begin{array}{c}\text { DF Across } \\
\text { Melter }\end{array}$ \\
\hline \multirow{29}{*}{ : } & Total $^{\text {s }}$ & 957667 & 2294 & 2206 & 2135 & 2212 & 433 \\
\hline & $\mathrm{Al}$ & 37518 & 29.00 & 24.24 & 22.72 & 25.32 & 1482 \\
\hline & As & 290 & 9.99 & 9.89 & 12.06 & 10.65 & 27 \\
\hline & $\mathrm{B}$ & 29721 & 41.58 & 32.59 & 18.07 & 30.75 & 967 \\
\hline & $\mathrm{Ba}$ & 343 & 0.16 & 0.14 & 0.14 & 0.14 & 2381 \\
\hline & $\mathrm{Ca}$ & 1711 & 2.93 & 3.75 & 3.40 & 3.36 & 509 \\
\hline & $\mathrm{Cd}$ & 3173 & 31.68 & 34.46 & 39.14 & 35.09 & 90 \\
\hline & $\mathrm{Cr}$ & 262 & 1.13 & 1.06 & 1.04 & 1.08 & 244 \\
\hline & $\mathrm{Cs}$ & 723 & 9.31 & 9.54 & 9.75 & 9.53 & 76 \\
\hline & $\mathrm{Cu}$ & 230 & 0.29 & 0.26 & 0.21 & 0.25 & 904 \\
\hline & $\mathrm{Fe}$ & 69576 & 62.93 & 53.39 & 45.52 & 53.95 & 1290 \\
\hline & I & 958 & $<0.06$ & $<0.06$ & $<0.06$ & $<0.06$ & $>15961$ \\
\hline & $\mathrm{K}$ & 1352 & 6.17 & 6.12 & 6.21 & 6.17 & 219 \\
\hline & $\mathrm{Li}$ & 26692 & 20.50 & 16.83 & 18.57 & 18.63 & 1432 \\
\hline & $\mathrm{Mg} \#$ & 6643 & 2.42 & 2.65 & 4.70 & 3.26 & 2038 \\
\hline & $\mathrm{Mn}$ & 22473 & 3.98 & 3.55 & 2.80 & 3.44 & 6528 \\
\hline & $\mathrm{Na}$ & 46824 & 59.23 & 51.83 & 56.70 & 55.92 & 837 \\
\hline & $\mathrm{Ni}$ & 4064 & 3.45 & 3.03 & 2.76 & 3.08 & 1319 \\
\hline & $\mathrm{P}$ & 544 & 1.58 & 0.73 & 0.77 & 1.02 & 531 \\
\hline & $\mathrm{Pb}$ & 1334 & 4.38 & 4.38 & 4.43 & 4.40 & 303 \\
\hline & $\mathrm{S}$ & 959 & 40.96 & 47.27 & 36.94 & 41.72 & 23 \\
\hline & $\mathrm{Sb}$ & 1514 & 3.23 & 3.20 & 3.52 & 3.31 & 457 \\
\hline & $\mathrm{Se}$ & 1022 & 516.16 & 547.76 & 622.23 & 562.05 & 2 \\
\hline & $\mathrm{Si}$ & 203827 & 75.65 & 59.88 & 52.92 & 62.82 & 3245 \\
\hline & $\mathrm{Sr}$ & 18787 & 8.73 & 6.40 & 6.77 & 7.30 & 2573 \\
\hline & $\mathrm{Te}$ & 1072 & 116.78 & 119.69 & 130.30 & 122.25 & 9 \\
\hline & $\mathrm{Ti}$ & 344 & 0.65 & 0.61 & 0.51 & 0.59 & 582 \\
\hline & $\mathrm{Zn}$ & 15388 & 8.58 & 7.57 & 5.81 & 7.32 & 2102 \\
\hline & $\mathrm{Zr}$ & 25239 & 6.08 & 4.08 & 3.69 & 4.62 & 5466 \\
\hline \multirow{6}{*}{$\mathscr{d}$} & $\mathrm{B}$ & 29721 & 262.81 & 270.37 & 345.19 & 292.79 & 102 \\
\hline & $\mathrm{Cl}$ & 96 & 91.67 & 98.49 & 93.60 & 94.59 & 1 \\
\hline & $\mathrm{F}$ & 383 & 219.57 & 277.70 & 299.92 & 265.73 & 1 \\
\hline & $I$ & 958 & 248.28 & 288.33 & 131.03 & 222.54 & 4 \\
\hline & $\mathrm{S}$ & 959 & 514.07 & 442.20 & 487.67 & 481.31 & 2 \\
\hline & $\mathrm{Se}$ & 1022 & 17.07 & 39.73 & 34.27 & 30.35 & 34 \\
\hline
\end{tabular}

NC - Not Calculated

$\$$ - From gravimetric analysis of filters and rinse dry-downs

\# - The analyzed $\mathrm{MgO}$ concentration of $1.15 \mathrm{wt} \%$ instead of the target value of $0.06 \mathrm{wt} \%$ was used to calculate the feed flux 
The Catholic University of America Vitreous State Laboratory
DuraMelter 1200 Tests with AZ-101HLW Simulants

Final Report, VSL-02R0100-2, Rev.I

Table 6.22. SBS Emission Rates for DM 1200 AZ101 Test 7.

\begin{tabular}{|c|c|c|c|c|c|c|c|}
\hline & $\begin{array}{c}\text { Average } \\
\text { Melter } \\
\text { Emissions } \\
\text { Flux } \\
\text { (mg/min) }\end{array}$ & $\begin{array}{c}\text { Run } 1 \\
(\mathrm{mg} / \mathrm{min})\end{array}$ & $\begin{array}{c}\text { Run } 2 \\
(\mathrm{mg} / \mathrm{min})\end{array}$ & $\begin{array}{c}\text { Run } 3 \\
(\mathrm{mg} / \mathrm{min})\end{array}$ & $\begin{array}{l}\text { Average } \\
(\mathrm{mg} / \mathrm{min})\end{array}$ & $\begin{array}{c}\text { DF Across } \\
\text { SBS }\end{array}$ \\
\hline \multirow{29}{*}{ 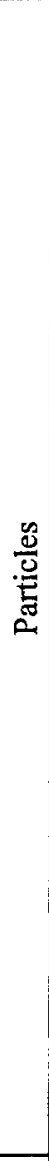 } & Total $^{\$}$ & 2212 & 320 & 568 & 496 & 461.3 & 5 \\
\hline & $\mathrm{Al}$ & 25.32 & $<0.06$ & 0.08 & $<0.06$ & $<0.07$ & $>362$ \\
\hline & As & 10.65 & 5.42 & 6.66 & 6.56 & 6.21 & 2 \\
\hline & $\mathrm{B}$ & 30.75 & 1.57 & 1.77 & 1.18 & 1.50 & 20 \\
\hline & $\mathrm{Ba}$ & 0.14 & $<0.06$ & $<0.06$ & $<0.06$ & $<0.06$ & $>2$ \\
\hline & $\mathrm{Ca}$ & 3.36 & 0.16 & 0.30 & 0.21 & 0.23 & 15 \\
\hline & $\mathrm{Cd}$ & 35.09 & 18.97 & 22.67 & 19.74 & 20.46 & 2 \\
\hline & $\mathrm{Cr}$ & 1.08 & 0.48 & 0.59 & 0.38 & 0.48 & 2 \\
\hline & $\mathrm{Cs}$ & 9.53 & 4.95 & 5.93 & 5.03 & 5.30 & 2 \\
\hline & $\mathrm{Cu}$ & 0.25 & 0.20 & 0.10 & $<0.06$ & $<0.12$ & $>2$ \\
\hline & $\mathrm{Fe}$ & 53.95 & 0.34 & 0.41 & 0.31 & 0.35 & 153 \\
\hline & $\mathrm{I}$ & $<0.06$ & $<0.06$ & $<0.06$ & $<0.06$ & $<0.06$ & $\mathrm{NC}$ \\
\hline & $\mathrm{K}$ & 6.17 & 2.32 & 2.95 & 2.22 & 2.50 & 2 \\
\hline & $\mathrm{Li}$ & 18.63 & 6.41 & 8.04 & 5.99 & 6.81 & 3 \\
\hline & $\mathrm{Mg}$ & 3.26 & $<0.06$ & $<0.06$ & 0.07 & $<0.06$ & $>54$ \\
\hline & $\mathrm{Mn}$ & 3.44 & $<0.06$ & $<0.06$ & 0.13 & $<0.08$ & $>43$ \\
\hline & $\mathrm{Na}$ & 55.92 & 20.04 & 23.67 & 18.67 & 20.79 & 3 \\
\hline & $\mathrm{Ni}$ & 3.08 & 0.10 & 0.14 & 0.07 & 0.10 & 30 \\
\hline & $\mathrm{P}$ & 1.02 & 0.26 & $<0.06$ & 0.08 & $<0.13$ & $>8$ \\
\hline & $\mathrm{Pb}$ & 4.40 & 2.14 & 2.63 & 1.92 & 2.23 & 2 \\
\hline & $\mathrm{S}$ & 41.72 & 18.31 & 20.30 & 17.72 & 18.77 & 2 \\
\hline & $\mathrm{Sb}$ & 3.31 & 1.63 & 1.99 & 1.77 & 1.80 & 2 \\
\hline & $\mathrm{Se}$ & 562.05 & 149.19 & 134.27 & 137.48 & 140.31 & 4 \\
\hline & $\mathrm{Si}$ & 62.82 & 1.43 & 1.64 & 1.54 & 1.53 & 41 \\
\hline & $\mathrm{Sr}$ & 7.30 & $<0.06$ & $<0.06$ & $<0.06$ & $<0.06$ & $>122$ \\
\hline & $\mathrm{Te}$ & 122.25 & 66.47 & 78.49 & 67.29 & 70.75 & 2 \\
\hline & $\mathrm{Ti}$ & 0.59 & $<0.06$ & $<0.06$ & 0.11 & 0.04 & 10 \\
\hline & $\mathrm{Zn}$ & 7.32 & 0.60 & 1.08 & 0.59 & 0.76 & 10 \\
\hline & $\mathrm{Zr}$ & 4.62 & $<0.06$ & $<0.06$ & $<0.06$ & $<0.06$ & $>77$ \\
\hline \multirow{6}{*}{ 心 } & $\mathrm{B}$ & 292.79 & 29.30 & 29.81 & 25.80 & 28.30 & 10 \\
\hline & $\mathrm{Cl}$ & 94.59 & 32.99 & 28.19 & 26.35 & 29.18 & 3 \\
\hline & $\mathrm{F}$ & 265.73 & 43.41 & 31.72 & 32.33 & 35.82 & 7 \\
\hline & I & 222.54 & 10.61 & 13.15 & 4.99 & 9.58 & 23 \\
\hline & $\mathrm{S}$ & 481.31 & 37.13 & 31.03 & 29.51 & 32.56 & 15 \\
\hline & $\mathrm{Se}$ & 30.35 & 37.90 & 38.86 & 37.50 & 38.09 & 1 \\
\hline
\end{tabular}

NC - Not Calculated

$\$$ - From gravimetric analysis of filters and rinse dry-downs 
The Catholic University of America Vitreous State Laboratory

Table 6.23. WESP Emission Rates for DM 1200 AZ101 Test 7.

\begin{tabular}{|c|c|c|c|c|c|c|c|c|}
\hline & $\begin{array}{l}\text { Average } \\
\text { Feed Flux } \\
(\mathrm{mg} / \mathrm{min})\end{array}$ & $\begin{array}{l}\text { Average } \\
\text { Melter } \\
\text { Emissions } \\
\text { Flux } \\
\text { (mg/min) }\end{array}$ & $\begin{array}{c}\text { Average } \\
\text { SBS } \\
\text { Emissions } \\
\text { Flux } \\
\text { (mg/min) }\end{array}$ & $\begin{array}{c}\text { WESP } \\
\text { Emissions } \\
\text { Flux } \\
(\mathrm{mg} / \mathrm{min})\end{array}$ & $\begin{array}{c}\text { DF } \\
\text { Across } \\
\text { WESP }\end{array}$ & $\begin{array}{l}\text { DF Across } \\
\text { SBS, WESP }\end{array}$ & $\begin{array}{c}\text { Cumulative DF } \\
\text { Across Melter, } \\
\text { SBS, WESP }\end{array}$ \\
\hline \multirow{29}{*}{$\frac{\mathscr{Q}}{0}$} & Total $^{\mathrm{S}}$ & 957667 & 2212 & 461.3 & 1.53 & 302 & 1446 & 593987 \\
\hline & $\mathrm{Al}$ & 37518 & 25.32 & 0.03 & $<0.06$ & $>0.4$ & $>422$ & $>625300$ \\
\hline & As & 290 & 10.65 & 6.21 & $<0.06$ & $>97.1$ & $>177$ & $>4835$ \\
\hline & $\mathrm{B}$ & 29721 & 30.75 & 1.50 & $<0.06$ & $>23.5$ & $>512$ & $>495345$ \\
\hline & $\mathrm{Ba}$ & 343 & 0.14 & $<0.06$ & $<0.06$ & $\mathrm{NC}$ & $>2$ & $>5718$ \\
\hline & $\mathrm{Ca}$ & 1711 & 3.36 & 0.23 & $<0.06$ & $>3.5$ & $>56$ & $>28522$ \\
\hline & $\mathrm{Cd}$ & 3173 & 35.09 & 20.46 & $<0.06$ & $>319.7$ & $>585$ & $>52880$ \\
\hline & $\mathrm{Cr}$ & 262 & 1.08 & 0.48 & $<0.06$ & $>7.6$ & $>18$ & $>4368$ \\
\hline & $\mathrm{Cs}$ & 723 & 9.53 & 5.30 & $<0.06$ & $>82.9$ & $>159$ & $>12045$ \\
\hline & $\mathrm{Cu}$ & 230 & 0.25 & 0.10 & $<0.06$ & $>1.5$ & $>4$ & $>3825$ \\
\hline & $\mathrm{Fe}$ & 69576 & 53.95 & 0.35 & $<0.06$ & $>5.5$ & $>899$ & $>1159605$ \\
\hline & $\mathrm{I}$ & 958 & $<0.06$ & $<0.06$ & $<0.06$ & $\mathrm{NC}$ & $\mathrm{NC}$ & $>15961$ \\
\hline & $\mathrm{K}$ & 1352 & 6.17 & 2.50 & $<0.06$ & $>39.0$ & $>103$ & $>22525$ \\
\hline & $\mathrm{Li}$ & 26692 & 18.63 & 6.81 & $<0.06$ & $>106.4$ & $>311$ & $>444860$ \\
\hline & $\mathrm{Mg \#}$ & 6643 & 3.26 & 0.02 & $<0.06$ & $>0.3$ & $>54$ & $>110717$ \\
\hline & $\mathrm{Mn}$ & 22473 & 3.44 & 0.04 & $<0.06$ & $>0.7$ & $>57$ & $>374544$ \\
\hline & $\mathrm{Na}$ & 46824 & 55.92 & 20.79 & $<0.06$ & $>324.9$ & $>932$ & $>780395$ \\
\hline & $\mathrm{Ni}$ & 4064 & 3.08 & 0.10 & $<0.06$ & $>1.6$ & $>51$ & $>67729$ \\
\hline & $\mathrm{P}$ & 544 & 1.02 & 0.11 & $<0.06$ & $>1.8$ & $>17$ & $>9060$ \\
\hline & $\mathrm{Pb}$ & 1334 & 4.40 & 2.23 & $<0.06$ & $>34.9$ & $>73$ & $>22225$ \\
\hline & $\mathrm{S}$ & 959 & 41.72 & 18.77 & $<0.06$ & $>293.3$ & $>695$ & $>15991$ \\
\hline & $\mathrm{Sb}$ & 1514 & 3.31 & 1.80 & $<0.06$ & $>28.1$ & $>55$ & $>25232$ \\
\hline & $\mathrm{Se}$ & 1022 & 562.05 & 140.31 & 0.10 & 1367 & 5478 & 9962 \\
\hline & $\mathrm{Si}$ & 203827 & 62.82 & 1.53 & 0.08 & 19 & 780 & 2532065 \\
\hline & $\mathrm{Sr}$ & 18787 & 7.30 & $<0.06$ & $<0.06$ & $\mathrm{NC}$ & $>122$ & $>313120$ \\
\hline & $\mathrm{Te}$ & 1072 & 122.25 & 70.75 & 0.09 & 814 & 1406 & 12330 \\
\hline & $\mathrm{Ti}$ & 344 & 0.59 & 0.04 & $<0.06$ & $>0.6$ & $>10$ & $>5741$ \\
\hline & $\mathrm{Zn}$ & 15388 & 7.32 & 0.76 & $<0.06$ & $>11.8$ & $>122$ & $>256460$ \\
\hline & $\mathrm{Zr}$ & 25239 & 4.62 & $<0.06$ & $<0.06$ & $\mathrm{NC}$ & $>77$ & $>420651$ \\
\hline \multirow{6}{*}{ : } & $\mathrm{B}$ & 29721 & 292.79 & 28.30 & 1.38 & 21 & 213 & 21613 \\
\hline & $\mathrm{Cl}$ & 96 & 94.59 & 29.18 & $<0.07$ & $>416.8$ & $>1576$ & $>1596$ \\
\hline & $\mathrm{F}$ & 383 & 265.73 & 35.82 & 1.91 & 19 & 139 & 201 \\
\hline & I & 958 & 222.54 & 9.58 & 1.26 & 8 & 176 & 759 \\
\hline & $\mathrm{S}$ & 959 & 481.31 & 32.56 & 12.53 & 3 & 38 & 77 \\
\hline & $\mathrm{Se}$ & 1022 & 30.35 & 38.09 & 0.61 & 62 & 50 & 1668 \\
\hline
\end{tabular}

NC - Not Calculated

$\$$ - From gravimetric analysis of filters and rinse dry-downs

\# - The analyzed $\mathrm{MgO}$ concentration of $1.15 \mathrm{wt} \%$ instead of the target value of $0.06 \mathrm{wt} \%$ was used to calculate the feed flux 
Table 6.24. Melter Emission Rates for DM 1200 AZ101 Test 8.

\begin{tabular}{|c|c|c|c|c|c|c|c|}
\hline & $\begin{array}{l}\text { Average } \\
\text { Feed Flux } \\
(\mathrm{mg} / \mathrm{min})\end{array}$ & $\begin{array}{c}\text { Run } 1 \\
(\mathrm{mg} / \mathrm{min})\end{array}$ & $\begin{array}{c}\text { Run } 2 \\
\text { (mg/min) }\end{array}$ & $\begin{array}{c}\text { Run } 3 \\
(\mathrm{mg} / \mathrm{min})\end{array}$ & $\begin{array}{l}\text { Average } \\
(\mathrm{mg} / \mathrm{min})\end{array}$ & $\begin{array}{c}\text { DF Across } \\
\text { Melter }\end{array}$ \\
\hline \multirow{29}{*}{$\frac{\mathscr{Q}}{\stackrel{O}{Z}}$} & Total $^{\mathrm{s}}$ & 285167 & 1354 & 1066 & 1453 & 1291 & 221 \\
\hline & $\mathrm{Al}$ & 11172 & 4.83 & 7.02 & 4.88 & 5.58 & 2004 \\
\hline & As & 86 & 1.08 & 1.16 & 1.99 & 1.41 & 61 \\
\hline & $\mathrm{B}$ & 8850 & 59.37 & 46.56 & 78.05 & 61.33 & 144 \\
\hline & $\mathrm{Ba}$ & 102 & 0.22 & 0.21 & 0.50 & 0.31 & 328 \\
\hline & $\mathrm{Ca}$ & 510 & 2.01 & 2.55 & 1.87 & 2.14 & 238 \\
\hline & $\mathrm{Cd}$ & 945 & 5.04 & 4.29 & 6.59 & 5.31 & 178 \\
\hline & $\mathrm{Cr}$ & 78 & 0.20 & 0.17 & 0.32 & 0.23 & 340 \\
\hline & $\mathrm{Cs}$ & 215 & 6.12 & 5.33 & 7.28 & 6.24 & 34 \\
\hline & $\mathrm{Cu}$ & 68 & 0.11 & 0.10 & 0.14 & 0.12 & 581 \\
\hline & $\mathrm{Fe}$ & 20718 & 10.88 & 15.20 & 11.55 & 12.54 & 1652 \\
\hline & $I$ & 285 & $<0.06$ & $<0.06$ & $<0.06$ & $<0.06$ & $>4753$ \\
\hline & $\mathrm{K}$ & 402 & 8.46 & 6.96 & 12.92 & 9.45 & 43 \\
\hline & $\mathrm{Li}$ & 7948 & 23.25 & 22.81 & 32.84 & 26.30 & 302 \\
\hline & $\mathrm{Mg \#}$ & 1978 & 2.44 & 3.02 & 2.79 & 2.75 & 719 \\
\hline & $\mathrm{Mn}$ & 6692 & 1.47 & 1.65 & 1.46 & 1.53 & 4384 \\
\hline & $\mathrm{Na}$ & 13943 & 135.21 & 103.26 & 180.51 & 139.66 & 100 \\
\hline & $\mathrm{Ni}$ & 1210 & 0.73 & 0.77 & 0.73 & 0.74 & 1628 \\
\hline & $\mathrm{P}-$ & 162 & 0.35 & 0.25 & 1.43 & 0.68 & 239 \\
\hline & $\mathrm{Pb}$ & 397 & 1.11 & 0.80 & 1.39 & 1.10 & 361 \\
\hline & $\mathrm{S}$ & 286 & 2.87 & 3.56 & 4.53 & 3.65 & 78 \\
\hline & $\mathrm{Sb}$ & 451 & 0.09 & 0.12 & 2.68 & 0.96 & 468 \\
\hline & $\mathrm{Se}$ & 304 & 28.47 & 28.15 & 31.22 & 29.28 & 10 \\
\hline & $\mathrm{Si}$ & 60694 & 13.96 & 24.80 & 1055.78 & 364.85 & 166 \\
\hline & $\mathrm{Sr}$ & 5594 & 5.37 & 6.50 & 6.86 & 6.25 & 896 \\
\hline & $\mathrm{Te}$ & 319 & 2.86 & 2.01 & 4.20 & 3.02 & 106 \\
\hline & $\mathrm{Ti}$ & 103 & 0.14 & 0.36 & 0.12 & 0.21 & 491 \\
\hline & $\mathrm{Zn}$ & 4582 & 2.57 & 3.19 & 2.93 & 2.90 & 1582 \\
\hline & $\mathrm{Zr}$ & 7515 & 2.29 & 2.90 & 2.25 & 2.48 & 3034 \\
\hline \multirow{6}{*}{ 胥 } & $\mathrm{B}$ & 8850 & 53.84 & 60.53 & 51.32 & 55.23 & 160 \\
\hline & $\mathrm{Cl}$ & 29 & $<0.07$ & $<0.07$ & $<0.07$ & $<0.07$ & $>475$ \\
\hline & $\mathrm{F}$ & 114 & $<0.07$ & $<0.07$ & $<0.07$ & $<0.07$ & $>1901$ \\
\hline & I & 285 & 12.97 & 17.97 & 14.64 & 15.19 & 19 \\
\hline & $\mathrm{S}$ & 286 & 19.40 & 12.97 & 18.90 & 17.09 & 17 \\
\hline & $\mathrm{Se}$ & 304 & 1.23 & 0.25 & 0.29 & 0.59 & 513 \\
\hline
\end{tabular}

NC - Not calculated

$\$$ - From gravimetric analysis of filters and rinse dry-downs

\# - The analyzed $\mathrm{MgO}$ concentration of $1.15 \mathrm{wt} \%$ instead of the target value of $0.06 \mathrm{wt} \%$ was used to calculate the feed flux 
The Catholic University of America Vitreous State Laboratory
DuraMelter 1200 Tests with AZ-101HLW Simulants

Final Report, VSL-02R0100-2, Rev.I

Table 6.25. SBS Emission Rates for DM 1200 AZ101 Test 8.

\begin{tabular}{|c|c|c|c|c|c|c|c|}
\hline & $\begin{array}{c}\text { Average } \\
\text { Melter Flux } \\
(\mathrm{mg} / \mathrm{min})\end{array}$ & $\begin{array}{c}\text { Run } 1 \\
(\mathrm{mg} / \mathrm{min})\end{array}$ & $\begin{array}{c}\text { Run } 2 \\
(\mathrm{mg} / \mathrm{min})\end{array}$ & $\begin{array}{c}\text { Run } 3 \\
(\mathrm{mg} / \mathrm{min})\end{array}$ & $\begin{array}{l}\text { Average } \\
(\mathrm{mg} / \mathrm{min})\end{array}$ & $\begin{array}{c}\text { DF } \\
\text { Across } \\
\text { SBS }\end{array}$ \\
\hline \multirow{29}{*}{$\frac{\mathscr{2}}{\frac{2}{2}}$} & Total $^{S}$ & 1291 & 218.8 & 182.6 & 213.3 & 205 & 6 \\
\hline & $\mathrm{Al}$ & 5.58 & 0.58 & $<0.06$ & 0.33 & 0.30 & 18 \\
\hline & As & 1.41 & 0.31 & 0.19 & 0.76 & 0.42 & 3 \\
\hline & $B$ & 61.33 & 0.32 & 1.98 & 2.87 & 1.72 & 36 \\
\hline & $\mathrm{Ba}$ & 0.31 & $<0.06$ & $<0.06$ & $<0.06$ & $<0.06$ & $>5$ \\
\hline & $\mathrm{Ca}$ & 2.14 & 0.34 & 0.21 & 0.64 & 0.40 & 5 \\
\hline & $\mathrm{Cd}$ & 5.31 & 1.82 & 1.42 & 1.46 & 1.56 & 3 \\
\hline & $\mathrm{Cr}$ & 0.23 & 0.07 & 0.06 & $<0.06$ & 0.04 & 4 \\
\hline & $\mathrm{Cs}$ & 6.24 & 1.97 & 1.30 & 1.38 & 1.55 & 4 \\
\hline & $\mathrm{Cu}$ & 0.12 & $<0.06$ & $<0.06$ & $<0.06$ & $<0.06$ & $>2$ \\
\hline & $\mathrm{Fe}$ & 12.54 & 0.36 & 0.07 & 0.30 & 0.24 & 52 \\
\hline & $\mathrm{I}$ & $<0.06$ & $<0.06$ & $<0.06$ & $<0.06$ & $<0.06$ & $\mathrm{NC}$ \\
\hline & $\mathrm{K}$ & 9.45 & 2.11 & 1.58 & 1.78 & 1.82 & 5 \\
\hline & $\mathrm{Li}$ & 26.30 & 4.01 & 3.08 & 3.35 & 3.48 & 8 \\
\hline & $\mathrm{Mg}$ & 2.75 & 0.20 & 0.13 & 0.20 & 0.18 & 16 \\
\hline & $\mathrm{Mn}$ & 1.53 & $<0.06$ & $<0.06$ & 0.12 & $<0.08$ & $>19$ \\
\hline & $\mathrm{Na}$ & 139.66 & 25.31 & 22.79 & 23.40 & 23.83 & 6 \\
\hline & $\mathrm{Ni}$ & 0.74 & 0.08 & $<0.06$ & $<0.06$ & $<0.07$ & $>11$ \\
\hline & $\mathrm{P}$ & 0.68 & $<0.06$ & 0.13 & $<0.06$ & $<0.08$ & $>9$ \\
\hline & $\mathrm{Pb}$ & 1.10 & 0.28 & 0.18 & 0.15 & 0.20 & 5 \\
\hline & $\mathrm{S}$ & 3.65 & 0.43 & 2.62 & 0.28 & 1.11 & 3 \\
\hline & $\mathrm{Sb}$ & 0.96 & $<0.06$ & 0.07 & $<0.06$ & $<0.06$ & $>16$ \\
\hline & $\mathrm{Se}$ & 29.28 & 5.16 & 5.80 & 5.74 & 5.57 & 5 \\
\hline & $\mathrm{Si}$ & 364.85 & 1.62 & 0.73 & 1.69 & 1.35 & 271 \\
\hline & $\mathrm{Sr}$ & 6.25 & 0.37 & 0.24 & 0.26 & 0.29 & 22 \\
\hline & $\mathrm{Te}$ & 3.02 & 1.29 & 0.89 & 0.86 & 1.01 & 3 \\
\hline & $\mathrm{Ti}$ & 0.21 & $<0.06$ & $<0.06$ & $<0.06$ & $<0.06$ & $>3$ \\
\hline & $\mathrm{Zn}$ & 2.90 & 0.26 & 0.14 & 0.26 & 0.22 & 13 \\
\hline & $\mathrm{Zr}$ & 2.48 & $<0.06$ & $<0.06$ & $<0.06$ & $<0.06$ & $>41$ \\
\hline \multirow{6}{*}{$\stackrel{\mathscr{8}}{6}$} & $\mathrm{~B}$ & 55.23 & 19.43 & 17.02 & 13.89 & 16.78 & 3 \\
\hline & $\mathrm{Cl}$ & $<0.06$ & $<0.07$ & $<0.07$ & $<0.07$ & $<0.06$ & $\mathrm{NC}$ \\
\hline & $F$ & $<0.06$ & $<0.07$ & $<0.07$ & $<0.07$ & $<0.06$ & $\mathrm{NC}$ \\
\hline & I & 15.19 & 5.88 & 2.44 & 2.84 & 3.72 & 4 \\
\hline & $S$ & 17.09 & 2.91 & 1.69 & 4.13 & 2.91 & 6 \\
\hline & $\mathrm{Se}$ & 0.59 & 0.21 & $<0.07$ & 0.57 & 0.26 & 2 \\
\hline
\end{tabular}

NC - Not Calculated

$\$$ - From gravimetric analysis of filters and rinse dry-downs 
The Catholic University of America

Vitreous State Laboratory

Table 6.26. WESP Emission Rates for DM 1200 AZ101 Test 8.

\begin{tabular}{|c|c|c|c|c|c|c|c|c|}
\hline & $\begin{array}{l}\text { Average } \\
\text { Feed Flux } \\
(\mathrm{mg} / \mathrm{min})\end{array}$ & $\begin{array}{c}\text { Average } \\
\text { Melter } \\
\text { Emissions } \\
\text { Flux } \\
\text { (mg/min) }\end{array}$ & $\begin{array}{l}\text { Average } \\
\text { SBS } \\
\text { Emissions } \\
\text { Flux } \\
\text { (mg/min) }\end{array}$ & $\begin{array}{l}\text { WESP } \\
\text { Emissions } \\
\text { Flux } \\
\text { (mg/min) }\end{array}$ & $\begin{array}{c}\text { DF } \\
\text { Across } \\
\text { WESP }\end{array}$ & $\begin{array}{l}\text { DF Across } \\
\text { SBS, WESP }\end{array}$ & $\begin{array}{c}\text { Cumulative DF } \\
\text { Across Melter, } \\
\text { SBS, WESP }\end{array}$ \\
\hline \multirow{29}{*}{$\frac{\mathscr{L}}{0}$} & Total $^{S}$ & 285167 & 1291 & 205 & 1.1 & 186 & 1174 & 259243 \\
\hline & $\mathrm{Al}$ & 11172 & 5.58 & 0.30 & $<0.06$ & $>4.7$ & $>93$ & $>186197$ \\
\hline & As & 86 & 1.41 & 0.42 & $<0.06$ & $>6.6$ & $>23$ & $>1440$ \\
\hline & $\mathrm{B}$ & 8850 & 61.33 & 1.72 & $<0.06$ & $>26.9$ & $>1022$ & $>147500$ \\
\hline & $\mathrm{Ba}$ & 102 & 0.31 & $<0.06$ & $<0.06$ & $\mathrm{NC}$ & $>5$ & $>1703$ \\
\hline & $\mathrm{Ca}$ & 510 & 2.14 & 0.40 & $<0.06$ & $>6.2$ & $>36$ & $>8493$ \\
\hline & $\mathrm{Cd}$ & 945 & 5.31 & 1.56 & $<0.06$ & $>24.5$ & $>88$ & $>15746$ \\
\hline & $\mathrm{Cr}$ & 78 & 0.23 & 0.04 & $<0.06$ & $>0.7$ & $>4$ & $>1301$ \\
\hline & $\mathrm{Cs}$ & 215 & 6.24 & 1.55 & $<0.06$ & $>24.2$ & $>104$ & $>3587$ \\
\hline & $\mathrm{Cu}$ & 68 & 0.12 & $<0.06$ & $<0.06$ & $\mathrm{NC}$ & $>2$ & $>1139$ \\
\hline & $\mathrm{Fe}$ & 20718 & 12.54 & 0.24 & $<0.06$ & $>3.8$ & $>209$ & $>345298$ \\
\hline & $\mathrm{I}$ & 285 & $<0.06$ & $<0.06$ & $<0.06$ & $\mathrm{NC}$ & $\mathrm{NC}$ & $>4753$ \\
\hline & $\mathrm{K}$ & 402 & 9.45 & 1.82 & $<0.06$ & $>28.5$ & $>157$ & $>6707$ \\
\hline & $\mathrm{Li}$ & 7948 & 26.30 & 3.48 & $<0.06$ & $>54.4$ & $>438$ & $>132467$ \\
\hline & Mg\# & 1978 & 2.75 & 0.18 & $<0.06$ & $>2.8$ & $>46$ & $>32967$ \\
\hline & $\mathrm{Mn}$ & 6692 & 1.53 & 0.04 & $<0.06$ & $>0.6$ & $>25$ & $>111529$ \\
\hline & $\mathrm{Na}$ & 13943 & 139.66 & 23.83 & $<0.06$ & $>372.4$ & $>2328$ & $>232380$ \\
\hline & $\mathrm{Ni}$ & 1210 & 0.74 & 0.03 & $<0.06$ & $>0.4$ & $>12$ & $>20168$ \\
\hline & $\mathrm{P}$ & 162 & 0.68 & 0.04 & $<0.06$ & $>0.7$ & $>11$ & $>2698$ \\
\hline & $\mathrm{Pb}$ & 397 & 1.10 & 0.20 & $<0.06$ & $>3.1$ & $>18$ & $>6618$ \\
\hline & $\mathrm{S}$ & 286 & 3.65 & 1.11 & $<0.06$ & $>17.3$ & $>61$ & $>4762$ \\
\hline & $\mathrm{Sb}$ & 451 & 0.96 & 0.02 & $<0.06$ & $>0.3$ & $>16$ & $>7513$ \\
\hline & $\mathrm{Se}$ & 304 & 29.28 & 5.57 & $<0.06$ & $>87.0$ & $>488$ & $>5073$ \\
\hline & $\mathrm{Si}$ & 60694 & 364.85 & 1.35 & 0.06 & 21 & 5652 & 940302 \\
\hline & $\mathrm{Sr}$ & 5594 & 6.25 & 0.29 & $<0.06$ & $>4.5$ & $>104$ & $>93238$ \\
\hline & $\mathrm{Te}$ & 319 & 3.02 & 1.01 & $<0.06$ & $>15.8$ & $>50$ & $>5320$ \\
\hline & $\mathrm{Ti}$ & 103 & 0.21 & $<0.06$ & $<0.06$ & $\mathrm{NC}$ & $>3$ & $>1710$ \\
\hline & $\mathrm{Zn}$ & 4582 & 2.90 & 0.22 & $<0.06$ & $>3.4$ & $>48$ & $>76367$ \\
\hline & $\mathrm{Zr}$ & 7515 & 2.48 & $<0.06$ & $<0.06$ & $\mathrm{NC}$ & $>41$ & $>125258$ \\
\hline \multirow{6}{*}{ 心 } & $\mathrm{B}$ & 8850 & 55.23 & 16.78 & 1.81 & 9 & 30 & 4883 \\
\hline & $\mathrm{Cl}$ & 29 & $<0.06$ & $<0.06$ & $<0.07$ & $\mathrm{NC}$ & NC & $>475$ \\
\hline & $\mathrm{F}$ & 114 & $<0.06$ & $<0.06$ & $<0.07$ & $\mathrm{NC}$ & $\mathrm{NC}$ & $>1901$ \\
\hline & I & 285 & 15.19 & 3.72 & 4.82 & 1 & 3 & 59 \\
\hline & $S$ & 286 & 17.09 & 2.91 & 2.37 & 1 & 7 & 120 \\
\hline & $\mathrm{Se}$ & 304 & 0.59 & 0.26 & 0.18 & 1 & 3 & 1659 \\
\hline
\end{tabular}

NC - Not Calculated

s - From gravimetric analysis of filters and rinse dry-downs

\# - The analyzed $\mathrm{MgO}$ concentration of $1.15 \mathrm{wt} \%$ instead of the target value of $0.06 \mathrm{wt} \%$ was used to calculate the feed flux 
Table 6.27. Melter Emission Rates for DM 1200 AZ101 Test 9.

\begin{tabular}{|c|c|c|c|c|c|c|c|}
\hline & Target Glass Temp & $1150^{\circ} \mathrm{C}$ & $1200^{\circ} \mathrm{C}$ & $1200^{\circ} \mathrm{C}$ & & \\
\hline & & $\begin{array}{l}\text { Average Feed Flux } \\
(\mathrm{mg} / \mathrm{min})\end{array}$ & $\begin{array}{c}\text { Run } 1 \\
(\mathrm{mg} / \mathrm{min})\end{array}$ & $\begin{array}{c}\text { Run } 2 \\
(\mathrm{mg} / \mathrm{min})\end{array}$ & $\begin{array}{c}\text { Run } 3 \\
(\mathrm{mg} / \mathrm{min})\end{array}$ & $\begin{array}{l}\text { Average } \\
(\mathrm{mg} / \mathrm{min})\end{array}$ & $\begin{array}{c}\text { DF } \\
\text { Across } \\
\text { Melter }\end{array}$ \\
\hline \multirow{29}{*}{$\underset{\mathscr{E}}{\frac{\mathscr{Z}}{0}}$} & Total $^{\text {s }}$ & 281833 & 354 & 560 & 693 & 536 & 526 \\
\hline & $\mathrm{Al}$ & 11041 & 2.50 & 2.57 & 1.69 & 2.25 & 4902 \\
\hline & As & 85 & 1.99 & 6.86 & 3.46 & 4.10 & 21 \\
\hline & $\mathrm{B}$ & 8747 & 2.56 & 6.34 & 3.55 & 4.15 & 2108 \\
\hline & $\mathrm{Ba}$ & 101 & $<0.06$ & $<0.06$ & $<0.06$ & $<0.06$ & $>1683$ \\
\hline & $\mathrm{Ca}$ & 504 & 0.46 & 0.75 & 0.55 & 0.59 & 859 \\
\hline & $\mathrm{Cd}$ & 934 & 14.20 & 48.19 & 33.96 & 32.12 & 29 \\
\hline & $\mathrm{Cr}$ & 77 & 0.10 & 0.07 & 0.11 & 0.09 & 824 \\
\hline & $\mathrm{Cs}$ & 213 & 0.87 & 2.40 & 1.25 & 1.50 & 141 \\
\hline & $\mathrm{Cu}$ & 68 & $<0.06$ & 0.23 & 0.06 & $<0.12$ & $>567$ \\
\hline & $\mathrm{Fe}$ & 20476 & 5.03 & 5.57 & 4.24 & 4.95 & 4140 \\
\hline & $\mathrm{I}$ & 282 & $<0.06$ & $<0.06$ & $<0.06$ & $<0.06$ & $>4697$ \\
\hline & $\mathrm{K}$ & 398 & 0.51 & 1.10 & 0.65 & 0.76 & 526 \\
\hline & $\mathrm{Li}$ & 7855 & 1.39 & 2.71 & 1.47 & 1.86 & 4227 \\
\hline & $\mathrm{Mg \#}$ & 1958 & 0.62 & 0.61 & 0.56 & 0.60 & 3264 \\
\hline & $\mathrm{Mn}$ & 6614 & 0.25 & 0.30 & 0.17 & 0.24 & 27815 \\
\hline & $\mathrm{Na}$ & 13780 & 6.37 & 11.12 & 7.23 & 8.24 & 1673 \\
\hline & $\mathrm{Ni}$ & 1196 & 0.33 & 0.34 & 0.21 & 0.29 & 4081 \\
\hline & $\mathrm{P}$ & 160 & 0.10 & 0.37 & $<0.06$ & $<0.18$ & $\geq 889$ \\
\hline & $\mathrm{Pb}$ & 392 & 1.19 & 3.55 & 2.74 & 2.49 & 157 \\
\hline & $S$ & 282 & 2.62 & 4.55 & 2.60 & 3.26 & 87 \\
\hline & $\mathrm{Sb}$ & 446 & 0.27 & 0.64 & 0.35 & 0.42 & 1060 \\
\hline & $\mathrm{Se}$ & 301 & 68.54 & 194.35 & 241.37 & 168.09 & 2 \\
\hline & $\mathrm{Si}$ & 59985 & 8.54 & 8.57 & 7.10 & 8.07 & 7434 \\
\hline & $\mathrm{Sr}$ & 5529 & 1.03 & 1.24 & 0.73 & 1.00 & 5540 \\
\hline & $\mathrm{Te}$ & 315 & 48.46 & 122.29 & 119.01 & 96.59 & 3 \\
\hline & $\mathrm{Ti}$ & 101 & $<0.06$ & $<0.06$ & $<0.06$ & $<0.06$ & $>1690$ \\
\hline & $\mathrm{Zn}$ & 4528 & 1.17 & 1.50 & 1.08 & 1.25 & 3620 \\
\hline & $\mathrm{Zr}$ & 7428 & 0.54 & 0.48 & 0.19 & 0.40 & 18529 \\
\hline \multirow{6}{*}{ 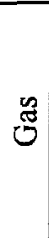 } & $\mathrm{B}$ & 8747 & 25.62 & 37.44 & 33.69 & 32.25 & 271 \\
\hline & $\mathrm{Cl}$ & 28 & 12.90 & 16.41 & 14.42 & 14.58 & 2 \\
\hline & $\mathrm{F}$ & 113 & 26.46 & 36.29 & 27.36 & 30.04 & 4 \\
\hline & $I$ & 282 & 60.87 & 127.18 & 107.75 & 98.60 & 3 \\
\hline & $\mathrm{S}$ & 282 & 77.97 & 148.19 & 131.49 & 119.22 & \\
\hline & $\mathrm{Se}$ & 301 & 5.40 & 19.19 & 5.94 & 10.18 & 30 \\
\hline
\end{tabular}

NC - Not Calculated

\$ - From gravimetric analysis of filters and rinse dry-downs

\# - The analyzed $\mathrm{MgO}$ concentration of $1.15 \mathrm{wt} \%$ instead of the target value of $0.06 \mathrm{wt} \%$ was used to calculate the feed flux 
Table 6.28. SBS Emission Rates for DM 1200 AZ101 Test 9.

\begin{tabular}{|c|c|c|c|c|c|c|c|}
\hline & Target Glass Temp. & $1150^{\circ} \mathrm{C}$ & $1200^{\circ} \mathrm{C}$ & $1200^{\circ} \mathrm{C}$ & & \\
\hline & & $\begin{array}{l}\text { Average Melter } \\
\text { Emissions Flux } \\
(\mathrm{mg} / \mathrm{min})\end{array}$ & $\begin{array}{c}\text { Run } 1 \\
(\mathrm{mg} / \mathrm{min})\end{array}$ & $\begin{array}{c}\text { Run } 2 \\
(\mathrm{mg} / \mathrm{min})\end{array}$ & $\begin{array}{c}\text { Run } 3 \\
(\mathrm{mg} / \mathrm{min})\end{array}$ & $\begin{array}{l}\text { Average } \\
(\mathrm{mg} / \mathrm{min})\end{array}$ & $\begin{array}{c}\text { DF } \\
\text { Across } \\
\text { SBS }\end{array}$ \\
\hline \multirow{29}{*}{$\frac{\mathscr{2}}{2}$} & Total $^{5}$ & 536 & 119 & 195 & 268 & 194 & 3 \\
\hline & $\mathrm{Al}$ & 2.25 & 0.17 & 0.46 & 0.28 & 0.30 & 7 \\
\hline & As & 4.10 & 1.50 & 2.71 & 1.42 & 1.87 & 2 \\
\hline & $\mathrm{B}$ & 4.15 & 0.11 & 0.39 & 0.12 & 0.21 & 20 \\
\hline & $\mathrm{Ba}$ & $<0.06$ & $<0.06$ & $<0.06$ & $<0.06$ & $<0.06$ & $\mathrm{NC}$ \\
\hline & $\mathrm{Ca}$ & 0.59 & 0.39 & 0.77 & 0.27 & 0.48 & 1 \\
\hline & $\mathrm{Cd}$ & 32.12 & 10.84 & 18.40 & 13.15 & 14.13 & 2 \\
\hline & $\mathrm{Cr}$ & 0.09 & $<0.06$ & $<0.06$ & $<0.06$ & $<0.06$ & $>2$ \\
\hline & $\mathrm{Cs}$ & 1.50 & 0.56 & 0.90 & 0.49 & 0.65 & 2 \\
\hline & $\mathrm{Cu}$ & 0.10 & $<0.06$ & 0.09 & $<0.06$ & $<0.07$ & $>1$ \\
\hline & $\mathrm{Fe}$ & 4.95 & 0.16 & 0.28 & 0.20 & 0.21 & 23 \\
\hline & I & $<0.06$ & $<0.06$ & $<0.06$ & $<0.06$ & $<0.06$ & $\mathrm{NC}$ \\
\hline & $\mathrm{K}$ & 0.76 & 0.27 & 0.55 & 0.25 & 0.36 & 2 \\
\hline & $\mathrm{Li}$ & 1.86 & 0.34 & 0.63 & 0.27 & 0.41 & 5 \\
\hline & $\mathrm{Mg}$ & 0.60 & 0.09 & 0.18 & 0.11 & 0.13 & 5 \\
\hline & $\mathrm{Mn}$ & 0.24 & $<0.06$ & $<0.06$ & $<0.06$ & $<0.06$ & $>4$ \\
\hline & $\mathrm{Na}$ & 8.24 & 1.90 & 3.51 & 1.66 & 2.36 & 3 \\
\hline & $\mathrm{Ni}$ & 0.29 & $<0.06$ & $<0.06$ & $<0.06$ & $<0.06$ & $>5$ \\
\hline & $\mathrm{P}$ & 0.15 & $<0.06$ & 0.50 & $<0.06$ & $<0.21$ & $\mathrm{NC}$ \\
\hline & $\mathrm{Pb}$ & 2.49 & 1.12 & 1.36 & 1.28 & 1.25 & 2 \\
\hline & $\mathrm{S}$ & 3.26 & 1.77 & 1.68 & 1.41 & 1.62 & 2 \\
\hline & $\mathrm{Sb}$ & 0.42 & 0.21 & 0.30 & 0.14 & 0.22 & 2 \\
\hline & $\mathrm{Se}$ & 168.09 & 16.05 & 41.55 & 87.68 & 48.43 & 3 \\
\hline & $\mathrm{Si}$ & 8.07 & 1.10 & 2.14 & 1.28 & 1.51 & 5 \\
\hline & $\mathrm{Sr}$ & 1.00 & $<0.06$ & $<0.06$ & $<0.06$ & $<0.06$ & $>17$ \\
\hline & $\mathrm{Te}$ & 96.59 & 29.61 & 46.96 & 42.81 & 39.79 & 2 \\
\hline & $\mathrm{Ti}$ & $<0.06$ & $<0.06$ & 0.08 & $<0.06$ & $<0.07$ & $\mathrm{NC}$ \\
\hline & $\mathrm{Zn}$ & 1.25 & 0.08 & 0.13 & 0.19 & 0.13 & 9 \\
\hline & $\mathrm{Zr}$ & 0.40 & $<0.06$ & $<0.06$ & $<0.06$ & $<0.06$ & $>7$ \\
\hline \multirow{6}{*}{$\stackrel{\mathscr{J}}{0}$} & B & 32.25 & 3.67 & 5.56 & 3.76 & 4.33 & 7 \\
\hline & $\mathrm{Cl}$ & 14.58 & 4.61 & 5.97 & 3.62 & 4.73 & 3 \\
\hline & $\mathbf{F}$ & 30.04 & 3.42 & 3.66 & 1.77 & 2.95 & 10 \\
\hline & $\mathrm{I}$ & 98.60 & 10.60 & 17.47 & 17.49 & 15.19 & 6 \\
\hline & $S$ & 119.22 & 0.14 & $<0.07$ & $<0.07$ & $<0.09$ & $>1325$ \\
\hline & $\mathrm{Se}$ & 10.18 & 10.28 & 19.16 & 2.19 & 10.54 & 1 \\
\hline
\end{tabular}

NC - Not Calculated

$\$$ - From gravimetric analysis of filters and rinse dry-downs 
The Catholic University of America Vitreous State Laboratory
DuraMelter 1200 Tests with AZ-101HLW Simulants Final Report, VSL-02R0100-2, Rev.I

Table 6.29 WESP Emission Rates for DM 1200 AZ101 Test 9

\begin{tabular}{|c|c|c|c|c|c|c|c|c|}
\hline & $\begin{array}{l}\text { Average } \\
\text { Feed Flux } \\
(\mathrm{mg} / \mathrm{min})\end{array}$ & $\begin{array}{c}\text { Average } \\
\text { Melter } \\
\text { Emissions } \\
\text { Flux } \\
\text { (mg/min) }\end{array}$ & $\begin{array}{c}\text { Average } \\
\text { SBS } \\
\text { Emissions } \\
\text { Flux } \\
(\mathrm{mg} / \mathrm{min})\end{array}$ & $\begin{array}{c}\text { WESP } \\
\text { Emissions } \\
\text { Flux } \\
(\mathrm{mg} / \mathrm{min})\end{array}$ & $\begin{array}{c}\text { DF } \\
\text { Across } \\
\text { WESP }\end{array}$ & $\begin{array}{c}\text { DF Across } \\
\text { SBS, } \\
\text { WESP }\end{array}$ & $\begin{array}{c}\text { Cumulative } \\
\text { DF Across } \\
\text { Melter, SBS, } \\
\text { WESP }\end{array}$ \\
\hline \multirow{29}{*}{ 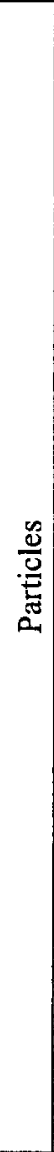 } & Total $^{\S}$ & 281833 & 536 & 194 & 1.7 & 114 & 315 & 165784 \\
\hline & $\mathrm{Al}$ & 11041 & 2.25 & 0.30 & $<0.06$ & $>4.7$ & $>38$ & $>184021$ \\
\hline & As & 85 & 4.10 & 1.87 & $<0.06$ & $>29.3$ & $>68$ & $>1423$ \\
\hline & $\mathrm{B}$ & 8747 & 4.15 & 0.21 & $<0.06$ & $>3.2$ & $>69$ & $>145776$ \\
\hline & $\mathrm{Ba}$ & 101 & $<0.06$ & $<0.06$ & $<0.06$ & $\mathrm{NC}$ & $\mathrm{NC}$ & $>1683$ \\
\hline & $\mathrm{Ca}$ & 504 & 0.59 & 0.48 & $<0.06$ & $>7.5$ & $>10$ & $>8394$ \\
\hline & $\mathrm{Cd}$ & 934 & 32.12 & 14.13 & $<0.06$ & $>220.8$ & $>535$ & $>15562$ \\
\hline & $\mathrm{Cr}$ & 77 & 0.09 & $<0.06$ & $<0.06$ & $\mathrm{NC}$ & $>2$ & $>1286$ \\
\hline & $\mathrm{Cs}$ & 213 & 1.50 & 0.65 & $<0.06$ & $>10.2$ & $>25$ & $>3545$ \\
\hline & $\mathrm{Cu}$ & 68 & 0.10 & 0.03 & $<0.06$ & $>0.5$ & $>2$ & $>1126$ \\
\hline & $\mathrm{Fe}$ & 20476 & 4.95 & 0.21 & $<0.06$ & $>3.3$ & $>82$ & $>341262$ \\
\hline & $I$ & 282 & $<0.06$ & $<0.06$ & $<0.06$ & $\mathrm{NC}$ & $\mathrm{NC}$ & $>4697$ \\
\hline & $\mathrm{K}$ & 398 & 0.76 & 0.36 & $<0.06$ & $>5.6$ & $>+3$ & $>6629$ \\
\hline & $\mathrm{Li}$ & 7855 & 1.86 & 0.41 & $<0.06$ & $>6.4$ & $>31$ & $>130919$ \\
\hline & $\mathrm{Mg \#}$ & 1958 & 0.60 & 0.13 & $<0.06$ & $>2.0$ & $>10$ & $>32633$ \\
\hline & $\mathrm{Mn}$ & 6614 & 0.24 & $<0.06$ & $<0.06$ & $\mathrm{NC}$ & $>4$ & $>110225$ \\
\hline & $\mathrm{Na}$ & 13780 & 8.24 & 2.36 & $<0.06$ & $>36.8$ & $>137$ & $>229664$ \\
\hline & $\mathrm{Ni}$ & 1196 & 0.29 & $<0.06$ & $<0.06$ & $\mathrm{NC}$ & $>5$ & $>19932$ \\
\hline & $\mathrm{P}$ & 160 & 0.15 & 0.17 & $<0.06$ & $>2.6$ & $>3$ & $>2666$ \\
\hline & $\mathrm{Pb}$ & 392 & 2.49 & 1.25 & $<0.06$ & $>19.6$ & $>42$ & $>6541$ \\
\hline & $S$ & 282 & 3.26 & 1.62 & $<0.06$ & $>25.3$ & $>54$ & $>4706$ \\
\hline & $\mathrm{Sb}$ & 446 & 0.42 & 0.22 & $<0.06$ & $>3.4$ & $>7$ & $>7426$ \\
\hline & $\mathrm{Se}$ & 301 & 168.09 & 48.43 & $<0.06$ & $>756.7$ & $>2801$ & $>5014$ \\
\hline & $\mathrm{Si}$ & 59985 & 8.07 & 1.51 & $<0.06$ & $>23.6$ & $>134$ & $>999743$ \\
\hline & $\mathrm{Sr}$ & 5529 & 1.00 & $<0.06$ & $<0.06$ & $\mathrm{NC}$ & $>17$ & $>92149$ \\
\hline & $\mathrm{Te}$ & 315 & 96.59 & 39.79 & 0.16 & 243 & 590 & 1928 \\
\hline & $\mathrm{Ti}$ & 101 & $<0.06$ & 0.03 & $<0.06$ & $>0.4$ & $\mathrm{NC}$ & $>1690$ \\
\hline & $\mathrm{Zn}$ & 4528 & 1.25 & 0.13 & $<0.06$ & $>2.1$ & $>21$ & $>75474$ \\
\hline & $\mathrm{Zr}$ & 7428 & 0.40 & $<0.06$ & $<0.06$ & $\mathrm{NC}$ & $>7$ & $>123794$ \\
\hline \multirow{6}{*}{ 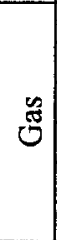 } & $\mathrm{B}$ & 8747 & 32.25 & 4.33 & 0.44 & 10 & 74 & 20011 \\
\hline & $\mathrm{Cl}$ & 28 & 14.58 & $4.7 \overline{3}$ & 1.37 & 3 & 11 & 21 \\
\hline & $\mathrm{F}$ & 113 & 30.04 & 2.95 & 0.24 & 13 & 128 & 479 \\
\hline & I & 282 & 98.60 & 15.19 & 1.52 & 10 & 65 & 186 \\
\hline & $\mathrm{S}$ & 282 & 119.22 & 0.05 & $<0.07$ & $>0.7$ & $>1987$ & $>4706$ \\
\hline & $\mathrm{Se}$ & 301 & 10.18 & 10.54 & 0.21 & 50 & 48 & 1434 \\
\hline
\end{tabular}

NC - Not Calculated

$\$$ - From gravimetric analysis of filters and rinse dry-downs

\# - The analyzed $\mathrm{MgO}$ concentration of $1.15 \mathrm{wt} \%$ instead of the target value of $0.06 \mathrm{wt} \%$ was used to calculate the feed flux 
Table 6.30. Test 1 Melter Outlet Particle Size Distribution Results.

\begin{tabular}{|c|c|c|c|c|c|}
\hline Date & $\begin{array}{c}\text { Impactor } \\
\text { Stage }\end{array}$ & Cutpoint, $\mu \mathrm{m}$ & Net Wt (mg) & $\begin{array}{c}\text { Concentration } \\
(\mathrm{mg} / \mathrm{dscf})\end{array}$ & Mass Percent \\
\hline \multirow{8}{*}{$\begin{array}{l}\bar{\wp} \\
\stackrel{\Im}{0}\end{array}$} & 1 & 15.43 & 15.3 & 32.4012 & 71.8 \\
\hline & 2 & 11.63 & 3.92 & 8.3015 & 18.4 \\
\hline & 3 & 4.50 & 1.25 & 2.6472 & 5.9 \\
\hline & 4 & 2.26 & 0.43 & 0.9106 & 2.0 \\
\hline & 5 & 1.30 & 0.05 & 0.1059 & 0.2 \\
\hline & 6 & 0.73 & 0.04 & 0.0847 & 0.2 \\
\hline & 7 & 0.43 & 0 & 0.0000 & 0.0 \\
\hline & 8 & $<0.43$ & 0.32 & 0.6777 & 1.5 \\
\hline \multirow{8}{*}{ 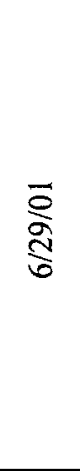 } & 1 & 15.87 & 0 & 0.0000 & 0.0 \\
\hline & 2 & 11.97 & 0.56 & 1.6607 & 51.4 \\
\hline & 3 & 4.63 & 0.27 & 0.8007 & 24.8 \\
\hline & 4 & 2.33 & 0.18 & 0.5338 & 16.5 \\
\hline & 5 & 1.34 & 0.08 & 0.2372 & 7.3 \\
\hline & 6 & 0.75 & 0 & 0.0000 & 0.0 \\
\hline & 7 & 0.44 & 0 & 0.0000 & 0.0 \\
\hline & 8 & $<0.44$ & 0 & 0.0000 & 0.0 \\
\hline \multirow{8}{*}{$\underset{\delta}{\delta}$} & 1 & 15.37 & 0.23 & 0.1939 & 7.0 \\
\hline & 2 & 11.59 & 0.87 & 0.7333 & 26.4 \\
\hline & 3 & 4.48 & 0.71 & 0.5985 & 21.5 \\
\hline & 4 & 2.25 & 0.57 & 0.4805 & 17.3 \\
\hline & 5 & 1.30 & 0.19 & 0.1602 & 5.8 \\
\hline & 6 & 0.73 & 0.13 & 0.1096 & 3.9 \\
\hline & 7 & 0.43 & 0.09 & 0.0759 & 2.7 \\
\hline & 8 & $<0.43$ & 0.51 & 0.4299 & 15.5 \\
\hline
\end{tabular}


Table 6.31. Test 2 Melter Outlet Particle Size Distribution Results.

\begin{tabular}{|c|c|c|c|c|c|}
\hline Date & $\begin{array}{c}\text { Impactor } \\
\text { Stage }\end{array}$ & Cutpoint, $\mu \mathrm{m}$ & Net Wt (mg) & $\begin{array}{c}\text { Concentration } \\
\text { (mg/dscf) }\end{array}$ & Mass Percent \\
\hline \multirow{8}{*}{ 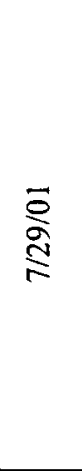 } & 1 & 16.34 & 4.42 & 0.8662 & 57.0 \\
\hline & 2 & 12.33 & 0.07 & 0.0137 & 0.9 \\
\hline & 3 & 4.76 & 0 & 0 & 0 \\
\hline & 4 & 2.40 & 0.16 & 0.0313 & 2.1 \\
\hline & 5 & 1.38 & 0.14 & 0.0274 & 1.8 \\
\hline & 6 & 0.77 & 0.38 & 0.0744 & 4.9 \\
\hline & 7 & 0.46 & 1.12 & 0.2194 & 14.4 \\
\hline & 8 & $<0.46$ & 1.47 & 0.2879 & 18.9 \\
\hline \multirow{8}{*}{$\underset{\wp}{\stackrel{\wp}{\curvearrowright}}$} & 1 & 15.54 & 6.99 & 10.6331 & 66.7 \\
\hline & 2 & 11.72 & 0.59 & 0.8975 & 5.6 \\
\hline & 3 & 4.53 & 0.32 & 0.4868 & 3.1 \\
\hline & 4 & 2.28 & 0.25 & 0.3803 & 2.4 \\
\hline & 5 & 1.31 & 0.47 & 0.7150 & 4.5 \\
\hline & 6 & 0.73 & 0.57 & 0.8671 & 5.4 \\
\hline & 7 & 0.43 & 0.59 & 0.8975 & 5.6 \\
\hline & 8 & $<0.43$ & 0.7 & 1.0648 & 6.7 \\
\hline \multirow{8}{*}{$\frac{\bar{g}}{2}$} & 1 & 15.87 & 7.13 & 16.633 & 66.7 \\
\hline & 2 & 11.97 & 0.59 & 1.3764 & 5.5 \\
\hline & 3 & 4.63 & 0.24 & 0.5599 & 2.2 \\
\hline & 4 & 2.33 & 0.23 & 0.5366 & 2.2 \\
\hline & 5 & 1.34 & 0.53 & 1.2364 & 5.0 \\
\hline & 6 & 0.75 & 0.735 & 1.7146 & 6.9 \\
\hline & 7 & 0.44 & 0.37 & 0.8632 & 3.5 \\
\hline & 8 & $<0.44$ & 0.86 & 2.0063 & 8.0 \\
\hline
\end{tabular}


The Catholic University of America

Vitreous State Laboratory
DuraMelter 1200 Tests with AZ-101HLW Simulants

Final Report, VSL-02R0100-2, Rev.1

Table 6.32. Test 3 Melter Outlet Particle Size Distribution Results.

\begin{tabular}{|c|c|c|c|c|c|}
\hline Date & $\begin{array}{l}\text { Impactor } \\
\text { Stage }\end{array}$ & Cutpoint, $\mu \mathrm{m}$ & Net Wt (mg) & $\begin{array}{l}\text { Concentration } \\
(\mathrm{mg} / \mathrm{dscf})\end{array}$ & Mass Percent \\
\hline \multirow{8}{*}{$\underset{\infty}{\infty}$} & 1 & 16.11 & 70.42 & 432.1870 & 73.7 \\
\hline & 2 & 12.15 & 5.07 & 31.1160 & 5.3 \\
\hline & 3 & 4.70 & 5.13 & 31.4842 & 5.4 \\
\hline & 4 & 2.36 & 4.59 & 28.1701 & 4.8 \\
\hline & 5 & 1.36 & 3.34 & 20.4985 & 3.5 \\
\hline & 6 & 0.76 & 2.09 & 12.8269 & 2.2 \\
\hline & 7 & 0.45 & 2.1 & 12.8883 & 2.2 \\
\hline & 8 & $<0.45$ & 2.86 & 17.5526 & 3.0 \\
\hline \multirow{8}{*}{ 官 } & 1 & 14.68 & 48.9 & 513.1448 & 77.4 \\
\hline & 2 & 11.07 & 3.54 & 37.1479 & 5.6 \\
\hline & 3 & 4.28 & 4.86 & 50.9997 & 7.7 \\
\hline & 4 & 2.15 & 2.48 & 26.0245 & 3.9 \\
\hline & 5 & 1.24 & 1.61 & 16.8950 & 2.5 \\
\hline & 6 & 0.70 & 0.79 & 8.2901 & 1.2 \\
\hline & 7 & 0.41 & 0.26 & 2.7284 & 0.4 \\
\hline & 8 & $<0.41$ & 0.77 & 8.0802 & 1.2 \\
\hline \multirow{8}{*}{$\underset{\infty}{\stackrel{\delta}{O}}$} & 1 & 14.48 & 14.48 & 118.9699 & 70.4 \\
\hline & 2 & 10.93 & 1.13 & 9.2843 & 5.5 \\
\hline & 3 & 4.22 & 0.86 & 7.0659 & 4.2 \\
\hline & 4 & 2.12 & 0.68 & 5.5870 & 3.3 \\
\hline & 5 & 1.22 & 0.5 & 4.1081 & 2.4 \\
\hline & 6 & 0.68 & 0.93 & 7.6410 & 4.5 \\
\hline & 7 & 0.41 & 0.83 & 6.8194 & 4.0 \\
\hline & 8 & $<0.41$ & 1.15 & 9.4486 & 5.6 \\
\hline
\end{tabular}


The Catholic University of America Vitreous State Laboratory
DuraMelter 1200 Tests with AZ-101 HLW Simulants

Final Report, VSL-02R0100-2, Rev.1

Table 6.33. Test 4 Melter Outlet Particle Size Distribution Results.

\begin{tabular}{|c|c|c|c|c|c|}
\hline Date & $\begin{array}{c}\text { Impactor } \\
\text { Stage }\end{array}$ & Cutpoint, $\mu \mathrm{m}$ & Net Wt (mg) & $\begin{array}{c}\text { Concentration } \\
(\mathrm{mg} / \mathrm{dsc})\end{array}$ & Mass Percent \\
\hline \multirow{8}{*}{ 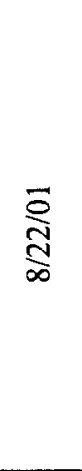 } & 1 & 12.5 & 16.9 & 2.345 & 65.3 \\
\hline & 2 & 9.4 & 2.43 & 0.336 & 9.4 \\
\hline & 3 & 3.63 & 0 & 0 & 0 \\
\hline & 4 & 1.82 & 1.45 & 0.201 & 5.6 \\
\hline & 5 & 1.05 & 0.79 & 0.109 & 3.05 \\
\hline & 6 & 0.59 & 0 & 0 & 0 \\
\hline & 7 & 0.36 & 2.170 & 0.300 & 8.35 \\
\hline & 8 & $<0.36$ & 2.160 & 0.299 & 8.30 \\
\hline \multirow{8}{*}{$\underset{\infty}{\vec{d}}$} & 1 & 14.4 & 34.3 & 6.538 & 65.3 \\
\hline & 2 & 10.9 & 3.060 & 0.583 & 5.82 \\
\hline & 3 & 4.21 & 3.960 & 0.754 & 7.50 \\
\hline & 4 & 2.12 & 3.210 & 0.611 & 6.11 \\
\hline & 5 & 1.22 & 1.840 & 0.350 & 3.50 \\
\hline & 6 & 0.68 & 1.620 & 0.309 & 3.08 \\
\hline & 7 & 0.41 & 2.110 & 0.402 & 4.01 \\
\hline & 8 & $<0.41$ & 2.460 & 0.468 & 4.68 \\
\hline \multirow{8}{*}{$\underset{\infty}{\stackrel{d}{d}}$} & 1 & 15.0 & 25.58 & 5.978 & 67.9 \\
\hline & 2 & 11.3 & 1.940 & 0.514 & 5.15 \\
\hline & 3 & 4.37 & 3.170 & 0.839 & 8.41 \\
\hline & 4 & 2.2 & 2.670 & 0.707 & 7.08 \\
\hline & 5 & 1.26 & 0.410 & 0.109 & 1.09 \\
\hline & 6 & 0.7 & 1.270 & 0.336 & 3.37 \\
\hline & 7 & 0.42 & 1.450 & 0.384 & 3.85 \\
\hline & 8 & $<0.42$ & 1.210 & 0.320 & 3.21 \\
\hline
\end{tabular}


The Catholic University of America

Vitreous State Laboratory
DuraMelter 1200 Tests with AZ-101HLW Simulants Final Report, VSL-02R0100-2, Rev.1

Table 6.34. Test 5 Melter Outlet Particle Size Distribution.

\begin{tabular}{|c|c|c|c|c|c|}
\hline Date & $\begin{array}{l}\text { Impactor } \\
\text { Stage }\end{array}$ & $\begin{array}{l}\text { Cutpoint } \\
\text { (microns) }\end{array}$ & $\begin{array}{l}\text { Net Mass } \\
\text { (mg) }\end{array}$ & $\begin{array}{c}\text { Concentration } \\
\text { (mg/dscf) }\end{array}$ & $\begin{array}{c}\text { Mass } \\
\text { Percent }\end{array}$ \\
\hline \multirow{8}{*}{$\begin{array}{l}\bar{\wp} \\
\stackrel{\infty}{\mathbf{d}} \\
\stackrel{\delta}{\delta}\end{array}$} & 1 & $>14.42$ & 13.3 & 1.076 & 56 \\
\hline & 2 & $10.88-14.42$ & 0.85 & 0.069 & 4 \\
\hline & 3 & $4.20-10.88$ & 1.29 & 0.104 & 5 \\
\hline & 4 & $2.12-4.20$ & 1.74 & 0.141 & 7 \\
\hline & 5 & $1.22-2.12$ & 2.37 & 0.192 & 10 \\
\hline & 6 & $0.68-1.22$ & 1.44 & 0.116 & 6 \\
\hline & 7 & $0.41-0.68$ & 0.79 & 0.064 & 3 \\
\hline & 8 & $<0.41$ & 1.83 & 0.148 & 8 \\
\hline \multirow{8}{*}{$\frac{\bar{g}}{\stackrel{\circ}{ু}}$} & 1 & $>14.16$ & 48.2 & 4.368 & 63 \\
\hline & 2 & $10.68-14.16$ & 2.60 & 0.236 & 3 \\
\hline & 3 & $4.13-10.68$ & 2.90 & 0.263 & 4 \\
\hline & 4 & $2.08-4.13$ & 5.1 & 0.462 & 7 \\
\hline & 5 & $1.19-2.08$ & 10.07 & 0.913 & 13 \\
\hline & 6 & $0.67-1.19$ & 3.82 & 0.346 & 5 \\
\hline & 7 & $0.40-0.67$ & 2.31 & 0.209 & 3 \\
\hline & 8 & $<0.40$ & 2.08 & 0.189 & 3 \\
\hline \multirow{8}{*}{$\frac{\vec{\rho}}{\frac{\rho}{g}}$} & 1 & $>13.91$ & 22.57 & 3.390 & 61 \\
\hline & 2 & $10.49-13.91$ & 2.96 & 0.445 & 8 \\
\hline & 3 & $4.06-10.49$ & 1.48 & 0.222 & 4 \\
\hline & 4 & $2.04-4.06$ & 2.23 & 0.335 & 6 \\
\hline & 5 & $1.17-2.04$ & 2.85 & 0.428 & 8 \\
\hline & 6 & $0.66-1.17$ & 2.39 & 0.359 & 6 \\
\hline & 7 & $0.39-0.66$ & 1.32 & 0.198 & 4 \\
\hline & 8 & $<0.39$ & 1.11 & 0.167 & 3 \\
\hline
\end{tabular}


The Catholic University of America Vitreous State Laboratory
DuraMelter 1200 Tests with AZ-101HLW Simulants Final Report, VSL-02R0100-2, Rev.1

Table 6.35. Test 6 Melter Outlet Particle Size Distribution.

\begin{tabular}{|c|c|c|c|c|c|}
\hline Date & $\begin{array}{c}\text { Impactor } \\
\text { Stage }\end{array}$ & $\begin{array}{l}\text { Cutpoint } \\
\text { (microns) }\end{array}$ & $\begin{array}{c}\text { Net Mass } \\
(\mathrm{mg})\end{array}$ & $\begin{array}{c}\text { Concentration } \\
\text { (mg/dscf })\end{array}$ & $\begin{array}{c}\text { Mass } \\
\text { Percent }\end{array}$ \\
\hline \multirow{8}{*}{$\frac{\vec{g}}{\frac{\delta}{0}}$} & 1 & $>13.58$ & 9.20 & 0.545 & 64 \\
\hline & 2 & $10.24-13.58$ & 1.32 & 0.079 & 9 \\
\hline & 3 & $3.96-10.24$ & 1.04 & 0.062 & 7 \\
\hline & 4 & $1.99-3.96$ & 0.510 & 0.030 & 4 \\
\hline & 5 & $1.15-1.99$ & 0.310 & 0.018 & 2 \\
\hline & 6 & $0.64-1.15$ & 0.310 & 0.021 & 2 \\
\hline & 7 & $0.38-0.64$ & 0.570 & 0.034 & 4 \\
\hline & 8 & $<0.38$ & 1.130 & 0.067 & 8 \\
\hline \multirow{8}{*}{$\frac{\bar{\delta}}{\stackrel{\delta}{\delta}}$} & 1 & $>16.44$ & 137.94 & 8.15 & 83 \\
\hline & 2 & $12.40-16.44$ & 11.90 & 0.699 & 7 \\
\hline & 3 & $4.79-12.40$ & 9.21 & 0.541 & 6 \\
\hline & 4 & $2.41-4.79$ & 2.58 & 0.152 & 2 \\
\hline & 5 & $1.39-2.41$ & 0.98 & 0.058 & 1 \\
\hline & 6 & $0.77-1.39$ & 1.17 & 0.069 & 1 \\
\hline & 7 & $0.46-0.77$ & 1.08 & 0.063 & 1 \\
\hline & 8 & $<0.46$ & 1.96 & 0.115 & 1 \\
\hline \multirow{8}{*}{$\underset{⿱ 亠}{\stackrel{D}{\sigma}}$} & 1 & $>14.87$ & 3.48 & 0.277 & 47 \\
\hline & 2 & $11.22-14.87$ & 0.550 & 0.044 & 7 \\
\hline & 3 & $4.34-11.22$ & 0.480 & 0.038 & 7 \\
\hline & 4 & $2.18-4.34$ & 0.400 & 0.032 & 5 \\
\hline & 5 & $1.25-2.18$ & 0.170 & 0.014 & 2 \\
\hline & 6 & $0.70-1.25$ & 0.0 & 0.000 & 0 \\
\hline & 7 & $0.41-0.70$ & 1.07 & 0.085 & 15 \\
\hline & 8 & $<0.41$ & 1.21 & 0.096 & 16 \\
\hline
\end{tabular}


The Catholic University of America

Vitreous State Laboratory
DuraMelter 1200 Tests with AZ-101HLW Simulants Final Report, VSL-02R0100-2, Rev.I

Table 6.36. Test 7 Melter Outlet Particle Size Distribution.

\begin{tabular}{|c|c|c|c|c|c|}
\hline Date & $\begin{array}{l}\text { Impactor } \\
\text { Stage }\end{array}$ & $\begin{array}{l}\text { Cutpoint } \\
\text { (microns) }\end{array}$ & $\begin{array}{l}\text { Net Mass } \\
\text { (mg) }\end{array}$ & $\begin{array}{c}\text { Concentration } \\
\text { (mg/dscf) }\end{array}$ & $\begin{array}{c}\text { Mass } \\
\text { Percent }\end{array}$ \\
\hline \multirow{8}{*}{ 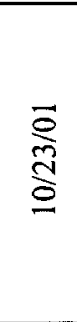 } & 1 & $>14.11$ & 25.56 & 4.79 & 55 \\
\hline & 2 & $10.64-14.11$ & 1.93 & 0.362 & 4 \\
\hline & 3 & $4.11-10.64$ & 1.15 & 0.216 & 2 \\
\hline & 4 & $2.07-4.11$ & 1.010 & 0.189 & 2 \\
\hline & 5 & $1.19-2.07$ & 0.840 & 0.157 & 2 \\
\hline & 6 & $0.67-1.19$ & 3.20 & 0.600 & 7 \\
\hline & 7 & 0.40 & 8.12 & 1.522 & 18 \\
\hline & 8 & $<0.40$ & 4.35 & 0.815 & 9 \\
\hline \multirow{8}{*}{ 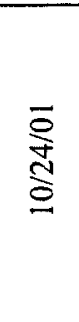 } & 1 & $>15.0$ & 23.50 & 4.766 & 47 \\
\hline & 2 & $11.31-15.0$ & 1.030 & 0.209 & 2 \\
\hline & 3 & $4.37-11.31$ & 1.110 & 0.225 & 2 \\
\hline & 4 & $2.20-4.37$ & 0.770 & 0.156 & 2 \\
\hline & 5 & $1.26-2.20$ & 0.730 & 0.148 & 1 \\
\hline & 6 & $0.71-1.26$ & 3.74 & 0.758 & 7 \\
\hline & 7 & $0.42-0.71$ & 10.63 & 2.156 & 21 \\
\hline & 8 & $<0.42$ & 8.53 & 1.730 & 17 \\
\hline \multirow{8}{*}{ 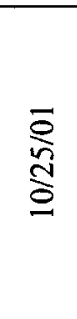 } & 1 & $>14.0$ & 9.24 & 1.639 & 36 \\
\hline & 2 & $10.56-14.0$ & 0.530 & 0.094 & 2 \\
\hline & 3 & $4.08-10.56$ & 0.510 & 0.090 & 2 \\
\hline & 4 & $2.50-4.08$ & 0.430 & 0.076 & 2 \\
\hline & 5 & $1.18-2.05$ & 0.180 & 0.032 & 1 \\
\hline & 6 & $0.66-1.18$ & 4.410 & 0.782 & 17 \\
\hline & 7 & $0.39-0.66$ & 4.630 & 0.822 & 18 \\
\hline & 8 & $<0.39$ & 5.470 & 0.971 & 22 \\
\hline
\end{tabular}


The Catholic University of America

Vitreous State Laboratory
DuraMelter 1200 Tests with AZ-101HLW Simulants Final Report, VSL-02R0100-2, Rev.1

Table 6.37. Test 8 Melter Outlet Particle Size Distribution.

\begin{tabular}{|c|c|c|c|c|c|}
\hline Date & $\begin{array}{l}\text { Impactor } \\
\text { Stage }\end{array}$ & $\begin{array}{l}\text { Cutpoint } \\
\text { (microns) }\end{array}$ & $\begin{array}{l}\text { Net Mass } \\
\text { (mg) }\end{array}$ & $\begin{array}{l}\text { Concentration } \\
\text { (mg/dscf) }\end{array}$ & $\begin{array}{c}\text { Mass } \\
\text { Percent }\end{array}$ \\
\hline \multirow{8}{*}{ 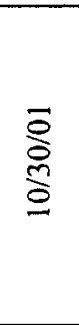 } & 1 & $>15.53$ & 5.6 & 1.073 & 20 \\
\hline & 2 & $11.72-15.53$ & 1.33 & 0.255 & 5 \\
\hline & 3 & $4.53-11.72$ & 0.72 & 0.138 & 3 \\
\hline & 4 & $2.28-4.53$ & 2.27 & 0.435 & 8 \\
\hline & 5 & $1.31-2.28$ & 3.13 & 0.600 & 11 \\
\hline & 6 & $0.73-1.31$ & 4.79 & 0.918 & 17 \\
\hline & 7 & $0.43-0.73$ & 4.75 & 0.91 & 17 \\
\hline & 8 & $<0.43$ & 4.96 & 0.95 & 18 \\
\hline \multirow{8}{*}{$\frac{\vec{\rho}}{\stackrel{\sigma}{\delta}}$} & 1 & $>14.26$ & 5.79 & 1.325 & 21 \\
\hline & 2 & $10.76-14.26$ & 1 & 0.229 & 4 \\
\hline & 3 & $4.16-10.76$ & 1.14 & 0.261 & 4 \\
\hline & 4 & $2.09-4.16$ & 2.3 & 0.526 & 8 \\
\hline & 5 & $1.20-2.09$ & 4.39 & 1.005 & 16 \\
\hline & 6 & $0.67-1.20$ & 5.68 & 1.3 & 21 \\
\hline & 7 & $0.40-0.67$ & 4.67 & 1.069 & 17 \\
\hline & 8 & $<0.40$ & 2.45 & 0.561 & 9 \\
\hline \multirow{8}{*}{$\equiv$} & 1 & $>15.68$ & 4.97 & 1.525 & 21 \\
\hline & 2 & $11.83-15.68$ & 0.69 & 0.212 & 3 \\
\hline & 3 & $4.57-11.83$ & 0.73 & 0.224 & 3 \\
\hline & 4 & $2.30-4.57$ & 2.62 & 0.804 & 11 \\
\hline & 5 & $1.32-2.30$ & 3.35 & 1.028 & 14 \\
\hline & 6 & $0.74-1.32$ & 4.82 & 1.479 & 21 \\
\hline & 7 & $0.43-0.74$ & 4.5 & 1.38 & 19 \\
\hline & 8 & $<0.43$ & 1.62 & 0.497 & 7 \\
\hline
\end{tabular}


The Catholic University of America

Table 6.38. Test 9 Melter Outlet Particle Size Distribution.

\begin{tabular}{|c|c|c|c|c|c|}
\hline Date & $\begin{array}{l}\text { Impactor } \\
\text { Stage }\end{array}$ & $\begin{array}{l}\text { Cutpoint } \\
\text { (microns) }\end{array}$ & $\begin{array}{l}\text { Net Mass } \\
\text { (mg) }\end{array}$ & $\begin{array}{c}\text { Concentration } \\
\text { (mg/dscf) }\end{array}$ & $\begin{array}{c}\text { Mass } \\
\text { Percent }\end{array}$ \\
\hline \multirow{8}{*}{ 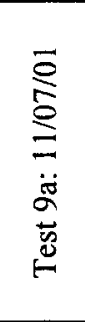 } & 1 & $>14.29$ & 4.69 & 1.733 & 56 \\
\hline & 2 & $10.78-14.29$ & 0.18 & 0.066 & 2 \\
\hline & 3 & $4.17-10.78$ & 0.09 & 0.033 & 1 \\
\hline & 4 & $2.10-4.17$ & 0.08 & 0.03 & 1 \\
\hline & 5 & $1.20-2.10$ & 0.09 & 0.033 & 1 \\
\hline & 6 & $0.67-1.20$ & 0.73 & 0.27 & 9 \\
\hline & 7 & $0.40-0.67$ & 0.78 & 0.288 & 9 \\
\hline & 8 & $<0.40$ & 1.68 & 0.621 & 20 \\
\hline \multirow{8}{*}{ 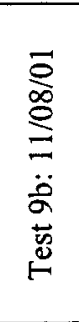 } & 1 & $>16.43$ & 5.94 & 2.575 & 51 \\
\hline & 2 & $12.39-16.43$ & 0.24 & 0.104 & 2 \\
\hline & 3 & $4.79-12.39$ & 0.30 & 0.13 & 3 \\
\hline & 4 & $2.41-4.79$ & 0.31 & 0.134 & 3 \\
\hline & 5 & $1.38-2.41$ & 0.36 & 0.156 & 3 \\
\hline & 6 & $0.77-1.38$ & 1.47 & 0.637 & 13 \\
\hline & 7 & $0.46-0.77$ & 1.35 & 0.585 & 12 \\
\hline & 8 & $<0.46$ & 1.59 & 0.689 & 14 \\
\hline \multirow{8}{*}{ 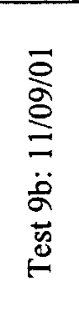 } & 1 & $>12.83$ & 3.19 & 1.124 & 36 \\
\hline & 2 & $9.68-12.83$ & 0.35 & 0.123 & 4 \\
\hline & 3 & $3.74-9.68$ & 0.04 & 0.014 & 2 \\
\hline & 4 & $1.88-3.74$ & 0.08 & 0.028 & 1 \\
\hline & 5 & $1.08-1.88$ & 0.20 & 0.07 & 2 \\
\hline & 6 & $0.61-1.08$ & 1.65 & 0.581 & 19 \\
\hline & 7 & $0.36-0.61$ & 2.03 & 0.715 & 23 \\
\hline & 8 & $<0.36$ & 1.27 & 0.447 & 14 \\
\hline
\end{tabular}


The Catholic University of America

Vitreous State Laboratory
DuraMelter 1200 Tests with AZ-101HLW Simulants Final Report, VSL-02R0100-2, Rev.1

Table 6.39. SBS Outlet Particle Size Distribution Results.

\begin{tabular}{|c|c|c|c|c|c|}
\hline Test & Impactor Stage & Cutpoint, $\mu \mathrm{m}$ & Net Wt (mg) & $\begin{array}{c}\text { Concentration } \\
\text { (mg/dscf) }\end{array}$ & Mass Percent \\
\hline \multirow{8}{*}{1} & 1 & 15.35 & 0 & 0.0000 & 0.0 \\
\hline & 2 & 11.58 & 0 & 0.0000 & 0.0 \\
\hline & 3 & 4.48 & 0 & 0.0000 & 0.0 \\
\hline & 4 & 2.25 & 7.77 & 2.6625 & 24.3 \\
\hline & 5 & 1.29 & 0 & 0.0000 & 0.0 \\
\hline & 6 & 0.72 & 2.76 & 0.9457 & 8.6 \\
\hline & 7 & 0.42 & 15.07 & 5.1639 & 47.1 \\
\hline & 8 & $<0.42$ & 6.41 & 2.1965 & 20.0 \\
\hline \multirow{8}{*}{2} & 1 & 14.03 & 0.17 & 0.0522 & 1.1 \\
\hline & 2 & 10.58 & 0.13 & 0.0399 & 0.8 \\
\hline & 3 & 4.09 & 0.06 & 0.0184 & 0.4 \\
\hline & 4 & 2.06 & 0.12 & 0.0369 & 0.8 \\
\hline & 5 & 1.18 & 0.55 & 0.1690 & 3.5 \\
\hline & 6 & 0.66 & 4.09 & 1.2568 & 26.2 \\
\hline & 7 & 0.39 & 4.92 & 1.5118 & 31.5 \\
\hline & 8 & $<0.39$ & 5.59 & 1.7177 & 35.8 \\
\hline \multirow{8}{*}{3} & 1 & 15.71 & 0 & 0 & 0 \\
\hline & 2 & 11.85 & 0 & 0 & 0 \\
\hline & 3 & 4.58 & 0 & 0 & 0 \\
\hline & 4 & 2.30 & 0 & 0 & 0 \\
\hline & 5 & 1.32 & 0 & 0 & 0 \\
\hline & 6 & 0.74 & 0.76 & 3.3763 & 3.6 \\
\hline & 7 & 0.43 & 19.45 & 86.4078 & 92.9 \\
\hline & 8 & $<0.43$ & 1.13 & 5.0201 & 5.4 \\
\hline \multirow{8}{*}{4} & 1 & 15.7 & 0.31 & 0.027 & 2.79 \\
\hline & 2 & 11.8 & 0.14 & 0.012 & 1.26 \\
\hline & 3 & 4.56 & 0.07 & 0.006 & 0.63 \\
\hline & 4 & 2.29 & 0 & 0 & 0 \\
\hline & 5 & 1.32 & 0.54 & 0.047 & 4.86 \\
\hline & 6 & 0.74 & 3.08 & 0.266 & 27.7 \\
\hline & 7 & 0.43 & 3.44 & 0.298 & 30.96 \\
\hline & 8 & $<0.43$ & 3.53 & 0.305 & 31.77 \\
\hline
\end{tabular}


Table 6.40. SBS Outlet Particle Size Distribution Results.

\begin{tabular}{|c|c|c|c|c|c|}
\hline Date & $\begin{array}{l}\text { Impactor } \\
\text { Stage }\end{array}$ & $\begin{array}{l}\text { Cutpoint } \\
\text { (microns) }\end{array}$ & $\begin{array}{l}\text { Net Mass } \\
\text { (mg) }\end{array}$ & $\begin{array}{l}\text { Concentration } \\
\text { (mg/dscf) }\end{array}$ & $\begin{array}{c}\text { Mass } \\
\text { Percent }\end{array}$ \\
\hline \multirow{8}{*}{ 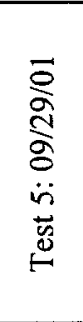 } & 1 & $>14.75$ & 0.56 & 0.040 & 5 \\
\hline & 2 & $11.13-14.75$ & 0.23 & 0.016 & 2 \\
\hline & 3 & $4.30-11.13$ & 0.21 & 0.015 & 2 \\
\hline & 4 & $2.16-4.30$ & 0.05 & 0.004 & 0 \\
\hline & 5 & $1.24-2.16$ & 0.28 & 0.020 & 2 \\
\hline & 6 & $0.69-1.24$ & 1.89 & 0.134 & 16 \\
\hline & 7 & $0.41-0.69$ & 3.41 & 0.241 & 29 \\
\hline & 8 & $<0.41$ & 5.20 & 0.368 & 44 \\
\hline \multirow{8}{*}{ 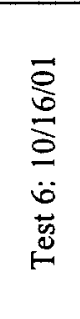 } & 1 & $>15.42$ & 0.08 & 0.002 & 1 \\
\hline & 2 & $11.63-15.42$ & 0.02 & 0.001 & 0 \\
\hline & 3 & $4.49-11.63$ & 0.00 & 0.000 & 0 \\
\hline & 4 & $2.26-4.49$ & 0.00 & 0.000 & 0 \\
\hline & 5 & $1.30-2.26$ & 0.23 & 0.006 & 3 \\
\hline & 6 & $0.73-1.30$ & 1.92 & 0.049 & 21 \\
\hline & 7 & $0.43-0.73$ & 2.89 & 0.074 & 32 \\
\hline & 8 & $<0.43$ & 4.02 & 0.103 & 44 \\
\hline \multirow{8}{*}{ 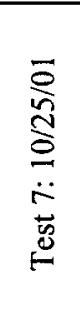 } & 1 & $>17.44$ & 1.47 & 0.264 & 8 \\
\hline & 2 & $13.15-17.44$ & 0.16 & 0.029 & 1 \\
\hline & 3 & $5.08-13.15$ & 0.09 & 0.016 & 1 \\
\hline & 4 & $2.56-5.08$ & 0.08 & 0.014 & 1 \\
\hline & 5 & $1.47-2.56$ & 0.31 & 0.056 & 2 \\
\hline & 6 & $0.82-1.47$ & 5.52 & 0.992 & 31 \\
\hline & 7 & $0.48-0.82$ & 6.66 & 1.197 & 37 \\
\hline & 8 & $<0.48$ & 3.48 & 0.625 & 20 \\
\hline \multirow{8}{*}{ 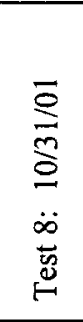 } & 1 & $>13.73$ & 1.06 & 0.457 & 17 \\
\hline & 2 & $10.36-13.73$ & 0.30 & 0.129 & 5 \\
\hline & 3 & $4.00-10.36$ & 0.11 & 0.047 & 2 \\
\hline & 4 & $2.01-4.00$ & 0.10 & 0.043 & 2 \\
\hline & 5 & $1.16-2.01$ & 0.32 & 0.138 & 5 \\
\hline & 6 & $0.65-1.16$ & 1.49 & 0.643 & 24 \\
\hline & 7 & $0.38-0.65$ & 1.4 & 0.604 & 23 \\
\hline & 8 & $<0.38$ & 1.31 & 0.565 & 22 \\
\hline \multirow{8}{*}{ 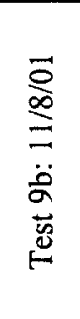 } & 1 & $>15.81$ & 2.29 & 0.573 & 33 \\
\hline & 2 & $11.93-15.81$ & 0.14 & 0.035 & 2 \\
\hline & 3 & $4.61-11.93$ & 0.04 & 0.010 & 1 \\
\hline & 4 & $2.32-4.61$ & 0.03 & 0.008 & 1 \\
\hline & 5 & $1.33-2.32$ & 0.14 & 0.035 & 2 \\
\hline & 6 & $0.74-1.33$ & 0.90 & 0.225 & 13 \\
\hline & 7 & $0.44-0.74$ & 2.06 & 0.515 & 30 \\
\hline & 8 & $<0.44$ & 1.24 & 0.31 & 18 \\
\hline
\end{tabular}


Table 6.41. Compositional Analysis by Particle Size: Test 1, Melter Emissions.

\begin{tabular}{|c|c|c|c|c|c|c|c|c|}
\hline $\begin{array}{l}\text { Particle Size } \\
\text { (microns) }\end{array}$ & $>15.4$ & $\begin{array}{c}11.6- \\
15.4\end{array}$ & $\begin{array}{l}4.5- \\
11.6\end{array}$ & $\begin{array}{c}2.3- \\
4.5\end{array}$ & $\begin{array}{c}1.3- \\
2.3\end{array}$ & $\begin{array}{c}0.7- \\
1.3\end{array}$ & $\begin{array}{c}0.4- \\
0.7\end{array}$ & $<0.4$ \\
\hline $\begin{array}{l}\text { Total Mass } \\
\text { (mg) }\end{array}$ & 0.23 & 0.87 & 0.71 & 0.57 & 0.19 & 0.13 & 0.09 & 0.51 \\
\hline $\begin{array}{c}\text { Mass Fraction } \\
(\%)\end{array}$ & $7 \%$ & $26 \%$ & $22 \%$ & $17 \%$ & $6 \%$ & $4 \%$ & $3 \%$ & $15 \%$ \\
\hline $\mathrm{Al}$ & $3.5 \%$ & $4.1 \%$ & $2.8 \%$ & $1.2 \%$ & $0.8 \%$ & $<0.05 \%$ & $<0.05 \%$ & $<0.05 \%$ \\
\hline$\overline{\mathrm{As}}$ & $<0.05 \%$ & $<0.05 \%$ & $<0.05 \%$ & $<0.05 \%$ & $<0.05 \%$ & $0.8 \%$ & $3.3 \%$ & $0.2 \%$ \\
\hline B & $1.7 \%$ & $2.5 \%$ & $3.9 \%$ & $2.9 \%$ & $1.8 \%$ & $1.2 \%$ & $0.6 \%$ & $<0.05 \%$ \\
\hline $\mathrm{Ba}$ & $<0.05 \%$ & $0.1 \%$ & $0.1 \%$ & $<0.05 \%$ & $<0.05 \%$ & $<0.05 \%$ & $<0.05 \%$ & $<0.05 \%$ \\
\hline $\mathrm{Ca}$ & $<0.05 \%$ & $0.2 \%$ & $0.1 \%$ & $0.4 \%$ & $2.9 \%$ & $3.8 \%$ & $1.1 \%$ & $<0.05 \%$ \\
\hline$\overline{\mathrm{Cd}}$ & $0.2 \%$ & $0.2 \%$ & $0.2 \%$ & $0.2 \%$ & $0.3 \%$ & $<0.05 \%$ & $0.6 \%$ & $0.5 \%$ \\
\hline $\mathrm{Cr}$ & $<0.05 \%$ & $0.1 \%$ & $0.1 \%$ & $0.1 \%$ & $<0.05 \%$ & $<0.05 \%$ & $0.6 \%$ & $1.0 \%$ \\
\hline $\mathrm{Cs}$ & $<0.05 \%$ & $0.6 \%$ & $<0.05 \%$ & $<0.05 \%$ & $2.6 \%$ & $<0.05 \%$ & $<0.05 \%$ & $2.4 \%$ \\
\hline $\mathrm{Cu}$ & $<0.05 \%$ & $<0.05 \%$ & $<0.05 \%$ & $<0.05 \%$ & $0.8 \%$ & $0.8 \%$ & $1.7 \%$ & $<0.05 \%$ \\
\hline $\mathrm{Fe}$ & $5.7 \%$ & $7.4 \%$ & $9.3 \%$ & $10.4 \%$ & $10.3 \%$ & $12.3 \%$ & $2.8 \%$ & $0.4 \%$ \\
\hline I & $\mathrm{NA}$ & $\mathrm{NA}$ & NA & $\mathrm{NA}$ & $\mathrm{NA}$ & NA & $\mathrm{NA}$ & $\mathrm{NA}$ \\
\hline $\mathrm{K}$ & $1.3 \%$ & $0.7 \%$ & $0.8 \%$ & $1.1 \%$ & $1.8 \%$ & $0.4 \%$ & $0.6 \%$ & $0.7 \%$ \\
\hline $\mathrm{Li}$ & $0.9 \%$ & $1.0 \%$ & $1.3 \%$ & $1.1 \%$ & $1.3 \%$ & $1.5 \%$ & $2.8 \%$ & $1.4 \%$ \\
\hline $\mathrm{Mg}$ & $0.7 \%$ & $0.6 \%$ & $0.8 \%$ & $1.0 \%$ & $1.1 \%$ & $1.2 \%$ & $0.6 \%$ & $0.1 \%$ \\
\hline $\mathrm{Mn}$ & $0.7 \%$ & $0.6 \%$ & $0.4 \%$ & $0.3 \%$ & $0.3 \%$ & $0.4 \%$ & $<0.05 \%$ & $0.1 \%$ \\
\hline $\mathrm{Na}$ & $5.4 \%$ & $4.8 \%$ & $6.5 \%$ & $8.4 \%$ & $7.4 \%$ & $8.8 \%$ & $21.1 \%$ & $7.8 \%$ \\
\hline $\mathrm{Ni}$ & $<0.05 \%$ & $0.6 \%$ & $0.7 \%$ & $0.4 \%$ & $2.4 \%$ & $2.3 \%$ & $5.0 \%$ & $<0.05 \%$ \\
\hline $\mathrm{P}$ & $<0.05 \%$ & $<0.05 \%$ & $0.3 \%$ & $<0.05 \%$ & $<0.05 \%$ & $<0.05 \%$ & $<0.05 \%$ & $<0.05 \%$ \\
\hline $\mathrm{Pb}$ & $<0.05 \%$ & $0.1 \%$ & $0.1 \%$ & $<0.05 \%$ & $<0.05 \%$ & $0.4 \%$ & $0.6 \%$ & $0.3 \%$ \\
\hline $\mathrm{S}$ & $<0.05 \%$ & $<0.05 \%$ & $<0.05 \%$ & $<0.05 \%$ & $<0.05 \%$ & $13.8 \%$ & $5.6 \%$ & $2.0 \%$ \\
\hline $\mathrm{Sb}$ & $<0.05 \%$ & $<0.05 \%$ & $<0.05 \%$ & $<0.05 \%$ & $<0.05 \%$ & $<0.05 \%$ & $<0.05 \%$ & $<0.05 \%$ \\
\hline $\mathrm{Se}$ & $10.4 \%$ & $7.2 \%$ & $10.9 \%$ & $16.9 \%$ & $21.8 \%$ & $22.7 \%$ & $31.1 \%$ & $16.6 \%$ \\
\hline $\mathrm{Si}$ & $12.6 \%$ & $11.0 \%$ & $9.7 \%$ & $5.4 \%$ & $3.2 \%$ & $2.7 \%$ & $<0.05 \%$ & $1.7 \%$ \\
\hline $\mathrm{Sr}$ & $1.3 \%$ & $1.3 \%$ & $1.7 \%$ & $1.8 \%$ & $1.6 \%$ & $1.2 \%$ & $0.6 \%$ & $0.1 \%$ \\
\hline $\mathrm{Te}$ & $0.7 \%$ & $0.8 \%$ & $0.1 \%$ & $0.2 \%$ & $1.6 \%$ & $0.8 \%$ & $10.6 \%$ & $9.0 \%$ \\
\hline $\mathrm{Ti}$ & $<0.05 \%$ & $0.1 \%$ & $<0.05 \%$ & $0.1 \%$ & $<0.05 \%$ & $<0.05 \%$ & $<0.05 \%$ & $<0.05 \%$ \\
\hline $\mathrm{Zn}$ & $1.3 \%$ & $1.4 \%$ & $1.8 \%$ & $1.9 \%$ & $1.8 \%$ & $1.2 \%$ & $1.1 \%$ & $0.2 \%$ \\
\hline $\mathrm{Zr}$ & $1.1 \%$ & $0.6 \%$ & $0.2 \%$ & $0.1 \%$ & $0.3 \%$ & $<0.05 \%$ & $<0.05 \%$ & $<0.05 \%$ \\
\hline $\begin{array}{c}\text { Mass Fraction } \\
\text { Sum }(\%)\end{array}$ & $47.4 \%$ & $45.9 \%$ & $51.8 \%$ & $53.8 \%$ & $63.9 \%$ & $76.2 \%$ & $90.0 \%$ & $44.4 \%$ \\
\hline
\end{tabular}

NC- Not Calculated

NA - Not Available 
The Catholic University of America

DuraMelter 1200 Tests with AZ-101HLW Simulants Vitreous State Laboratory

Final Report, VSL-02R0100-2, Rev.1

Table 6.42. Compositional Analysis by Particle Size: Test 2, Melter Emissions.

\begin{tabular}{|c|c|c|c|c|c|c|c|c|}
\hline $\begin{array}{c}\text { Particle Size } \\
\text { (microns) }\end{array}$ & $>15.5$ & $\begin{array}{c}11.7- \\
15.5 \\
\end{array}$ & $\begin{array}{l}4.5- \\
11.7 \\
\end{array}$ & $\begin{array}{c}2.3- \\
4.5 \\
\end{array}$ & $\begin{array}{c}1.3- \\
2.3 \\
\end{array}$ & $\begin{array}{c}0.7- \\
1.3 \\
\end{array}$ & $0.4-0.7$ & $<0.4$ \\
\hline $\begin{array}{l}\text { Total Mass } \\
(\mathrm{mg})\end{array}$ & 6.99 & 0.59 & 0.32 & 0.25 & 0.47 & 0.57 & 0.59 & 0.70 \\
\hline $\begin{array}{c}\text { Mass Fraction } \\
(\%)\end{array}$ & $67 \%$ & $6 \%$ & $3 \%$ & $2 \%$ & $4 \%$ & $5 \%$ & $6 \%$ & $7 \%$ \\
\hline $\mathrm{Al}$ & $0.8 \%$ & $<0.05 \%$ & $<0.05 \%$ & $0.4 \%$ & $0.7 \%$ & $1.5 \%$ & $2.8 \%$ & $3.4 \%$ \\
\hline As & $0.3 \%$ & $1.5 \%$ & $0.9 \%$ & $1.0 \%$ & $0.5 \%$ & $0.6 \%$ & $0.3 \%$ & $0.1 \%$ \\
\hline $\mathrm{B}$ & $0.1 \%$ & $1.7 \%$ & $4.5 \%$ & $5.0 \%$ & $4.0 \%$ & $4.5 \%$ & $2.9 \%$ & $3.1 \%$ \\
\hline $\mathrm{Ba}$ & $<0.05 \%$ & $<0.05 \%$ & $<0.05 \%$ & $<0.05 \%$ & $<0.05 \%$ & $<0.05 \%$ & $<0.05 \%$ & $<0.05 \%$ \\
\hline $\mathrm{Ca}$ & $0.3 \%$ & $0.3 \%$ & $<0.05 \%$ & $0.4 \%$ & $0.1 \%$ & $<0.05 \%$ & $<0.05 \%$ & $<0.05 \%$ \\
\hline $\mathrm{Cd}$ & $0.9 \%$ & $9.7 \%$ & $6.1 \%$ & $1.6 \%$ & $1.1 \%$ & $0.7 \%$ & $0.8 \%$ & $0.6 \%$ \\
\hline $\mathrm{Cr}$ & $<0.05 \%$ & $<0.05 \%$ & $<0.05 \%$ & $<0.05 \%$ & $<0.05 \%$ & $<0.05 \%$ & $<0.05 \%$ & $<0.05 \%$ \\
\hline $\mathrm{Cs}$ & $<0.05 \%$ & $0.9 \%$ & $<0.05 \%$ & $<0.05 \%$ & $1.1 \%$ & $<0.05 \%$ & $0.9 \%$ & $<0.05 \%$ \\
\hline $\mathrm{Cu}$ & $<0.05 \%$ & $0.5 \%$ & $0.3 \%$ & $<0.05 \%$ & $<0.05 \%$ & $0.1 \%$ & $2.3 \%$ & $<0.05 \%$ \\
\hline $\mathrm{Fe}$ & $1.2 \%$ & $0.2 \%$ & $2.7 \%$ & $7.8 \%$ & $8.7 \%$ & $7.9 \%$ & $7.4 \%$ & $7.1 \%$ \\
\hline$I$ & $\mathrm{NA}$ & $\mathrm{NA}$ & NA & $\mathrm{NA}$ & $\mathrm{NA}$ & NA & NA & NA \\
\hline $\mathrm{K}$ & $0.1 \%$ & $1.1 \%$ & $1.3 \%$ & $1.6 \%$ & $1.0 \%$ & $0.9 \%$ & $0.8 \%$ & $0.6 \%$ \\
\hline $\mathrm{Li}$ & $0.7 \%$ & $0.8 \%$ & $0.9 \%$ & $1.0 \%$ & $1.1 \%$ & $1.1 \%$ & $1.0 \%$ & $1.0 \%$ \\
\hline $\mathrm{Mg}$ & $0.4 \%$ & $<0.05 \%$ & $0.2 \%$ & $0.8 \%$ & $0.9 \%$ & $0.7 \%$ & $0.6 \%$ & $0.6 \%$ \\
\hline $\mathrm{Mn}$ & $0.1 \%$ & $<0.05 \%$ & $<0.05 \%$ & $0.4 \%$ & $0.1 \%$ & $0.4 \%$ & $0.3 \%$ & $0.5 \%$ \\
\hline $\mathrm{Na}$ & $3.7 \%$ & $5.4 \%$ & $5.0 \%$ & $7.6 \%$ & $7.1 \%$ & $5.7 \%$ & $4.6 \%$ & $4.7 \%$ \\
\hline $\mathrm{Ni}$ & $0.1 \%$ & $1.1 \%$ & $1.6 \%$ & $0.6 \%$ & $0.5 \%$ & $0.7 \%$ & $4.7 \%$ & $0.1 \%$ \\
\hline $\mathrm{P}$ & $0.1 \%$ & $<0.05 \%$ & $<0.05 \%$ & $<0.05 \%$ & $<0.05 \%$ & $<0.05 \%$ & $<0.05 \%$ & $<0.05 \%$ \\
\hline $\mathrm{Pb}$ & $<0.05 \%$ & $0.6 \%$ & $0.5 \%$ & $0.2 \%$ & $0.1 \%$ & $0.1 \%$ & $0.1 \%$ & $0.1 \%$ \\
\hline $\mathrm{S}$ & $<0.05 \%$ & $<0.05 \%$ & $<0.05 \%$ & $<0.05 \%$ & $<0.05 \%$ & $<0.05 \%$ & $<0.05 \%$ & $<0.05 \%$ \\
\hline $\mathrm{Sb}$ & $0.3 \%$ & $<0.05 \%$ & $<0.05 \%$ & $<0.05 \%$ & $<0.05 \%$ & $<0.05 \%$ & $<0.05 \%$ & $<0.05 \%$ \\
\hline $\mathrm{Se}$ & $11.2 \%$ & $10.0 \%$ & $14.2 \%$ & $16.4 \%$ & $12.0 \%$ & $10.1 \%$ & $10.5 \%$ & $11.9 \%$ \\
\hline $\mathrm{Si}$ & $1.7 \%$ & $<0.05 \%$ & $<0.05 \%$ & $2.8 \%$ & $4.6 \%$ & $6.4 \%$ & $8.8 \%$ & $10.8 \%$ \\
\hline $\mathrm{Sr}$ & $0.8 \%$ & $0.1 \%$ & $0.5 \%$ & $1.2 \%$ & $1.6 \%$ & $1.4 \%$ & $1.3 \%$ & $1.4 \%$ \\
\hline $\mathrm{Te}$ & $3.6 \%$ & $38.6 \%$ & $29.5 \%$ & $6.6 \%$ & $4.1 \%$ & $2.7 \%$ & $2.5 \%$ & $2.3 \%$ \\
\hline $\mathrm{Ti}$ & $<0.05 \%$ & $<0.05 \%$ & $0.2 \%$ & $<0.05 \%$ & $0.1 \%$ & $<0.05 \%$ & $0.1 \%$ & $<0.05 \%$ \\
\hline $\mathrm{Zn}$ & $0.7 \%$ & $0.1 \%$ & $0.3 \%$ & $1.2 \%$ & $1.5 \%$ & $1.4 \%$ & $1.3 \%$ & $1.4 \%$ \\
\hline $\mathrm{Zr}$ & $0.1 \%$ & $<0.05 \%$ & $<0.05 \%$ & $<0.05 \%$ & $0.1 \%$ & $0.2 \%$ & $0.3 \%$ & $0.6 \%$ \\
\hline $\begin{array}{l}\text { Mass Fraction } \\
\text { Sum }(\%)\end{array}$ & $27.2 \%$ & $72.6 \%$ & $68.6 \%$ & $56.6 \%$ & $51.1 \%$ & $46.9 \%$ & $54.2 \%$ & $50.4 \%$ \\
\hline
\end{tabular}

NC- Not Calculated

NA - Not Available 
Table 6.43. Compositional Analysis by Particle Size: Test 3, Melter Emissions.

\begin{tabular}{|c|c|c|c|c|c|c|c|c|}
\hline $\begin{array}{c}\text { Particle Size } \\
\text { (microns) }\end{array}$ & $>16.1$ & $\begin{array}{c}12.2- \\
16.1 \\
\end{array}$ & $\begin{array}{l}4.7- \\
12.2 \\
\end{array}$ & $\begin{array}{c}2.4- \\
4.7 \\
\end{array}$ & $\begin{array}{c}1.4- \\
2.4\end{array}$ & $\begin{array}{c}0.8- \\
1.4\end{array}$ & $0.5-0.8$ & $<0.5$ \\
\hline $\begin{array}{l}\text { Total Mass } \\
\text { (mg) }\end{array}$ & 70.42 & 5.07 & 5.13 & 4.59 & 3.34 & 2.09 & 2.10 & 2.86 \\
\hline $\begin{array}{c}\text { Mass Fraction } \\
(\%)\end{array}$ & $74 \%$ & $5 \%$ & $5 \%$ & $5 \%$ & $3 \%$ & $2 \%$ & $2 \%$ & $3 \%$ \\
\hline $\mathrm{Al}$ & $4.3 \%$ & $3.5 \%$ & $2.0 \%$ & $1.2 \%$ & $0.4 \%$ & $0.1 \%$ & $<0.05 \%$ & $<0.05 \%$ \\
\hline As & $0.1 \%$ & $0.2 \%$ & $<0.05 \%$ & $0.1 \%$ & $0.1 \%$ & $0.4 \%$ & $0.5 \%$ & $0.6 \%$ \\
\hline $\mathrm{B}$ & $2.7 \%$ & $2.7 \%$ & $2.9 \%$ & $3.7 \%$ & $1.9 \%$ & $0.4 \%$ & $<0.05 \%$ & $<0.05 \%$ \\
\hline $\mathrm{Ba}$ & $<0.05 \%$ & $<0.05 \%$ & $<0.05 \%$ & $<0.05 \%$ & $<0.05 \%$ & $<0.05 \%$ & $<0.05 \%$ & $<0.05 \%$ \\
\hline $\mathrm{Ca}$ & $0.2 \%$ & $0.1 \%$ & $0.2 \%$ & $0.2 \%$ & $0.1 \%$ & $<0.05 \%$ & $<0.05 \%$ & $<0.05 \%$ \\
\hline $\mathrm{Cd}$ & $0.3 \%$ & $0.2 \%$ & $0.3 \%$ & $0.6 \%$ & $0.7 \%$ & $1.1 \%$ & $1.2 \%$ & $1.4 \%$ \\
\hline $\mathrm{Cr}$ & $0.1 \%$ & $<0.05 \%$ & $0.1 \%$ & $0.2 \%$ & $0.2 \%$ & $0.3 \%$ & $0.3 \%$ & $0.4 \%$ \\
\hline Cs & $<0.05 \%$ & $0.1 \%$ & $0.2 \%$ & $0.3 \%$ & $0.3 \%$ & $1.2 \%$ & $1.5 \%$ & $1.8 \%$ \\
\hline $\mathrm{Cu}$ & $<0.05 \%$ & $<0.05 \%$ & $<0.05 \%$ & $0.4 \%$ & $<0.05 \%$ & $<0.05 \%$ & $<0.05 \%$ & $<0.05 \%$ \\
\hline $\mathrm{Fe}$ & $6.8 \%$ & $5.6 \%$ & $7.6 \%$ & $13.4 \%$ & $9.7 \%$ & $2.7 \%$ & $0.3 \%$ & $0.1 \%$ \\
\hline $\mathrm{I}$ & NA & $\mathrm{NA}$ & $\mathrm{NA}$ & NA & $\mathrm{NA}$ & NA & $\mathrm{NA}$ & $\mathrm{NA}$ \\
\hline $\mathrm{K}$ & $0.1 \%$ & $0.1 \%$ & $0.2 \%$ & $0.2 \%$ & $0.3 \%$ & $0.7 \%$ & $0.9 \%$ & $1.0 \%$ \\
\hline $\mathrm{Li}$ & $1.3 \%$ & $1.1 \%$ & $1.2 \%$ & $1.6 \%$ & $1.3 \%$ & $2.0 \%$ & $2.0 \%$ & $2.5 \%$ \\
\hline $\mathrm{Mg}$ & $0.8 \%$ & $0.6 \%$ & $0.8 \%$ & $1.2 \%$ & $0.9 \%$ & $0.5 \%$ & $0.1 \%$ & $0.1 \%$ \\
\hline $\mathrm{Mn}$ & $0.5 \%$ & $0.5 \%$ & $0.2 \%$ & $0.2 \%$ & $0.1 \%$ & $0.1 \%$ & $<0.05 \%$ & $<0.05 \%$ \\
\hline $\mathrm{Na}$ & $3.9 \%$ & $3.3 \%$ & $3.7 \%$ & $5.1 \%$ & $4.5 \%$ & $8.8 \%$ & $9.1 \%$ & $10.6 \%$ \\
\hline $\mathrm{Ni}$ & $0.5 \%$ & $0.4 \%$ & $0.5 \%$ & $1.3 \%$ & $0.2 \%$ & $0.1 \%$ & $<0.05 \%$ & $<0.05 \%$ \\
\hline $\mathrm{P}$ & $0.1 \%$ & $0.2 \%$ & $<0.05 \%$ & $<0.05 \%$ & $0.1 \%$ & $<0.05 \%$ & $<0.05 \%$ & $<0.05 \%$ \\
\hline $\mathrm{Pb}$ & $0.1 \%$ & $0.1 \%$ & $0.1 \%$ & $0.2 \%$ & $0.1 \%$ & $0.2 \%$ & $0.2 \%$ & $0.2 \%$ \\
\hline$S$ & $0.1 \%$ & $0.6 \%$ & $0.1 \%$ & $1.0 \%$ & $1.4 \%$ & $8.0 \%$ & $9.2 \%$ & $11.5 \%$ \\
\hline $\mathrm{Sb}$ & $0.1 \%$ & $<0.05 \%$ & $<0.05 \%$ & $0.1 \%$ & $<0.05 \%$ & $<0.05 \%$ & $<0.05 \%$ & $0.1 \%$ \\
\hline $\mathrm{Se}$ & $5.0 \%$ & $3.6 \%$ & $4.2 \%$ & $8.6 \%$ & $9.0 \%$ & $10.4 \%$ & $8.1 \%$ & $10.6 \%$ \\
\hline $\mathrm{Si}$ & $10.3 \%$ & $9.2 \%$ & $6.5 \%$ & $6.2 \%$ & $2.9 \%$ & $0.8 \%$ & $<0.05 \%$ & $<0.05 \%$ \\
\hline $\mathrm{Sr}$ & $1.5 \%$ & $1.2 \%$ & $1.5 \%$ & $2.4 \%$ & $1.4 \%$ & $0.5 \%$ & $0.1 \%$ & $<0.05 \%$ \\
\hline $\mathrm{Te}$ & $1.2 \%$ & $1.2 \%$ & $1.9 \%$ & $5.0 \%$ & $6.4 \%$ & $6.9 \%$ & $5.9 \%$ & $6.5 \%$ \\
\hline $\mathrm{Ti}$ & $<0.05 \%$ & $<0.05 \%$ & $<0.05 \%$ & $<0.05 \%$ & $<0.05 \%$ & $<0.05 \%$ & $<0.05 \%$ & $<0.05 \%$ \\
\hline $\mathrm{Zn}$ & $1.3 \%$ & $1.2 \%$ & $1.2 \%$ & $1.9 \%$ & $1.2 \%$ & $0.6 \%$ & $0.2 \%$ & $0.2 \%$ \\
\hline $\mathrm{Zr}$ & $0.7 \%$ & $0.6 \%$ & $0.2 \%$ & $0.1 \%$ & $0.1 \%$ & $<0.05 \%$ & $<0.05 \%$ & $<0.05 \%$ \\
\hline $\begin{array}{l}\text { Mass Fraction } \\
\text { Sum }(\%)\end{array}$ & $42.2 \%$ & $36.5 \%$ & $35.6 \%$ & $55.3 \%$ & $43.3 \%$ & $45.7 \%$ & $39.6 \%$ & $47.6 \%$ \\
\hline
\end{tabular}

NC- Not Calculated

NA - Not Available 
The Catholic University of America

Table 6.44. Compositional Analysis by Particle Size: Test 4, Melter Emissions.

\begin{tabular}{|c|c|c|c|c|c|c|c|c|}
\hline $\begin{array}{l}\text { Particle Size } \\
\text { (microns) }\end{array}$ & $>12.5$ & $\begin{array}{l}9.4- \\
12.5 \\
\end{array}$ & $\begin{array}{c}3.6- \\
9.4 \\
\end{array}$ & $\begin{array}{c}1.8- \\
3.6 \\
\end{array}$ & $\begin{array}{c}1.1- \\
1.8\end{array}$ & $\begin{array}{c}0.6- \\
1.1 \\
\end{array}$ & $0.4-0.6$ & $<0.4$ \\
\hline $\begin{array}{l}\text { Total Mass } \\
\text { (mg) }\end{array}$ & 16.90 & 2.43 & 0.00 & 1.45 & 0.79 & 0.00 & 2.17 & 2.16 \\
\hline $\begin{array}{c}\text { Mass Fraction } \\
(\%)\end{array}$ & $65 \%$ & $9 \%$ & $0 \%$ & $6 \%$ & $3 \%$ & $0 \%$ & $8 \%$ & $8 \%$ \\
\hline $\mathrm{Al}$ & $3.4 \%$ & $3.4 \%$ & $\mathrm{NC}$ & $1.3 \%$ & $0.8 \%$ & $\mathrm{NC}$ & $<0.05 \%$ & $0.3 \%$ \\
\hline As & $<0.05 \%$ & $0.1 \%$ & $\mathrm{NC}$ & $<0.05 \%$ & $<0.05 \%$ & $\mathrm{NC}$ & $0.2 \%$ & $0.3 \%$ \\
\hline $\mathrm{B}$ & $1.8 \%$ & $2.2 \%$ & $\mathrm{NC}$ & $2.4 \%$ & $1.4 \%$ & $\mathrm{NC}$ & $<0.05 \%$ & $0.1 \%$ \\
\hline $\mathrm{Ba}$ & $<0.05 \%$ & $<0.05 \%$ & $\mathrm{NC}$ & $<0.05 \%$ & $0.1 \%$ & $\mathrm{NC}$ & $<0.05 \%$ & $<0.05 \%$ \\
\hline $\mathrm{Ca}$ & $0.2 \%$ & $0.6 \%$ & $\mathrm{NC}$ & $0.8 \%$ & $0.7 \%$ & $\mathrm{NC}$ & $0.2 \%$ & $0.2 \%$ \\
\hline $\mathrm{Cd}$ & $0.2 \%$ & $0.2 \%$ & $\mathrm{NC}$ & $0.3 \%$ & $0.4 \%$ & $\mathrm{NC}$ & $0.8 \%$ & $0.6 \%$ \\
\hline $\mathrm{Cr}$ & $<0.05 \%$ & $<0.05 \%$ & $\mathrm{NC}$ & $0.1 \%$ & $0.1 \%$ & $\mathrm{NC}$ & $0.3 \%$ & $0.2 \%$ \\
\hline $\mathrm{Cs}$ & $<0.05 \%$ & $<0.05 \%$ & $\mathrm{NC}$ & $<0.05 \%$ & $0.7 \%$ & $\mathrm{NC}$ & $1.5 \%$ & $<0.05 \%$ \\
\hline $\mathrm{Cu}$ & $<0.05 \%$ & $<0.05 \%$ & $\mathrm{NC}$ & $<0.05 \%$ & $<0.05 \%$ & $\mathrm{NC}$ & $<0.05 \%$ & $<0.05 \%$ \\
\hline $\mathrm{Fe}$ & $5.1 \%$ & $5.2 \%$ & $\mathrm{NC}$ & $10.3 \%$ & $9.8 \%$ & $\mathrm{NC}$ & $0.3 \%$ & $0.4 \%$ \\
\hline $\mathrm{I}$ & $\mathrm{NA}$ & $\mathrm{NA}$ & NA & NA & NA & NA & NA & NA \\
\hline $\mathrm{K}$ & $0.1 \%$ & $0.1 \%$ & $\mathrm{NC}$ & $0.2 \%$ & $0.3 \%$ & $\mathrm{NC}$ & $0.6 \%$ & $0.4 \%$ \\
\hline $\mathrm{Li}$ & $1.4 \%$ & $1.0 \%$ & $\mathrm{NC}$ & $1.4 \%$ & $1.5 \%$ & $\mathrm{NC}$ & $1.7 \%$ & $1.0 \%$ \\
\hline $\mathrm{Mg}$ & $0.6 \%$ & $0.6 \%$ & $\mathrm{NC}$ & $1.1 \%$ & $1.1 \%$ & $\mathrm{NC}$ & $0.1 \%$ & $0.1 \%$ \\
\hline $\mathrm{Mn}$ & $0.3 \%$ & $0.4 \%$ & $\mathrm{NC}$ & $0.2 \%$ & $0.2 \%$ & $\mathrm{NC}$ & $<0.05 \%$ & $0.1 \%$ \\
\hline $\mathrm{Na}$ & $4.1 \%$ & $3.3 \%$ & $\mathrm{NC}$ & $5.4 \%$ & $6.8 \%$ & $\mathrm{NC}$ & $7.6 \%$ & $4.8 \%$ \\
\hline $\mathrm{Ni}$ & $0.3 \%$ & $0.4 \%$ & $\mathrm{NC}$ & $0.3 \%$ & $0.2 \%$ & $\mathrm{NC}$ & $<0.05 \%$ & $0.1 \%$ \\
\hline $\mathrm{P}$ & $<0.05 \%$ & $0.3 \%$ & $\mathrm{NC}$ & $0.3 \%$ & $1.8 \%$ & $\mathrm{NC}$ & $0.2 \%$ & $<0.05 \%$ \\
\hline $\mathrm{Pb}$ & $0.1 \%$ & $0.1 \%$ & $\mathrm{NC}$ & $0.1 \%$ & $0.2 \%$ & $\mathrm{NC}$ & $0.2 \%$ & $0.1 \%$ \\
\hline$S$ & $0.1 \%$ & $0.7 \%$ & $\mathrm{NC}$ & $<0.05 \%$ & $3.9 \%$ & $\mathrm{NC}$ & $7.7 \%$ & $4.5 \%$ \\
\hline $\mathrm{Sb}$ & $0.1 \%$ & $<0.05 \%$ & $\mathrm{NC}$ & $<0.05 \%$ & $<0.05 \%$ & $\mathrm{NC}$ & $<0.05 \%$ & $<0.05 \%$ \\
\hline $\mathrm{Se}$ & $14.3 \%$ & $4.5 \%$ & $\mathrm{NC}$ & $9.6 \%$ & $12.5 \%$ & $\mathrm{NC}$ & $11.8 \%$ & $10.0 \%$ \\
\hline $\mathrm{Si}$ & $8.5 \%$ & $9.3 \%$ & $\mathrm{NC}$ & $6.0 \%$ & $4.4 \%$ & $\mathrm{NC}$ & $<0.05 \%$ & $0.5 \%$ \\
\hline $\mathrm{Sr}$ & $1.0 \%$ & $0.9 \%$ & $\mathrm{NC}$ & $1.5 \%$ & $1.3 \%$ & $\mathrm{NC}$ & $<0.05 \%$ & $0.1 \%$ \\
\hline $\mathrm{Te}$ & $0.9 \%$ & $0.7 \%$ & $\mathrm{NC}$ & $3.3 \%$ & $5.4 \%$ & $\mathrm{NC}$ & $8.8 \%$ & $6.0 \%$ \\
\hline $\mathrm{Ti}$ & $0.2 \%$ & $<0.05 \%$ & $\mathrm{NC}$ & $<0.05 \%$ & $0.1 \%$ & $\mathrm{NC}$ & $<0.05 \%$ & $<0.05 \%$ \\
\hline $\mathrm{Zn}$ & $1.1 \%$ & $1.0 \%$ & $\mathrm{NC}$ & $1.7 \%$ & $1.6 \%$ & $\mathrm{NC}$ & $0.2 \%$ & $0.2 \%$ \\
\hline $\mathrm{Zr}$ & $0.6 \%$ & $0.6 \%$ & $\mathrm{NC}$ & $0.2 \%$ & $0.3 \%$ & $\mathrm{NC}$ & $<0.05 \%$ & $0.1 \%$ \\
\hline $\begin{array}{l}\text { Mass Fraction } \\
\text { Sum }(\%)\end{array}$ & $44.5 \%$ & $35.7 \%$ & $\mathrm{NC}$ & $46.5 \%$ & $55.5 \%$ & $\mathrm{NC}$ & $42.3 \%$ & $29.9 \%$ \\
\hline
\end{tabular}

NC- Not Calculated

NA - Not Available 
Table 6.45. Compositional Analysis by Particle Size: Test 5, Melter Emissions.

\begin{tabular}{|c|c|c|c|c|c|c|c|c|}
\hline $\begin{array}{l}\text { Particle Size } \\
\text { (microns) }\end{array}$ & $>14.2$ & $\begin{array}{c}10.7- \\
14.2\end{array}$ & $\begin{array}{l}4.1- \\
10.7\end{array}$ & $\begin{array}{c}2.1- \\
4.1\end{array}$ & $\begin{array}{c}1.2- \\
2.1\end{array}$ & $0.7-1.2$ & $0.4-0.7$ & $<0.4$ \\
\hline $\begin{array}{l}\text { Total Mass } \\
(\mathrm{mg})\end{array}$ & 48.20 & 2.60 & 2.90 & 5.10 & 10.07 & 3.82 & 2.31 & 2.08 \\
\hline $\begin{array}{c}\text { Mass Fraction } \\
(\%)\end{array}$ & $63 \%$ & $3 \%$ & $4 \%$ & $7 \%$ & $13 \%$ & $5 \%$ & $3 \%$ & $3 \%$ \\
\hline $\mathrm{Al}$ & $1.5 \%$ & $2.4 \%$ & $1.4 \%$ & $0.4 \%$ & $<0.05 \%$ & $<0.05 \%$ & $<0.05 \%$ & $<0.05 \%$ \\
\hline As & $0.1 \%$ & $0.3 \%$ & $<0.05 \%$ & $0.1 \%$ & $<0.05 \%$ & $0.1 \%$ & $0.2 \%$ & $0.6 \%$ \\
\hline $\mathrm{B}$ & $1.5 \%$ & $2.4 \%$ & $3.1 \%$ & $4.0 \%$ & $2.2 \%$ & $4.8 \%$ & $4.8 \%$ & $2.0 \%$ \\
\hline $\mathrm{Ba}$ & $<0.05 \%$ & $<0.05 \%$ & $<0.05 \%$ & $<0.05 \%$ & $<0.05 \%$ & $<0.05 \%$ & $<0.05 \%$ & $<0.05 \%$ \\
\hline $\mathrm{Ca}$ & $0.1 \%$ & $0.2 \%$ & $0.2 \%$ & $0.1 \%$ & $<0.05 \%$ & $0.1 \%$ & $<0.05 \%$ & $<0.05 \%$ \\
\hline $\mathrm{Cd}$ & $0.1 \%$ & $0.2 \%$ & $0.2 \%$ & $0.2 \%$ & $0.1 \%$ & $0.4 \%$ & $0.6 \%$ & $0.5 \%$ \\
\hline $\mathrm{Cr}$ & $<0.05 \%$ & $<0.05 \%$ & $<0.05 \%$ & $<0.05 \%$ & $<0.05 \%$ & $<0.05 \%$ & $<0.05 \%$ & $0.1 \%$ \\
\hline $\mathrm{Cs}$ & NA & $\mathrm{NA}$ & $\mathrm{NA}$ & NA & $\mathrm{NA}$ & NA & NA & $\mathrm{NA}$ \\
\hline $\mathrm{Cu}$ & $<0.05 \%$ & $<0.05 \%$ & $<0.05 \%$ & $<0.05 \%$ & $<0.05 \%$ & $<0.05 \%$ & $<0.05 \%$ & $<0.05 \%$ \\
\hline $\mathrm{Fe}$ & $2.4 \%$ & $3.9 \%$ & $4.8 \%$ & $3.7 \%$ & $0.8 \%$ & $0.5 \%$ & $0.1 \%$ & $0.2 \%$ \\
\hline $\mathrm{I}$ & $\mathrm{NA}$ & NA & NA & $\mathrm{NA}$ & $\mathrm{NA}$ & $\mathrm{NA}$ & $\mathrm{NA}$ & NA \\
\hline $\mathrm{K}$ & $0.1 \%$ & $0.2 \%$ & $0.2 \%$ & $0.4 \%$ & $0.2 \%$ & $0.6 \%$ & $0.6 \%$ & $0.6 \%$ \\
\hline $\mathrm{Li}$ & $1.2 \%$ & $1.9 \%$ & $2.3 \%$ & $2.5 \%$ & $1.2 \%$ & $2.5 \%$ & $2.3 \%$ & $1.9 \%$ \\
\hline $\mathrm{Mg}$ & $0.3 \%$ & $0.5 \%$ & $0.6 \%$ & $0.5 \%$ & $0.2 \%$ & $0.2 \%$ & $0.1 \%$ & $0.1 \%$ \\
\hline $\mathrm{Mn}$ & $0.2 \%$ & $0.4 \%$ & $0.3 \%$ & $0.2 \%$ & $0.1 \%$ & $0.1 \%$ & $<0.05 \%$ & $0.1 \%$ \\
\hline $\mathrm{Na}$ & $2.9 \%$ & $4.3 \%$ & $6.0 \%$ & $8.2 \%$ & $4.8 \%$ & $10.9 \%$ & $12.4 \%$ & $9.7 \%$ \\
\hline $\mathrm{Ni}$ & $0.2 \%$ & $0.3 \%$ & $0.3 \%$ & $0.1 \%$ & $<0.05 \%$ & $<0.05 \%$ & $<0.05 \%$ & $<0.05 \%$ \\
\hline $\mathrm{P}$ & $0.1 \%$ & $<0.05 \%$ & $<0.05 \%$ & $<0.05 \%$ & $<0.05 \%$ & $<0.05 \%$ & $<0.05 \%$ & $<0.05 \%$ \\
\hline $\mathrm{Pb}$ & $0.1 \%$ & $0.1 \%$ & $0.1 \%$ & $<0.05 \%$ & $<0.05 \%$ & $0.1 \%$ & $<0.05 \%$ & $<0.05 \%$ \\
\hline$S$ & $<0.05 \%$ & $<0.05 \%$ & $0.3 \%$ & $0.3 \%$ & $0.1 \%$ & $0.9 \%$ & $0.8 \%$ & $<0.05 \%$ \\
\hline $\mathrm{Sb}$ & $<0.05 \%$ & $<0.05 \%$ & $<0.05 \%$ & $<0.05 \%$ & $<0.05 \%$ & $<0.05 \%$ & $<0.05 \%$ & $<0.05 \%$ \\
\hline $\mathrm{Se}$ & $2.2 \%$ & $3.6 \%$ & $4.5 \%$ & $4.0 \%$ & $2.4 \%$ & $5.9 \%$ & $6.7 \%$ & $6.8 \%$ \\
\hline $\mathrm{Si}$ & $3.7 \%$ & $5.3 \%$ & $3.8 \%$ & $1.1 \%$ & $0.1 \%$ & $<0.05 \%$ & $<0.05 \%$ & $<0.05 \%$ \\
\hline $\mathrm{Sr}$ & $0.7 \%$ & $1.1 \%$ & $1.2 \%$ & $0.7 \%$ & $0.2 \%$ & $0.4 \%$ & $0.2 \%$ & $0.1 \%$ \\
\hline $\mathrm{Te}$ & $<0.05 \%$ & $<0.05 \%$ & $<0.05 \%$ & $<0.05 \%$ & $<0.05 \%$ & $<0.05 \%$ & $<0.05 \%$ & $<0.05 \%$ \\
\hline $\mathrm{Ti}$ & $<0.05 \%$ & $<0.05 \%$ & $<0.05 \%$ & $<0.05 \%$ & $<0.05 \%$ & $<0.05 \%$ & $<0.05 \%$ & $<0.05 \%$ \\
\hline $\mathrm{Zn}$ & $0.5 \%$ & $0.9 \%$ & $0.8 \%$ & $0.5 \%$ & $0.1 \%$ & $0.2 \%$ & $0.1 \%$ & $0.1 \%$ \\
\hline $\mathrm{Zr}$ & $0.6 \%$ & $1.0 \%$ & $1.1 \%$ & $0.8 \%$ & $0.2 \%$ & $0.1 \%$ & $<0.05 \%$ & $<0.05 \%$ \\
\hline $\begin{array}{l}\text { Mass Fraction } \\
\text { Sum }(\%)\end{array}$ & $18.5 \%$ & $28.9 \%$ & $31.0 \%$ & $27.9 \%$ & $12.9 \%$ & $27.9 \%$ & $29.2 \%$ & $23.0 \%$ \\
\hline
\end{tabular}

NC- Not Calculated

NA - Not Available 
The Catholic University of America Vitreous State Laboratory
DuraMelter 1200 Tests with AZ-101HLW Simulants

Final Report, VSL-02R0100-2, Rev.I

Table 6.46 Average Concentrations (ppmv) of Selected Species in Off-Gas Measured by FTIR Spectroscopy for DM 1200 AZ-101 Tests.

\begin{tabular}{|c|c|c|c|c|c|c|c|c|c|c|c|}
\hline \multirow{2}{*}{ Test \# } & \multicolumn{3}{|c|}{$\begin{array}{c}1 \\
6 / 25 / 01-6 / 28 / 01\end{array}$} & \multicolumn{3}{|c|}{$\begin{array}{c}2 \\
7 / 24 / 01-8 / 1 / 01\end{array}$} & \multicolumn{2}{|c|}{$\begin{array}{c}3 \\
8 / 7 / 01-8 / 10 / 01\end{array}$} & \multicolumn{3}{|c|}{$\begin{array}{c}4 \\
8 / 21 / 01-8 / 24 / 01\end{array}$} \\
\hline & $\begin{array}{l}\text { Melter } \\
\text { Outlet }\end{array}$ & $\begin{array}{l}\text { SBS } \\
\text { Outlet }\end{array}$ & $\begin{array}{l}\text { WESP } \\
\text { Outlet }\end{array}$ & $\begin{array}{l}\text { Melter } \\
\text { Outlet }\end{array}$ & $\begin{array}{c}\text { SBS } \\
\text { Outlet }\end{array}$ & $\begin{array}{l}\text { WESP } \\
\text { Outlet }\end{array}$ & $\begin{array}{l}\text { Melter } \\
\text { Outlet }\end{array}$ & $\begin{array}{c}\text { SBS } \\
\text { Outlet }\end{array}$ & $\begin{array}{l}\text { Melter } \\
\text { Outlet }\end{array}$ & $\begin{array}{c}\text { SBS } \\
\text { Outlet }\end{array}$ & $\begin{array}{l}\text { WESP } \\
\text { Outlet }\end{array}$ \\
\hline Total Sampling Time (hr) & 7.2 & 5.6 & 3.1 & 18.8 & 17.0 & 8.3 & 17.9 & 12.4 & 8.3 & 8.6 & 1.9 \\
\hline Acetonitrile & 1.7 & $<1.0$ & $<1.0$ & $<1.0$ & 1.1 & $<1.0$ & 8.5 & 1.6 & 6.3 & 2.1 & $<1.0$ \\
\hline Acrylonitrile & $<1.0$ & $<1.0$ & $<1.0$ & $<1.0$ & $<1.0$ & $<1.0$ & $<1.0$ & $<1.0$ & $<1.0$ & $<1.0$ & $<1.0$ \\
\hline $\mathrm{CO}_{2}$ & 3960 & 5590 & 6500 & 3880 & 3960 & 4670 & 8380 & 8440 & 6320 & 7390 & 5720 \\
\hline $\mathrm{CO}$ & $<1.0$ & 1.9 & 2.1 & 18.6 & 18.2 & 23.3 & 24.5 & 73.2 & $<1.0$ & 1.4 & 3.6 \\
\hline $\mathrm{H}_{2} \mathrm{O}[\%]$ & 11.8 & 3.4 & 3.8 & 8.3 & 2.6 & 3.4 & 34.0 & 5.8 & 24.6 & 5.6 & 5.9 \\
\hline $\mathrm{HCl}$ & $<1.0$ & $<1.0$ & $<1.0$ & $<1.0$ & $<1.0$ & $<1.0$ & $<1.0$ & $<1.0$ & 1.7 & $<1.0$ & $<1.0$ \\
\hline $\mathrm{HCN}$ & $<1.0$ & $<1.0$ & $<1.0$ & $<1.0$ & $<1.0$ & $<1.0$ & $<1.0$ & 1.2 & $<1.0$ & $<1.0$ & $<1.0$ \\
\hline $\mathrm{HF}$ & $<1.0$ & $<1.0$ & $<1.0$ & $<1.0$ & $<1.0$ & $<1.0$ & 5.2 & 1.9 & 2.6 & $<1.0$ & $<1.0$ \\
\hline $\mathrm{HNO}_{2}$ & $<1.0$ & $<1.0$ & $<1.0$ & $<1.0$ & $<1.0$ & $<1.0$ & $<1.0$ & $<1.0$ & $<1.0$ & $<1.0$ & $<1.0$ \\
\hline $\mathrm{HNO}_{3}$ & $<1.0$ & $<1.0$ & $<1.0$ & $<1.0$ & $<1.0$ & $<1.0$ & $<1.0$ & $<1.0$ & $<1.0$ & $<1.0$ & $<1.0$ \\
\hline $\mathrm{N}_{2} \mathrm{O}$ & 9.0 & 15.4 & 17.5 & 5.2 & 5.1 & 5.9 & 19.4 & 16.0 & 11.5 & 15.5 & 12.4 \\
\hline $\mathrm{NH}_{3}$ & $<1.0$ & $<1.0$ & $<1.0$ & 20.7 & 8.5 & 5.5 & 7.5 & 4.9 & 3.1 & $<1.0$ & $<1.0$ \\
\hline NO & 158 & 238 & 266 & 26.7 & 28.0 & 31.2 & 461 & 334 & 323 & 350 & 238 \\
\hline $\mathrm{NO}_{2}$ & 16.6 & 24.1 & 34.4 & $<1.0$ & $<1.0$ & 3.2 & 18.1 & 67.8 & 10.6 & 27.4 & 23.7 \\
\hline $\mathrm{SO}_{2}$ & $<1.0$ & $<1.0$ & $<1.0$ & $<1.0$ & $<1.0$ & $<1.0$ & $<1.0$ & $<1.0$ & 1.0 & $<1.0$ & $<1.0$ \\
\hline
\end{tabular}

*Note: Measurements at the three locations (Melter, SBS, WESP) were not made simultaneously; each data set corresponds to a different test time interval. 
The Catholic University of America Vitreous State Laboratory
DuraMelter 1200 Tests with AZ-101HLW Simulants Final Report, VSL-02R0100-2, Rev.1

Table 6.46. Average Concentrations (ppmv) of Selected Off-Gas Species Measured by FTIR Spectroscopy (continued).

\begin{tabular}{|l|r|r|r|r|r|r|r|r|r|}
\hline \multicolumn{1}{|c|}{ Test \# } & \multicolumn{3}{|c|}{5} & \multicolumn{3}{c|}{6} & \multicolumn{3}{c|}{$\begin{array}{c}7 \\
(10 / 11-10 / 17)\end{array}$} \\
\hline Port* & $\begin{array}{c}\text { Melter } \\
\text { Outlet }\end{array}$ & $\begin{array}{c}\text { SBS } \\
\text { Outlet }\end{array}$ & $\begin{array}{r}\text { WESP } \\
\text { Outlet }\end{array}$ & $\begin{array}{r}\text { Melter } \\
\text { Outlet }\end{array}$ & $\begin{array}{c}\text { SBS } \\
\text { Outlet }\end{array}$ & $\begin{array}{r}\text { WESP } \\
\text { Outlet }\end{array}$ & $\begin{array}{r}\text { Melter } \\
\text { Outlet }\end{array}$ & $\begin{array}{c}\text { SBS } \\
\text { Outlet }\end{array}$ & $\begin{array}{r}\text { WESP } \\
\text { Outlet }\end{array}$ \\
\hline Acetonitrile & $<1.0$ & $<1.0$ & $<1.0$ & $<1.0$ & $<1.0$ & 1.4 & 147 & $<1.0$ & $<1.0$ \\
\hline Acrylonitrile & $<1.0$ & $<1.0$ & $<1.0$ & $<1.0$ & $<1.0$ & $<1.0$ & $<1.0$ & $<1.0$ & 5.2 \\
\hline $\mathrm{CO}_{2}$ & 5740 & 6100 & 6490 & 1170 & 1380 & 2040 & 2120 & 4650 & 3200 \\
\hline $\mathrm{CO}$ & 209 & 197 & 242 & 26.2 & 27.0 & 57.4 & 75.1 & 150 & 62.9 \\
\hline $\mathrm{H}_{2} \mathrm{O}[\%]$ & 8.4 & 4.3 & 4.3 & 7.9 & 3.0 & 4.7 & 17.2 & 7.7 & 2.1 \\
\hline $\mathrm{HCl}$ & $<1.0$ & $<1.0$ & $<1.0$ & $<1.0$ & $<1.0$ & $<1.0$ & $<1.0$ & $<1.0$ & $<1.0$ \\
\hline $\mathrm{HCN}$ & 6.9 & 7.2 & 8.2 & $<1.0$ & $<1.0$ & $<1.0$ & 1.2 & 4.3 & 3.9 \\
\hline $\mathrm{HF}$ & $<1.0$ & $<1.0$ & $<1.0$ & $<1.0$ & $<1.0$ & $<1.0$ & 7.4 & 3.0 & $<1.0$ \\
\hline $\mathrm{HNO}_{2}$ & 8.3 & 11.8 & 17.8 & $<1.0$ & $<1.0$ & $<1.0$ & 5.5 & $<1.0$ & $<1.0$ \\
\hline $\mathrm{HNO}_{3}$ & $<1.0$ & $<1.0$ & $<1.0$ & $<1.0$ & $<1.0$ & $<1.0$ & 5.6 & $<1.0$ & $<1.0$ \\
\hline $\mathrm{N}_{2} \mathrm{O}$ & 123 & 126 & 131 & 4.8 & 6.3 & 8.0 & 11.0 & 24.5 & 21.0 \\
\hline $\mathrm{NH}_{3}$ & 13.3 & 1.8 & $<1.0$ & 3.6 & 3.0 & 1.8 & 2.7 & 4.9 & $<1.0$ \\
\hline $\mathrm{NO}^{2.0}$ & 2680 & 2470 & 2360 & 95.8 & 122 & 203 & 213 & 488 & 304 \\
\hline $\mathrm{NO}_{2}$ & 1040 & 993 & 1540 & 2.1 & 3.3 & 12.8 & 188 & 14.0 & 6.2 \\
\hline $\mathrm{SO}_{2}$ & 2.3 & $<1.0$ & $<1.0$ & $<1.0$ & $<1.0$ & $<1.0$ & $<1.0$ & $<1.0$ & $<1.0$ \\
\hline
\end{tabular}

*Note: Measurements at the three locations (Melter, SBS, WESP) were not made simultaneously; each data set corresponds to a different test time interval.

\begin{tabular}{|l|r|r|r|r|r|}
\hline \multicolumn{1}{|c|}{ Test \# } & \multicolumn{2}{|c|}{$\begin{array}{c}\text { 8 } \\
(10 / 29-11 / 2)\end{array}$} & \multicolumn{2}{c|}{$\begin{array}{c}\text { 9a } \\
(11 / 6-11 / 7)\end{array}$} & $\begin{array}{c}\text { 9b } \\
(11 / 8-11 / 9)\end{array}$ \\
\hline Port* & $\begin{array}{c}\text { SBS } \\
\text { Outlet }\end{array}$ & WESP Outlet & $\begin{array}{c}\text { SBS } \\
\text { Outlet }\end{array}$ & WESP Outlet & $\begin{array}{c}\text { SBS } \\
\text { Outlet }\end{array}$ \\
\hline Acetonitrile & $<1.0$ & $<1.0$ & $<1.0$ & $<1.0$ & $<1.0$ \\
\hline Acrylonitrile & $<1.0$ & $<1.0$ & $<1.0$ & $<1.0$ & $<1.0$ \\
\hline $\mathrm{CO}_{2}$ & 10,080 & 11,800 & 4330 & 4340 & 5060 \\
\hline $\mathrm{CO}$ & 345 & 437 & 19.7 & 19.9 & 23.6 \\
\hline $\mathrm{H}_{2} \mathrm{O}[\%]$ & 3.0 & 3.3 & 2.5 & 2.9 & 2.7 \\
\hline $\mathrm{HCl}$ & $<1.0$ & $<1.0$ & $<1.0$ & $<1.0$ & $<1.0$ \\
\hline $\mathrm{HCN}$ & 10.2 & 10.3 & $<1.0$ & $<1.0$ & $<1.0$ \\
\hline $\mathrm{HF}$ & $<1.0$ & $<1.0$ & $<1.0$ & $<1.0$ & $<1.0$ \\
\hline $\mathrm{HNO}_{2}$ & 10.1 & 15.0 & $<1.0$ & $<1.0$ & $<1.0$ \\
\hline $\mathrm{HNO}_{3}$ & $<1.0$ & $<1.0$ & $<1.0$ & $<1.0$ & $<1.0$ \\
\hline $\mathrm{N}_{2} \mathrm{O}$ & 192 & 215 & 6.2 & 6.4 & 7.2 \\
\hline $\mathrm{NH}_{3}$ & $<1.0$ & $<1.0$ & 3.8 & $<1.0$ & 6.0 \\
\hline $\mathrm{NO}$ & 2780 & 2580 & 40.4 & 39.4 & 46.7 \\
\hline $\mathrm{NO}_{2}$ & 1060 & 1690 & $<1.0$ & 1.2 & $<1.0$ \\
\hline $\mathrm{SO}_{2}$ & $<1.0$ & $<1.0$ & $<1.0$ & $<1.0$ & 1.5 \\
\hline
\end{tabular}

*Note: Measurements at the three locations (Melter, SBS, WESP) were not made simultaneously; each data set corresponds to a different test time interval. 
The Catholic University of America

DuraMelter 1200 Tests with AZ-101HLW Simulants Vitreous State Laboratory

Table 6.47. Average NO $\mathrm{N}_{\mathrm{x}}$ Emission Fluxes as Measured by FTIR Spectroscopy.

\begin{tabular}{|c|c|c|c|}
\hline Test & NO [mole/h] & $\mathrm{NO}_{2}[\mathrm{~mole} / \mathrm{h}]$ & Feed $[\mathrm{mole} / \mathrm{h}]$ \\
\hline 1 & $\begin{array}{l}2.06 \text { (Melter outlet) } \\
3.12 \text { (SBS outlet) } \\
3.26 \text { (WESP outlet) }\end{array}$ & $\begin{array}{l}0.22 \text { (Melter outlet) } \\
0.33 \text { (SBS outlet) } \\
0.44 \text { (WESP outlet) }\end{array}$ & 2.18 \\
\hline 2 & $\begin{array}{l}0.36 \text { (Melter outlet) } \\
0.44 \text { (SBS outlet) } \\
0.48 \text { (WESP outlet) }\end{array}$ & $\begin{array}{l}0.01 \text { (Melter outlet) } \\
0.01 \text { (SBS outlet) } \\
0.05 \text { (WESP outlet) }\end{array}$ & 1.94 \\
\hline 3 & $\begin{array}{l}7.14 \text { (Melter outlet) } \\
5.27 \text { (SBS outlet) }\end{array}$ & $\begin{array}{l}0.32 \text { (Melter outlet) } \\
1.09 \text { (SBS outlet) }\end{array}$ & 7.81 \\
\hline 4 & $\begin{array}{l}4.97 \text { (Melter outlet) } \\
5.59 \text { (SBS outlet) }\end{array}$ & $\begin{array}{l}0.16 \text { (Melter outlet) } \\
0.44 \text { (SBS outlet) }\end{array}$ & 4.49 \\
\hline 5 & $\begin{array}{l}40.60 \text { (Melter outlet) } \\
43.70 \text { (SBS outlet) } \\
39.47 \text { (WESP outlet) }\end{array}$ & $\begin{array}{l}15.95 \text { (Melter outlet) } \\
18.12 \text { (SBS outlet) } \\
25.64 \text { (WESP outlet) }\end{array}$ & 77.85 \\
\hline 6 & $\begin{array}{l}1.39 \text { (Melter outlet) } \\
1.89 \text { (SBS outlet) } \\
3.61 \text { (WESP outlet) }\end{array}$ & $\begin{array}{l}0.03 \text { (Melter outlet) } \\
0.05 \text { (SBS outlet) } \\
0.23 \text { (WESP outlet) }\end{array}$ & 1.98 \\
\hline 7 & $\begin{array}{l}3.38 \text { (Melter outlet) } \\
10.71 \text { (SBS outlet) } \\
5.33 \text { (WESP outlet) }\end{array}$ & $\begin{array}{l}3.34 \text { (Melter outlet) } \\
0.28 \text { (SBS outlet) } \\
0.11 \text { (WESP outlet) }\end{array}$ & 7.67 \\
\hline 8 & $\begin{array}{l}43.20 \text { (SBS outlet) } \\
41.91 \text { (WESP outlet) }\end{array}$ & $\begin{array}{l}16.53 \text { (SBS outlet) } \\
27.60 \text { (WESP outlet) }\end{array}$ & 77.85 \\
\hline $9 a$ & $\begin{array}{l}0.63 \text { (SBS outlet) } \\
0.60 \text { (WESP outlet) }\end{array}$ & $\begin{array}{l}0.01 \text { (SBS outlet) } \\
0.02 \text { (WESP outlet) }\end{array}$ & 1.82 \\
\hline $9 b$ & 0.68 (SBS outlet) & 0.01 (SBS outlet) & 2.39 \\
\hline
\end{tabular}


The Catholic University of America Vitreous State Laboratory
DuraMelter 1200 Tests with AZ-101HLW Simulants Final Report, VSL-02R0100-2, Rev.1

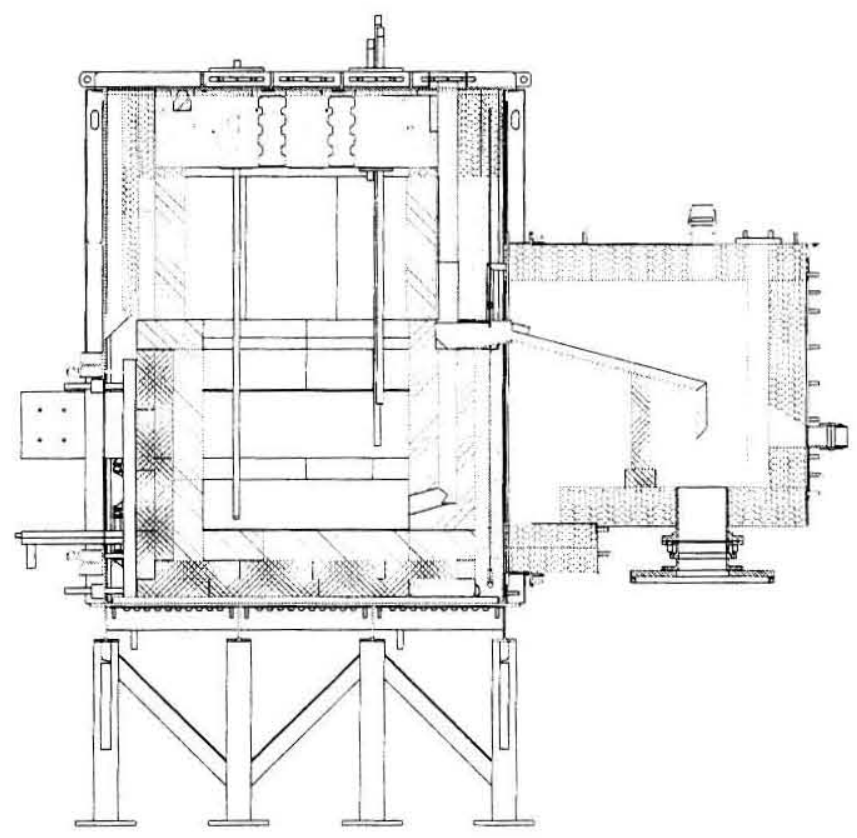

Figure 1.1 Cross-section of the DM1200 melter through the discharge chamber.

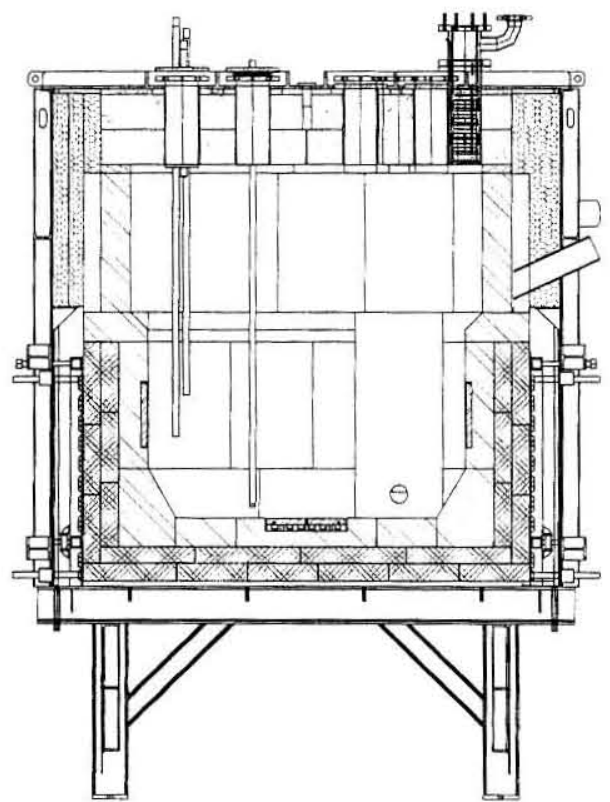

Figure 1.2. Cross-section of the DM1200 melter showing electrodes. 

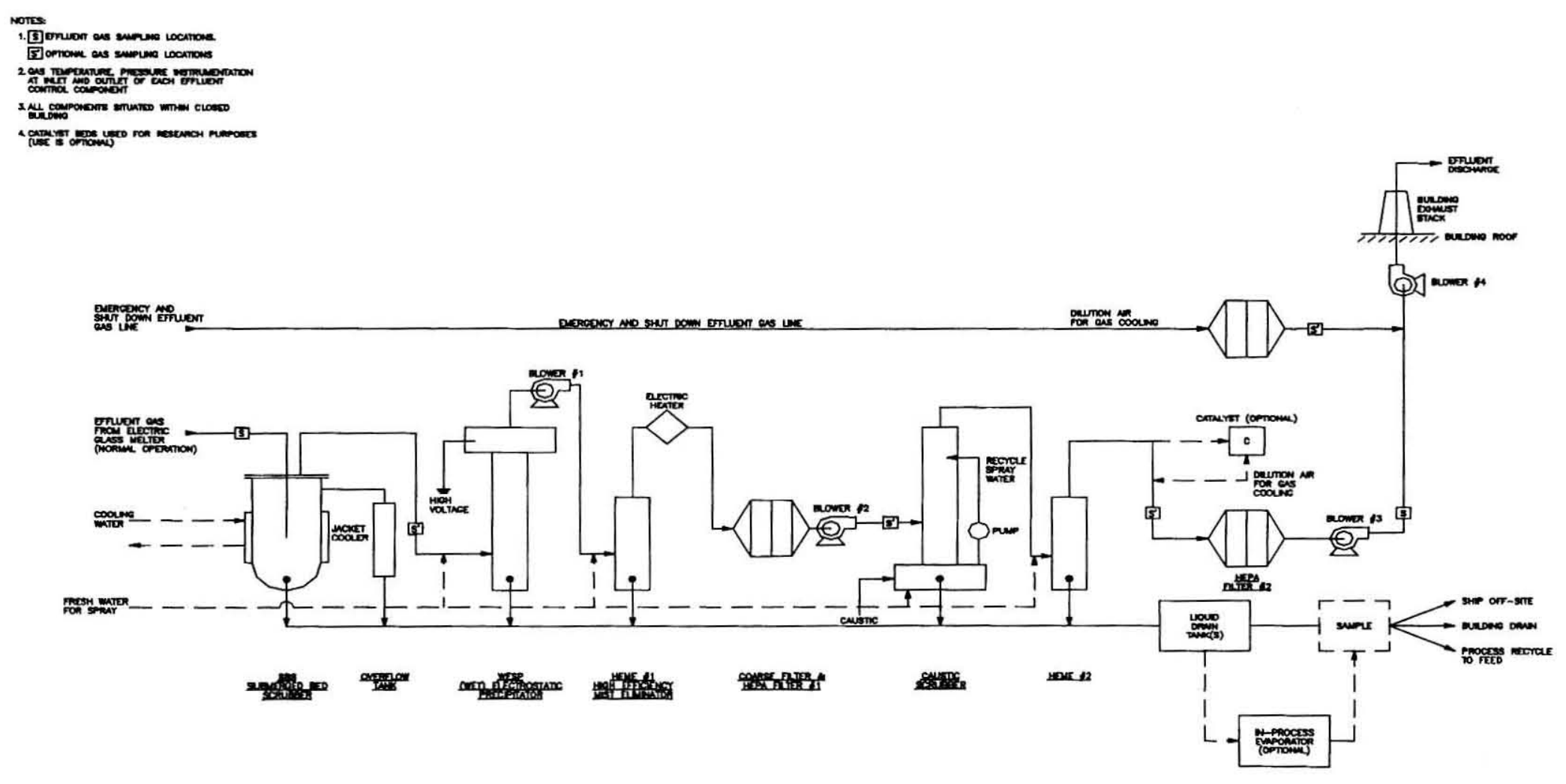

Figure 1.3. Schematic diagram of the DM1200 off-gas system used for these tests. 
The Catholic University of America

Vitreous State Laboratory
DuraMelter 1200 Tests with AZ-I01HLW Simulants Final Report, VSL-02R0100-2, Rev.

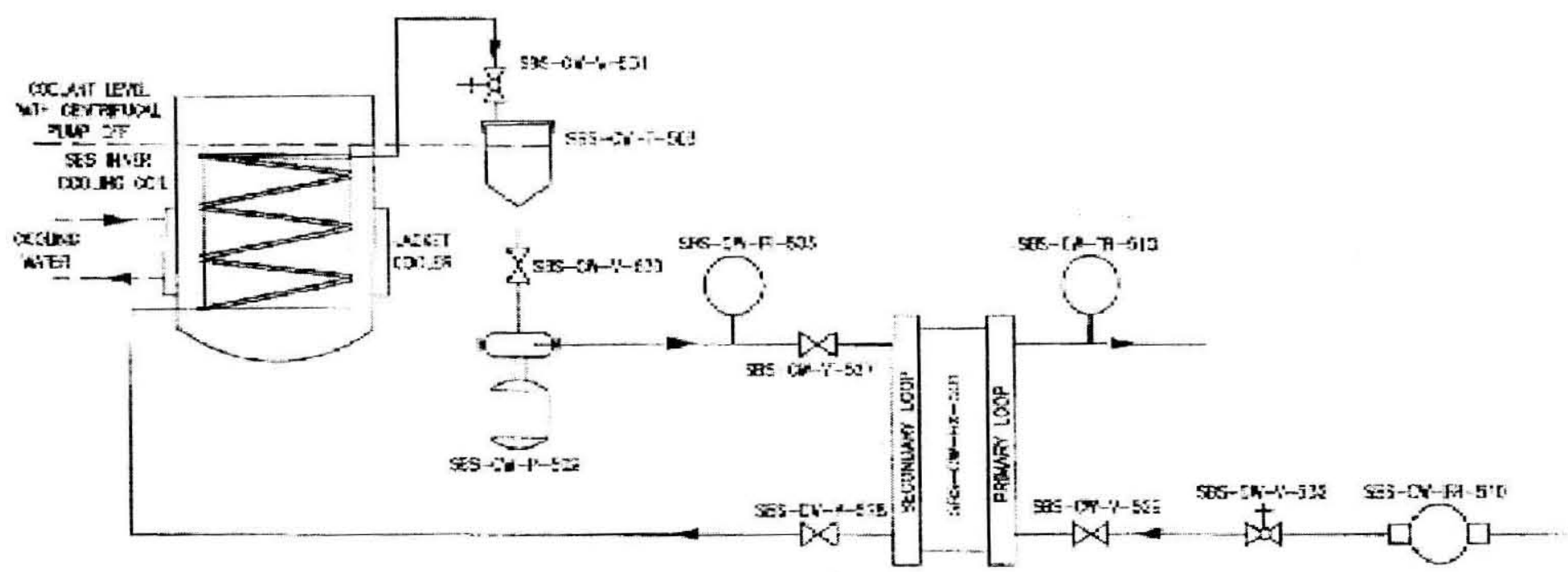

Figure 1.4. Process flow diagram of the modified SBS cooling system. 
The Catholic University of America Vitreous State Laboratory
DuraMelter 1200 Tests with AZ-101HLW Simulants

Final Report, VSL-02R0100-2, Rev.1

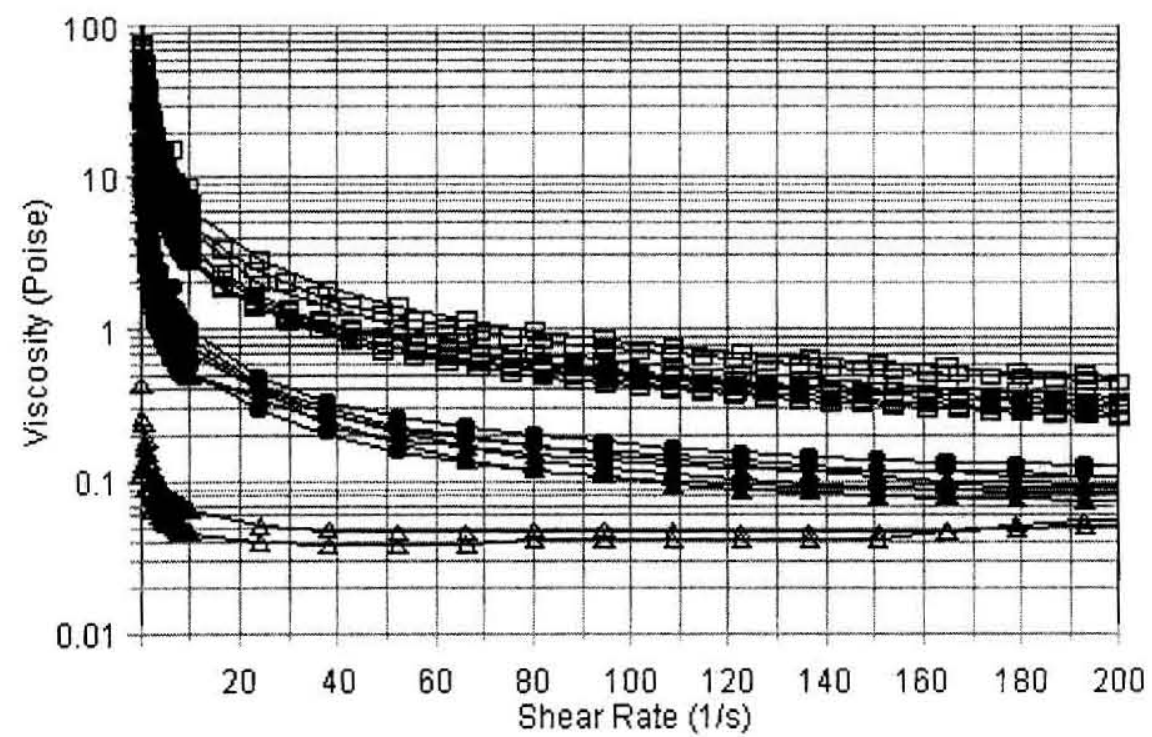

$$
\square \text { Nominal } \quad \text { Frit } \rightarrow \text { High Water } ₫ \text { Nitrated }
$$

Figure 2.1. Rheograms comparing AZ-101 melter feeds from DM1200 melter tests.

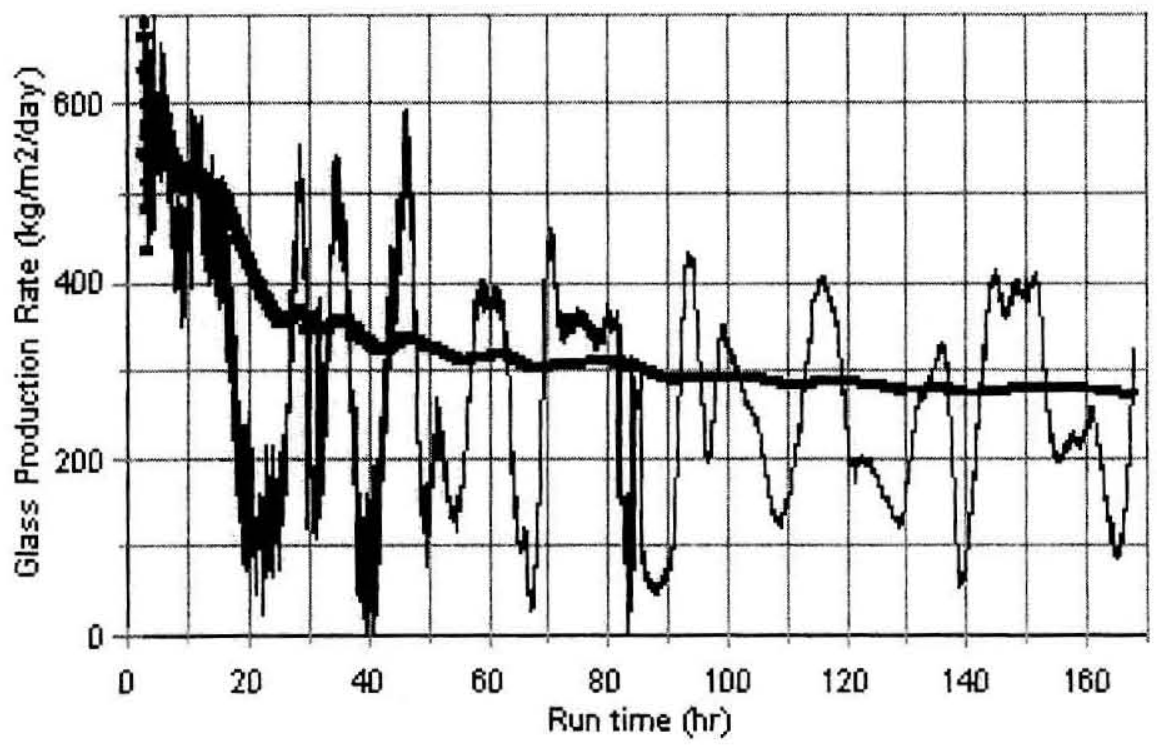

$$
2 \mathrm{hr} \text { Moving Aurg. } \rightarrow-\text { Cumulative }
$$

Figure 3.1. Glass production rates for DM1200 Test 1 (570 g/l with no bubbling). 
The Catholic University of America Vitreous State Laboratory
DuraMelter 1200 Tests with AZ-101HLW Simulants

Final Report, VSL-02R0100-2, Rev.1

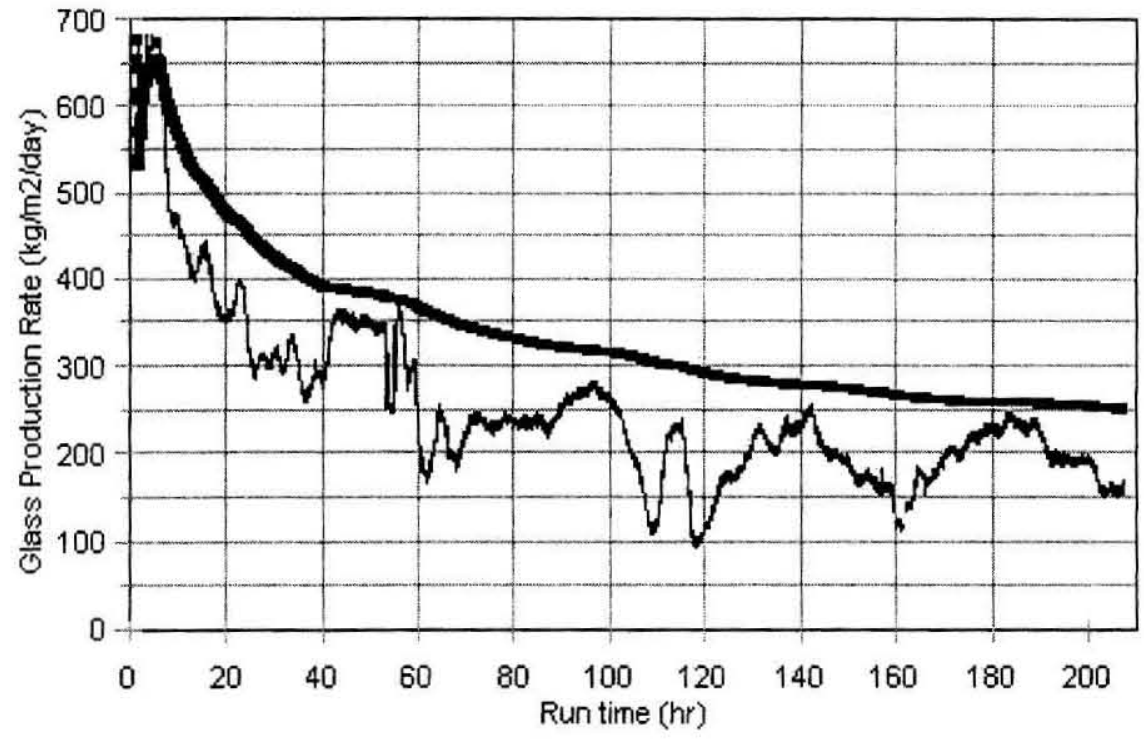

$2 \mathrm{hr}$ Moving Avg. $\rightarrow-$ Cumulative

Figure 3.2. Glass production rates for DM1200 Test $2(570 \mathrm{~g} / \mathrm{l}+10 \mathrm{~g}$ sugar/l with no bubbling).

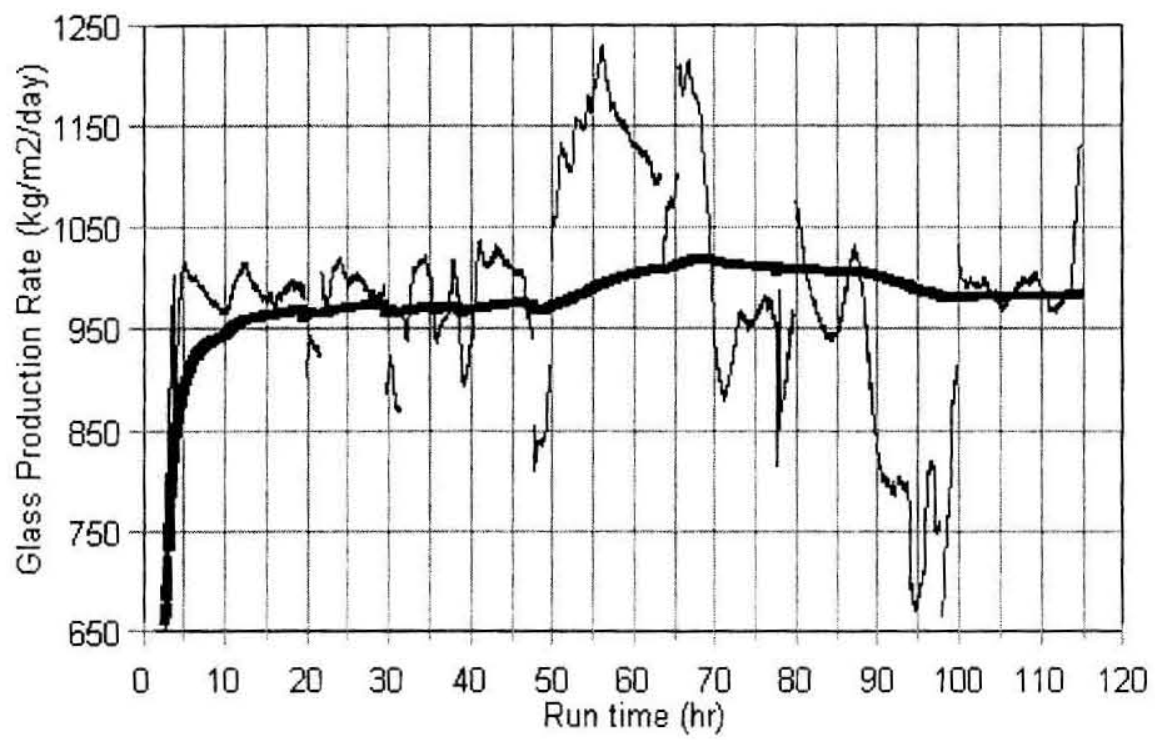

$2 \mathrm{hr}$ Moving Avg. - Cumulative

Figure 3.3. Glass production rates for DM1200 Test 3 (400 g/l with deep bubbling). 
The Catholic University of America Vitreous State Laboratory
DuraMelter 1200 Tests with AZ-101HLW Simulants Final Report, VSL-02R0100-2, Rev.1

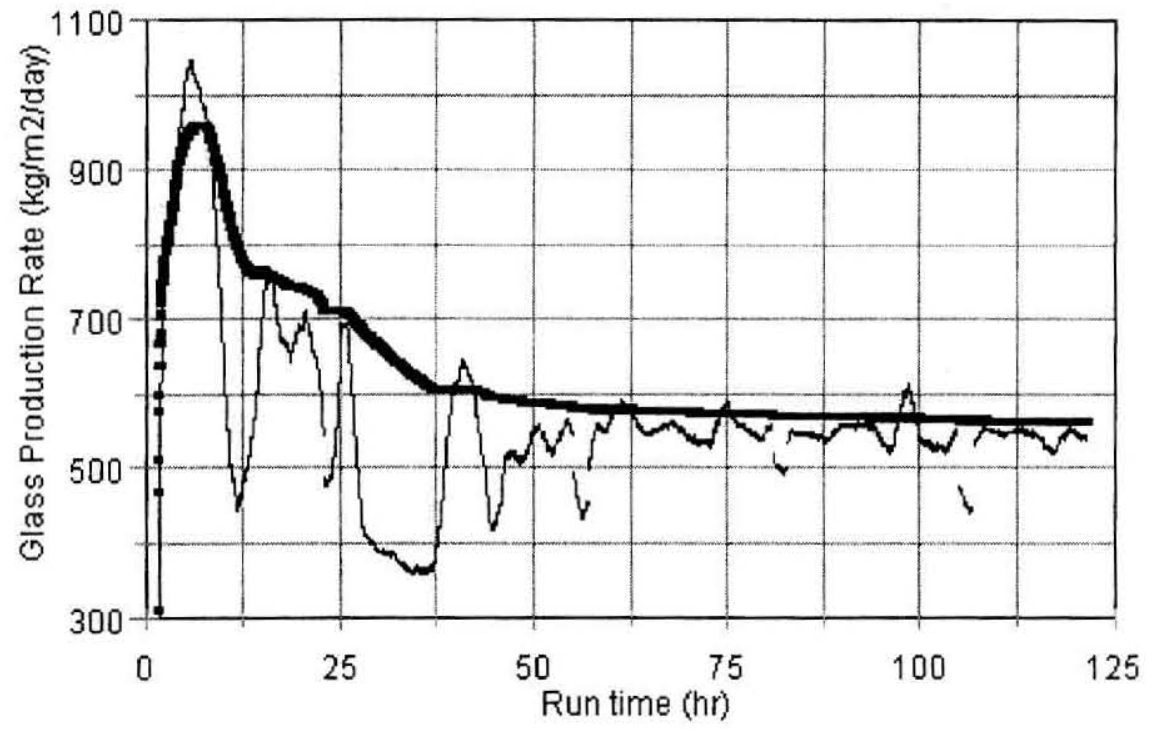

$2 \mathrm{hr}$ Moving Avg. $\rightarrow$ Cumulative

Figure 3.4. Glass production rates for DM1200 Test 4 (400 g/l with shallow bubbling).

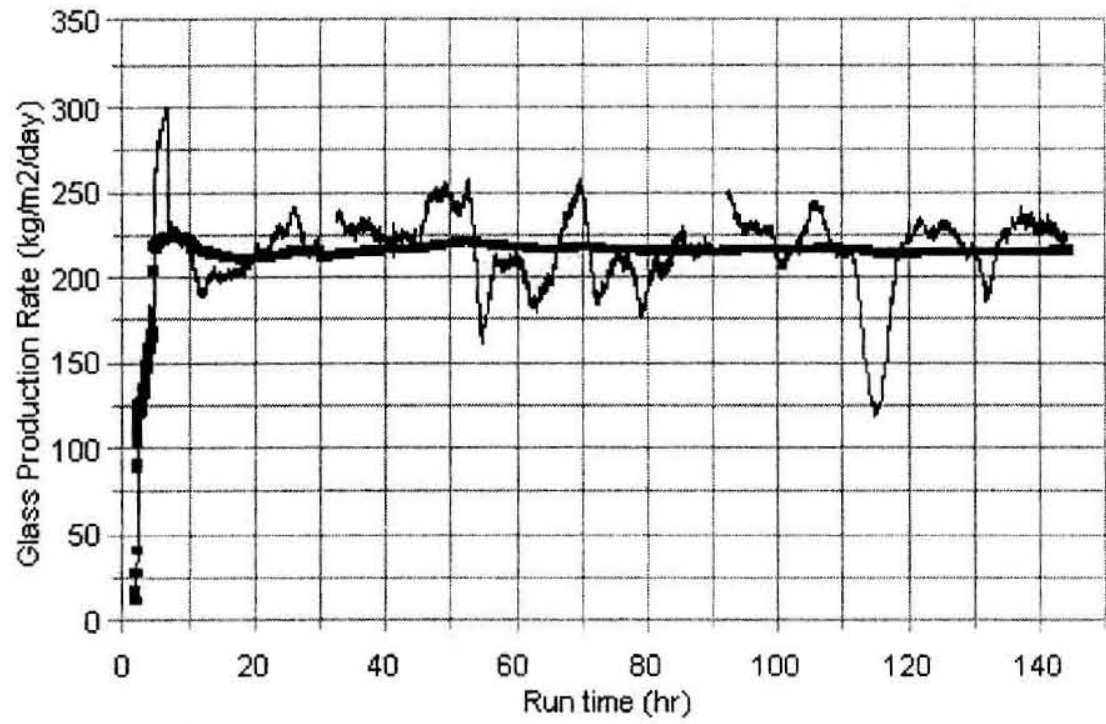

$\mathrm{hr}$ Moving Avg. - Cumulative

Figure 3.5. Glass production rates for DM1200 Test 5 (nitrated feed, sugar ratio = $0.5,420 \mathrm{~g} / \mathrm{l}$ with no bubbling). 
The Catholic University of America Vitreous State Laboratory
DuraMelter 1200 Tests with AZ-101HLW Simulants Final Report, VSL-02R0100-2, Rev.1

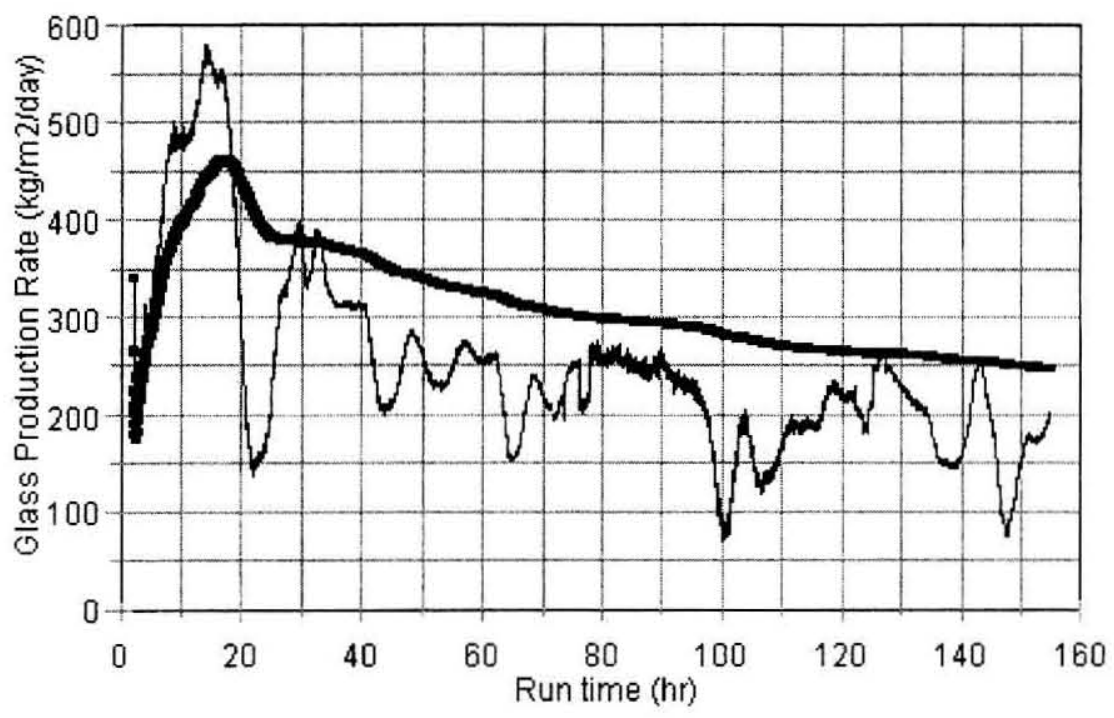

\section{$2 \mathrm{hr}$ Moving Avg. - Cumulative}

Figure 3.6. Glass production rates for DM1200 Test 6 (frit additives, $7.5 \mathrm{~g}$ sugar/l, $480 \mathrm{~g} / \mathrm{l}$ with no bubbling).

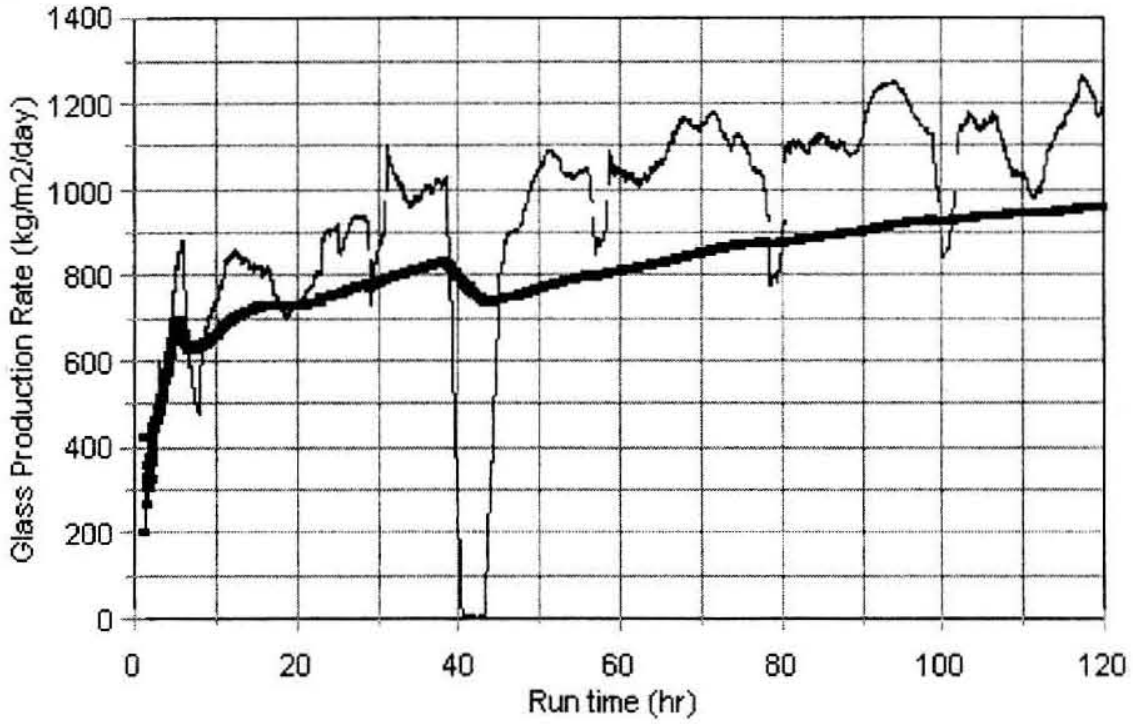

$2 \mathrm{hr}$ Moving Avg. $\rightarrow$ Cumulative

Figure 3.7. Glass production rates for DM1200 Test 7 (frit additives, $7.5 \mathrm{~g}$ sugar/l, $480 \mathrm{~g} / \mathrm{l}$, with bubbling). 
The Catholic University of America Vitreous State Laboratory
DuraMelter 1200 Tests with AZ-101HLW Simulants Final Report, VSL-02R0100-2, Rev.1

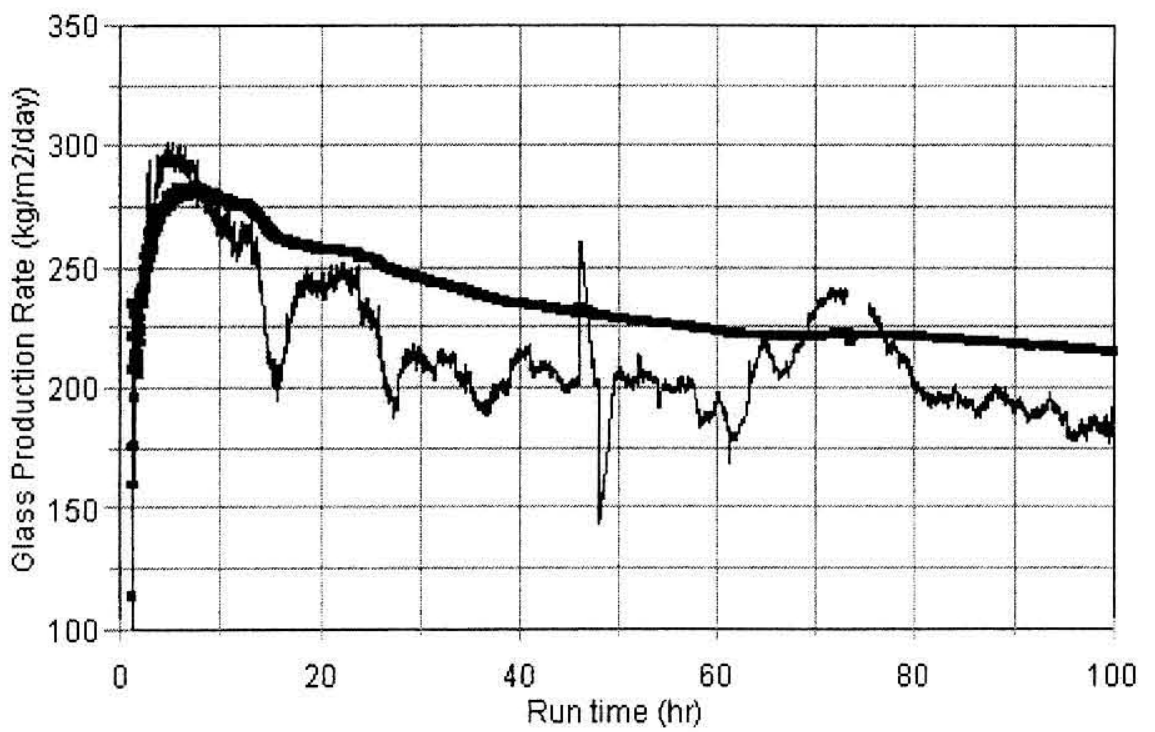

$2 \mathrm{hr}$ Moving Avg. $\rightarrow-$ Cumulative

Figure 3.8. Glass production rates for DM1200 Test 8 (nitrated feed, sugar ratio $=0.7$, with no bubbling).

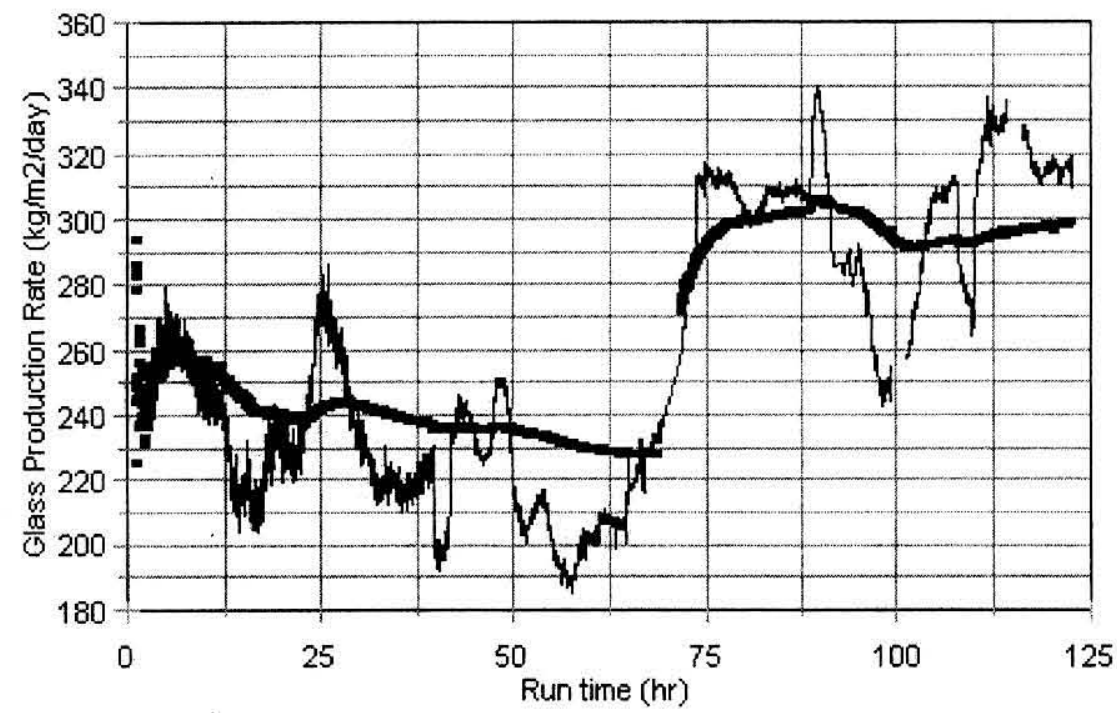

$2 \mathrm{hr}$ Moving Avg. - Cumulative

Figure 3.9. Glass production rates for DM1200 Test $9(570 \mathrm{~g} / \mathrm{l}, 10 \mathrm{~g}$ sugar feeding with peristaltic feeding). Note that glass temperature was increased for the second half of this test. 
The Catholic University of America Vitreous State Laboratory
DuraMelter 1200 Tests with AZ-101HLW Simulants Final Report, VSL-02R0100-2, Rev.1

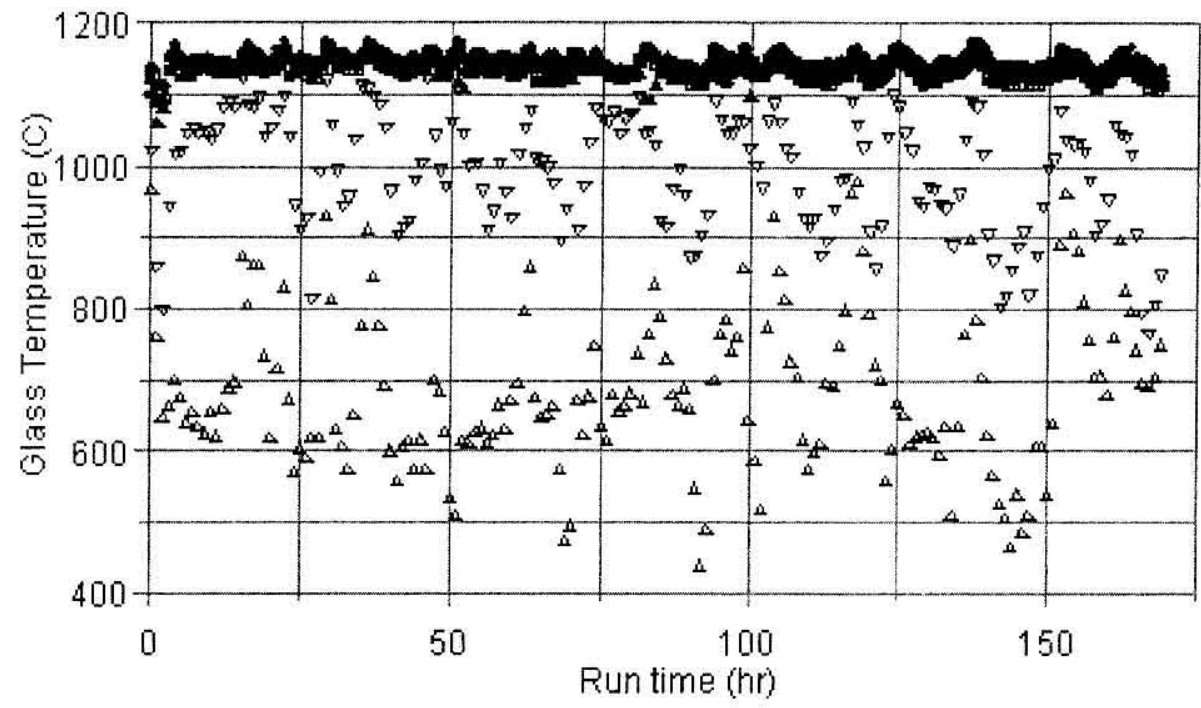

- 1" from floor a 13" from floor * $18^{\prime \prime}$ from floor " $27^{\prime \prime}$ from floor

- 9" from floor = $18^{\prime \prime}$ from floor * 24 "from floor a 30 "from floor

Figure 3.10. Glass temperature for DM1200 Test 1 (570 g/l with no bubbling).

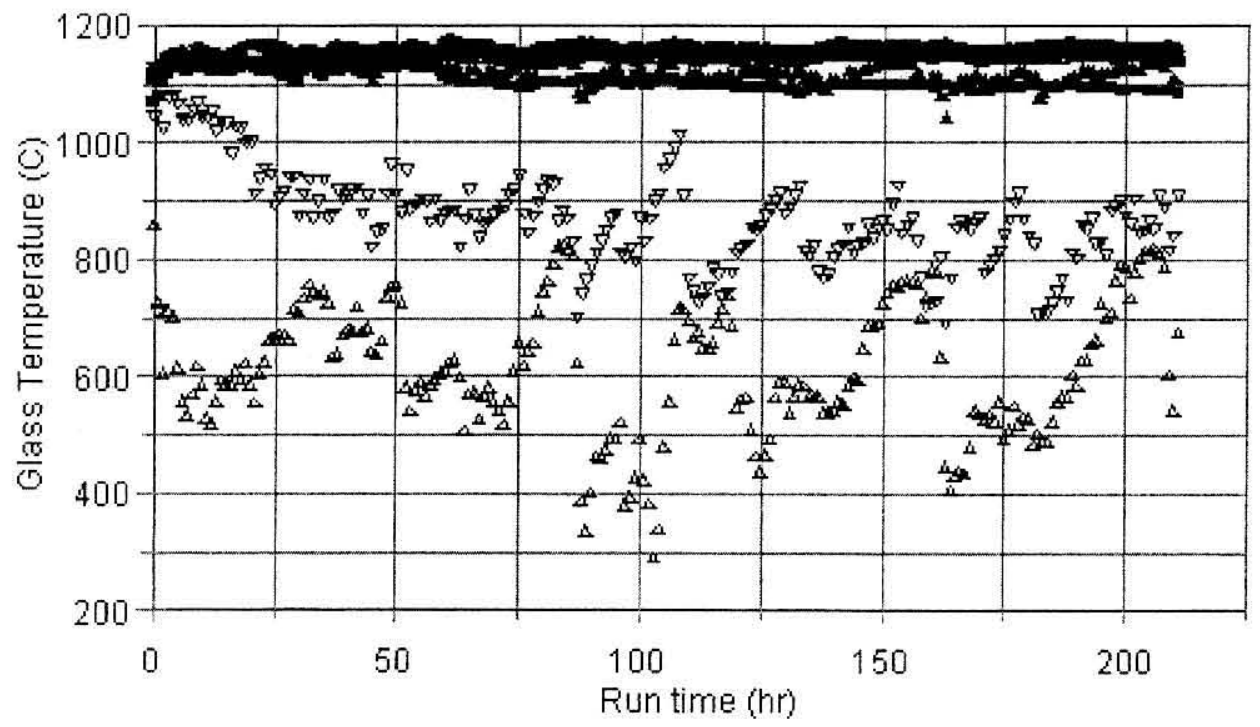
- 1 "from floor
- $13^{\prime \prime}$ from floor
- 18 "from floor
* $27^{\prime \prime}$ from floor
- g" from floor $^{\prime \prime}$
18 " from floor
- 24" from floor
* $30^{\prime \prime}$ from floor

Figure 3.11. Glass temperature for DM1200 Test $2(570 \mathrm{~g} / \mathrm{l}+10 \mathrm{~g}$ sugar/l with no bubbling). 
The Catholic University of America Vitreous State Laboratory
DuraMelter 1200 Tests with AZ-101HLW Simulants

Final Report, VSL-02R0100-2, Rev.I

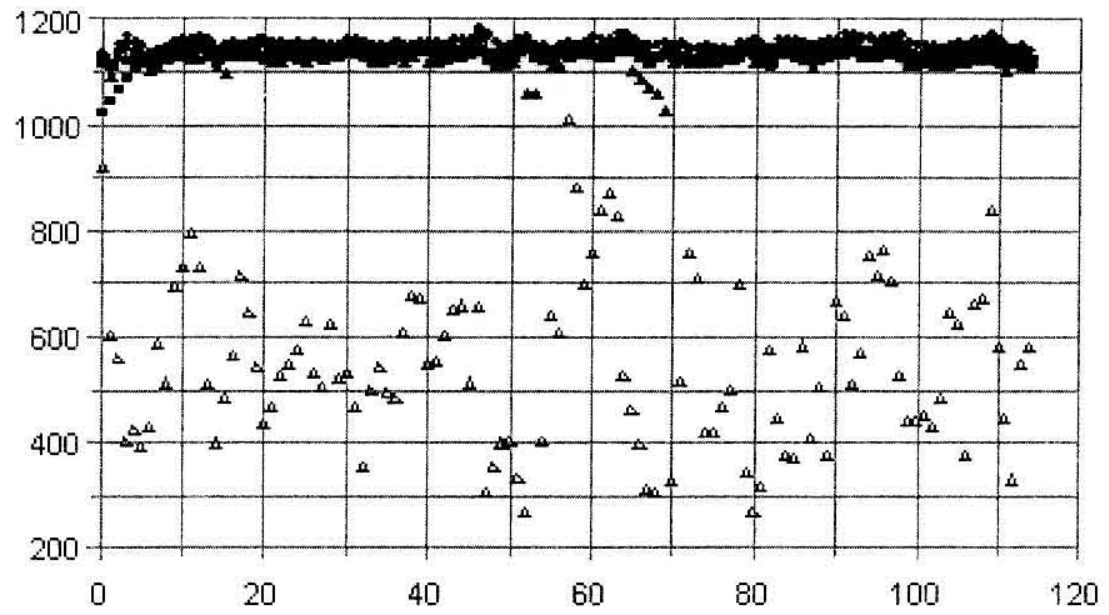

- 1" from floor a 13" from floor " $18^{\prime \prime}$ from floor $\quad 27$ " from floo

- 9" from floor * 18" from floor * 24" from floor \& 30 "from floor

Figure 3.12. Glass temperature for DM1200 Test 3 (400 g/l with deep bubbling).

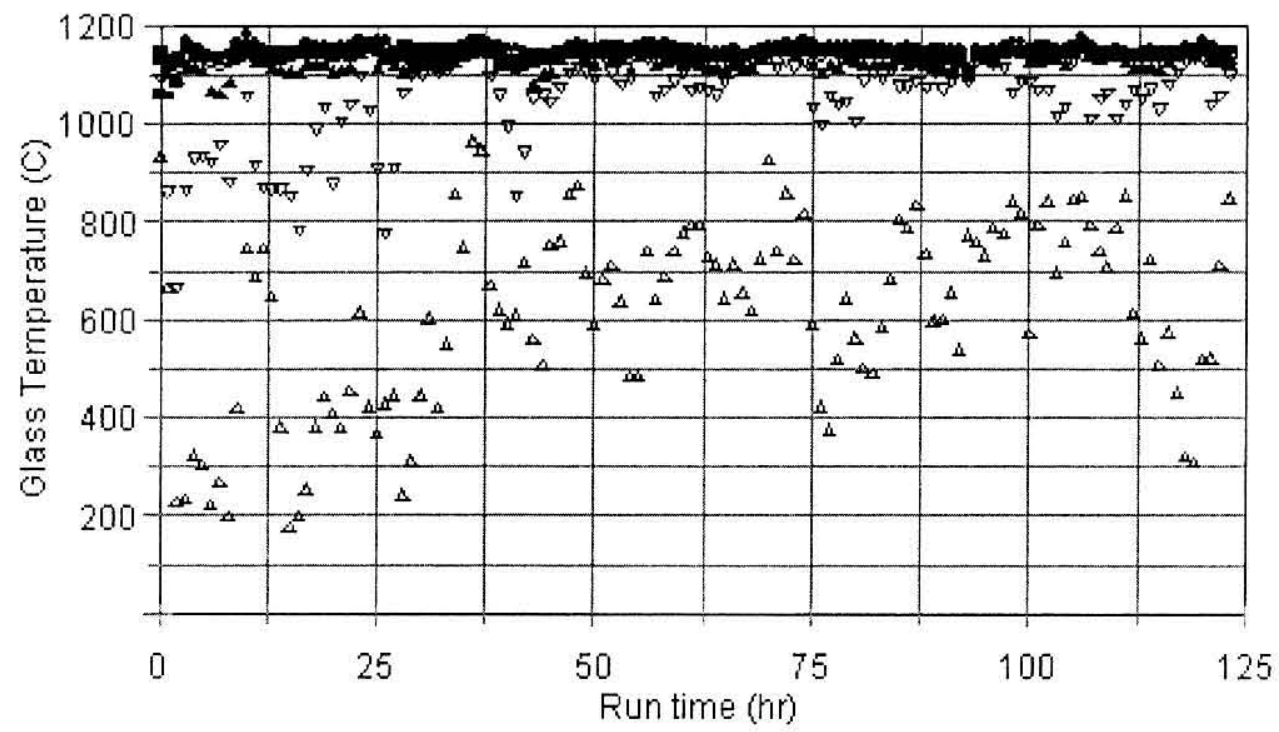

- 1" from floor a $13^{\prime \prime}$ from floor $\quad 18^{\prime \prime}$ from floor $\quad \nabla 27^{\prime \prime}$ from floor

- g" from floor - 18 " from floor $\quad$ * 24 " from floor \& 30 "from floor

Figure 3.13. Glass temperature for DM1200 Test 4 (400 g/l with shallow bubbling). 
The Catholic University of America Vitreous State Laboratory

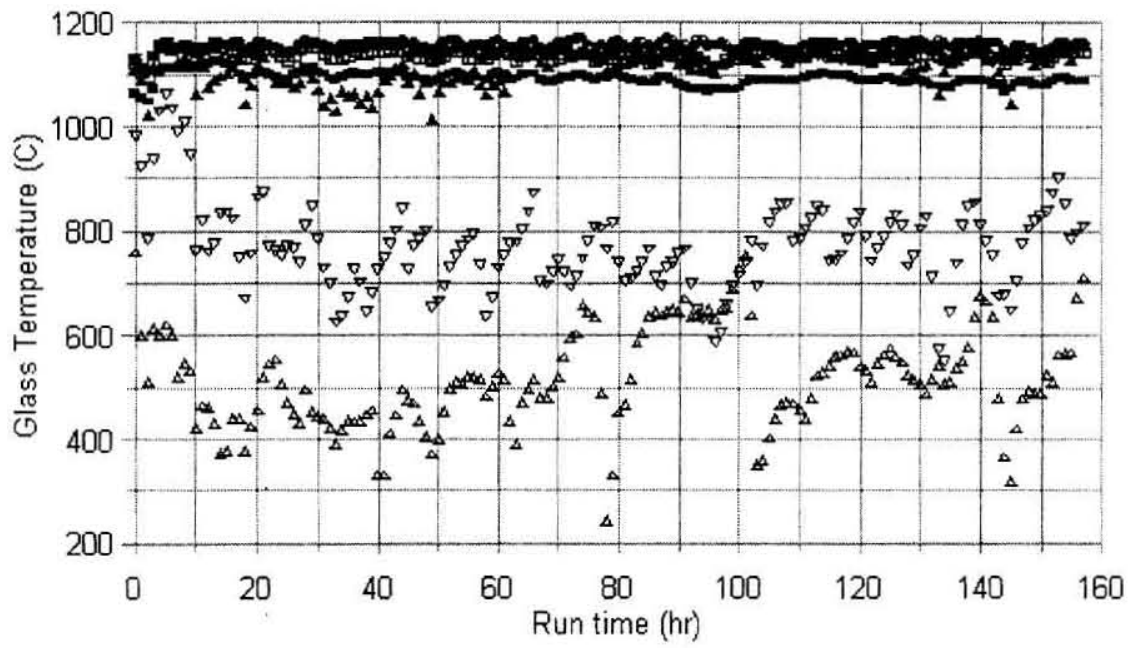
- 1" from floor
- $13^{\prime \prime}$ from floor
- 18 " from floor
$\nabla 27^{\prime \prime}$ from floor
- 9" from floor
- 18 " from floor
- 24 " from floor
- 30" from floor

Figure 3.14. Glass temperature for DM1200 Test 5 (nitrated feed, sugar ratio $=0.5,420 \mathrm{~g} / \mathrm{l}$ with no bubbling.

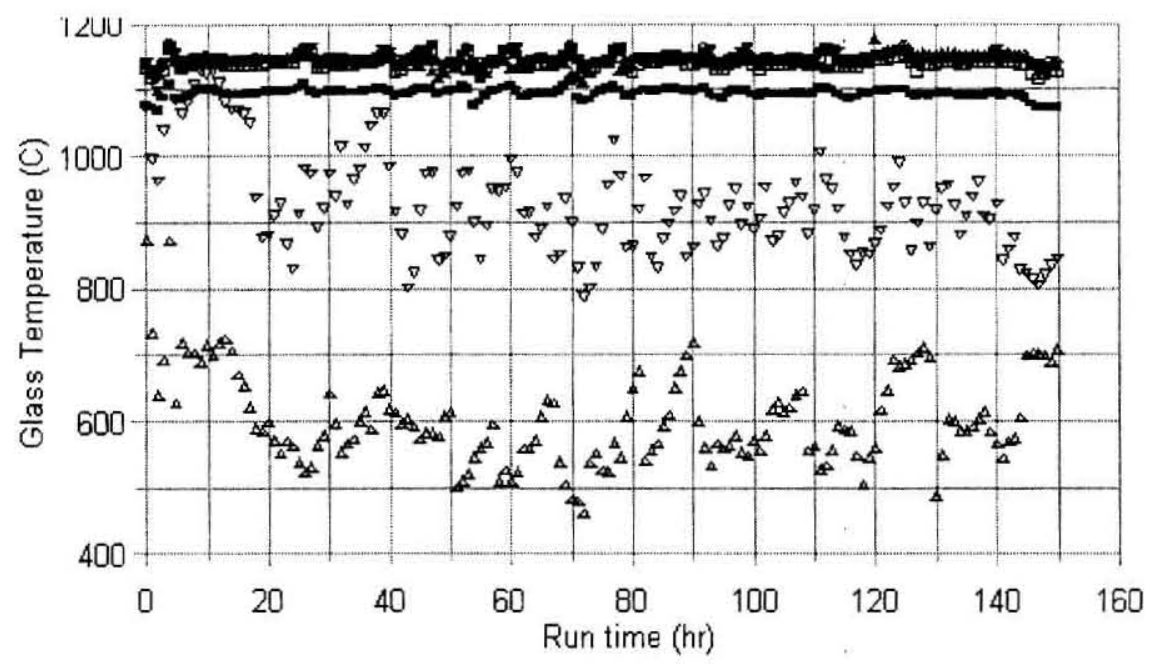
- 1 " from floor
- $13^{\prime \prime}$ from floor
$18^{\prime \prime}$ from floor
- 27" from floor
- 9" from floor
- $18^{\prime \prime}$ from floor
4 24 " from floor
- 30 " from floor

Figure 3.15. Glass temperature for DM1200 Test 6 (frit additives, $7.5 \mathrm{~g}$ sugar/l, 480 g/l with no bubbling). 
The Catholic University of America Vitreous State Laboratory
DuraMelter 1200 Tests with AZ-101HLW Simulants Final Report, VSL-02R0100-2, Rev.I

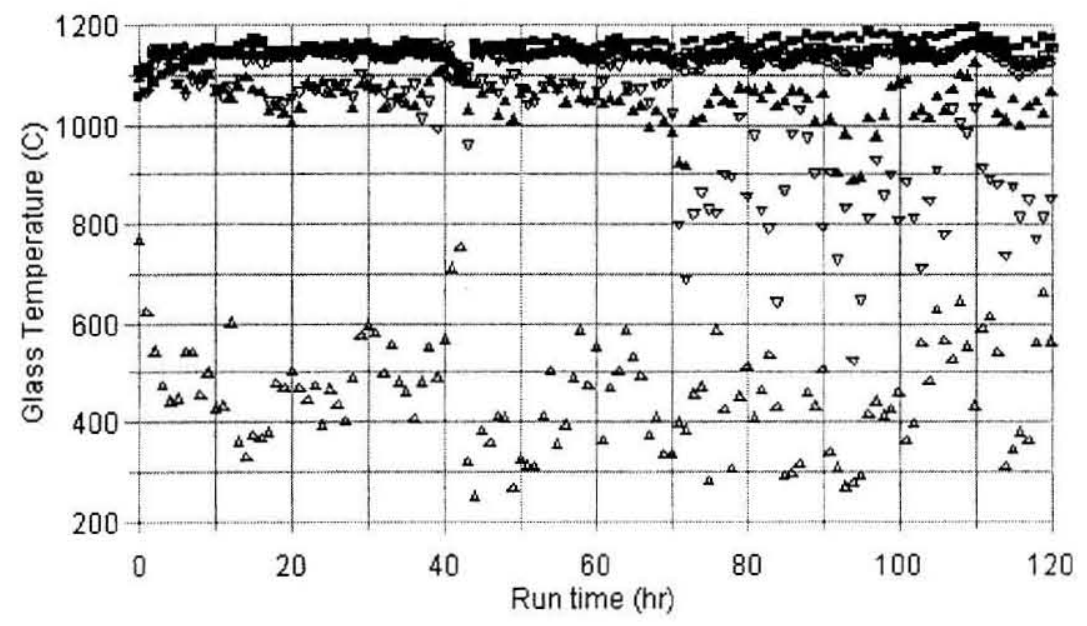

\begin{tabular}{|c|c|c|c|c|}
\hline 1 "from floor & 0 & $13^{\prime \prime}$ from floor & & $18^{\prime \prime}$ from floor \\
\hline$g^{\prime \prime}$ from floor & 。 & $18^{\prime \prime}$ from floor & 2 & 24 " from floor \\
\hline
\end{tabular}

Figure 3.16. Glass temperature for DM1200 Test 7 (frit additives, $7.5 \mathrm{~g}$ sugar/l, $480 \mathrm{~g} / \mathrm{l}$ with bubbling).

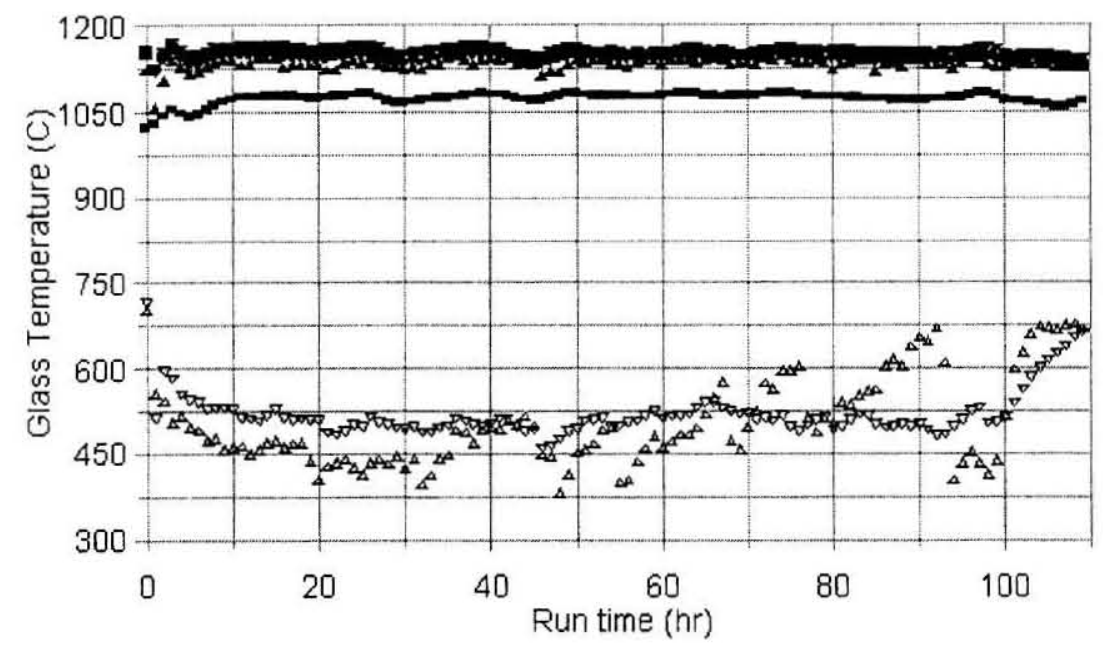
- 1 " from floor
- $13^{\prime \prime}$ from floor
18 "from floor $\quad \nabla \quad 27^{\prime \prime}$ from floor
- 9" from floor
- 18 " from floor
24 " from floor $\triangle 30$ " from floor

Figure 3.17. Glass temperature for DM1200 Test 8 (nitrated feed, sugar ratio $=0.7$, with no bubbling). 
The Catholic University of America Vitreous State Laboratory
DuraMelter 1200 Tests with AZ-101HLW Simulants

Final Report, VSL-02R0100-2, Rev.1

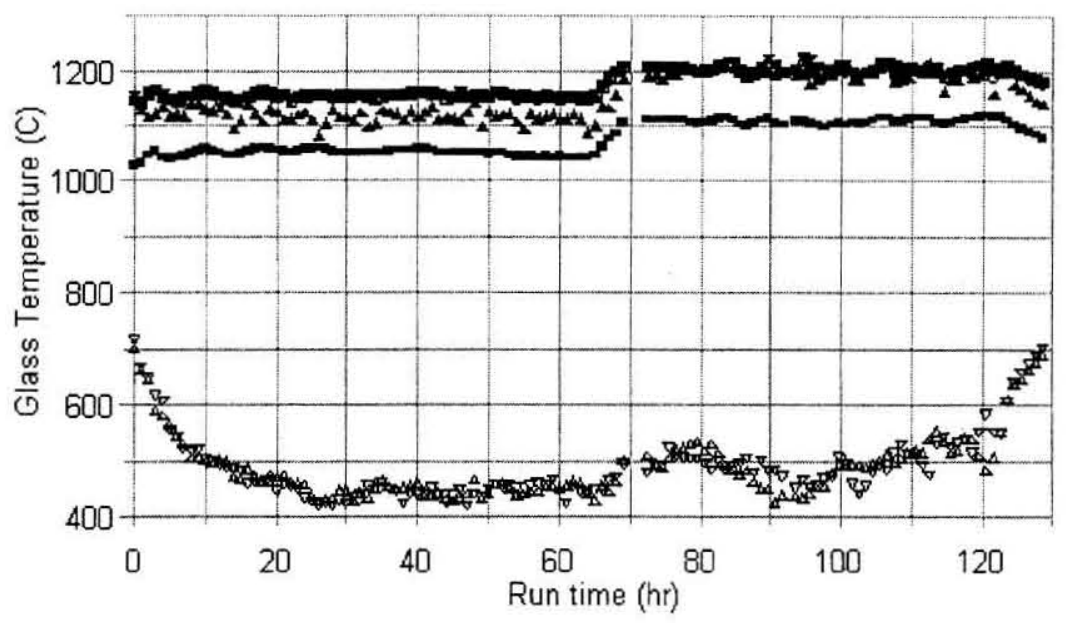

$$
\begin{aligned}
& \text { - } 1 \text { "from floor a } 13^{\prime \prime} \text { from floor } ~-18 \text { "from floor v } 27^{\prime \prime} \text { from floor } \\
& \text { - 9" from floor - } 18^{\prime \prime} \text { from floor - } 24 \text { "from floor a } 30 \text { " from floor }
\end{aligned}
$$

Figure 3.18. Glass temperatures for DM1200 Test $9(570 \mathrm{~g} / \mathrm{l}, 10 \mathrm{~g}$ sugar/l, continuous feeding).

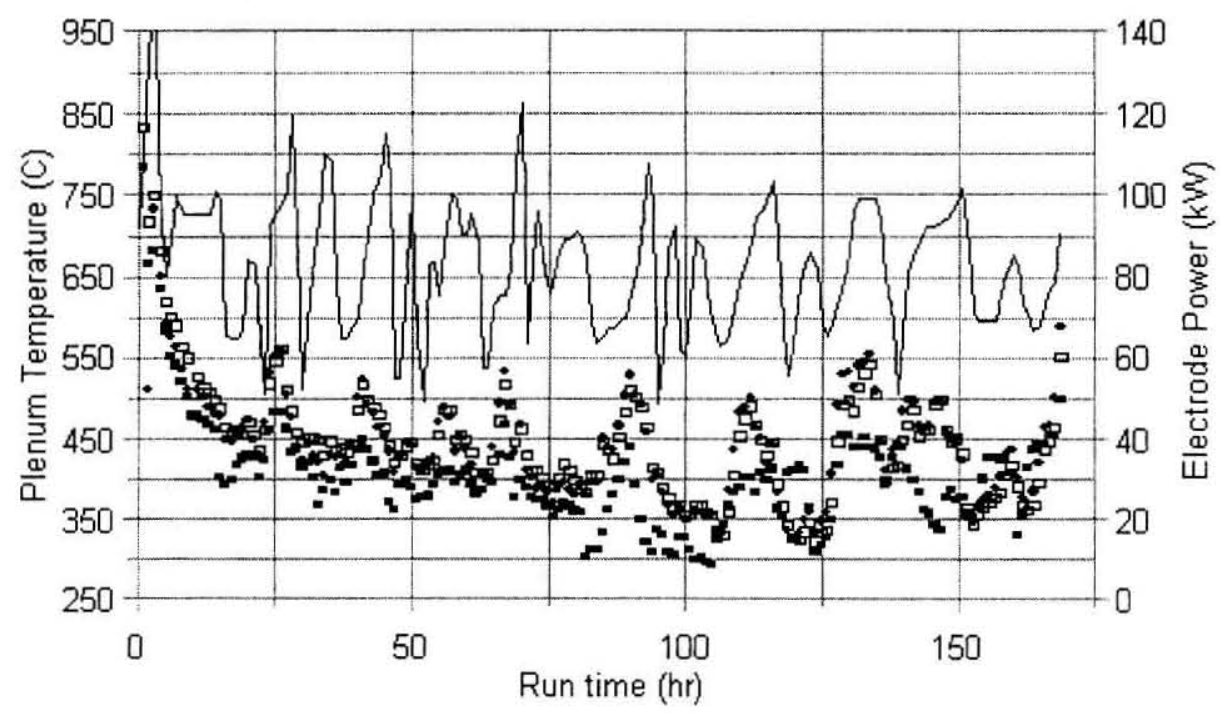

Figure 3.19. Plenum temperatures and electrode power for DM1200 Test 1 (570 g/l with no bubbling). 
The Catholic University of America Vitreous State Laboratory
DuraMelter 1200 Tests with AZ-101HLW Simulants Final Report, VSL-02R0100-2, Rev.I

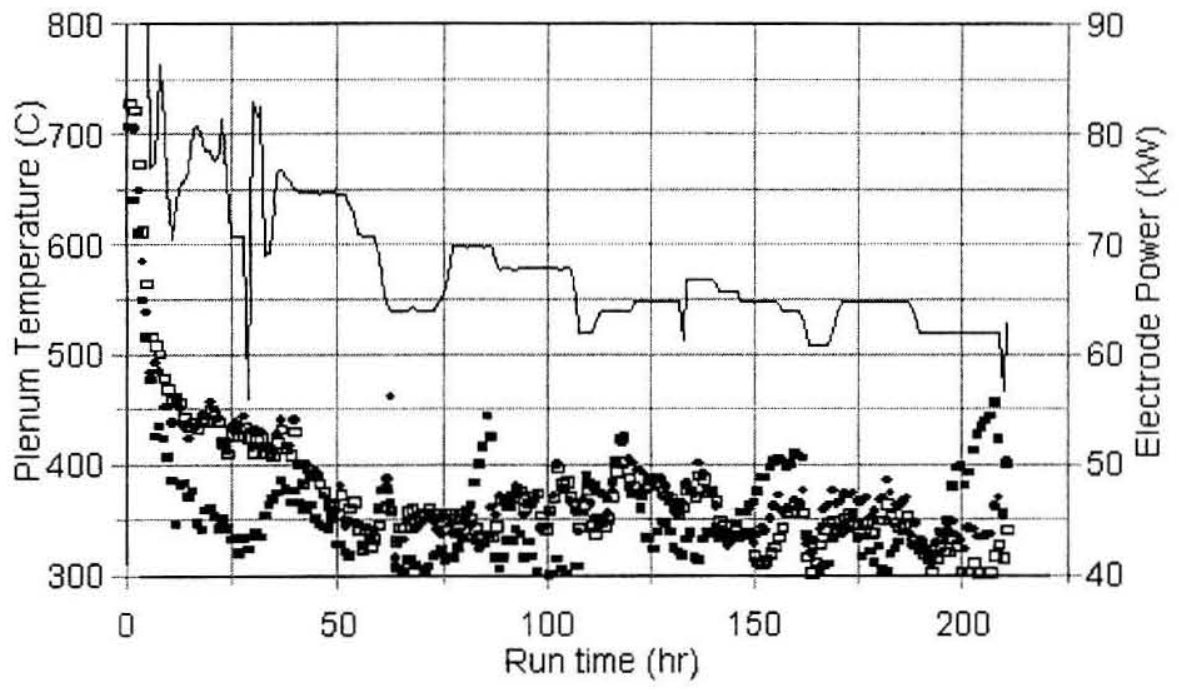

$$
\begin{aligned}
& \text { - 8" below ceiling - } 17^{\prime \prime} \text { below ceiling } \\
& \text { - Exposed } \quad \text { Electrode power }
\end{aligned}
$$

Figure 3.20. Plenum temperatures and electrode power for DM1200 Test $2(570 \mathrm{~g} / \mathrm{l}$ and $10 \mathrm{~g}$ sugar/l with no bubbling).

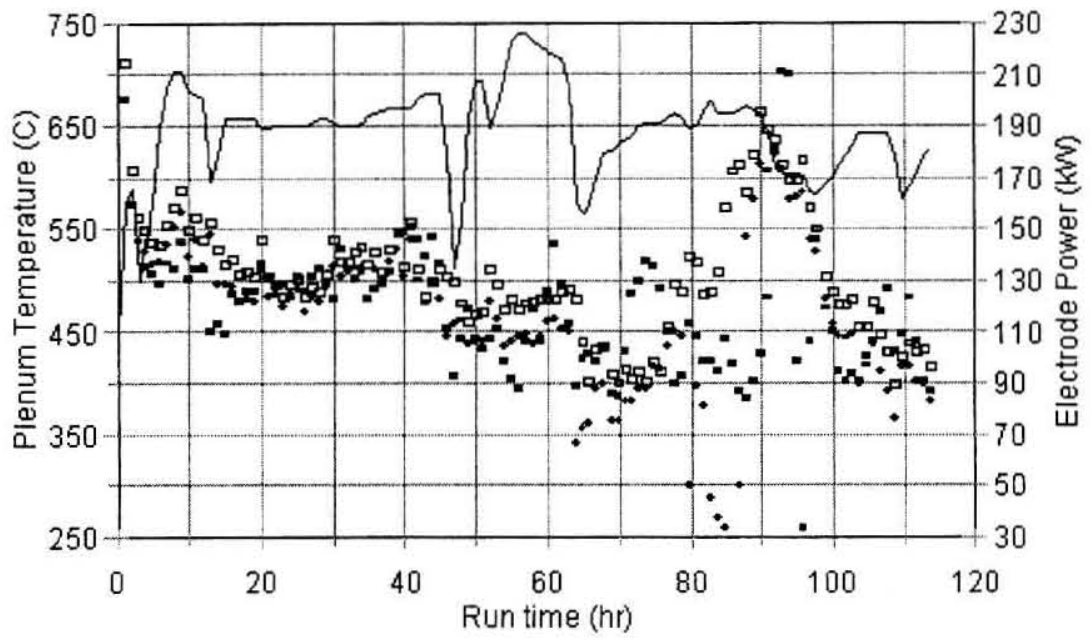

$$
\begin{aligned}
& \text { - } 8^{\prime \prime} \text { below ceiling - } 17^{\prime \prime} \text { below ceiling } \\
& \text { - Exposed - Electrode power }
\end{aligned}
$$

Figure 3.21. Plenum temperatures and electrode power for DM1200 Test 3 (400 g/l with deep bubbling). 
The Catholic University of America Vitreous State Laboratory
DuraMelter 1200 Tests with AZ-101HLW Simulants

Final Report, VSL-02R0100-2, Rev.1

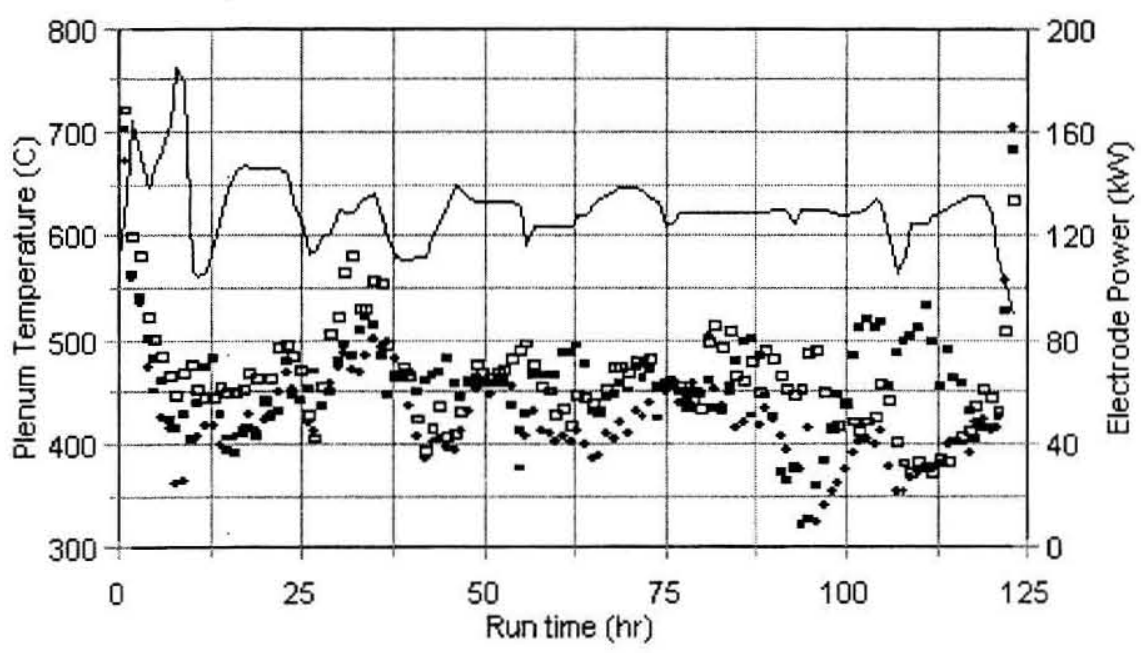
- 8 " below ceiling
- $17^{\prime \prime}$ below ceiling
- Exposed
Electrode power

Figure 3.22. Plenum temperatures and electrode power for DM1200 Test 4 (400 g/l with shallow bubbling).

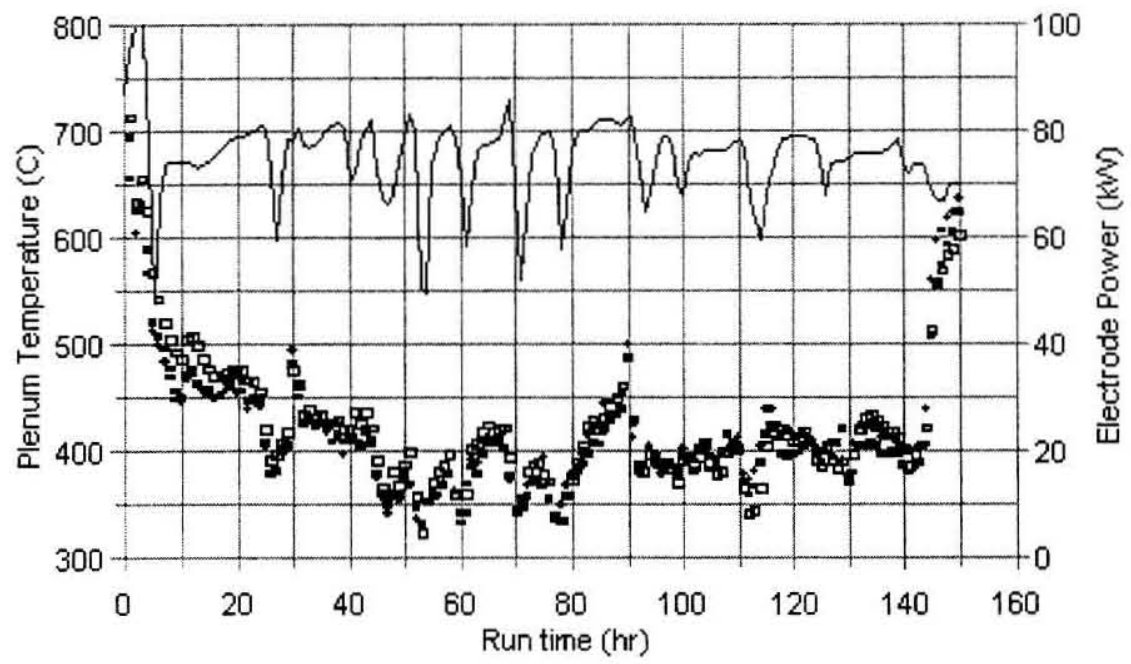

- 8 " below ceiling - $17^{\text {" below ceiling }}$

- Exposed Electrode power

Figure 3.23. Plenum temperatures and electrode power for DM1200 Test 5 (nitrated feed, sugar ratio $=0.5,420 \mathrm{~g} / \mathrm{l}$ with no bubbling). 
The Catholic University of America

Vitreous State Laboratory
DuraMelter 1200 Tests with AZ-101HLW Simulants Final Report, VSL-02R0100-2, Rev.I

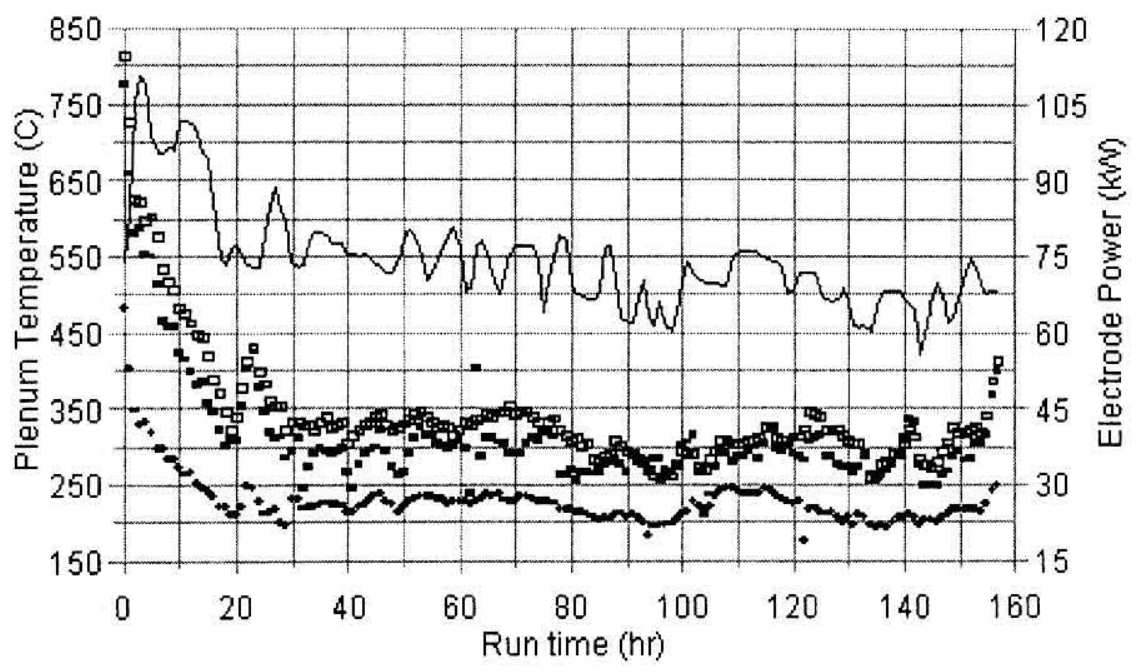

$$
\begin{aligned}
& \text { - 8" below ceiling - } 17^{\prime \prime} \text { below ceiling } \\
& \text { - Exposed } \quad \text { Electrode power }
\end{aligned}
$$

Figure 3.24. Plenum temperatures and electrode power for DM1200 Test 6 (frit additives, $7.5 \mathrm{~g}$ sugar/l, $480 \mathrm{~g} / \mathrm{l}$ with no bubbling).

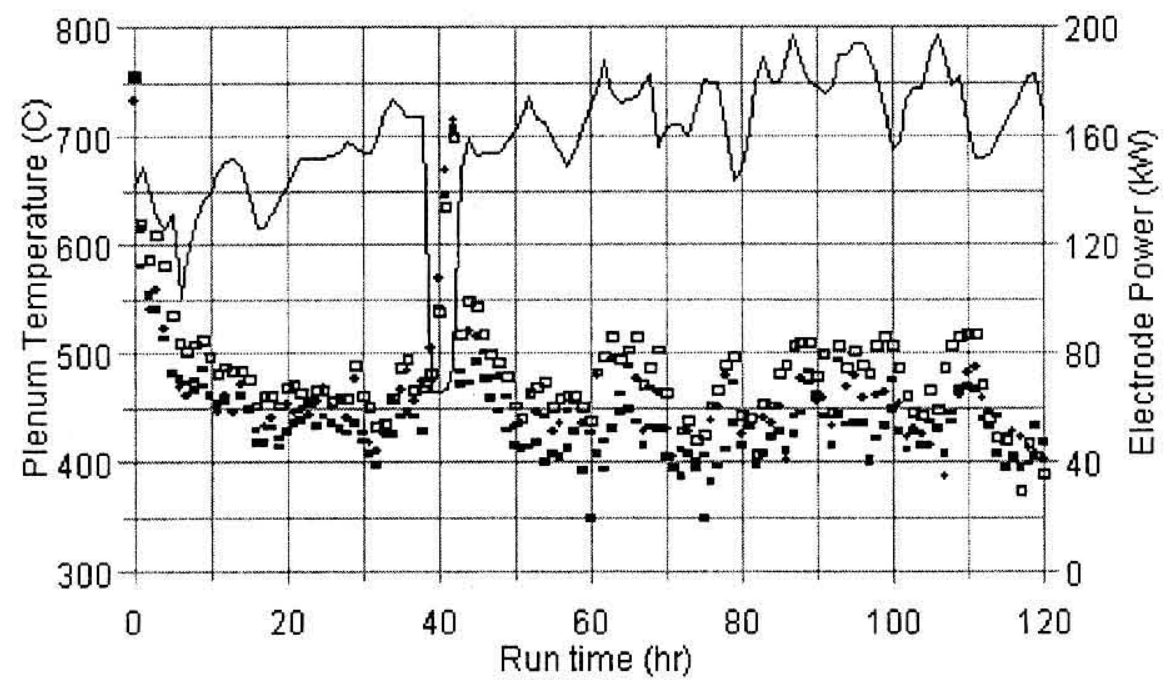

$$
\begin{aligned}
& \text { - 8" below ceiling - } 17^{\prime \prime} \text { below ceiling } \\
& \text { - Exposed - Electrode power }
\end{aligned}
$$

Figure 3.25. Plenum temperatures and electrode power for DM1200 Test 7 (frit additives, $7.5 \mathrm{~g}$ sugar/l, $480 \mathrm{~g} / \mathrm{I}$ with bubbling). 
The Catholic University of America Vitreous State Laboratory
DuraMelter 1200 Tests with AZ-101HLW Simulants Final Report, VSL-02R0100-2, Rev.1

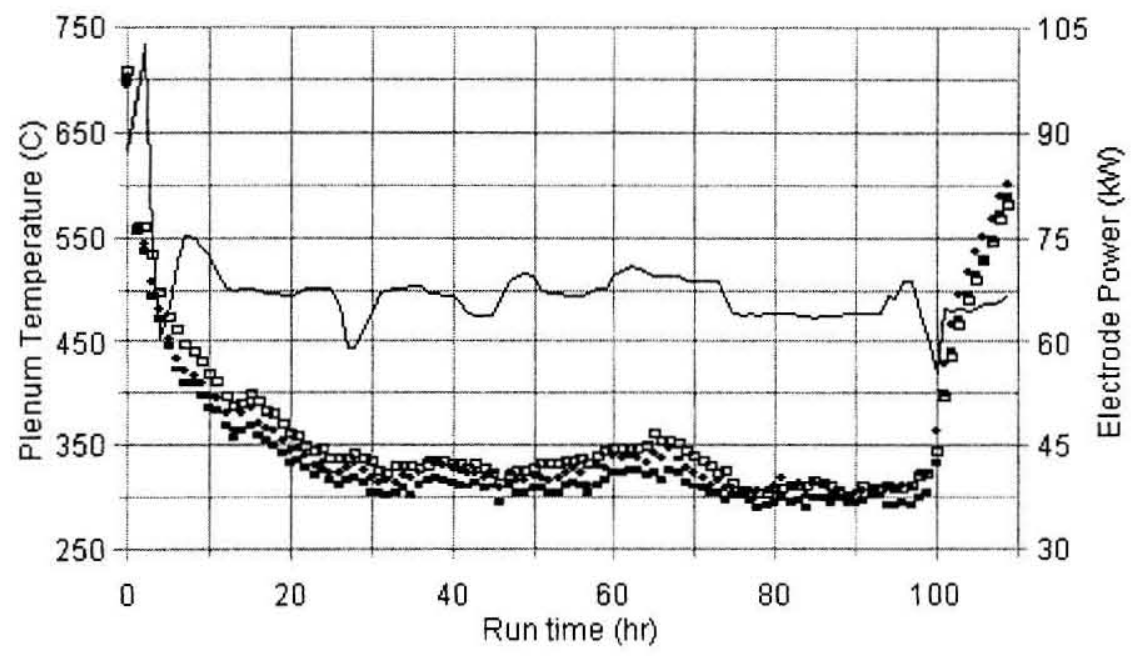

$$
\begin{aligned}
& \text { - } 8 \text { " below ceiling - } 17^{\prime \prime} \text { below ceiling } \\
& \text { - Exposed } \quad-\text { Electrode power }
\end{aligned}
$$

Figure 3.26. Plenum temperatures and electrode power for DM1200 Test 8 (nitrated feed, sugar ratio $=0.7$, with no bubbling).

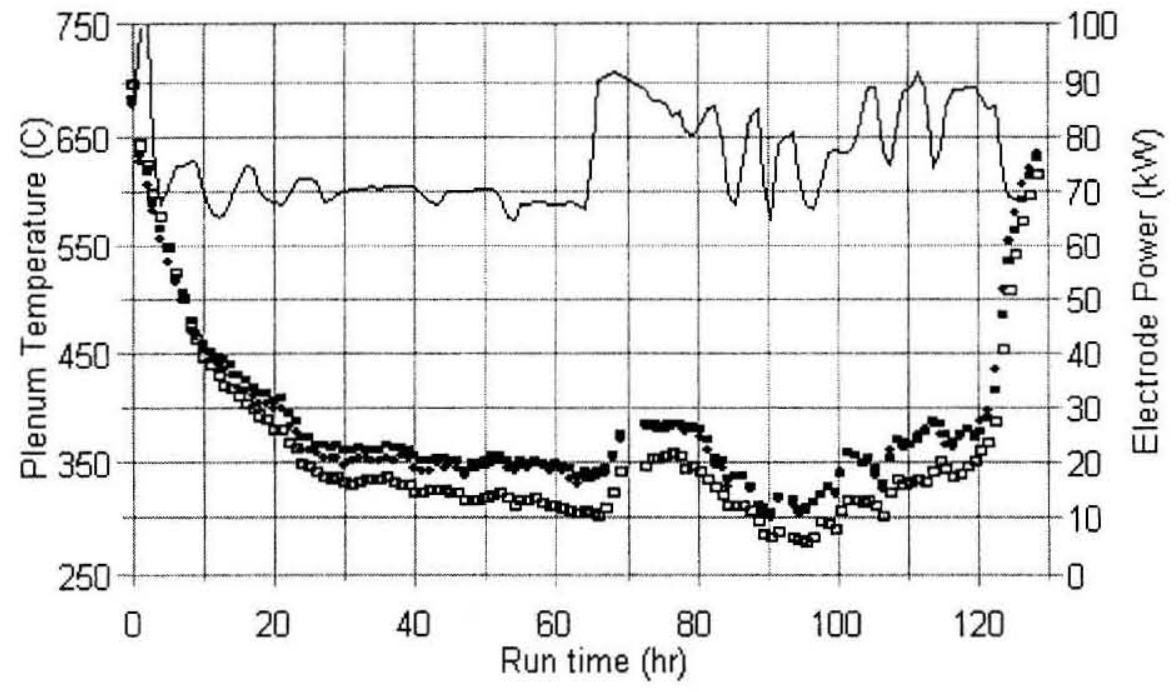

$$
\begin{aligned}
& \text { - 8" below ceiling - } 17^{\prime \prime} \text { below ceiling } \\
& \text { - Exposed - Electrode power }
\end{aligned}
$$

Figure 3.27. Plenum temperatures and electrode power DM1200 Test $9(570 \mathrm{~g} / \mathrm{l}$, $10 \mathrm{~g}$ sugar/l, continuous feeding). 
The Catholic University of America

Vitreous State Laboratory
DuraMelter 1200 Tests with AZ-101HLW Simulants Final Report, VSL-02R0100-2, Rev.1

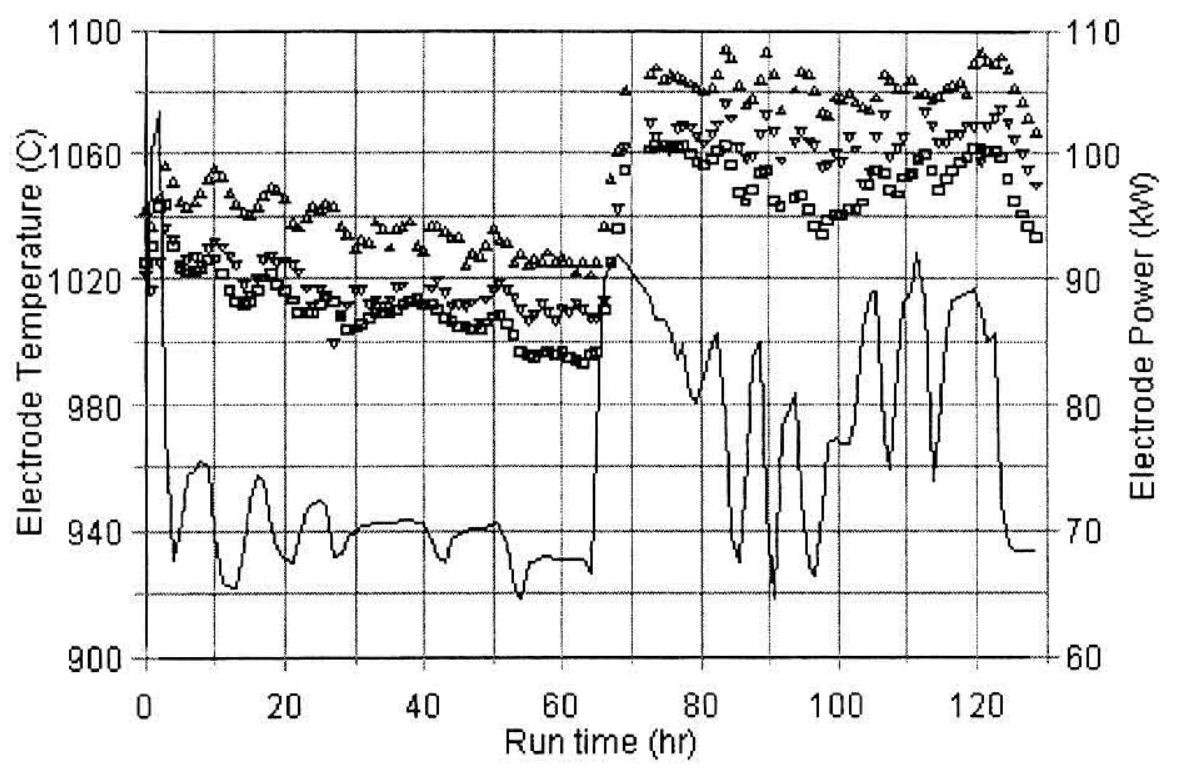

$\triangle$ East $\backsim$ West a Bottom- Power

Figure 3.28. Electrode temperatures and power for DM1200 Test 9. 
The Catholic University of America Vitreous State Laboratory
ORP-51438, Rev. 0

DuraMelter 1200 Tests with AZ-101HLW Simulants Final Report, VSL-02R0100-2, Rev.1

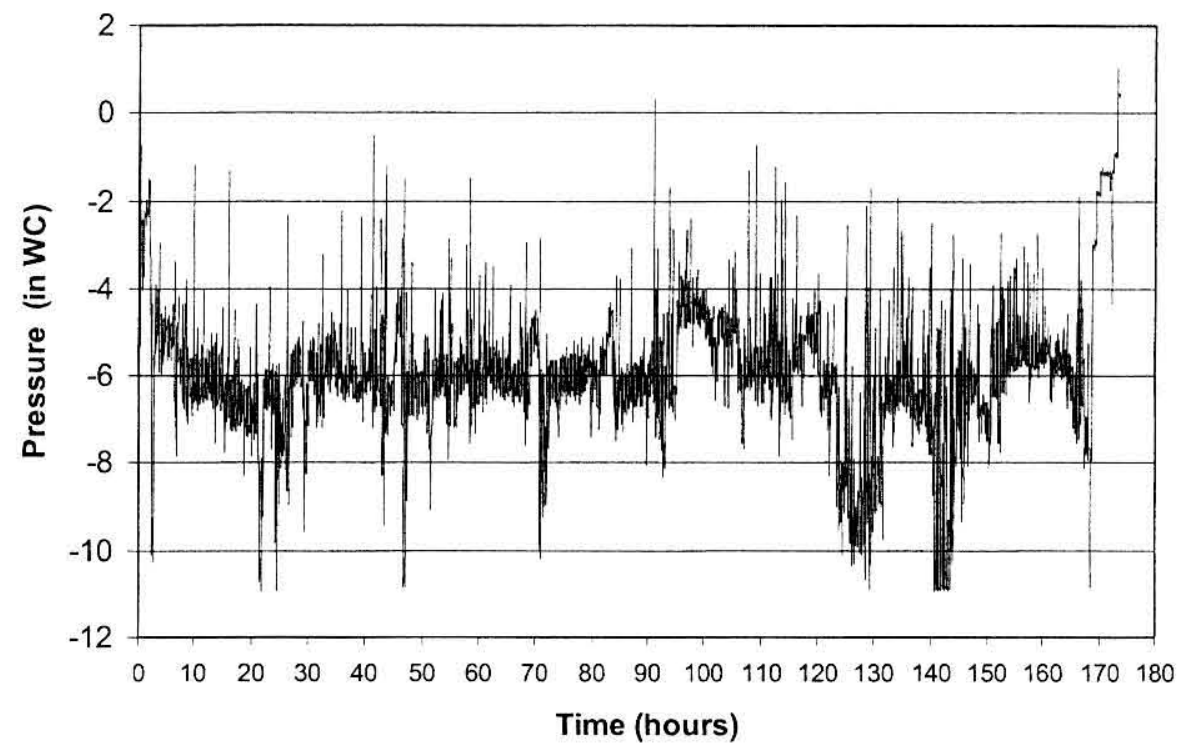

Figure 4.1. Melter pressure during Test 1 , as logged at 2 minute intervals.

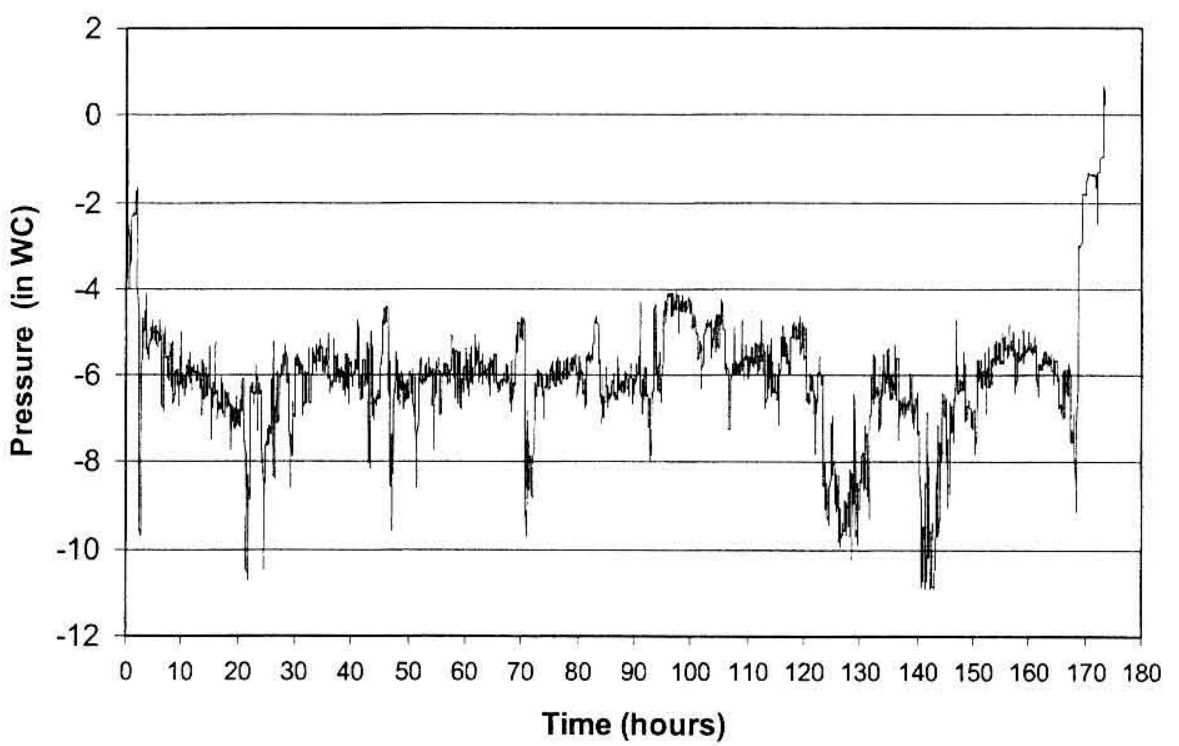

Figure 4.2. Melter pressure during Test 1 , smoothed by averaging over 5 measurements. 
The Catholic University of America Vitreous State Laboratory
DuraMelter 1200 Tests with AZ-101HLW Simulants

Final Report, VSL-02R0100-2, Rev.1

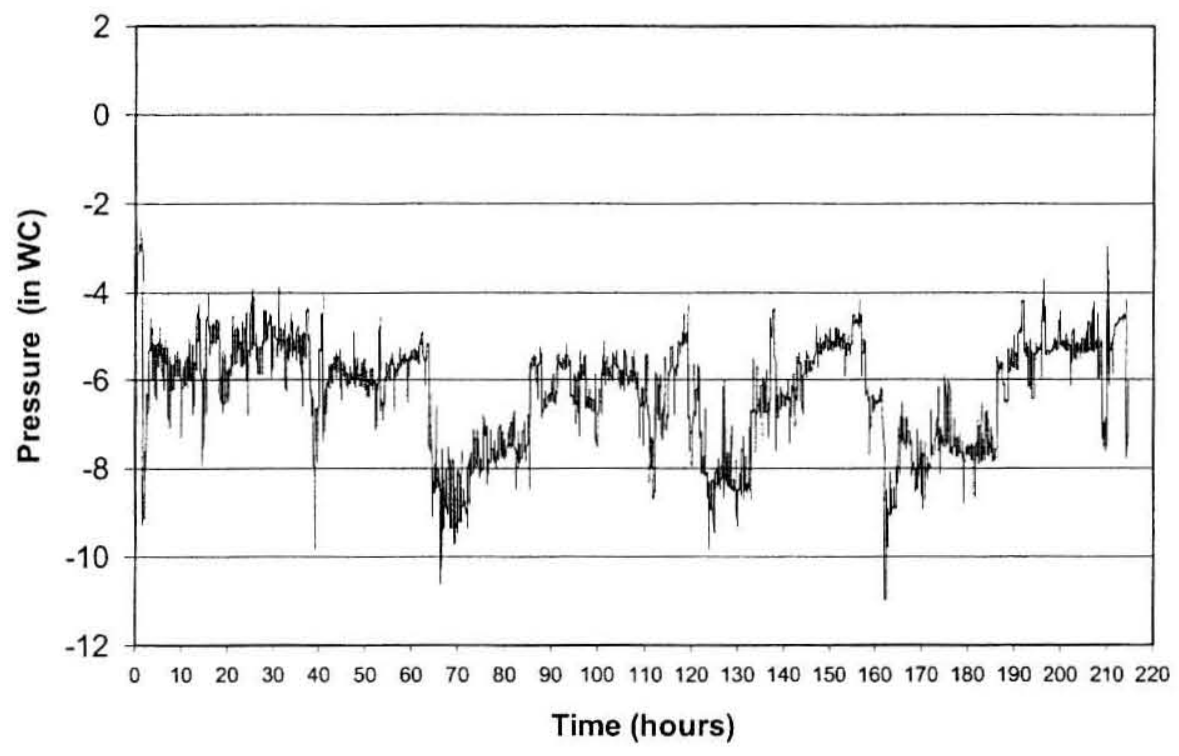

Figure 4.3. Ten-minute time-averaged melter pressure during Test 2 .

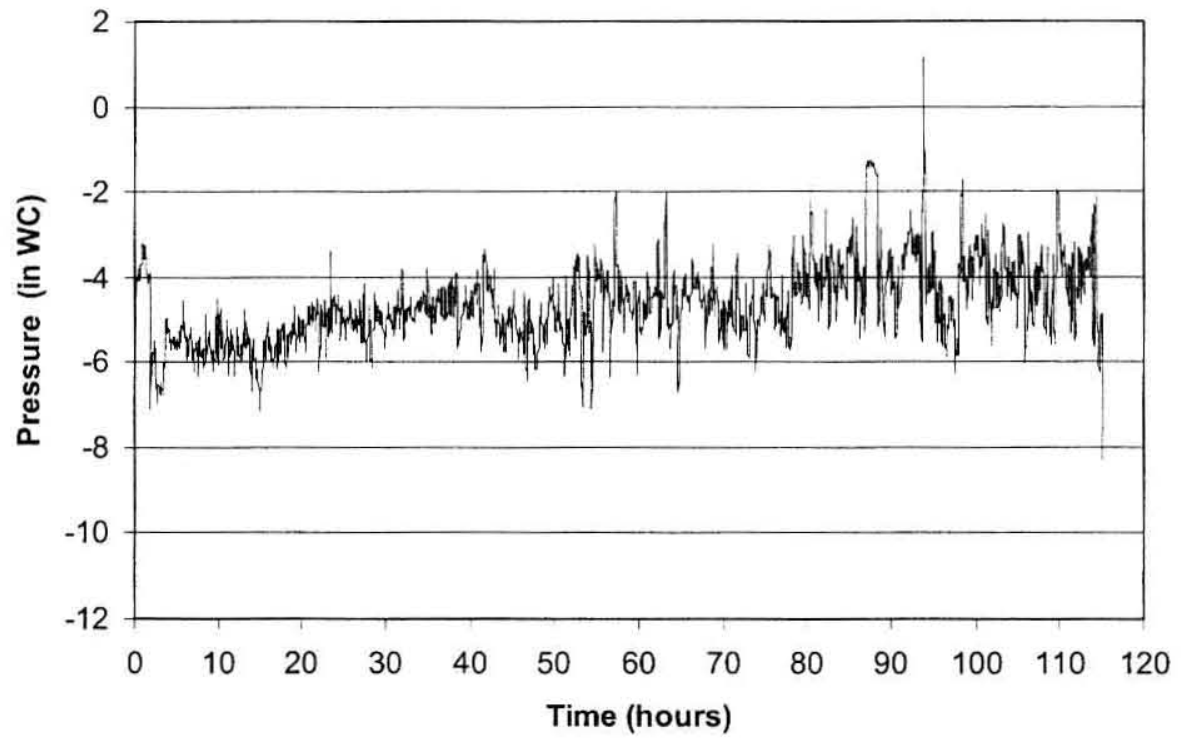

Figure 4.4. Ten-minute time-averaged melter pressure during Test 3. 
The Catholic University of America Vitreous State Laboratory
DuraMelter 1200 Tests with AZ-101HLW Simulants

Final Report, VSL-02R0100-2, Rev.1

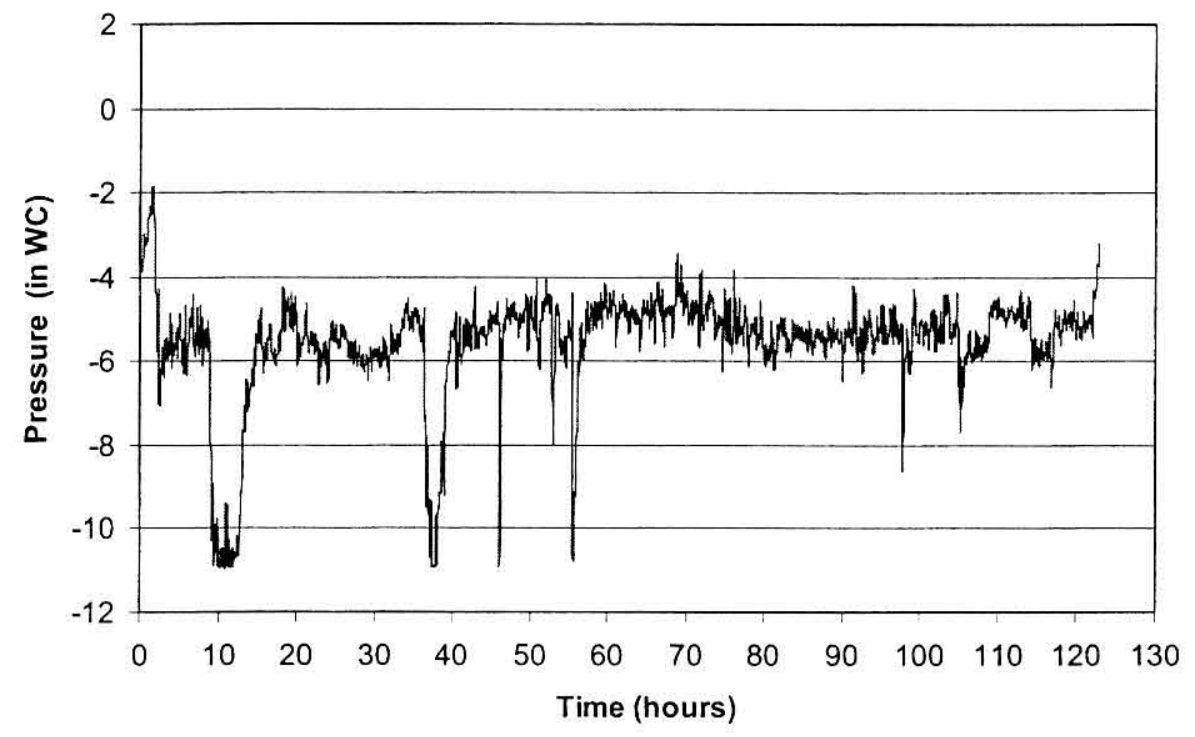

Figure 4.5. Ten-minute time-averaged melter pressure during Test 4 .

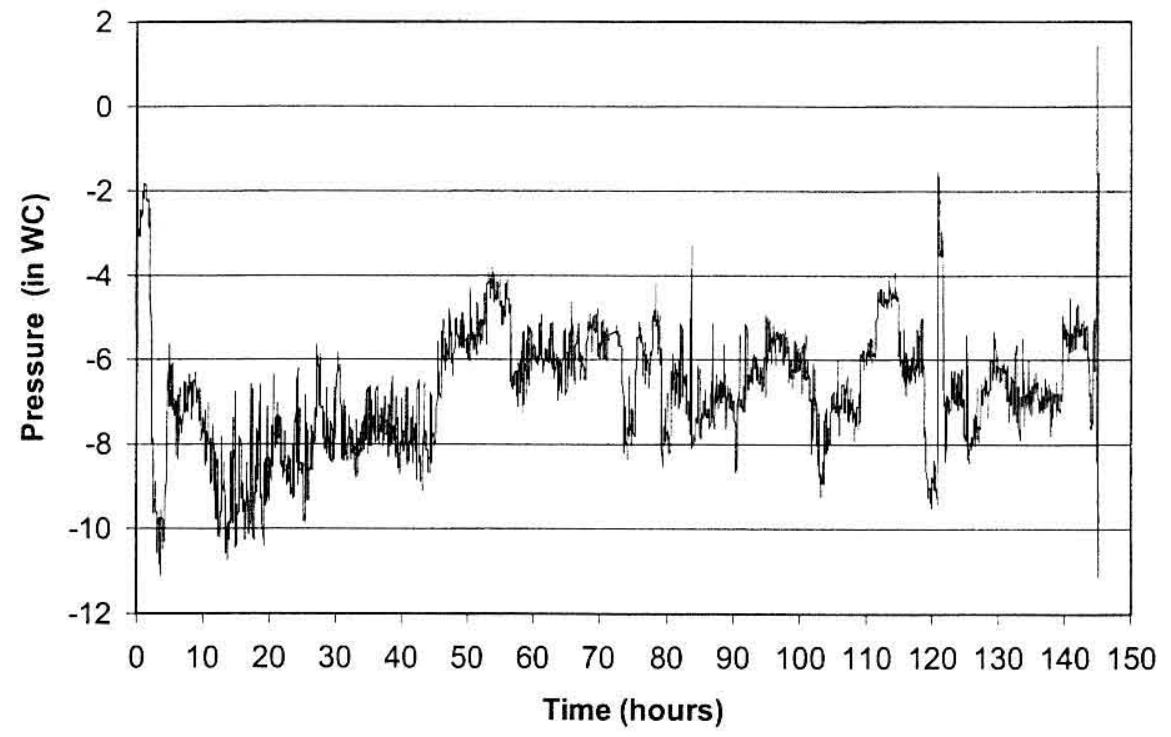

Figure 4.6. Ten-minute time-averaged melter pressure during Test 5. 
The Catholic University of America Vitreous State Laboratory
DuraMelter 1200 Tests with AZ-101HLW Simulants Final Report, VSL-02R0100-2, Rev.1

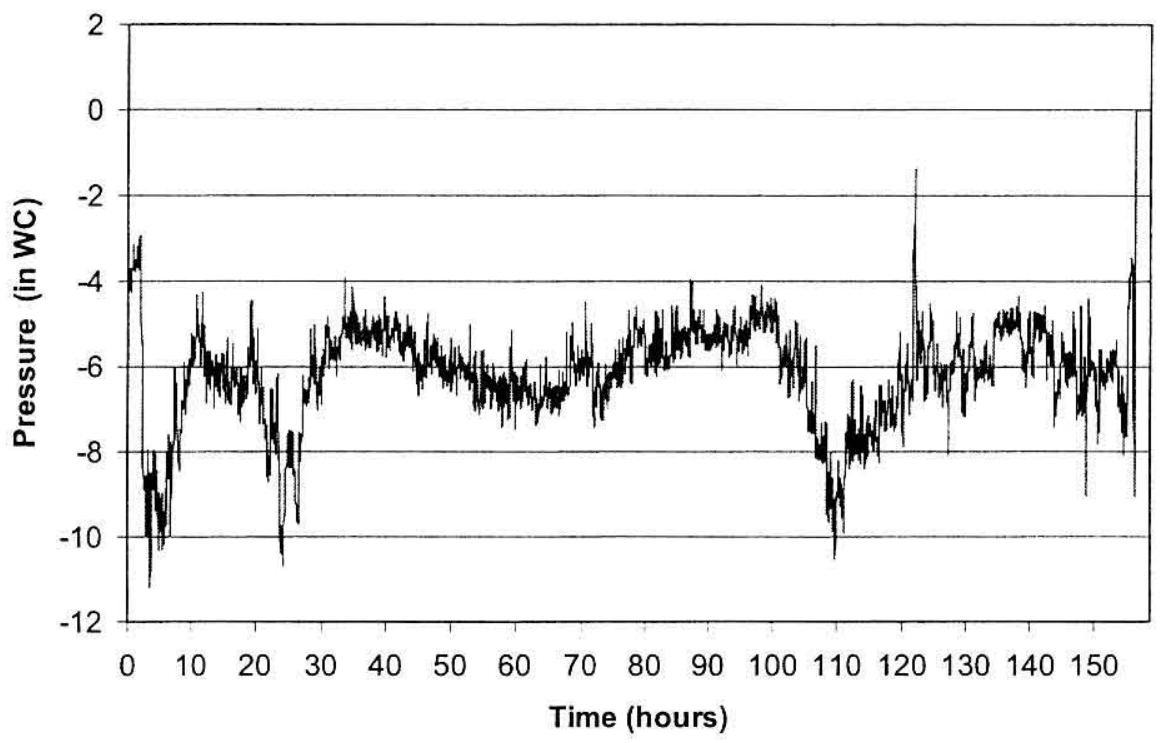

Figure 4.7. Ten-minute time-averaged melter pressure during Test 6.

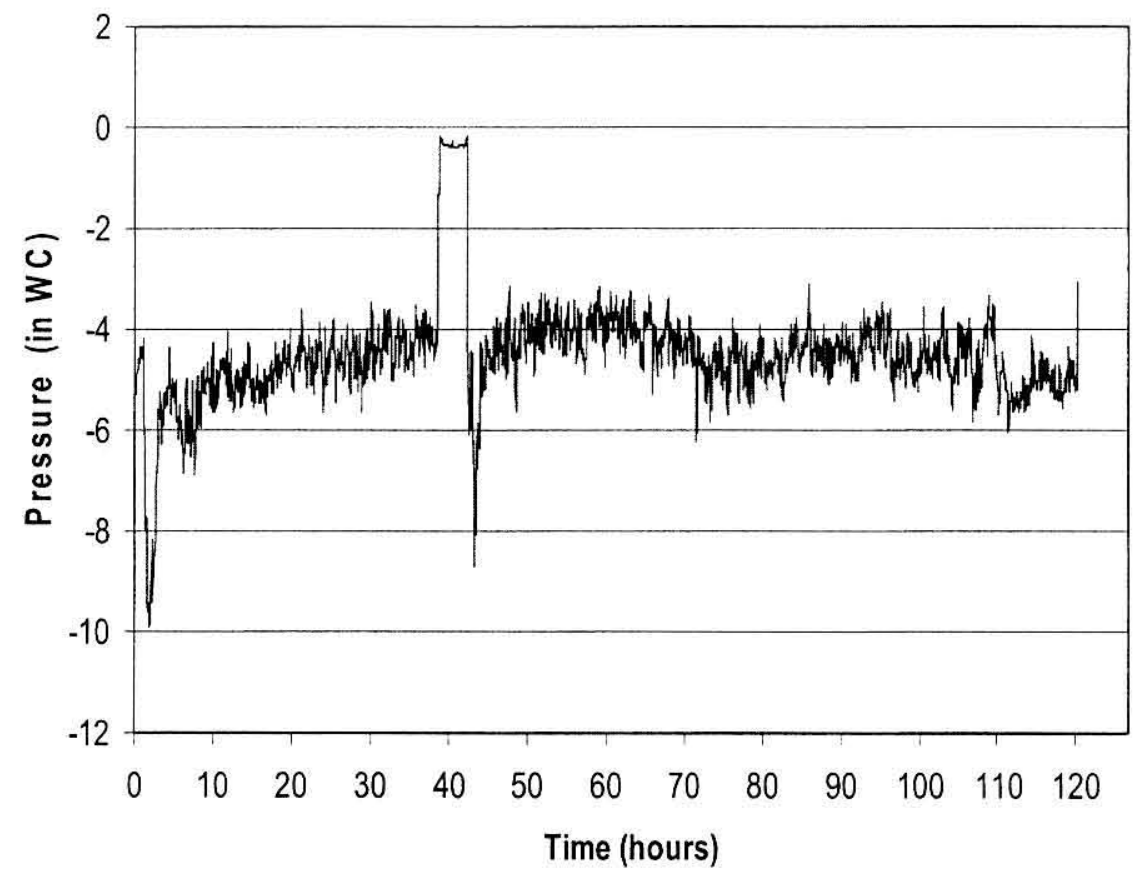

Figure 4.8. Ten-minute time-averaged melter pressure during Test 7 . 


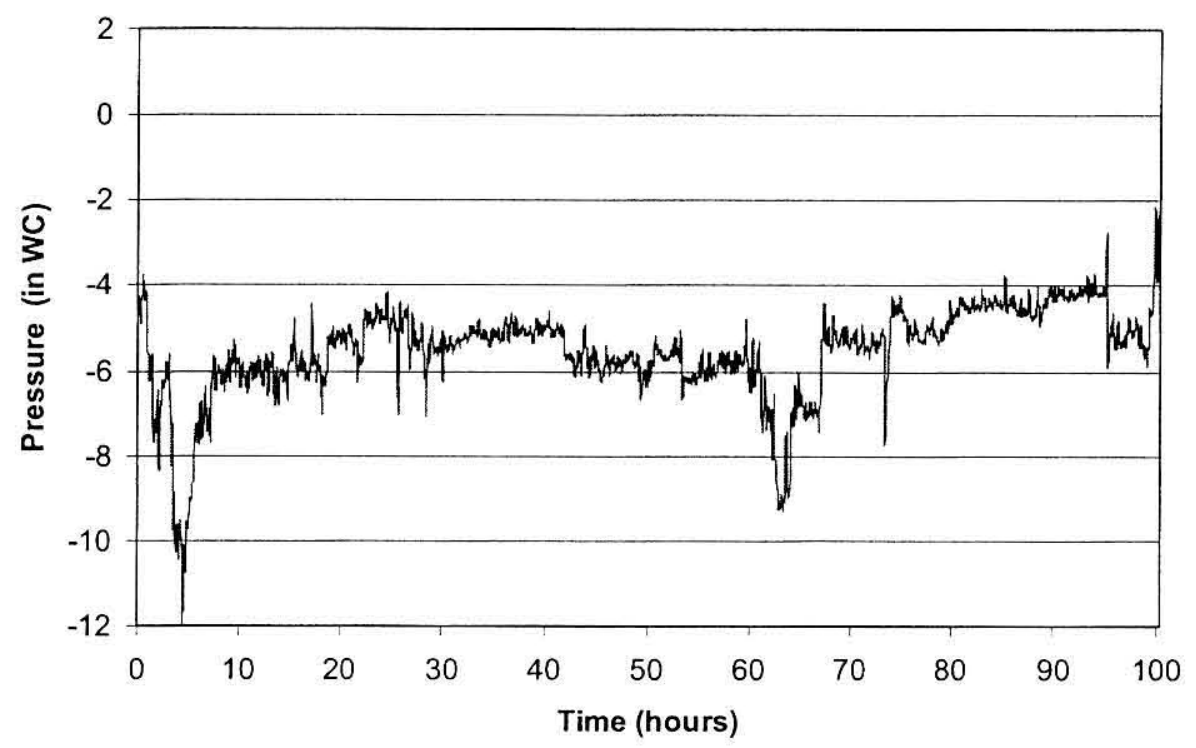

Figure 4.9. Ten-minute time-averaged melter pressure during Test 8.

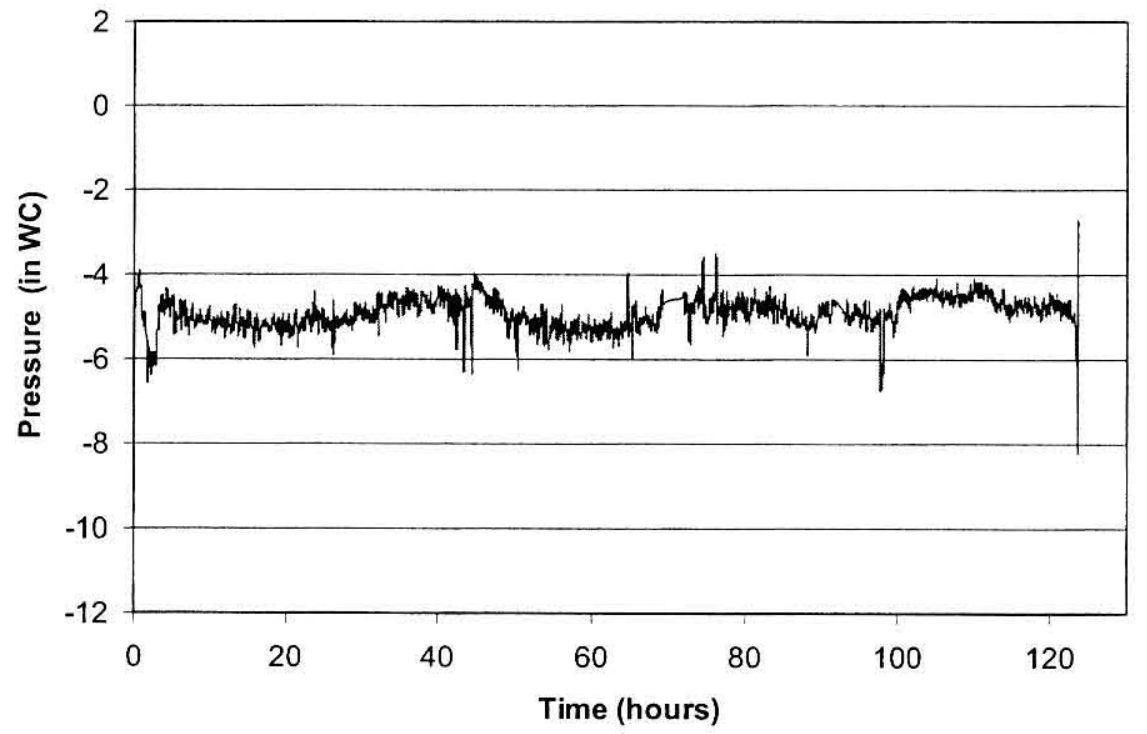

Figure 4.10. Ten-minute time-averaged melter pressure during Test 9. 


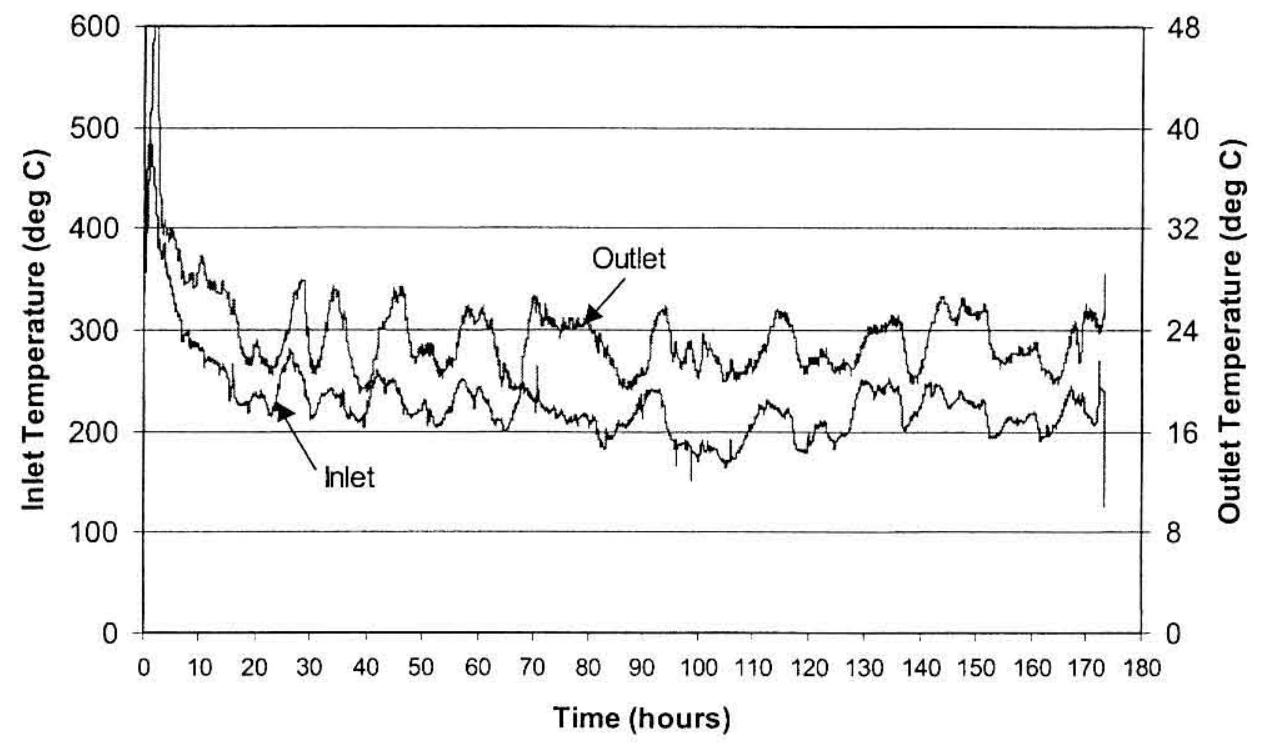

Figure 4.11. SBS gas inlet and outlet temperatures during Test 1.

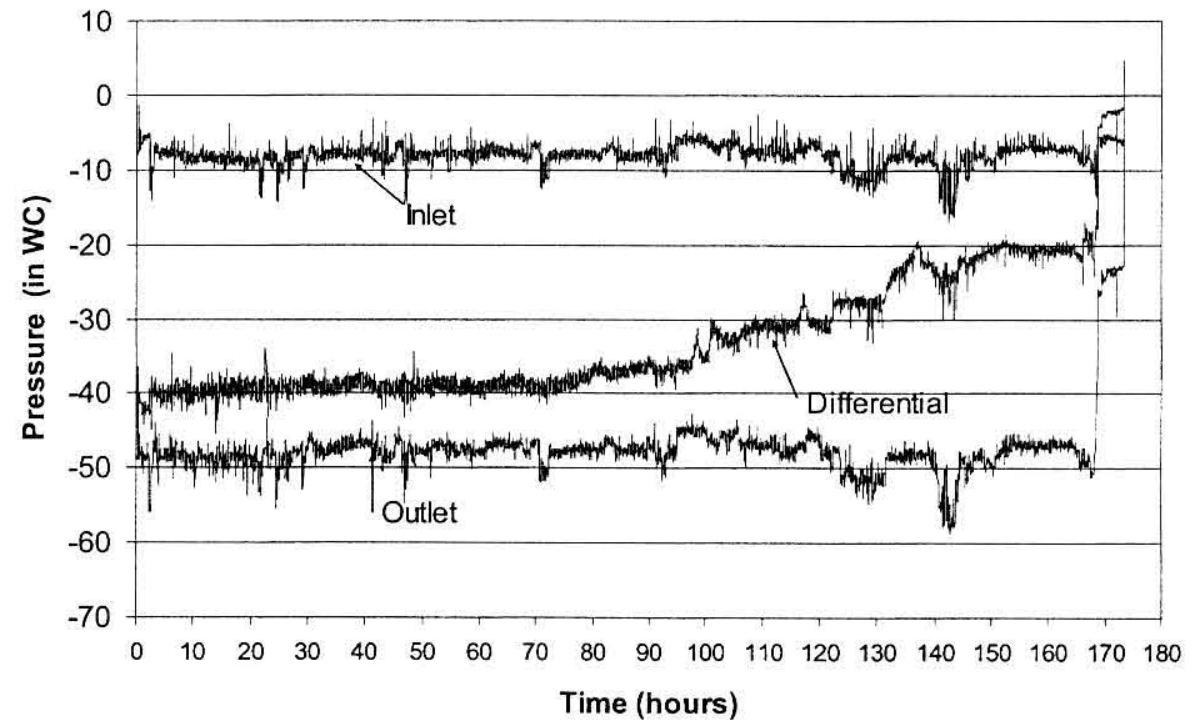

Figure 4.12. SBS inlet, outlet and differential pressures during Test 1 (note that differential pressure sensor clogged after 100 hours). 
The Catholic University of America

Vitreous State Laboratory
DuraMelter 1200 Tests with AZ-101HLW Simulants Final Report, VSL-02R0100-2, Rev.1

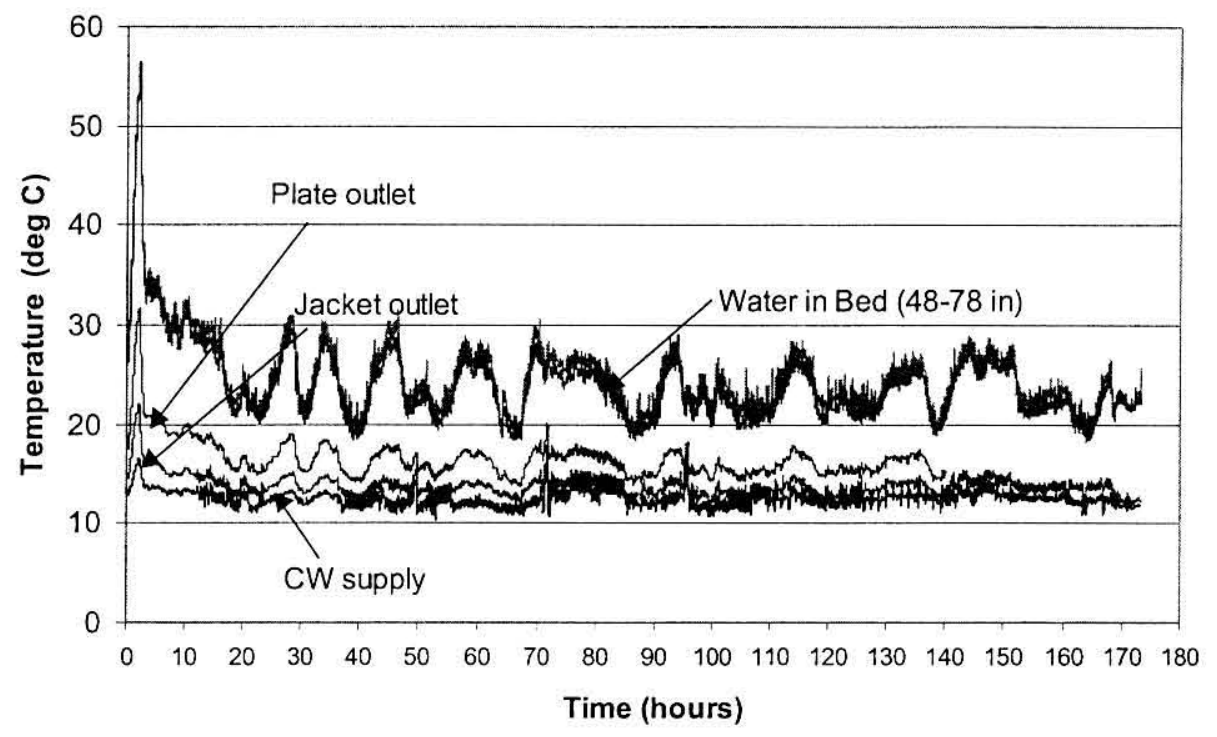

Figure 4.13. SBS cooling water and bed temperatures for Test 1 (cooling water data averaged over 10 minutes ( 5 measurements)).

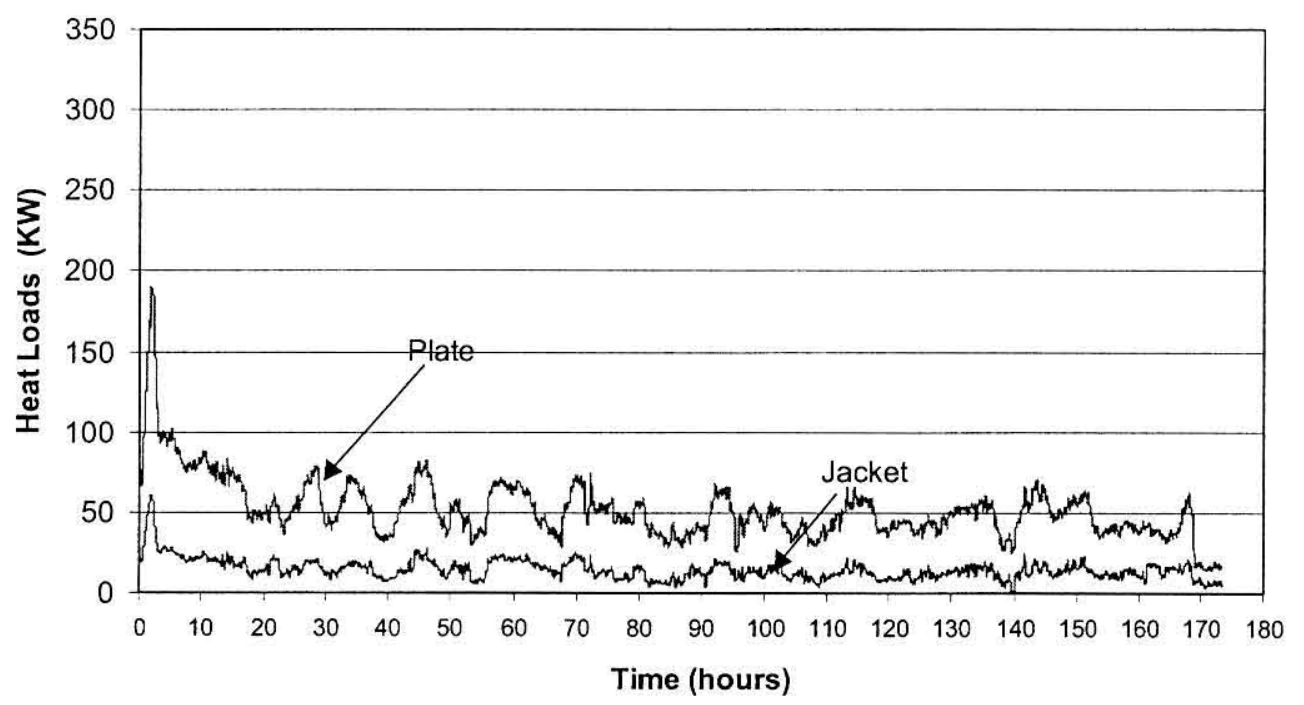

Figure 4.14. Calculated heat loads on the cooling water jacket and plate heat exchanger for Test 1. 


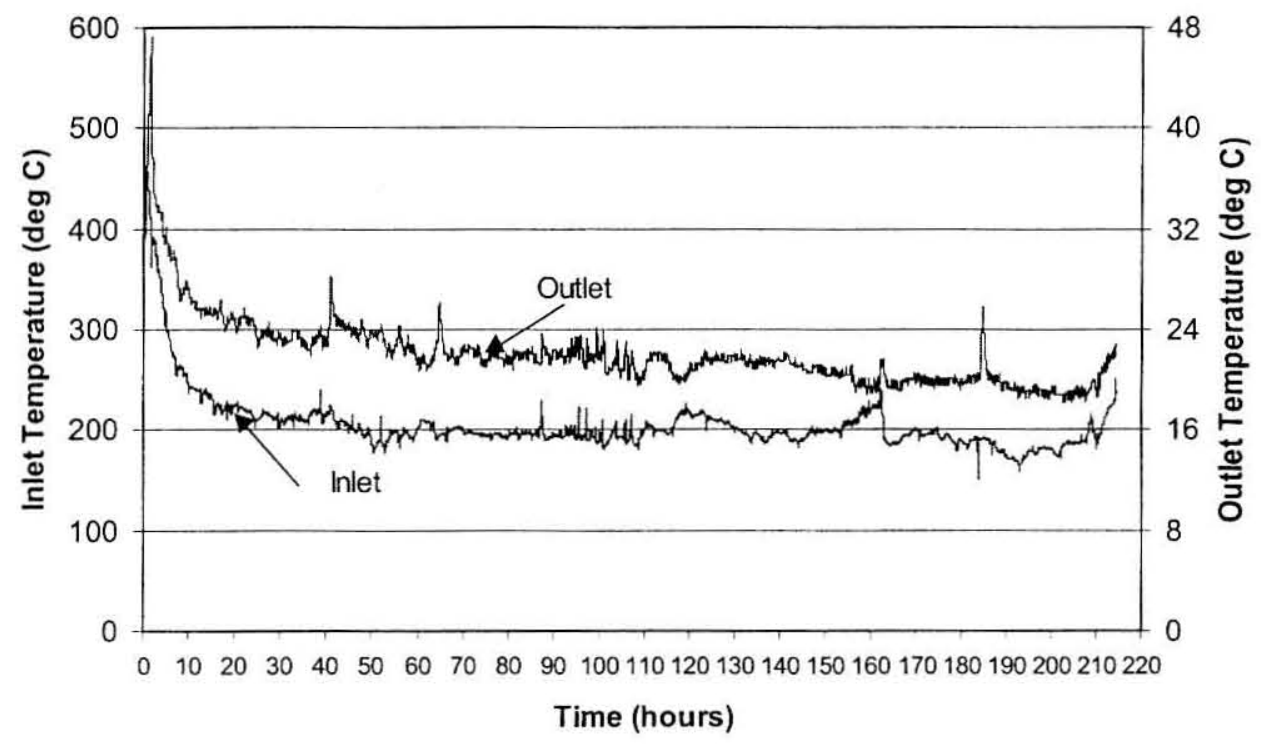

Figure 4.15. SBS gas inlet and outlet temperatures during Test 2.

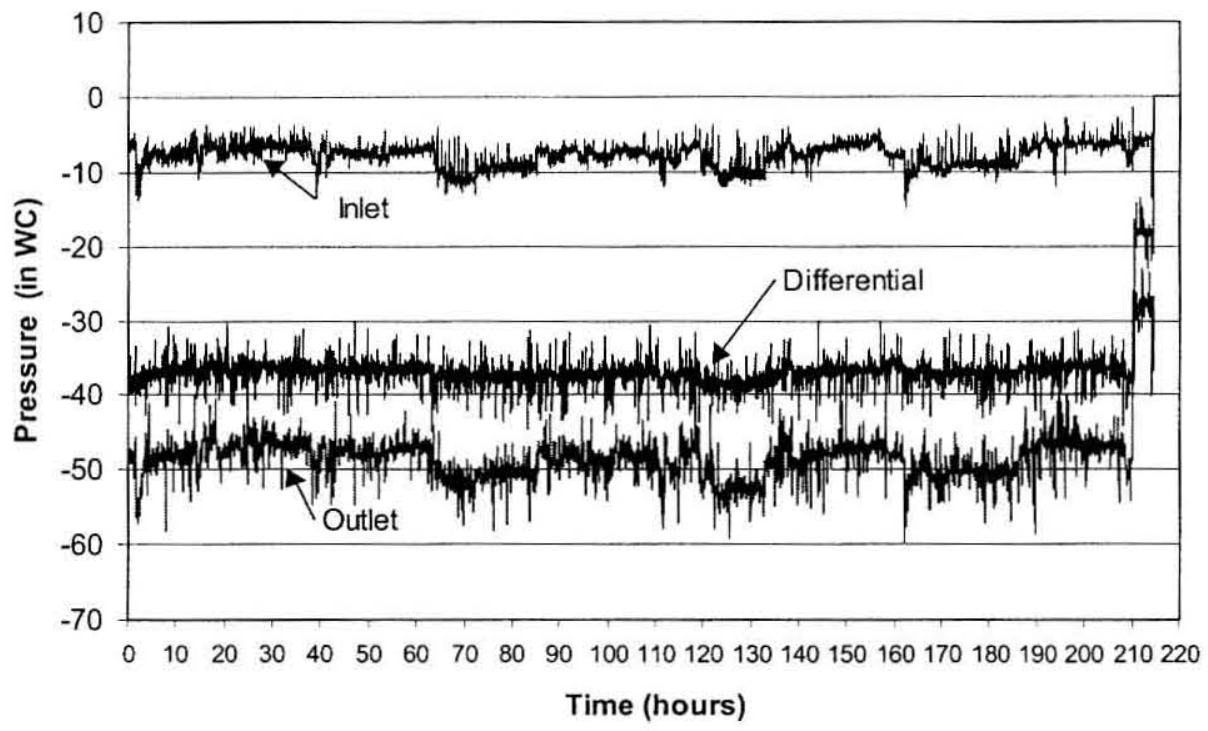

Figure 4.16. SBS inlet, outlet and differential pressures during Test 2. 
The Catholic University of America Vitreous State Laboratory
DuraMelter 1200 Tests with AZ-101HLW Simulants Final Report, VSL-02R0100-2, Rev.1

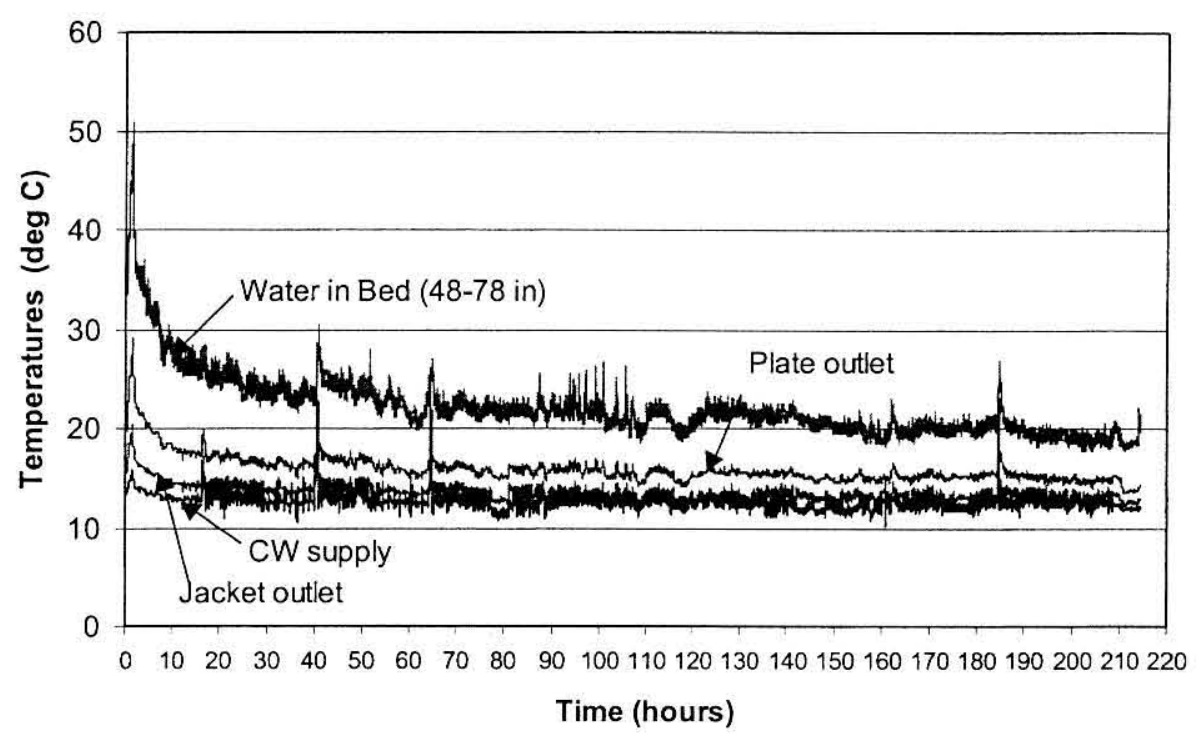

Figure 4.17. SBS cooling water and bed temperatures for Test 2 (cooling water data averaged over 10 minutes ( 5 measurements)).

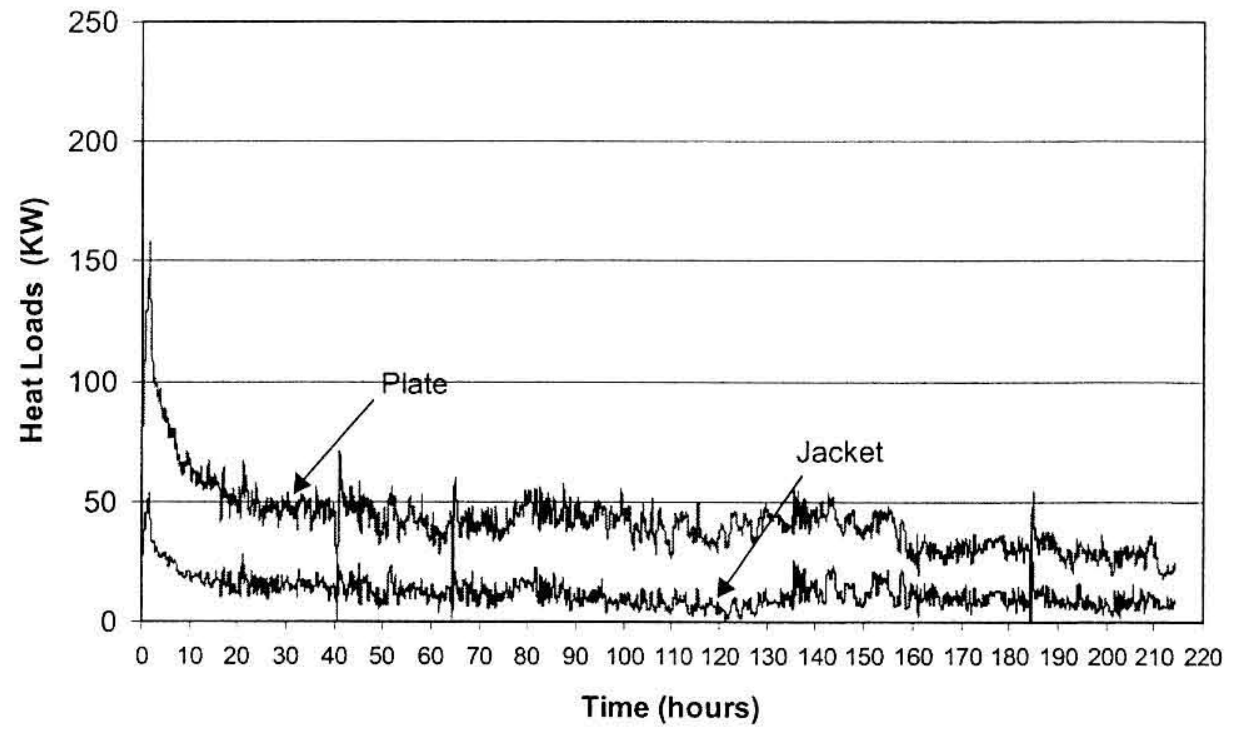

Figure 4.18. Calculated heat loads on the cooling water jacket and plate heat exchanger for Test 2. 
The Catholic University of America Vitreous State Laboratory
DuraMelter 1200 Tests with AZ-101HLW Simulants Final Report, VSL-02R0100-2, Rev.1

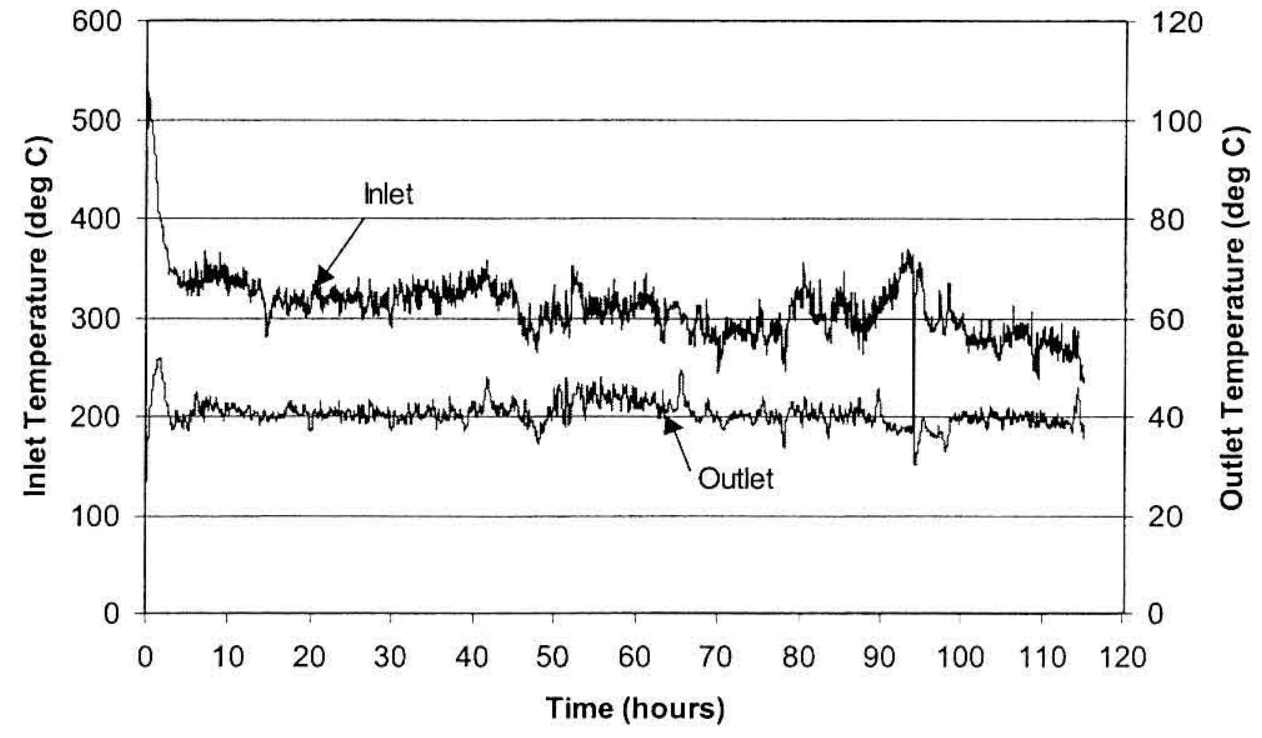

Figure 4.19. SBS gas inlet and outlet temperatures during Test 3.

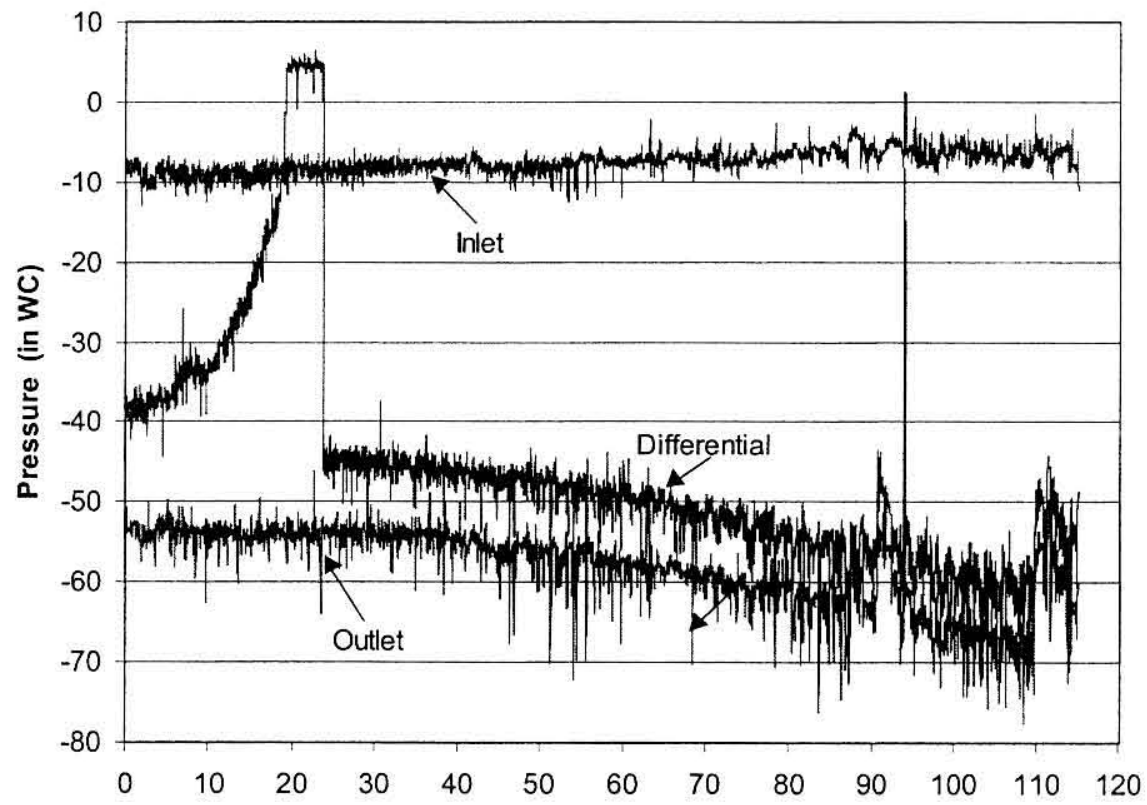

Figure 4.20. SBS inlet, outlet, and differential pressures during Test 3. Note: Between 10 and 22 hours of operations, differential pressure sensor was clogged. 
The Catholic University of America Vitreous State Laboratory
DuraMelter 1200 Tests with AZ-101HLW Simulants Final Report, VSL-02R0100-2, Rev.1

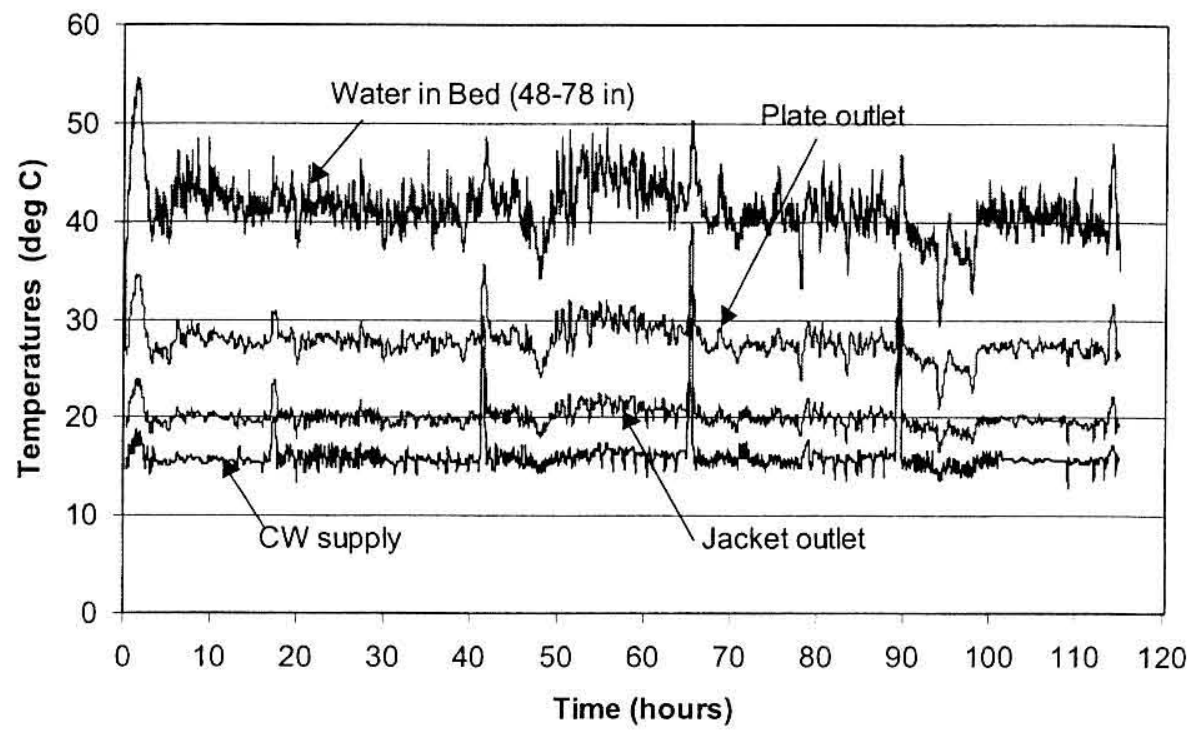

Figure 4.21. SBS cooling water and bed temperatures for Test 3 (cooling water data averaged over 10 minutes ( 5 measurements)).

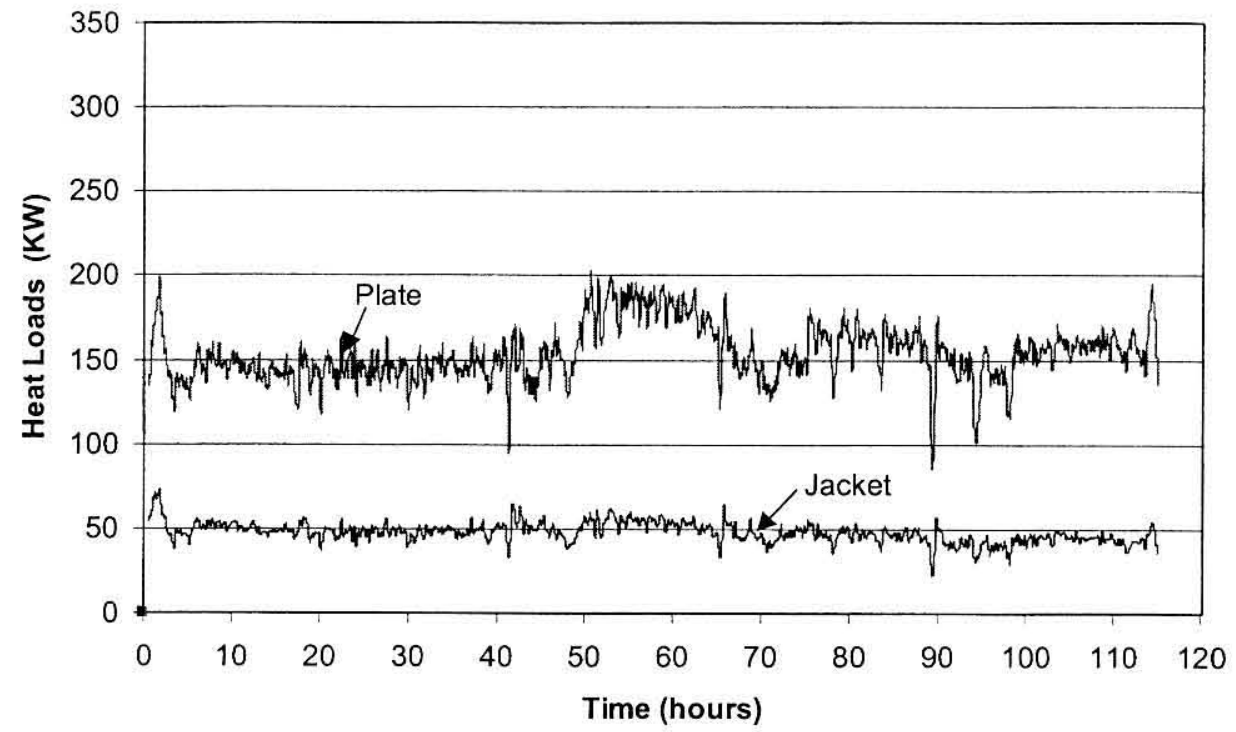

Figure 4.22. Calculated heat loads on the cooling water jacket and plate heat exchanger for Test 3 . 
The Catholic University of America Vitreous State Laboratory
DuraMelter 1200 Tests with AZ-101HLW Simulants Final Report, VSL-02R0100-2, Rev.1

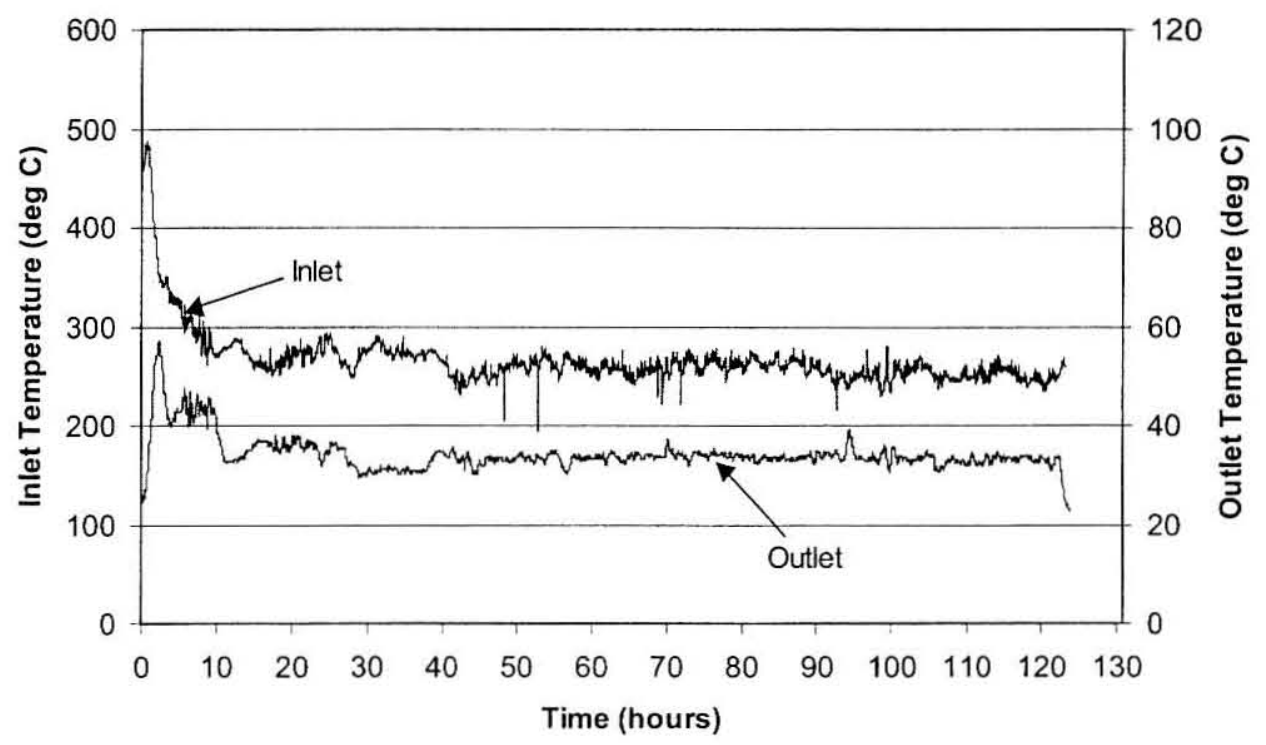

Figure 4.23. SBS gas inlet and outlet temperatures during Test 4.

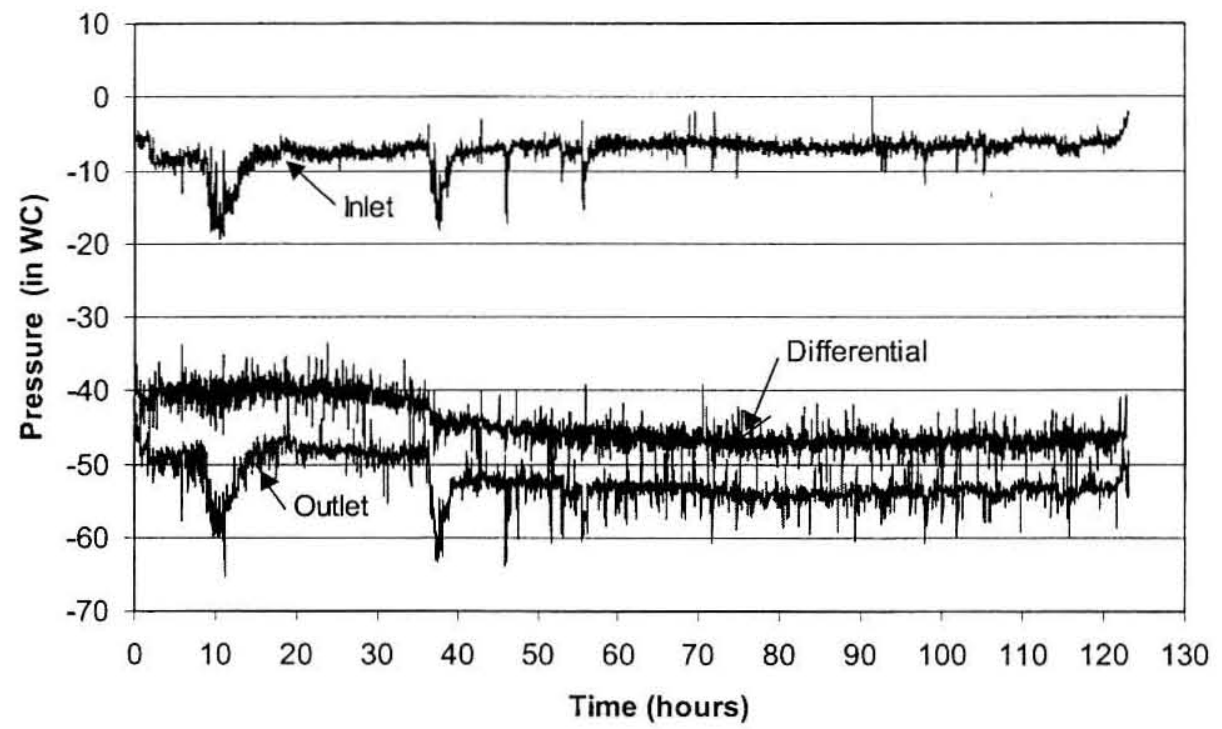

Figure 4.24. SBS inlet, outlet, and differential pressures during Test 4 . 
The Catholic University of America Vitreous State Laboratory
ORP-51438, Rev. 0

DuraMelter 1200 Tests with AZ-101HLW Simulants

Final Report, VSL-02R0100-2, Rev.1

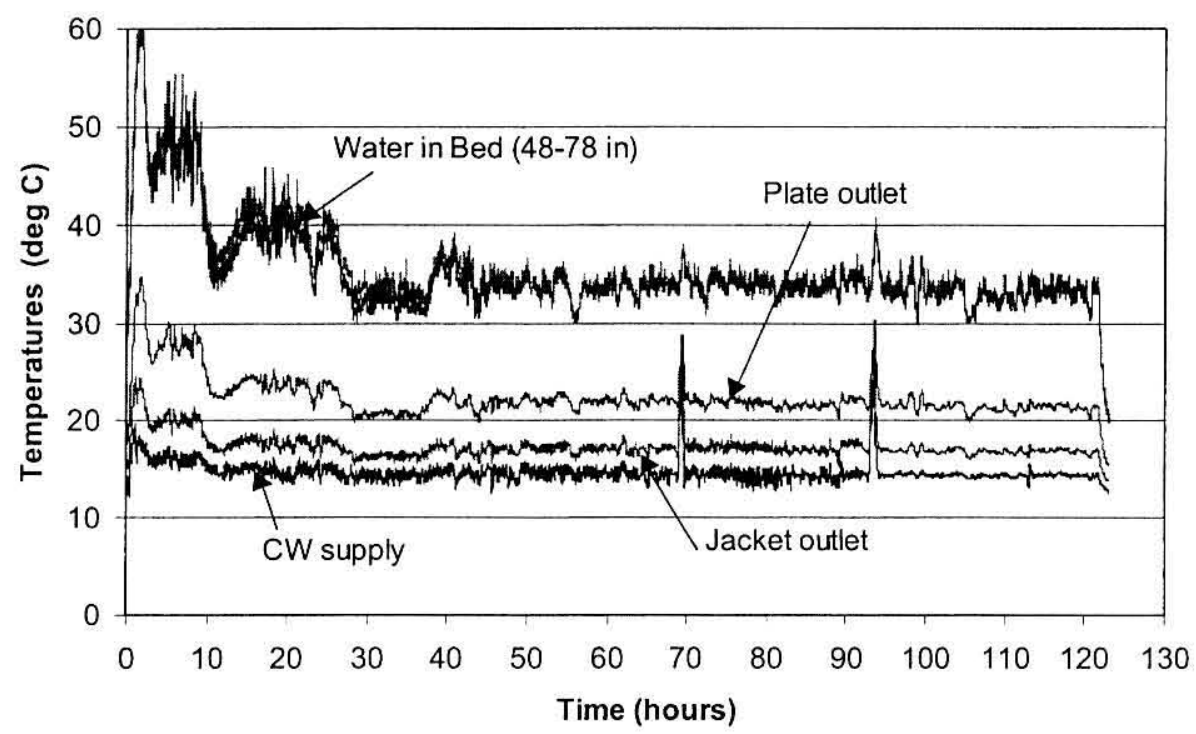

Figure 4.25. SBS cooling water and bed temperatures for Test 4 (cōling water data averaged over 10 minutes (5 measurements)).

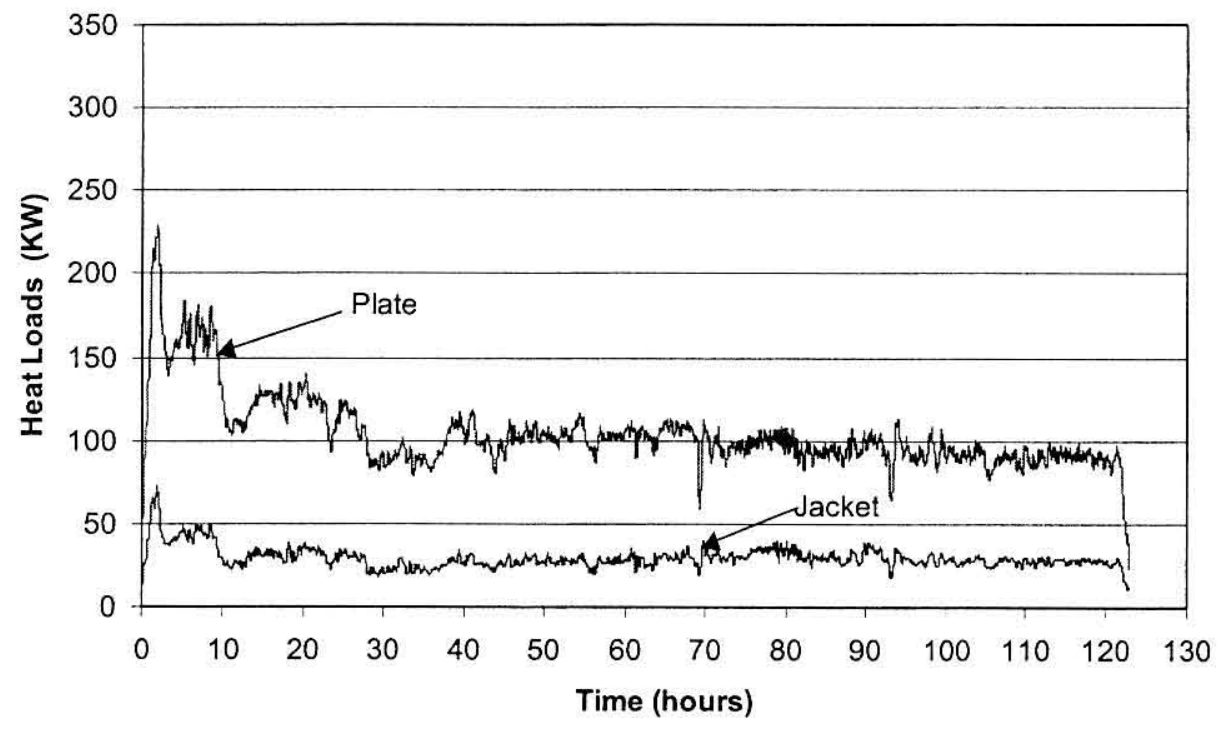

Figure 4.26. Calculated heat loads on the cooling water jacket and plate heat exchanger for Test 4. 


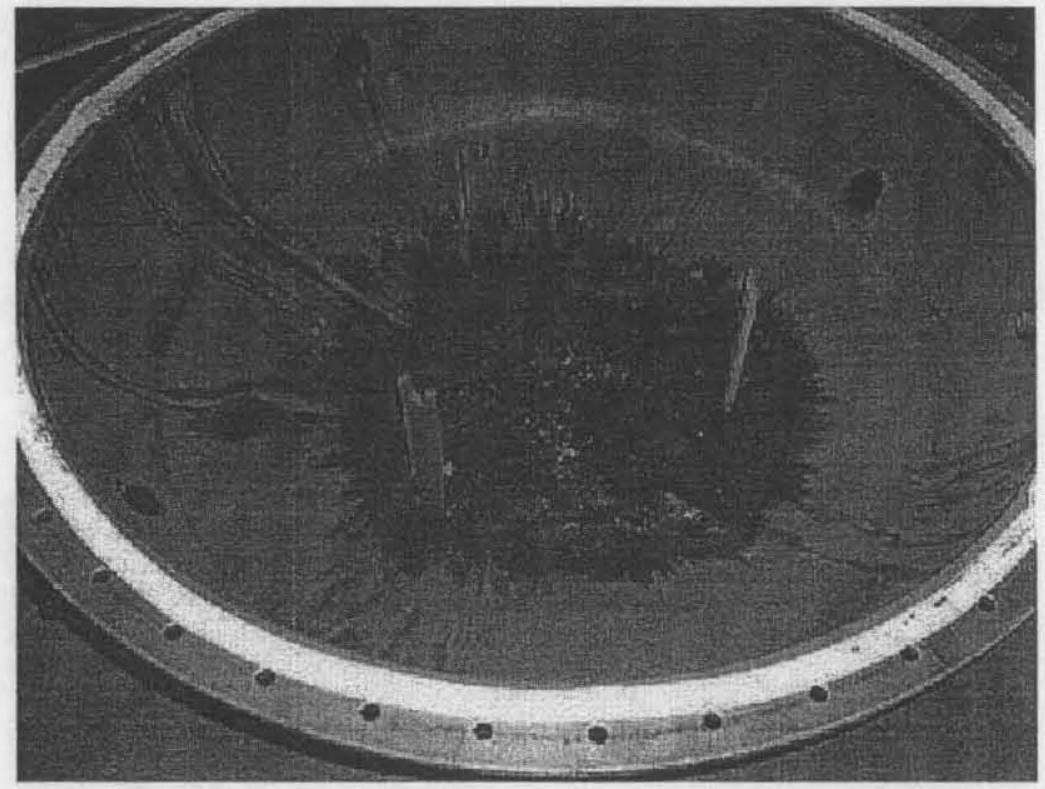

(a)

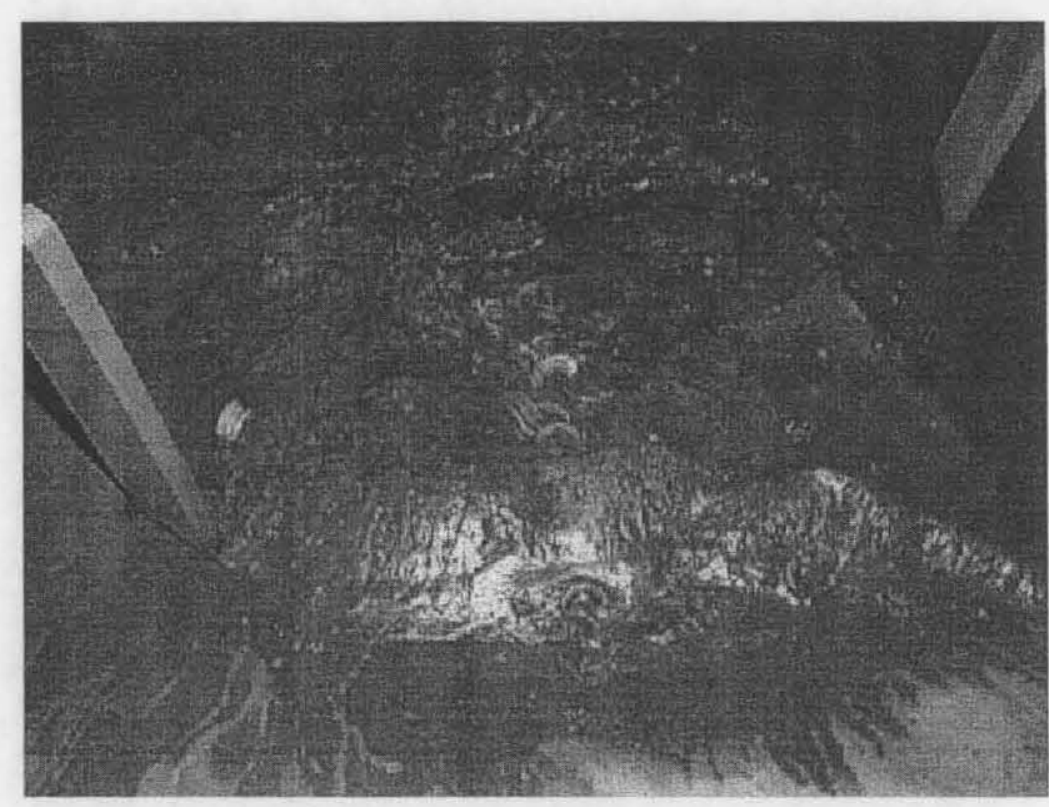

(b)

Figure 4.27. a. Deposits in SBS bowl,

b. Close-up of deposits in SBS bowl (Test 4). 


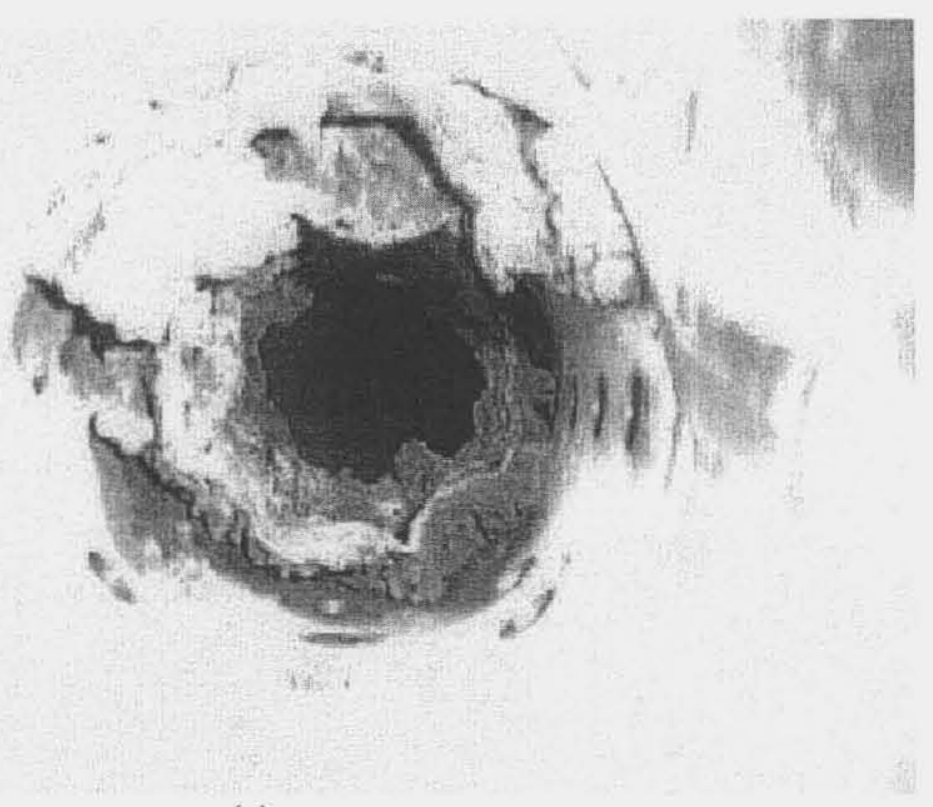

(a)

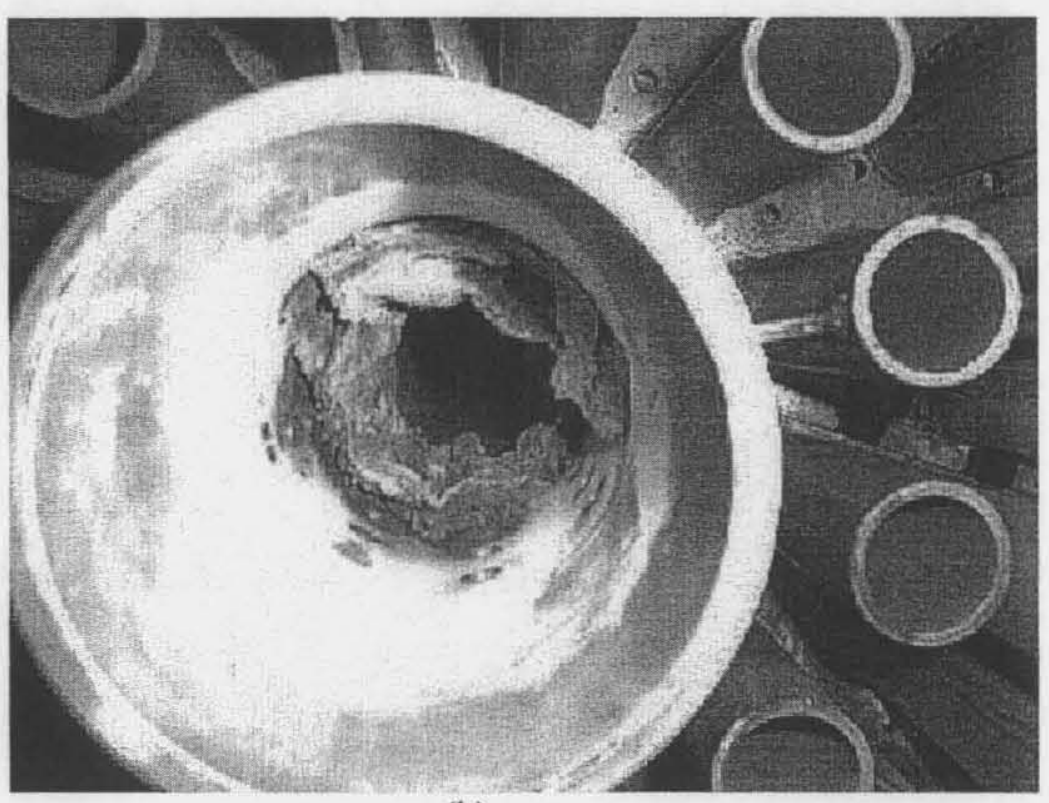

(b)

Figure 4.28. a. SBS down-comer deposits - close-up b. SBS down-comer deposits (Test 4). 


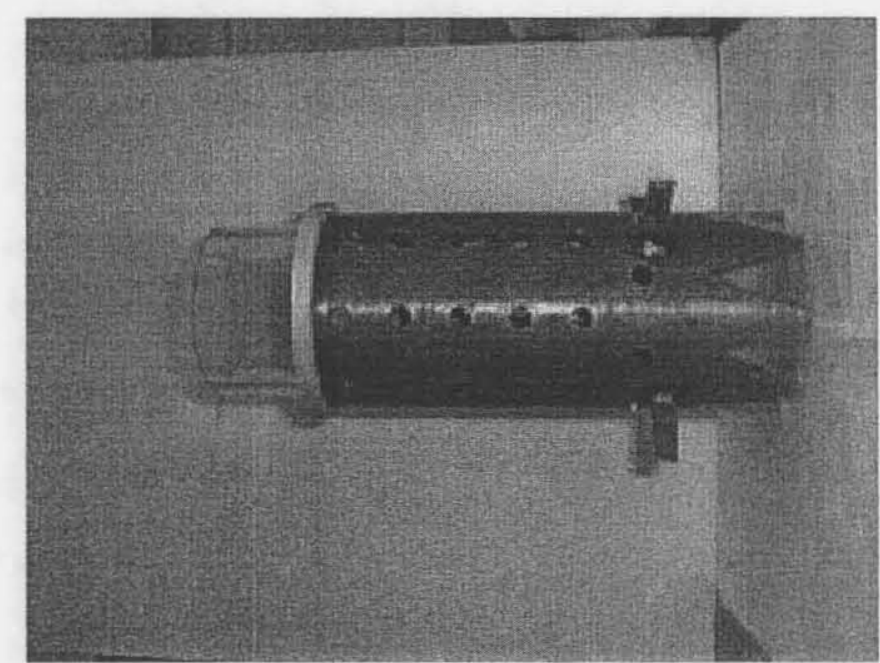

(a)

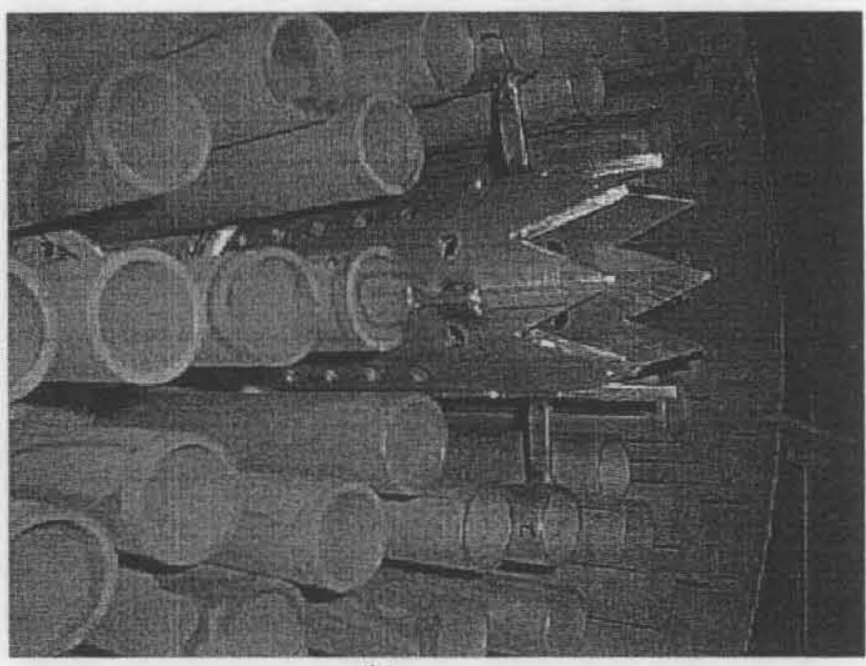

(b)

Figure 4.29. a) Modified SBS down-comer gas diffuser with Teflon gasket and centering tool

b) Installed gasket and downcomer (Test 4). 


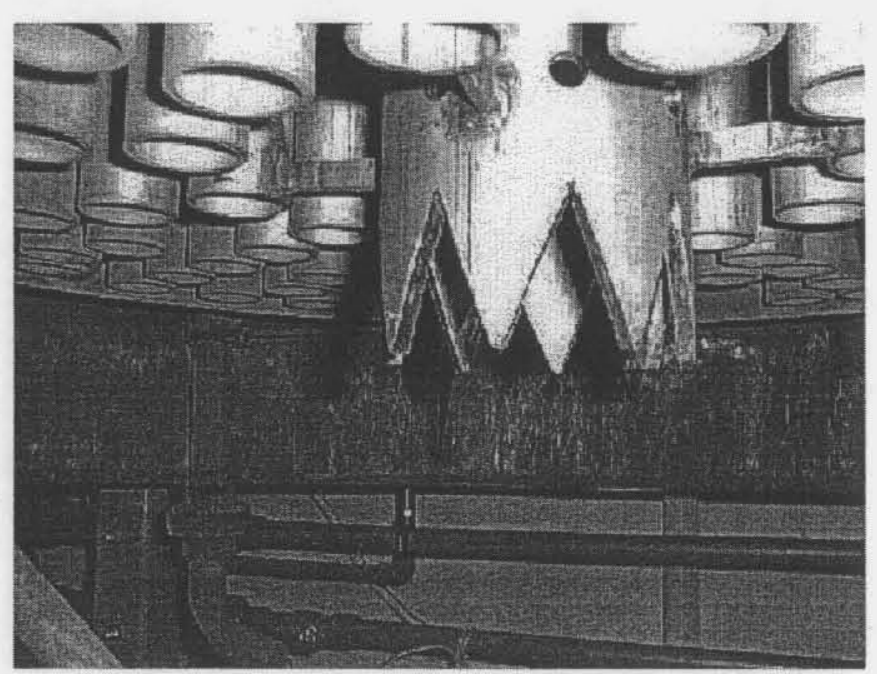

(a)

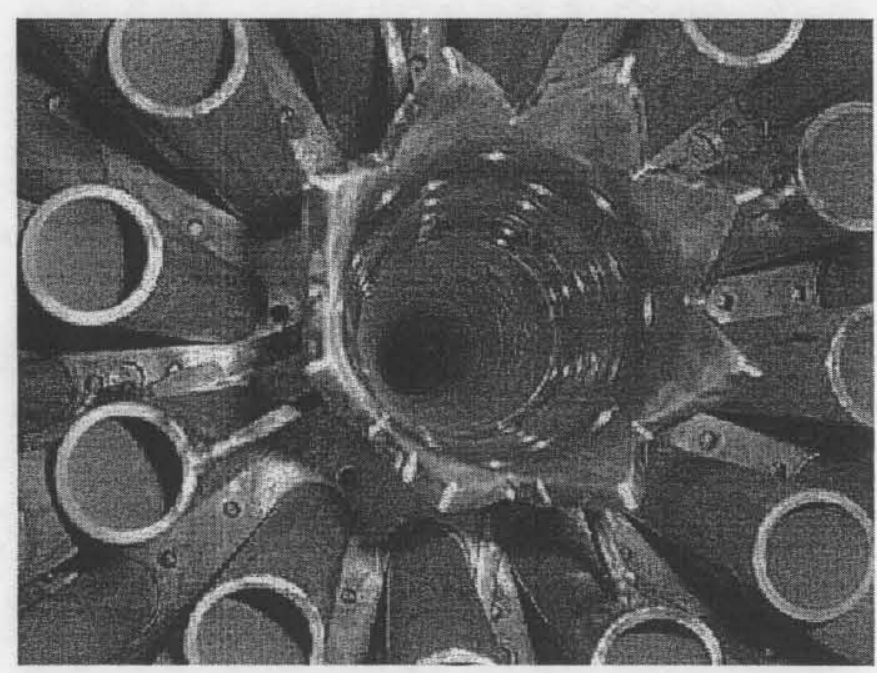

(b)

Figure 4.30. (a) Installed SBS down-comer (side view)

(b) Installed SBS down-comer (bottom view) (Test 4). 
The Catholic University of America Vitreous State Laboratory
DuraMelter 1200 Tests with AZ-101HLW Simulants Final Report, VSL-02R0100-2, Rev.1

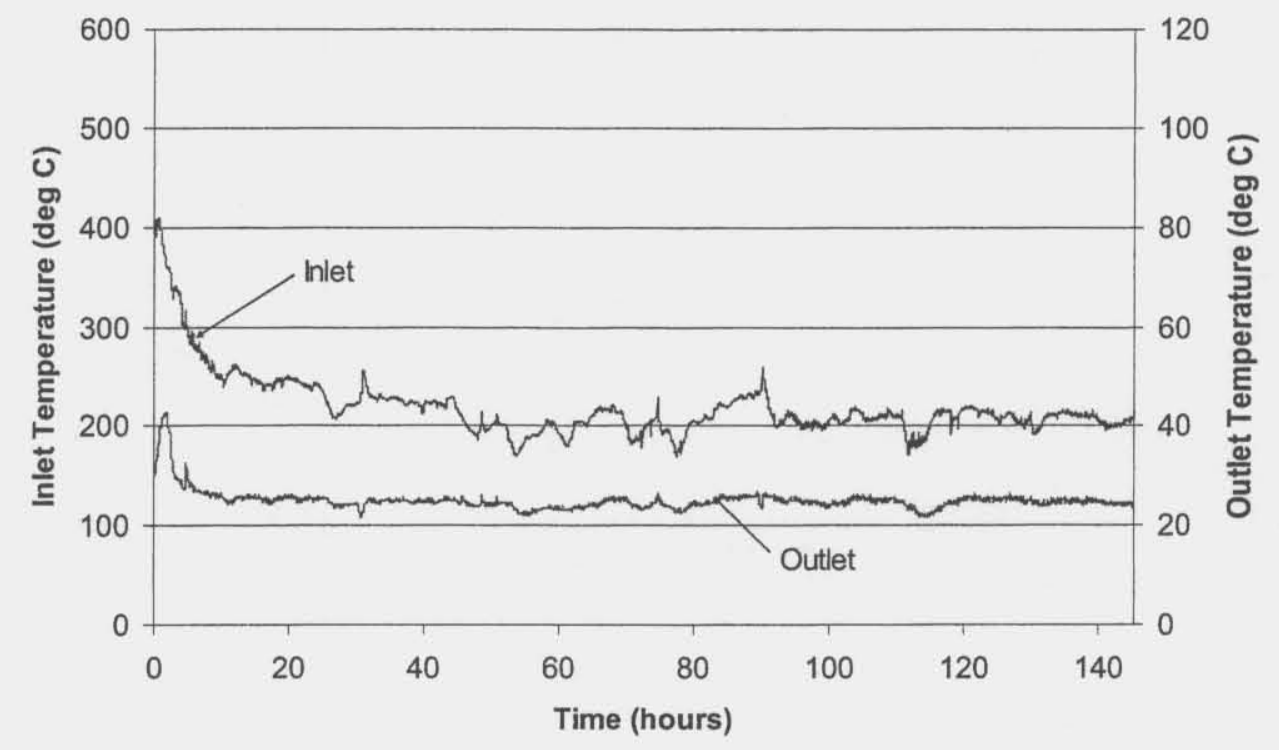

Figure 4.31. SBS gas inlet and outlet temperatures during Test 5.

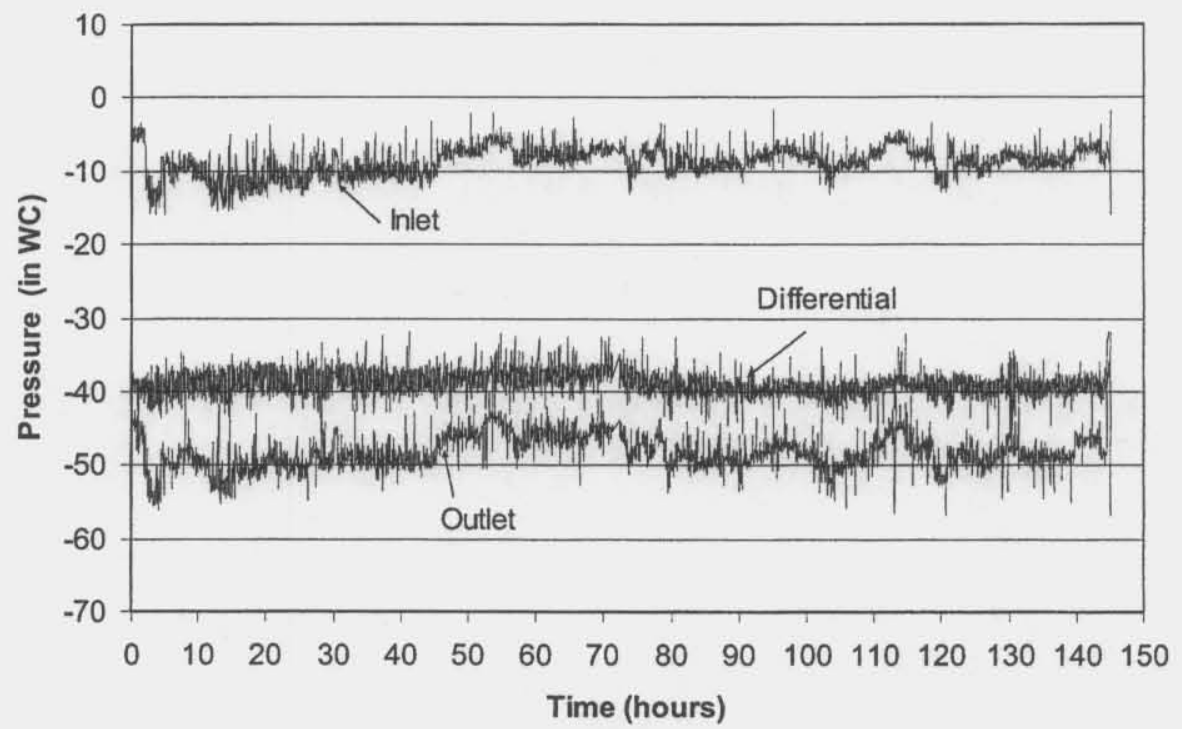

Figure 4.32. SBS inlet, outlet and differential pressures during Test 5. 
The Catholic University of America

Vitreous State Laboratory
DuraMelter 1200 Tests with AZ-101HLW Simulants Final Report, VSL-02R0100-2, Rev.1

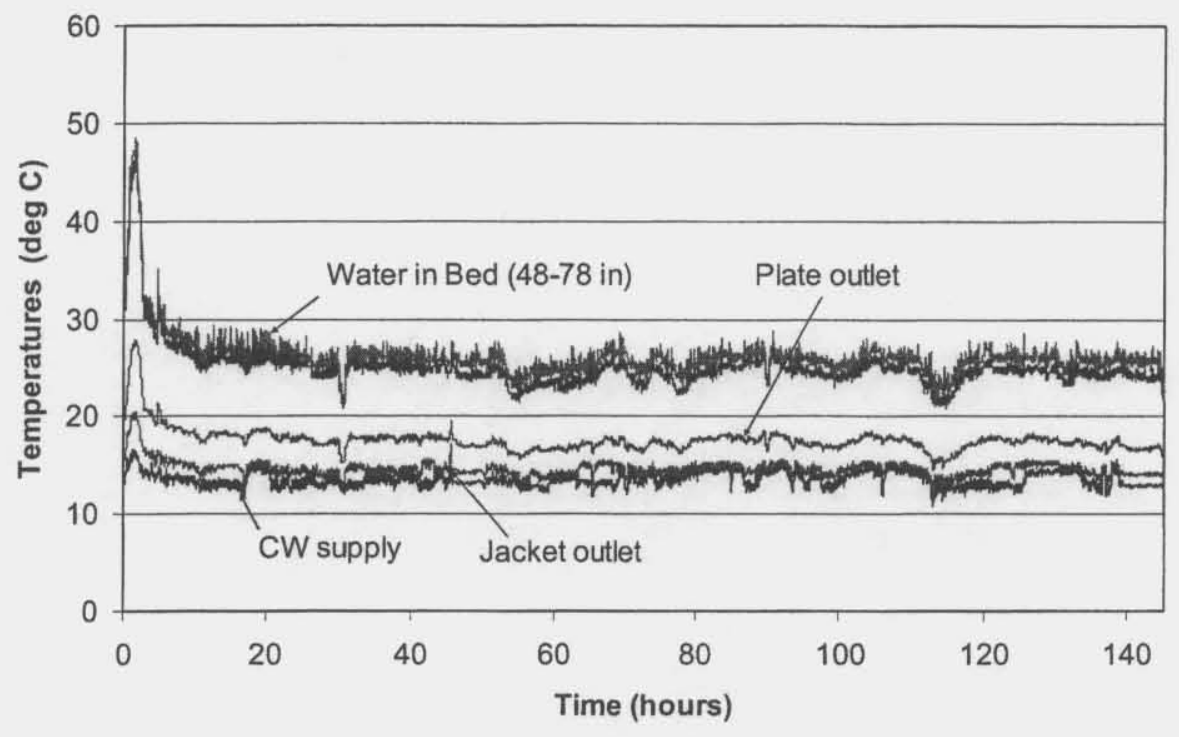

Figure 4.33. SBS cooling water and bed temperatures for Test 5 (cooling water data averaged over 10 minutes (5 measurements)).

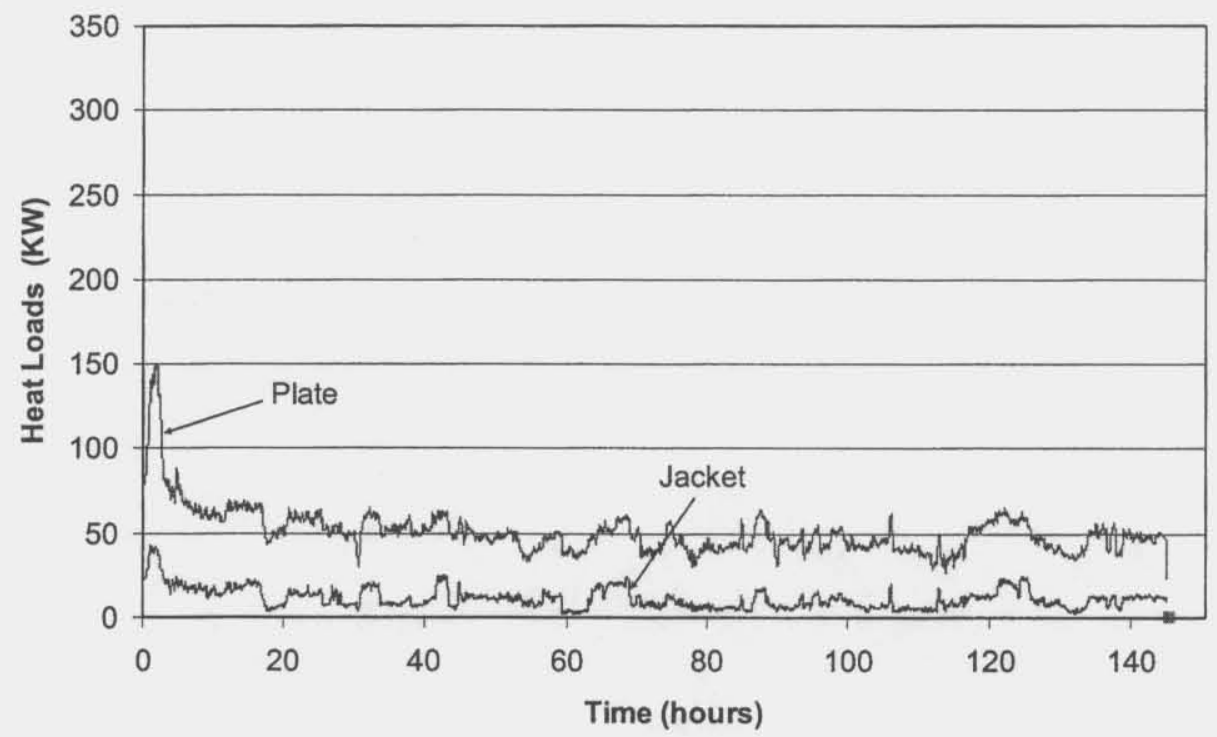

Figure 4.34. Calculated heat loads on the cooling water jacket and plate heat exchanger for Test 5. 


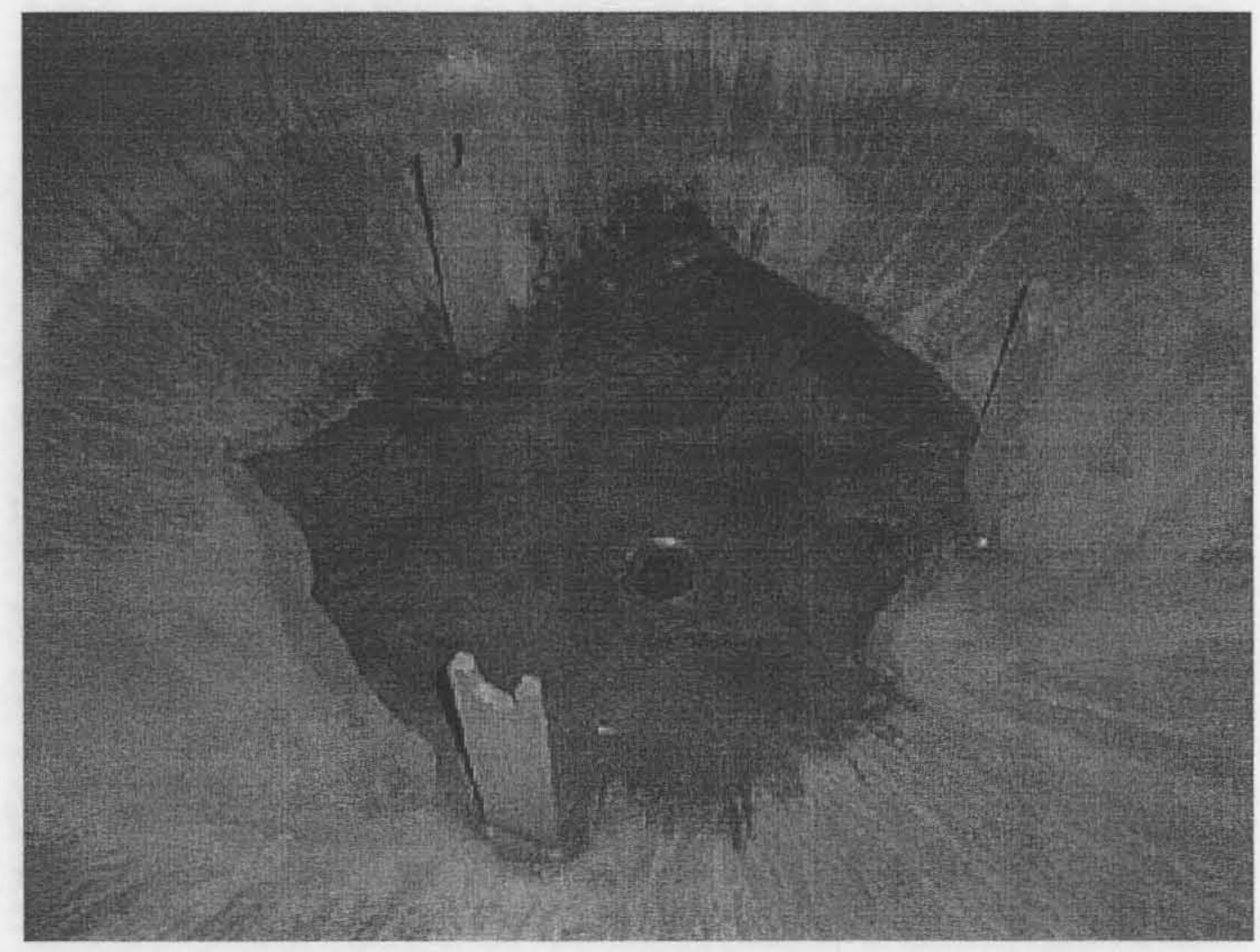

Figure 4.35. SBS bottom dish deposits showing square deposition pattern caused by sparge lances (Test 5). 

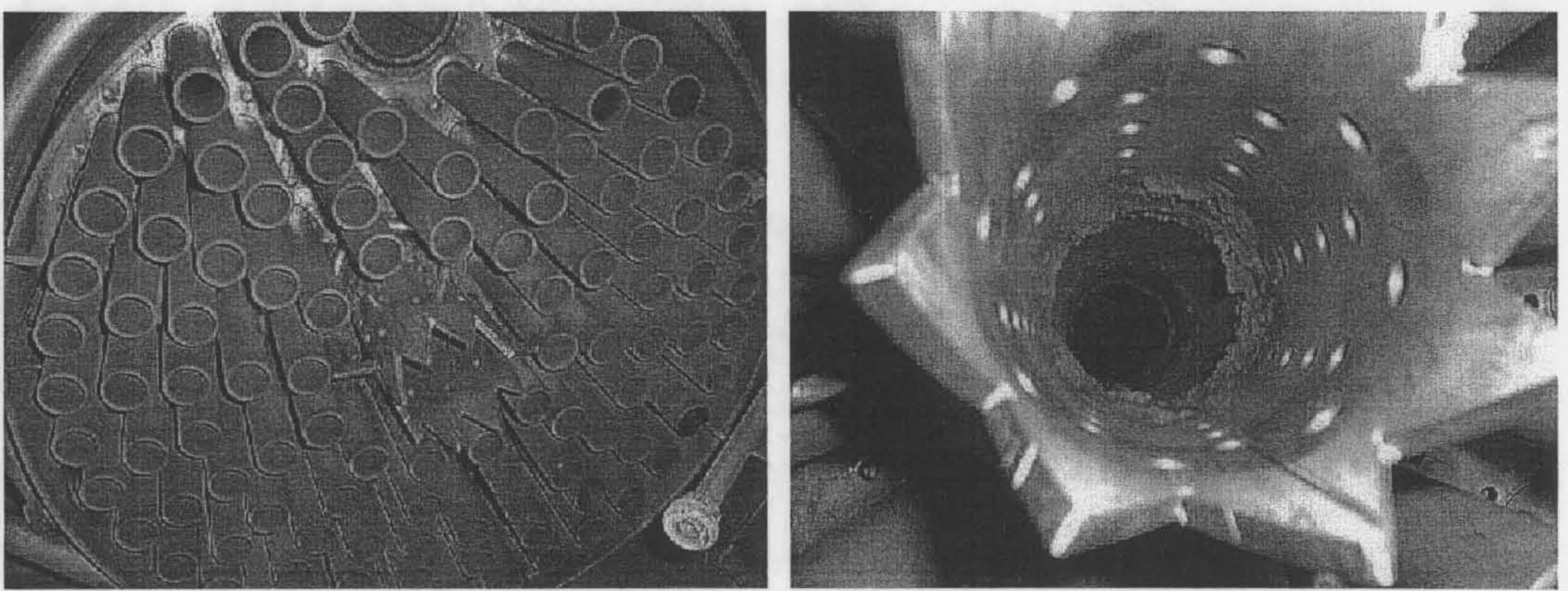

Figure 4.36. General view of the SBS weir tubes and deposits inside the down-comer pipe (Test 5). 


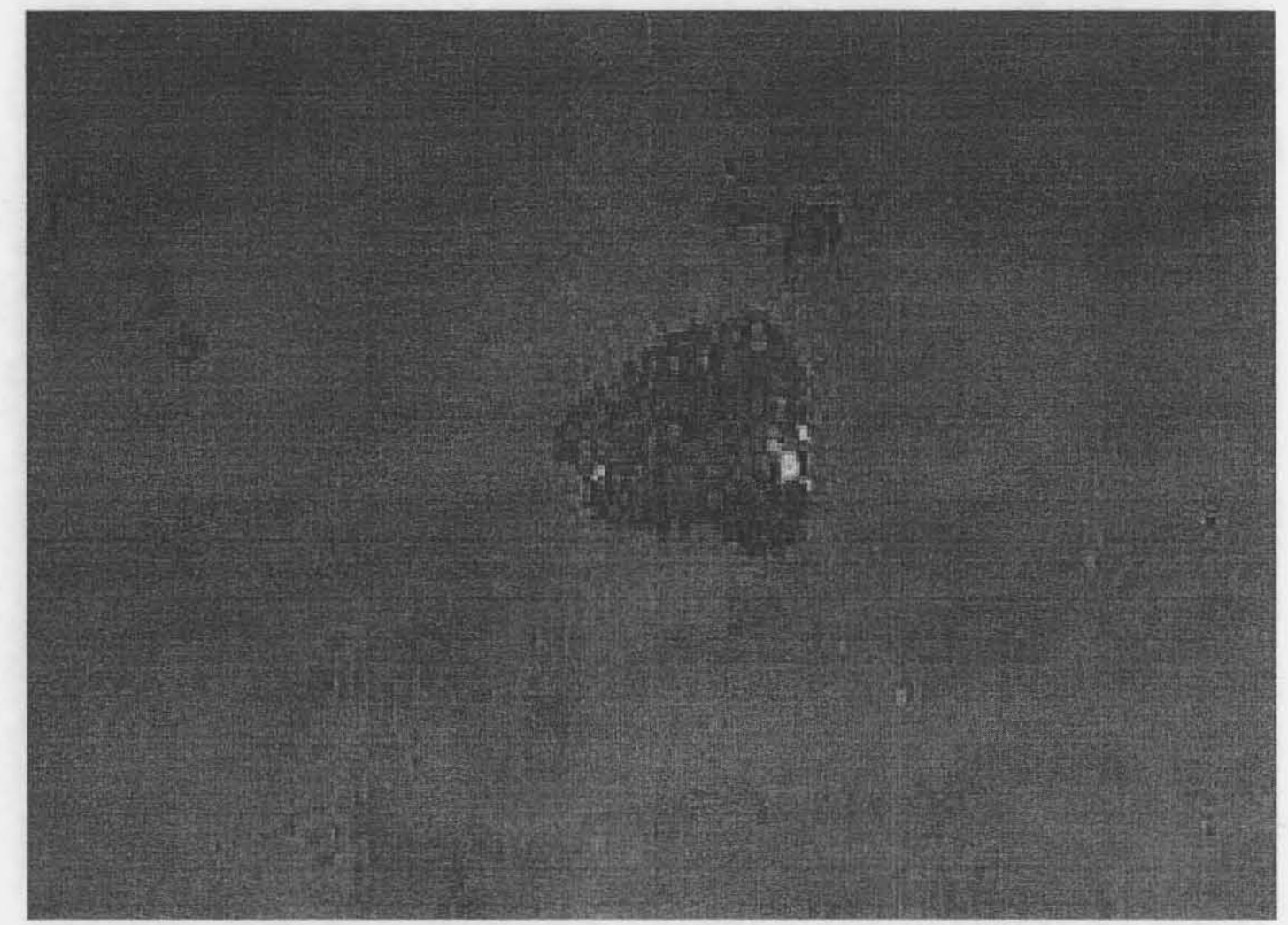

Figure 4.37. Deposits dislodged from the SBS down-comer pipe (Test 5). 
The Catholic University of America

Vitreous State Laboratory
DuraMelter 1200 Tests with AZ-101HLW Simulants Final Report, VSL-02R0100-2, Rev.1

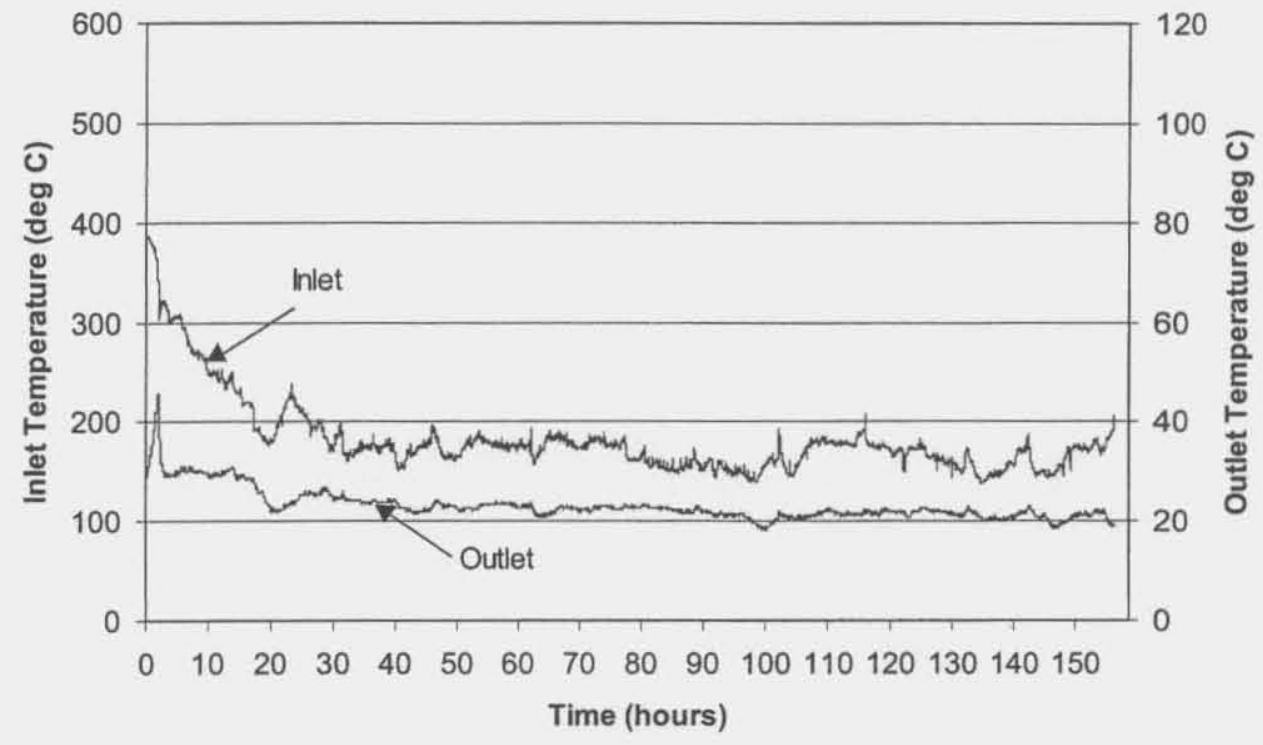

Figure 4.38. SBS gas inlet and outlet temperatures during Test 6 .

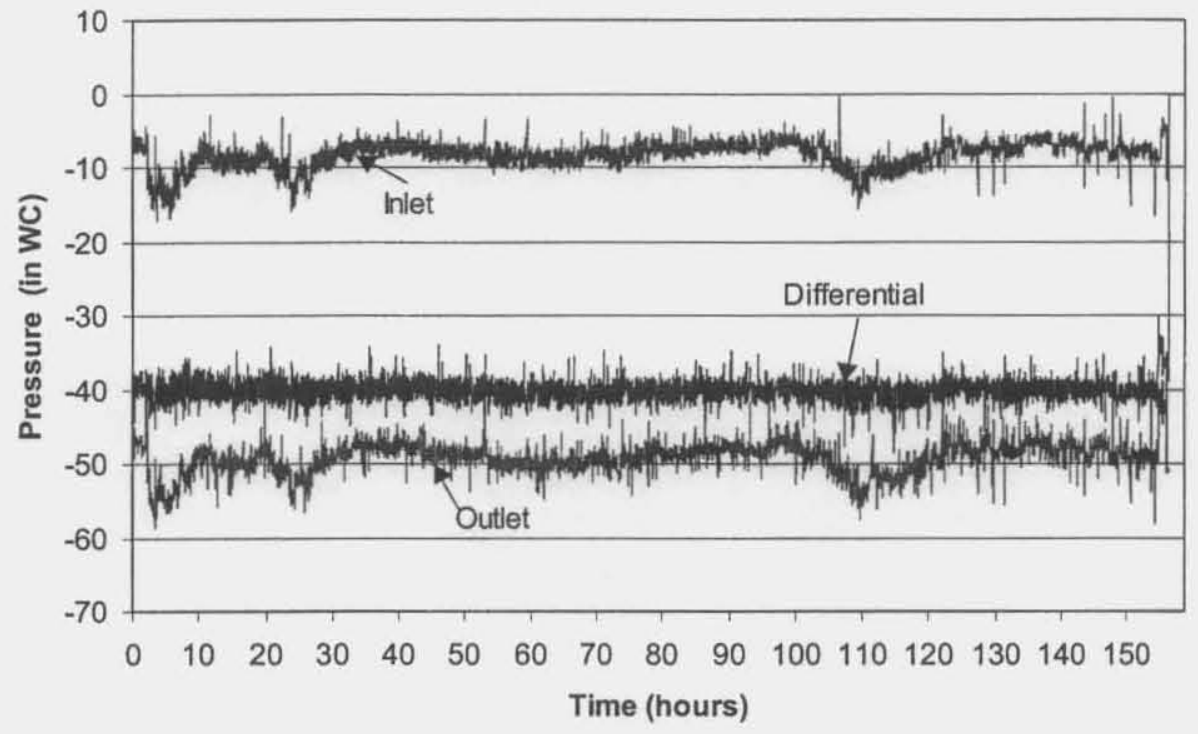

Figure 4.39. SBS inlet, outlet, and differential pressures during Test 6. 


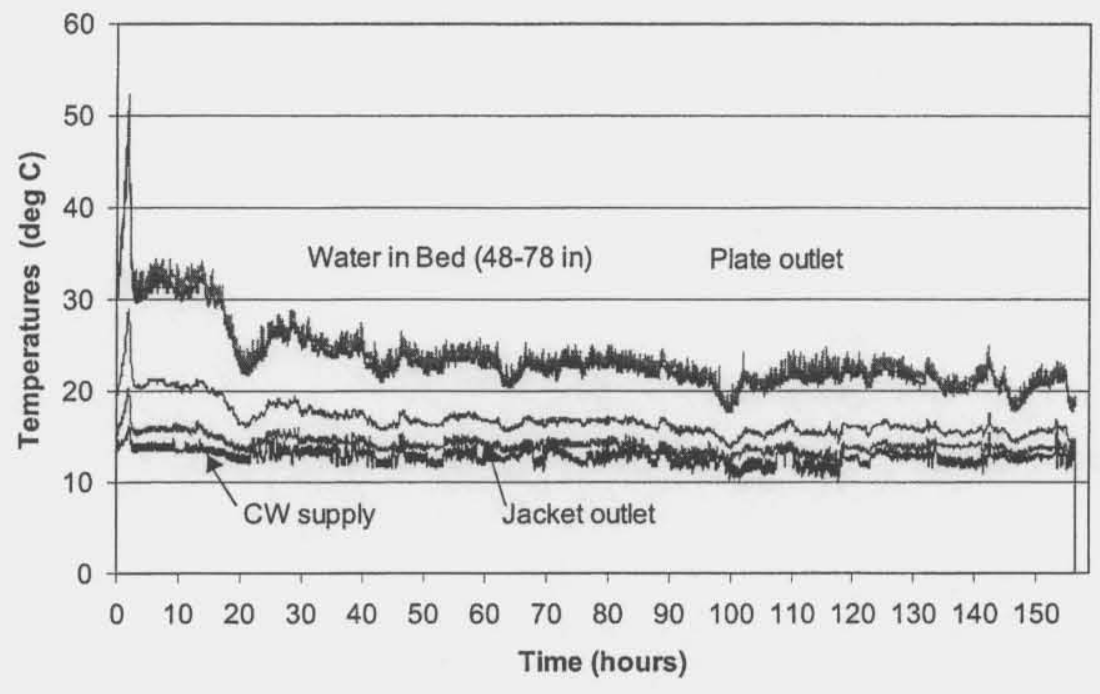

Figure 4.40. SBS cooling water and bed temperatures for Test 6 (cooling water data averaged over 10 minutes ( 5 measurements)).

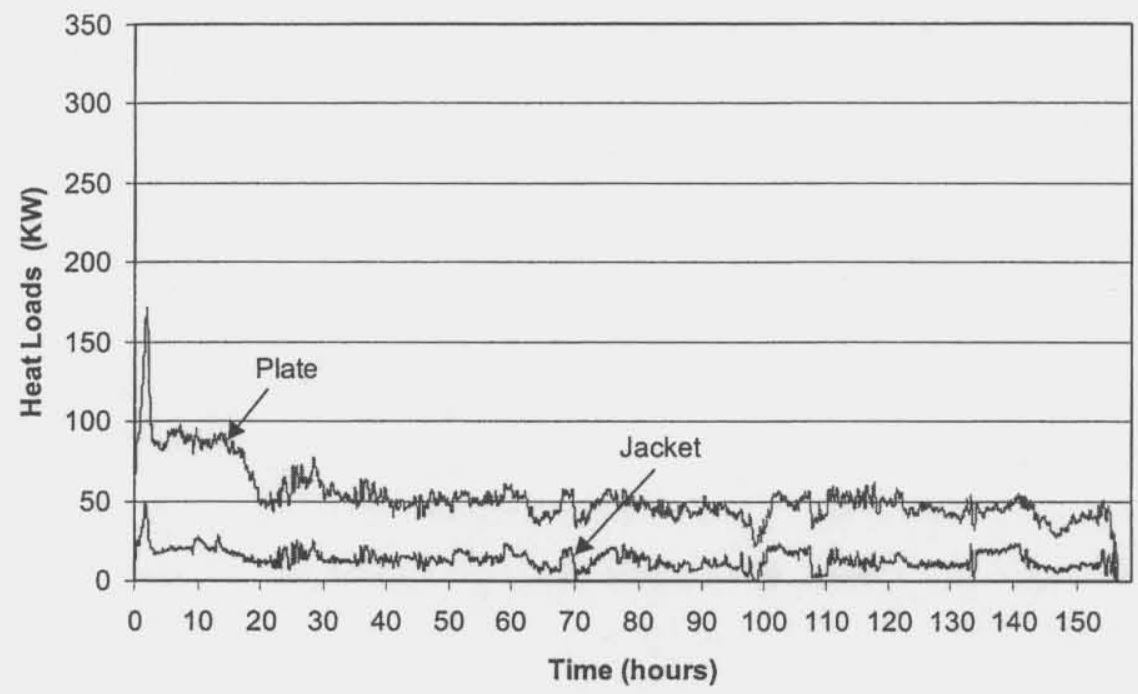

Figure 4.41. Calculated heat loads on the cooling water jacket and plate heat exchanger for Test 6. 

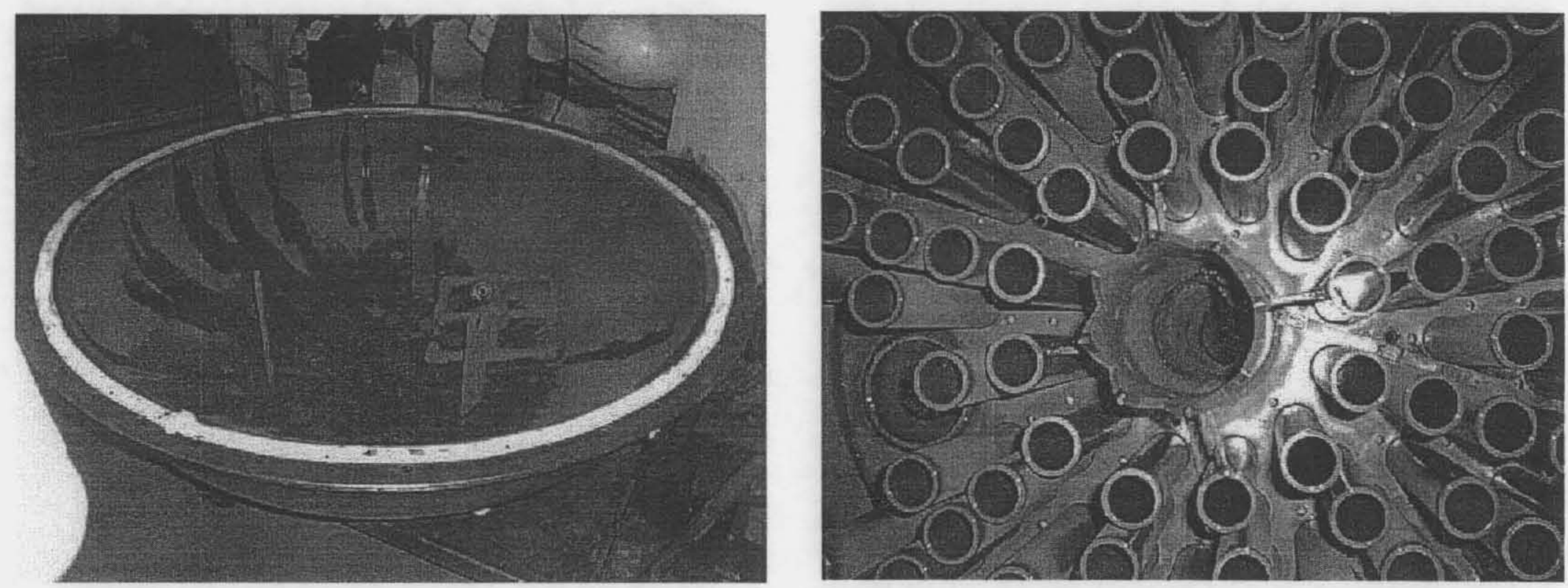

Figure 4.42. Deposits in the SBS bowl and bottom view of the weir tubes with down-comer pipe (Test 6). 
The Catholic University of America

Vitreous State Laboratory
DuraMelter 1200 Tests with AZ-101 HLW Simulants

Final Report, VSL-02R0100-2, Rev.1

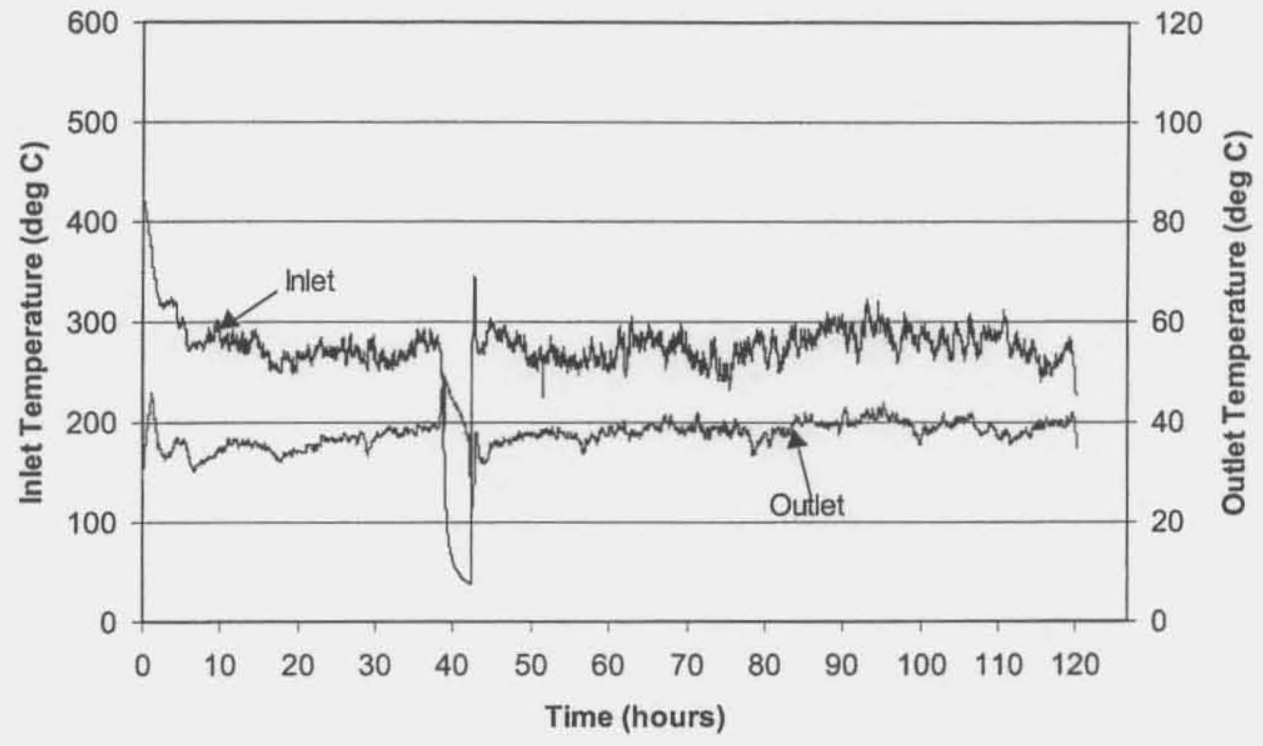

Figure 4.43. SBS gas inlet and outlet temperatures during Test 7.

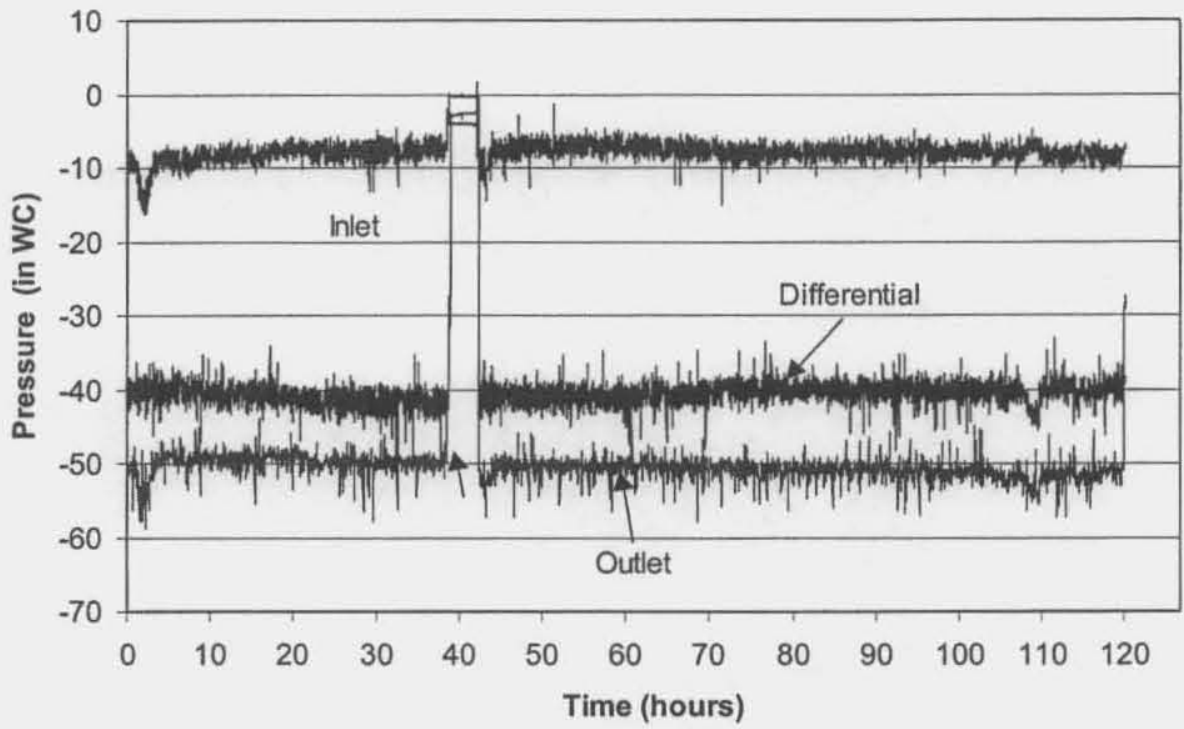

Figure 4.44. SBS inlet, outlet, and differential pressures during Test 7. Note: Feed was interrupted from hours 38 to 43 . 
The Catholic University of America Vitreous State Laboratory
DuraMelter 1200 Tests with AZ-101HLW Simulants Final Report, VSL-02R0100-2, Rev.1

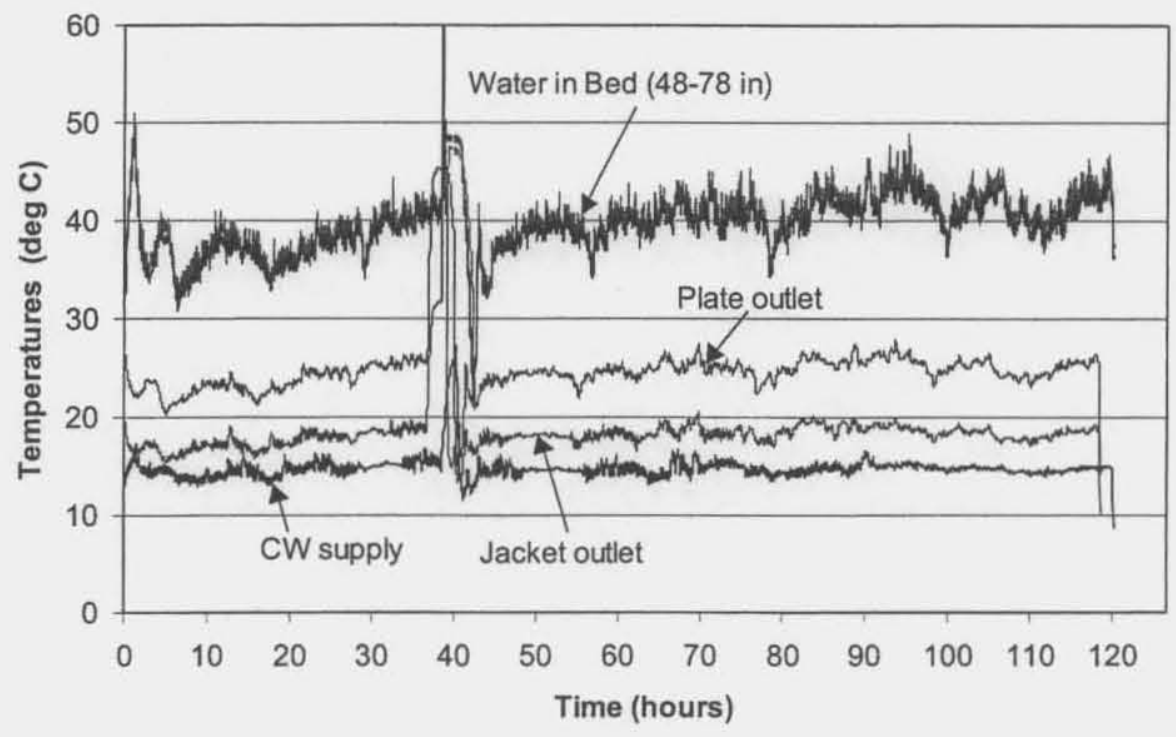

Figure 4.45. SBS cooling water and bed temperatures during Test 7 (cooling water data averaged over 10 min. ( 5 measurements)). Note: Feed was interrupted from hours 38 to 43.

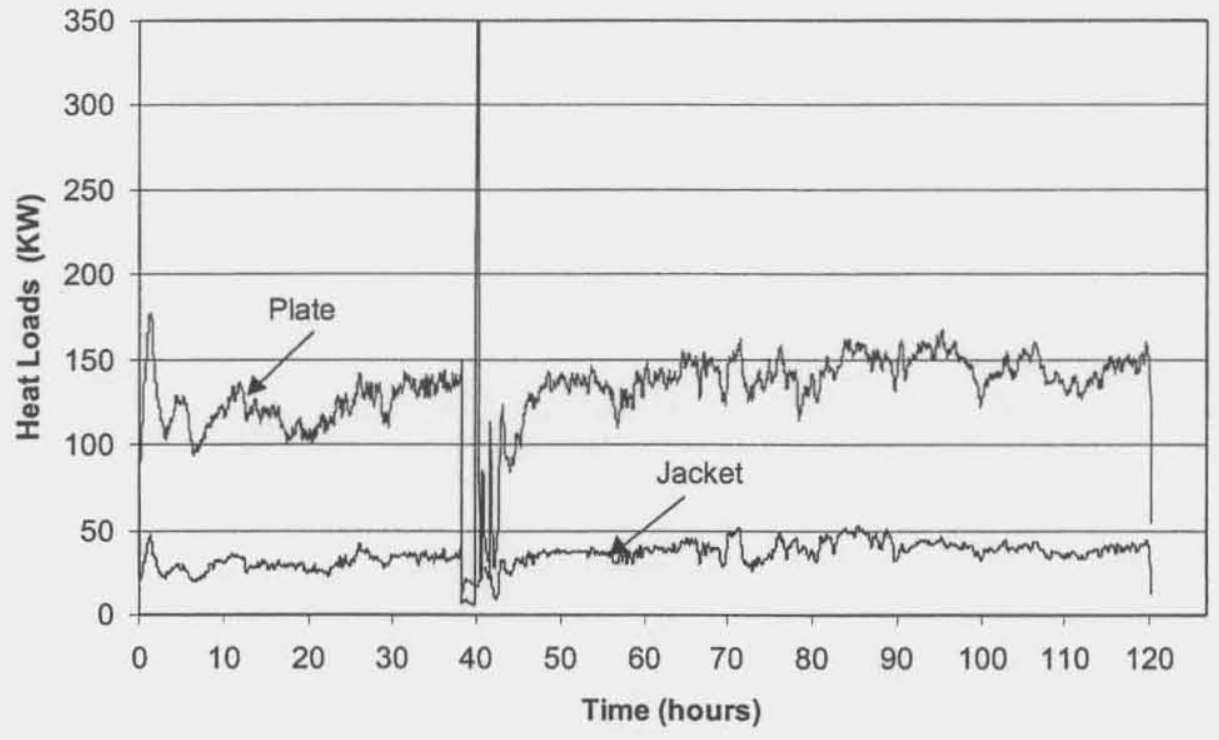

Figure 4.46. Calculated heat loads on the cooling water jacket and plate heat exchanger for Test 7. Note: Feed was interrupted from hours 38 to 43. 

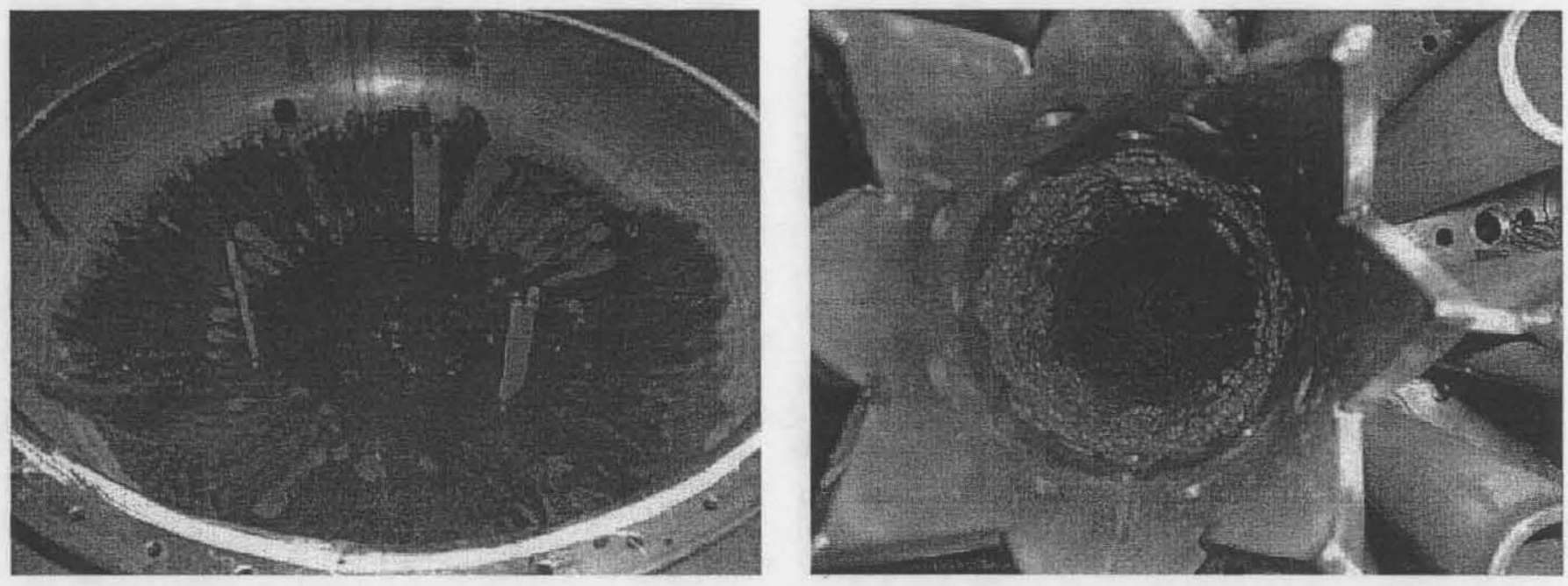

Figure 47. Deposits in the SBS bowl and bottom view of the down-comer pipe showing deposits (Test \#7). 


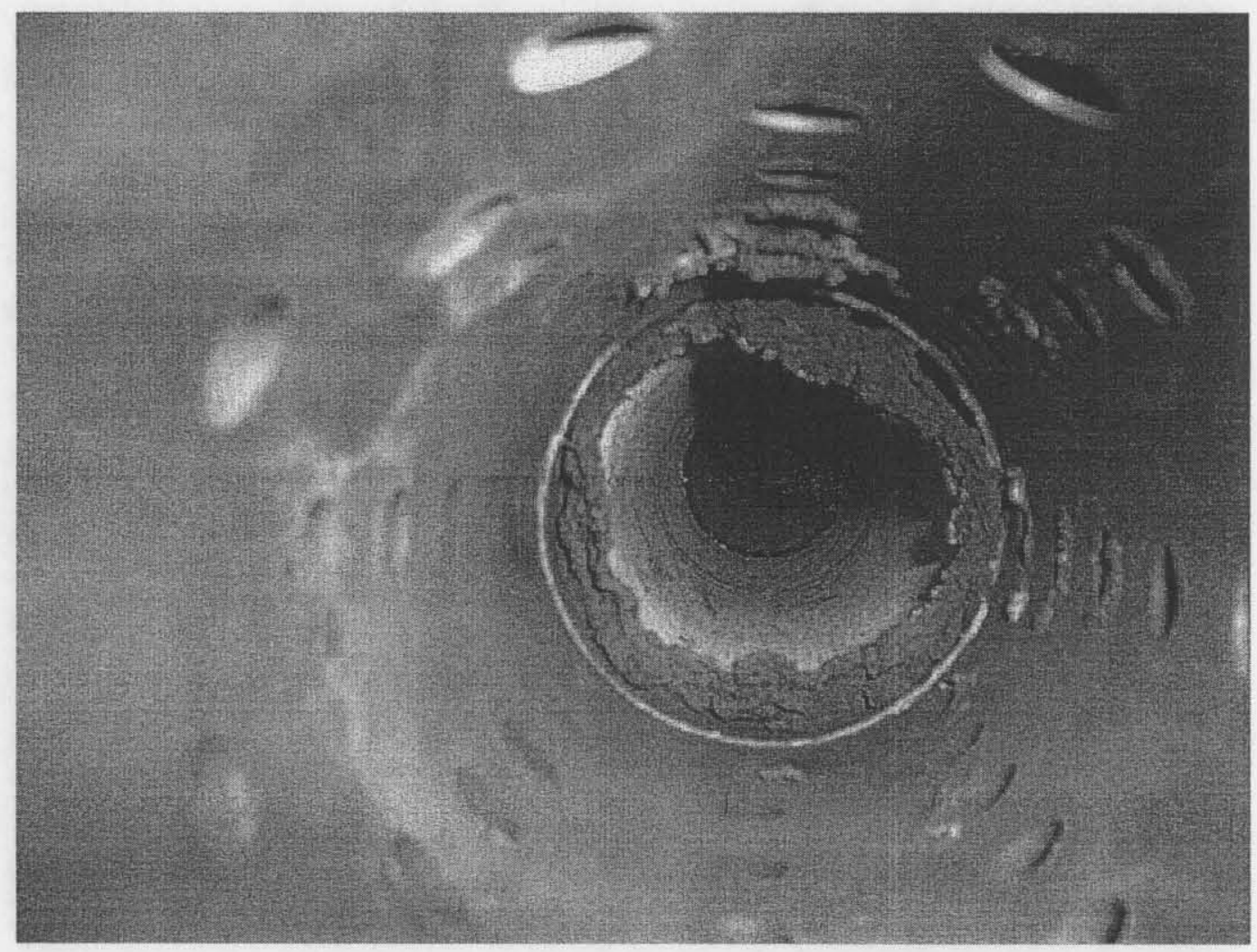

Figure 4.48. Deposits in the SBS down-comer pipe (Test 7). 


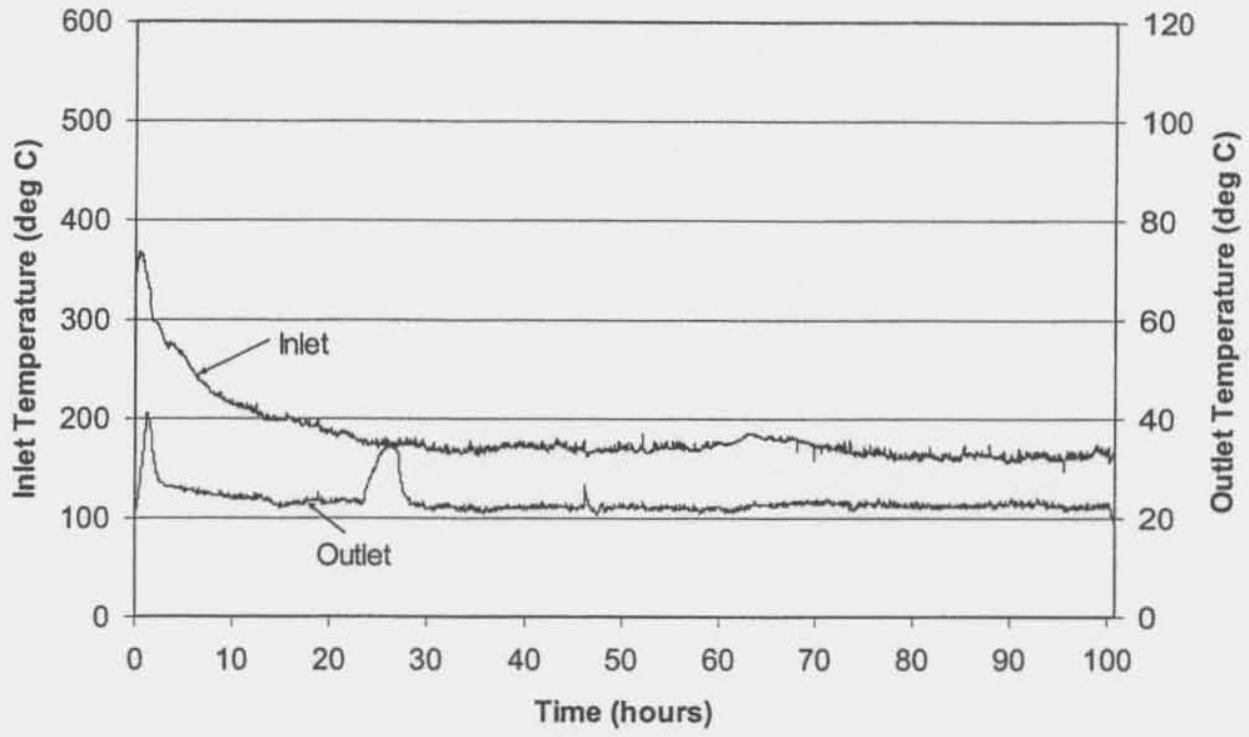

Figure 4.49. SBS gas inlet and outlet temperatures during Test 8. Note: Between 23 and 28 hours of operations, water circulation in cooling coil was interrupted.

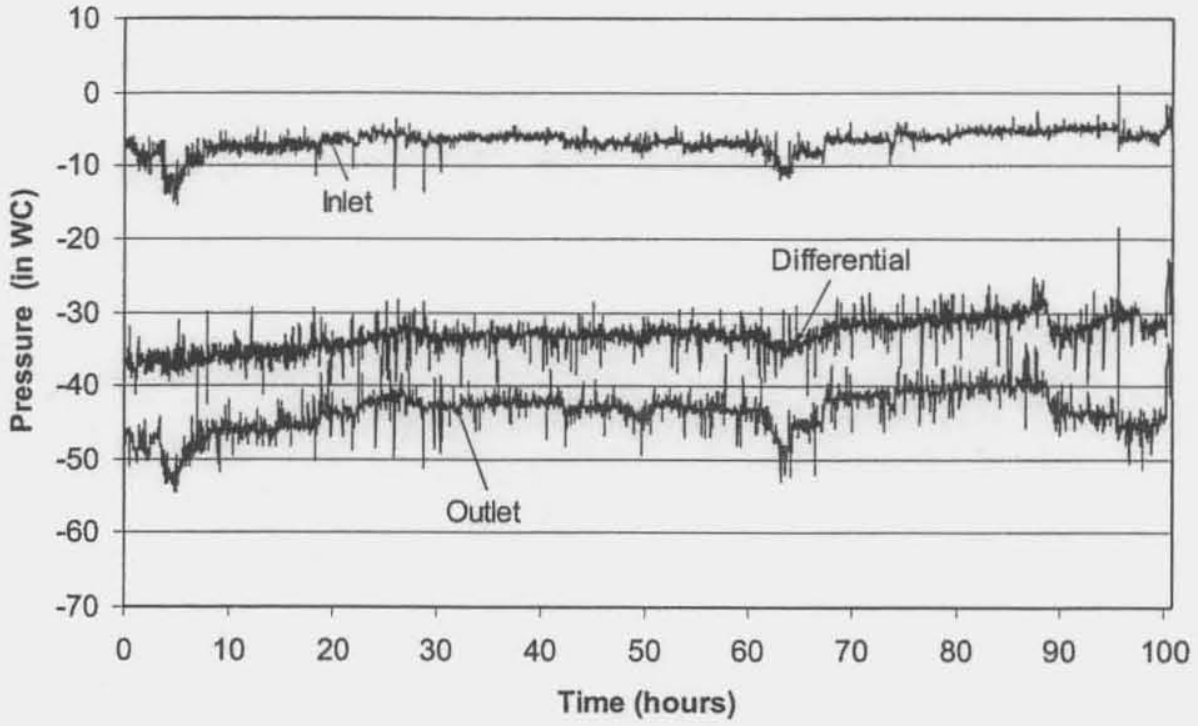

Figure 4.50. SBS inlet, outlet, and differential pressures during Test 8 . 
The Catholic University of America Vitreous State Laboratory

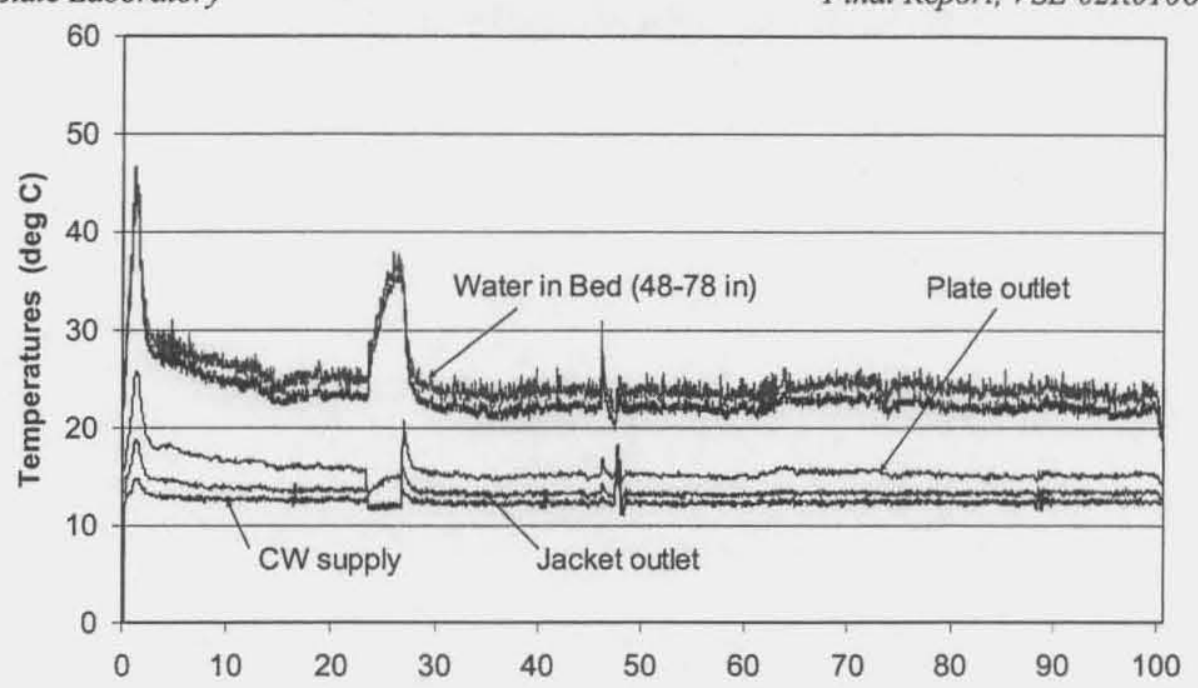

Figure 4.51. SBS cooling water and bed temperatures for Test 8 (cooling water data averaged over 10 minutes ( 5 measurements)).

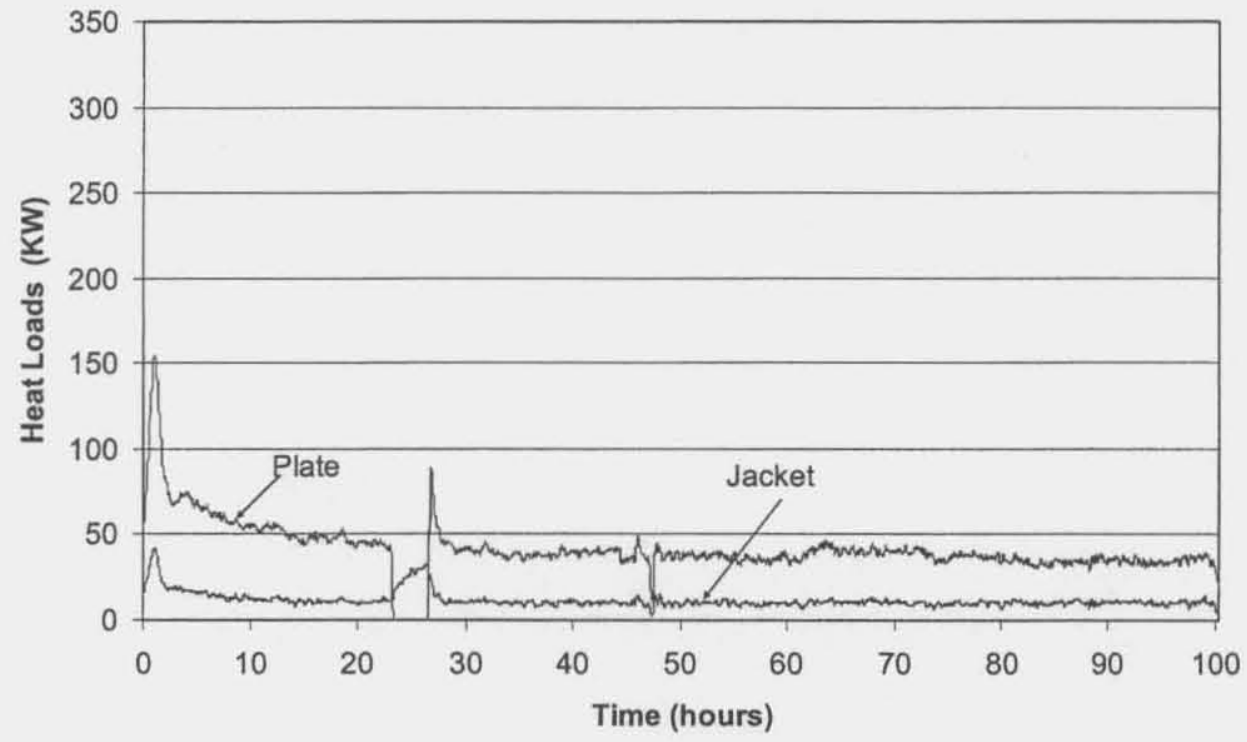

Figure 4.52. Calculated heat loads on the cooling water jacket and plate heat exchanger for Test 8. Note: Between 23 and 28 hours of operations, water circulation in SBS cooling coil was interrupted. 
The Catholic University of America Vitreous State Laboratory
DuraMelter 1200 Tests with AZ-101HLW Simulants Final Report, VSL-02R0100-2, Rev.1

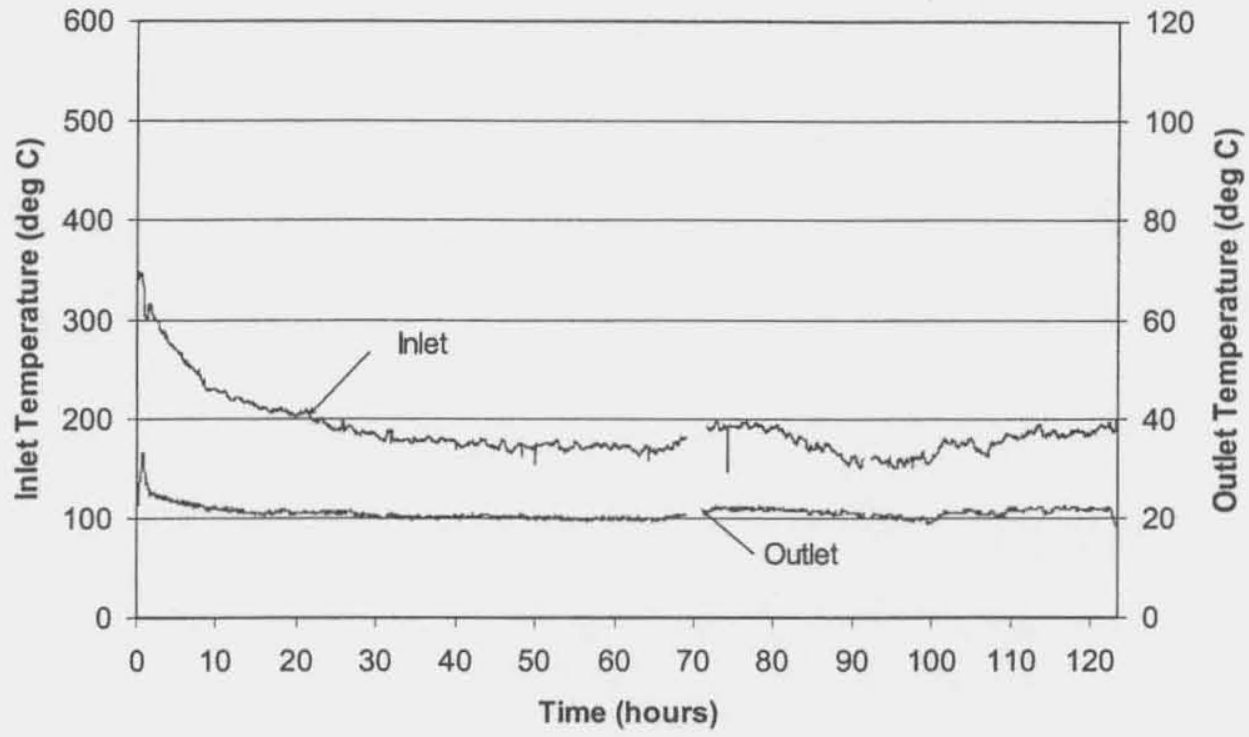

Figure 4.53. SBS gas inlet and outlet temperatures during Test 9.

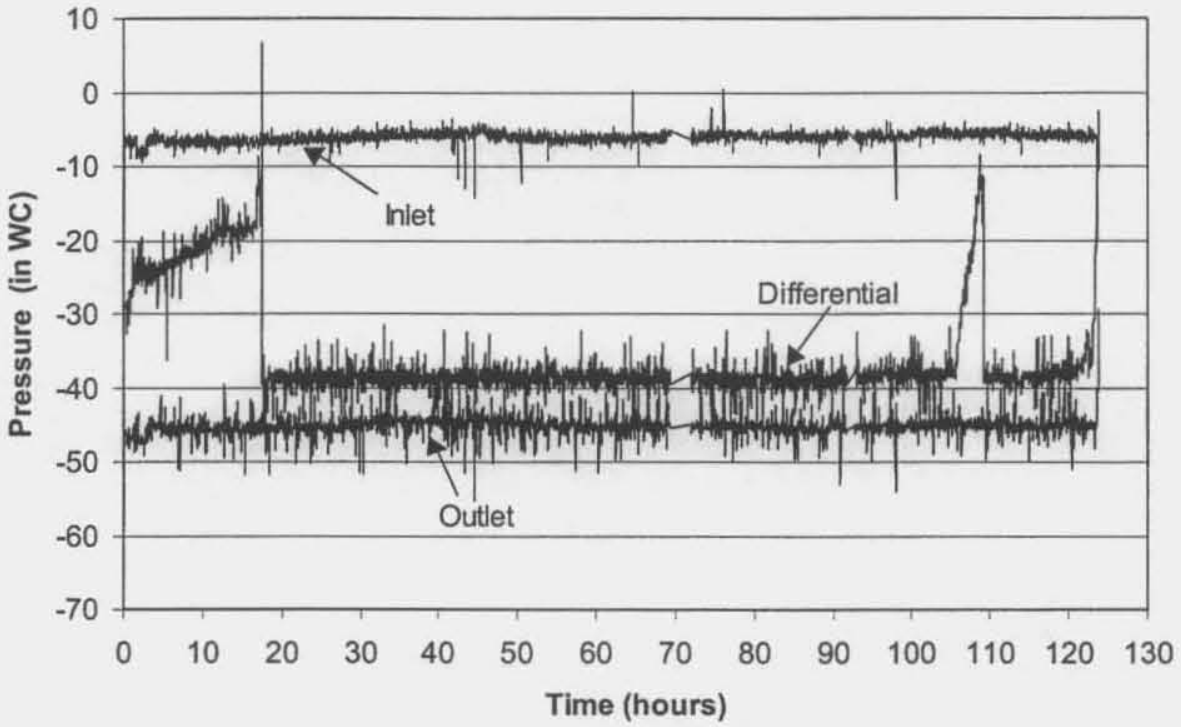

Figure 4.54. SBS inlet, outlet, and differential pressures during Test 9. Note: Differential pressure sensor was clogged for the first 17.5 hours and from hours 106 to 109. 
The Catholic University of America

Vitreous State Laboratory
DuraMelter 1200 Tests with AZ-101HLW Simulants Final Report, VSL-02R0100-2, Rev.1

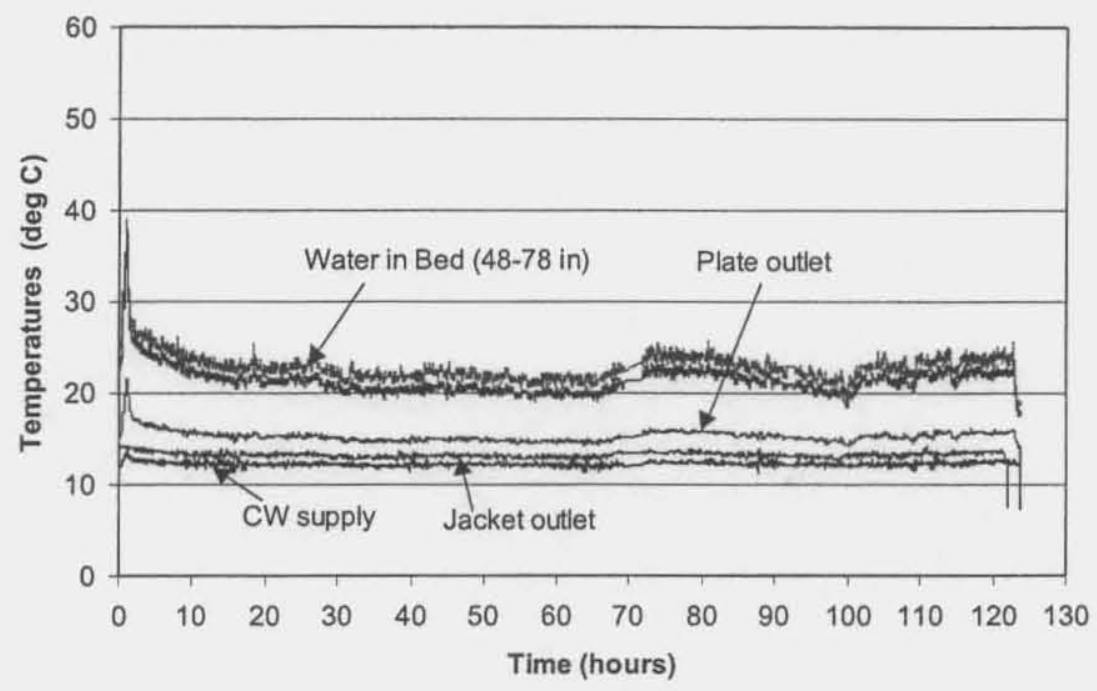

Figure 4.55. SBS cooling water and bed temperatures for Test 9 (cooling water data averaged over 10 minutes ( 5 measurements)).

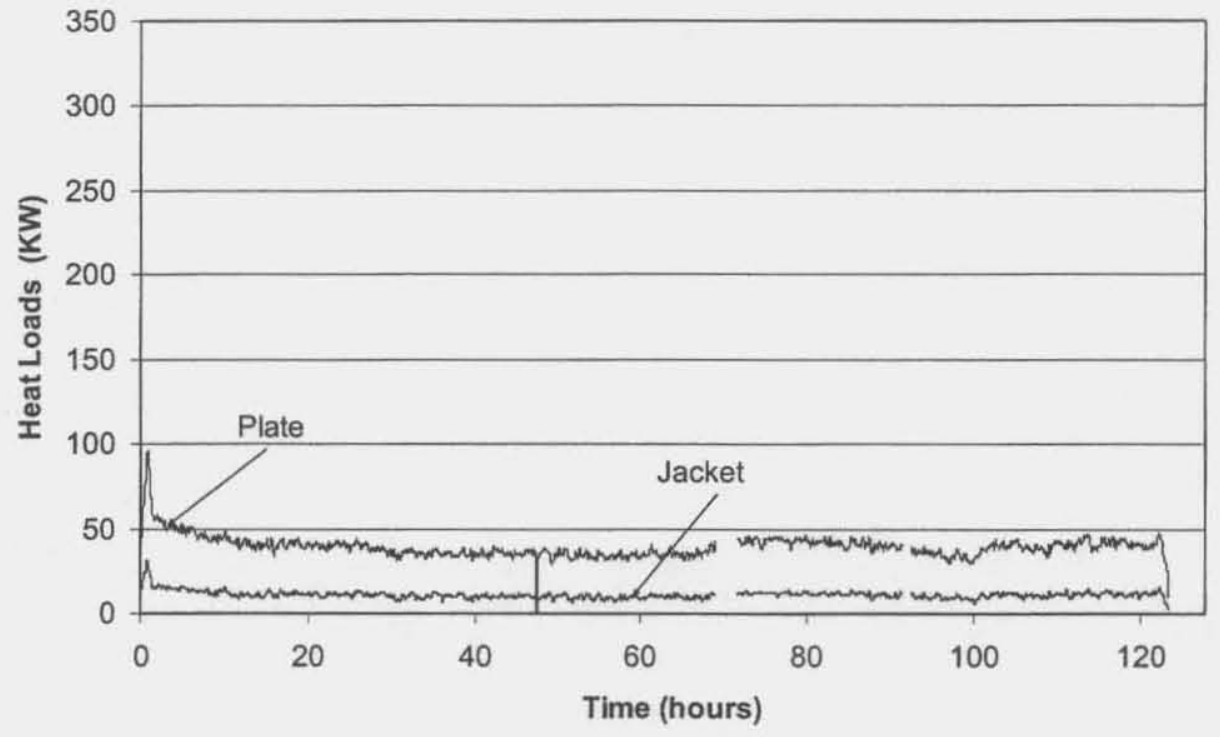

Figure 4.56. Calculated heat loads on the cooling water jacket and plate heat exchanger for Test 9. 


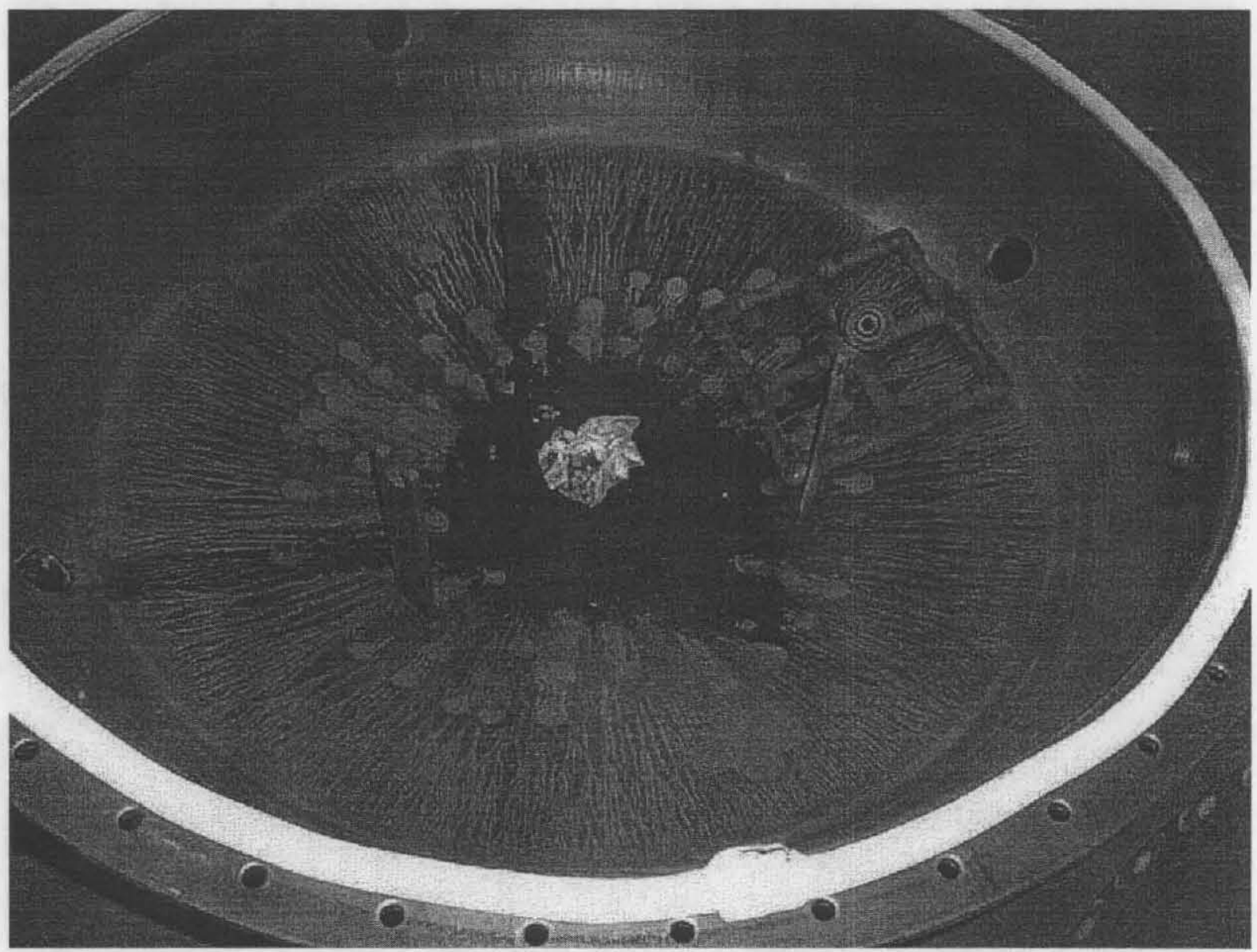

Figure 4.57. Deposits in the SBS bottom dish (Test 9). 


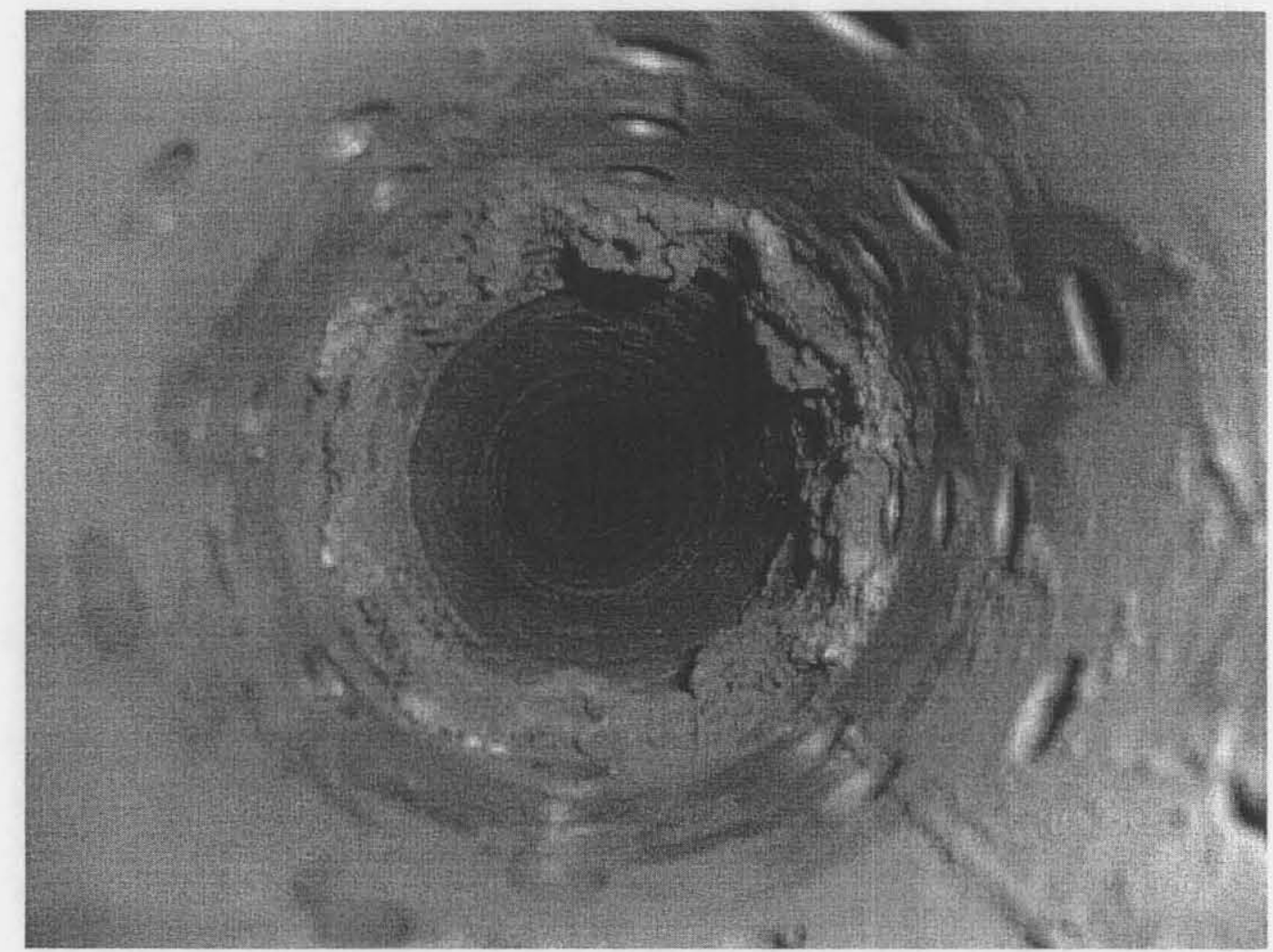


The Catholic University of America Vitreous State Laboratory
DuraMelter 1200 Tests with AZ-101HLW Simulants Final Report, VSL-02R0100-2, Rev.1

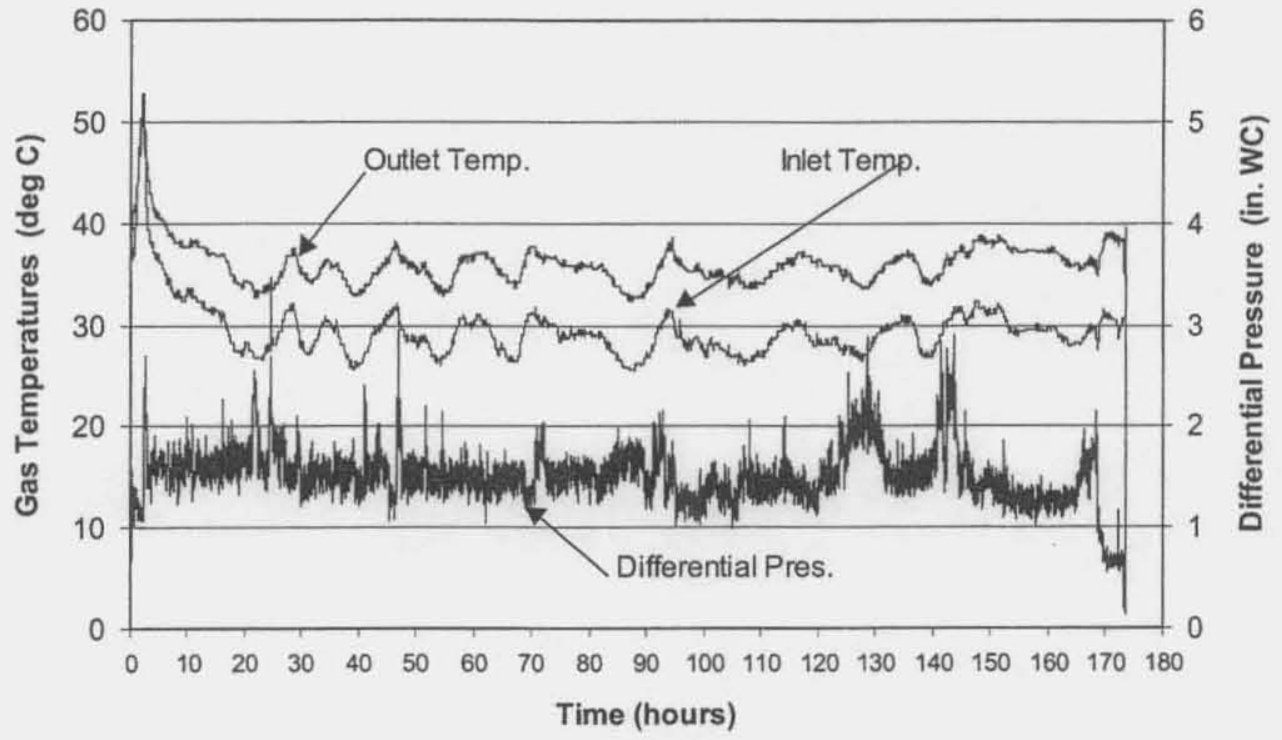

Figure 4.59. WESP inlet and outlet temperatures and differential pressure across the WESP measured during Test 1.

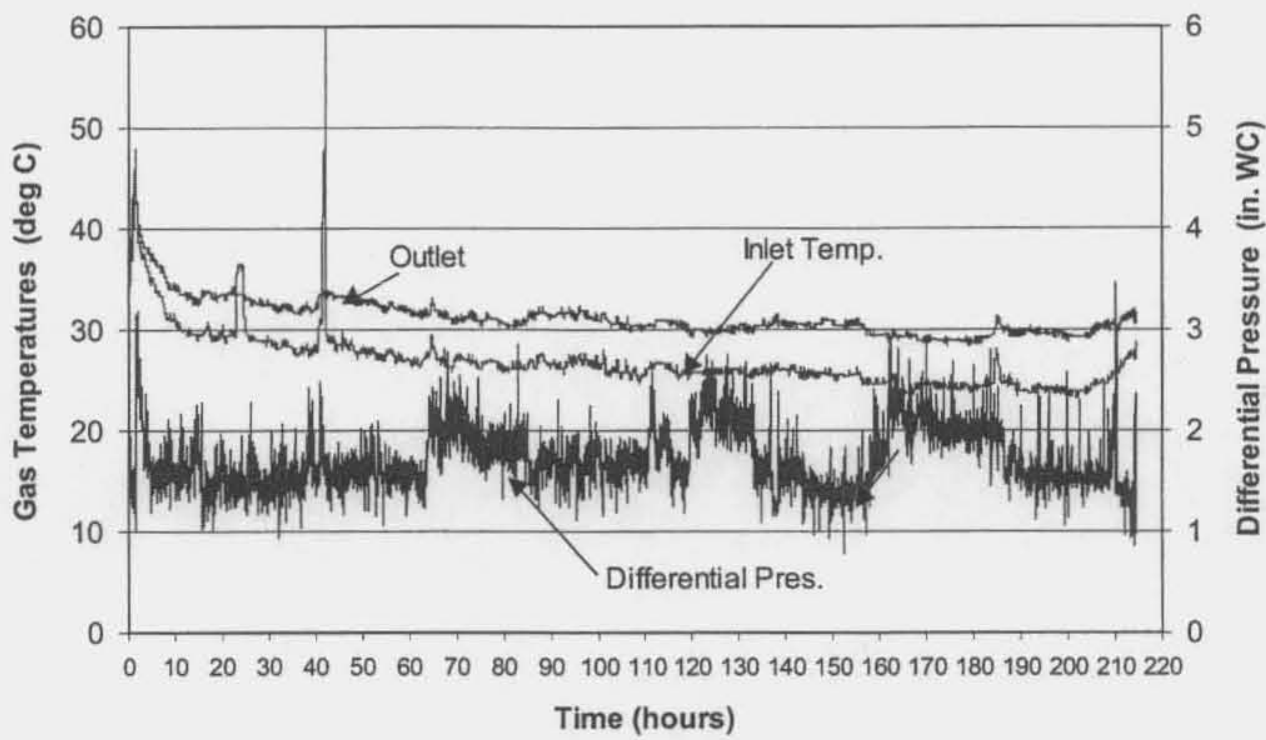

Figure 4.60. WESP inlet and outlet temperatures and differential pressure across the WESP measured during Test 2. 
The Catholic University of America Vitreous State Laboratory
DuraMelter 1200 Tests with AZ-101HLW Simulants Final Report, VSL-02R0100-2, Rev.1

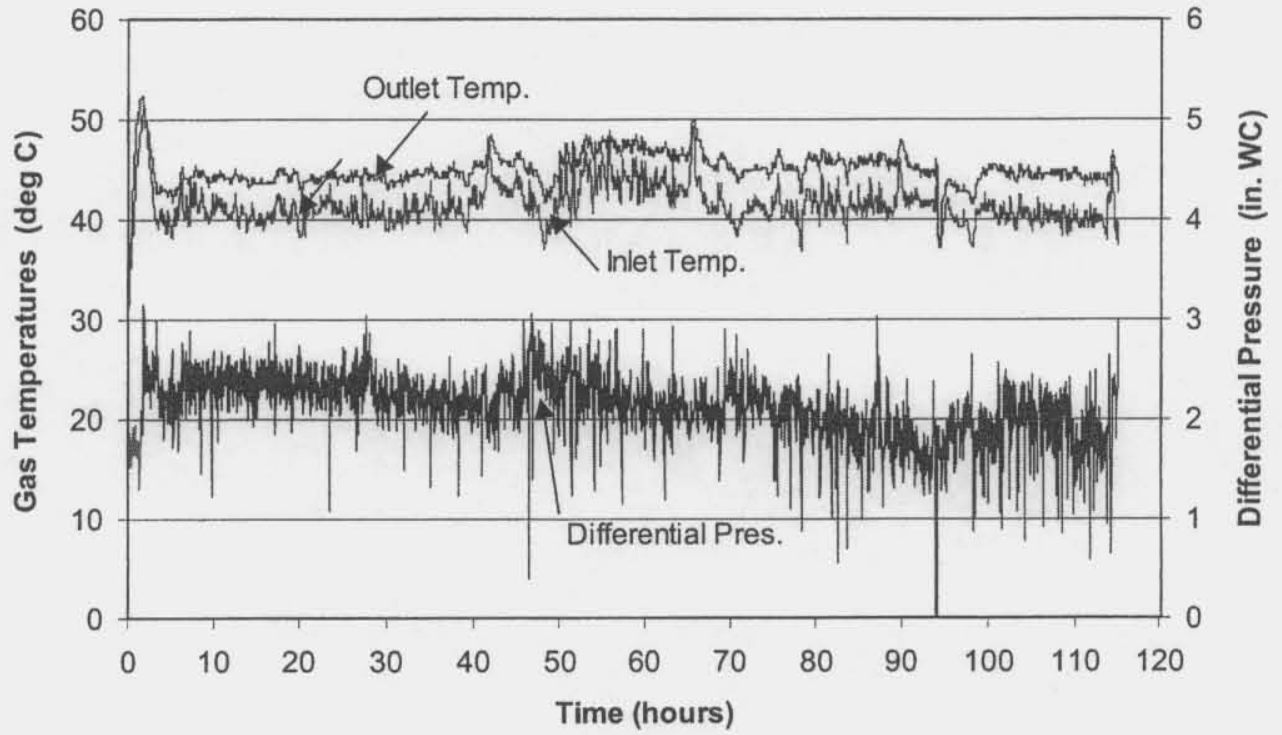

Figure 4.61. WESP inlet and outlet temperatures and differential pressure across the WESP measured during Test 3 .

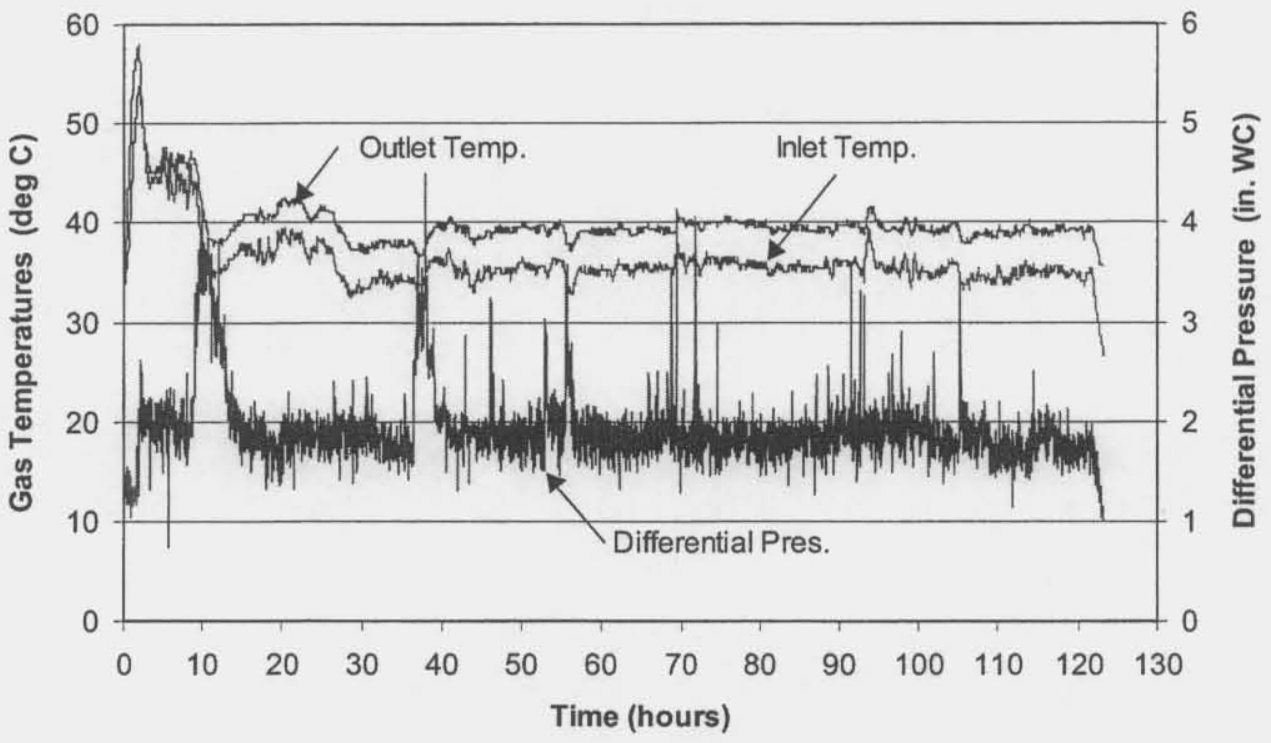

Figure 4.62. WESP inlet and outlet temperatures and differential pressure across the WESP measured during Test 4. 

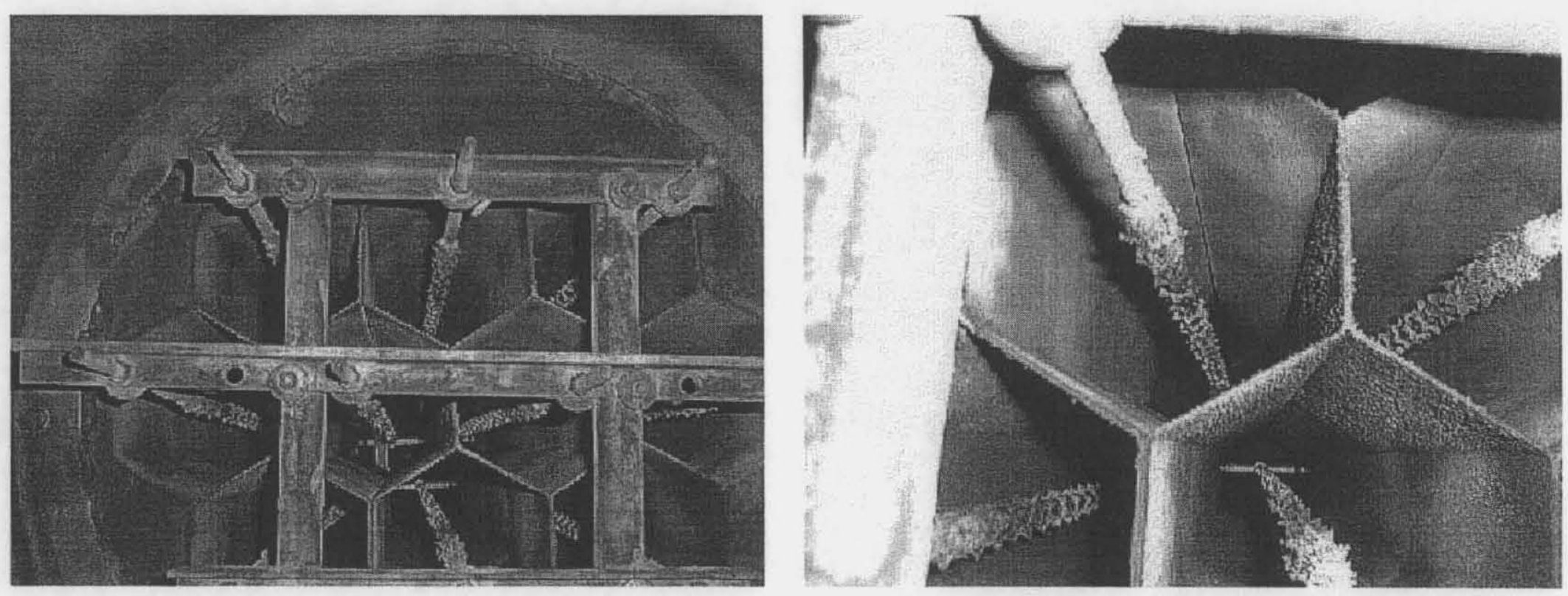

Figure 4.63. Views of WESP collector plates and rods before wash-down at the end of Test 4 . 
The Catholic University of America Vitreous State Laboratory
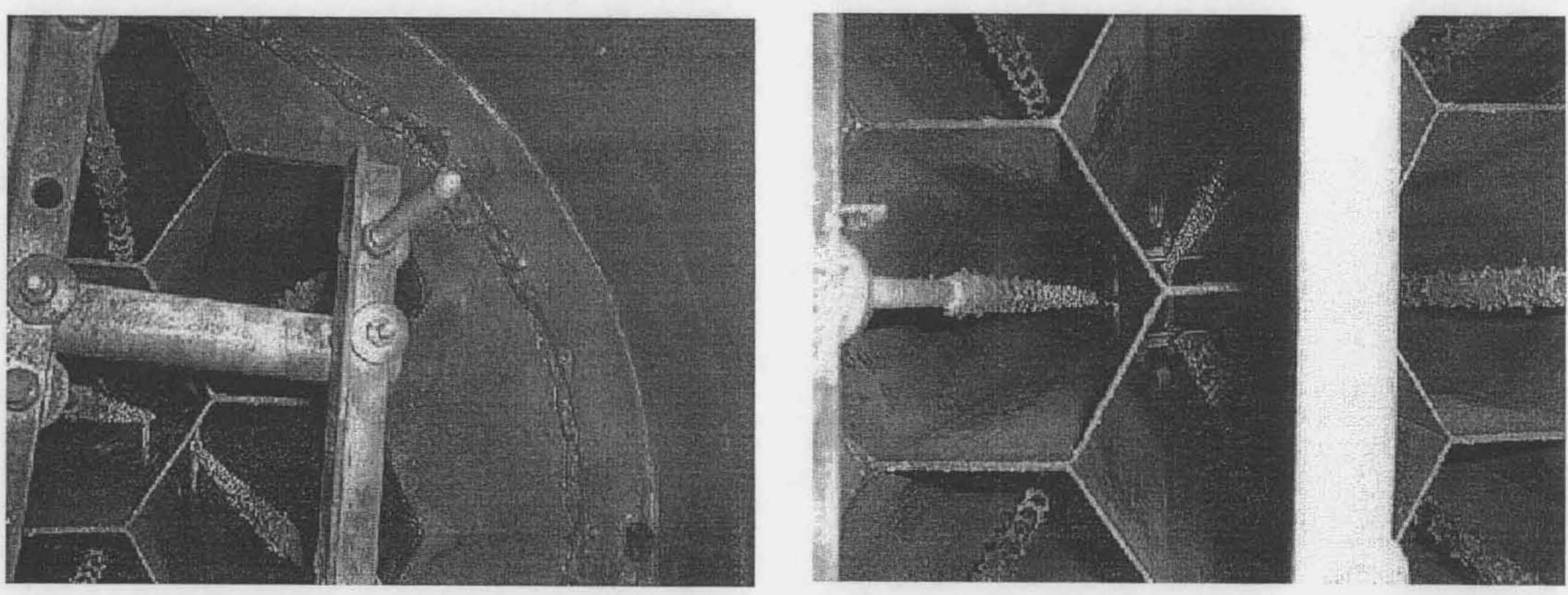

Figure 4.64. Views of WESP collector plates and rods after wash-down at the end of Test 4 . 
The Catholic University of America Vitreous State Laboratory
DuraMelter 1200 Tests with AZ-101HLW Simulants Final Report, VSL-02R0100-2, Rev.1

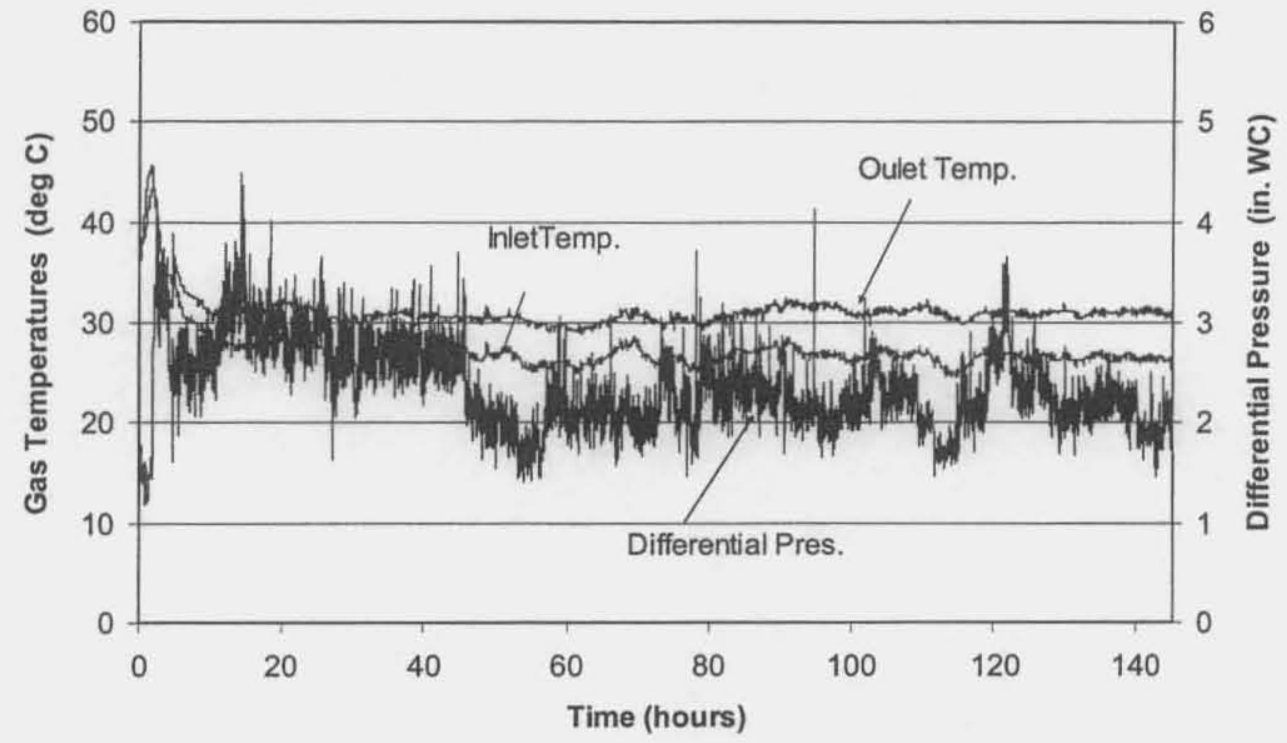

Figure 4.65. WESP inlet and outlet temperatures and differential pressure across the WESP measured during Test 5.

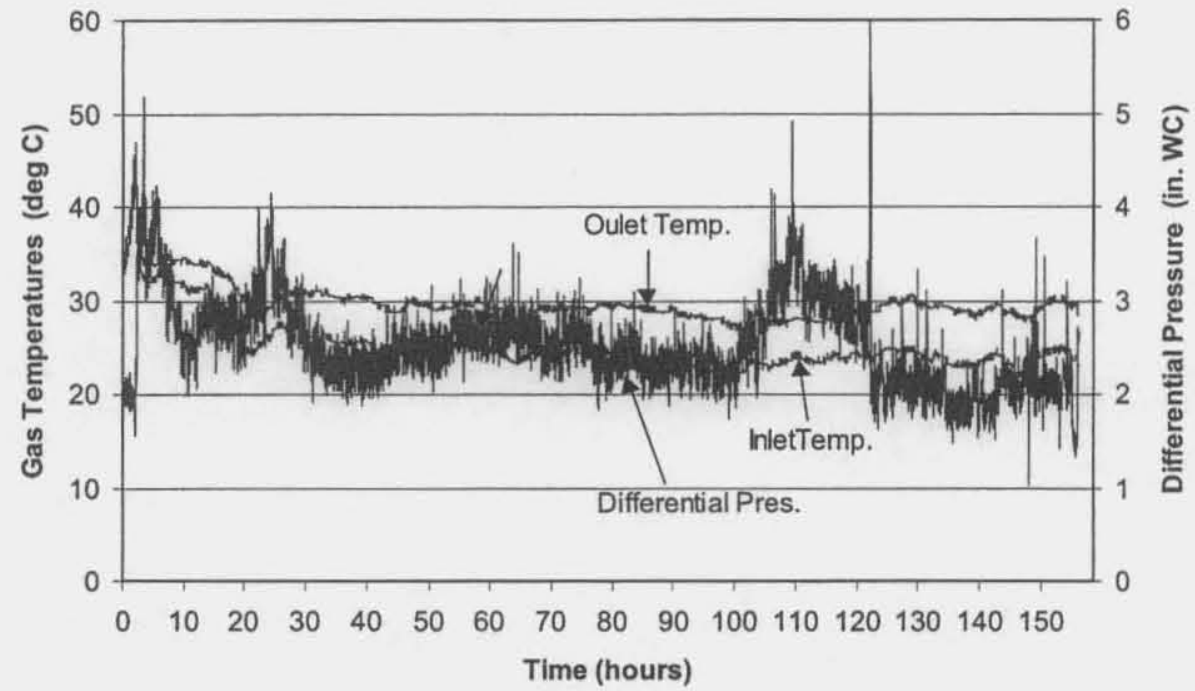

Figure 4.66. WESP inlet and outlet temperatures and differential pressure across the WESP measured during Test 6. 
The Catholic University of America

Vitreous State Laboratory
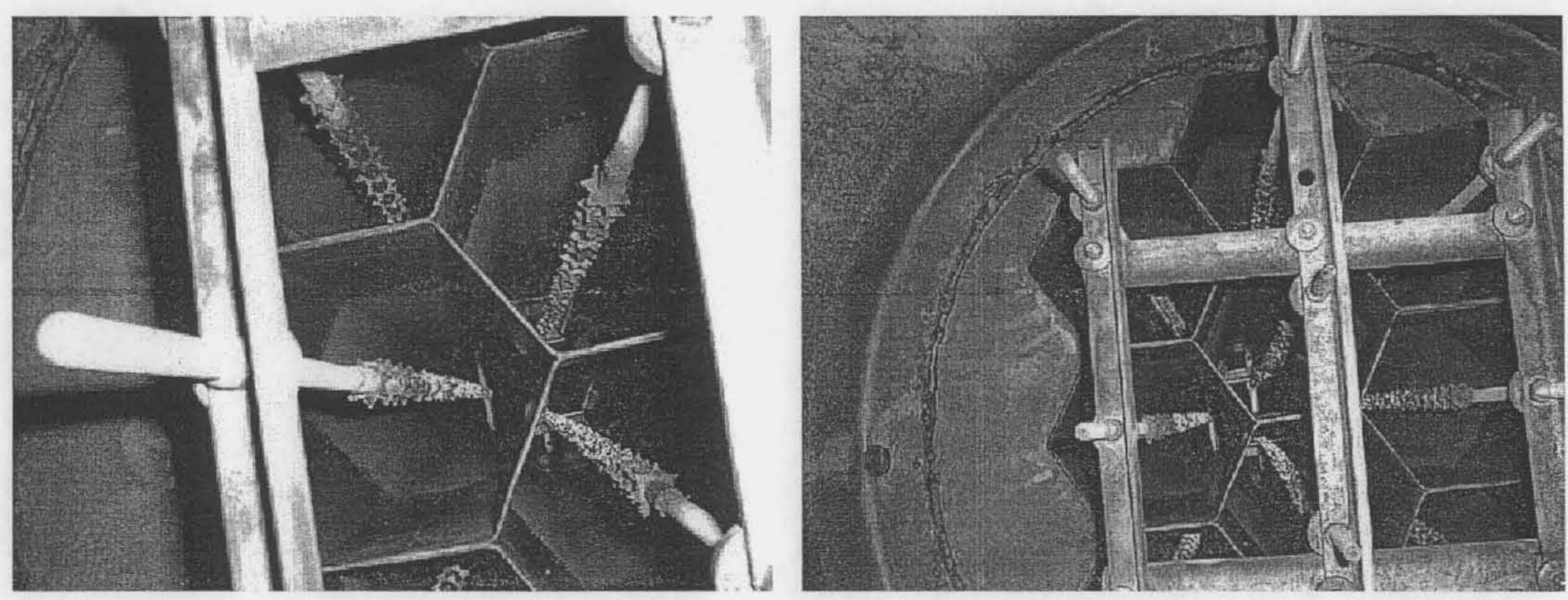

Figure 4.67. Views of WESP collector plates and rods before wash-down at the end of Test 6. 

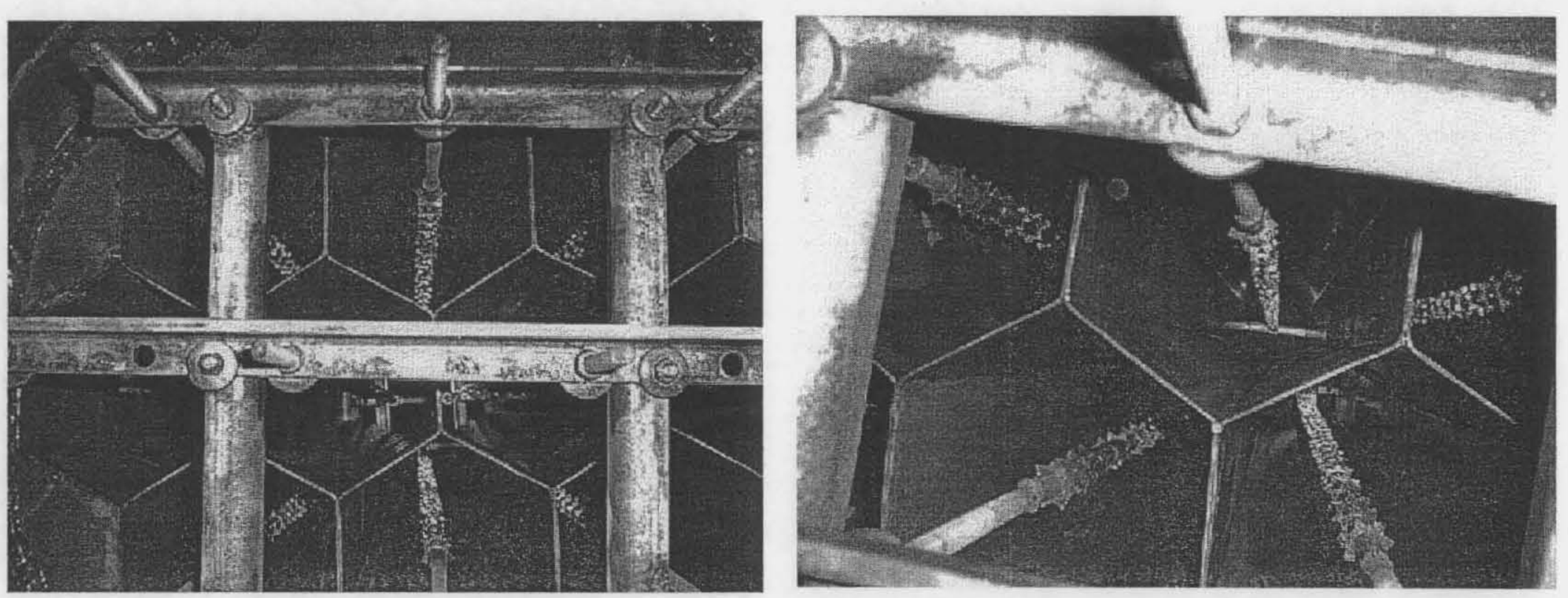

Figure 4.68. Views of WESP collector plates and rods after wash-down at the end of Test 6. 
The Catholic University of America Vitreous State Laboratory
DuraMelter 1200 Tests with AZ-101HLW Simulants Final Report, VSL-02R0100-2, Rev.1

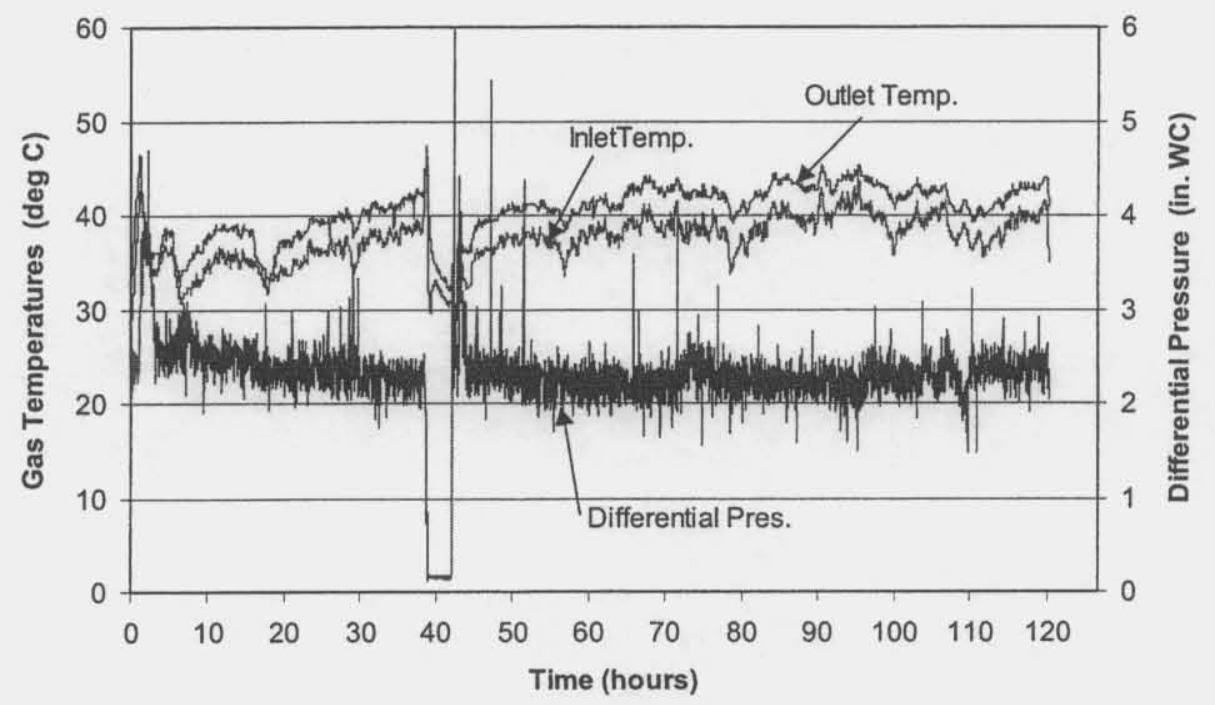

Figure 4.69. WESP inlet and outlet temperatures and differential pressure across the WESP measured during Test 7. Note: A cooling water line break necessitated interruption of feeding and diversion of off-gas to bypass line between 38th and 43th hours of operations. 

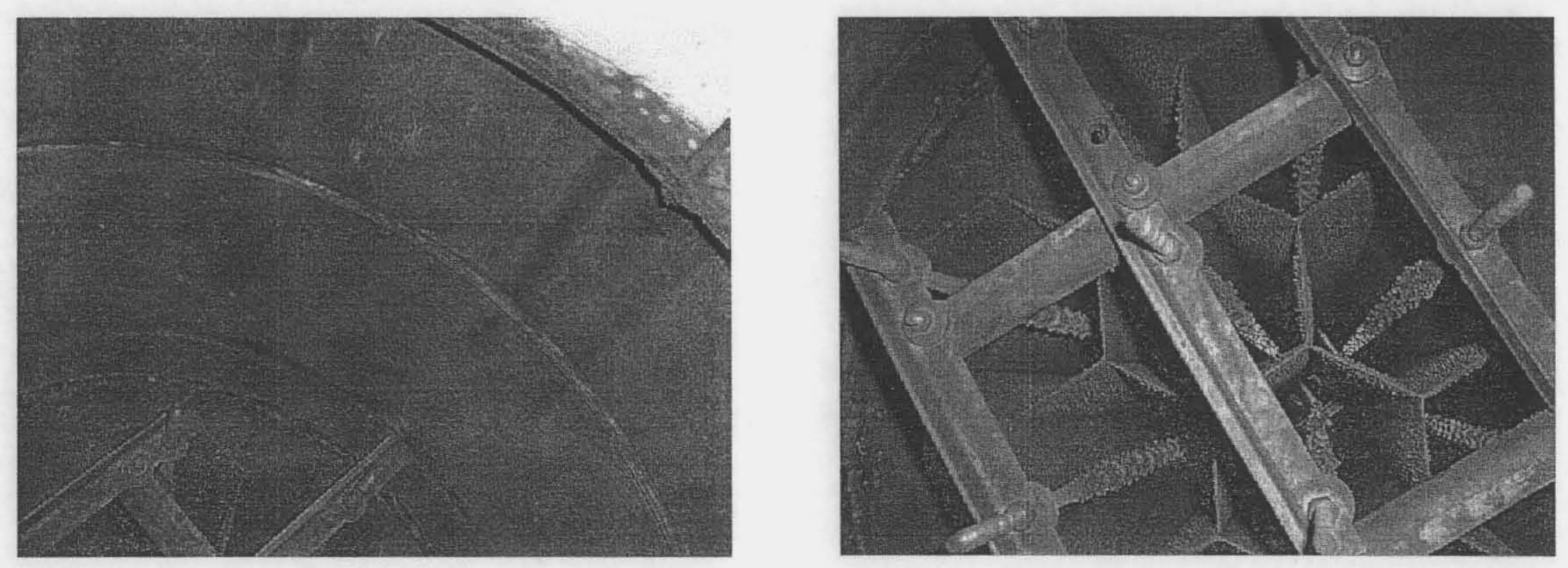

Figure 4.70. Views of WESP collector plates and rods before wash-down at the end of Test 7. 

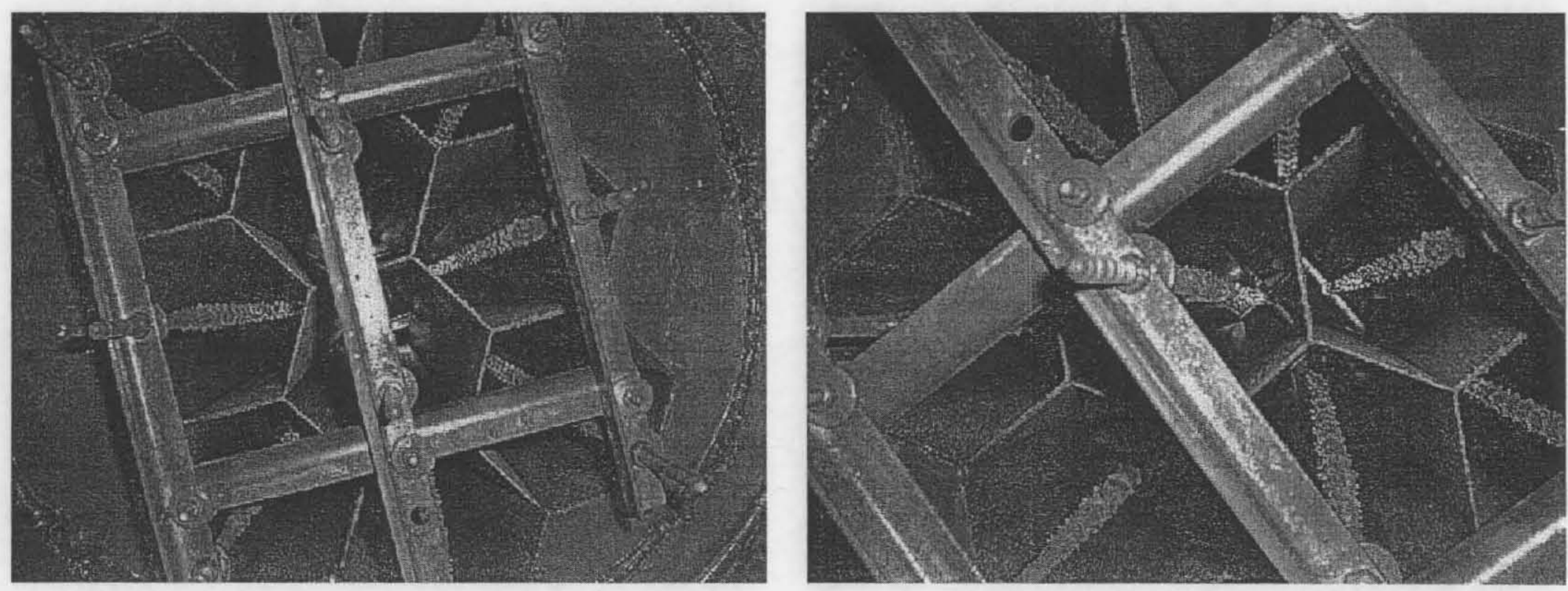

Figure 4.71. Views of WESP collector plates and rods after wash-down at the end of Test 7. 
The Catholic University of America Vitreous State Laboratory
DuraMelter 1200 Tests with AZ-101HLW Simulants Final Report, VSL-02R0100-2, Rev.1

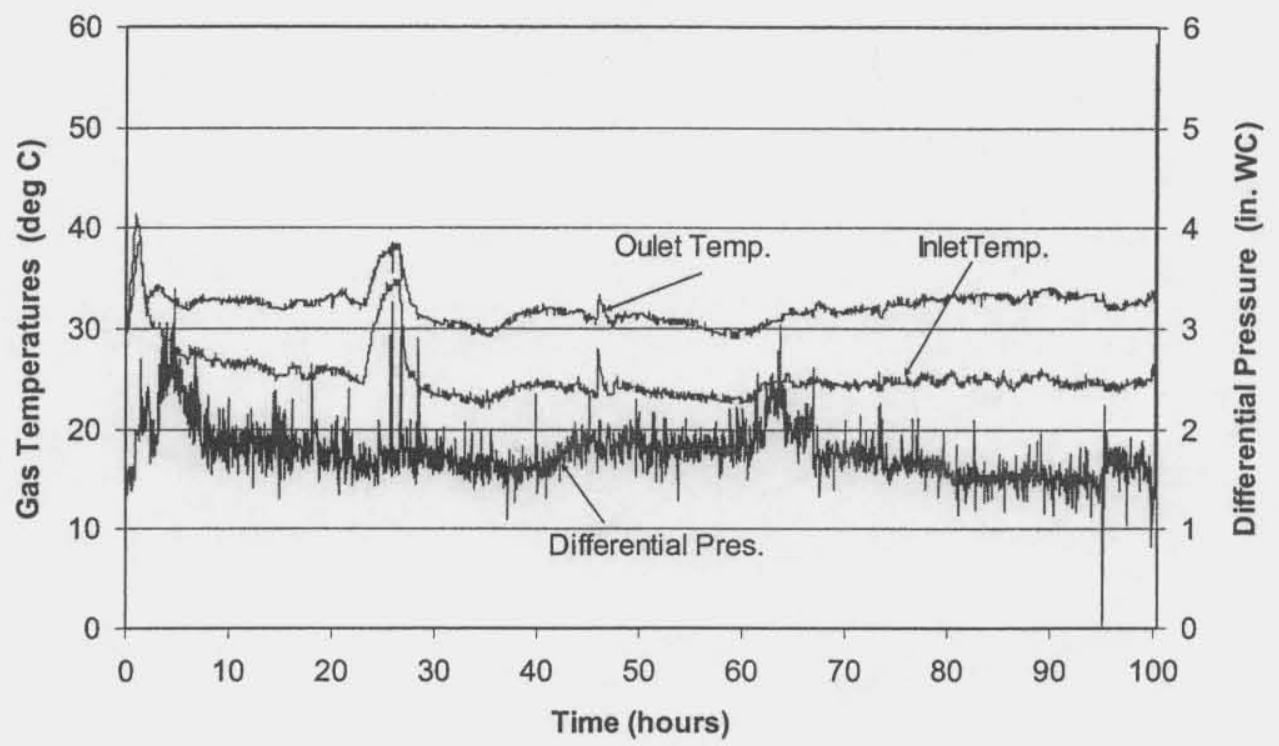

Figure 4.72. WESP inlet and outlet temperatures and differential pressure across the WESP measured during Test 8. Note: Between 23 and 28 hours of operations, water circulation in SBS cooling coil was interrupted. 


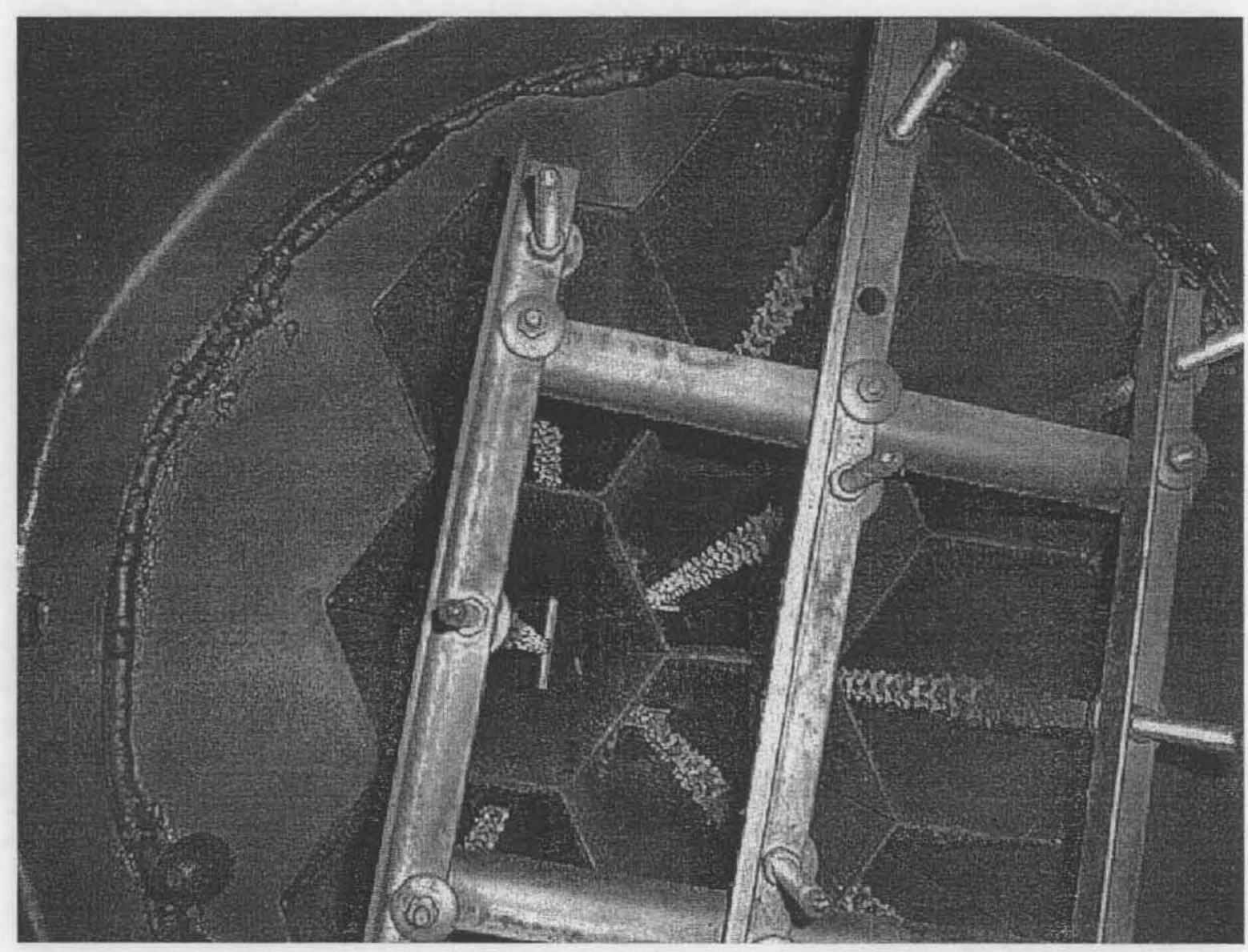

Figure 4.73. View of WESP collector plates and rods before wash-down at the end of Test 8. 


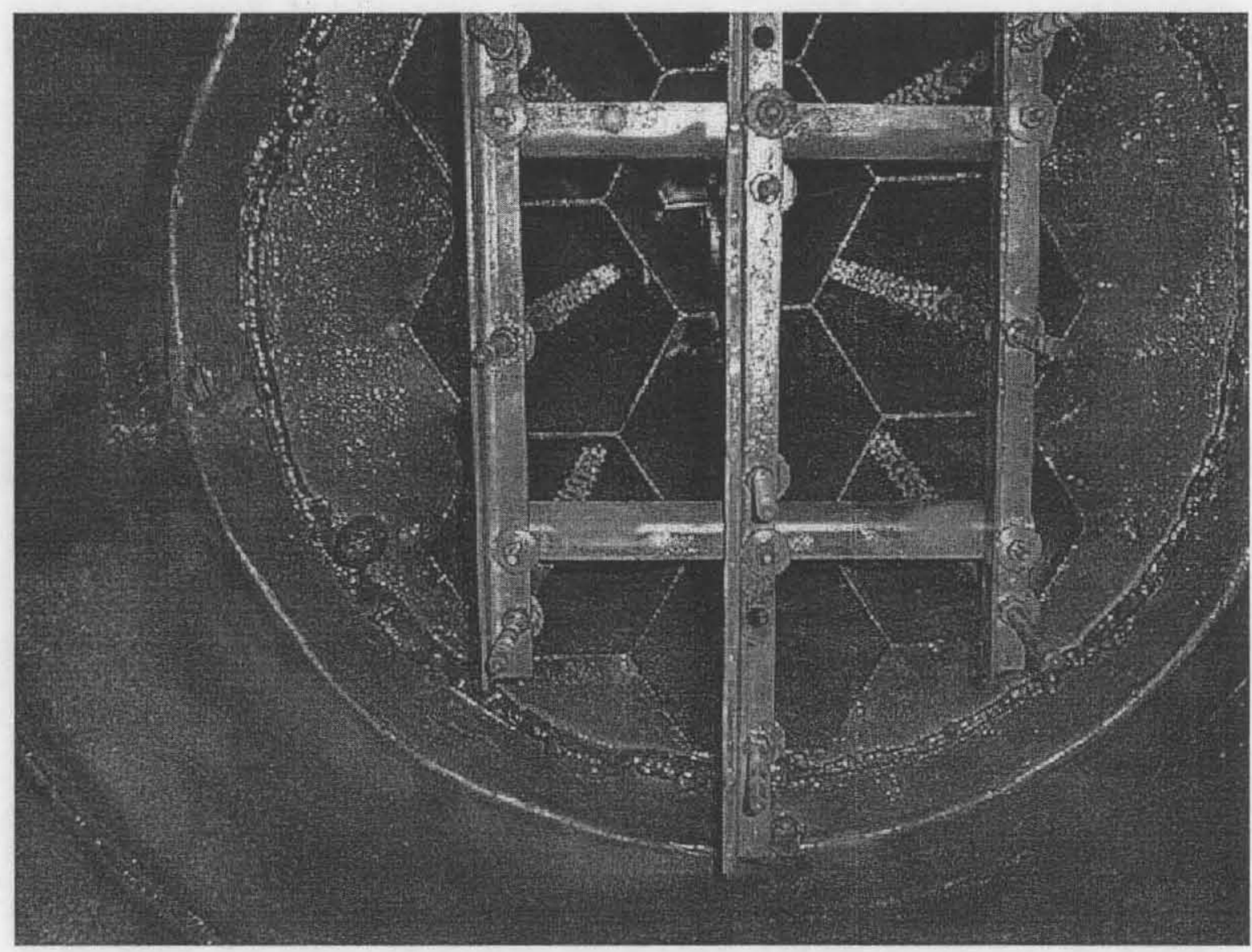

Figure 4.74. View of WESP collector plates and rods after wash-down at the end of Test 8. 
The Catholic University of America Vitreous State Laboratory
DuraMelter 1200 Tests with AZ-101HLW Simulants Final Report, VSL-02R0100-2, Rev.1

TEST \#7 - WESP GAS TEMPERATURES AND PRESSURE

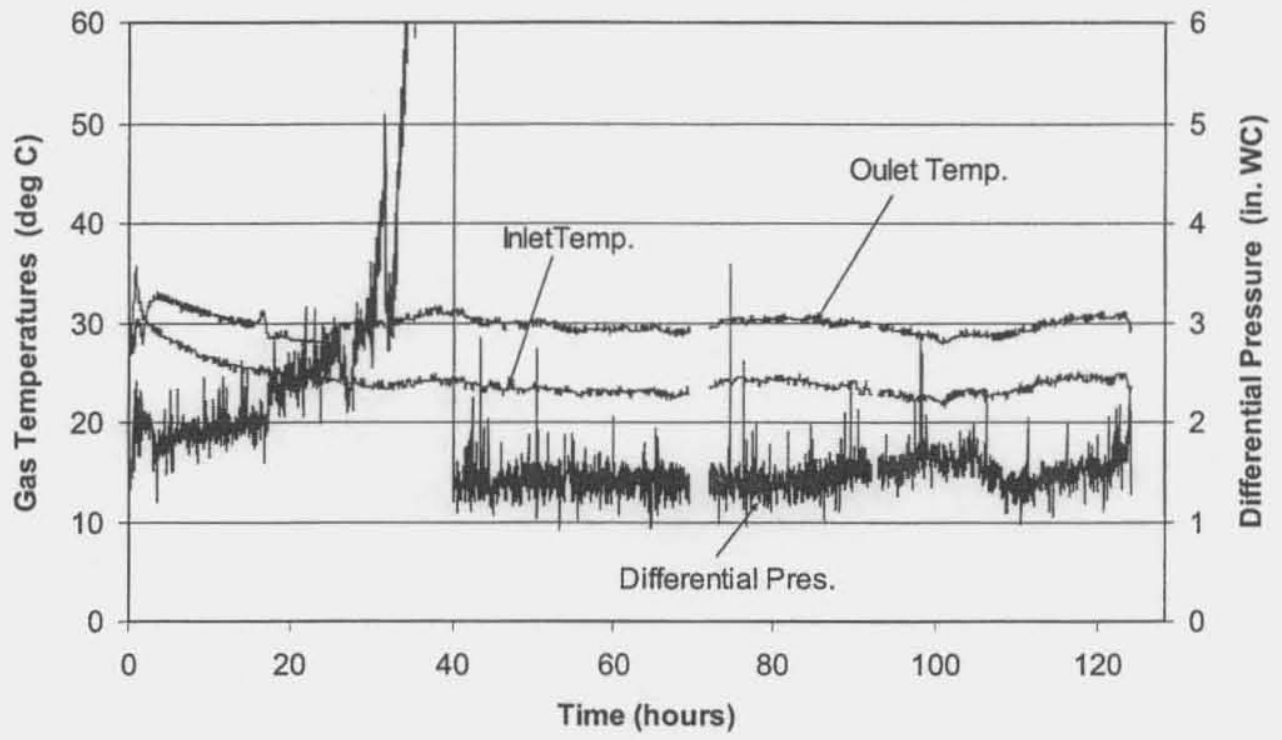

Figure 4.75. WESP inlet and outlet temperatures and differential pressure across the WESP measured during Test 9. (Differential pressure sensor clogged at $\mathbf{3 0}$ hours). 


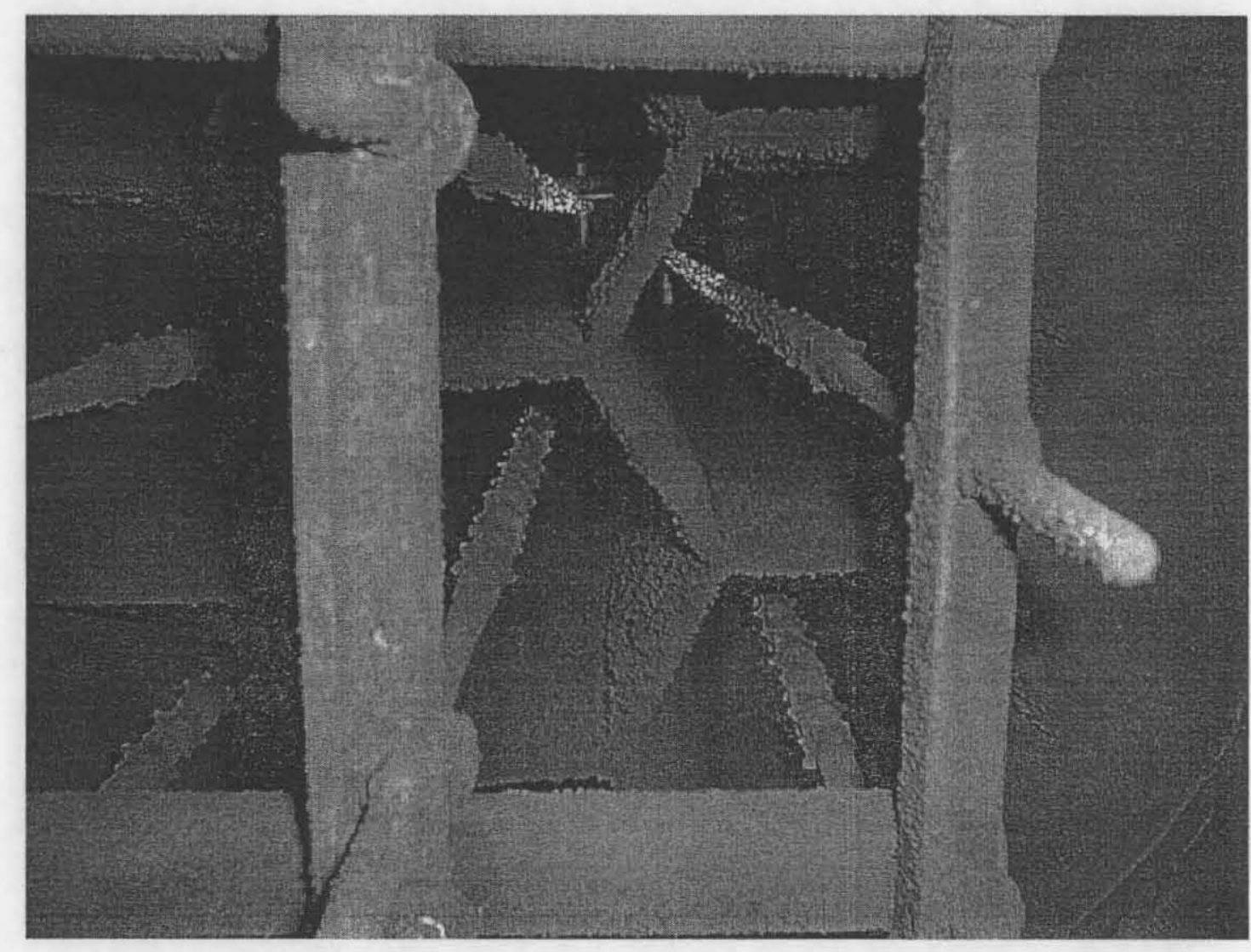

Figure 4.76. View of WESP collector plates and rods before wash-down at the end of Test 9. 


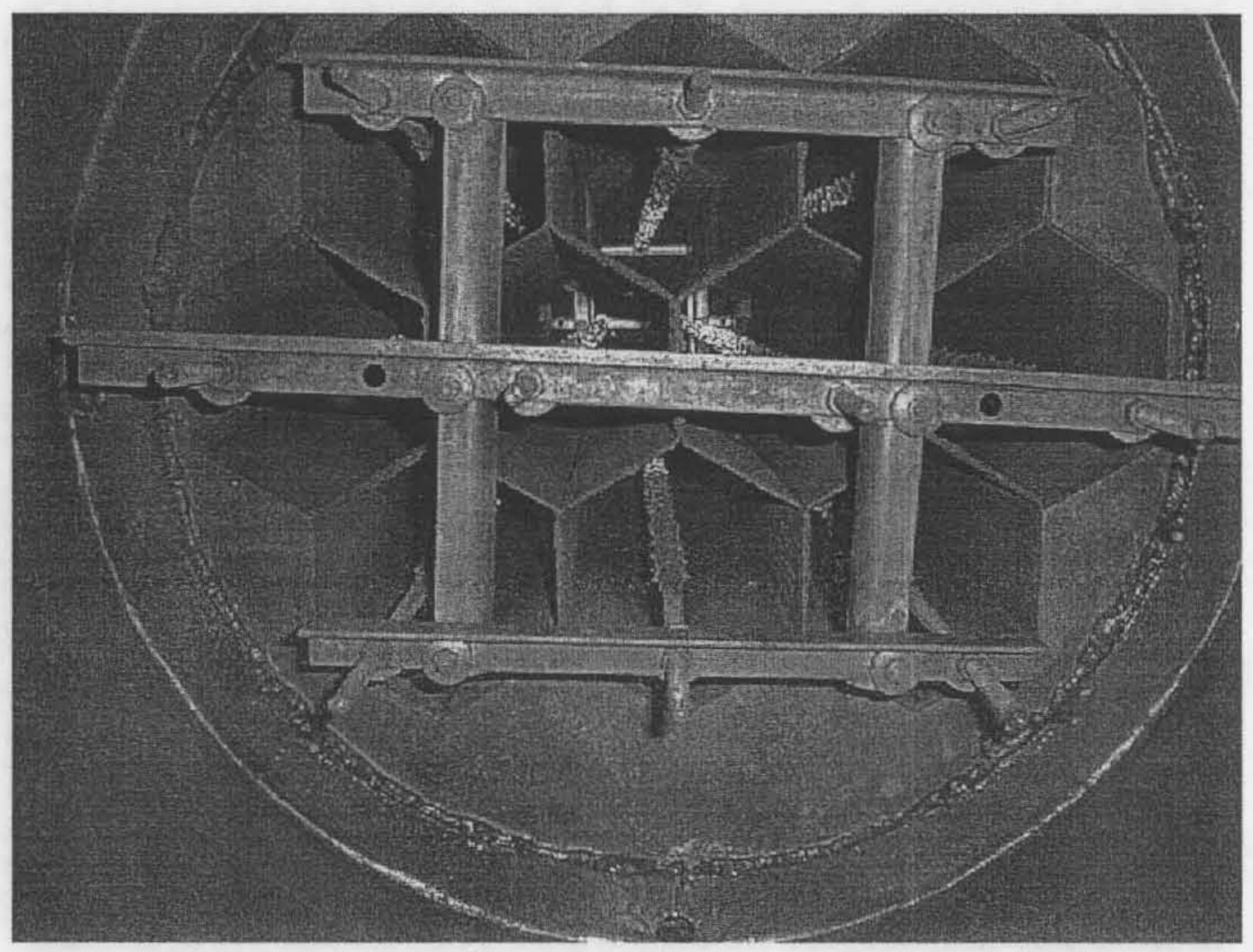

Figure 4.77. View of WESP collector plates and rods after wash-down at the end of Test 9. 


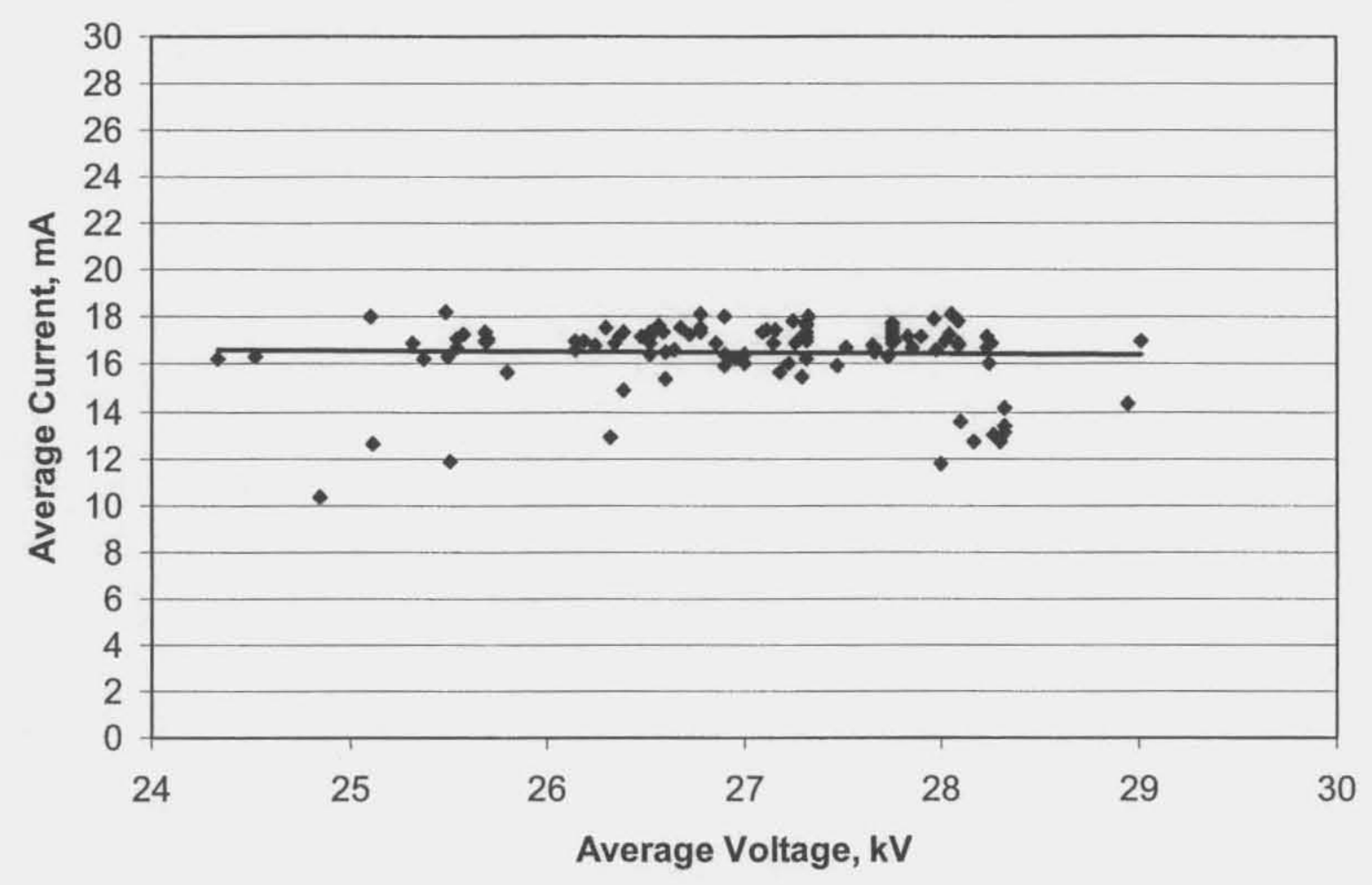

Figure 4.78. Variation of average WESP current with average WESP voltage over all tests. 
The Catholic University of America Vitreous State Laboratory
ORP-51438, Rev. 0

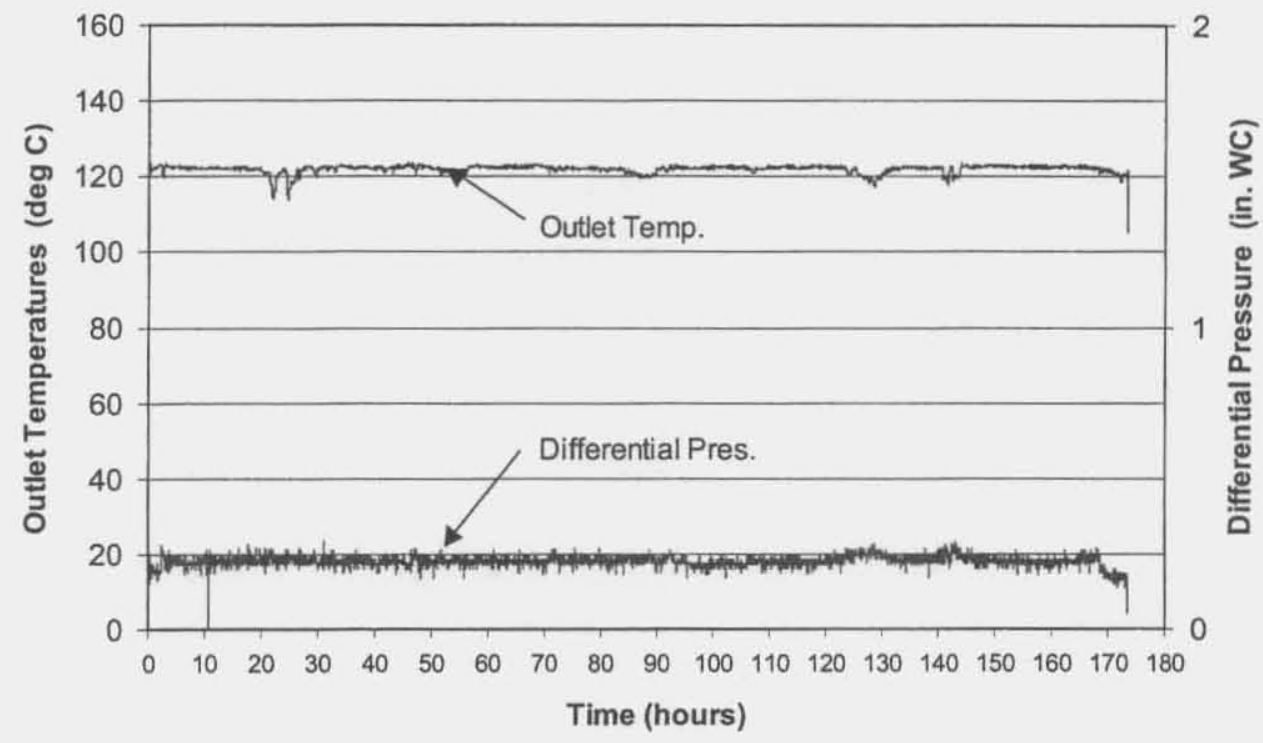

Figure 4.79. Outlet temperature and differential pressure for HEPA \#1 during Test 1.

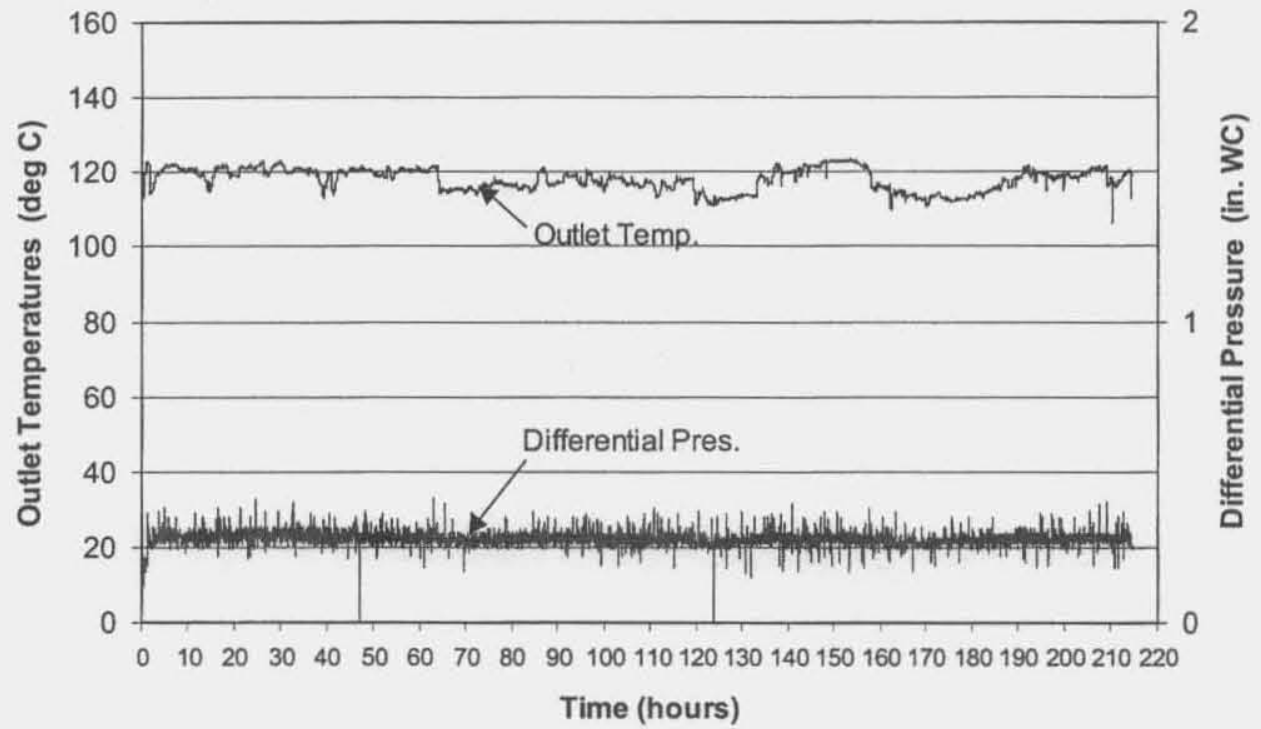

Figure 4.80. Outlet temperature and differential pressure for HEPA \#1 during Test 2. 
The Catholic University of America Vitreous State Laboratory
DuraMelter 1200 Tests with AZ-101HLW Simulants Final Report, VSL-02R0100-2, Rev.1

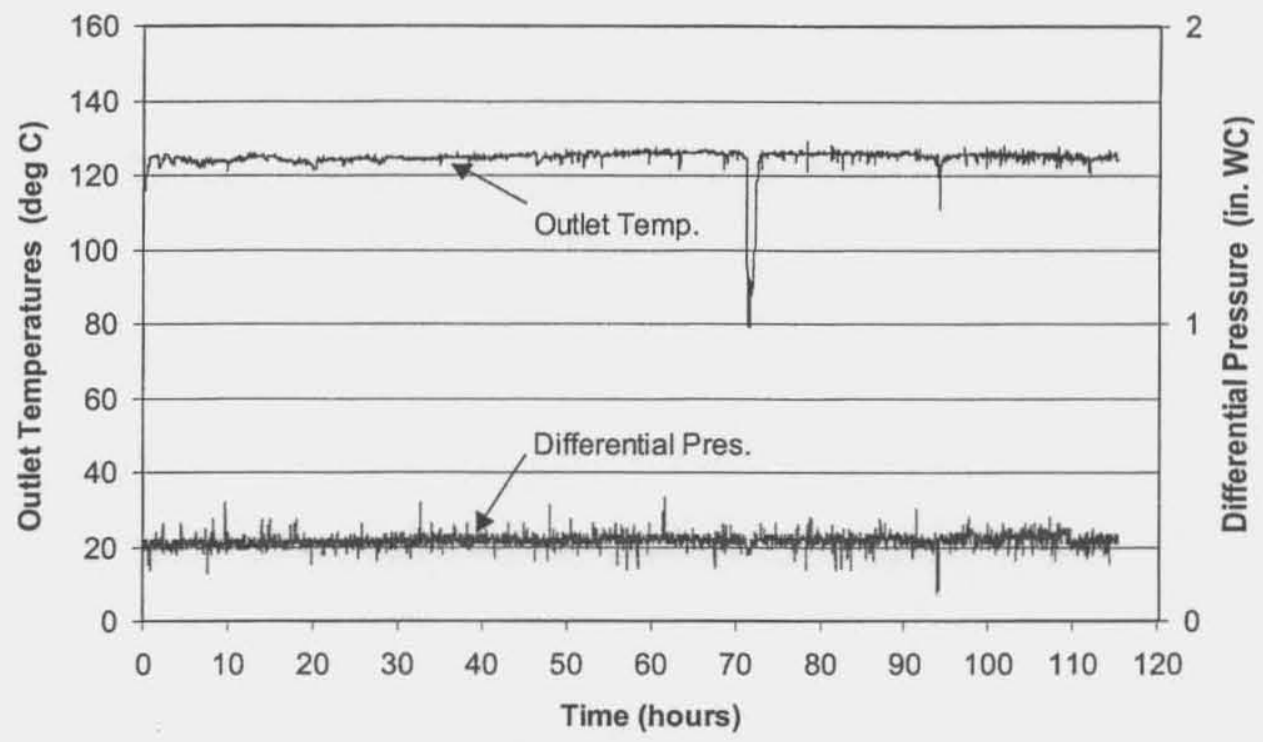

Figure 4.81. Outlet temperature and differential pressure for HEPA \#1 during Test 3.

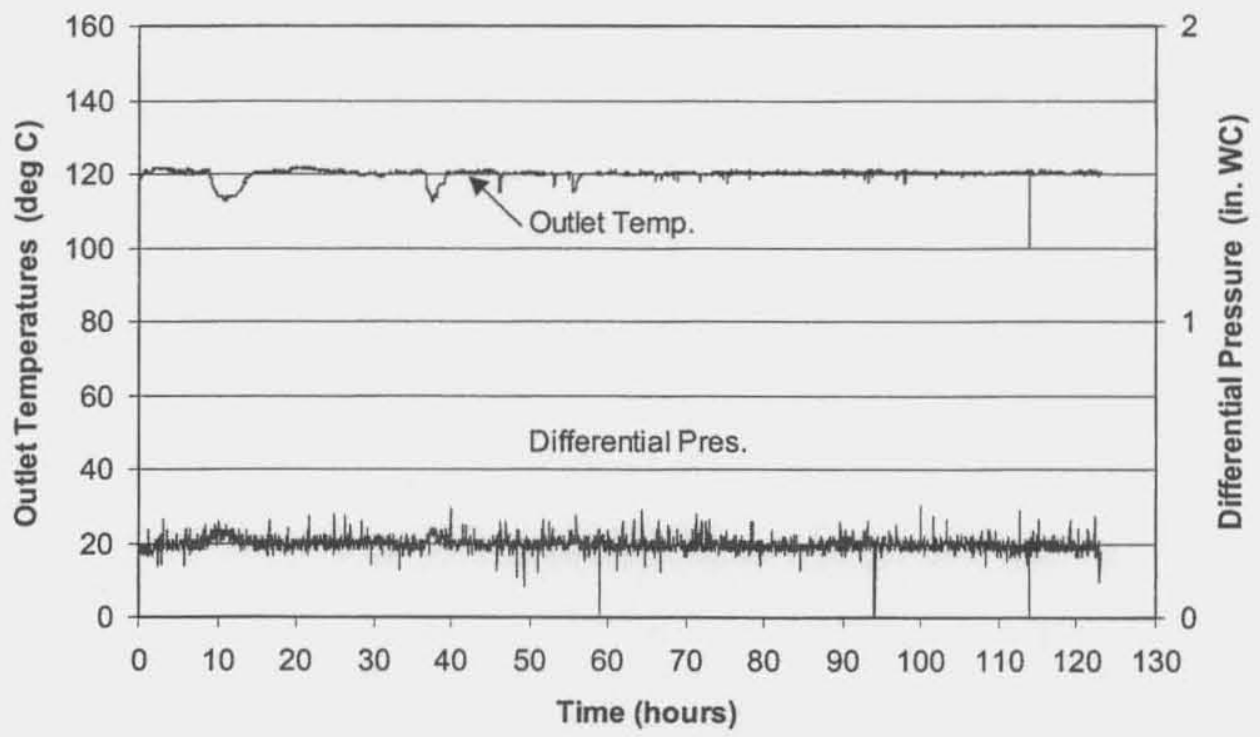

Figure 4.82. Outlet temperature and differential pressure for HEPA \#1 during Test 4. 
The Catholic University of America Vitreous State Laboratory
DuraMelter 1200 Tests with AZ-101HLW Simulants

Final Report, VSL-02R0100-2, Rev.1

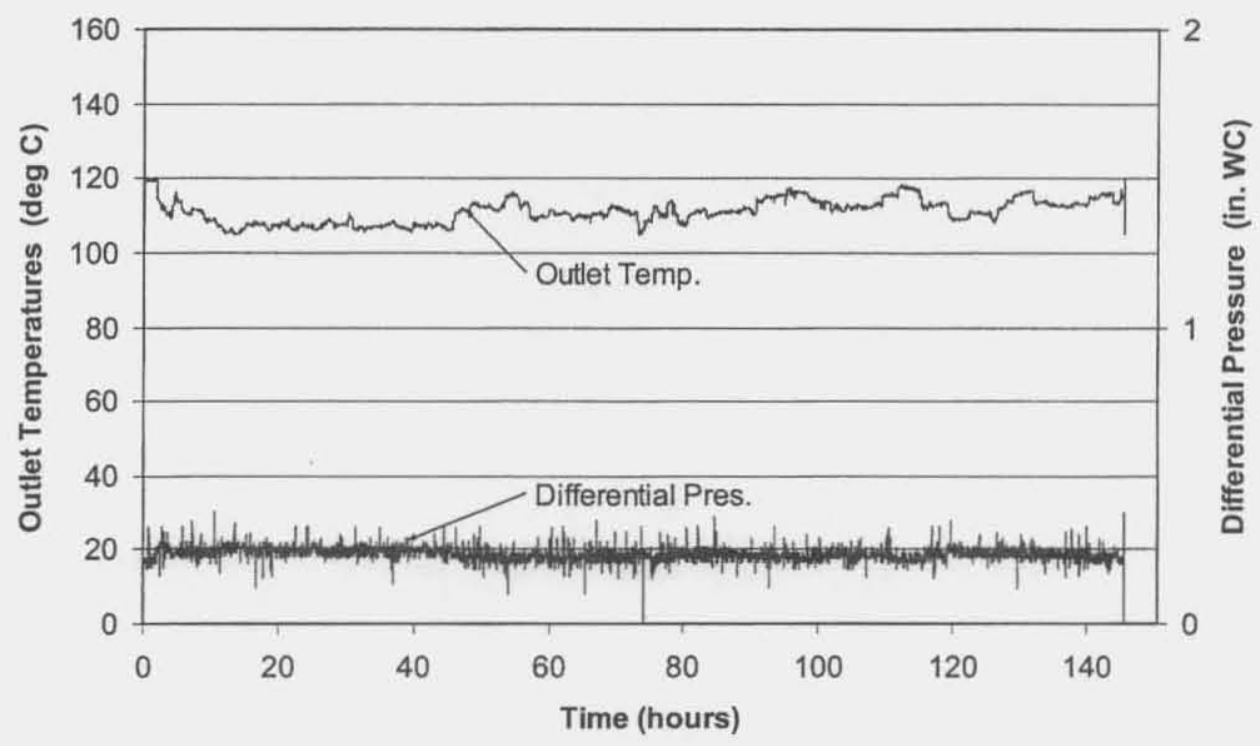

Figure 4.83. Outlet temperature and differential pressure for HEPA \#1 during Test 5.

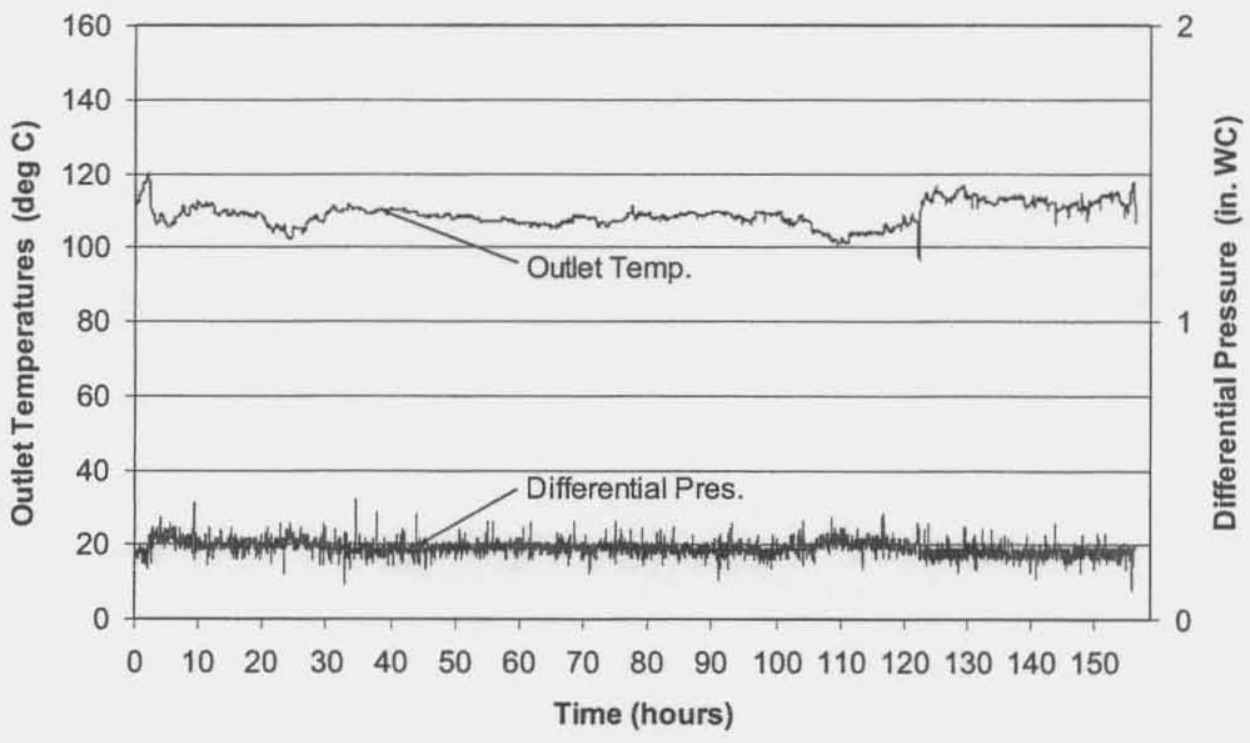

Figure 4.84. Outlet temperature and differential pressure for HEPA \#1 during Test 6 . 
The Catholic University of America Vitreous State Laboratory
ORP-51438, Rev. 0

DuraMelter 1200 Tests with AZ-101HLW Simulants

Final Report, VSL-02R0100-2, Rev.1

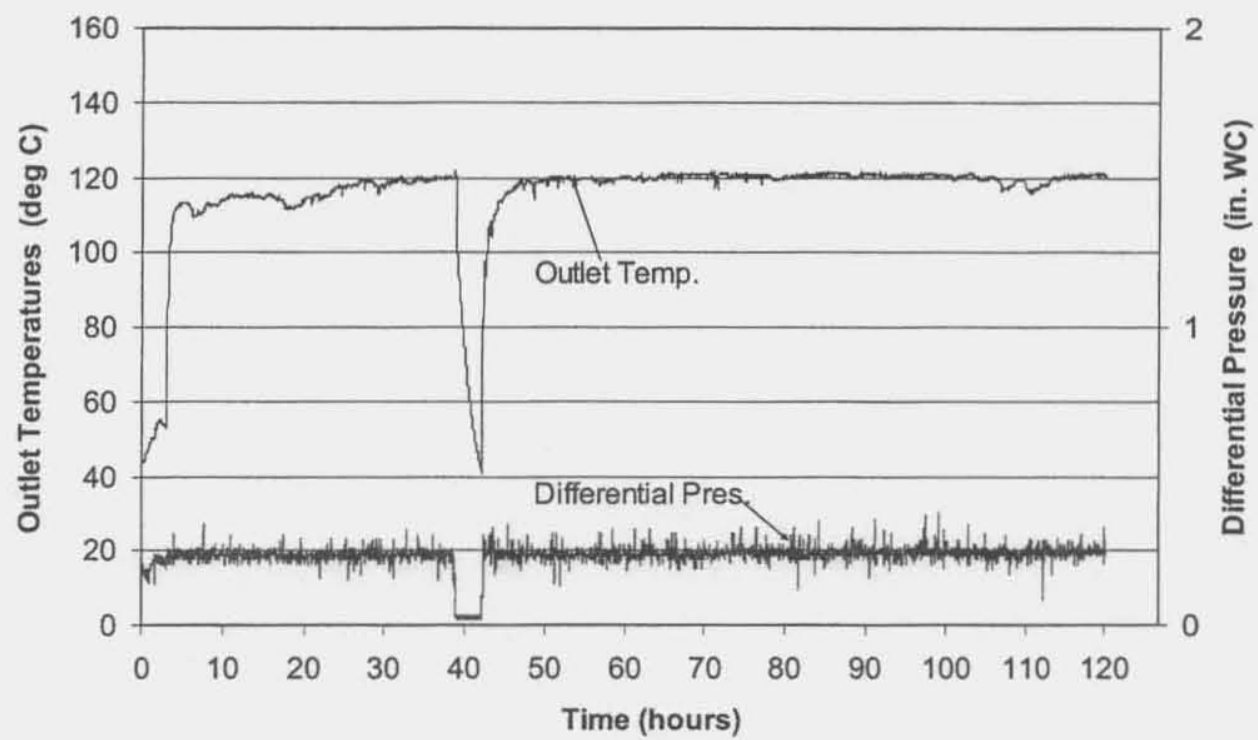

Figure 4.85. Outlet temperature and differential pressure for HEPA \#1 during Test 7. Note: Feed was interrupted from hours 38 to 43.

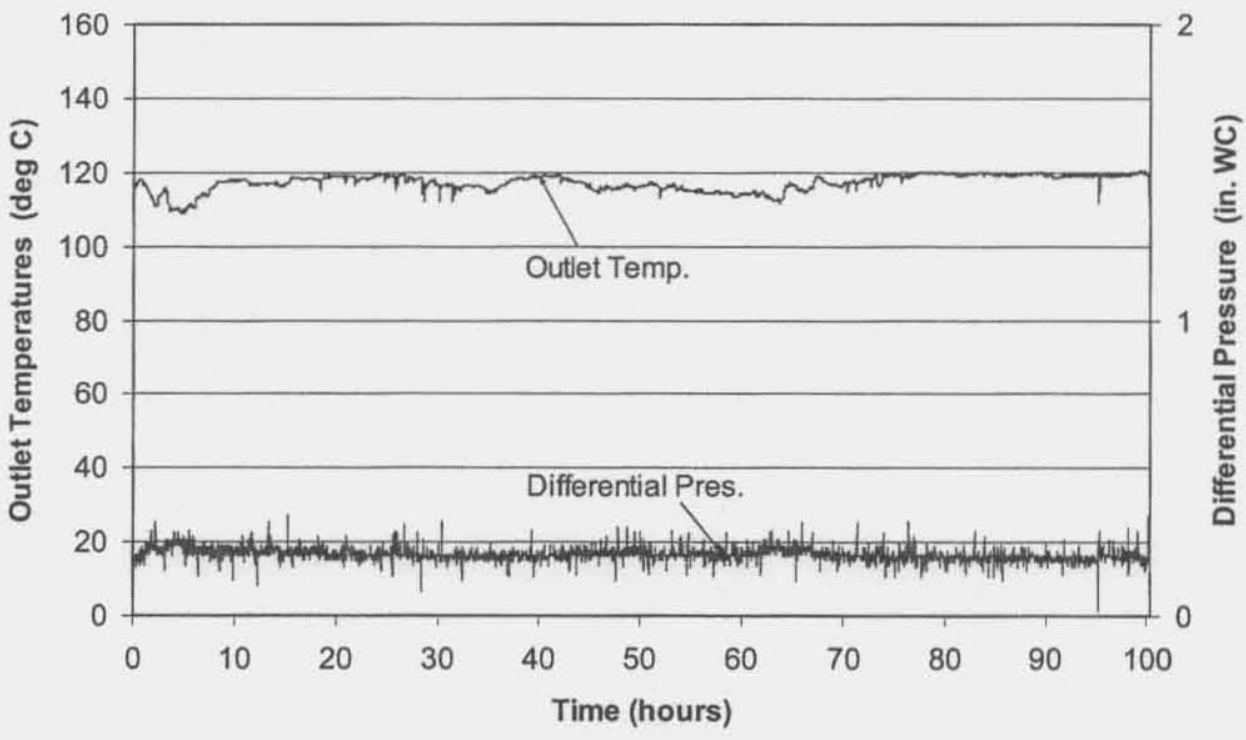

Figure 4.86. Outlet temperature and differential pressure for HEPA \#1 during Test 8. 


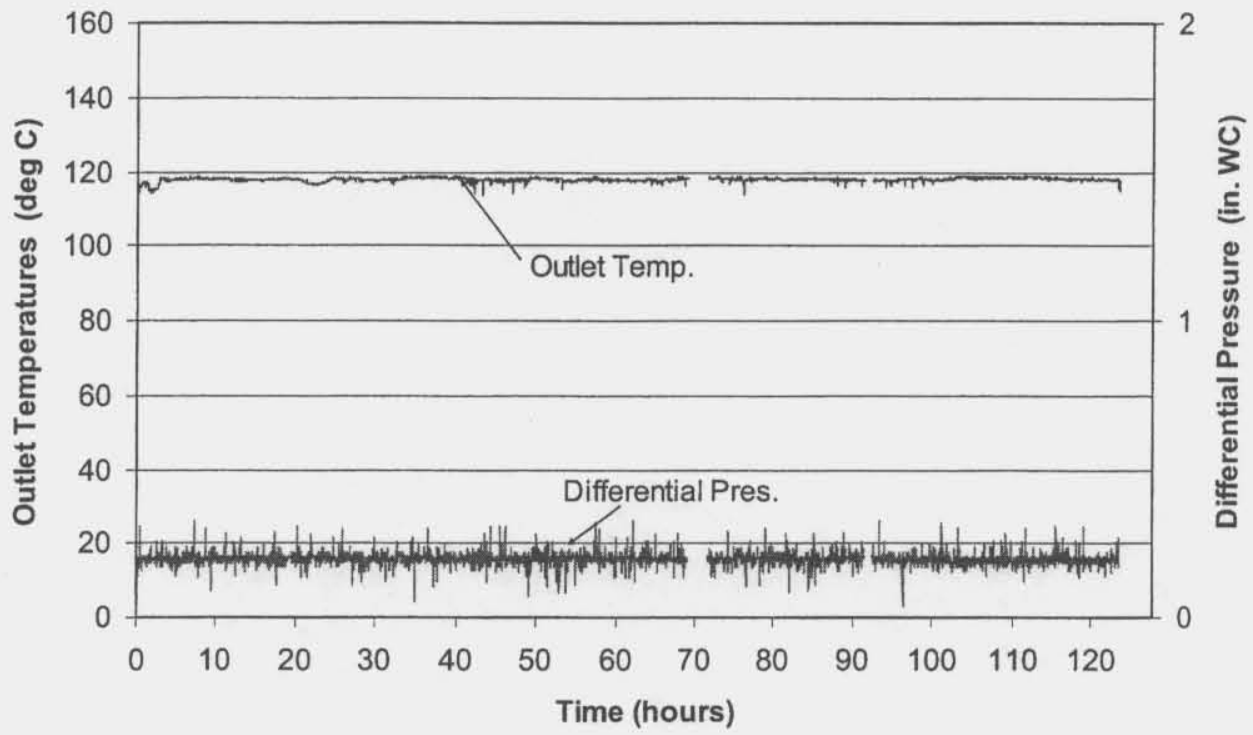

Figure 4.87. Outlet temperature and differential pressure for HEPA \#1 during Test 9.

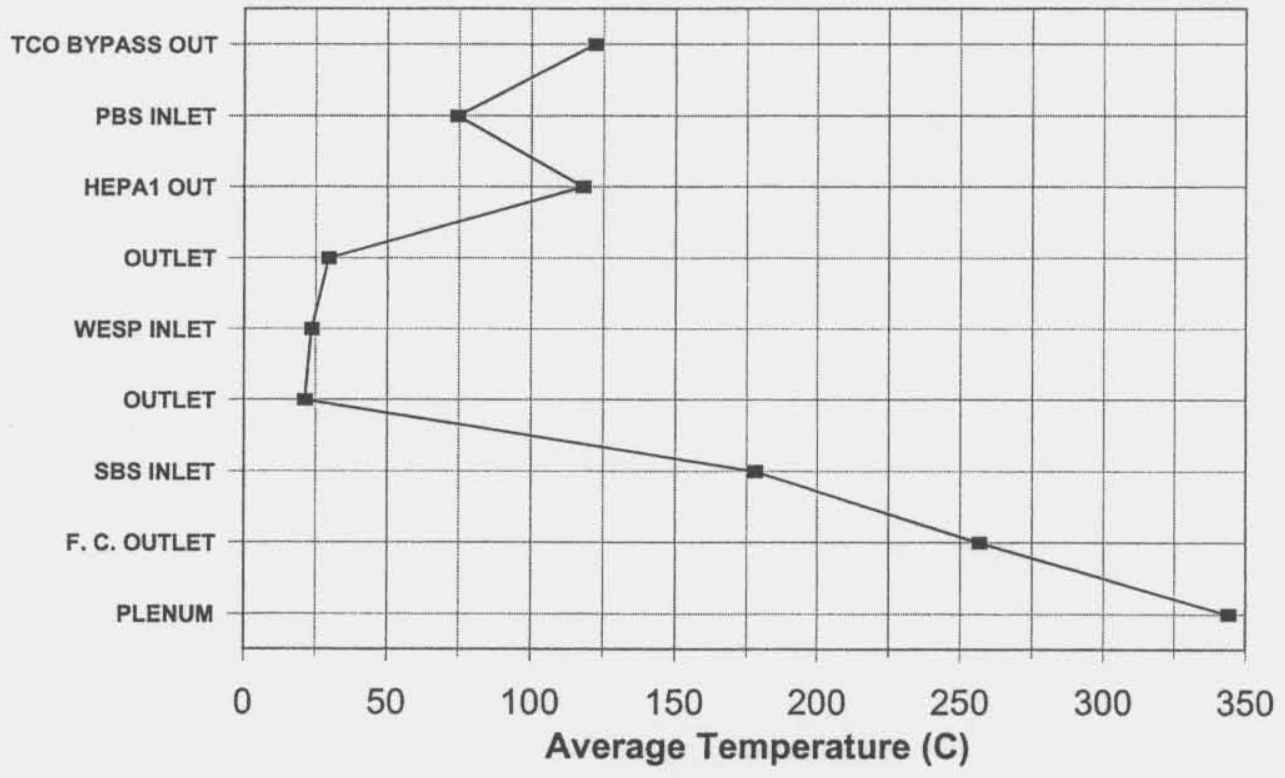

Figure 4.88. Average off-gas temperatures along the off-gas train during Test $9 \mathrm{~b}$. 
The Catholic University of America Vitreous State Laboratory
ORP-51438, Rev. 0

DuraMelter 1200 Tests with AZ-101HLW Simulants

Final Report, VSL-02R0100-2, Rev.1

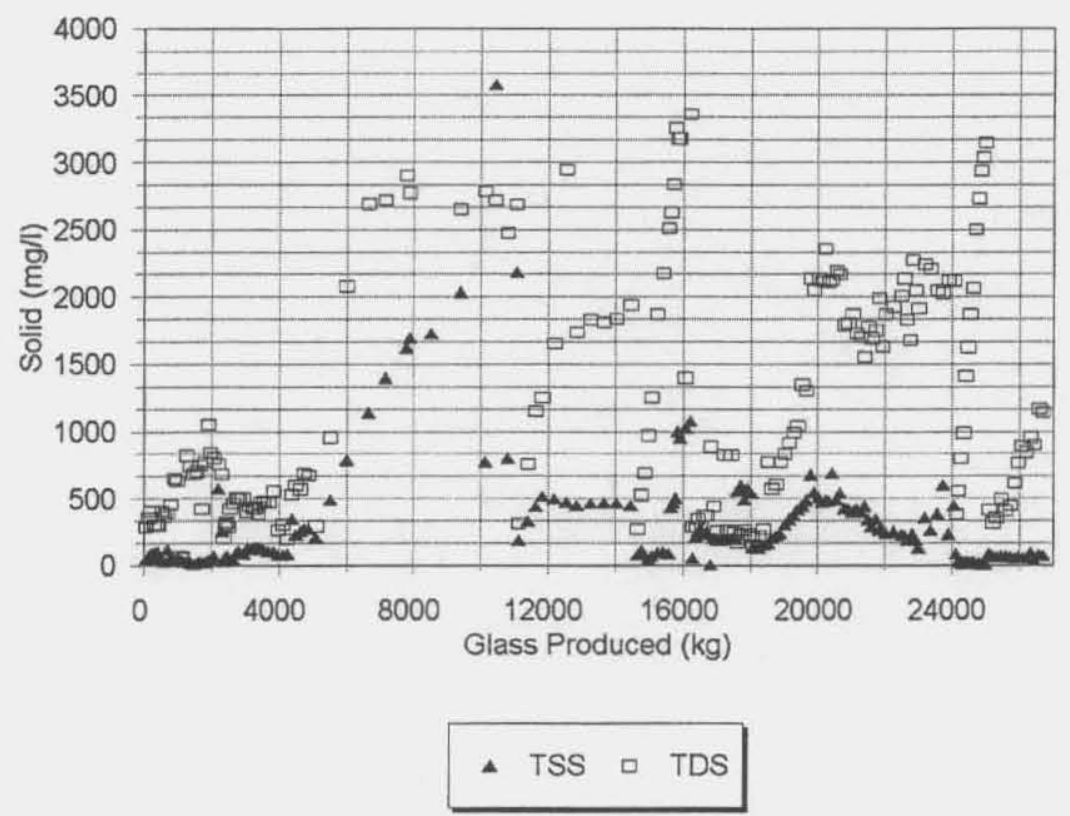

Figure 4.89. Total suspended solids (TSS) and total dissolved solids (TDS) in SBS blow-down solutions.

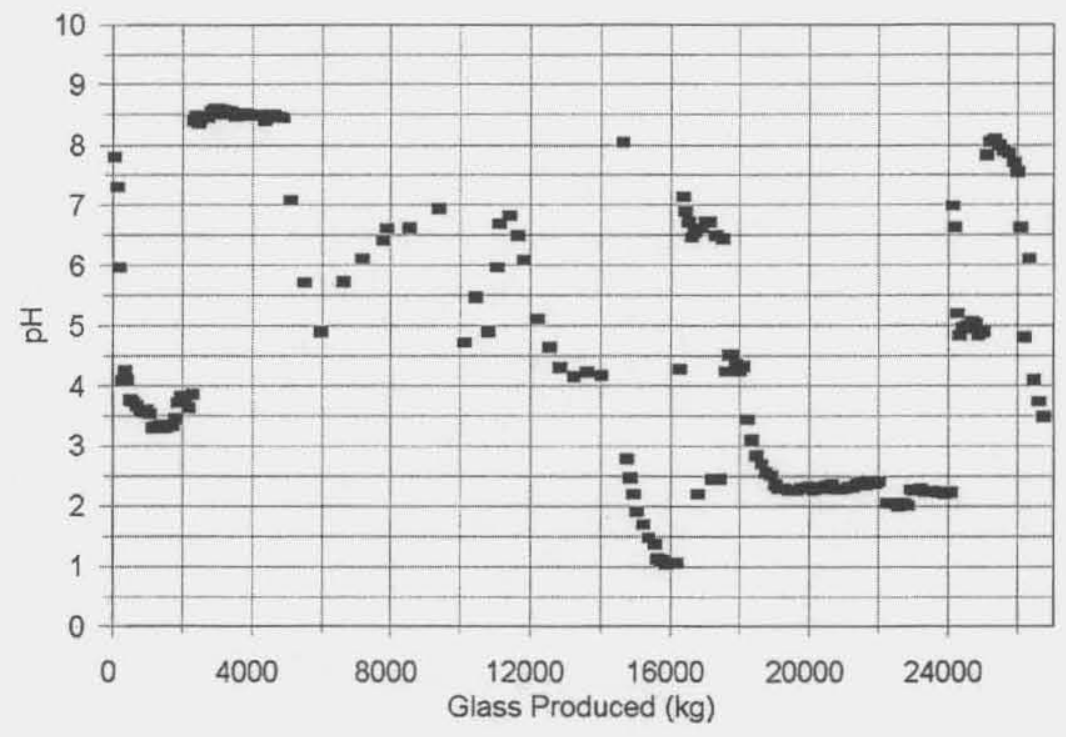

Figure 4.90. pH of SBS blow-down solutions. 
The Catholic University of America Vitreous State Laboratory
DuraMelter 1200 Tests with AZ-101HLW Simulants Final Report, VSL-02R0100-2, Rev.1

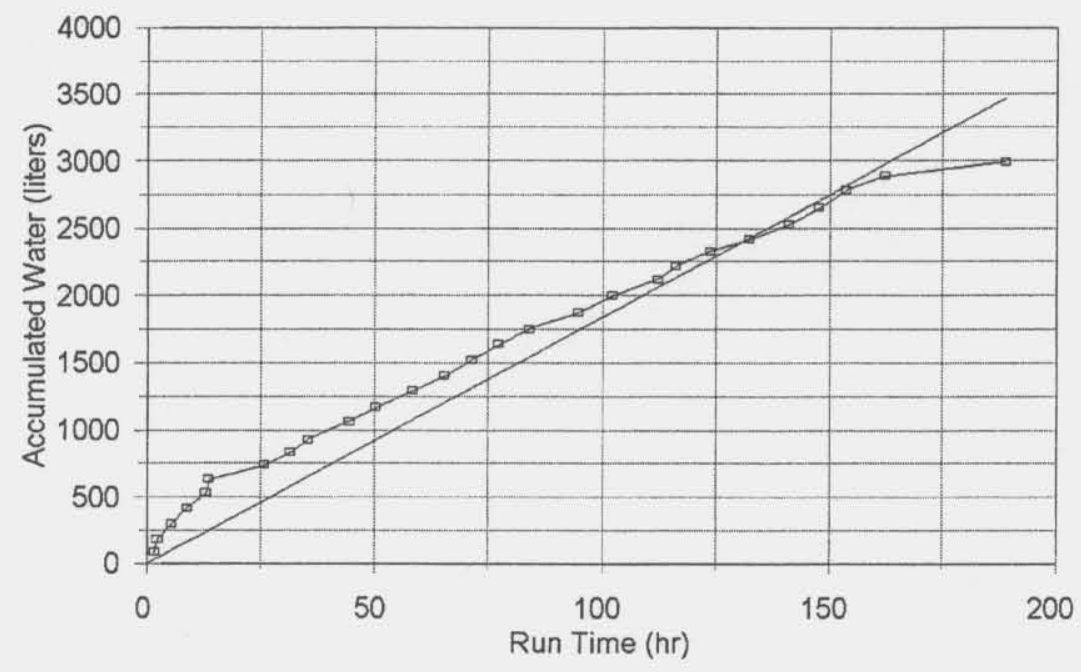

- SBS BLOWDOWNS - AVERAGE FEED

Figure 4.91. Test 1 accumulated SBS blow-down volume and average accumulated feed water.

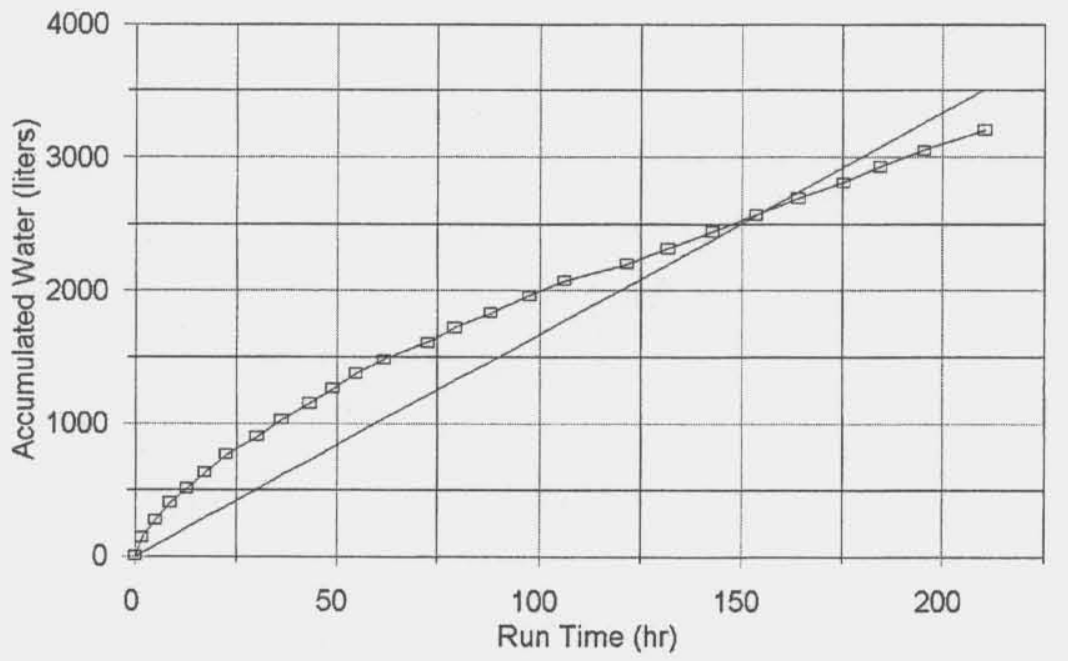

SBS BLOWDOWNS - AVERAGE FEED

Figure 4.92. Test 2 accumulated SBS blow-down volume and average accumulated feed water. 
The Catholic University of America Vitreous State Laboratory
ORP-51438, Rev. 0

DuraMelter 1200 Tests with AZ-101HLW Simulants Final Report, VSL-02R0100-2, Rev.1

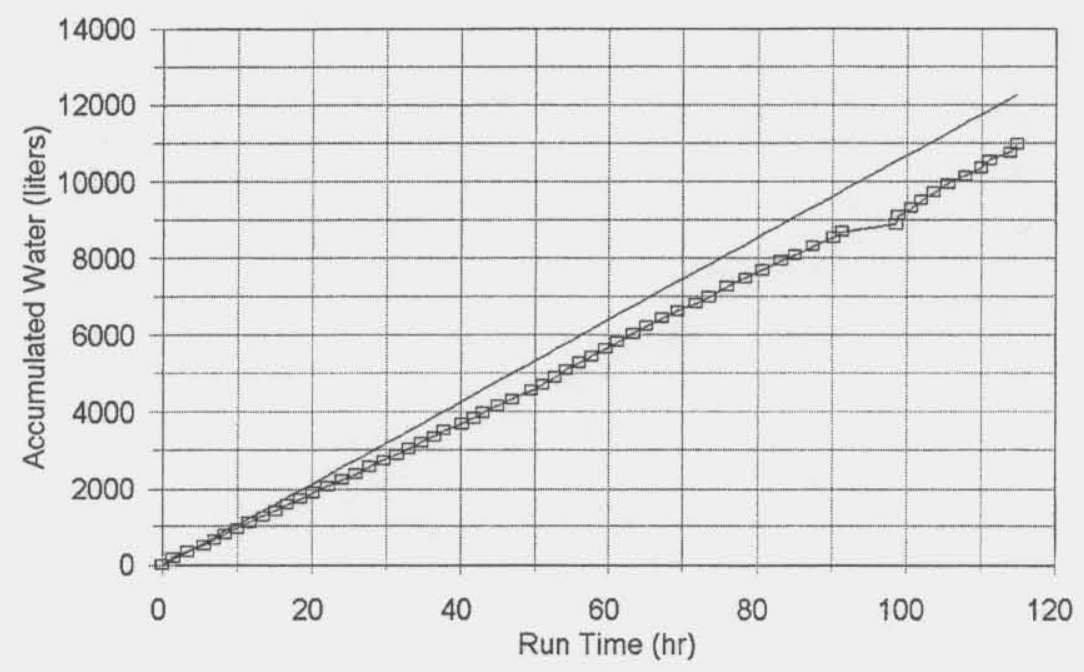

$\because$ SBS BLOWDOWNS — AVERAGE FEED

Figure 4.93. Test 3 accumulated SBS blow-down volume and average accumulated feed water.

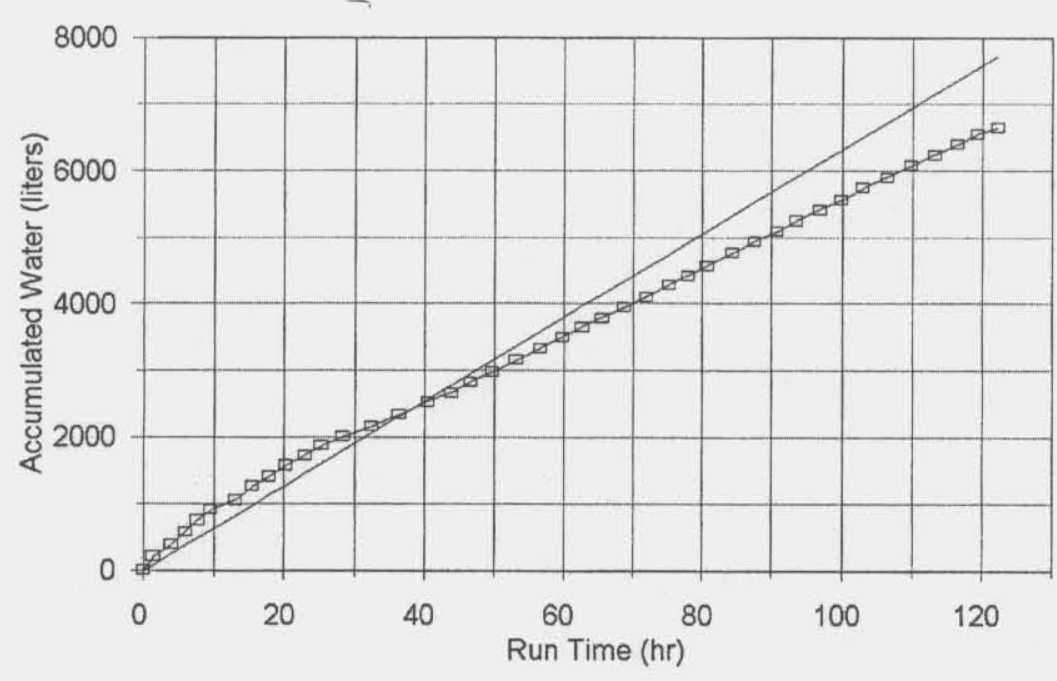

$\rightarrow$ SBS BLOWDOWNS — AVERAGE FEED

Figure 4.94. Test 4 accumulated SBS blow-down volume and average accumulated feed water. 
The Catholic University of America Vitreous State Laboratory

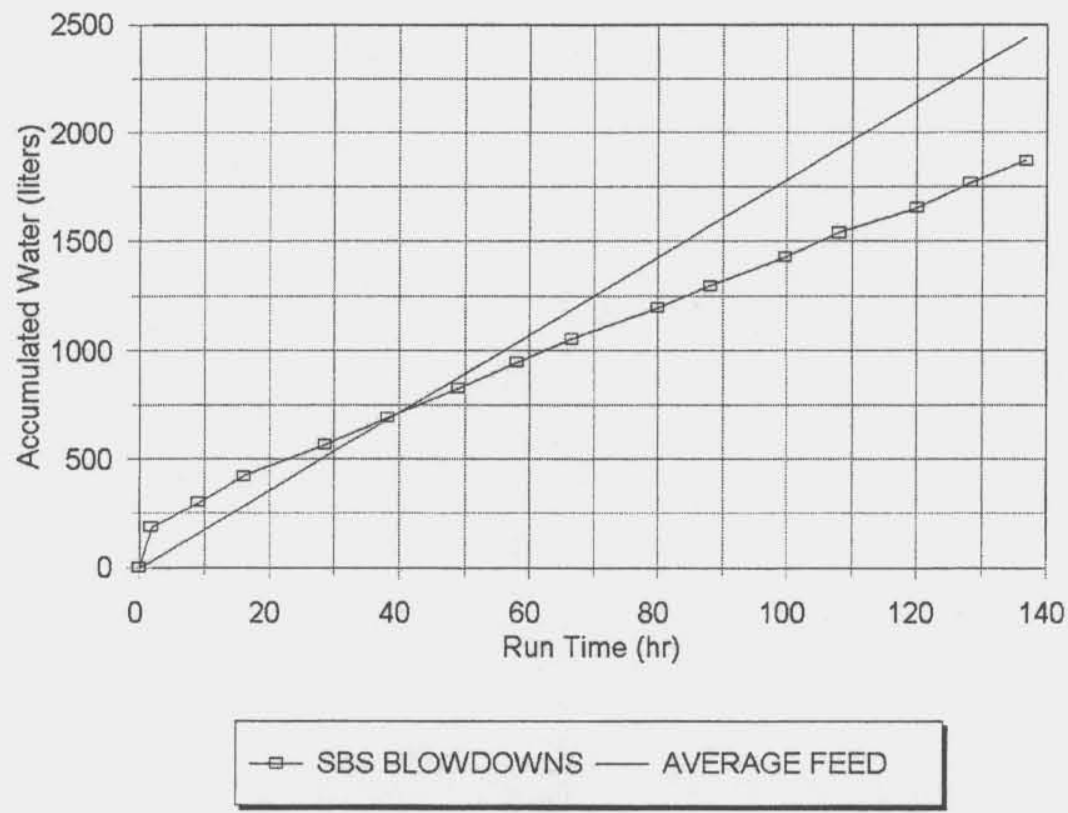

Figure 4.95. Test 5 accumulated SBS blow-down volume and average accumulated feed water.

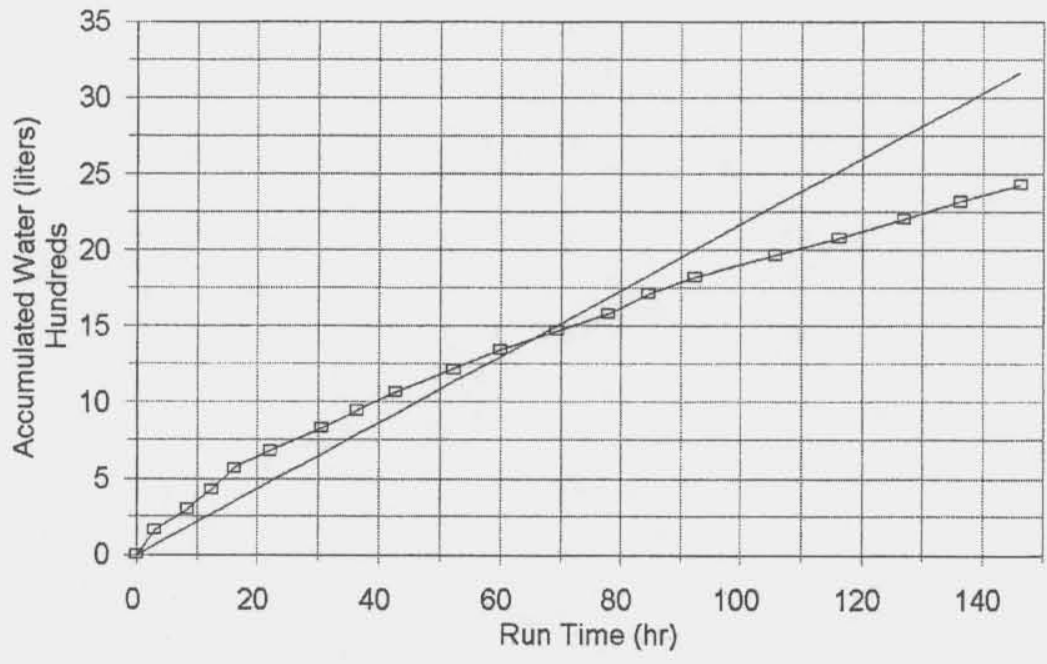

$\because$ SBS BLOWDOWNS - AVERAGE FEED

Figure 4.96. Test 6 accumulated SBS blow-down volume and average accumulated feed water. 


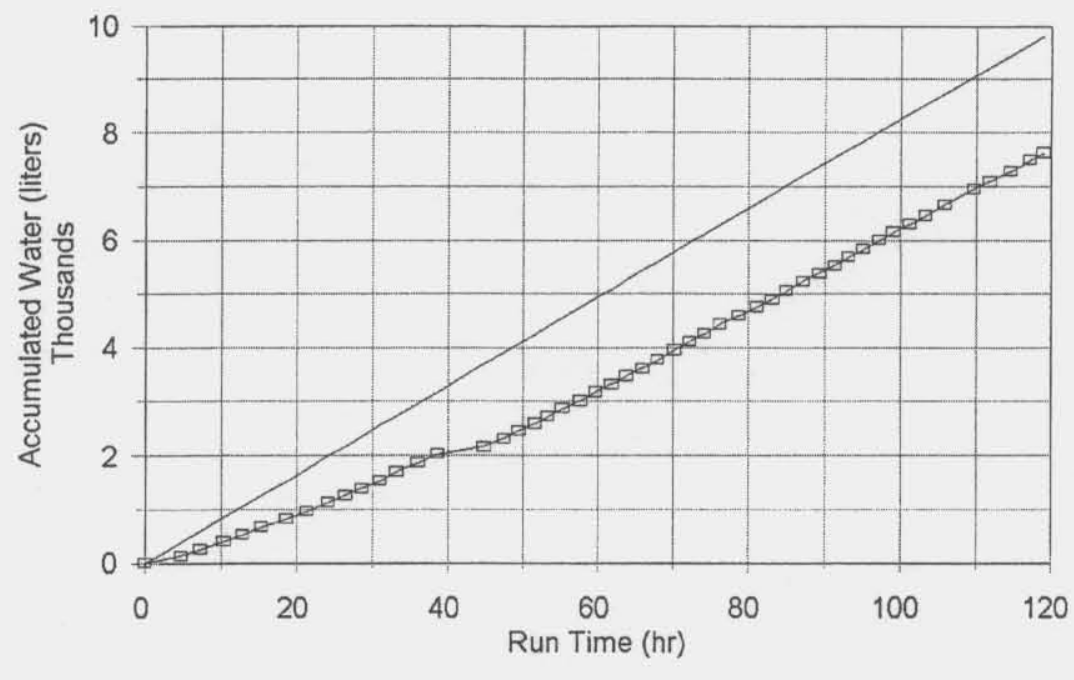

$$
\text { - SBS BLOWDOWNS — AVERAGE FEED }
$$

Figure 4.97. Test 7 accumulated SBS blow-down volume and average accumulated feed water.

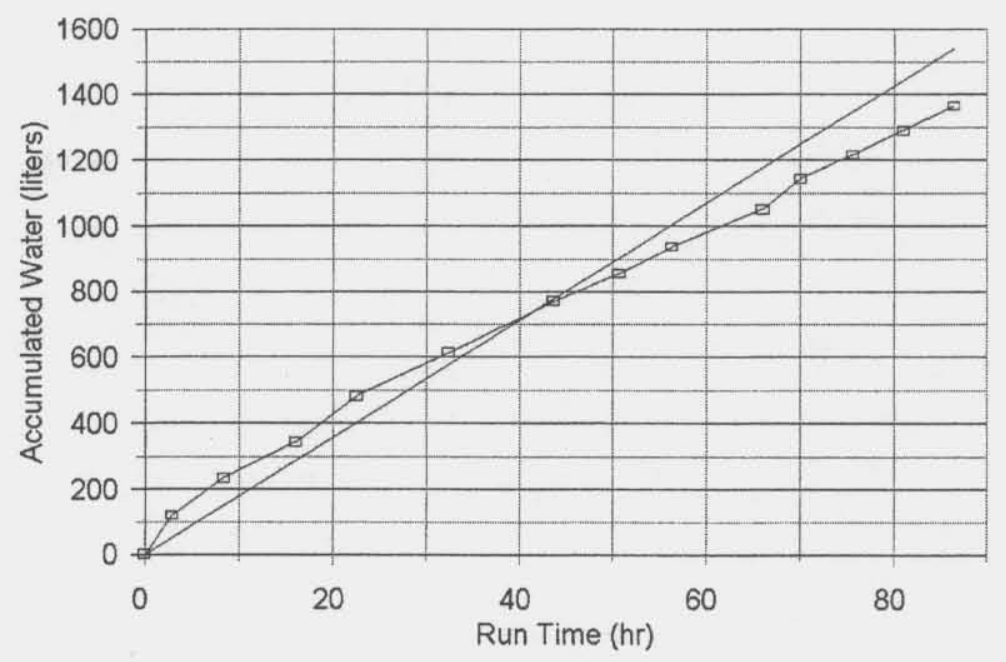

$$
\text { SBS BLOWDOWNS — AVERAGE FEED }
$$

Figure 4.98. Test 8 accumulated SBS blow-down volume and average accumulated feed water. 
The Catholic University of America Vitreous State Laboratory
DuraMelter 1200 Tests with AZ-101HLW Simulants Final Report, VSL-02R0100-2, Rev.1

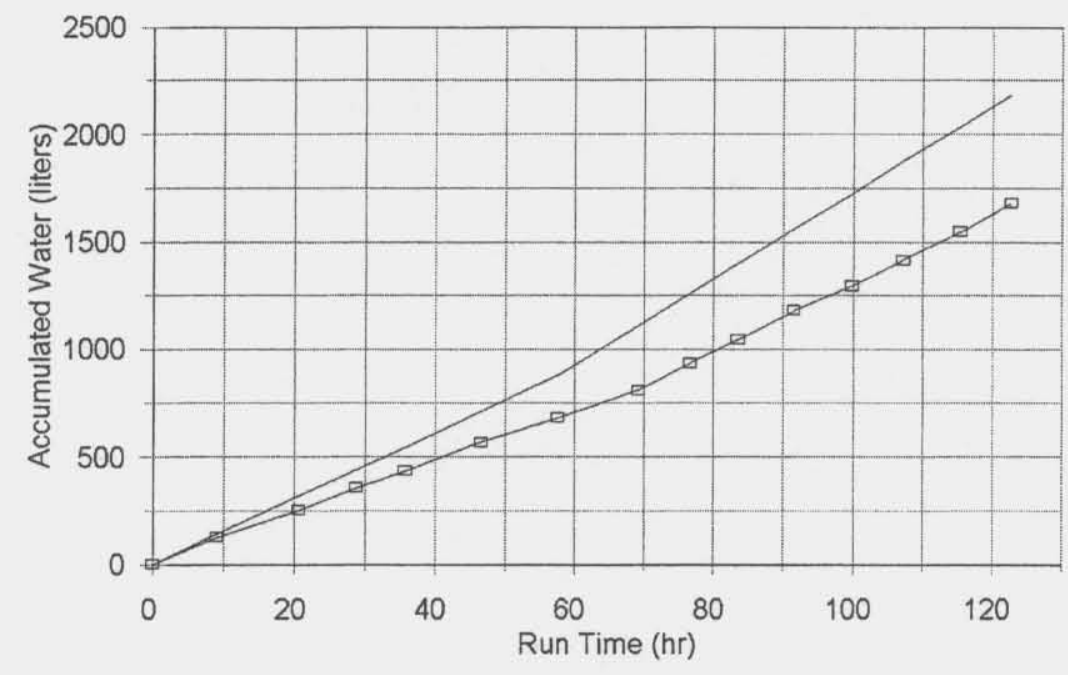

$\rightarrow$ SBS BLOWDOWNS — AVERAGE FEED

Figure 4.99. Test 9 accumulated SBS blow-down volume and average accumulated feed water.

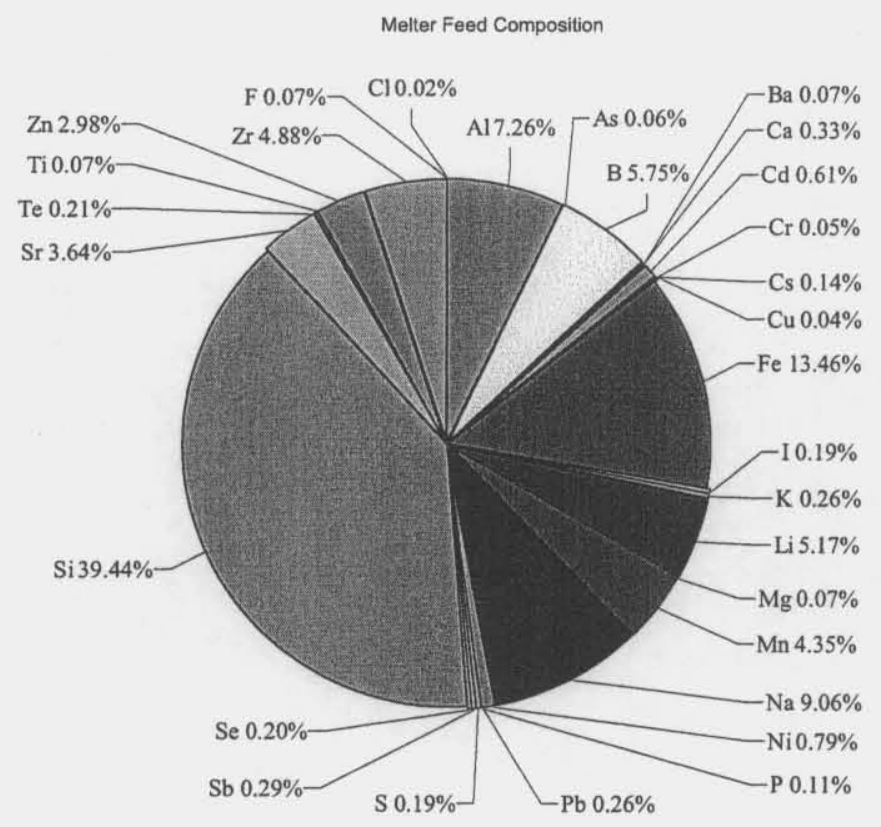

Figure 4.100. Feed composition (excludes oxygen, nitrogen species, and organic compounds). 
The Catholic University of America Vitreous State Laboratory
DuraMelter 1200 Tests with AZ-101HLW Simulants Final Report, VSL-02R0100-2, Rev.1

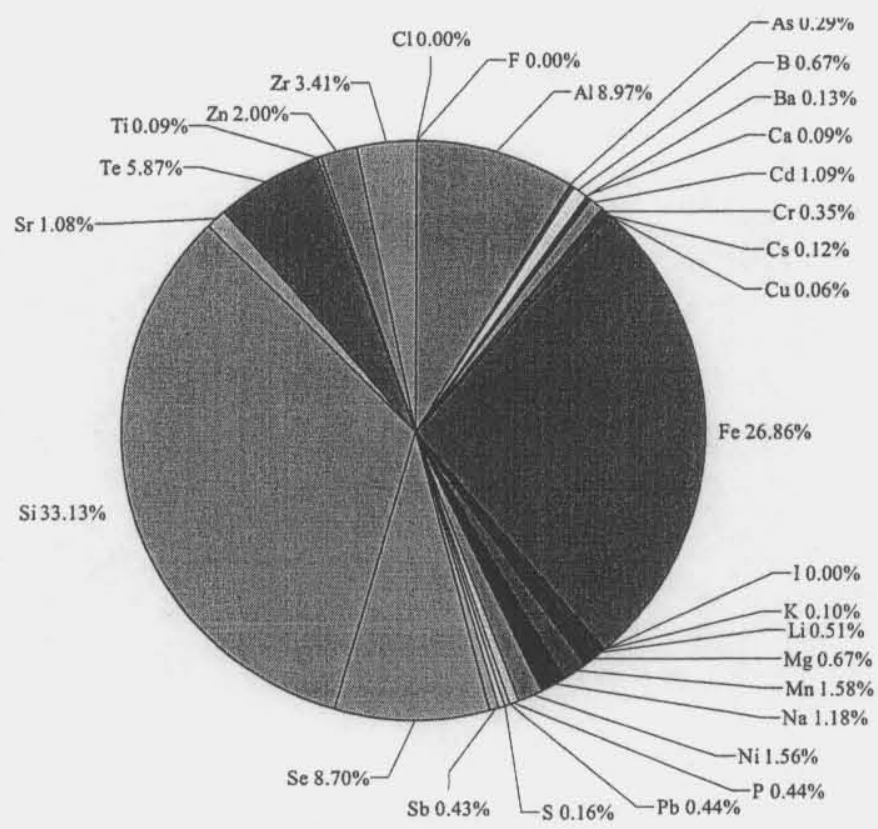

Figure 4.101. SBS suspended solids composition from the end of Test 3 (excludes oxygen, nitrogen species, organic compounds, chlorine, and fluorine.

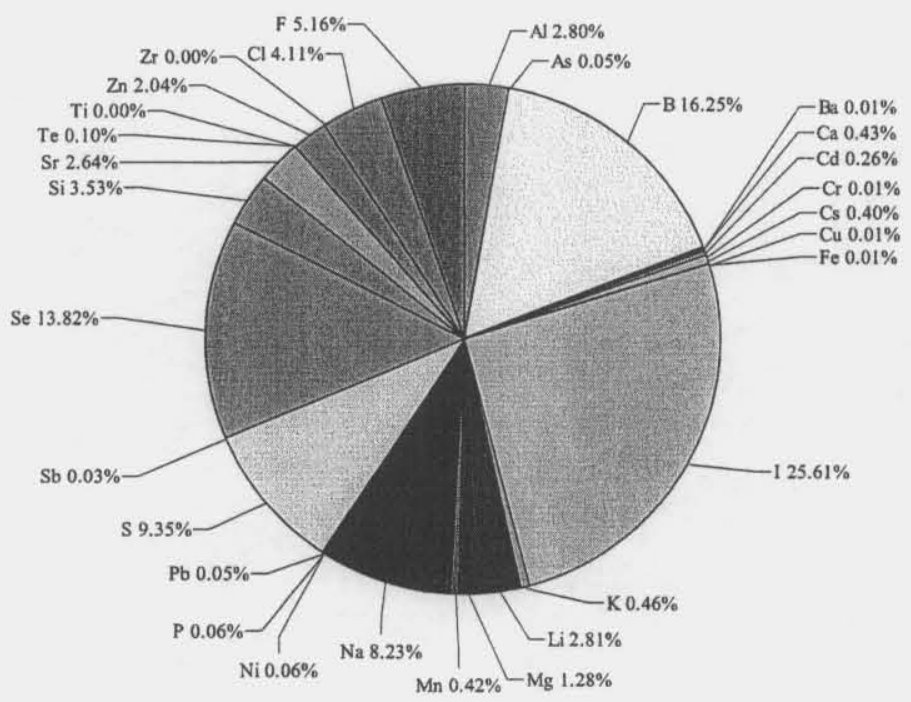

Figure 4.102. SBS dissolved solids composition from the end of Test 3 (excludes oxygen, nitrogen species and organic compounds). 
The Catholic University of America

Vitreous State Laboratory
DuraMelter 1200 Tests with AZ-101HLW Simulants Final Report, VSL-02R0100-2, Rev.1

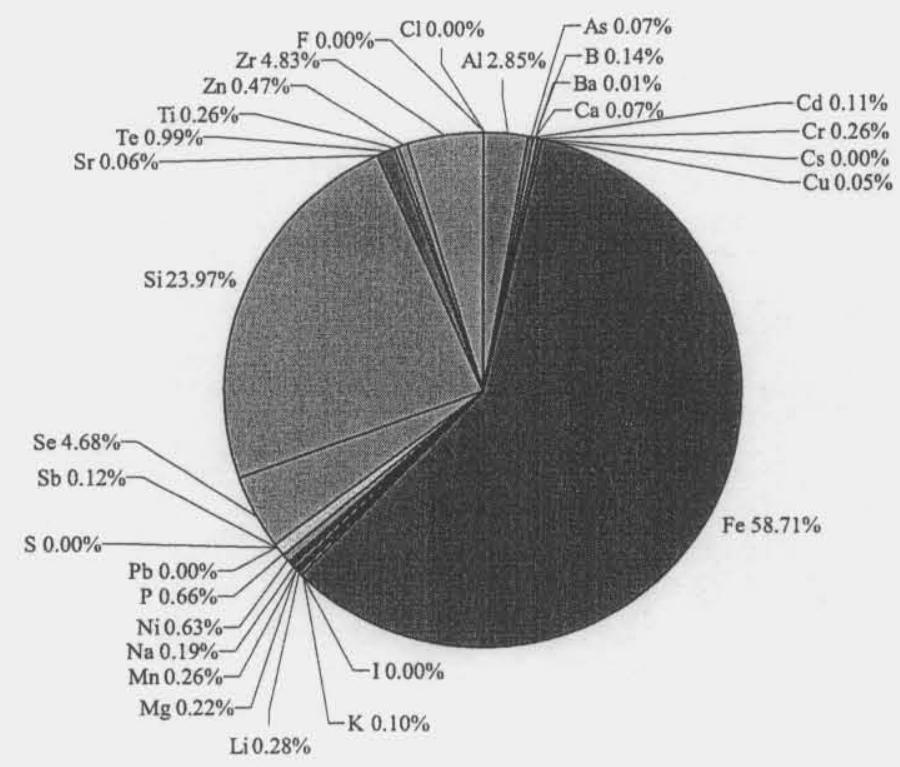

Figure 4.103. SBS suspended solids composition from the end of Test 5 (excludes oxygen, nitrogen specie, organic compounds, chlorine and fluorine).

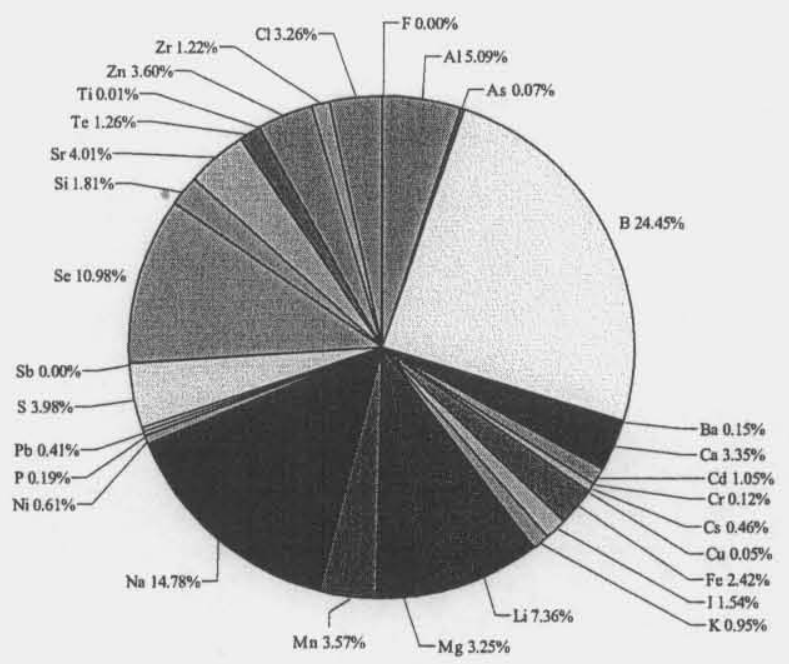

Figure 4.104. SBS dissolved solids composition from the end of Test 5 (excludes oxygen, nitrogen species and organic compounds). 
The Catholic University of America Vitreous State Laboratory

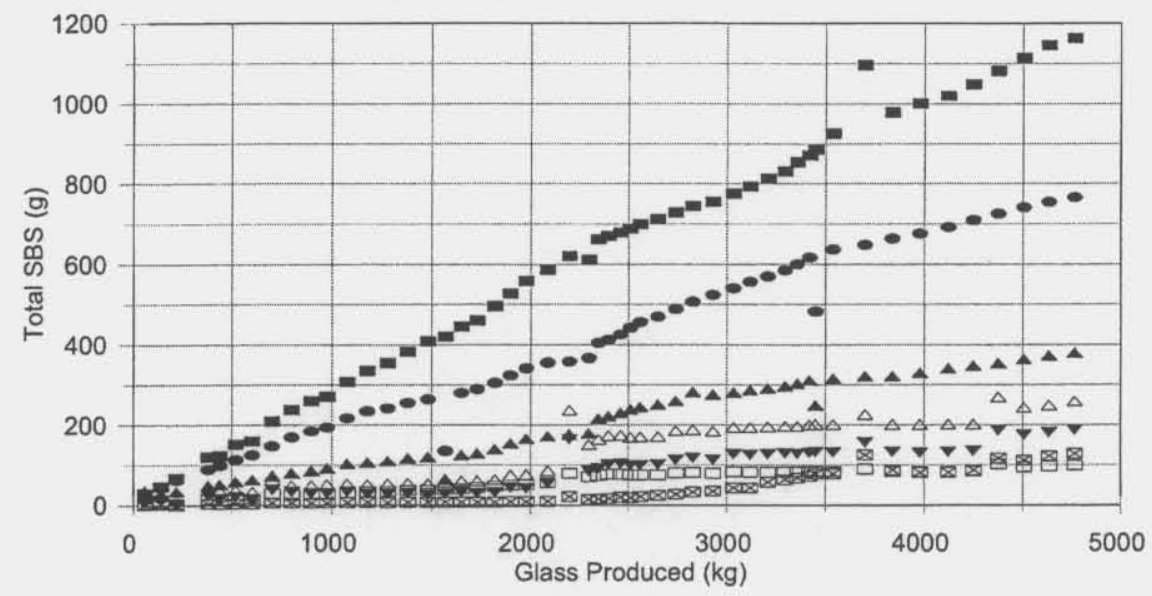

ㅁl $-\mathrm{B} \sim \mathrm{Fe} \triangle \mathrm{Na}=\mathrm{Se} \Delta \mathrm{Si} \otimes \mathrm{Te}$

Figure 4.105. Accumulations of major constituents in SBS fluids during Tests 1 and 2.

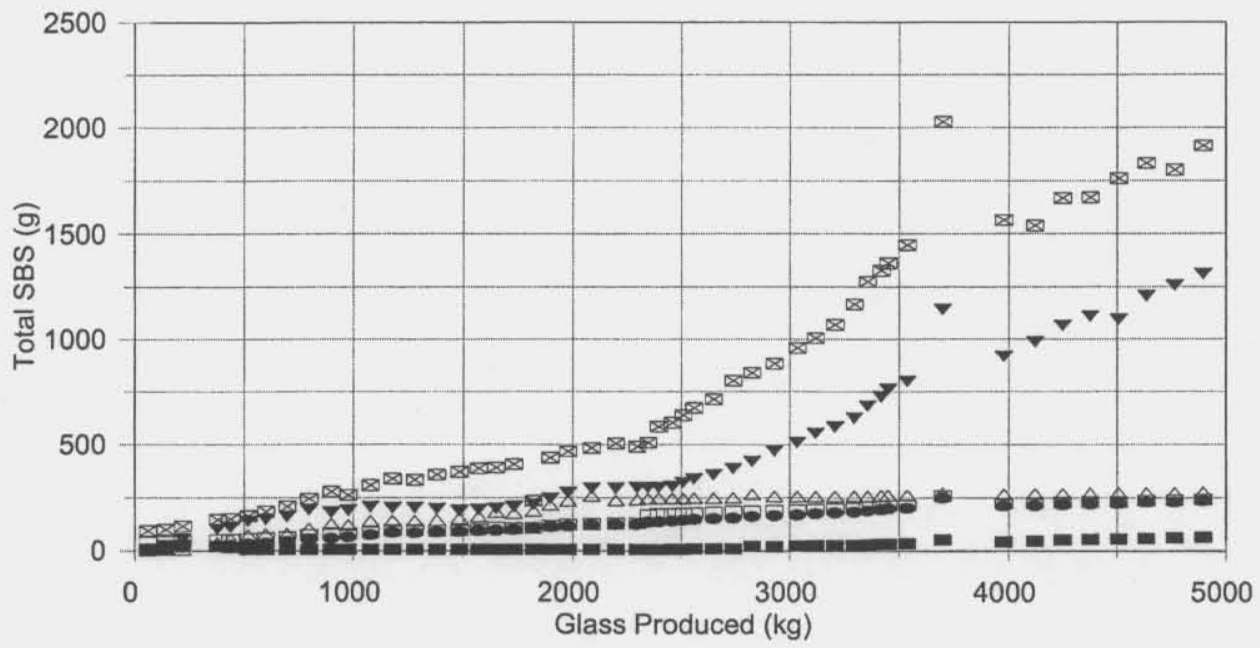
ㅁ $\mathrm{Cl}$
- $\mathrm{F}$
$\checkmark 1$
$\triangle$ Nitrate
- Nitrite
$\bowtie \quad$ Sulfate

Figure 4.106. Accumulations of anions in SBS fluids during Tests 1 and 2. 
The Catholic University of America

Vitreous State Laboratory
DuraMelter 1200 Tests with AZ-101HLW Simulants

Final Report, VSL-02R0100-2, Rev.I

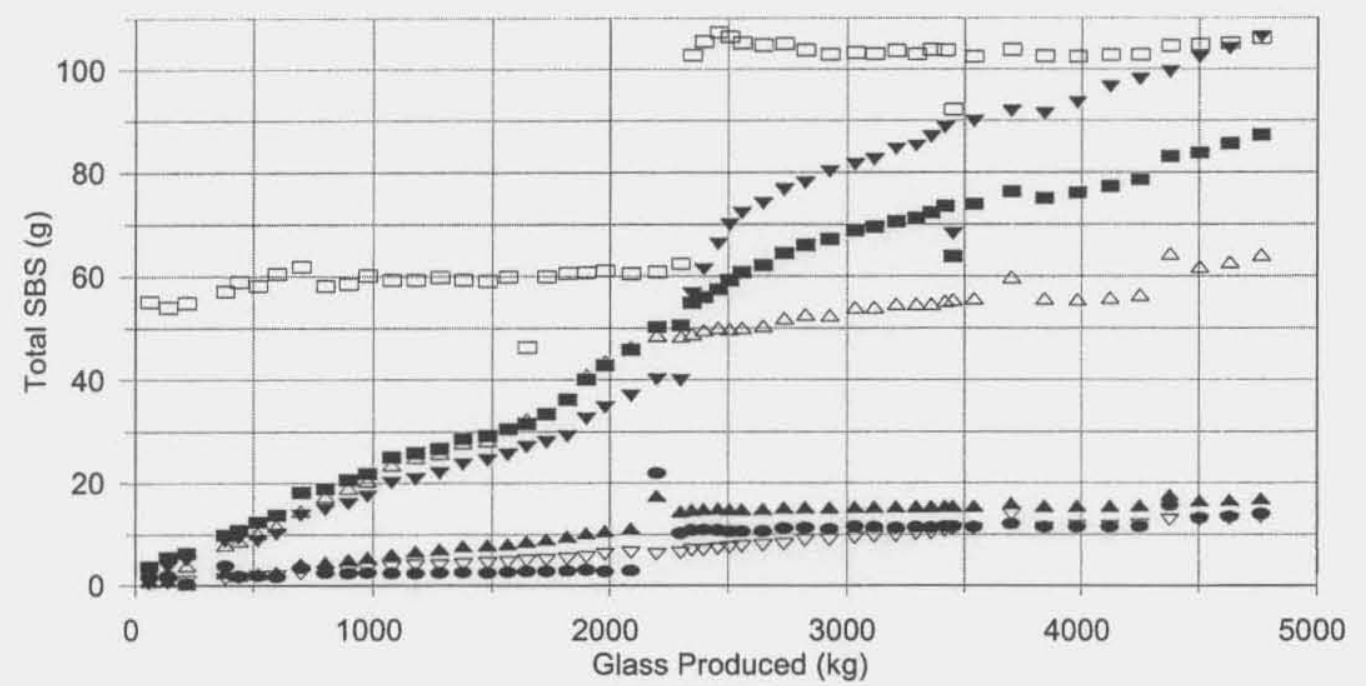

$$
\square \mathrm{Ca} \nabla \mathrm{Cs} \nabla \mathrm{Li} \wedge \mathrm{Mn}=\mathrm{Sr} \Delta \mathrm{Zn} \cdot \mathrm{Zr}
$$

Figure 4.107. Accumulations of select constituents in SBS fluids during Tests 1 and 2.

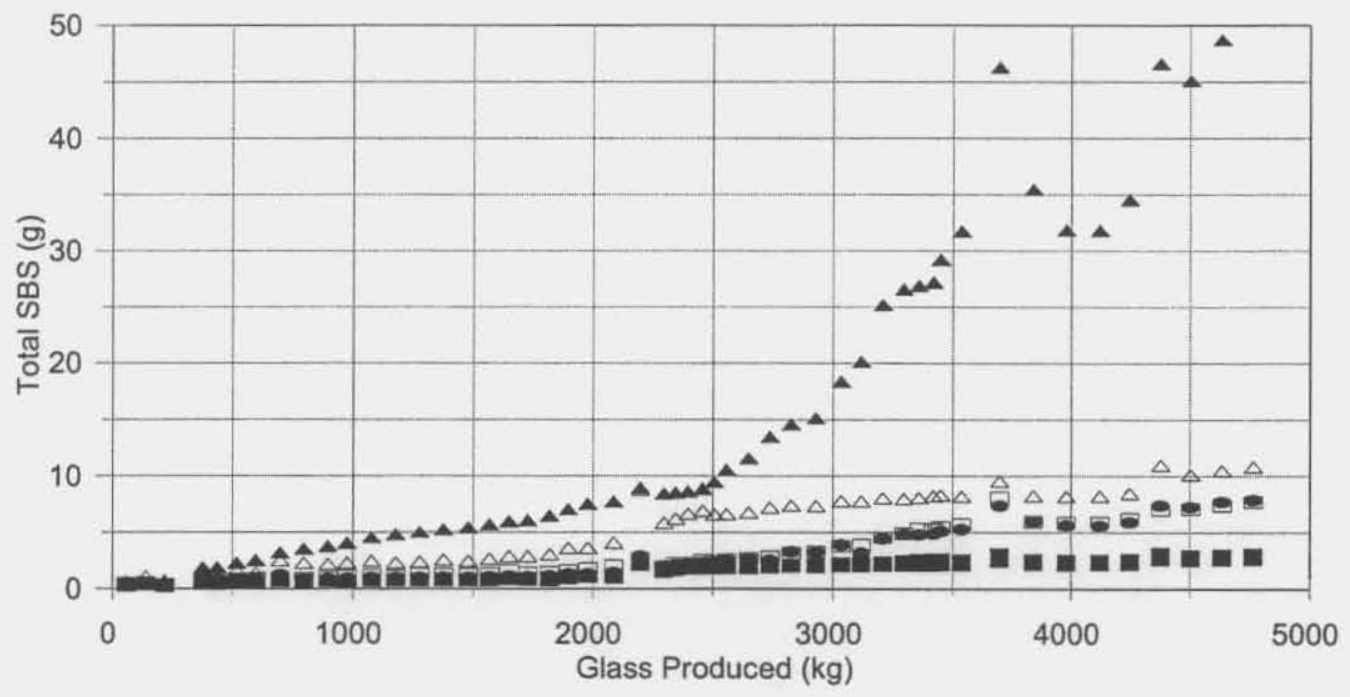

$$
\square \mathrm{As} \triangle \mathrm{Cd}=\mathrm{Cr} \Delta \mathrm{Ni} \bullet \mathrm{Pb}=\mathrm{Sb}
$$

Figure 4.108. Accumulations of RCRA metals in SBS fluids during Tests 1 and 2. 
The Catholic University of America Vitreous State Laboratory
DuraMelter 1200 Tests with AZ-101HLW Simulants Final Report, VSL-02R0100-2, Rev.1

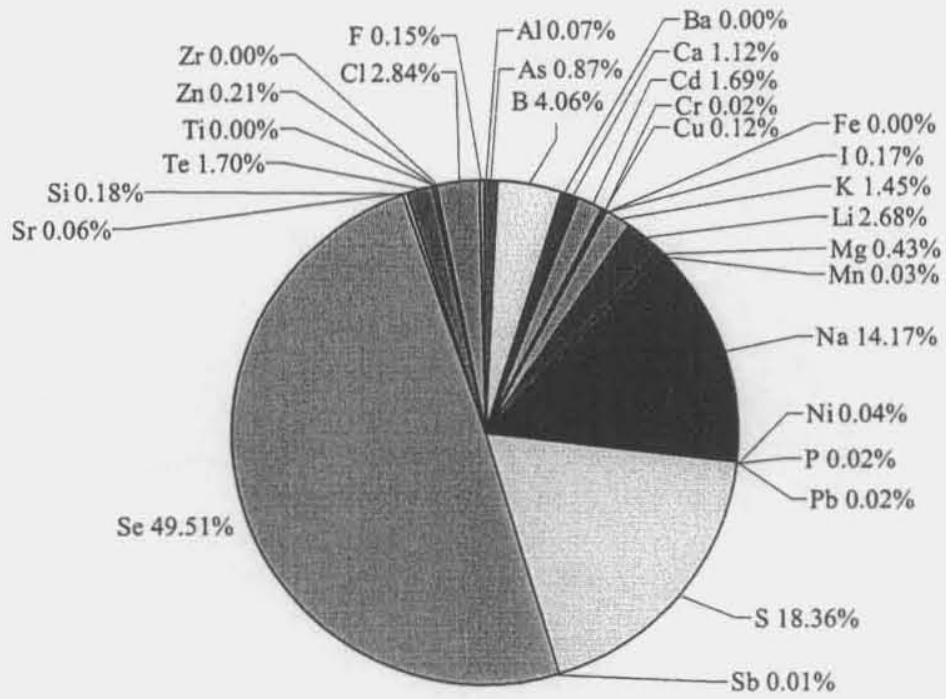

Figure 4.109. WESP dissolved solids composition from the end of Test 3 (excludes oxygen, nitrogen species, and organic compounds).

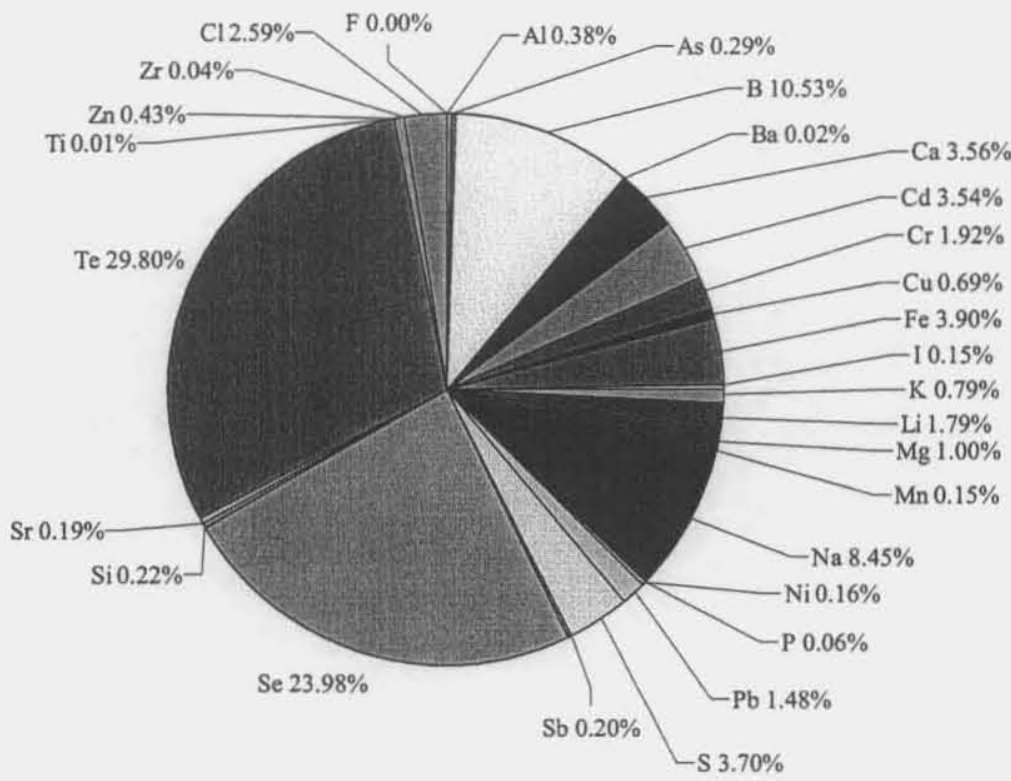

Figure 4.110. WESP dissolved solids composition from the end of Test 5 (excludes oxygen, nitrogen species, and organic compounds). 
The Catholic University of America Vitreous State Laboratory
DuraMelter 1200 Tests with AZ-101 HLW Simulants

Final Report, VSL-02R0100-2, Rev.1
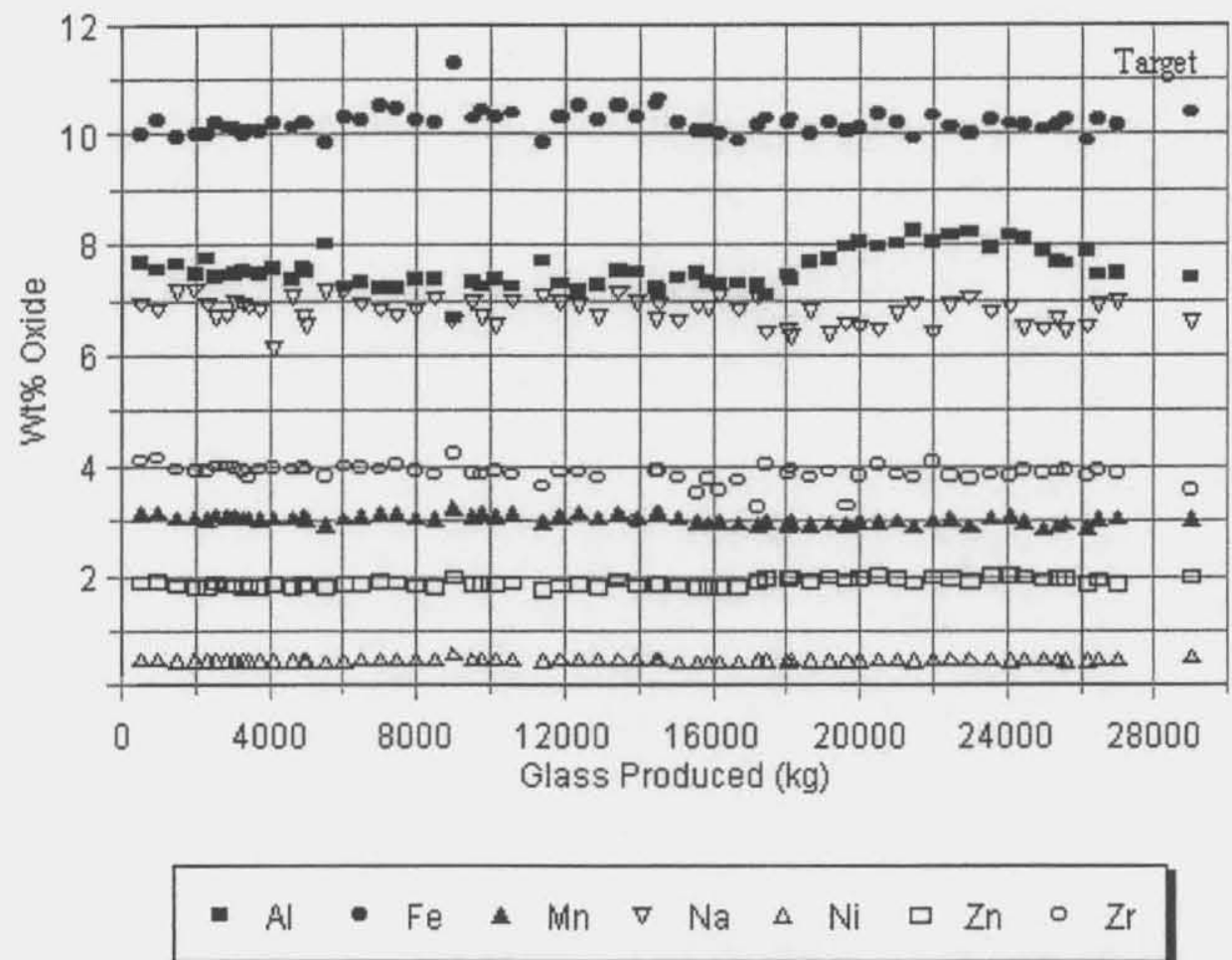

Figure 5.1. XRF analysis of selected oxides in melter glasses.

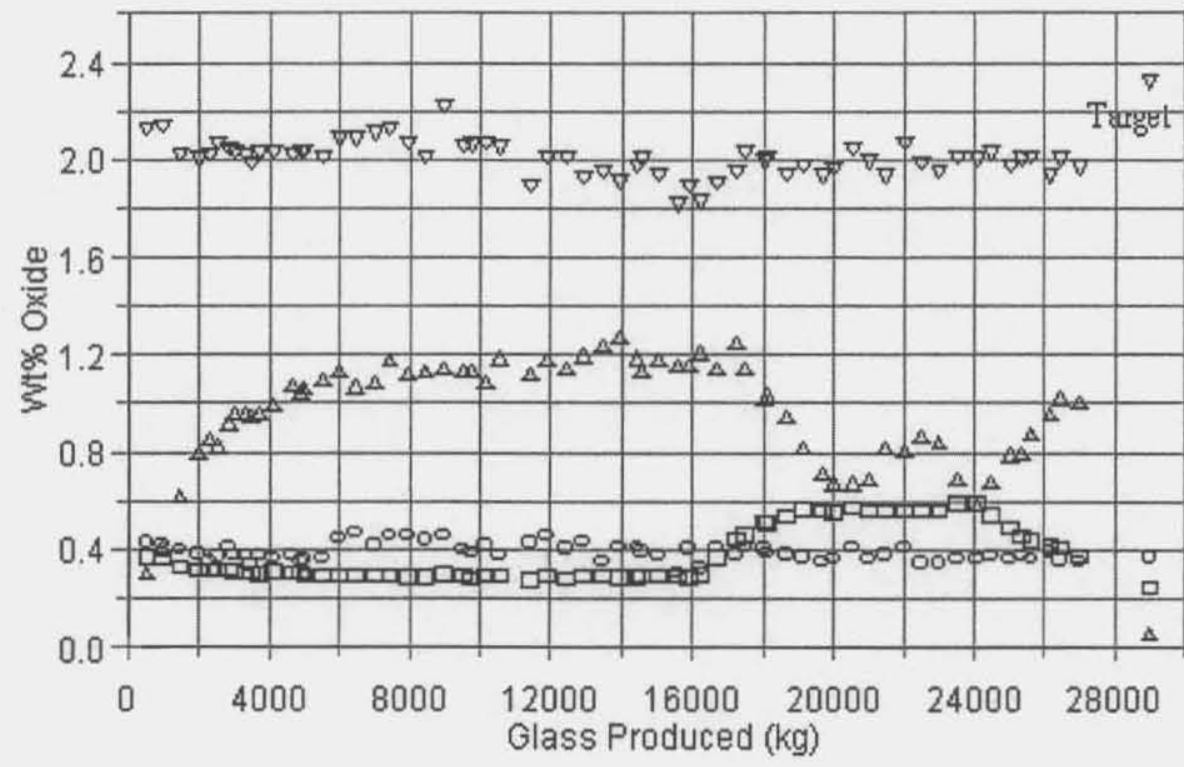

$\square \mathrm{Ca} \circ \mathrm{Cd} \triangle \mathrm{Mg} \nabla \mathrm{Sr}$

Figure 5.2. XRF analysis of selected minor oxides in melter glasses. 


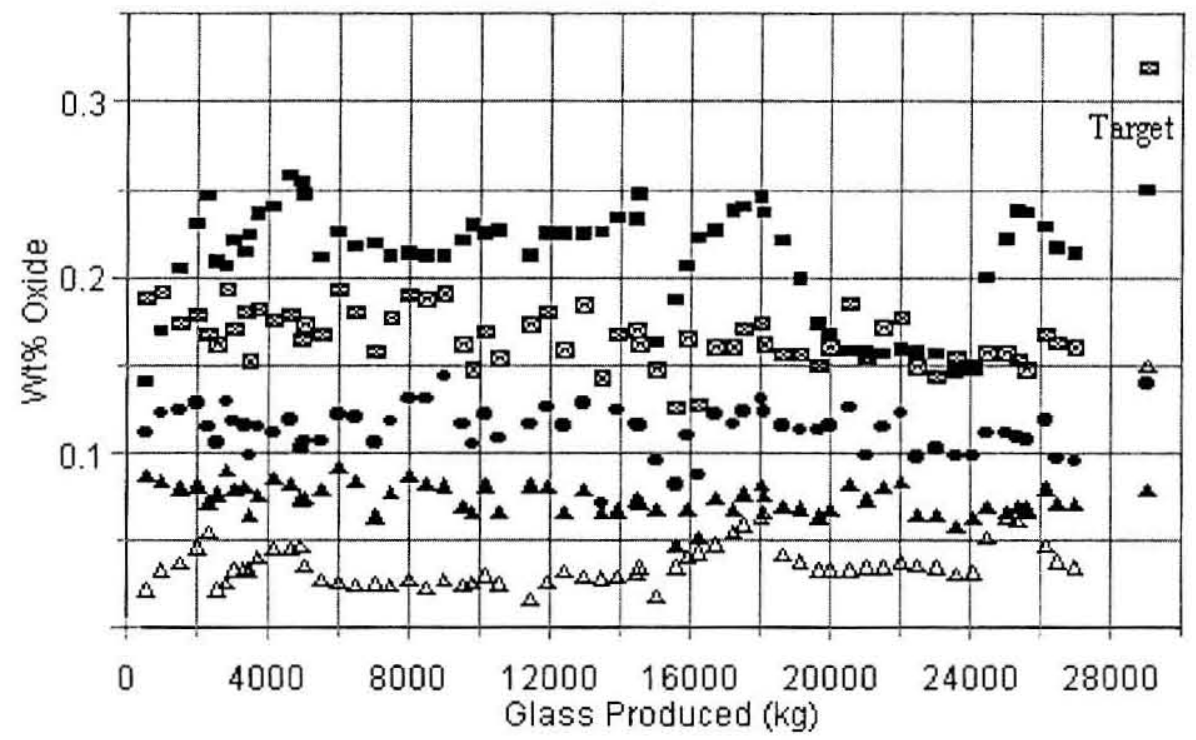

- $\mathrm{CS}$ - $\mathrm{Sb}=\mathrm{S} \Delta \mathrm{Se} \cdot \mathrm{Te}$

Figure 5.3. XRF analysis of volatile elements in melter glasses.

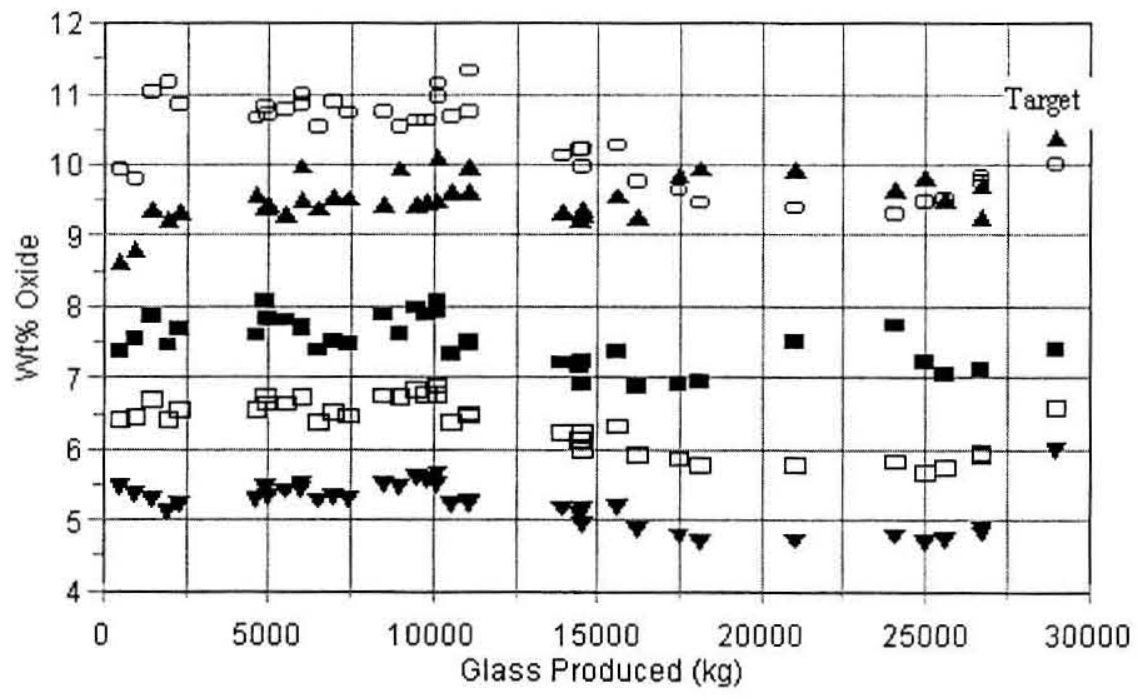

- Al 0 B $\triangle \mathrm{Fe} \boldsymbol{\mathrm { Li }} \square \mathrm{Na}$

Figure 5.4. DCP analysis of major oxides in melter glasses. 
The Catholic University of America Vitreous State Laboratory
DuraMelter 1200 Tests with AZ-101HLW Simulants

Final Report, VSL-02R0100-2, Rev.1

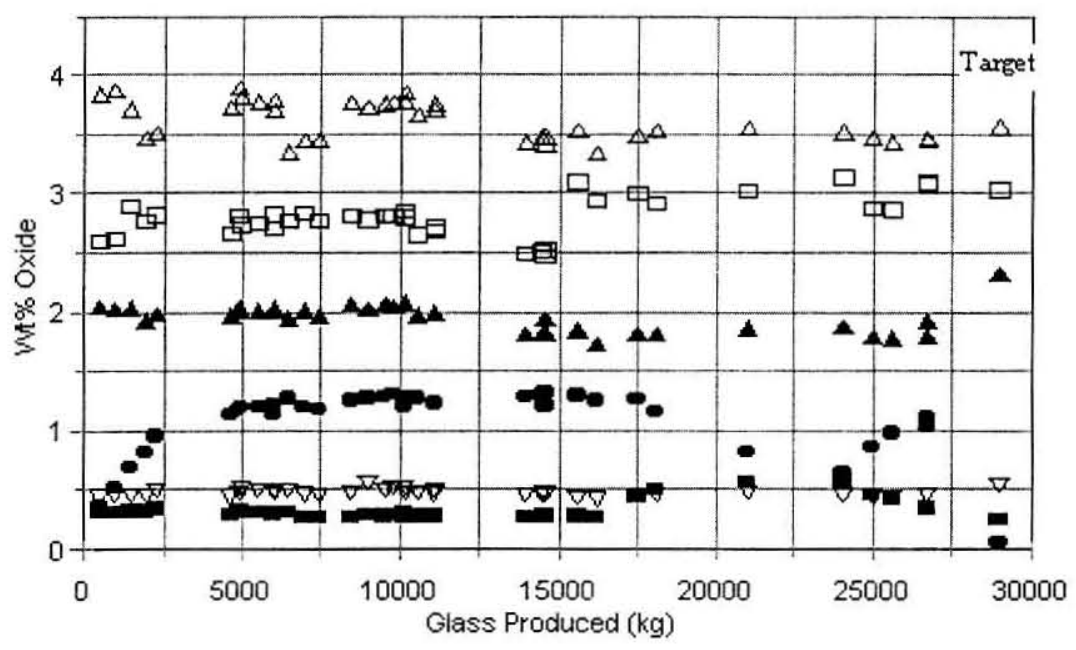

- $\mathrm{Ca}$ - Mg $\square \mathrm{Mn} \nabla \mathrm{Ni}$ \& Sr $\Delta \mathrm{Zr}$

Figure 5.5. DCP analysis of selected minor oxides in melter glasses.

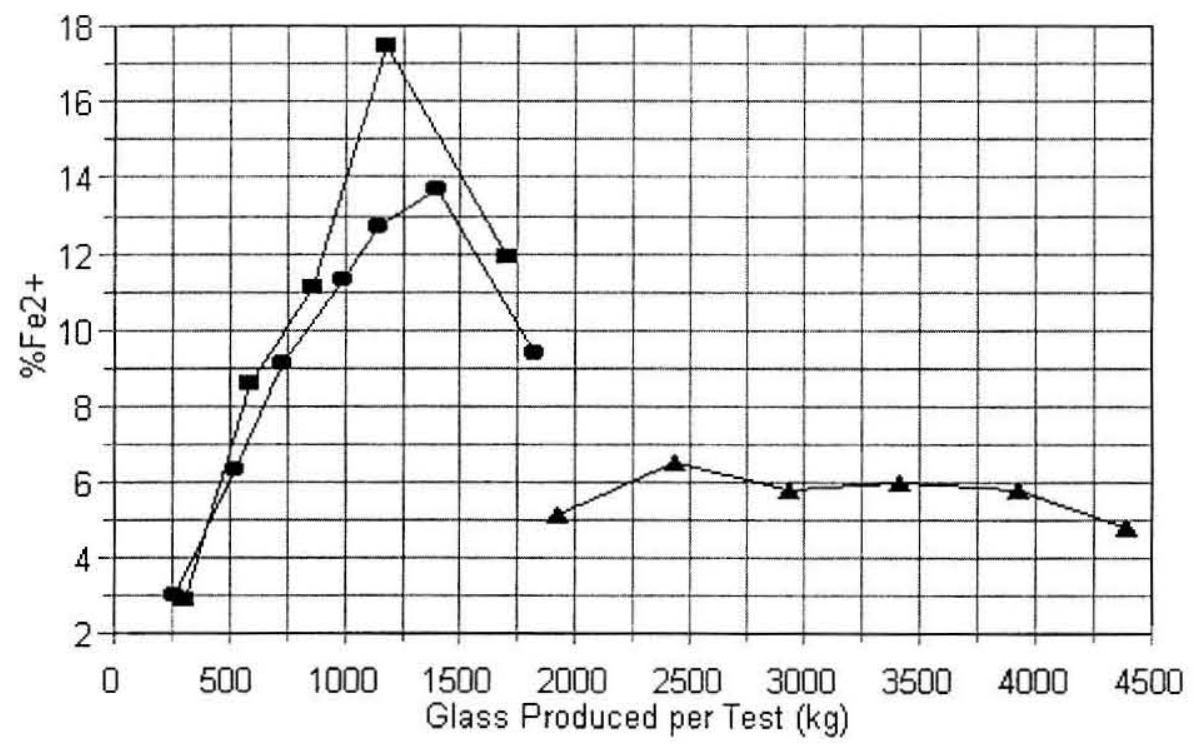

Test\#2 - Test\#7 7 - Test\#9

Figure 5.6. Divalent iron as a percentage of total $\mathrm{Fe}$ in melter glasses from select tests. 
The Catholic University of America

Vitreous State Laboratory
DuraMelter 1200 Tests with AZ-101HLW Simulants Final Report, VSL-01R0100-2, Rev.1

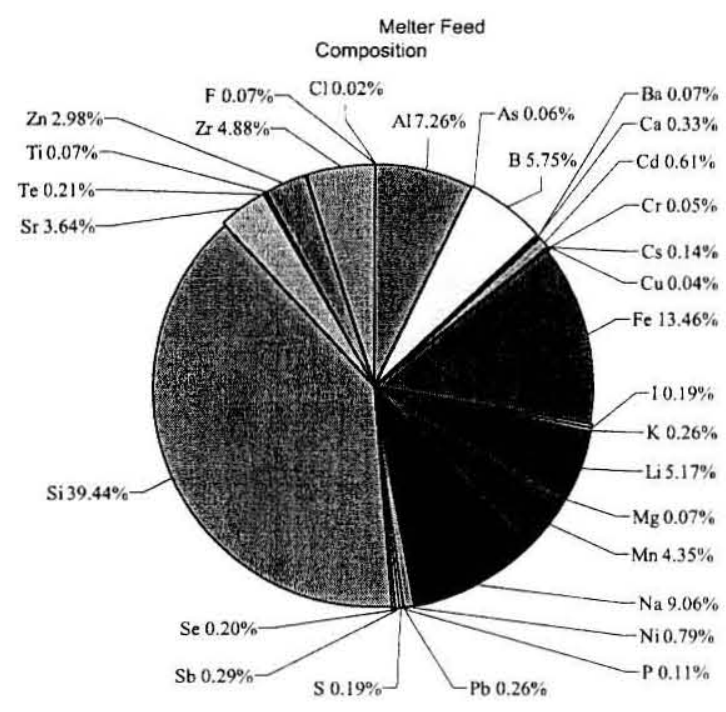

Figure 6.1. Feed composition (excludes oxygen, nitrogen species, and organic compounds).

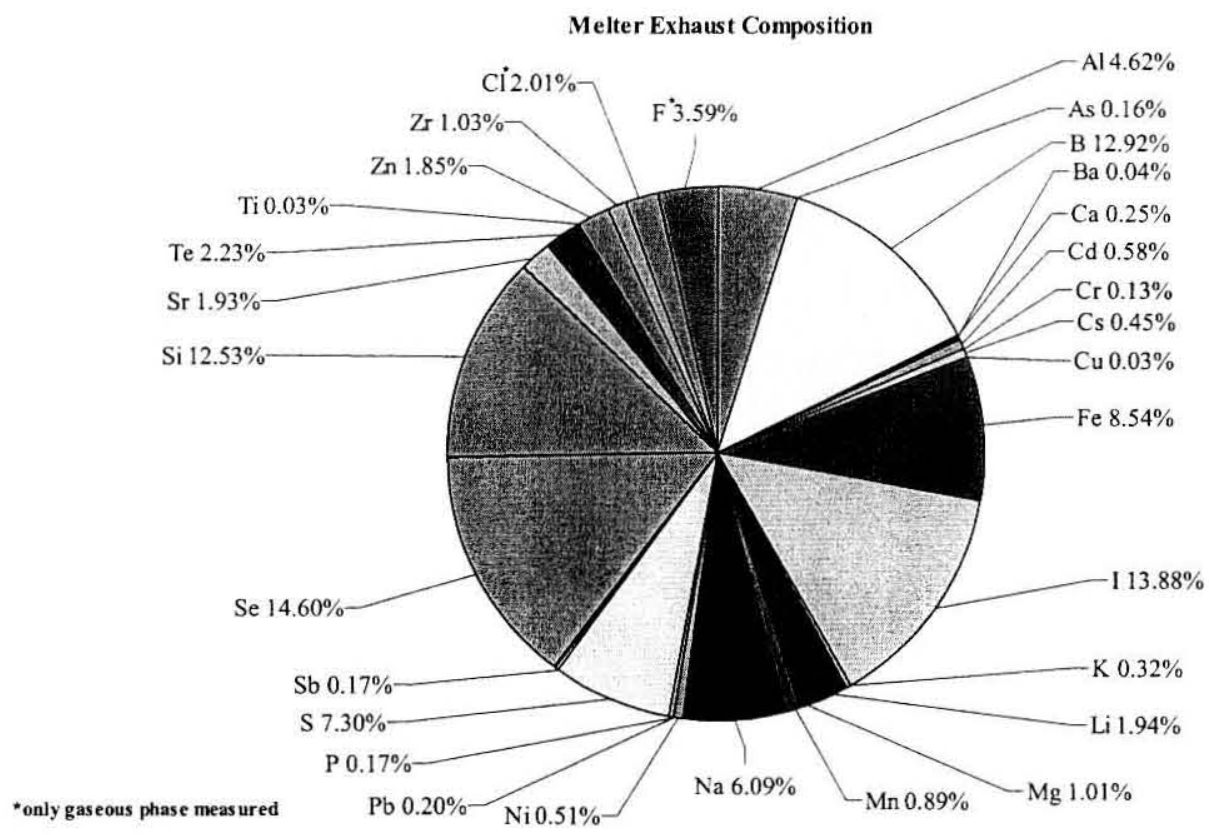

Figure 6.2. Test 3 melter exhaust composition (excludes oxygen, nitrogen species, organic compounds). 
The Catholic University of America Vitreous State Laboratory
DuraMelter 1200 Tests with AZ-101HLW Simulants Final Report, VSL-01R0100-2, Rev.1

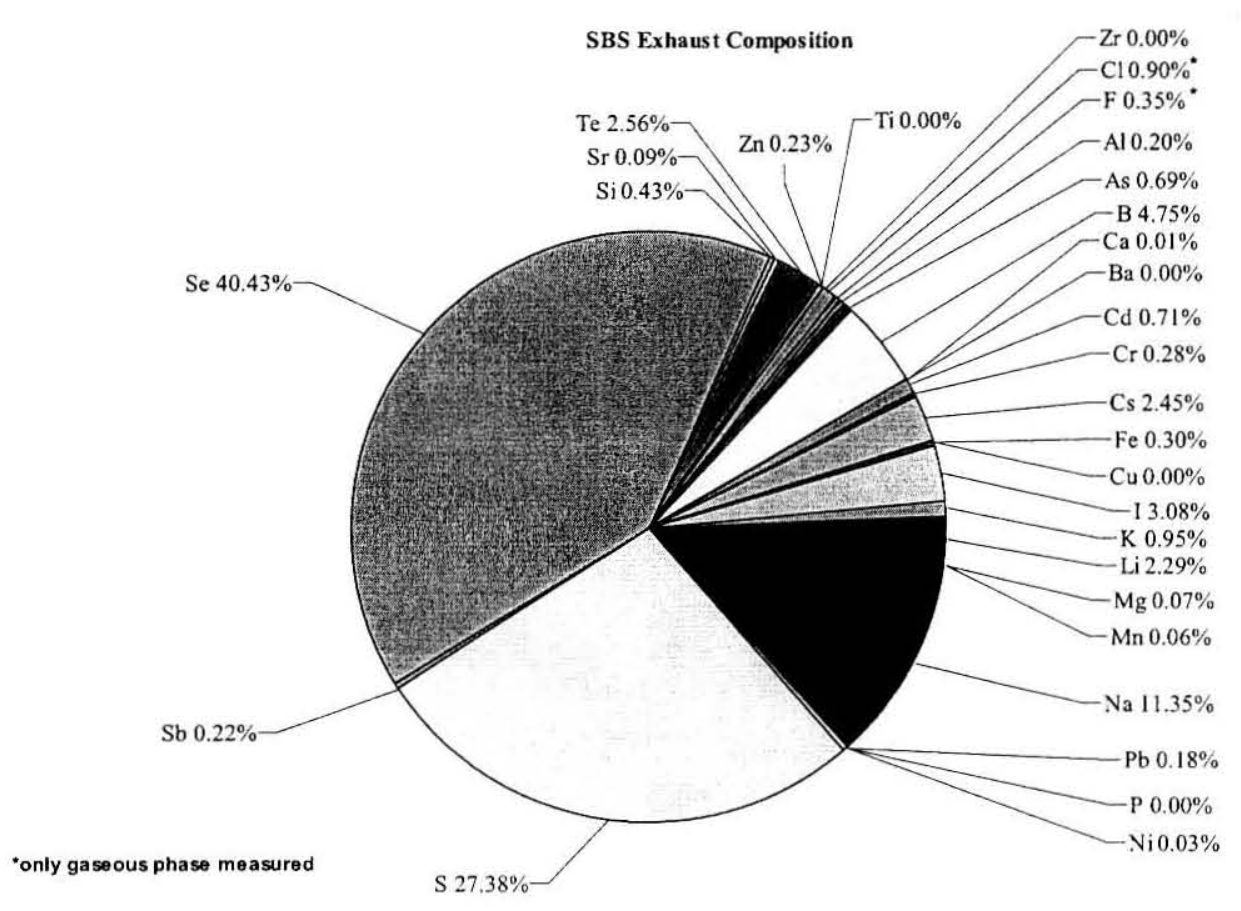

Figure 6.3. Test 3 SBS exhaust composition (excludes oxygen, nitrogen species, organic compounds).

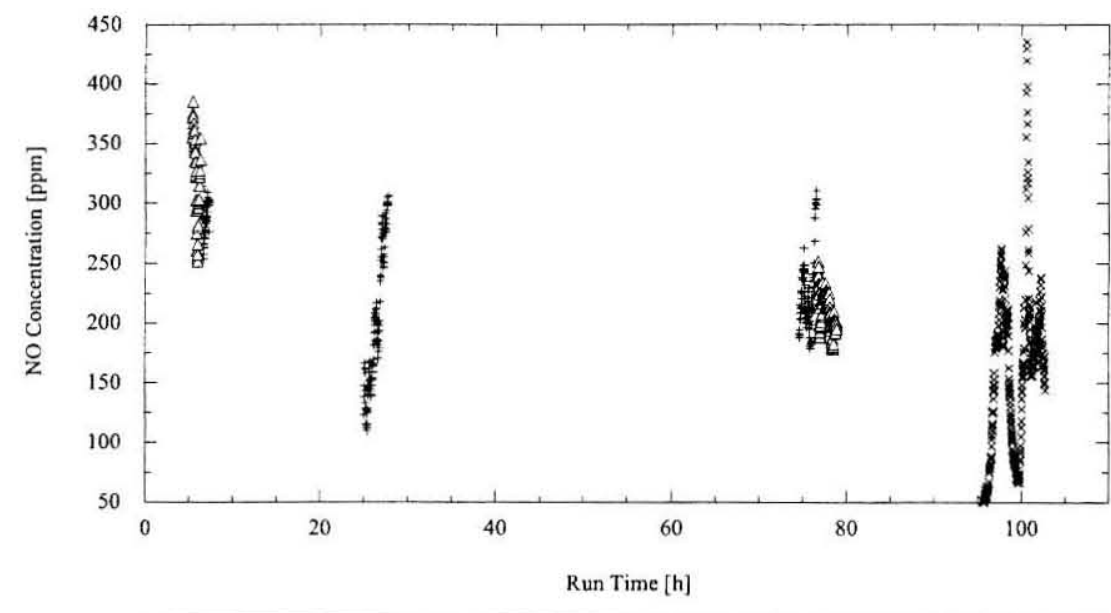

\begin{tabular}{|l|ll|}
\hline$\times$ NO (melter outlet) & + NO (sbs outlet) & NO (wesp outlet) \\
\hline
\end{tabular}

Figure 6.4. Off-gas concentration of NO measured by FTIR spectroscopy at intervals during Test 1. 
The Catholic University of America

Vitreous State Laboratory
DuraMelter 1200 Tests with AZ-101HLW Simulants Final Report, VSL-01R0100-2, Rev.1

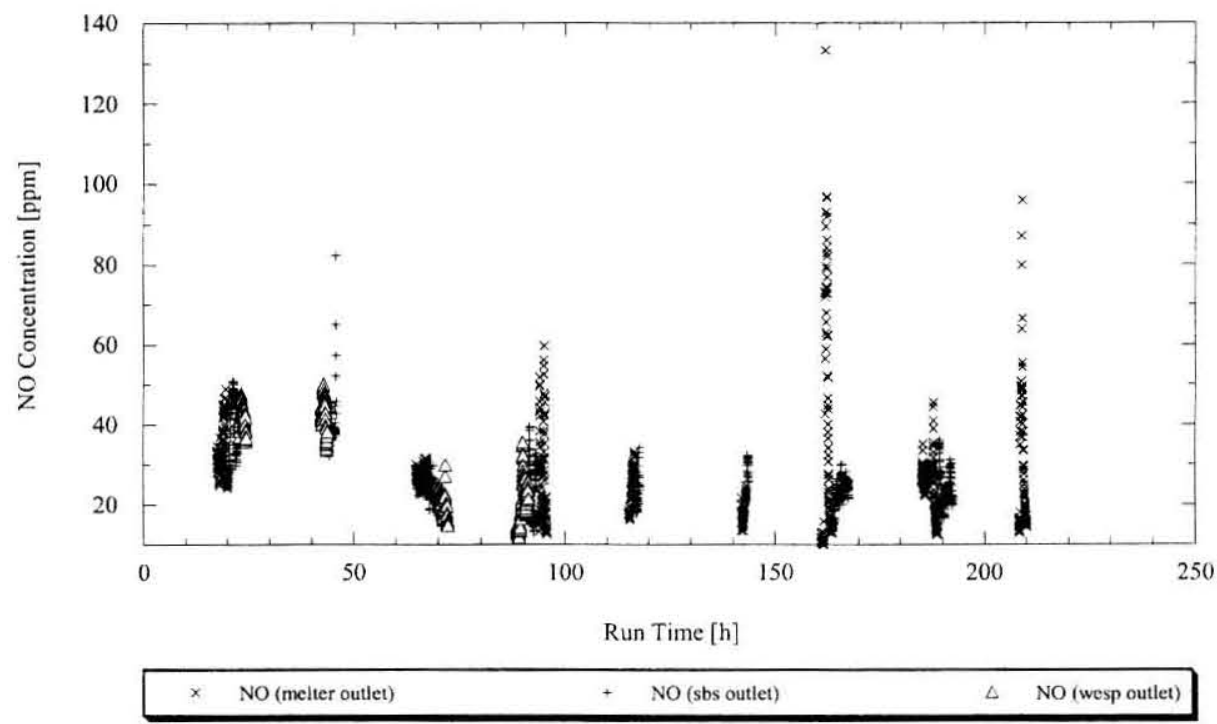

Figure 6.5. Off-gas concentration of NO measured by FTIR spectroscopy at intervals during Test 2 .

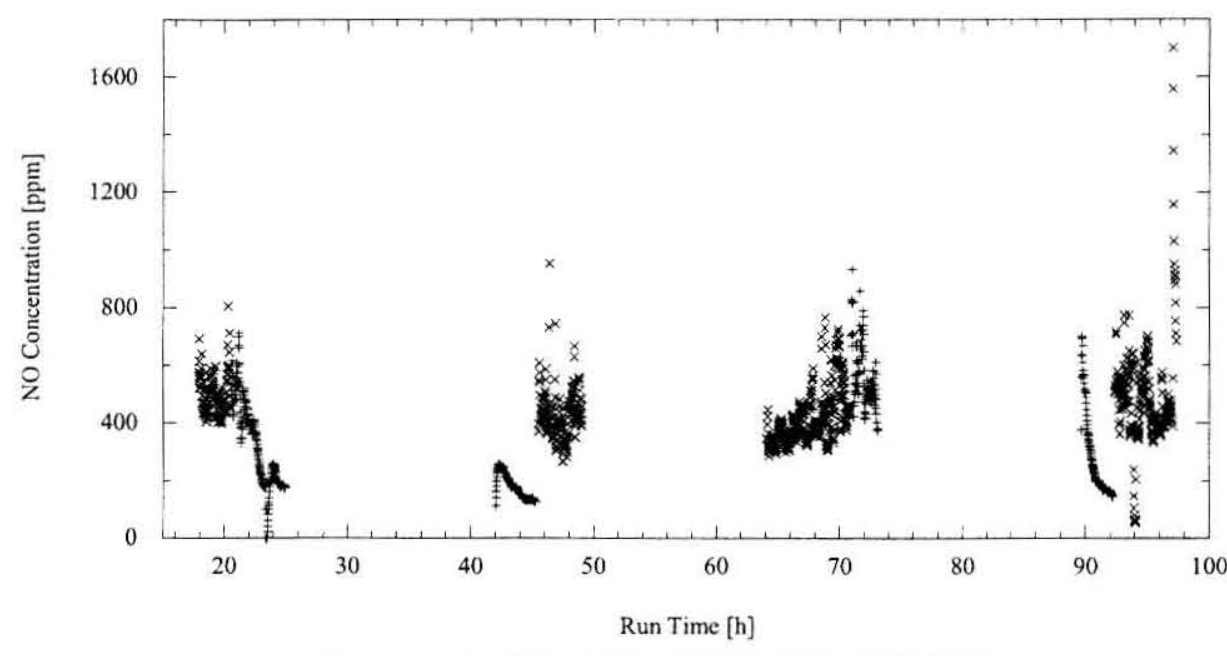

NO (melter outlet)

NO (sbs outlet)

Figure 6.6. Off-gas concentration of NO measured by FTIR spectroscopy at intervals during Test 3.

F-92 
The Catholic University of America Vitreous State Laboratory
DuraMelter 1200 Tests with AZ-101HLW Simulants

Final Report, VSL-01R0100-2, Rev.1

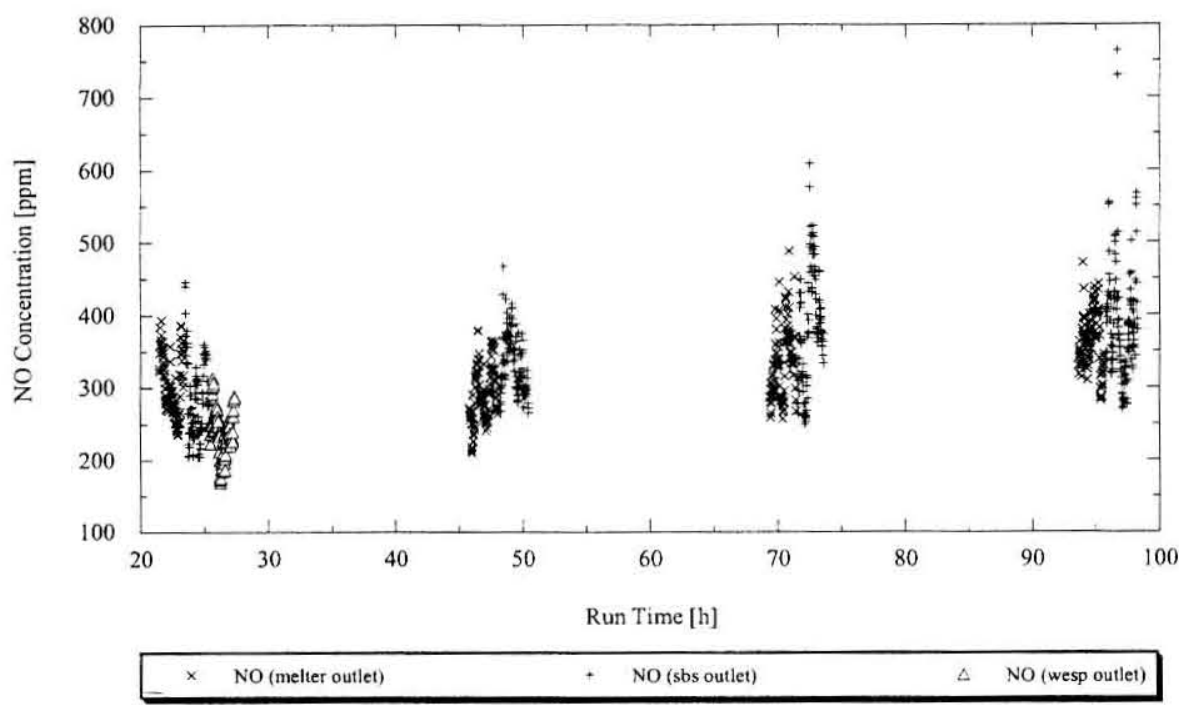

Figure 6.7. Off-gas concentration of measured by FTIR spectroscopy at intervals during Test 4 .

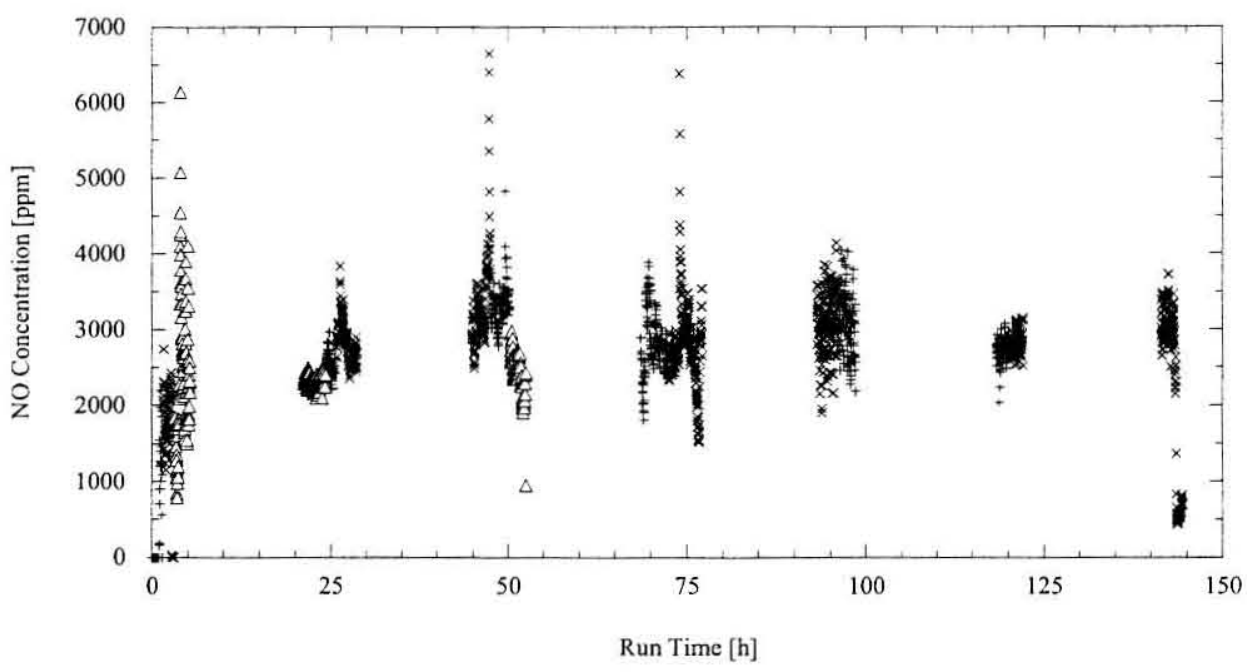

NO (melter outlet)

NO (sbs outlet)

$\triangle \quad$ NO (wesp outlet)

Figure 6.8. Off-gas concentration of NO measured by FTIR spectroscopy at intervals during Test 5. 
The Catholic University of America Vitreous State Laboratory
DuraMelter 1200 Tests with AZ-101HLW Simulants Final Report, VSL-01R0100-2, Rev.I

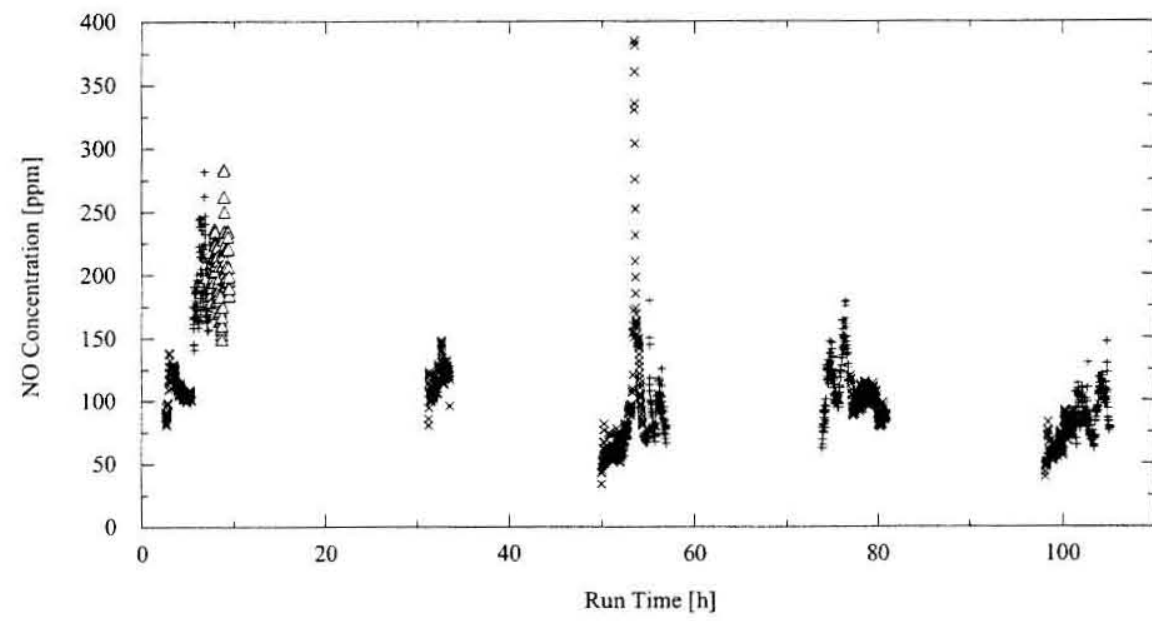

$\times \quad$ NO (melter outlet)

NO (sbs outlet)

NO (wesp outlet)

Figure 6.9. Off-gas concentration of NO measured by FTIR spectroscopy at intervals during Test 6.

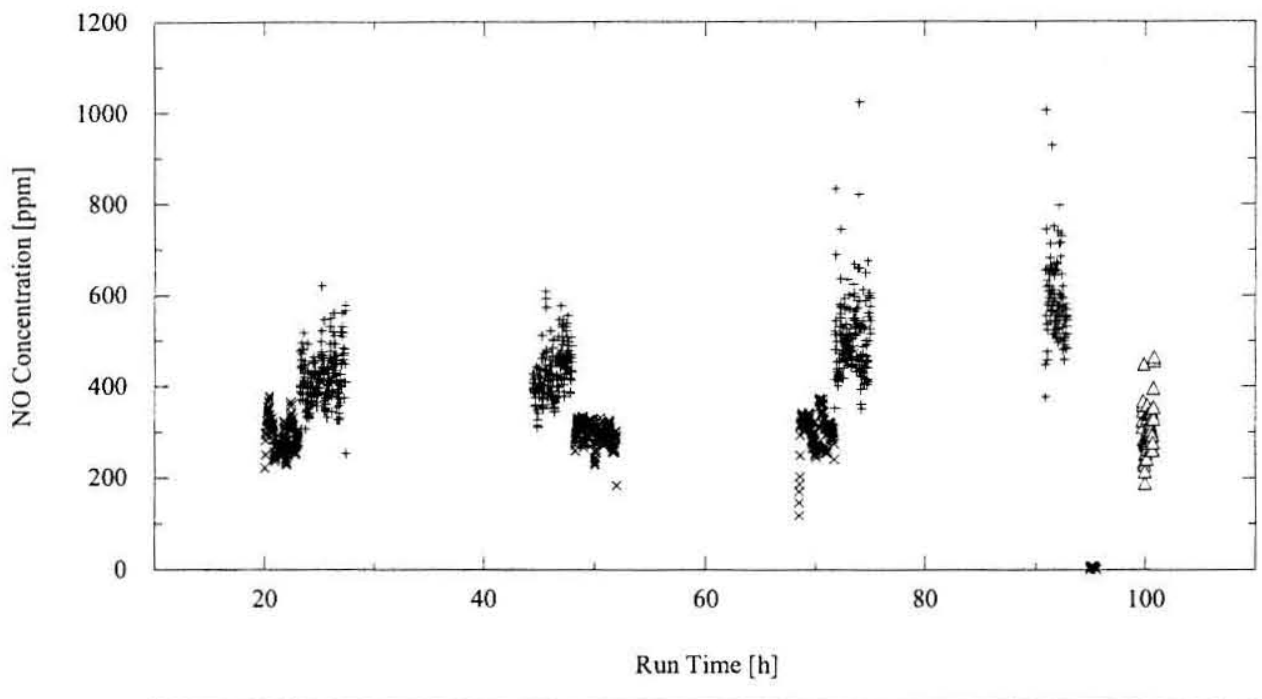

$\times \quad$ NO (melter outlet)

NO (sbs outlet)

$\triangle \quad N O$ (wesp outlet)

Figure 6.10. Off-gas concentration of NO measured by FTIR at intervals during Test 7 . 
The Catholic University of America Vitreous State Laboratory
DuraMelter 1200 Tests with AZ-101HLW Simulants Final Report, VSL-01R0100-2, Rev.1

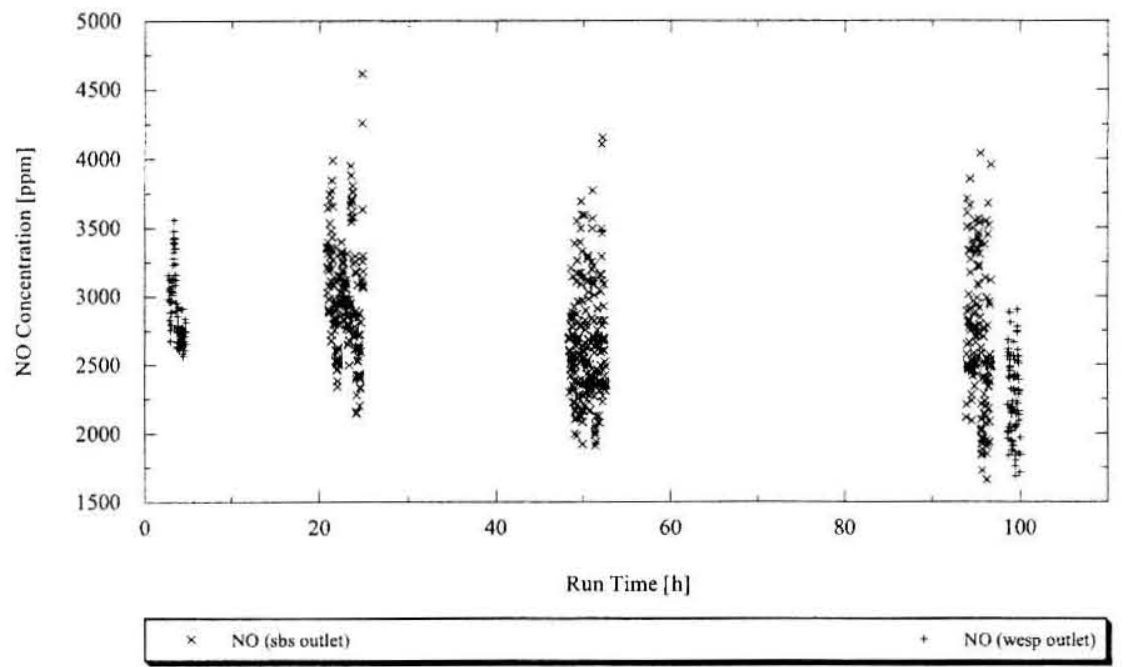

Figure 6.11. Off-gas concentration of NO measured by FTIR spectroscopy at intervals during Test 8.

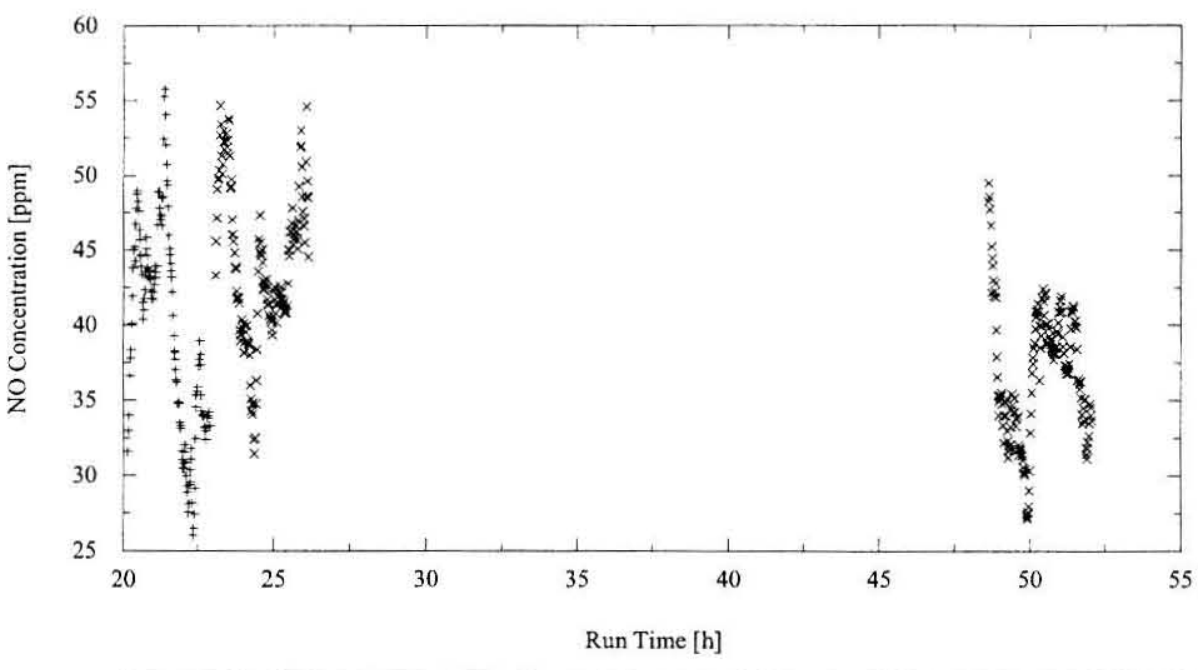

NO (sbs outlet)

NO (wesp outlet)

Figure 6.12. Off-gas concentration of NO measured by FTIR spectroscopy at intervals during Test 9a.

F-95 
The Catholic University of America Vitreous State Laboratory
DuraMelter 1200 Tests with AZ-101HLW Simulants Final Report, VSL-01R0100-2, Rev.1

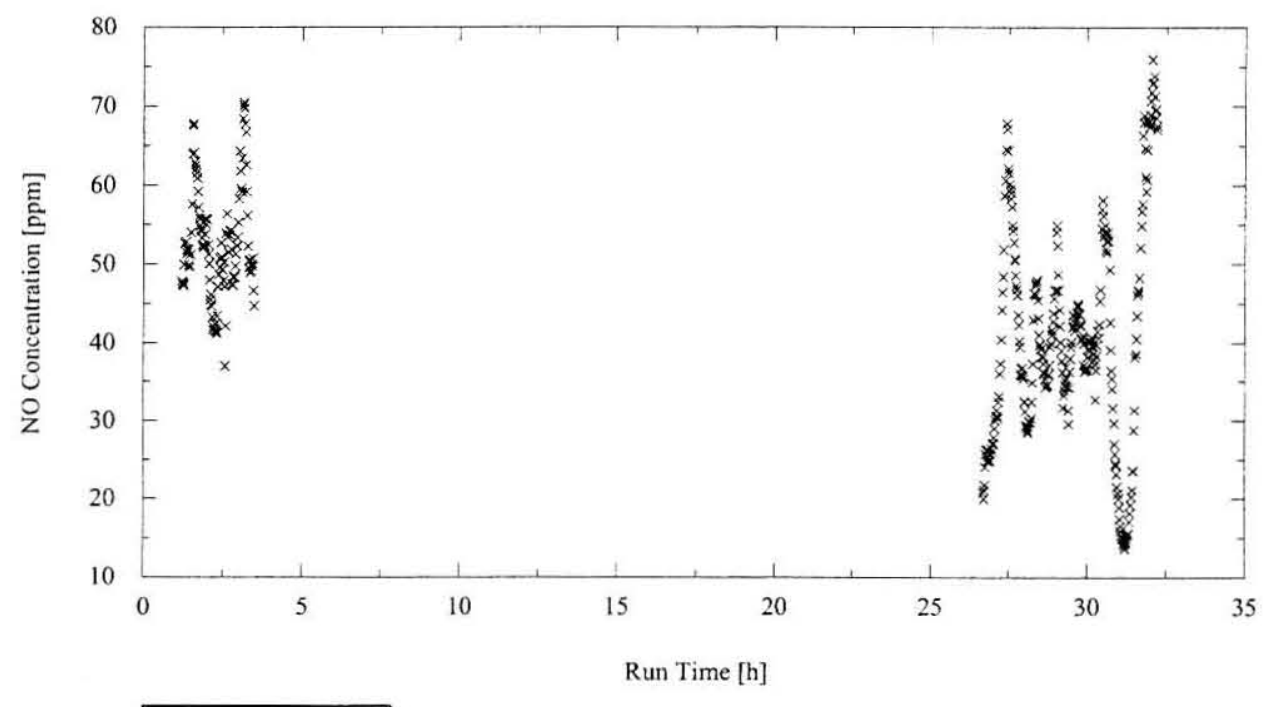

Figure 6.13. Off-gas concentration of NO measured by FTIR spectroscopy at intervals during Test $9 \mathrm{~b}$.

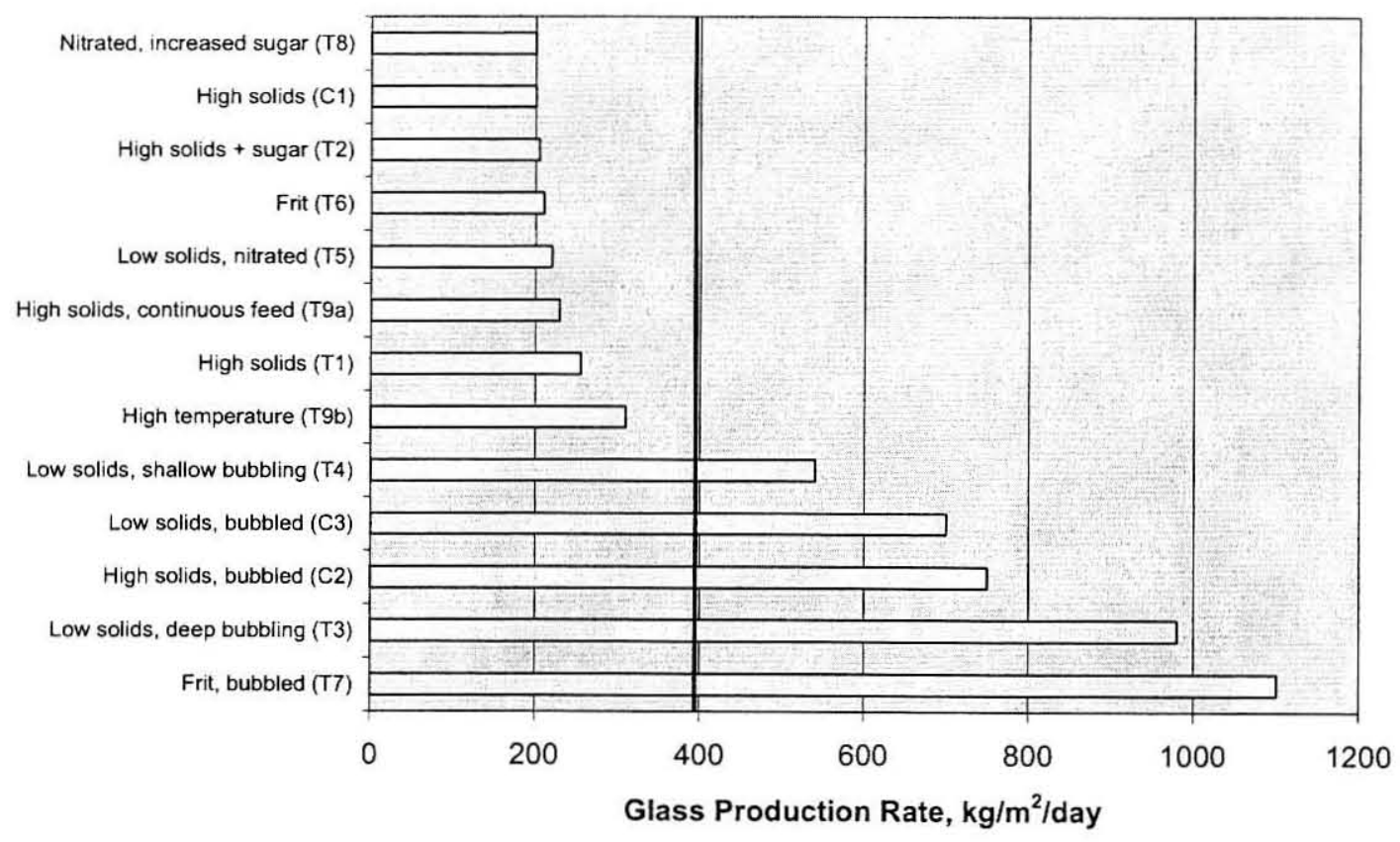

Figure 7.1. Summary of DM1200 glass production rates for each test; $T x=$ present tests, $\mathbf{C x}=$ commissioning tests (reference 5 ). 
The Catholic University of America Vitreous State Laboratory
DuraMelter 1200 Tests with AZ-101HLW Simulants Final Report, VSL-01R0100-2, Rev.1

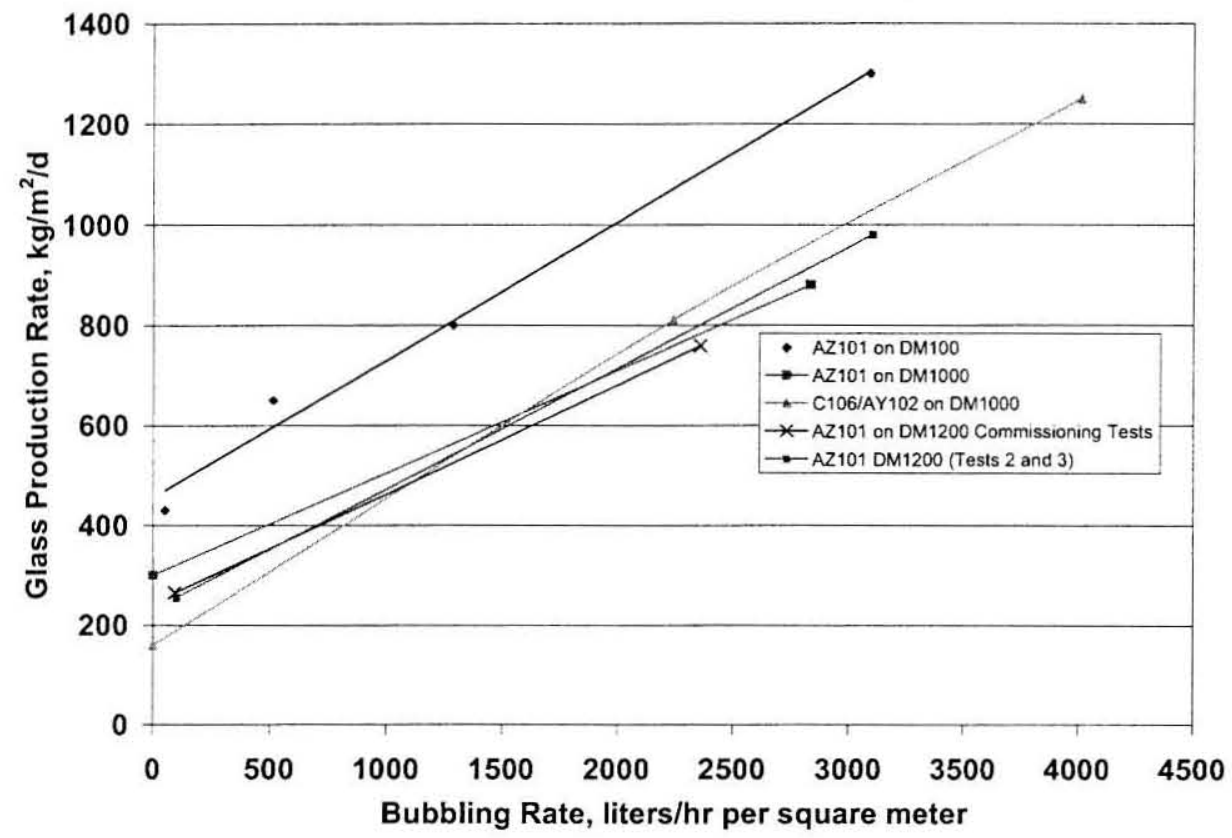

Figure 7.2. Summary of glass production rates as a function of total bubbler flow rate per unit melt surface area from DM100, DM1000, and DM1200 tests. (Note: Solids contents for Test 2 and 3 are different.) 


\section{APPENDIX A}

\section{ANALYSIS OF OFF-GAS SYSTEM DEPOSITS}

Components in the off-gas system were inspected throughout testing to provide additional information on component performance. In many instances, deposits that were found were sampled and analyzed. Provided in this Appendix are masses, analyzed compositions, SEM micrographs, EDS spectra, and back-scattered electron (BSE) images of the solid and sludge samples from the SBS bowl, SBS down-comer, WESP, and the off-gas blowers. Descriptions and a summary of the results for all of the samples are provided in Table A.1.

Post-test inspections were conducted on off-gas system components as follows:

\section{Test 1:}

- WESP collector plates, rods, and WESP bottom.

\section{Test 2:}

- WESP collector plates, rods, and WESP bottom.

\section{Test 3:}

- SBS bowl, diffuser plate, down-comer, and overflow tank.

- WESP collector plates, rods, and WESP bottom.

- Paxton blower.

\section{Test 4:}

- Film cooler.

- SBS bowl, diffuser plate, down-comer, and overflow tank.

- WESP collector plates, rods, and WESP bottom.

\section{Test 5:}

- SBS bowl, diffuser plate, down-comer, and overflow tank.

- WESP collector plates, rods, and WESP bottom.

\section{Test 6:}

- Film cooler.

- SBS bowl, diffuser plate, down-comer, and overflow tank. 
The Catholic University of America

Vitreous State Laboratory
DuraMelter 1200 Tests with AZ-101HLW Simulants Final Report, VSL-02R0100-2, Rev.1

- WESP collector plates, rods, and WESP bottom.

\section{Test 7:}

- SBS bowl, diffuser plate, down-comer, and overflow tank.

- WESP collector plates, rods, and WESP bottom.

\section{Test 8:}

- Film cooler.

- SBS bowl, diffuser plate, down-comer, and overflow tank.

- WESP collector plates and rods and WESP bottom.

\section{Test 9:}

- SBS bowl, diffuser plate, down-comer, and overflow tank.

- WESP collector plates, rods, and WESP bottom.

- Paxton blower.

No observation of color changes or solid depositions in the HEME were made during any of the tests.

\section{Tests 1 and 2}

At the end of each of the first two tests, solid samples from the WESP internals were obtained. DCP analysis (Table A.1) of a sample taken after Test 1 (12G-W-136A) indicated that the deposits consist mainly of iron, selenium, and tellurium, with lesser amounts of cadmium, chromium, and sulfur. SEM BSE images and EDS spectra of sample 12G-W-136A are given in Figures A1-A.3. SEM/EDS analysis of this material revealed agglomerated particles primarily consisting of $\mathrm{Fe}$ oxide and Se- and Te-containing phases. Some sulfur-containing regions were also observed and many other elements were present in trace amounts.

BSE images and EDS spectra of five regions from a sample taken after Test 2 (12J-W13B) are presented in Figures A.4 through Figure A.9. Material was selected on the basis of color and texture, mounted directly to an SEM stub with carbon tape, and left uncoated. BSE images were taken of representative areas and EDS spectra were collected. SEM/EDS analysis of the sample revealed significant variations in iron, tellurium, and cadmium concentrations. Other elements detected were sulfur, chlorine, and chromium. It should be noted that the cesium peak annotated on the spectra is buried beneath the tellurium peaks and may not be as significant as indicated in the EDS analysis results. 
University of America ste Laboratory
DuraMelter 1200 Tests with AZ-101HLW Simulants

Final Report, VSL-02R0100-2, Rev.1

DCP analysis (Table A.1) of the sludge samples from the SBS overflow tank (12K-S-6A and $12 \mathrm{~K}-\mathrm{S}-6 \mathrm{~B}$ ) show compositions that are similar to each other, and to that of the melter feed for many components (e.g., $\mathrm{SiO}_{2}$ between 42 and $46 \mathrm{wt} \%, \mathrm{Fe}_{2} \mathrm{O}_{3}$ about $10 \%$ ). Sample $12 \mathrm{~K}-\mathrm{S}-$ 10ARe was prepared from $12 \mathrm{~K}-\mathrm{S}-10 \mathrm{~A}$ and SEM/EDS analysis revealed a variety of materials; the corresponding spectra and point-by-point EDS results are given in Figures A.10 - A.15.

Deposits from the down-comer (12K-S-10B) were analyzed using BSE imaging and EDS spectra, as shown in Figures A.16 and A.17. Material was selected on the basis of color and texture, mounted directly to an SEM stub with carbon tape, and left uncoated. SEM/EDS analysis revealed significant variations in iron, selenium, and silicon concentrations. Representative BSE images show two primary regions: one region showed a stratified material, the other was a reddish, agglomerated material. Sulfur, fluorine, calcium, and zinc were also detected. Dense pockets of fluorine were observed periodically in the stratified material.

Figures A.18 - A21 show BSE images and EDS spectra of a solid sample (12K-W-6B) that was taken from WESP collector plate and rods. Material was selected from this sample on the basis of color and texture, mounted directly to an SEM stub with carbon tape, and left uncoated. SEM/EDS analysis revealed significant variations in iron, selenium, and tellurium concentrations; sulfur, cadmium, and chromium were also detected.

DCP analysis (Table A.1) of solids taken from the surface of Paxton blower rotor blades and outlet pipe (12K-O-10A) and filtered solids from washing these components (12K-O-10B) show the most abundant constituent to be selenium, with significant amounts of aluminum, nickel, sodium, and tellurium. BSE images and EDS spectra of the same samples are given in Figures A.22 and A.23. SEM/EDS analysis revealed agglomerated particles primarily consisting of $\mathrm{Se}$ and $\mathrm{Ni}$ deposits. In addition, pockets of $\mathrm{S}, \mathrm{Fe}, \mathrm{Al}, \mathrm{F}, \mathrm{Na}$, and Te were observed along with trace amounts of I. Point 1 on the BSE image (Figure A.22) was most representative of material throughout sample; Region 4 is some type of plastic material with the above-mentioned deposits on the surface.

\section{Test 4}

Figures A.24 - A.29 show BSE images and EDS spectra for samples of down-comer deposits (12K-O-144B) collected at the end of this test. SEM/EDS analysis revealed large regions of agglomerated material, including regions of crystallization. Major elements observed were $\mathrm{Al}, \mathrm{Mg}, \mathrm{Ca}, \mathrm{Mn}, \mathrm{Zn}, \mathrm{Se}, \mathrm{Sr}$, and $\mathrm{F}$.

DCP analysis (Table A.1) of the filtered solids from solutions generated by washing the WESP electrode rods (12K-O-144C) indicate the material to be rich in $\mathrm{Te}$ and $\mathrm{Se}$, with lesser amounts of $\mathrm{As}, \mathrm{Cd}, \mathrm{Cr}, \mathrm{Fe}, \mathrm{Pb}, \mathrm{Sb}$ and $\mathrm{Si}$.

Deposited solids of less than half an inch in thickness were observed on the walls of the film cooler at the end of this test but samples were not collected. 


\section{Test 5}

Approximately $1.4 \mathrm{~kg}$ of solids was removed from the SBS bowl at the end of this test. Deposits were removed from the WESP collector plates and rods. The WESP collector plate and rods were very clean after the wash-down.

\section{Test 6}

No solids build-up was observed in the film cooler; however, only a small part of the film cooler could be seen during the inspection.

A slight amount of corrosion was observed on the star-tips of the WESP collector rods.

The mass of the solids removed from the SBS bowl was $0.028 \mathrm{~kg}$ with very little solids build-up observed on the SBS down-comer.

\section{Test 7}

DCP analysis (Table A.1) of two separate samples of sludge from the SBS bowl (12N-O$133 \mathrm{~A}$ ) both indicate the material to be similar in composition to the melter feed (e.g., about $45 \mathrm{wt} \% \mathrm{SiO}_{2}$ and about $11 \mathrm{wt} \% \mathrm{Fe}_{2} \mathrm{O}_{3}$ ) with enrichments of certain volatile constituents ( $\mathrm{Se}$ and $\mathrm{Te})$.

Figures A.30 - A.32 show SEM/EDS analysis results of down-comer solids, which revealed a variety of agglomerated materials composed primarily of $\mathrm{Se}, \mathrm{Te}, \mathrm{Fe}$, and $\mathrm{Si}$. Large regions of sodium overlapping with fluorine (presumably sodium fluoride) were evident.

\section{Test 8}

DCP analysis (Table A.1) of the solids from the SBS overflow tank (12O-O-70A) and solids from the SBS bowl (120-O-75A) are similar to each other with respect to selenium. Material in the overflow tank contains more insoluble constituents, such as iron and silicon, whereas material from the SBS bowl contains more soluble constituents, such as boron and tellurium.

Before the wash-down of the WESP, large amounts of solids were observed on the rods. However, the wash-down was effective in removing the accumulated solids.

At the end of the test, inspection of the film cooler revealed a layer of solid deposits less than $1 / 2$ " thick primarily near the bottom on one side. The rest of the pipe was clean up to the transition pipe. Above the transition pipe, dry powdery solids were present. 


\section{Test 9}

The masses of solids removed from SBS bowl and SBS down-comer were $0.44 \mathrm{~kg}$ and $0.044 \mathrm{~kg}$, respectively.

Before the WESP wash-down, both the collector plates and rods were caked with solids. The plates and rods were covered with solids from the bottom up to about 12" up from the bottom. The wash-down was effective in removing the solids from this area but there were some solids left on the collector rods and plates from approximately 24 " from the bottom towards the top.

Solids obtained from the Paxton blower (12Q-O-119B) only partially dissolved using the standard dissolution method (a nitric/hydrofluoric acid mixture with microwave heating); the major component of the dissolved fraction was iron. 
The Catholic University of America

Table A.1. DCP Analyzed Compositions (wt\% Oxide Basis) of Samples Taken During DM 1200 Off-Gas System Inspections.

\begin{tabular}{|c|c|c|c|c|}
\hline Test \# & 1 & 2 & 3 & 3 \\
\hline Date/Time & $7 / 05 / 01,10: 00$ & $8 / 02 / 01,10: 15$ & $8 / 13 / 01,13: 20$ & $8 / 13 / 01,13: 20$ \\
\hline Sample Name & $12 \mathrm{G}-\mathrm{W}-136 \mathrm{~A}$ & $12 \mathrm{~J}-\mathrm{W}-13 \mathrm{~B}$ & $12 \mathrm{~K}-\mathrm{S}-6 \mathrm{~A}$ & $12 \mathrm{~K}-\mathrm{S}-6 \mathrm{~B}$ \\
\hline Figures & A.1-A.3 & A.4-A.9 & - & - \\
\hline Mass (kg) & $\mathrm{NA}$ & NA & 7.644 & 2.296 \\
\hline $\begin{array}{l}\text { Sample } \\
\text { Description }\end{array}$ & $\begin{array}{l}\text { WESP solids from } \\
\text { internal rods and } \\
\text { diffuser plate }\end{array}$ & $\begin{array}{l}\text { Dry crust on } \\
\text { WESP upper } \\
\text { internals. }\end{array}$ & $\begin{array}{l}\text { Sludge at the } \\
\text { bottom of SBS } \\
\text { overflow tank. }\end{array}$ & $\begin{array}{l}\text { Sludge at the } \\
\text { bottom of SBS } \\
\text { overflow tank }\end{array}$ \\
\hline $\begin{array}{l}\mathrm{Wt} \% \text { water in } \\
\text { original sample }\end{array}$ & NA & NA & 36.22 & 37.67 \\
\hline $\begin{array}{l}\text { Dried at } 110^{\circ} \mathrm{C} \\
\text { prior to analysis? }\end{array}$ & No & No & Yes & Yes \\
\hline $\mathrm{Al}_{2} \mathrm{O}_{3}$ & 0.04 & \multirow{28}{*}{$\begin{array}{l}\text { Main constituents } \\
\text { determined by } \\
\text { SEM/EDS are } \\
\text { Te, Fe, Cd; also } \\
\text { present were S, } \\
\mathrm{Cl}, \mathrm{Cr} \text { and Cs, as } \\
\text { shown in EDS } \\
\text { spectra (Figures } \\
\text { A.4-A.9). }\end{array}$} & 10.63 & 9.69 \\
\hline $\mathrm{As}_{2} \mathrm{O}_{3}$ & 0.13 & & 0.09 & 0.08 \\
\hline $\mathrm{B}_{2} \mathrm{O}_{3}$ & 0.36 & & 1.69 & 1.43 \\
\hline $\mathrm{BaO}$ & 0.00 & & 0.04 & 0.04 \\
\hline $\mathrm{CaO}$ & 0.05 & & 0.14 & 0.14 \\
\hline $\mathrm{CdO}$ & 1.24 & & 0.41 & 0.39 \\
\hline $\mathrm{CoO}$ & $\mathrm{ND}$ & & 0.03 & 0.01 \\
\hline $\mathrm{Cr}_{2} \mathrm{O}_{3}$ & 1.42 & & 0.08 & 0.08 \\
\hline $\mathrm{CuO}$ & 0.03 & & 0.03 & 0.03 \\
\hline $\mathrm{Fe}_{2} \mathrm{O}_{3}$ & 37.72 & & 9.61 & 9.91 \\
\hline $\mathrm{K}_{2} \mathrm{O}$ & 0.23 & & 0.05 & 0.04 \\
\hline $\mathrm{Li}_{2} \mathrm{O}$ & 0.31 & & 0.62 & 0.54 \\
\hline $\mathrm{MgO}$ & 0.02 & & 0.49 & 0.45 \\
\hline $\mathrm{MnO}_{2}$ & 0.09 & & 2.61 & 2.42 \\
\hline $\mathrm{Na} 2 \mathrm{O}$ & 0.97 & & 1.17 & 1.01 \\
\hline $\mathrm{NiO}$ & 0.07 & & 0.61 & 0.62 \\
\hline $\mathrm{P}_{2} \mathrm{O}_{5}$ & $<0.01$ & & 0.43 & 0.41 \\
\hline $\mathrm{PbO}$ & 0.96 & & 0.18 & 0.19 \\
\hline $\mathrm{SO}_{3}$ & 1.23 & & ND & ND \\
\hline $\mathrm{Sb}_{2} \mathrm{O}_{3}$ & 0.86 & & 0.29 & 0.25 \\
\hline $\mathrm{SeO}_{2}$ & 15.66 & & 2.79 & 2.96 \\
\hline $\mathrm{SiO}_{2}$ & 0.47 & & 42.28 & 45.94 \\
\hline $\mathrm{SrO}$ & 0.01 & & 1.03 & 0.85 \\
\hline $\mathrm{TeO}_{2}$ & 23.76 & & 1.40 & 1.55 \\
\hline $\mathrm{TiO}_{2}$ & 0.03 & & 0.10 & 0.09 \\
\hline $\mathrm{ZnO}$ & 0.16 & & 1.83 & 1.55 \\
\hline $\mathrm{ZrO}_{2}$ & 0.01 & & 5.16 & 5.06 \\
\hline Sum & 85.84 & & 83.87 & 85.74 \\
\hline
\end{tabular}

ND-Not detected

NA-Not analyzed 
The Catholic University of America Vitreous State Laboratory
DuraMelter 1200 Tests with AZ-101 HLW Simulants Final Report, VSL-02R0100-2, Rev.1

\section{Table A.1. DCP Analyzed Compositions (wt \% Oxide Basis) of Samples Taken During DM1200 Off-Gas System Inspections (Continued).}

\begin{tabular}{|c|c|c|c|c|}
\hline Test \# & 3 & 3 & 3 & 3 \\
\hline Date/Time & $8 / 14 / 01,21: 30$ & $8 / 16 / 01,13: 27$ & $8 / 13 / 01,13: 55$ & $8 / 15 / 01,11: 24$ \\
\hline Sample Number & $12 \mathrm{~K}-\mathrm{S}-10 \mathrm{~A}$ & $12 \mathrm{~K}-\mathrm{S}-10 \mathrm{~B}$ & $12 \mathrm{~K}-\mathrm{W}-6 \mathrm{~B}$ & $12 \mathrm{~K}-\mathrm{O}-10 \mathrm{~A}$ \\
\hline Figures & A.10-A.15 & A.16-A.17 & A.18-A.21 & A.22-A.23 \\
\hline Mass (kg) & 9.12 & 0.572 & $\mathrm{NA}$ & 0.00145 \\
\hline $\begin{array}{l}\text { Sample } \\
\text { Description }\end{array}$ & $\begin{array}{l}\text { Sludge from SBS } \\
\text { bowl. }\end{array}$ & $\begin{array}{l}\text { Solids from SBS- } \\
\text { down-comer pipe. }\end{array}$ & $\begin{array}{l}\text { Solids scraped from } \\
\text { WESP collector } \\
\text { plate and rods. }\end{array}$ & $\begin{array}{l}\text { Solids from surface } \\
\text { of rotor blades and } \\
\text { outlet pipe of } \\
\text { Paxton blower. }\end{array}$ \\
\hline $\begin{array}{l}\text { Wt \% water of } \\
\text { original sample }\end{array}$ & NA & NA & NA & NA \\
\hline $\begin{array}{l}\text { Dried at } 110^{\circ} \mathrm{C} \\
\text { prior to analysis? }\end{array}$ & Yes & No & No & No \\
\hline $\mathrm{Al}_{2} \mathrm{O}_{3}$ & \multirow{26}{*}{$\begin{array}{l}\text { (Sample \#12K- } \\
\text { S-10ARe). } \\
\text { Main constituents } \\
\text { determined by } \\
\text { SEM/EDS for } \\
20 \text { points on the } \\
\text { sample can be } \\
\text { seen in Figures } \\
\text { A.11 through } \\
\text { Figure A.15. }\end{array}$} & \multirow{26}{*}{$\begin{array}{l}\text { Main constituents } \\
\text { determined by } \\
\text { SEM/EDS are Fe, } \\
\text { Se, Si; other } \\
\text { elements present } \\
\text { were S, F, Ca, } \mathrm{Zn} \\
\text { and F, as shown in } \\
\text { EDS spectra } \\
\text { (Figures A.16- } \\
\text { A.17). }\end{array}$} & \multirow{26}{*}{$\begin{array}{l}\text { Main constituents } \\
\text { determined by } \\
\text { SEM/EDS are Fe, } \\
\text { Se, Te; other } \\
\text { elements present } \\
\text { were S, Cd, Cr and } \\
\text { Cs, as shown in } \\
\text { EDS spectra } \\
\text { (Figures A.18- } \\
\text { A.21). }\end{array}$} & 7.51 \\
\hline $\mathrm{As}_{2} \mathrm{O}_{3}$ & & & & 0.08 \\
\hline $\mathrm{B}_{2} \mathrm{O}_{3}$ & & & & 0.37 \\
\hline $\mathrm{BaO}$ & & & & 0.01 \\
\hline $\mathrm{CaO}$ & & & & 0.31 \\
\hline $\mathrm{CdO}$ & & & & 0.33 \\
\hline $\mathrm{Cr}_{2} \mathrm{O}_{3}$ & & & & 0.52 \\
\hline $\mathrm{CuO}$ & & & & ND \\
\hline $\mathrm{Fe}_{2} \mathrm{O}_{3}$ & & & & 3.05 \\
\hline $\mathrm{K}_{2} \mathrm{O}$ & & & & 1.09 \\
\hline $\mathrm{Li}_{2} \mathrm{O}$ & & & & 0.96 \\
\hline $\mathrm{MgO}$ & & & & 0.09 \\
\hline $\mathrm{MnO}$ & & & & 0.09 \\
\hline $\mathrm{Na}_{2} \mathrm{O}$ & & & & 5.17 \\
\hline $\mathrm{NiO}$ & & & & 8.49 \\
\hline $\mathrm{P}_{2} \mathrm{O}_{5}$ & & & & 0.32 \\
\hline $\mathrm{PbO}$ & & & & 0.13 \\
\hline $\mathrm{Sb}_{2} \mathrm{O}_{3}$ & & & & 0.07 \\
\hline $\mathrm{SeO}_{2}$ & & & & 30.82 \\
\hline $\mathrm{SiO}_{2}$ & & & & 1.84 \\
\hline $\mathrm{SrO}$ & & & & 0.02 \\
\hline $\mathrm{TeO}_{2}$ & & & & 2.87 \\
\hline $\mathrm{TiO}_{2}$ & & & & 0.07 \\
\hline $\mathrm{ZnO}$ & & & & 0.51 \\
\hline $\mathrm{ZrO}_{2}$ & & & & 0.02 \\
\hline Sum & & & & 67.89 \\
\hline
\end{tabular}

ND-Not detected

NA-Not analyzed 
The Catholic University of America

Vitreous State Laboratory

Table A.1. DCP Analyzed Compositions (wt\% Oxide Basis) of Samples Taken During DM1200 Off-Gas System Inspections (Continued).

\begin{tabular}{|c|c|c|c|c|}
\hline Test \# & 3 & 4 & 4 & 4 \\
\hline Date/Time & $8 / 15 / 01,11: 24$ & $8 / 29 / 01,15: 10$ & $8 / 30 / 01,08: 18$ & $8 / 30 / 01,11: 26$ \\
\hline Sample Number & $12 \mathrm{~K}-\mathrm{O}-10 \mathrm{~B}$ & $12 \mathrm{~K}-\mathrm{S}-144 \mathrm{~A}$ & $12 \mathrm{~K}-\mathrm{O}-144 \mathrm{~B}$ & $12 \mathrm{~K}-\mathrm{O}-144 \mathrm{C}$ \\
\hline Figures & - & - & A.24-A.29 & - \\
\hline Mass (kg) & $\mathrm{NA}$ & 5.986 & 0.328 & NA \\
\hline $\begin{array}{l}\text { Sample } \\
\text { Description }\end{array}$ & $\begin{array}{l}\text { Filtered solids } \\
\text { from solution } \\
\text { after flushing } \\
\text { Paxton outlet } \\
\text { pipe. }\end{array}$ & $\begin{array}{l}\text { Sludge from SBS } \\
\text { bowl solids. }\end{array}$ & $\begin{array}{l}\text { Solids collected } \\
\text { from SBS down- } \\
\text { comer pipe. }\end{array}$ & $\begin{array}{l}\text { Filtered solids from } \\
\text { solution after } \\
\text { washing the WESP } \\
\text { electrode rods. }\end{array}$ \\
\hline $\begin{array}{l}\mathrm{Wt} \% \text { water of } \\
\text { original sample }\end{array}$ & NA & 39.93 & NA & NA \\
\hline $\begin{array}{l}\text { Dried at } 110^{\circ} \mathrm{C} \\
\text { prior to analysis? }\end{array}$ & $\begin{array}{l}\text { Yes, after } \\
\text { filtering. }\end{array}$ & No & - & Yes, after filtering. \\
\hline $\mathrm{Al}_{2} \mathrm{O}_{3}$ & 7.50 & \multirow{26}{*}{$\begin{array}{l}\mathrm{pH}=6.90 \\
\text { Density }=1.72 \\
\mathrm{~g} / \mathrm{ml} .\end{array}$} & \multirow{26}{*}{$\begin{array}{l}\text { Major constituents } \\
\text { determined by } \\
\mathrm{SEM} / \mathrm{EDS} \text { are } \mathrm{Al} \text {, } \\
\mathrm{Mg}, \mathrm{Ca}, \mathrm{Mn}, \mathrm{Zn} \text {, } \\
\mathrm{Se}, \mathrm{Sr} \text { and } \mathrm{F} \text {, as } \\
\text { shown in } \mathrm{EDS} \\
\text { spectra (Figures } \\
\text { A.25-A.29). }\end{array}$} & 0.21 \\
\hline $\mathrm{As}_{2} \mathrm{O}_{3}$ & 0.09 & & & 1.37 \\
\hline $\mathrm{B}_{2} \mathrm{O}_{3}$ & 0.11 & & & 0.28 \\
\hline $\mathrm{BaO}$ & 0.02 & & & 0.00 \\
\hline $\mathrm{CaO}$ & 1.32 & & & 0.07 \\
\hline $\mathrm{CdO}$ & 1.58 & & & 2.66 \\
\hline $\mathrm{Cr}_{2} \mathrm{O}_{3}$ & 0.76 & & & 4.85 \\
\hline $\mathrm{CuO}$ & 0.42 & & & 0.05 \\
\hline $\mathrm{Fe}_{2} \mathrm{O}_{3}$ & 3.28 & & & 7.31 \\
\hline $\mathrm{K}_{2} \mathrm{O}$ & 0.58 & & & 0.07 \\
\hline $\mathrm{Li}_{2} \mathrm{O}$ & 0.13 & & & 0.06 \\
\hline $\mathrm{MgO}$ & 0.17 & & & 0.05 \\
\hline $\mathrm{MnO}$ & 0.14 & & & 0.13 \\
\hline $\mathrm{Na}_{2} \mathrm{O}$ & 1.43 & & & 0.16 \\
\hline $\mathrm{NiO}$ & 11.31 & & & 0.12 \\
\hline $\mathrm{P}_{2} \mathrm{O}_{5}$ & 0.51 & & & 0.08 \\
\hline $\mathrm{PbO}$ & 0.34 & & & 1.98 \\
\hline $\mathrm{Sb}_{2} \mathrm{O}_{3}$ & 0.09 & & & 2.49 \\
\hline $\mathrm{SeO}_{2}$ & 31.64 & & & 19.48 \\
\hline $\mathrm{SiO}_{2}$ & 2.14 & & & 2.85 \\
\hline $\mathrm{SrO}$ & 0.04 & & & 0.01 \\
\hline $\mathrm{TeO}_{2}$ & 7.47 & & & 41.68 \\
\hline $\mathrm{TiO}_{2}$ & 0.12 & & & 0.04 \\
\hline $\mathrm{ZnO}$ & 0.90 & & & 0.24 \\
\hline $\mathrm{ZrO}_{2}$ & 0.04 & & & 0.23 \\
\hline Sum & 72.13 & & & 86.48 \\
\hline
\end{tabular}

NA-Not analyzed 
Table A.1. DCP Analyzed Compositions (wt\% Oxide Basis) of Samples Taken During DM1200 Off-Gas System Inspections (Continued).

\begin{tabular}{|c|c|c|c|c|}
\hline Test \# & 7 & 7 & 7 & 8 \\
\hline Date/Time & $10 / 28 / 01,05: 11$ & 10/28/01,05:11 & $10 / 28 / 01,11: 59$ & $11 / 02 / 01,20: 00$ \\
\hline Sample Number & $12 \mathrm{~N}-\mathrm{O}-133 \mathrm{~A}$ & $12 \mathrm{~N}-\mathrm{O}-133 \mathrm{~A}$ & $12 \mathrm{~N}-\mathrm{O}-133 \mathrm{~B}$ & $12 \mathrm{O}-\mathrm{O}-70 \mathrm{~A}$ \\
\hline Figures & - & - & A.30-A.32 & - \\
\hline Mass (kg) & NA & NA & 0.058 & 1.01 \\
\hline $\begin{array}{l}\text { Sample } \\
\text { Description }\end{array}$ & $\begin{array}{l}\text { Sludge from } \\
\text { bottom of SBS } \\
\text { bowl. } \\
\text { (Sample \#1) }\end{array}$ & $\begin{array}{l}\text { Sludge from } \\
\text { bottom of SBS } \\
\text { bowl. } \\
\text { (Sample \#2) }\end{array}$ & $\begin{array}{l}\text { Solids from the } \\
\text { SBS down-comer } \\
\text { pipe. }\end{array}$ & $\begin{array}{l}\text { Leftover solids } \\
\text { after blow-down of } \\
\text { SBS overflow } \\
\text { tank. }\end{array}$ \\
\hline $\begin{array}{l}\text { Wt \% water of } \\
\text { original sample }\end{array}$ & 48.28 & 48.28 & NA & NA \\
\hline $\begin{array}{l}\text { Dried at } 110^{\circ} \mathrm{C} \\
\text { prior to analysis? }\end{array}$ & Yes & Yes & - & Yes, after filtering. \\
\hline $\mathrm{Al}_{2} \mathrm{O}_{3}$ & 3.03 & 3.13 & \multirow{26}{*}{$\begin{array}{l}\text { Major constituents } \\
\text { determined by } \\
\text { SEM/EDS are Se, } \\
\mathrm{Te}, \mathrm{Fe} \text { and } \mathrm{Si} \text {, as } \\
\text { shown in EDS } \\
\text { spectra (Figure } \\
\text { A.32). }\end{array}$} & 3.96 \\
\hline $\mathrm{As}_{2} \mathrm{O}_{3}$ & 0.09 & 0.04 & & $<0.01$ \\
\hline $\mathrm{B}_{2} \mathrm{O}_{3}$ & 5.04 & 4.98 & & 1.20 \\
\hline $\mathrm{BaO}$ & 0.03 & 0.03 & & 0.03 \\
\hline $\mathrm{CaO}$ & 0.26 & 0.25 & & 0.13 \\
\hline $\mathrm{CdO}$ & 0.18 & 0.17 & & 0.25 \\
\hline $\mathrm{Cr}_{2} \mathrm{O}_{3}$ & 0.07 & 0.07 & & 0.16 \\
\hline $\mathrm{CuO}$ & 0.01 & 0.01 & & 0.05 \\
\hline $\mathrm{Fe}_{2} \mathrm{O}_{3}$ & 11.10 & 10.69 & & 25.97 \\
\hline $\mathrm{K}_{2} \mathrm{O}$ & 0.08 & 0.09 & & 0.07 \\
\hline $\mathrm{Li}_{2} \mathrm{O}$ & 2.52 & 2.64 & & 0.83 \\
\hline $\mathrm{MgO}$ & 0.37 & 0.37 & & 0.24 \\
\hline $\mathrm{MnO}$ & 0.97 & 0.94 & & 0.16 \\
\hline $\mathrm{Na}_{2} \mathrm{O}$ & 2.95 & 2.96 & & 0.73 \\
\hline $\mathrm{NiO}$ & 0.47 & 0.47 & & 1.16 \\
\hline $\mathrm{P}_{2} \mathrm{O}_{5}$ & 0.24 & 0.23 & & 0.27 \\
\hline $\mathrm{PbO}$ & 0.20 & 0.20 & & 0.26 \\
\hline $\mathrm{Sb}_{2} \mathrm{O}_{3}$ & 0.34 & 0.26 & & 0.22 \\
\hline $\mathrm{SeO}_{2}$ & 10.60 & 11.20 & & 15.04 \\
\hline $\mathrm{SiO}_{2}$ & 45.76 & 44.80 & & 31.11 \\
\hline $\mathrm{SrO}$ & 0.65 & 0.62 & & 0.16 \\
\hline $\mathrm{TeO}_{2}$ & 2.68 & 2.63 & & 2.08 \\
\hline $\mathrm{TiO}_{2}$ & 0.22 & 0.20 & & 0.26 \\
\hline $\mathrm{ZnO}$ & 1.27 & 1.30 & & 0.54 \\
\hline $\mathrm{ZrO}_{2}$ & 1.45 & 1.36 & & 2.03 \\
\hline Sum & 90.5 & 89.63 & & 86.92 \\
\hline
\end{tabular}

ND-Not detected

NA-Not analyzed 
Table A.1. DCP Analyzed Compositions (wt\% Oxide Basis) of Samples Taken During DM1200 Off-Gas System Inspections (Continued).

\begin{tabular}{|c|c|c|c|}
\hline Test \# & 8 & 8 & 9 \\
\hline Date/Time & $11 / 03 / 01,08: 30$ & $11 / 03 / 01,08: 30$ & $12 / 17 / 01,16: 30$ \\
\hline Sample Number & $120-0-75 \mathrm{~A}$ & $12 \mathrm{O}-\mathrm{O}-75 \mathrm{~A}$ & 12Q-O-119B \\
\hline Figures & - & - & - \\
\hline Mass (kg) & 0.324 & 0.324 & 0.038 \\
\hline $\begin{array}{c}\text { Sample } \\
\text { Description }\end{array}$ & $\begin{array}{l}\text { Solids from SBS } \\
\text { bowl. } \\
\text { (Sample \#1). }\end{array}$ & $\begin{array}{c}\text { Solids from SBS } \\
\text { bowl. } \\
\text { (Sample \#2). }\end{array}$ & $\begin{array}{c}\text { Solids from Paxton } \\
\text { blower. }\end{array}$ \\
\hline $\begin{array}{l}\mathrm{Wt} \% \text { water of } \\
\text { original sample }\end{array}$ & 26.90 & 26.90 & NA \\
\hline $\begin{array}{l}\text { Dried at } 110^{\circ} \mathrm{C} \\
\text { prior to analysis? }\end{array}$ & Yes & Yes & - \\
\hline $\mathrm{Al}_{2} \mathrm{O}_{3}$ & 0.02 & 0.02 & \multirow{27}{*}{$\begin{array}{l}\text { Since the total } \\
\text { recovery was only } \\
17.55 \% \text {, the results } \\
\text { are not reported. } \\
\text { However, of that } \\
\text { fraction, the main } \\
\text { component was } \\
\mathrm{Fe}_{2} \mathrm{O}_{3} \text {. }\end{array}$} \\
\hline $\mathrm{As}_{2} \mathrm{O}_{3}$ & 0.02 & 0.03 & \\
\hline $\mathrm{B}_{2} \mathrm{O}_{3}$ & 18.75 & 18.62 & \\
\hline $\mathrm{BaO}$ & 0.00 & 0.00 & \\
\hline $\mathrm{CaO}$ & 0.01 & 0.02 & \\
\hline $\mathrm{CdO}$ & 0.34 & 0.35 & \\
\hline $\mathrm{Cr}_{2} \mathrm{O}_{3}$ & 0.10 & 0.10 & \\
\hline $\mathrm{CuO}$ & 0.02 & 0.01 & \\
\hline $\mathrm{Fe}_{2} \mathrm{O}_{3}$ & 1.23 & 1.18 & \\
\hline $\mathrm{K}_{2} \mathrm{O}$ & 0.26 & 0.26 & \\
\hline $\mathrm{Li} 2 \mathrm{O}$ & 0.94 & 0.91 & \\
\hline $\mathrm{MgO}$ & 0.02 & 0.03 & \\
\hline $\mathrm{MnO}$ & 0.01 & 0.01 & \\
\hline $\mathrm{MnO}_{2}$ & ND & ND & \\
\hline $\mathrm{Na}_{2} \mathrm{O}$ & 3.89 & 3.93 & \\
\hline $\mathrm{NiO}$ & 0.01 & 0.01 & \\
\hline $\mathrm{P}_{2} \mathrm{O}_{5}$ & 0.01 & 0.07 & \\
\hline $\mathrm{PbO}$ & 0.11 & 0.13 & \\
\hline $\mathrm{Sb}_{2} \mathrm{O}_{3}$ & 0.80 & 0.82 & \\
\hline $\mathrm{SeO}_{2}$ & 9.43 & 9.21 & \\
\hline $\mathrm{SiO}_{2}$ & 0.19 & 0.22 & \\
\hline $\mathrm{SrO}$ & 0.04 & 0.04 & \\
\hline $\mathrm{TeO}_{2}$ & 32.26 & 33.61 & \\
\hline $\mathrm{TiO}_{2}$ & 0.01 & 0.01 & \\
\hline $\mathrm{ZnO}$ & 0.03 & 0.03 & \\
\hline $\mathrm{ZrO}_{2}$ & 0.02 & 0.02 & \\
\hline Sum & 68.53 & 69.62 & \\
\hline
\end{tabular}

ND-Not detected

NA-Not analyzed 
The Catholic University of America Vitreous State Laboratory
DuraMelter 1200 Tests with AZ-101HLW Simulants Final Report, VSL-02R0100-2, Rev.I

Table A.2. Mass of Solids Removed from SBS Bowl and SBS Down-Comer.

\begin{tabular}{|c|c|c|c|c|}
\hline \multirow{3}{*}{$\begin{array}{l}\text { Before installation } \\
\text { of SBS down- } \\
\text { comer with } \\
\text { serrated bottom. }\end{array}$} & \multirow{2}{*}{ Test \# } & $\begin{array}{l}\text { Glass } \\
\text { Produced }\end{array}$ & $\begin{array}{l}\text { Solids from } \\
\text { SBS bowl. }\end{array}$ & $\begin{array}{l}\text { Solids from SBS down- } \\
\text { comer pipe }\end{array}$ \\
\hline & & Mass $(\mathrm{kg})$ & Mass $(\mathrm{kg})$ & Mass $(\mathrm{kg})$ \\
\hline & $1-3$ & 11075 & 9.12 & 0.572 \\
\hline \multirow{6}{*}{$\begin{array}{l}\text { After installation } \\
\text { of SBS down- } \\
\text { comer with } \\
\text { serrated bottom. }\end{array}$} & 4 & 3465 & 5.99 & 0.328 \\
\hline & 5 & 1659 & 1.4 & Solids present; cleaned. \\
\hline & 6 & 1901 & 0.028 & No visible solids build-up. \\
\hline & 7 & 5976 & $<\sim 0.5$ & 0.058 \\
\hline & 8 & 944 & 0.324 & $\begin{array}{l}1 / 4 " \text { thick build-up, up to } \\
\sim 4 " \text { from end; cleaned. }\end{array}$ \\
\hline & 9 & 1706 & 0.44 & 0.044 \\
\hline
\end{tabular}


The Catholic University of America Vitreous State Laboratory
DuraMelter 1200 Tests with AZ-101HLW Simulants

Final Report, VSL-02R0100-2, Rev.1

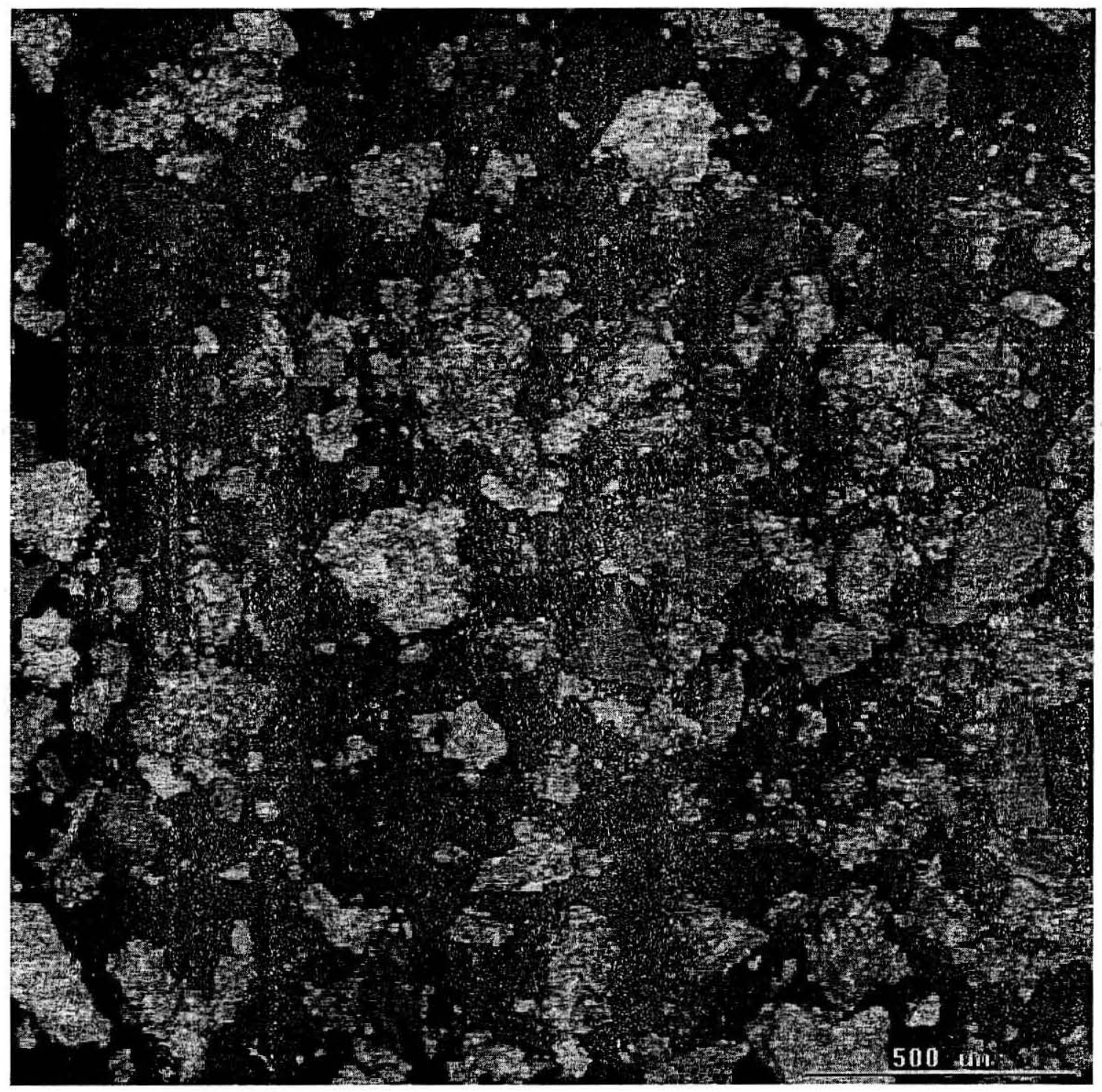

Figure A.1: BSE image of particles. The brighter, more agglomerated materials typically have high concentrations of Se and Te. The darker, more homogeneous materials are predominantly Fe oxide, incorporating varying amounts of Se. (Sample \#12G-W-136A). 
The Catholic University of America Vitreous State Laboratory
DuraMelter 1200 Tests with AZ-101 HLW Simulants Final Report, VSL-02R0100-2, Rev.1

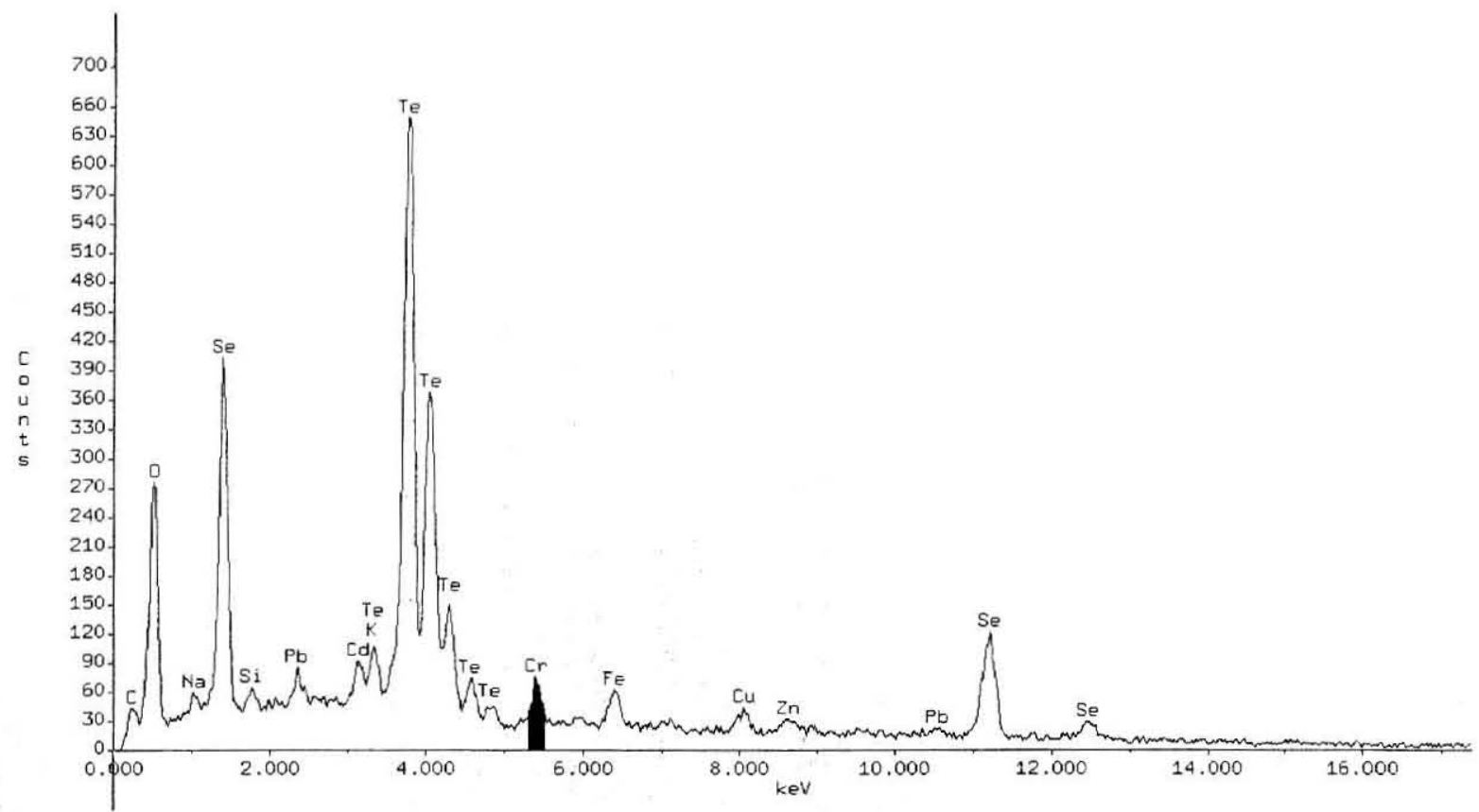

Figure A.2. General EDS spectrum of agglomerated material, (Sample \#12G-136A).

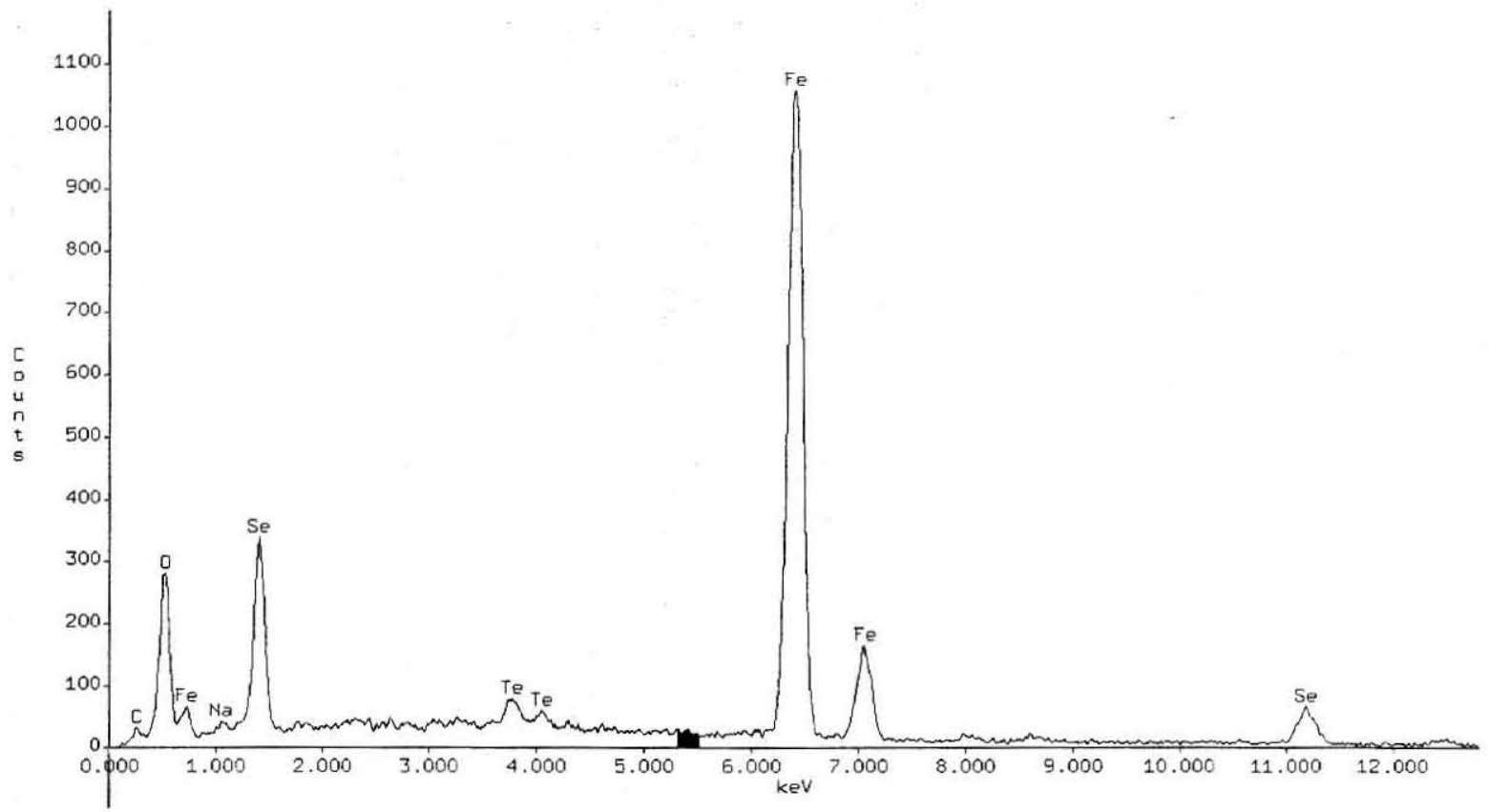

Figure A.3. General EDS spectrum of relatively homogeneous particles, (Sample \#12G-W-136A). 
The Catholic University of America

Vitreous State Laboratory
DuraMelter 1200 Tests with AZ-101HLW Simulants Final Report, VSL-02R0100-2, Rev.1

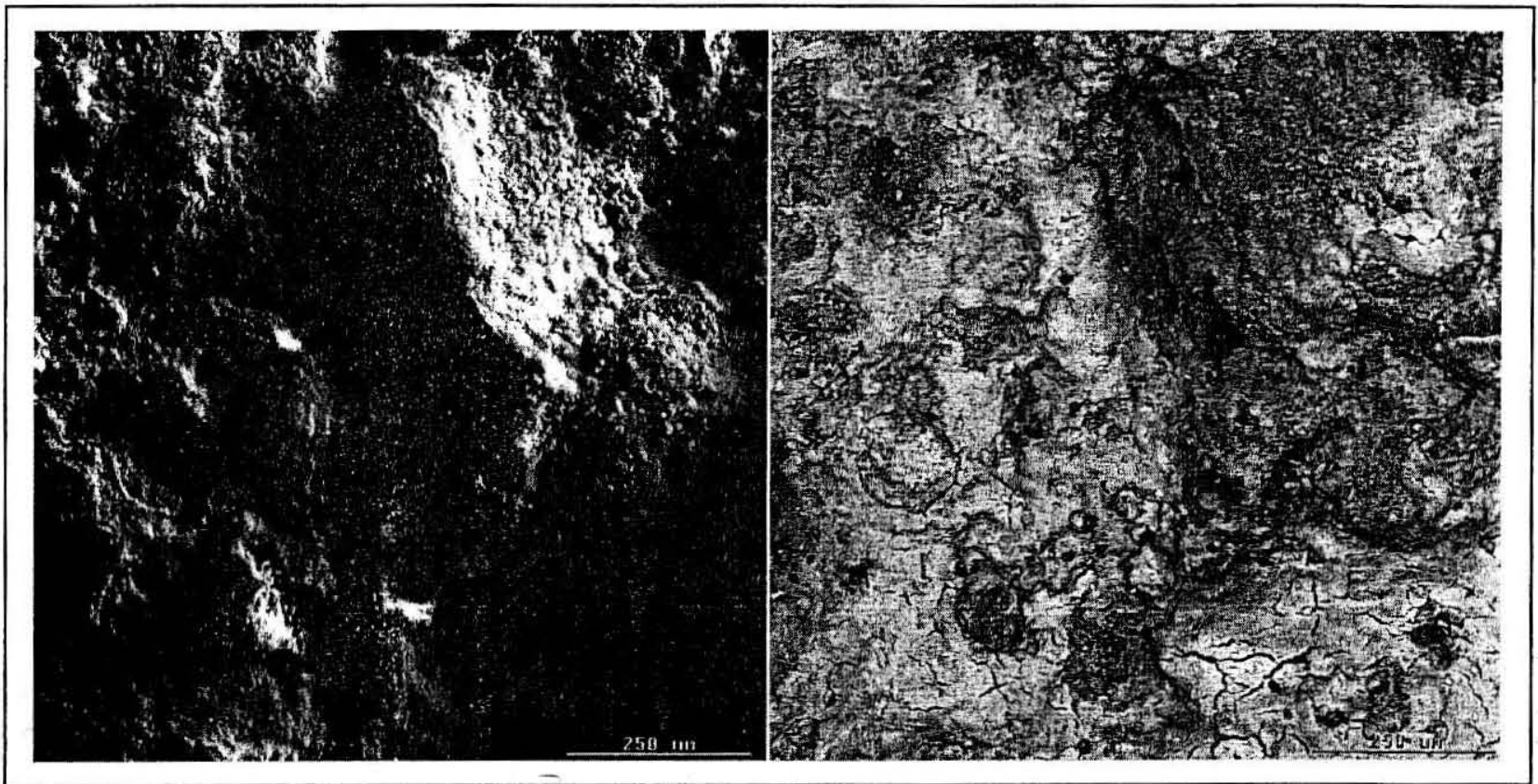

Accelerating Voltage: $25 \mathrm{KeV}$

Resolution:
Magnification: 100

Pixel Size: 0.975702 microns

Region \#1

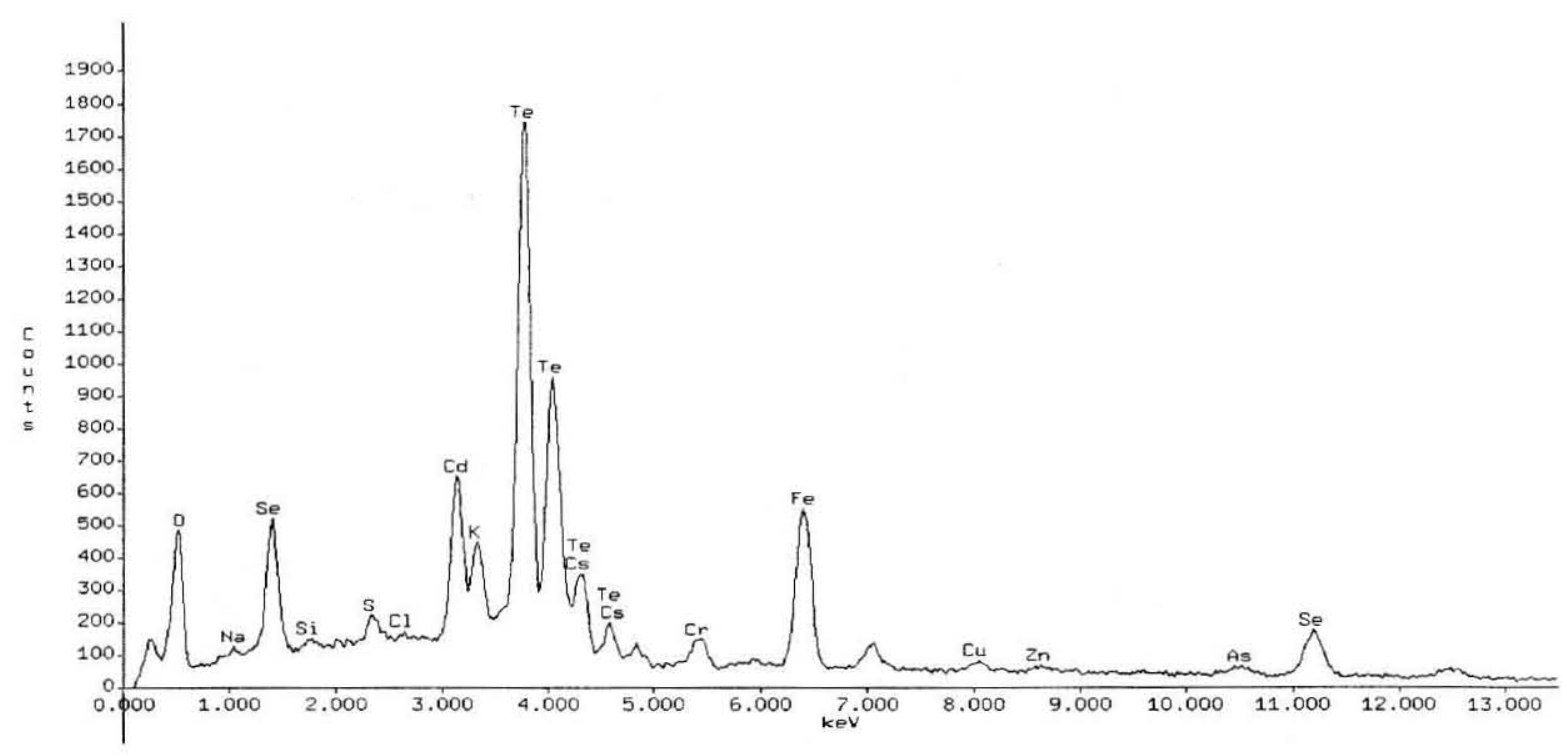

Region \#1

Figure A.4. BSE image and EDS analysis of Region \#1 in sample 12J-W-13B. 
The Catholic University of America Vitreous State Laboratory
DuraMelter 1200 Tests with AZ-101HLW Simulants Final Report, VSL-02R0100-2, Rev.1

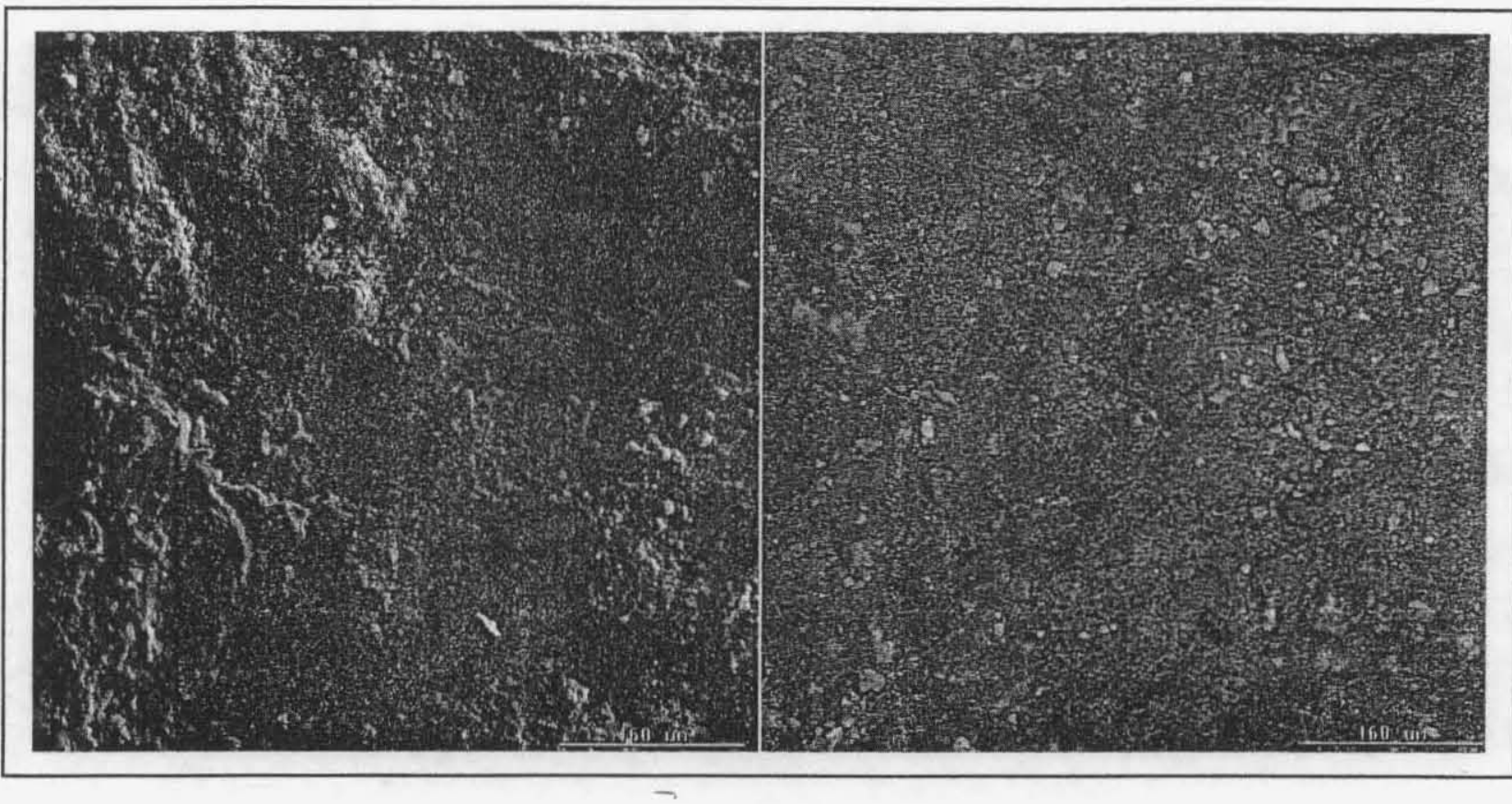

Accelerating Voltage: $25 \mathrm{KeV}$

Resolution: $\quad 1024 \times 1024$
Magnification: 160

Pixel Size: $\quad 0.609814$ microns Region \#2

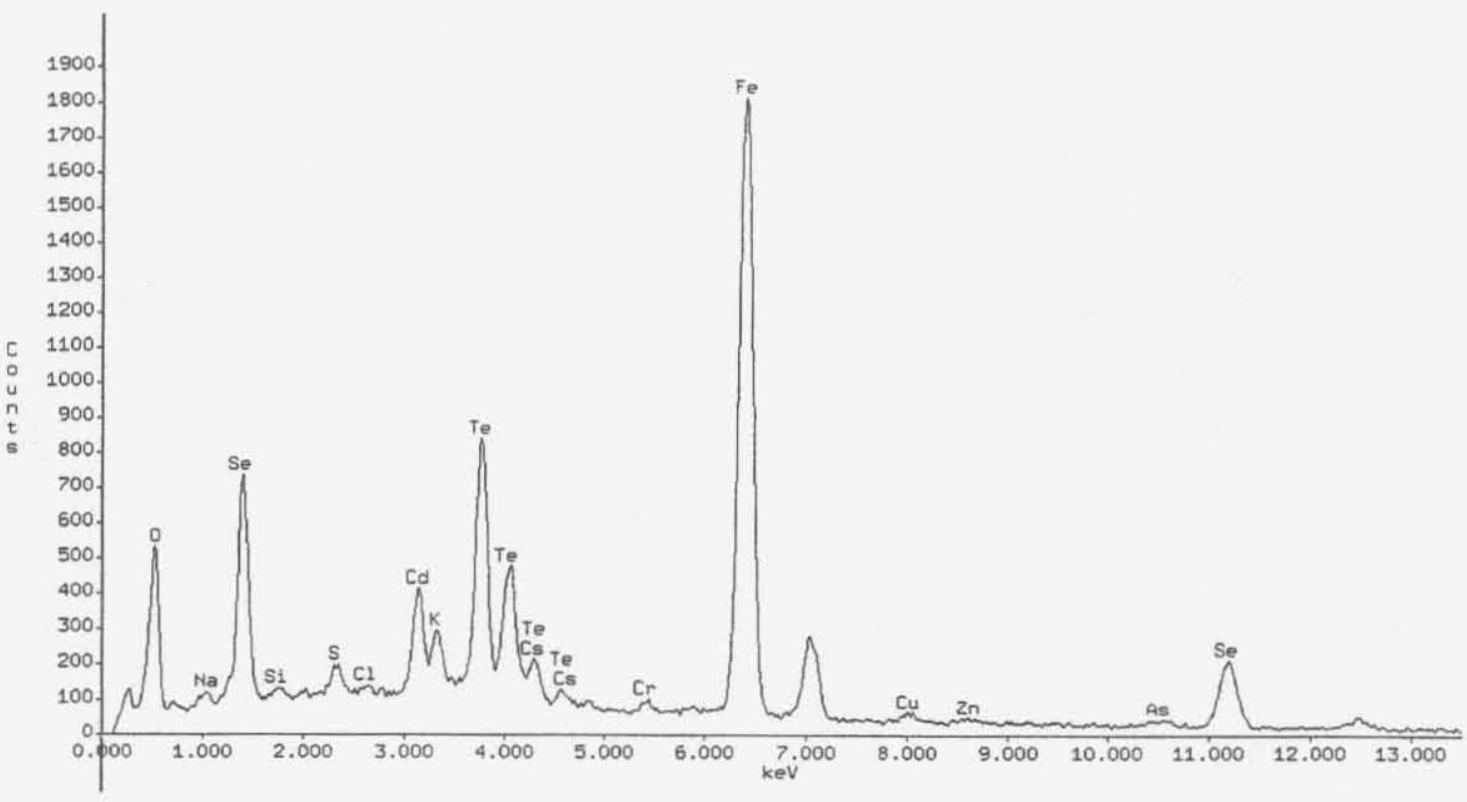

Figure A.5. BSE image and EDS analysis of Region \#2 in sample 12J-W-13B. 
The Catholic University of America Vitreous State Laboratory
DuraMelter 1200 Tests with AZ-101HLW Simulants Final Report, VSL-02R0100-2, Rev.1

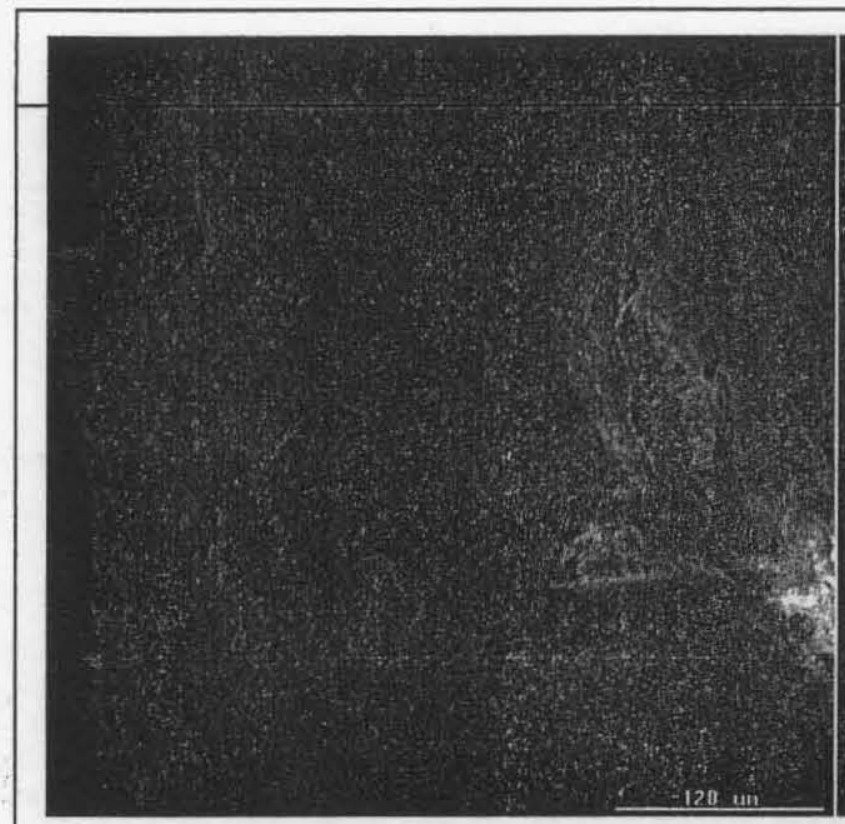

Accelerating Voltage: $25 \mathrm{KeV}$

Resolution:

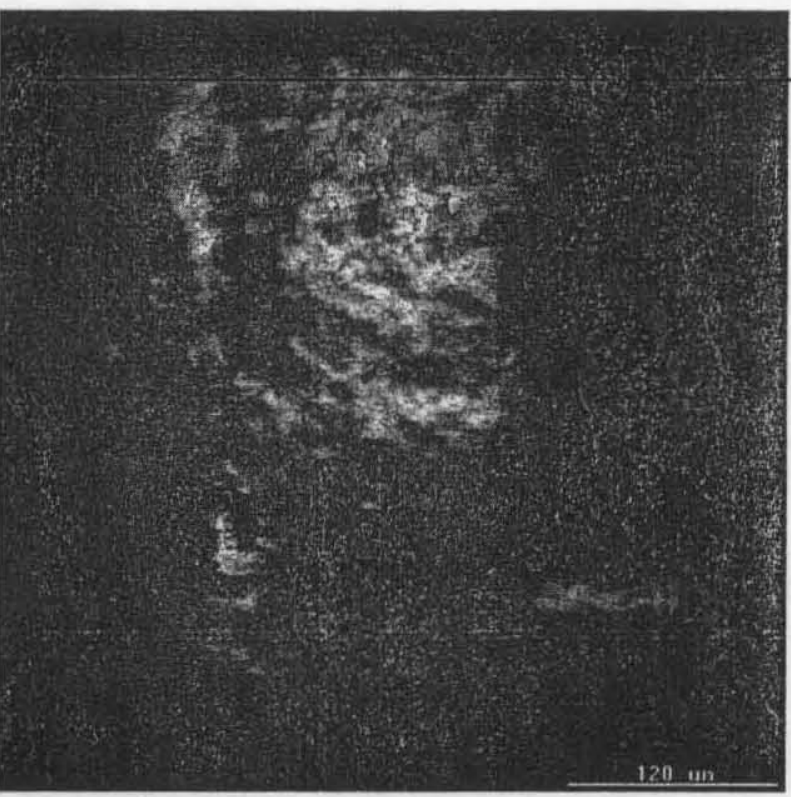

Magnification: 220

Pixel Size: $\quad 0.443501$ microns

Region \#3

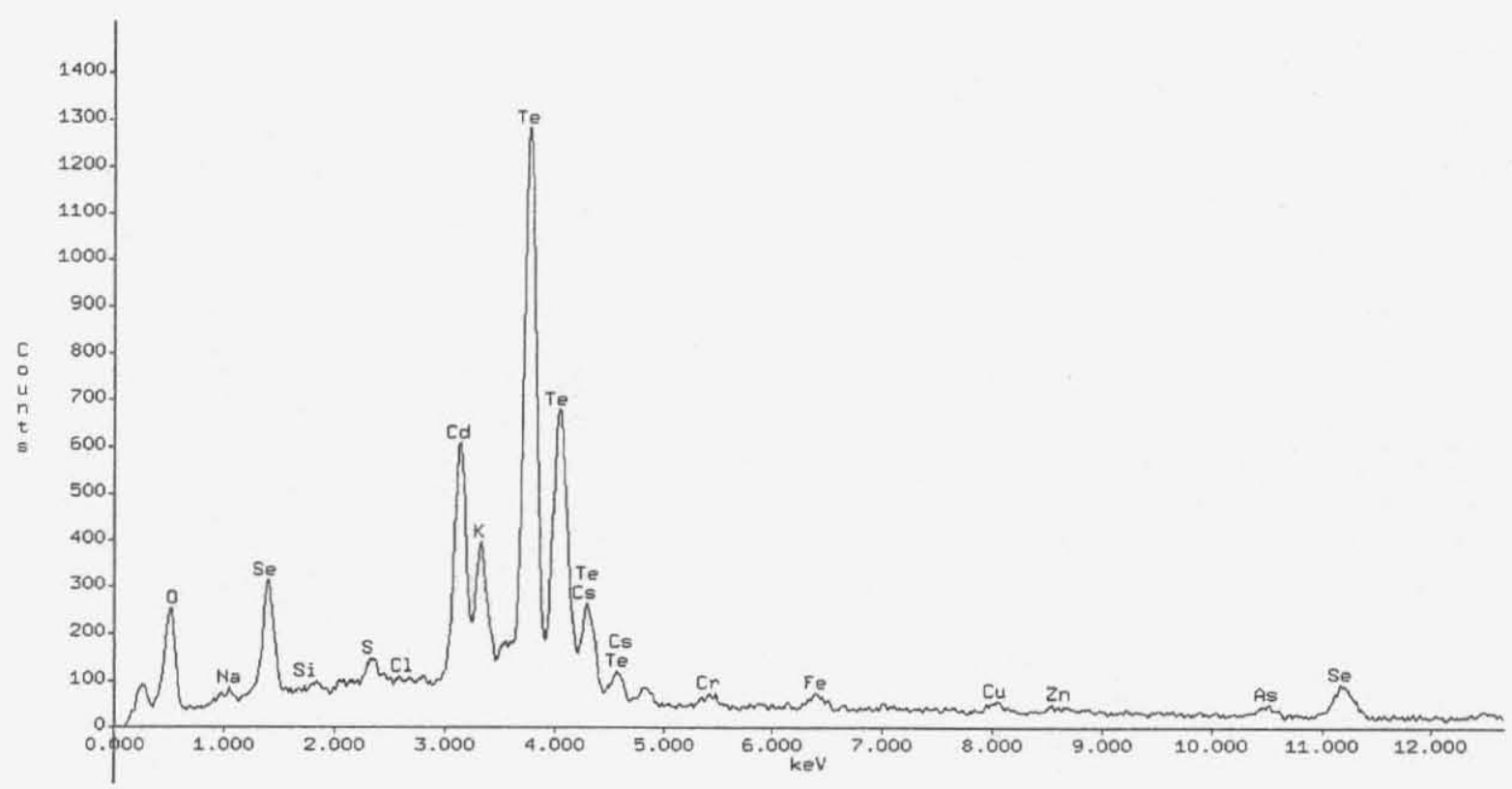

Figure A.6. BSE image and EDS analysis of Region \#3 in sample 12J-W-13B. 
The Catholic University of America

Vitreous State Laboratory
DuraMelter 1200 Tests with AZ-101HLW Simulants

Final Report, VSL-02R0100-2, Rev.1

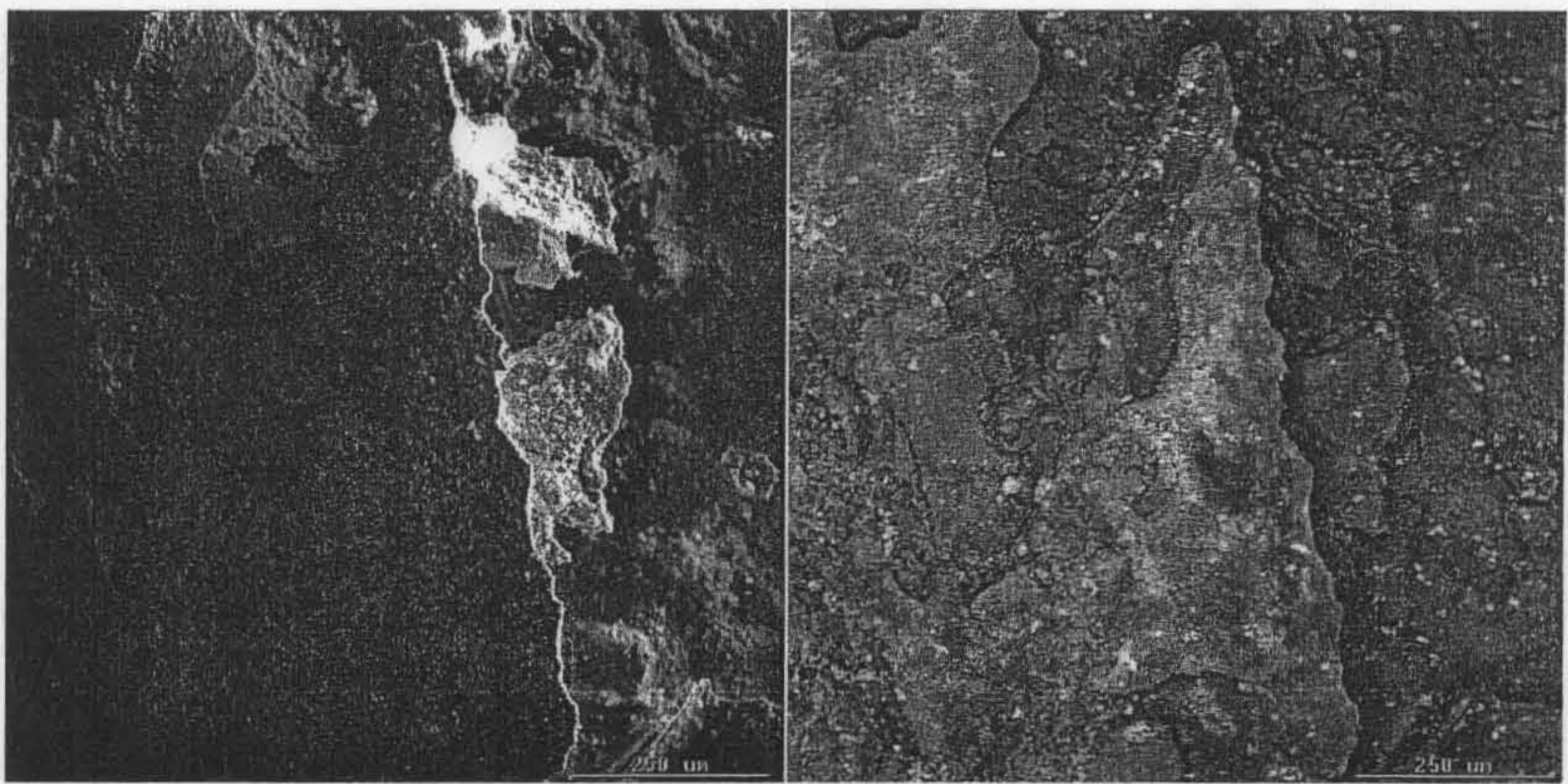

Accelerating Voltage: $25 \mathrm{KeV}$

Resolution:

$1024 \times 1024$

Magnification: 100

Pixel Size:

0.975702 microns

Region \#4

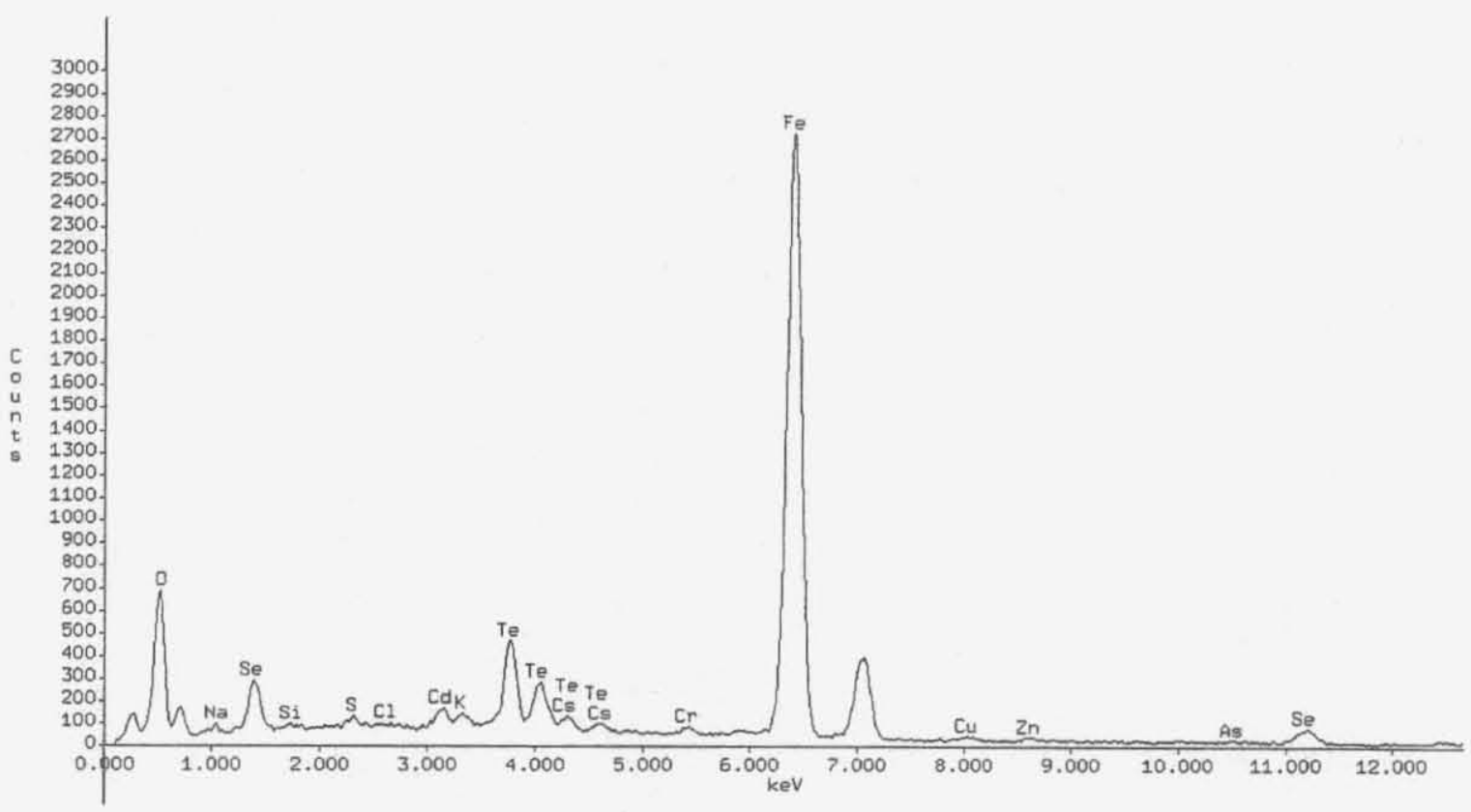

Figure A.7. BSE image and EDS analysis of Region \#4 in sample 12J-W-13B. 
The Catholic University of America Vitreous State Laboratory
DuraMelter 1200 Tests with AZ-101HLW Simulants Final Report, VSL-02R0100-2, Rev.1

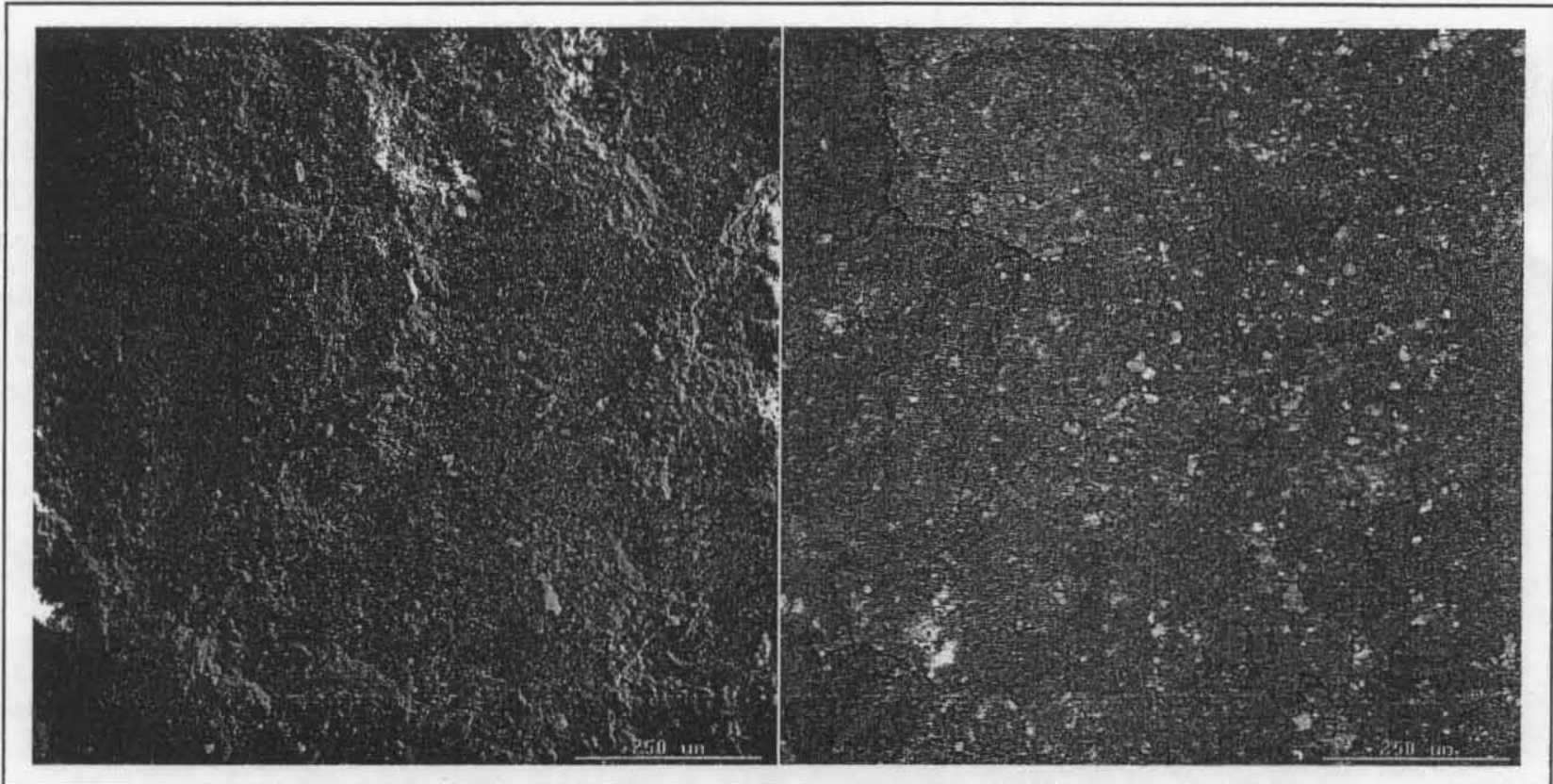

Accelerating Voltage: $25 \mathrm{KeV}$

Resolution: $\quad 1024 \times 1024$
Magnification: 100

Pixel Size: $\quad 0.975702$ microns

Region \#5

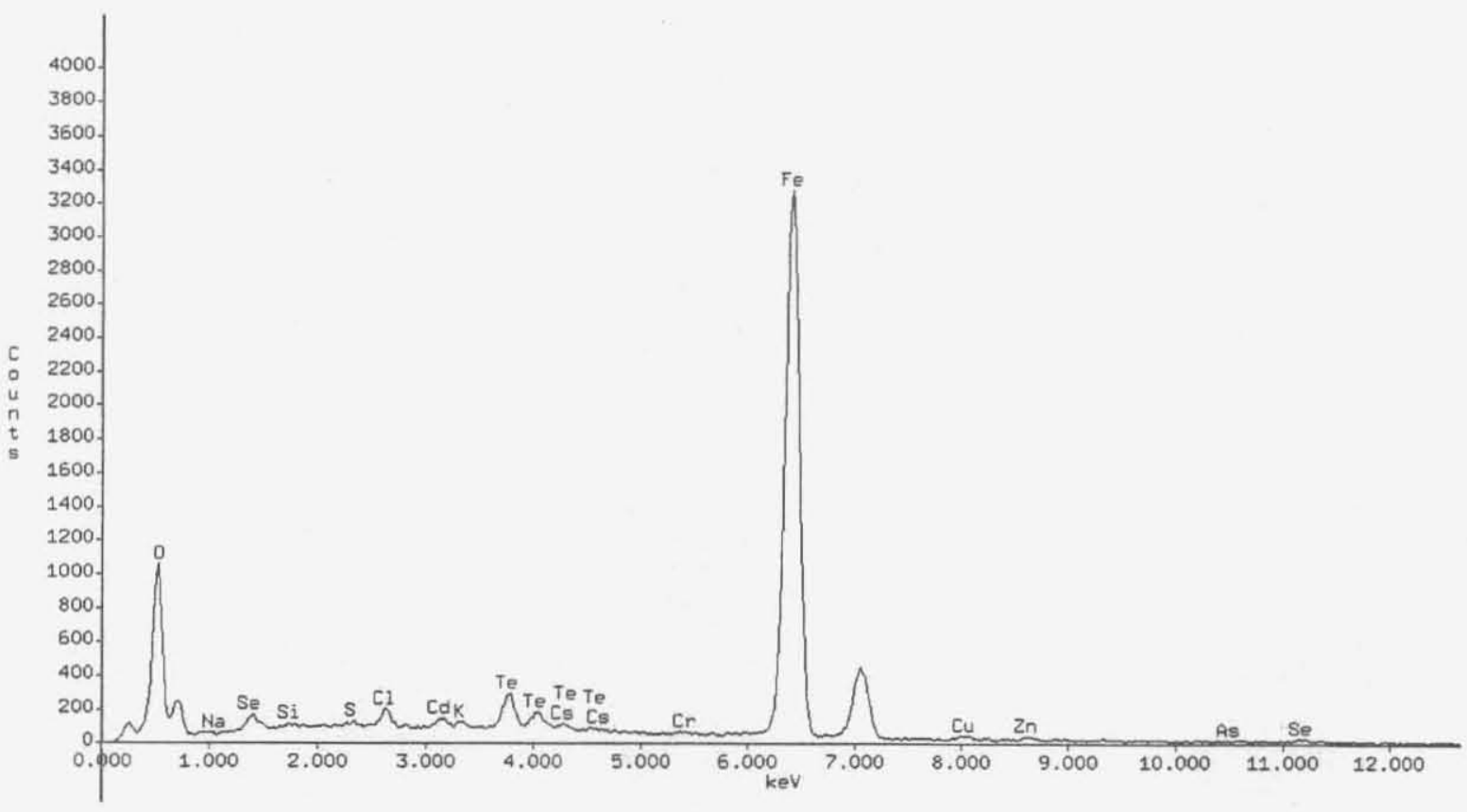

Figure A.8. BSE image and EDS analysis of Region \#5 in sample 12J-W-13B. 
The Catholic University of America

Vitreous State Laboratory
DuraMelter 1200 Tests with AZ-101HLW Simulants

Final Report, VSL-02R0100-2, Rev.1

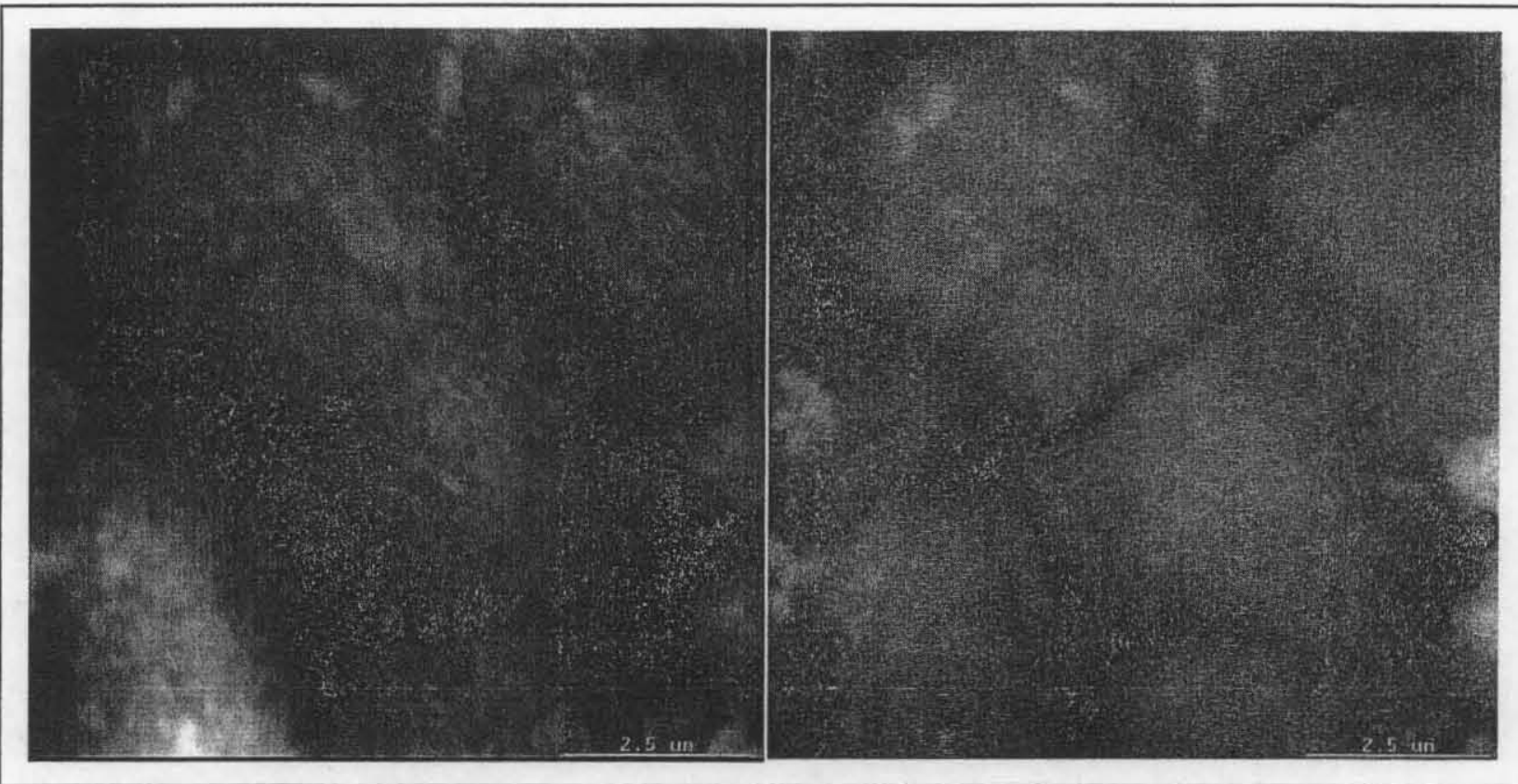

Accelerating Voltage: $25 \mathrm{KeV}$ Resolution: $1024 \times 1024$

Magnification: 100

Pixel Size: $\quad 0.00975702$ microns

Region \#5 Close-up

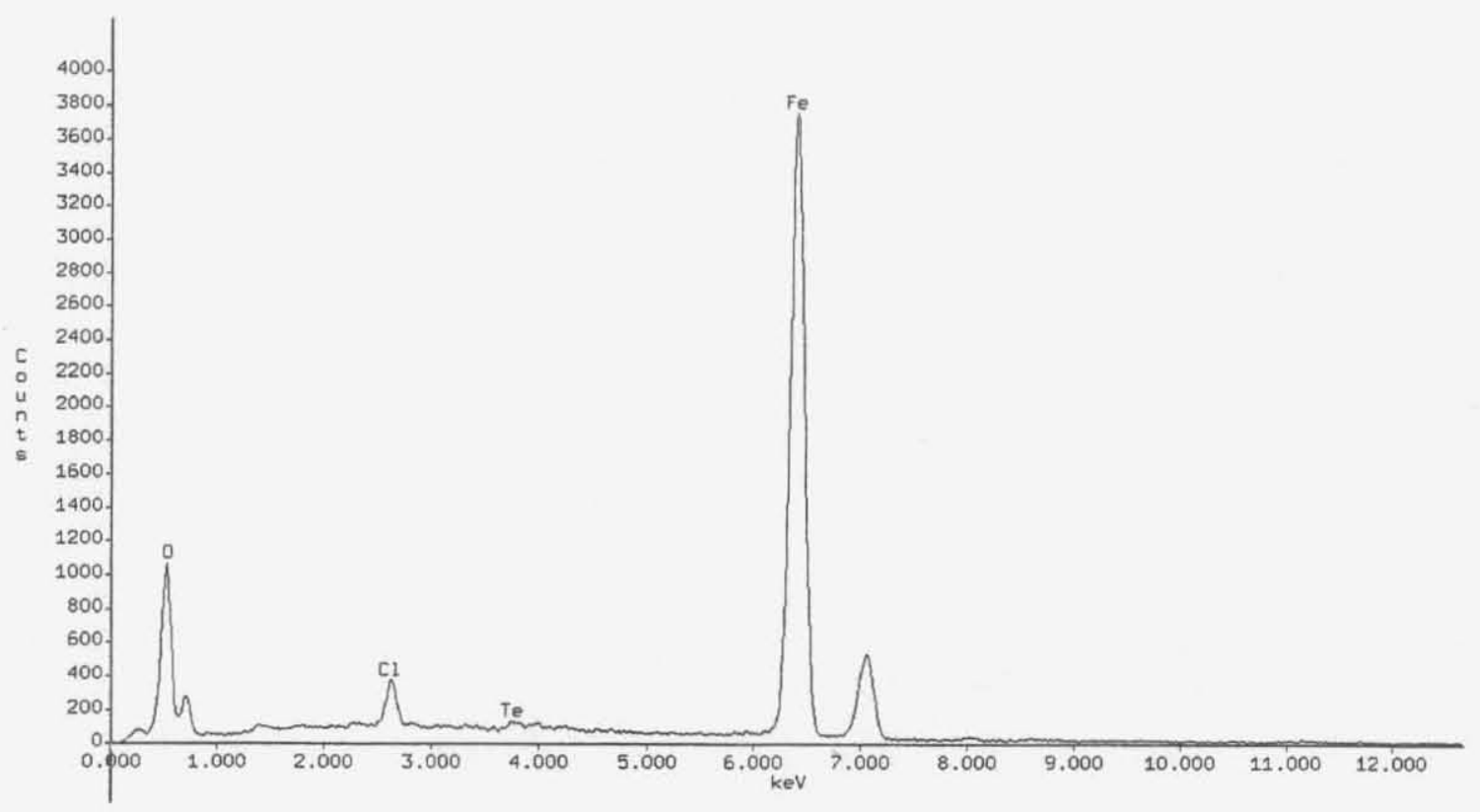

Figure A.9. BSE image and EDS analysis of Region \#5 (close-up) in sample 12J-W-13B. 
The Catholic University of America

Vitreous State Laboratory
DuraMelter 1200 Tests with AZ-101HLW Simulants Final Report, VSL-02R0100-2, Rev.1

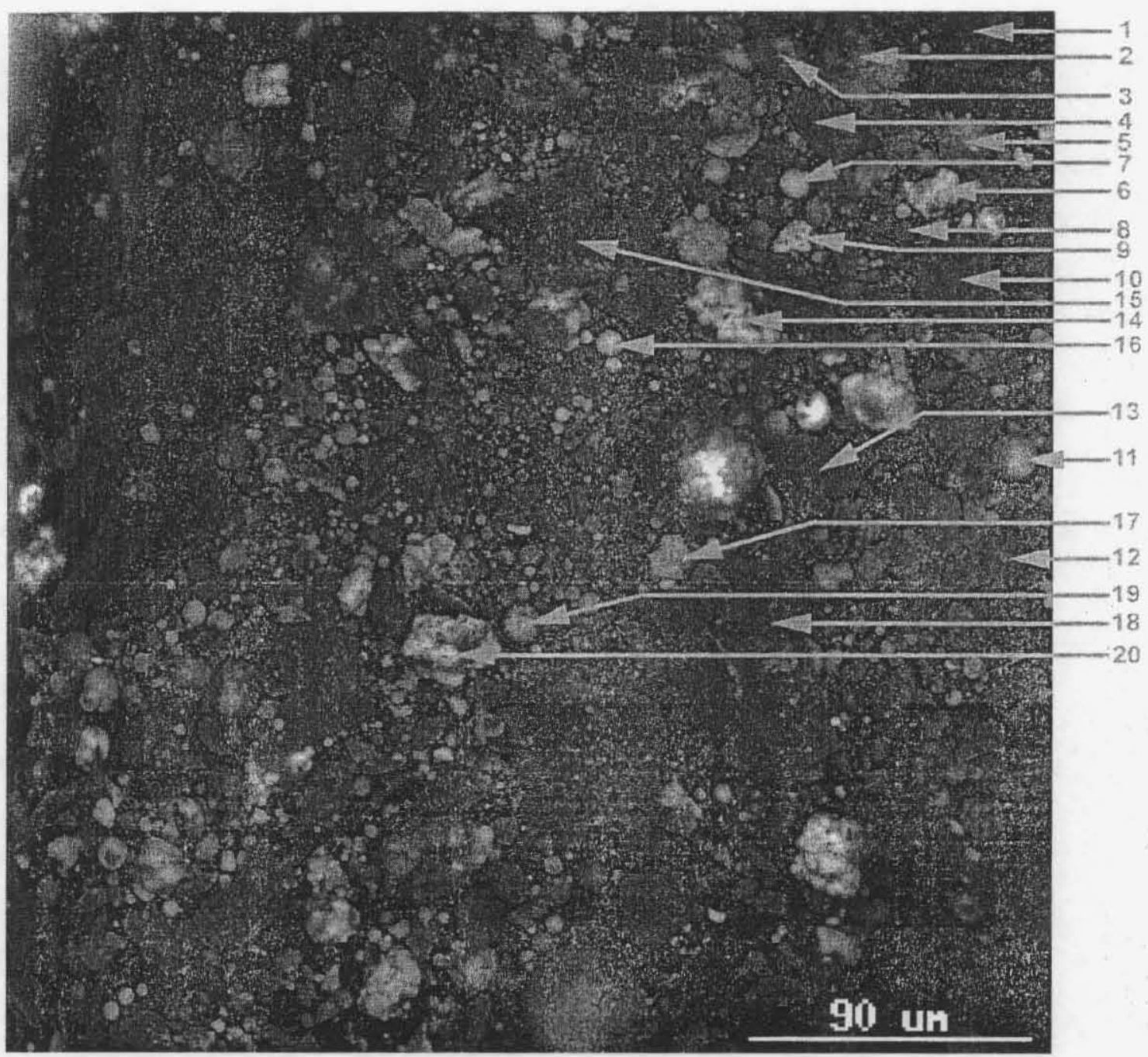

Figure A.10. Point-by-point EDS analysis of particles (Sample \#12K-S-10ARe). 
The Catholic University of America Vitreous State Laboratory
DuraMelter 1200 Tests with AZ-101HLW Simulants Final Report, VSL-02R0100-2, Rev.I

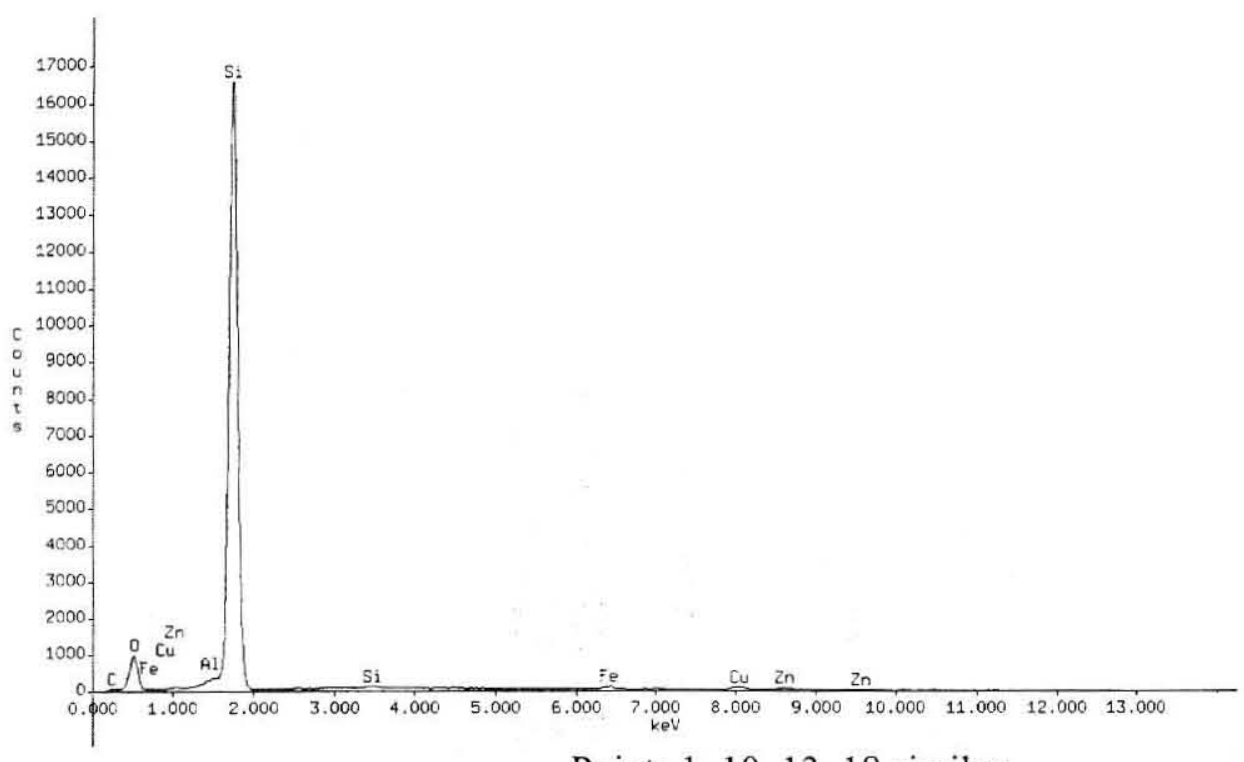

Points 1, 10, 13, 18 similar.

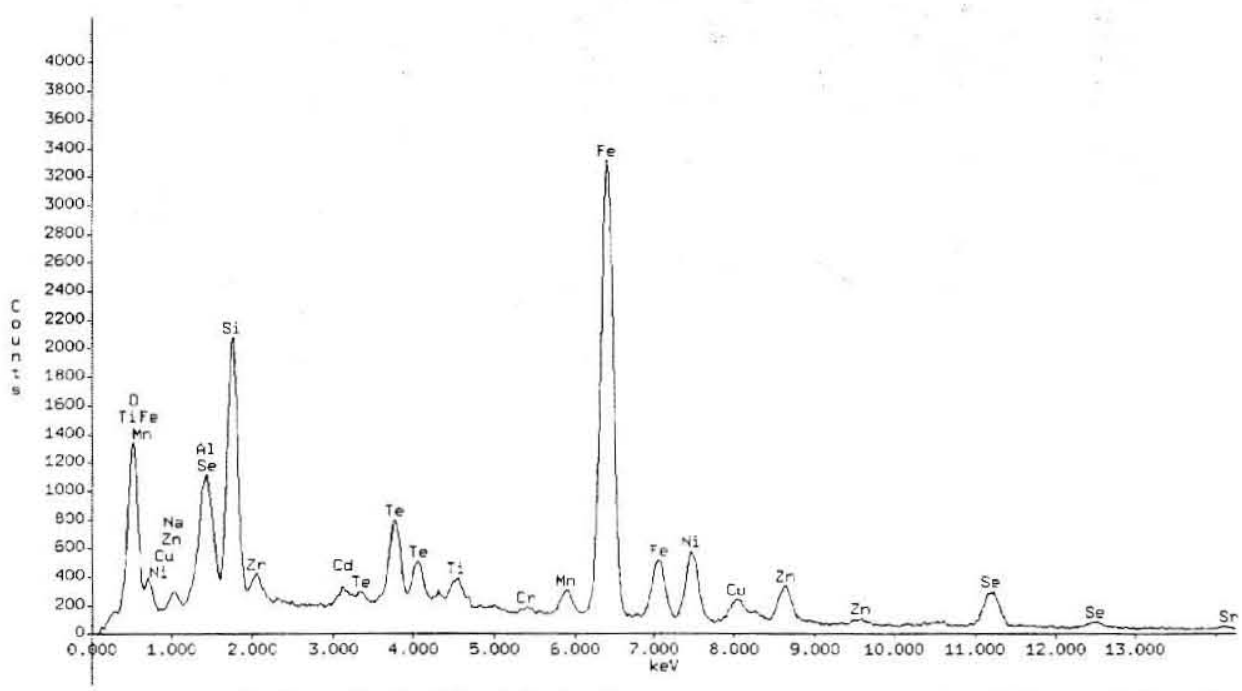

Points 2, 5, 12, 16 similar. Varying amounts of Te and Se observed.

Figure A.11. EDS analysis of Regions \#1, 10, 13, 18, 2, 5, 12, and 16 in sample 12K-S-10Are. 
The Catholic University of America Vitreous State Laboratory
DuraMelter 1200 Tests with AZ-101HLW Simulants Final Report, VSL-02R0100-2, Rev.1
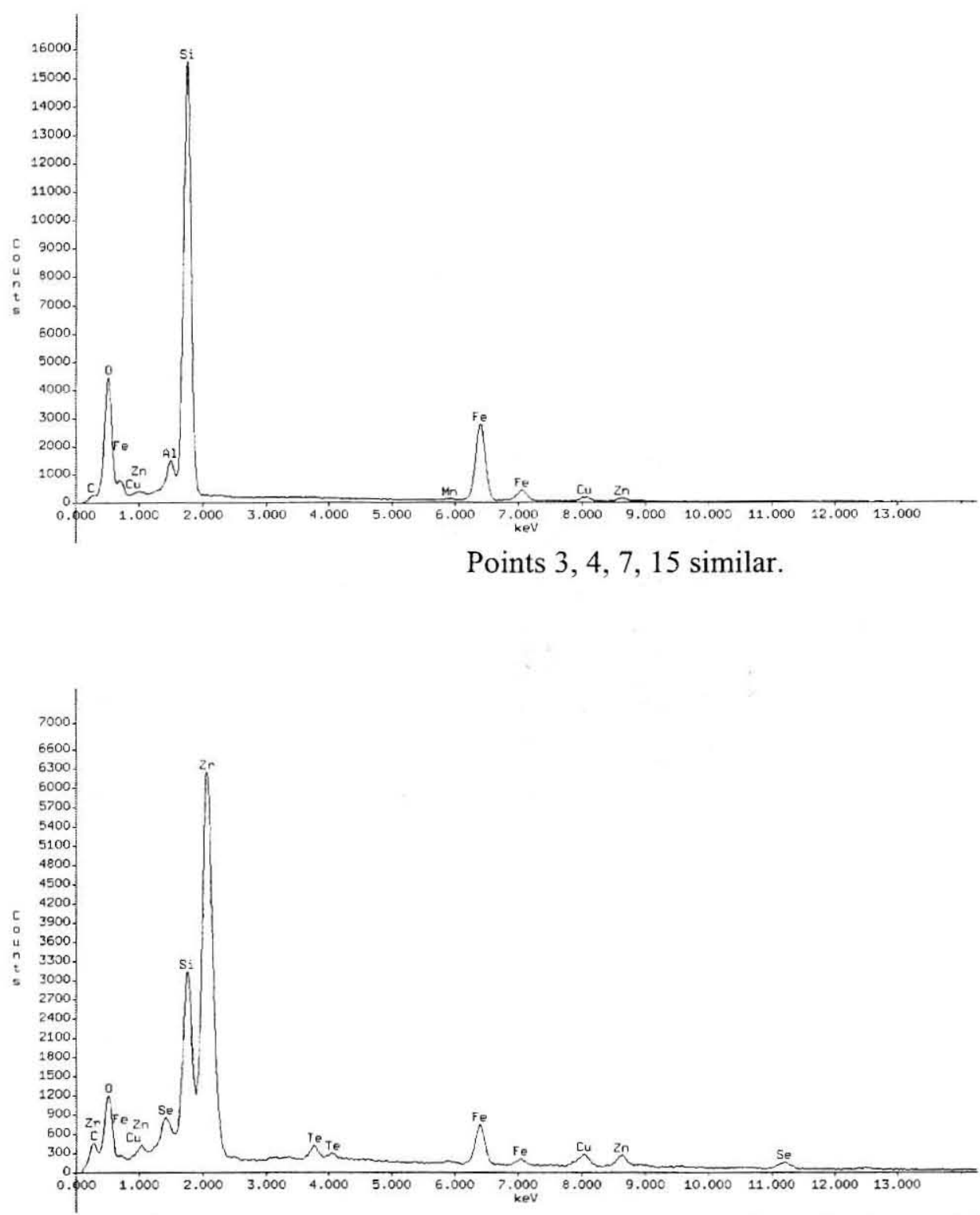

Points 6, 9, 14, 19, 20 similar. Varying amounts of Te, Se, Cu, and $\mathrm{Zn}$ observed.

Figure A.12. EDS analysis of Regions \#3, 4, 7, 15, 6, 9, 14, 19, and 20 in sample 12K-S-10Are. 
The Catholic University of America Vitreous State Laboratory
DuraMelter 1200 Tests with AZ-101HLW Simulants Final Report, VSL-02R0100-2, Rev.1
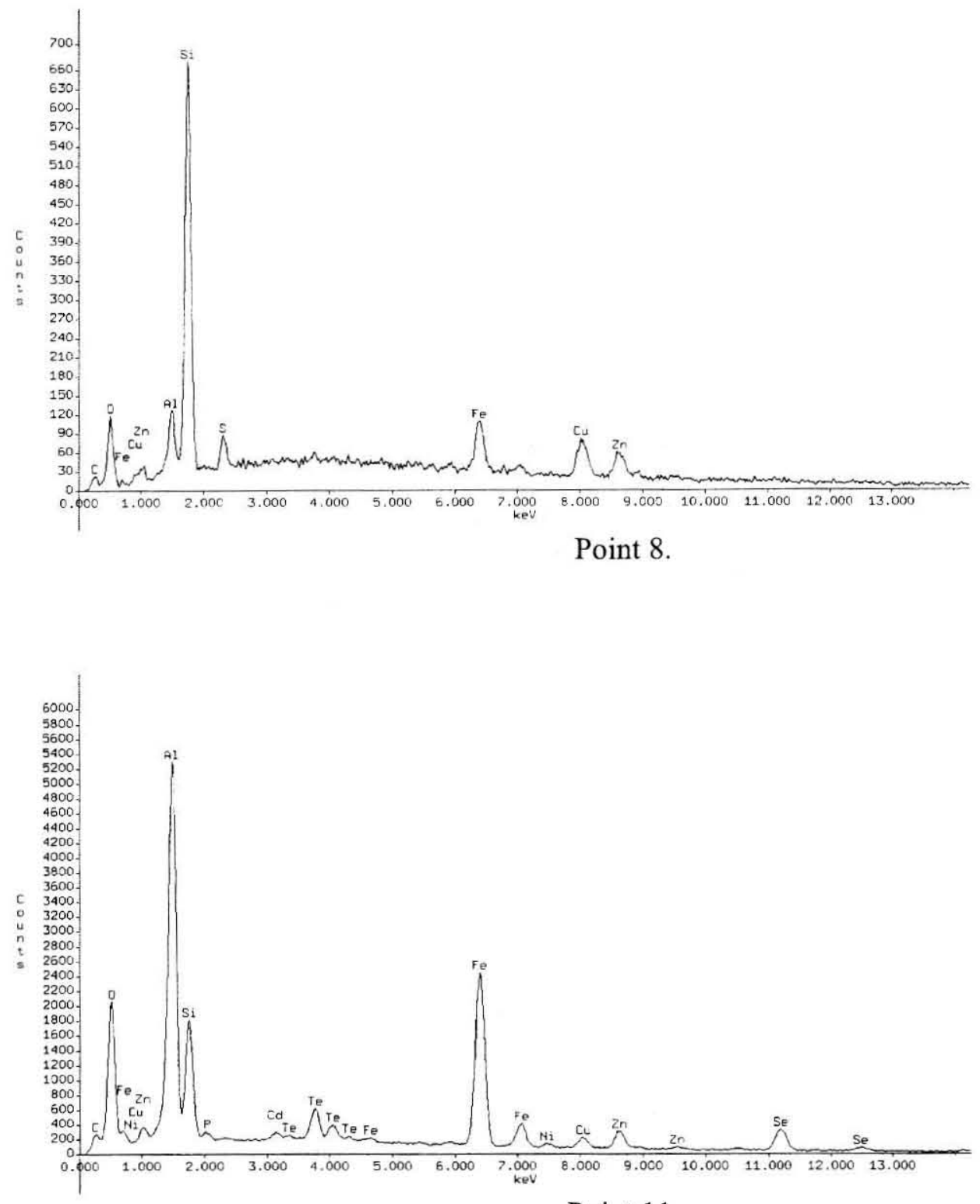

Point 11 .

Figure A.13. EDS analysis of Regions \#8 and 11 in sample 12K-S-10ARe. 
The Catholic University of America Vitreous State Laboratory
DuraMelter 1200 Tests with AZ-101HLW Simulants Final Report, VSL-02R0100-2, Rev.1

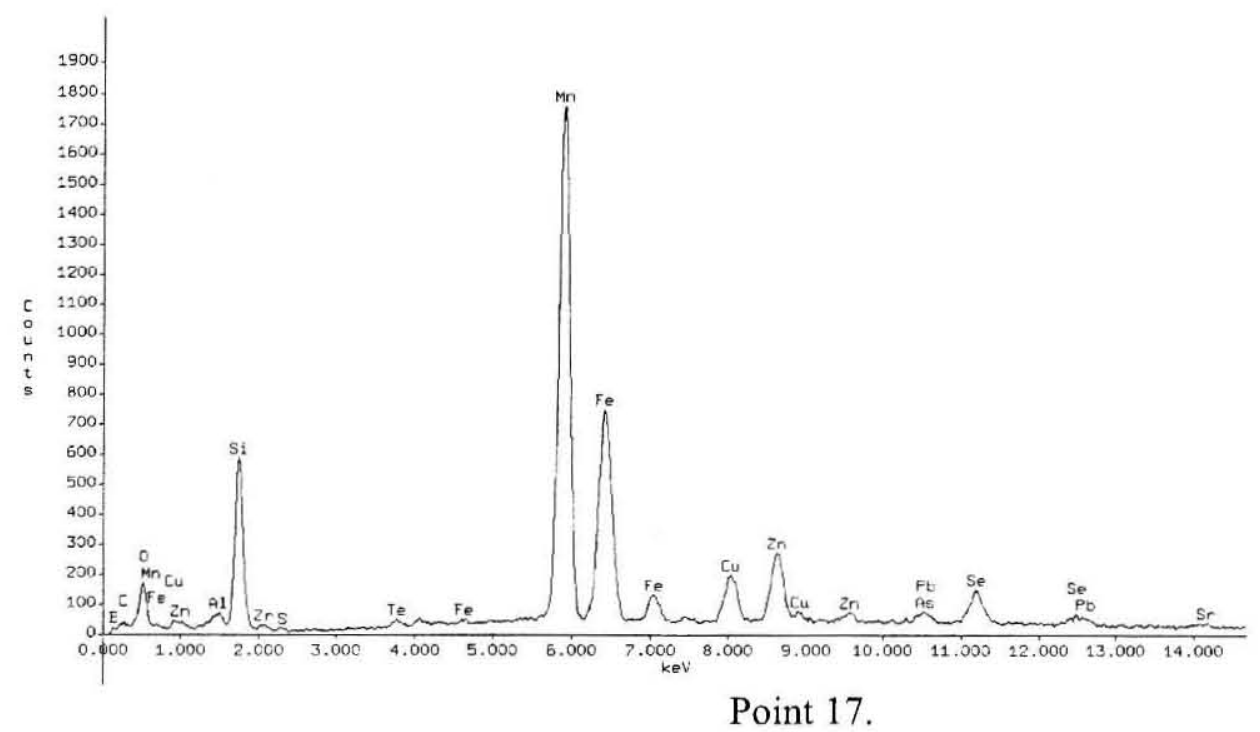

Figure A.14. EDS analysis of Region \#17 in sample 12K-S-10ARe. 
The Catholic University of America Vitreous State Laboratory
DuraMelter 1200 Tests with AZ-101HLW Simulants

Final Report, VSL-02R0100-2, Rev.1
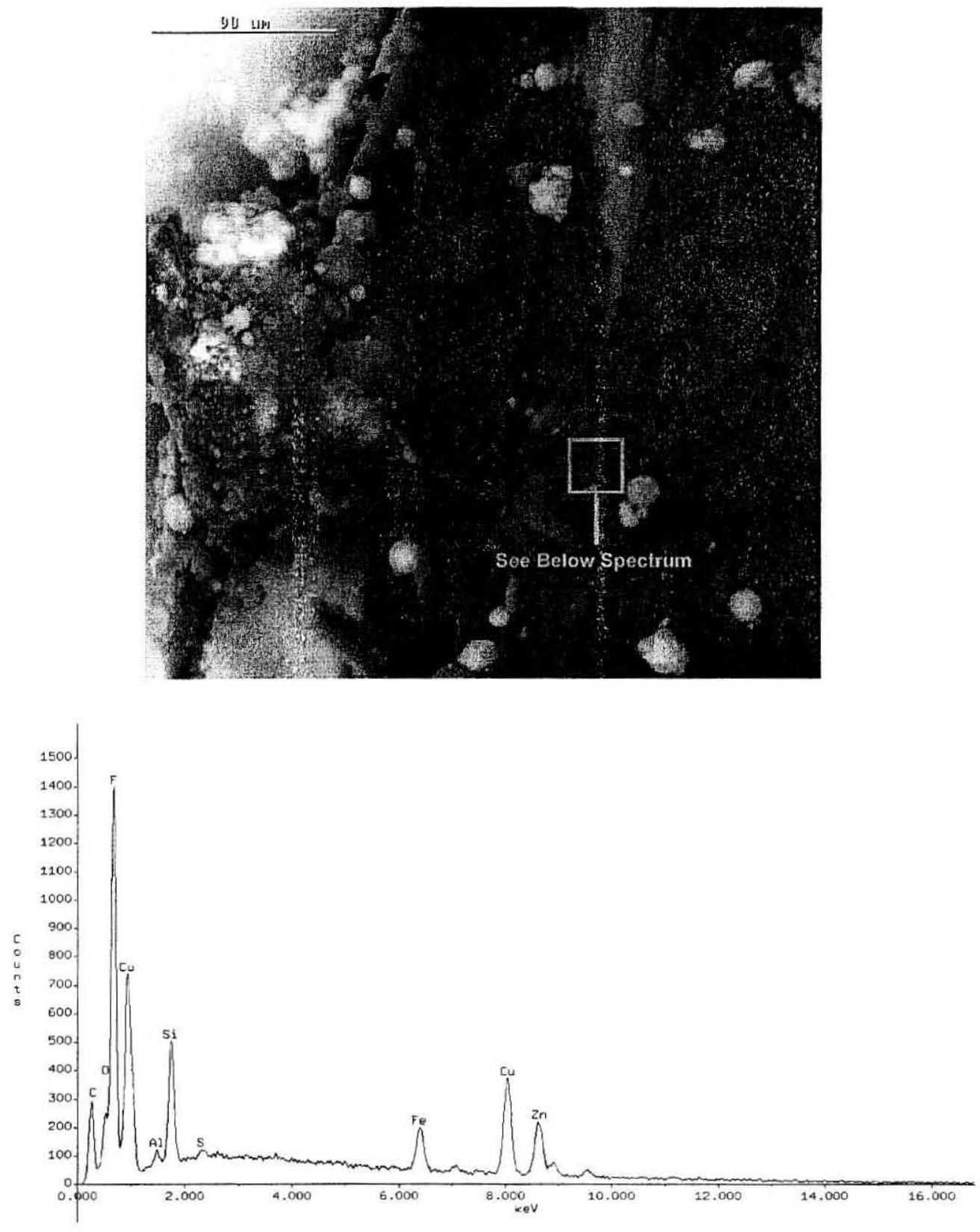

Figure A.15. A significant fluorine peak was observed in the material shown above (Sample12K-S-10ARe). 
The Catholic University of America Vitreous State Laboratory
DuraMelter 1200 Tests with AZ-101HLW Simulants Final Report, VSL-02R0100-2, Rev.I
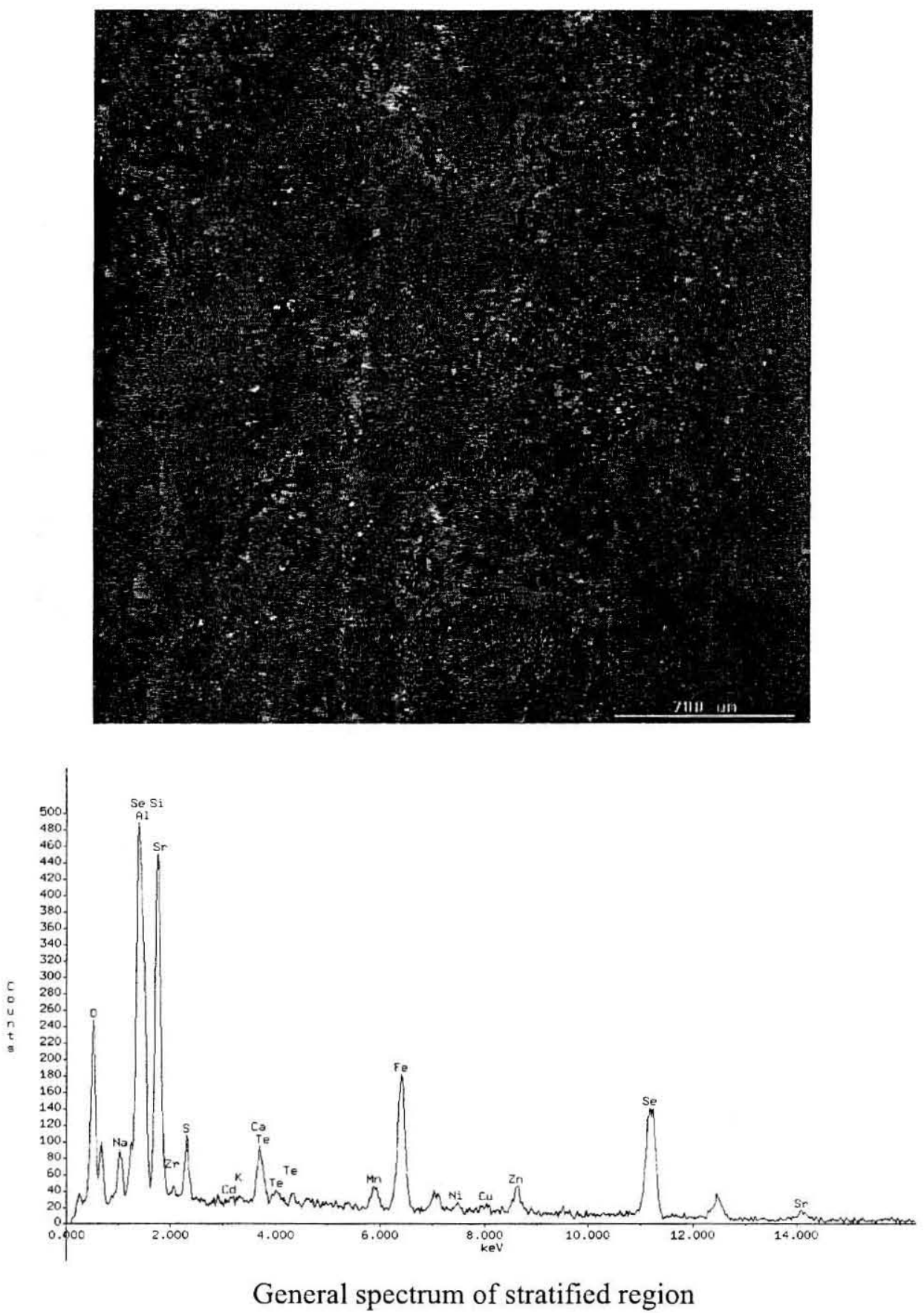

Figure A.16. BSE image and EDS analysis of stratified region (Sample 12K-S-10B). 
The Catholic University of America Vitreous State Laboratory
DuraMelter 1200 Tests with AZ-101HLW Simulants Final Report, VSL-02R0100-2, Rev.1

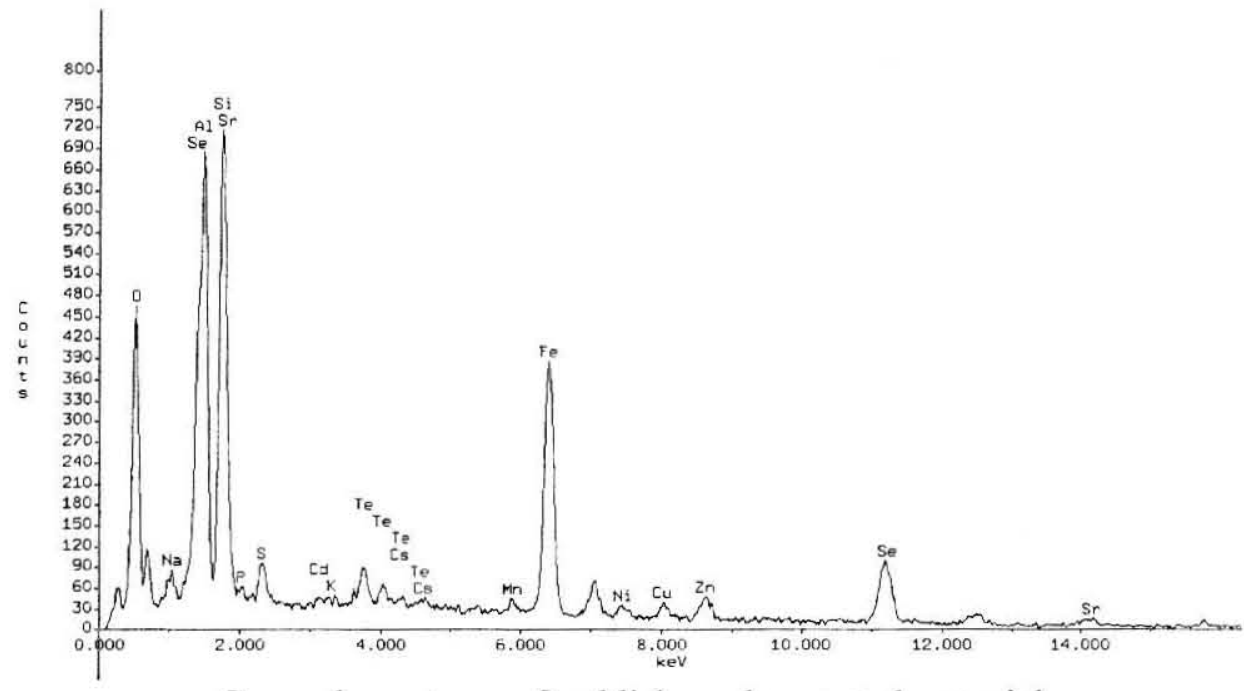

General spectrum of reddish agglomerated material

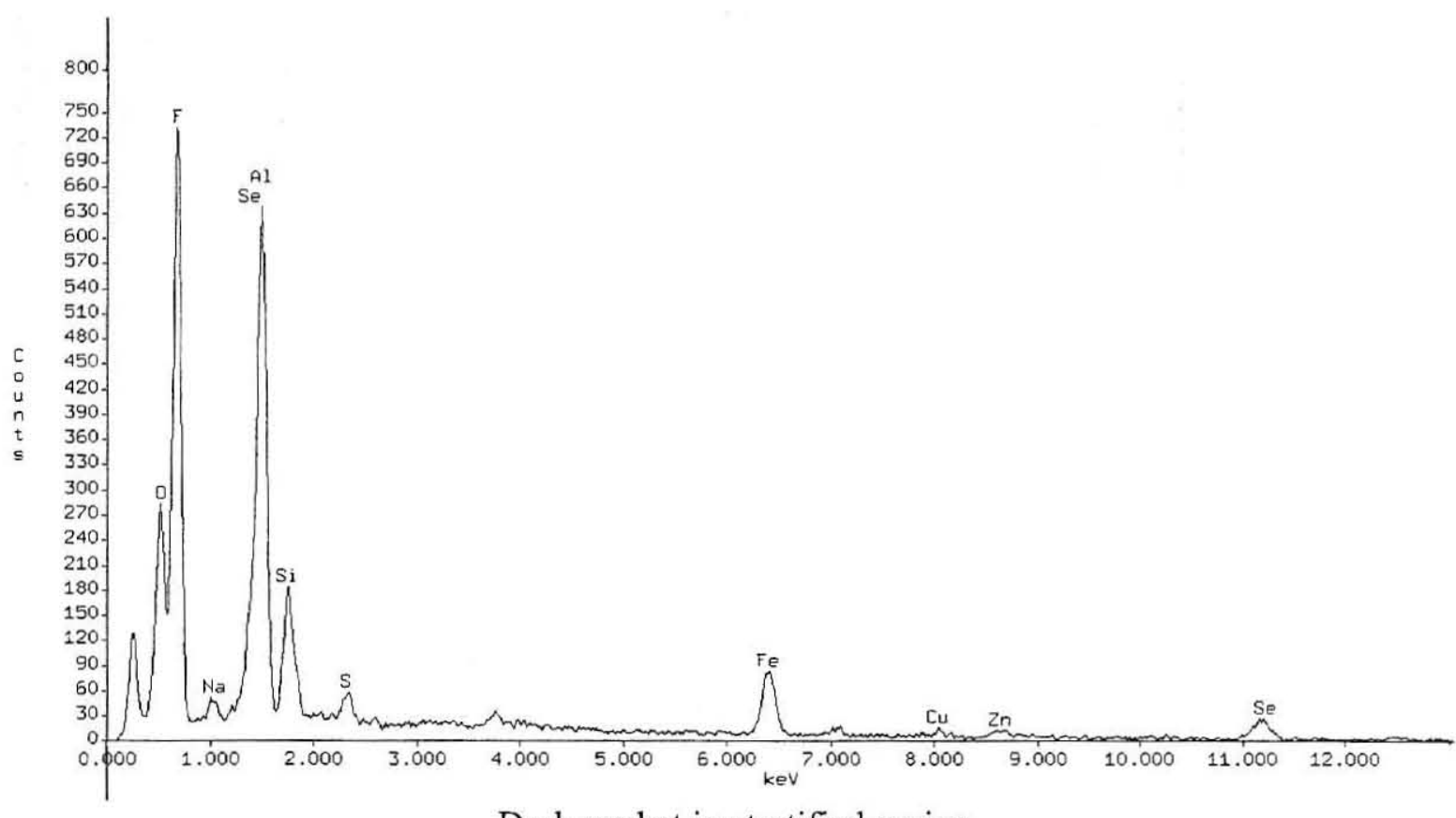

Dark pocket in stratified region

Figure A.17. BSE image and EDS analysis of reddish agglomerated region and dark pocket in stratified region (Sample 12K-S-10B). 
The Catholic University of America

Vitreous State Laboratory
DuraMelter 1200 Tests with AZ-101HLW Simulants

Final Report, VSL-02R0100-2, Rev.1

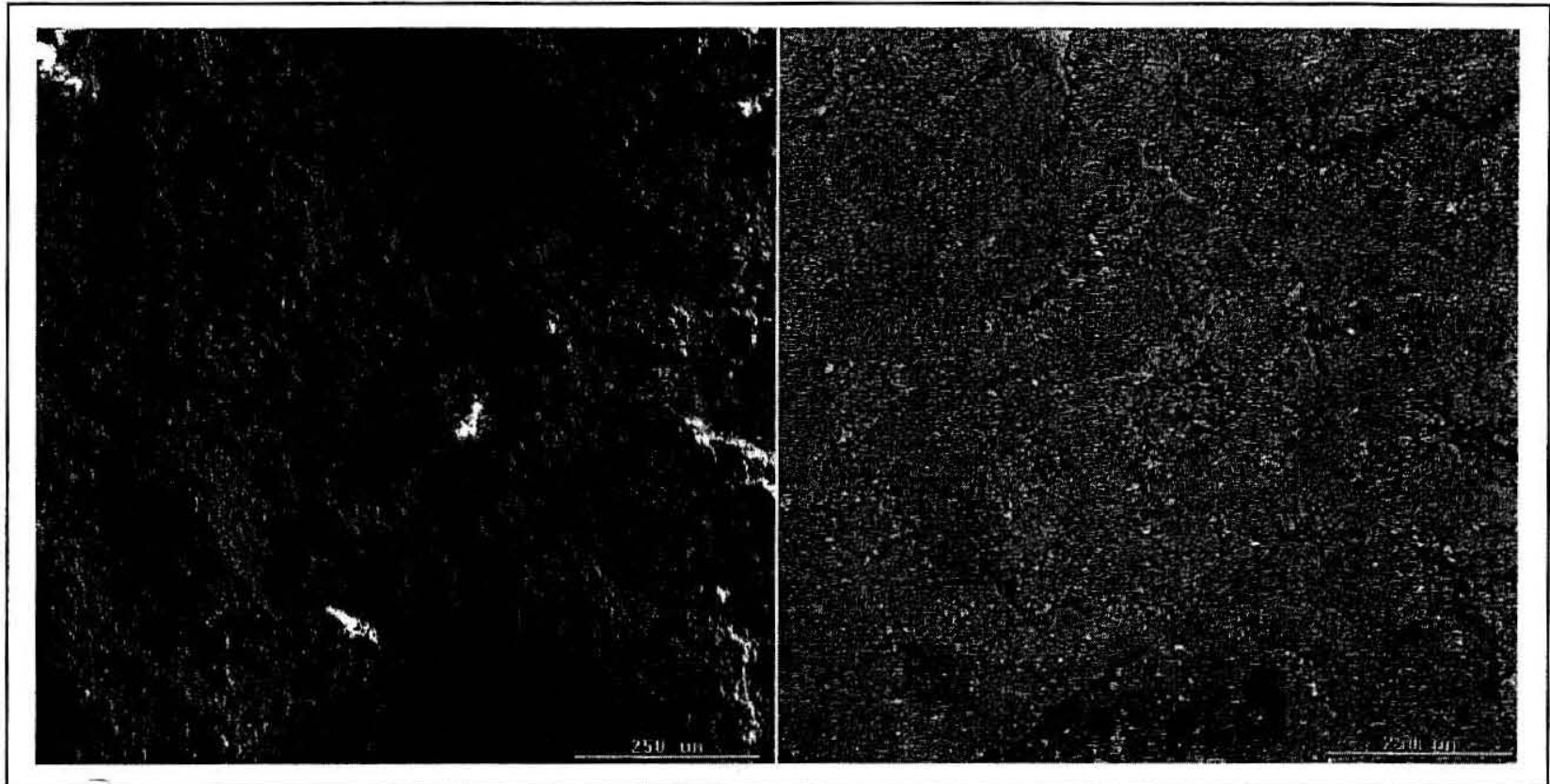

Accelerating Voltage: $25 \mathrm{KeV}$

Resolution:

$1024 \times 1024$

Magnification: 100

Pixel Size: $\quad 0.975702$ microns

Region \#1

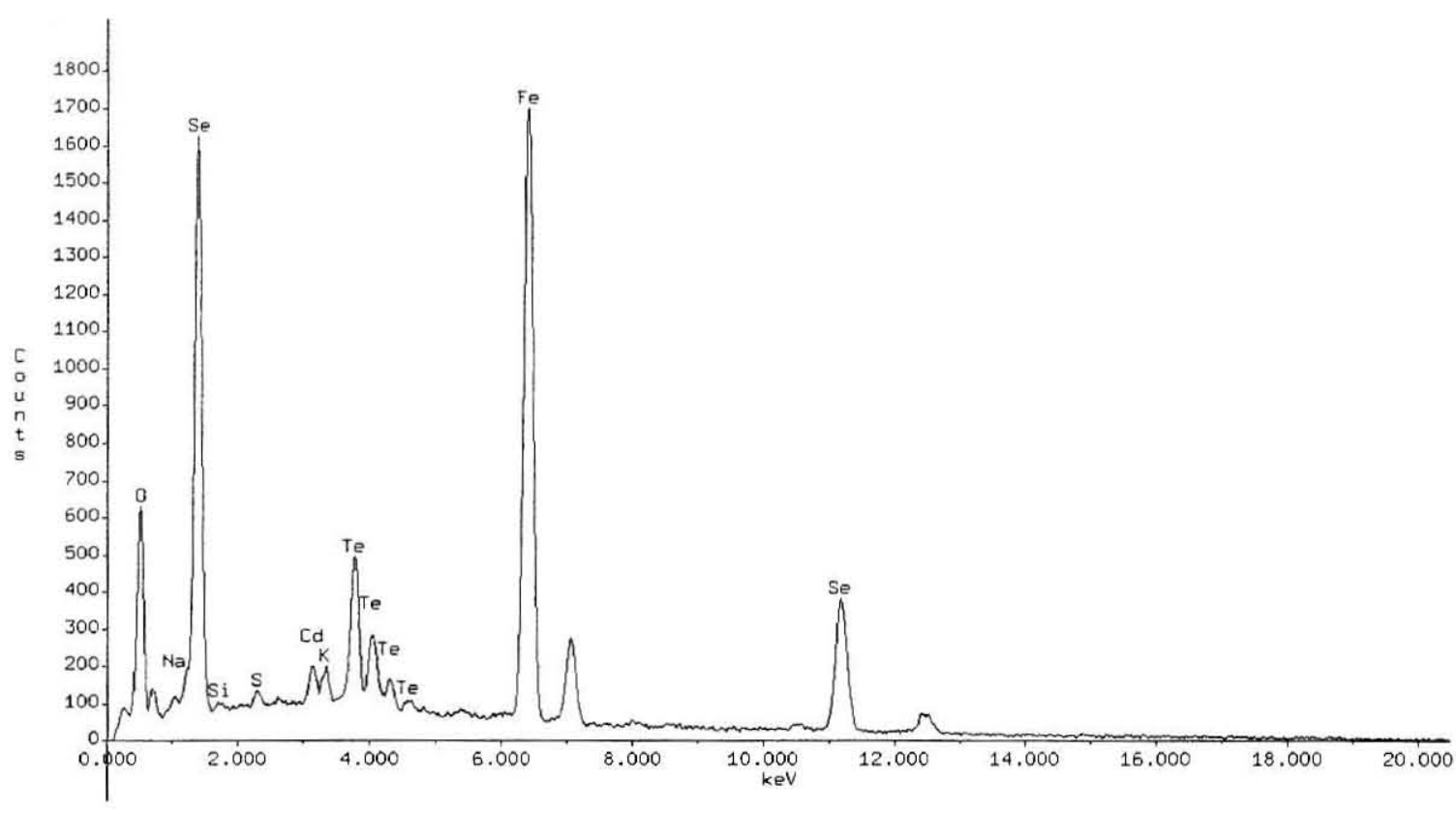

Figure A.18. BSE image and EDS analysis of Region \#1 in sample 12K-W-6B. 
The Catholic University of America

Vitreous State Laboratory
DuraMelter 1200 Tests with AZ-101HLW Simulants

Final Report, VSL-02R0100-2, Rev.1

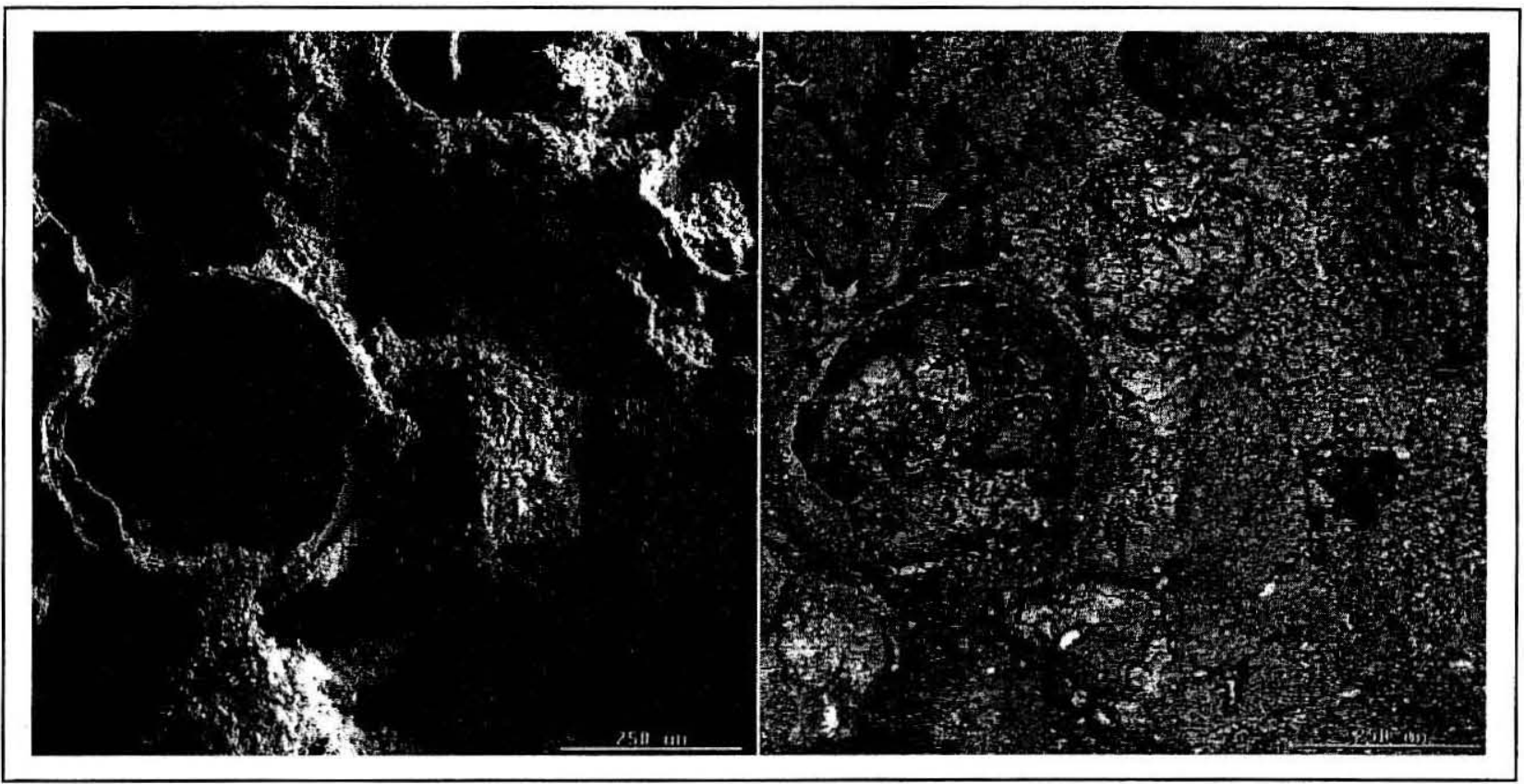

Accelerating Voltage: $25 \mathrm{KeV}$

Resolution:

$1024 \times 1024$
Magnification: 330

Pixel Size: $\quad 0.975702$ microns

Region \#2

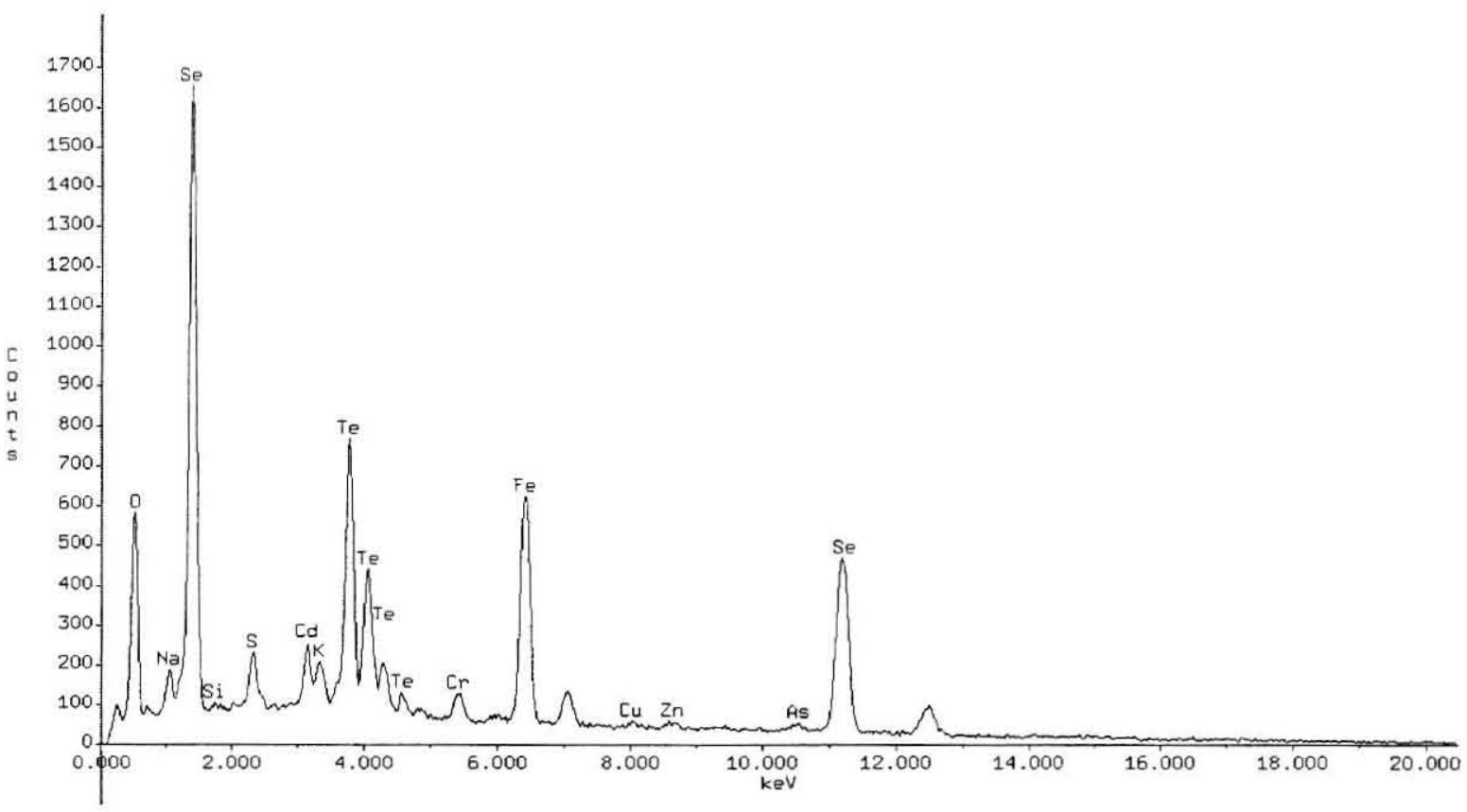

Figure A.19. BSE image and EDS analysis of Region \#2 in sample 12K-W-6B. 
The Catholic University of America Vitreous State Laboratory
DuraMelter 1200 Tests with AZ-101HLW Simulants Final Report, VSL-02R0100-2, Rev.1

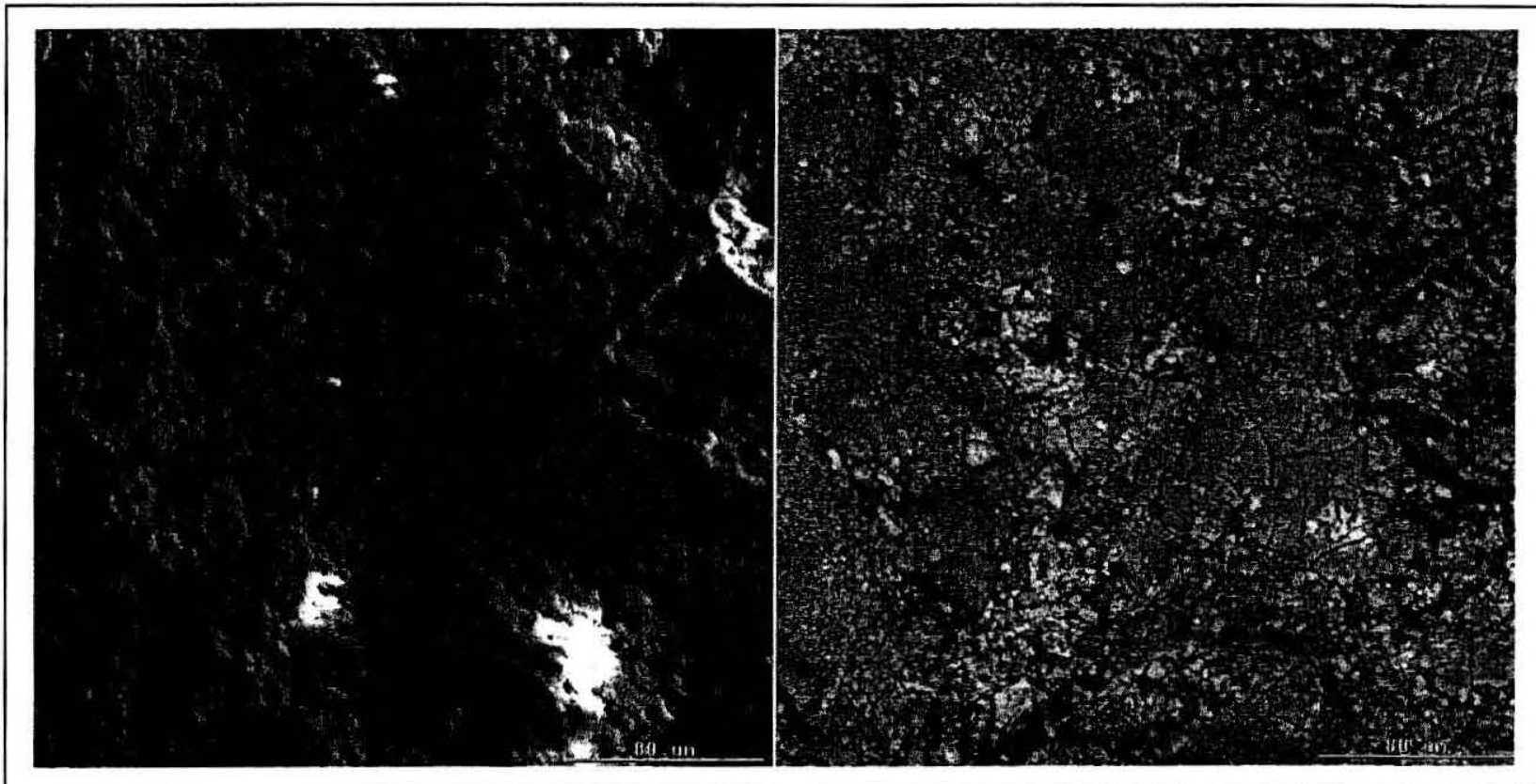

Accelerating Voltage: $25 \mathrm{KeV}$

Resolution:

$1024 \times 1024$

Magnification: 330

Pixel Size: 0.295667 microns

Region \#3

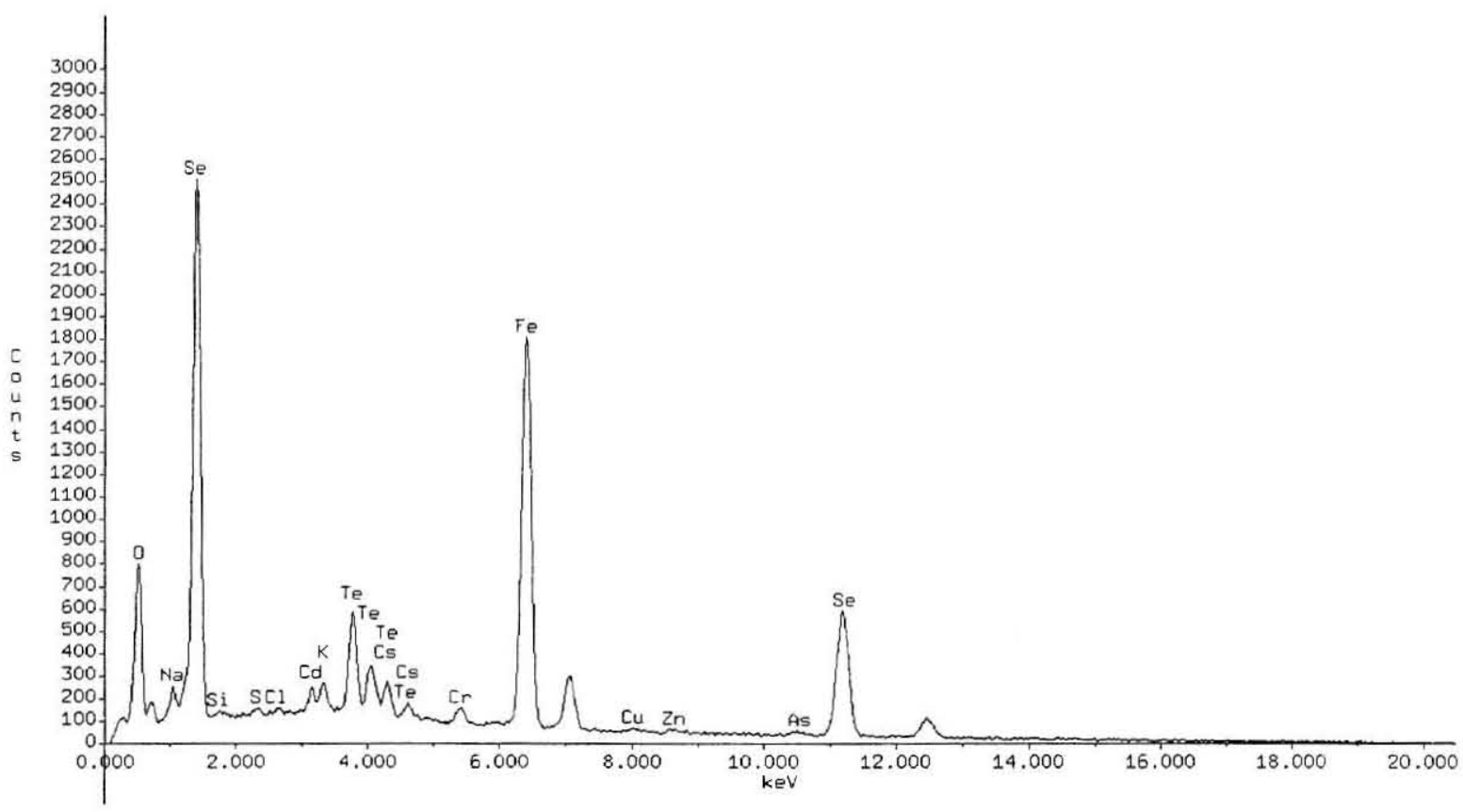

Figure A.20. BSE image and EDS analysis of Region \#3 in sample 12K-W-6B. 
The Catholic University of America

Vitreous State Laboratory
DuraMelter 1200 Tests with AZ-101HLW Simulants

Final Report, VSL-02R0100-2, Rev.1

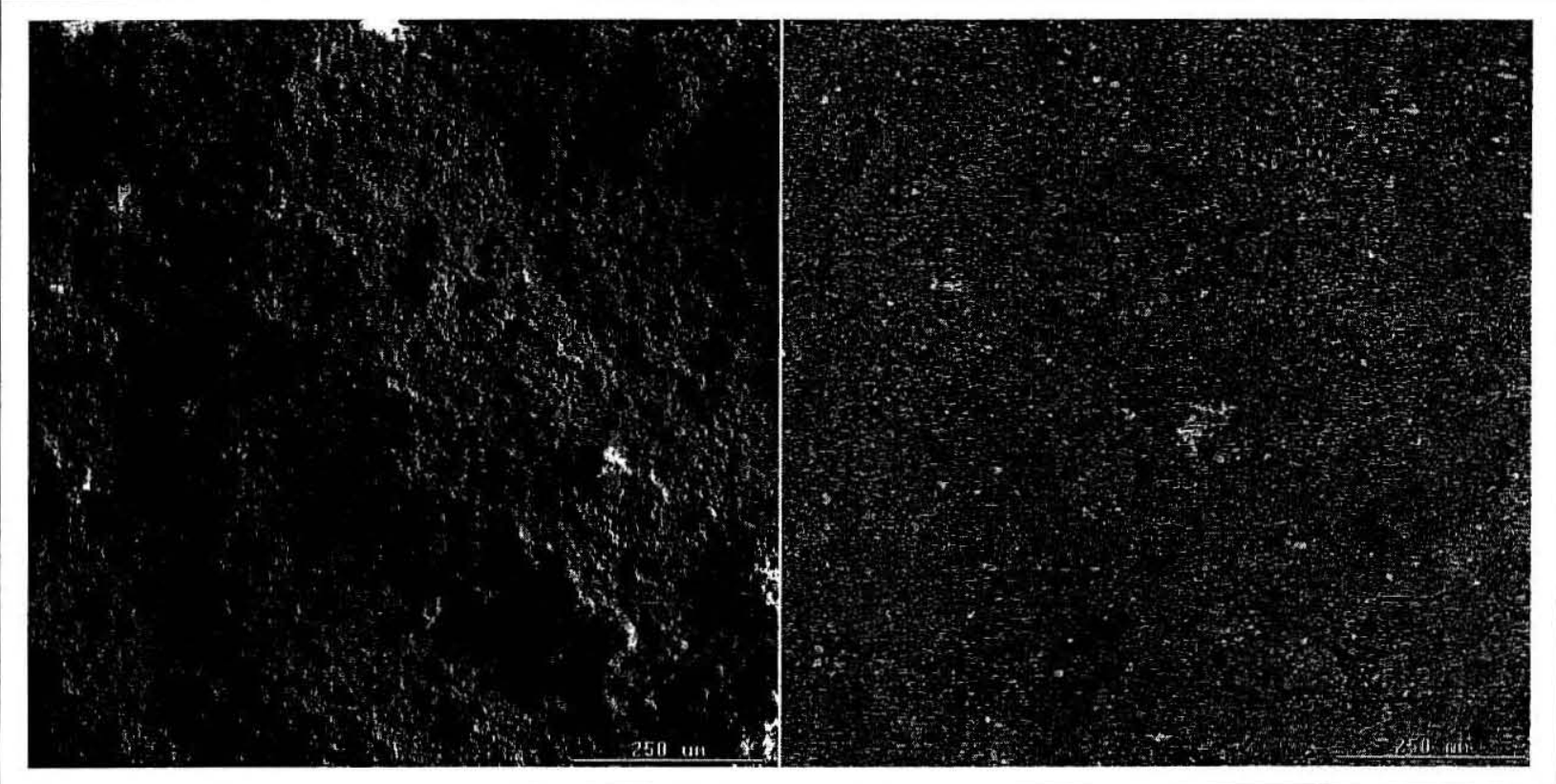

Accelerating Voltage: $25 \mathrm{KeV}$

Resolution:
Magnification: 100

Pixel Size: $\quad 0.975702$ microns

Region \#4

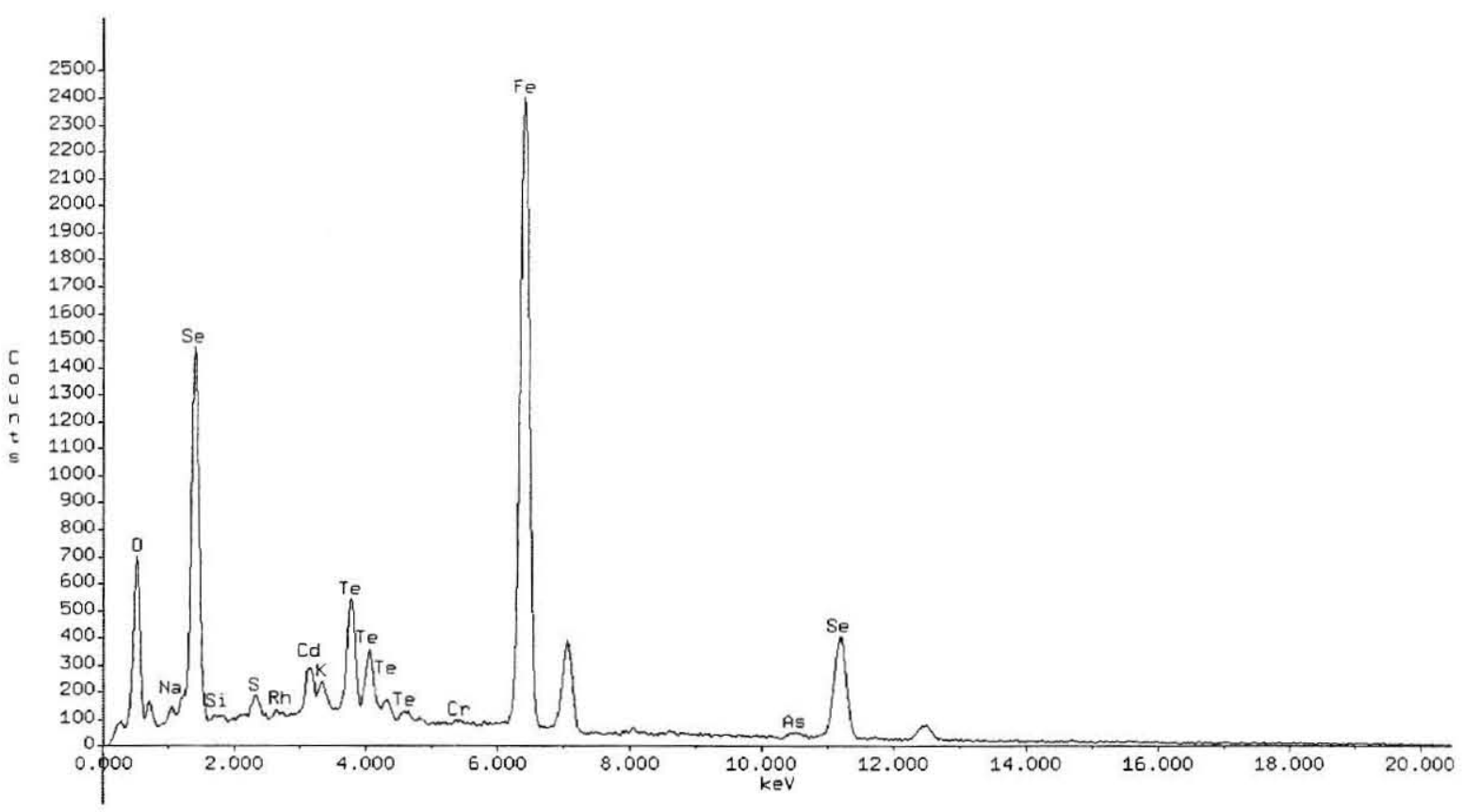

Figure A.21. BSE image and EDS analysis of Region \#4 in sample 12K-W-6B. 
The Catholic University of America Vitreous State Laboratory
DuraMelter 1200 Tests with AZ-101HLW Simulants Final Report, VSL-02R0100-2, Rev.1

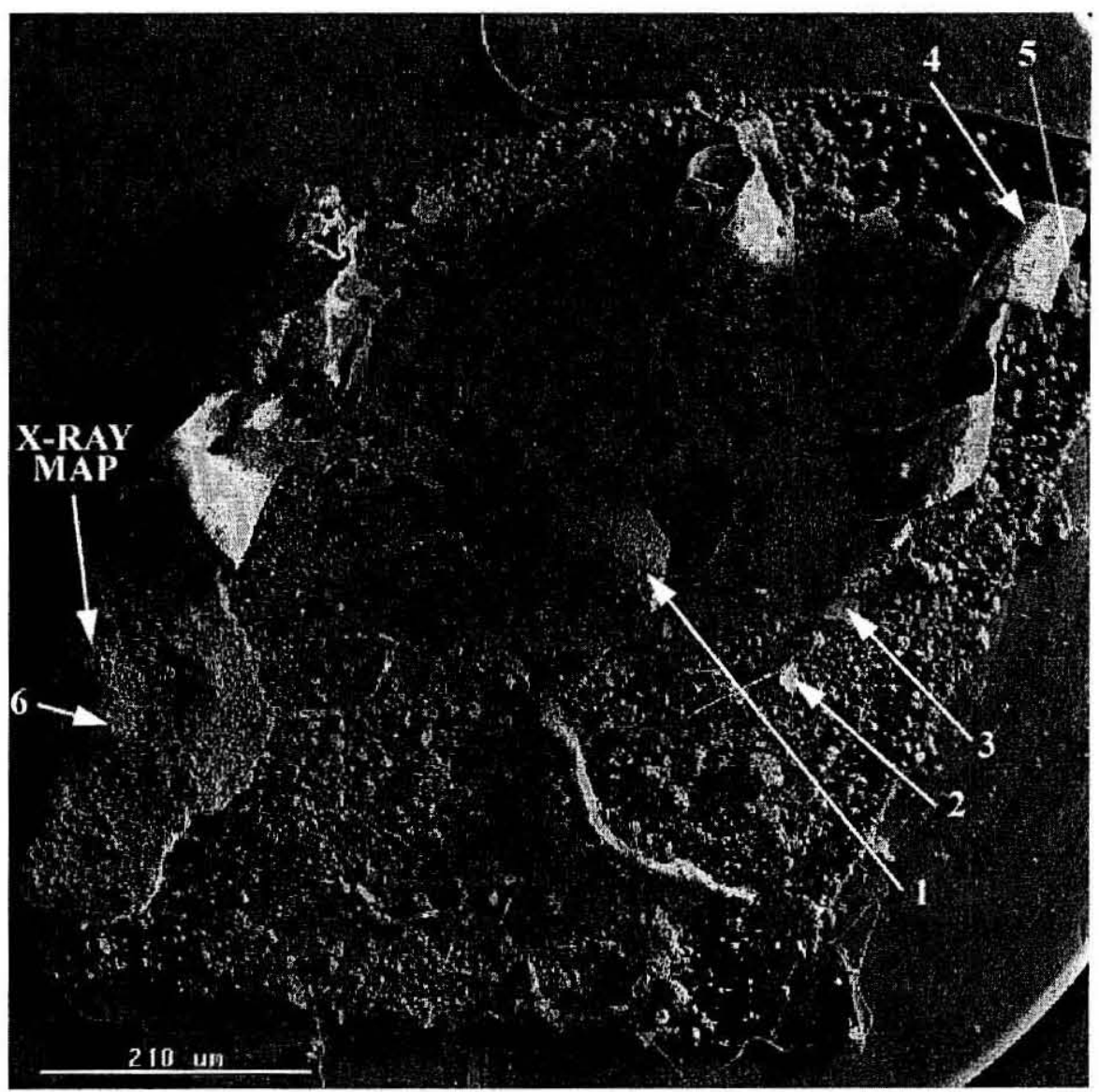

Figure A.22. Points numbered 1-6 relate to the EDS spectra in the following figures. The region labeled "X-RAY MAP" is the general region that $\mathrm{x}$-ray mapping was conducted (Sample \# 12K-O-10A). 
The Catholic University of America Vitreous State Laboratory
DuraMelter 1200 Tests with AZ-101 HLW Simulants

Final Report, VSL-02R0100-2, Rev.1

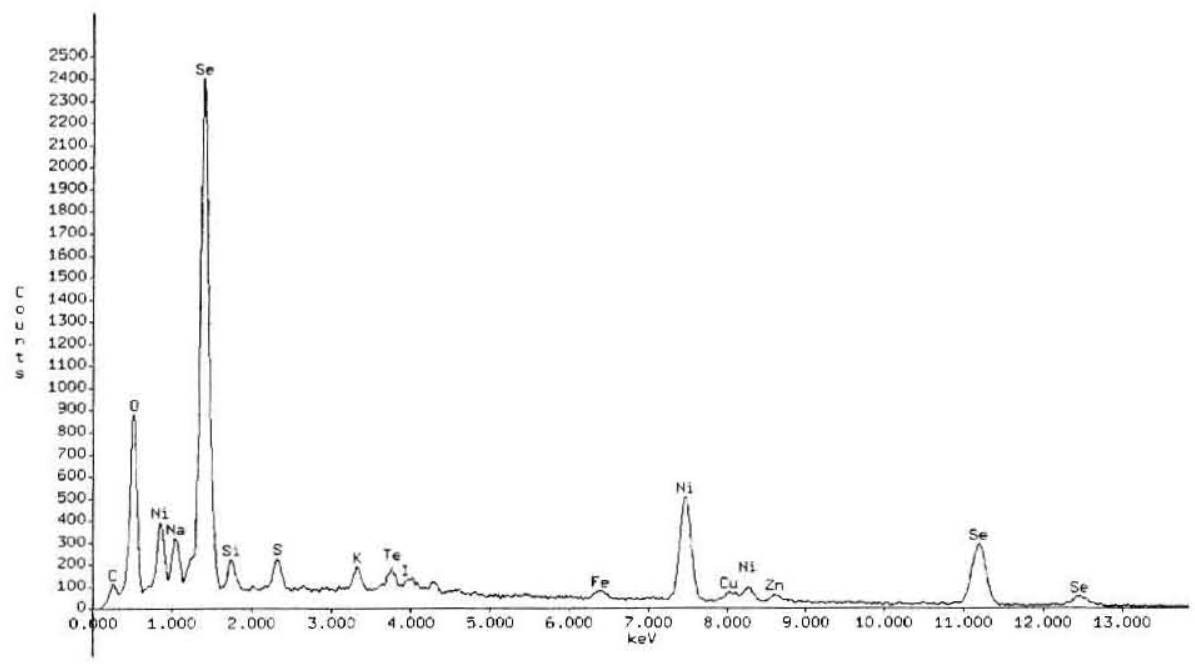

(Region \#1)

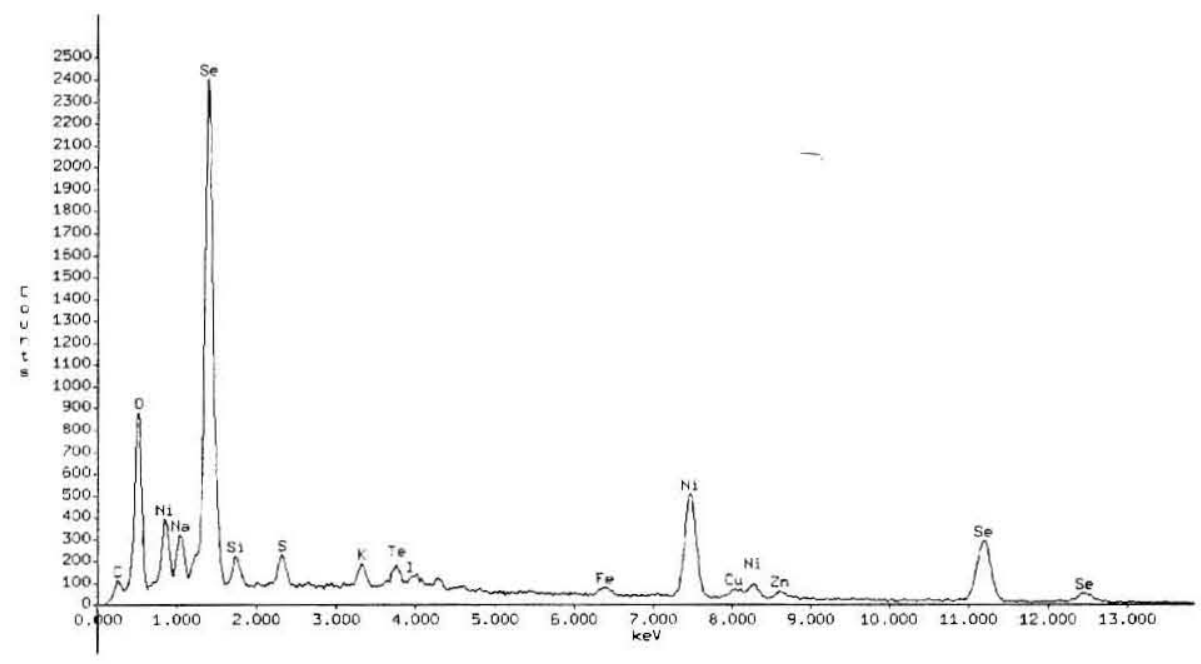

(Region \#2)

Figure A.23. EDS spectra of two sample regions (Regions \#1 and \#2) indicated in Figure A.22 (Sample \# 12K-0-10A). 
The Catholic University of America Vitreous State Laboratory
DuraMelter 1200 Tests with AZ-101HLW Simulants Final Report, VSL-02R0100-2, Rev.1

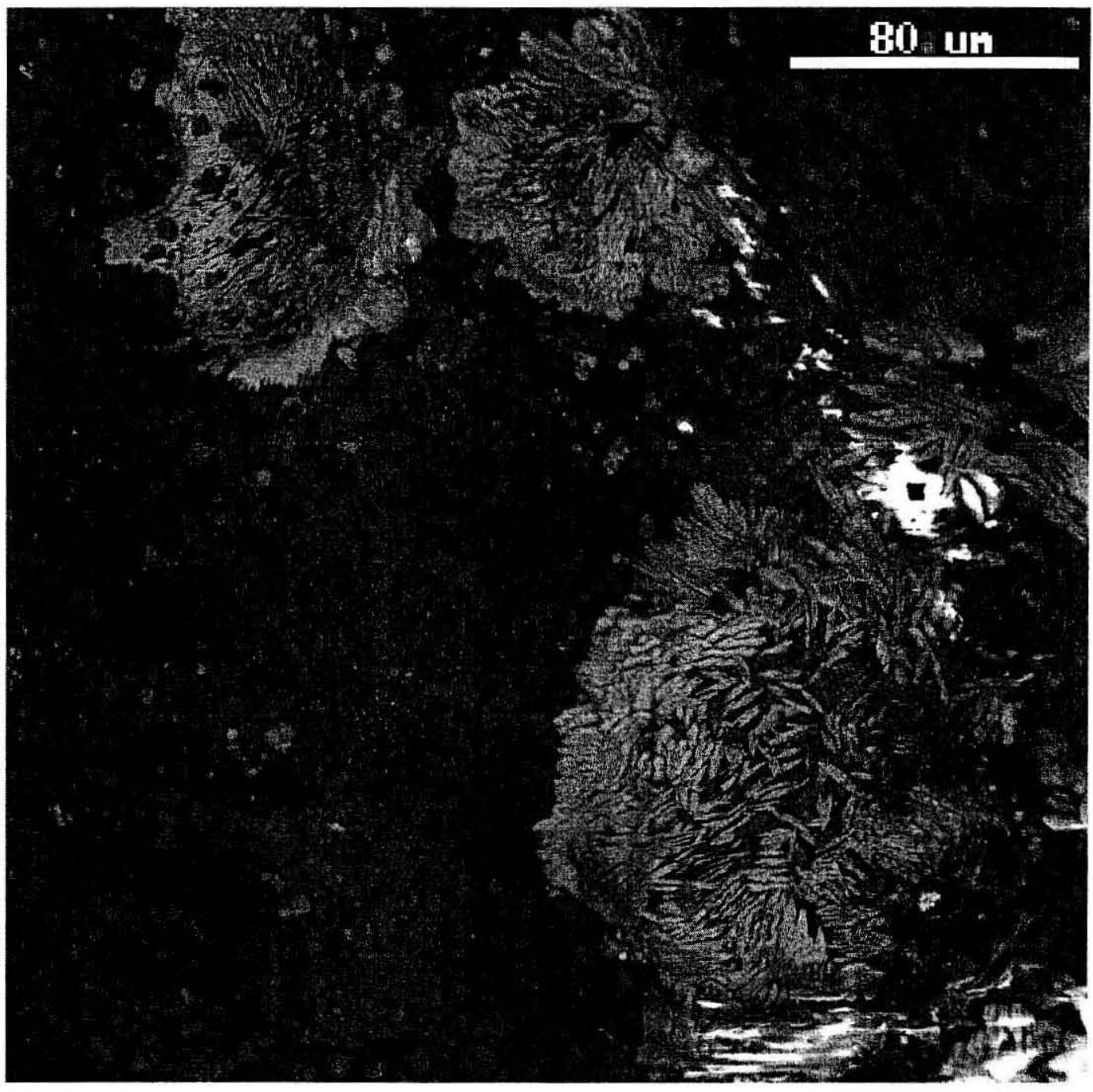

Figure A.24. BSE image of agglomerated material (despite carbon coating, charging was still problematic). (Sample \# 12K-O-144B). 
The Catholic University of America Vitreous State Laboratory
DuraMelter 1200 Tests with AZ-101HLW Simulants

Final Report, VSL-02R0100-2, Rev.1

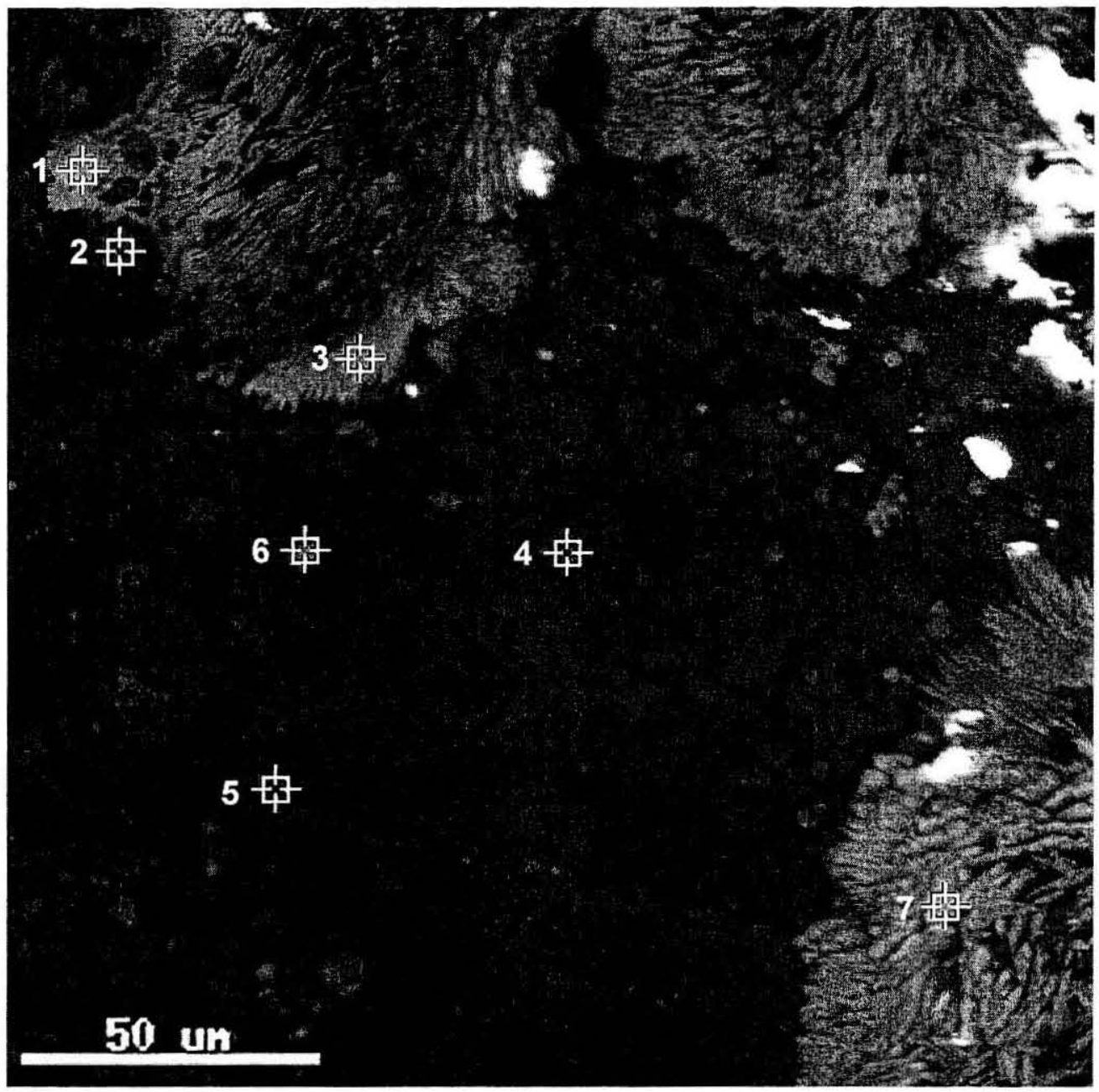

Figure A.25. Numbered regions in the agglomerated material refer to EDS spectra in the following figures. (Sample \# 12K-O-144B). 
The Catholic University of America

Vitreous State Laboratory
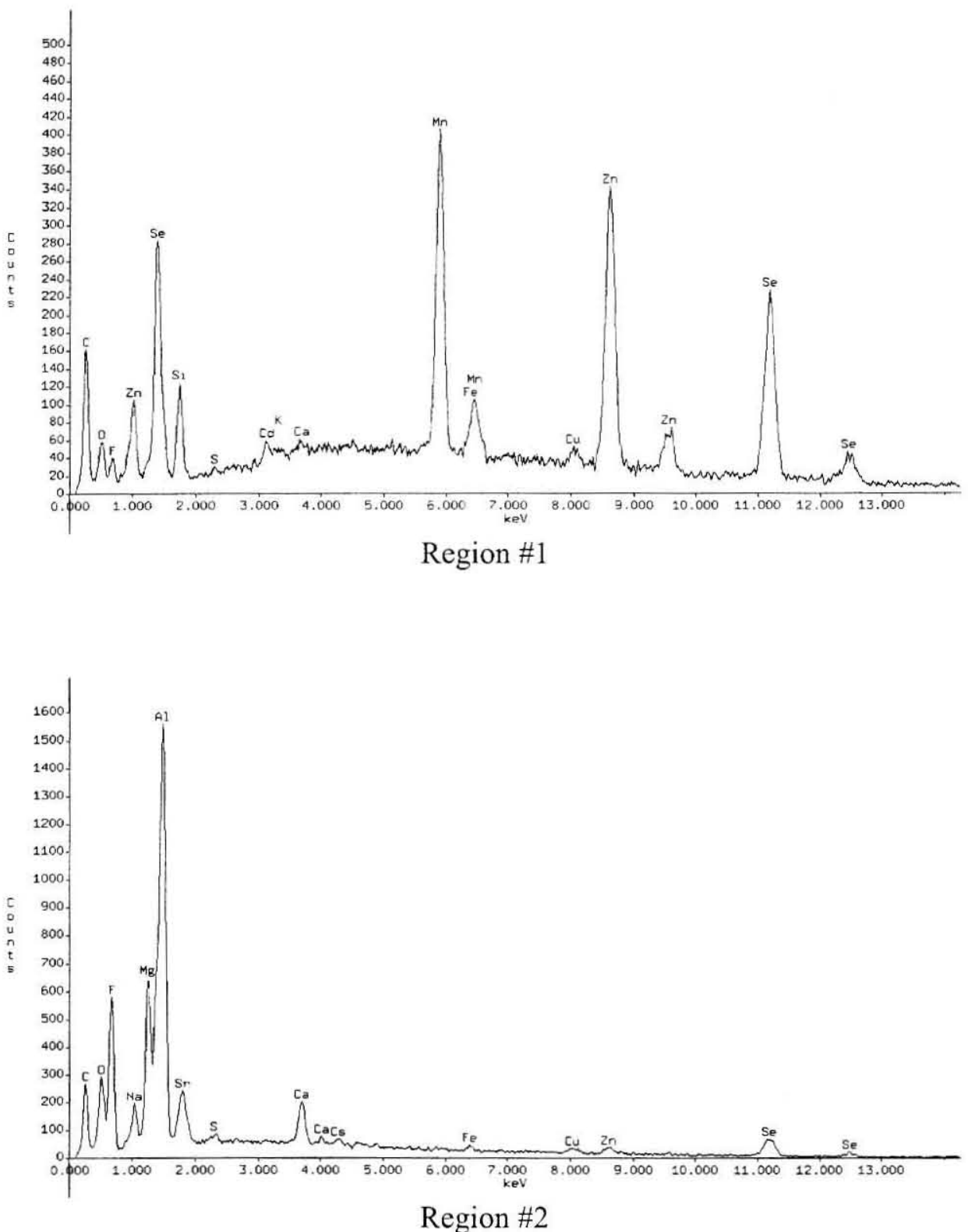

Figure A.26. EDS spectra of two sample regions (Regions \#1 and \#2) indicated in Figure A.25 (Sample \# 12K-O-144B). 
The Catholic University of America

Vitreous State Laboratory
DuraMelter 1200 Tests with AZ-101HLW Simulants Final Report, VSL-02R0100-2, Rev.1
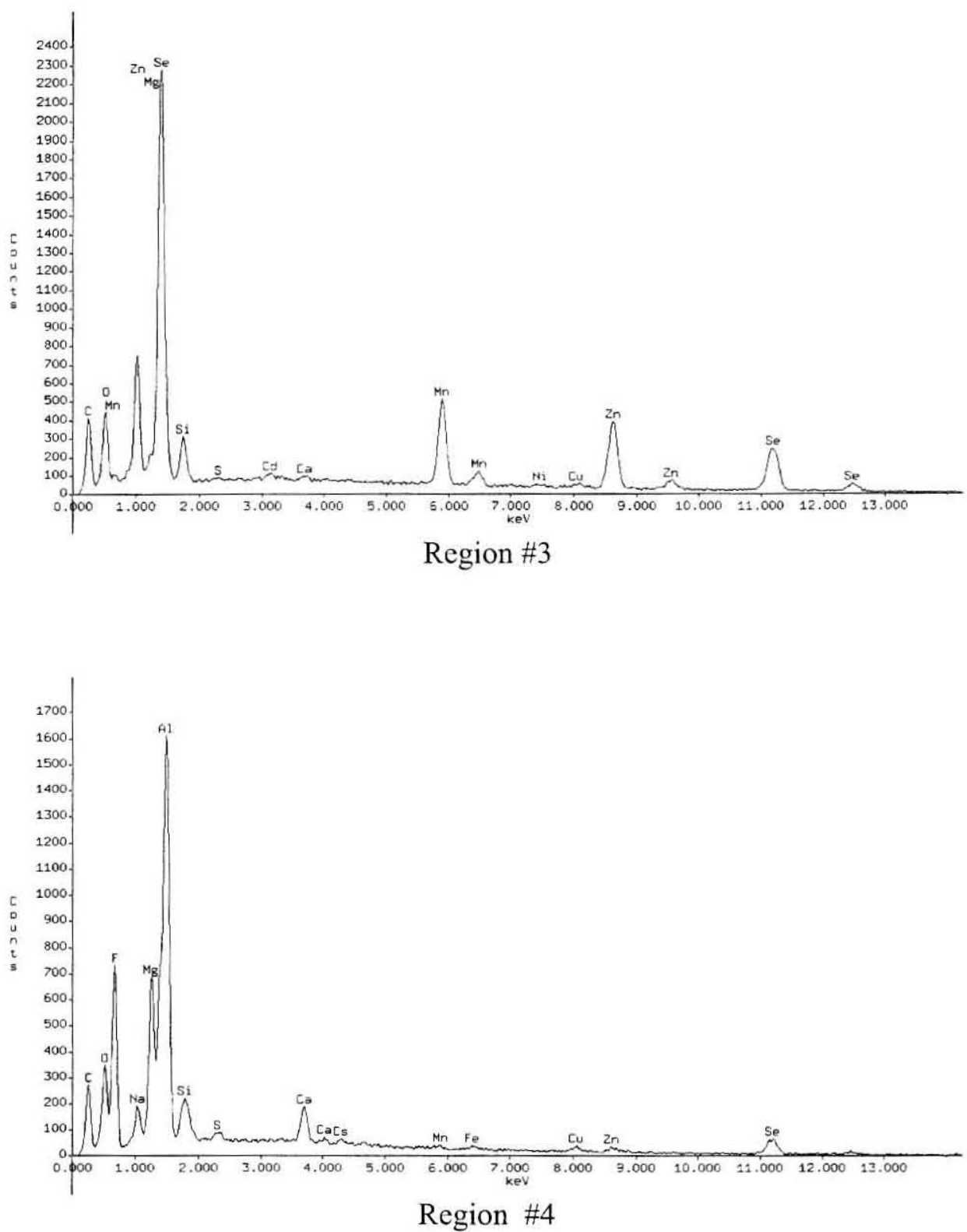

Figure A.27. EDS spectra of two sample regions (Regions \#3 and \#4) indicated in Figure A.25 (Sample \# 12K-O-144B). 
The Catholic University of America

Vitreous State Laboratory
DuraMelter 1200 Tests with AZ-101HLW Simulants Final Report, VSL-02R0100-2, Rev.1

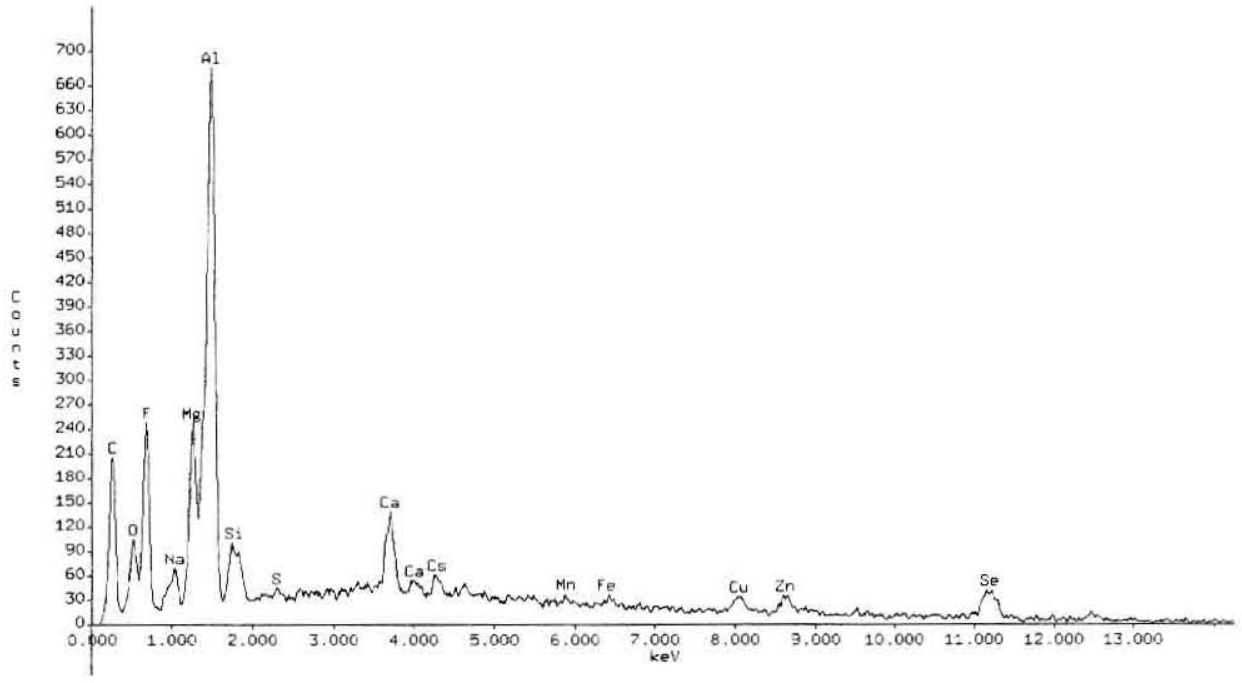

Region \#5

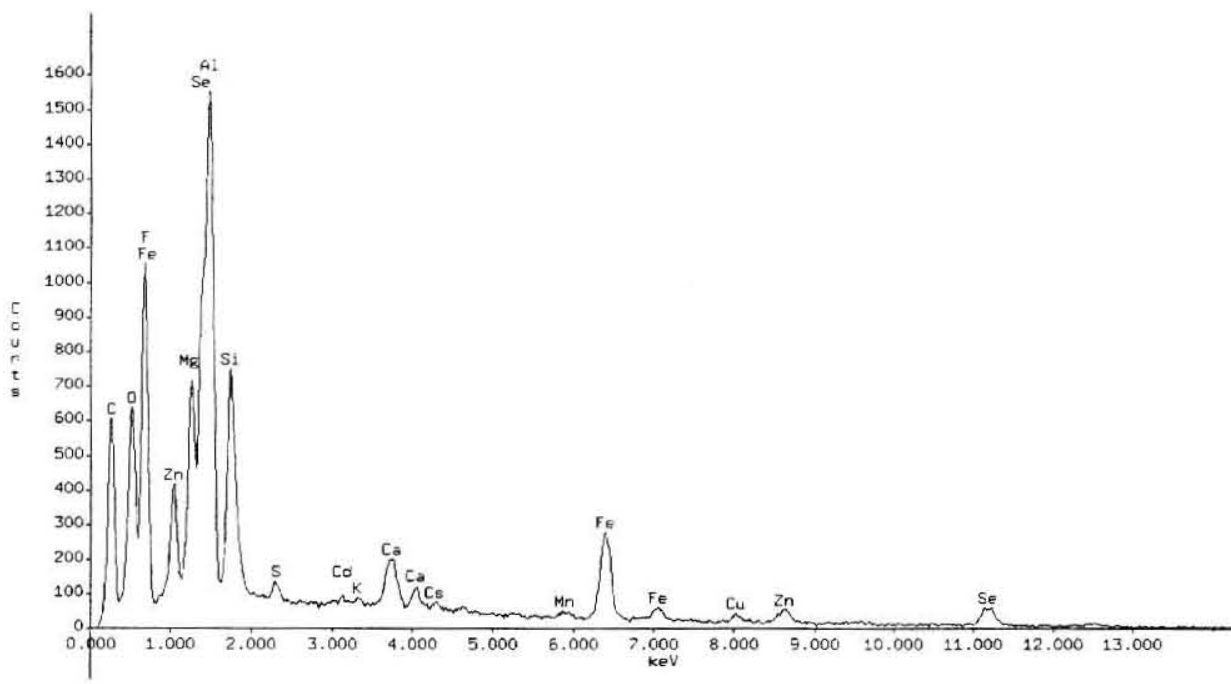

Region \#6

Figure A.28. EDS spectra of two sample regions (Regions \#5 and \#6) indicated in Figure A.25 (Sample \# 12K-O-144B). 
The Catholic University of America Vitreous State Laboratory
DuraMelter 1200 Tests with AZ-101 HLW Simulants Final Report, VSL-02R0100-2, Rev.1

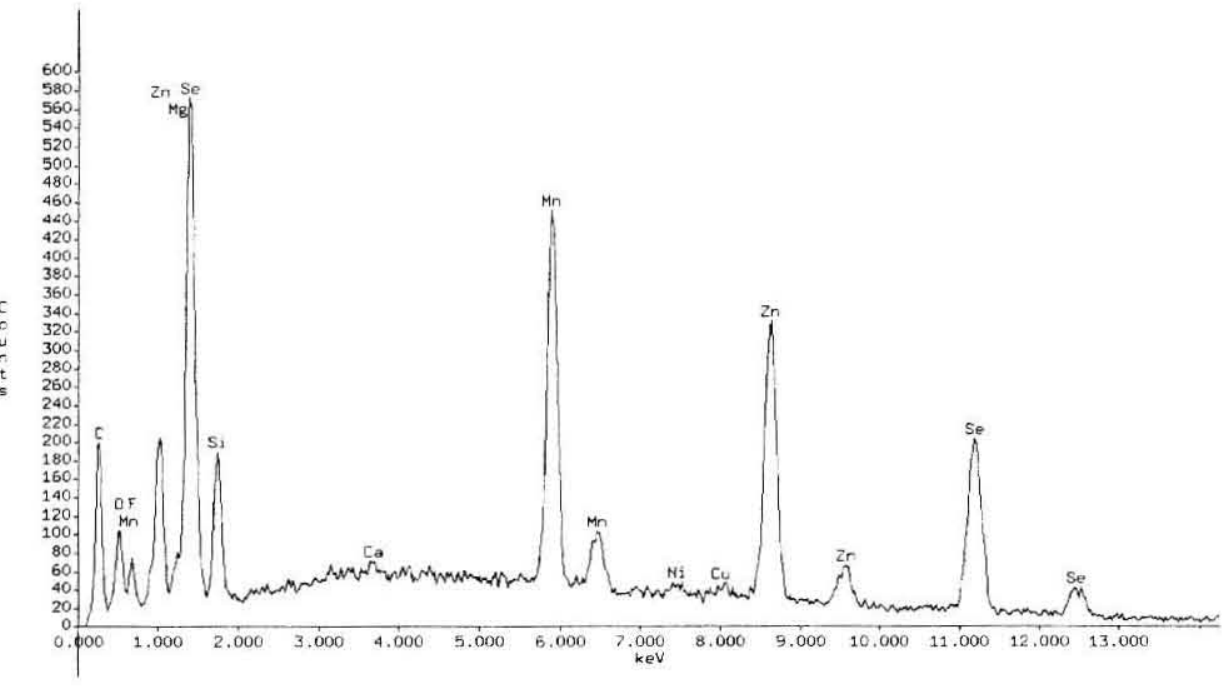

Region \#7

Figure A.29. EDS spectra of sample region (Region \#7) indicated in Figure A.25 (Sample \# 12K-O-144B). 
The Catholic University of America Vitreous State Laboratory
DuraMelter 1200 Tests with AZ-101HLW Simulants

Final Report, VSL-02R0100-2, Rev.1

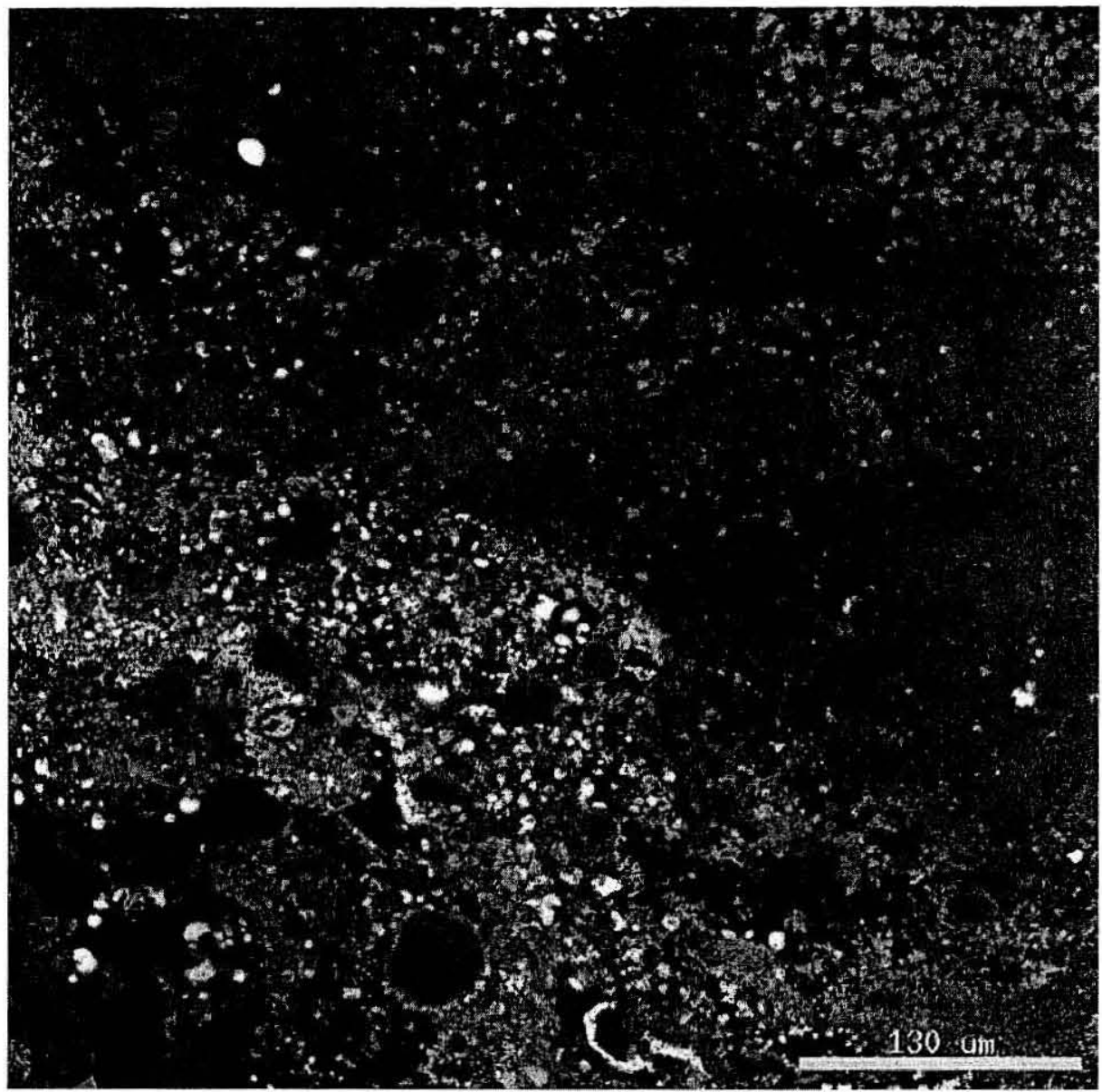

Figure A.30. BSE image of sample cross-section (Sample \#12N-O-133B). 
The Catholic University of America Vitreous State Laboratory
DuraMelter 1200 Tests with AZ-101HLW Simulants Final Report, VSL-02R0100-2, Rev.1

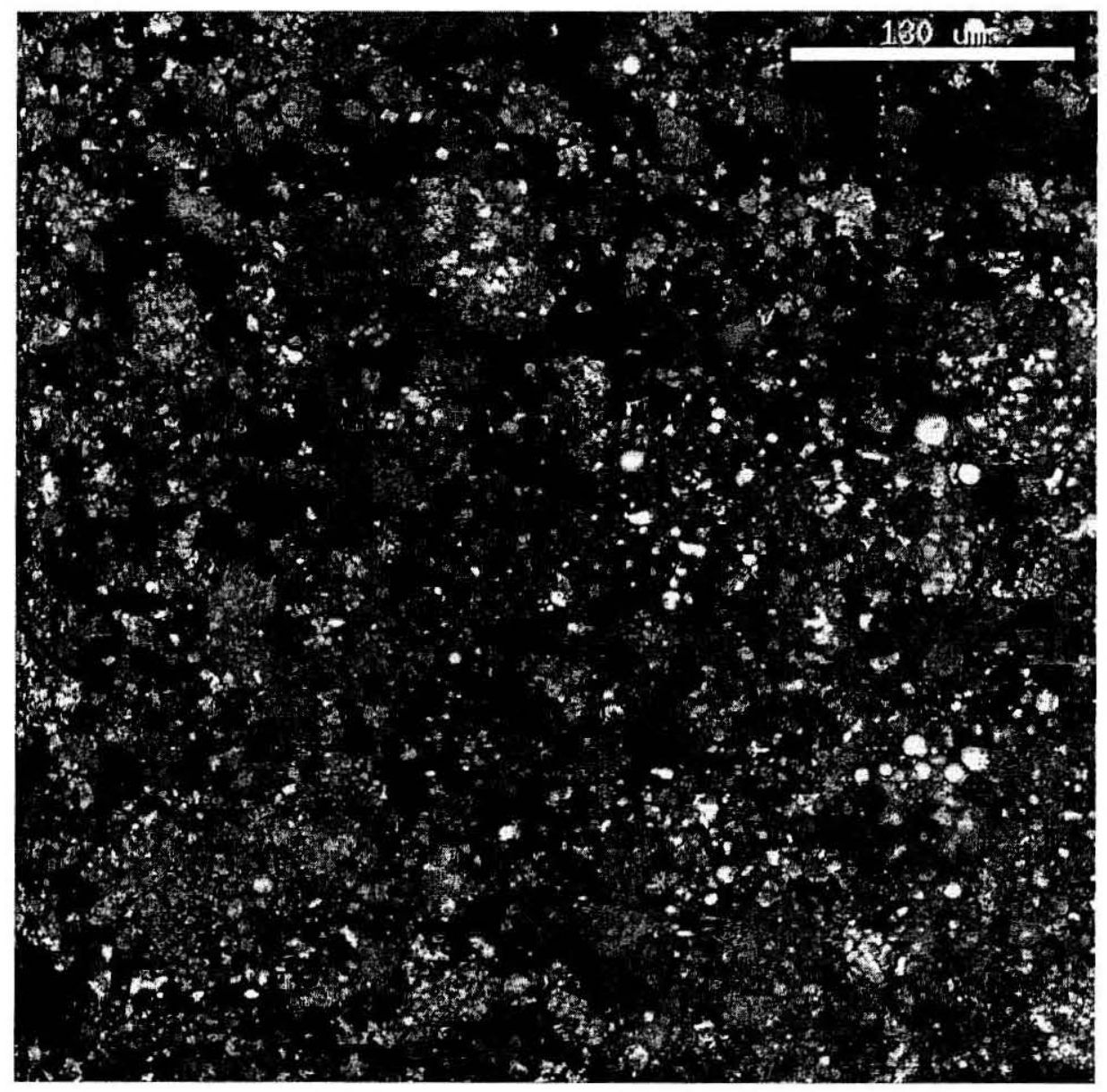

Figure A.31. BSE image of surface mounted specimen shown in Figure A.30 (Sample \# 12N-O-133B). 
The Catholic University of America Vitreous State Laboratory
DuraMelter 1200 Tests with AZ-101HLW Simulants Final Report, VSL-02R0100-2, Rev.1

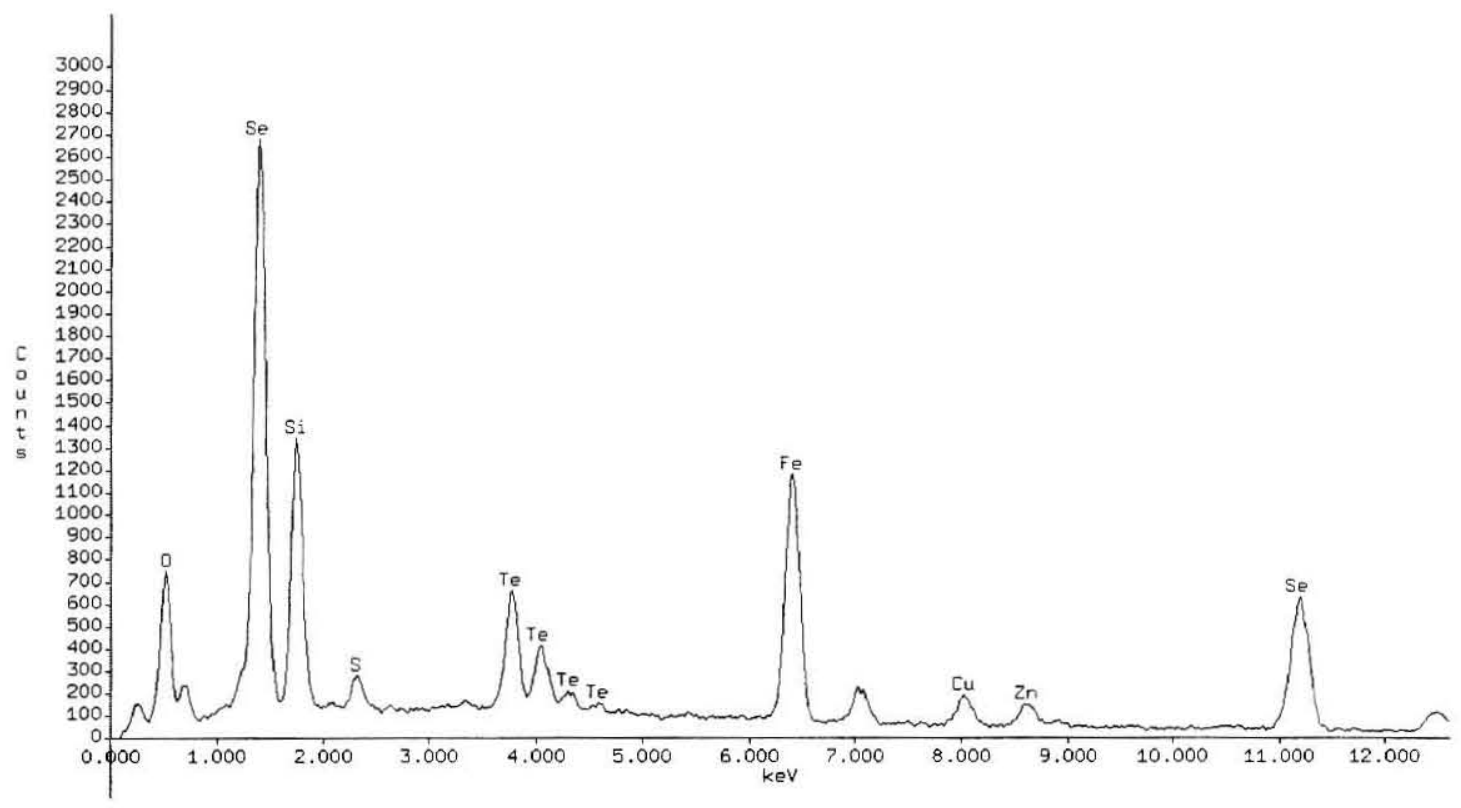

Figure A.32. EDS spectrum revealing general elemental composition. Fluorine is not shown here (Sample \# 12N-O-133B). 


\section{APPENDIX B}

\section{VISCOSITY AND PARTICLE SIZE DISTRIBUTION OF SBS FLUIDS}

Viscosities and particle size distributions were determined for six samples of DM1200 SBS fluids. The samples were selected from six different tests, as shown in Table B.1. The viscosities of the fluids were essentially the same as that of water.

SBS blown-down liquids were characterized for particle size distribution using a Microtrac S-3000 particle size analyzer. The particle size distribution measurement range of the instrument was 0.243 to $1408 \mu \mathrm{m}$. For these measurements, an aliquot of well-shaken SBS fluid was dispersed in deionized water. The measured particle size distributions are shown in Figures B.1-B.6. Table B.1 provides a summary of the particle size data in terms of mean sizes with respect to volume, number, and surface area statistics.

The mean size in terms of volume statistics ranges from 2 to $13 \mu \mathrm{m}$. The particle size at which the volume of suspended solids exceeds $50 \%$ of the total cumulative volume ranges from 1 to $7.5 \mu \mathrm{m}$. The particle size increases with increasing $\mathrm{pH}$ of the SBS liquids, as would be expected based on increased dissolution at low $\mathrm{pH}$. The use of nitrated melter feed resulted in the most acidic SBS fluids and, in turn, the lowest particle sizes for the SBS suspended solids. The sample from Test 3 deviates from this general $\mathrm{pH}$ trend, presumably due to agglomeration resulting from the high TSS concentration.

Melter exhaust particle size distribution data are compiled in Tables 30-40 of this report. Based on these results, in term of mass statistics, particles larger than roughly $14 \mu \mathrm{m}$ in size contribute over $65 \%$ of the total mass in melter exhaust particles. In comparison, the suspended solids in the SBS fluids are composed of much smaller particles. This difference may result from a combination of the disassociation of agglomerated particles emitted from the melter when they contact the SBS fluid, as well as attrition and dissolution of larger particles. 
Table B.1. Measured Properties of SBS Liquids.

\begin{tabular}{|c|c|c|c|c|c|c|c|c|c|c|}
\hline \multirow{2}{*}{ Test } & \multirow{2}{*}{$\begin{array}{l}\text { Sampling } \\
\text { Date }\end{array}$} & \multirow{2}{*}{$\begin{array}{l}\text { Sample } \\
\text { Name }\end{array}$} & \multirow{2}{*}{$\begin{array}{c}\text { TSS } \\
(\mathrm{mg} / \mathrm{l})\end{array}$} & \multirow{2}{*}{$\begin{array}{r}\text { TDS } \\
(\mathrm{mg} / \mathrm{l})\end{array}$} & \multirow[b]{2}{*}{$\mathrm{pH}$} & \multicolumn{4}{|c|}{ Particle Characteristics } & \multirow{2}{*}{$\begin{array}{c}\text { Viscosity } \\
\text { (Poise) } \\
(a)>100 / \mathrm{s}\end{array}$} \\
\hline & & & & & & $\begin{array}{c}\mathrm{mv} \\
(\mu \mathrm{m})\end{array}$ & $\begin{array}{c}\mathrm{mn} \\
(\mu \mathrm{m})\end{array}$ & $\begin{array}{c}\mathrm{ma} \\
(\mu \mathrm{m})\end{array}$ & $\begin{array}{c}\text { ps } \\
@ 50 \%\end{array}$ & \\
\hline 2 & $8 / 1 / 01$ & $12 \mathrm{I}-\mathrm{S}-88 \mathrm{~A}$ & 268 & 672 & 8.45 & 8.01 & 0.69 & 3.23 & 5.43 & $<0.02$ \\
\hline 3 & $8 / 11 / 01$ & $12 \mathrm{~J}-\mathrm{S}-139 \mathrm{~A}$ & 3584 & 2720 & 5.47 & 12.90 & 0.55 & 3.06 & 7.47 & $<0.02$ \\
\hline 4 & $8 / 25 / 01$ & $12 \mathrm{~K}-\mathrm{S}-136 \mathrm{~A}$ & 448 & 1936 & 4.57 & 8.25 & 0.64 & 2.96 & 5.35 & $<0.02$ \\
\hline 5 & $10 / 1 / 01$ & $12 \mathrm{~L}-\mathrm{S}-116 \mathrm{~A}$ & 1076 & 3356 & 1.05 & 2.12 & 0.63 & 1.43 & 2.07 & $<0.02$ \\
\hline 7 & $10 / 26 / 01$ & $12 \mathrm{~N}-\mathrm{S}-126 \mathrm{~B}$ & 448 & 2120 & 2.22 & 3.49 & 0.51 & 1.73 & 2.55 & $<0.02$ \\
\hline 9 & $11 / 10 / 01$ & $12 \mathrm{P}-\mathrm{S}-41 \mathrm{~A}$ & 72 & 1144 & 3.48 & 3.36 & 0.44 & 0.94 & 1.17 & $<0.02$ \\
\hline \multicolumn{3}{|c|}{ Deionized Water } & $\mathrm{NA}$ & $\overline{N A}$ & $\overline{N A}$ & $\mathrm{NA}$ & NA & NA & NA & $<0.02$ \\
\hline
\end{tabular}

NA: not analyzed

mv: mean size in terms of volume statistics

$\mathrm{mn}$ : mean size in terms of number statistics

ma: mean size in terms of surface area statistics

ps@50\%: particle size at 50\% volume of particles passed. 
The Catholic University of America Vitreous State Laboratory
DuraMelter 1200 Tests with AZ-101HLW Simulants Final Report, VSL-02R0100-2, Rev.1

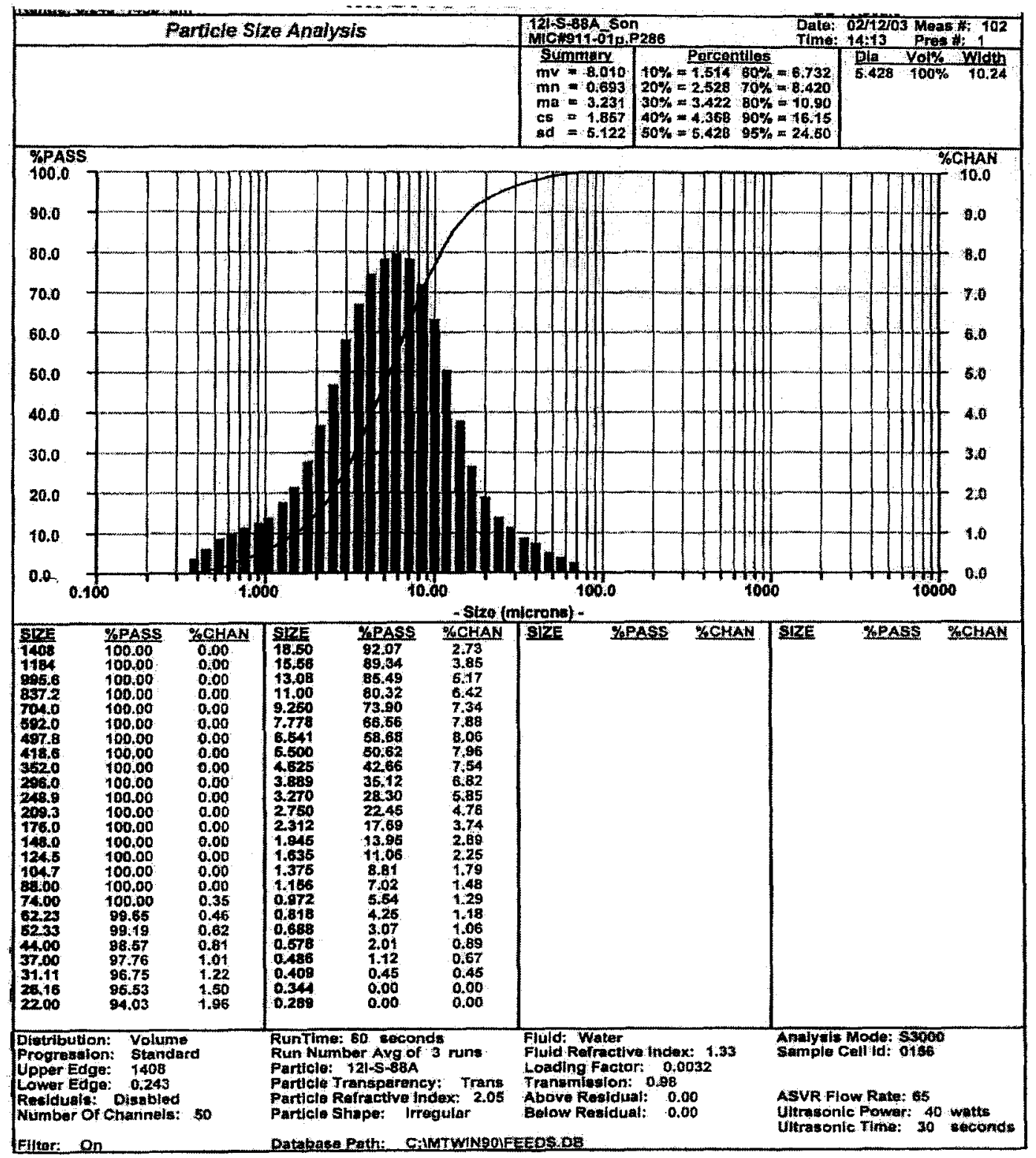

Figure B.1. Particle size distribution of suspended solids in 12I-S-88A. 
The Catholic University of America Vitreous State Laboratory
DuraMelter 1200 Tests with AZ-101HLW Simulants

Final Report, VSL-02R0100-2, Rev.I

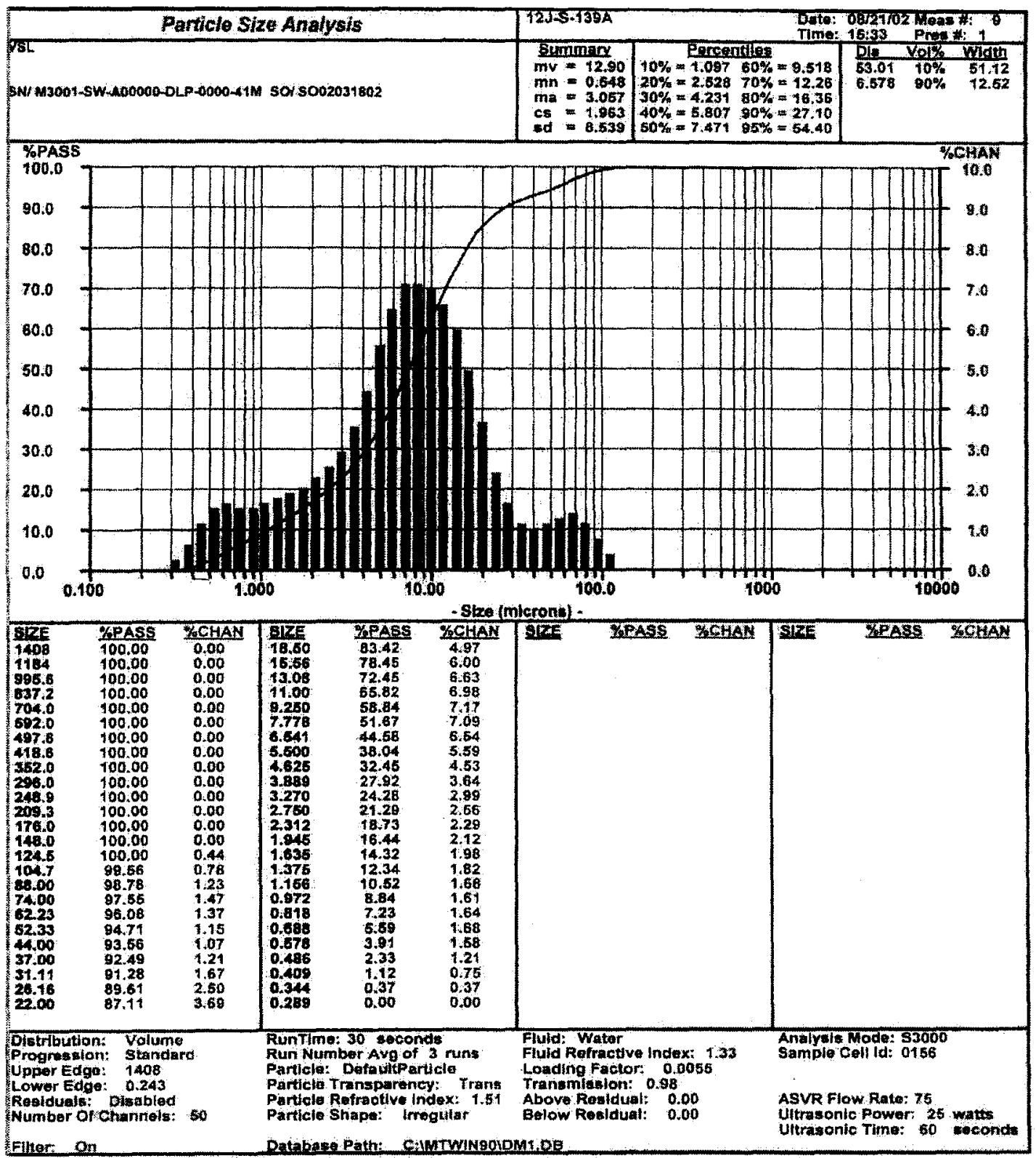

Figure B.2. Particle size distribution of suspended solids in 12J-S-139A. 
The Catholic University of America Vitreous State Laboratory
DuraMelter 1200 Tests with AZ-101HLW Simulants

Final Report, VSL-02R0100-2, Rev.I

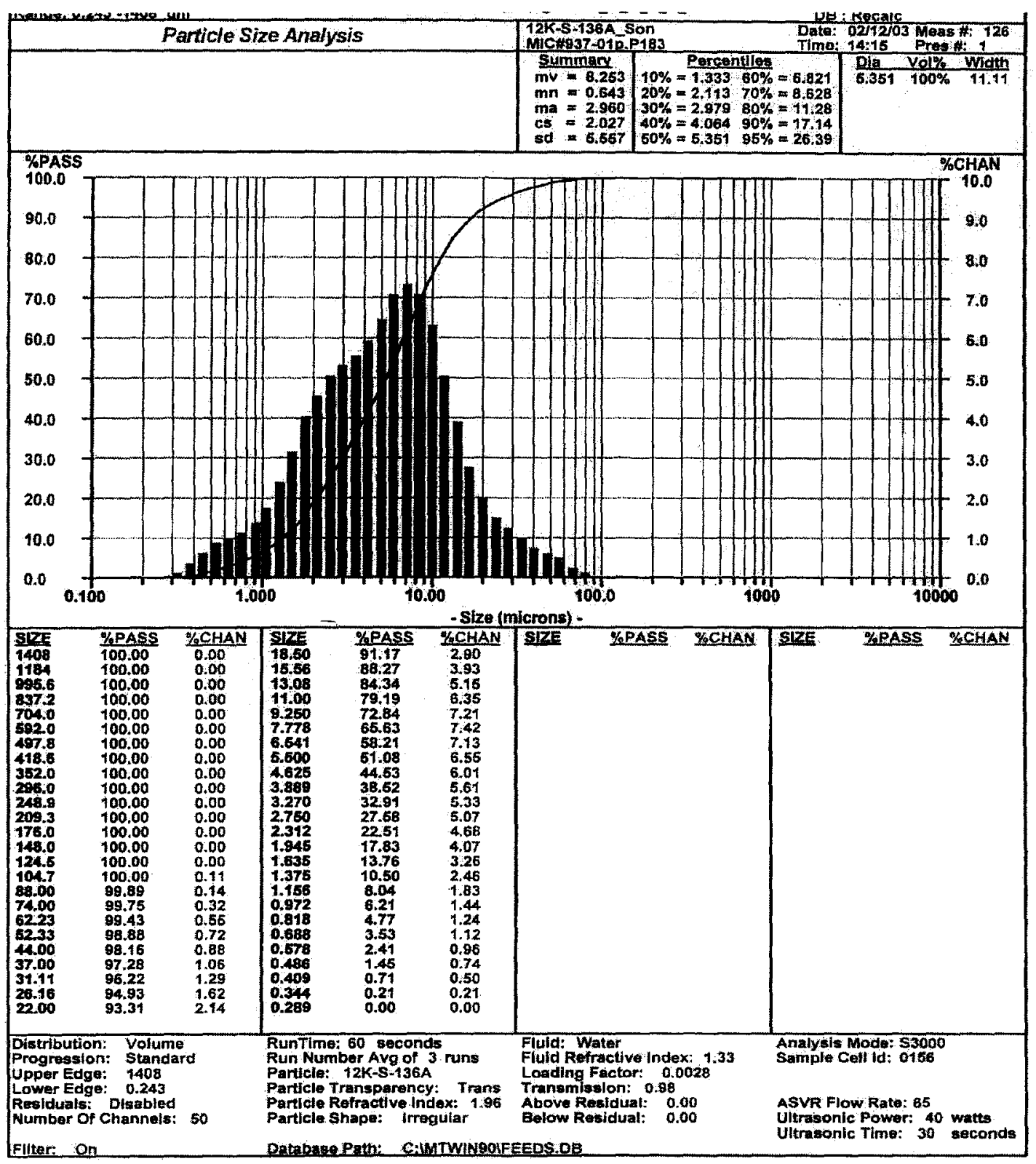

Figure B.3. Particle size distribution of suspended solids in 12K-S-136A. 
The Catholic University of America Vitreous State Laboratory
DuraMelter 1200 Tests with AZ-101HLW Simulants

Final Report, VSL-02R0100-2, Rev.1

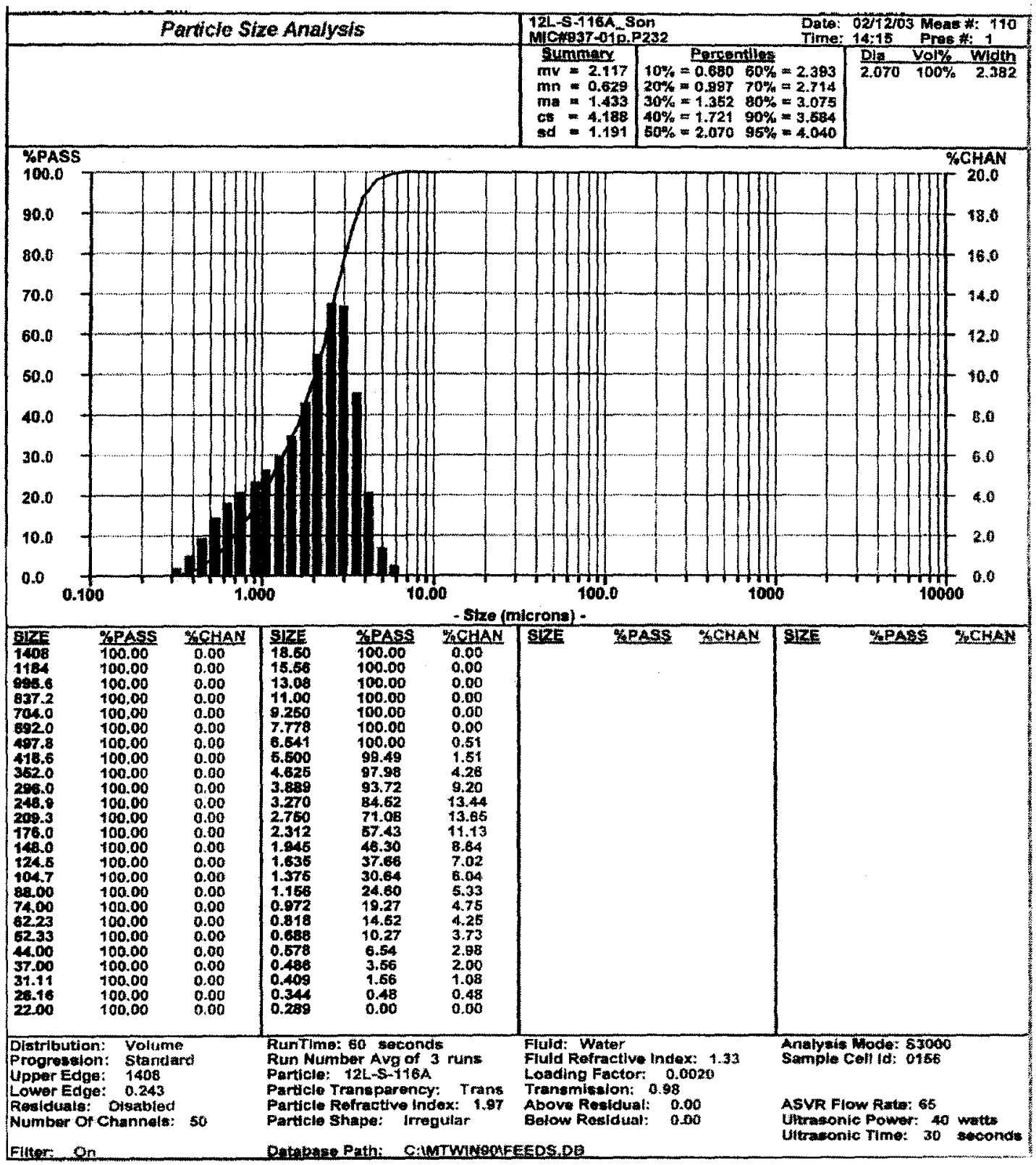

Figure B.4. Particle size distribution of suspended solids in 12L-S-116A. 
The Catholic University of America Vitreous State Laboratory
DuraMelter 1200 Tests with AZ-101HLW Simulants Final Report, VSL-02R0100-2, Rev.1

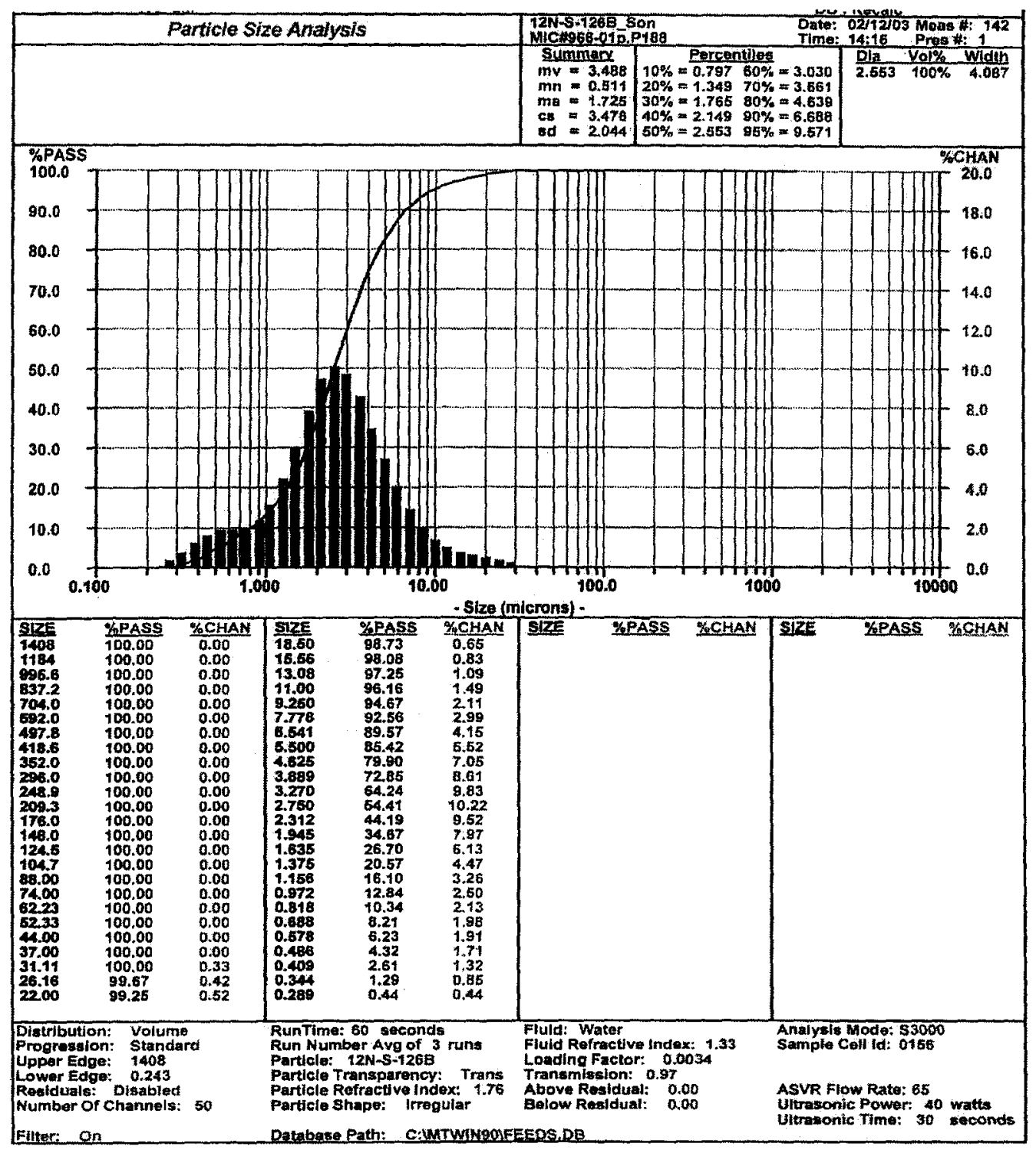

Figure B.5. Particle size distribution of suspended solids in 12N-S-126B. 
The Catholic University of America Vitreous State Laboratory
DuraMelter 1200 Tests with AZ-101HLW Simulants Final Report, VSL-02R0100-2, Rev.1

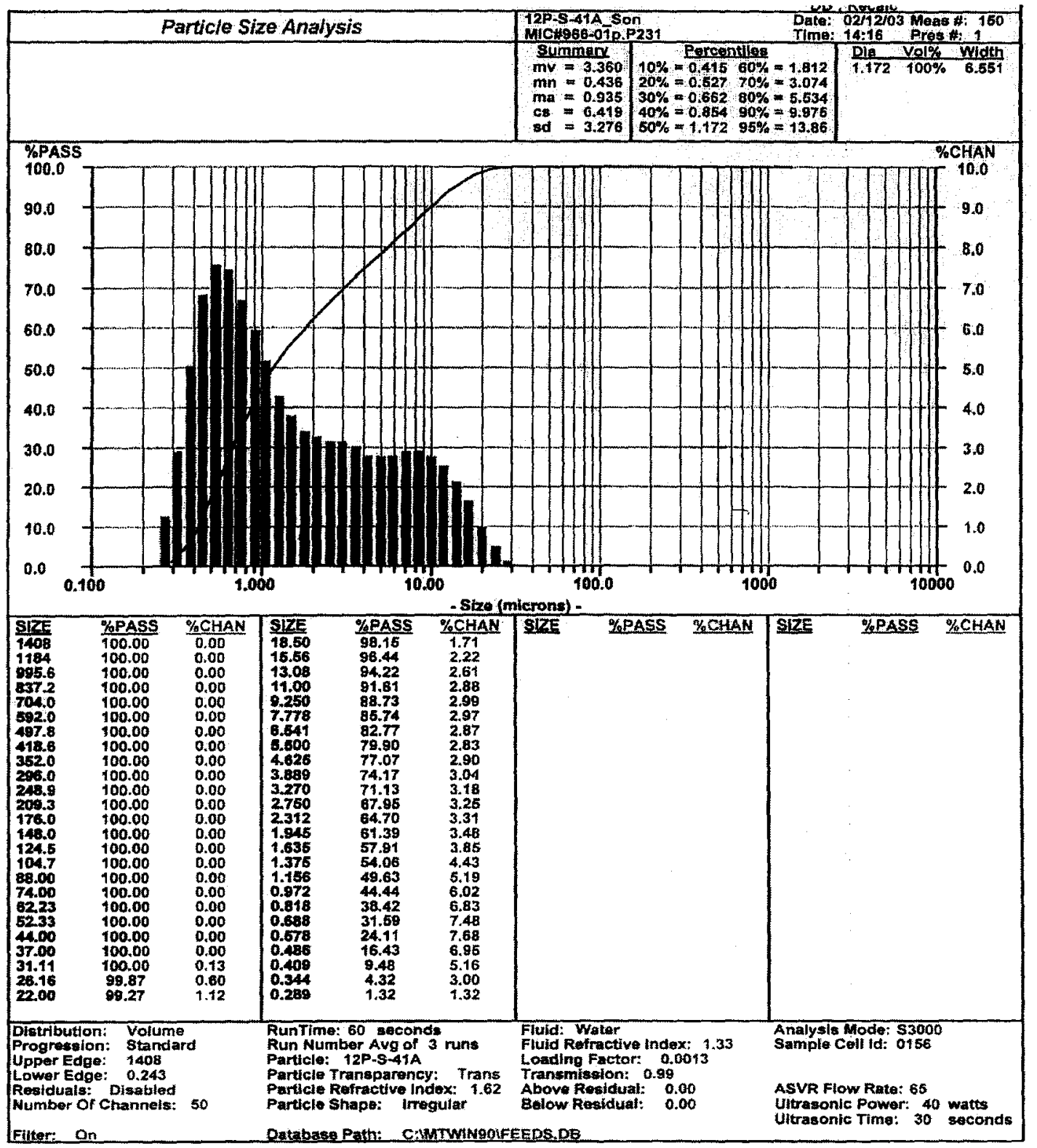

Figure B.6. Particle size distribution of suspended solids in 12P-S-41A. 
Bechtel National, Inc.

WTP PDC, Submittal Coordinator

2435 Stevens Center Place

MSIN H4-02/MS11-B

Richland, WA 99352

Attn: Mr. L. Scot Jenkins

H4-02 (MS14-3A)

SUBCONTRACT NO. 24590-101-TSA-W000-0009

DURATEK FEDERAL SERVICES, INC. RESEARCH AND TECHNOLOGY SUPPORT, TRANSMITTAL OF FINAL REPORT, APPROVED BY BNI R\&T MANAGER FOR USE.

Dear Mr. Jenkins:

Please find attached the following Final Report approved by BNI R\&T Manger for use on July 31 , 2003:

- Final Report - Tests on the DuraMelter 1200 HLW Pilot Melter System Using AZ-101 HLW Simulants VSL-02R0100-2, Revision 1, dated 2/17/2003

Should you have any questions and/or concerns, please contact the undersigned on 376-9942.

Sincerely,

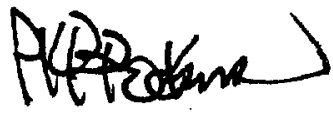

P. K. Brockman

Duratek Federal Services, Inc.

Vice President / RPP Project Manager

Attachments

cc: Steve Barnes w/o att

Karen Hornbuckle w/o att

Joe Perez w/o att

Chris Musick w/o att

Brad Bowan w/o att

Glenn Diener w/o att

Ron Garretsen w/o att

Chris Chapman w/o att

Document Control file w/o att

P. K. Brockman LB
H4-02 (MS1-B)

H4-02 (MS1-B)

$\mathrm{H} 4-02$ (MS1-B)

H4-02 (MS1-B)

Columbia

Columbia

H4-02 (MS14-3A)

$\mathrm{H} 1-11$

Columbia
Jeannette Doyle w/o att

Karen Petro w/o att

H4-02 (MS1-B)

Scot Jenkins w/o att

H4-02 (MS14-3A) 
BECHTEL NATIONAL INC Fax:5093718346, ORP-51438, Rev.0 Jun 112003 10:22 P.04

\section{MASTER DISTRIBUTION SCHEDULE \\ Sheer 1 of 1}

\begin{tabular}{|c|c|}
\hline Document Number & Rev \\
\hline 24590-101-TSA-W000-0009-34-83 & $00 \mathrm{C}$ \\
\hline
\end{tabular}

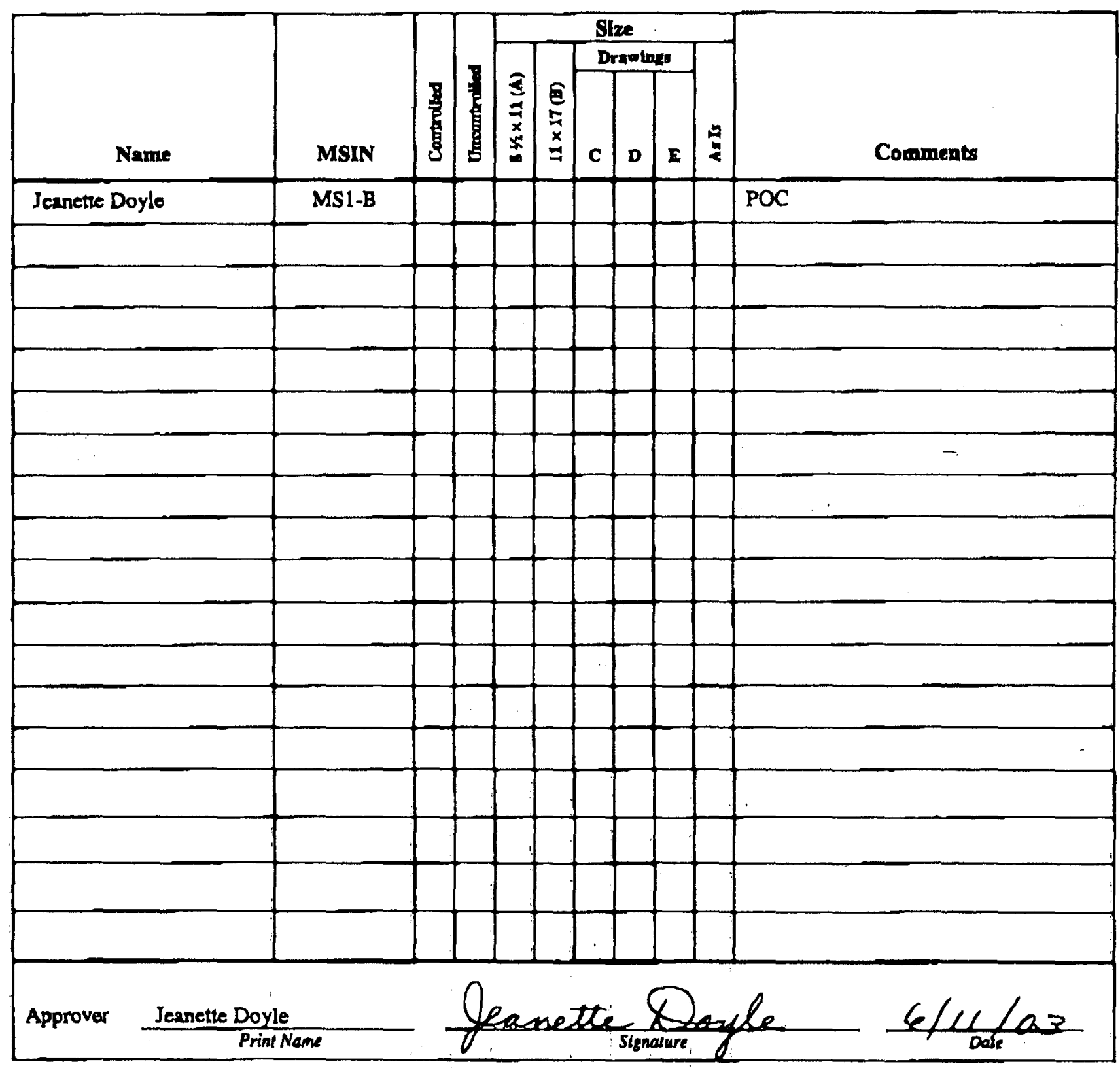

$$
2^{20}
$$




\section{MASTER DISTRIBUTION SCHEDULE}

Sheet 1 of 1

\begin{tabular}{|c|c|}
\hline Document Number & Rev \\
\hline VSL-02R0100-2, Tests on the DuraMelter 1200 HLW & \\
Pilot Melter System Using HLW AZ-101 Simulants & 1 \\
\hline
\end{tabular}

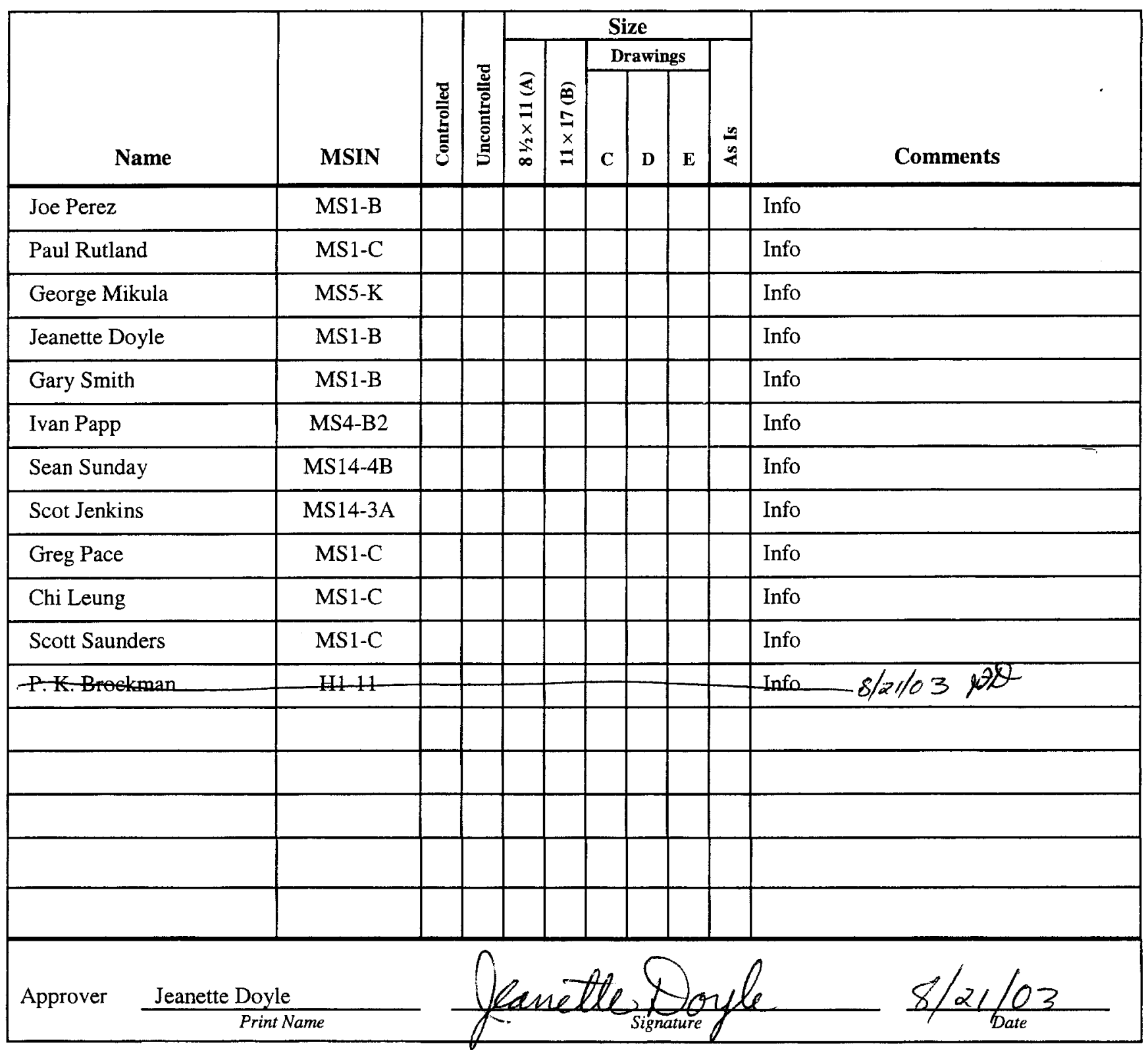




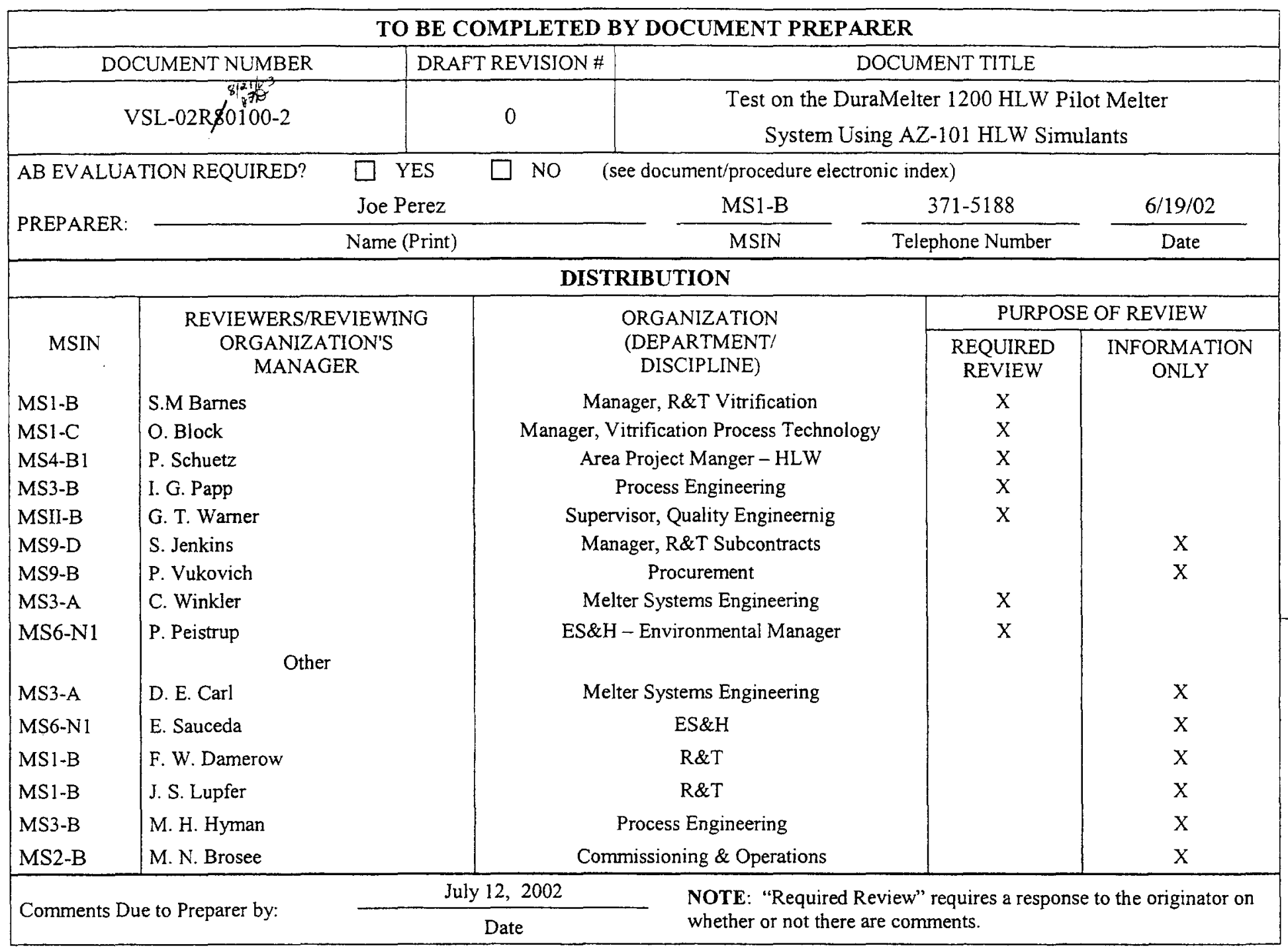

TO BE COMPLETED BY REVIEWER

REVIEWER'S NAME/ORGANIZATION: J.m. Cerez $f_{\text {or }}$ steveBarves /R+T Vitrification DATE: July

This document was reviewed per 24590-WTP-GPP-PADC-003, Internal Review and Approval of Documents, section 3.0, and is: ACCEPTED, NO COMMENTS $\square \quad$ COMMENTS ON DOCUMENT $\square \quad$ COMMENTS ATTACHED

Document maintains AB consistency? $\square$ Yes $\square$ No

Document maintains QAM compliance? $\square$ Yes $\square$ No

INITIAL, ES\&H

INITIAL, QA

If the document is not compliant with the $A B$, an $A B C N$ shall be approved and either the $A B$ documents revised or a $D T D$ approved before the document can be issued.

AB revised (date) $\quad$ ABCN No.

Approval Date:

Comments Resolved:

Approval Date:

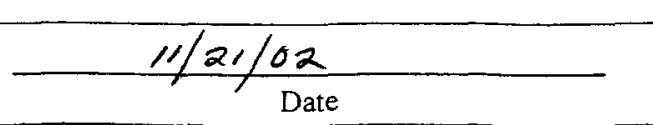




\section{DOCUMENT REVIEW RECORD}

Sheet 1 of 10

Return to: Joe Perez

Comments Due: July12, 2002

\begin{tabular}{|ll|l|l|l|l|}
\hline Document Title: & $\begin{array}{l}\text { Test on the DuraMelter 1200 HLW Pilot } \\
\text { Melter System Using AZ-101 HLW Simulants }\end{array}$ & $\begin{array}{l}\text { Document No. } \\
\text { VSL-02R0100-2 }\end{array}$ & $\begin{array}{c}\text { Revision: } \\
0\end{array}$ \\
\hline Reviewer: & Date: & Response by: & Date: & Comments Resolved; \\
JM Perez & $8-15-02$ & KS Matlack & $11 / 12 / 02$ & June 11, 2002 \\
\hline
\end{tabular}

\begin{tabular}{|c|c|c|c|c|c|c|}
\hline Item No. & Section/Paragraph & Comment & Response & Significance $^{2}$ & Resolution & Incorporated? \\
\hline 1 & General & $\begin{array}{l}\text { 1. The status of equipment inspections and } \\
\text { performance are generally not well } \\
\text { documented, i.e., film cooler, feed } \\
\text { nozzle, overflow, bubbler, T/C and } \\
\text { level probes in the glass, lid and glass } \\
\text { contact refractory, off-gas jumper and } \\
\text { WESP. } \\
\text { 2. Many key data and analyses, e.g., pH, } \\
\text { plenum pressure would be strengthened } \\
\text { by adding an average and std. dev. for } \\
\text { data so variability can be readily } \\
\text { discerned. R\&T and VSL will work } \\
\text { together to identify key data for added } \\
\text { data treatment on future reports. }\end{array}$ & $\begin{array}{l}\text { The Project's desire for inspection data has } \\
\text { grown over time. The Test Plan for this } \\
\text { work specified only the film cooler, } \\
\text { transition line, and the SBS and WESP } \\
\text { internals, which are discussed in the report. } \\
\text { Any other available data will be added } \\
\text { where possible. } \\
\text { OK. }\end{array}$ & $\mathrm{M}$ & & \\
\hline 2 & Pg. 6 & $\begin{array}{l}\text { Footnote is not consistent with text of last } \\
\text { objective regarding use of "L" versus } \\
\text { simplified bubbler. This also pertains to the } \\
\text { 2nd para. of page } 8 \text {. }\end{array}$ & $\begin{array}{l}\text { True, but this is correct. The Project elected } \\
\text { not to run the tests associated with the last } \\
\text { objective so none were performed. We will } \\
\text { delete this objective from the list to avoid } \\
\text { confusion. }\end{array}$ & $\mathrm{M}$ & & \\
\hline 3 & Pg. 7, first line & $\begin{array}{l}\text { The phrase in () does not make sense. It } \\
\text { may belong with the proceeding sentence. }\end{array}$ & Agreed. & M & & \\
\hline 4 & $\begin{array}{l}\text { Pg. } 7, \text { Sec. } 1.3 .1,1 \mathrm{st} \\
\text { para., 3rd sent. }\end{array}$ & $\begin{array}{l}\text { Describe after which test the baffles were } \\
\text { installed in the feed tank. I don't remember }\end{array}$ & Agreed. & M & & \\
\hline
\end{tabular}




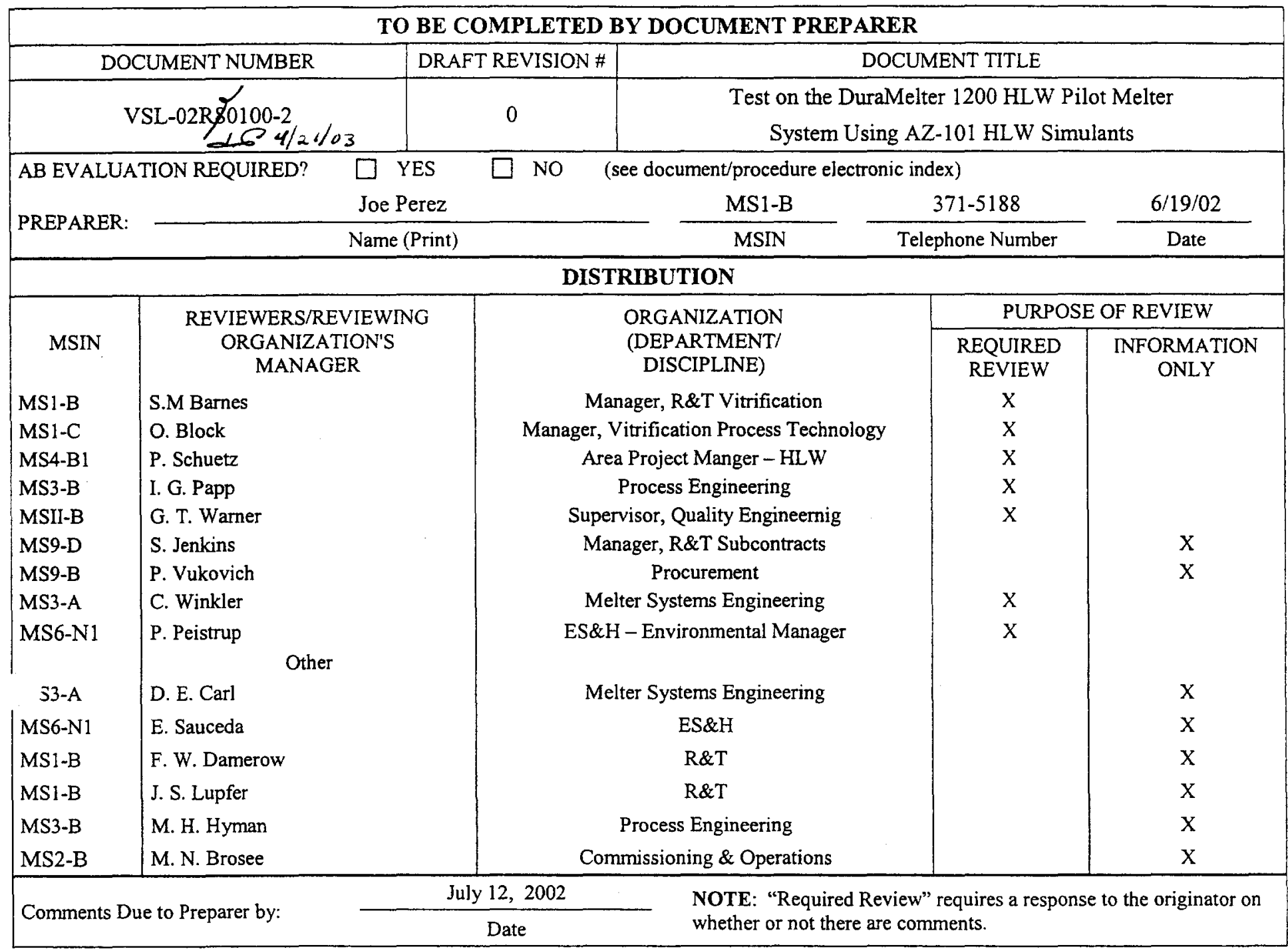

\section{TO BE COMPLETED BY REVIEWER}

REVIEWER'S NAME/ORGANIZATION: D. Carl/C. Winkler/Melter Systems

DATE: 27JUN02

This document was reviewed per 24590-WTP-GPP-PADC-003, Internal Review and Approval of Documents, section 3.0, and is:

ACCEPTED, NO COMMENTS

Document maintains $A B$ consistency?

Document maintains QAM compliance?

\section{COMMENTS ON DOCUMENT \\ COMMENTS ATTACHED \}

If the document is not compliant with the $A B$, an $A B C N$ shall be approved and either the $A B$ documents revised or a $D T D$ approved before the document can be issued.

ABCN No.

3 revised (date):
DTD No.
Approval Date:

Approval Date:
Comments Resolved: 24590-F00016 Rev $2(03 / 05 / 02)$
I $2 \mathrm{ded} 2$ Date

Ref: 24590-WTP-GPP-PADC-003 
From:

Hyman, Marve

Sent:

Monday, April 28, 2003 11:06 AM

To:

Perez, Joseph

Cc:

Blodgett, Stephanie; Kaye, James $\mathrm{H}$

Subject:

RE: Concurrance - Comments on DM1200 AZ-101 Report VSL-02R0100-2 Rev 0

On behalf of I Papp/Engineering, I concur with VSI-02R0100-2 Rev 0 , "Tests on the Duramelter $1200 \mathrm{HLW}$ Pilot Melter system Using AZ-101 HLW simulants."

Marve

-.---Original Message-.-.

From: Perez, Joseph

Sent: Tuesday, April 22, 2003 5:37 PM

To: Hyman, Marve

Cc: Blodgett, Stephanie

Subject: RE: Responses to Comments on DM1200 AZ-101 Report

Marv, I was putting a report approval packet together and I couldn't resurrect what should have been the final e:mail from you or the coordinator on this comment set. Do you have a record of it. I usually get the signed hard copy back from you. It would have been something you would have done in early December or so.

Sorry for the imposition.

Thanks,

Joe Perez

$\mathrm{R} \& \mathrm{~T} / \mathrm{WTP}$

Ph.: 509.371 .8444

Fax: 509.371 .8346

----original Message-----

From: Hyman, Marve

Sent: Tuesday, November 12, $20023: 21$ PM

To: Perez, Joseph

Cc: Blodgett, Stephanie; Howell, John F.; Rouse, James

Subject: FW: Responses to Comments on DM1200 AZ-101 Report

Joe: Here are some replies to the comment responses.

General: Can we assume that the edits and improvements that were suggested will be incorporated as appropriate?

Item No. MH7: If the temperature readings are not suspect, then the conjecture is suspect, especially considering the decrease in temperature for Test 7 . The conjecture needs to be qualified or replaced by another surmise.

Item No. MH8: Where it is mentioned that the scrubber was bypassed, add the words ". . . because there is no HLW offgas train." [Insert which scrubber; there is an SBS.]

Item No. MH14: A response to the comment should be given. inadvertently left out the "M" in the significance column.)

Cheers, Marve

-..--original Message-....

From: Perez, Joseph

Sent: Tuesday, November 12, $20022: 23$ PM

To: Hyman, Marve; Rouse, James; Larson, Donald

Subject: FW: Responses to Comments on DM1200 AZ-101 Report 
Please review your respective comments and tell me and Marve if they are acceptable. Need feedback by Friday if possible.

thanks

Joe Perez

$\mathrm{R} \& \mathrm{~T} / \mathrm{WTP}$

Ph.: 509.371 .8444

Fax: 509.371 .8346

-..--Original Message-..--

From: Ian L. Pegg [mailto:ianp@vsl.cua.edu]

Sent: Tuesday, November 12, 2002 9:04 AM

To: Perez, Joseph

Cc: ijoseph@duratekinc.com; bbowan@duratekinc.com

Subject: Responses to Comments on DM1200 AZ-I01 Report

Joe :

Please find attached the subject comment responses.

Regards,

Ian. 


\section{DOCUMENT REVIEW REQUEST}

Sheet 1 of 1

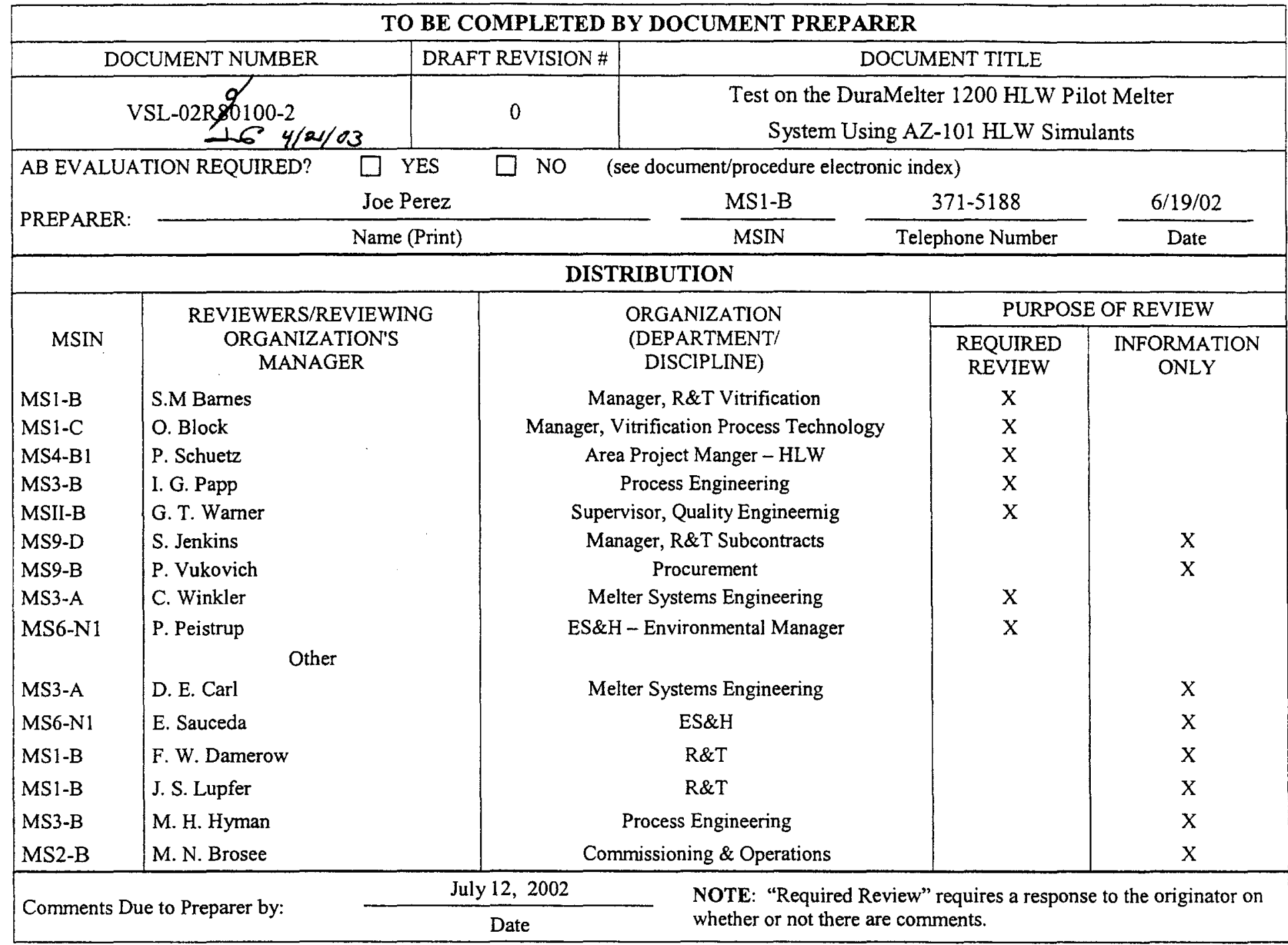

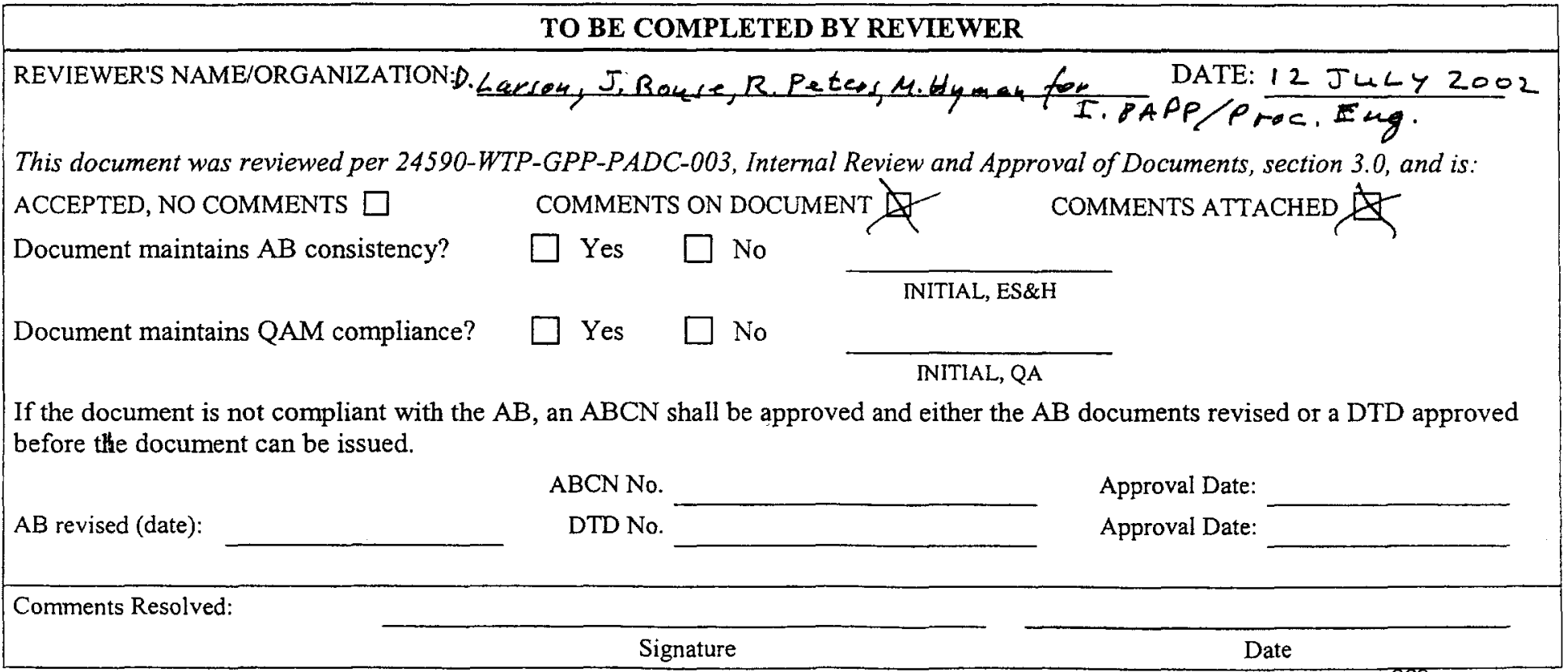


Note to report, VSL-02R0100-2, "Tests on the DuraMelter 1200 HLW Pilot Melter System Using AZ-101 HLW Simulants" Rev 0

Phil Schuetz (a required reviewer) sent an e:mail stating he would have no comments. However, the report review coordinator misplaced the e:mail replay from Mr. Schuetz.

Joe Perez, R\&T document review coordinator

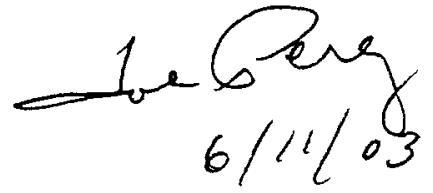




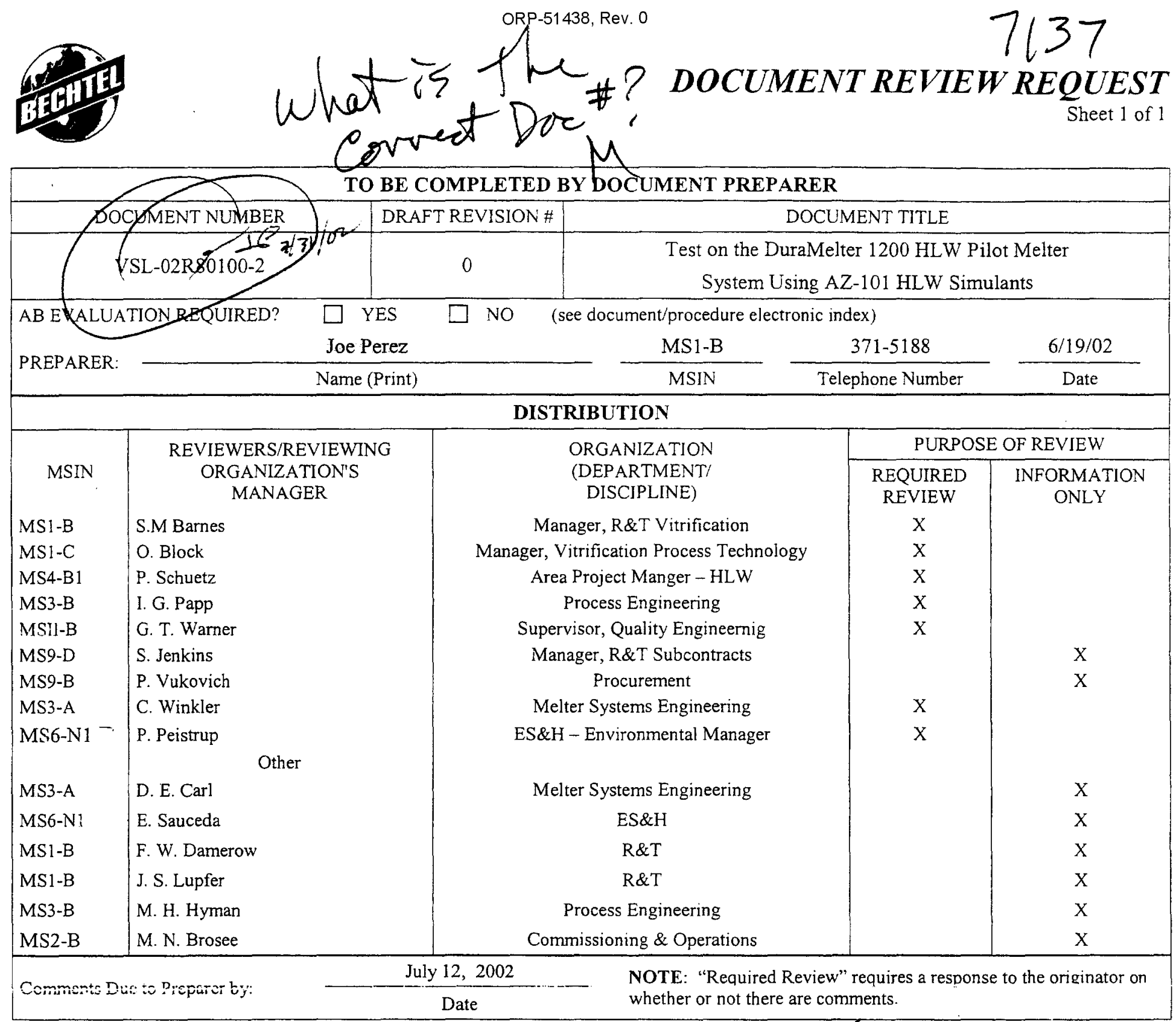

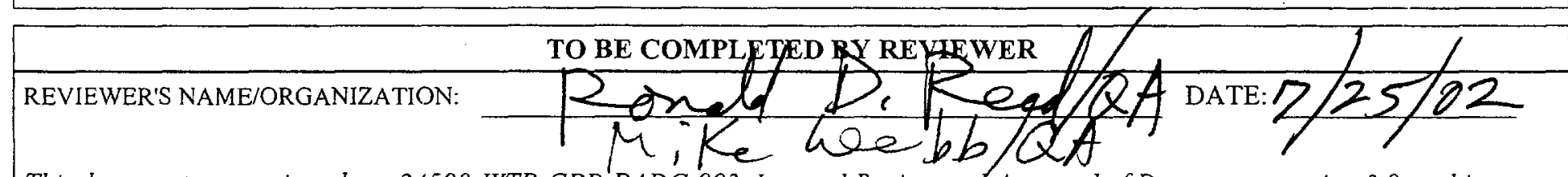

This document was reviewed per 24590-WTP-GPP-PADC-003, Internal Review and Approval of Documents, section 3.0, and is:

ACCEPTED, NO COMMENTS

Document maintains $\mathrm{AB}$ consistency?

Document maintains QAM compliance?
COMMENTS ATTACHED

\section{INITIAL, ES\&H}

If the document is not compliant with the $\mathrm{AB}$, an $\mathrm{ABCN}$ shall be approved and either the $\mathrm{AB}$ documents revised or a $\mathrm{DTD}$ approved before the document can be issued.

\begin{tabular}{lrl} 
AB revised (date): & ABCN No. & Approval Date: \\
& DTD No. & Approval Date: \\
\hline
\end{tabular}

Comments Resolved: $\frac{\text { Comments recened after review }}{\text { Signature }}$ 


\section{Perez, Joseph}

From:

Sent:

To:

Cc:

Subject:
Perez, Joseph

Monday, July 22, 2002 1:21 PM

Warner, Gregory T; Block, Oliver U; Peistrup, Philip

Sauceda, Ermelinda; WTP QA

Test on the DuraMelter 1200 HLW Pilot Melter, VSL-02R80100-2, rev. 0

You were identified as a required reviewer for the subject document.

Comments have not been received by the due date. Issue of the document will proceed without your comments. If comments are subsequently submitted, they will be addressed, resolved and incorporated (as appropriate) in the next revision of the document.

If you do not have comments, please respond as such to this message. In addition, by procedure your signature is required on the Attached DRR. Please complete the form and send it to Joe Perez, R\&T, mail stop: MS1-B

Thank-you

Joe Perez

R\&T:WTP

Ph.: 509.371 .5188

Fax: 509.371 .5242 


\section{Research and Technology Completion Form}

R\&T Scoping Statement(s): VH-4 - HLW Melter Tests Pilot Scale

Test Specification Number/Title:

24590-HLW-TSP-RT-01-001, HLW Melter Attainment Studies and Equipment Performance Evaluations

Test Plan Number/Title:

a). VSL-01T0100-2, Test Plan - Tests on the DuraMelter 1200 HLW Pilot Melter System Using HLW AZ-101 Simulants

b). VSL-01T0100-3, Test Plan - Throughput Tests on the DuraMelter $1200 \mathrm{HLW}$ Pilot Melter System Using HLW Simulants

Test Report Number/Title:

VSL-02R0100-2, Rev. 1, Tests on the DuraMelter 1200 HLW Pilot Melter System Using AZ-101 HLW Simulants

Prepared by: JM Perez, Jr.

Date: April, 21, 2003

List Test Objectives:

1) Determine processing rates without bubbling on the DM1200 system in longer-duration tests than were performed on the DM1000 system and at a larger scale than the tests performed on the DM100 system.
State how objectives were met:

Nine tests were performed on the DM1200 pilot melter system generating a total of $26,725 \mathrm{~kg}$ of glass, as summarized below:

- Test 1 - High-solids feed ( $570 \mathrm{~g}$ glass/liter), without bubbling (<4 lpm); 168 hours, $2301 \mathrm{~kg}$ glass produced.

- Test 2 - High-solids feed ( 570 g glass/liter $)+10$ g sugar /liter, without bubbling ( $<4 \mathrm{lpm}) ; 216$ hours, $2594 \mathrm{~kg}$ glass produced.

- Test 3 - Low-solids feed ( $400 \mathrm{~g}$ glass/liter), with deep bubbling 1 (nominal $60 \mathrm{lpm}$ ); 115 hours, $6180 \mathrm{~kg}$ glass produced.

- Test 4 - Low-solids feed ( 400 g glass/liter), with shallow bubbling (nominal $60 \mathrm{lpm}$ ); 122 hours, $3465 \mathrm{~kg}$ glass produced.

- Test 5 - Nitrated feed ( 420 g glass/liter) with sugar ratio $=$ 0.5 , without bubbling $(<4 \mathrm{lpm}) ; 144$ hours, $1659 \mathrm{~kg}$ glass produced.

- Test 6 - Frit-based feed ( $480 \mathrm{~g}$ glass/liter), without bubbling $(<4 \mathrm{lpm}) ; 155$ hours, $1901 \mathrm{~kg}$ glass produced.

- Test 7 - Frit-based feed ( $480 \mathrm{~g}$ glass/liter), deep bubbling (60 lpm); 120 hours, $5976 \mathrm{~kg}$ glass produced.

- Test 8 - Nitrated feed ( 420 g glass/liter) with sugar ratio $=$ 0.7 , without bubbling $(<4 \mathrm{lpm}) ; 100$ hours, $944 \mathrm{~kg}$ glass produced.

- Test $9 \mathrm{a}-$ High-solids feed $(570 \mathrm{~g}$ glass/liter $)+10 \mathrm{~g}$ sugar /liter, without bubbling $(<4 \mathrm{lpm})$; continuous feeding (as opposed to pulsed feeding) from two feed tubes instead of one, 69 hours, $859 \mathrm{~kg}$ glass produced.

- Test $9 \mathrm{~b}$ - High-solids feed ( $570 \mathrm{~g}$ glass/ liter) $+10 \mathrm{~g}$ sugar /liter, without bubbling $(<4 \mathrm{lpm})$; continuous feeding (as opposed to pulse) from two feed tubes instead of one, $1200^{\circ} \mathrm{C}$ glass temperature, 54 hours, $847 \mathrm{~kg}$ glass produced.

Test results are discussed in Section 3, Melter Operations. The DM1200 is approximately $10 \mathrm{X}$ larger than the DM100 system. Test durations were nominally 5 days in duration but varied depending on the test objective and whether or not bubblers were used (tests with bubblers achieve steady state more quickly than when bubblers are not used. 


\section{Research and Technology Completion Form}

R\&T Scoping Statement(s):

Test Specification Number/Title:

Test Plan Number/Title:

Test Report Number/Title:
VH-4 - HLW Melter Tests Pilot Scale

24590-HLW-TSP-RT-01-001, HLW Melter Attainment Studies and Equipment Performance Evaluations

a). VSL-01T0100-2, Test Plan - Tests on the DuraMelter 1200 HLW Pilot Melter System Using HLW AZ-101 Simulants

b). VSL-01T0100-3, Test Plan - Throughput Tests on the DuraMelter 1200 HLW Pilot Melter System Using HLW Simulants

VSL-02R0100-2, Rev. 1, Tests on the DuraMelter 1200 HLW Pilot Melter System Using AZ-101 HLW Simulants

\section{List Test Objectives:}

2) If the design-basis rate of $400 \mathrm{~kg} / \mathrm{m}^{2} / \mathrm{d}$ is achieved without bubbling with a high-solids feed, determine the effect of solids content on processing rates from a similar test but with the nominal solids loading in the melter feed (this corresponds to $20 \mathrm{wt} \%$ solids in the Envelope D material from pretreatment).

3) Determine the effect of continuous versus pulsed feed from two feed tubes instead of one on feed spreading and processing rates.

\section{State how objectives were met:}

The design-basis rate was not achieved without bubblers. As a result, this objective was not executed.
The change to continuous feeding with two feed tubes using a peristaltic pump as opposed to pulsed feeding from a single feed tube using a simulated ADS pump did not result in an increase in production rates, which is evident when comparing the results to comparable tests discussed above (Test 9a: 230 $\mathrm{kg} / \mathrm{m}^{2} /$ day vs. $205-255 \mathrm{~kg} / \mathrm{m}^{2} /$ day). As a result it was concluded that the method and location of feeding the DM1200 did not have a measurable affect on production rate.
4) Obtain processing data for a simulated, acidified AZ-101 HLW feed (Test 1 only), as employed at WVDP, using the DM1200 system at the Project nominal melter feed concentration ( $20 \mathrm{wt} \%$ solids from pretreatment, which corresponds to approximately 400 grams of oxides per liter of feed), without bubbling, to determine the effect of nitrated melter feed on feed handling, foaming, coldcap characteristics, feed spreading, and processing rates.

5) Obtain processing data for a simulated AZ-101 HLW feed with frit instead of glass forming chemicals using the DM1200 system, without bubbling, to determine the effect on feed handling, foaming, cold-cap characteristics, and processing rates.
Non-bubbled tests $\# 5$ and \#8 were conducted on $9 / 25 / 01$ and $10 / 29 / 01$, respectively. Test $\# 5$ used a carbon to nitrate ratio of 0.5 and test \#8 was conducted with a ratio of 0.7 .

Maximum steady state processing rates for test \#5 and \#8 were 220 and $200 \mathrm{~kg} / \mathrm{m}^{2} / \mathrm{d}$, respectively. The melter feed was nitrated through the addition of nitric acid to mimic the West Valley feed properties. Melter feed oxide concentration was $420 \mathrm{~g} / \mathrm{L}$. The $\mathrm{pH}$ of the test $\# 5$ and $\# 8$ melter feeds averaged 5.6 and 4.9 , respectively. Although the cold cap was reported to appear more fluid than the alkaline-based feeds; it did not result in higher throughput rate attainment.

Test \#6 was conducted and obtained a steady-state production rate of $210 \mathrm{~kg} / \mathrm{m}^{2} / \mathrm{d}$. Throughput rate and processing conditions were not significantly different than the baseline. 


\section{Research and Technology Completion Form}

R\&T Scoping Statement(s):

Test Specification Number/Title:

Test Plan Number/Title:

Test Report Number/Title:
VH-4 - HLW Melter Tests Pilot Scale

24590-HLW-TSP-RT-01-001, HLW Melter Attainment Studies and Equipment

Performance Evaluations

a). VSL-01T0100-2, Test Plan - Tests on the DuraMelter 1200 HLW Pilot Melter System Using HLW AZ-101 Simulants

b). VSL-01T0100-3, Test Plan - Throughput Tests on the DuraMelter 1200 HLW Pilot Melter System Using HLW Simulants

VSL-02R0100-2, Rev. 1, Tests on the DuraMelter $1200 \mathrm{HLW}$ Pilot Melter System Using AZ-101 HLW Simulants
List Test Objectives:

6) Determine the amount of reductant (sugar) to be added to the feed to maintain part of the iron (5 to $15 \%)$ in the $\mathrm{Fe}^{2+}$ state in order to prevent foaming incidents and determine the processing rate with this feed.
State how objectives were met:

Test \#2 was conducted with incrementally increased sugar levels until a measurable change in the $\mathrm{Fe}^{+2}: \mathrm{Fe}^{\text {total }}$ ratio of glass was observed (but not to exceed $20 \% \mathrm{Fe}^{+2}$ ). It was determined that $10 \mathrm{~g}$ sugar per liter of melter feed was sufficient to alter the $\mathrm{F}^{+2}: \mathrm{Fe}^{\text {total }}$ from $\sim 1 \% \mathrm{Fe}^{+2}$ to $\sim 12 \%$ $\mathrm{Fe}^{+2}$. Table 5.4 summarizes the iron redox from all nine tests. Observable cold cap foaming, presumed to be due to reduction of manganese; was eliminated with the use of sugar. This surface foaming did not appear to hamper production during tests using bubblers. Therefore, sugar addition was thought to not be automatically assumed essential for HLW processing with bubblers.

7) Obtain processing data for a simulated AZ-101 HLW feed using the DM1200 system, without bubbling, at an operating temperature of between 1175 and $1200{ }^{\circ} \mathrm{C}$, to determine the effect on feed handling, foaming, cold-cap characteristics, and processing rates.

8) Evaluate off-gas system component performance by sampling exhaust at various points in the off-gas system as well as sump solutions from the various off-gas system components.

9) Collect data on mass balance across the melter.
Test \#9b was conducted with the target glass temperature increased to $\sim 1200^{\circ} \mathrm{C}$. Actual average temperatures for thermocouples in the glass melt averaged between $1199^{\circ} \mathrm{C}$ and $1208^{\circ} \mathrm{C}$. Glass production rate increased $\sim 50 \%$ to 310 $\mathrm{kg} / \mathrm{m}^{2} / \mathrm{d}$. It is noteworthy that the melter, SBS and WESP DFs were each lower during test \#9 (combined 9a [1 sample period] and $9 \mathrm{~b}$ [ 2 sample periods]) than the baseline, test $\# 2$. Table 6.2 summarizes these results.

Section 6 presents results of significant sampling and analyses of off-gas system component performance. Sample condensates and emissions sampling were both carried out.

Feed, glass and off-gas emissions were obtained to support mass balance calculations. Flux rates of feed and glass were in agreement within $89 \%$ to $100 \%$, depending on the test conducted (Tables 1.2, 1.3 and 1.4). Off-gas flux rates are reported in Table 6.2. Off-gas emissions ranged from $\sim 0.2$ to $\sim 5 \mathrm{~kg} / \mathrm{m}^{2} / \mathrm{d}$. These values were not large enough to close the mass balance in all cases. 


\section{Research and Technology Completion Form}

R\&T Scoping Statement(s):

Test Specification Number/Title:

Test Plan Number/Title:

Test Report Number/Title:
VH-4 - HLW Melter Tests Pilot Scale

24590-HLW-TSP-RT-01-001, HLW Melter Attainment Studies and Equipment Performance Evaluations

a). VSL-01T0100-2, Test Plan - Tests on the DuraMelter 1200 HLW Pilot Melter System Using HLW AZ-101 Simulants

b). VSL-01T0100-3, Test Plan - Throughput Tests on the DuraMelter $1200 \mathrm{HLW}$ Pilot Melter System Using HLW Simulants

VSL-02R0100-2, Rev. 1, Tests on the DuraMelter 1200 HLW Pilot Melter System Using AZ-101 HLW Simulants
List Test Objectives:

10) Identify possible processing problems such as foaming, secondary phase formation, and poor cold cap characteristics, particularly in comparison to previous small-scale and DM1000 melter tests.
State how objectives were met:

Section 7, Summary and Conclusions compares DM1200 results with previous DM1000 and DM100 results. Results were generally consistent with DM1000. DM100 test results typically over-estimated larger scale results (see Figure 7.2).

Process rates were typically restricted by cold cap solids accumulation. That is, the cold cap thickness was determined to be increasing over time indicating that the melt rates were not keeping up with the feed rate. This necessitated reduction in feed rates.

Foam formation at the cold cap/glass interface was judged by $\mathrm{R} \& \mathrm{~T}$ to be due to $\mathrm{MnO}_{2}$ or $\mathrm{Mn}_{2} \mathrm{O}_{3}$ decomposition to $\mathrm{MnO}$ liberating oxygen. This was effectively eliminated with $8 \mathrm{~g} / \mathrm{L}$ sugar. Later testing with bubblers also demonstrated that at bubbler air rates set to achieve optimum (maximum) throughput, foam accumulation was eliminated.

11) Collect data on product glass composition and product quality.

Section 5 presents results of glass composition and redox analyses. Glass properties for the target glass were obtained during laboratory studies and documented in the test plan.

12) Collect operating data with a simulated air-displacement slurry (ADS) pump melter feed system (i.e., intermittent, pulsed flow of feed to the The majority of tests were conducted with the simulated ADS pump. Figure 4.1 depicts the effect of the intermittent feed system on plenum pressure. Plenum pressure typically ranged between -2 to -8 in. WC.

melter), particularly with respect to off-gas transients.

13) Determine the effect of bubbling and the positioning of the bubblers (deep or shallow) on processing rates.

Deep bubbling (bubbler located 6 in. from floor) resulted in throughput rate of $980 \mathrm{~kg} / \mathrm{m}^{2} / \mathrm{d}$ with a feed containing $400 \mathrm{~g}$ ox/L (Test \#3). Shallow bubbling (bubbler located 13.5 in. from floor) resulted in throughput rate of $540 \mathrm{~kg} / \mathrm{m}^{2} / \mathrm{d}$ with a feed containing $400 \mathrm{~g}$-ox/L (Test \#4).

14) Obtain processing data for a simulated AZ-101 HLW feed with frit instead of glass forming chemicals using the DM1200 system, with bubbling (standard "L" shaped bubbler design), to determine the effect on feed handling, foaming, cold-cap characteristics, and processing rates.
Test \#7 resulted in a throughput rate of $1100 \mathrm{~kg} / \mathrm{m}^{2} / \mathrm{d}$ when processing AZ-101 simulant with frit. Feeding and cold cap processing characteristics were similar to the baseline feed. 


\section{Research and Technology Completion Form}

R\&T Scoping Statement(s):

Test Specification Number/Title:

Test Plan Number/Title:

Test Report Number/Title:
VH-4 - HLW Melter Tests Pilot Scale

24590-HLW-TSP-RT-01-001, HLW Melter Attainment Studies and Equipment Performance Evaluations

a). VSL-01T0100-2, Test Plan - Tests on the DuraMelter 1200 HLW Pilot Melter System Using HLW AZ-101 Simulants

b). VSL-01 T0100-3, Test Plan - Throughput Tests on the DuraMelter 1200 HLW Pilot Melter System Using HLW Simulants

VSL-02R0100-2, Rev. 1, Tests on the DuraMelter 1200 HLW Pilot Melter System Using AZ-101 HLW Simulants

\begin{tabular}{|c|c|}
\hline List any Test Exceptions: & Did exceptions impact the objective? $\square$ Yes $\square$ No (Explain) \\
\hline $\begin{array}{l}\text { 1) 24590-101-TEF-W000-0009-38-02 Test } \\
\text { Exception \#1 }\end{array}$ & $\begin{array}{l}\text { Directed VSL to perform SW- } 846 \text { method sampling of off-gas as a } \\
\text { "dress rehearsal" for future DM } 1200 \text { testing. }\end{array}$ \\
\hline List Success Criteria & Did the test meet the criteria? \\
\hline $\begin{array}{l}\text { 1) Conduct testing per the approved test } \\
\text { matrix and obtain steady state throughput } \\
\text { data sufficient to assess the effect of the } \\
\text { test variables on glass production rate. }\end{array}$ & $\begin{array}{l}\text { As described above the test matrix was successfully executed. } \\
\text { Adequate data was obtained with which to measure steady state } \\
\text { throughput rates for each variable. }\end{array}$ \\
\hline $\begin{array}{l}\text { 2) Operate the integrated off-gas treatment } \\
\text { system }\end{array}$ & $\begin{array}{l}\text { The complete integrated system was operated during testing. } \\
\text { Multiple sampling periods occurred during each test to develop } \\
\text { emissions estimates and decontamination factors for each unit } \\
\text { operation. }\end{array}$ \\
\hline List QA Requirements: & $\begin{array}{l}\text { Did the subcontractor meet the requirements? } \quad \text { Yes } \square \text { No } \\
\text { (Explain) }\end{array}$ \\
\hline 1) NQA-1 & $\begin{array}{l}\text { Work was conducted under an NQA-1 based quality assurance } \\
\text { program that is in place at VSL. This program is supplemented by a } \\
\text { Quality Assurance Project Plan for RPP-WTP work [11] that is } \\
\text { conducted at VSL, which includes the correlation of the VSL QA } \\
\text { program with } 10-C F R-830.120 \text {. Work conducted prior to } 8 / 6 / 01 \\
\text { (includes execution of Tests \#1 and \#2) was performed in } \\
\text { compliance with the requirements of NQA-1 (1994). Work } \\
\text { conducted after 8/6/01 was done in compliance with the } \\
\text { requirements of NQA-1 (1989) and NQA-2a (1990). }\end{array}$ \\
\hline List R\&T Test Conditions: & Were test conditions followed? \\
\hline $\begin{array}{l}\text { 1) Feed oxide concentrations: } \\
\text { - "high solids" }=570 \mathrm{~g} / \mathrm{L} \\
\text { - "high water" }=400 \mathrm{~g} / \mathrm{L} \\
\text { - nitrated feed }=420 \mathrm{~g} / \mathrm{L} \\
\text { - feed } \mathrm{w} / \text { frit }=480 \mathrm{~g} / \mathrm{L}\end{array}$ & $\begin{array}{l}\text { Feeds were within } 9 \text { to } 20 \text { g-oxide per liter of target values. These } \\
\text { are provided in Tables } 2.4 \text { and } 2.5 \text {. }\end{array}$ \\
\hline
\end{tabular}




\section{Research and Technology Completion Form}

R\&T Scoping Statement(s):

Test Specification Number/Title:

Test Plan Number/Title:

Test Report Number/Title:
VH-4 - HLW Melter Tests Pilot Scale

24590-HLW-TSP-RT-01-001, HLW Melter Attainment Studies and Equipment Performance Evaluations

a). VSL-01T0100-2, Test Plan - Tests on the DuraMelter 1200 HLW Pilot Melter System Using HLW AZ-101 Simulants

b). VSL-01T0100-3, Test Plan - Throughput Tests on the DuraMelter 1200 HLW Pilot Melter System Using HLW Simulants

VSL-02R0100-2, Rev. 1, Tests on the DuraMelter 1200 HLW Pilot Melter System Using AZ-101 HLW Simulants
2) Operate melter glass temperature nominally at $1150^{\circ} \mathrm{C}$; except for high temperature test, Test \# $9 \mathrm{~b}$ which is to be operated at $1200^{\circ} \mathrm{C}$

3) Glass composition

4) Cold cap coverage

5) Plenum temperature maintained between $400^{\circ} \mathrm{C}$ and $600^{\circ} \mathrm{C}$
The average value of five thermocouples positioned between nine inch and eighteen inches from the tank floor are used to adjust electrode power input to maintain the temperature at $1150^{\circ} \mathrm{C}, \pm 25^{\circ} \mathrm{C}$. Operators are also allowed to used judgement in changing the power input set point if any one thermocouple "significantly" exceeds the temperature limit. Table 3.2 reports the average, minimum and maximum values for each test for each thermocouple. The minimum value is a little misleading as the data monitoring includes the initial startup of the melter. However, as seen in Figures 3.10 through 3.18 the tight cluster of data points provides visual evidence of consistently maintaining the average bulk glass temperature around the $1150^{\circ} \mathrm{C}$ setpoint.

The specified AZ-101 composition was maintained throughout the testing period. Feed sample analyses are documented in Table 2.6. Product glass analyses are documented in Tables 5.1 and 5.2.

Goal was to maintain full glass surface coverage to the extent possible. Based on visual inspection of cold cap coverage less than full coverage could be maintained during some periods due to the propensity for the feed to accumulate in mounds rather than spreading across the glass surface. This is due to feed properties, and therefore, was acceptable.

Average temperatures remained below $600^{\circ} \mathrm{C}$ for all tests. In about half the tests the average temperature was below $400^{\circ} \mathrm{C}$. Because plenum temperature is responsive to cold cap coverage and cold cap conditions, plenum temperature is not independently controlled. Condition was considered fulfilled considering need to maximize feeding and throughput. Table 3.2 reports the average, minimum and maximum values for each test. Figures $3.19-3.27$, inclusive, provides data plots of plenum temperature versus time.

Was testing performed with simulants? If yes, discuss how results compare to radioactive tests. $\bigotimes$ Yes $\square$ No Testing was performed with a simulant specified prior to the BNI contract period. Simulant was required to be held constant in order to be able to assess throughput effects as specific parameters were changed. Review of the report, "WTP-RPT-075, Rev. A, "Development of LAW and HLW Vitrification Physical Property Bounding Conditions and Simulant Verification Criteria" did not identify or document suitable radioactive AZ-101 data. Particle size data showed the simulant to have a mean particle diameter volume distribution $130 \%$ larger than an AZ-101 waste core sample data reported in PNL-1 1025. However, the mean particle diameter number distribution of the simulant was $27 \%$ smaller. Particle size can affect fluid properties and melting properties. The effect of GFC particle sizes is expected to significantly affect the rate of melting. Comparing limited HLW radioactive data to simulant data it is currently thought that the VSL simulant may represent the lower end of yield stress and consistency fields. As a result, the actual radioactive waste may be expected to result in lower processing throughput results due to difficulties in cold cap flow. If this is true the test results were conservative in that they overpredict expected production rate and 


\section{Research and Technology Completion Form}

R\&T Scoping Statement(s):

Test Specification Number/Title:

Test Plan Number/Title:

Test Report Number/Title:
VH-4 - HLW Melter Tests Pilot Scale

24590-HLW-TSP-RT-01-001, HLW Melter Attainment Studies and Equipment Performance Evaluations

a). VSL-01T0100-2, Test Plan - Tests on the DuraMelter 1200 HLW Pilot Melter System Using HLW AZ-101 Simulants

b). VSL-01T0100-3, Test Plan - Throughput Tests on the DuraMelter 1200 HLW Pilot Melter System Using HLW Simulants

VSL-02R0100-2, Rev. 1, Tests on the DuraMelter 1200 HLW Pilot Melter System Using AZ-101 HLW Simulants

bubblers would still be recommended for the HL W melter.

Are all discrepancies resolved? If no, explain

$\triangle$ Yes $\square$ No

Are all subcontractor signoffs completed?

This work is acceptable to complete the indicated: If Other, please explain what the report completes.

$\bigotimes$ Test Plan(s)

$\bigotimes$ Yes $\square$ No

Does the Testing or Report suggest any follow-on work? If yes, describe the suggested activity $\triangle$ Yes $\square$ No and, if appropriate, attach a Request for Technology Development (RTD).

1. Testing has resulted in the recommendation that bubblers be incorporated into the WTP HLW melter design in order to meet the throughput requirement of $3.0 \mathrm{MT} / \mathrm{d}$ base plate production requirement. At the time testing was performed the based plate production requirement was $1.5 \mathrm{MT} / \mathrm{d}$. Bubblers were required to meeting this original lower rate as well.

2. The erratic performance of the WESP indicated serious defects in either its physical or its electrical power supply design. Continued assessment and troubleshooting ultimately determined the defects to be physical; i.e., electrical shorting due to poorly designed isolation, corrosion of clamping components due to the aggressive off gas environment, and lack of sufficient drainage capability to prevent the accumulation of standing water. These defects were corrected in 2002.

3. Solids accumulation of the SBS downcomer near the entrance to the distribution plate dictate that continued assessment and engineering scrutiny be applied to this area.

4. The simulated ADS pump arrangement was a poor representation of the ADS. The installation and use of the ADS pump should be a priority. Note that the ADS pump was installed in 2002.

Additional comments:

\section{Approved by R\&T Manager or pessignee:}

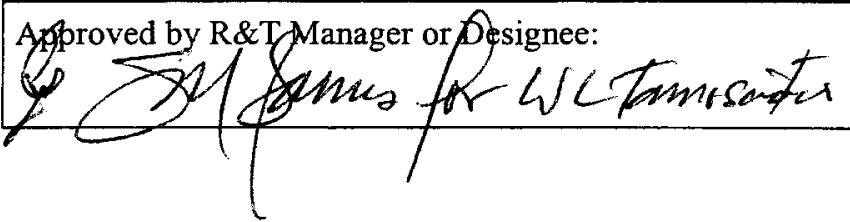

Date:

$$
7 / 31 / 2003
$$

